\title{
Interoperability Analysis of Non-Contact Fingerprinting Devices vs. Contact-Based Fingerprinting Devices
}

Emily Biller

Follow this and additional works at: https://researchrepository.wvu.edu/etd

\section{Recommended Citation}

Biller, Emily, "Interoperability Analysis of Non-Contact Fingerprinting Devices vs. Contact-Based Fingerprinting Devices" (2017). Graduate Theses, Dissertations, and Problem Reports. 5210. https://researchrepository.wvu.edu/etd/5210

This Thesis is protected by copyright and/or related rights. It has been brought to you by the The Research Repository @ WVU with permission from the rights-holder(s). You are free to use this Thesis in any way that is permitted by the copyright and related rights legislation that applies to your use. For other uses you must obtain permission from the rights-holder(s) directly, unless additional rights are indicated by a Creative Commons license in the record and/ or on the work itself. This Thesis has been accepted for inclusion in WVU Graduate Theses, Dissertations, and Problem Reports collection by an authorized administrator of The Research Repository @ WVU. For more information, please contact researchrepository@mail.wvu.edu. 


\title{
Interoperability Analysis of Non-Contact Fingerprinting Devices vs. Contact-Based Fingerprinting Devices
}

\author{
Emily Biller
}

\author{
Thesis submitted to the \\ Benjamin M. Statler College of Engineering and Mineral Resources At \\ West Virginia University
}

In partial fulfillment of the requirements for the degree of

Master of Science in Electrical Engineering

Jeremy Dawson, Ph.D., Chair

Donald Adjeroh, Ph.D.

Thirimachos Bourlai Ph.D.

Lane Department of Computer Science and Electrical Engineering Morgantown, WV

2017

Keywords: Fingerprint Matching, Fingerprints, Contactless, Contact-Based, Ten-prints Copyright 2017 Emily Biller 


\title{
Abstract \\ Interoperability Analysis of Non-Contact Fingerprinting Devices vs. Contact-Based Fingerprinting Devices
}

\author{
Emily Biller
}

Fingerprints are an essential biometric identifier that have been used for centuries due to the high diversity among individuals. Contactless fingerprint systems possess advantages over conventional contact-based systems, such as increased throughput in check point screening scenarios. Increased acceptance of contactless fingerprinting technologies requires careful operational validation due to the inherent differences in their capture techniques. To leverage the maturation of contactless technologies, reliability and data interoperability with legacy fingerprint datasets must be considered. The goal of this study is to evaluate the interoperability of contactless fingerprinting devices with contact-based fingerprinting devices. To achieve this goal, the fingerprint dataset of the ManTech Non-Contact Multi-Sensor Fingerprint Collection Phase II collected by West Virginia University, has been employed.

The main objectives this study aims to achieve are:

- Assessing the match performance of the contactless versus contact-based fingerprint devices.

- Statistical match performance evaluation using match score statistics.

- Assessing the match performance of demographic strata in comparison to the total dataset.

Following the generation of genuine and imposter match score distributions, Receiver Operating Characteristic Curves were plotted to compare the match performance of each device. The Kullback Leibler Divergence measurements have been calculated which signify the amount of variation between match score distributions as well as the Equal Error Rates for the genuine and imposter distributions. With the help of these procedures, the interoperability of contactless devices and contact-based devices has been examined and analyzed. Results obtained from the devices, representing a 'snapshot' of capability during their development cycle, indicate that Rank1 accuracy is between 74.33 and 98.68 percent with contact based methods. The Rank 1 accuracy is between 74.66 and 97.27 percent with other contactless devices. 


\section{Table of Contents}

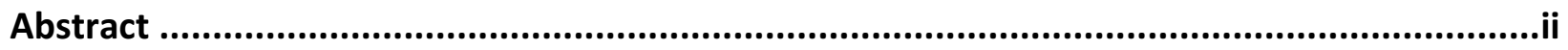

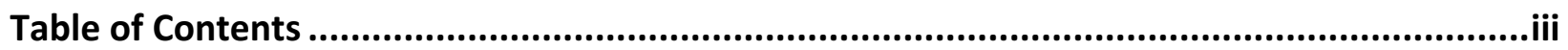

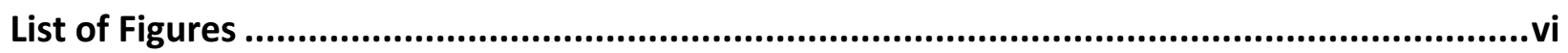

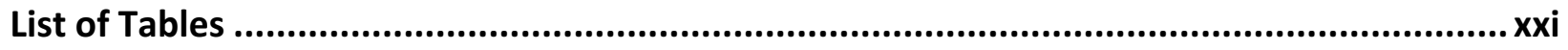

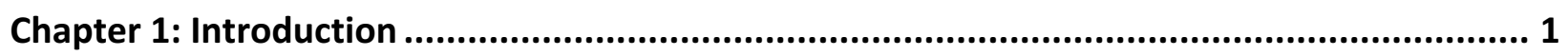

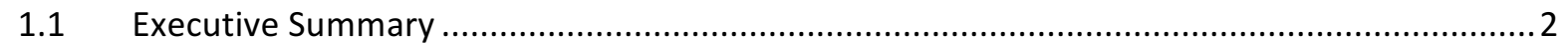

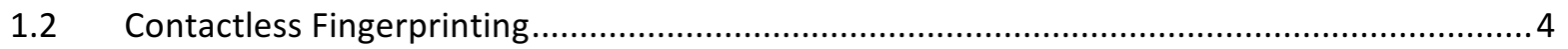

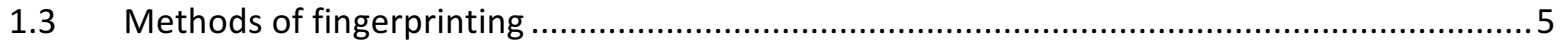

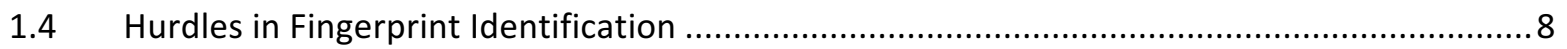

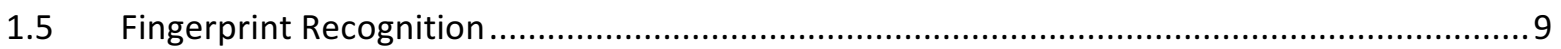

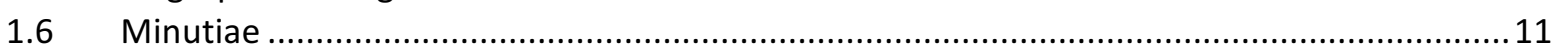

1.7 Advantages and Disadvantages of Contact and Contactless Fingerprinting ......................... 12

1.8 Challenges involved with Contactless Fingerprint technology ........................................... 14

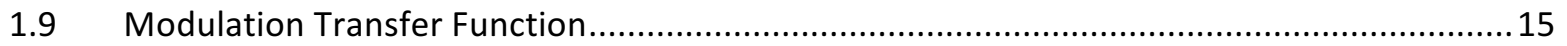

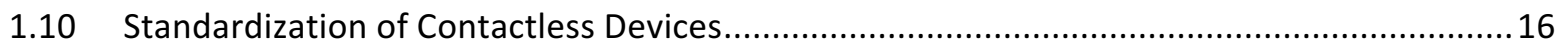

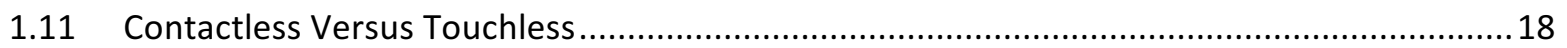

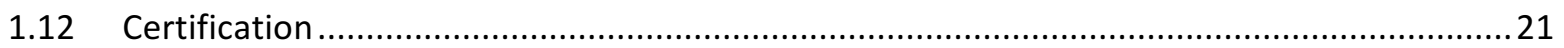

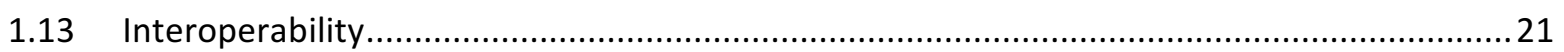

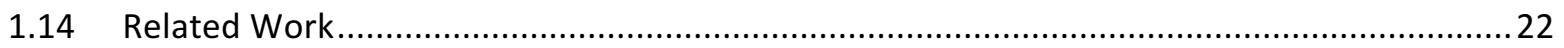

1.15 Problem Statement and Thesis Organization ............................................................ 24

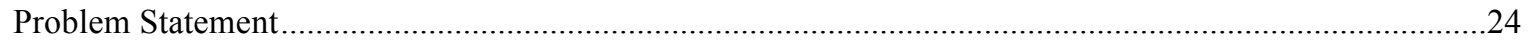

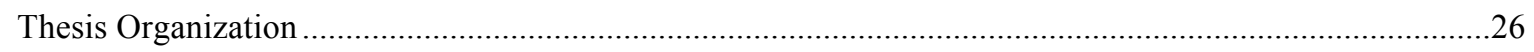

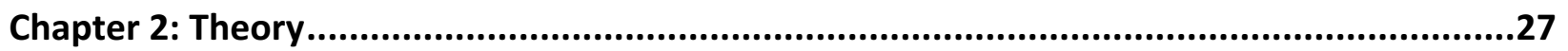

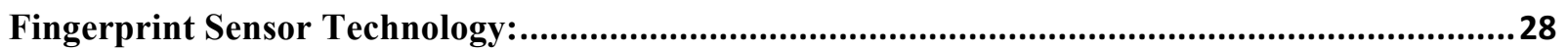

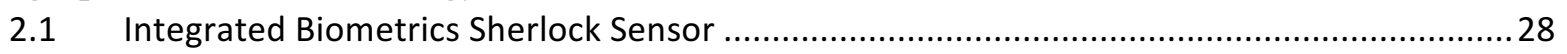

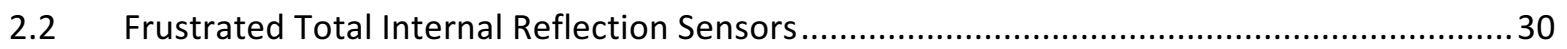

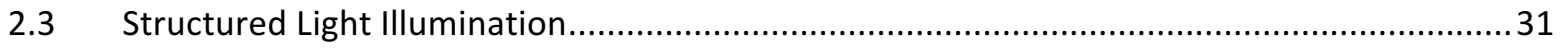

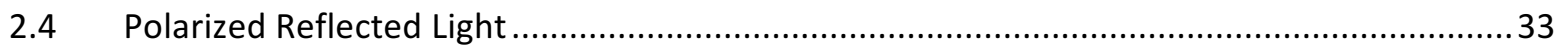

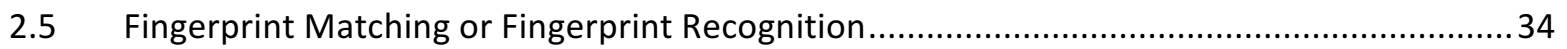

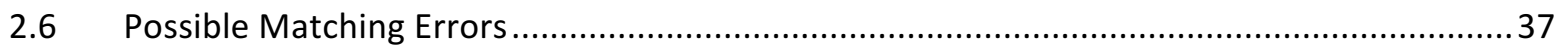

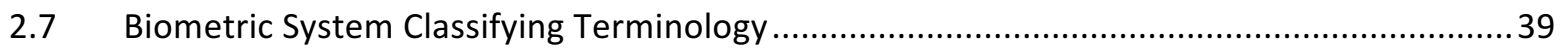

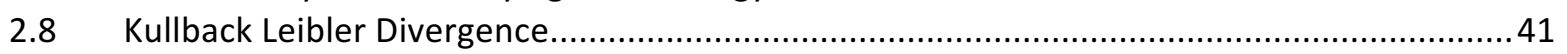

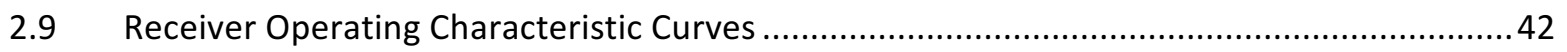

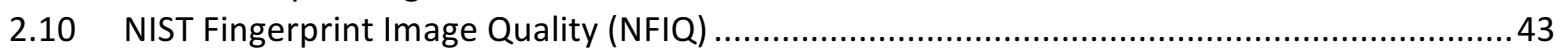

Chapter 3: Data Collection \& Preparation .........................................................................44

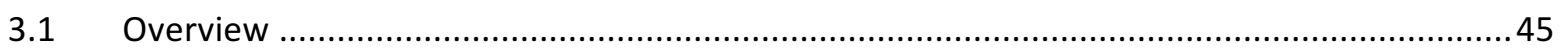

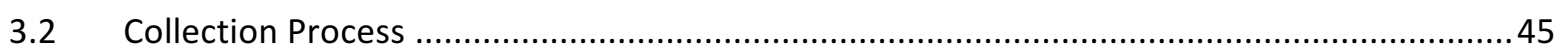

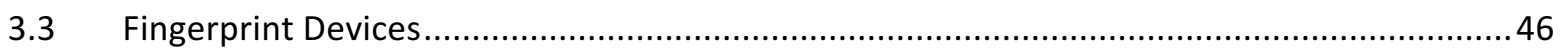

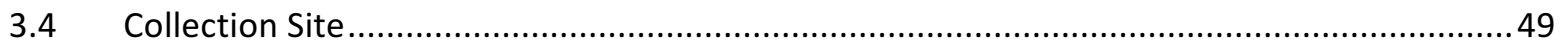




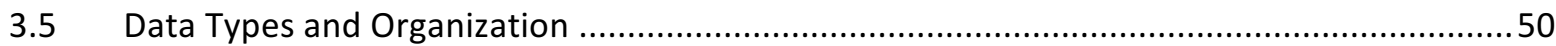

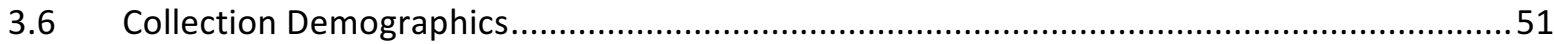

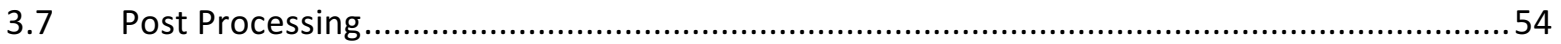

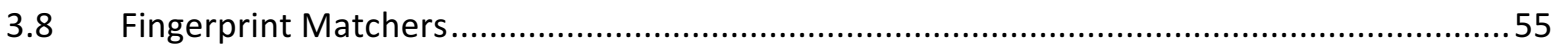

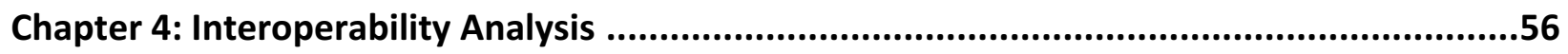

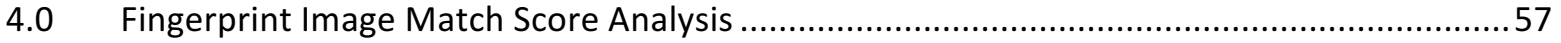

4.1 Cross-Examined Genuine and Imposter Score Distributions ............................................69

4.1.1 NBIS Cross-Examined Genuine/Imposter Distributions ..........................................................69

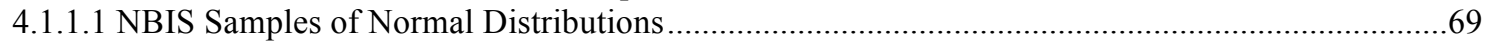

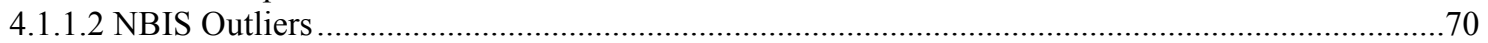

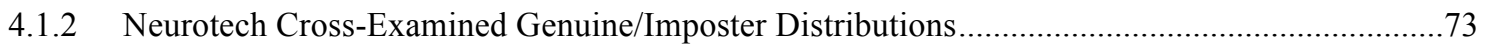

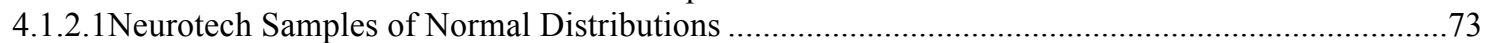

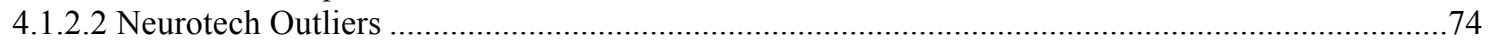

4.1.3 SourceAFIS Cross-Examined Genuine/Imposter Distributions ...................................................75

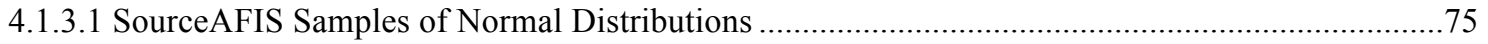

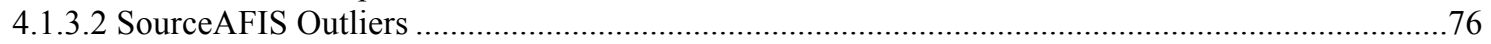

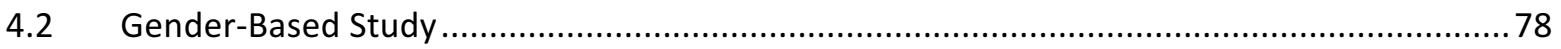

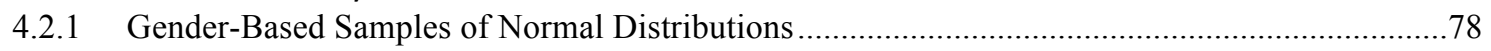

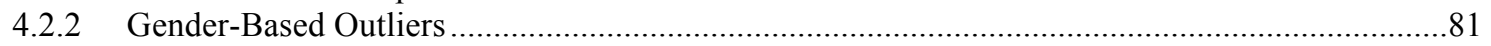

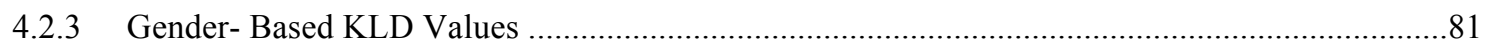

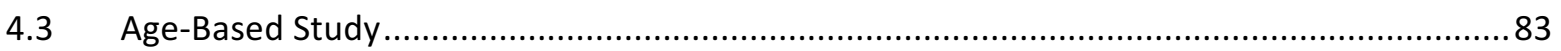

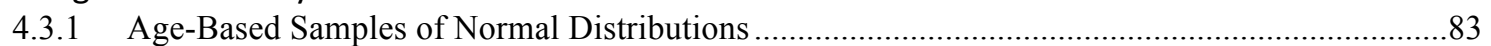

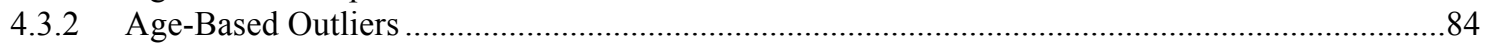

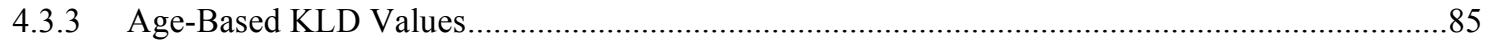

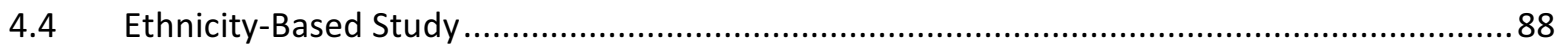

4.4.1 Ethnicity-Based Study Sample Genuine/Imposter Distributions ................................................8

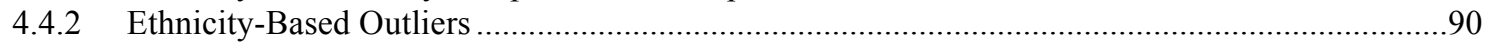

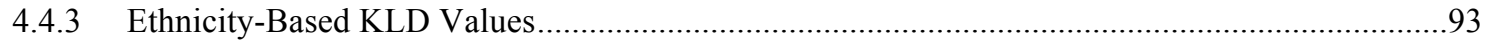

4.5 Issues Faced with Devices and Matchers Which can be Observed in the Results................101

Chapter 5: Conclusion...........................................................................................................102

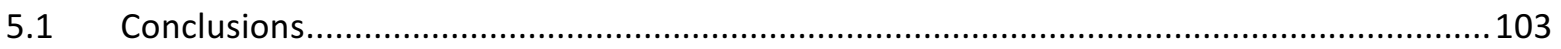

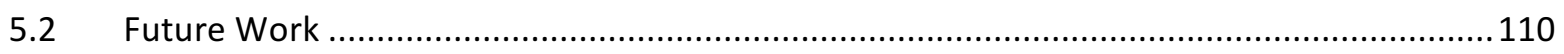

Appendix A General Study of All Data.......................................................................111

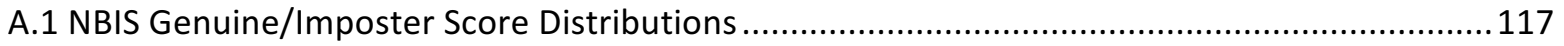

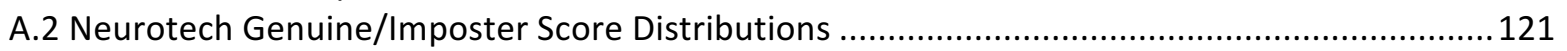

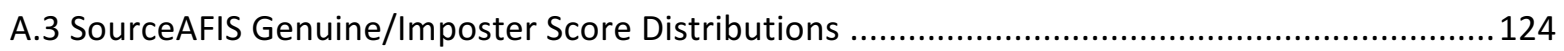

A.4 NBIS Cross-Examined Genuine/Imposter Distributions ...................................................... 128

A.5 Neurotech Cross-Examined Genuine/Imposter Distributions ................................................ 138

A.6 SourceAFIS Cross-Examined Genuine/Imposter Score Distributions .................................... 148

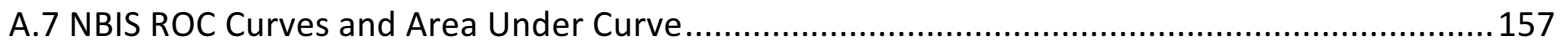

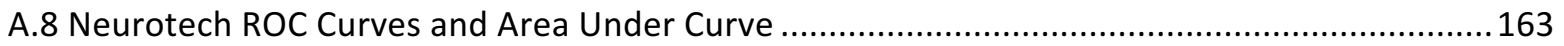

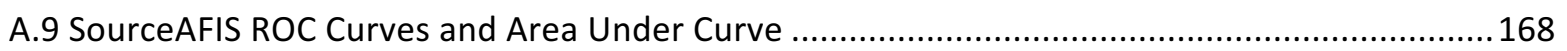

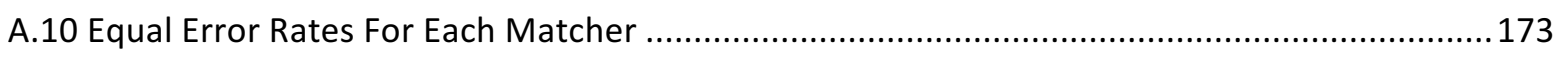

Appendix B Gender Based Study .....................................................................177

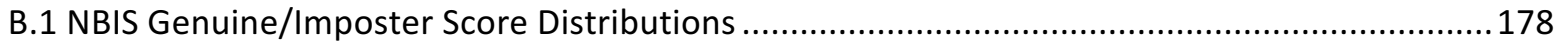

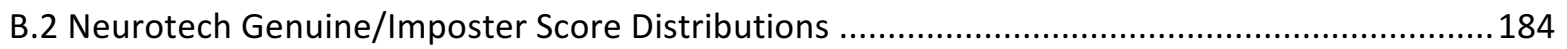




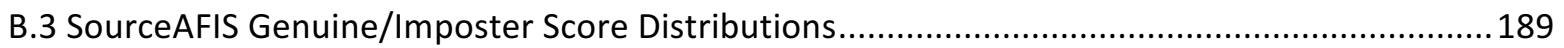

B.4 NBIS ROC Curves and Area Under Curve ....................................................................... 194

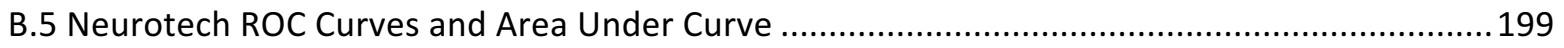

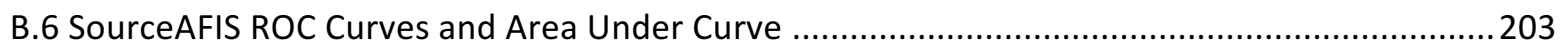

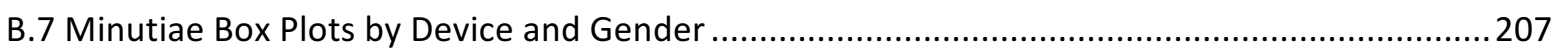

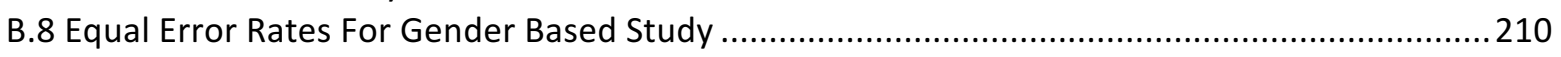

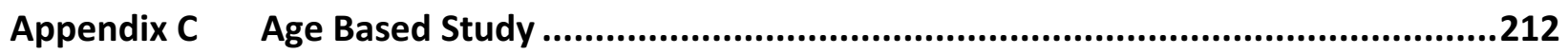

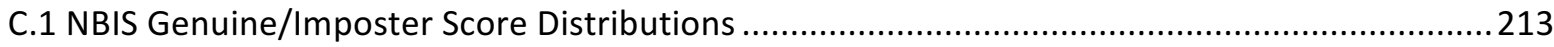

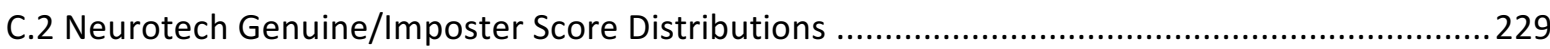

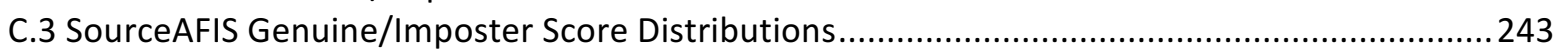

C.4 NBIS ROC Curves and Area Under Curve ......................................................................... 258

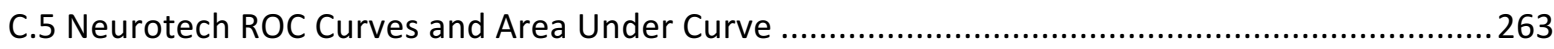

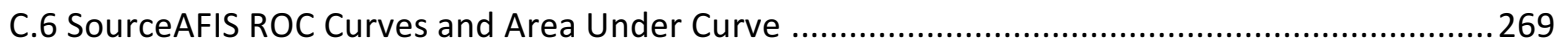

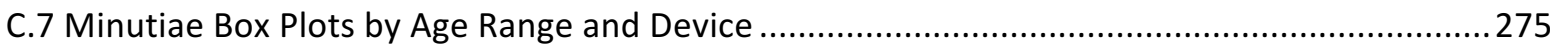

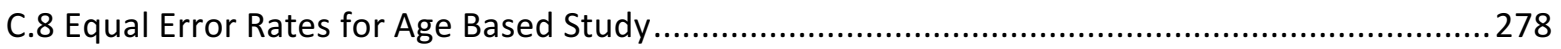

Appendix D Ethnicity Based Study .....................................................................280

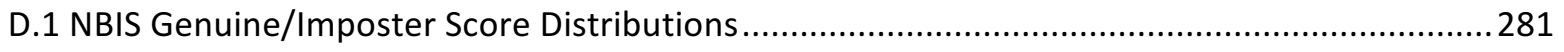

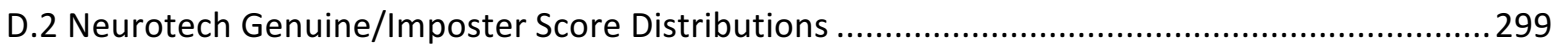

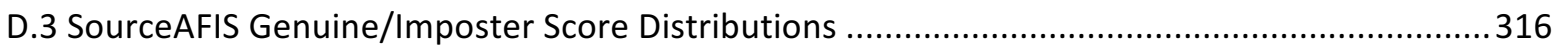

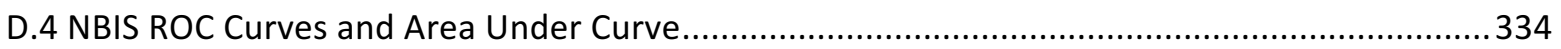

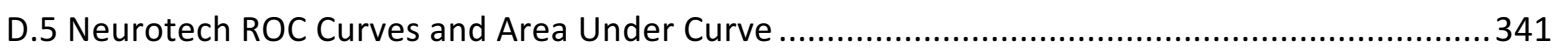

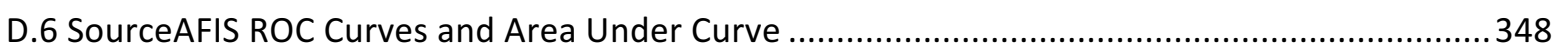

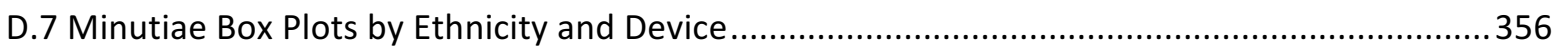




\section{List of Figures}

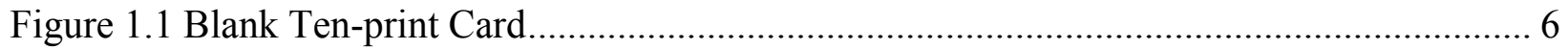

Figure 1.2 Ten print card with fingerprints........................................................................... 7

Figure 1.3 Example of Livescan Fingerprint Capture [2] ...................................................... 8

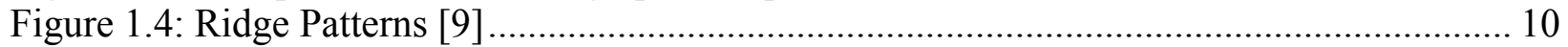

Figure 1.5: (Left)Fingerprint Minutiae types [13] (Right) Fingerprint minutiae marked by

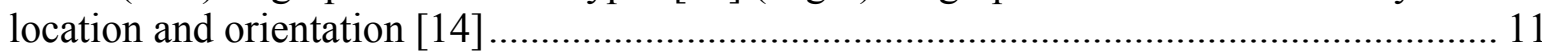

Figure 1.6: Images of various finger conditions: (a) normal finger; (b) dry finger; (c) wet finger;

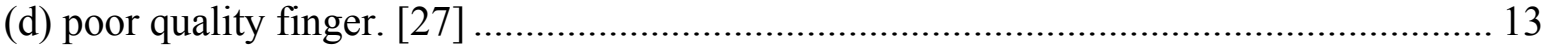

Figure 1.7: Visualizing compatibility between (a) a touchless fingerprint from line-scan sensor using the proposed nonparametric unwrapping (b) the corresponding ink-on-paper rolled

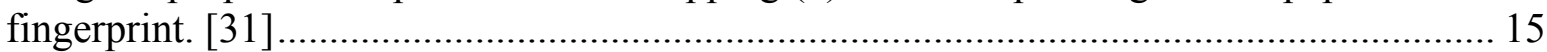

Figure 1.8 Difference between contactless (left) and touchless (right) [1] .............................. 18 Figure 1.9 (a) ANDI On-The-Go (OTG) [43] (b) MorphoWave or Morpho Finger-On-The-Fly (FOTF) [39] (c) TBS Touchless Ten Printer [1] (d) AIRprint Standoff Fingerprint Scanner [1] (e) TBS 3D Enroll [40] (f) IDair ONEprint [41] (g) NEC HS100-10 hybrid contactless

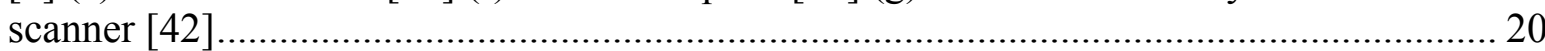

Figure 2.1 Explanation of Sherlock sensor by Integrated Biometrics [50]............................. 29

Figure 2.2 Sherlock Sensor [51] ............................................................................... 30

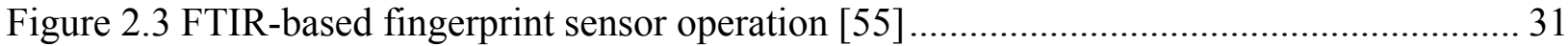

Figure 2.4 Diagram of how structured light illumination sees a finger [58] ............................ 33

Figure 2.5 General block diagram for a Fingerprint Identification System. [68] ....................... 35

Figure 2.6 Typical Minutiae Matching Algorithm [64] ...................................................... 37

Figure 2.7 Examples of False Non-Matches or False Rejects [69] ........................................... 38

Figure 2.8 Examples of False Matches or False Accepts [69] ................................................ 38

Figure 2.9 Representation of a typical genuine and imposter score distribution [70] ................ 41

Figure 2.10 A Sample ROC Curve [72] ........................................................................ 42

Figure 3.1: Fingerprint devices (from top left): CrossMatch Guardian R2, L1/Morpho TouchPrint 5300, CrossMatch SEEK Avenger, Northrup Grumman BioSled, MorphoTrak MorphoIDent, Advanced Optical Systems (AOS) ANDI On-The-Go, MorphoTrak Fingeron-the-Fly. The IDair InnerID (on iPhone) system was not available for photograph. [2] .. 47

Figure 3.2 Sample images from a single individual for each method of capture (a) Tenprint scan of rolled print (b) Tenprint Scan of Four Finger Slap (c) Livescan rolled print image (d) Livescan Four Finger Slap image (e) ANDI OTG Right Index Finger Capture (f) Morpho

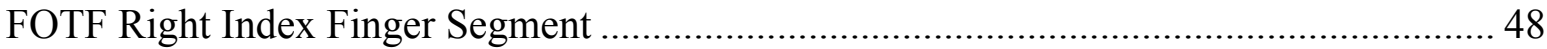

Figure 3.3 Collection laboratory and station arrangement. [2] ............................................. 49

Figure 3.4 Number of participants by month .............................................................. 51

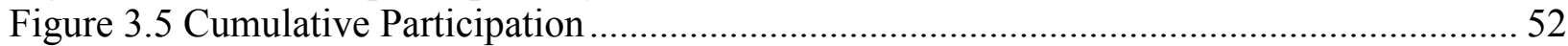

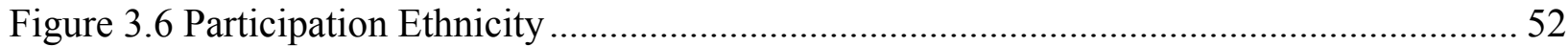

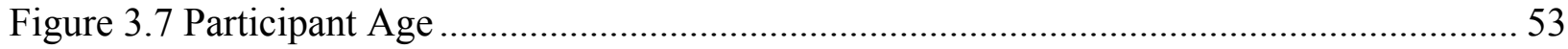

Figure 3.8 Breakdown of gender \& ethnicity .................................................................. 53

Figure 4.1 Normalized NFIQ Score Distributions for All Devices ........................................ 65

Figure 4.2 Normalized NFIQ Score Distributions for All Devices - Rolls ............................... 66

Figure 4.3 Normalized NFIQ Score Distributions for All Contact-based Devices .................... 67 
Figure 4.4 Normalized NFIQ Score Distributions for all Contactless Devices (Excluding InnerID).

Figure 4.5 (Left) NBIS 10print - Crossmatch Seek Avenger (Right) NBIS ANDI Binary - FP II

Guardian. 69

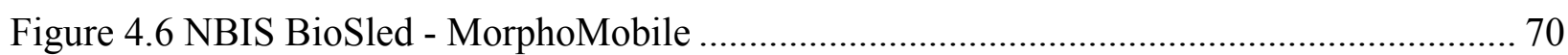

Figure 4.7 (Left) NBIS 10print - ANDI Greyscale (Right) NBIS ANDI Greyscale - Morpho FOTF

Figure 4.8 (Left) NBIS ANDI Greyscale - MorphoMobile (Right) NBIS 10print - InnerID .... 71

Figure 4.9 (Left) NBIS ANDI Binary - InnerID (Right) NBIS ANDI Greyscale - InnerID ....... 71

Figure 4.10 (Left) NBIS BioSled - InnerID (Right) NBIS Crossmatch SEEK AVENGER -

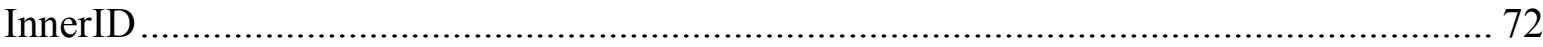

Figure 4.11 (Left) NBIS FP II Guardian (Right) NBIS InnerID - L1 .................................... 72

Figure 4.12 (Left) NBIS InnerID - Morpho FOTF (Right) NBIS InnerID - MorphoMobile...... 72

Figure 4.13 (Left) Neurotech 10print - Morpho FOTF (Right) Neurotech ANDI Binary - BioSled

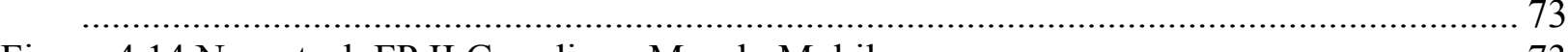

Figure 4.14 Neurotech FP II Guardian - MorphoMobile.................................................... 73

Figure 4.15 (Left) Neurotech ANDI Binary - InnerID (Right) Neurotech ANDI Greyscale InnerID 74

Figure 4.16 (Left) Neurotech BioSled - InnerID (Right) Neurotech Crossmatch SEEK

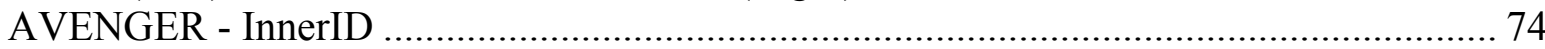

Figure 4.17 (Left) Neurotech FP II Guardian - InnerID (Right) Neurotech InnerID - L1 ......... 75

Figure 4.18 (Left) Neurotech InnerID - Morpho FOTF (Right) Neurotech InnerID -

MorphoMobile 75

Figure 4.19 (Left) SourceAFIS 10print - BioSled (Right) SourceAFIS L1 - MorphoMobile ..... 76

Figure 4.20 SourceAFIS Morpho FOTF - MorphoMobile................................................... 76

Figure 4.21 (Left) SourceAFIS 10print - InnerID (Right) SourceAFIS ANDI Binary - InnerID 77

Figure 4.22 (Left) SourceAFIS ANDI Greyscale - InnerID (Right) SourceAFIS BioSled -

InnerID 77

Figure 4.23 (Left) SourceAFIS Crossmatch SEEK AVENGER - InnerID (Right) SourceAFIS FP

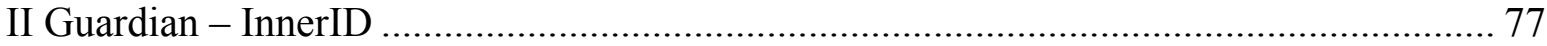

Figure 4.24 (Left) SourceAFIS InnerID - L1 (Right) SourceAFIS InnerID - Morpho FOTF .... 78

Figure 4.25 SourceAFIS InnerID - MorphoMobile ................................................................ 78

Figure 4.26 (Left) NBIS ANDI Binary Male (Right) NBIS Crossmatch Seek Avenger Male .... 79

Figure 4.27 (Left) NBIS ANDI Greyscale Female (Right) FP II Guardian Female................... 79

Figure 4.28 (Left) Neurotech ANDI Greyscale Male (Right) Neurotech BioSled Male............. 80

Figure 4.29 (Left) Neurotech BioSled Rolls Female (Right) Neurotech Morpho FOTF Female 80

Figure 4.30 (Left) SourceAFIS Morpho FOTF Male (Right) SourceAFIS MorphoMobile Male 80

Figure 4.31(Left) ANDI Binary Female (Right) SourceAFIS FP II Guardian Female .............. 81

Figure 4.32 (a) Neurotech BioSled Rolls for ages 18-19 (b) SourceAFIS FP II Guardian Ages 20 $-29$.

(c) SourceAFIS Morpho FOTF for ages 30 - 39 (d)Neurotech ANDI Binary for Ages 40 and up

Figure 4.33 (Left) NBIS Crossmatch Seek Avenger Rolls for ages 40 and up (Right) NBIS

BioSled Rolls for ages 40 and up. 85

Figure 4.34 (Left) Neurotech FP II Guardian for ages 40 and up (Right) Neurotech L1 for ages 40 and up. 85 
Figure 4.35 (Left) NBIS ANDI Binary for the African Ethnicity (Right) NBIS ANDI Binary for the Middle Eastern Ethnicity

Figure 4.36 (Left) NBIS ANDI Greyscale for the Asian Ethnicity (Right) NBIS BioSled for the Asian Indian Ethnicity 89

Figure 4.37 (Left) Neurotech BioSled for the Other Ethnicity (Right) Neurotech FP II Guardian for the Hispanic Ethnicity 90

Figure 4.38 (Left) SourceAFIS Crossmatch Seek Avenger Rolls For the African American Ethncity (Right) SourceAFIS MorphoMobile for the Caucasian Ethnicity........................... 90

Figure 4.39 (Left) NBIS FP II Rolls For the American Indian Ethnicity (Right) NBIS

Crossmatch Seek Avenger for the American Indian Ethnicity.... 91

Figure 4.40 (Left) NBIS Crossmatch Seek Avenger Rolls for the American Indian Ethnicity (Right) Neurotech BioSled for the American Indian Ethnicity

Figure 4.41 (Left) Neurotech Crossmatch Seek Avenger for the American Indian Ethnicity

(Right) Neurotech Crossmatch Seek Avenger Rolls for the American Indian Ethnicity ..... 92

Figure 4.42 (Left) SourceAFIS FP II Guardian for the American Indian Ethnicity (Right)

SourceAFIS Crossmatch Seek Avenger for the American Indian Ethnicity ........................ 92

Figure 4.43 (Left) SourceAFIS Crossmatch Seek Avenger Rolls for the American Indian Ethnicity (Right) SourceAFIS MorphoMobile for the American Indian Ethnicity .............. 93

Figure A.1.1 (Left) NBIS ANDI Binary (Right) NBIS ANDI Greyscale .................................. 117

Figure A.1.2 (Left) NBIS BioSled (Right) NBIS BioSled Rolls ............................................. 118

Figure A.1.3 (Left) NBIS FP II Guardian (Right) NBIS FPII Guardian Rolls.......................... 118

Figure A.1.4 (Left) NBIS Crossmatch Seek Avenger (Right) NBIS Crossmatch Seek Avenger Rolls.... 118

Figure A.1.5 (Left) NBIS InnerID (Right) NBIS L1 ……................................................... 119

Figure A.1.6 (Left) NBIS L1 Rolls (Right) NBIS Morpho FOTF.............................................. 119

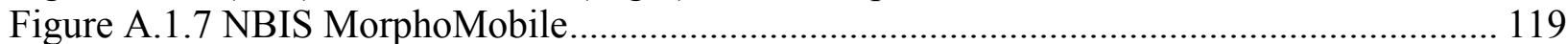

Figure A.2.1 (Left) Neurotech ANDI Binary (Right) Neurotech ANDI Greyscale ................... 121

Figure A.2.2 (Left) Neurotech BioSled (Right) Neurotech BioSled Rolls.................................. 121

Figure A.2.3 (Left) Neurotech FP II Guardian (Right) Neurotech FP II Guardian Rolls........... 122

Figure A.2.4 (Left) Neurotech Crossmatch Seek Avenger (Right) Neurotech Crossmatch Seek

Avenger Rolls

122

Figure A.2.5 (Left) Neurotech InnerID (Right) Neurotech L1 .......................................... 122

Figure A.2.6 (Left) Neurotech Morpho FOTF (Right) Neurotech MorphoMobile.................... 123

Figure A.3.1 (Left) SourceAFIS ANDI Binary (Right) SourceAFIS ANDI Greyscale.............. 124

Figure A.3.2 (Left) SourceAFIS BioSled (Right) SourceAFIS BioSled Rolls............................ 124

Figure A.3.3 (Left) SourceAFIS FP II Guardian (Right) SourceAFIS FP II Guardian Rolls .... 125

Figure A.3.4 (Left) SourceAFIS Crossmatch Seek Avenger (Right) SourceAFIS Crossmatch

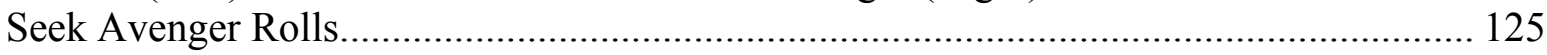

Figure A.3.5 (Left) SourceAFIS InnerID (Right) SourceAFIS L1 ........................................... 125

Figure A.3.6 (Left) SourceAFIS L1 Rolls (Right) SourceAFIS Morpho FOTF ........................ 126

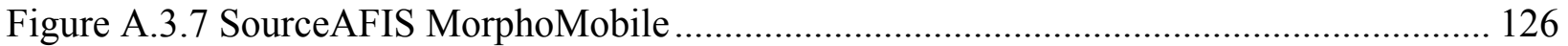

Figure A.4.1 (Left) NBIS 10print Rolls - BioSled Rolls (Right) NBIS 10print Rolls - Crossmatch

Seek Avenger Rolls.................................................................................................. 128

Figure A.4.2 (Left) NBIS 10print Rolls - FP II Guardian Rolls (Right) NBIS 10print Rolls - L1

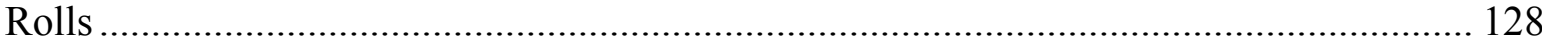

Figure A.4.3 (Left) NBIS 10print - ANDI Binary (Right) NBIS 10print - ANDI Greyscale ... 129 
Figure A.4.4 (Left) NBIS 10print - BioSled (Right) NBIS 10print - Crossmatch Seek Avenger

Figure A.4.5 (Left) NBIS 10print - FP II Guardian (Right) NBIS 10print - L1.................... 129

Figure A.4.6 (Left) NBIS 10print - Morpho FOTF (Right) NBIS 10print - MorphoMobile .... 130

Figure A.4.7 (Left) NBIS ANDI Binary - ANDI Greyscale (Right) NBIS ANDI Binary -

BioSled.

Figure A.4.8 (Left) NBIS ANDI Binary - Crossmatch Seek Avenger (Right) NBIS ANDI Binary - FP II Guardian.

Figure A.4.9 (Left) NBIS ANDI Binary - L1 (Right) NBIS ANDI Binary - Morpho FOTF ... 131

Figure A.4.10 (Left) NBIS ANDI Binary - MorphoMobile (Right) NBIS ANDI Greyscale BioSled.

Figure A.4.11 (Left) NBIS ANDI Greyscale - Crossmatch Seek Avenger (Right) NBIS ANDI Greyscale - FP II Guardian.

Figure A.4.12 (Left) NBIS ANDI Greyscale - L1 (Right) NBIS ANDI Greyscale - Morpho FOTF

Figure A.4.13 (Left) NBIS ANDI Greyscale - MorphoMobile (Right) NBIS BioSled Rolls Crossmatch Seek Avenger Rolls

Figure A.4.14 (Left) NBIS BioSled Rolls - FP II Guardian Rolls (Right) NBIS BioSled Rolls -

L1 Rolls

Figure A.4.15 (Left) NBIS BioSled - Crossmatch Seek Avenger (Right) NBIS BioSled - FP II Guardian.

Figure A.4.16 (Left) NBIS BioSled - L1 (Right) NBIS BioSled - Morpho FOTF 133

Figure A.4.17 (Left) NBIS BioSled - MorphoMobile (Right) NBIS Crossmatch Seek Avenger Rolls - FP Guardian Rolls

Figure A.4.18 (Left) NBIS Crossmatch Seek Avenger Rolls - L1 Rolls (Right) NBIS

Crossmatch Seek Avenger - FP II Guardian

Figure A.4.19 (Left) NBIS Crossmatch Seek Avenger - L1 (Right) NBIS Crossmatch Seek

Avenger - Morpho FOTF

Figure A.4.20 (Left) NBIS Crossmatch Seek Avenger - MorphoMobile (Right) NBIS FP II

Guardian Rolls - L1 Rolls

Figure A.4.21 (Left) NBIS FP II Guardian - L1 (Right) NBIS FP II Guardian - Morpho FOTF

Figure A.4.22 (Left) NBIS FP II Guardian - MorphoMobile (Right) NBIS L1 - Morpho FOTF

Figure A.4.23 (Left) NBIS L1 - MorphoMobile (Right) NBIS Morpho FOTF - MorphoMobile

Figure A.5.1 (Left) Neurotech 10print - ANDI Binary (Right) Neurotech 10print - ANDI

Greyscale 138

Figure A.5.2 (Left) Neurotech 10print - BioSled (Right) Neurotech 10print - Crossmatch Seek

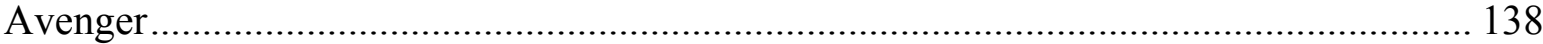

Figure A.5.3 (Left) Neurotech 10print - FP II Guardian (Right) Neurotech 10print - L1 ....... 139

Figure A.5.4 (Left) Neurotech 10print - Morpho FOTF (Right) Neurotech 10print MorphoMobile.....

Figure A.5.5 (Left) Neurotech 10print Rolls - BioSled Rolls (Right) Neurotech 10print Rolls Crossmatch Seek Avenger Rolls. 
Figure A.5.6 (Left) Neurotech 10print Rolls - FP II Guardian Rolls (Right) Neurotech ANDI Binary - ANDI Greyscale. 140

Figure A.5.7 (Left) Neurotech ANDI Binary - BioSled (Right) Neurotech ANDI Binary Crossmatch Seek Avenger.

Figure A.5.8 (Left) Neurotech ANDI Binary - FP II Guardian (Right) Neurotech ANDI Binary L1

Figure A.5.9 (Left) Neurotech ANDI Binary - Morpho FOTF (Right) Neurotech ANDI Binary MorphoMobile

Figure A.5.10 (Left) Neurotech ANDI Greyscale - BioSled (Right) Neurotech ANDI Greyscale

- Crossmatch Seek Avenger

Figure A.5.11 (Left) Neurotech ANDI Greyscale - FP II Guardian (Right) Neurotech ANDI Greyscale - L1

Figure A.5.12 (Left) Neurotech ANDI Greyscale - Morpho FOTF (Right) Neurotech ANDI Greyscale - MorphoMobile

Figure A.5.13 (Left) Neurotech BioSled - Crossmatch Seek Avenger (Right) Neurotech BioSled - FP II Guardian. 142

Figure A.5.14 (Left) Neurotech BioSled - L1 (Right) Neurotech BioSled - Morpho FOTF .... 142 Figure A.5.15 (Left) Neurotech BioSled - MorphoMobile (Right) Neurotech BioSled Rolls Crossmatch Seek Avenger Rolls. 143

Figure A.5.16 (Left) Neurotech BioSled Rolls- FP II Guardian Rolls (Right) Neurotech

Crossmatch Seek Avenger - FP II Guardian

Figure A.5.17 (Left) Neurotech Crossmatch Seek Avenger - L1 (Right) Neurotech Crossmatch Seek Avenger - Morpho FOTF 144

Figure A.5.18 (Left) Neurotech Crossmatch Seek Avenger - MorphoMobile (Right) Neurotech

Crossmatch Seek Avenger Rolls - FP II Guardian Rolls 144

Figure A.5.19 (Left) Neurotech FP II Guardian - L1 (Right) Neurotech FP II Guardian - Morpho FOTF 144

Figure A.5.20 (Left) Neurotech FP II Guardian - MorphoMobile (Right) Neurotech L1 Morpho FOTF 145

Figure A.5.21 (Left) Neurotech L1 - MorphoMobile (Right) Neurotech L1 Rolls - 10print Rolls

Figure A.5.22 (Left) Neurotech L1 Rolls - BioSled Rolls (Right) Neurotech L1 Rolls 145 Crossmatch Seek Avenger Rolls. 145

Figure A.5.23 (Left) NeurotechL1 Rolls - FP II Guardian Rolls (Right) Neurotech Morpho FOTF - MorphoMobile

Figure A.6.1 (Left) SourceAFIS 10print - ANDI Binary (Right) SourceAFIS 10print - ANDI Greyscale.

Figure A.6.2 (Left) SourceAFIS 10print - BioSled (Right) SourceAFIS 10print - Crossmatch Seek Avenger

Figure A.6.3 (Left) SourceAFIS 10print - FP II Guardian (Right) SourceAFIS 10print - L1 .. 149

Figure A.6.4 (Left) SourceAFIS 10print - Morpho FOTF (Right) SourceAFIS 10print MorphoMobile 149

Figure A.6.5 (Left) SourceAFIS 10print Rolls - BioSled Rolls (Right) SourceAFIS 10print Rolls - Crossmatch Seek Avenger Rolls 149

Figure A.6.6 (Left) SourceAFIS 10print Rolls - FP II Guardian Rolls (Right) SourceAFIS 10print Rolls - L1 Rolls 
Figure A.6.7 (Left) SourceAFIS ANDI Binary - ANDI Greyscale (Right) SourceAFIS ANDI Binary - BioSled

Figure A.6.8 (Left) SourceAFIS ANDI Binary - Crossmatch Seek Avenger (Right) SourceAFIS

ANDI Binary - FP II Guardian.

Figure A.6.9 (Left) SourceAFIS ANDI Binary - L1 (Right) SourceAFIS ANDI Binary - Morpho FOTF

Figure A.6.10 (Left) SourceAFIS ANDI Binary - MorphoMobile (Right) SourceAFIS ANDI Greyscale - BioSled.....

Figure A.6.11 (Left) SourceAFIS ANDI Greyscale - Crossmatch Seek Avenger (Right)

SourceAFIS ANDI Greyscale - FP II Guardian.

Figure A.6.12 (Left) SourceAFIS ANDI Greyscale - L1 (Right) SourceAFIS ANDI Greyscale Morpho FOTF

Figure A.6.13 (Left) SourceAFIS ANDI Greyscale - MorphoMobile (Right) SourceAFIS

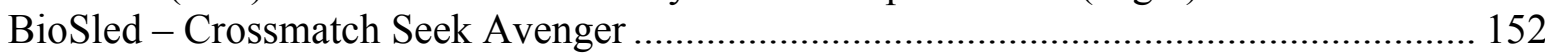

Figure A.6.14 (Left) SourceAFIS BioSled - FP II Guardian (Right) BioSled - L1 .................. 152

Figure A.6.15 (Left) SourceAFIS BioSled - Morpho FOTF (Right) SourceAFIS BioSled MorphoMobile

Figure A.6.16 (Left) SourceAFIS BioSled Rolls - Crossmatch Seek Avenger Rolls (Right)

SourceAFIS BioSled Rolls - FP II Guardian Rolls

Figure A.6.17 (Left) SourceAFIS BioSled Rolls - L1 Rolls (Right) SourceAFIS Crossmatch

Seek Avenger - FP II Guardian

Figure A.6.18 (Left) SourceAFIS Crossmatch Seek Avenger- L1 (Right) SourceAFIS

Crossmatch Seek Avenger - Morpho FOTF 154

Figure A.6.19 (Left) SourceAFIS Crossmatch Seek Avenger - MorphoMobile (Right)

SourceAFIS Crossmatch Seek Avenger Rolls - FP II Guardian Rolls

Figure A.6.20 (Left) SourceAFIS Crossmatch Seek Avenger Rolls - L1 Rolls (Right)

SourceAFIS FP II Guardian - L1

Figure A.6.21 (Left) SourceAFIS FP II Guardian - Morpho FOTF (Right) SourceAFIS FP II

Guardian - MorphoMobile.

Figure A.6.22 (Left) SourceAFIS FP II Guardian Rolls - L1 Rolls (Right) SourceAFIS L1 -

Morpho FOTF

Figure A.6.23 (Left) SourceAFIS L1 - MorphoMobile (Right) SourceAFIS Morpho FOTF -

MorphoMobile 156

Figure A.7.1 NBIS ROC Curves for each device 157

Figure A.7.2 Zoomed In NBIS ROC Curves for each device 158

Figure A.7.3 (Left) NBIS ANDI Binary ROC Curve (Right) NBIS ANDI Greyscale ROC Curve

Figure A.7.4 (Left) NBIS BioSled ROC Curve (Right) NBIS BioSled Rolls ROC Curve........ 159

Figure A.7.5 (Left) NBIS FP II Guardian ROC Curve (Right) NBIS FP II Guardian Rolls ROC

Curve

Figure A.7.6 (Left) NBIS Crossmatch Seek Avenger ROC Curve (Right) NBIS Crossmatch Seek

Avenger Rolls ROC Curve 160

Figure A.7.7 (Left) NBIS InnerID ROC Curve (Right) NBIS L1 Curve ..................................... 161

Figure A.7.8 (Left) NBIS L1 Rolls ROC Curve (Right) NBIS Morpho FOTF ROC Curve...... 161

Figure A.7.9 NBIS MorphoMobile ROC Curve. 162

Figure A.8.1 Neurotech ROC Curves for each Device. 163 
Figure A.8.2 Zoomed In Neurotech ROC Curves for each Device 164

Figure A.8.3 (Left) Neurotech ANDI Binary ROC Curve (Right) Neurotech ANDI Greyscale ROC Curve. 165

Figure A.8.4 (Left) Neurotech BioSled ROC Curve (Right) Neurotech BioSled Rolls ROC Curve 165

Figure A.8.5 (Left) Neurotech FP II Guardian ROC Curve (Right) Neurotech FP II Guardian Rolls ROC Curve ............................................................................................... 166

Figure A.8.6 (Left) Neurotech Crossmatch Seek Avenger ROC Curve (Right) Neurotech Crossmatch Seek Avenger Rolls ROC Curve.................................................................. 166

Figure A.8.7 (Left) Neurotech InnerID ROC Curve (Right) Neurotech L1 ROC Curve ............ 167

Figure A.8.8 (Left) Neurotech Morpho FOTF ROC Curve (Right) Neurotech MorphoMobile

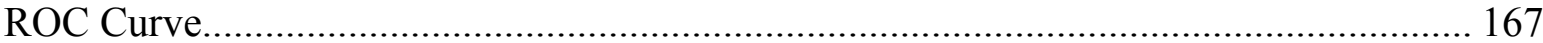

Figure A.9.1 SourceAFIS ROC Curves For each Device......................................................... 168

Figure A.9.2 Zoomed In SourceAFIS ROC Curves For each Device ...................................... 169

Figure A.9.3 (Left) SourceAFIS ANDI Binary ROC Curve (Right) SourceAFIS ANDI Greyscale ROC Curve.......................................................................................................... 170

Figure A.9.4 (Left) SourceAFIS BioSled ROC Curve (Right) SourceAFIS BioSled Rolls ROC Curve 170

Figure A. 9.5(Left) SourceAFIS FP II Guardian ROC Curve (Right) SourceAFIS FP II Guardian Rolls ROC Curve 171

Figure A.9.6 (Left) SourceAFIS Crossmatch Seek Avenger ROC Curve (Right) SourceAFIS Crossmatch Seek Avenger Rolls ROC Curve.

Figure A.9.7 (Left) SourceAFIS InnerID ROC Curve (Right) SourceAFIS L1 ROC Curve..... 172 Figure A.9.8 (Left) SourceAFIS L1 Rolls ROC Curve (Right) SourceAFIS Morpho FOTF ROC Curve 172

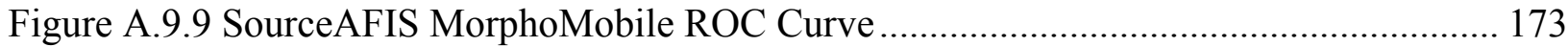

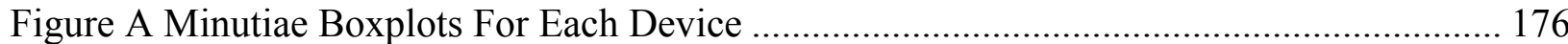

Figure B.1.1 NBIS ANDI Binary Male (Left) Female (Right)................................................. 179

Figure B.1.2 NBIS ANDI Greyscale Male (Left) Female (Right) ……………………........... 180

Figure B.1.3 NBIS BioSled Male (Left) Female (Right).......................................................... 180

Figure B.1.4 NBIS BioSled Rolls Male (Left) Female (Right) ................................................ 180

Figure B.1.5 NBIS FP II Guardian Male (Left) Female (Right) …………………………......... 181

Figure B.1.6 NBIS FP II Guardian Rolls Male (Left) Female (Right) ..................................... 181

Figure B.1.7 NBIS Crossmatch Seek Avenger Male (Left) Female (Right) ............................... 181

Figure B.1.8 NBIS Crossmatch Seek Avenger Rolls Male (Left) Female (Right).................... 182

Figure B.1.9 NBIS InnerID Male (Left) Female (Right) ...................................................... 182

Figure B.1.10 NBIS L1 Male (Left) Female (Right) ……................................................. 182

Figure B.1.11 NBIS L1 Rolls Male (Left) Female (Right)..................................................... 183

Figure B.1.12 NBIS Morpho FOTF Rolls Male (Left) Female (Right) ...................................... 183

Figure B.1.13 NBIS MorphoMobile Rolls Male (Left) Female (Right)...................................... 183

Figure B.2.1 Neurotech ANDI Binary Male (Left) Female (Right) ………………………...... 185

Figure B.2.2 Neurotech ANDI Greyscale Male (Left) Female (Right) ..................................... 185

Figure B.2.3 Neurotech BioSled Male (Left) Female (Right) .................................................. 185

Figure B.2.4 Neurotech BioSled Rolls Male (Left) Female (Right)......................................... 186

Figure B.2.5 Neurotech FP II Guardian Male (Left) Female (Right) ……………..................... 186

Figure B.2.6 Neurotech FP II Guardian Rolls Male (Left) Female (Right) ................................. 186 
Figure B.2.7 Neurotech Crossmatch Seek Avenger Male (Left) Female (Right) ................... 187

Figure B.2.8 Neurotech Crossmatch Seek Avenger Rolls Male (Left) Female (Right) ............ 187

Figure B.2.9 Neurotech InnerID Male (Left) Female (Right) ............................................. 187

Figure B.2.10 Neurotech L1 Male (Left) Female (Right) ................................................. 188

Figure B.2.11 Neurotech Morpho FOTF Male (Left) Female (Right) ................................. 188

Figure B.2.12 Neurotech MorphoMobile Male (Left) Female (Right) ................................. 188

Figure B.3.1 SourceAFIS ANDI Binary Male (Left) Female (Right) .................................. 190

Figure B.3.2 SourceAFIS ANDI Greyscale Male (Left) Female (Right) .............................. 190

Figure B.3.3 SourceAFIS BioSled Male (Left) Female (Right) ............................................ 190

Figure B.3.4 SourceAFIS BioSled Rolls Male (Left) Female (Right) .................................. 191

Figure B.3.5 SourceAFIS FP II Guardian Male (Left) Female (Right).................................. 191

Figure B.3.6 SourceAFIS FP II Guardian Male (Left) Female (Right)................................. 191

Figure B.3.7 SourceAFIS Crossmatch Seek Avenger Male (Left) Female (Right) .................. 192

Figure B.3.8 SourceAFIS Crossmatch Seek Avenger Rolls Male (Left) Female (Right) ......... 192

Figure B.3.9 SourceAFIS InnerID Male (Left) Female (Right) ............................................ 192

Figure B.3.10 SourceAFIS L1 Male (Left) Female (Right) ............................................... 193

Figure B.3.11 SourceAFIS L1 Rolls Male (Left) Female (Right) ........................................ 193

Figure B.3.12 SourceAFIS Morpho FOTF Male (Left) Female (Right) ................................. 193

Figure B.3.13 SourceAFIS MorphoMobile Male (Left) Female (Right) ................................ 194

Figure B.4.1 NBIS ANDI Binary Gender ROC Curves (Left) NBIS ANDI Greyscale Gender

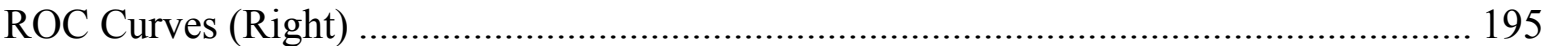

Figure B.4.2 NBIS BioSled Gender ROC Curves (Left) NBIS BioSled Rolls Gender ROC Curves (Right) ................................................................................................. 195

Figure B.4.3 NBIS FP II Guardian Gender ROC Curves (Left) NBIS FP II Guardian Rolls

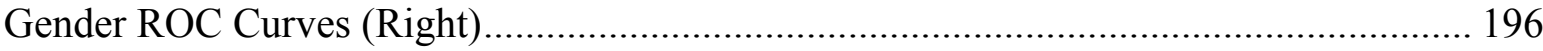

Figure B.4.4 NBIS Crossmatch Seek Avenger Gender ROC Curves (Left) NBIS Crossmatch Seek Avenger Rolls Gender ROC Curves (Right)........................................................ 196

Figure B.4.5 NBIS InnerID Gender ROC Curves (Left) NBIS L1 Gender ROC Curves (Right) 197

Figure B.4.6 NBIS L1 Rolls Gender ROC Curves (Left) NBIS Morpho FOTF Gender ROC

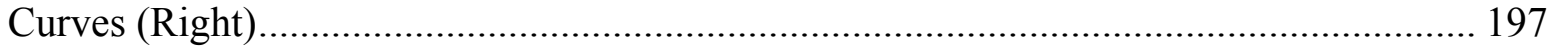

Figure B.4.7 NBIS MorphoMobile Gender ROC Curves....................................................... 198

Figure B.5.1 Neurotech ANDI Binary Gender ROC Curves (Left) Neurotech ANDI Greyscale Gender ROC Curves (Right) ................................................................................... 199

Figure B.5.2 Neurotech BioSled Gender ROC Curves (Left) Neurotech BioSled Rolls Gender

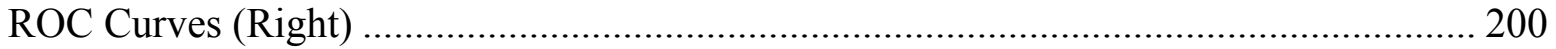

Figure B.5.3 Neurotech FP II Guardian Gender ROC Curves (Left) Neurotech FP II Guardian Rolls Gender ROC Curves (Right) ........................................................................ 200

Figure B.5.4 Neurotech Crossmatch Seek Avenger Gender ROC Curves (Left) Neurotech Crossmatch Seek Avenger Rolls Gender ROC Curves (Right) ..................................... 201

Figure B.5.5 Neurotech InnerID Gender ROC Curves (Left) Neurotech L1 Gender ROC Curves (Right) .................................................................................................. 201

Figure B.5.6 Neurotech Morpho FOTF Gender ROC Curves (Left) Neurotech MorphoMobile

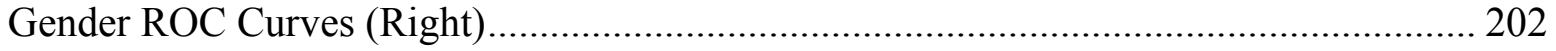

Figure B.6.1 SourceAFIS ANDI Binary Gender ROC Curves (Left) SourceAFIS ANDI Greyscale Gender ROC Curves (Right) ............................................................... 203 
Figure B.6.2 SourceAFIS BioSled Gender ROC Curves (Left) SourceAFIS BioSled Rolls Gender ROC Curves (Right) 204

Figure B.6.3 SourceAFIS FP II Guardian Gender ROC Curves (Left) SourceAFIS FP II Guardian Rolls Gender ROC Curves (Right) 204

Figure B.6.4 SourceAFIS Crossmatch Seek Avenger Gender ROC Curves (Left) SourceAFIS Crossmatch Seek Avenger Gender ROC Curves (Right) .... 205

Figure B.6.5 SourceAFIS InnerID Gender ROC Curves (Left) SourceAFIS L1 Gender ROC

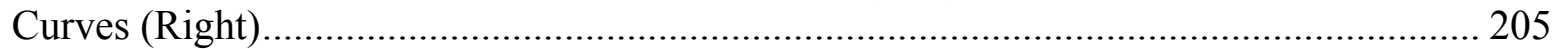

Figure B.6.6 SourceAFIS L1 Rolls Gender ROC Curves (Left) SourceAFIS Morpho FOTF

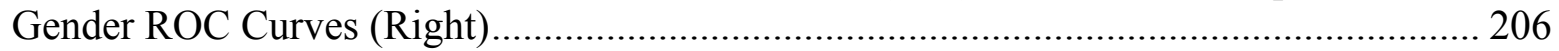

Figure B.6.7 SourceAFIS MorphoMobile Gender ROC Curves ........................................... 206

Figure B.7.1 Minutiae Box Plot for ANDI Binary by Gender (Left) Minutiae Box Plot for ANDI Greyscale by Gender (Right) ............................................................................... 207

Figure B.7.2 Minutiae Box Plot for BioSled by Gender (Left) Minutiae Box Plot for BioSled Rolls by Gender (Right) ........................................................................................ 207

Figure B.7.3 Minutiae Box Plot for FP II Guardian by Gender (Left) Minutiae Box Plot for FP II Guardian Rolls by Gender (Right) ........................................................................... 208

Figure B.7.4 Minutiae Box Plot for Crossmatch Seek Avenger by Gender (Left) Minutiae Box Plot for Crossmatch Seek Avenger Rolls by Gender (Right) ....................................... 208

Figure B.7.5 Minutiae Box Plot for InnerID by Gender (Left) Minutiae Box Plot for L1 by

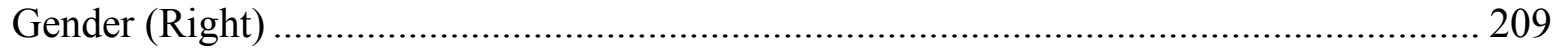

Figure B.7.6 Minutiae Box Plot for L1 Rolls by Gender (Left) Minutiae Box Plot for Morpho FOTF by Gender (Right) ................................................................................... 209

Figure B.7.7 Minutiae Box Plot for MorphoMobile by Gender ............................................ 210 Figure C.1.1 NBIS ANDI Binary (a) Ages 18-19 (b) Ages 20-29 (c) Ages 30-39 (d) Ages 40 and

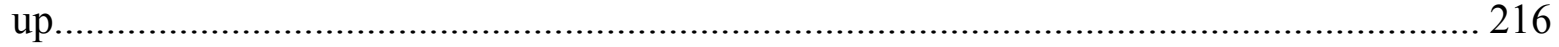

Figure C.1.2 NBIS ANDI Greyscale (a) Ages 18-19 (b) Ages 20-29 (c) Ages 30-39 (d) Ages 40

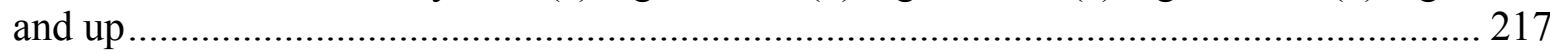

Figure C.1.3 NBIS BioSled (a) Ages 18-19 (b) Ages 20-29 (c) Ages 30-39 (d) Ages 40 and up

Figure C.1.4 NBIS BioSled Rolls (a) Ages 18-19 (b) Ages 20-29 (c) Ages 30-39 (d) Ages 40 and

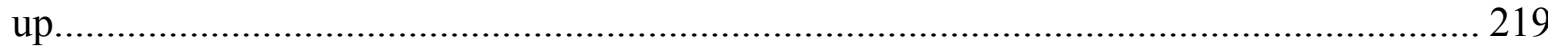

Figure C.1.5 NBIS FP II Guardian (a) Ages 18-19 (b) Ages 20-29 (c) Ages 30-39 (d) Ages 40

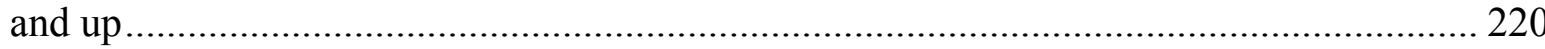

Figure C.1.6 NBIS FP II Guardian Rolls (a) Ages 18-19 (b) Ages 20-29 (c) Ages 30-39 (d) Ages

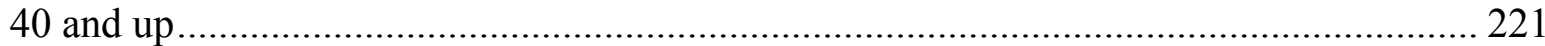

Figure C.1.7 NBIS Crossmatch Seek Avenger (a) Ages 18-19 (b) Ages 20-29 (c) Ages 30-39 (d) Ages 40 and up............................................................................................. 222

Figure C.1.8 NBIS Crossmatch Seek Avenger Rolls (a) Ages 18-19 (b) Ages 20-29 (c) Ages 3039 (d) Ages 40 and up...................................................................................... 223

Figure C.1.9 NBIS InnerID (a) Ages 18-19 (b) Ages 20-29 (c) Ages 30-39 (d) Ages 40 and up 224

Figure C.1.10 NBIS L1 (a) Ages 18-19 (b) Ages 20-29 (c) Ages 30-39 (d) Ages 40 and up... 225 Figure C.1.11 NBIS L1 Rolls (a) Ages 18-19 (b) Ages 20-29 (c) Ages 30-39 (d) Ages 40 and up 226 
Figure C.1.12 NBIS Morpho FOTF (a) Ages 18-19 (b) Ages 20-29 (c) Ages 30-39 (d) Ages 40 and up....

Figure C.1.13 NBIS MorphoMobile (a) Ages 18-19 (b) Ages 20-29 (c) Ages 30-39 (d) Ages 40

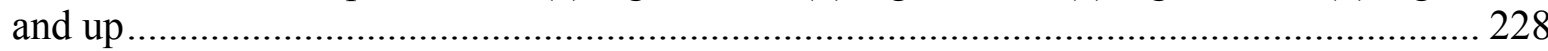

Figure C.2.1 Neurotech ANDI Binary (a) Ages 18-19 (b) Ages 20-29 (c) Ages 30-39 (d) Ages 40 and up 231

Figure C.2.2 Neurotech ANDI Greyscale (a) Ages 18-19 (b) Ages 20-29 (c) Ages 30-39 (d) Ages 40 and up

Figure C.2.3 Neurotech BioSled (a) Ages 18-19 (b) Ages 20-29 (c) Ages 30-39 (d) Ages 40 and up.....

Figure C.2.4 Neurotech BioSled Rolls (a) Ages 18-19 (b) Ages 20-29 (c) Ages 30-39 (d) Ages 40 and up 234

Figure C.2.5 Neurotech FP II Guardian (a) Ages 18-19 (b) Ages 20-29 (c) Ages 30-39 (d) Ages 40 and up 235

Figure C.2.6 Neurotech FP II Guardian Rolls (a) Ages 18-19 (b) Ages 20-29 (c) Ages 30-39 (d) Ages 40 and up. 236

Figure C.2.7 Neurotech Crossmatch Seek Avenger (a) Ages 18-19 (b) Ages 20-29 (c) Ages 3039 (d) Ages 40 and up... 237

Figure C.2.8 Neurotech Crossmatch Seek Avenger Rolls (a) Ages 18-19 (b) Ages 20-29 (c) Ages 30-39 (d) Ages 40 and up. 238

Figure C.2.9 Neurotech InnerID (a) Ages 18-19 (b) Ages 20-29 (c) Ages 30-39 (d) Ages 40 and up (Not Available) 239

Figure C.2.10 Neurotech L1 (a) Ages 18-19 (b) Ages 20-29 (c) Ages 30-39 (d) Ages 40 and up 240

Figure C.2.11 Neurotech Morpho FOTF (a) Ages 18-19 (b) Ages 20-29 (c) Ages 30-39 (d) Ages 40 and up. 241

Figure C.2.12 Neurotech MorphoMobile (a) Ages 18-19 (b) Ages 20-29 (c) Ages 30-39 (d) Ages 40 and up. 242

Figure C.3.1 SourceAFIS ANDI Binary (a) Ages 18-19 (b) Ages 20-29 (c) Ages 30-39 (d) Ages 40 and up. .245

Figure C.3.2 SourceAFIS ANDI Greyscale (a) Ages 18-19 (b) Ages 20-29 (c) Ages 30-39 (d) Ages 40 and up. 246

Figure C.3.3 SourceAFIS BioSled (a) Ages 18-19 (b) Ages 20-29 (c) Ages 30-39 (d) Ages 40 and up....

Figure C.3.4 SourceAFIS BioSled Rolls (a) Ages 18-19 (b) Ages 20-29 (c) Ages 30-39 (d) Ages 40 and up 248

Figure C.3.5 SourceAFIS FP II Guardian (a) Ages 18-19 (b) Ages 20-29 (c) Ages 30-39 (d) Ages 40 and up. 249

Figure C.3.6 SourceAFIS FP II Guardian Rolls (a) Ages 18-19 (b) Ages 20-29 (c) Ages 30-39 (d) Ages 40 and up 250

Figure C.3.7 SourceAFIS Crossmatch Seek Avenger (a) Ages 18-19 (b) Ages 20-29 (c) Ages 3039 (d) Ages 40 and up. 251

Figure C.3.8 SourceAFIS Crossmatch Seek Avenger Rolls (a) Ages 18-19 (b) Ages 20-29 (c) Ages 30-39 (d) Ages 40 and up 252

Figure C.3.9 SourceAFIS InnerID (a) Ages 18-19 (b) Ages 20-29 (c) Ages 30-39 (d) Ages 40 and up..... 
Figure C.3.10 SourceAFIS L1 (a) Ages 18-19 (b) Ages 20-29 (c) Ages 30-39 (d) Ages 40 and up 254

Figure C.3.11 SourceAFIS L1 Rolls (a) Ages 18-19 (b) Ages 20-29 (c) Ages 30-39 (d) Ages 40 and up

Figure C.3.12 SourceAFIS Morpho FOTF (a) Ages 18-19 (b) Ages 20-29 (c) Ages 30-39 (d)

Ages 40 and up. 255

Figure C.3.13 SourceAFIS MorphoMobile (a) Ages 18-19 (b) Ages 20-29 (c) Ages 30-39 (d) Ages 40 and up 256

Figure C.4.1 NBIS ANDI Binary Age Demographic ROC Curves (Left) NBIS ANDI Greyscale

Age Demographic ROC Curves (Right) ....................................................................... 260

Figure C.4.2 NBIS BioSled Age Demographic ROC Curves (Left) NBIS BioSled Rolls Age

Demographic ROC Curves (Right).......................................................................... 260

Figure C.4.3 NBIS FP II Guardian Age Demographic ROC Curves (Left) NBIS FP II Guardian

Rolls Age Demographic ROC Curves (Right)........................................................... 261

Figure C.4.4 NBIS Crossmatch Seek Avenger Age Demographic ROC Curves (Left) NBIS

Crossmatch Seek Avenger Rolls Age Demographic ROC Curves (Right) ...................... 261

Figure C.4.5 NBIS InnerID Age Demographic ROC Curves (Left) NBIS L1 Age Demographic

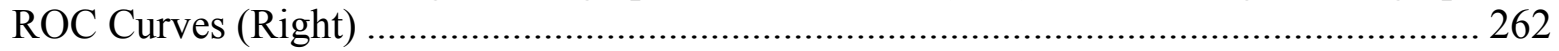

Figure C.4.6 NBIS L1 Rolls Age Demographic ROC Curves (Left) NBIS Morpho FOTF Age

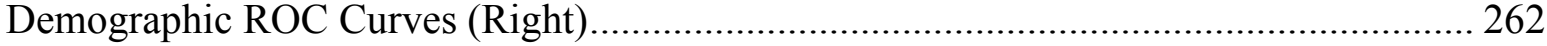

Figure C.4.7 NBIS MorphoMobile Age Demographic ROC Curves ...................................... 263

Figure C.5.1 Neurotech ANDI Binary Age Demographic ROC Curves (Left) Neurotech ANDI

Greyscale Age Demographic ROC Curves (Right) .................................................... 266

Figure C.5.2 Neurotech BioSled Age Demographic ROC Curves (Left) Neurotech BioSled Rolls

Age Demographic ROC Curves (Right) .................................................................. 266

Figure C.5.3 Neurotech FP II Guardian Age Demographic ROC Curves (Left) Neurotech FP II

Guardian Rolls Age Demographic ROC Curves (Right)............................................... 267

Figure C.5.4 Neurotech Crossmatch Seek Avenger Age Demographic ROC Curves (Left)

Neurotech Crossmatch Seek Avenger Rolls Age Demographic ROC Curves (Right) ...... 267

Figure C.5.5 Neurotech L1 Age Demographic ROC Curves (Left) Neurotech Morpho FOTF Age

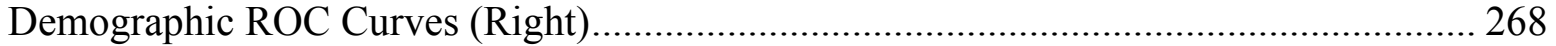

Figure C.5.6 Neurotech MorphoMobile Age Demographic ROC Curves .............................. 268

Figure C.6.1 SourceAFIS ANDI Binary Age Demographic ROC Curves (Left) SourceAFIS

ANDI Greyscale Age Demographic ROC Curves (Right) .......................................... 271

Figure C.6.2 SourceAFIS BioSled Age Demographic ROC Curves (Left) SourceAFIS BioSled

Rolls Age Demographic ROC Curves (Right)............................................................ 271

Figure C.6.3 SourceAFIS FP II Guardian Age Demographic ROC Curves (Left) SourceAFIS FP

II Guardian Rolls Age Demographic ROC Curves (Right) ........................................ 272

Figure C.6.4 SourceAFIS Crossmatch Seek Avenger Age Demographic ROC Curves (Left)

SourceAFIS Crossmatch Seek Avenger Age Demographic ROC Curves (Right)............ 272

Figure C.6.5 SourceAFIS InnerID Age Demographic ROC Curves (Left) SourceAFIS L1 Age

Demographic ROC Curves (Right)...................................................................... 273

Figure C.6.6 SourceAFIS L1 Rolls Age Demographic ROC Curves (Left) SourceAFIS Morpho

FOTF Age Demographic ROC Curves (Right) ......................................................... 273

Figure C.6.7 SourceAFIS MorphoMobile Age Demographic ROC Curves ........................... 274

Figure C.7.1 (Left)AND Binary Box Plot (Right) ANDI Greyscale Box Plot......................... 275 
Figure C.7.2 (Left) BioSled Rolls Box Plot (Right) BioSled Box Plot ................................. 275

Figure C.7.3 (Left)FP II Guardian Rolls Box Plot (Right) FP II Guardian Box Plot................ 276

Figure C.7.4 (Left)Crossmatch Seek Avenger Rolls Box Plot (Right) Crossmatch Seek Avenger

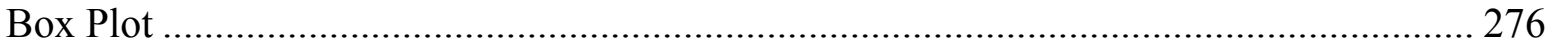

Figure C.7.5 (Left)InnerID Box Plot (Right) L1 Rolls Box Plot........................................... 277

Figure C.7.6 (Left)L1 Box Plot (Right) Morpho FOTF Box Plot ........................................ 277

Figure C.7.7 MorphoMobile Box Plots ............................................................................. 278

Figure D.1.1 NBIS ANDI Binary (a) African (b) African American (c) American Indian (d)

Asian (e) Asian Indian (f) Caucasian (g) Hispanic (h) Middle Eastern (i) Other.............. 286

Figure D.1.2 NBIS ANDI Greyscale (a) African (b) African American (c) American Indian (d)

Asian (e) Asian Indian (f) Caucasian (g) Hispanic (h) Middle Eastern (i) Other.............. 287

Figure D.1.3 NBIS BioSled (a) African (b) African American (c) American Indian (d) Asian (e)

Asian Indian (f) Caucasian (g) Hispanic (h) Middle Eastern (i) Other............................. 288

Figure D.1.4 NBIS BioSled Rolls (a) African (b) African American (c) American Indian (d)

Asian (e) Asian Indian (f) Caucasian (g) Hispanic (h) Middle Eastern (i) Other.............. 289

Figure D.1.5 NBIS FP II Guardian (a) African (b) African American (c) American Indian (d)

Asian (e) Asian Indian (f) Caucasian (g) Hispanic (h) Middle Eastern (i) Other.............. 290

Figure D.1.6 NBIS FP II Guardian Rolls (a) African (b) African American (c) American Indian

(d) Asian (e) Asian Indian (f) Caucasian (g) Hispanic (h) Middle Eastern (i) Other ......... 291

Figure D.1.7 NBIS Crossmatch Seek Avenger (a) African (b) African American (c) American

Indian (d) Asian (e) Asian Indian (f) Caucasian (g) Hispanic (h) Middle Eastern (i) Other

Figure D.1.8 Crossmatch Seek Avenger Rolls (a) African (b) African American (c) American Indian (d) Asian (e) Asian Indian (f) Caucasian (g) Hispanic (h) Middle Eastern (i) Other

Figure D.1.9 NBIS InnerID (a) African (b) African American (c) American Indian (Not

Available due to InnerID being removed from collection midway through) (d) Asian (e)

Asian Indian (f) Caucasian (g) Hispanic (h) Middle Eastern (i) Other...

Figure D.1.10 NBIS L1 (a) African (b) African American (c) American Indian (d) Asian (e)

Asian Indian (f) Caucasian (g) Hispanic (h) Middle Eastern (i) Other............................. 295

Figure D.1.11 L1 Rolls (a) African (b) African American (c) American Indian (d) Asian (e)

Asian Indian (f) Caucasian (g) Hispanic (h) Middle Eastern (i) Other............................. 296

Figure D.1.12 NBIS Morpho FOTF (a) African (b) African American (c) American Indian (d)

Asian (e) Asian Indian (f) Caucasian (g) Hispanic (h) Middle Eastern (i) Other.............. 297

Figure D.1.13 NBIS MorphoMobile (a) African (b) African American (c) American Indian (d)

Asian (e) Asian Indian (f) Caucasian (g) Hispanic (h) Middle Eastern (i) Other.............. 298

Figure D.2.1 Neurotech ANDI Binary (a) African (b) African American (c) American Indian (d)

Asian (e) Asian Indian (f) Caucasian (g) Hispanic (h) Middle Eastern (i) Other.............. 304

Figure D.2.2 Neurotech ANDI Greyscale (a) African (b) African American (c) American Indian

(d) Asian (e) Asian Indian (f) Caucasian (g) Hispanic (h) Middle Eastern (i) Other ......... 305

Figure D.2.3 Neurotech BioSled (a) African (b) African American (c) American Indian (d) Asian

(e) Asian Indian (f) Caucasian (g) Hispanic (h) Middle Eastern (i) Other ........................ 306

Figure D.2.4 Neurotech BioSled Rolls (a) African (b) African American (c) American Indian (d)

Asian (e) Asian Indian (f) Caucasian (g) Hispanic (h) Middle Eastern (i) Other.............. 307

Figure D.2.5 Neurotech FP II Guardian (a) African (b) African American (c) American Indian

(d) Asian (e) Asian Indian (f) Caucasian (g) Hispanic (h) Middle Eastern (i) Other ......... 308 
Figure D.2.6 Neurotech FP II Guardian Rolls (a) African (b) African American (c) American Indian (d) Asian (e) Asian Indian (f) Caucasian (g) Hispanic (h) Middle Eastern (i) Other

Figure D.2.7 Neurotech Crossmatch Seek Avenger (a) African (b) African American (c)

American Indian (d) Asian (e) Asian Indian (f) Caucasian (g) Hispanic (h) Middle Eastern

(i) Other.............................................................................................................. 310

Figure D.1.8 Neurotech Crossmatch Seek Avenger Rolls (a) African (b) African American (c) American Indian (d) Asian (e) Asian Indian (f) Caucasian (g) Hispanic (h) Middle Eastern

(i) Other 311

Figure D.2.9 Neurotech InnerID (a) African (b) African American (c) American Indian (d) Asian

(e) Asian Indian (f) Caucasian (g) Hispanic (h) Middle Eastern (i) Other 312

(Some Results aren't shown because of this device's low quality images and stopping operation midway through collection) 312

Figure D.2.10 Neurotech L1 (a) African (b) African American (c) American Indian (d) Asian (e)

Asian Indian (f) Caucasian (g) Hispanic (h) Middle Eastern (i) Other. 313

Figure D.2.11 Neurotech Morpho FOTF (a) African (b) African American (c) American Indian (d) Asian (e) Asian Indian (f) Caucasian (g) Hispanic (h) Middle Eastern (i) Other ......... 314 (This device had trouble capturing darker complexions and the Neurotech matcher removed images that were not up to a certain quality so that is why this device could not show distributions for the ethnicities African and African American. 314

Figure D.2.12 Neurotech MorphoMobile (a) African (b) African American (c) American Indian

(d) Asian (e) Asian Indian (f) Caucasian (g) Hispanic (h) Middle Eastern (i) Other ......... 315

Figure D.3.1 SourceAFIS ANDI Binary (a) African (b) African American (c) American Indian

(d) Asian (e) Asian Indian (f) Caucasian (g) Hispanic (h) Middle Eastern (i) Other ......... 321

Figure D.3.2 SourceAFIS ANDI Greyscale (a) African (b) African American (c) American Indian (d) Asian (e) Asian Indian (f) Caucasian (g) Hispanic (h) Middle Eastern (i) Other

Figure D.3.3 SourceAFIS BioSled (a) African (b) African American (c) American Indian (d)

Asian (e) Asian Indian (f) Caucasian (g) Hispanic (h) Middle Eastern (i) Other.

Figure D.3.4 SourceAFIS BioSled Rolls (a) African (b) African American (c) American Indian

(d) Asian (e) Asian Indian (f) Caucasian (g) Hispanic (h) Middle Eastern (i) Other ......... 324

Figure D.3.5 SourceAFIS FP II Guardian (a) African (b) African American (c) American Indian

(d) Asian (e) Asian Indian (f) Caucasian (g) Hispanic (h) Middle Eastern (i) Other ......... 325

Figure D.3.6 SourceAFIS FP II Guardian Rolls (a) African (b) African American (c) American Indian (d) Asian (e) Asian Indian (f) Caucasian (g) Hispanic (h) Middle Eastern (i) Other

Figure D.3.7 SourceAFIS Crossmatch Seek Avenger (a) African (b) African American (c)

American Indian (d) Asian (e) Asian Indian (f) Caucasian (g) Hispanic (h) Middle Eastern

(i) Other. 327

Figure D.3.8 Crossmatch Seek Avenger Rolls (a) African (b) African American (c) American Indian (d) Asian (e) Asian Indian (f) Caucasian (g) Hispanic (h) Middle Eastern (i) Other 328

Figure D.3.9 SourceAFIS InnerID (a) African (b) African American (c) American Indian (d)

Asian (e) Asian Indian (f) Caucasian (g) Hispanic (h) Middle Eastern (i) Other. 329

Figure D.3.10 SourceAFIS L1 (a) African (b) African American (c) American Indian (d) Asian

(e) Asian Indian (f) Caucasian (g) Hispanic (h) Middle Eastern (i) Other ........................... 330 
Figure D.3.11 SourceAFIS L1 Rolls (a) African (b) African American (c) American Indian (d)

Asian (e) Asian Indian (f) Caucasian (g) Hispanic (h) Middle Eastern (i) Other.............. 331

Figure D.3.12 SourceAFIS Morpho FOTF (a) African (b) African American (c) American Indian

(d) Asian (e) Asian Indian (f) Caucasian (g) Hispanic (h) Middle Eastern (i) Other ......... 332

Figure D.3.13 SourceAFIS MorphoMobile (a) African (b) African American (c) American Indian (d) Asian (e) Asian Indian (f) Caucasian (g) Hispanic (h) Middle Eastern (i) Other

Figure D.4.1 NBIS ANDI Binary Ethnicity Based ROC Curves ............................................ 334

Figure D.4.2 NBIS ANDI Greyscale Ethnicity Based ROC Curves ...................................... 335

Figure D.4.3 NBIS BioSled Ethnicity Based ROC Curves ................................................ 335

Figure D.4.4 NBIS BioSled Rolls Ethnicity Based ROC Curves .......................................... 336

Figure D.4.5 NBIS FP II Guardian Ethnicity Based ROC Curves ....................................... 336

Figure D.4.6 NBIS FP II Guardian Rolls Ethnicity Based ROC Curves................................ 337

Figure D.4.7 NBIS Crossmatch Seek Avenger Ethnicity Based ROC Curves......................... 337

Figure D.4.8 NBIS Crossmatch Seek Avenger Rolls Ethnicity Based ROC Curves ................ 338

Figure D.4.9 NBIS InnerID Ethnicity Based ROC Curves ................................................. 338

Figure D.4.10 NBIS L1 Ethnicity Based ROC Curves...................................................... 339

Figure D.4.11 NBIS L1 Rolls Ethnicity Based ROC Curves ................................................ 339

Figure D.4.12 NBIS Morpho FOTF Ethnicity Based ROC Curves ...................................... 340

Figure D.4.13 NBIS MorphoMobile Ethnicity Based ROC Curves........................................ 340

Figure D.5.1 Neurotech ANDI Binary Ethnicity Based ROC Curves .................................... 341

Figure D.5.2 Neurotech ANDI Greyscale Ethnicity Based ROC Curves............................... 342

Figure D.5.3 Neurotech BioSled Ethnicity Based ROC Curves............................................ 342

Figure D.5.4 Neurotech BioSled Rolls Ethnicity Based ROC Curves .................................... 343

Figure D.5.5 Neurotech FP II Guardian Ethnicity Based ROC Curves................................... 343

Figure D.5.6 Neurotech FP II Guardian Rolls Ethnicity Based ROC Curves .......................... 344

Figure D.5.7 Neurotech Crossmatch Seek Avenger Ethnicity Based ROC Curves .................. 344

Figure D.5.8 Neurotech Crossmatch Seek Avenger Rolls Ethnicity Based ROC Curves......... 345

Figure D.5.9 Neurotech InnerID Ethnicity Based ROC Curves ............................................ 345

Figure D.5.10 Neurotech L1 Ethnicity Based ROC Curves .................................................. 346

Figure D.5.11 Neurotech Morpho FOTF Ethnicity Based ROC Curves ................................. 346

Figure D.5.12 Neurotech MorphoMobile Ethnicity Based ROC Curves ................................. 347

Figure D.6.1 SourceAFIS ANDI Binary Ethnicity Based ROC Curves.................................. 349

Figure D.6.2 SourceAFIS ANDI Greyscale Ethnicity Based ROC Curves ............................ 349

Figure D.6.3 SourceAFIS BioSled Ethnicity Based ROC Curves......................................... 350

Figure D.6.4 SourceAFIS BioSled Rolls Ethnicity Based ROC Curves ................................ 350

Figure D.6.5 SourceAFIS FP II Guardian Ethnicity Based ROC Curves ............................... 351

Figure D.6.6 SourceAFIS FP II Guardian Rolls Ethnicity Based ROC Curves ....................... 351

Figure D.6.7 SourceAFIS Crossmatch Seek Avenger Ethnicity Based ROC Curves ............... 352

Figure D.6.8 SourceAFIS Crossmatch Seek Avenger Rolls Ethnicity Based ROC Curves....... 352

Figure D.6.9 SourceAFIS InnerID Ethnicity Based ROC Curves ......................................... 353

Figure D.6.10 SourceAFIS L1 Ethnicity Based ROC Curves ............................................. 353

Figure D.6.11 SourceAFIS L1 Rolls Ethnicity Based ROC Curves....................................... 354

Figure D.6.12 SourceAFIS Morpho FOTF Ethnicity Based ROC Curves.............................. 354

Figure D.6.13 SourceAFIS MorphoMobile Ethnicity Based ROC Curves ............................. 355

Figure D.7.1 ANDI Binary Ethnicity Based Minutiae Box Plots........................................... 356 
Figure D.7.2 ANDI Greyscale Ethnicity Based Minutiae Box Plots.................................... 357

Figure D.7.3 BioSled Ethnicity Based Minutiae Box Plots .................................................. 357

Figure D.7.4 BioSled Rolls Ethnicity Based Minutiae Box Plots .......................................... 358

Figure D.7.5 FP II Guardian Ethnicity Based Minutiae Box Plots......................................... 358

Figure D.7.6 FP II Guardian Rolls Ethnicity Based Minutiae Box Plots ................................ 359

Figure D.7.7 Crossmatch Seek Avenger Ethnicity Based Minutiae Box Plots ........................ 359

Figure D.7.8 Crossmatch Seek Avenger Rolls Ethnicity Based Minutiae Box Plots ................ 360

Figure D.7.9 InnerID Ethnicity Based Minutiae Box Plots ................................................ 360

Figure D.7.10 L1 Ethnicity Based Minutiae Box Plots ........................................................ 361

Figure D.7.11 L1 Rolls Ethnicity Based Minutiae Box Plots.............................................. 361

Figure D.7.12 Morpho FOTF Ethnicity Based Minutiae Box Plots ....................................... 362

Figure D.7.13 MorphoMobile Ethnicity Based Minutiae Box Plots ...................................... 362 


\section{List of Tables}

Table 1.1 Advantages and Disadvantages to Contact-Based Fingerprinting............................ 14

Table 1.2: Advantages and Disadvantages to Contactless Fingerprinting................................ 15

Table 1.3: Contactless Devices with the devices used in this research project highlighted in red $[1]$

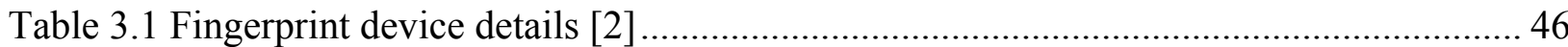

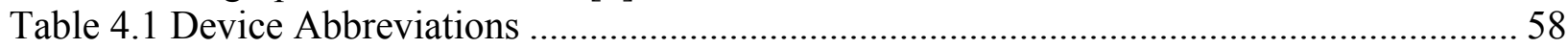

Table 4.2 ManTech Table Comparison to Thesis Results ....................................................... 58

Table 4.3 NBIS TMR1 Table............................................................................................ 60

Blue shading $=$ Contactless vs Contactless, Orange Shading $=$ Contactless vs Contact-Based,

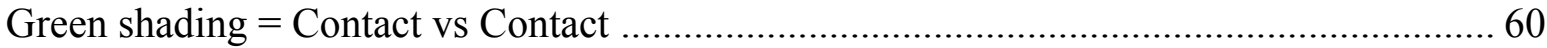

Table 4.4 Neurotech TMR1 Table .................................................................................... 61

Blue shading $=$ Contactless vs Contactless, Orange Shading $=$ Contactless vs Contact-Based,

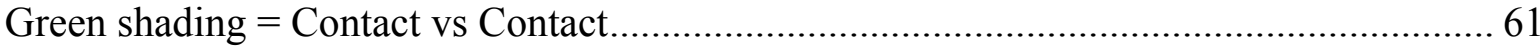

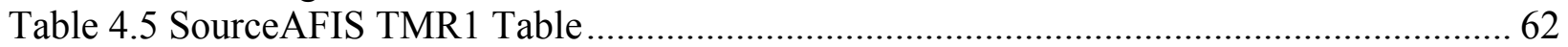

Blue shading $=$ Contactless vs Contactless, Orange Shading $=$ Contactless vs Contact-Based,

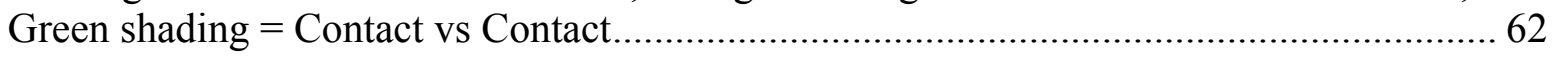

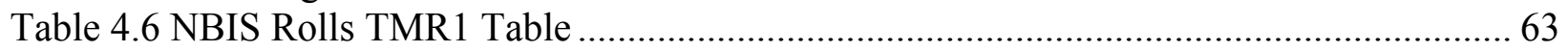

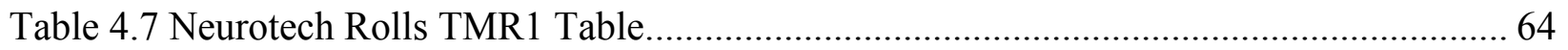

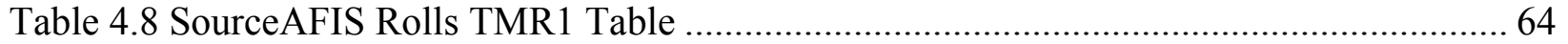

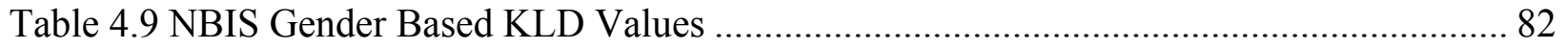

Table 4.10 Neurotech Gender Based KLD Values ................................................................... 82

Table 4.11 SourceAFIS Gender Based KLD Values ......................................................... 83

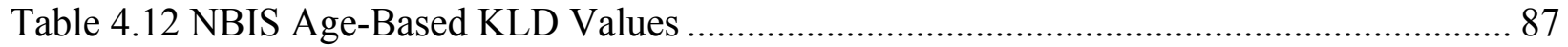

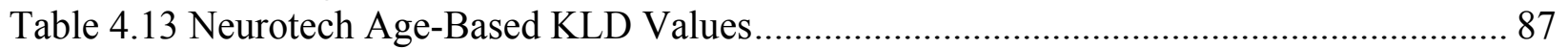

Table 4.14 SourceAFIS Age-Based KLD Values................................................................ 88

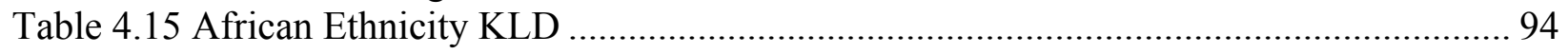

Table 4.16 African Ethnicity KLD ............................................................................... 94

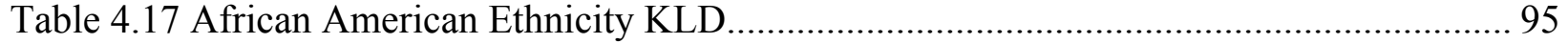

Table 4.18 American Indian Ethnicity KLD ..................................................................... 96

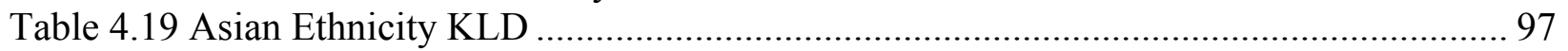

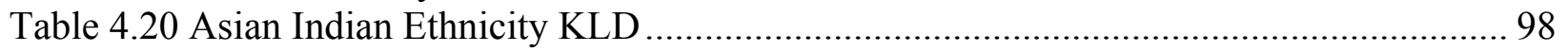

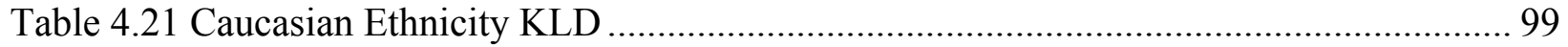

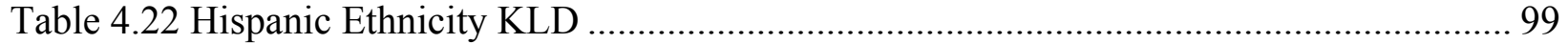

Table 4.23 Middle Eastern Ethnicity KLD ......................................................................... 100

Table 4.24 Other Ethnicity KLD .................................................................................... 100

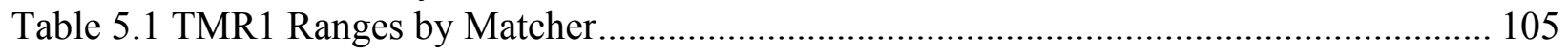

Table 5.2 Range of TMR1 Rates between Matchers ….................................................... 106

Table 5.3 Equal Error Rates for Each Matching Run ...................................................... 108

Table 5.4 Equal Error Rates For Each Device ................................................................... 109

Table 5.5 Equal Error Rates For Each Rolled Matching Run................................................ 109

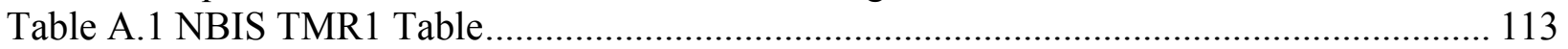

Table A.2 Neurotech TMR1 Table ................................................................................... 114

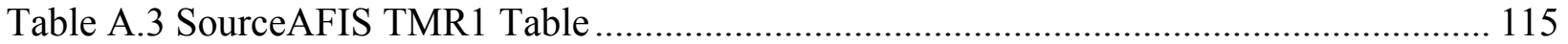

Table A.4 NBIS Rolls TMR1 Table ............................................................................... 116 


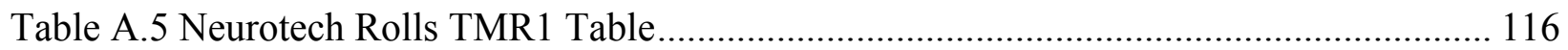

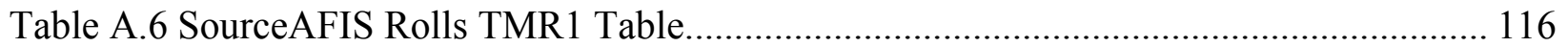

Table A.7 NBIS Maximum and Minimum Score Values........................................................ 117

Table A.8 Neurotech Maximum and Minimum Score Values ..................................................... 120

Table A.9 SourceAFIS Maximum and Minimum Score Values ............................................. 123

Table A.10 NBIS Cross-Examined Maximum and Minimum Score Values ............................... 127

Table A.11 Neurotech Cross-Examined Maximum and Minimum Score Values....................... 137

Table A.12 SourceAFIS Cross-Examined Maximum and Minimum Score Values .................. 147

Table A.13 NBIS Area Under the Curve for ROC graphs. .................................................. 158

Table A.14 Neurotech Area Under the Curve for ROC graphs................................................. 164

Table A.15 SourceAFIS Area Under the Curve for ROC graphs............................................. 169

Table A.16 Equal Error Rates for Each Matching Run .......................................................... 174

Table A.17 Equal Error Rates For Each Device ………...................................................... 175

Table A.18 Equal Error Rates For Each Rolled Matching Run............................................ 175

Table B.1 NBIS Male Genuine and Imposter KLD, Minimum and Maximum Scores............... 178

Table B.2 NBIS Female Genuine and Imposter KLD, Minimum and Maximum Scores .......... 179

Table B.3 Neurotech Male Genuine and Imposter KLD, Minimum and Maximum Scores ...... 184

Table B.4 Neurotech Female Genuine and Imposter KLD, Minimum and Maximum Scores .. 184

Table B.5 SourceAFIS Male Genuine and Imposter KLD, Minimum and Maximum Scores ... 189

Table B.6 SourceAFIS Female Genuine and Imposter KLD, Minimum and Maximum Scores 189

Table B.7 NBIS Area Under the Curve for ROC Plots .......................................................... 194

Table B.8 Neurotech Area Under the Curve for ROC Plots....................................................... 199

Table B.9 SourceAFIS Area Under the Curve for ROC Plots...................................................... 203

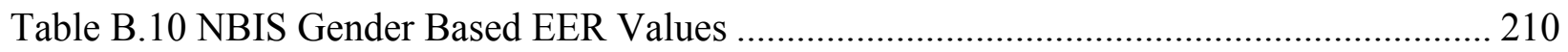

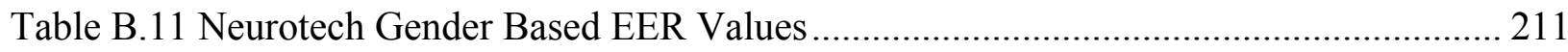

Table B.12 SourceAFIS Gender Based EER Values............................................................... 211

Table C.1 NBIS 18-19 Genuine and Imposter KLD, Minimum and Maximum Scores ............. 213

Table C.2 NBIS 20-29 Genuine and Imposter KLD, Minimum and Maximum Scores ............. 214

Table C.3 NBIS 30-39 Genuine and Imposter KLD, Minimum and Maximum Scores ............ 214

Table C.4 NBIS 40 and up Genuine and Imposter KLD, Minimum and Maximum Scores ...... 215

Table C.5 Neurotech 18-19 Genuine and Imposter KLD, Minimum and Maximum Scores ..... 229

Table C.6 Neurotech 20-29 Genuine and Imposter KLD, Minimum and Maximum Scores..... 229

Table C.7 NBIS 30-39 Genuine and Imposter KLD, Minimum and Maximum Scores ............ 230

Table C. 8 NBIS 40 and up Genuine and Imposter KLD, Minimum and Maximum Scores ...... 230

Table C.9 SourceAFIS 18-19 Genuine and Imposter KLD, Minimum and Maximum Scores.. 243

Table C.10 SourceAFIS 20-29 Genuine and Imposter KLD, Minimum and Maximum Scores 243

Table C.11 SourceAFIS 30-39 Genuine and Imposter KLD, Minimum and Maximum Scores 244

Table C.12 SourceAFIS 40 and up Genuine and Imposter KLD, Minimum and Maximum Scores

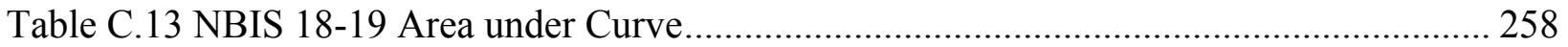

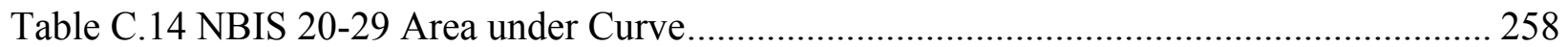

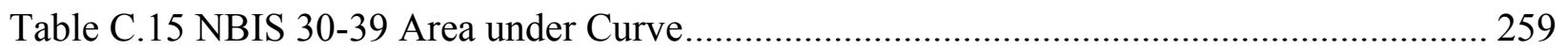

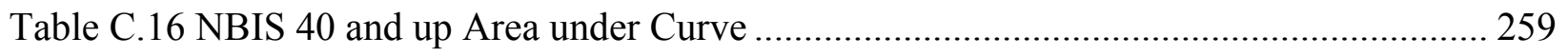

Table C.17 Neurotech 18-19 Area under Curve ………............................................................ 263

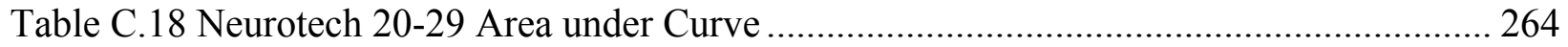

Table C.19 Neurotech 30-39 Area under Curve …………....................................................... 264 
Table C.20 Neurotech 40 and up Area under Curve......................................................... 265

Table C.21 SourceAFIS 18-19 Area under Curve ................................................................... 269

Table C.22 SourceAFIS 20-29 Area under Curve ............................................................... 269

Table C.23 SourceAFIS 30-39 Area under Curve ............................................................. 270

Table C.24 SourceAFIS 40 and up Area under Curve........................................................ 270

Table C.25 NBIS Equal Error Rates For Age Based Study.................................................. 278

Table C.26 Neurotech Equal Error Rates For Age Based Study ........................................... 279

Table C.27 SourceAFIS Equal Error Rates For Age Based Study ........................................ 279

Table D.1 NBIS African Genuine and Imposter KLD, Maximum and Minimum Scores ........ 281

Table D.2 NBIS African American Genuine and Imposter KLD, Maximum and Minimum Scores 282

Table D.3 NBIS American Indian Genuine and Imposter KLD, Maximum and Minimum Scores

Table D.4 NBIS Asian Genuine and Imposter KLD, Maximum and Minimum Scores

Table D.5 NBIS Asian Indian Genuine and Imposter KLD, Maximum and Minimum Scores . 283

Table D.6 NBIS Caucasian Genuine and Imposter KLD, Maximum and Minimum Scores .... 284

Table D.7 NBIS Hispanic Genuine and Imposter KLD, Maximum and Minimum Scores ....... 284

Table D.8 NBIS Middle Eastern Genuine and Imposter KLD, Maximum and Minimum Scores

Table D 9 NBIS Other Genuine and Imposter KID, Maximum and Minimum Scores

Table D.10 Neurotech African Genuine and Imposter KLD, Maximum and Minimum Scores 299

Table D.11 Neurotech African American Genuine and Imposter KLD, Maximum and Minimum

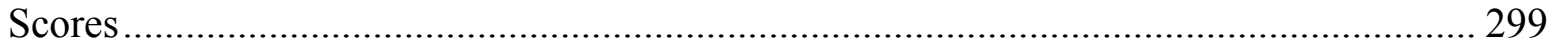

Table D.12 Neurotech American Indian Genuine and Imposter KLD, Maximum and Minimum

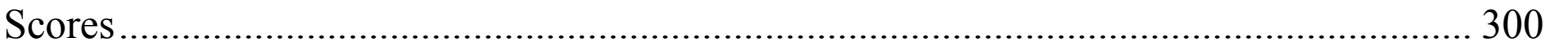

Table D.13 Neurotech Asian Genuine and Imposter KLD, Maximum and Minimum Scores... 300

Table D.14 Neurotech Asian Indian Genuine and Imposter KLD, Maximum and Minimum

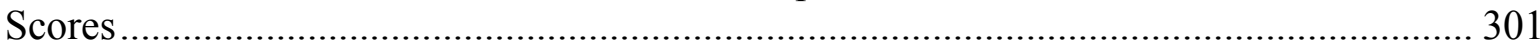

Table D.15 Neurotech Caucasian Genuine and Imposter KLD, Maximum and Minimum Scores

Table D.16 Neurotech Hispanic Genuine and Imposter KLD, Maximum and Minimum Scores 301

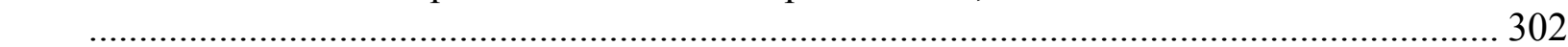

Table D.17 Neurotech Middle Eastern Genuine and Imposter KLD, Maximum and Minimum

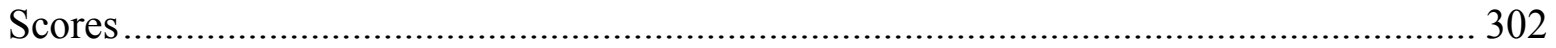

Table D.18 Neurotech Other Genuine and Imposter KLD, Maximum and Minimum Scores ... 303

Table D.19 SourceAFIS African Genuine and Imposter KLD, Maximum and Minimum Scores

Table D.20 SourceAFIS African American Genuine and Imposter KLD, Maximum and

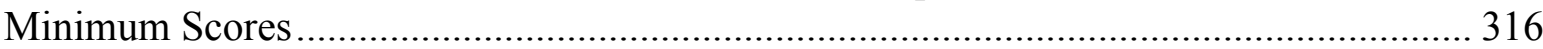

Table D.21 SourceAFIS American Indian Genuine and Imposter KLD, Maximum and Minimum

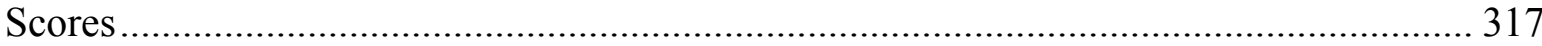

Table D.22 SourceAFIS Asian Genuine and Imposter KLD, Maximum and Minimum Scores 317

Table D.23 SourceAFIS Asian Indian Genuine and Imposter KLD, Maximum and Minimum

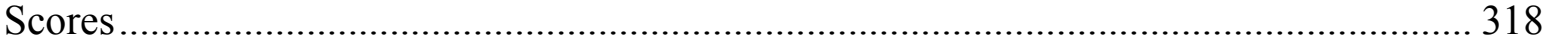

Table D.24 SourceAFIS Caucasian Genuine and Imposter KLD, Maximum and Minimum

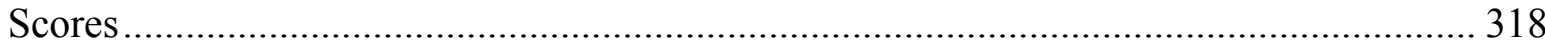


Table D.25 SourceAFIS Hispanic Genuine and Imposter KLD, Maximum and Minimum Scores

Table D.26 SourceAFIS Middle Eastern Genuine and Imposter KLD, Maximum and Minimum

Scores. 319

Table D.27 SourceAFIS Other Genuine and Imposter KLD, Maximum and Minimum Scores 320

Table D.28 NBIS Area Under Curve for Each Ethnicity

Table D.29 Neurotech Area Under Curve for Each Ethnicity

341

Table D.30 SourceAFIS Area Under Curve for Each Ethnicity..... 348 
Chapter 1:

Introduction 


\subsection{Executive Summary}

Fingerprints have been used as a biometric identifier for decades due to a high degree of uniqueness, even among large populations. Fingerprint technologies have evolved significantly since the early days of fingerprinting. Starting with wet-ink ten-print cards, to the digitizing of these cards, then livescan devices, arriving at the present situation of contactless fingerprinting devices, fingerprinting has developed consistently to become one of the most used forms of identification. The two most widely used methods of collecting fingerprints are wet-ink and livescan devices. The wet-ink technique requires the subject to cover their fingers in ink with a trained individual subsequently pressing and rolling their prints onto a ten-print card. Livescan devices omit the use of ink and other materials, requiring the user to roll and press their fingers against a platen to acquire their fingerprint information. Both of these techniques have drawbacks inherent to the contact-based nature of these methods.

Although the fingerprint scanners that are primarily used in civil, law enforcement and military applications are mature technologies, there still exists challenges in operational scenarios, including contamination from previous user, inconsistencies due to operator error, low throughput, distortion due to uncontrollable pressure used by individuals, and inability to capture prints from decomposed flesh of deceased individuals. These issues can be drastically reduced or eliminated by the use of non-contact fingerprinting devices. Contactless fingerprinting devices allow for forensics analysts to be able to capture the prints from deceased individuals without the possibility of decomposed flesh being disturbed. Contactless devices can potentially offer equal or higher quality images (compared to traditional contact-based livescan), faster capture times, less operator training, reduced likelihood of the spread of contaminants, and unattended fingerprint collection. 
Contactless devices still have some issues that need to be addressed before they can be widely used. Standardization of these technologies pose one of the biggest challenges that face contactless devices. Standardizing these devices comes with a high level of difficulty due to the many ways that a print can be captured without contact. Proving that contactless devices are fully backwards compatible with the images produced by previous contact-based devices or methods is extremely crucial to the advancement of these technologies. NIST is leading efforts in standardizing these kinds of devices by creating the CRADA program, also known as the Contactless Fingerprint Capture Device Measurement Research Program, which builds upon the NIST's research in developing methodologies for measuring the image fidelity of contactless fingerprint capture devices. Another important factor in creating contactless technology is the presence of interoperability challenges, including edge detection, lack of deformation, and threedimensional image unwrapping. Additionally, the technology involved in contactless imaging generally has a higher cost than well-established contact-based sensors.

The goal of the research described herein is to investigate the interoperability and matching performance of contact and contactless fingerprint scanners. This data used in this research project was collected in Phase 2 of a Non-Contact Fingerprint Evaluation project sponsored by ManTech International Corp. and the U.S. Dept. of Justice. The collection took place between December 2014 and April 2015 with 450 participants providing data (IRB \#1309097716). Rolled-ink fingerprint cards were collected, as well as commercial and prototype contactless and contact-based fingerprint devices. For this research effort, fingerprint data was evaluated from wet-ink ten-print cards and traditional contact-based legacy fingerprint devices versus fingerprint data generated by next-generation contactless fingerprint scanners. The feasibility and performance of contactless fingerprint databases was investigated. To investigate 
the matching performance and interoperability of these devices three different matching algorithms were utilized, including Neurotechnology MegaMatcher, open source NIST Biometric Image Software (NBIS) and open source fingerprint recognition toolkit by SourceAFIS. Matching runs were performed on the right index finger and thumb data from each device against themselves and all other devices, as well as the 500 dpi scans from the wet-ink fingerprint cards. Matching runs were also evaluated for genuine and imposter match score distributions. The stratifications of gender, ethnicity, and age for contactless and contact-based devices was also investigated. Based on this snapshot during the development of contactless fingerprints, Rank1 accuracy is between 74.33 and 98.68 percent with contact based methods. The Rank 1 accuracy is between 74.66 and 97.27 percent with other contactless devices.

\subsection{Contactless Fingerprinting}

Quickly identifying a person by waving their hand in front of a scanner could seem futuristic, but this is exactly what contactless fingerprinting devices do. Contactless fingerprinting devices can capture all fingers on one hand in one quick motion instead of the minutes that it can take on some traditional contact-based scanners or wet-ink cards. Non-contact fingerprinting is quite self-explanatory as it is capturing images of fingerprints without making contact with a device or surface. This capture can be accomplished in many ways with many different cameras and techniques. Contactless fingerprinting technology aims to speed up the process of authorizing access through high-traffic areas such as border controls, airports, and access control points. 


\subsection{Methods of fingerprinting}

There are currently two other widely used techniques to acquire fingerprint images, wetink and livescan. The wet-ink method requires a subject to cover their fingers in ink with an individual/operator to aid them in pressing and rolling their prints onto a ten-print fingerprint card. This method requires materials to be replaced by the operator, and clean-up for both the subject and operator. The issues of clean-up and materials are addressed by livescan devices but these sensors still require an individual to press or roll their fingers against a platen to acquire the fingerprint information. Livescan technologies mimic the method of wet-ink ten-print cards, producing flat images of the three-dimensional finger [1]. An example of a livescan capture is shown in Figure 1.3. Both of these techniques create distortions of the three-dimensional fingerprint, whereas contactless devices create an image without any distortions due to pressure or other contact-based factors. 


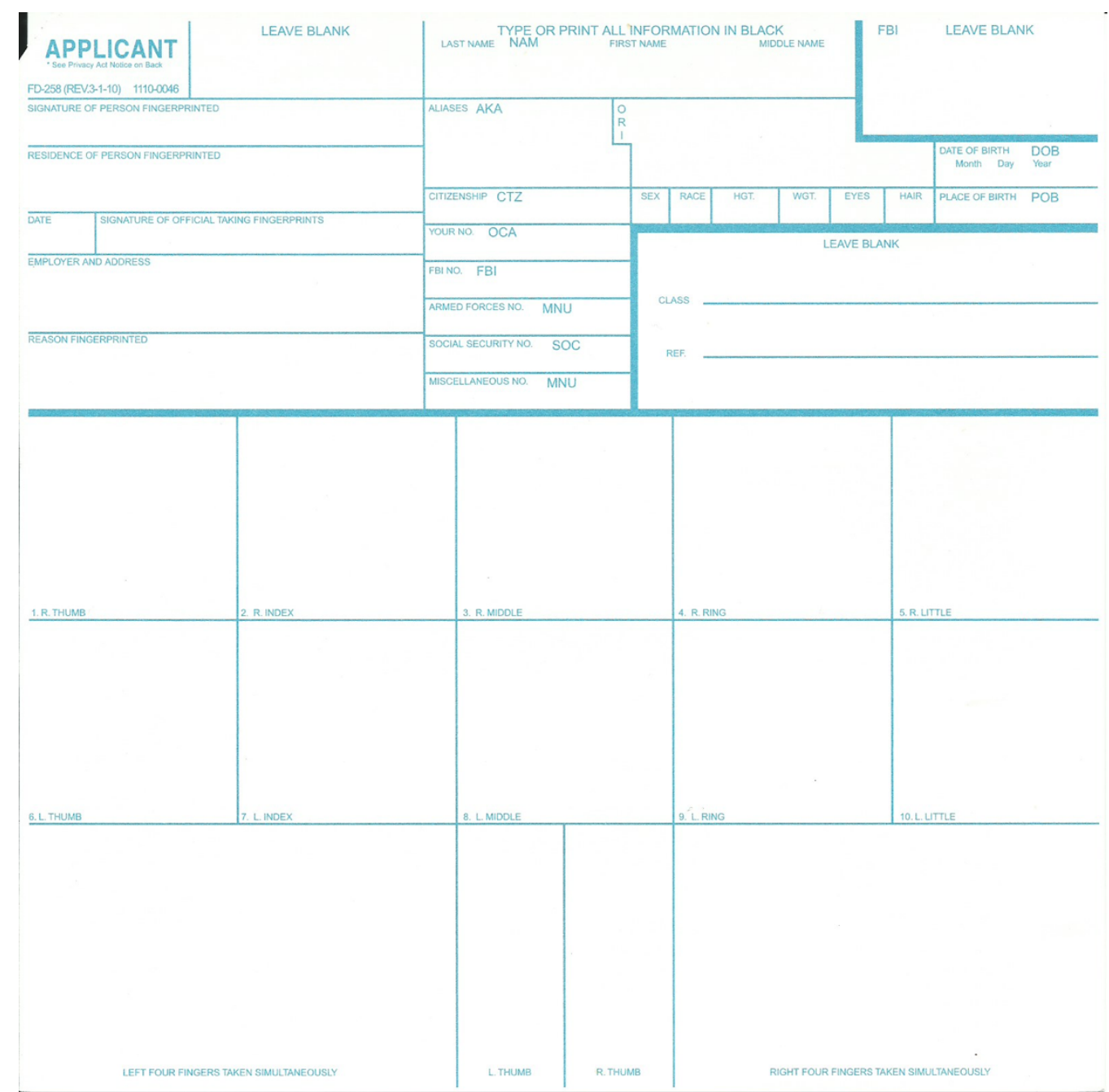

Figure 1.1 Blank Ten-print Card 


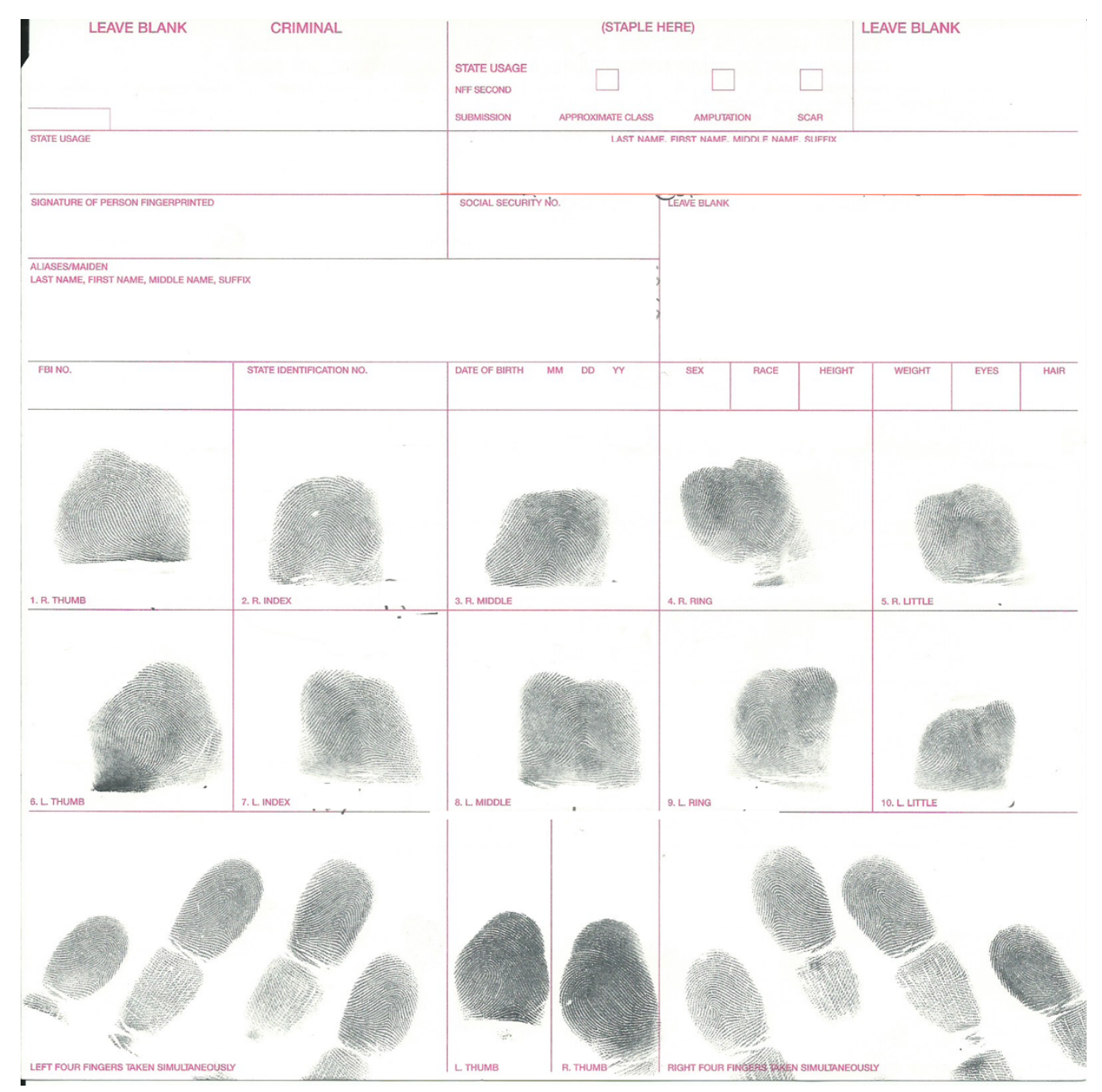

Figure 1.2 Ten print card with fingerprints 


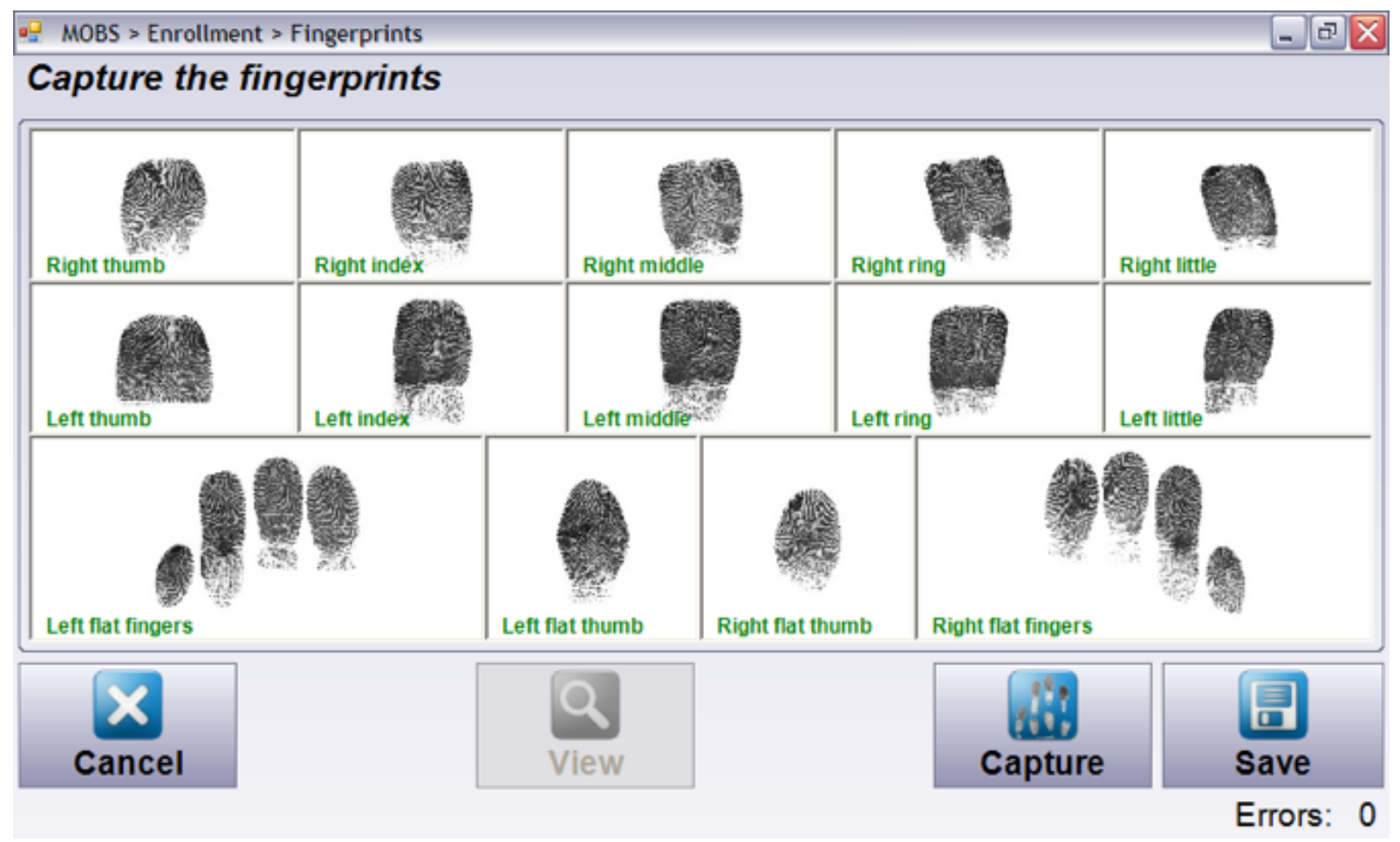

Figure 1.3 Example of Livescan Fingerprint Capture [2]

\subsection{Hurdles in Fingerprint Identification}

Fingerprint Identification has overcome numerous hurdles to get to the mature level of technology that we have now. The first standardized way to identify persons by their fingerprints was through wet-ink ten-print cards. These, as stated above are messy, and require properly trained personnel to accurately collect fingerprint images that are not smudged or blurred. These physical cards were sufficient until other agencies outside of the FBI needed them, which brought about the need for digitization of these cards into electronic fingerprint transmission files or EFTs, to easily share these files between different agencies or law enforcement offices.

Digitization of these cards refers to the scanning of ten-print cards with FBI certified scanners to meet the required compliance with the FBI's EFTS IQS (Electronic Fingerprint Transmission Specification - Image Quality Specification) [3]. EFTS IQS requires a standard to be met when working with ten-print cards so that different agencies can share and utilize files. After 
successful adaption of standards and digitization of ten-print cards, livescan devices were developed which acquire fingerprints digitally to eliminate the process of scanning ten-print cards in to digitize them. Livescan devices make for a much quicker transaction with less cleanup and materials than wet ink prints, but these devices still create distortions due to pressure against a flat surface. Livescan devices can employ a number of sensing mechanisms, including but not limited to: optical, capacitive, thermal, pressure-based, and ultrasound, although some of these methods do not provide images that contain the same representation of detail necessary for some latent fingerprint comparisons [4]. These devices are still being used today in the majority of establishments, but technology has advanced favorably whereby devices can be created that can acquire fingerprints without making any contact with the hand, eliminating distortion from pressure. The final hurdle to address is the issue of interoperability between devices of contactbased nature with contactless fingerprinting devices. Interoperability is the characteristic of a device or system to communicate, exchange data, and use the information that has been exchanged [5]. For this effort, interoperability is a term used to show that images of the same subject's print from contactless and contact-based devices are comparable and will produce a match.

\subsection{Fingerprint Recognition}

Fingerprint recognition or fingerprint authentication is the procedure of verifying a match between two fingerprints. There are three different styles of fingerprint matching: pattern-based (or Image-based) matching, correlation based matching, and minutiae matching. Pattern-based matching compares the similarities of two images, often used for determining if there are duplicates. Correlation based matching involves aligning two fingerprint images and computing the correlation for each corresponding pixel, for all possible alignments [6]. Minutiae matching 
is primarily based on the comparison of features of the fingerprint minutiae pattern location and direction. The three basic fingerprint patterns that happen in fingerprint ridges are an arch, loop, and whorl, loops are sometimes classified by right or left [7]. An arch is a pattern where the ridges enter from one side of the finger, rise in the center forming an arc, and then exit the other side of the finger [8] [9]. A loop is where the ridges enter from one side of the finger, form a curve and then exit on the same side [8] [9]. A whorl is when ridges form circularly around a central point on the finger. [8] [9]

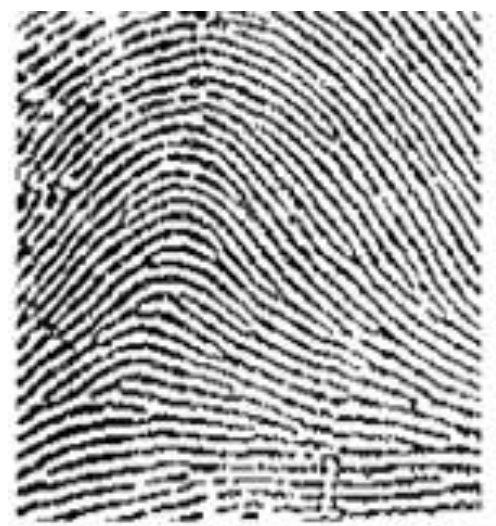

Arch

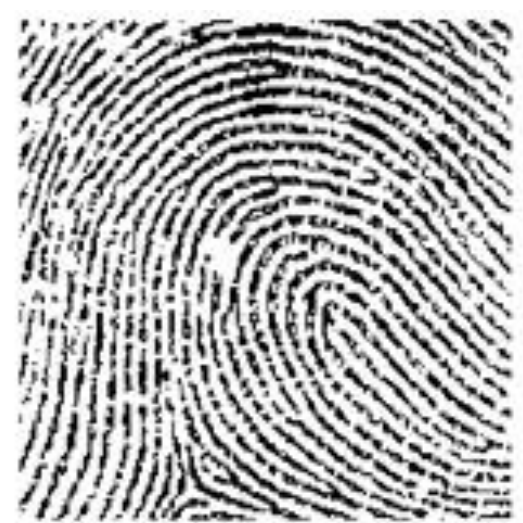

Loop

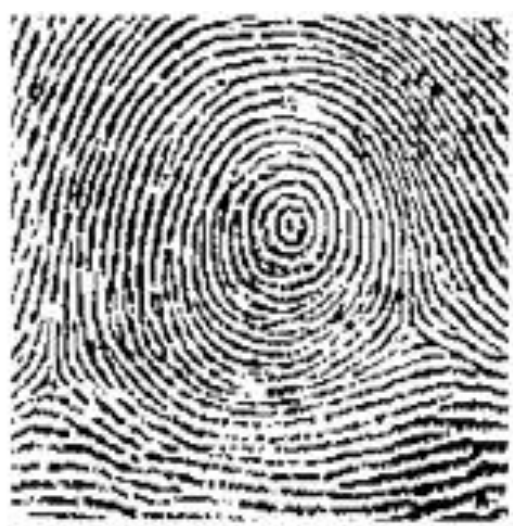

Whorl

Figure 1.4: Ridge Patterns [9]

An AFIS, or Automated Fingerprint Identification System, is designed to interpret the flow of overall ridges to assign a fingerprint classification and then extract the minutiae detail [10]. The detail extracted is a subset of the total amount of information available, but enough information to search a large repository effectively [11]. AFIS systems are the primary identification tool for most law enforcement agencies in the United States and the rest of the world [10]. 


\subsection{Minutiae}

An important feature of fingerprints are minutiae. Minutiae are the major features of fingerprints that are used to compare one fingerprint to another. The different types of minutiae include core, delta, crossover or bridge, spur, ridge enclosure, island, short ridge, ridge bifurcation, and ridge ending. Minutiae are the basis upon which the matching template is formed. The precise locations of the minutiae are recorded as numerical spatial ( $\mathrm{x}$ and $\mathrm{y}$ ) coordinates, for each fingerprint image. The orientation of the minutiae are also recorded as vector angles which denote the direction of each minutia characteristic (shown in Figure 1.6). It is important to note that minutiae identified by human fingerprint examiners can vary from those identified by an automated system [12].
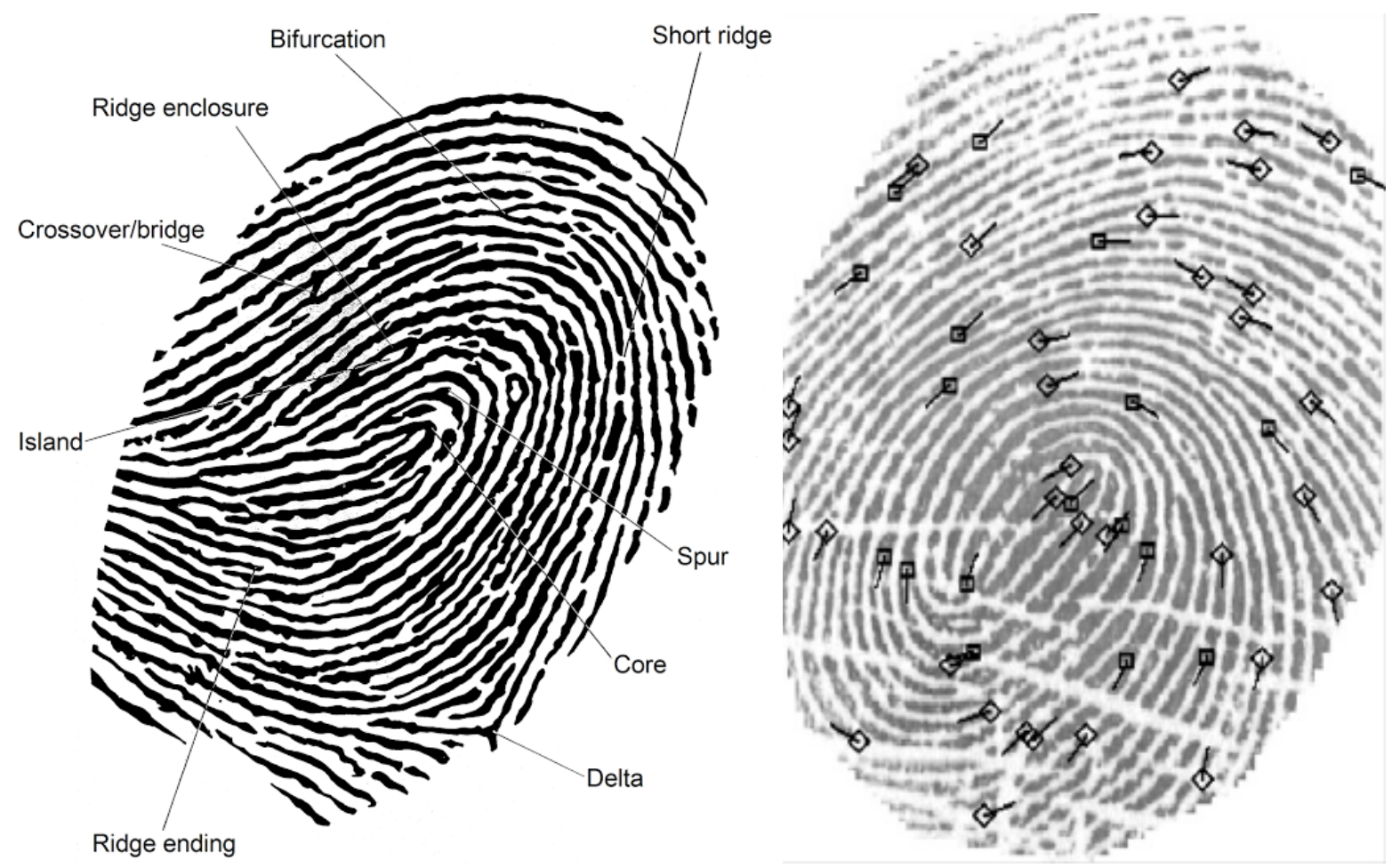

Figure 1.5: (Left)Fingerprint Minutiae types [13] (Right) Fingerprint minutiae marked by location and orientation [14] 


\subsection{Advantages and Disadvantages of Contact and Contactless}

\section{Fingerprinting}

Traditional fingerprint devices and wet-ink ten-print cards, though being implemented and used for decades, still have many disadvantages which were the motivation for the development of contactless sensors. Wet-ink ten-print cards require operators to have a moderate amount of training and even with training, the same subject's prints can come out completely different in two different collection attempts. Different operators can change the outcome as well. With the contact-based style of traditional scanners, hygiene can be a major issue [15] [16] [17]. With many hands all touching one scanner, it's practically impossible to keep the scanner clean and disinfected while still moving subjects through in a timely manner. To the same degree, oils can be left on the scanning surface, which can distort the next subject's prints [15] [16] [18] [19]. Additionally, in applications where fingerprint scanners are used, such as border controls and airports, diseases and/or viruses could potentially contaminate scanners and spread quickly with every person passing through touching this device. The next biggest issue is that the contact made and pressure used by the subject is uncontrollable [16] [20] [21] [22]. Since pressure used is uncontrollable, prints from the same person captured at two different times can look completely different [23]. Some traditional scanners cannot collect fingers that are extremely dry, wrinkly, or very scarred and worn fingers [15] [22] [24]. The elasticity of skin is also uncontrollable with temperature, hydration and genetics, changing the elasticity of each subject's skin [25] [26]. Contact-based scanners can be an inconvenience to capture images from a forensics standpoint when it comes to skin degradation Contactless fingerprinting devices allow for forensics analysts to be able to capture the prints from deceased individuals without the possibility of decomposed flesh being disturbed. Another problem with contact-based devices is 
the operator needing to invade the subject's personal space to show or help them roll their prints. Along with this issue arises the problem of training operators. Training operators for contactless devices is very minimal compared to contact-based devices. All the issues discussed above can be solved or at least improved with non-contact fingerprint devices. Additionally, contactless devices capture equal or higher quality images (compared to traditional contact-based livescan), with faster capture times. Some contactless devices do not require a human operator at all and they drastically reduce spread of contaminants.

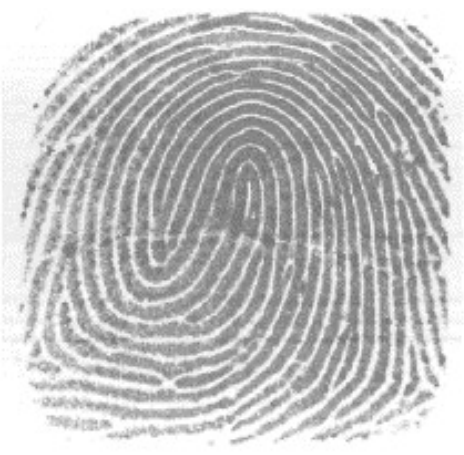

(a)

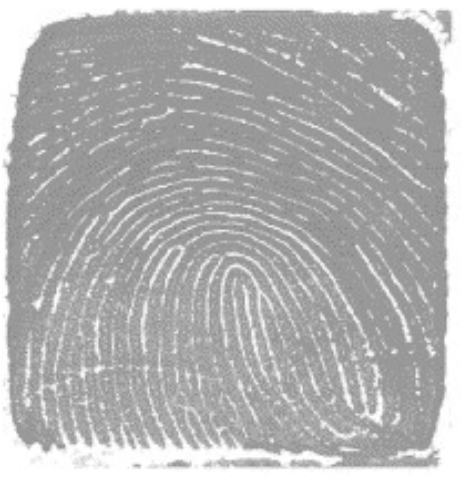

(c)

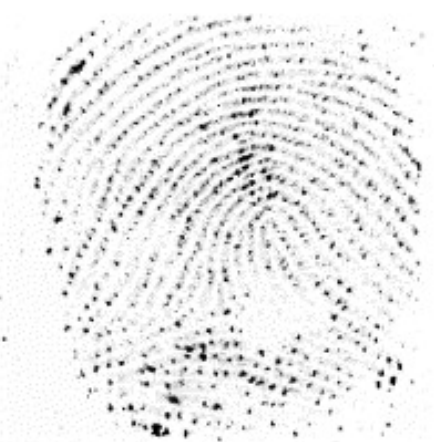

(b)

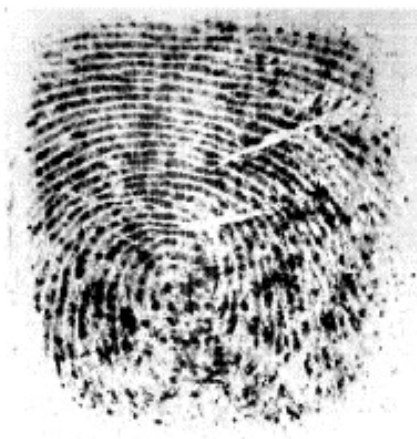

(d)

Figure 1.6: Images of various finger conditions: (a) normal finger; (b) dry finger; (c) wet finger; (d) poor quality finger. [27] 
Table 1.1 Advantages and Disadvantages to Contact-Based Fingerprinting Advantages and Disadvantages to Contact-Based Fingerprinting

\begin{tabular}{|c|c|}
\hline Advantages & Disadvantages \\
\hline $\begin{array}{l}\text { Technology is cheaper than that of contactless } \\
\text { devices }\end{array}$ & Operator Training Required \\
\hline $\begin{array}{l}\text { Images created have deformation, increasing } \\
\text { the compatibility with databases already in } \\
\text { use. }\end{array}$ & $\begin{array}{l}\text { Inability to capture dry, wrinkly, worn, and } \\
\text { scarred prints }\end{array}$ \\
\hline \multirow[t]{5}{*}{ Mature Technologies } & Clean up required for each participant \\
\hline & Materials Needed (Ten-prints) \\
\hline & Uncontrollable Amount of Pressure \\
\hline & Residue/Contamination from prior subjects \\
\hline & Inability or difficulty capturing forensics data \\
\hline
\end{tabular}

\subsection{Challenges involved with Contactless Fingerprint technology}

Contactless fingerprint scanners are significantly different from contact-based scanners.

Therefore, images produced by contactless devices are fundamentally different from conventional scanned wet-ink ten-print cards and livescan fingerprints, which creates the problem of recognition between devices and prints. The technology needed to produce contactless devices tends to be costly. Though contactless scanners capture true un-deformed images of the finger, it's a detriment because no existing livescan or touch-based fingerprint database is absent of this deformation, creating perhaps the biggest challenge for interoperability [20] [28]. All things equal (cost being irrelevant for comparison), in a 1-to-1 comparison, same resolution, contrast, and quality, an image from a contactless device will never have the deformation that a contact-based image will, which poses the issue of interoperability [29]. A possible fix to this problem could be a measurement/estimation of deformation due to contact [30]. Images created by contactless devices must be backwards compatible with the deformed images created by contact-based fingerprinting. Sometimes the images created by contactless 
devices can be three-dimensional. If so, an algorithm must be applied that unwraps the threedimensional image into a two-dimensional image for compatibility [20].

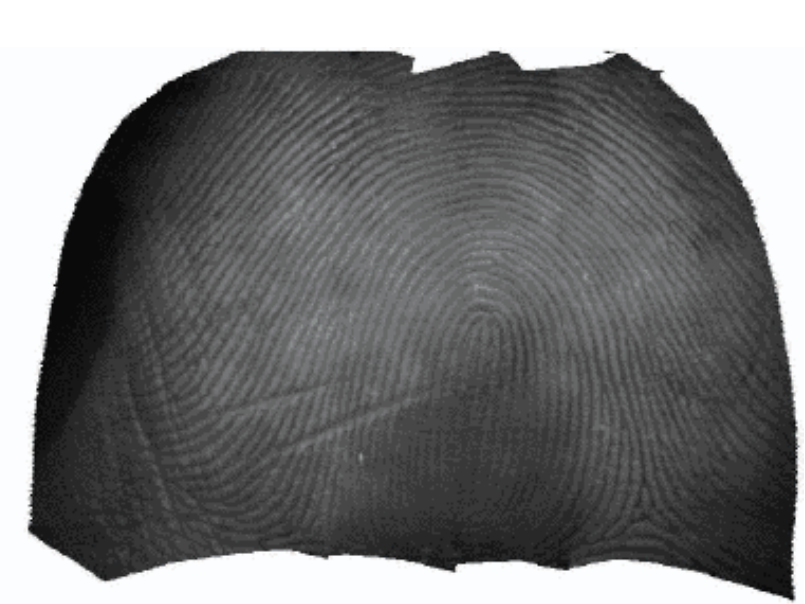

(a)

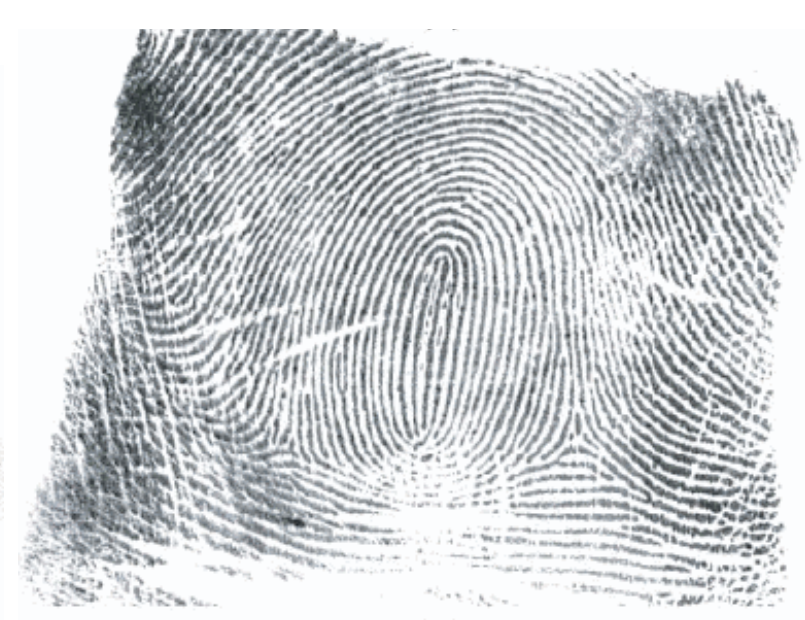

(b)

Figure 1.7: Visualizing compatibility between (a) a touchless fingerprint from line-scan sensor using the proposed nonparametric unwrapping (b) the corresponding ink-on-paper rolled fingerprint. [31]

Table 1.2: Advantages and Disadvantages to Contactless Fingerprinting

\begin{tabular}{|l|l|}
\hline \multicolumn{1}{|c|}{ Advantages and Disadvantages to Contactless Fingerprinting } \\
\hline Advantages & Disadvantages \\
\hline Hygienically Sound & Lack of Deformation \\
\hline Operator Training reduced & Cost \\
\hline Ability to capture forensics data & 3D Image Unwrapping \\
\hline Pressure is irrelevant & Edge Detection \\
\hline $\begin{array}{l}\text { Ability to capture dry, wrinkled, } \\
\text { scarred/worn fingers }\end{array}$ & \\
\hline Residue obscuring images is irrelevant & \\
\hline Reduces Spread of Contaminants & \\
\hline
\end{tabular}

\subsection{Modulation Transfer Function}

A major component that must be considered occurs in modern methods of fingerprinting is the modulation transfer function of the device (or scanner for wet-ink ten-print cards). Modulation transfer function, or MTF, is used to describe the performance of a camera lens, scanning sensor, or any transfer of information from one point to another through a system in 
terms of imaging performance. It is a measure of the transfer of modulation (or contrast) from the subject to the image that is produced. In other words, it measures how faithfully the lens reproduces detail from the object to the captured image. MITRE developed the MTF application to support performance analysis of fingerprint capture devices and printers for the FBI's Integrated Automated Fingerprint Identification System (IAFIS). MITRE's MTF application is used to compute the modulation transfer function of an imaging system [32]. The general modulation transfer function that applies to several contact-based devices is shown below. This equation is generated from multiplying the MTF of the Lens, CCD image sensor MTF and the Electronic processing MTF to get the MTF of the whole system [33]. Coming from the Equation 1.1, the MTF of digital wet-ink ten-print cards is solely based on the MTF of the scanner or the electronic processing MTF. Using this same equation, the MTF of contact-based scanners can be found by multiplying, the electronic processing MTF and the MTF of the CCD image sensor. The MTF of contactless fingerprinting devices would be developed with every variable of equation 1.1.

\section{$M T F=M T F_{L} \bullet M T F_{C C D} \bullet M T F_{e}$}

[33] Equation 1.1

\subsection{Standardization of Contactless Devices}

With the development of new fingerprint scanners and databases arose a need for standards for fingerprint devices and the images that they produce. Since the different devices that are available all use different techniques to capture fingerprint images, standardization is critical. Additionally, all the images produced are different, considering that some devices can create a two-dimensional image and others, a three-dimensional image. Even within the 
collection of devices used to capture the data used for this research, there were many different techniques including: moving and fixed structured light illumination, digital camera images, polarized reflected light, composite digital camera images and line scanning optical cameras. Considering all these different techniques and their capability to produce different types of images, standards must be created for compatibility between all devices.

NIST headed up the effort of developing standards for these types of devices. In January of 2015, The National Institute of Standards and Technology (NIST) developed a research program for research in developing methodology for measuring the image fidelity of contactless fingerprint capturing devices. This program is called the Contactless Fingerprint Capture Device Measurement Research Program, or CRADA, and focuses on producing open testing methods, metrics, and artifacts that will support the future certification of devices for inclusion on the Government Certified Products Lists [34]. In this context, artifacts refer to an object that can be used for testing fingerprint devices, with known geometric attributes, employed for comparison with a captured image to establish fidelity and repeatability, and measure error [35]. Participants of this program are requested to provide their contactless fingerprint devices, software and nonproprietary documentation, on loan, to NIST for testing [34]. Each device must be able to sense contactless fingerprints reliably in a contactless manner and produce resulting images in a standard or open file format. NIST will test the devices with controlled artifacts and will measure/analyze properties of the images captured. [34]

Most recently in March of 2017, NIST published the "Contactless Fingerprint Devices Usability Test" [36]. This study deals with the usability of contactless and contact-based devices, looking specifically into the efficiency, effectiveness and user satisfaction of each device. This study is similar to our own, as they also look into the similarity scores for each device. 


\subsection{Contactless Versus Touchless}

"Contactless fingerprinting" has different meanings within the biometrics industry. "Contactless" can refer solely to the sensor of a device or the entirety of the device or system. Contactless devices can have a varying level of contact, where the subject may have part of their hand or finger resting on a surface in order for the sensor to capture the remaining part of the hand or finger in a non-contact still manner. If the whole device is contactless, then there is absolutely no contact made with any surface or sensor. These devices can be referred to as touchless. Figure 1.9 shows the difference between contactless and touchless devices. Table 2 provides a list of current contactless technologies [1]. The three contactless devices that were used to collect the data evaluated in in this research project are touchless devices. Shown below is a table of current contactless technologies (Table 2) as well as pictures of some of these devices in Figure 1.10.
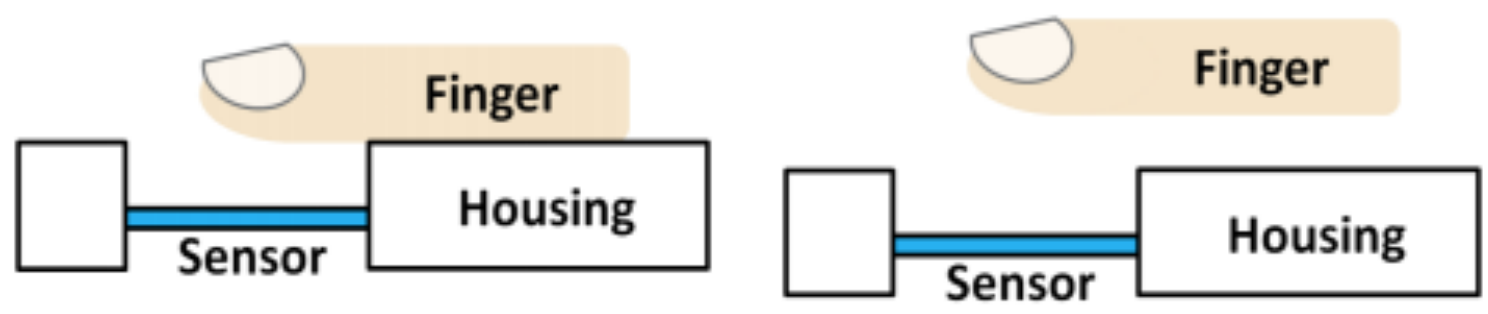

Figure 1.8 Difference between contactless (left) and touchless (right) [1] 
Table 1.3: Contactless Devices with the devices used in this research project highlighted in red [1].

\begin{tabular}{|c|c|c|c|c|}
\hline Device & Scanning Technique & Scanning Resolution & Touchless & Maturity Level \\
\hline $\begin{array}{l}\text { Advanced Optical Systems Inc. } \\
\text { (AOS) AIRprint [37] }\end{array}$ & $\begin{array}{l}\text { Polarized Reflected } \\
\text { light }\end{array}$ & 500 ppi & Yes & Pre-commercialization \\
\hline $\begin{array}{l}\text { Carnegie Mellon University, } \\
\text { Handshot, LLC Handshot ID }\end{array}$ & $\begin{array}{l}\text { Composite Digital } \\
\text { Camera Images }\end{array}$ & $1000 \mathrm{ppi}$ & Yes & $\mathrm{R} \& \mathrm{D}$ \\
\hline Handshot HandID & Digital Camera Images & 1000 ppi & Yes & $\mathrm{R} \& \mathrm{D}$ \\
\hline $\begin{array}{l}\text { University of Massachusetts } \\
\text { Lowell single-finger Rolled } \\
\text { Equivalent Scanner [38] }\end{array}$ & $\begin{array}{l}\text { Line Scanning Optical } \\
\text { Camera }\end{array}$ & $\begin{array}{l}\text { Single Finger: } 700 \text { ppi } \\
\text { (Up-Sampled to } 1000 \\
\text { dpi) } \\
\text { 4-slap/Palm: } 72 \text { ppi }\end{array}$ & No & Prototype \\
\hline $\begin{array}{l}\text { General Electric Tenprint } \\
\text { Scanner }\end{array}$ & $\begin{array}{l}\text { Multi-focus Digital } \\
\text { images combined into a } \\
\text { 3D global finger shape }\end{array}$ & $1000 \mathrm{ppi}$ & Yes & Pre-commercialization \\
\hline $\begin{array}{l}\text { AFIS and Biometrics Consulting, } \\
\text { Inc. } \\
\text { Fingerprint-on-the-Move }\end{array}$ & $\begin{array}{l}\text { High Resolution Digital } \\
\text { Camera }\end{array}$ & $500 \mathrm{ppi}$ & Yes & Pre-commercialization \\
\hline $\begin{array}{l}\text { FlashScan3D Single-finger } \\
\text { Scanner }\end{array}$ & $\begin{array}{l}\text { Moving Structured Light } \\
\text { Illumination }\end{array}$ & 500 ppi & No & Commercialization \\
\hline FlashScan3D Tenprint Scanner & $\begin{array}{l}\text { Moving Structured Light } \\
\text { Illumination }\end{array}$ & $500 \mathrm{ppi}$ & Yes & Prototype \\
\hline $\begin{array}{l}\text { FlashScan3D Single-finger Rolled- } \\
\text { Equivalent Scanner }\end{array}$ & $\begin{array}{l}\text { Fixed Structured Light } \\
\text { illumination on moving } \\
\text { finger }\end{array}$ & $1000 \mathrm{ppi}$ & No & $\mathrm{R} \& \mathrm{D}$ \\
\hline $\begin{array}{l}\text { FlashScan3D Full-hand } \\
\text { Contactless 3D Scanner }\end{array}$ & $\begin{array}{l}\text { Fixed Structured Light } \\
\text { illumination on moving } \\
\text { hand }\end{array}$ & $500 \mathrm{ppi}$ & No & Pre-commercialization \\
\hline $\begin{array}{l}\text { TBS Touchless Ten Printer (TTP) } \\
\text { [1] }\end{array}$ & $\begin{array}{l}\text { Composite Digital } \\
\text { Camera Images with } \\
\text { structured light for 3D } \\
\text { modeling }\end{array}$ & $1000 \mathrm{ppi}$ & No & Prototype \\
\hline $\begin{array}{l}\text { MorphoTrak Finger-on-the-Fly } \\
\text { [39] }\end{array}$ & $\begin{array}{l}\text { Structured Light } \\
\text { Illumination }\end{array}$ & $500 \mathrm{ppi}$ & Yes & Pre-commercialization \\
\hline $\begin{array}{l}\text { Lumidigm Contactless } \\
\text { Fingerprint Sensor }\end{array}$ & $\begin{array}{l}\text { Stereoscopic digital } \\
\text { images using multiple } \\
\text { color illumination to } \\
\text { capture 3D } \\
\text { representation }\end{array}$ & Undetermined & Yes & Prototype \\
\hline TBSGuard 3D-Line [40] & $\begin{array}{l}\text { Composite Digital } \\
\text { Camera Images }\end{array}$ & $500 \mathrm{ppi}$ & Yes & Commercial \\
\hline TST BiRD $3 / 4[1]$ & $\begin{array}{l}\text { Digital Camera image } \\
\text { with multispectral } \\
\text { imaging for liveness } \\
\text { detection }\end{array}$ & $500 \mathrm{ppi}$ & Yes & Commercial \\
\hline $\begin{array}{l}\text { Advanced Optical Systems Inc. } \\
\text { (AOS) ONEprint [41] }\end{array}$ & Digital Camera Image & $500 \mathrm{ppi}$ & Yes & Commercial \\
\hline NEC HS100-10 [42] & Digital Camera Image & $600 \mathrm{ppi}$ & No & Commercial \\
\hline $\begin{array}{l}\text { Advanced Optical Systems Inc. } \\
\text { (AOS) ANDI-on-the-go (OTG) } \\
{[43]}\end{array}$ & Digital Camera Image & $500 \mathrm{ppi}$ & Yes & Prototype \\
\hline
\end{tabular}




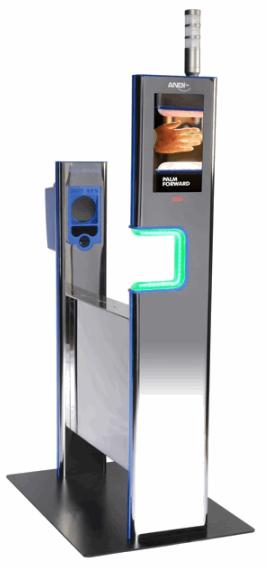

(a)

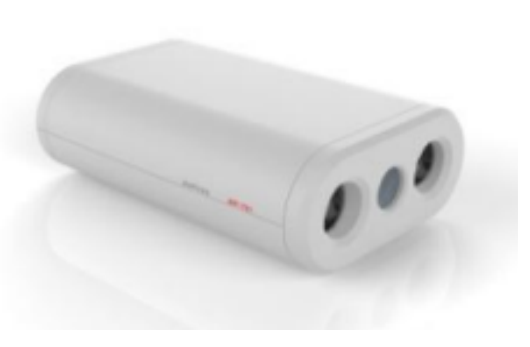

(d)

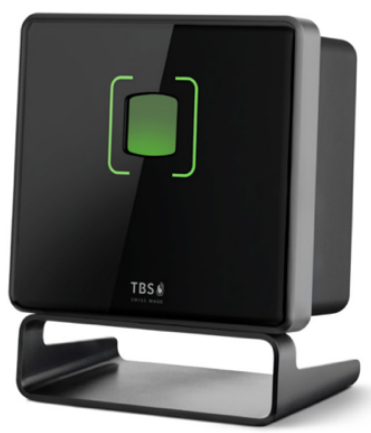

(e)

(b)

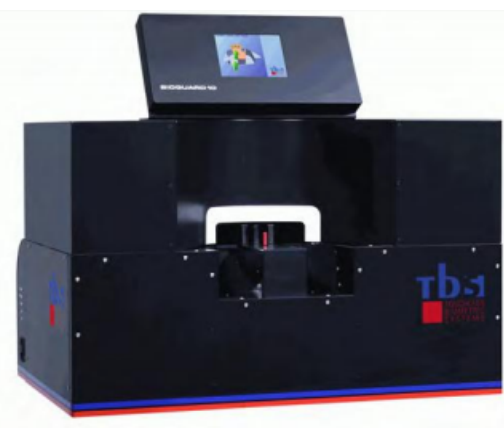

(c)

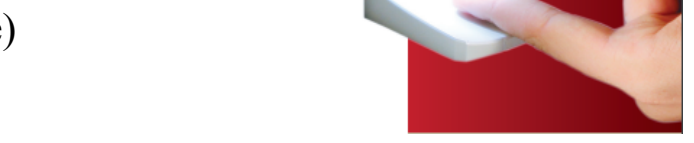

(f)

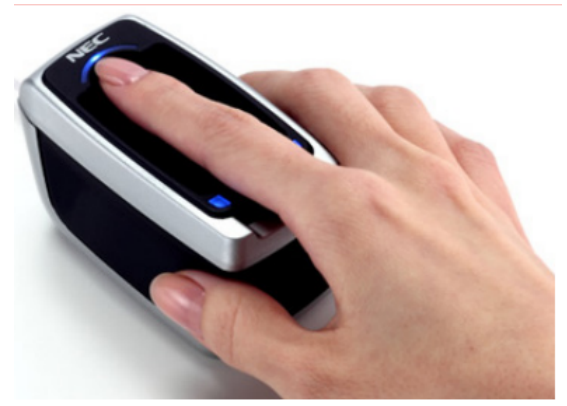

(g)

Figure 1.9 (a) ANDI On-The-Go (OTG) [43] (b) MorphoWave or Morpho Finger-On-The-Fly (FOTF) [39] (c) TBS Touchless Ten Printer [1] (d) AIRprint Standoff Fingerprint Scanner [1] (e) TBS 3D Enroll [40] (f) IDair ONEprint [41] (g) NEC HS100-10 hybrid contactless scanner [42] 


\subsection{Certification}

Since the second contactless fingerprint collection ended, some of the contactless devices have been certified. The FBI added the Advanced Optical Systems ANDI On-The-Go to the agency's certified product list (CPL) on November 27, 2015 [44]. After meeting the Personal Identity Verification (PIV) Image quality specifications standard, the FBI also certified the Morpho (Safran) MorphoWave Desktop contactless fingerprint scanner on January 5, 2016, which is the commercial version of the Morpho Finger-on-the-fly [45].

\subsection{Interoperability}

Interoperability is the most important issue facing the widespread adoption of contactless fingerprinting devices, considering the databases created by these systems must be completely backward-compatible with images captured from traditional scanners. The applications of these devices requires full interoperability with legacy systems. Contactless fingerprinting technology aims to address the issues experienced with previous techniques while also providing more information and fingerprint detail. Interoperability calls for careful design of lighting, resolution, and three-dimensional shape estimation. These devices also call for independent certification, Personal Identity Verification (PIV), and testing due to the diversity in these contactless devices compared to contact-based interaction. It's important to note that interoperability could mean different things. Jain and Ross considered interoperability as a biometrics systems ability to adapt to variability in images produced by different sensor technologies [46]. Interoperability could also refer to the usability between fingerprinting devices, as described in [36].

Interoperability has been evaluated for every different method of fingerprinting device throughout history to investigate the ability of the system to work with a diverse set of 
fingerprinting devices or methods. For this effort, interoperability is a term used to show that images of the same subject's print from contactless and contact-based devices are comparable and will produce a match. Interoperability could also refer to the usability between devices.

\subsection{Related Work}

Research in contactless fingerprint recognition has increased in recent years. However, related work is still limited. One technical report that is related to contactless fingerprinting devices belongs to the US Department of Justice and ManTech [1]. They used 13 Contactless Fingerprinting devices that were still in the research and development stage and 4 commercial livescan devices to evaluate the efforts that were being made in government, industry, and academia in pursuing contactless fingerprint collection technologies. The researchers within this study identified that, at the time, there were no prior concerted efforts to compare contact versus contactless fingerprinting. Another study that examined contactless fingerprinting devices was performed by the University of Massachusetts, this study explored methods that would allow for cross examination of 10prints, livescan and contactless devices [30].

Another study that considered the interoperability between fingerprinting devices not particularly with contactless devices but other contact-based fingerprinting devices was researched at WVU [47]. The approach they proposed reduced the match error rates between devices by several levels but each performance gain depended on the matching algorithm used. A similar study that considered the interoperability of fingerprinting devices, but once again only looked at livescan devices and ten-print cards [48]. This study looked at data from 494 participants using 4 contact-based devices. They found that match score similarity between two same subject samples were generally higher when both images were captured from the same device. They also found higher match score sensitivity due to image quality when using two 
different devices.

A case study by Arun Ross and Anil Jain also considered the interoperability of biometric sensors, capacitive and optical [46]. This study showed the influence of different fingerprint sensors on match performance. They experienced an EER of $23.13 \%$ for images matched between two different sensors, while the EERs for the other cases between images from the same sensors were $6.14 \%$ and $10.39 \%$.

NIST led the effort of developing standards for contactless devices. In January of 2015, The National Institute of Standards and Technology (NIST) developed a research program for research in developing methodology for measuring the image fidelity of contactless fingerprint capturing devices. This program is called the Contactless Fingerprint Capture Device Measurement Research Program, or CRADA, and focuses on producing open testing methods, metrics, and artifacts that will support the future certification of devices for inclusion on the Government Certified Products Lists [34].

The dataset used in the research described in this theses was compiled for the effort reported in [1]. A second technical report by ManTech evaluated interoperability among contact and contactless systems for only a selection of 200 participants of this data [2]. Some of their findings are displayed in Table 4.2 with comparison to the findings of this thesis. They used only the participants out of the first 200 subjects that had data for every device. The study here used similar methods to acquire the results for the full dataset.

Most recently in March of 2017, NIST published the "Contactless Fingerprint Devices Usability Test" [36]. This study deals with the usability of contactless and contact-based devices, looking specifically into the efficiency, effectiveness and user satisfaction of each device. This study is like that presented here, as they also evaluated the similarity scores for each device and 
found at least two of the touchless devices exhibited match scores well above an imposter baseline for matches of the same subject from contact-based device images [36]. Other papers have also been referenced throughout this thesis.

\subsection{Problem Statement and Thesis Organization}

\section{Problem Statement}

The main goal of this research is to investigate the interoperability of contactless fingerprinting devices with the currently used livescan legacy fingerprinting devices and techniques. Fingerprint data will be evaluated from wet-ink ten-print cards and traditional contact-based legacy fingerprint devices versus fingerprint data generated by next-generation contactless fingerprint scanners. The feasibility and performance of contactless fingerprint databases will be explored. To investigate the matching performance and interoperability of these devices, three different matching algorithms were utilized, including: Neurotechnology MegaMatcher, open source NIST Biometric Image Software (NBIS) and open source fingerprint recognition toolkit by SourceAFIS. Matching runs were performed on the right index finger and right thumb data from each device against themselves and all other devices, as well as the 500 dpi scans from the wet-ink fingerprint cards (thumb data results are not shown as they did not involve the contactless devices). After identifying the interoperability, it is desired to discover the stratification of contactless devices to see if a person's demographics share a relationship with the match capabilities of their fingerprints. The main tasks associated with these goals are:

1. Data Collection: Guiding 450 participants through the collection on each fingerprint device as well as rolling his or her prints with wet ink ten-print cards. 
2. Image Pre-processing: Isolating each fingerprint image with segmentation programs as well as extracting images that are doubles or erroneous.

3. Match Score Generation: Data from each device was ran through each matching algorithm against every other device's data to determine similarity scores as well as ran against itself to determine genuine and imposter scores.

4. Data Analysis: Kullback-Leibler divergence was performed on the similarity scores generated from the matching runs as well as producing the score distributions. Genuine and imposter match score distributions were produced. EER values were also generated from the genuine and imposter distributions.

Knowing that contactless technologies have many advantageous qualities over traditional fingerprinting methods, this work will aid in determining if contactless databases are comparable to those currently in use is critical to the advancement of contactless fingerprinting.

The contributions of this research effort are:

- It shows fingerprints collected under ideal conditions from contactless and contact-based devices can be matched against each other in a statistically meaningful way. Data collection and analysis can be employed to determine the comparative match performance of contact-based and contactless fingerprinting devices.

- This dataset enables the investigation of performance and interoperability of contactless and contact-based fingerprint devices. Few biometric dataset resources exist that cover as many devices capturing the same subject pool. This research can escalate the understanding, performance, and adoption of contactless fingerprint systems, as well as inter-device matching. 
- Evaluation of demographic stratification of contactless devices.

\section{Thesis Organization}

Chapter 2 explains the art of contactless fingerprinting and the technologies that are involved in the process. Chapter 3 describes in detail the process of data collection and the process that occurred in preparation for the matching algorithms. Chapter 4 covers the interoperability analysis; this section will display the results of this research project. The conclusions section, or Chapter 5, deals with the summary of the results from the interoperability analysis. 
Chapter 2: Theory 


\section{Fingerprint Sensor Technology: \\ 2.1 Integrated Biometrics Sherlock Sensor}

The Sherlock sensor is a contact-based mobile fingerprint reader designed for stand-alone or embedded module usage [49]. This compact reader is manufactured by Integrated Biometrics and is used in two devices from the CFPv2 collection, the CrossMatch SEEK Avenger and Northrop Grumman BioSled. This sensor and the Integrated Biometrics Watson sensor were the first non-optical FAP 45, appendix F fingerprint sensors [50]. This sensor captures a livescan equivalent to a ten-print card. The FBI has granted the Sherlock with PIV-071006, Mobile ID IQS FAP 45, GSA FIPS 201, and Appendix F certifications [49] [51]. This platen has the ability to capture single or dual flat prints, or single rolled prints [49]. It utilizes Integrated Biometrics' Light Emitting Sensor (LES) technology along with a thin film transistor (TFT) [49]. The LES technology, proprietary to Integrated Biometrics, employs the use of an electroluminescence film, which emits a high resolution image when an electric field is applied to the film [52]. This reader uses a hybrid method of capture, utilizing both capacitive and optical sensor technology [49].

The Sherlock enables fingerprint capture in standalone applications, smartphones, or tablets, with its ideal use being in mobile computers, hand-held devices, smartphones and tablets [51]. The Northrop Grumman BioSled used a Samsung Galaxy S5 Android Smartphone housed in rugged case that strategically positions the Sherlock fingerprint sensor for fast and easy capture [53]. The CrossMatch SEEK Avenger delivers an all in one design with large touchscreen and keyboard with Sherlock Sensor built directly into the device [54].

The LES film provides the image, the thin film transistor captures the image and a USB interface allows the customer to integrate into their device [52]. When an electric field is applied 
across the LES film, and a finger is placed on the film, the EL particles suspended in the film glow beneath the finger resulting in a high resolution image of the fingerprint [50]. In the case of the Sherlock sensor, this film is laminated directly to a thin film transistor (TFT) camera [50]. The Sherlock sensor is smaller, lighter and thinner than most sensors with reduced power requirements, which makes it desirable compared to optical sensors [50]. This sensor is also not affected by bright lights or direct sunlight, allowing it to be used in severe conditions for field collection [50]. The Sherlock sensor is also spoof resistant, as the finger touching the film must be conductive, restricting users from capturing artificial silicon fingers for prints [50].

\section{Light Emitting Film and Thin Film Transistor Camera}

\section{The properties of LE film (Thickness and Lighting sources)}

The phosphor laver emits lighting in the LE film. So there is no need for external light source on the TrT image array sensor.

Fingerprint

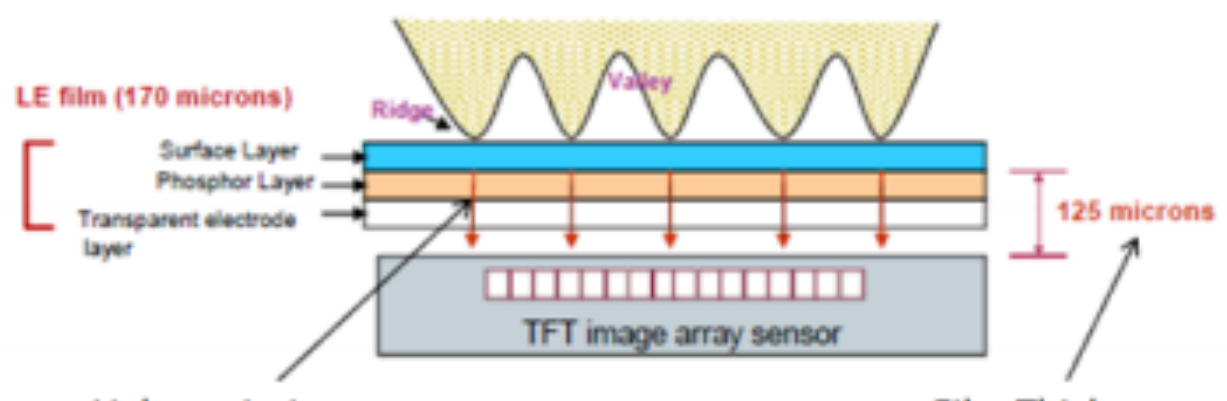

Light emission

Film Thickness

\section{Mounting the LES film directly on the TFT Camera eliminates the need for lens, image scaling and optical distortion correction}

Figure 2.1 Explanation of Sherlock sensor by Integrated Biometrics [50] 


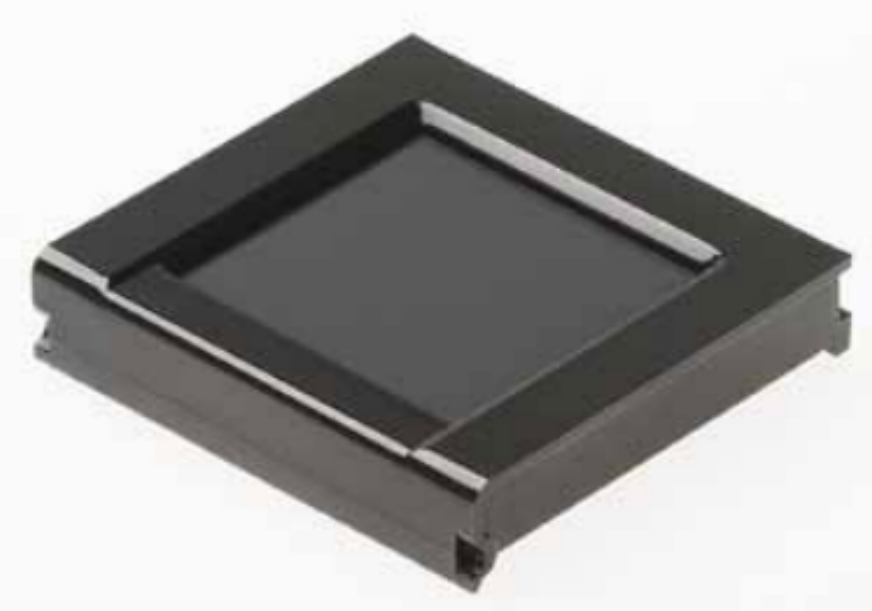

Figure 2.2 Sherlock Sensor [51]

\subsection{Frustrated Total Internal Reflection Sensors}

Frustrated total internal reflection or FTIR, is the oldest and most used livescan technique [55]. A contact image sensor with FTIR projects high contrast images onto a linear array sensor by the Frustrated total internal reflection of the fingerprint being projected by a GRIN (GRadient INdex of refraction) lens array onto the linear array sensor [56].

The embodiment is a contact image sensor with an optically transparent platen in which light must be introduced inside the platen [56] . Light is introduced using either a flat or curved surface, which acts as a lens to help collimate the light so that it forms a flat sheet of light the length of the sensor [56] [57]. Once introduced, the light is directed inside the platen using total internal reflection (TIR) or reflections from a mirror-like surface or surfaces [56]. The reflections inside the platen allow for the light to placed more conveniently within the device and allow for the device to be more compact [56]. The reflective surfaces or TIR surfaces can be slightly rough to partially diffuse the light beam to send more light onto the fingerprint [56]. 
If viewed through the transparent platen at an oblique angle, a high contrast image can be obtained [56]. The image is then focused by the GRIN lens array onto a linear sensor array [56]. Light is directed at an angle to the top interior surface of the platen, typically 45 degrees or more to a line normal to the surface of the platen, where it is reflected by total internal reflection if no fingerprint is present [56]. When light is not reflected, fingers are on the sensor, and due to frustrated total internal reflection, the light is absorbed, resulting in a dark pattern for the fingerprint ridges and bright light for the fingerprint valleys [56] [55].

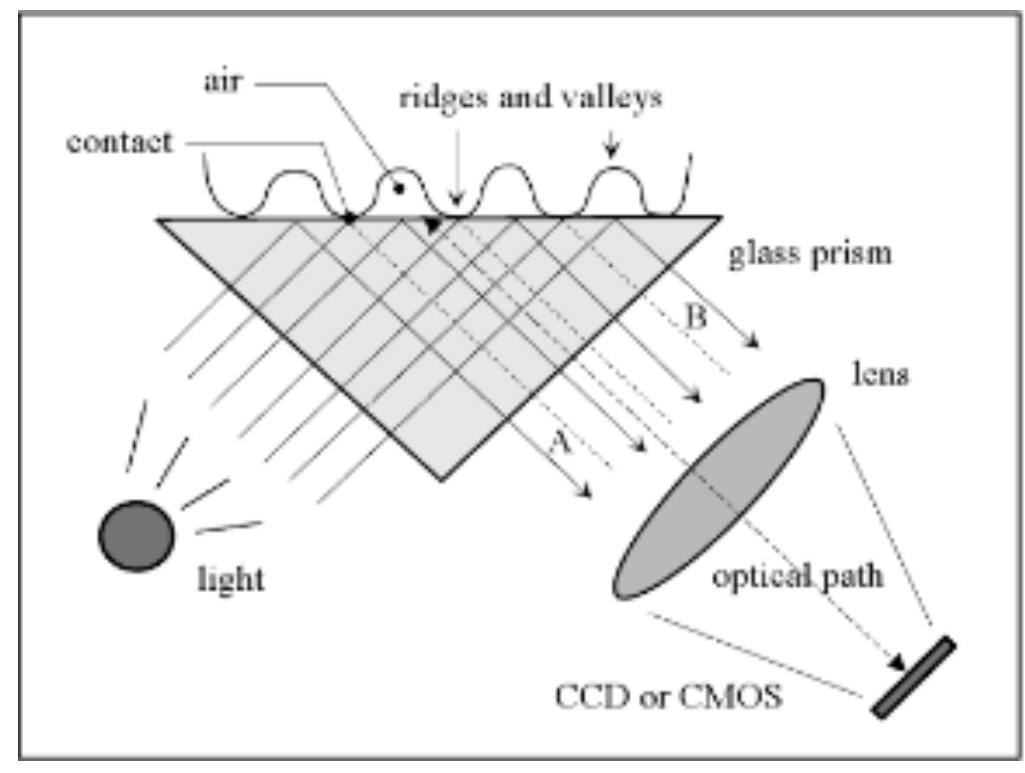

Figure 2.3 FTIR-based fingerprint sensor operation [55]

\subsection{Structured Light Illumination}

Structured light illumination is a method to capture images in a contactless manner. To execute this system, fine-grid-structured lights from different directions are projected to the target object and the images are captured from cameras at different angles [58]. The captured images are processed to create either a three-dimensional or hybrid two-dimensional model of the target object [58]. The process of capturing an image obtains all or most of the fingerprint information at once. The multiple angles of the cameras allow for the capture of worn finger 
ridges and fine features which are difficult to capture with just one camera or conventional scanners [58]. Structured Light Illumination involves at least one projection unit for projecting a structured light pattern onto a hand positioned in the scan volume on or in front of the backdrop pattern [59]. These systems also include at least one camera for capturing multiple images of the hand, these images consist of at least a portion of the hand and the backdrop pattern [59]. The embodiment of these systems further includes a processing unit for calculating three dimensional coordinates of the hand from the images using the predetermined coordinates of the backdrop pattern to align the plurality of images and mapping the three-dimensional coordinates to a twodimensional flat surface to create a two-dimensional representation equivalent to that of a wet ink rolled fingerprint [59]. One way that the images created can be segmented to remove the background and shadows is shown in Equation 2.1, where $I_{F}(x, y)$ is the original image, $I_{S}(x, y)$ is the binary segmentation mask, $t_{1}$ and $t_{h}$ are two experimentally estimated threshold values [60].

$$
I_{S}(x, y)= \begin{cases}1, & t_{1}<I_{F}(x, y)<t_{h} \\ 0, & \text { otherwise }\end{cases}
$$




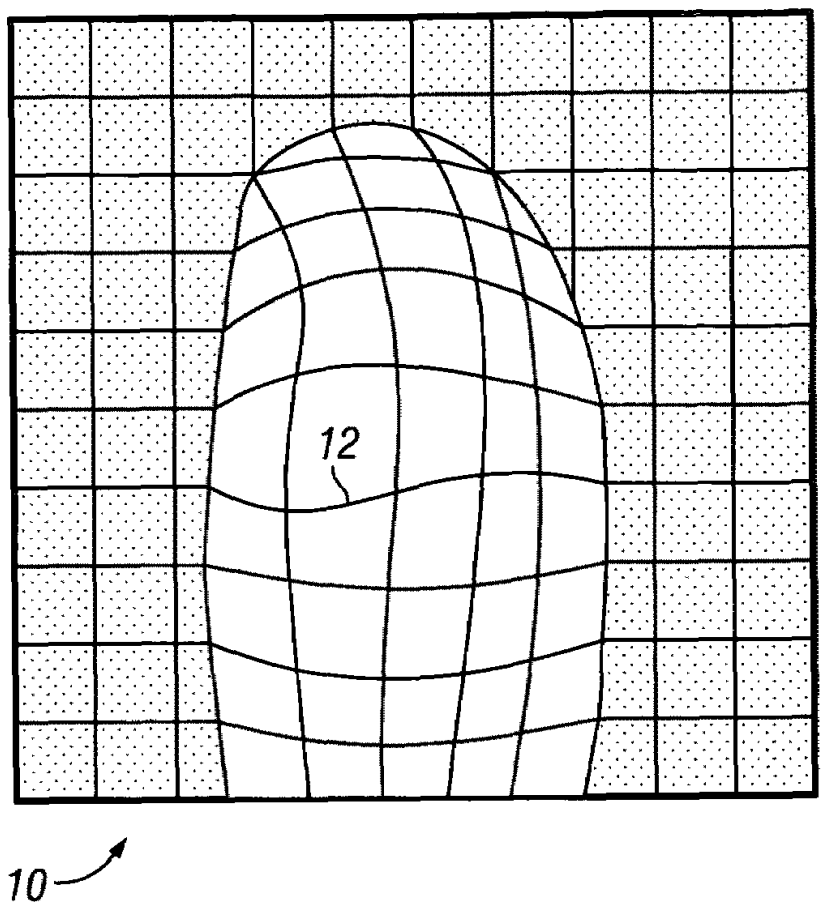

Figure 2.4 Diagram of how structured light illumination sees a finger [58]

\subsection{Polarized Reflected Light}

This technique provides a contactless method of capturing a three-dimensional fingerprint using polarization rotation to get different angles of reflected light. This approach is comprised of illuminating a fingerprint and directing a reflected light from the fingerprint through an imaging system [61]. This imaging system comprises an image capture device, a liquid crystal panel (LCP) and a birefringent element wherein the LCP and the birefringent element are positioned between the fingerprint and the image capture device such that the reflected light from the fingerprint passes through the LCP and birefringent element [61]. In other words, this system can be described as a polarizer added between the imaging capturing device and the finger surface [62]. This technique captures a plurality of rotation angles of the linearly polarized images of the fingerprint to comprise each of the steps of polarizing the reflected light [61]. The polarization filters could have angles of 0,45 , and 90 , although other polarization angles can be 
used. [62] At least one image is captured from each of the polarization orientation states of the LCP, registering these images to create a fused image [61]. Next step is calculating a depth of structural features using a depth from focus algorithm and generating a two dimensional rolled image of the fingerprint using the calculated depth of structural features and the fused image using an algorithm to reverse the projection of the fingerprint surface on to a two-dimensional projection [61].

\subsection{Fingerprint Matching or Fingerprint Recognition}

Fingerprint matching refers to determining the similarity between two given fingerprint images [63]. Fingerprint matching is used by law enforcement agencies for two main reasons, establish the identity of a suspect (or victim) based on partial prints, or latents, left at a crime scene and identifying repeat offenders based on prints of all their fingers [64]. A fingerprint recognition system can be used for verification and identification. Verification compares an input fingerprint to the an "enrolled" fingerprint to see if they are from the same finger or a one-to-one match [64]. For identification, the system compares an input fingerprint with all of the prints that are enrolled in the database or gallery also known as a one-to-many match or one-to-N match [64]. Identification is used in cases to determine if a person is already known under a duplicated or false identity [64]. There are a couple different methods of fingerprint matching but minutiae based fingerprint matching is the most popular method of fingerprint matching [65] [64] [66] [67] [68]. 


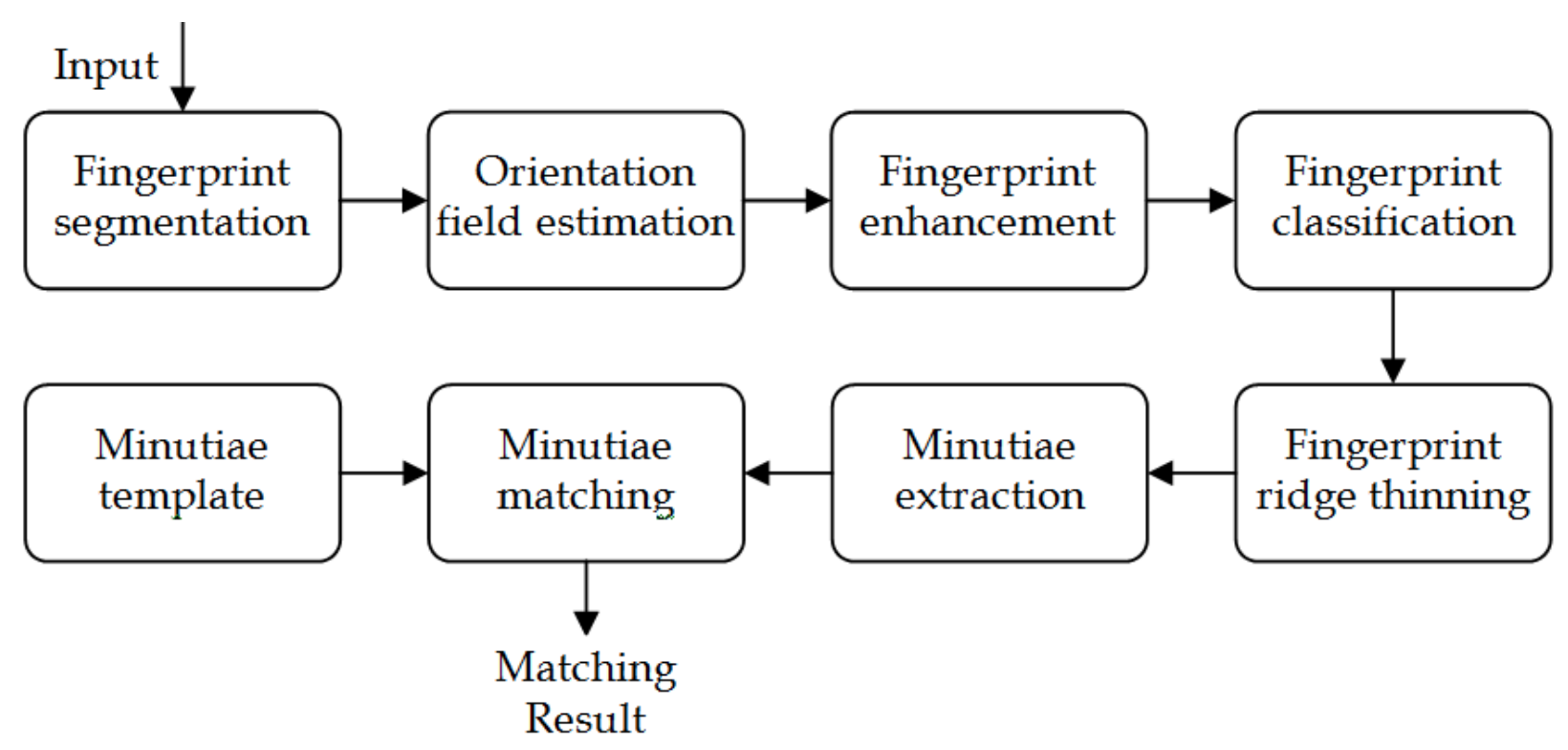

Figure 2.5 General block diagram for a Fingerprint Identification System. [68]

Fingerprint matching can be broken down into four primary sections, sensor, feature extraction, matcher, and system database. The sensor module captures the fingerprint data of the individual [66]. Some sensors are described earlier in this chapter. Feature extraction is the process in which the acquired data is processed to extract a set of discriminatory features [66]. During this process the position and orientation of minutiae points (local ridge and valley singularities) in a fingerprint image are extracted [66]. Features extracted from a fingerprint image are generally categorized into three levels [64]. Level 1 features capture macrodetails such as friction ridge flow, pattern type, and singular points [64]. Level 2 features refer to minutiae such as ridge bifurcations and endings [64]. Level 3 features include all dimensional attributes of the ridge such as ridge path deviation, width, shape, pores, edge contour, and other details, including incipient ridges, creases, and scars [64].

The next section of recognition is the matcher, in which the features extracted during recognition are compared against the stored templates to generate matching scores [66]. The 
number of matching minutiae between the input and the template fingerprint images is determined and a matching or similarity score is reported [66]. The similarity or match scores should be high for fingerprints from the same finger and low for those of different fingers [64]. The matching process can be difficult due to large intraclass variations or variations in fingerprint images of the same finger and large interclass similarity (similarity between fingerprint images from different fingers [64]. The final section of fingerprint matching is the system database module, which is used by the matcher to store the fingerprint templates of the enrolled users [66].

Minutiae based matching uses local minutiae structures to quickly find a coarse alignment between two fingerprints and then consolidate the local matching results at a global level [64]. Minutiae based algorithms typically consists of four steps shown in Figure 2.6. The algorithm starts by computing the pairwise similarity between minutiae of two fingerprints by comparing minutiae descriptors that are invariant to rotation and translation [64] [68]. The next step is to align the two fingerprints according to the most similar minutiae pair [64]. Next the algorithm establishes minutiae correspondence, minutiae that are close enough both in location and direction are deemed to be mated or corresponding minutiae [64]. The last component of the algorithm is the computation of a similarity score that reflects the degree of match between the two prints [64]. The score is computed based on number of matching minutiae, the percentage of matching minutiae in the overlapping area of two fingerprints, and the consistency of ridge count between matching minutiae [64]. 


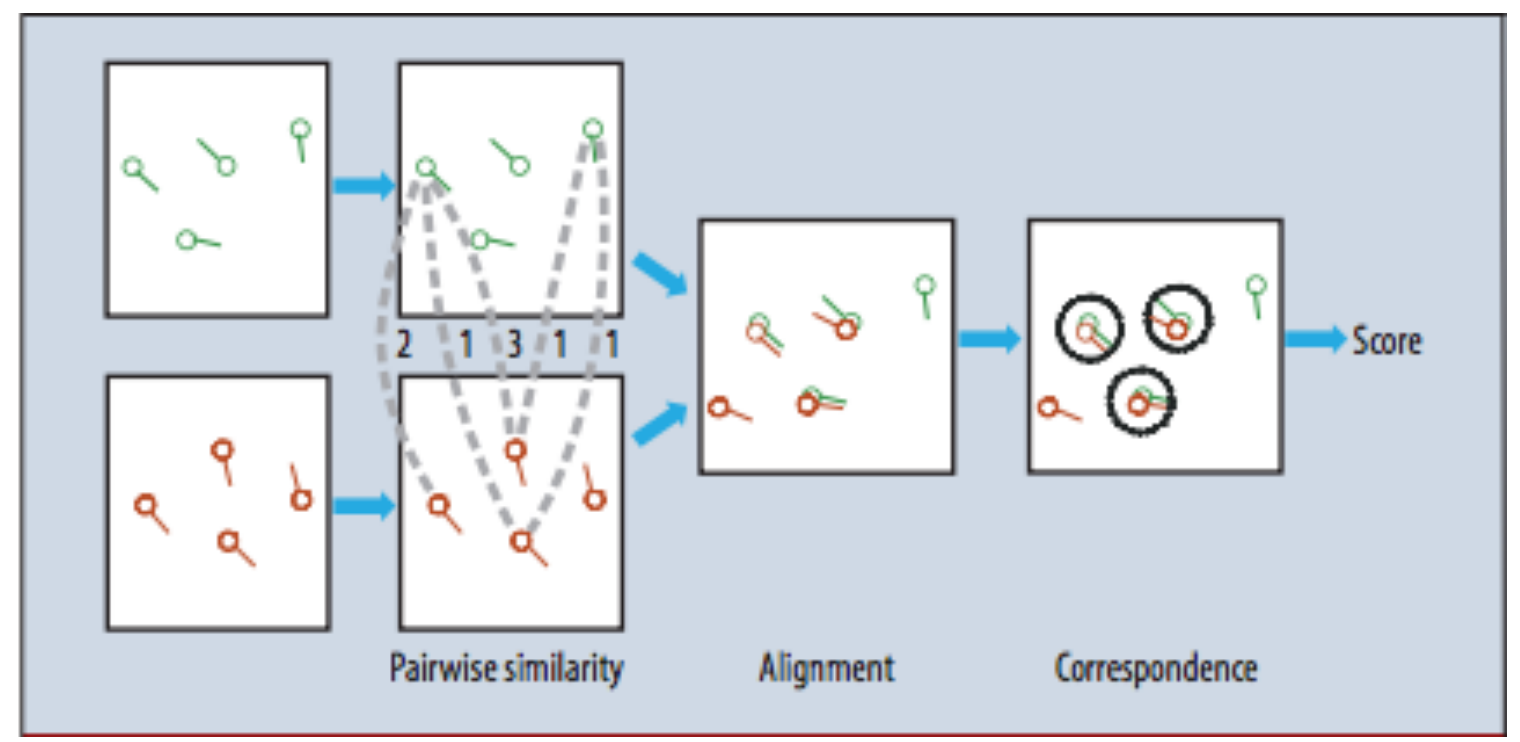

Figure 2.6 Typical Minutiae Matching Algorithm [64]

\subsection{Possible Matching Errors}

A matcher has two main types of errors that it can make: a false match and a false nonmatch [64] [66]. A false match is when the matcher mistakes a match from two different fingers to be the same finger [64] [66]. A false non-match is when the matcher mistakes two prints from the same person to be from two different persons [64] [66]. A false match is normally called a false accept and a false non-match can also be called a false reject [64] [66]. Examples of false non-matches or false rejects are shown in Figure 2.7 with the images on the left creating a false non-match with the images on the right, with the corresponding images being from the same finger. Examples of false matches or false accepts are shown in Figure 2.8 with the images on the left producing a false match with the images right, the corresponding images being from different fingers. 

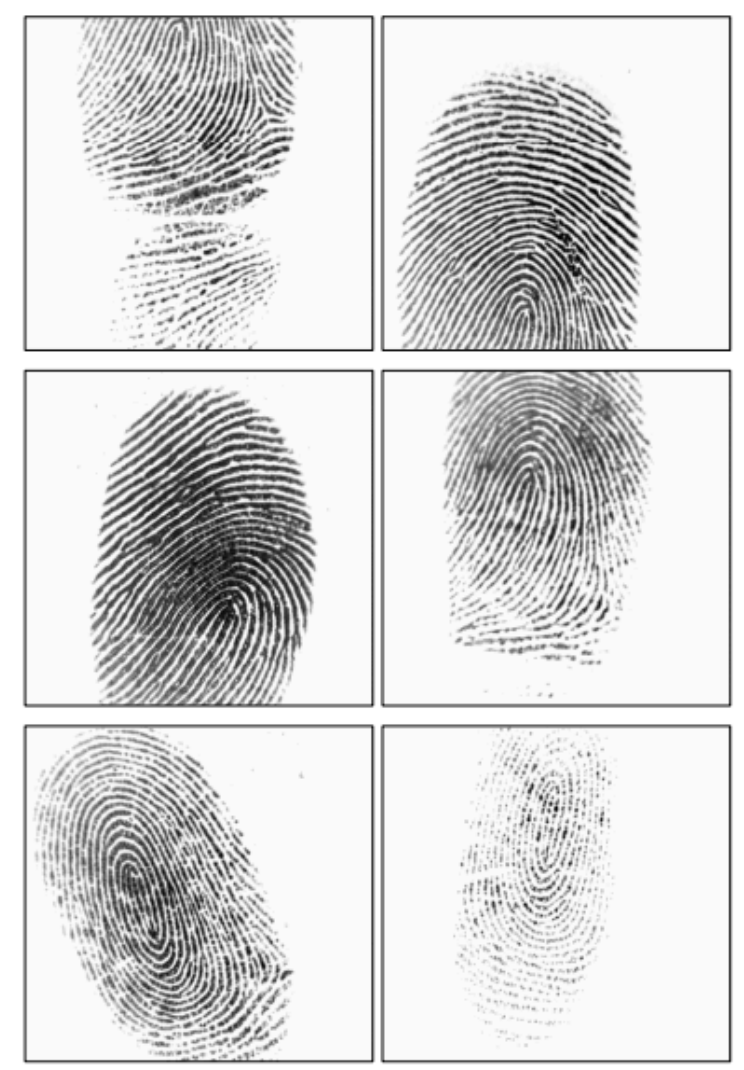

Figure 2.7 Examples of False Non-Matches or False Rejects [69]

Each row in Figure 2.7 shows a pair of impressions from the same finger which were falsely non-matched by an algorithm
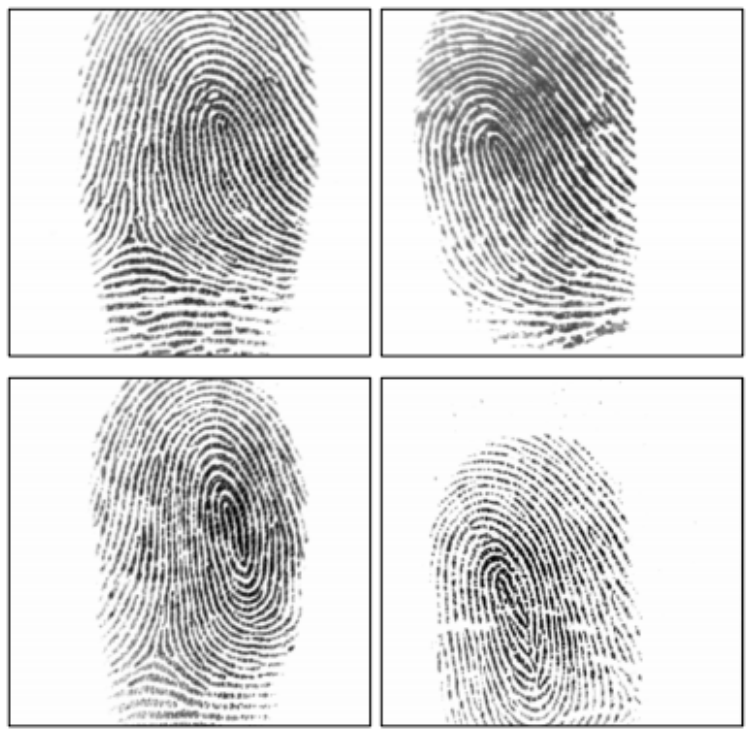

Figure 2.8 Examples of False Matches or False Accepts [69] 
Each row of Figure 2.8 shows a pair of impressions of different fingers that were falsely matched by an algorithm.

\subsection{Biometric System Classifying Terminology}

Genuine Match

A genuine match indicates the similarity between two mate samples, i.e. a genuine match score is generated with a pair of samples from the same user and same finger.

Imposter Match

An imposter match or a false accept score, measures the similarity between two non-mate samples, i.e. an imposter match score is generated with a pair of samples from two different users or two different fingers.

Genuine Acceptance Rate (GAR)

The Genuine Acceptance Rate is defined as the probability at which a correct match or genuine match is made in a data set. It is the number of genuine matches made divided by the number of attempts. The GAR can also be described as the true match rate (TMR).

False Acceptance Rate (FAR)

The false acceptance rate or false match rate, is defined as the probability at which imposters or false accept errors are made in a data set. It is the number of false acceptances divided by the number of imposter attempts.

False Rejection Rate (FRR) 
The False Rejection rate is the probability that a genuine match will be mistaken for an imposter match.

True Match Rank 1 (TMR1)

True Matches or Genuine Matches at rank 1. Rank 1 is defined as the highest similarity score in a one to many probe to gallery match.

False Match Rank 1 (FMR1)

False Matches or Imposter Matches at rank 1. Rank 1 is defined as the highest similarity score in a one to many probe to gallery match.

True Match Rank 1 Rate (TMR1 Rate)

The Rate at which a True Match Rank 1 happens within a matching run.

False Match Rank 1 Rate (FMR1 Rate)

The Rate at which a False Match Rank 1 happens within a matching run.

Equal Error Rate (EER)

The Equal Error Rate is a determined from the False Acceptance Rate and the False Rejection Rate. The Equal Error Rate is where FAR and FRR cross, or their intersection point. 


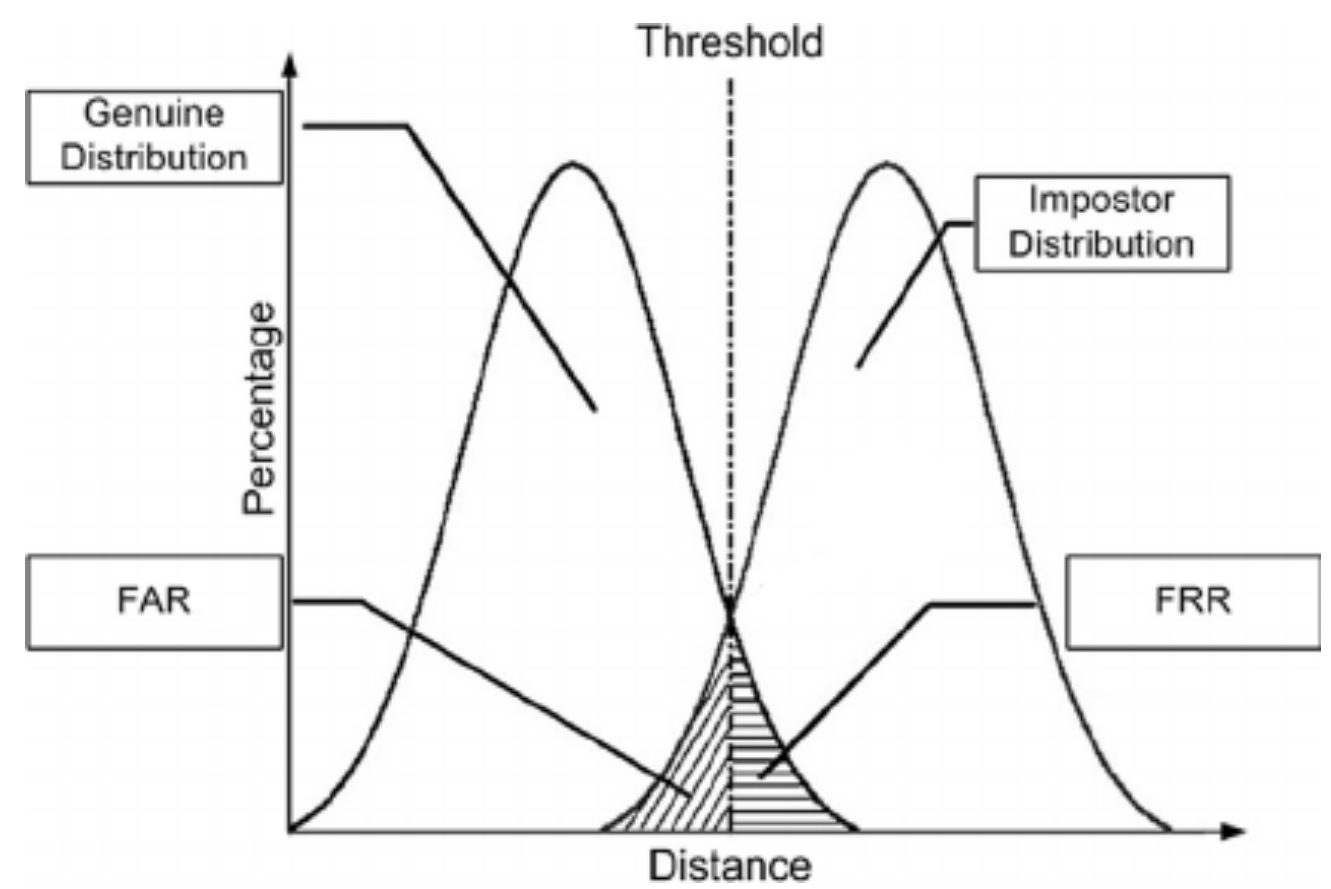

Figure 2.9 Representation of a typical genuine and imposter score distribution [70]

\subsection{Kullback Leibler Divergence}

Kullback-Leibler Divergence (KLD) is used to measure the difference between two probability distributions over the same variable $\mathrm{x}$ [71]. KLD is a non-symmetric measure of the difference between two probability distributions $\mathrm{p}(\mathrm{x})$ and $\mathrm{q}(\mathrm{x})$ [71]. Specifically, KLD is a measure of the information lost when $\mathrm{q}(\mathrm{x})$ is used to approximate $\mathrm{p}(\mathrm{x})$ [71]. The KL divergence of the probability distributions $\mathrm{P}, \mathrm{Q}$, and a finite set $\mathrm{X}$ is defined as shown in Equation 2.1.

$$
D_{K L}(P \| Q)=\sum_{x \in X} P(x) \log \frac{P(x)}{Q(x)}
$$

Typically, $\mathrm{p}(\mathrm{x})$ represents the "true" distribution of data, in our case that is the genuine or imposter match distribution of the overall dataset. Q(x) usually represents a theory, model, description or approximation of $\mathrm{p}(\mathrm{x})$, in this case it represents the genuine or imposter match distribution of a certain demographic group. 


\subsection{Receiver Operating Characteristic Curves}

A receiver operating characteristic curve or ROC curve is a graphical plot that illustrated genuine acceptance rate against false acceptance rate. An ROC curve is a way to represent the performance of a matching or classification system [72]. It is created by plotting the GAR vs. the FAR at various threshold settings. The closer the curve follows the left-hand border and the top boarder of the ROC space, the more accurate the test [73]. The slope of the tangent line at any given threshold, gives the likelihood ratio for that value of the test [73]. An example of an ROC curve is shown in Figure 2.9.

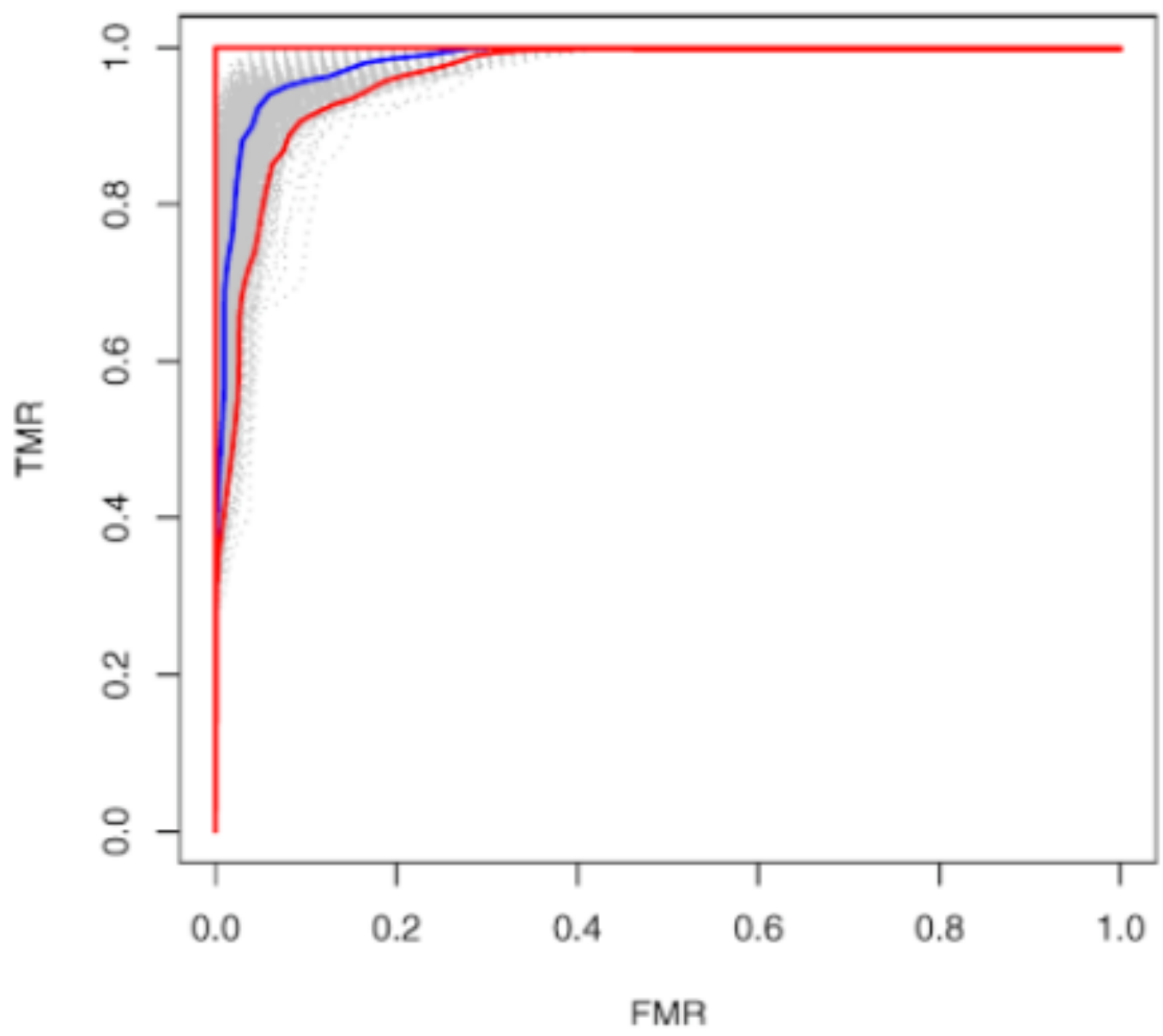

Figure 2.10 A Sample ROC Curve [72]. 


\subsection{NIST Fingerprint Image Quality (NFIQ)}

NFIQ stands for NIST Fingerprint Image Quality, this algorithm assigns a quality value to fingerprint images. NFIQ was developed in 2004 by NIST, it was created to produce a value from a fingerprint image which is directly predictive of the expected matching performance [74]. The values that can be assigned range from 1 to 5,1 being the highest quality and 5 being the lowest quality. NIST is currently developing NFIQ 2.0, which would be better optimized for contactless fingerprints [75]. 


\section{Chapter 3: Data \\ Collection \& \\ Preparation}




\subsection{Overview}

This data collection was Phase 2 of a Non-Contact Fingerprint Evaluation project sponsored by ManTech International Corp. and the U.S. Dept. of Justice (CFPv2) (IRB\# 1309097716). The main goal of this research was to obtain data to enable the evaluation of contactless fingerprint devices. The target number of participants for this collection was 400 . Data was collected from 450 participants while the collection took place between 12/1/2014 and 4/2/2015. In 2012, The NIJ SSBT CoE performed a biometric collection of fingerprint data from traditional scanners and contactless devices, named Contactless Fingerprint Collection; Round 1 (CFPv1). Due to the success of the CFPv1 and its value to the biometrics research community, a second collection of contact and non-contact fingerprint data has been performed (CFPv2). [2]

\subsection{Collection Process}

Data Collection began December $1^{\text {st }}$ of 2014 and ended April $2^{\text {nd }}$ of 2015 . Participants were contacted via email, based on previous studies where they opted for future contact about new data collections, where a website and phone number were provided so that they could schedule an appointment or call in to make an appointment. Data collection was performed on the WVU Evansdale Campus, in an indoor laboratory space as the collection area, with all sensors and rolled-ink impressions collected in the same area. Data was collected from 450 individuals, providing two sequential sessions of fingerprints for each sensor. The collection utilized livescan and non-contact fingerprint devices provided by ManTech with the addition of wet ink ten-print cards. [2] 


\subsection{Fingerprint Devices}

Seven different fingerprinting devices were used for data collection. Both livescan and contactless devices were used as well as rolled ink impressions on a standard 10-print card. Table 3.1 lists the devices used in this data collection and the data that was collected from each device, while Figure 3.1 shows pictures of each of these devices.

Table 3.1 Fingerprint device details [2]

\begin{tabular}{|l|l|l|l|}
\hline Vendor & Sensor & Collection Type & Data Collected \\
\hline 1. CrossMatch & Guardian R2 & Livescan, contact & Left \& right hands, slaps \& rolls \\
\hline 2. L1/Morpho & TouchPrint 5300 & Livescan, contact & Left \& right hands, slaps \& rolls \\
\hline 3. CrossMatch & SEEK Avenger & Mobile, contact & Livescan equivalent to 10-print card \\
\hline 4. Northrup Grumman & BioSled & Mobile, contact & Livescan equivalent to 10-print card \\
\hline 5. MorphoTrak & MorphoIDent & Mobile, Contact & Left \& right hands, slaps only \\
\hline $\begin{array}{l}\text { 6. Advanced Optical } \\
\text { Systems (AOS) }\end{array}$ & ANDI On-The-Go & Portal, contactless & 4 fingers on right hands only \\
\hline 7. MorphoTrak & Finger-on-the-Fly & Livescan, contactless & Left \& right hands, 4 fingers only \\
\hline 8. IDair & InnerID (on iPhone) & Mobile, contactless & Left \& right hands, finger photos \\
\hline
\end{tabular}



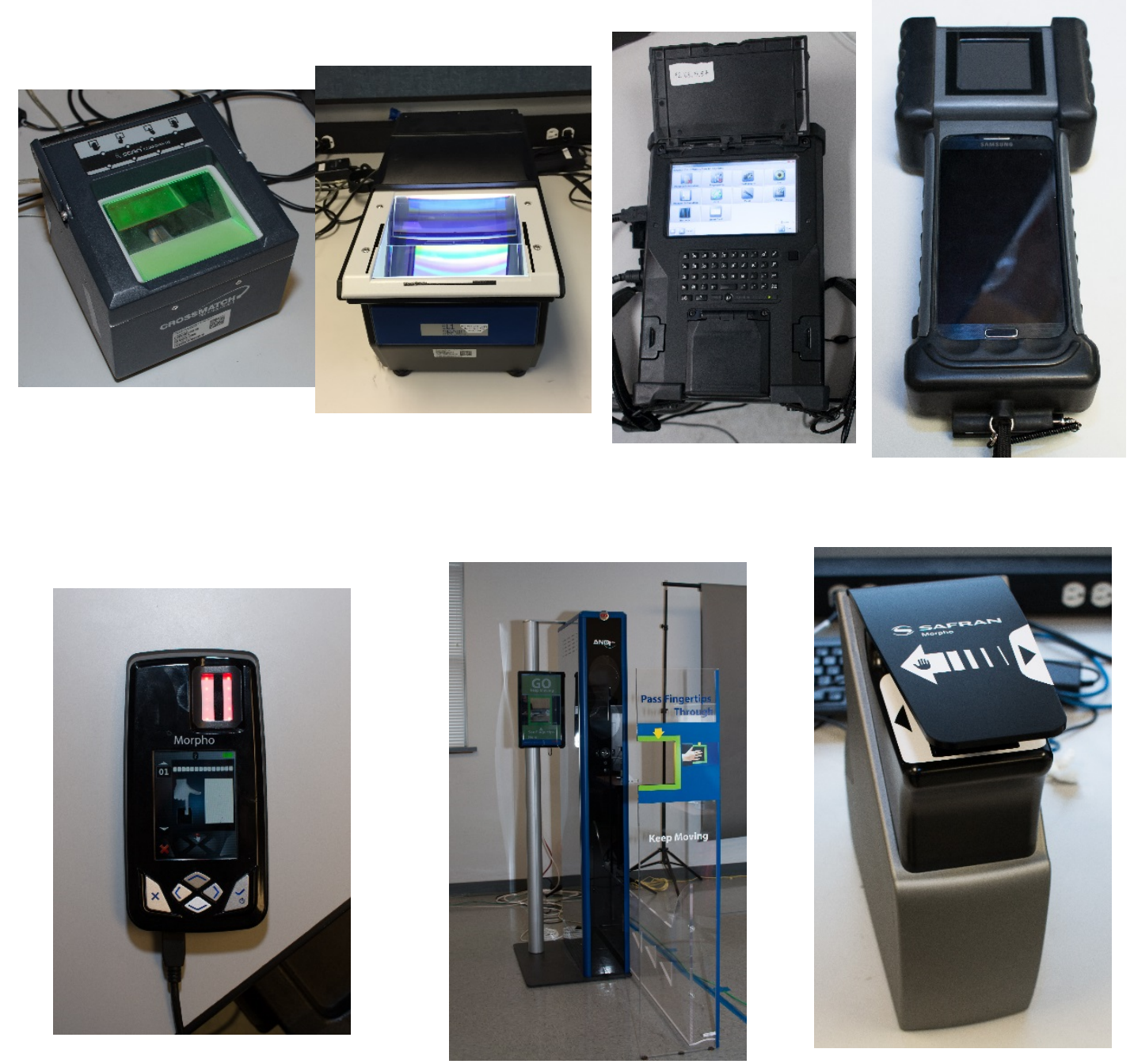

Figure 3.1: Fingerprint devices (from top left): CrossMatch Guardian R2, L1/Morpho TouchPrint 5300, CrossMatch SEEK Avenger, Northrup Grumman BioSled, MorphoTrak MorphoIDent, Advanced Optical Systems (AOS) ANDI On-The-Go, MorphoTrak Finger-on-the-Fly. The IDair InnerID (on iPhone) system was not available for photograph. [2] 


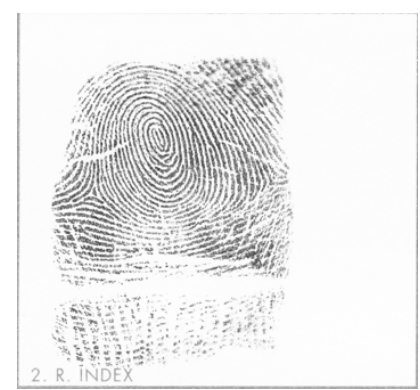

(a)

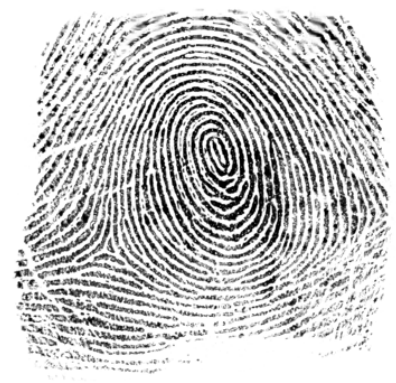

(c)

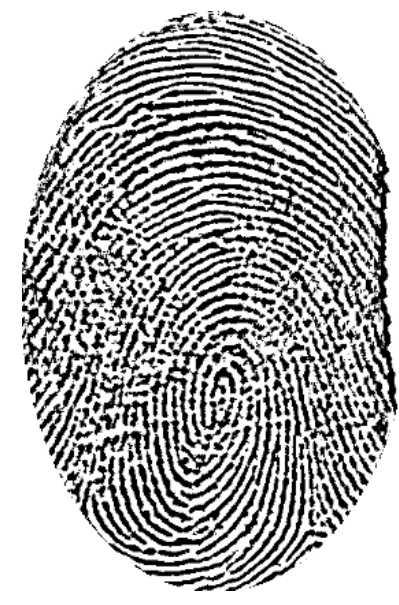

(e)

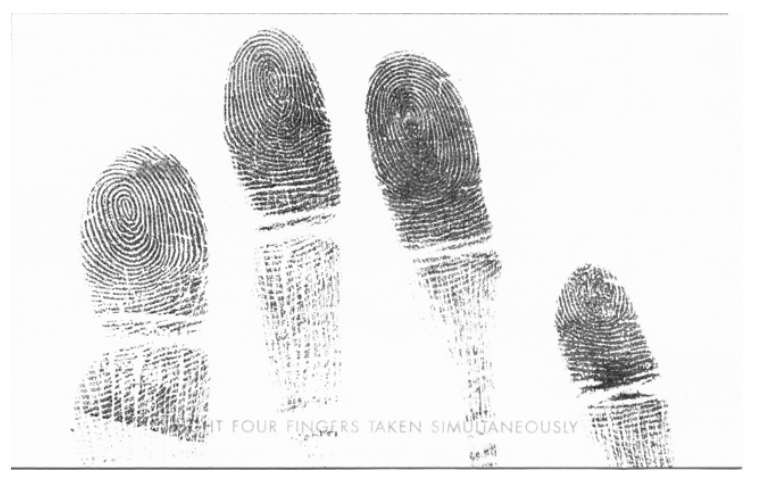

(b)
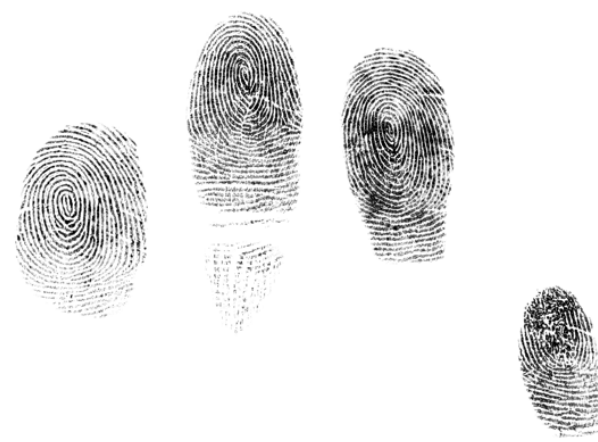

(d)

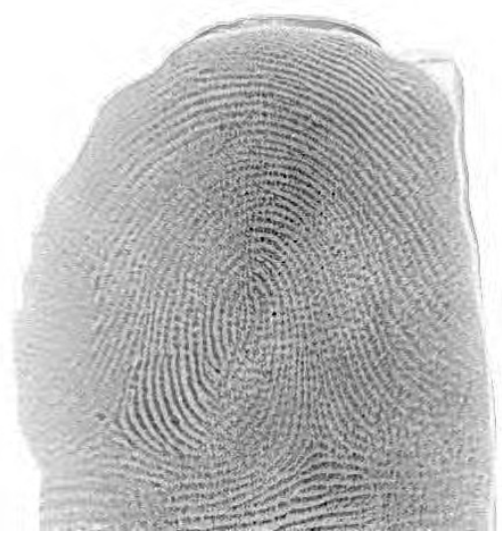

(f)

Figure 3.2 Sample images from a single individual for each method of capture (a) Tenprint scan of rolled print (b) Tenprint Scan of Four Finger Slap (c) Livescan rolled print image (d) Livescan Four Finger Slap image (e) ANDI OTG Right Index Finger Capture (f) Morpho FOTF Right Index Finger Segment 


\subsection{Collection Site}

The laboratory space that housed the various collection equipment was approximately $24 \times 24 \mathrm{ft}$. The collection area used approximately $12 \times 24 \mathrm{ft}$ of this space. Three different benches were used to acquire the data from the devices which were table top or mobile devices, while the ANDI OTG was a freestanding device. The first bench housed the MorphoIDent, BioSled, and InnerID devices, as well as a laptop for data transfer from these devices. The second bench was used to house the SEEK Avenger and Finger-On-The-Fly (FOTF) devices, and the control laptop for the FOTF system. The third bench housed the Guardian and Touchprint devices and their control laptop, as well as the control laptop for the ANDI On-The-Go (OTG) system. The OTG system was located in the middle of the collection area to allow space for the participant to gain a walking start before interaction with the system. A station for fingerprint impression collection was located on a nearby counter surface. A sink was available for wet ink cleanup after the fingerprint impression station. Figure 3.2 illustrates the laboratory layout for data collection. [2]

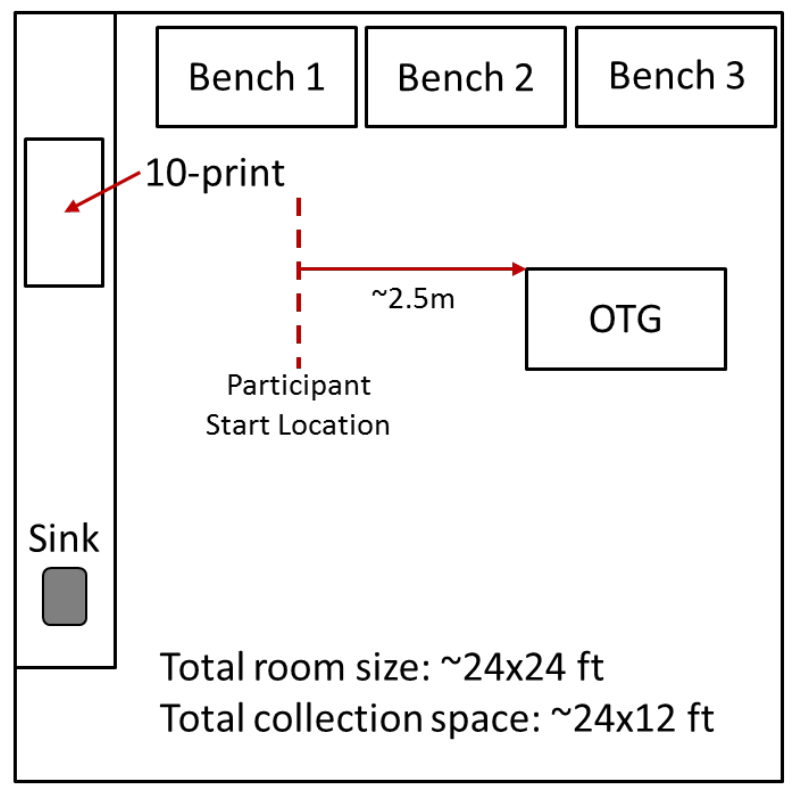

Bench 1:

- MorpholDent

- InnerID

- BioSled

- Laptop

Bench 2:

- SEEK Avenger

- FOTF

- Laptop

Bench 3:

- Guardian

- TouchPrint

- Laptop x2

Figure 3.3 Collection laboratory and station arrangement. [2] 


\subsection{Data Types and Organization}

Each participant provided two sequential sessions of fingerprints for each sensor. These sessions were collected within $30-45$ minutes of each other, so they show little variation due to collection time. The wet-ink prints were only collected once and were scanned at 500 and 1000ppi. The file structure for all of the electronically collected images is as follows:

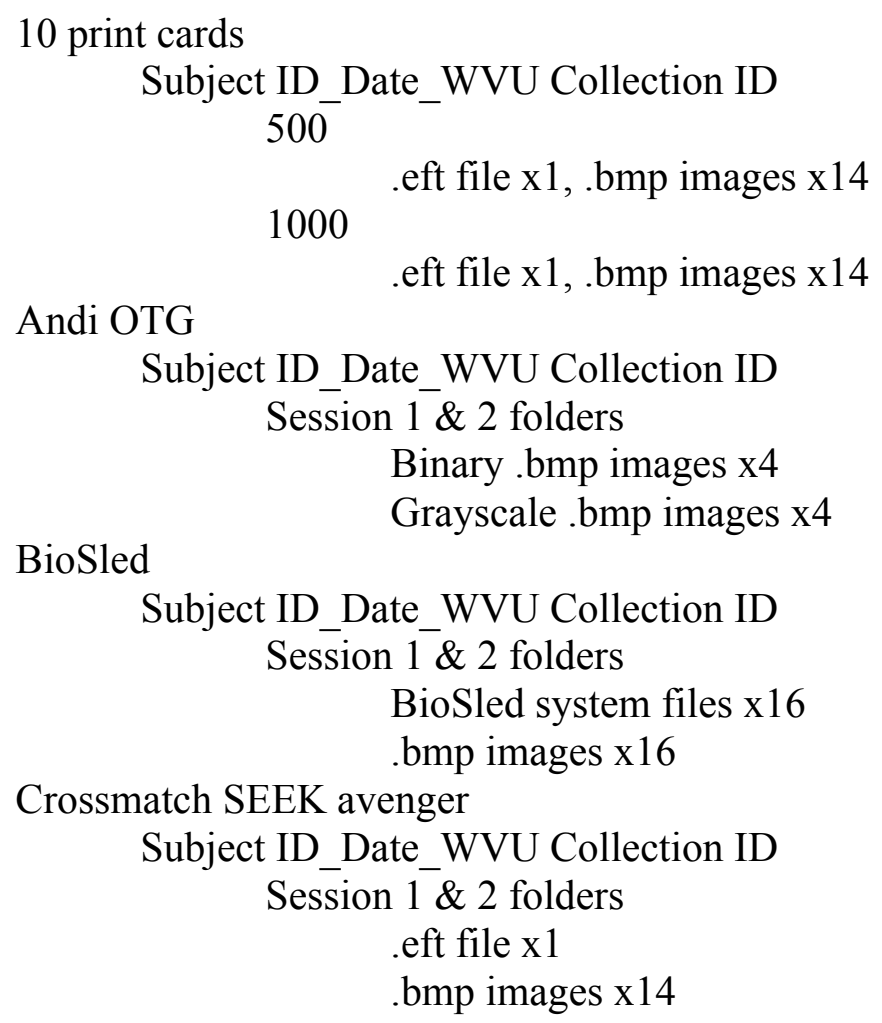

Subject ID_Date_WVU Collection ID

Session $1 \& 2$ folders BioSled system files x 16

.bmp images x16

Crossmatch SEEK avenger

Subject ID_Date_WVU Collection ID

Session $1 \& 2$ folders .eft file $\mathrm{x} 1$ .bmp images $\mathrm{x} 14$

FPII Guardian

Subject ID_Date_WVU Collection ID

Session $1 \& 2$ folders

Sensor date folder

innerID .bmp images $\mathrm{x} 13$

Subject ID_Date_WVU Collection ID

Session $1 \& 2$ folders .bmp images $\mathrm{x} 10$

L1 TouchPrint

Subject ID_Date_WVU Collection ID

Session $1 \& 2$ folders .bmp images $\mathrm{x} 13$ 


\author{
Morpho FOTF \\ Subject ID_Date_WVU Collection ID \\ Session $1 \& 2$ folders \\ Unsegmented .bmp images $\mathrm{x} 4$ \\ MorphoMobile \\ Segmented .bmp images x16 \\ Subject ID_Date_WVU Collection ID \\ Session $1 \& 2$ folders \\ .bmp images $\mathrm{x} 10$
}

The syntax of each fingerprint image filename is as follows:

SubjectRID_CollectionDate_CollectionNumber_SensorName_Slap/RollIdentifier_SessionNumber_Finger/SlapIdentifier.EXT

\title{
3.6 Collection Demographics
}

Figures $3.3-3.7$ provide information on cumulative participation in the data collection and a breakdown of ethnicity, age, and gender.

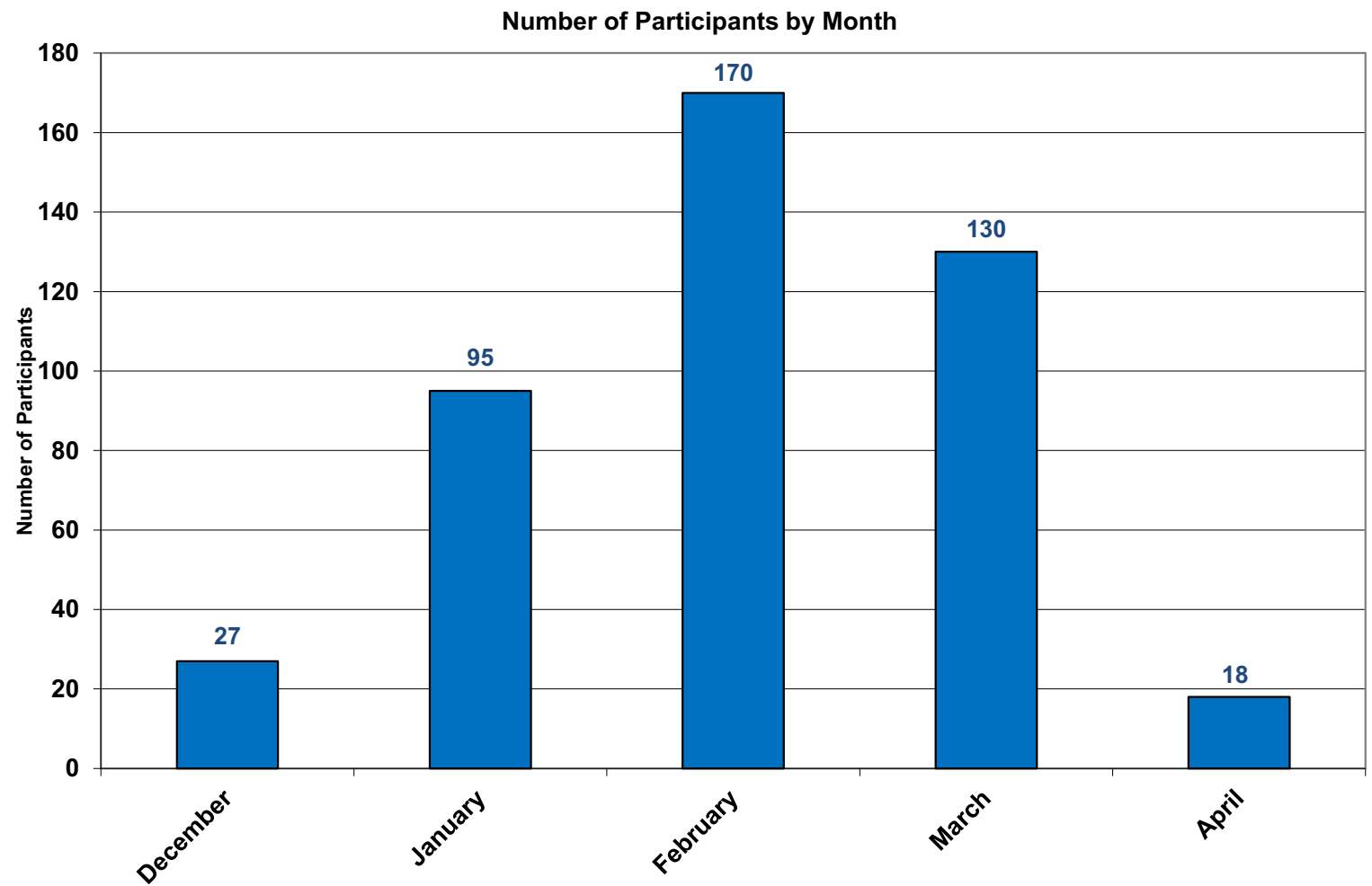

Figure 3.4 Number of participants by month 


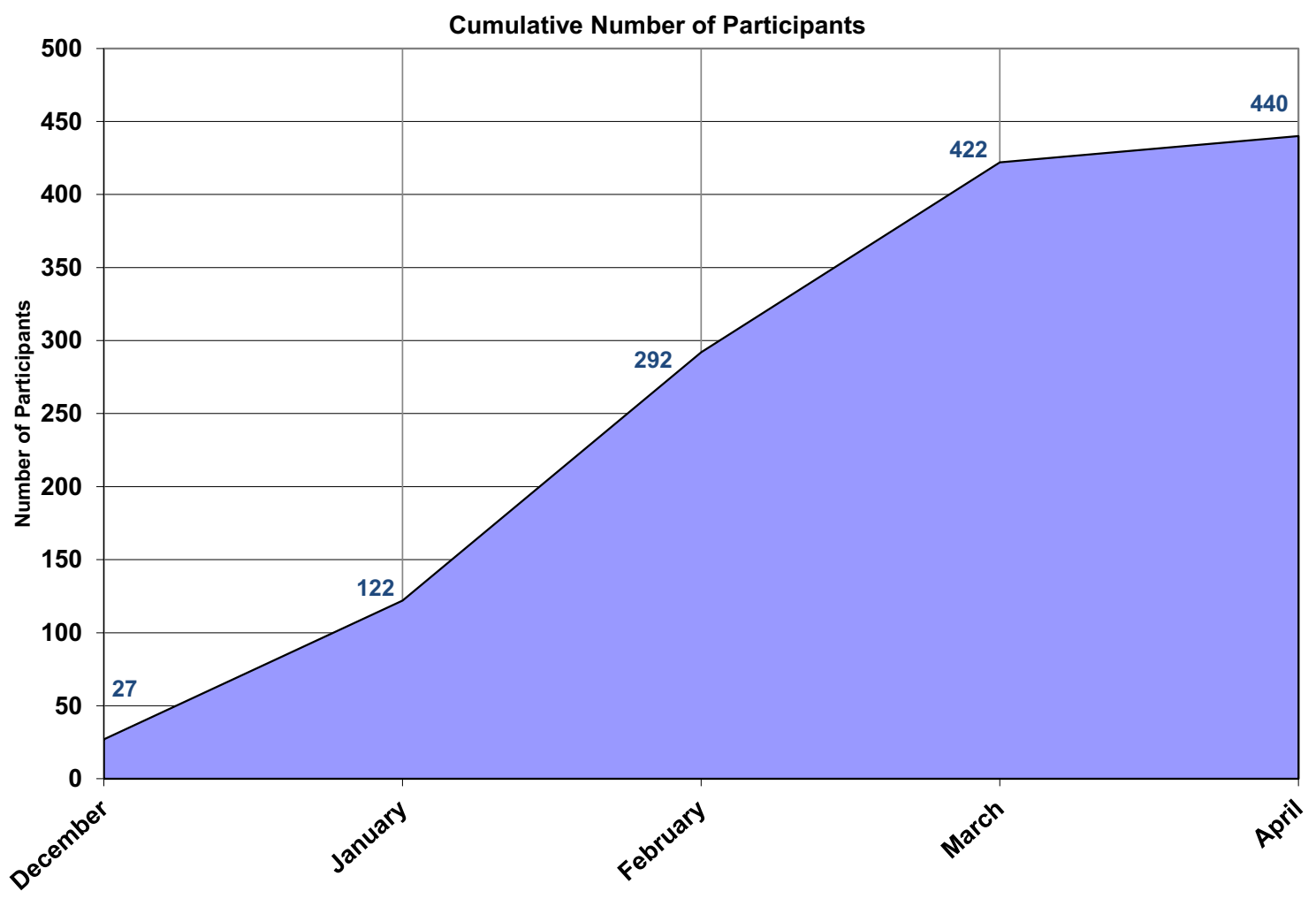

Figure 3.5 Cumulative Participation

\section{Participants by Ethnicity Group (\%)}

\begin{tabular}{|l|}
\hline Caucasian \\
$\square$ Asian \\
$\square$ Asian Indian \\
$\square$ African American \\
$\square$ African \\
$\square$ Middle Eastern \\
$\square$ Hispanic \\
$\square$ Other \\
$\square$ American Indian \\
$\square$ Unknown \\
\hline
\end{tabular}

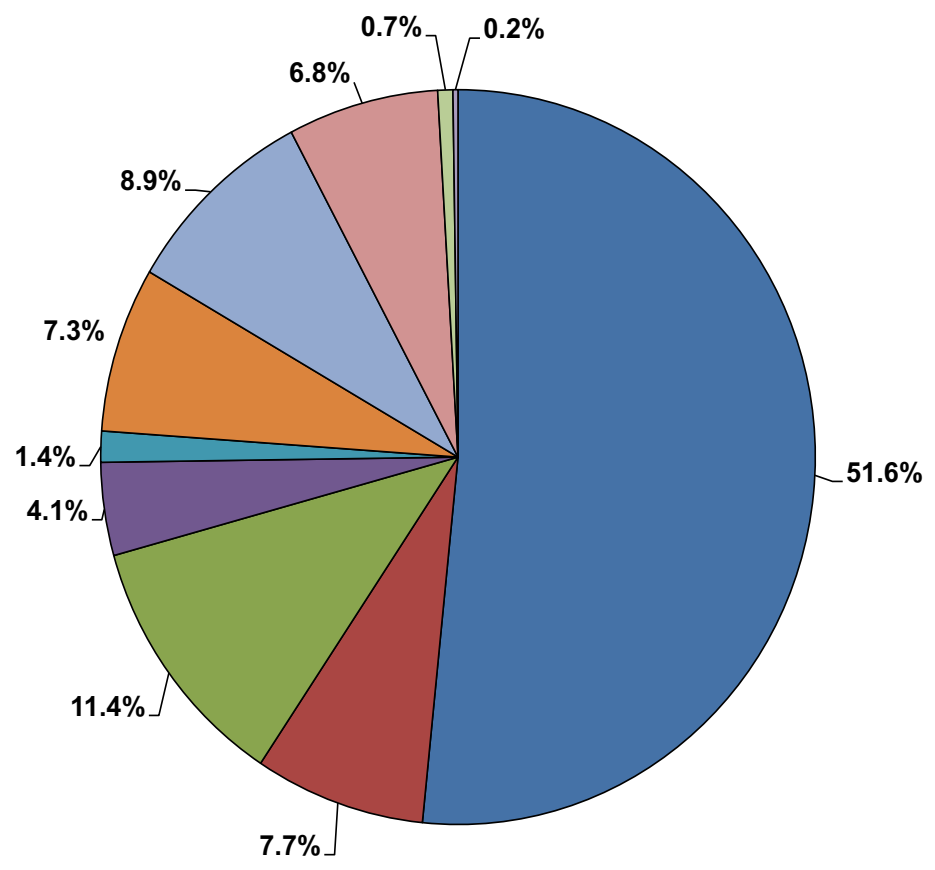

Figure 3.6 Participation Ethnicity 


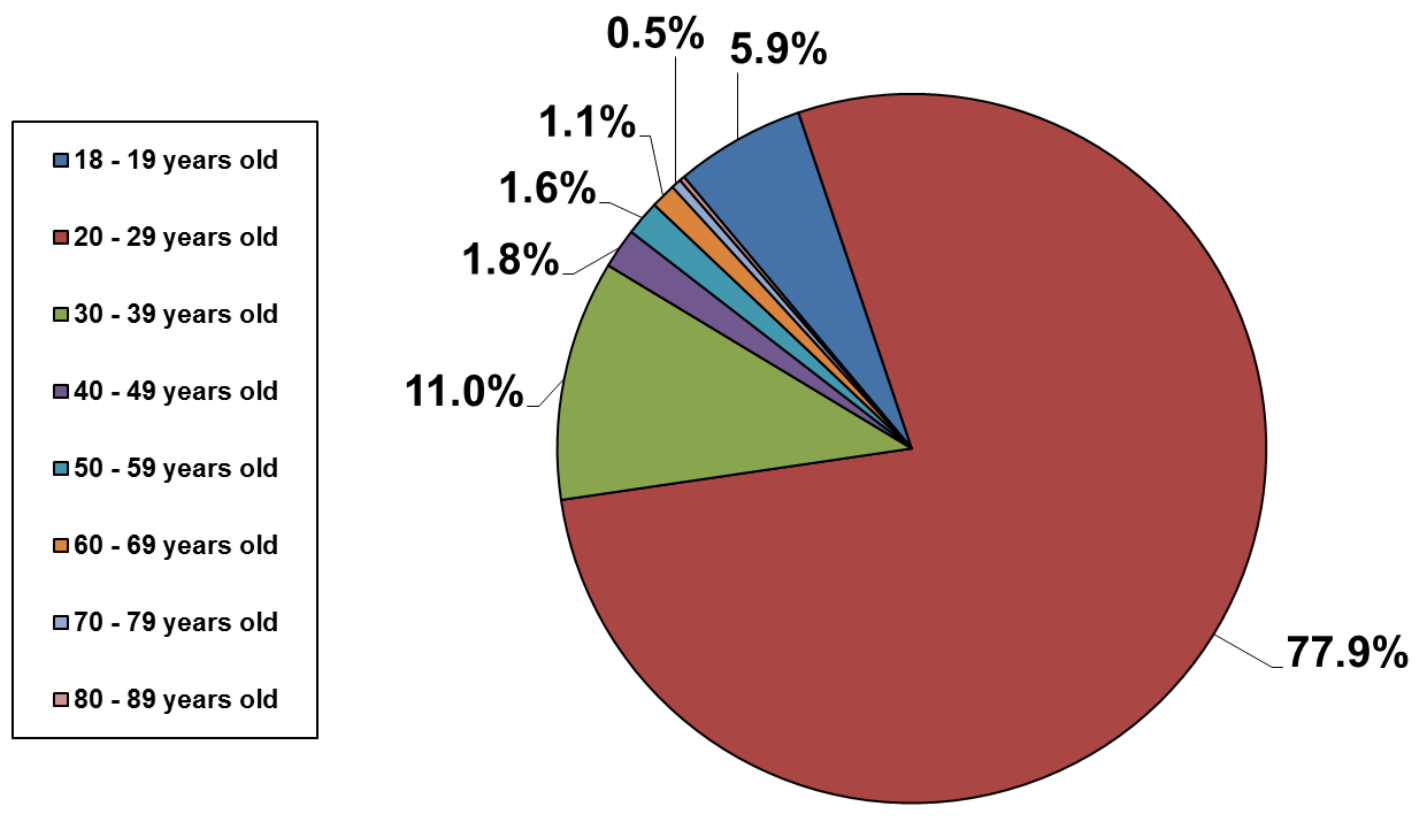

Figure 3.7 Participant Age

Demographics by Gender and Ethnicity

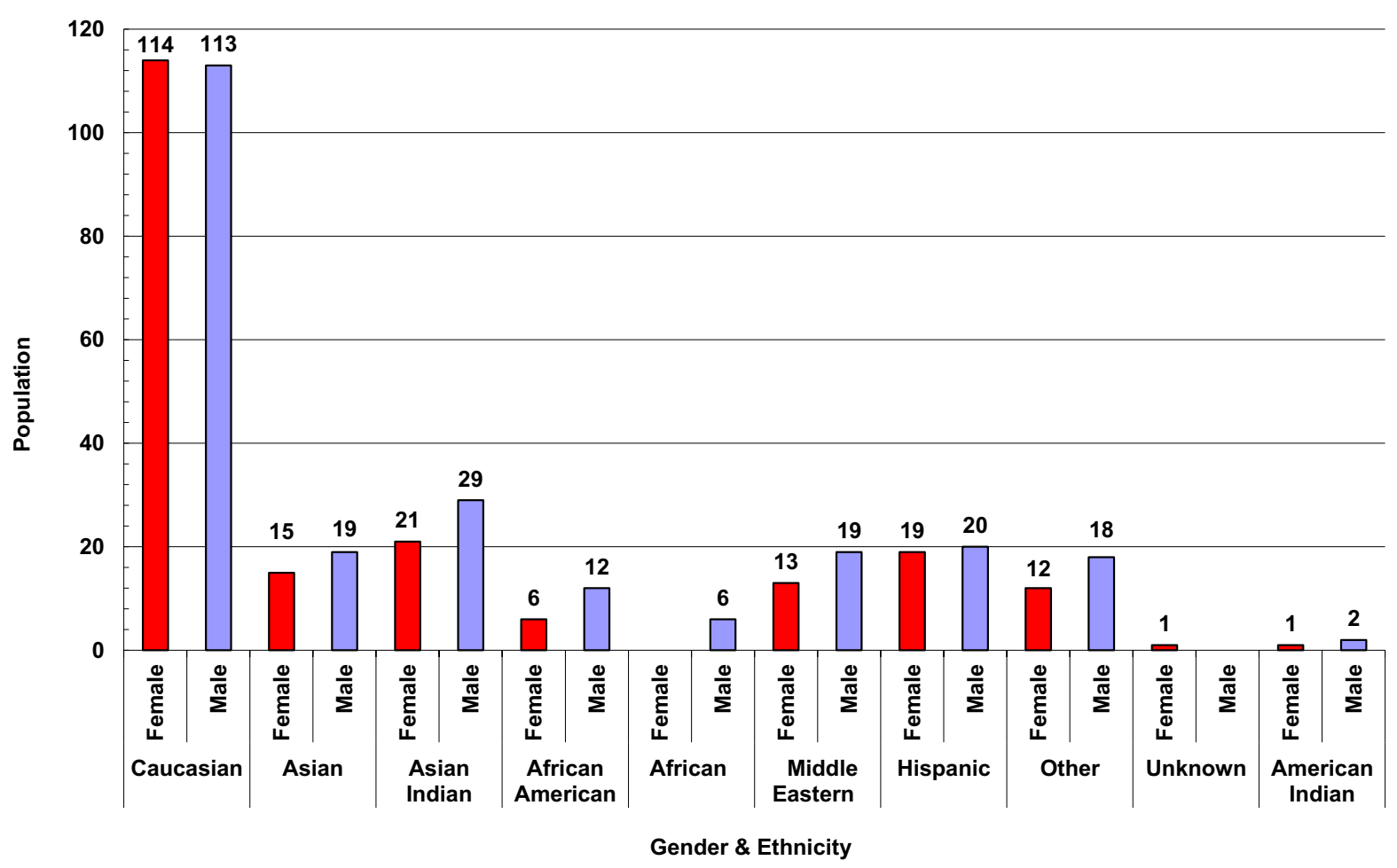

Figure 3.8 Breakdown of gender \& ethnicity 


\subsection{Post Processing}

The data collected was stored on each station's laptop computer where it was then compiled onto the storage server in weekly backup operations. With all data compiled, a script was written to rename and re-order the data according to the hierarchy listed in section 3.5. Once transferred, the fingerprint images were stored in .nst files which can be opened with NISTPack's TransactionEdit software. To extract these image files, the data was split among 4 workstations and each file was exported manually from the .nst files into .bmp format. Once all the files had been exported, a Matlab script was used to recognize and rename the files according to the format listed in section 3.5.

Data was delivered to ManTech in two releases. One took place after the first 200 participants, and the second after the final total 450 was achieved. Electronic records of the tenprint cards were delivered to the FBI due to IRB restrictions on data transfer. ManTech supplied WVU with an FBI-certified flat-bed scanner (Epson Perfection V700) and Aware Accuscan card scanning software to create the electronic records. Cards were scanned at both 500 and 1000ppi, and an .eft record and individual .bmp images were created for each participant in both resolutions.

Due to an error in the software of the ANDI OTG, with some participants, fingers were recorded erroneously, causing there to be duplicate images of the same finger on one hand. To accommodate this problem, all images used for this research were ran through the fingerprint matcher to find duplicates within one hand. The duplicates were then removed from the dataset. 


\subsection{Fingerprint Matchers}

Three matching algorithms were utilized in this research project; Neurotechnology MegaMatcher, National Institute of Standards and Technology (NIST) Biometric Image Software (NBIS), and SourceAFIS Fingerprint Recognition Toolkit. The Neurotechnology MegaMatcher comes in an SDK that is developed by Neurotechnology. It is a large-scale AFIS that was introduced back in 2005 and its used for multi-biometric identification [76]. It is compliant with rolled, flat, and latent fingerprints. NIST has recognized MegaMatcher fingerprint algorithm as MINEX compliant and suitable for use in personal identity verification (PIV) program applications [76]. The MegaMatcher software also includes fingerprint image quality determination [76]. The Neurotech MegaMatcher also claims to be tolerant to fingerprint translation, rotation, and deformation.

The next matching algorithm was developed by NIST and is called NBIS. This software is open source and available to download via NIST Biometric Open Souce Server [77]. For this research project, the NBIS.NET adaptation was used. This software has the capability to generate the NIST Fingerprint Image Quality of the images or the NFIQ score of the image. It also has the capability to deliver the number of minutia found in the image. The SourceAFIS fingerprint recognition toolkit is an open source SDK that utilizes a minutia based matching technique [78]. It incorporates some of the same components used in the NIST NBIS software.

For a more in depth report of this data collection, refer to "Evaluation of Contactless versus Contact Fingerprint Data, Phase 2" [2] 
Chapter 4:

Interoperability

Analysis 


\subsection{Fingerprint Image Match Score Analysis}

Each of the three matchers (NIST NBIS, Neurotech MegaMatcher, and SourceAFIS) were used to produce genuine and imposter scores as well as the matching scores between devices. These groups were then broken down into demographic strata to compare the results. Each of the matchers works similarly as a probe image is matched against the set of images in the gallery. To generate the genuine scores, a probe image is matched against a gallery which consist of other images from the same subject. To generate imposter scores, a probe image is matched against a gallery which consist of images from all the other subjects within the dataset. In both cases, the probe image is not included in the gallery. Each matching run resulted in a genuine and imposter match score list in the form of a comma separated variable (csv) file. These files were then imported into Matlab to generate the imposter and match score distributions as well as the KLD scores and ROC Curves. The Neurotech and SourceAFIS matchers output only the match scores, but the NIST NBIS matcher was also used to acquire the minutiae counts and NFIQ scores for each image, these results were used to create the minutia box plots that are shown in Appendix A and the NIFQ score Distributions(shown in Figures $4.1-4.4$ ). Table 4.2 shows the results of the ManTech Study Compared to ours. Tables $4.3-4.8$ show the number of rank 1 true and false matches as well as the rate rank 1 rates for each matching run. The outliers and some of the normal genuine and imposter distributions are shown, all graphs/results can be viewed in Appendix A. The results to the demographic strata tests are shown in Appendix B, C and D. Appendix B deals with the gender-based study, Appendix C shows the age-based study and Appendix D displays the ethnicity-based study. Table 4.1 shows the abbreviations used in results tables in this chapter and chapter 5. 
Table 4.1 Device Abbreviations

\begin{tabular}{|l|l|}
\hline Code & Definition \\
\hline $10 \mathrm{p}$ & Ten Print Card (Scanned at 1000 \& 500 dpi) \\
\hline IID & InnerID (iPhone App) \\
\hline MFF & Safran Morpho Finger on the Fly \\
\hline CSA & CrossMatch SEEK Avenger \\
\hline BIO & Northrup Grumman BioSled \\
\hline ANDI BIN & ANDI On the Go (OTG) Binary Images \\
\hline ANDI GRY & ANDI On the Go (OTG) Greyscale Images \\
\hline L1 & L-1 Scanner \\
\hline CG2 & CrossMatch Guardian 2 \\
\hline SMM & Safran Morpho Mobile MorphoIDent \\
\hline
\end{tabular}

Table 4.2 ManTech Table Comparison to Thesis Results

\begin{tabular}{|l|l|l|l|l|l|}
\hline Probe & Gallery & ManTech & NBIS & Neurotech & SourceAFIS \\
\hline CG2 R & CG2 R & $\mathbf{1 0 0}$ & 100 & 100 & 100 \\
\hline MFF & MFF & $\mathbf{9 9 . 8}$ & 100 & 100 & 100 \\
\hline CSA R & CG2 R & $\mathbf{9 7 . 8}$ & 97.75 & 99.5 & 97.75 \\
\hline CSA & CG2 R & $\mathbf{9 5}$ & 98.1 & 99.61 & 98.01 \\
\hline SMM & CG2 R & $\mathbf{9 6 . 5}$ & 95.63 & 99.4 & 95.63 \\
\hline L1 R & CG2 R & $\mathbf{9 7}$ & 98.74 & 99.09 & 98.74 \\
\hline L1 & CG2 R & $\mathbf{9 6 . 7}$ & 97.47 & 99.19 & 97.47 \\
\hline BioSled R & CG2 R & $\mathbf{9 6 . 3}$ & 95.11 & 98.4 & 95.11 \\
\hline BioSled & CG2 R & $\mathbf{9 4 . 2}$ & 95.35 & 97.2 & 95.35 \\
\hline ANDI GRY & CG2 R & $\mathbf{7 6}$ & 74.33 & 97.19 & 74.33 \\
\hline ANDI BIN & CG2 R & $\mathbf{8 3 . 6}$ & 86.66 & 97.02 & 86.65 \\
\hline MFF & CG2 R & $\mathbf{9 2 . 4}$ & 93.22 & 98.68 & 93.21 \\
\hline CG2 R & MFF & $\mathbf{9 2}$ & 93.22 & 98.68 & 93.21 \\
\hline CSA & MFF & $\mathbf{8 7 . 2}$ & 95.93 & 98.69 & 95.93 \\
\hline ANDI GRY & MFF & $\mathbf{7 9 . 5}$ & 74.66 & 97.27 & 74.66 \\
\hline ANDI BIN & MFF & $\mathbf{8 3 . 2}$ & 85.10 & 94.89 & 83.86 \\
\hline IID & MFF & $\mathbf{2}$ & .32 & 1.94 & .32 \\
\hline
\end{tabular}

Table 4.2 shows that our results compared to those obtained in the ManTech study detailed in [2]. The values highlighted in red represent the TMR1 accuracies that increased when the full dataset was considered in the interoperability assessment. ManTech used the FP II Guardian Rolls (CG2 R) as the baseline comparison to compare all the other devices to. Most of the results presented here indicate a higher accuracy. This is noteworthy since, typically, when more data is added, overall rank 1 accuracy is expected to decline. This could have been caused 
by ManTech using only the participants that had data for all devices, as some subjects that didn't have data for every device could have produced higher results than those that had data for every device. Another possibility of why this occurred is that by the time second part of the collection was happening the operators would have had more experience and training and been able to better use each of the devices. 
Table 4.3 NBIS TMR1 Table

Blue shading $=$ Contactless vs Contactless, Orange Shading $=$ Contactless vs Contact-Based,

Green shading $=$ Contact vs Contact

\begin{tabular}{|c|c|c|c|c|c|}
\hline Match & Total & TMR1 & FMR1 & TMR1 Rate & FMR1 Rate \\
\hline ANDI BIN - ANDI BIN & 830 & 830 & 0 & 100 & 0 \\
\hline ANDI BIN - ANDI GRY & 830 & 829 & 1 & 99.88 & 0.12 \\
\hline ANDI BIN - IID & 316 & 5 & 311 & 1.58 & 98.42 \\
\hline ANDI BIN - MFF & 812 & 691 & 121 & 85.1 & 14.9 \\
\hline ANDI GRY - ANDI GRY & 831 & 831 & 0 & 100 & 0 \\
\hline ANDI GRY - IID & 316 & 5 & 311 & 1.58 & 98.42 \\
\hline ANDI GRY - MFF & 813 & 607 & 206 & 74.66 & 25.34 \\
\hline IID - IID & 319 & 319 & 0 & 100 & 0 \\
\hline IID - MFF & 315 & 1 & 314 & 0.32 & 99.68 \\
\hline MFF - MFF & 1734 & 1734 & 0 & 100 & 0 \\
\hline $10 p-A N D I B I N$ & 381 & 296 & 85 & 77.69 & 22.31 \\
\hline 10p - ANDI GRY & 381 & 260 & 121 & 68.24 & 31.76 \\
\hline $10 p-I I D$ & 144 & 3 & 141 & 2.08 & 97.92 \\
\hline $10 p-M F F$ & 395 & 336 & 59 & 85.06 & 14.94 \\
\hline ANDI BIN - BIO & 767 & 656 & 111 & 85.53 & 14.47 \\
\hline ANDI BIN - CSA & 753 & 679 & 74 & 90.17 & 9.83 \\
\hline ANDI BIN - CG2 & 811 & 738 & 73 & 91 & 9 \\
\hline ANDI BIN - L1 & 820 & 721 & 99 & 87.93 & 12.07 \\
\hline ANDI BIN - SMM & 800 & 700 & 100 & 87.5 & 12.5 \\
\hline ANDI GRY - BIO & 768 & 540 & 228 & 70.31 & 29.69 \\
\hline ANDI GRY - CSA & 754 & 584 & 170 & 77.45 & 22.55 \\
\hline ANDI GRY - CG2 & 812 & 623 & 189 & 76.72 & 23.28 \\
\hline ANDI GRY - L1 & 821 & 614 & 207 & 74.79 & 25.21 \\
\hline ANDI GRY - SMM & 801 & 580 & 221 & 72.41 & 27.59 \\
\hline BIO - IID & 297 & 6 & 291 & 2.02 & 97.98 \\
\hline $\mathrm{BIO}$ - MFF & 785 & 709 & 76 & 90.32 & 9.68 \\
\hline CSA - IID & 289 & 7 & 282 & 2.42 & 97.58 \\
\hline CSA - MFF & 762 & 731 & 31 & 95.93 & 4.07 \\
\hline CG2 - IID & 306 & 3 & 303 & 0.98 & 99.02 \\
\hline CG2 - MFF & 847 & 809 & 38 & 95.51 & 4.49 \\
\hline IID - L1 & 315 & 0 & 315 & 0 & 100 \\
\hline IID - SMM & 313 & 1 & 312 & 0.32 & 99.68 \\
\hline L1 - MFF & 861 & 821 & 40 & 95.35 & 4.65 \\
\hline MFF - SMM & 1666 & 1458 & 208 & 87.52 & 12.48 \\
\hline $10 p-10 p$ & 405 & 405 & 0 & 100 & 0 \\
\hline $10 p-B I O$ & 375 & 330 & 45 & 88 & 12 \\
\hline $10 p-C S A$ & 369 & 337 & 32 & 91.33 & 8.67 \\
\hline $10 p-C G 2$ & 398 & 364 & 34 & 91.46 & 8.54 \\
\hline $10 p-L 1$ & 401 & 368 & 33 & 91.77 & 8.23 \\
\hline $10 p-S M M$ & 388 & 339 & 49 & 87.37 & 12.63 \\
\hline $\mathrm{BIO}-\mathrm{BIO}$ & 806 & 806 & 0 & 100 & 0 \\
\hline BIO - CSA & 738 & 709 & 29 & 96.07 & 3.93 \\
\hline $\mathrm{B} 1 \mathrm{O}-\mathrm{CG} 2$ & 792 & 758 & 34 & 95.71 & 4.29 \\
\hline BIO - L1 & 801 & 769 & 32 & 96 & 4 \\
\hline BIO - SMM & 778 & 746 & 32 & 95.89 & 4.11 \\
\hline CSA - CSA & 784 & 784 & 0 & 100 & 0 \\
\hline CSA - CG2 & 771 & 763 & 8 & 98.96 & 1.04 \\
\hline CSA - L1 & 778 & 770 & 8 & 98.97 & 1.03 \\
\hline CSA - SMM & 758 & 753 & 5 & 99.34 & 0.66 \\
\hline CG2 - CG2 & 870 & 870 & 0 & 100 & 0 \\
\hline CG2 - L1 & 860 & 846 & 14 & 98.37 & 1.63 \\
\hline CG2 - SMM & 838 & 817 & 21 & 97.49 & 2.51 \\
\hline L1 - L1 & 883 & 883 & 0 & 100 & 0 \\
\hline L1 - SMM & 849 & 832 & 17 & 98 & 2 \\
\hline SMM - SMM & 859 & 859 & 0 & 100 & 0 \\
\hline
\end{tabular}


Table 4.4 Neurotech TMR1 Table

Blue shading $=$ Contactless vs Contactless, Orange Shading $=$ Contactless vs Contact-Based,

Green shading $=$ Contact vs Contact

\begin{tabular}{|c|c|c|c|c|c|}
\hline Neurotech & Total & TMR1 & FMR1 & TMR1 Rate & FMR1 Rate \\
\hline \multirow{2}{*}{$\begin{array}{l}\text { ANDI BIN - ANDI BIN } \\
\text { ANDI BIN - ANDI GRY }\end{array}$} & 825 & 825 & 0 & 100 & 0 \\
\hline & 804 & 802 & 2 & 99.75 & 0.25 \\
\hline \multirow{8}{*}{$\begin{array}{r}\text { ANDI BIN - IID } \\
\text { ANDI BIN - MFF } \\
\text { ANDI GRY - ANDI GRY } \\
\text { ANDI GRY - IID } \\
\text { ANDI GRY - MFF } \\
\text { IID - IID } \\
\text { IID - MFF } \\
\text { MFF - MFF }\end{array}$} & 319 & 9 & 310 & 2.82 & 97.18 \\
\hline & 822 & 780 & 42 & 94.89 & 5.11 \\
\hline & 796 & 796 & 0 & 100 & 0 \\
\hline & 287 & 5 & 282 & 1.74 & 98.26 \\
\hline & 768 & 747 & 21 & 97.27 & 2.73 \\
\hline & 273 & 273 & 0 & 100 & 0 \\
\hline & 309 & 6 & 303 & 1.94 & 98.06 \\
\hline & 1626 & 1626 & 0 & 100 & 0 \\
\hline \multirow{3}{*}{$\begin{array}{r}10 p-\text { ANDI BIN } \\
10 p-\text { ANDI GRY } \\
10 p-M F F\end{array}$} & 381 & 372 & 9 & 97.64 & 2.36 \\
\hline & 355 & 347 & 8 & 97.75 & 2.25 \\
\hline & 396 & 381 & 15 & 96.21 & 3.79 \\
\hline \multirow{2}{*}{$\begin{array}{l}\text { ANDI BIN - BIO } \\
\text { ANDI BIN - CSA }\end{array}$} & 778 & 730 & 48 & 93.83 & 6.17 \\
\hline & 764 & 732 & 32 & 95.81 & 4.19 \\
\hline $\begin{array}{l}\text { ANDI BIN - CG2 } \\
\text { ANDI BIN - I1 }\end{array}$ & 822 & 788 & 34 & 95.86 & 4.14 \\
\hline \multirow{2}{*}{$\begin{array}{r}\text { ANDI BIN - L1 } \\
\text { ANDI BIN - SMM }\end{array}$} & 832 & 794 & 38 & 95.43 & 4.57 \\
\hline & 810 & 770 & 40 & 95.06 & 4.94 \\
\hline ANDI GRY - BIO & 735 & 694 & 41 & 94.42 & 5.58 \\
\hline ANDI GRY - CSA & 721 & 698 & 23 & 96.81 & 3.19 \\
\hline ANDI GRY - CG2 & 783 & 756 & 27 & 96.55 & 3.45 \\
\hline \multirow{2}{*}{ ANDI GRY - L1 } & 790 & 767 & 23 & 97.09 & 2.91 \\
\hline & 767 & 739 & 28 & 96.35 & 3.65 \\
\hline $\begin{array}{r}\text { ANDI GRY - SMM } \\
\text { BIO - IID }\end{array}$ & 297 & 5 & 292 & 1.68 & 98.32 \\
\hline BIO - MFF & 785 & 758 & 27 & 96.56 & 3.44 \\
\hline CSA - IID & 287 & 6 & 281 & 2.09 & 97.91 \\
\hline CSA - MFF & 762 & 752 & 10 & 98.69 & 1.31 \\
\hline CG2 - IID & 304 & 3 & 301 & 0.99 & 99.01 \\
\hline CG2 - MFF & 847 & 831 & 16 & 98.11 & 1.89 \\
\hline \multirow{2}{*}{$\begin{array}{r}\text { IID - L1 } \\
\text { IID - SMM }\end{array}$} & 311 & 4 & 307 & 1.29 & 98.71 \\
\hline & 307 & 2 & 305 & 0.65 & 99.35 \\
\hline L1 - MFF & 861 & 847 & 14 & 98.37 & 1.63 \\
\hline MFF - SMM & 1662 & 1638 & 24 & 98.56 & 1.44 \\
\hline $10 p-10 p$ & 388 & 388 & 0 & 100 & 0 \\
\hline $10 p-B I O$ & 376 & 362 & 14 & 96.28 & 3.72 \\
\hline $10 p-C S A$ & 370 & 367 & 3 & 99.19 & 0.81 \\
\hline $10 p-C G 2$ & 399 & 396 & 3 & 99.25 & 0.75 \\
\hline $10 p-L 1$ & 402 & 400 & 2 & 99.5 & 0.5 \\
\hline $10 p-S M M$ & 389 & 380 & 9 & 97.69 & 2.31 \\
\hline $\mathrm{BIO}-\mathrm{BIO}$ & 802 & 802 & 0 & 100 & 0 \\
\hline BIO - CSA & 738 & 717 & 21 & 97.15 & 2.85 \\
\hline $\mathrm{B} 1 \mathrm{O}-\mathrm{CG} 2$ & 792 & 770 & 22 & 97.22 & 2.78 \\
\hline BIO - L1 & 801 & 780 & 21 & 97.38 & 2.62 \\
\hline BIO - SMM & 778 & 756 & 22 & 97.17 & 2.83 \\
\hline CSA - CSA & 783 & 783 & 0 & 100 & 0 \\
\hline CSA - CG2 & 771 & 767 & 4 & 99.48 & 0.52 \\
\hline CSA - L1 & 778 & 776 & 2 & 99.74 & 0.26 \\
\hline CSA - SMM & 758 & 757 & 1 & 99.87 & 0.13 \\
\hline CG2 - CG2 & 867 & 867 & 0 & 100 & 0 \\
\hline CG2 - L1 & 860 & 849 & 11 & 98.72 & 1.28 \\
\hline CG2 - SMM & 838 & 828 & 10 & 98.81 & 1.19 \\
\hline L1 - L1 & 883 & 883 & 0 & 100 & 0 \\
\hline L1 - SMM & 849 & 843 & 6 & 99.29 & 0.71 \\
\hline SMM - SMM & 855 & 855 & 0 & 100 & 0 \\
\hline
\end{tabular}


Table 4.5 SourceAFIS TMR1 Table

Blue shading $=$ Contactless vs Contactless, Orange Shading $=$ Contactless vs Contact-Based,

Green shading $=$ Contact vs Contact

\begin{tabular}{|c|c|c|c|c|c|}
\hline SourceAFIS & Total & TMR1 & FMR1 & TMR1 Rate & FMR1 Rate \\
\hline ANDI BIN - ANDI BIN & 830 & 830 & 0 & 100 & 0 \\
\hline ANDI BIN - ANDI GRY & 830 & 829 & 1 & 99.88 & 0.12 \\
\hline ANDI BIN - IID & 321 & 5 & 311 & 1.56 & 96.88 \\
\hline ANDI BIN - MFF & 824 & 691 & 121 & 83.86 & 14.68 \\
\hline ANDI GRY - ANDI GRY & 831 & 831 & 0 & 100 & 0 \\
\hline ANDI GRY - IID & 316 & 5 & 311 & 1.58 & 98.42 \\
\hline ANDI GRY - MFF & 813 & 607 & 206 & 74.66 & 25.34 \\
\hline IID - IID & 319 & 319 & 0 & 100 & 0 \\
\hline IID - MFF & 315 & 1 & 314 & 0.32 & 99.68 \\
\hline MFF - MFF & 1734 & 1734 & 0 & 100 & 0 \\
\hline $10 p-A N D I$ BIN & 380 & 296 & 85 & 77.89 & 22.37 \\
\hline $10 p-A N D I G R Y$ & 381 & 260 & 121 & 68.24 & 31.76 \\
\hline $10 p-I I D$ & 144 & 3 & 141 & 2.08 & 97.92 \\
\hline $10 p-M F F$ & 395 & 336 & 59 & 85.06 & 14.94 \\
\hline ANDI BIN - BIO & 778 & 656 & 111 & 84.32 & 14.27 \\
\hline ANDI BIN - CSA & 764 & 679 & 74 & 88.87 & 9.69 \\
\hline ANDI BIN - CG2 & 822 & 738 & 73 & 89.78 & 8.88 \\
\hline ANDI BIN - L1 & 832 & 721 & 99 & 86.66 & 11.9 \\
\hline ANDI BIN - SMM & 800 & 700 & 100 & 87.5 & 12.5 \\
\hline ANDI GRY - BIO & 768 & 540 & 228 & 70.31 & 29.69 \\
\hline ANDI GRY - CSA & 754 & 584 & 170 & 77.45 & 22.55 \\
\hline ANDI GRY - CG2 & 812 & 623 & 189 & 76.72 & 23.28 \\
\hline ANDI GRY - L1 & 821 & 614 & 207 & 74.79 & 25.21 \\
\hline ANDI GRY - SMM & 801 & 580 & 221 & 72.41 & 27.59 \\
\hline BIO - IID & 297 & 6 & 291 & 2.02 & 97.98 \\
\hline $\mathrm{BIO}-\mathrm{MFF}$ & 785 & 709 & 76 & 90.32 & 9.68 \\
\hline CSA - IID & 289 & 7 & 282 & 2.42 & 97.58 \\
\hline CSA - MFF & 762 & 731 & 31 & 95.93 & 4.07 \\
\hline CG2 - IID & 306 & 3 & 303 & 0.98 & 99.02 \\
\hline CG2 - MFF & 847 & 809 & 38 & 95.51 & 4.49 \\
\hline IID - L1 & 315 & 0 & 315 & 0 & 100 \\
\hline IID - SMM & 313 & 1 & 312 & 0.32 & 99.68 \\
\hline L1 - MFF & 861 & 821 & 40 & 95.35 & 4.65 \\
\hline MFF - SMM & 1666 & 1458 & 208 & 87.52 & 12.48 \\
\hline $10 p-10 p$ & 406 & 405 & 0 & 99.75 & 0 \\
\hline $10 p-B I O$ & 375 & 330 & 45 & 88 & 12 \\
\hline $10 p-C S A$ & 369 & 337 & 32 & 91.33 & 8.67 \\
\hline $10 p-C G 2$ & 398 & 364 & 34 & 91.46 & 8.54 \\
\hline 10p - L1 & 401 & 368 & 33 & 91.77 & 8.23 \\
\hline $10 p-S M M$ & 388 & 339 & 49 & 87.37 & 12.63 \\
\hline $\mathrm{BIO}-\mathrm{BIO}$ & 806 & 806 & 0 & 100 & 0 \\
\hline BIO - CSA & 738 & 709 & 29 & 96.07 & 3.93 \\
\hline $\mathrm{BIO}-\mathrm{CG} 2$ & 792 & 758 & 34 & 95.71 & 4.29 \\
\hline BIO - L1 & 801 & 769 & 32 & 96 & 4 \\
\hline BIO - SMM & 778 & 746 & 32 & 95.89 & 4.11 \\
\hline CSA - CSA & 784 & 784 & 0 & 100 & 0 \\
\hline CSA - CG2 & 771 & 763 & 8 & 98.96 & 1.04 \\
\hline CSA - L1 & 778 & 770 & 8 & 98.97 & 1.03 \\
\hline CSA - SMM & 758 & 753 & 5 & 99.34 & 0.66 \\
\hline CG2 - CG2 & 870 & 870 & 0 & 100 & 0 \\
\hline CG2 - L1 & 860 & 846 & 14 & 98.37 & 1.63 \\
\hline CG2 - SMM & 838 & 817 & 21 & 97.49 & 2.51 \\
\hline L1 - L1 & 883 & 883 & 0 & 100 & 0 \\
\hline L1 - SMM & 849 & 832 & 17 & 98 & 2 \\
\hline SMM - SMM & 859 & 859 & 0 & 100 & 0 \\
\hline
\end{tabular}


The Total column in Tables 4.3 - 4.5 shows the total number of rank 1 matches acquired. The TMR1 and FMR1 columns denote the number of True Match Rank 1 and False Match Rank 1 matches out of the total number of matches. The True Match Rank 1 Rate is the number of TMR1 Matches divided by the total number of matches. The TMR1 values that are highest, or share the highest value of the three matchers, are denoted in red. The blue shading in Tables 4.3 4.5, denote the matches between contactless devices, the orange denotes matches between contactless and contact-based devices and the green is for contact-based devices only. The total number of matches may vary within the Neurotech TMR1 Table 4.4 compared to the NBIS and SourceAFIS totals. This is because the Neurotech matcher removed images of bad quality from the gallery, which led to fewer matches. This is most likely why the Neurotech matcher produced the best TMR1 accuracy for almost every test. The matching algorithms used were not optimized for use with images obtained from contactless methods of capture, this may account for the lower matching accuracies when contactless methods of capture were involved. The TMR1 accuracy Ranges between matchers can better viewed in Table 5.2.

Table 4.6 NBIS Rolls TMR1 Table

\begin{tabular}{|c|c|c|c|c|c|c|}
\hline NBIS Rolls & Match & Total & TMR1 & FMR1 & TMR1 Rate & FMR1 Rate \\
\hline & $10 p R-10 p R$ & 417 & 417 & 0 & 100.00 & 0.00 \\
\hline & $10 p R-B I O R$ & 389 & 342 & 47 & 87.92 & 12.08 \\
\hline & $10 p R-C S A R$ & 383 & 349 & 34 & 91.12 & 8.88 \\
\hline & $10 p R-C G 2 R$ & 412 & 369 & 43 & 89.56 & 10.44 \\
\hline & $10 p R-L 1 R$ & 415 & 372 & 43 & 89.64 & 10.36 \\
\hline & $B I O R-B I O R$ & 828 & 828 & 0 & 100.00 & 0.00 \\
\hline & $B I O R-C S A R$ & 765 & 735 & 30 & 96.08 & 3.92 \\
\hline & $B 1 O R-C G 2 R$ & 818 & 778 & 40 & 95.11 & 4.89 \\
\hline & $B I O R-L 1 R$ & 824 & 785 & 39 & 95.27 & 4.73 \\
\hline & $C S A R-C S A R$ & 811 & 811 & 0 & 100.00 & 0.00 \\
\hline & $C S A R-C G 2 R$ & 799 & 781 & 18 & 97.75 & 2.25 \\
\hline & $C S A R-L 1 R$ & 809 & 795 & 14 & 98.27 & 1.73 \\
\hline & $C G 2 R-C G 2 R$ & 877 & 877 & 0 & 100.00 & 0.00 \\
\hline & $C G 2 R-L 1 R$ & 871 & 860 & 11 & 98.74 & 1.26 \\
\hline & $L 1 R-L 1 R$ & 893 & 893 & 0 & 100.00 & 0.00 \\
\hline
\end{tabular}


Table 4.7 Neurotech Rolls TMR1 Table

\begin{tabular}{|c|c|c|c|c|c|}
\hline Neurotech Rolls & Total & TMR1 & FMR1 & TMR1 Rate & FMR1 Rate \\
\hline $10 p R-10 p R$ & 360 & 360 & 0 & 100.00 & 0.00 \\
\hline $10 p R-B I O R$ & 390 & 375 & 15 & 96.15 & 3.85 \\
\hline $10 p R-C S A R$ & 383 & 377 & 6 & 98.43 & 1.57 \\
\hline $10 p R-C G 2 R$ & 411 & 404 & 7 & 98.30 & 1.70 \\
\hline$B I O R-B I O R$ & 815 & 815 & 0 & 100.00 & 0.00 \\
\hline$B I O R-C S A R$ & 757 & 743 & 14 & 98.15 & 1.85 \\
\hline$B 1 O R-C G 2 R$ & 810 & 797 & 13 & 98.40 & 1.60 \\
\hline$C S A R-C S A R$ & 804 & 804 & 0 & 100.00 & 0.00 \\
\hline$C S A R-C G 2 R$ & 796 & 792 & 4 & 99.50 & 0.50 \\
\hline$C G 2 R-C G 2 R$ & 862 & 862 & 0 & 100.00 & 0.00 \\
\hline$L 1 R-10 p R$ & 828 & 807 & 21 & 97.46 & 2.54 \\
\hline$\angle 1 R-B I O R$ & 835 & 819 & 16 & 98.08 & 1.92 \\
\hline$L 1 R-C S A R$ & 820 & 811 & 9 & 98.90 & 1.10 \\
\hline$L 1 R-C G 2 R$ & 878 & 870 & 8 & 99.09 & 0.91 \\
\hline
\end{tabular}

Table 4.8 SourceAFIS Rolls TMR1 Table

\begin{tabular}{|c|c|c|c|c|c|c|}
\hline SourceAFIS Rolls & Match & Total & TMR1 & FMR1 & TMR1 Rate & FMR1 Rate \\
\hline & $10 p R-10 p R$ & 418 & 417 & 0 & 99.76 & 0.00 \\
\hline & $10 p R-B I O R$ & 389 & 342 & 47 & 87.92 & 12.08 \\
\hline & $10 p R-C S A R$ & 383 & 349 & 34 & 91.12 & 8.88 \\
\hline & $10 p R-C G 2 R$ & 411 & 369 & 43 & 89.78 & 10.46 \\
\hline & $10 p R-L 1 R$ & 415 & 372 & 43 & 89.64 & 10.36 \\
\hline & $B I O R-B I O R$ & 828 & 828 & 0 & 100.00 & 0.00 \\
\hline & $B I O R-C S A R$ & 765 & 735 & 30 & 96.08 & 3.92 \\
\hline & $B I O R-C G 2 R$ & 818 & 778 & 40 & 95.11 & 4.89 \\
\hline & $B I O R-L 1 R$ & 824 & 785 & 39 & 95.27 & 4.73 \\
\hline & CSA R - CSA R & 811 & 811 & 0 & 100.00 & 0.00 \\
\hline & $C S A R-C G 2 R$ & 799 & 781 & 18 & 97.75 & 2.25 \\
\hline & $C S A R-L 1 R$ & 809 & 795 & 14 & 98.27 & 1.73 \\
\hline & $C G 2 R-C G 2 R$ & 877 & 877 & 0 & 100.00 & 0.00 \\
\hline & $C G 2 R-L 1 R$ & 871 & 860 & 11 & 98.74 & 1.26 \\
\hline & $L 1 R-L 1 R$ & 893 & 893 & 0 & 100.00 & 0.00 \\
\hline
\end{tabular}

The TMR1 Accuracy shown in Tables 4.6 - 4.8 are relatively high compared to the other TMR1 accuracy tables. These high values are because all tests are between rolled prints images that were produced on contact-based devices. The values that are highest, or share the highest value of the three matchers, are denoted in red. The total number of matches may vary within the Neurotech TMR1 Rolls Table compared to the NBIS and SourceAFIS totals. Again, this is because the Neurotech matcher removed images of bad quality from the gallery, which led to fewer matches being performed. Again, this is most likely why the Neurotech matcher produced the best TMR1 accuracy for almost every rolls test. 


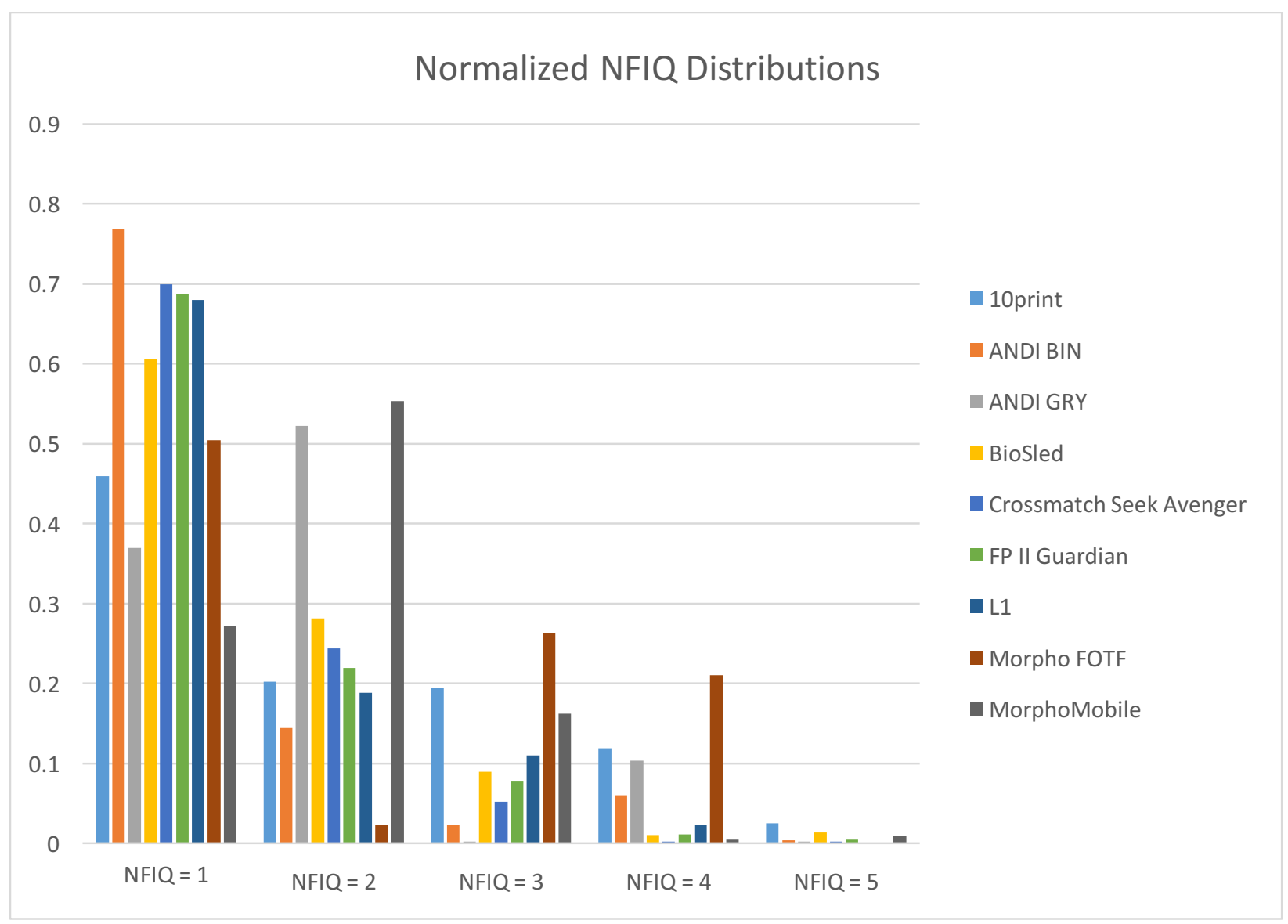

Figure 4.1 Normalized NFIQ Score Distributions for All Devices 


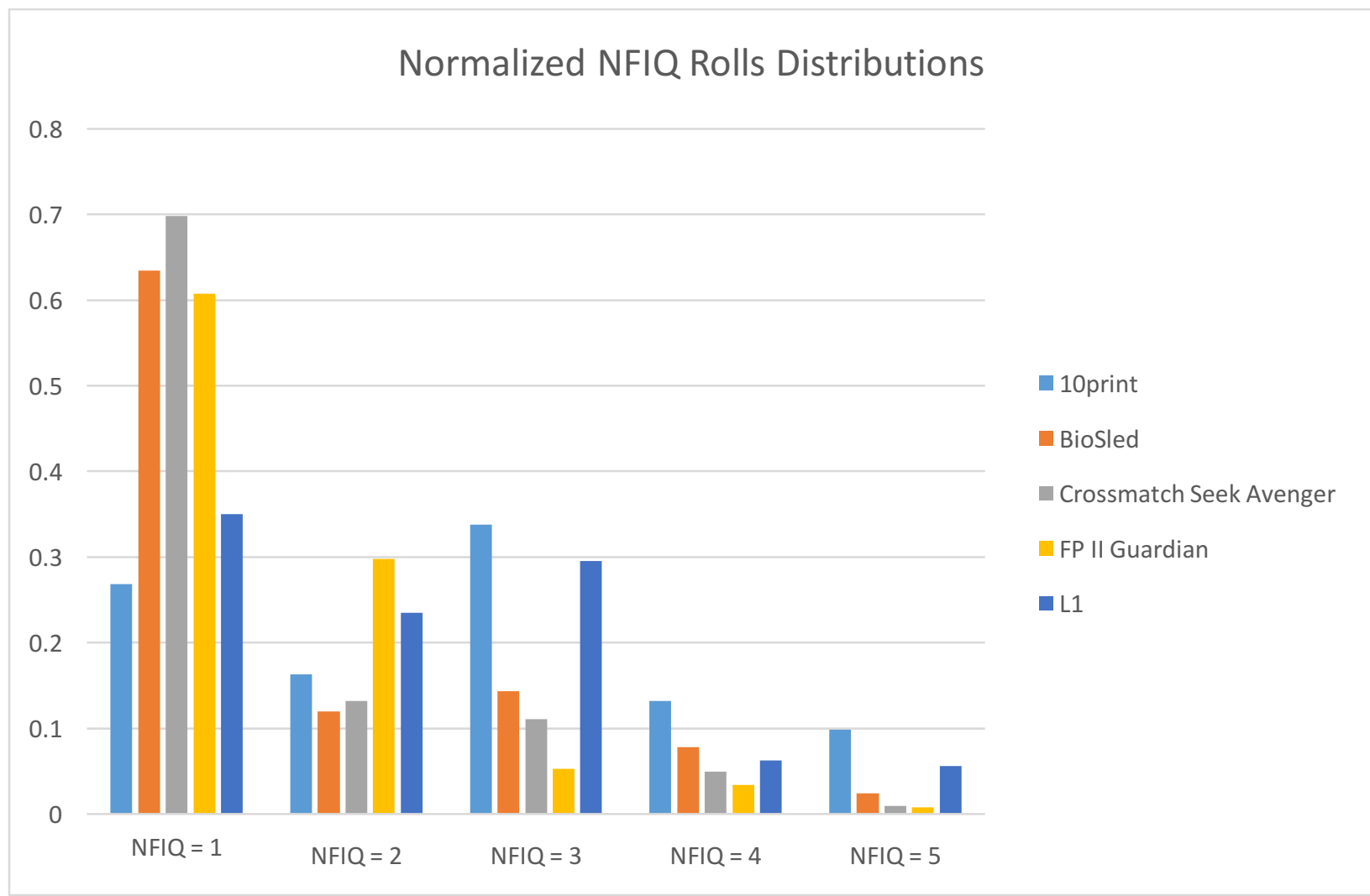

Figure 4.2 Normalized NFIQ Score Distributions for All Devices - Rolls 


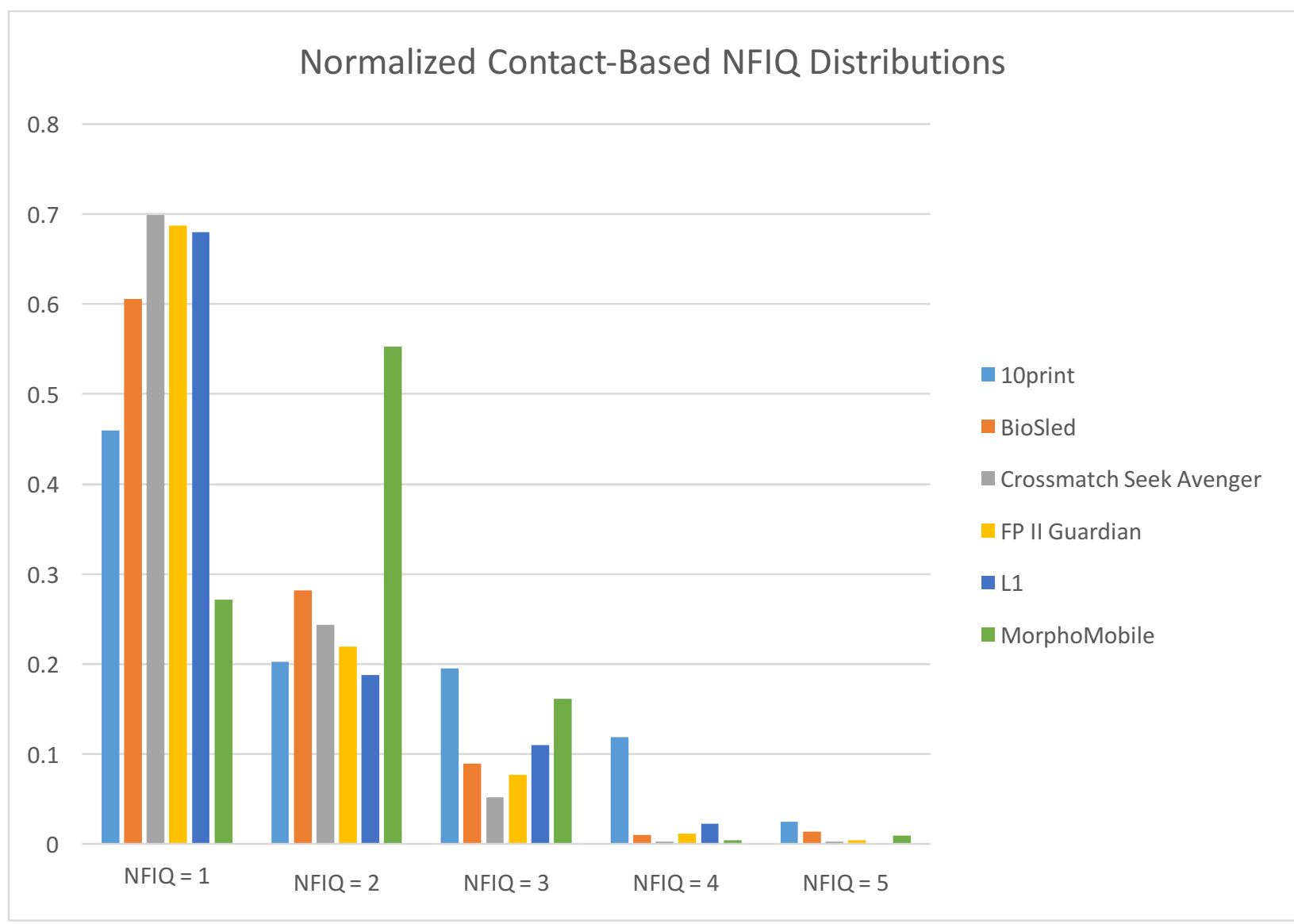

Figure 4.3 Normalized NFIQ Score Distributions for All Contact-based Devices 


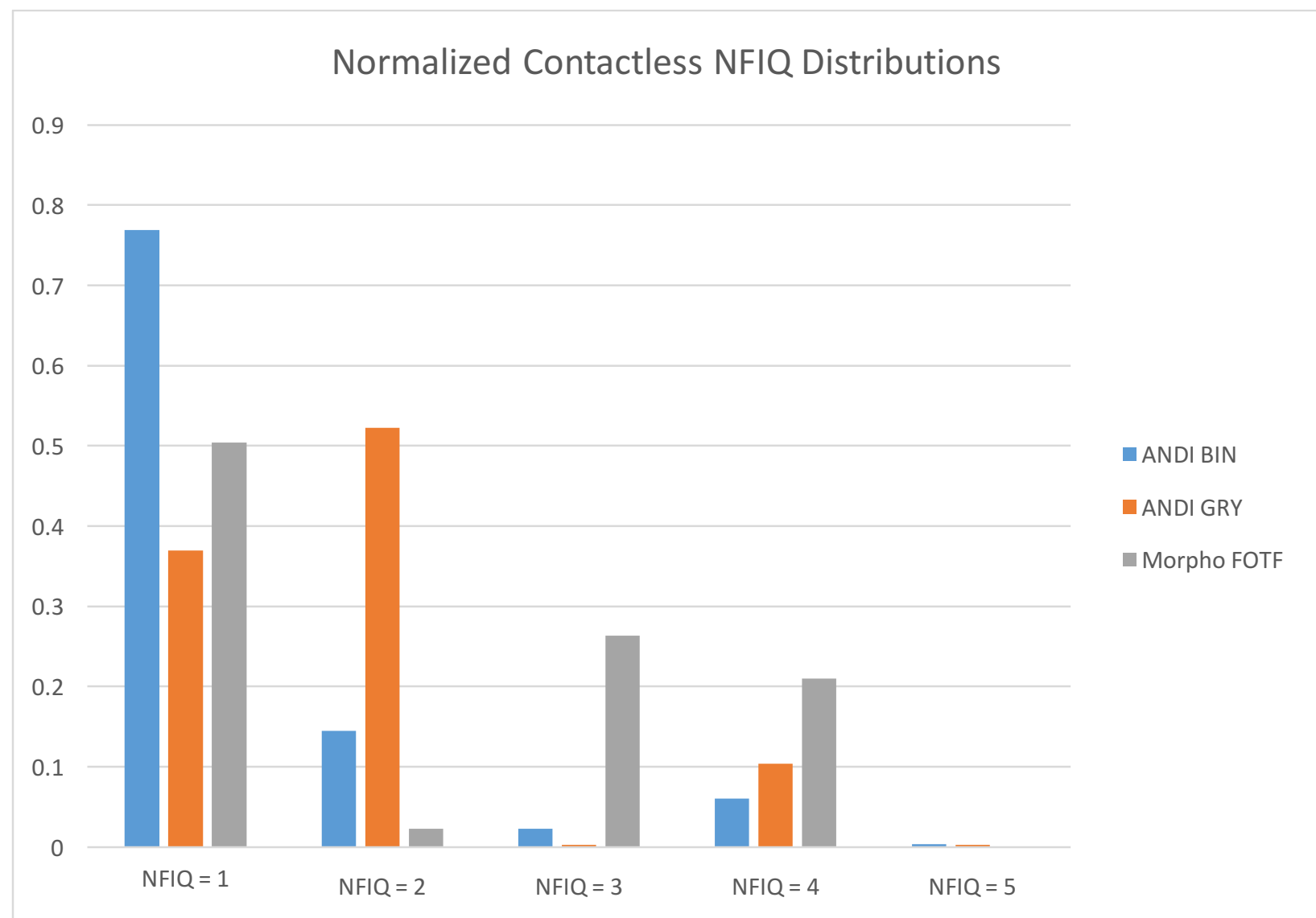

Figure 4.4 Normalized NFIQ Score Distributions for all Contactless Devices (Excluding InnerID)

These NFIQ scores were output from the NBIS matching algorithm. NFIQ is explained in Section 2.10. The same number of images was not produced by each device. One device that stands out is the Morpho FOTF, which had almost double that of the other devices. The ten-print NFIQ distributions seem lower than most, this could be due to low operator experience in collecting ten-print cards.

Figure 4.1 shows all the devices distributions together, while Figures $4.3-4.4$ show these same distributions displayed by method of capture. Figure 4.2 shows the distributions for rolled images only and the devices that acquired them. For the contact-based devices, these distributions agree with the rest of the results, as they show that devices with better image quality 
distributions produced better results than those with poor image NFIQ distributions. The NFIQ algorithm is not optimized for contactless images, that is why these results do not really agree with the rest of the results

\subsection{Cross-Examined Genuine and Imposter Score Distributions}

\subsubsection{NBIS Cross-Examined Genuine/Imposter Distributions}

\subsubsection{NBIS Samples of Normal Distributions}

The genuine/imposter distributions shown in Figures $4.5-4.6$ are sample genuine/imposter match score distributions obtained using the NBIS matcher. They have been chosen to illustrate the typical genuine/imposter score distributions of each type of device used in this study, as well as the ten-print cards. The rest of the distributions have been provided in Appendix A.
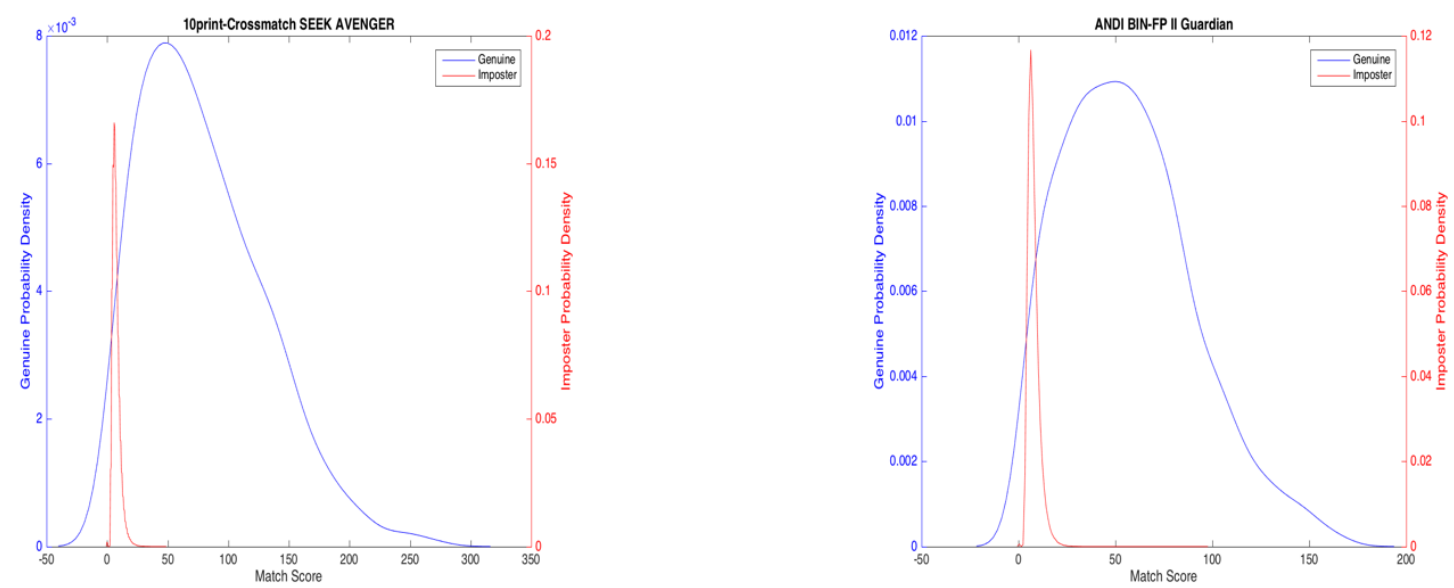

Figure 4.5 (Left) NBIS 10print - Crossmatch Seek Avenger (Right) NBIS ANDI Binary - FP II Guardian 


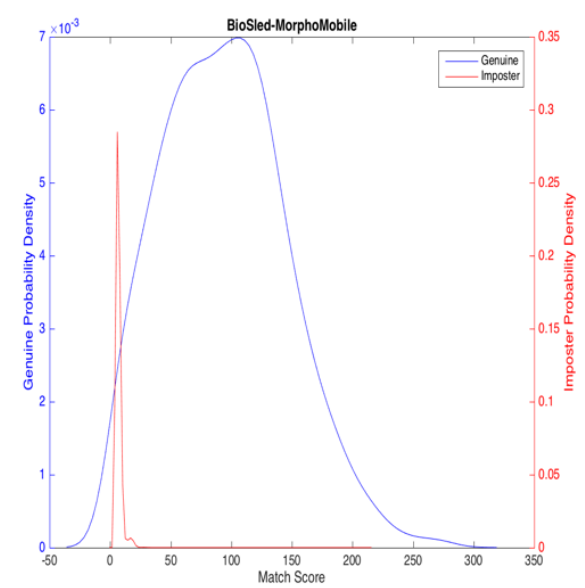

Figure 4.6 NBIS BioSled - MorphoMobile

\subsubsection{NBIS Outliers}

Due to the InnerID running into technical problems during the collection, it was removed from the collection midway through. While still in operation, the InnerID produced low quality images, this statement is proven with the genuine/imposter distributions shown below. Each test shown in Figures 4.8(Right) - 4.18 involved the InnerID device. The genuine/imposter distributions shown in Figures $4.7-4.8$ (left) show outliers that do not involve the InnerID device. The reason these differ slightly from the typical genuine/imposer distribution is due to the match involving the ANDI Greyscale images. Considering the TMR1 scores involving the ANDI greyscale images, they do not perform as well as most of the other matches with these three match scenarios achieving TMR1 accuracy of $68.24,74.66$, and 72.41 , respectively for this particular matcher. 

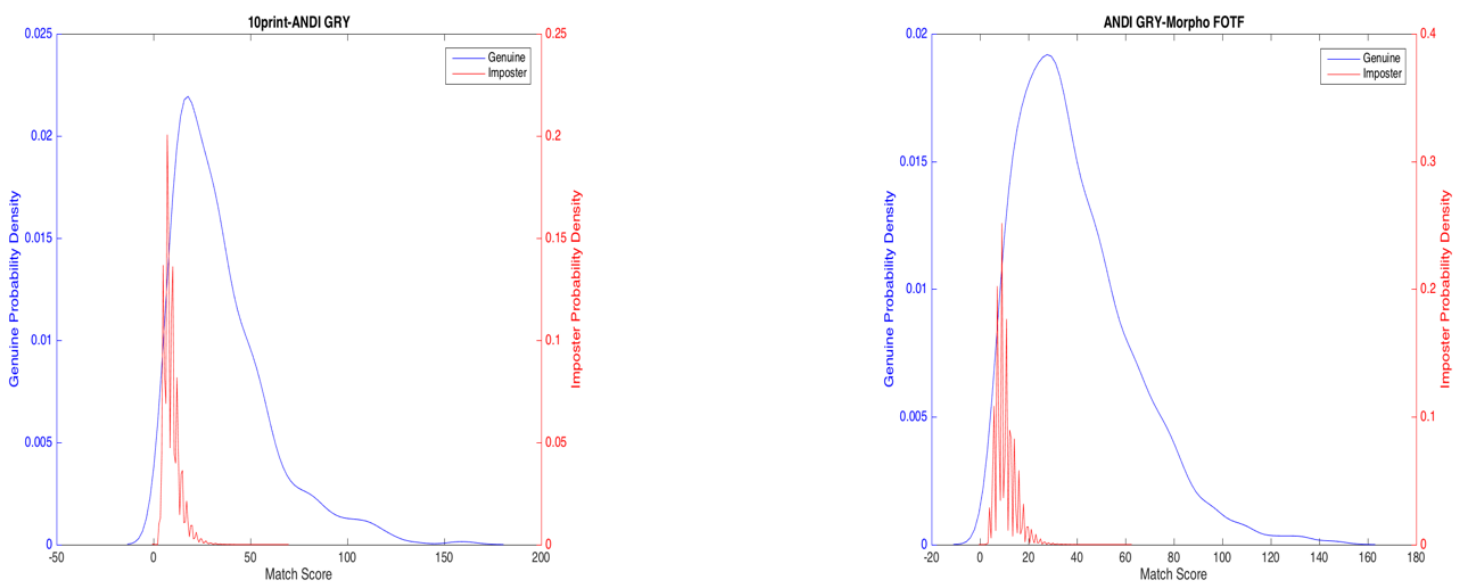

Figure 4.7 (Left) NBIS 10print - ANDI Greyscale (Right) NBIS ANDI Greyscale - Morpho FOTF
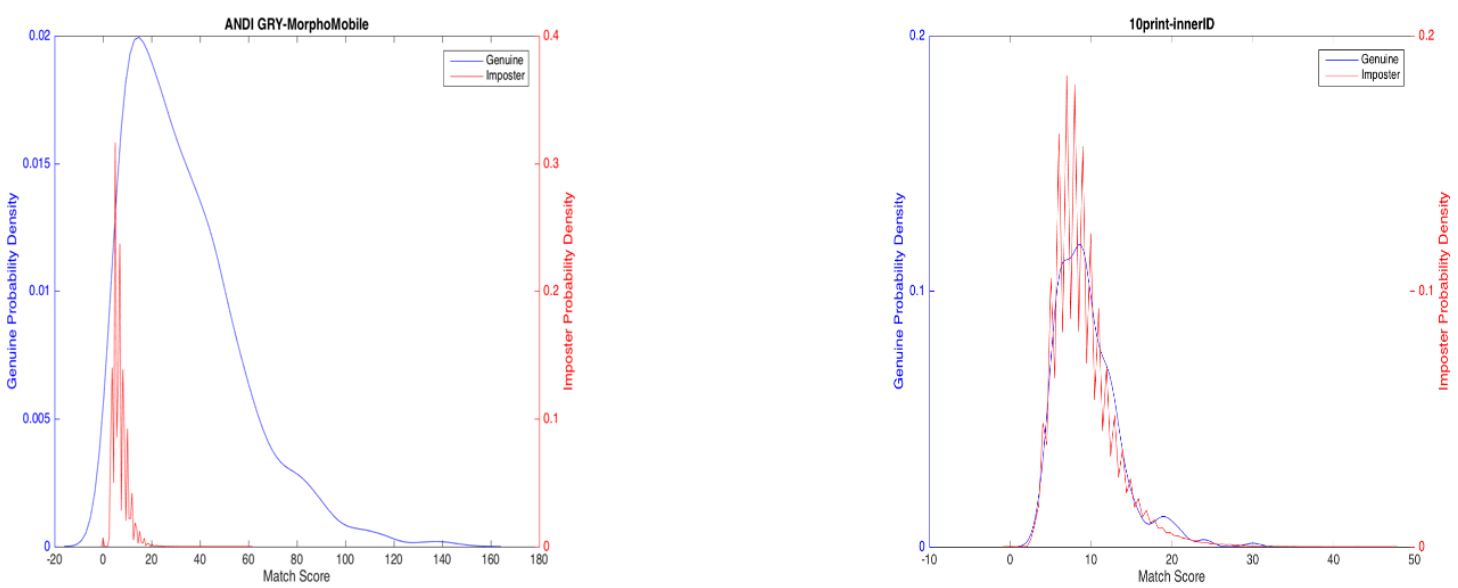

Figure 4.8 (Left) NBIS ANDI Greyscale - MorphoMobile (Right) NBIS 10print - InnerID
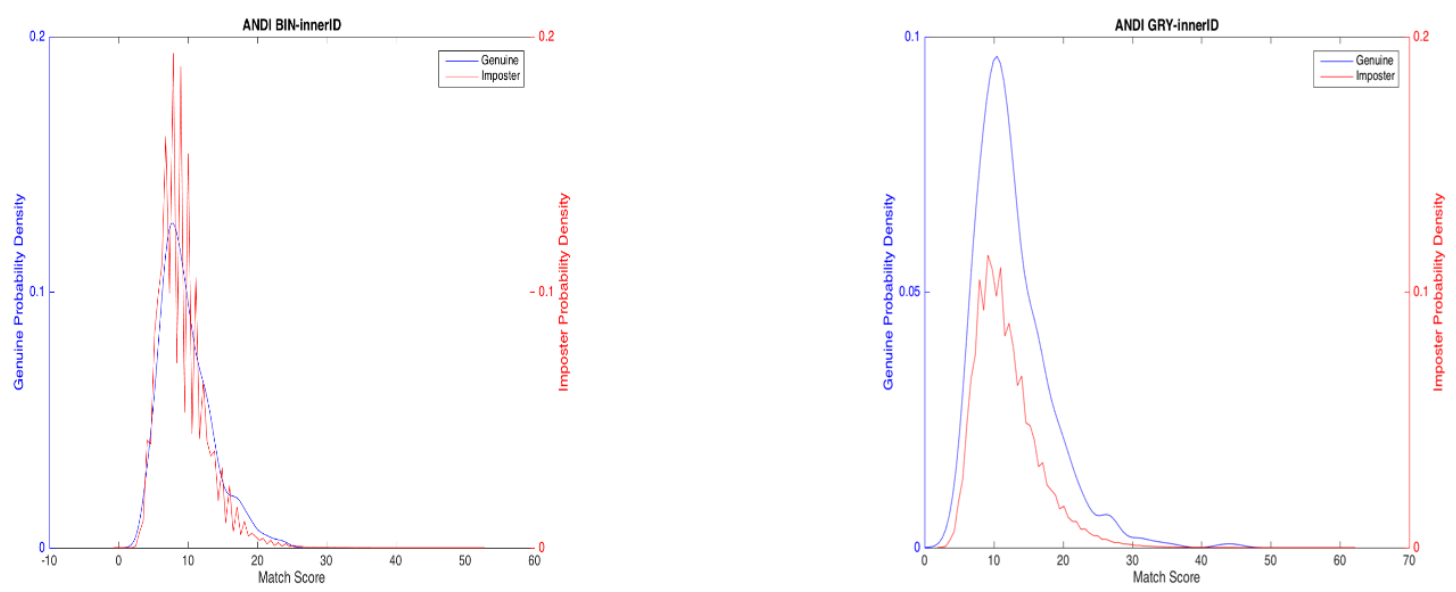

Figure 4.9 (Left) NBIS ANDI Binary - InnerID (Right) NBIS ANDI Greyscale - InnerID 

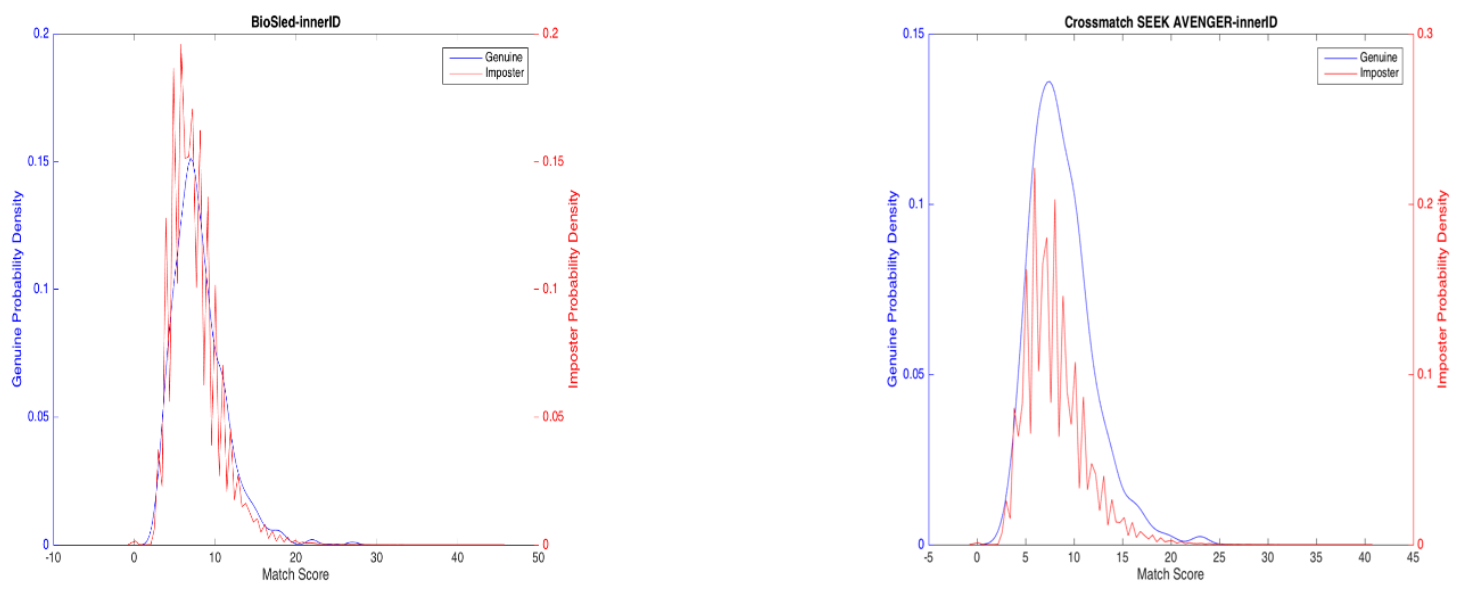

Figure 4.10 (Left) NBIS BioSled - InnerID (Right) NBIS Crossmatch SEEK AVENGER - InnerID
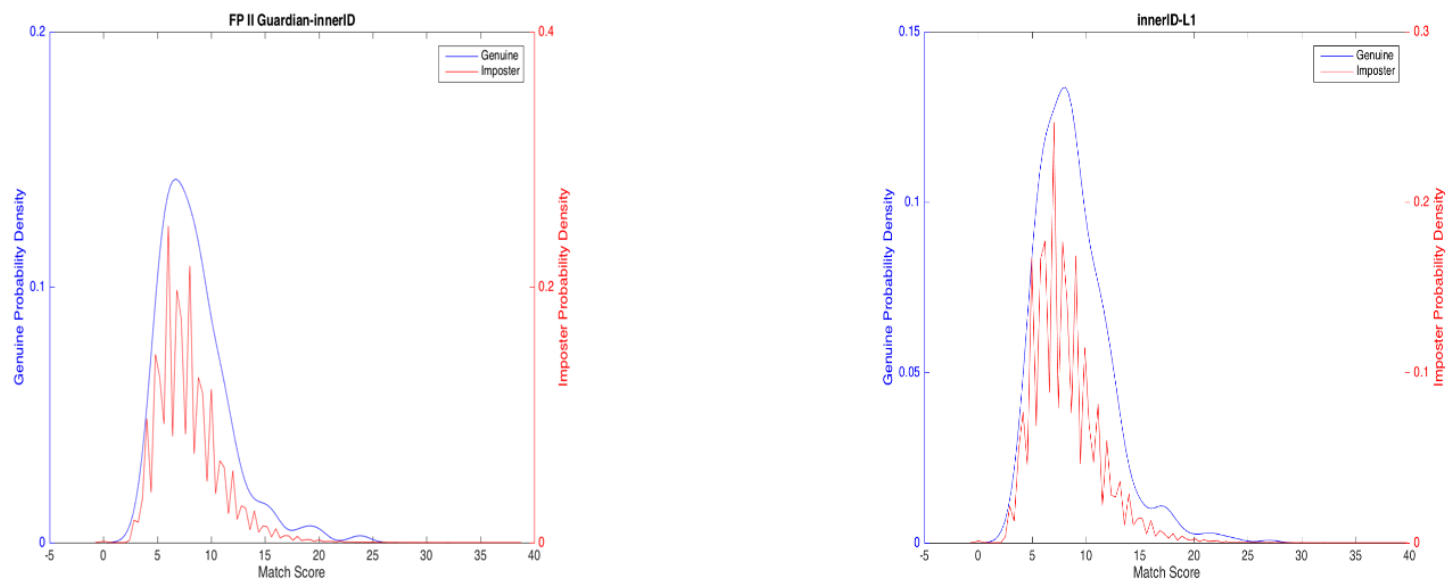

Figure 4.11 (Left) NBIS FP II Guardian (Right) NBIS InnerID - L1
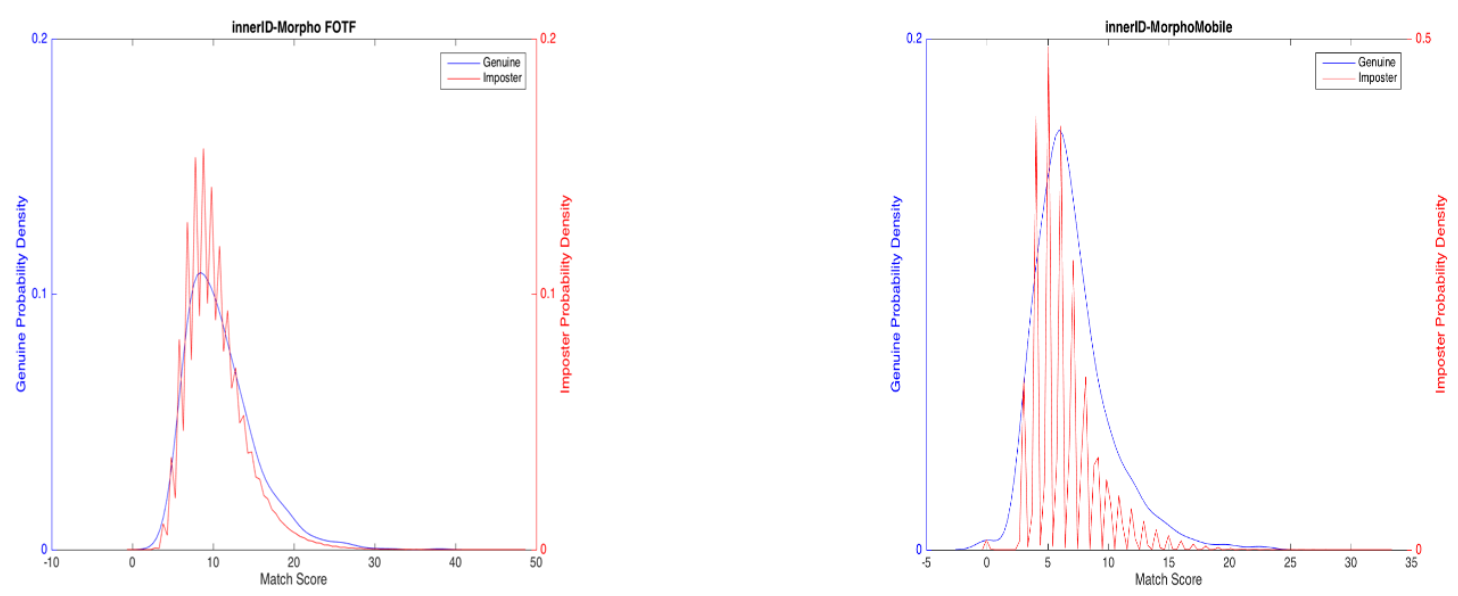

Figure 4.12 (Left) NBIS InnerID - Morpho FOTF (Right) NBIS InnerID - MorphoMobile 


\subsubsection{Neurotech Cross-Examined Genuine/Imposter Distributions}

\subsubsection{Neurotech Samples of Normal Distributions}

The genuine/imposter distributions shown in Figures $4.13-4.14$ are sample genuine/imposter match score distributions obtained using the Neurotech matcher. They have been chosen to illustrate the typical genuine/imposter score distributions of each type of device used in this study, as well as the ten-print cards. The rest of the distributions have been provided in Appendix A.
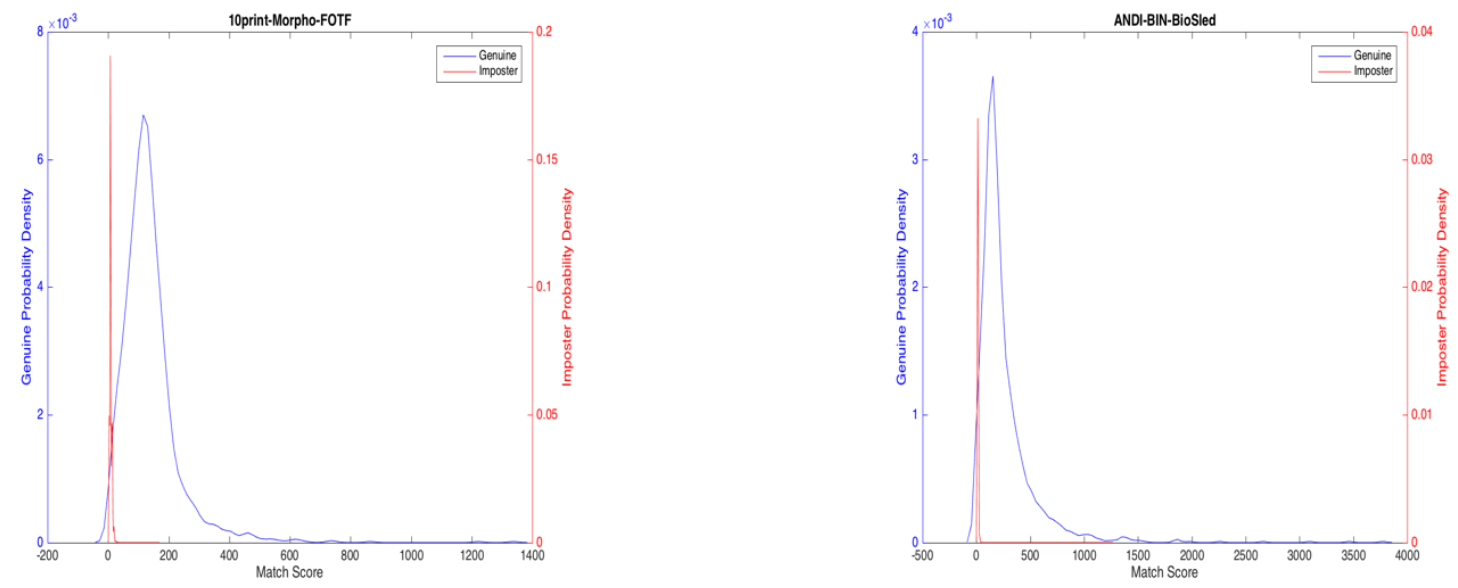

Figure 4.13 (Left) Neurotech 10print - Morpho FOTF (Right) Neurotech ANDI Binary - BioSled

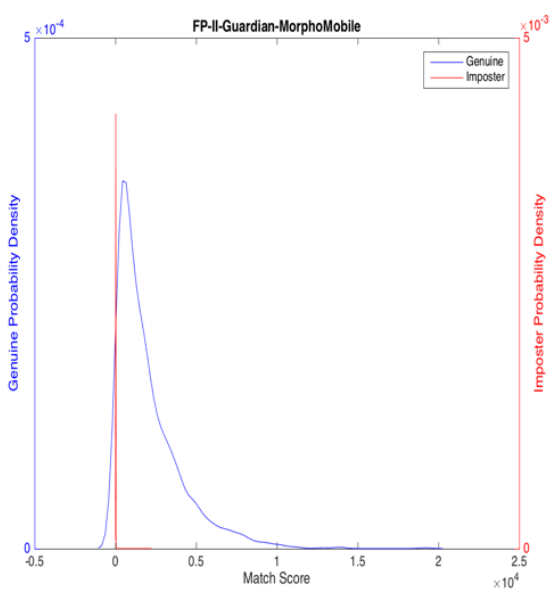

Figure 4.14 Neurotech FP II Guardian - MorphoMobile 


\subsubsection{Neurotech Outliers}

As described in section 4.1.1.1.
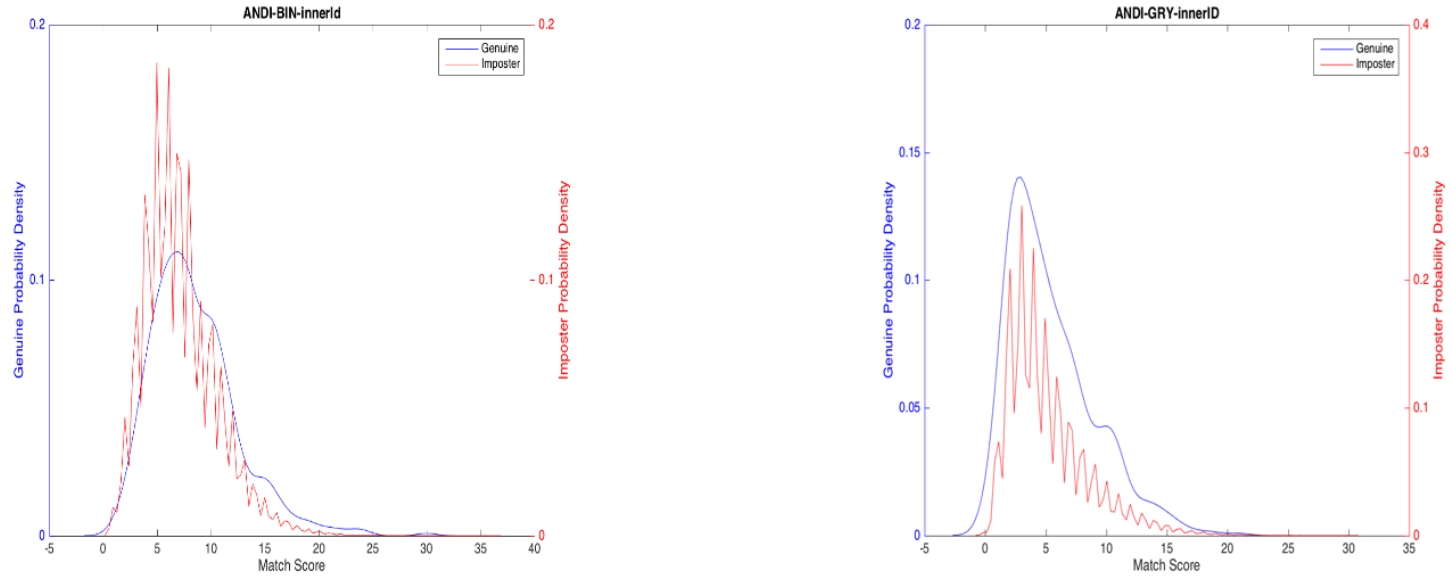

Figure 4.15 (Left) Neurotech ANDI Binary - InnerID (Right) Neurotech ANDI Greyscale - InnerID
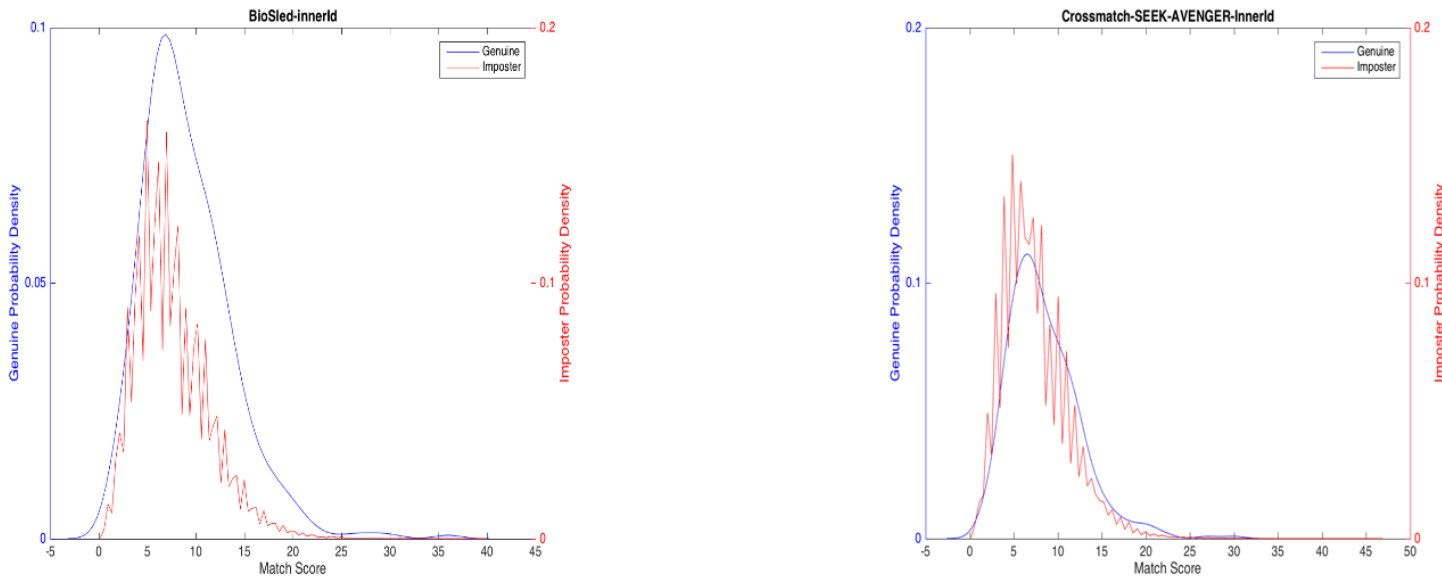

Figure 4.16 (Left) Neurotech BioSled - InnerID (Right) Neurotech Crossmatch SEEK AVENGER - InnerID 

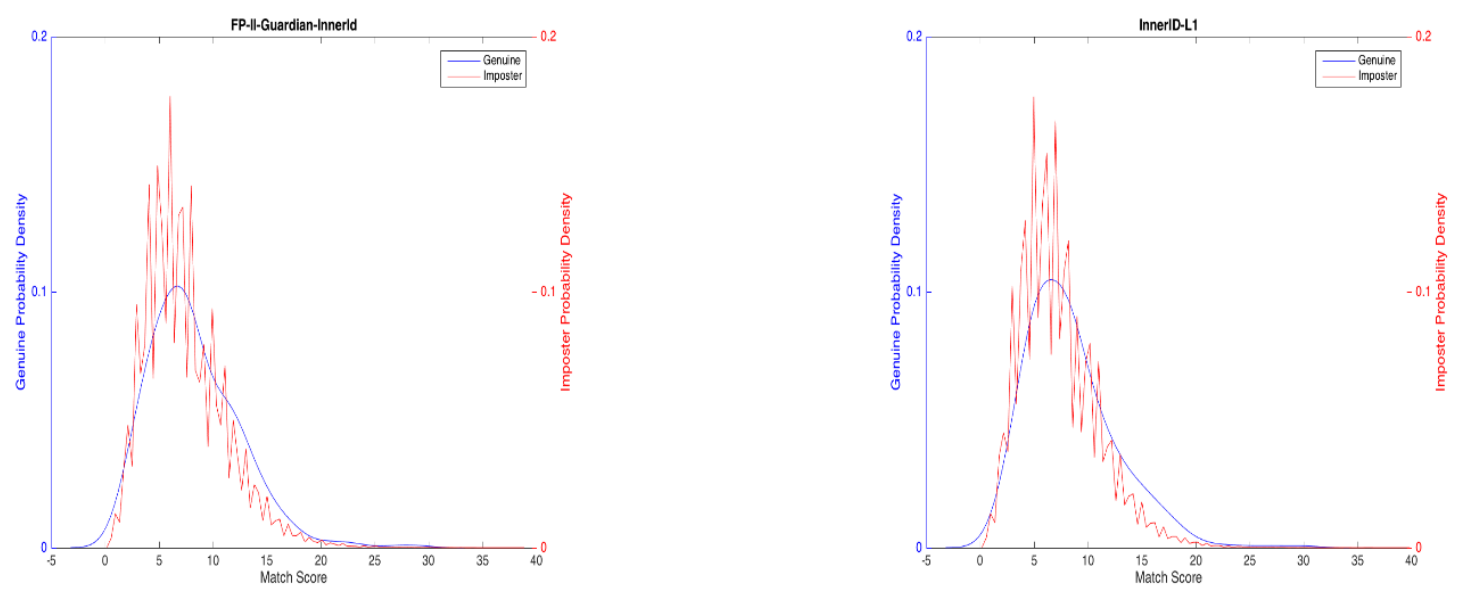

Figure 4.17 (Left) Neurotech FP II Guardian - InnerID (Right) Neurotech InnerID - L1
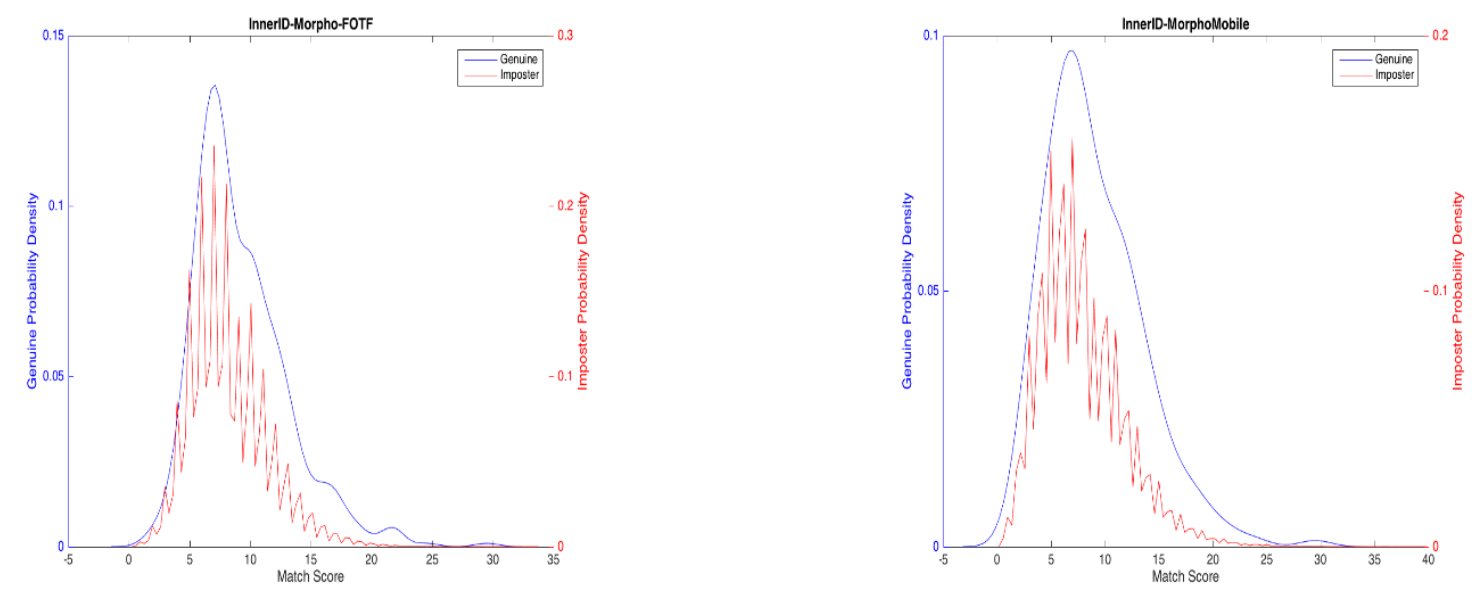

Figure 4.18 (Left) Neurotech InnerID - Morpho FOTF (Right) Neurotech InnerID - MorphoMobile

\subsubsection{SourceAFIS Cross-Examined Genuine/Imposter Distributions}

\subsubsection{SourceAFIS Samples of Normal Distributions}

The genuine/imposter distributions shown in Figures $4.19-4.20$ are sample genuine/imposter match score distributions obtained using the SourceAFIS matcher. They have been chosen to illustrate the typical genuine/imposter score distributions of each type of device used in this study, as well as the ten-print cards. The rest of the distributions have been provided in Appendix A. 

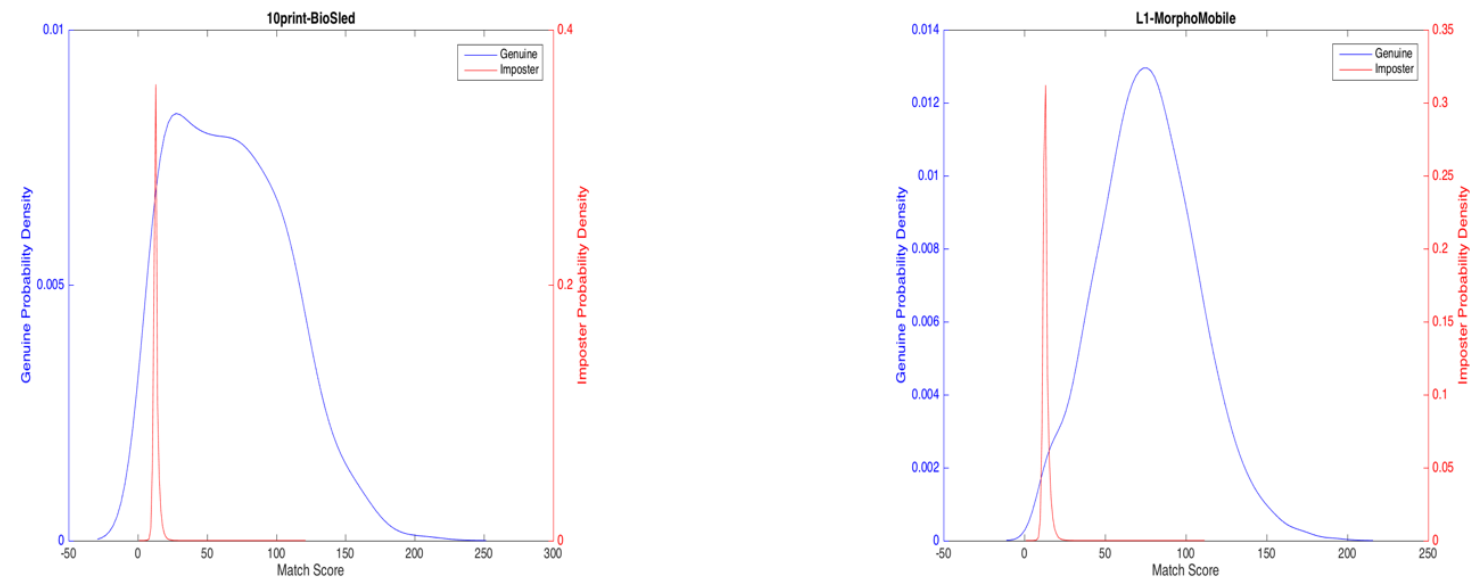

Figure 4.19 (Left) SourceAFIS 10print - BioSled (Right) SourceAFIS L1 - MorphoMobile

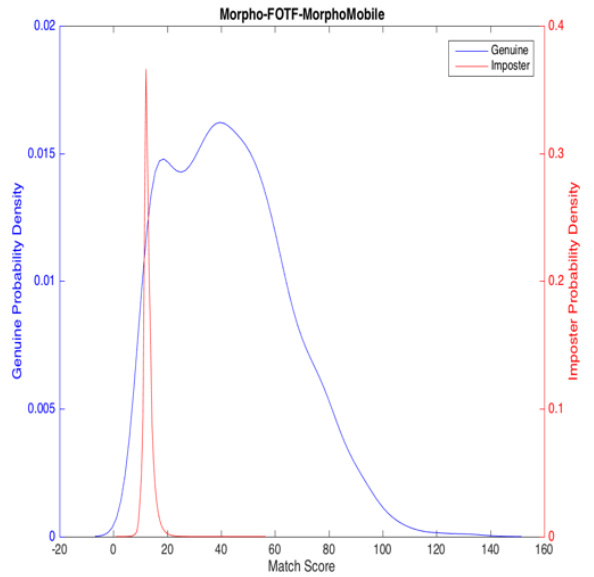

Figure 4.20 SourceAFIS Morpho FOTF - MorphoMobile

\subsubsection{SourceAFIS Outliers}

As described in section 4.1.1.1. 

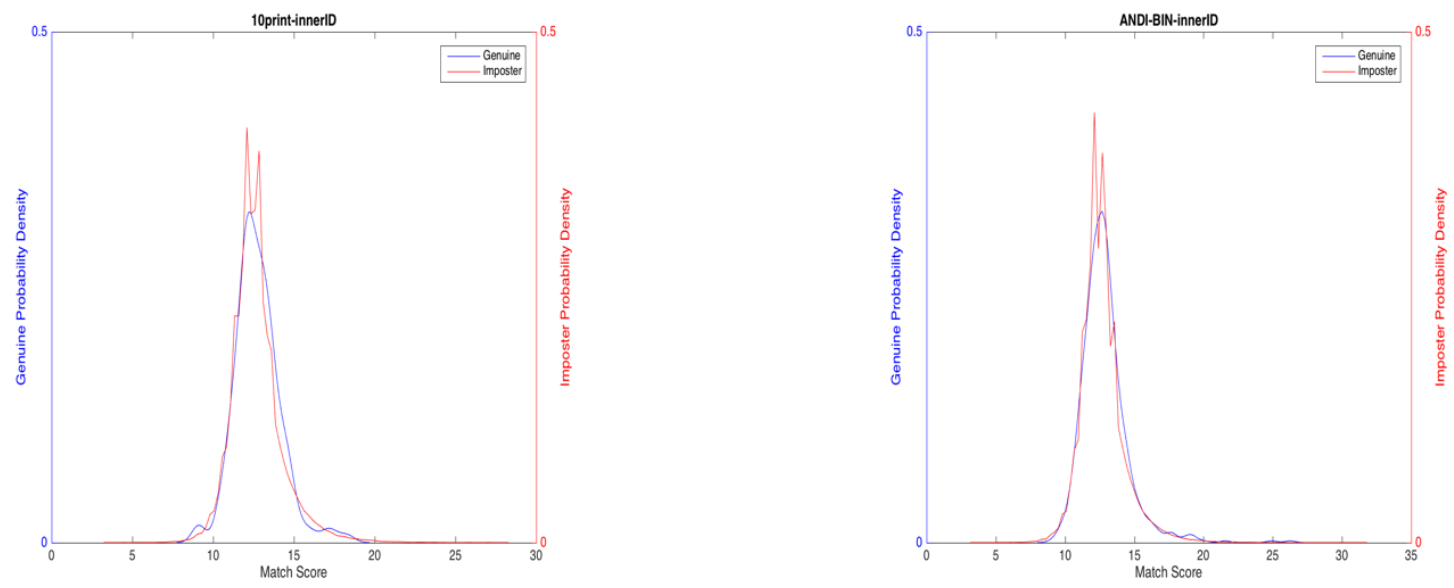

Figure 4.21 (Left) SourceAFIS 10print - InnerID (Right) SourceAFIS ANDI Binary - InnerID
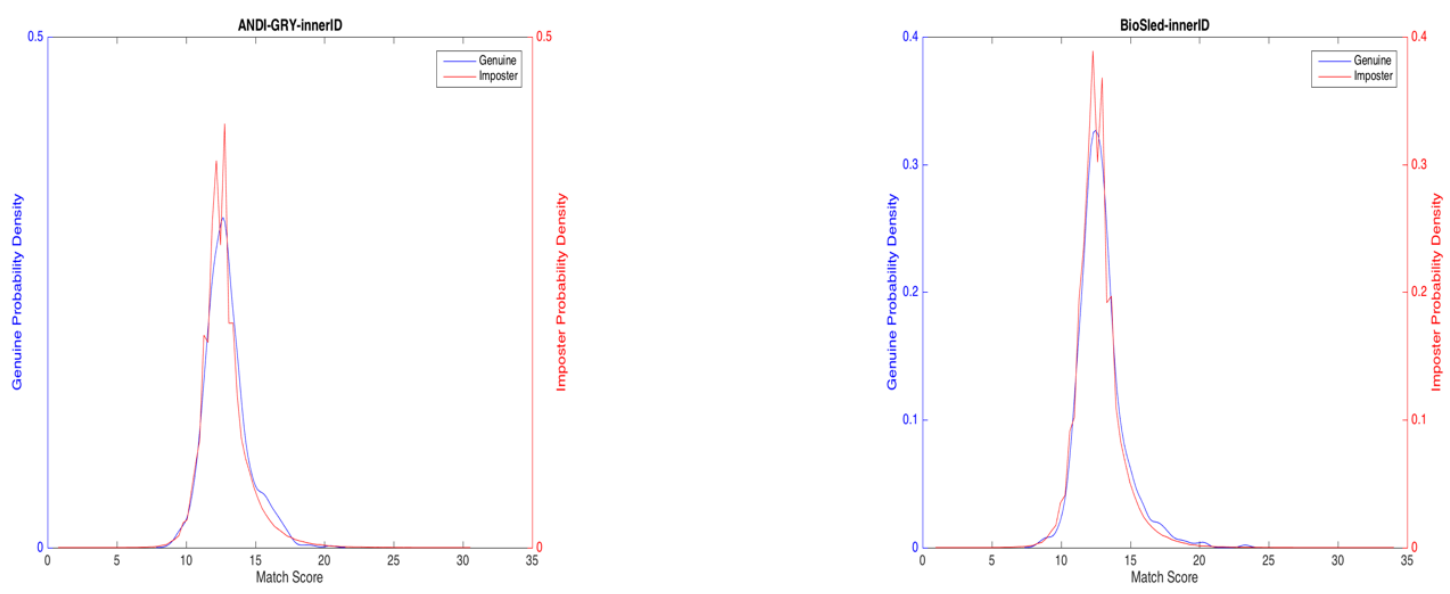

Figure 4.22 (Left) SourceAFIS ANDI Greyscale - InnerID (Right) SourceAFIS BioSled - InnerID
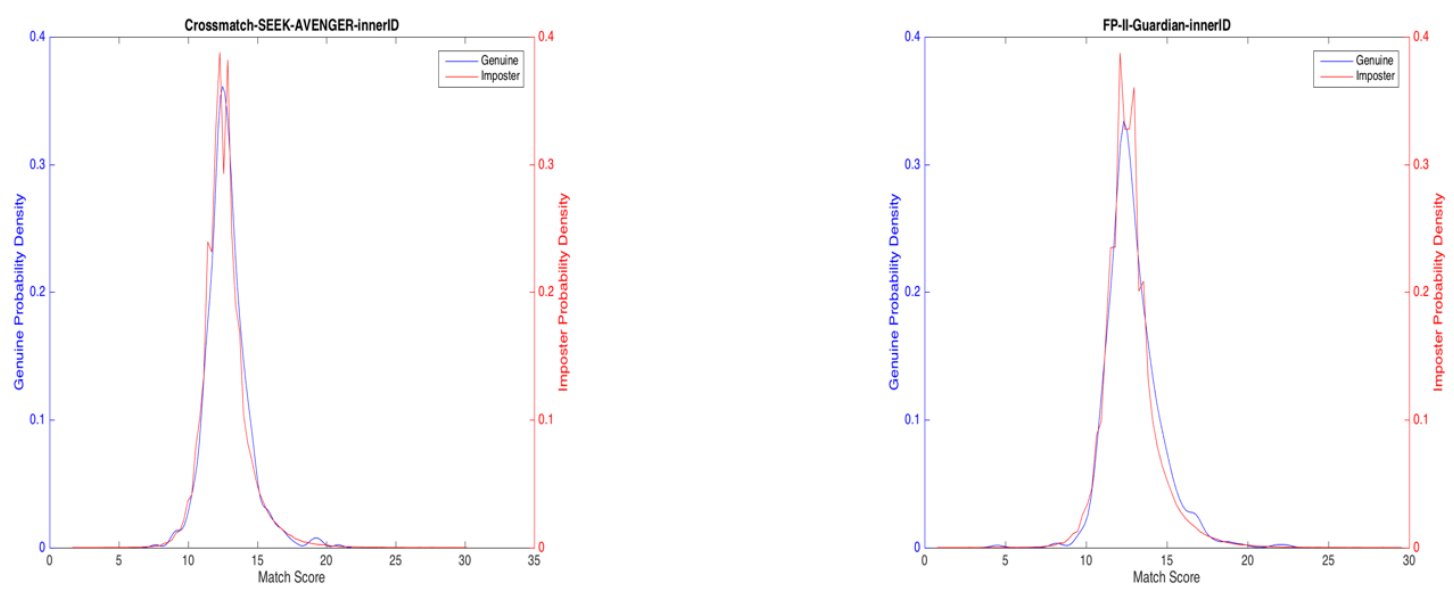

Figure 4.23 (Left) SourceAFIS Crossmatch SEEK AVENGER - InnerID (Right) SourceAFIS FP II Guardian InnerID 

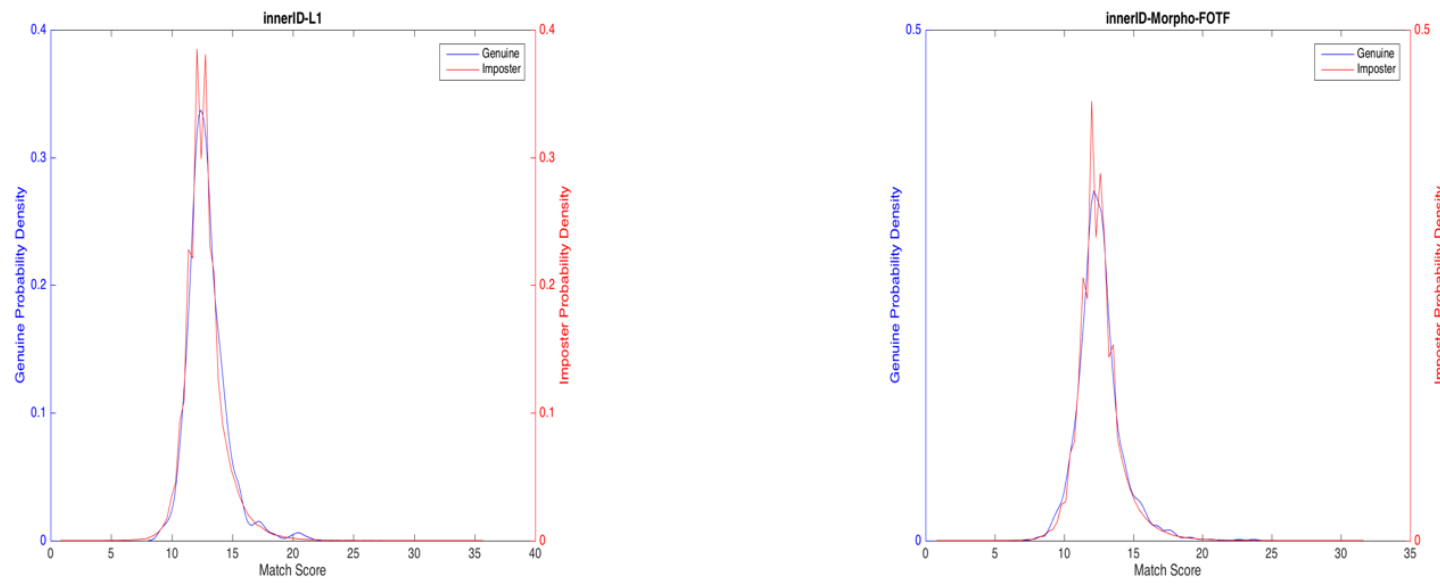

Figure 4.24 (Left) SourceAFIS InnerID - L1 (Right) SourceAFIS InnerID - Morpho FOTF

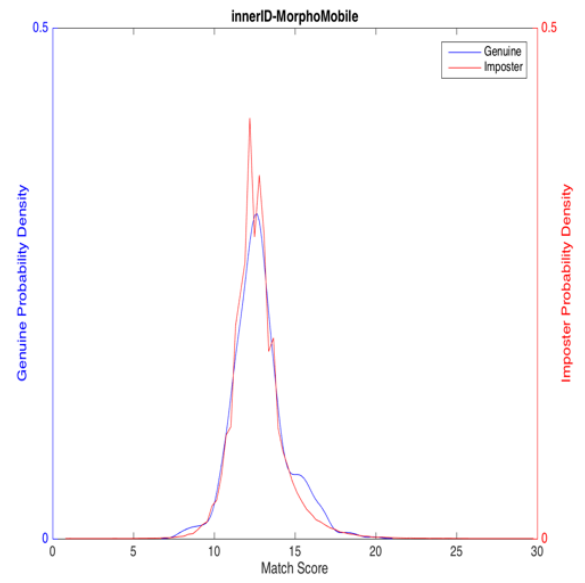

Figure 4.25 SourceAFIS InnerID - MorphoMobile

The full set of results for the general study can be seen in Appendix A.

\subsection{Gender-Based Study}

\subsubsection{Gender-Based Samples of Normal Distributions}

The genuine/imposter distributions shown in Figures $4.26-4.31$ are sample genuine/imposter match score distributions obtained using the each of the three matching algorithms for male and female strata. They have been chosen to illustrate the typical genuine/imposter score distributions of each type of device and gender used in this study. Figures 4.26, 4.28, and 4.30 represent male genuine/imposter distributions for each of the 
matchers and Figures 4.27, 4.29, and 4.31 represent female genuine/imposter distributions for each matcher. There are no noticeable trends shown within the distributions. The rest of the distributions have been provided in Appendix B.
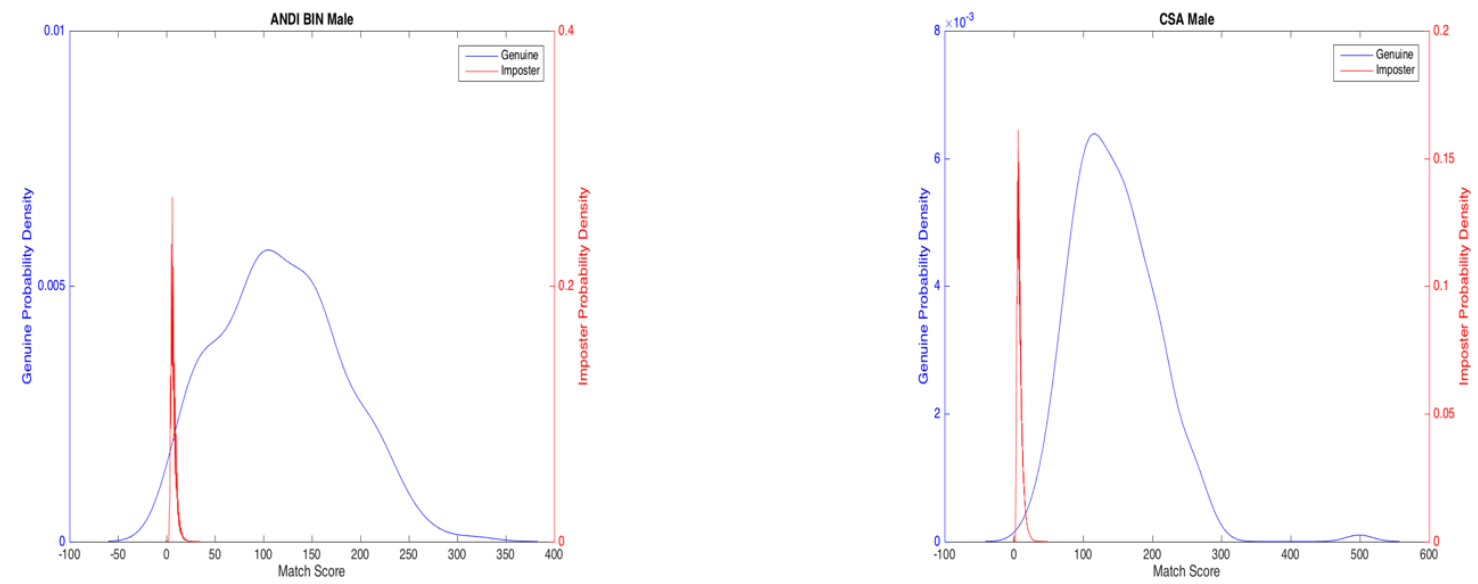

Figure 4.26 (Left) NBIS ANDI Binary Male (Right) NBIS Crossmatch Seek Avenger Male
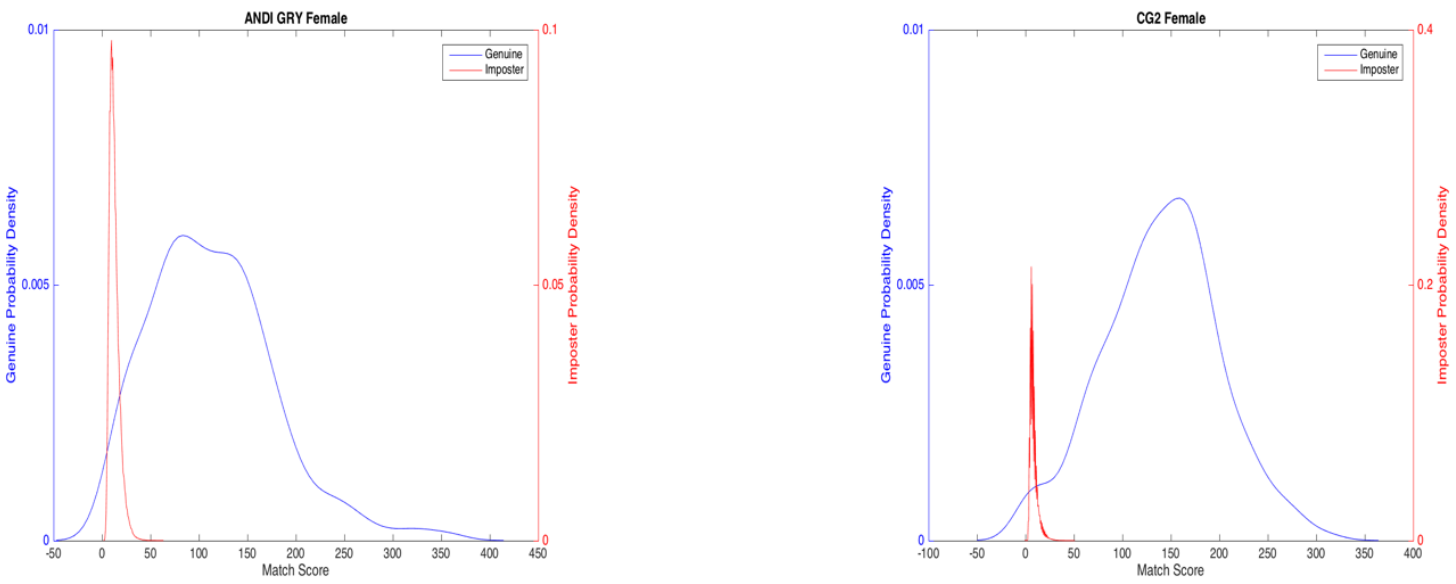

Figure 4.27 (Left) NBIS ANDI Greyscale Female (Right) FP II Guardian Female 

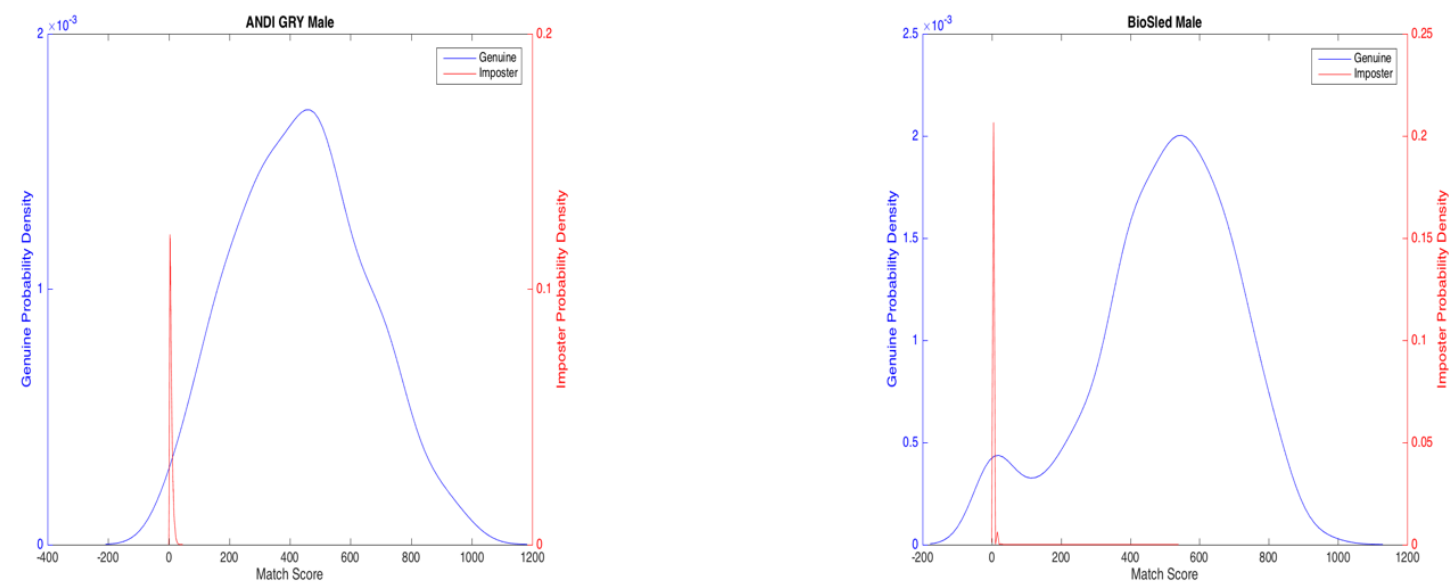

Figure 4.28 (Left) Neurotech ANDI Greyscale Male (Right) Neurotech BioSled Male
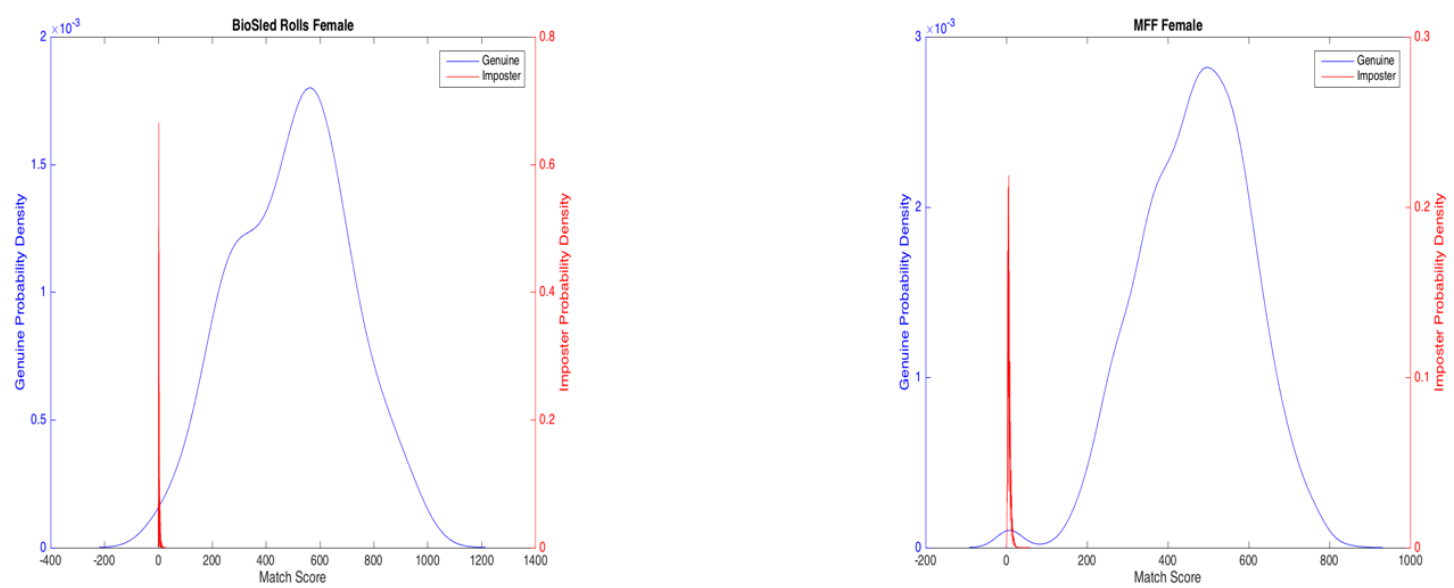

Figure 4.29 (Left) Neurotech BioSled Rolls Female (Right) Neurotech Morpho FOTF Female
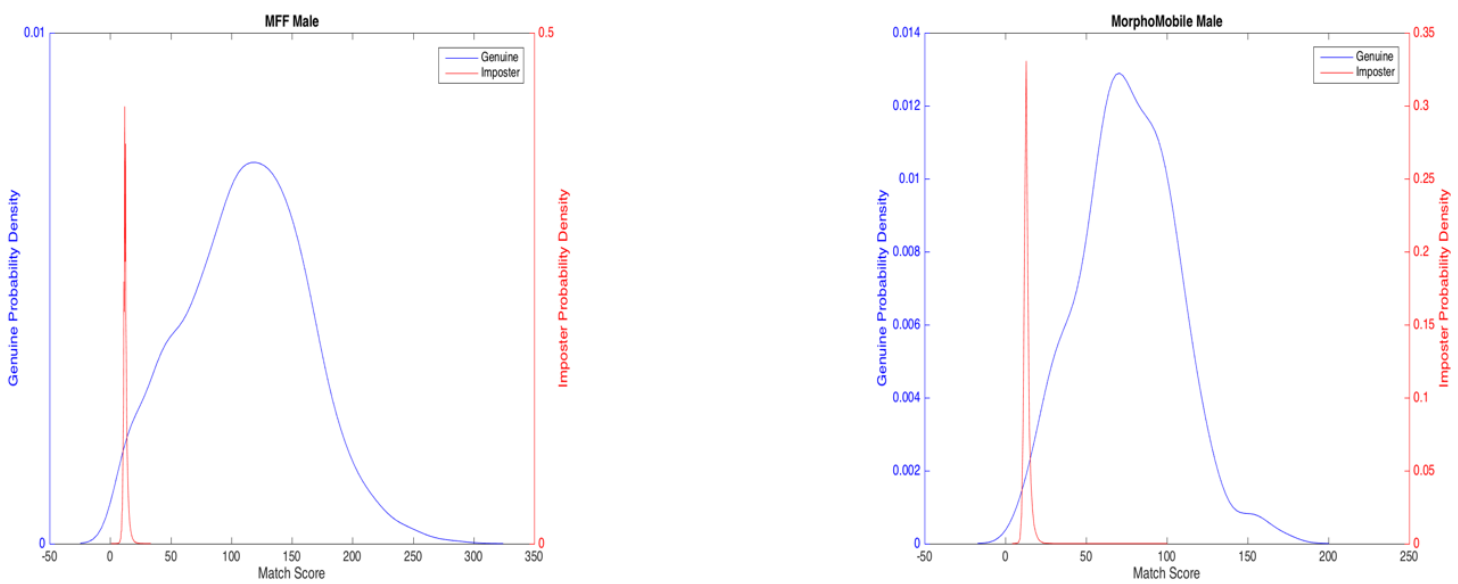

Figure 4.30 (Left) SourceAFIS Morpho FOTF Male (Right) SourceAFIS MorphoMobile Male 

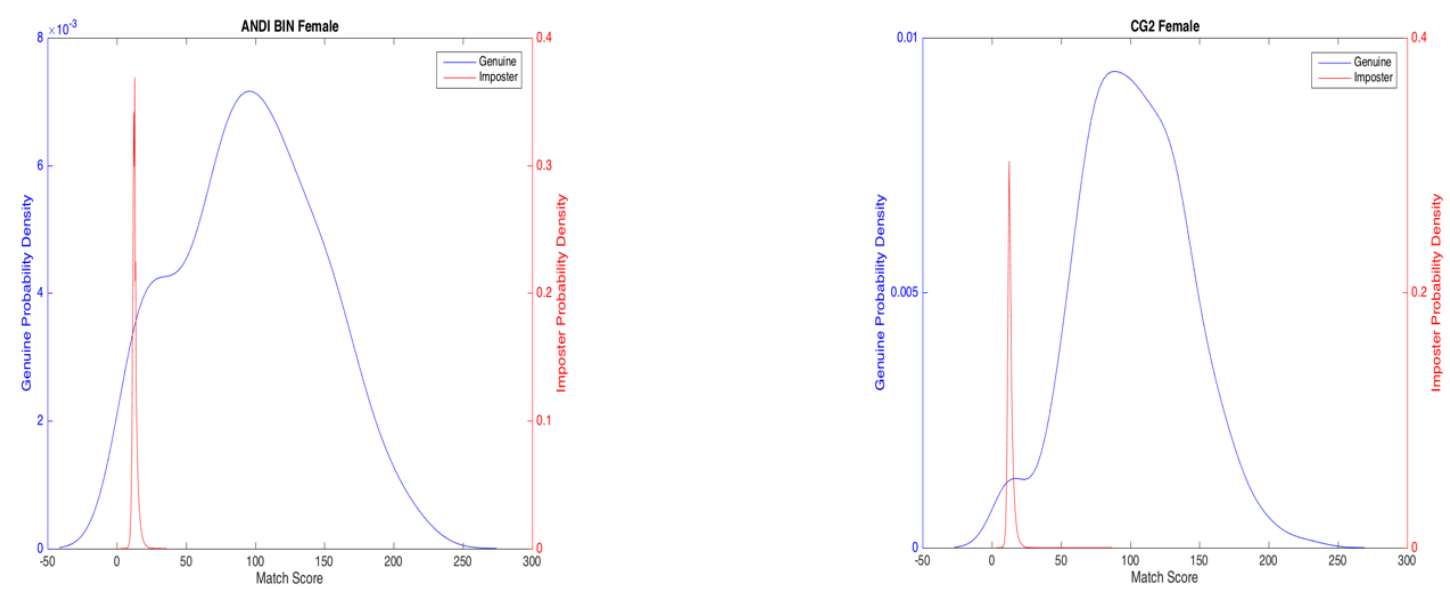

Figure 4.31(Left) ANDI Binary Female (Right) SourceAFIS FP II Guardian Female

\subsubsection{Gender-Based Outliers}

There were not any outliers based on the genuine/imposter distributions for this matcher using these stratifications.

\subsubsection{Gender- Based KLD Values}

The values shown in Tables $4.9-4.11$ show the Kullback Leibler Divergence measures of the genuine and imposter distributions for both genders on each matcher. The genuine KLD values produced for males showed little variation with the genuine average being 0.0245 and an imposter KLD average of 1.0595. For females the values varied showed more but still were relatively low with a genuine KLD average of 0.0417 and an imposter KLD average of 3.8321 . These values reveal the similarity to the original dataset and show little to no performance variation between genders. The outlying values for Tables $4.9-4.11$ show no trend as they don't show the same variations across all three matcher algorithms results. The KLD values for this study were inconclusive as they showed little to no variation from the general study of the whole dataset. 
Table 4.9 NBIS Gender Based KLD Values

\begin{tabular}{|r|l|l|l|l|}
\hline NBIS KLD Values & \multicolumn{2}{|c|}{ Male } & \multicolumn{2}{c|}{ Female } \\
\hline Device & Genuine & Imposter & Genuine & Imposter \\
\cline { 2 - 5 } ANDI BIN & 0.0282 & 1.6236 & 0.0316 & 0.7008 \\
\cline { 2 - 5 } ANDI GRY & 0.0373 & 0.0893 & 0.0214 & 0.2379 \\
BioSled & 0.0328 & 0.0836 & 0.0434 & 3.8267 \\
\cline { 2 - 5 } BioSled Rolls & 0.0327 & 0.4227 & 0.0176 & 5.4024 \\
\cline { 2 - 5 } CG2 & 0.0272 & 0.1348 & 0.0356 & 7.8629 \\
\cline { 2 - 5 } CG2 Rolls & 0.0163 & 0.9924 & 0.0388 & 2.2804 \\
${ } }$ & 0.0148 & 1.0866 & 0.1378 & 0.3865 \\
\cline { 2 - 5 } CSA Rolls & 0.0217 & 6.4243 & 0.0614 & 3.5796 \\
L1 & 0.0219 & 0.1464 & 0.0221 & 9.0137 \\
\cline { 2 - 5 } L1 Rolls & 0.0155 & 0.2235 & 0.0394 & 6.0952 \\
MFF & 0.0543 & 0.5960 & 0.0169 & 1.1263 \\
\cline { 2 - 5 } & 0.0248 & 0.1028 & 0.0461 & 1.7152 \\
\hline MorphoMobile & & & & \\
\hline
\end{tabular}

Table 4.10 Neurotech Gender Based KLD Values

\begin{tabular}{|r|l|l|l|l|}
\hline Neurotech KLD Values & \multicolumn{2}{|c|}{ Male } & \multicolumn{2}{c|}{ Female } \\
\hline Device & Genuine & Imposter & Genuine & Imposter \\
\cline { 2 - 5 } ANDI BIN & 0.0073 & 5.9406 & 0.0102 & 3.1744 \\
ANDI GRY & 0.0044 & 0.1447 & 0.0041 & 0.2866 \\
\cline { 2 - 5 } BioSled & 0.0148 & 0.0063 & 0.0251 & 0.3071 \\
BioSled Rolls & 0.0080 & 0.0110 & 0.0073 & 0.0299 \\
CG2 & 0.0041 & 0.0099 & 0.0094 & 0.0807 \\
CG2 Rolls & 0.0051 & 0.0052 & 0.0103 & 0.0210 \\
CSA & 0.0363 & 5.0927 & 0.0641 & 0.6460 \\
CSA Rolls & 0.0046 & 0.8327 & 0.0309 & 1.2743 \\
L1 & 0.0052 & 0.0005 & 0.0054 & 0.0032 \\
MFF & 0.0087 & 2.2364 & 0.0218 & 0.6446 \\
\cline { 2 - 5 } MorphoMobile & 0.0144 & 0.0235 & 0.0116 & 0.2484 \\
\hline & & & & \\
\hline
\end{tabular}


Table 4.11 SourceAFIS Gender Based KLD Values

\begin{tabular}{|r|l|l|l|l|}
\hline SourceAFIS KLD Values & \multicolumn{2}{|c|}{ Male } & \multicolumn{2}{c|}{ Female } \\
\hline Device & Genuine & Imposter & Genuine & Imposter \\
ANDI BIN & 0.0328 & 0.0849 & 0.0309 & 2.5337 \\
ANDI GRY & 0.0269 & 2.5543 & 0.0737 & 0.1235 \\
BioSled & 0.0359 & 1.0288 & 0.1505 & 6.9133 \\
\cline { 2 - 5 } BioSled Rolls & 0.0265 & 0.0113 & 0.0275 & 42.0973 \\
CG2 & 0.0491 & 0.0202 & 0.0228 & 1.4555 \\
\cline { 2 - 5 } CG2 Rolls & 0.0255 & 0.3119 & 0.0294 & 16.7425 \\
CSA & 0.0516 & 1.8634 & 0.1705 & 0.4403 \\
\cline { 2 - 5 } CSA Rolls & 0.0204 & 1.6716 & 0.0719 & 0.1603 \\
L1 & 0.0511 & 0.0240 & 0.0621 & 6.6097 \\
Rolls & 0.0202 & 1.2847 & 0.0205 & 1.6524 \\
MFF & 0.0313 & 1.9772 & 0.0492 & 0.0939 \\
\cline { 2 - 5 } MorphoMobile & 0.0440 & 0.0222 & 0.0364 & 6.4280 \\
\hline & & & &
\end{tabular}

\subsection{Age-Based Study}

\subsubsection{Age-Based Samples of Normal Distributions}

The genuine/imposter distributions shown in Figure 4.32 are sample genuine/imposter match score distributions obtained using the NBIS matcher for male and female strata. They have been chosen to illustrate the typical genuine/imposter score distributions of each type of device and age range used in this study. This study is biased as a large majority of the subjects were between the ages of 20-29. There are no noticeable trends shown within the distributions. The rest of the distributions have been provided in Appendix C. 


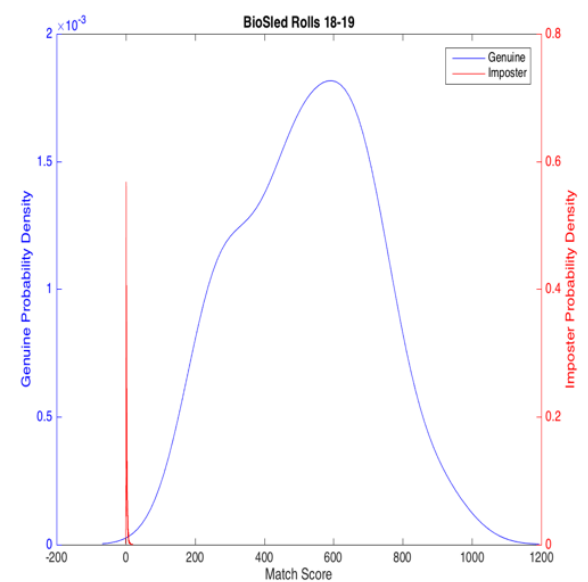

(a)

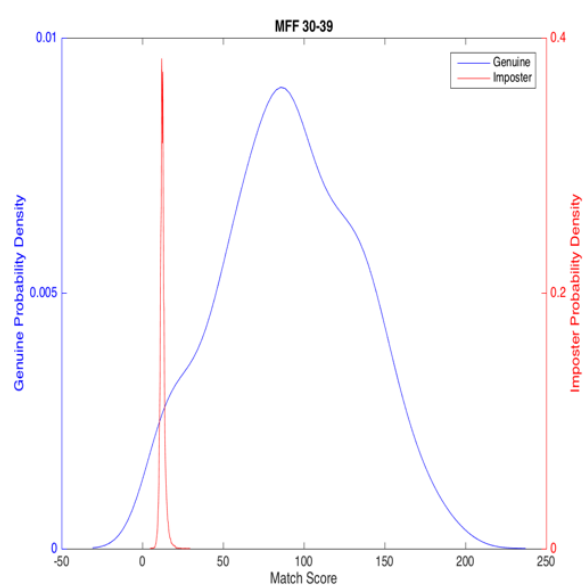

(c)

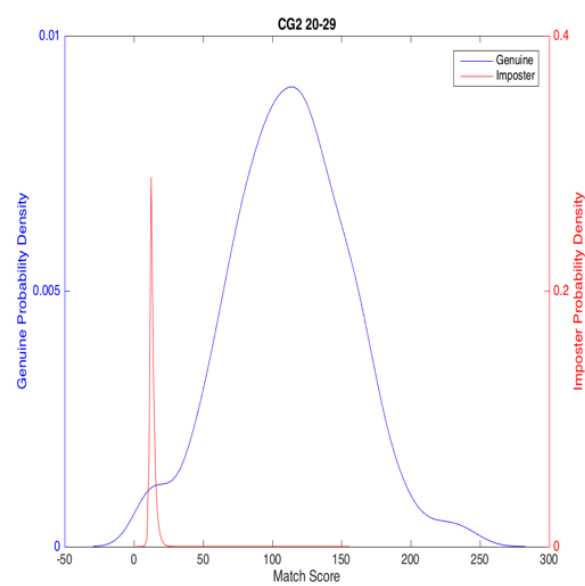

(b)

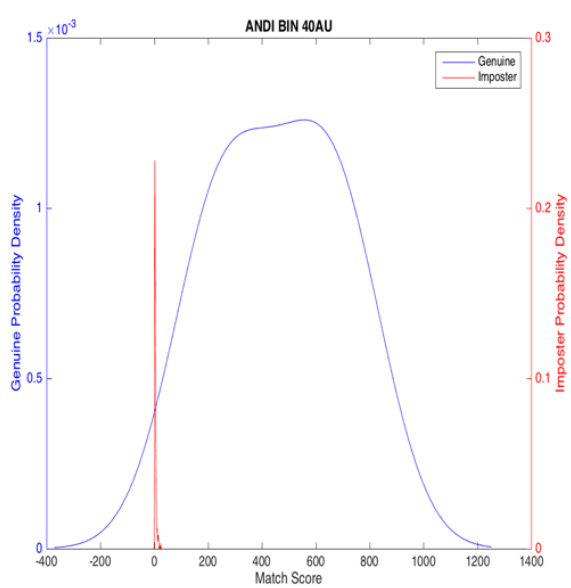

(d)

Figure 4.32 (a) Neurotech BioSled Rolls for ages 18-19 (b) SourceAFIS FP II Guardian Ages 20 - 29

(c) SourceAFIS Morpho FOTF for ages 30 - 39 (d)Neurotech ANDI Binary for Ages 40 and up

\subsubsection{Age-Based Outliers}

Figures $4.33-4.34$ show the outlying distributions from the age-based study. These figures show a slight trend considering each of them are from the age range of 40 years and older, this is most likely caused by older subjects' hands being more worn and wrinkled than those of younger subjects, causing devices to produce lower quality images. 

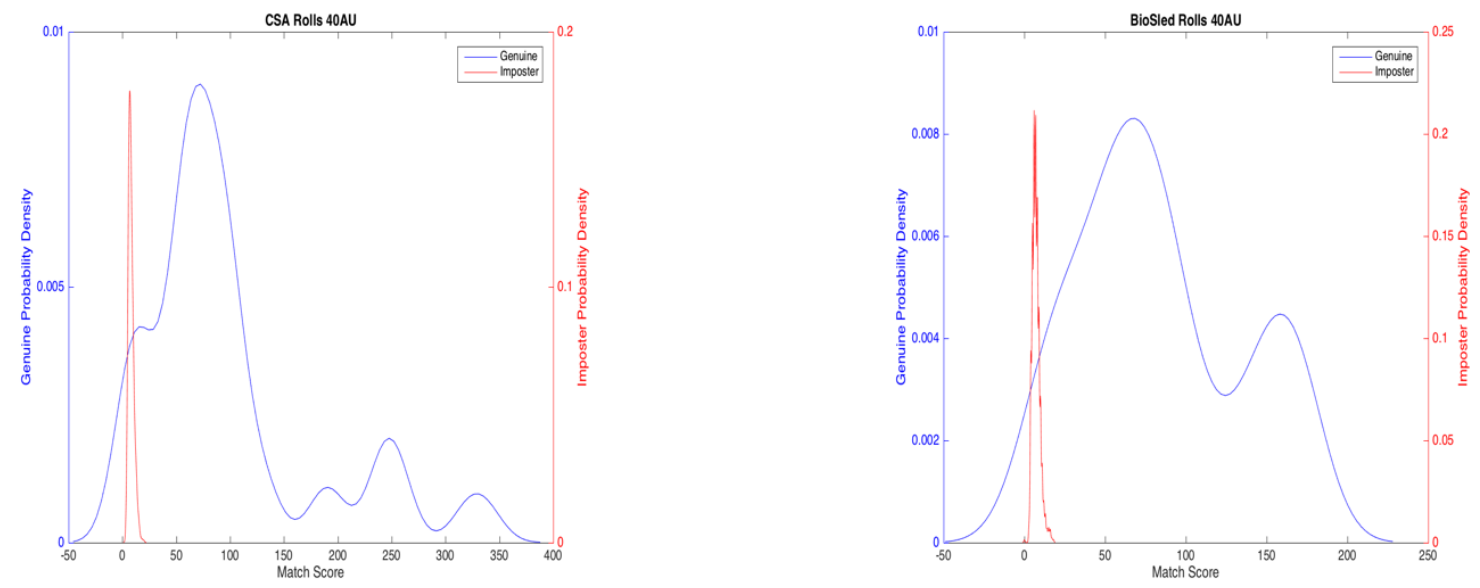

Figure 4.33 (Left) NBIS Crossmatch Seek Avenger Rolls for ages 40 and up (Right) NBIS BioSled Rolls for ages 40 and up
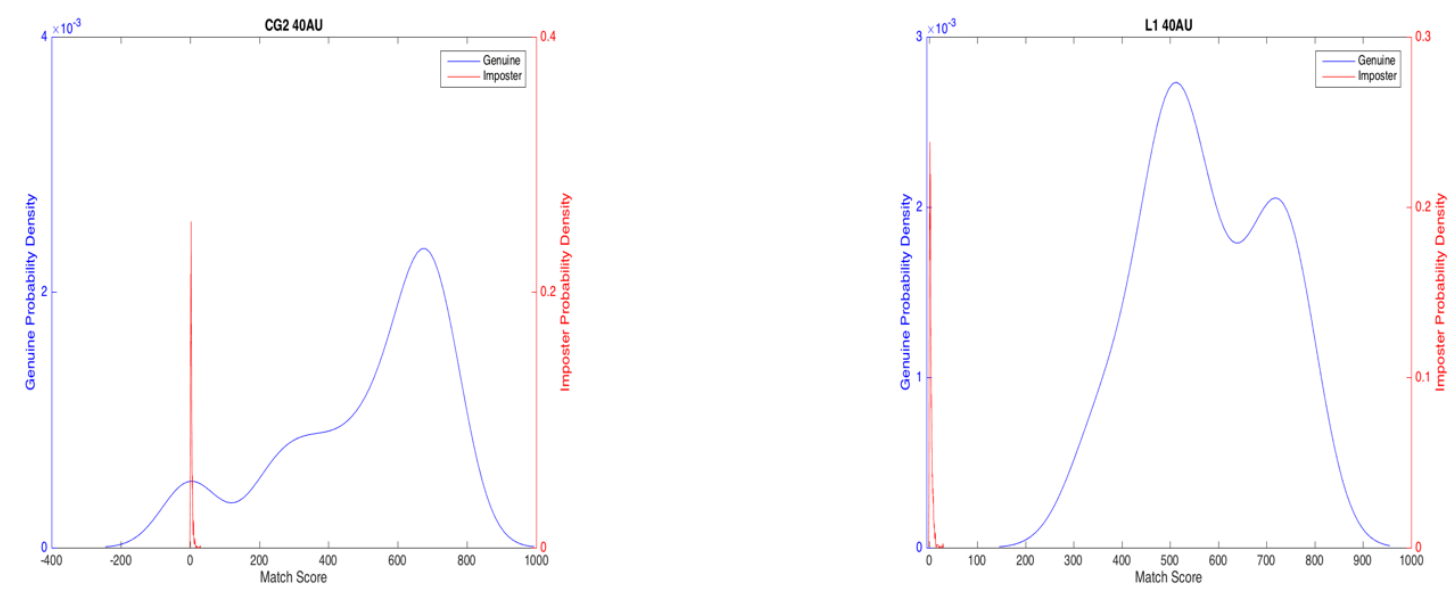

Figure 4.34 (Left) Neurotech FP II Guardian for ages 40 and up (Right) Neurotech L1 for ages 40 and up

\subsubsection{Age-Based KLD Values}

The values shown in Tables $4.12-4.14$ show the Kullback Leibler Divergence measures of the genuine and imposter distributions for the different age ranges on each matcher. The genuine KLD values produced for each age range showed little variation with the genuine KLD averages ranging from 0.0207 to 0.0807 and the imposter KLD averages ranging between 1.604 to 2.7602 between age ranges and matchers. The imposter KLD values show slightly more variation which is likely caused by each one to many match having many more imposter 
matchers as it does genuine matches. These values reveal the similarity to the general study of the complete dataset and show little to no performance variation across age ranges. 
Table 4.12 NBIS Age-Based KLD Values

\begin{tabular}{|r|l|l|l|l|l|l|l|l|}
\hline NBIS KLD Values & \multicolumn{2}{|c|}{$\mathbf{1 8 - 1 9}$} & \multicolumn{2}{c|}{$\mathbf{2 0 - 2 9}$} & \multicolumn{2}{c|}{$\mathbf{3 0 - 3 9}$} & \multicolumn{2}{c|}{$\mathbf{4 0}$ and up } \\
\hline Device & Gen & Imp & Gen & Imp & Gen & Imp & Gen & Imp \\
ANDI BIN & 0.0838 & 0.8833 & 0.0114 & 0.1265 & 0.0489 & 1.0251 & 0.1035 & 0.9506 \\
ANDI GRY & 0.0343 & 0.6518 & 0.0131 & 0.2460 & 0.0730 & 0.8623 & 0.1147 & 0.9976 \\
BioSled & 0.0408 & 1.9944 & 0.0065 & 0.0128 & 0.0554 & 1.9477 & 0.0398 & 1.6229 \\
\cline { 2 - 9 } BioSled Rolls & 0.0437 & 3.4163 & 0.0136 & 0.6659 & 0.0471 & 1.4452 & 0.0861 & 2.5945 \\
CG2 & 0.1168 & 3.1837 & 0.0132 & 0.0120 & 0.0786 & 1.2275 & 0.0984 & 2.7650 \\
\cline { 2 - 9 } CG2 Rolls & 0.0633 & 2.1104 & 0.0071 & 0.0106 & 0.0552 & 0.9662 & 0.0985 & 1.6706 \\
CSA & 0.0394 & 1.7727 & 0.1228 & 0.8538 & 0.2237 & 3.2299 & 0.0485 & 5.4184 \\
\cline { 2 - 9 } CSA Rolls & 0.0348 & 4.3977 & 0.0452 & 7.4043 & 0.0762 & 3.0186 & 0.0991 & 3.9052 \\
L1 & 0.0604 & 3.4227 & 0.0092 & 0.0190 & 0.0559 & 4.0166 & 0.0718 & 2.9361 \\
L1 Rolls & 0.0529 & 2.4702 & 0.0258 & 0.0337 & 0.1650 & 2.7238 & 0.0499 & 4.7252 \\
MFF & 0.0320 & 1.8875 & 0.0137 & 0.1676 & 0.1250 & 1.0947 & 0.0436 & 1.1245 \\
\cline { 2 - 8 } MorphoMobile & 0.0427 & 0.8768 & 0.0373 & 3.5694 & 0.1000 & 0.7262 & 0.1182 & 0.6724 \\
\hline
\end{tabular}

Table 4.13 Neurotech Age-Based KLD Values

\begin{tabular}{|r|l|l|l|l|l|l|l|l|}
\hline Neurotech & \multicolumn{2}{|c|}{$\mathbf{1 8 - 1 9}$} & \multicolumn{2}{c|}{$\mathbf{2 0 - 2 9}$} & \multicolumn{2}{c|}{$30-39$} & \multicolumn{2}{c|}{ 40 and up } \\
\hline Device & Gen & Imp & Gen & Imp & Gen & Imp & Gen & Imp \\
\cline { 2 - 8 } ANDI BIN & 0.0230 & 3.6518 & 0.0022 & 0.1658 & 0.0150 & 3.4460 & 0.0232 & 3.8684 \\
\cline { 2 - 9 } ANDI GRY & 0.0174 & 1.2327 & 0.0029 & 0.9697 & 0.0101 & 0.6944 & 0.0334 & 3.1249 \\
\cline { 2 - 9 } BioSled & 0.0138 & 0.4548 & 0.0033 & 0.0120 & 0.0300 & 0.9620 & 0.0140 & 1.2292 \\
\cline { 2 - 9 } BioS Rolls & 0.0167 & 0.0106 & 0.0073 & 0.0012 & 0.0434 & 0.0183 & 0.0079 & 0.0277 \\
CG2 & 0.0118 & 0.1391 & 0.0036 & 0.0090 & 0.0170 & 0.2309 & 0.0388 & 0.3270 \\
CG2 Rolls & 0.0109 & 0.0156 & 0.0028 & 0.0015 & 0.0324 & 0.0102 & 0.0178 & 0.0164 \\
\cline { 2 - 9 } CSA & 0.0617 & 2.1182 & 0.0153 & 5.0395 & 0.1189 & 1.4025 & 0.0193 & 5.4521 \\
CSA Rolls & 0.0115 & 4.1534 & 0.0377 & 9.8309 & 0.0207 & 6.1675 & 0.0175 & 9.6163 \\
L1 & 0.0203 & 0.0037 & 0.0034 & 0.0002 & 0.0362 & 0.0039 & 0.0289 & 0.0048 \\
MFF & 0.0224 & 2.8592 & 0.0059 & 0.9825 & 0.0252 & 1.9548 & 0.0292 & 2.4726 \\
\cline { 2 - 8 } & 0.0139 & 0.1080 & 0.0044 & 0.4012 & 0.0191 & 0.3839 & 0.0229 & 0.4160 \\
\hline
\end{tabular}


Table 4.14 SourceAFIS Age-Based KLD Values

\begin{tabular}{|c|c|c|c|c|c|c|c|c|}
\hline \multirow{2}{*}{$\begin{array}{r}\text { SourceAFIS KLD } \\
\text { Values } \\
\text { Device }\end{array}$} & \multicolumn{2}{|c|}{$18-19$} & \multicolumn{2}{|c|}{$20-29$} & \multicolumn{2}{|c|}{ 30-39 } & \multicolumn{2}{|c|}{40 and up } \\
\hline & Gen & Imp & Gen & $\operatorname{Imp}$ & Gen & Imp & Gen & Imp \\
\hline ANDI BIN & 0.0360 & 1.1649 & 0139 & 3.7860 & 0.0889 & 5.4711 & 0.0760 & 2.7526 \\
\hline ANDI GRY & 0.0584 & 6.9912 & 5 & 3.2 & & 0.9 & & 1.7703 \\
\hline Biosled & 0.0944 & 3.9 & 0.0 & 0.4 & & & & 58 \\
\hline led Rolls & 0.0369 & 4.31 & & 00 & & & 01 & 43 \\
\hline CG2 & 0.1343 & 4.4908 & 0.0 & 1.2 & & & 89 & 8069 \\
\hline CG2 Rolls & 0.0708 & 8.6481 & & & & & & \\
\hline CSA & 0.0368 & & & & & & & \\
\hline CSA Rolls & 0.02 & & & & & & & \\
\hline$\angle 1$ & 0.0755 & 3.0207 & 0.0 & 0.4659 & 15 & & & \\
\hline L1 Rolls & 0.1159 & & & & 43 & & & 1.5156 \\
\hline MFF & 0.0866 & 1.1763 & 0.0243 & 1.9716 & 043 & 0.9298 & 0.0611 & 2.3292 \\
\hline MorphoMobile & 0.1229 & 4.1186 & 0.0235 & 10.5725 & 0.1931 & 5.1173 & 0.1420 & 14.1331 \\
\hline
\end{tabular}

\subsection{Ethnicity-Based Study}

\subsubsection{Ethnicity-Based Study Sample Genuine/Imposter Distributions}

The genuine/imposter distributions shown in Figures 4.35 - 4.38 are sample genuine/imposter match score distributions obtained using the each of the three matching algorithms for each possible ethnicity from this collection. They have been chosen to illustrate the typical genuine/imposter score distributions of each type of device and ethnicity used in this study. This study is biased as a large majority of the subjects were of the Caucasian ethnicity. The ethnicities of "Other Pacific Islander" and "Unknown" data were not used in this study as they did not have enough results to provide distributions or analysis. There are no noticeable trends shown within the distributions. The rest of the distributions have been provided in Appendix D. 

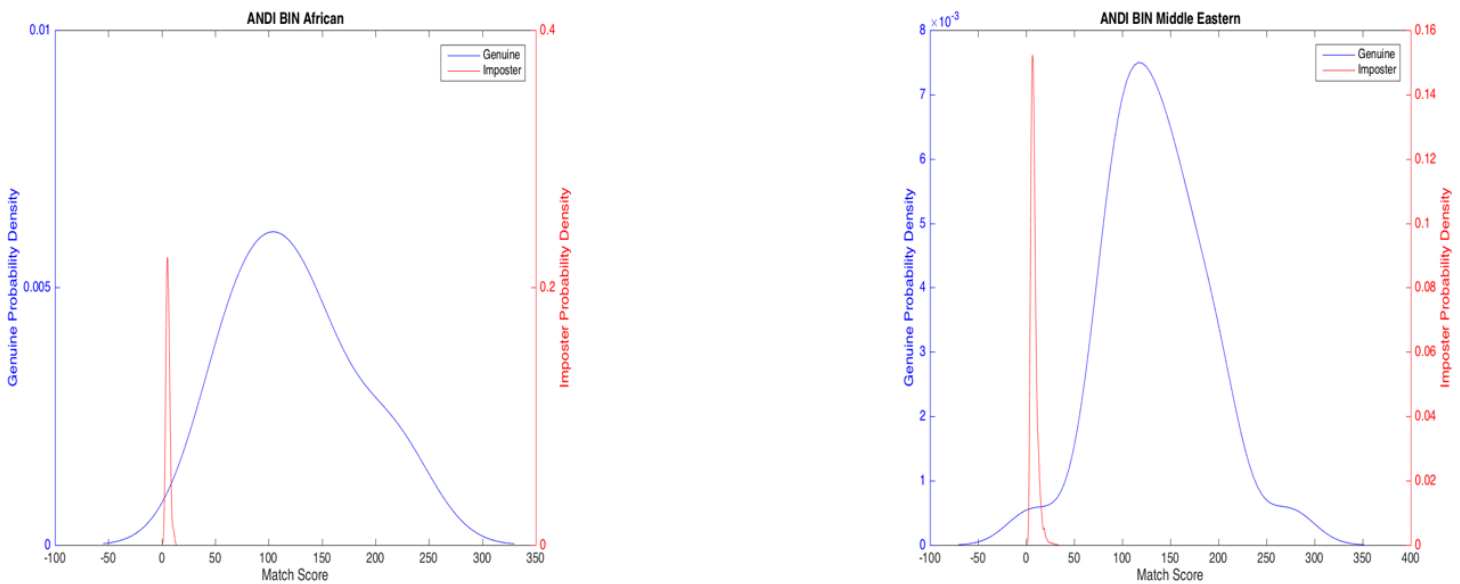

Figure 4.35 (Left) NBIS ANDI Binary for the African Ethnicity (Right) NBIS ANDI Binary for the Middle Eastern Ethnicity
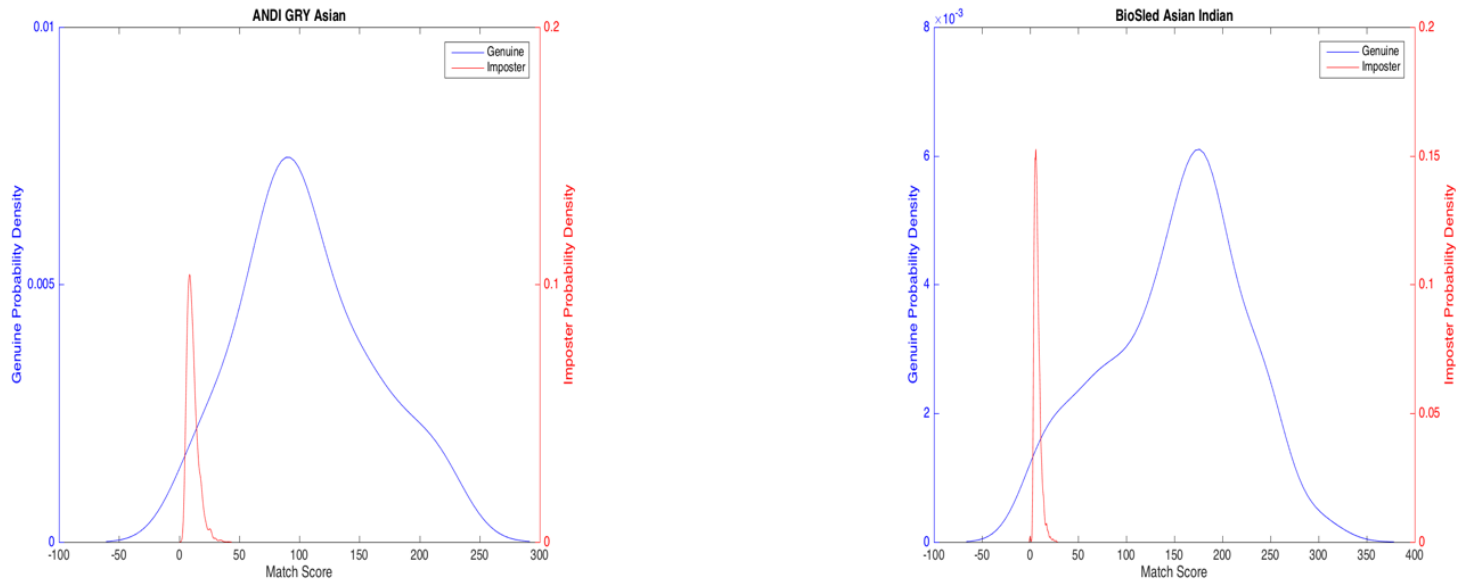

Figure 4.36 (Left) NBIS ANDI Greyscale for the Asian Ethnicity (Right) NBIS BioSled for the Asian Indian Ethnicity 

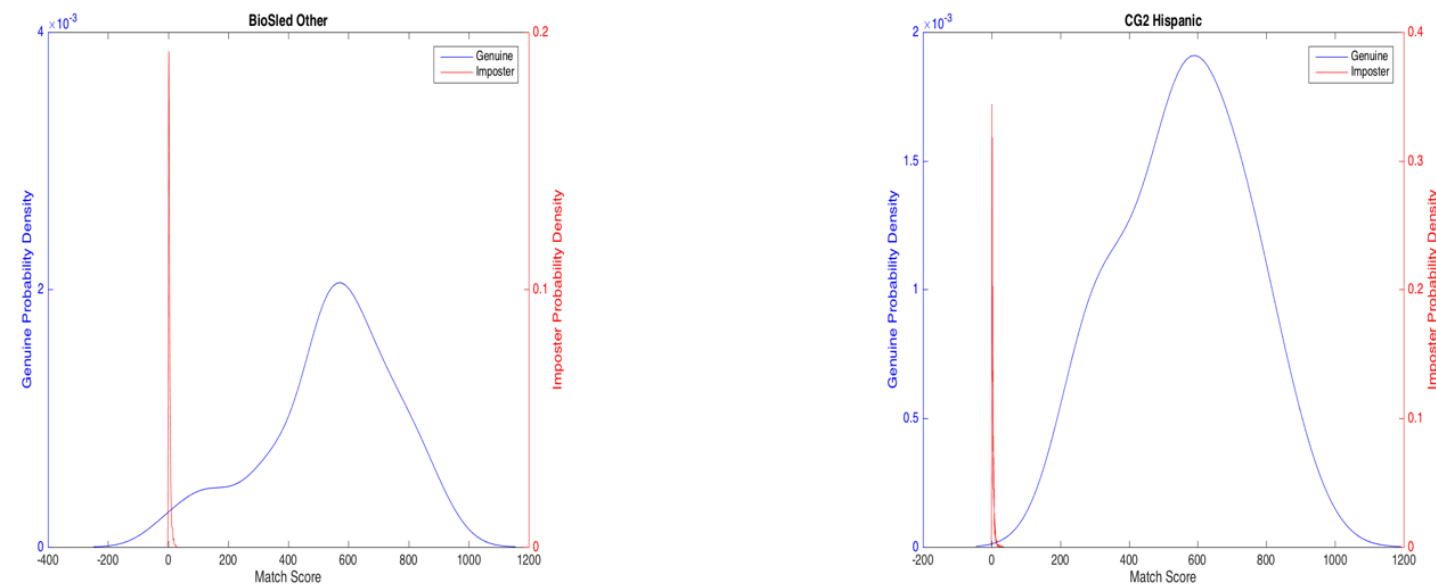

Figure 4.37 (Left) Neurotech BioSled for the Other Ethnicity (Right) Neurotech FP II Guardian for the Hispanic Ethnicity
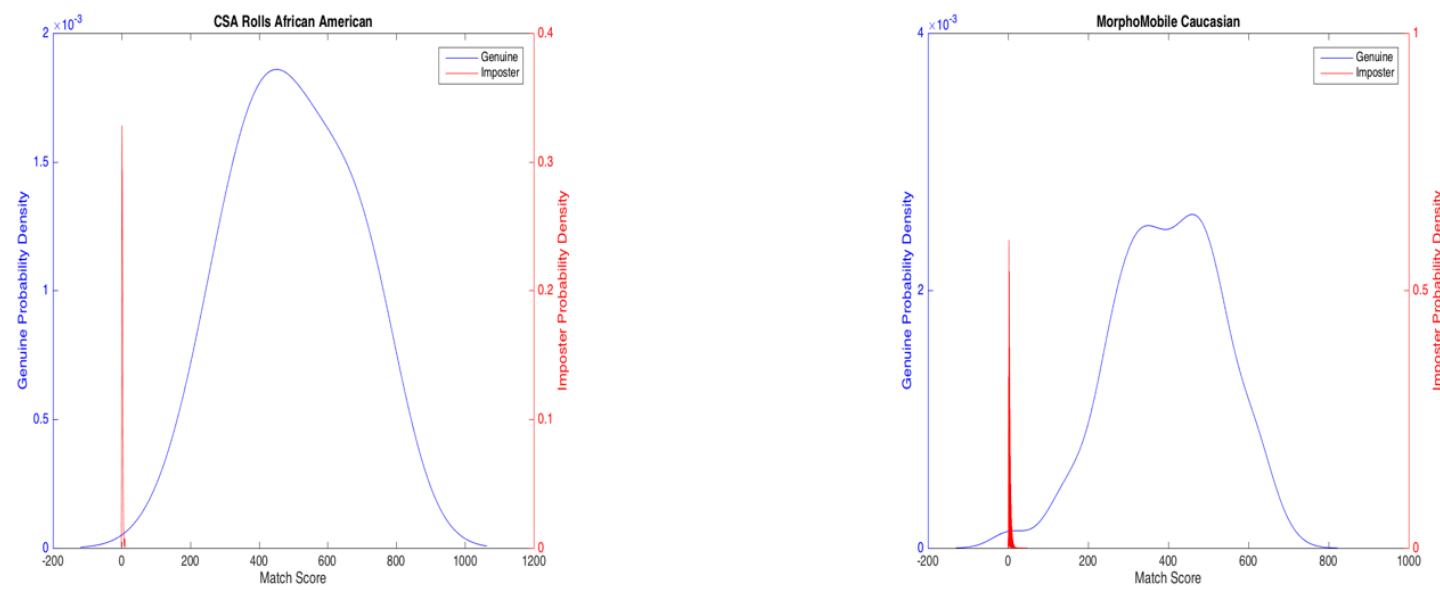

Figure 4.38 (Left) SourceAFIS Crossmatch Seek Avenger Rolls For the African American Ethncity (Right) SourceAFIS MorphoMobile for the Caucasian Ethnicity

\subsubsection{Ethnicity-Based Outliers}

The outliers that were produced for this study all involved the American Indian Ethnicity. Considering Figure 3.5, these graphs are most likely caused by the low number of subjects identified as American Indian. The large variation in these graphs also had an effect on the KLD values for this ethnicity, shown in Table 4.18. 

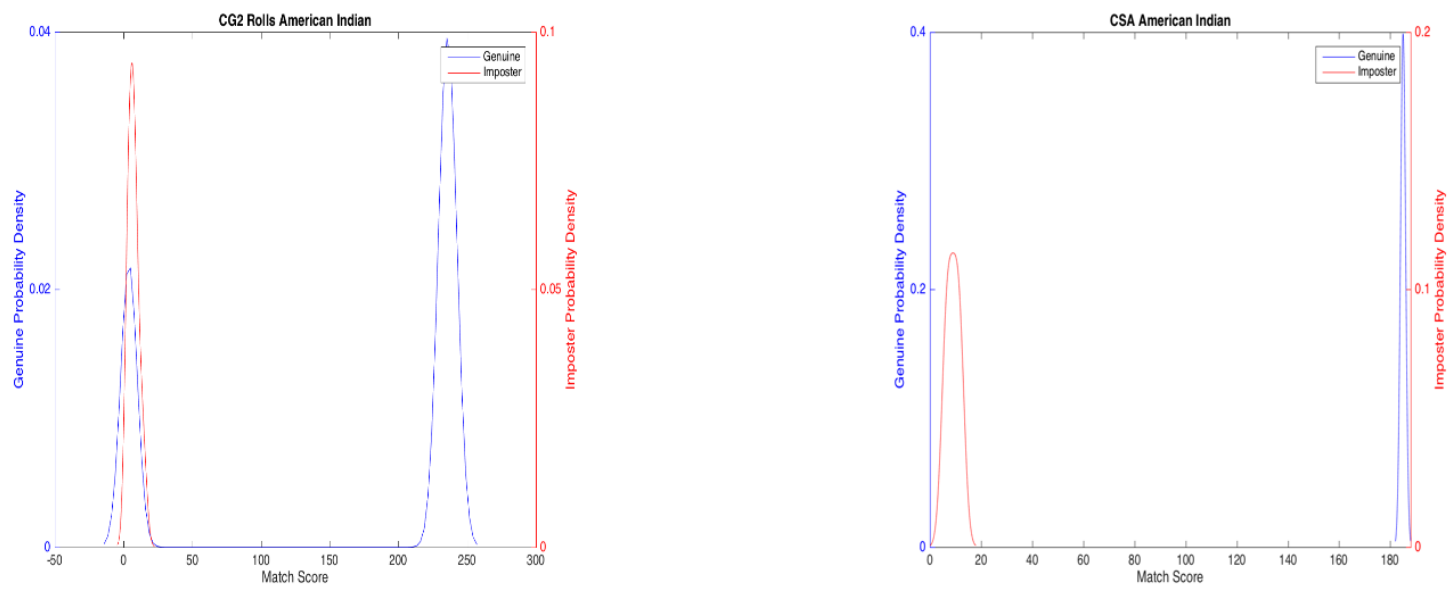

Figure 4.39 (Left) NBIS FP II Rolls For the American Indian Ethnicity (Right) NBIS Crossmatch Seek Avenger for the American Indian Ethnicity
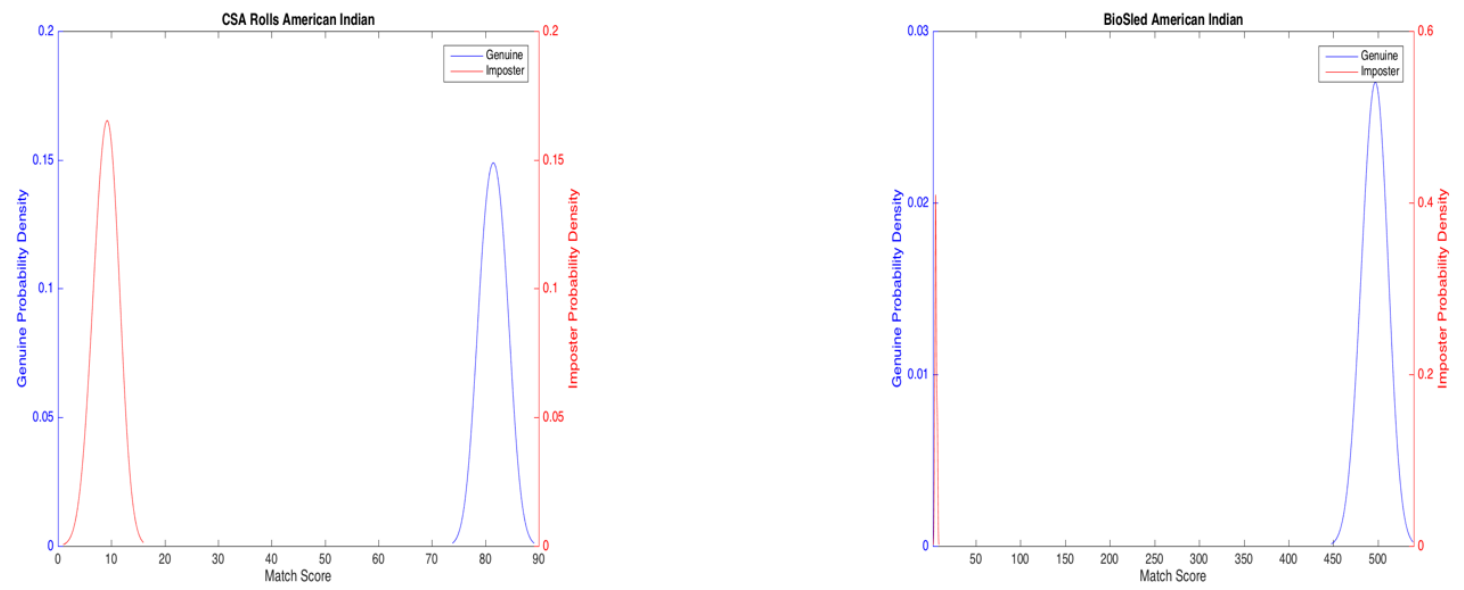

Figure 4.40 (Left) NBIS Crossmatch Seek Avenger Rolls for the American Indian Ethnicity (Right) Neurotech BioSled for the American Indian Ethnicity 

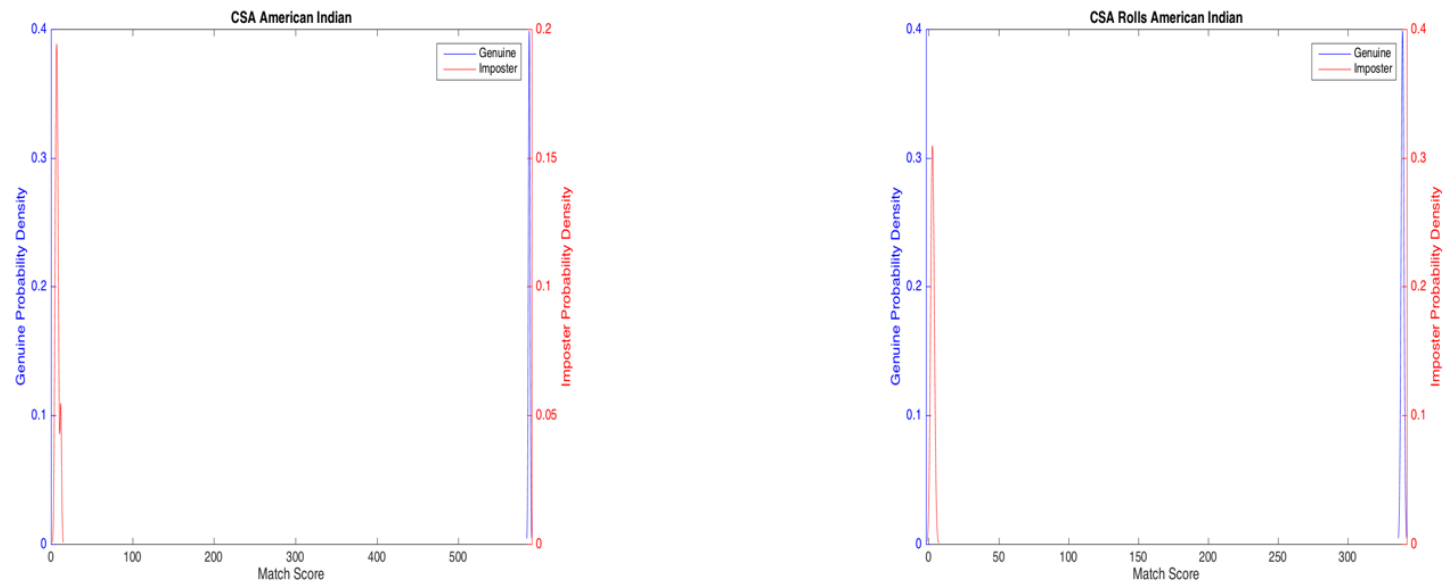

Figure 4.41 (Left) Neurotech Crossmatch Seek Avenger for the American Indian Ethnicity (Right) Neurotech Crossmatch Seek Avenger Rolls for the American Indian Ethnicity
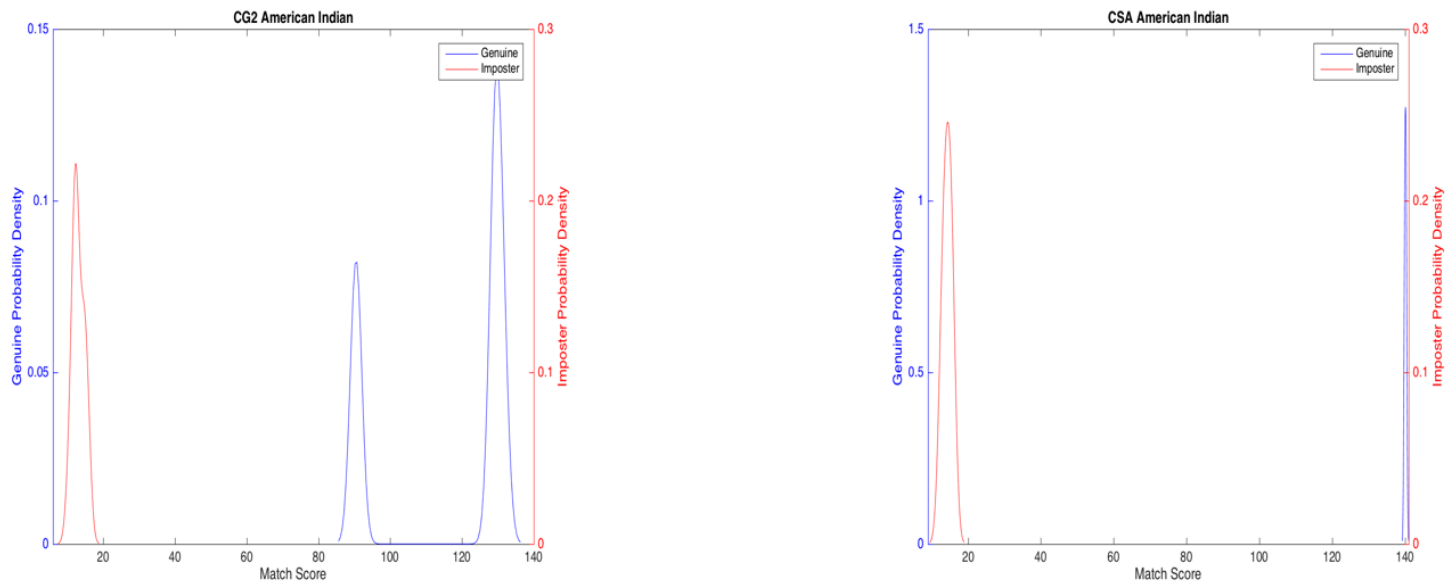

Figure 4.42 (Left) SourceAFIS FP II Guardian for the American Indian Ethnicity (Right) SourceAFIS Crossmatch Seek Avenger for the American Indian Ethnicity 

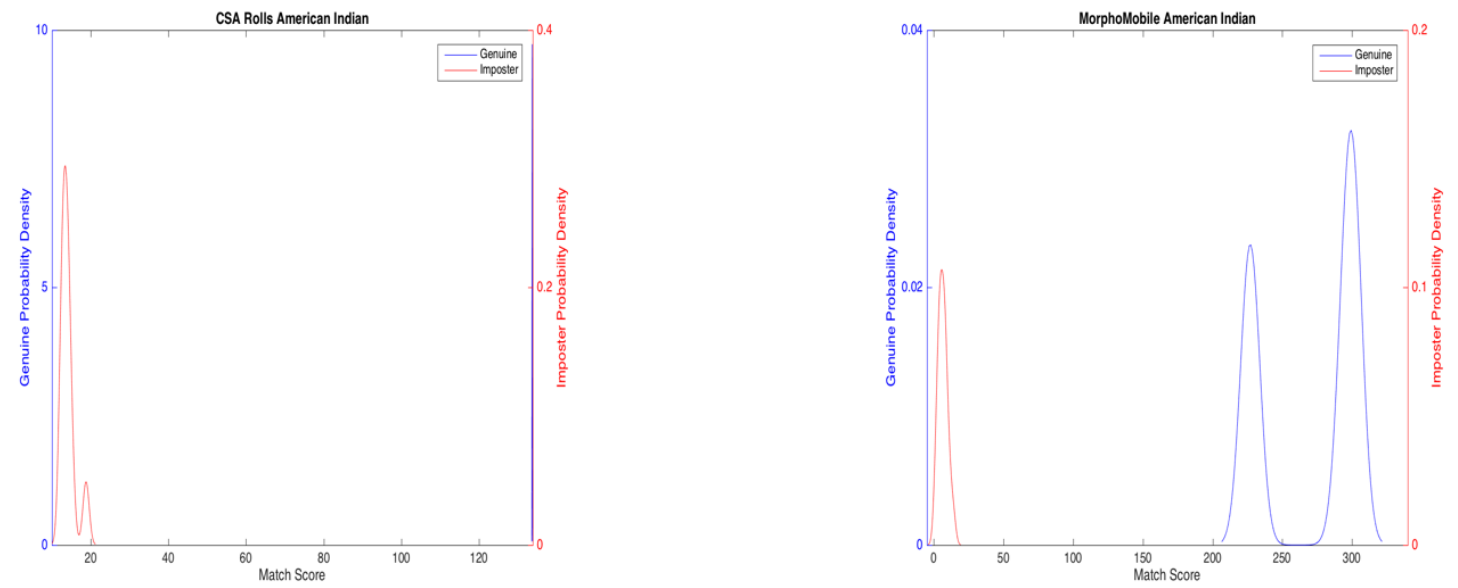

Figure 4.43 (Left) SourceAFIS Crossmatch Seek Avenger Rolls for the American Indian Ethnicity (Right) SourceAFIS MorphoMobile for the American Indian Ethnicity

\subsubsection{Ethnicity-Based KLD Values}

The KLD values for the ethnicities involved in this study showed little variation with averages ranging from 0.0655 to 1.2355 for genuine distributions and 2.0559 to 3.7915 for the imposter distributions. Table 4.15 shows the averages KLD values among devices for each ethnicity. Tables $4.16-4.24$ show the KLD values produced by each matcher and device by ethnicity. These values reveal the similarity to the original dataset and show little to no performance variation across ethnicities. The only genuine outlier and highest KLD value, the American Indian ethnicity averaged a value of 1.2355 , the main reason for this variation is because this ethnicity occupied a very small subset of the data as well as the distributions shown in Figures 4.39 - 4.43. 
Table 4.15 African Ethnicity KLD

\begin{tabular}{|l|r|r|}
\hline \multicolumn{3}{|c|}{ Average KLD Value per Ethnicity } \\
\hline & Genuine & Imposter \\
\hline African & 0.1489 & 2.6386 \\
\hline African American & 0.0940 & 2.1271 \\
\hline American Indian & 1.2355 & 2.8202 \\
\hline Asian & 0.0736 & 2.3542 \\
\hline Asian Indian & 0.0849 & 3.7915 \\
\hline Caucasian & 0.1290 & 2.7291 \\
\hline Hispanic & 0.1761 & 2.9911 \\
\hline Middle Eastern & 0.1090 & 2.2396 \\
\hline Other & 0.0655 & 2.0559 \\
\hline
\end{tabular}

Table 4.16 African Ethnicity KLD

\begin{tabular}{|r|c|r|r|r|r|r|}
\hline & \multicolumn{2}{|c|}{ NBIS } & \multicolumn{2}{c|}{ Neurotech } & \multicolumn{2}{c|}{ SourceAFIS } \\
\hline Device & Genuine & Imposter & Genuine & Imposter & Genuine & Imposter \\
\cline { 2 - 7 } ANDI BIN & 0.0783 & 3.7148 & 0.0097 & 11.8608 & 0.2958 & 2.7067 \\
\cline { 2 - 7 } ANDI GRY & 0.0510 & 1.7698 & 0.0311 & 2.5344 & 0.4007 & 1.5936 \\
\cline { 2 - 7 } BioSled & 1.1091 & 1.3129 & 0.1777 & 1.294 & 0.4054 & 2.0859 \\
\cline { 2 - 7 } BioSled Rolls & 0.0779 & 1.8666 & 0.0744 & 0.0389 & 0.0877 & 1.3681 \\
CG2 & 0.2033 & 1.9118 & 0.0442 & 0.382 & 0.1495 & 3.1481 \\
\cline { 2 - 7 } CG2 Rolls & 0.0749 & 1.6157 & 0.0462 & 0.0045 & 0.1186 & 1.4545 \\
CSA & 0.0744 & 1.1948 & 0.074 & 8.7876 & 0.1544 & 1.8807 \\
\cline { 2 - 7 } CSA Rolls & 0.1097 & 4.4832 & 0.0204 & 11.2825 & 0.0627 & 1.3722 \\
L1 & 0.1288 & 2.8118 & 0.158 & 0.0027 & 0.1761 & 1.9177 \\
\cline { 2 - 7 } L1 Rolls & 0.1106 & 3.7102 & & & 0.0652 & 2.1790 \\
MFF & 0.1435 & 2.3449 & & & 0.0991 & 3.9406 \\
\cline { 2 - 7 } SMM & 0.0581 & 0.4241 & 0.0151 & 0.4388 & 0.1763 & 2.2796 \\
\hline
\end{tabular}

As stated earlier the Neurotech matcher removed images of bad quality and that is why there are blank spots in this table as there were not enough results to process the KLD values. There are no genuine outlying values and the outlying values of the imposters are most likely caused by the fact that for every match there are more imposter matches than there are genuine 
matches, so imposter values should show more variation. These KLD values are inconclusive as they show little to no performance variation.

Table 4.17 African American Ethnicity KLD

\begin{tabular}{|r|r|r|r|r|r|r|}
\hline & \multicolumn{2}{|c|}{ NBIS } & \multicolumn{2}{c|}{ Neurotech } & \multicolumn{2}{c|}{ SourceAFIS } \\
\hline Device & Genuine & Imposter & Genuine & Imposter & Genuine & Imposter \\
\cline { 2 - 7 } ANDI BIN & 0.0958 & 2.1134 & 0.0149 & 5.2858 & 0.1160 & 1.6159 \\
\cline { 2 - 7 } ANDI GRY & 0.0829 & 0.8404 & 0.012 & 2.4253 & 0.1484 & 1.7079 \\
\cline { 2 - 7 } BioSled & 0.0744 & 1.6203 & 0.019 & 0.9989 & 0.1706 & 2.0052 \\
\cline { 2 - 7 } BioSled Rolls & 0.0757 & 1.9739 & 0.0172 & 0.0317 & 0.0599 & 1.8500 \\
\cline { 2 - 7 } CG2 & 0.1037 & 1.4777 & 0.0316 & 0.0974 & 0.2349 & 0.4904 \\
CG2 Rolls & 0.0621 & 0.9826 & 0.0208 & 0.0052 & 0.0525 & 1.0825 \\
\cline { 2 - 7 } CSA & 0.1006 & 1.3257 & 0.0186 & 5.251 & 0.2122 & 1.8233 \\
CSA Rolls & 0.0769 & 3.1626 & 0.0244 & 9.1188 & 0.1688 & 1.5454 \\
\cline { 2 - 7 } L1 & 0.1638 & 3.5862 & 0.0152 & 0.0029 & 0.1865 & 3.9513 \\
\cline { 2 - 7 } L1 Rolls & 0.1393 & 2.2078 & & & 0.0430 & 1.5083 \\
\cline { 2 - 7 } MFF & 0.0915 & 1.0985 & & & 0.0971 & 6.1422 \\
\cline { 2 - 7 } SMM & 0.1340 & 0.5541 & 0.0405 & 0.3605 & 0.2903 & 4.0777 \\
\hline
\end{tabular}

As stated above the Neurotech matcher removed images of bad quality and that is why there are blank spots in this table as there were not enough results to process the KLD values. There are no genuine outlying values and the outlying values of the imposters are most likely caused by the fact that for every match there are more imposter matches than there are genuine matches, so imposter values will show more variation. These KLD values are inconclusive as they show little to no performance variation. 
Table 4.18 American Indian Ethnicity KLD

\begin{tabular}{|r|r|r|r|r|r|r|}
\hline & \multicolumn{2}{|c|}{ NBIS } & \multicolumn{2}{c|}{ Neurotech } & \multicolumn{2}{c|}{ SourceAFIS } \\
\hline Device & Genuine & Imposter & Genuine & Imposter & Genuine & Imposter \\
\cline { 2 - 7 } ANDI BIN & 0.1323 & 3.4101 & 0.008 & 10.1086 & 0.1132 & 4.7527 \\
\cline { 2 - 7 } ANDI GRY & 0.4058 & 2.3173 & 0.0299 & 2.9429 & 0.2532 & 1.3756 \\
\cline { 2 - 7 } BioSled & 0.2299 & 1.6737 & 0.2007 & 0.9628 & 1.6077 & 1.8946 \\
\cline { 2 - 7 } BioSled Rolls & 0.1608 & 1.8898 & 0.3719 & 0.0142 & 0.1340 & 1.8281 \\
\cline { 2 - 7 } CG2 & 0.1522 & 1.6136 & 0.1273 & 0.4346 & 11.5257 & 3.1644 \\
\cline { 2 - 7 } CG2 Rolls & 16.2262 & 1.5360 & 0.0367 & 0.0117 & 0.2110 & 1.2978 \\
\cline { 2 - 7 } CSA & 0.6285 & 3.3994 & 0.3452 & 7.3243 & 1.4274 & 2.5726 \\
\cline { 2 - 7 } CSA Rolls & 0.4880 & 7.7471 & 0.256 & 13.0463 & 1.1587 & 1.6133 \\
\cline { 2 - 7 } L1 & 0.0855 & 2.4008 & 0.042 & 0.0021 & 0.3275 & 2.1223 \\
\cline { 2 - 7 } L1 Rolls & 1.0198 & 3.2321 & & & 0.3340 & 1.8069 \\
\cline { 2 - 7 } MFF & 0.1508 & 2.7805 & 0.0272 & 4.5010 & 0.2095 & 1.8234 \\
\cline { 2 - 7 } SMM & 0.7334 & 0.5920 & 0.4359 & 0.405 & 3.6469 & 2.1081 \\
\hline
\end{tabular}

As stated above the Neurotech matcher removed images of bad quality and that is why there are blank spots in this table as there were not enough results to process the KLD values. The outlying values for genuine matches show variation for the FP II Guardian and this is most likely caused from there being such a small number of subjects identifying as American Indian that participated in this collection as shown in Figures 3.6 and 3.8 point out. 
Table 4.19 Asian Ethnicity KLD

\begin{tabular}{|r|c|r|r|r|r|r|}
\hline & \multicolumn{2}{|c|}{ NBIS } & \multicolumn{2}{c|}{ Neurotech } & \multicolumn{2}{c|}{ SourceAFIS } \\
\hline Device & Genuine & Imposter & Genuine & Imposter & Genuine & Imposter \\
ANDI BIN & 0.0840 & 0.9661 & 0.0171 & 3.073 & 0.0840 & 4.7585 \\
\cline { 2 - 7 } ANDI GRY & 0.1544 & 0.7247 & 0.0139 & 0.7672 & 0.1145 & 5.1985 \\
\cline { 2 - 7 } BioSled & 0.0697 & 2.2829 & 0.0071 & 0.7864 & 0.1331 & 2.9401 \\
\cline { 2 - 7 } BioSled Rolls & 0.0348 & 1.6884 & 0.0343 & 0.023 & 0.0268 & 1.5402 \\
\cline { 2 - 7 } CG2 & 0.0359 & 3.6145 & 0.0219 & 0.29 & 0.1013 & 4.2584 \\
\cline { 2 - 7 } CG2 Rolls & 0.0609 & 1.0781 & 0.0156 & 0.0118 & 0.0552 & 2.3071 \\
\cline { 2 - 7 } CSA & 0.1235 & 0.7760 & 0.0267 & 4.9202 & 0.2142 & 1.0064 \\
\cline { 2 - 7 } CSA Rolls & 0.0646 & 3.4451 & 0.0184 & 5.9803 & 0.0797 & 4.0234 \\
\cline { 2 - 7 } L1 & 0.0797 & 4.1767 & 0.0476 & 0.0046 & 0.2285 & 3.3654 \\
\cline { 2 - 7 } Lolls & 0.0824 & 3.1502 & & & 0.0987 & 1.2757 \\
\cline { 2 - 7 } MFF & 0.0782 & 1.1225 & 0.0438 & 3.1822 & 0.1283 & 2.5673 \\
\cline { 2 - 7 } SMM & 0.0992 & 0.8352 & 0.0219 & 0.1911 & 0.0760 & 6.0662 \\
\hline
\end{tabular}

As stated above the Neurotech matcher removed images of bad quality and that is why there are blank spots in this table as there were not enough results to process the KLD values. There are no genuine outlying values and the outlying values of the imposters are most likely caused by the fact that for every match there are more imposter matches than there are genuine matches, so imposter values will show more variation. These KLD values are inconclusive as they show little to no performance variation. 
Table 4.20 Asian Indian Ethnicity KLD

\begin{tabular}{|r|c|r|r|r|r|r|}
\hline & \multicolumn{2}{|c|}{ NBIS } & \multicolumn{2}{c|}{ Neurotech } & \multicolumn{2}{c|}{ SourceAFIS } \\
\hline Device & Genuine & Imposter & Genuine & Imposter & Genuine & Imposter \\
\cline { 2 - 7 } ANDI BIN & 0.1264 & 1.1123 & 0.0179 & 3.4657 & 0.0501 & 3.6349 \\
\cline { 2 - 7 } ANDI GRY & 0.1199 & 0.6402 & 0.0115 & 1.506 & 0.1811 & 1.6904 \\
\cline { 2 - 7 } BioSled & 0.0495 & 1.6303 & 0.029 & 0.6308 & 0.1587 & 3.2662 \\
\cline { 2 - 7 } BioSled Rolls & 0.0640 & 1.1785 & 0.0062 & 0.0119 & 0.0287 & 59.8285 \\
\cline { 2 - 7 } CG2 & 0.0854 & 3.7023 & 0.0242 & 0.1915 & 0.1493 & 2.9030 \\
\cline { 2 - 7 } CG2 Rolls & 0.0377 & 0.7534 & 0.0166 & 0.0147 & 0.0658 & 3.8008 \\
\cline { 2 - 7 } CSA & 0.1555 & 4.0159 & 0.0206 & 2.4215 & 0.2086 & 1.0524 \\
\cline { 2 - 7 } CSA Rolls & 0.1786 & 4.2146 & 0.0805 & 4.7043 & 0.1789 & 1.6509 \\
\cline { 2 - 7 } L1 & 0.0133 & 4.0681 & 0.0309 & 0.0042 & 0.1051 & 2.0692 \\
L1 Rolls & 0.0592 & 2.9283 & & & 0.0603 & 2.1540 \\
\cline { 2 - 7 } MFF & 0.1142 & 1.1213 & 0.0325 & 2.7939 & 0.0915 & 0.9591 \\
\cline { 2 - 7 } SMM & 0.1598 & 0.7430 & 0.0186 & 0.1396 & 0.2404 & 7.7016 \\
\hline
\end{tabular}

For Tables 4.20 through 4.22 as already stated, the Neurotech matcher removed images of bad quality and that is why there are blank spots in this table as there were not enough results to process the KLD values. There are no genuine outlying values and the outlying values of the imposters are most likely caused by the fact that for every match there are more imposter matches than there are genuine matches, so imposter values will show more variation. These KLD values are inconclusive as they show little to no performance variation. 
Table 4.21 Caucasian Ethnicity KLD

\begin{tabular}{|r|c|c|c|c|c|c|}
\hline & \multicolumn{2}{|c|}{ NBIS } & \multicolumn{2}{c|}{ Neurotech } & \multicolumn{2}{c|}{ SourceAFIS } \\
\hline Device & Genuine & Imposter & Genuine & Imposter & Genuine & Imposter \\
\cline { 2 - 7 } ANDI BIN & 0.0451 & 1.2735 & 0.0054 & 4.9975 & 0.0217 & 4.9814 \\
\cline { 2 - 7 } ANDI GRY & 0.0177 & 0.2685 & 0.0051 & 0.1466 & 0.0348 & 5.4394 \\
\cline { 2 - 7 } BioSled & 0.0126 & 0.0685 & 0.0086 & 0.1891 & 0.0809 & 0.5678 \\
\cline { 2 - 7 } BioSled Rolls & 0.0147 & 0.4063 & 0.0042 & 0.0155 & 0.0195 & 0.3038 \\
\cline { 2 - 7 } CG2 & 0.0188 & 0.5531 & 0.0066 & 0.1977 & 0.0453 & 1.9521 \\
\cline { 2 - 7 } CG2 Rolls & 0.0203 & 1.6604 & 0.0187 & 0.0238 & 0.0223 & 6.5154 \\
\cline { 2 - 7 } CSA & 0.0130 & 0.7696 & 0.0305 & 5.8025 & 0.0317 & 0.6262 \\
\cline { 2 - 7 } CSA Rolls & 0.0114 & 10.4262 & 0.0049 & 0.8022 & 0.0125 & 1.1713 \\
\cline { 2 - 7 } L1 & 0.0191 & 0.1701 & 0.009 & 0.0005 & 0.0174 & 1.1054 \\
\cline { 2 - 7 } L1 Rolls & 0.0127 & 0.2256 & & & 0.0356 & 2.1613 \\
\cline { 2 - 7 } MFF & 0.0133 & 2.6603 & 0.3185 & 16.0444 & 0.0555 & 0.4224 \\
\cline { 2 - 7 } SMM & 0.0220 & 1.7941 & 3.4609 & 8.4185 & 0.0440 & 13.3578 \\
\hline
\end{tabular}

Table 4.22 Hispanic Ethnicity KLD

\begin{tabular}{|r|c|r|r|r|r|r|}
\hline & \multicolumn{2}{|c|}{ NBIS } & \multicolumn{2}{c|}{ Neurotech } & \multicolumn{2}{c|}{ SourceAFIS } \\
\hline Device & Genuine & Imposter & Genuine & Imposter & Genuine & Imposter \\
\cline { 2 - 7 } ANDI BIN & 0.1275 & 3.9449 & 0.0159 & 3.4466 & 0.0701 & 1.9296 \\
ANDI GRY & 0.1196 & 0.5169 & 0.0162 & 0.5137 & 0.0884 & 1.1892 \\
\cline { 2 - 7 } BioSled & 0.0451 & 1.8945 & 0.0298 & 0.0173 & 0.1920 & 1.8107 \\
BioSled Rolls & 0.0863 & 0.2200 & 0.0167 & 0.0226 & 0.0685 & 0.1880 \\
\cline { 2 - 7 } CG2 & 0.0451 & 1.5253 & 0.0383 & 0.1664 & 0.0971 & 6.6522 \\
CG2 Rolls & 0.0720 & 2.0993 & 0.0163 & 0.016 & 0.0714 & 3.2236 \\
\cline { 2 - 7 } CSA & 0.1817 & 1.2669 & 0.0166 & 2.2627 & 0.1413 & 2.8328 \\
CSA Rolls & 0.0300 & 3.1587 & 0.0163 & 4.6099 & 0.0894 & 2.3991 \\
\cline { 2 - 7 } L1 & 0.0292 & 3.6985 & 0.024 & 0.0034 & 0.1326 & 2.5505 \\
\hline L1 Rolls & 0.0485 & 2.9663 & & & 0.0691 & 2.0646 \\
\cline { 2 - 7 } MFF & 0.1302 & 1.1028 & 0.3185 & 16.0444 & 0.1329 & 3.5195 \\
\cline { 2 - 7 } SMM & 0.0447 & 0.7097 & 3.4609 & 8.4185 & 0.0796 & 17.7029 \\
\hline
\end{tabular}


Table 4.23 Middle Eastern Ethnicity KLD

\begin{tabular}{|r|c|r|r|r|r|r|}
\hline & \multicolumn{2}{|c|}{ NBIS } & \multicolumn{2}{c|}{ Neurotech } & \multicolumn{2}{c|}{ SourceAFIS } \\
\hline Device & Genuine & Imposter & Genuine & Imposter & Genuine & Imposter \\
\cline { 2 - 7 } ANDI BIN & 0.1897 & 0.9501 & 0.0567 & 2.7733 & 0.1821 & 1.6499 \\
\cline { 2 - 7 } ANDI GRY & 0.1799 & 0.4859 & 0.0509 & 1.7713 & 0.2166 & 3.6323 \\
\cline { 2 - 7 } BioSled & 0.0942 & 1.3721 & 0.0299 & 0.7524 & 0.2231 & 2.9841 \\
\cline { 2 - 7 } BioSled Rolls & 0.0573 & 2.0614 & 0.0219 & 0.0228 & 0.1069 & 2.4911 \\
\cline { 2 - 7 } CG2 & 0.0991 & 3.0393 & 0.0211 & 0.2659 & 0.1805 & 3.7760 \\
\cline { 2 - 7 } CG2 Rolls & 0.0197 & 1.0196 & 0.0274 & 0.0091 & 0.0553 & 1.8371 \\
\cline { 2 - 7 } CSA & 0.2012 & 3.0568 & 0.1126 & 3.3431 & 0.2585 & 1.2818 \\
\cline { 2 - 7 } CSA Rolls & 0.1629 & 3.0872 & 0.0375 & 7.193 & 0.1423 & 1.1311 \\
\cline { 2 - 7 } L1 & 0.0284 & 1.2655 & 0.0399 & 0.0036 & 0.1101 & 3.7773 \\
\cline { 2 - 7 } Lolls & 0.0442 & 2.7705 & & & 0.0589 & 1.3476 \\
\cline { 2 - 7 } MFF & 0.1744 & 1.1906 & 0.0636 & 1.9447 & 0.1368 & 1.1941 \\
\cline { 2 - 7 } SMM & 0.1195 & 0.6415 & 0.0162 & 0.1793 & 0.2942 & 14.0849 \\
\hline
\end{tabular}

Table 4.24 Other Ethnicity KLD

\begin{tabular}{|r|c|c|c|c|c|r|}
\hline & \multicolumn{2}{|c|}{ NBIS } & \multicolumn{2}{c|}{ Neurotech } & \multicolumn{2}{c|}{ SourceAFIS } \\
\hline Device & Genuine & Imposter & Genuine & Imposter & Genuine & Imposter \\
\cline { 2 - 7 } ANDI BIN & 0.1411 & 1.1652 & 0.0309 & 2.9675 & 0.0872 & 1.1178 \\
\cline { 2 - 7 } ANDI GRY & 0.1260 & 0.9902 & 0.0302 & 0.4649 & 0.1441 & 1.4404 \\
\cline { 2 - 7 } BioSled & 0.0306 & 0.8462 & 0.0166 & 1.2776 & 0.0888 & 2.9397 \\
\cline { 2 - 7 } BioSled Rolls & 0.0979 & 2.8849 & 0.0145 & 0.0199 & 0.1010 & 1.4833 \\
CG2 & 0.0582 & 3.5313 & 0.0203 & 0.2719 & 0.0951 & 3.8648 \\
\cline { 2 - 7 } CG2 Rolls & 0.1028 & 1.0031 & 0.0222 & 0.0103 & 0.0355 & 1.9505 \\
\cline { 2 - 7 } CSA & 0.0718 & 0.5612 & 0.0237 & 3.0206 & 0.0519 & 0.8744 \\
CSA Rolls & 0.0518 & 3.2520 & 0.0349 & 4.8668 & 0.0609 & 0.9662 \\
\cline { 2 - 7 } L1 & 0.0657 & 3.3504 & 0.0379 & 0.0041 & 0.0997 & 2.0550 \\
\cline { 2 - 7 } L1 Rolls & 0.0783 & 3.0633 & & & 0.0847 & 4.0564 \\
\cline { 2 - 7 } MFF & 0.0935 & 1.3848 & 0.0556 & 2.8755 & 0.0638 & 1.3916 \\
\cline { 2 - 7 } SMM & 0.0762 & 0.6609 & 0.0192 & 0.2111 & 0.0784 & 11.1336 \\
\hline
\end{tabular}

For Tables 4.23 through 4.24 as previously stated, the Neurotech matcher removed images of bad quality and that is why there are blank spots in this table as there were not enough results to process the KLD values. There are no genuine outlying values and the outlying values 
of the imposters are most likely caused by the fact that for every match there are more imposter matches than there are genuine matches, so imposter values will show more variation. These KLD values are inconclusive as they show little to no performance variation.

\subsection{Issues Faced with Devices and Matchers Which can be Observed in the Results}

The L1 rolled images sometimes caused complications from the matchers, so in some cases there are no results from this specific category. Neurotech's Matcher removed images that were of bad quality, and this resulted in some tests having fewer images, or non at all. This became more of an issue once the data was subdivided into demographic strata. Another obstacle that was faced, involved the Morpho Finger-On-The-Fly, this device tended not to allow for capture of subjects that had a darker complexion, which once again only became an issue once the data was broken into demographic strata. The InnerID was removed from the collection midway through when it stopped working. The images that were produced are low in quality, and stand out among the other results. 
Chapter 5:
Conclusion 


\subsection{Conclusions}

The main goal of this research effort was to investigate the interoperability of contactless fingerprinting devices with the currently used livescan legacy fingerprinting devices and techniques. This goal was accomplished through performing these tasks; data collection, image pre-processing, match score generation and data analysis. The collection of data was achieved as 450 participant's data was collected and each participant was guided through the collection successfully. Our pre-processing involved doing segmentation of the images that involved more than one print and extraction was performed on duplicates and erroneously captured prints. Preprocessing also involved developing the matching programs from the software development kits, the programs were each able to run all of the data through to produce match score csv files. Succession of the goal of Data Analysis is shown in all of the results tables, these tables include TMR1 rates, EER values, and genuine/imposter distributions were also produced. The goal of investigation into stratification of contactless devices to see if a person's demographics share a relationship with the capabilities of their fingerprints, was examined and was inconclusive. Its important to note that this dataset is biased as a large amount of the subjects were between the ages of 20 to 29 and of the Caucasian ethnicity. With the similarity shown by the Kullback Leibler Divergence measurements, from this small data set, there was little to no performance variation across demographics. Other statistical analysis could have displayed these results differently and have been more conclusive, such as using the Weighted Hamming Distance instead off the KLD. If this dataset were larger, a larger variation could be experienced, possibly showing devices that favored certain demographics or conversely worked unfavorably with certain demographics. 
These Results are to be taken as a snapshot of the technology in terms of the interoperability with legacy based methods. Based on this snapshot during the development of contactless fingerprints Rank1 accuracy is between 74.33 and 98.68 percent with contact based methods. The contactless fingerprint Rank 1 accuracy is between 74.66 and 97.27 percent with other contactless devices. We think the lower values may be because incompatibility with the matchers, low quality images, and lack of deformation. The results shown depict the accuracy of contactless devices in comparison to the current widely used contact-based fingerprinting technology. Table 5.1 shows the TMR1 Ranges for each device type combination. Table 5.2 displays the ranges of TMR1 accuracy across all the matchers. The Equal Error Rates can be found in Tables 5.3 through 5.5 for all matching runs with each matcher.

Due to the growing number of contactless devices that are currently available, interoperability between contactless devices and contact-based devices is more significant now than it has ever been however, without the availability of a larger dataset, it cannot be conclusively proven that contact-based devices and non-contact devices are completely compatible and interoperable 
Table 5.1 TMR1 Ranges by Matcher

\begin{tabular}{|c|c|c|}
\hline \multicolumn{2}{|r|}{ Device } & TMR1 Range \\
\hline \multirow[t]{9}{*}{$N B I S$} & 10print - Contact & $87.37-91.77$ \\
\hline & 10print - Contactless W/O InnerID & $68.24-85.06$ \\
\hline & 10print - Contactless W InnerID & $2.08-85.08$ \\
\hline & Contact - Contact & $95.71-100$ \\
\hline & $\begin{array}{l}\text { Contactless - Contactless W/O } \\
\text { InnerID }\end{array}$ & $74.66-100$ \\
\hline & Contactless - Contactless W Inner & $0.32-100$ \\
\hline & Contact - Contactless W/O Inner ID & $70.31-95.93$ \\
\hline & Contact - Contactless W InnerID & $0-95.93$ \\
\hline & Contact Rolls - Contact Rolls & $87.92-100$ \\
\hline \multirow{9}{*}{ Neurotech } & 10print - Contact & $96.28-99.5$ \\
\hline & 10print - Contactless W/O InnerID & \\
\hline & 10print - Contactless W InnerID & $96.21-97.75$ \\
\hline & Contact - Contact & $97.15-100$ \\
\hline & $\begin{array}{l}\text { Contactless - Contactless W/O } \\
\text { InnerID }\end{array}$ & $94.89-100$ \\
\hline & Contactless - Contactless Inner & $1.74-100$ \\
\hline & Contact - Contactless W/O Inner ID & $93.83-98.69$ \\
\hline & Contact - Contactless W InnerID & $0.65-98.69$ \\
\hline & Contact Rolls - Contact Rolls & $96.15-100$ \\
\hline \multirow[t]{9}{*}{ SourceAFIS } & 10print - Contact & $87.37-91.77$ \\
\hline & 10print - Contactless W/O InnerID & $68.24-85.06$ \\
\hline & 10print - Contactless W InnerID & $2.08-85.06$ \\
\hline & Contact - Contact & $95.71-100$ \\
\hline & $\begin{array}{l}\text { Contactless - Contactless W/O } \\
\text { InnerID }\end{array}$ & $74.66-100$ \\
\hline & $\begin{array}{l}\text { Contactless - Contactless W } \\
\text { InnerID }\end{array}$ & $0.32-100$ \\
\hline & Contact - Contactless W/O Inner ID & $70.31-95.93$ \\
\hline & Contact - Contactless W InnerID & $0-95.93$ \\
\hline & Contact Rolls - Contact Rolls & $87.92-100$ \\
\hline
\end{tabular}


Table 5.2 Range of TMR1 Rates between Matchers

\begin{tabular}{|c|c|c|c|}
\hline \multicolumn{4}{|c|}{ Range of TMR1 Rates between matchers } \\
\hline $10 p-10 p$ & $99.75-100$ & BIOSLED - CSA & $96.07-97.15$ \\
\hline $10 p-A N D I B I N$ & $77.69-97.64$ & BIOSLED - CG2 & $95.71-97.22$ \\
\hline $10 p-A N D I G R Y$ & $68.24-97.75$ & BIOSLED - INNERID & $1.68-2.02$ \\
\hline $10 p-B I O S L E D$ & $88-96.28$ & BIOSLED - L1 & $96-97.38$ \\
\hline $10 p-C S A$ & $91.33-99.19$ & BIOSLED - SMM & $95.89-97.17$ \\
\hline $10 p-C G 2$ & $91.46-99.25$ & BIOSLED - MFF & $90.32-96.56$ \\
\hline $10 p$ - INNERID & $2.08-2.08$ & CSA - CSA & $100-100$ \\
\hline $10 p-L 1$ & $91.77-99.5$ & CSA - CG2 & $98.96-99.48$ \\
\hline $10 p-S M M$ & $87.37-97.69$ & CSA - INNERID & $2.09-2.42$ \\
\hline $10 p-M F F$ & $85.06-96.21$ & CSA - L1 & $98.97-99.74$ \\
\hline ANDI BIN - ANDI BIN & $100-100$ & CSA - SMM & $99.34-99.87$ \\
\hline ANDI BIN - ANDI GRY & $99.75-99.88$ & CSA - MFF & $95.93-98.69$ \\
\hline$A N D I B I N$ - BIOSLED & $84.32-93.83$ & CG2 - CG2 & $100-100$ \\
\hline$A N D I B I N$ - CSA & $88.87-95.81$ & CG2 - INNERID & $0.98-0.99$ \\
\hline ANDI BIN - CG2 & $89.78-95.86$ & CG2 - L1 & $98.37-98.72$ \\
\hline ANDI BIN - INNERID & $1.56-2.82$ & CG2 - SMM & $97.49-98.81$ \\
\hline$A N D I B I N-L 1$ & $86.66-95.43$ & CG2 - MFF & $95.51-98.11$ \\
\hline ANDI BIN - SMM & $87.5-95.06$ & INNERID - INNERID & $100-100$ \\
\hline$A N D I B I N-M F F$ & $83.86-94.89$ & INNERID - L1 & $0-1.29$ \\
\hline ANDI GRY - ANDI GRY & $100-100$ & INNERID - SMM & $0.32-0.65$ \\
\hline ANDI GRY - BIOSLED & $70.31-94.42$ & INNERID - MFF & $0.32-1.94$ \\
\hline ANDI GRY - CSA & $77.45-96.81$ & $L 1-L 1$ & $100-100$ \\
\hline ANDI GRY - CG2 & $76.72-96.55$ & L1 - SMM & $98-99.29$ \\
\hline ANDI GRY - INNERID & $1.58-1.74$ & L1 - MFF & $95.35-98.37$ \\
\hline ANDI GRY - L1 & $74.79-97.09$ & SMM - SMM & $100-100$ \\
\hline ANDI GRY - SMM & $72.41-96.35$ & MFF - SMM & $87.52-98.56$ \\
\hline ANDI GRY - MFF & $74.66-97.27$ & MFF - MFF & $100-100$ \\
\hline BIOSLED - BIOSLED & $100-100$ & & \\
\hline
\end{tabular}

Tables 5.1 - 5.2 show the summarized TMR1 Accuracy for each of the matching runs and collection methods. The InnerID produced very low match accuracy with both contact and contactless devices. As expected, the matching accuracy generally was highest when a device was matched against its same type. The ANDI OTG binary images produced higher accuracy results than that of the ANDI OTG greyscale images. The matching accuracy between ten-print card images and contactless devices was lower than that with contact-based devices, this most likely is caused by the fact there was only one image for each subject for this dataset compared to at least two sessions of images for every other device. It could also have to do with the modulation transfer of the ten-print images from the scanner. The matching accuracy was better for the ANDI OTG Binary images when it was against the contact-based devices than against the 
Morpho FOTF, the way these devices translate their images into an image that is usable with contact-based images may be different causing different features to be extracted from similar images. The ANDI Greyscale images performed the worst in terms of match accuracy amongst the contactless devices (excluding the InnerID), this may have been caused because the feature extraction process for the matchers used may be different for greyscale images. The Morpho FOTF performed best out of the contactless devices against contact-based methods, may have occurred due to the Morpho FOTF collecting double the amount of images compared to most the other devices.

It is important to note that, if this dataset was much larger, the results of this analysis could improve and be more conclusive and the statistical variations could be reduced. 
Table 5.3 Equal Error Rates for Each Matching Run

\begin{tabular}{|c|c|c|c|}
\hline Match & NBIS EER & Neurotech EER & SourceAFIS EER \\
\hline $10 p-10 p$ & 0.0000 & 0.0000 & 0.0000 \\
\hline 10p-ANDI BIN & 0.1207 & 0.0411 & 0.1500 \\
\hline 10p-ANDI GRY & 0.1495 & 0.0309 & 0.2401 \\
\hline $10 p-B / O$ & 0.0778 & 0.0316 & 0.1034 \\
\hline $10 p-C S A$ & 0.0587 & 0.0123 & 0.0660 \\
\hline $10 p-C G 2$ & 0.0558 & 0.0107 & 0.0911 \\
\hline $10 p-L 1$ & 0.0561 & 0.0100 & 0.0748 \\
\hline $10 p-M F F$ & 0.0980 & 0.0269 & 0.1829 \\
\hline $10 p-S M M$ & 0.0806 & 0.0273 & 0.1111 \\
\hline $10 p-\| D$ & 0.4660 & & 0.4861 \\
\hline$A N D I B I N-A N D I B I N$ & 0.0231 & 0.0171 & 0.0309 \\
\hline$A N D I B I N-A N D I G R Y$ & 0.0401 & 0.0171 & 0.0782 \\
\hline$A N D I B I N-B I O$ & 0.0997 & 0.0479 & 0.1105 \\
\hline$A N D I B I N-C S A$ & 0.0723 & 0.0280 & 0.0782 \\
\hline$A N D I B I N-C G 2$ & 0.0769 & 0.0328 & 0.0899 \\
\hline$A N D I B I N-L 1$ & 0.0878 & 0.0335 & 0.0996 \\
\hline$A N D I B I N-M F F$ & 0.1048 & 0.0377 & 0.1641 \\
\hline$A N D I B I N-S M M$ & 0.0919 & 0.0370 & 0.0859 \\
\hline$A N D I B I N-\| I D$ & 0.4714 & 0.4298 & 0.4767 \\
\hline ANDI GRY-ANDI GRY & 0.0345 & 0.0166 & 0.0612 \\
\hline$A N D I G R Y-B I O$ & 0.1549 & 0.0491 & 0.1698 \\
\hline$A N D I G R Y-C S A$ & 0.1178 & 0.0281 & 0.1420 \\
\hline ANDI GRY-CG2 & 0.1155 & 0.0352 & 0.1487 \\
\hline ANDI GRY-L1 & 0.1249 & 0.0304 & 0.1640 \\
\hline ANDI GRY-MFF & 0.1302 & 0.0260 & 0.2232 \\
\hline ANDI GRY-SMM & 0.1366 & 0.0357 & 0.1523 \\
\hline$A N D I G R Y-\| I D$ & 0.4727 & 0.4841 & 0.4667 \\
\hline$B I O-B I O$ & 0.0256 & 0.0151 & 0.0238 \\
\hline$B I O-C S A$ & 0.0378 & 0.0227 & 0.0359 \\
\hline$B 1 O-C G 2$ & 0.0483 & 0.0229 & 0.0387 \\
\hline$B I O-L 1$ & 0.0452 & 0.0238 & 0.0392 \\
\hline$B I O-M F F$ & 0.0950 & 0.0251 & 0.1007 \\
\hline BIO-SMM & 0.0466 & 0.0232 & 0.0438 \\
\hline$B I O-I I D$ & 0.4622 & 0.4342 & 0.4748 \\
\hline CSA-CSA & 0.0049 & 0.0013 & 0.0020 \\
\hline CSA-CG2 & 0.0154 & 0.0059 & 0.0137 \\
\hline$C S A-L 1$ & 0.0181 & 0.0032 & 0.0107 \\
\hline CSA-MFF & 0.0645 & 0.0134 & 0.0706 \\
\hline CSA-SMM & 0.0219 & 0.0053 & 0.0166 \\
\hline$C S A-\| D$ & 0.4490 & 0.4507 & 0.4798 \\
\hline CG2-CG2 & 0.0172 & 0.0161 & 0.0189 \\
\hline$C G 2-L 1$ & 0.0217 & 0.0129 & 0.0192 \\
\hline CG2-MFF & 0.0566 & 0.0159 & 0.0785 \\
\hline CG2-SMM & 0.0339 & 0.0114 & 0.0299 \\
\hline$C G 2-I I D$ & 0.4700 & 0.4572 & 0.4886 \\
\hline$L 1-L 1$ & 0.0134 & 0.0112 & 0.0112 \\
\hline L1-MFF & 0.0622 & 0.0129 & 0.0877 \\
\hline L1-SMM & 0.0291 & 0.0089 & 0.0265 \\
\hline$M F F-M F F$ & 0.0111 & 0.0078 & 0.0295 \\
\hline MFF-SMM & 0.0837 & 0.0166 & 0.0970 \\
\hline$S M M-S M M$ & 0.0175 & 0.0070 & 0.0108 \\
\hline$I I D-L 1$ & 0.4477 & 0.4446 & 0.4817 \\
\hline$I I D-M F F$ & 0.4816 & 0.4601 & 0.4960 \\
\hline IID-SMM & 0.4778 & 0.4591 & 0.4810 \\
\hline$\|I D-\| D$ & 0.1392 & 0.0678 & 0.1654 \\
\hline
\end{tabular}


Table 5.4 Equal Error Rates For Each Device

\begin{tabular}{|l|lll|}
\hline \multicolumn{4}{|c|}{ EER VALUES FOR THE GENUINE/IMPOSTER RUNS OF EACH DEVICE } \\
\hline MATCH & NBIS & Neurotech & SourceAFIS \\
Andi Binary & 0.0429 & 0.0346 & 0.0587 \\
Andi Greyscale & 0.0594 & 0.0336 & 0.1159 \\
BioSled & 0.0506 & 0.0307 & 0.0479 \\
BioSled Rolls & 0.0398 & 0.0201 & 0.0744 \\
CG2 & 0.0342 & 0.0298 & 0.0376 \\
CG2 Rolls & 0.0137 & 0.0070 & 0.0194 \\
CSA & 0.0088 & 0.0027 & 0.0027 \\
CSA Rolls & 0.0251 & 0.0076 & 0.0363 \\
InnerID & 0.2308 & 0.1239 & 0.2770 \\
L1 & 0.0267 & 0.0223 & 0.0222 \\
Morpho FOTF & 0.0145 & 0.0123 & 0.0405 \\
MorphoMobile & 0.0326 & 0.0141 & 0.0164 \\
\hline
\end{tabular}

Table 5.5 Equal Error Rates For Each Rolled Matching Run

\begin{tabular}{|l|lll|}
\hline \multicolumn{4}{|c|}{ EER VALUES FOR EACH ROLLS MATCHING RUN } \\
\hline \multicolumn{1}{|c|}{ MATCH } & NBIS & Neurotech & SourceAFIS \\
10p Rolls-10p Rolls & 0.0024 & 0.0000 & 0.0000 \\
10p Rolls-BIO Rolls & 0.0848 & 0.0232 & 0.1567 \\
10p Rolls-CSA Rolls & 0.0678 & 0.0136 & 0.1381 \\
10p Rolls-CG2 Rolls & 0.0753 & 0.0099 & 0.1254 \\
10p Rolls-L1 Rolls & 0.0755 & 0.0127 & 0.1300 \\
BIO Rolls-BIO Rolls & 0.0206 & 0.0099 & 0.0430 \\
BIO Rolls-CSA Rolls & 0.0462 & 0.0149 & 0.0707 \\
BIO Rolls-CG2 Rolls & 0.0452 & 0.0127 & 0.0662 \\
BIO Rolls-L1 Rolls & 0.0469 & 0.0142 & 0.0856 \\
CSA Rolls-CSA Rolls & 0.0130 & 0.0038 & 0.0174 \\
CSA Rolls-CG2 Rolls & 0.0230 & 0.0038 & 0.0396 \\
CSA Rolls-L1 Rolls & 0.0244 & 0.0056 & 0.0529 \\
CG2 Rolls-CG2 Rolls & 0.0080 & 0.0035 & 0.0096 \\
CG2 Rolls-L1 Rolls & 0.0245 & 0.0058 & 0.0456 \\
L1 Rolls-L1 Rolls & 0.0162 & & 0.0259 \\
\hline
\end{tabular}

Tables $5.3-5.5$ depict the equal error rates of each matching run. The lower the EER, the higher the accuracy of the matching run. The EER values highlighted in red indicate the InnerID values which, as previously discussed, produced the lowest matching accuracy obtained in this study, also produced highest EER values ( 0.4). The overall average cross-examined Equal Error Rate was 0.1240 , the values above this average are denoted in yellow, the values below the 
average are denoted in black. The overall average EER value was 0.0463 among all the devices for Table 5.4 values above this average are denoted in yellow.

\subsection{Future Work}

All the conclusions that have been listed above are based on the match score values that have been obtained from only 3 different contactless fingerprinting devices, more devices should be explored to get a better range of results. A larger dataset would also aid in the research on contactless devices. A larger dataset consisting of thousands of subjects would yield more conclusive results. Further study could also include using the match score distributions for the left-hand fingers to check for similarities in match performance. Different statistical analysis could be performed on the data that could better display the results. Future work could also include using the current versions of the devices used in this study and comparing the data produced with our data. A comparison could be performed on the data from this collection and the data from the phase 1 collection for the recurring subjects. Further Study could also include running the data on a different matching algorithm than the three already used. A larger dataset with more contactless devices would make the biggest impact to contactless fingerprinting research community. Another study could be done with less bias towards age ranges and ethnicities, with there being a larger variety of age ranges and ethnicities data being collected. If this study were to be done again, the two sessions could be spanned over a few weeks to create more variation between sessions. 
Appendix A

General Study of

All Data 
This Appendix shows the results from the general study using all of the data without being divided up into any demographic strata. The TMR1 Tables included in this section, show the TMR1 Results from every matching run that was performed. The genuine and imposter distributions for each matching run are shown in this section as well as the distributions each device ran against itself. The Receiver Operating Characteristic Curves or ROC Curves are shown for each of the devices used in this collection, a table of values is shown to identify the area under the curve for each ROC curve. All of the results stated above are shown for each of the three matchers used. The EER values are shown for each matcher for all matching runs. At the end of this appendix there are box plots that represent the number of minutiae found in images from each device, this data came from the NBIS matcher. 
Table A.1 NBIS TMR1 Table

\begin{tabular}{|c|c|c|c|c|c|}
\hline Match & Total & TMR1 & FMR1 & TMR1 Rate & FMR1 Rate \\
\hline $10 p-10 p$ & 405 & 405 & 0 & 100.00 & 0.00 \\
\hline $10 p-A N D I B I N$ & 381 & 296 & 85 & 77.69 & 22.31 \\
\hline $10 p-A N D I G R Y$ & 381 & 260 & 121 & 68.24 & 31.76 \\
\hline $10 p-B I O$ & 375 & 330 & 45 & 88.00 & 12.00 \\
\hline $10 p-C S A$ & 369 & 337 & 32 & 91.33 & 8.67 \\
\hline $10 p-C G 2$ & 398 & 364 & 34 & 91.46 & 8.54 \\
\hline $10 p-I I D$ & 144 & 3 & 141 & 2.08 & 97.92 \\
\hline $10 p-L 1$ & 401 & 368 & 33 & 91.77 & 8.23 \\
\hline $10 p-S M M$ & 388 & 339 & 49 & 87.37 & 12.63 \\
\hline $10 p-M F F$ & 395 & 336 & 59 & 85.06 & 14.94 \\
\hline$A N D I B I N$ - ANDI BIN & 830 & 830 & 0 & 100.00 & 0.00 \\
\hline$A N D I B I N$ - ANDI GRY & 830 & 829 & 1 & 99.88 & 0.12 \\
\hline$A N D I B I N-B I O$ & 767 & 656 & 111 & 85.53 & 14.47 \\
\hline$A N D I B I N-C S A$ & 753 & 679 & 74 & 90.17 & 9.83 \\
\hline$A N D I B I N$ - CG2 & 811 & 738 & 73 & 91.00 & 9.00 \\
\hline$A N D I B I N-I I D$ & 316 & 5 & 311 & 1.58 & 98.42 \\
\hline$A N D I B I N-L 1$ & 820 & 721 & 99 & 87.93 & 12.07 \\
\hline$A N D I B I N-S M M$ & 800 & 700 & 100 & 87.50 & 12.50 \\
\hline$A N D I B I N-M F F$ & 812 & 691 & 121 & 85.10 & 14.90 \\
\hline$A N D I G R Y-A N D I G R Y$ & 831 & 831 & 0 & 100.00 & 0.00 \\
\hline$A N D I G R Y-B I O$ & 768 & 540 & 228 & 70.31 & 29.69 \\
\hline ANDI GRY - CSA & 754 & 584 & 170 & 77.45 & 22.55 \\
\hline$A N D I G R Y-C G 2$ & 812 & 623 & 189 & 76.72 & 23.28 \\
\hline$A N D I G R Y-I I D$ & 316 & 5 & 311 & 1.58 & 98.42 \\
\hline$A N D I G R Y-L 1$ & 821 & 614 & 207 & 74.79 & 25.21 \\
\hline$A N D I G R Y-S M M$ & 801 & 580 & 221 & 72.41 & 27.59 \\
\hline$A N D I G R Y-M F F$ & 813 & 607 & 206 & 74.66 & 25.34 \\
\hline$B I O-B I O$ & 806 & 806 & 0 & 100.00 & 0.00 \\
\hline$B I O-C S A$ & 738 & 709 & 29 & 96.07 & 3.93 \\
\hline$B I O-C G 2$ & 792 & 758 & 34 & 95.71 & 4.29 \\
\hline$B I O-I I D$ & 297 & 6 & 291 & 2.02 & 97.98 \\
\hline$B I O-L 1$ & 801 & 769 & 32 & 96.00 & 4.00 \\
\hline$B I O-S M M$ & 778 & 746 & 32 & 95.89 & 4.11 \\
\hline$B I O-M F F$ & 785 & 709 & 76 & 90.32 & 9.68 \\
\hline$C S A-C S A$ & 784 & 784 & 0 & 100.00 & 0.00 \\
\hline$C S A-C G 2$ & 771 & 763 & 8 & 98.96 & 1.04 \\
\hline$C S A-I I D$ & 289 & 7 & 282 & 2.42 & 97.58 \\
\hline$C S A-L 1$ & 778 & 770 & 8 & 98.97 & 1.03 \\
\hline$C S A-S M M$ & 758 & 753 & 5 & 99.34 & 0.66 \\
\hline$C S A-M F F$ & 762 & 731 & 31 & 95.93 & 4.07 \\
\hline$C G 2$ - CG2 & 870 & 870 & 0 & 100.00 & 0.00 \\
\hline$C G 2-I I D$ & 306 & 3 & 303 & 0.98 & 99.02 \\
\hline$C G 2-L 1$ & 860 & 846 & 14 & 98.37 & 1.63 \\
\hline CG2 - SMM & 838 & 817 & 21 & 97.49 & 2.51 \\
\hline$C G 2-M F F$ & 847 & 809 & 38 & 95.51 & 4.49 \\
\hline$I I D-I I D$ & 319 & 319 & 0 & 100.00 & 0.00 \\
\hline$I I D-L 1$ & 315 & 0 & 315 & 0.00 & 100.00 \\
\hline$I I D-S M M$ & 313 & 1 & 312 & 0.32 & 99.68 \\
\hline$I I D-M F F$ & 315 & 1 & 314 & 0.32 & 99.68 \\
\hline$L 1-L 1$ & 883 & 883 & 0 & 100.00 & 0.00 \\
\hline$L 1-S M M$ & 849 & 832 & 17 & 98.00 & 2.00 \\
\hline$L 1-M F F$ & 861 & 821 & 40 & 95.35 & 4.65 \\
\hline$S M M-S M M$ & 859 & 859 & 0 & 100.00 & 0.00 \\
\hline$M F F-S M M$ & 1666 & 1458 & 208 & 87.52 & 12.48 \\
\hline$M F F-M F F$ & 1734 & 1734 & 0 & 100.00 & 0.00 \\
\hline
\end{tabular}


Table A.2 Neurotech TMR1 Table

\begin{tabular}{|c|c|c|c|c|c|}
\hline Neurotech & Total & TMR1 & FMR1 & TMR1 Rate & FMR1 Rate \\
\hline $10 p-10 p$ & 388 & 388 & 0 & 100.00 & 0.00 \\
\hline $10 p-A N D I B I N$ & 381 & 372 & 9 & 97.64 & 2.36 \\
\hline $10 p-A N D I G R Y$ & 355 & 347 & 8 & 97.75 & 2.25 \\
\hline $10 p-B I O$ & 376 & 362 & 14 & 96.28 & 3.72 \\
\hline $10 p-C S A$ & 370 & 367 & 3 & 99.19 & 0.81 \\
\hline $10 p-C G 2$ & 399 & 396 & 3 & 99.25 & 0.75 \\
\hline $10 p-L 1$ & 402 & 400 & 2 & 99.50 & 0.50 \\
\hline $10 p-S M M$ & 389 & 380 & 9 & 97.69 & 2.31 \\
\hline $10 p-M F F$ & 396 & 381 & 15 & 96.21 & 3.79 \\
\hline$A N D I B I N-A N D I B I N$ & 825 & 825 & 0 & 100.00 & 0.00 \\
\hline ANDI BIN - ANDI GRY & 804 & 802 & 2 & 99.75 & 0.25 \\
\hline$A N D I B I N-B I O$ & 778 & 730 & 48 & 93.83 & 6.17 \\
\hline ANDI BIN - CSA & 764 & 732 & 32 & 95.81 & 4.19 \\
\hline$A N D I B I N$ - CG2 & 822 & 788 & 34 & 95.86 & 4.14 \\
\hline$A N D I B I N-I I D$ & 319 & 9 & 310 & 2.82 & 97.18 \\
\hline$A N D I B I N-L 1$ & 832 & 794 & 38 & 95.43 & 4.57 \\
\hline ANDI BIN - SMM & 810 & 770 & 40 & 95.06 & 4.94 \\
\hline$A N D I B I N-M F F$ & 822 & 780 & 42 & 94.89 & 5.11 \\
\hline$A N D I G R Y-A N D I G R Y$ & 796 & 796 & 0 & 100.00 & 0.00 \\
\hline$A N D I G R Y-B I O$ & 735 & 694 & 41 & 94.42 & 5.58 \\
\hline$A N D I G R Y-C S A$ & 721 & 698 & 23 & 96.81 & 3.19 \\
\hline$A N D I G R Y$ - CG2 & 783 & 756 & 27 & 96.55 & 3.45 \\
\hline$A N D I G R Y-I I D$ & 287 & 5 & 282 & 1.74 & 98.26 \\
\hline$A N D I G R Y-L 1$ & 790 & 767 & 23 & 97.09 & 2.91 \\
\hline$A N D I G R Y-S M M$ & 767 & 739 & 28 & 96.35 & 3.65 \\
\hline ANDI GRY - MFF & 768 & 747 & 21 & 97.27 & 2.73 \\
\hline$B I O-B I O$ & 802 & 802 & 0 & 100.00 & 0.00 \\
\hline$B I O-C S A$ & 738 & 717 & 21 & 97.15 & 2.85 \\
\hline$B 1 O-C G 2$ & 792 & 770 & 22 & 97.22 & 2.78 \\
\hline$B I O-I I D$ & 297 & 5 & 292 & 1.68 & 98.32 \\
\hline$B I O-L 1$ & 801 & 780 & 21 & 97.38 & 2.62 \\
\hline$B I O-S M M$ & 778 & 756 & 22 & 97.17 & 2.83 \\
\hline$B I O-M F F$ & 785 & 758 & 27 & 96.56 & 3.44 \\
\hline CSA - CSA & 783 & 783 & 0 & 100.00 & 0.00 \\
\hline$C S A-C G 2$ & 771 & 767 & 4 & 99.48 & 0.52 \\
\hline$C S A-I I D$ & 287 & 6 & 281 & 2.09 & 97.91 \\
\hline$C S A-L 1$ & 778 & 776 & 2 & 99.74 & 0.26 \\
\hline CSA - SMM & 758 & 757 & 1 & 99.87 & 0.13 \\
\hline$C S A-M F F$ & 762 & 752 & 10 & 98.69 & 1.31 \\
\hline$C G 2-C G 2$ & 867 & 867 & 0 & 100.00 & 0.00 \\
\hline$C G 2-I I D$ & 304 & 3 & 301 & 0.99 & 99.01 \\
\hline$C G 2-L 1$ & 860 & 849 & 11 & 98.72 & 1.28 \\
\hline CG2 - SMM & 838 & 828 & 10 & 98.81 & 1.19 \\
\hline$C G 2-M F F$ & 847 & 831 & 16 & 98.11 & 1.89 \\
\hline$I I D-I I D$ & 273 & 273 & 0 & 100.00 & 0.00 \\
\hline$I I D-L 1$ & 311 & 4 & 307 & 1.29 & 98.71 \\
\hline IID - SMM & 307 & 2 & 305 & 0.65 & 99.35 \\
\hline$I I D-M F F$ & 309 & 6 & 303 & 1.94 & 98.06 \\
\hline$L 1-L 1$ & 883 & 883 & 0 & 100.00 & 0.00 \\
\hline$L 1-S M M$ & 849 & 843 & 6 & 99.29 & 0.71 \\
\hline$L 1-M F F$ & 861 & 847 & 14 & 98.37 & 1.63 \\
\hline$S M M-S M M$ & 855 & 855 & 0 & 100.00 & 0.00 \\
\hline$M F F-S M M$ & 1662 & 1638 & 24 & 98.56 & 1.44 \\
\hline$M F F-M F F$ & 1626 & 1626 & 0 & 100.00 & 0.00 \\
\hline
\end{tabular}


Table A.3 SourceAFIS TMR1 Table

\begin{tabular}{|c|c|c|c|c|c|}
\hline SourceAFIS & Total & TMR1 & FMR1 & TMR1 Rate & FMR1 Rate \\
\hline $10 p-10 p$ & 406 & 405 & 0 & 99.75 & 0.00 \\
\hline $10 p-A N D I B I N$ & 380 & 296 & 85 & 77.89 & 22.37 \\
\hline $10 p-A N D I G R Y$ & 381 & 260 & 121 & 68.24 & 31.76 \\
\hline $10 p-B I O$ & 375 & 330 & 45 & 88.00 & 12.00 \\
\hline $10 p-C S A$ & 369 & 337 & 32 & 91.33 & 8.67 \\
\hline $10 p-C G 2$ & 398 & 364 & 34 & 91.46 & 8.54 \\
\hline $10 p-I I D$ & 144 & 3 & 141 & 2.08 & 97.92 \\
\hline $10 p-L 1$ & 401 & 368 & 33 & 91.77 & 8.23 \\
\hline $10 p-S M M$ & 388 & 339 & 49 & 87.37 & 12.63 \\
\hline $10 p-M F F$ & 395 & 336 & 59 & 85.06 & 14.94 \\
\hline$A N D I B I N-A N D I B I N$ & 830 & 830 & 0 & 100.00 & 0.00 \\
\hline$A N D I B I N-A N D I G R Y$ & 830 & 829 & 1 & 99.88 & 0.12 \\
\hline$A N D I B I N-B I O$ & 778 & 656 & 111 & 84.32 & 14.27 \\
\hline$A N D I B I N$ - CSA & 764 & 679 & 74 & 88.87 & 9.69 \\
\hline$A N D I B I N-C G 2$ & 822 & 738 & 73 & 89.78 & 8.88 \\
\hline$A N D I B I N-I I D$ & 321 & 5 & 311 & 1.56 & 96.88 \\
\hline$A N D I B I N-L 1$ & 832 & 721 & 99 & 86.66 & 11.90 \\
\hline$A N D I B I N-S M M$ & 800 & 700 & 100 & 87.50 & 12.50 \\
\hline$A N D I B I N-M F F$ & 824 & 691 & 121 & 83.86 & 14.68 \\
\hline$A N D I G R Y$ - ANDI GRY & 831 & 831 & 0 & 100.00 & 0.00 \\
\hline$A N D I G R Y-B I O$ & 768 & 540 & 228 & 70.31 & 29.69 \\
\hline ANDI GRY - CSA & 754 & 584 & 170 & 77.45 & 22.55 \\
\hline ANDI GRY - CG2 & 812 & 623 & 189 & 76.72 & 23.28 \\
\hline$A N D I G R Y-I I D$ & 316 & 5 & 311 & 1.58 & 98.42 \\
\hline ANDI GRY - L1 & 821 & 614 & 207 & 74.79 & 25.21 \\
\hline$A N D I G R Y$ - SMM & 801 & 580 & 221 & 72.41 & 27.59 \\
\hline$A N D I G R Y-M F F$ & 813 & 607 & 206 & 74.66 & 25.34 \\
\hline$B I O-B I O$ & 806 & 806 & 0 & 100.00 & 0.00 \\
\hline$B I O-C S A$ & 738 & 709 & 29 & 96.07 & 3.93 \\
\hline$B I O-C G 2$ & 792 & 758 & 34 & 95.71 & 4.29 \\
\hline$B I O-I I D$ & 297 & 6 & 291 & 2.02 & 97.98 \\
\hline$B I O-L 1$ & 801 & 769 & 32 & 96.00 & 4.00 \\
\hline$B I O-S M M$ & 778 & 746 & 32 & 95.89 & 4.11 \\
\hline$B I O-M F F$ & 785 & 709 & 76 & 90.32 & 9.68 \\
\hline CSA - CSA & 784 & 784 & 0 & 100.00 & 0.00 \\
\hline CSA - CG2 & 771 & 763 & 8 & 98.96 & 1.04 \\
\hline$C S A-I I D$ & 289 & 7 & 282 & 2.42 & 97.58 \\
\hline$C S A-\angle 1$ & 778 & 770 & 8 & 98.97 & 1.03 \\
\hline$C S A-S M M$ & 758 & 753 & 5 & 99.34 & 0.66 \\
\hline$C S A-M F F$ & 762 & 731 & 31 & 95.93 & 4.07 \\
\hline$C G 2$ - $C G 2$ & 870 & 870 & 0 & 100.00 & 0.00 \\
\hline$C G 2-I I D$ & 306 & 3 & 303 & 0.98 & 99.02 \\
\hline$C G 2-L 1$ & 860 & 846 & 14 & 98.37 & 1.63 \\
\hline CG2 - SMM & 838 & 817 & 21 & 97.49 & 2.51 \\
\hline$C G 2-M F F$ & 847 & 809 & 38 & 95.51 & 4.49 \\
\hline$I I D-I I D$ & 319 & 319 & 0 & 100.00 & 0.00 \\
\hline$I I D-L 1$ & 315 & 0 & 315 & 0.00 & 100.00 \\
\hline$I I D-S M M$ & 313 & 1 & 312 & 0.32 & 99.68 \\
\hline$I I D-M F F$ & 315 & 1 & 314 & 0.32 & 99.68 \\
\hline$L 1-L 1$ & 883 & 883 & 0 & 100.00 & 0.00 \\
\hline$L 1-S M M$ & 849 & 832 & 17 & 98.00 & 2.00 \\
\hline$L 1-M F F$ & 861 & 821 & 40 & 95.35 & 4.65 \\
\hline$S M M-S M M$ & 859 & 859 & 0 & 100.00 & 0.00 \\
\hline$M F F-S M M$ & 1666 & 1458 & 208 & 87.52 & 12.48 \\
\hline$M F F-M F F$ & 1734 & 1734 & 0 & 100.00 & 0.00 \\
\hline
\end{tabular}


Table A.4 NBIS Rolls TMR1 Table

\begin{tabular}{|c|c|c|c|c|c|c|}
\hline NBIS Rolls & Match & Total & TMR1 & FMR1 & TMR1 Rate & FMR1 Rate \\
\hline & $10 p R-10 p R$ & 417 & 417 & 0 & 100.00 & 0.00 \\
\hline & $10 p R-B I O R$ & 389 & 342 & 47 & 87.92 & 12.08 \\
\hline & $10 p R-C S A R$ & 383 & 349 & 34 & 91.12 & 8.88 \\
\hline & $10 p R-C G 2 R$ & 412 & 369 & 43 & 89.56 & 10.44 \\
\hline & $10 p R-L 1 R$ & 415 & 372 & 43 & 89.64 & 10.36 \\
\hline & $B I O R-B I O R$ & 828 & 828 & 0 & 100.00 & 0.00 \\
\hline & $B I O R-C S A R$ & 765 & 735 & 30 & 96.08 & 3.92 \\
\hline & $B I O R-C G 2 R$ & 818 & 778 & 40 & 95.11 & 4.89 \\
\hline & $B I O R-L 1 R$ & 824 & 785 & 39 & 95.27 & 4.73 \\
\hline & $C S A R-C S A R$ & 811 & 811 & 0 & 100.00 & 0.00 \\
\hline & $C S A R-C G 2 R$ & 799 & 781 & 18 & 97.75 & 2.25 \\
\hline & $C S A R-L 1 R$ & 809 & 795 & 14 & 98.27 & 1.73 \\
\hline & $C G 2 R-C G 2 R$ & 877 & 877 & 0 & 100.00 & 0.00 \\
\hline & $C G 2 R-L 1 R$ & 871 & 860 & 11 & 98.74 & 1.26 \\
\hline & $L 1 R-L 1 R$ & 893 & 893 & 0 & 100.00 & 0.00 \\
\hline
\end{tabular}

Table A.5 Neurotech Rolls TMR1 Table

\begin{tabular}{|c|c|c|c|c|c|}
\hline Neurotech Rolls & Total & TMR1 & FMR1 & TMR1 Rate & FMR1 Rate \\
\hline $10 p R-10 p R$ & 360 & 360 & 0 & 100.00 & 0.00 \\
\hline $10 p R-B I O R$ & 390 & 375 & 15 & 96.15 & 3.85 \\
\hline $10 p R-C S A R$ & 383 & 377 & 6 & 98.43 & 1.57 \\
\hline $10 p R-C G 2 R$ & 411 & 404 & 7 & 98.30 & 1.70 \\
\hline$B I O R-B I O R$ & 815 & 815 & 0 & 100.00 & 0.00 \\
\hline$B I O R-C S A R$ & 757 & 743 & 14 & 98.15 & 1.85 \\
\hline$B I O R-C G 2 R$ & 810 & 797 & 13 & 98.40 & 1.60 \\
\hline CSA R - CSA R & 804 & 804 & 0 & 100.00 & 0.00 \\
\hline$C S A R-C G 2 R$ & 796 & 792 & 4 & 99.50 & 0.50 \\
\hline$C G 2 R-C G 2 R$ & 862 & 862 & 0 & 100.00 & 0.00 \\
\hline$L 1 R-10 p R$ & 828 & 807 & 21 & 97.46 & 2.54 \\
\hline$L 1 R-B I O R$ & 835 & 819 & 16 & 98.08 & 1.92 \\
\hline$L 1 R-C S A R$ & 820 & 811 & 9 & 98.90 & 1.10 \\
\hline$L 1 R-C G 2 R$ & 878 & 870 & 8 & 99.09 & 0.91 \\
\hline
\end{tabular}

Table A.6 SourceAFIS Rolls TMR1 Table

\begin{tabular}{|c|c|c|c|c|c|c|}
\hline SourceAFIS Rolls & Match & Total & TMR1 & FMR1 & TMR1 Rate & FMR1 Rate \\
\hline & $10 p R-10 p R$ & 418 & 417 & 0 & 99.76 & 0.00 \\
\hline & $10 p R-B I O R$ & 389 & 342 & 47 & 87.92 & 12.08 \\
\hline & $10 p R-C S A R$ & 383 & 349 & 34 & 91.12 & 8.88 \\
\hline & $10 p R-C G 2 R$ & 411 & 369 & 43 & 89.78 & 10.46 \\
\hline & $10 p R-L 1 R$ & 415 & 372 & 43 & 89.64 & 10.36 \\
\hline & $B I O R-B I O R$ & 828 & 828 & 0 & 100.00 & 0.00 \\
\hline & $B I O R-C S A R$ & 765 & 735 & 30 & 96.08 & 3.92 \\
\hline & $B 1 O R-C G 2 R$ & 818 & 778 & 40 & 95.11 & 4.89 \\
\hline & $B I O R-L 1 R$ & 824 & 785 & 39 & 95.27 & 4.73 \\
\hline & CSA R - CSA R & 811 & 811 & 0 & 100.00 & 0.00 \\
\hline & CSA R - CG2 R & 799 & 781 & 18 & 97.75 & 2.25 \\
\hline & $C S A R-L 1 R$ & 809 & 795 & 14 & 98.27 & 1.73 \\
\hline & $C G 2 R-C G 2 R$ & 877 & 877 & 0 & 100.00 & 0.00 \\
\hline & $C G 2 R-L 1 R$ & 871 & 860 & 11 & 98.74 & 1.26 \\
\hline & $L 1 R-L 1 R$ & 893 & 893 & 0 & 100.00 & 0.00 \\
\hline
\end{tabular}


Table A.7 NBIS Maximum and Minimum Score Values

\begin{tabular}{|c|c|c|c|c|}
\hline \multicolumn{5}{|l|}{ NBIS } \\
\hline Sensor & $\begin{array}{l}\text { Genuine } \\
\text { Maximum } \\
\text { Score }\end{array}$ & $\begin{array}{l}\text { Genuine } \\
\text { Minimum } \\
\text { Score }\end{array}$ & $\begin{array}{l}\text { Imposter } \\
\text { Maximum } \\
\text { Score }\end{array}$ & $\begin{array}{l}\text { Imposter } \\
\text { Minimum } \\
\text { Score }\end{array}$ \\
\hline ANDI BIN & 319 & 4 & 44 & 0 \\
\hline ANDIGRY & 358 & 8 & 70 & 0 \\
\hline Biosled & 304 & 4 & 245 & 0 \\
\hline BIO Rolls & 317 & 5 & 184 & 0 \\
\hline CG2 & 358 & 4 & 159 & 0 \\
\hline CG2 Rolls & 452 & 4 & 262 & 0 \\
\hline CSA & 499 & 8 & 63 & 0 \\
\hline CSA Rolls & 506 & 4 & 31 & 0 \\
\hline InnerID & 176 & 7 & 62 & 4 \\
\hline$L 1$ & 328 & 4 & 137 & 0 \\
\hline L1 Rolls & 449 & 0 & 143 & 0 \\
\hline MFF & 360 & 5 & 48 & 0 \\
\hline MorphoMobile & 278 & 3 & 201 & 0 \\
\hline
\end{tabular}

\section{A.1 NBIS Genuine/Imposter Score Distributions}
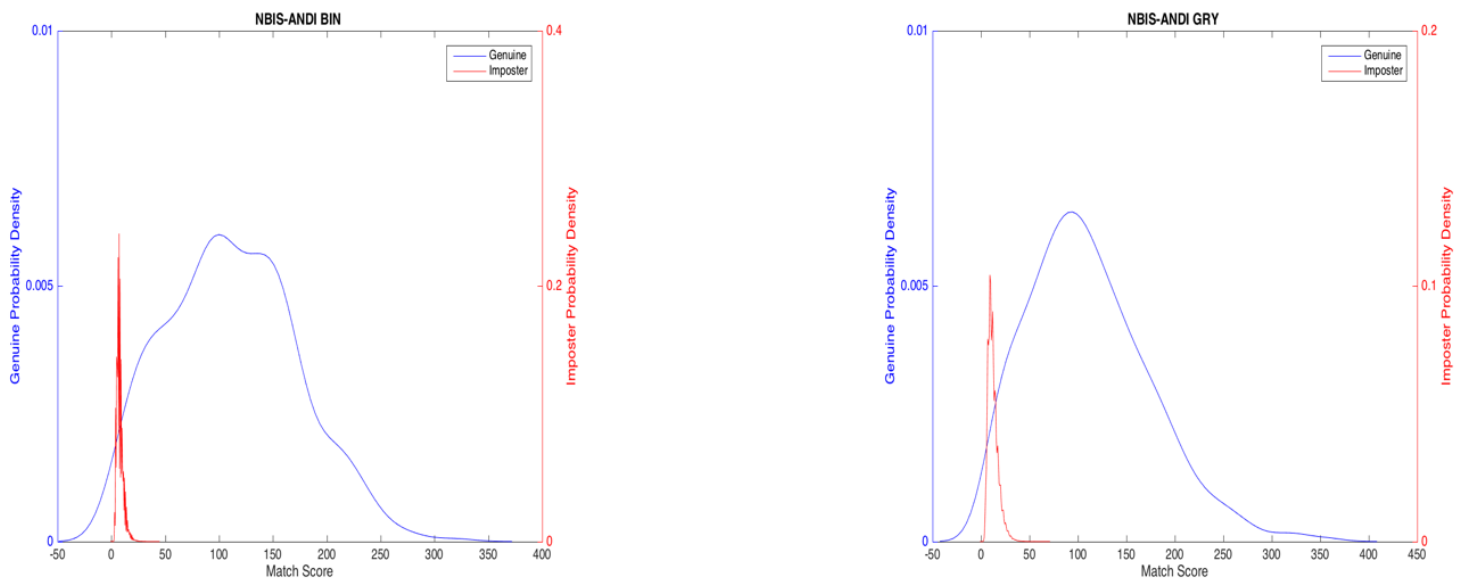

Figure A.1.1 (Left) NBIS ANDI Binary (Right) NBIS ANDI Greyscale 

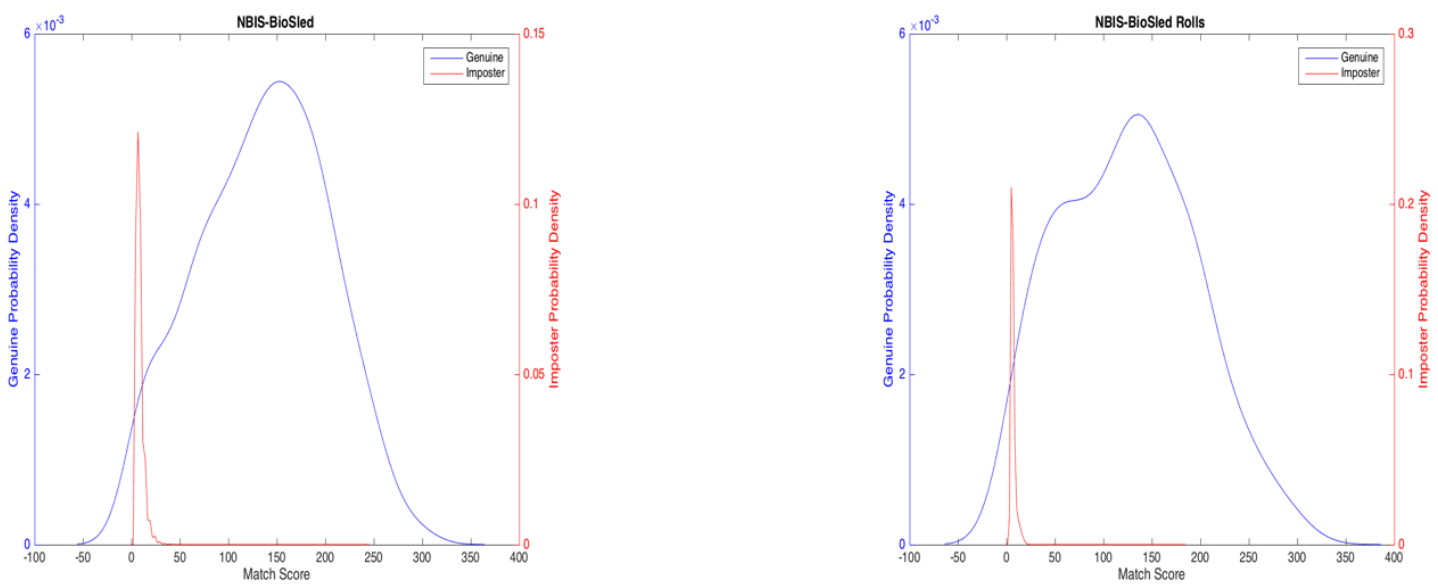

Figure A.1.2 (Left) NBIS BioSled (Right) NBIS BioSled Rolls
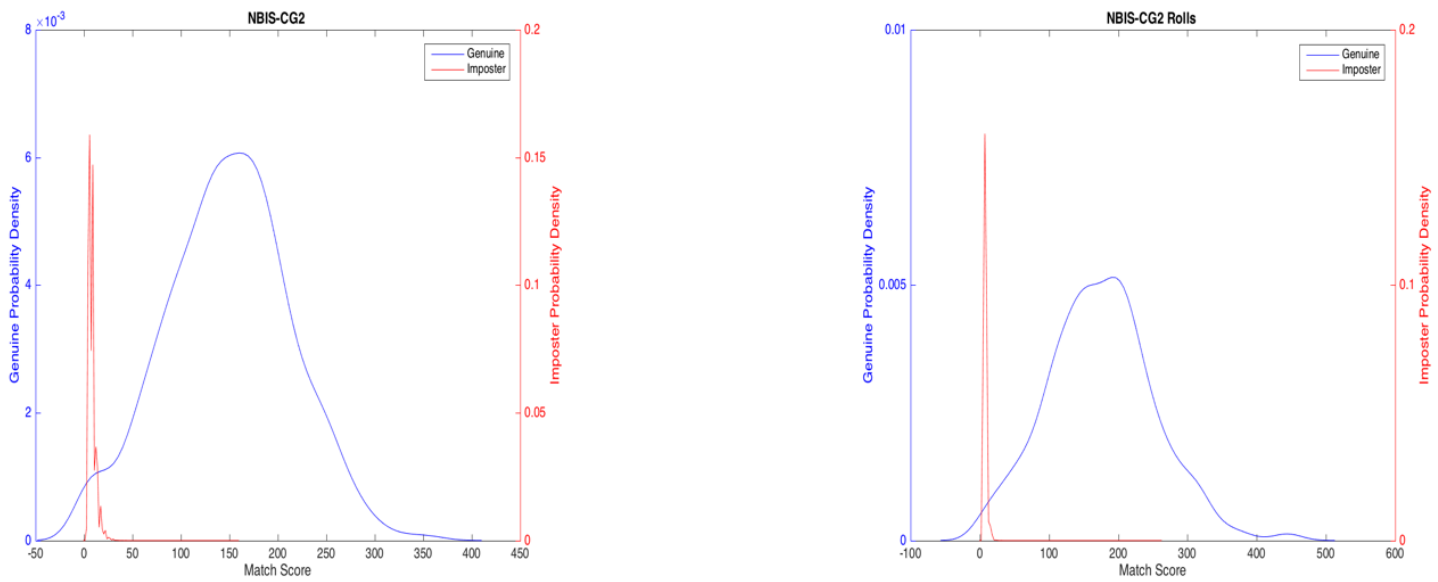

Figure A.1.3 (Left) NBIS FP II Guardian (Right) NBIS FPII Guardian Rolls
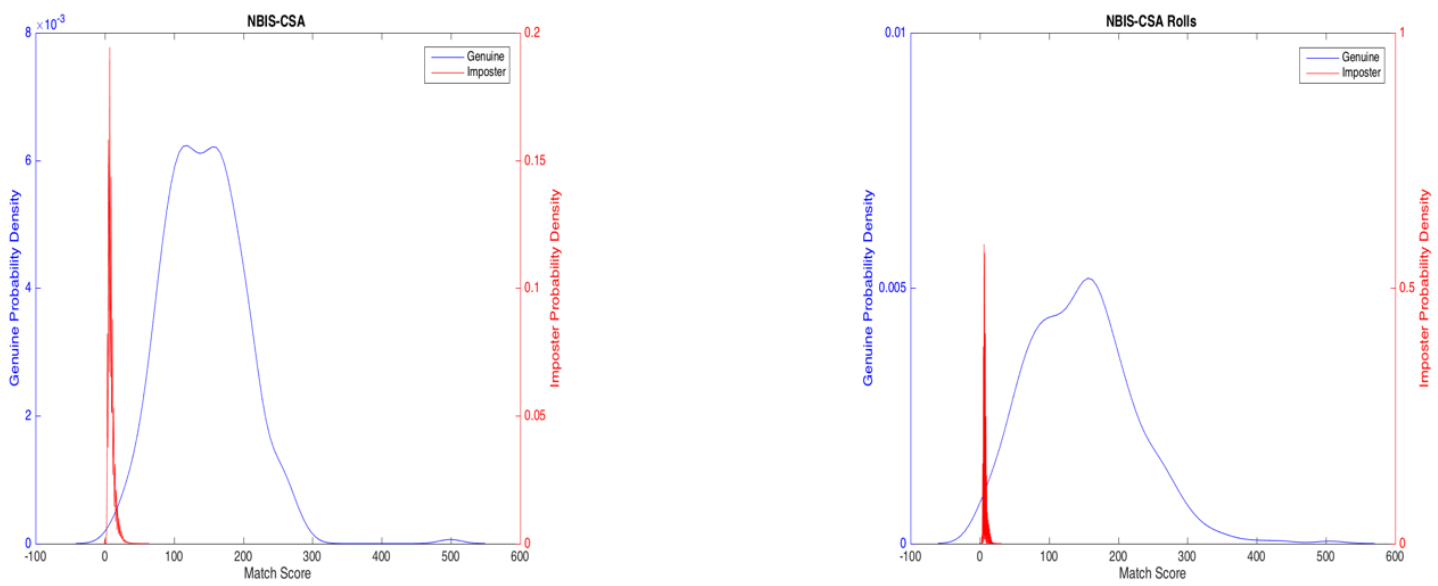

Figure A.1.4 (Left) NBIS Crossmatch Seek Avenger (Right) NBIS Crossmatch Seek Avenger Rolls 

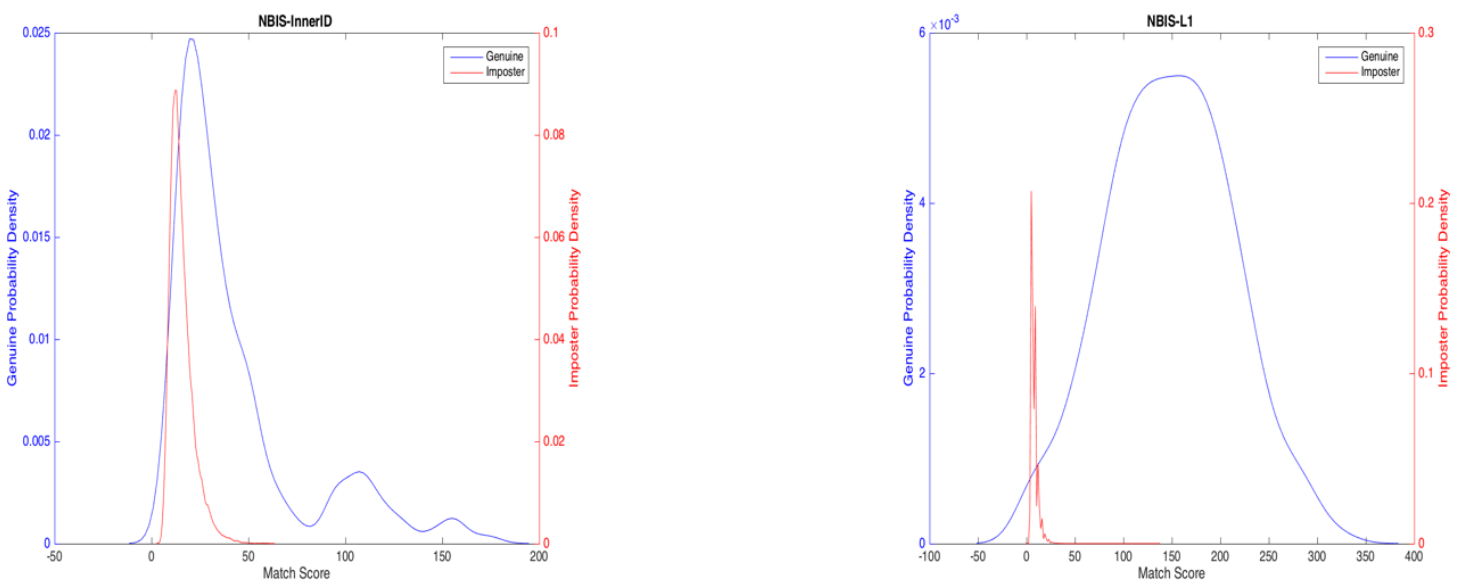

Figure A.1.5 (Left) NBIS InnerID (Right) NBIS L1
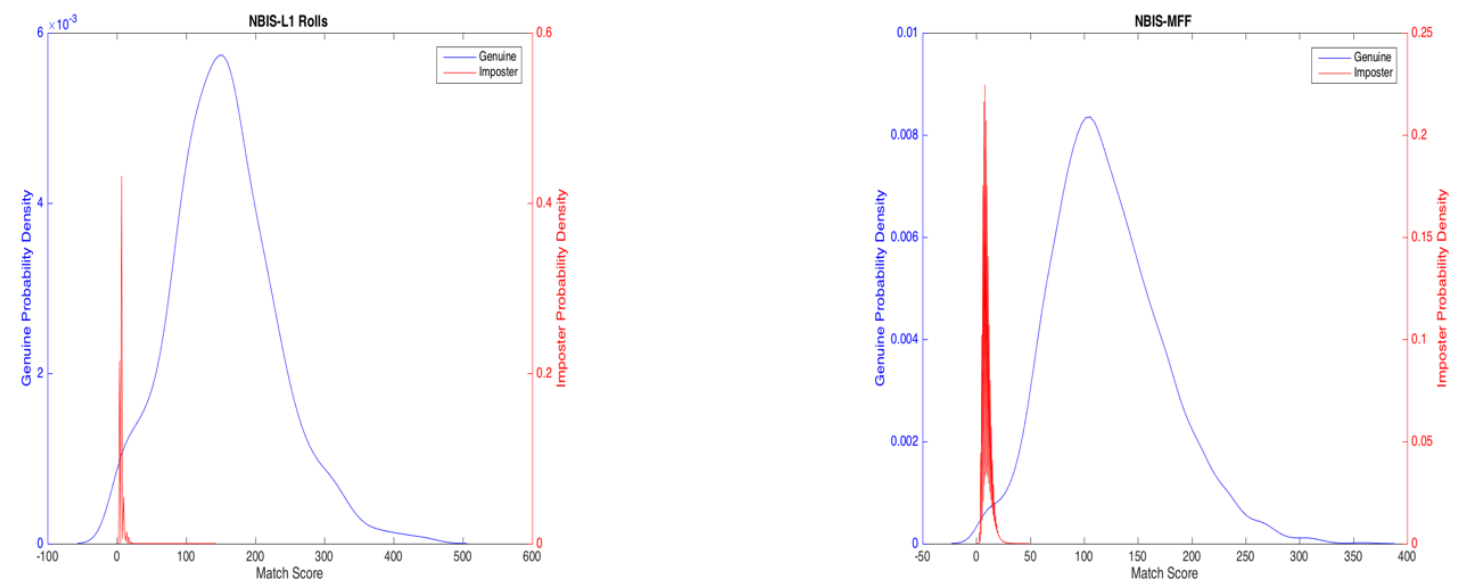

Figure A.1.6 (Left) NBIS L1 Rolls (Right) NBIS Morpho FOTF

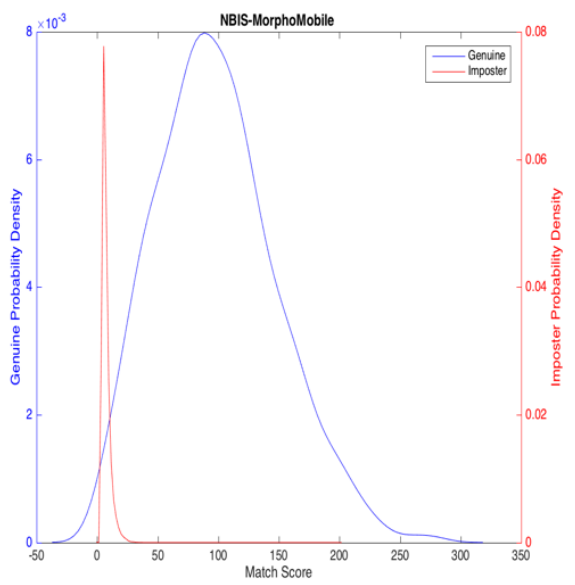

Figure A.1.7 NBIS MorphoMobile 
Table A. 8 Neurotech Maximum and Minimum Score Values

\begin{tabular}{|c|c|c|c|c|}
\hline \multicolumn{5}{|c|}{ Neurotech } \\
\hline Sensor & $\begin{array}{l}\text { Genuine } \\
\text { Maximum } \\
\text { Score }\end{array}$ & $\begin{array}{l}\text { Genuine } \\
\text { Minimum } \\
\text { Score }\end{array}$ & $\begin{array}{l}\text { Imposter } \\
\text { Maximum } \\
\text { Score }\end{array}$ & $\begin{array}{l}\text { Imposter } \\
\text { Minimum } \\
\text { Score }\end{array}$ \\
\hline \multirow{2}{*}{$\begin{array}{l}\text { ANDI BIN } \\
\text { ANDI GRY }\end{array}$} & 986 & 0 & 41 & 0 \\
\hline & 1003 & 3 & 45 & 0 \\
\hline \multirow{2}{*}{$\begin{array}{l}\text { BioSled } \\
\text { BIO Rolls }\end{array}$} & 951 & 0 & 539 & 0 \\
\hline & 993 & 0 & 656 & 0 \\
\hline \multirow{2}{*}{$\begin{array}{l}\text { CG2 } \\
\text { CG2 Rolls }\end{array}$} & 1000 & 1 & 641 & 0 \\
\hline & 1188 & 2 & 658 & 0 \\
\hline CSA & 1267 & 1 & 58 & 0 \\
\hline \multirow{2}{*}{$\begin{array}{l}\text { CSA Rolls } \\
\text { InnerID }\end{array}$} & 1682 & 0 & 26 & 0 \\
\hline & 508 & 2 & 35 & 0 \\
\hline \multirow{2}{*}{$\begin{array}{l}\text { L1 } \\
\text { L1 Rolls }\end{array}$} & 925 & 0 & 681 & 0 \\
\hline & & & & \\
\hline \multirow{2}{*}{$\begin{array}{l}\text { MFF } \\
\text { MorphoMobile }\end{array}$} & 952 & 2 & 58 & 0 \\
\hline & 705 & 1 & 465 & 0 \\
\hline
\end{tabular}




\section{A.2 Neurotech Genuine/Imposter Score Distributions}
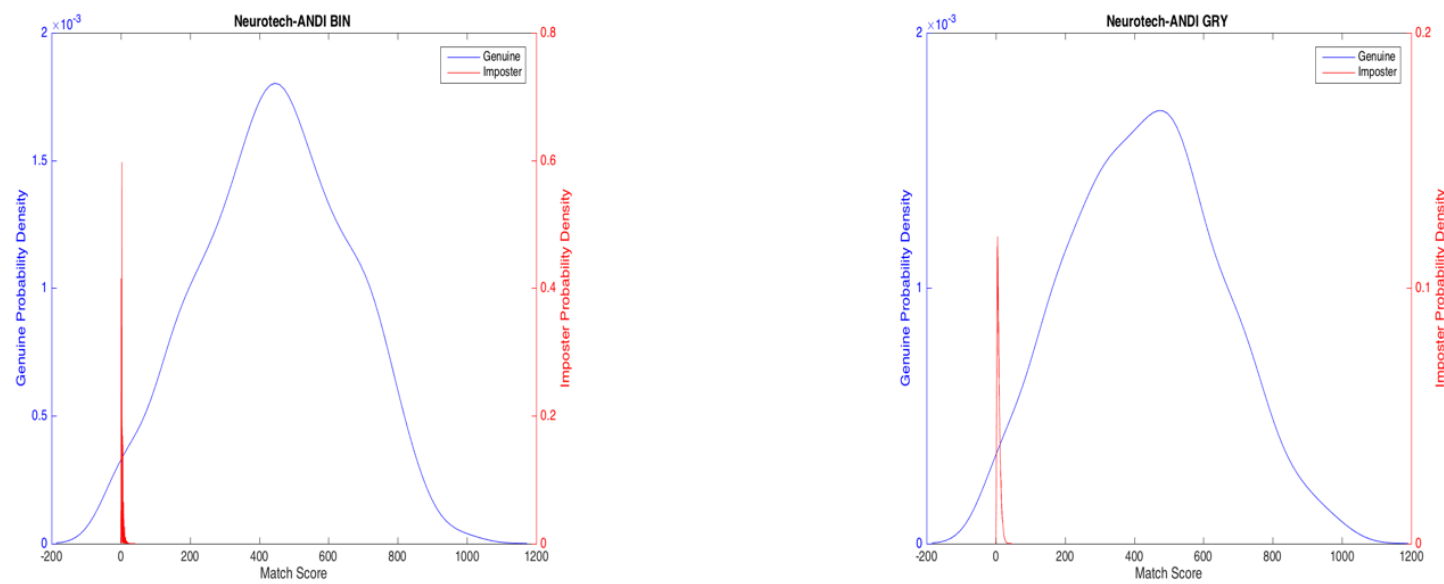

Figure A.2.1 (Left) Neurotech ANDI Binary (Right) Neurotech ANDI Greyscale
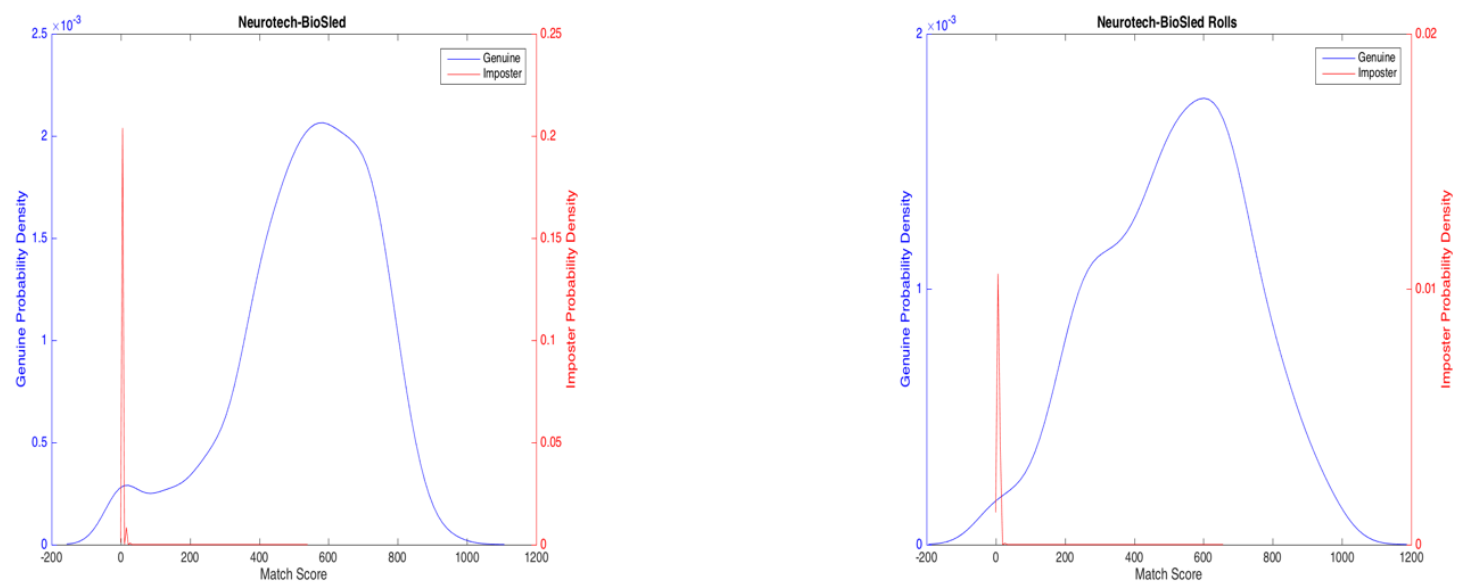

Figure A.2.2 (Left) Neurotech BioSled (Right) Neurotech BioSled Rolls 

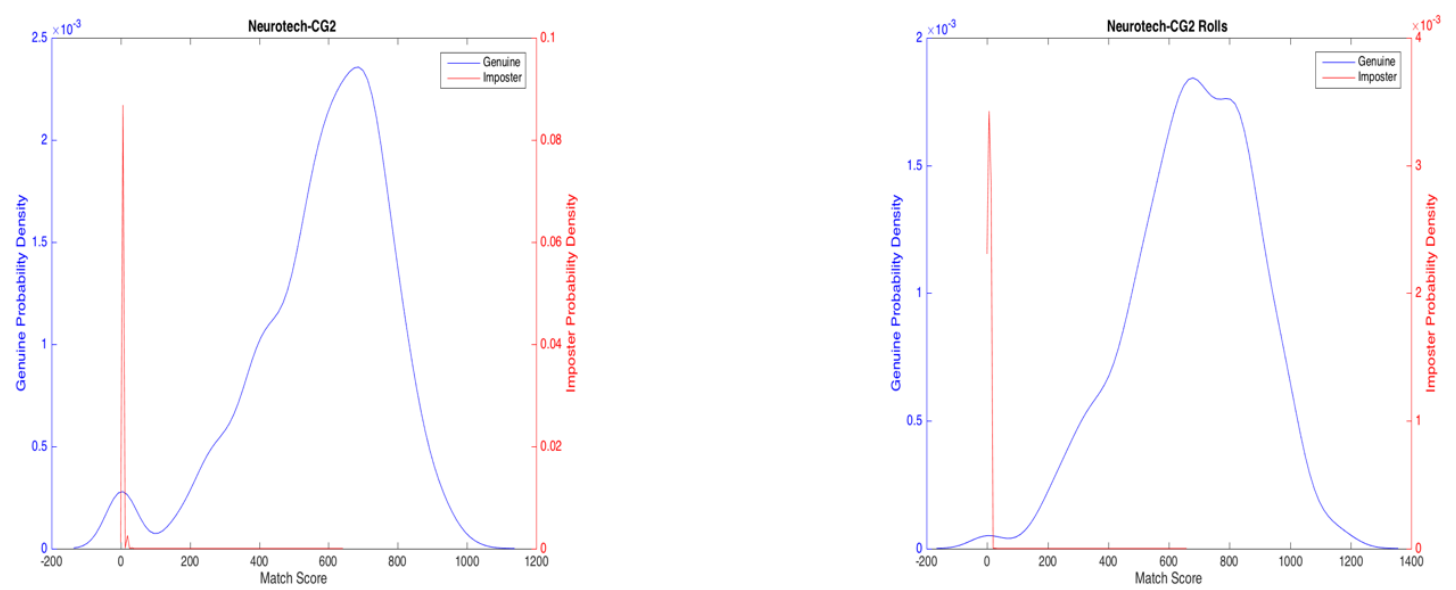

Figure A.2.3 (Left) Neurotech FP II Guardian (Right) Neurotech FP II Guardian Rolls
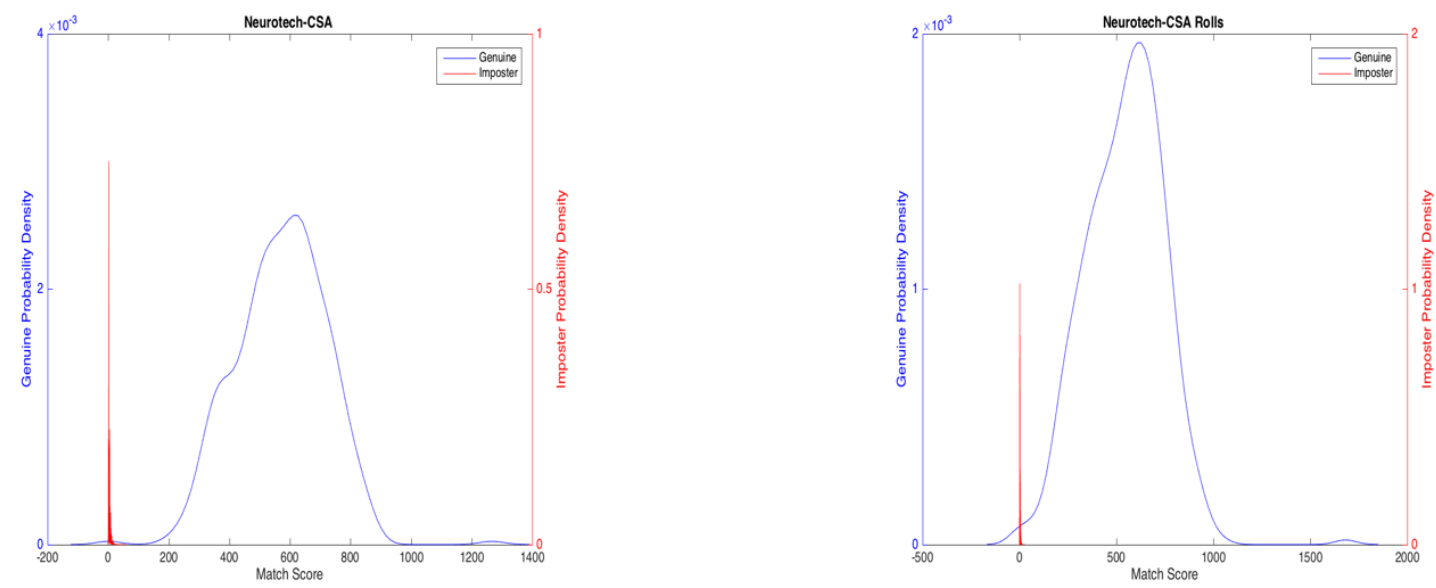

Figure A.2.4 (Left) Neurotech Crossmatch Seek Avenger (Right) Neurotech Crossmatch Seek Avenger Rolls
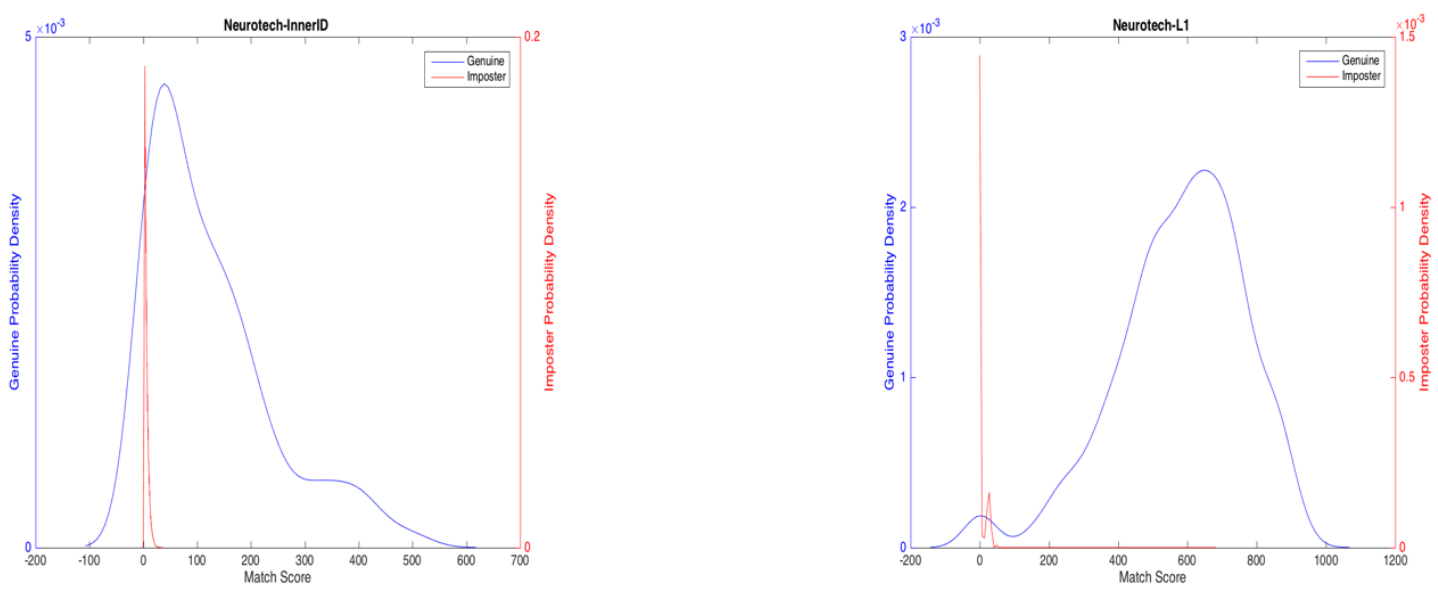

Figure A.2.5 (Left) Neurotech InnerID (Right) Neurotech L1 

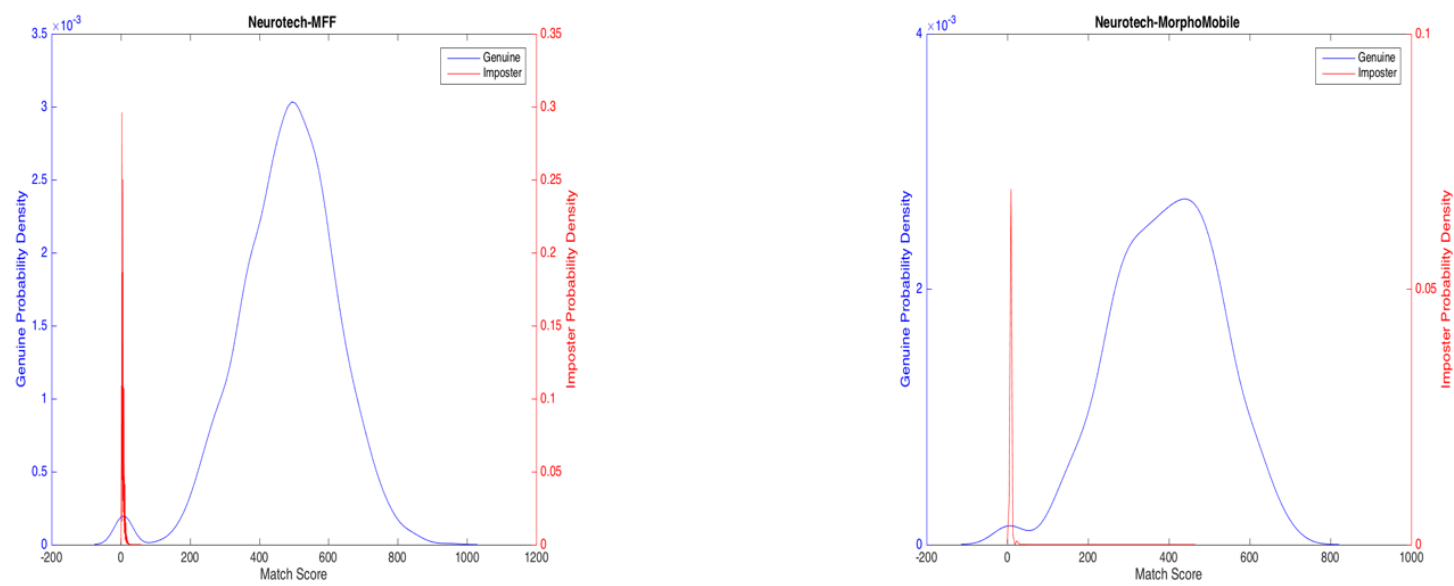

Figure A.2.6 (Left) Neurotech Morpho FOTF (Right) Neurotech MorphoMobile

Table A.9 SourceAFIS Maximum and Minimum Score Values

\begin{tabular}{|c|c|c|c|c|}
\hline \multicolumn{5}{|c|}{ SourceAFIS } \\
\hline Sensor & $\begin{array}{l}\text { Genuine } \\
\text { Maximum } \\
\text { Score }\end{array}$ & $\begin{array}{l}\text { Genuine } \\
\text { Minimum } \\
\text { Score }\end{array}$ & $\begin{array}{l}\text { Imposter } \\
\text { Maximum } \\
\text { Score }\end{array}$ & $\begin{array}{l}\text { Imposter } \\
\text { Minimum } \\
\text { Score }\end{array}$ \\
\hline ANDI BIN & 258.72 & 10.45 & 41.22 & 2.40 \\
\hline$A N D I G R Y$ & 227.51 & 8.98 & 35.99 & 1.05 \\
\hline Biosled & 248.36 & 9.96 & 141.30 & 1.13 \\
\hline BIO Rolls & 331.39 & 4.48 & 219.31 & 1.02 \\
\hline CG2 & 248.38 & 10.82 & 120.65 & 2.58 \\
\hline CG2 Rolls & 364.86 & 11.06 & 214.73 & 1.02 \\
\hline CSA & 284.96 & 13.24 & 35.92 & 2.56 \\
\hline CSA Rolls & 517.80 & 9.01 & 35.01 & 1.03 \\
\hline InnerID & 148.15 & 8.88 & 32.62 & 3.67 \\
\hline L1 & 288.08 & 9.57 & 89.70 & 1.21 \\
\hline L1 Rolls & 341.77 & 0 & 35.18 & 0.00 \\
\hline MFF & 289.72 & 9.18 & 36.04 & 1.02 \\
\hline MorphoMobile & 172.81 & 9.08 & 99.94 & 4.47 \\
\hline
\end{tabular}




\section{A.3 SourceAFIS Genuine/Imposter Score Distributions}
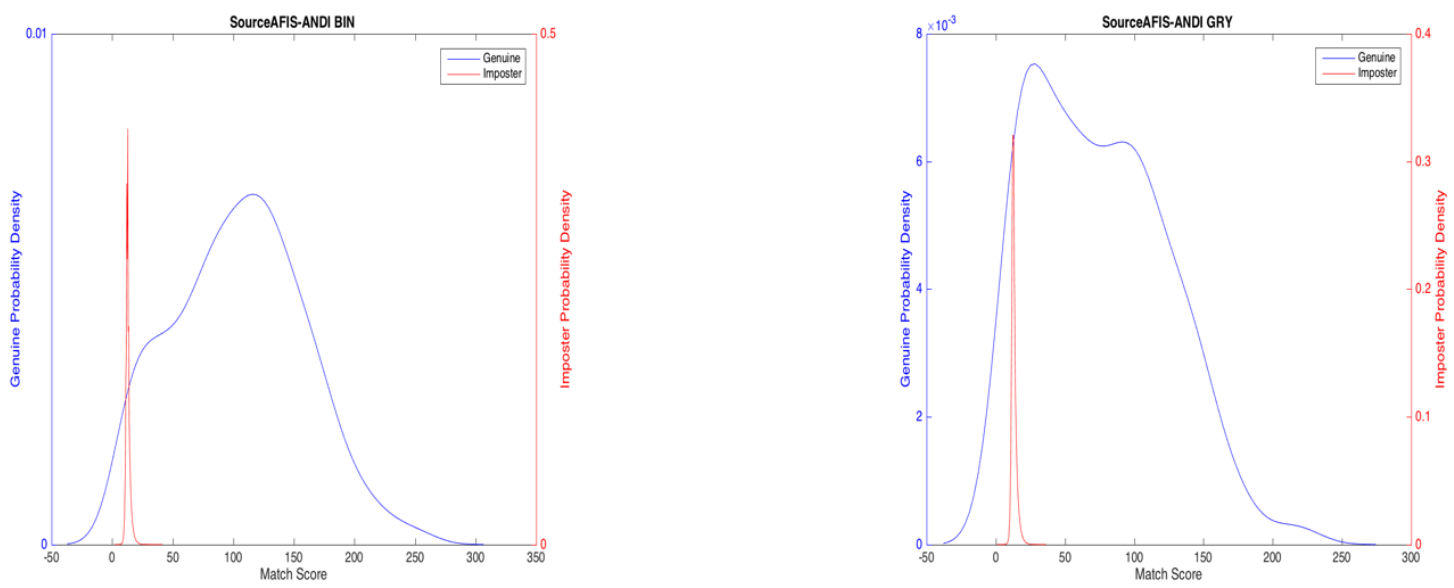

Figure A.3.1 (Left) SourceAFIS ANDI Binary (Right) SourceAFIS ANDI Greyscale
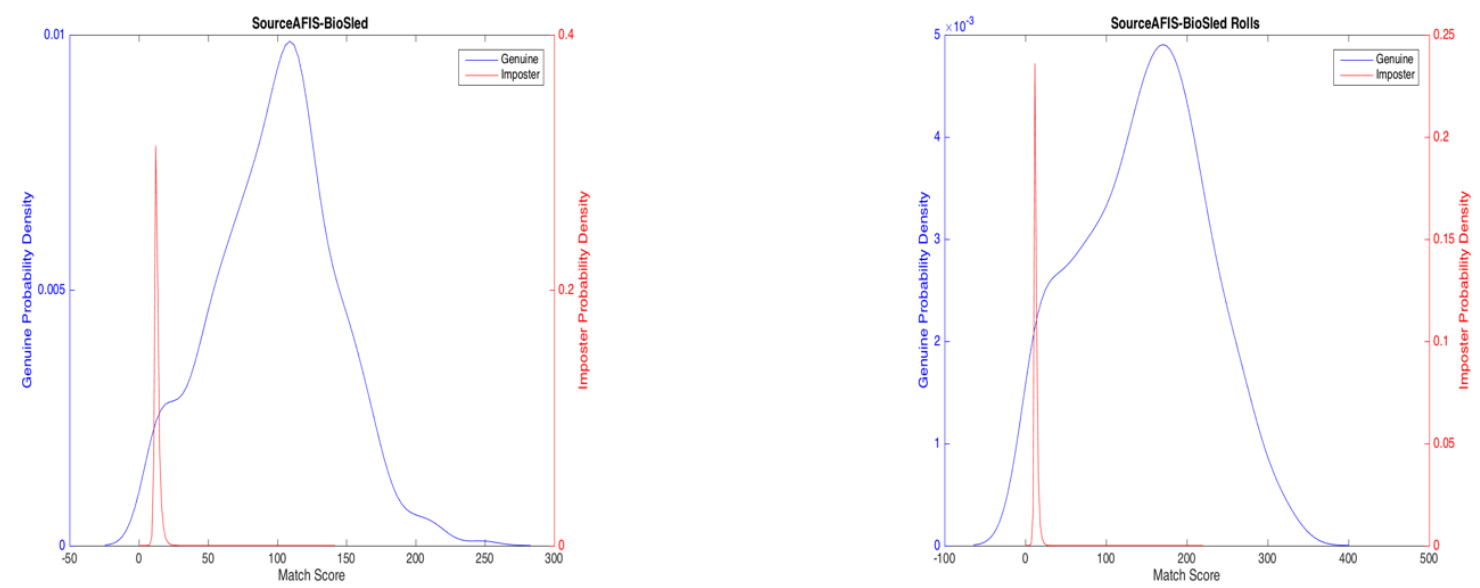

Figure A.3.2 (Left) SourceAFIS BioSled (Right) SourceAFIS BioSled Rolls 

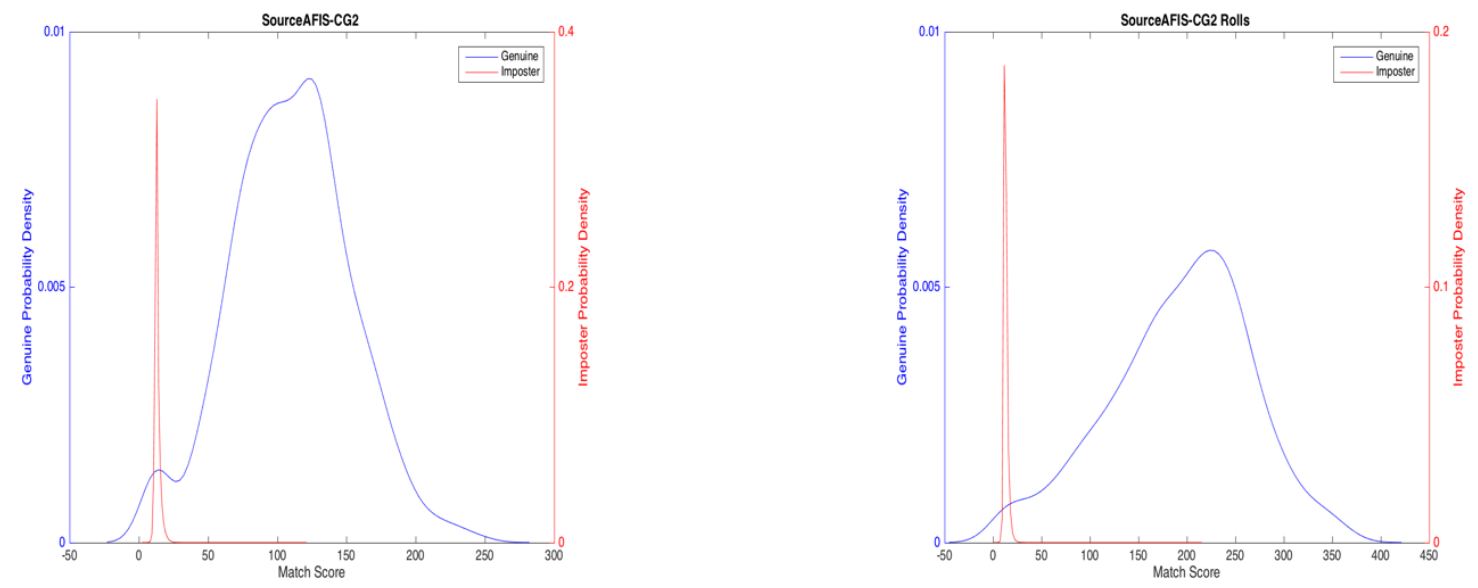

Figure A.3.3 (Left) SourceAFIS FP II Guardian (Right) SourceAFIS FP II Guardian Rolls
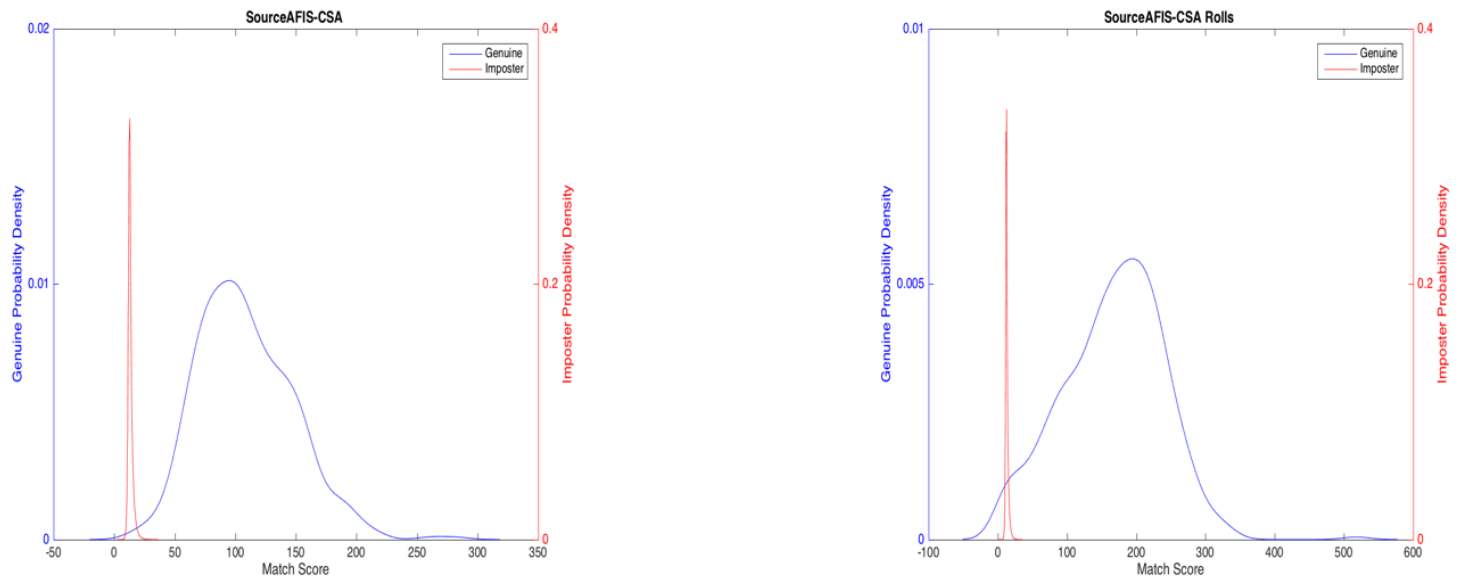

Figure A.3.4 (Left) SourceAFIS Crossmatch Seek Avenger (Right) SourceAFIS Crossmatch Seek Avenger Rolls
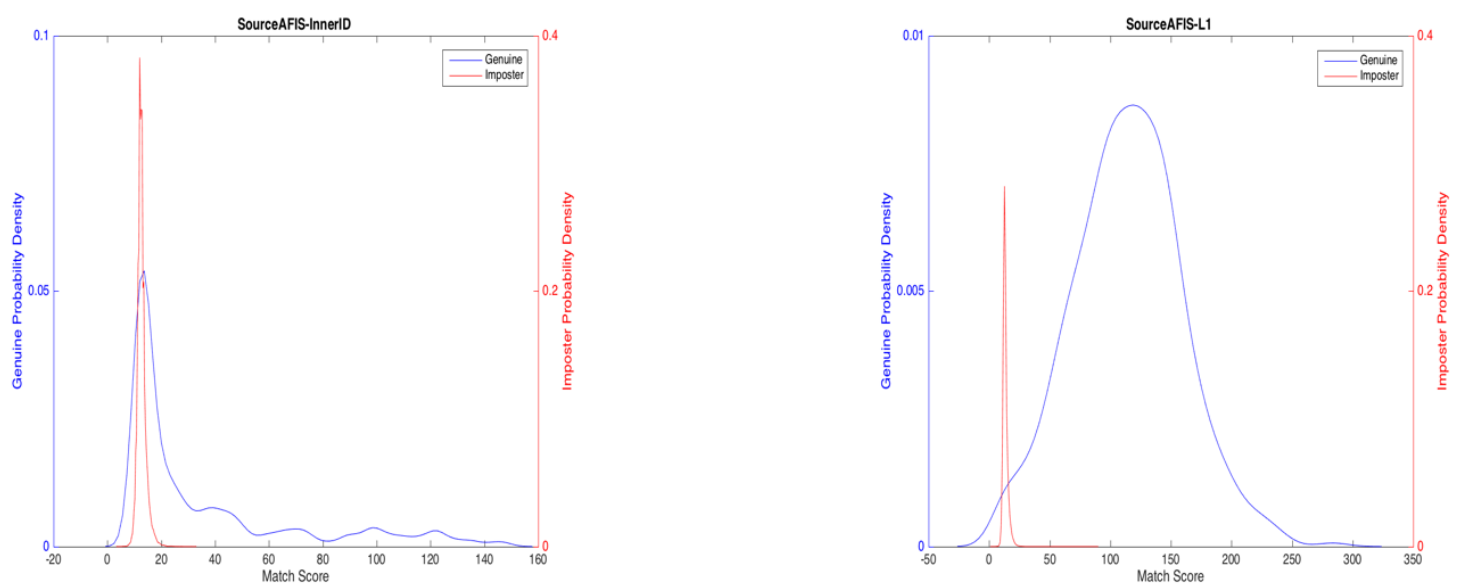

Figure A.3.5 (Left) SourceAFIS InnerID (Right) SourceAFIS L1 

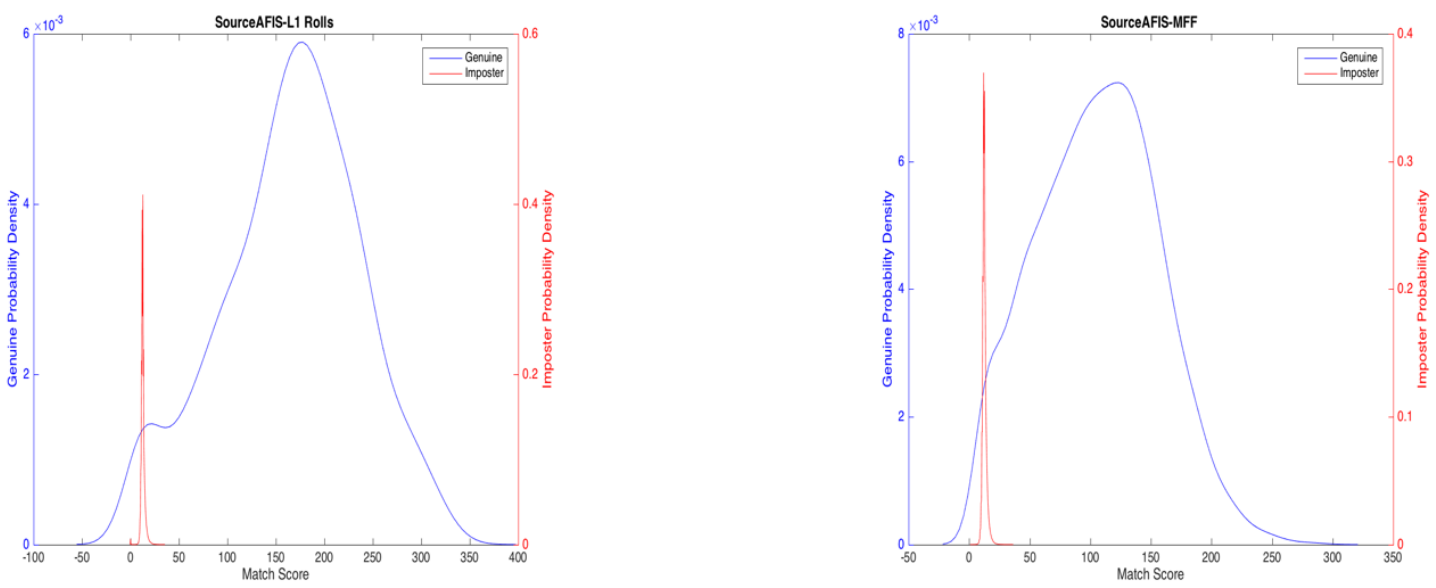

Figure A.3.6 (Left) SourceAFIS L1 Rolls (Right) SourceAFIS Morpho FOTF

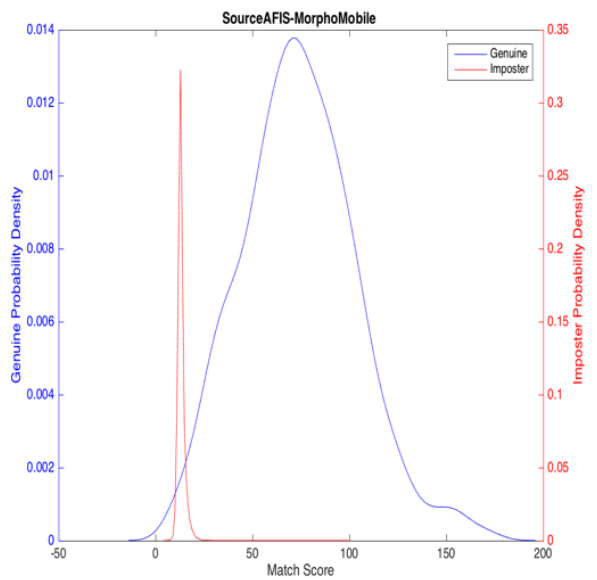

Figure A.3.7 SourceAFIS MorphoMobile 
Table A.10 NBIS Cross-Examined Maximum and Minimum Score Values

\begin{tabular}{|c|c|c|c|c|}
\hline Devices & Max Genuine & Min Genuine & Max Imposter & Min Imposter \\
\hline $10 p R-B I O R$ & 316 & 0 & 140 & 0 \\
\hline $10 p R-C S A R$ & 261 & 0 & 36 & 0 \\
\hline $10 p R-C G 2 R$ & 308 & 0 & 57 & 0 \\
\hline $10 p R-L 1 R$ & 322 & 0 & 115 & 0 \\
\hline $10 p-A N D I B I N$ & 167 & 4 & 50 & 0 \\
\hline $10 p-A N D I G R Y$ & 163 & 4 & 69 & 0 \\
\hline $10 p-B I O$ & 232 & 3 & 180 & 0 \\
\hline $10 p-C S A$ & 272 & 4 & 48 & 0 \\
\hline $10 p-C G 2$ & 303 & 4 & 47 & 0 \\
\hline $10 p-L 1$ & 266 & 3 & 125 & 0 \\
\hline $10 p-M F F$ & 164 & 4 & 64 & 0 \\
\hline $10 p-S M M$ & 235 & 3 & 96 & 0 \\
\hline $10 p-I I D$ & 30 & 4 & 47 & 0 \\
\hline$A N D I B I N-A N D I G R Y$ & 293 & 5 & 70 & 0 \\
\hline$A N D I B I N-B I O$ & 191 & 3 & 130 & 0 \\
\hline$A N D I B I N$ - CSA & 168 & 0 & 39 & 0 \\
\hline$A N D I B I N$ - CG2 & 169 & 3 & 97 & 0 \\
\hline$A N D I B I N-L 1$ & 172 & 3 & 51 & 0 \\
\hline$A N D I B I N-M F F$ & 169 & 3 & 57 & 0 \\
\hline ANDI BIN - SMM & 158 & 0 & 74 & 0 \\
\hline$A N D I B I N-I I D$ & 24 & 3 & 52 & 0 \\
\hline$A N D I G R Y-B I O$ & 146 & 3 & 89 & 0 \\
\hline ANDI GRY - CSA & 181 & 3 & 50 & 0 \\
\hline$A N D I G R Y-C G 2$ & 212 & 3 & 95 & 0 \\
\hline$A N D I G R Y-L 1$ & 185 & 3 & 59 & 0 \\
\hline ANDI GRY - MFF & 149 & 3 & 62 & 0 \\
\hline ANDI GRY - SMM & 148 & 0 & 61 & 0 \\
\hline$A N D I G R Y-I I D$ & 45 & 4 & 61 & 3 \\
\hline$B I O R-C S A R$ & 444 & 4 & 232 & 0 \\
\hline$B 1 O R-C G 2 R$ & 416 & 3 & 220 & 0 \\
\hline$B I O R-L 1 R$ & 453 & 0 & 193 & 0 \\
\hline$B I O-C S A$ & 316 & 3 & 236 & 0 \\
\hline$B 1 O-C G 2$ & 336 & 0 & 185 & 0 \\
\hline$B I O-L 1$ & 285 & 3 & 175 & 0 \\
\hline$B I O-M F F$ & 185 & 0 & 101 & 0 \\
\hline$B I O-S M M$ & 280 & 3 & 215 & 0 \\
\hline$B I O-I I D$ & 27 & 3 & 45 & 0 \\
\hline$C S A R-C G 2 R$ & 443 & 4 & 82 & 0 \\
\hline CSA R - L1R & 447 & 0 & 112 & 0 \\
\hline CSA - CG2 & 291 & 3 & 164 & 0 \\
\hline$C S A-\angle 1$ & 349 & 3 & 105 & 0 \\
\hline$C S A-M F F$ & 205 & 0 & 49 & 0 \\
\hline$C S A-S M M$ & 258 & 3 & 134 & 0 \\
\hline$C S A-I I D$ & 23 & 3 & 40 & 0 \\
\hline$C G 2 R-L 1 R$ & 436 & 0 & 209 & 0 \\
\hline$C G 2-L 1$ & 353 & 3 & 142 & 0 \\
\hline$C G 2-M F F$ & 209 & 0 & 86 & 0 \\
\hline CG2 - SMM & 256 & 0 & 126 & 0 \\
\hline$C G 2-\| I D$ & 24 & 3 & 38 & 0 \\
\hline$L 1-M F F$ & 188 & 3 & 88 & 0 \\
\hline$L 1-S M M$ & 277 & 0 & 176 & 0 \\
\hline$M F F-S M M$ & 159 & 0 & 80 & 0 \\
\hline$I I D-L 1$ & 27 & 3 & 39 & 0 \\
\hline$I I D-M F F$ & 38 & 3 & 48 & 0 \\
\hline$I I D-S M M$ & 23 & 0 & 33 & 0 \\
\hline
\end{tabular}




\section{A.4 NBIS Cross-Examined Genuine/Imposter Distributions}
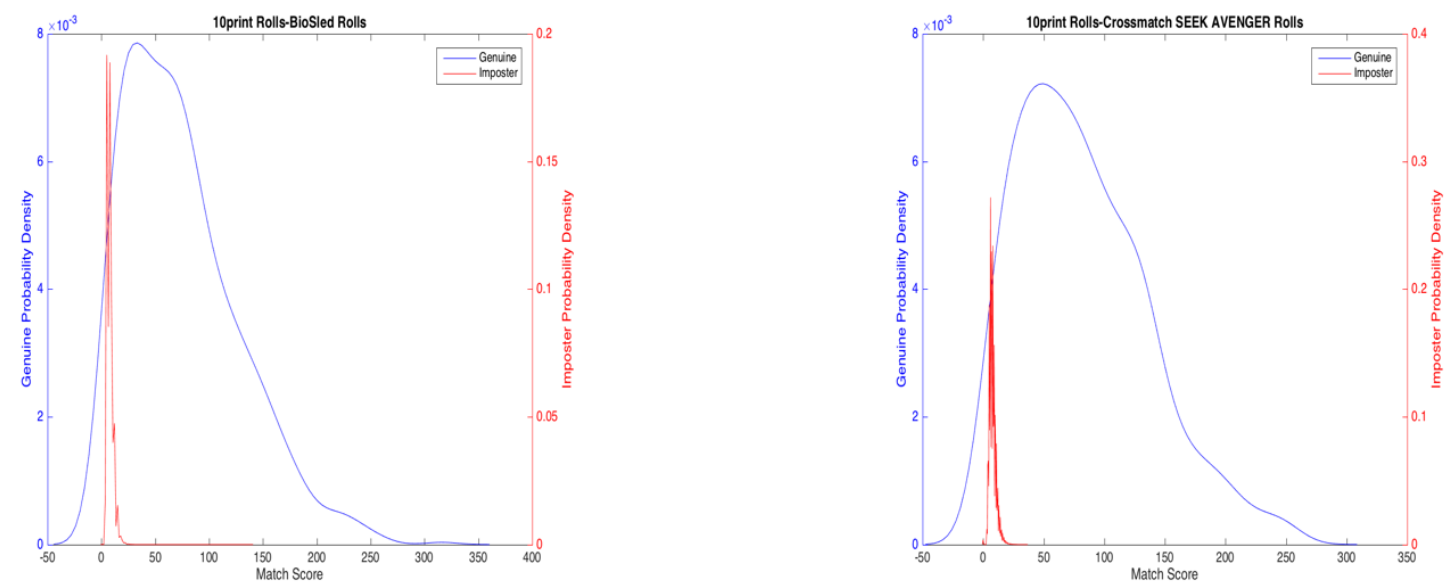

Figure A.4.1 (Left) NBIS 10print Rolls - BioSled Rolls (Right) NBIS 10print Rolls - Crossmatch Seek Avenger Rolls
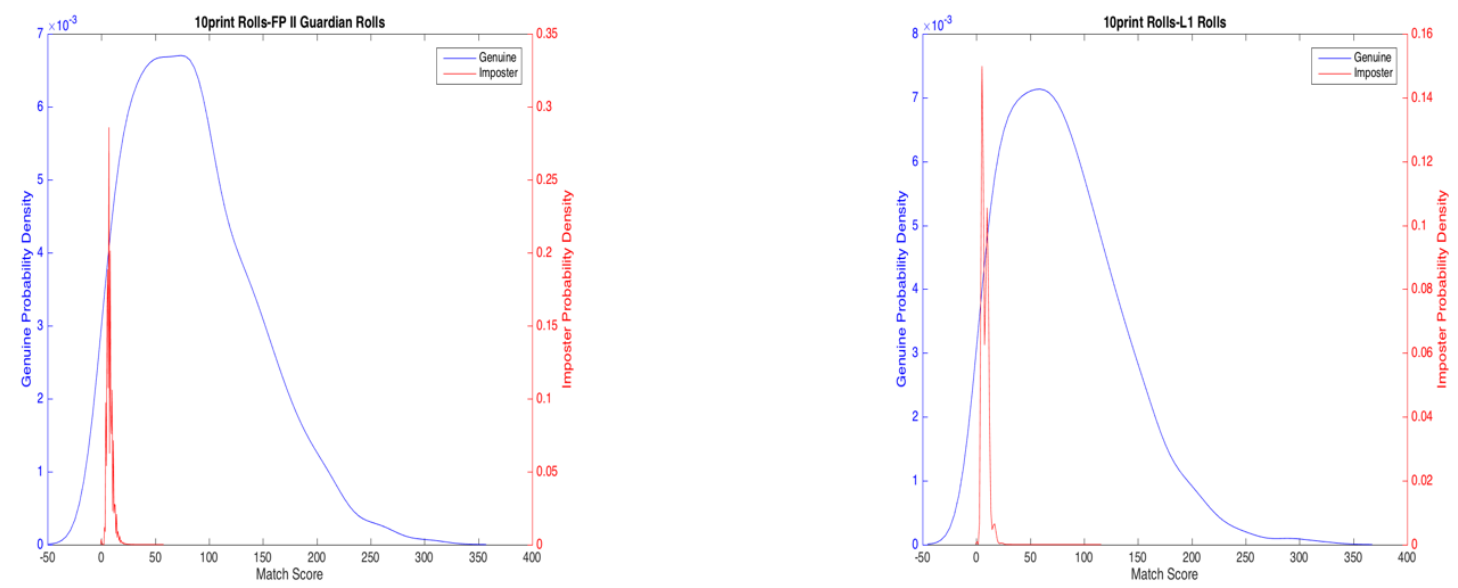

Figure A.4.2 (Left) NBIS 10print Rolls - FP II Guardian Rolls (Right) NBIS 10print Rolls - L1 Rolls 

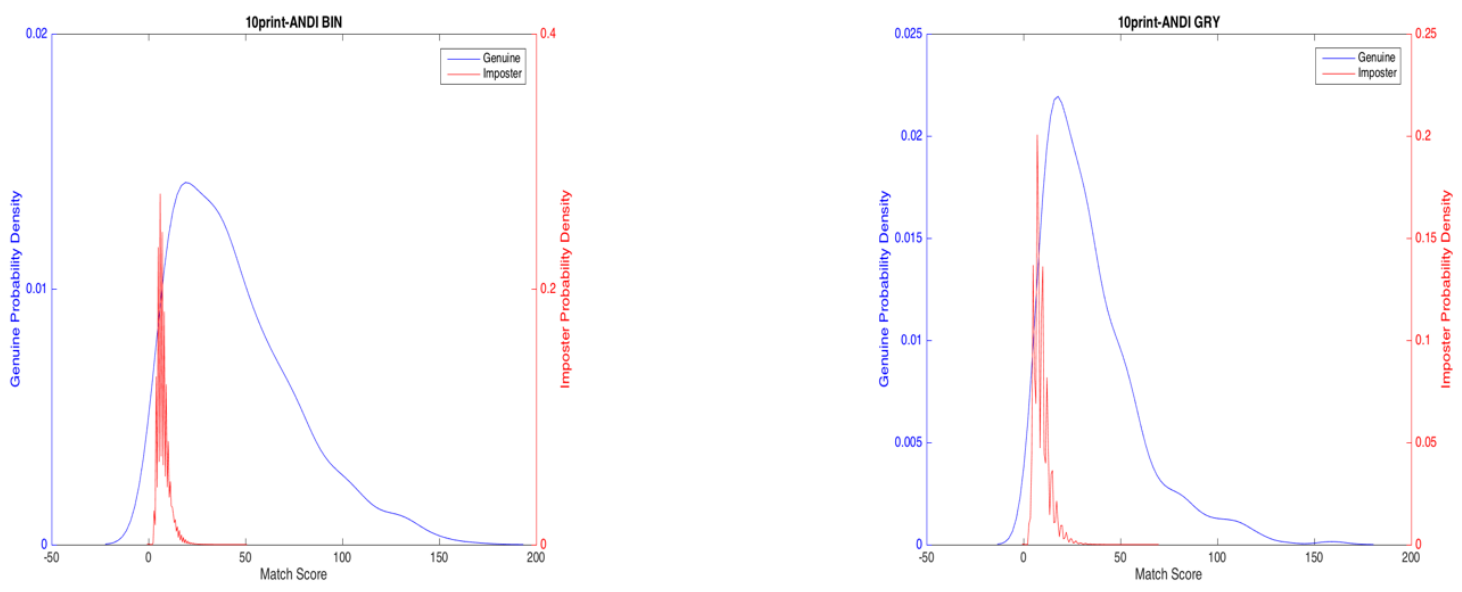

Figure A.4.3 (Left) NBIS 10print - ANDI Binary (Right) NBIS 10print - ANDI Greyscale
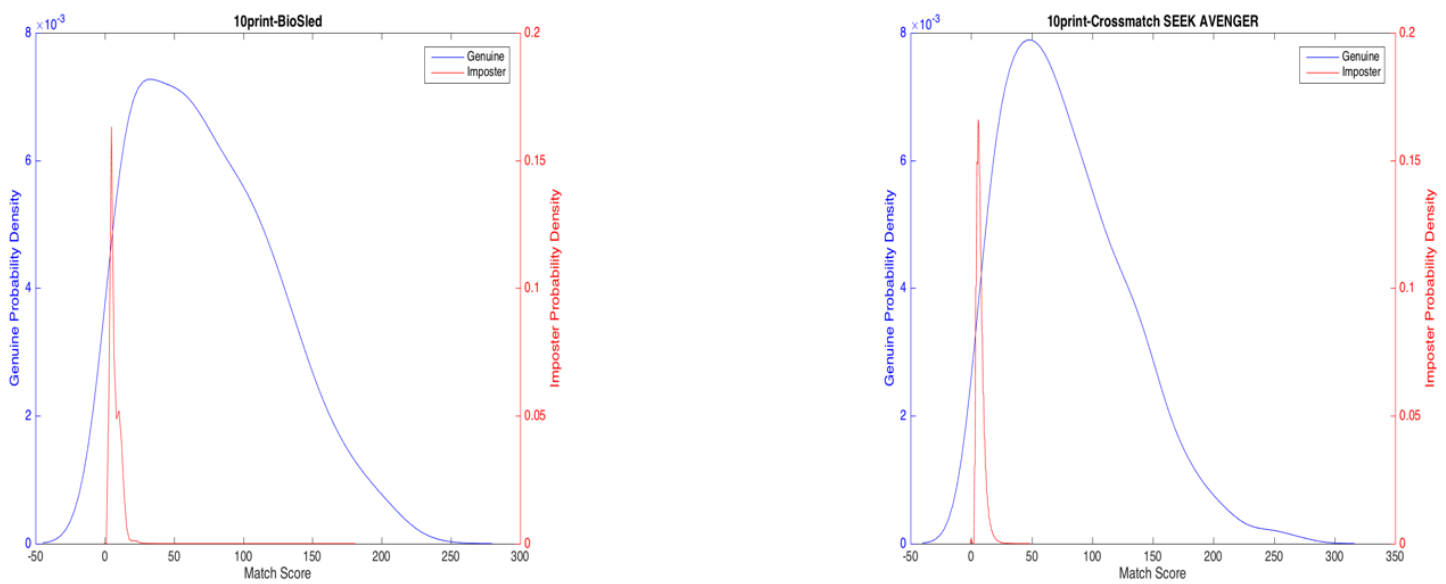

Figure A.4.4 (Left) NBIS 10print - BioSled (Right) NBIS 10print - Crossmatch Seek Avenger
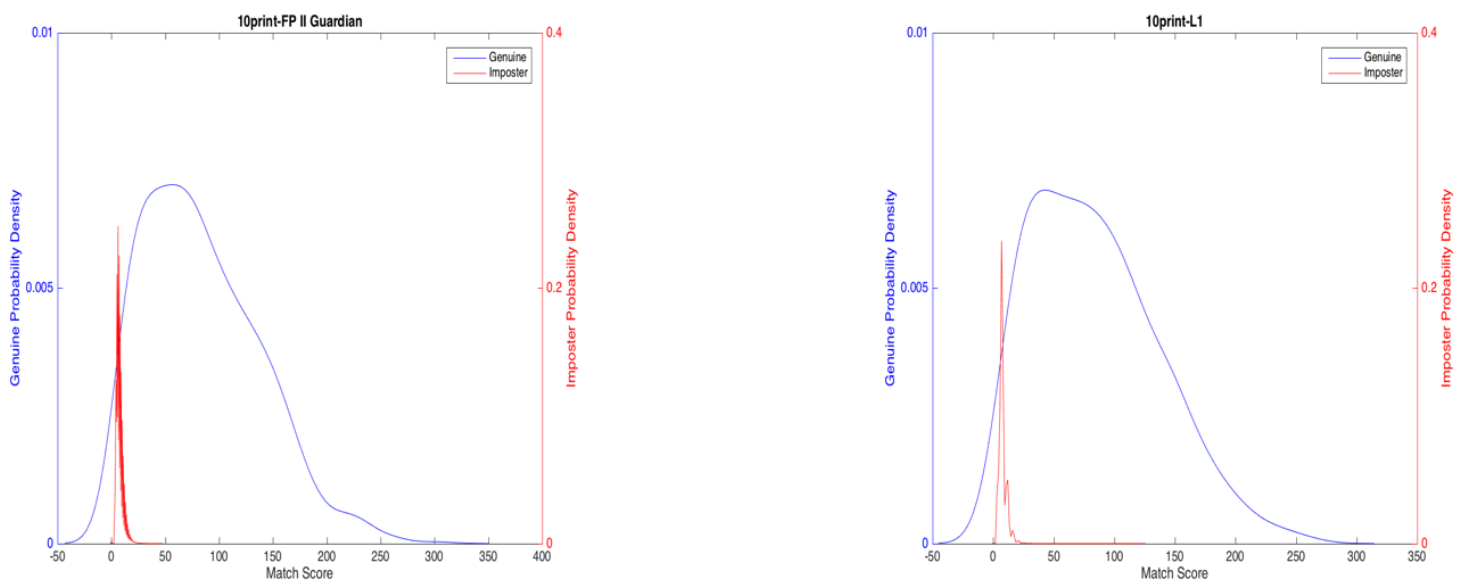

Figure A.4.5 (Left) NBIS 10print - FP II Guardian (Right) NBIS 10print - L1 

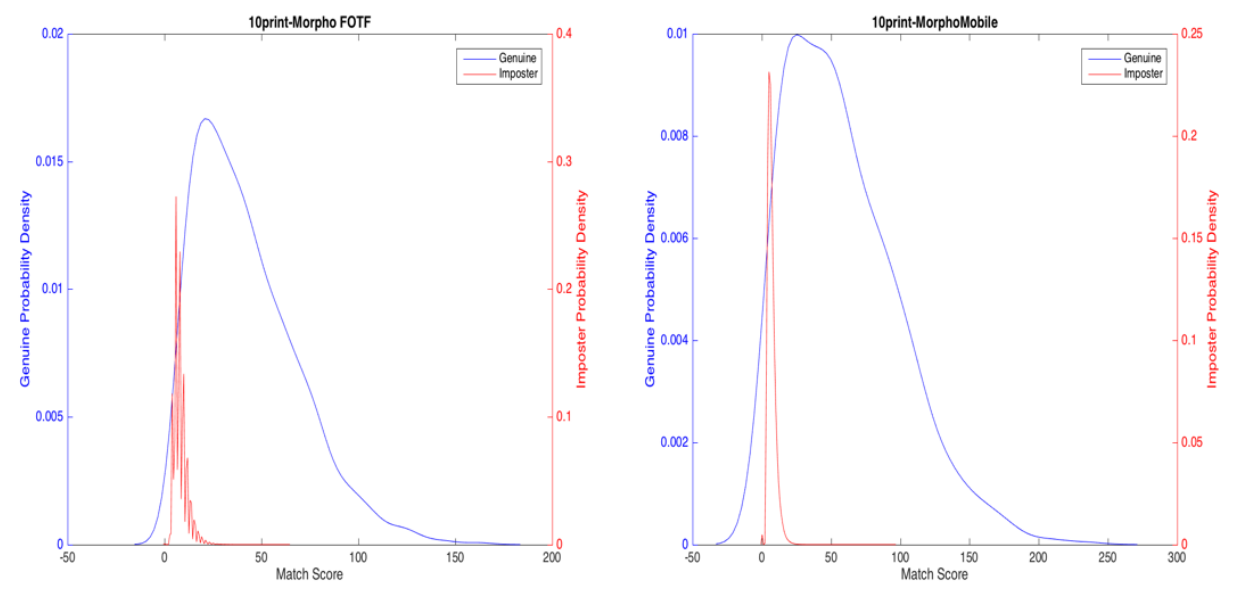

Figure A.4.6 (Left) NBIS 10print - Morpho FOTF (Right) NBIS 10print - MorphoMobile
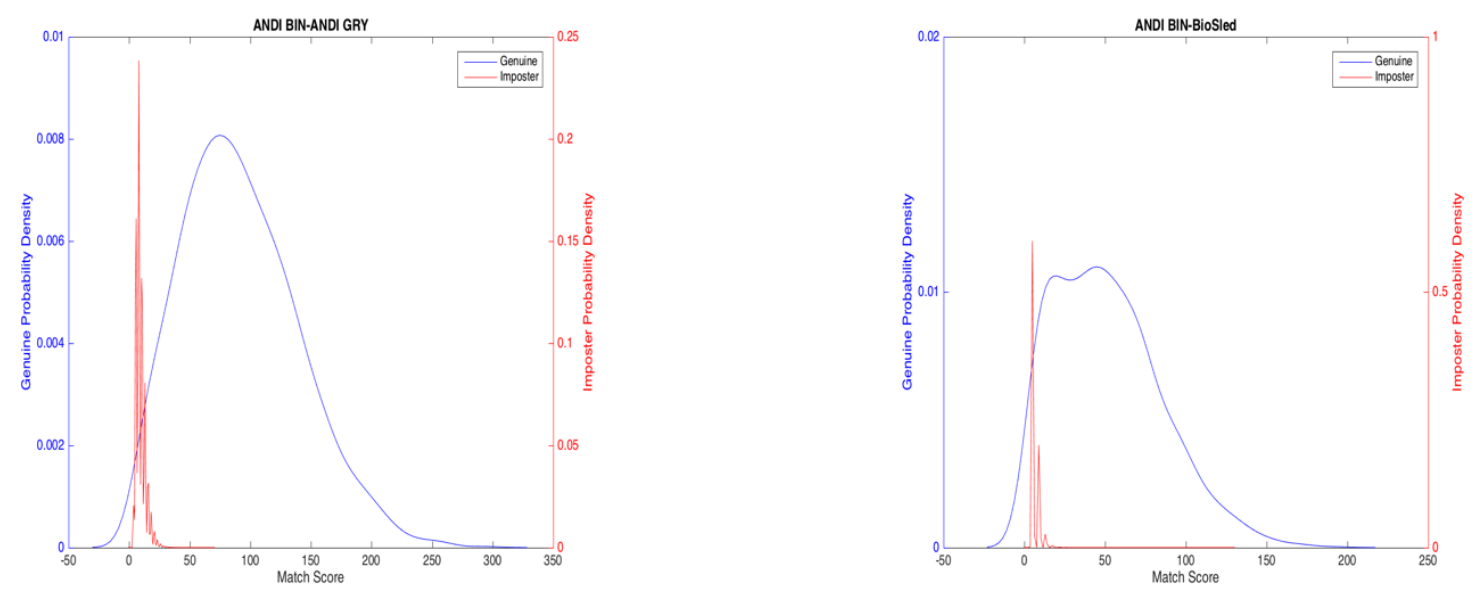

Figure A.4.7 (Left) NBIS ANDI Binary - ANDI Greyscale (Right) NBIS ANDI Binary - BioSled
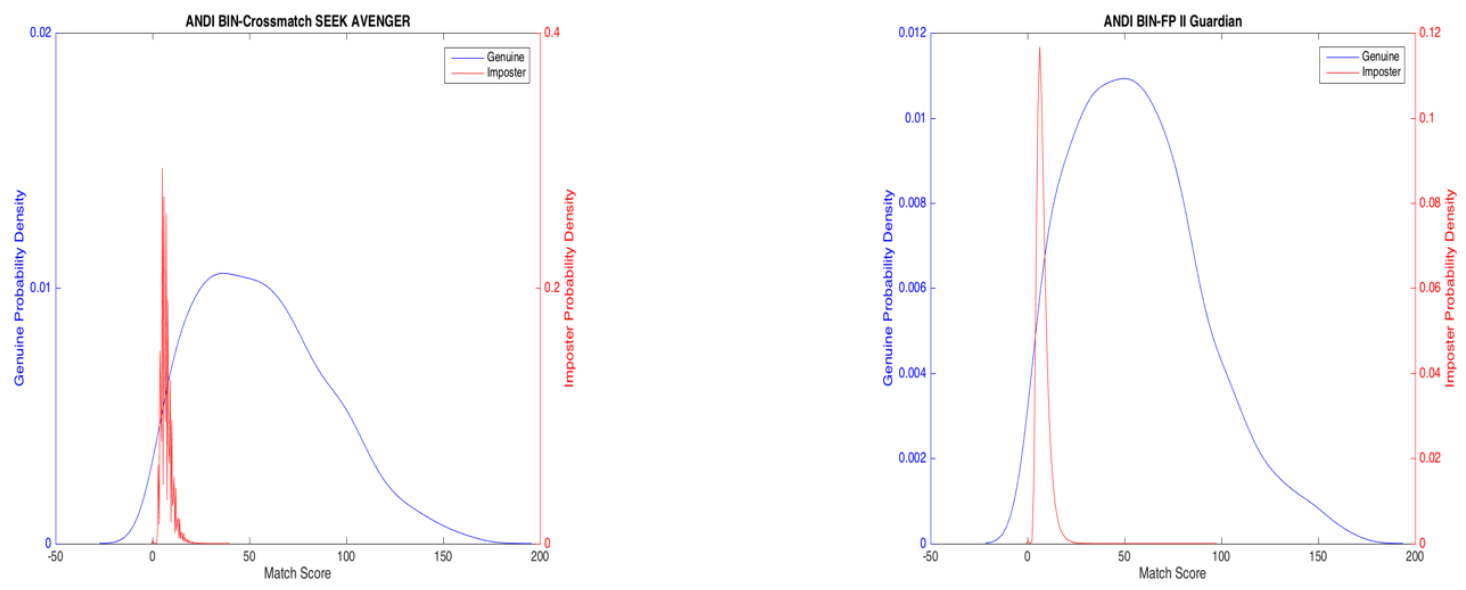

Figure A.4.8 (Left) NBIS ANDI Binary - Crossmatch Seek Avenger (Right) NBIS ANDI Binary - FP II Guardian 

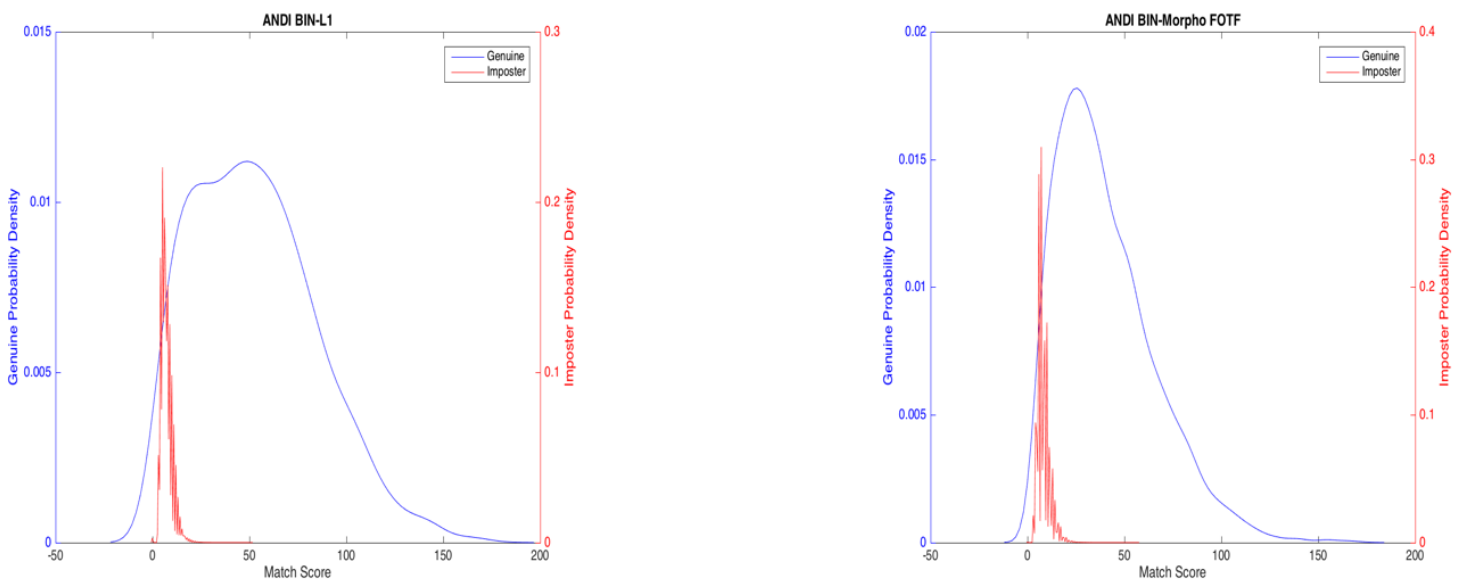

Figure A.4.9 (Left) NBIS ANDI Binary - L1 (Right) NBIS ANDI Binary - Morpho FOTF
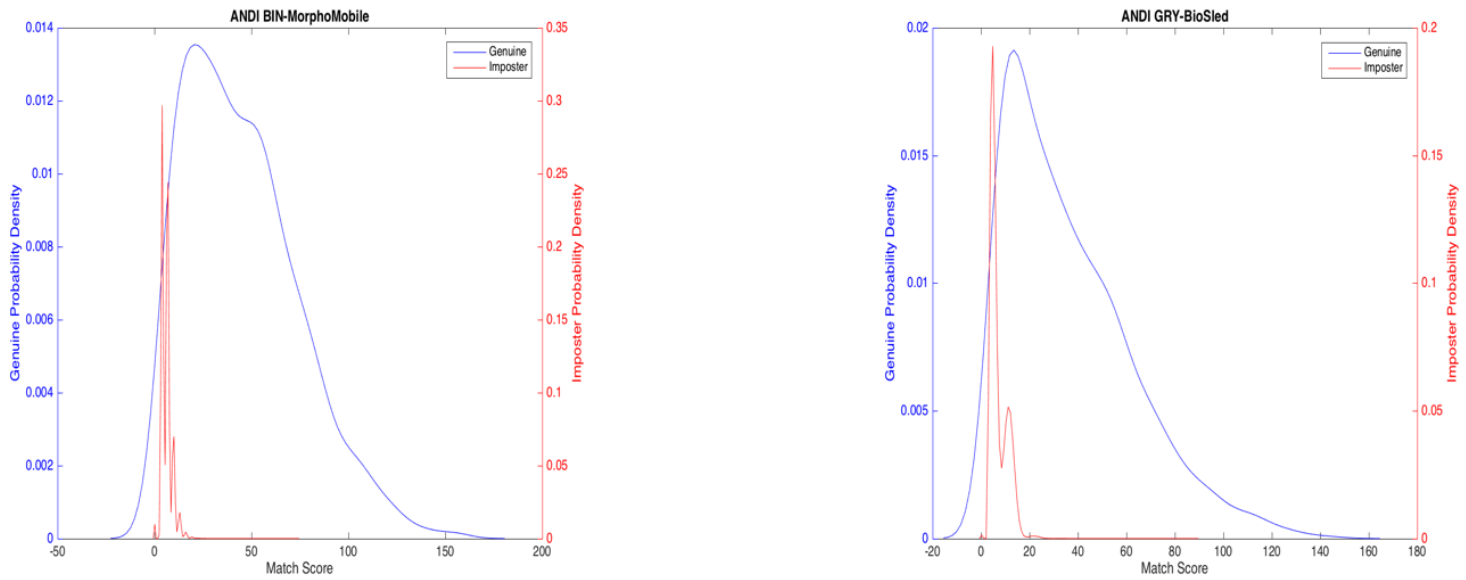

Figure A.4.10 (Left) NBIS ANDI Binary - MorphoMobile (Right) NBIS ANDI Greyscale - BioSled
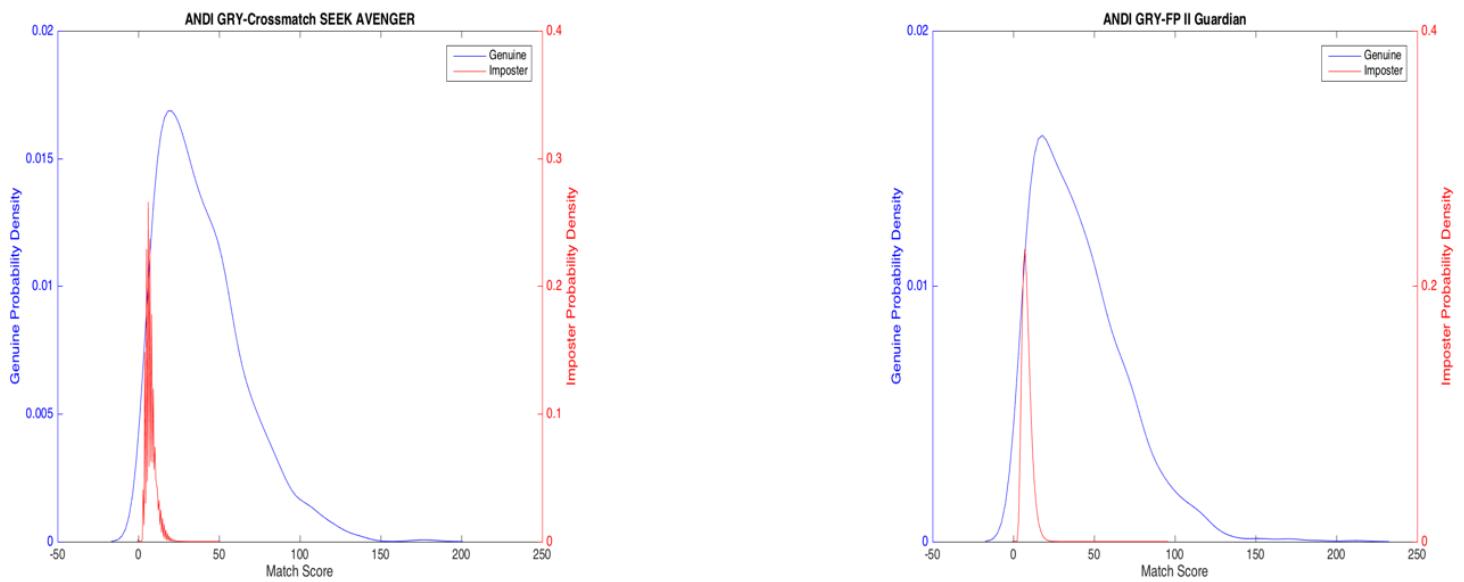

Figure A.4.11 (Left) NBIS ANDI Greyscale - Crossmatch Seek Avenger (Right) NBIS ANDI Greyscale - FP II Guardian 

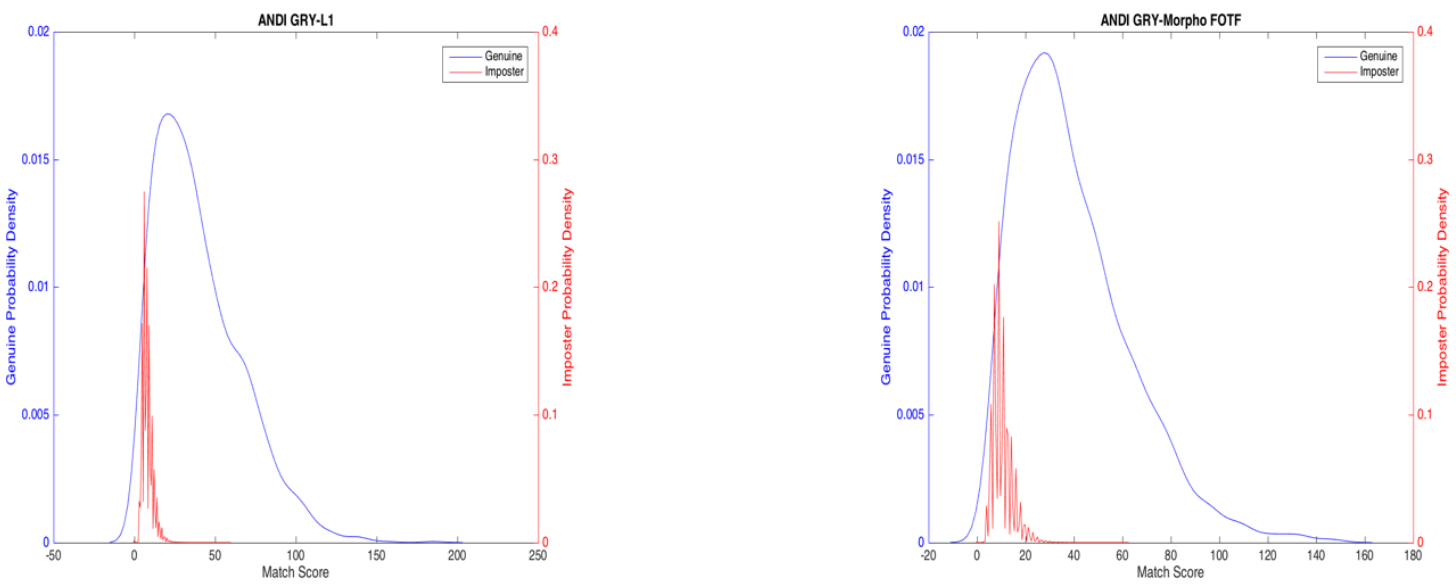

Figure A.4.12 (Left) NBIS ANDI Greyscale - L1 (Right) NBIS ANDI Greyscale - Morpho FOTF
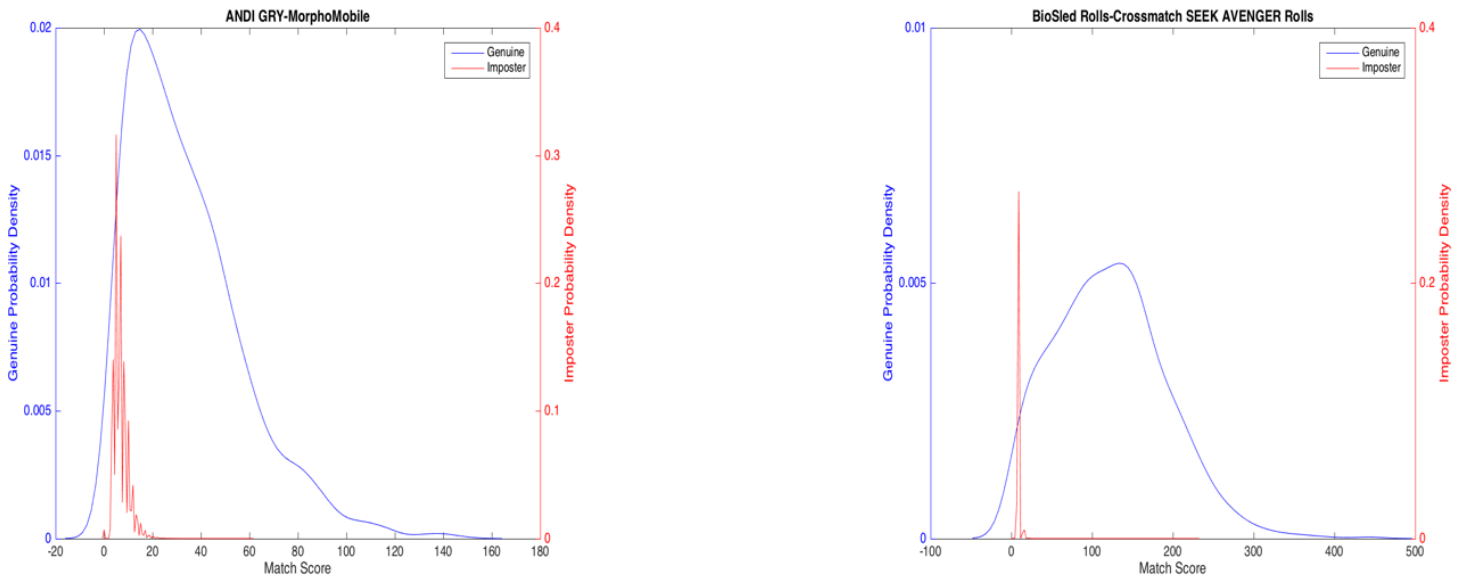

Figure A.4.13 (Left) NBIS ANDI Greyscale - MorphoMobile (Right) NBIS BioSled Rolls - Crossmatch Seek Avenger Rolls
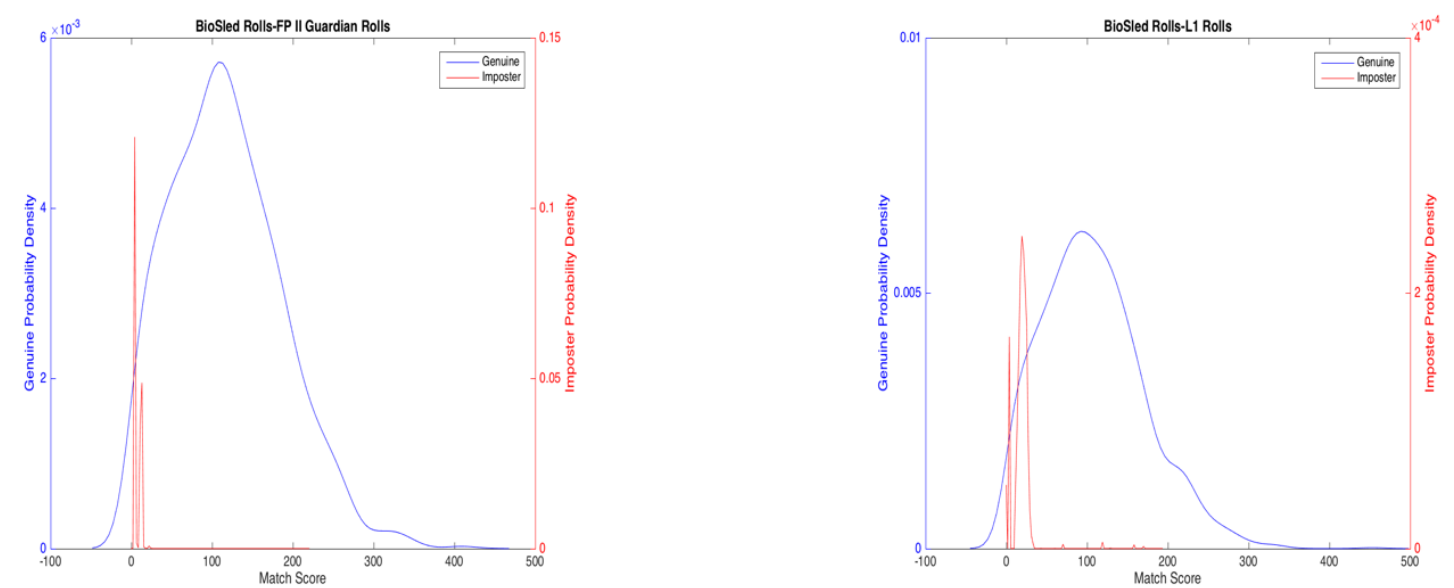

Figure A.4.14 (Left) NBIS BioSled Rolls - FP II Guardian Rolls (Right) NBIS BioSled Rolls - L1 Rolls 

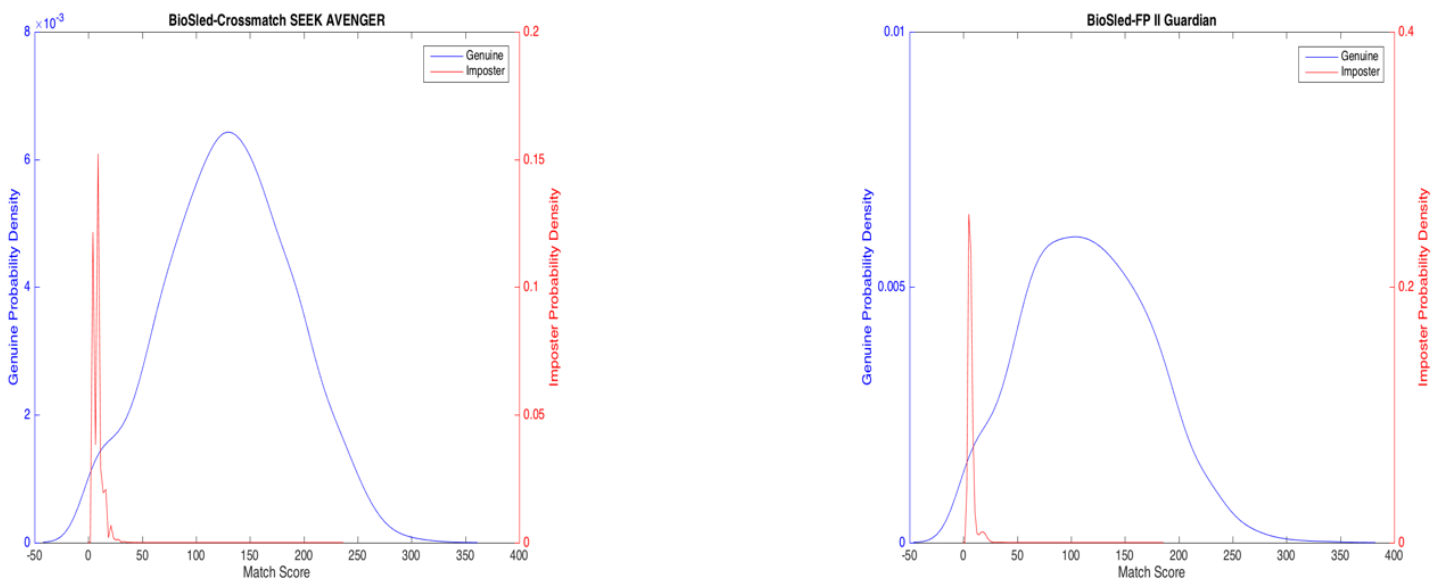

Figure A.4.15 (Left) NBIS BioSled - Crossmatch Seek Avenger (Right) NBIS BioSled - FP II Guardian
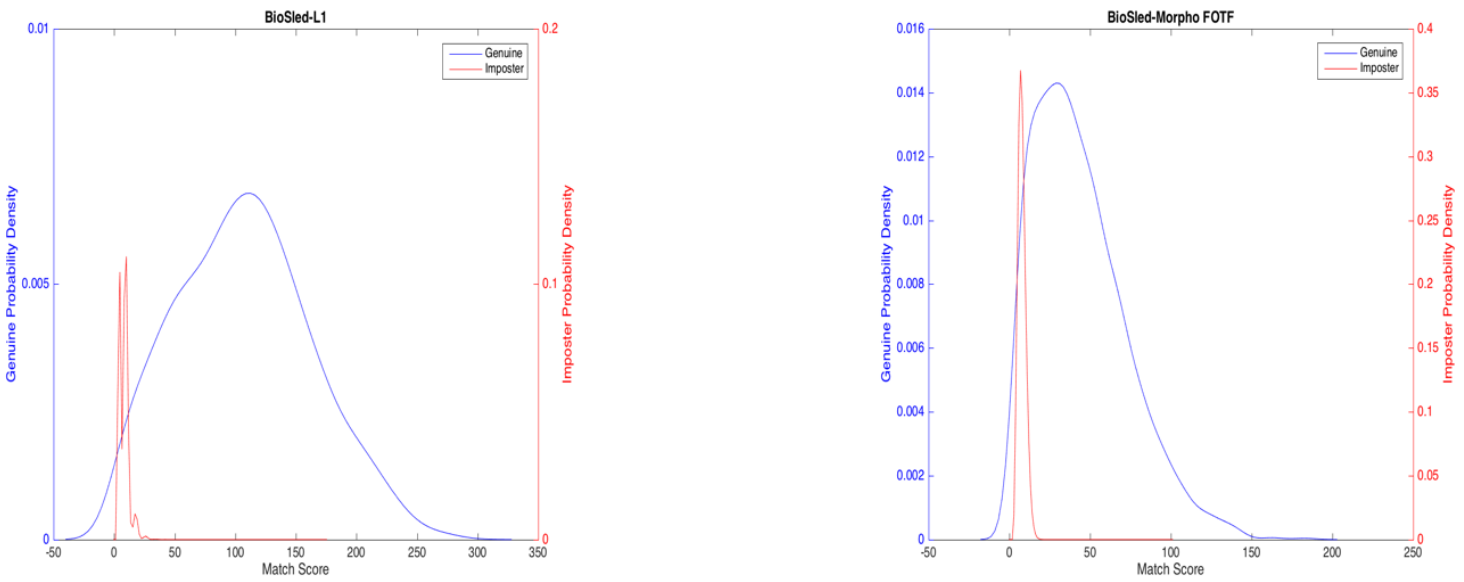

Figure A.4.16 (Left) NBIS BioSled - L1 (Right) NBIS BioSled - Morpho FOTF
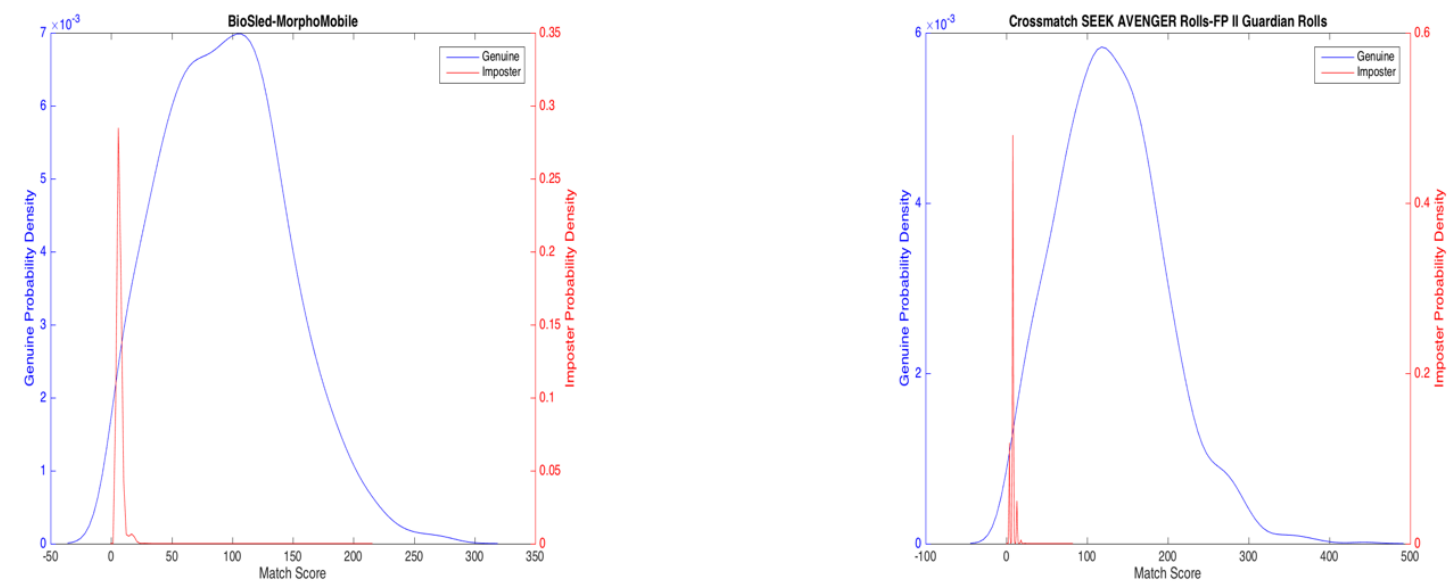

Figure A.4.17 (Left) NBIS BioSled - MorphoMobile (Right) NBIS Crossmatch Seek Avenger Rolls - FP Guardian Rolls 

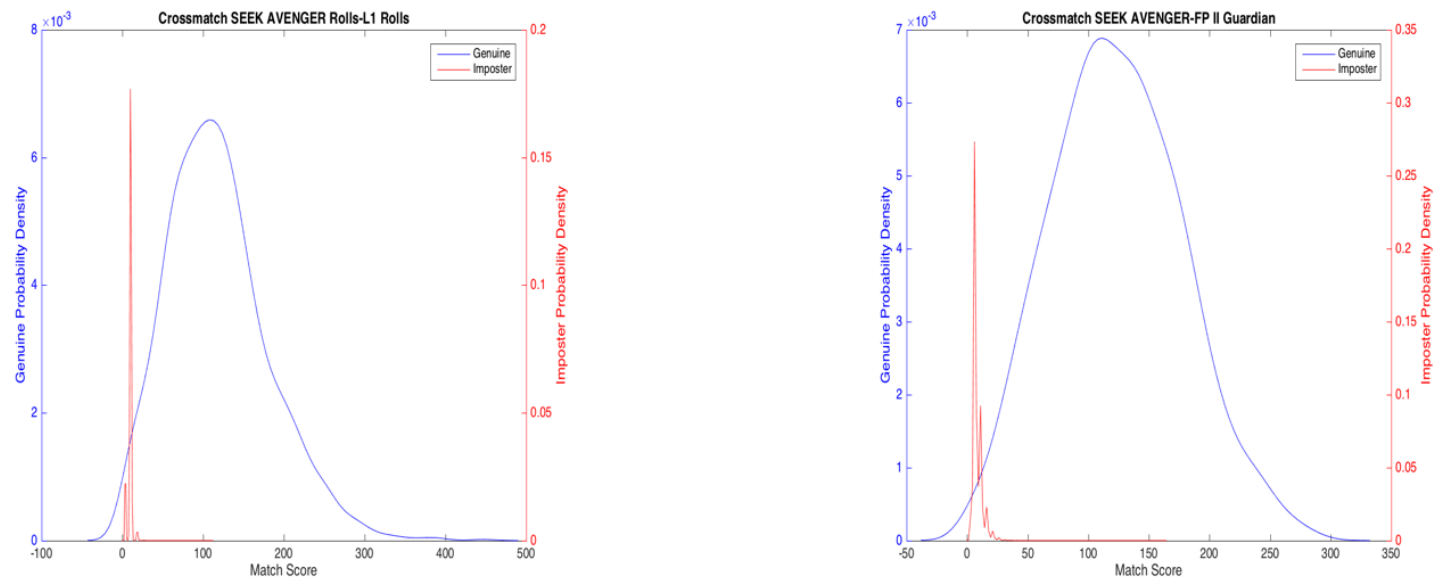

Figure A.4.18 (Left) NBIS Crossmatch Seek Avenger Rolls - L1 Rolls (Right) NBIS Crossmatch Seek Avenger FP II Guardian
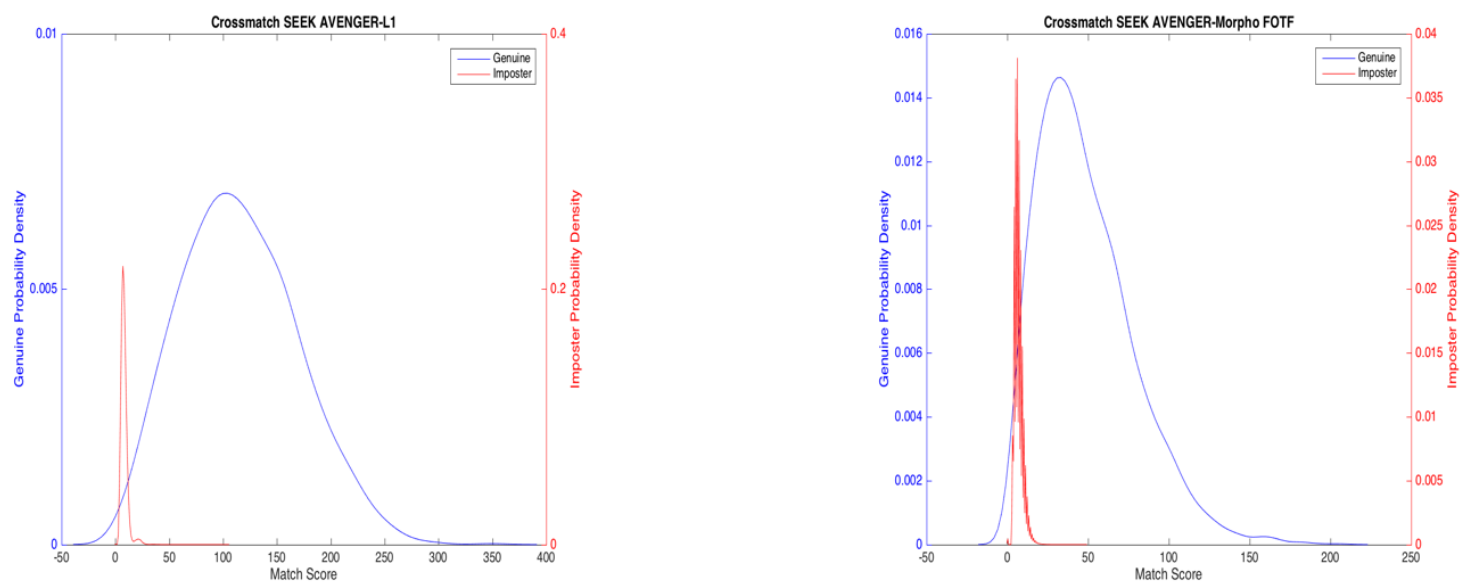

Figure A.4.19 (Left) NBIS Crossmatch Seek Avenger - L1 (Right) NBIS Crossmatch Seek Avenger - Morpho FOTF 

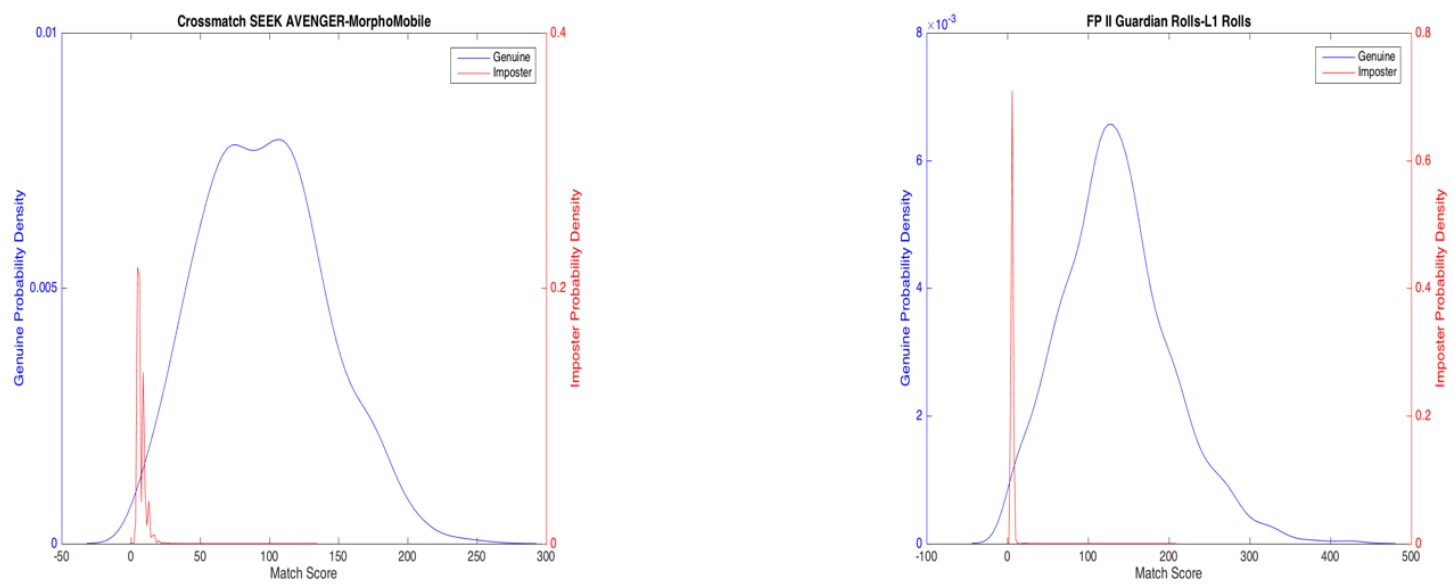

Figure A.4.20 (Left) NBIS Crossmatch Seek Avenger - MorphoMobile (Right) NBIS FP II Guardian Rolls - L1 Rolls
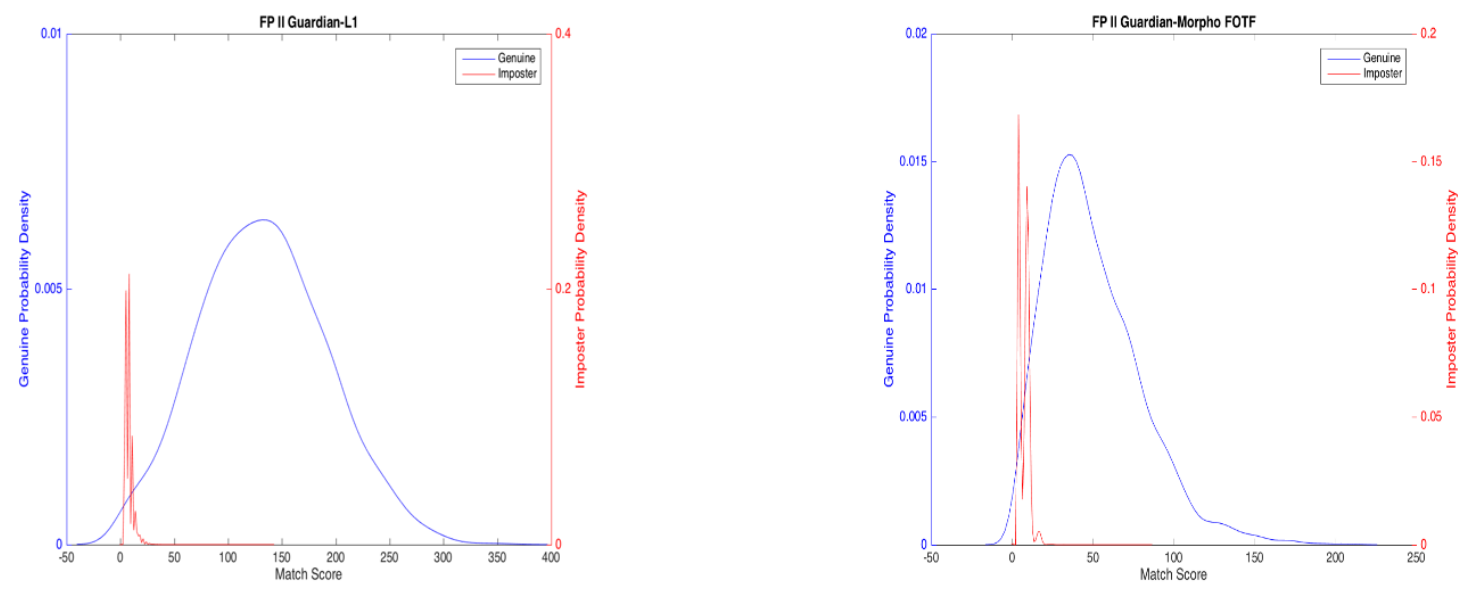

Figure A.4.21 (Left) NBIS FP II Guardian - L1 (Right) NBIS FP II Guardian - Morpho FOTF
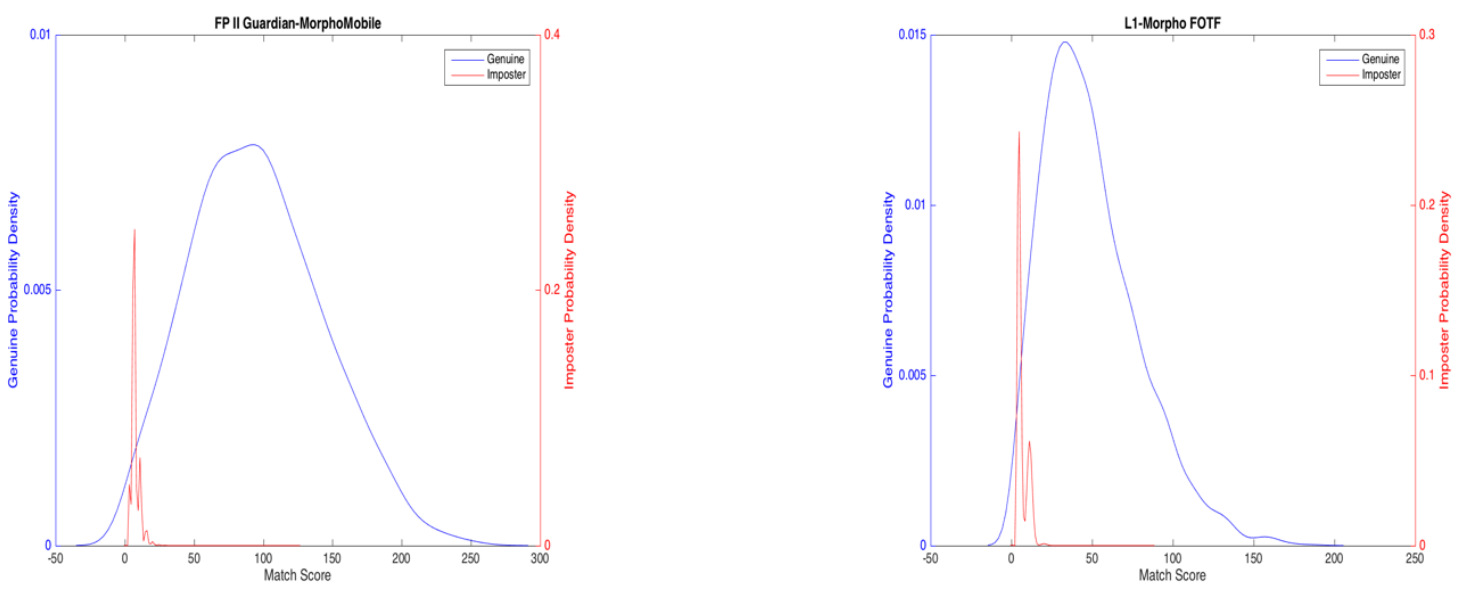

Figure A.4.22 (Left) NBIS FP II Guardian - MorphoMobile (Right) NBIS L1 - Morpho FOTF 

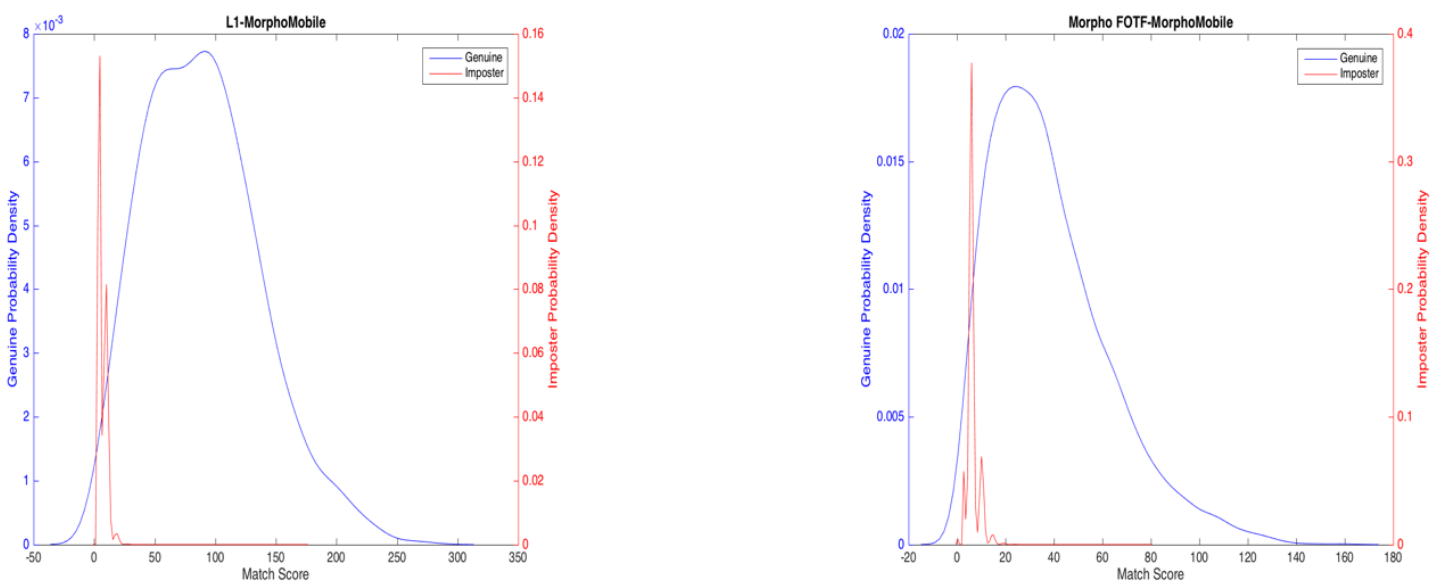

Figure A.4.23 (Left) NBIS L1 - MorphoMobile (Right) NBIS Morpho FOTF - MorphoMobile 
Table A.11 Neurotech Cross-Examined Maximum and Minimum Score Values

\begin{tabular}{|c|c|c|c|c|}
\hline Neurotech & Max Genuine & Min Genuine & Max Imposter & Min Imposter \\
\hline \multirow{2}{*}{$\begin{array}{l}10 p-A N D I B I N \\
10 p-A N D I G R Y\end{array}$} & 2274 & 3 & 54 & 1 \\
\hline & 414 & 2 & 44 & 0 \\
\hline $10 p-B I O$ & 15133 & 1 & 3380 & 1 \\
\hline $10 p-C S A$ & 12502 & 4 & 67 & 1 \\
\hline $10 p-C G 2$ & 22403 & 3 & 268 & 1 \\
\hline $10 p-L 1$ & 16829 & 7 & 3710 & 1 \\
\hline \multirow{2}{*}{$\begin{array}{r}10 p-S M M \\
10 p-M F F\end{array}$} & 8661 & 4 & 590 & 1 \\
\hline & 1338 & 3 & 169 & 1 \\
\hline $10 p R-B I O R$ & 5013 & 3 & 1077 & 1 \\
\hline $10 p R-C S A R$ & 5407 & 3 & 39 & 1 \\
\hline $10 p R-C G 2 R$ & 5966 & 2 & 141 & 1 \\
\hline$A N D I B I N-A N D I G R Y$ & 825 & 2 & 43 & 0 \\
\hline$A N D I B I N-B I O$ & 3771 & 3 & 1265 & 1 \\
\hline$A N D I B I N-C S A$ & 2535 & 2 & 58 & 1 \\
\hline$A N D I B I N$ - CG2 & 2690 & 2 & 717 & 1 \\
\hline ANDI BIN - L1 & 2613 & 1 & 230 & 1 \\
\hline ANDI BIN - SMM & 2254 & 2 & 557 & 1 \\
\hline ANDI BIN - MFF & 1021 & 3 & 96 & 1 \\
\hline$A N D I B I N-I I D$ & 30 & 1 & 36 & 1 \\
\hline$A N D I G R Y-B I O$ & 447 & 0 & 351 & 0 \\
\hline ANDI GRY - CSA & 455 & 1 & 45 & 0 \\
\hline$A N D I G R Y$ - CG2 & 447 & 1 & 247 & 0 \\
\hline$A N D I G R Y-L 1$ & 423 & 0 & 153 & 0 \\
\hline$A N D I G R Y-S M M$ & 410 & 0 & 169 & 0 \\
\hline$A N D I G R Y-M F F$ & 413 & 2 & 74 & 2 \\
\hline$A N D I G R Y-I I D$ & 21 & 0 & 30 & 0 \\
\hline$B I O-C S A$ & 24719 & 5 & 18663 & 1 \\
\hline$B 1 O-C G 2$ & 23638 & 2 & 6572 & 1 \\
\hline$B I O-L 1$ & 23100 & 2 & 5679 & 1 \\
\hline$B I O-S M M$ & 15269 & 4 & 6152 & 1 \\
\hline$B I O-M F F$ & 1616 & 3 & 748 & 1 \\
\hline$B I O R$ - CSA R & 14386 & 1 & 4758 & 1 \\
\hline$B I O R-C G 2 R$ & 10015 & 2 & 2978 & 1 \\
\hline$B I O-I I D$ & 36 & 1 & 39 & 1 \\
\hline$C S A-C G 2$ & 22034 & 1 & 4443 & 1 \\
\hline$C S A-I I D$ & 30 & 1 & 46 & 1 \\
\hline$C S A-\angle 1$ & 19946 & 4 & 2776 & 1 \\
\hline CSA - SMM & 14426 & 3 & 896 & 1 \\
\hline$C S A-M F F$ & 1388 & 3 & 165 & 1 \\
\hline$C S A R-C G 2 R$ & 9281 & 3 & 135 & 1 \\
\hline$C G 2-I I D$ & 29 & 1 & 38 & 1 \\
\hline$C G 2-L 1$ & 25511 & 3 & 5504 & 1 \\
\hline CG2 - SMM & 19192 & 2 & 2237 & 1 \\
\hline$C G 2-M F F$ & 1273 & 3 & 504 & 1 \\
\hline$I I D-L 1$ & 30 & 1 & 39 & 1 \\
\hline IID - SMM & 30 & 1 & 39 & 1 \\
\hline$I I D-M F F$ & 30 & 1 & 33 & 1 \\
\hline$L 1-S M M$ & 18846 & 3 & 4406 & 1 \\
\hline$L 1-M F F$ & 1516 & 3 & 224 & 1 \\
\hline$L 1 R-10 p R$ & 5042 & 2 & 1282 & 1 \\
\hline$\angle 1 R-B I O R$ & 9172 & 2 & 2597 & 1 \\
\hline$L 1 R-C S A R$ & 7047 & 1 & 416 & 1 \\
\hline$L 1 R-C G 2 R$ & 12847 & 1 & 3455 & 1 \\
\hline$M F F-S M M$ & 1305 & 3 & 183 & 1 \\
\hline
\end{tabular}




\section{A.5 Neurotech Cross-Examined Genuine/Imposter Distributions}
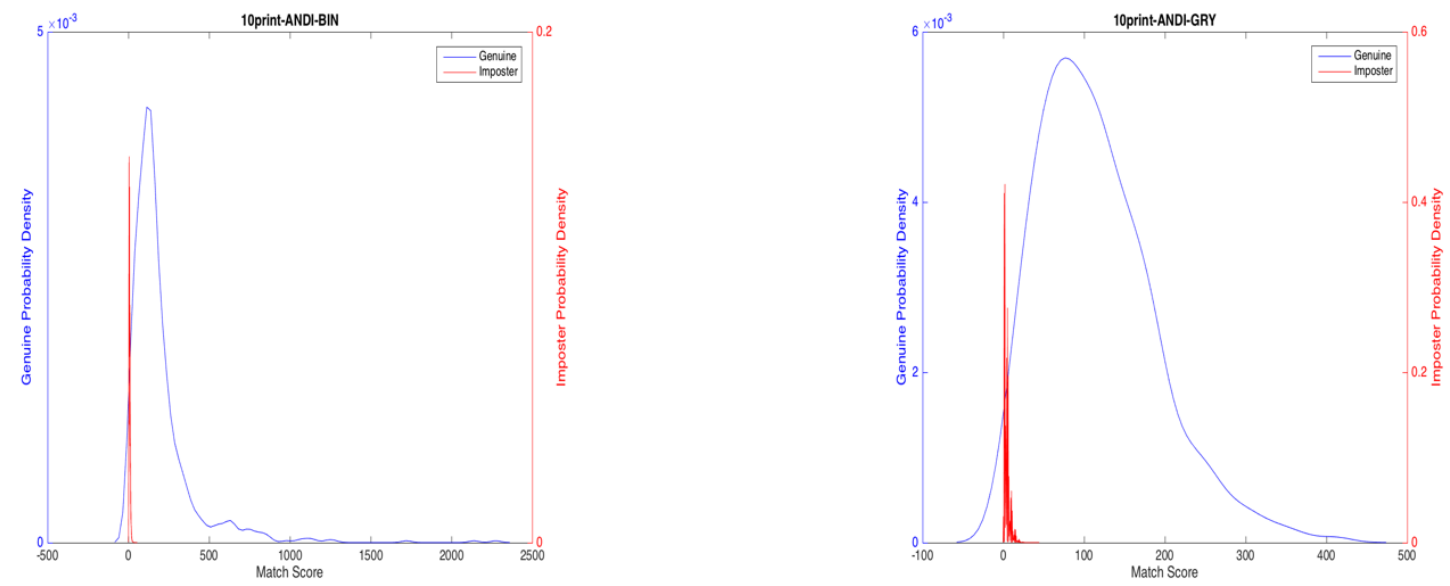

Figure A.5.1 (Left) Neurotech 10print - ANDI Binary (Right) Neurotech 10print - ANDI Greyscale
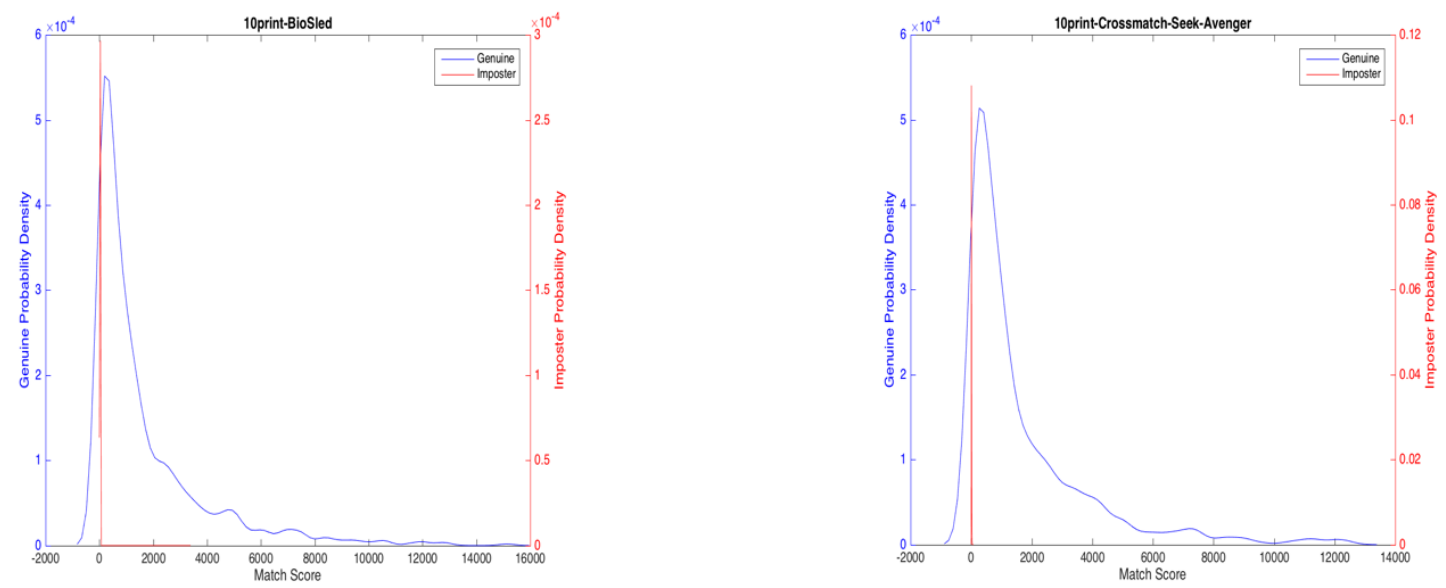

Figure A.5.2 (Left) Neurotech 10print - BioSled (Right) Neurotech 10print - Crossmatch Seek Avenger
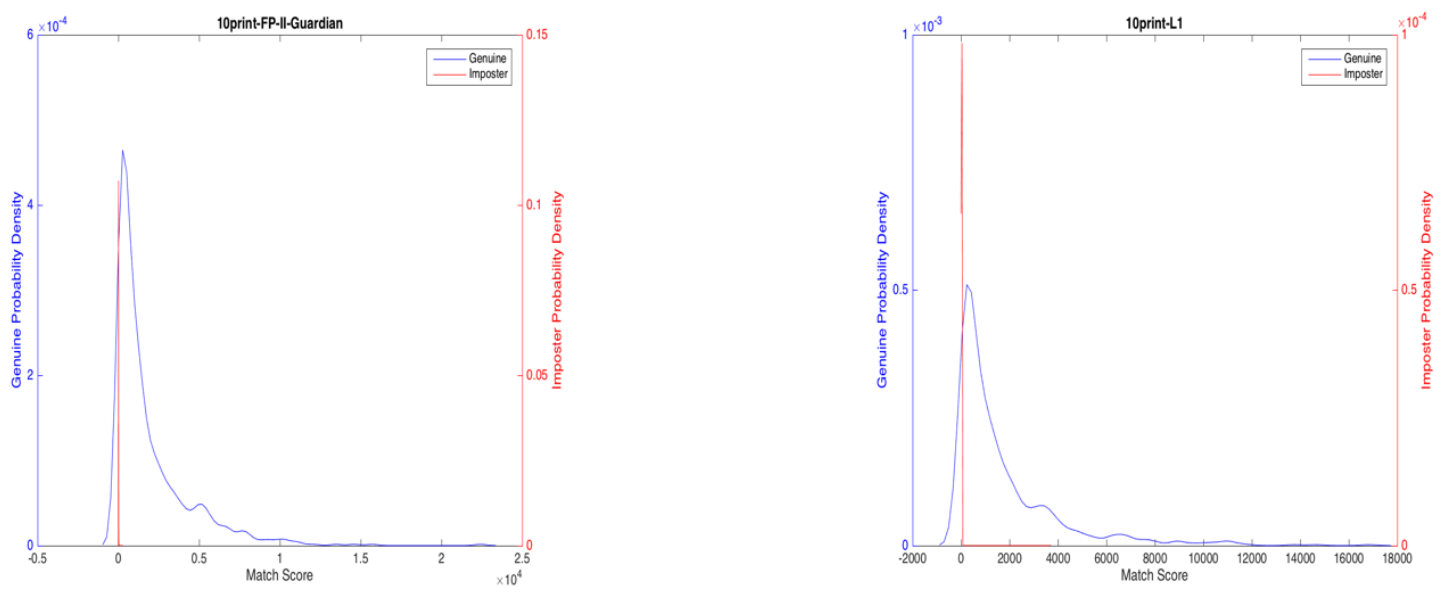
Figure A.5.3 (Left) Neurotech 10print - FP II Guardian (Right) Neurotech 10print - L1
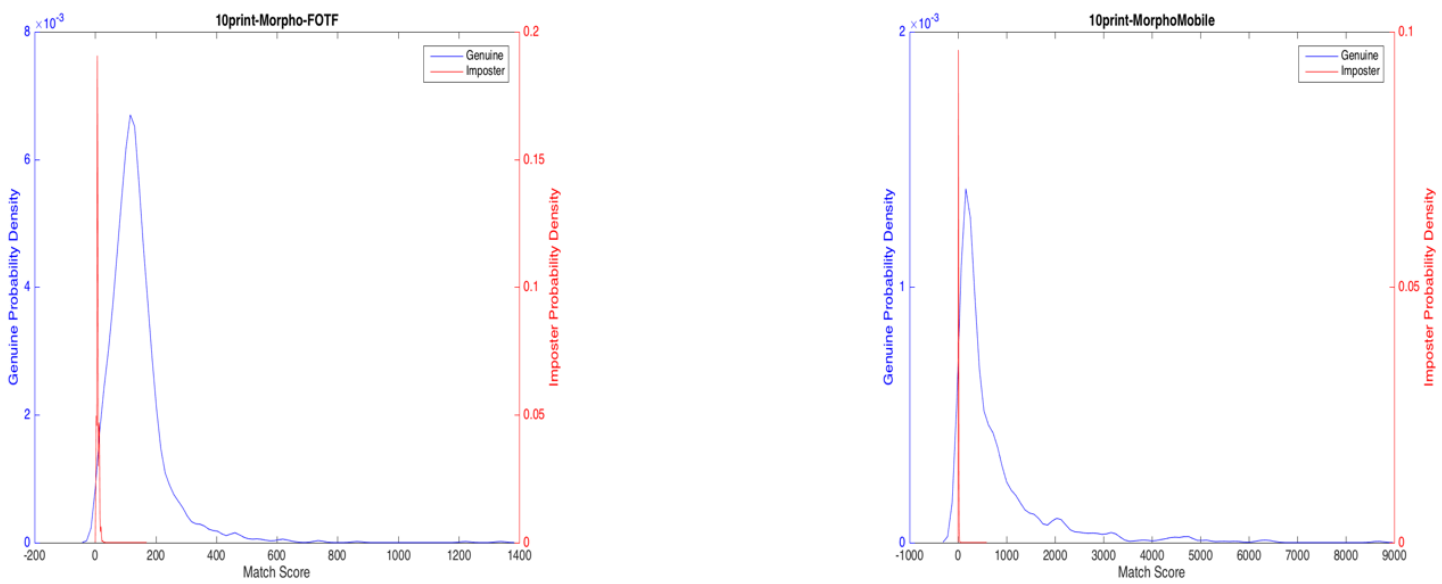

Figure A.5.4 (Left) Neurotech 10print - Morpho FOTF (Right) Neurotech 10print - MorphoMobile
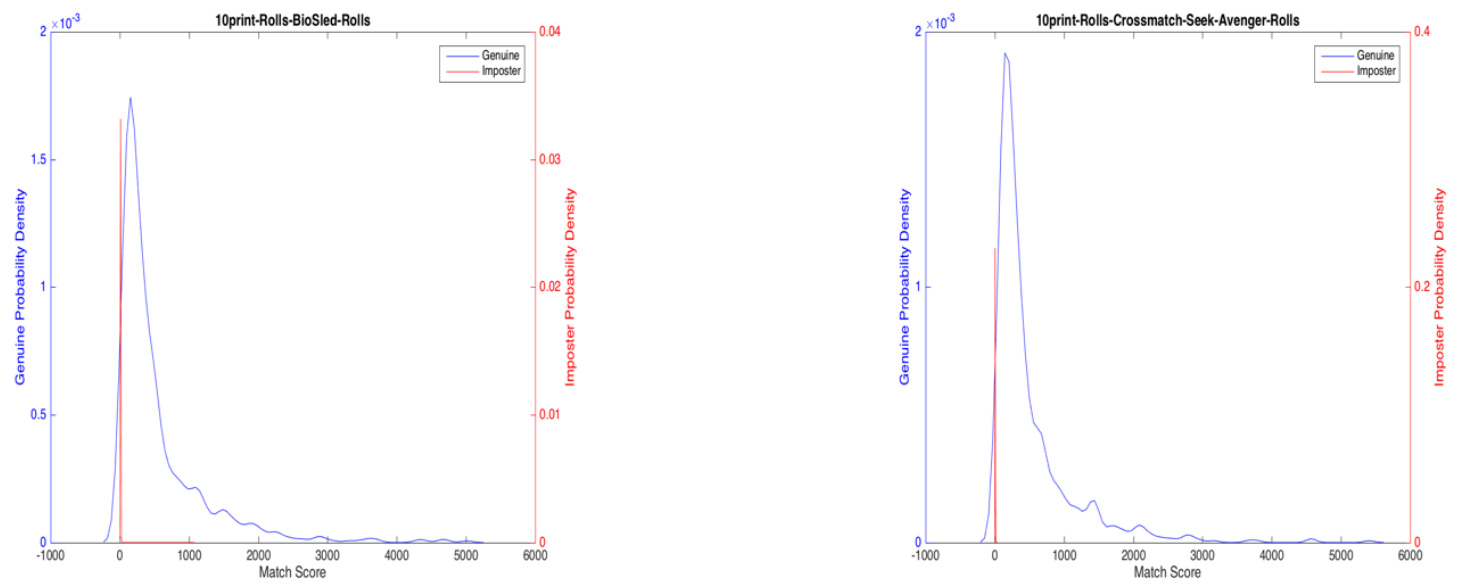

Figure A.5.5 (Left) Neurotech 10print Rolls - BioSled Rolls (Right) Neurotech 10print Rolls - Crossmatch Seek Avenger Rolls 

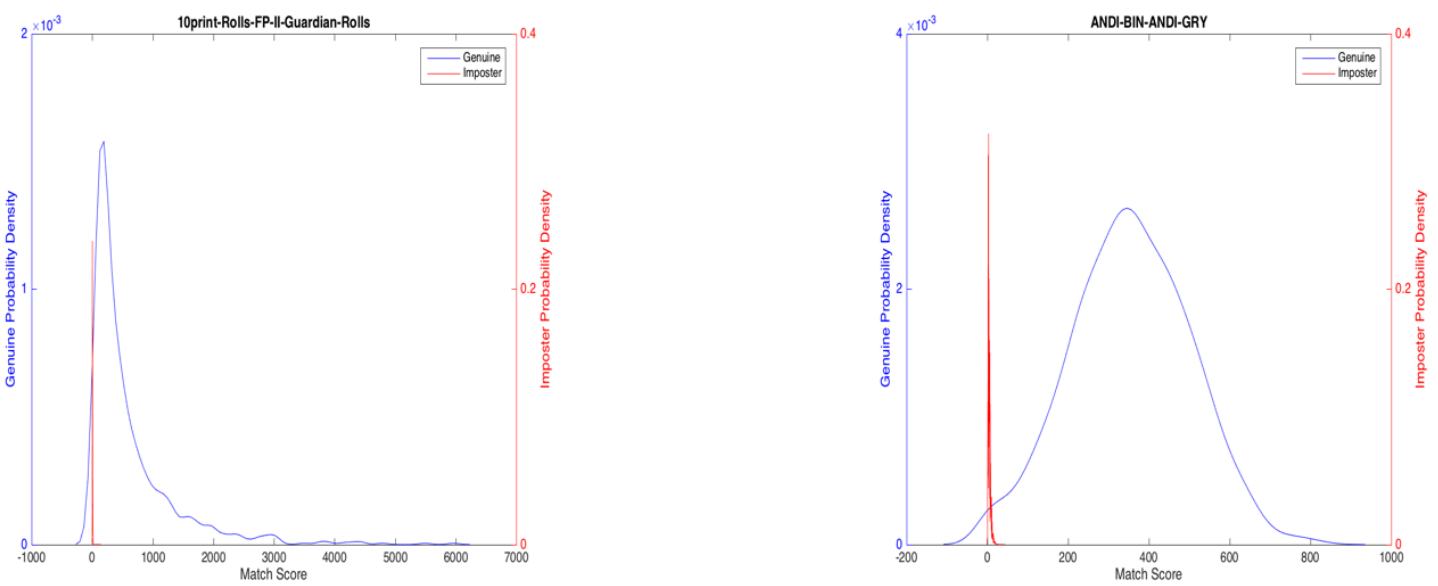

Figure A.5.6 (Left) Neurotech 10print Rolls - FP II Guardian Rolls (Right) Neurotech ANDI Binary - ANDI Greyscale
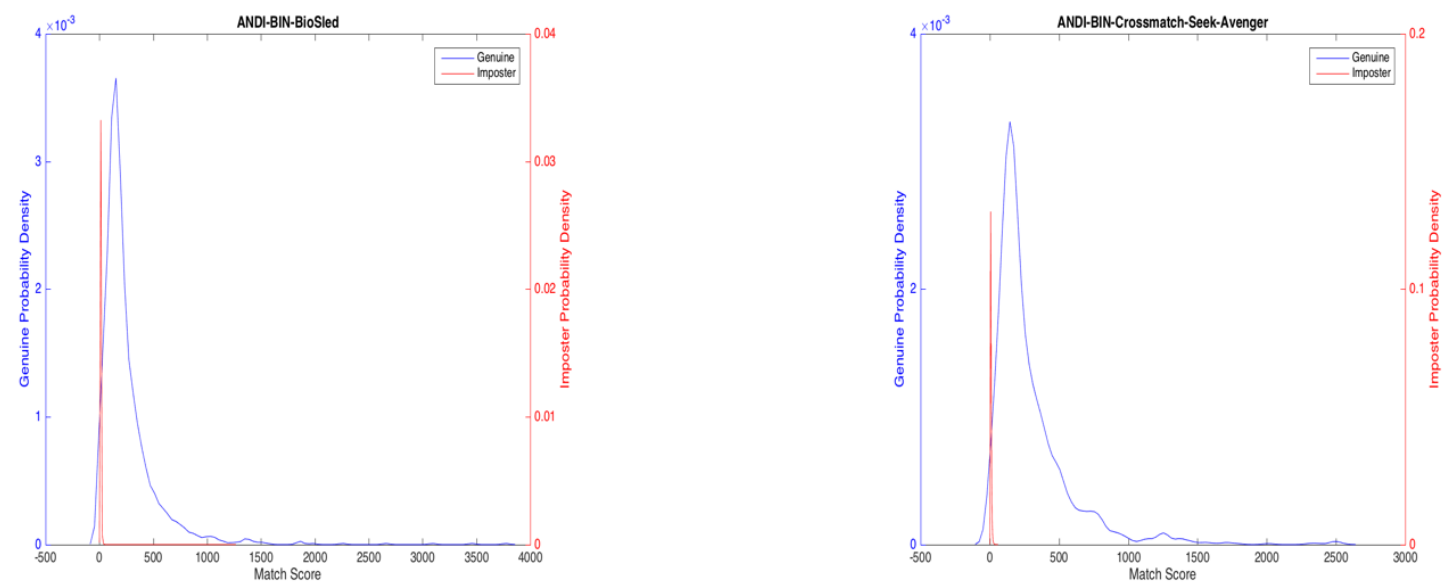

Figure A.5.7 (Left) Neurotech ANDI Binary - BioSled (Right) Neurotech ANDI Binary - Crossmatch Seek Avenger
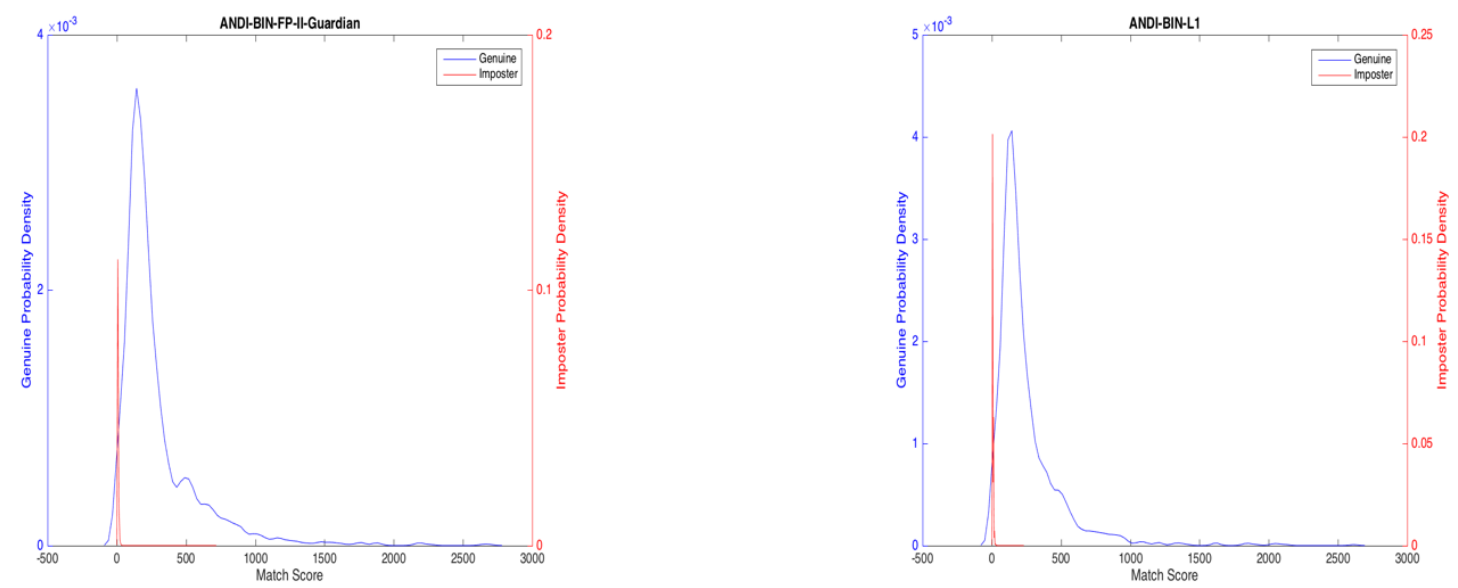

Figure A.5.8 (Left) Neurotech ANDI Binary - FP II Guardian (Right) Neurotech ANDI Binary - L1 

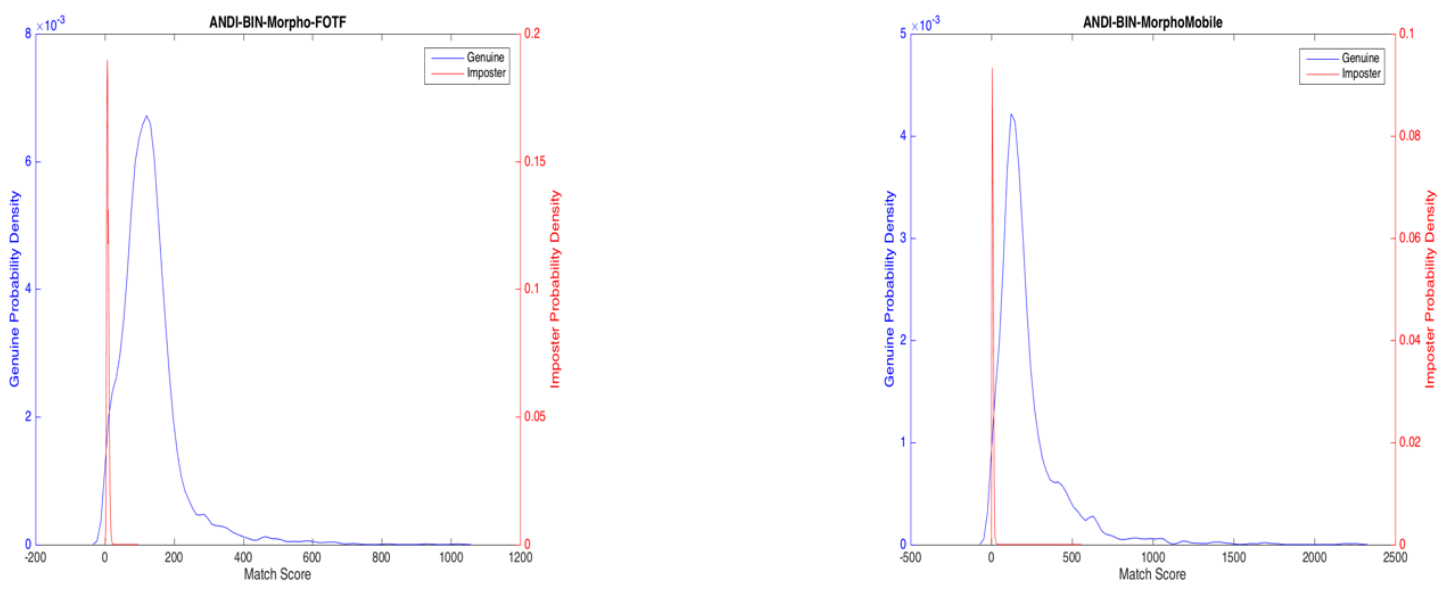

Figure A.5.9 (Left) Neurotech ANDI Binary - Morpho FOTF (Right) Neurotech ANDI Binary - MorphoMobile
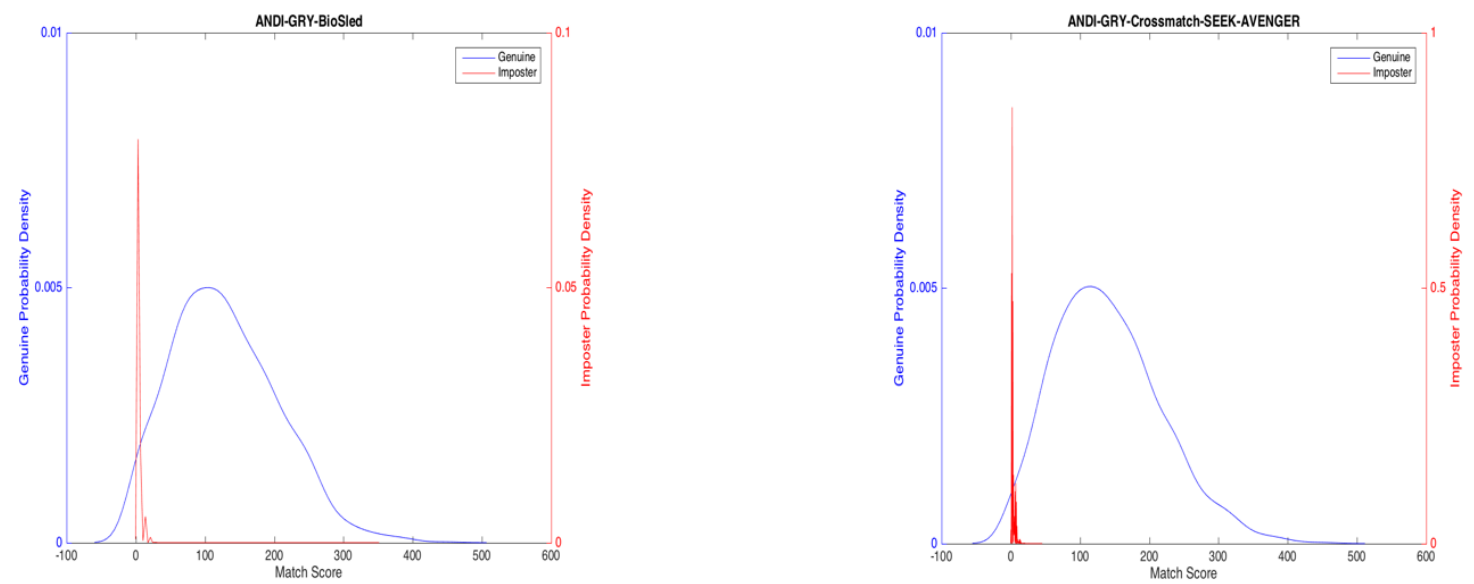

Figure A.5.10 (Left) Neurotech ANDI Greyscale - BioSled (Right) Neurotech ANDI Greyscale - Crossmatch Seek Avenger
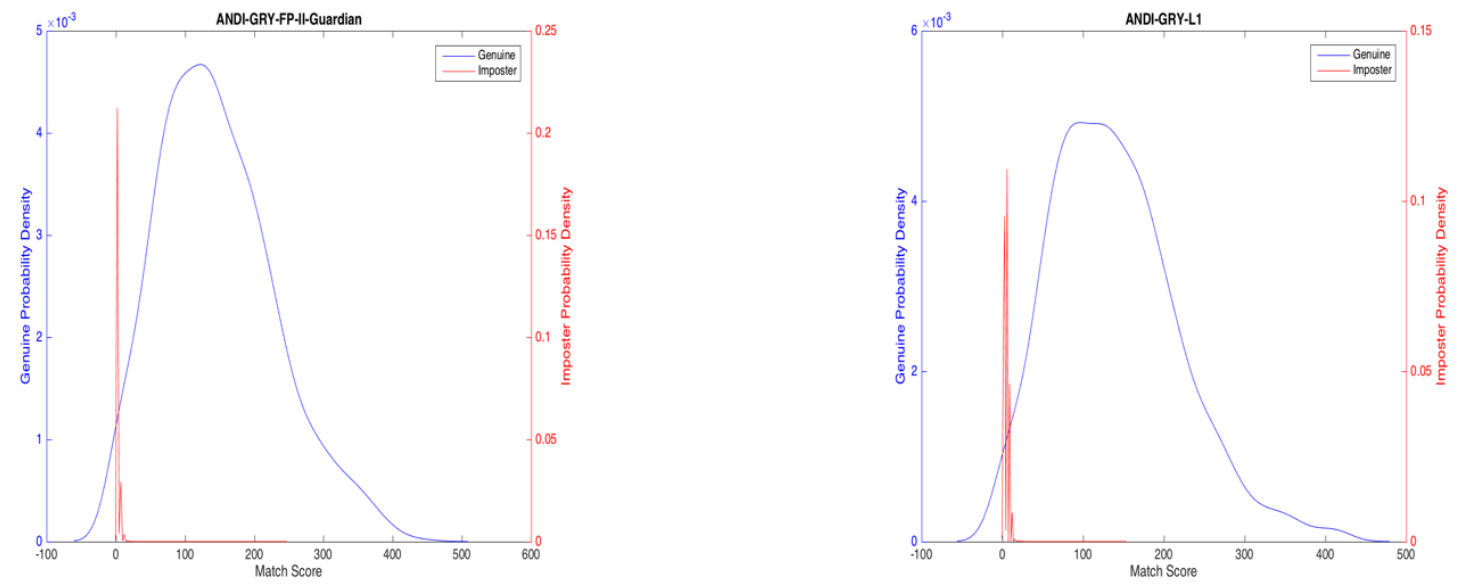

Figure A.5.11 (Left) Neurotech ANDI Greyscale - FP II Guardian (Right) Neurotech ANDI Greyscale - L1 

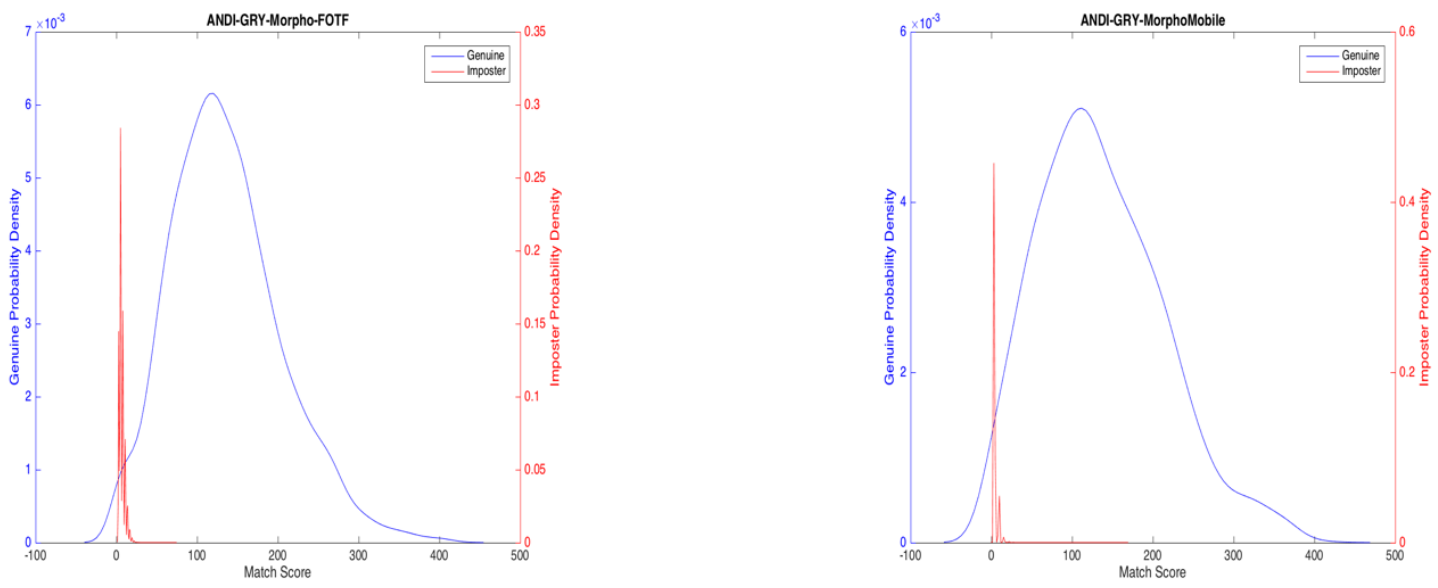

Figure A.5.12 (Left) Neurotech ANDI Greyscale - Morpho FOTF (Right) Neurotech ANDI Greyscale MorphoMobile
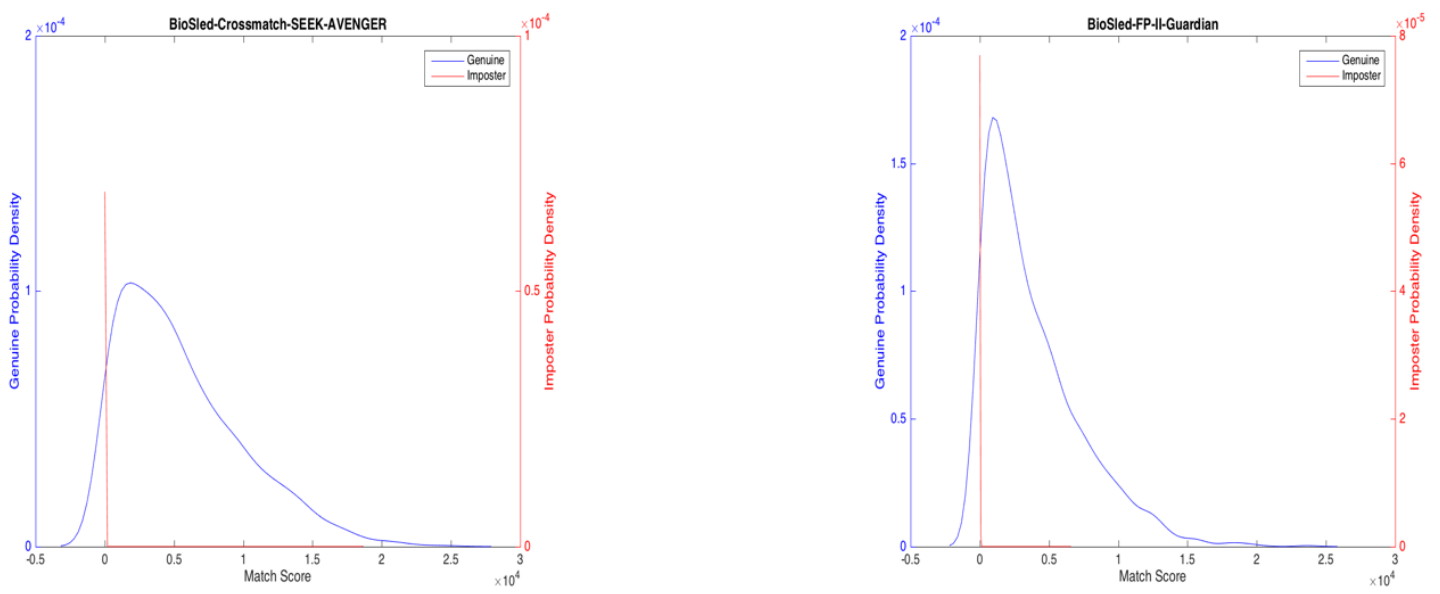

Figure A.5.13 (Left) Neurotech BioSled - Crossmatch Seek Avenger (Right) Neurotech BioSled - FP II Guardian
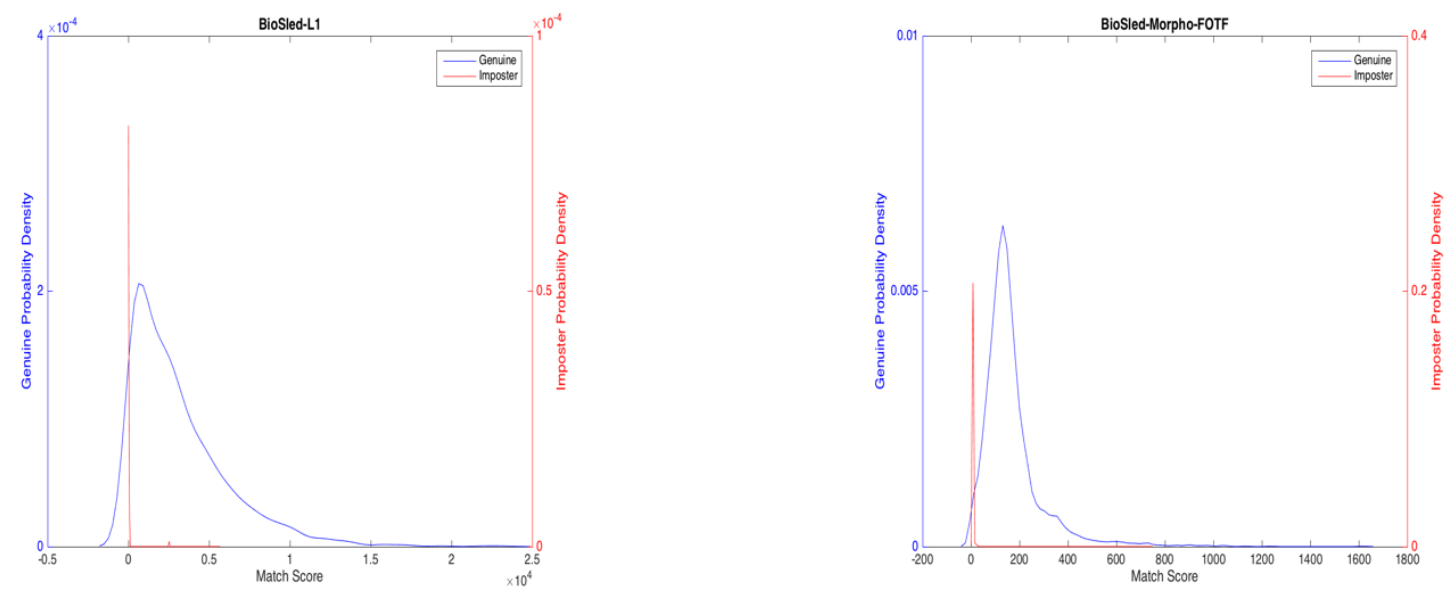

Figure A.5.14 (Left) Neurotech BioSled - L1 (Right) Neurotech BioSled - Morpho FOTF 

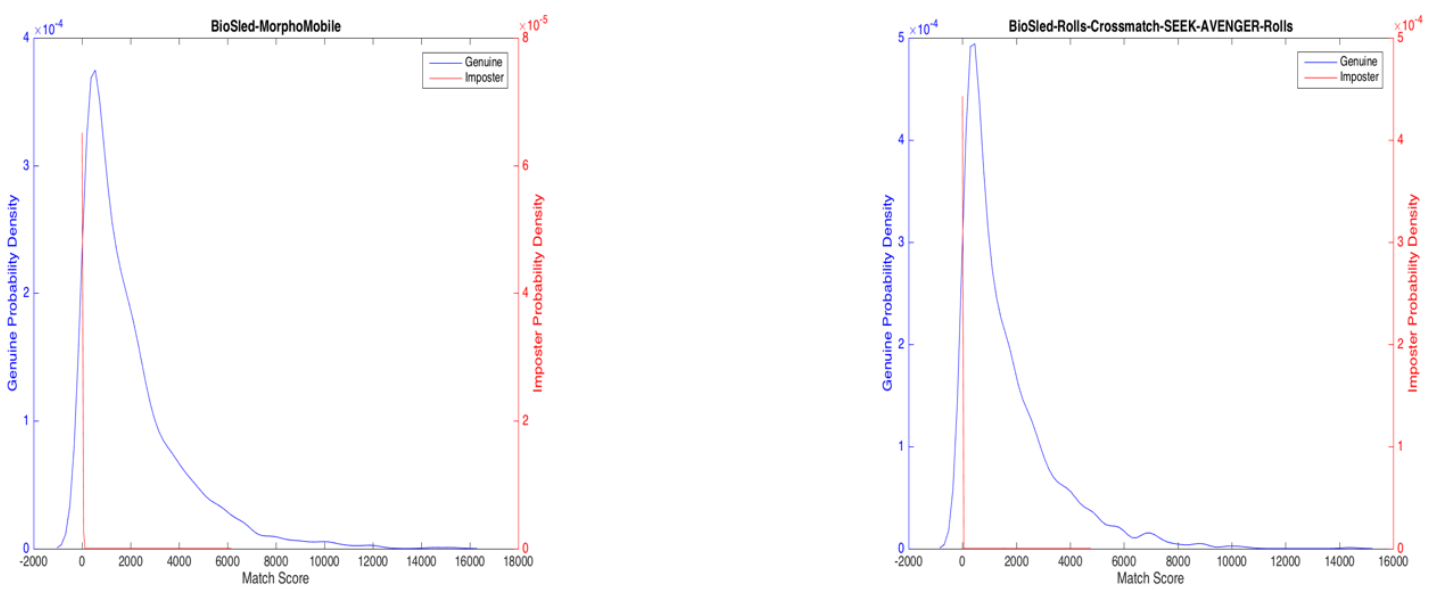

Figure A.5.15 (Left) Neurotech BioSled - MorphoMobile (Right) Neurotech BioSled Rolls - Crossmatch Seek Avenger Rolls
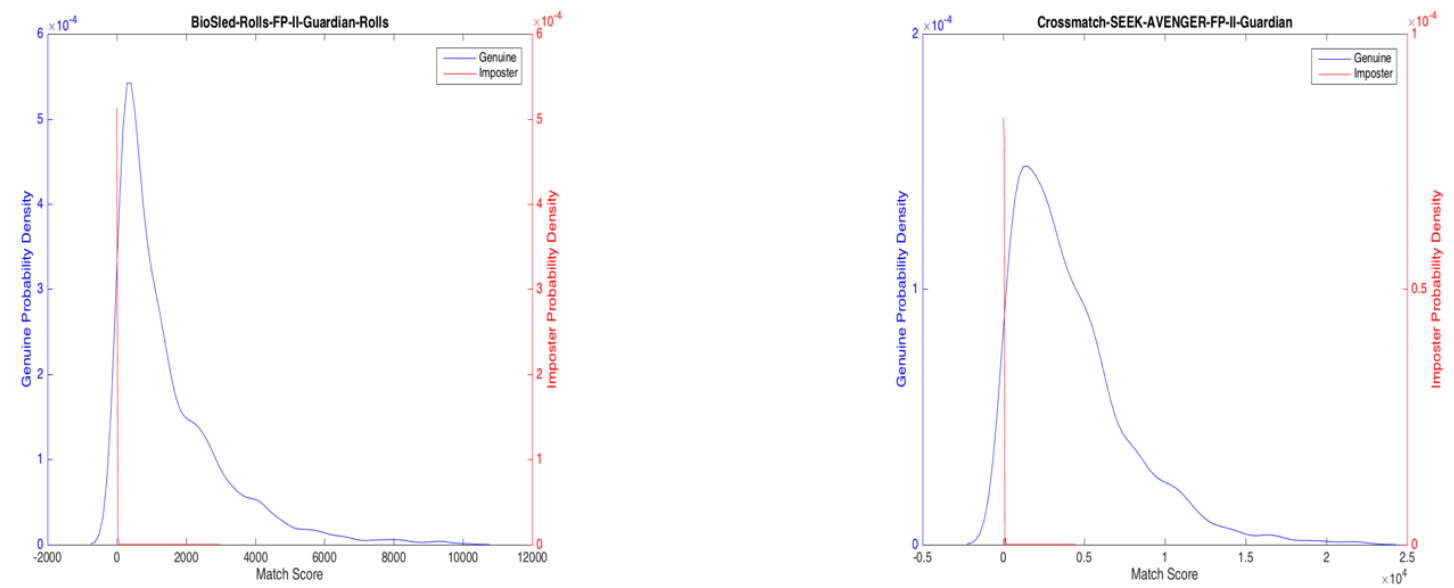

Figure A.5.16 (Left) Neurotech BioSled Rolls- FP II Guardian Rolls (Right) Neurotech Crossmatch Seek AvengerFP II Guardian 

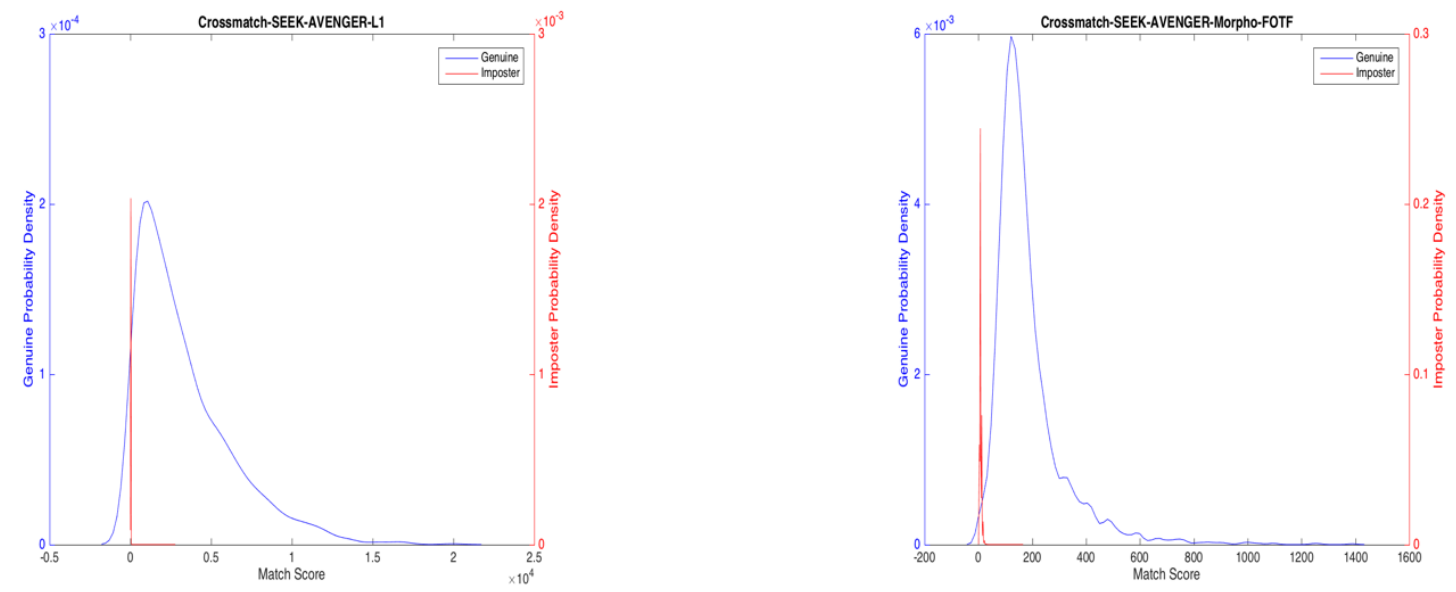

Figure A.5.17 (Left) Neurotech Crossmatch Seek Avenger - L1 (Right) Neurotech Crossmatch Seek Avenger Morpho FOTF
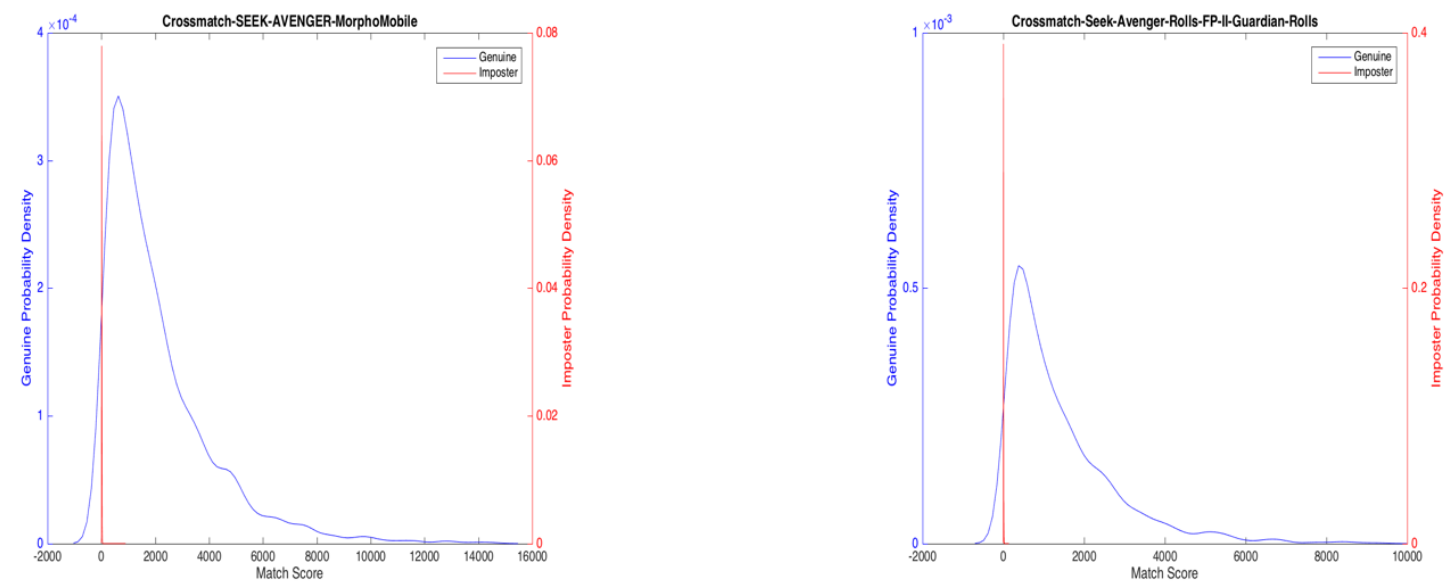

Figure A.5.18 (Left) Neurotech Crossmatch Seek Avenger - MorphoMobile (Right) Neurotech Crossmatch Seek Avenger Rolls - FP II Guardian Rolls
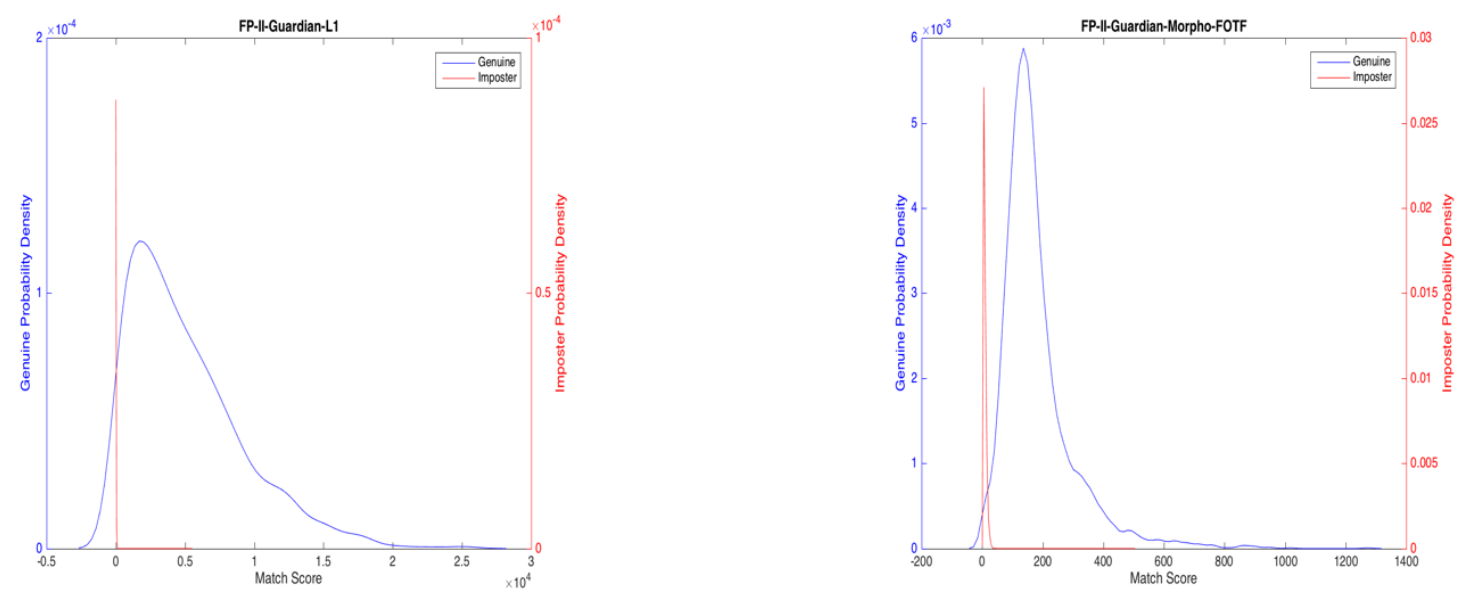

Figure A.5.19 (Left) Neurotech FP II Guardian - L1 (Right) Neurotech FP II Guardian - Morpho FOTF 

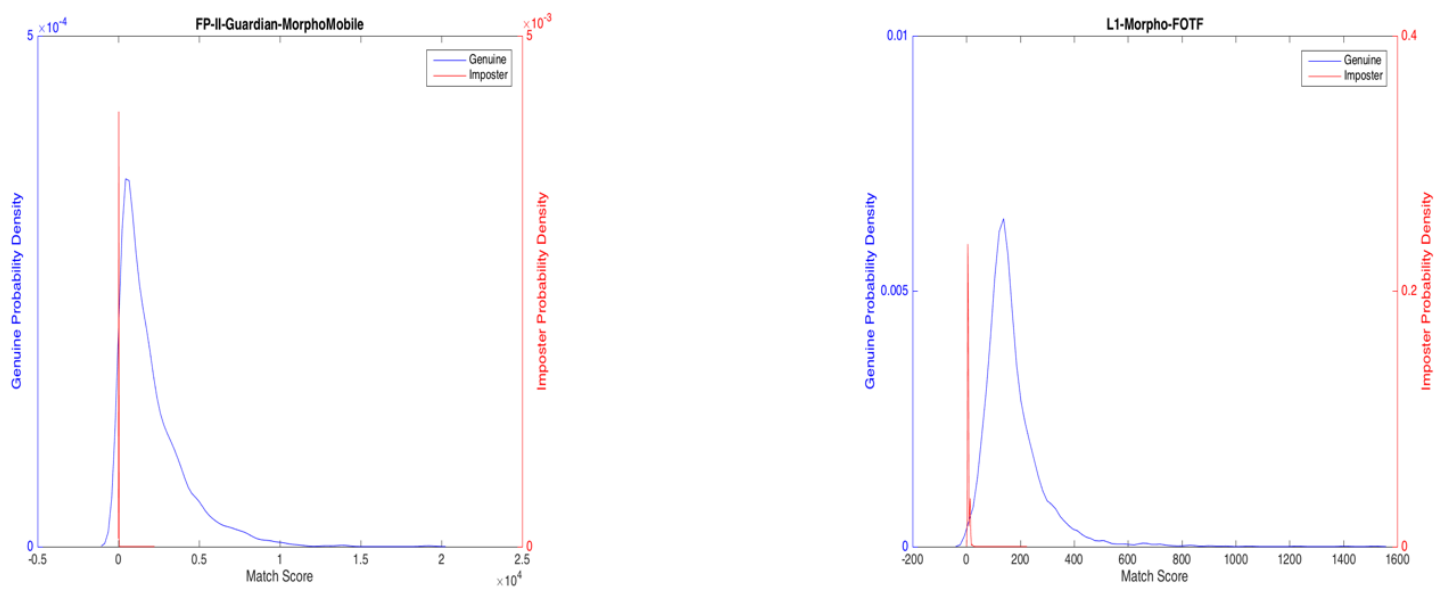

Figure A.5.20 (Left) Neurotech FP II Guardian - MorphoMobile (Right) Neurotech L1 - Morpho FOTF
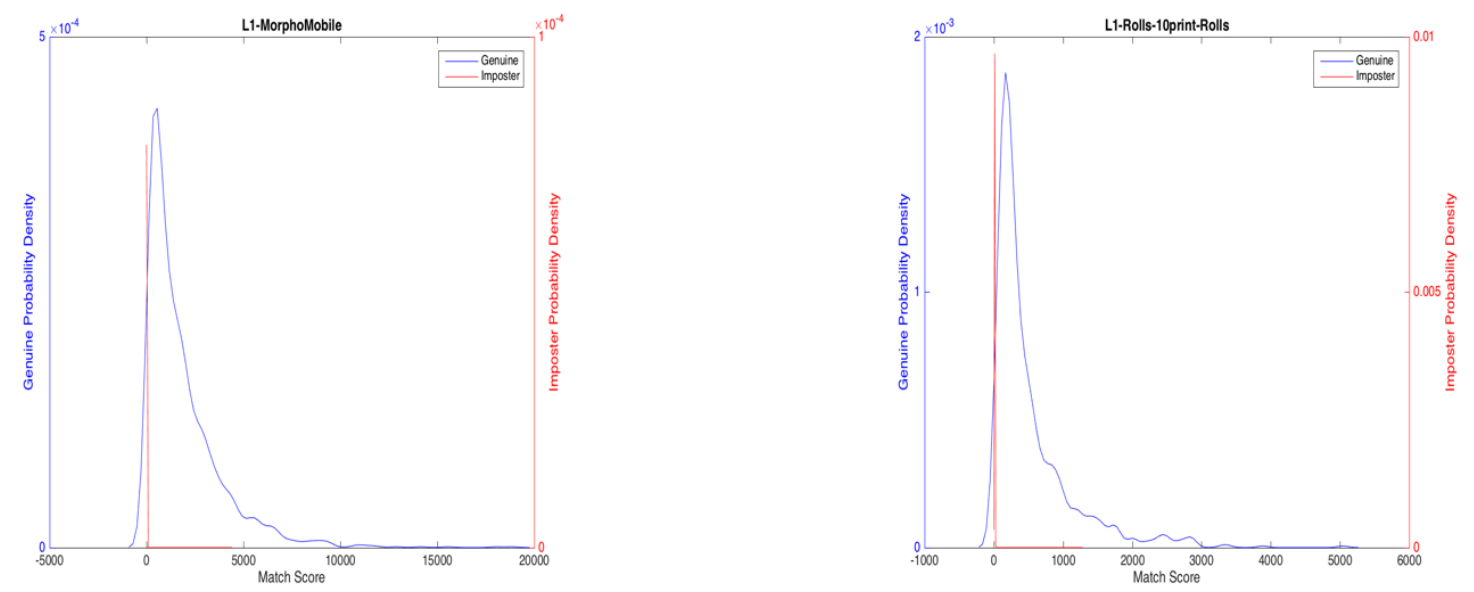

Figure A.5.21 (Left) Neurotech L1 - MorphoMobile (Right) Neurotech L1 Rolls - 10print Rolls
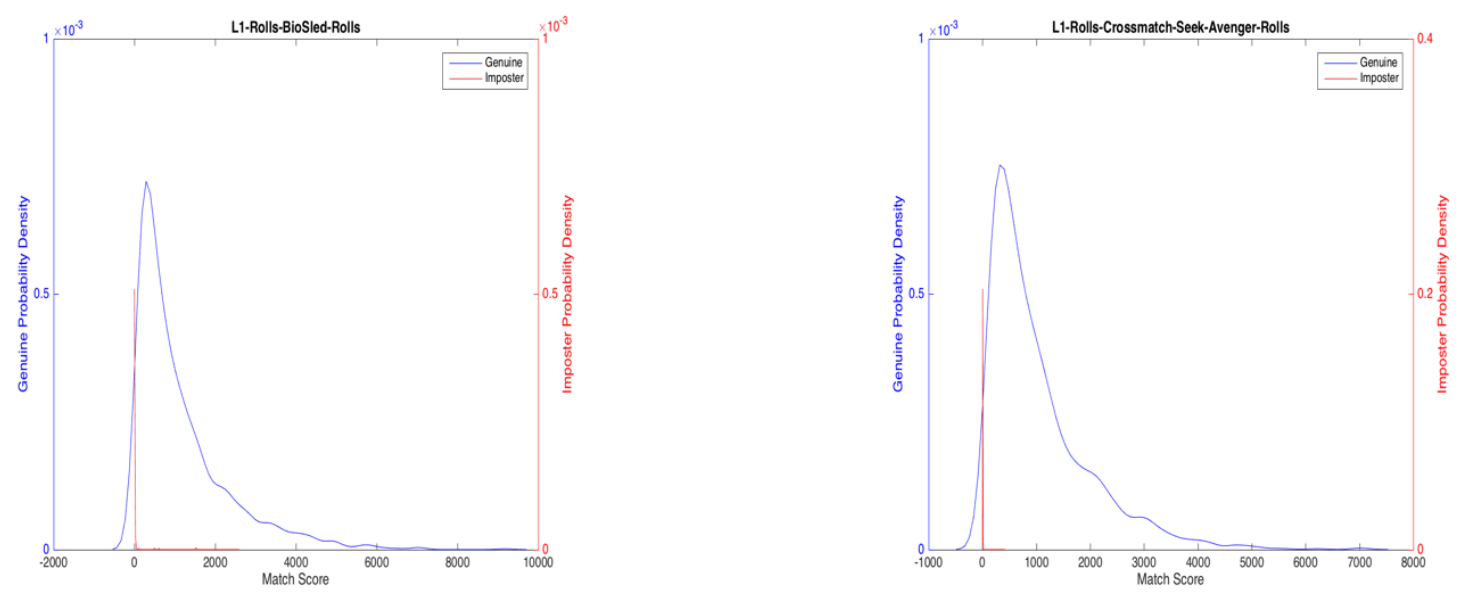

Figure A.5.22 (Left) Neurotech L1 Rolls - BioSled Rolls (Right) Neurotech L1 Rolls - Crossmatch Seek Avenger Rolls 

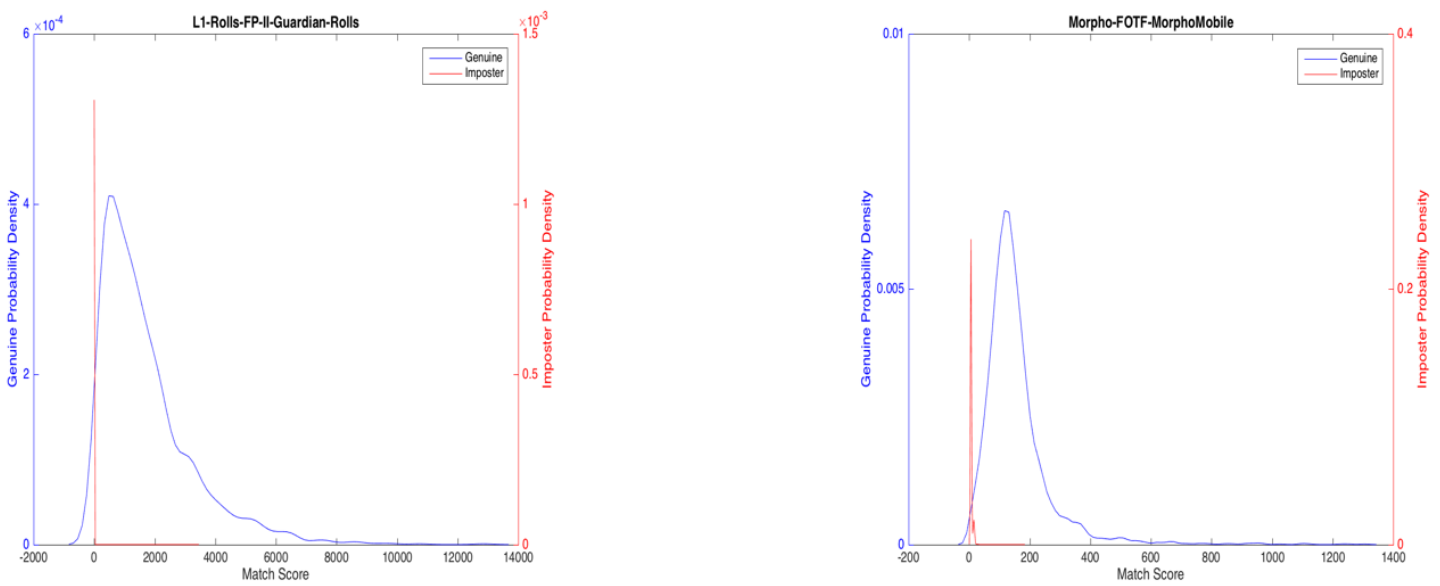

Figure A.5.23 (Left) NeurotechL1 Rolls - FP II Guardian Rolls (Right) Neurotech Morpho FOTF - MorphoMobile 
Table A.12 SourceAFIS Cross-Examined Maximum and Minimum Score Values

\begin{tabular}{|c|c|c|c|c|}
\hline SourceAFIS & Max Genuine & Min Genuine & Max Imposter & Min Imposter \\
\hline \multirow{2}{*}{$\begin{array}{l}10 p-A N D I B I N \\
10 p-A N D I G R Y\end{array}$} & 146.27 & 9.67 & 36.36 & 1.15 \\
\hline & 115.10 & 8.67 & 35.21 & 1.07 \\
\hline $10 p-B I O$ & 211.68 & 10.67 & 120.76 & 1.17 \\
\hline $10 p-C S A$ & 225.33 & 11.04 & 34.61 & 3.05 \\
\hline $10 p-C G 2$ & 231.38 & 9.41 & 62.39 & 1.08 \\
\hline $10 p-L 1$ & 220.90 & 9.87 & 167.78 & 1.11 \\
\hline $10 p-M F F$ & 170.37 & 9.91 & 53.03 & 1.09 \\
\hline $10 p-S M M$ & 159.79 & 9.27 & 64.65 & 1.15 \\
\hline $10 p R-B I O R$ & 270.40 & 9.61 & 171.60 & 1.02 \\
\hline $10 p R-C S A R$ & 303.44 & 8.89 & 31.84 & 1.02 \\
\hline $10 p R-C G 2 R$ & 293.25 & 10.30 & 43.02 & 1.02 \\
\hline $10 p R-L 1 R$ & 287.39 & 0.00 & 129.25 & 0.00 \\
\hline $10 p-I I D$ & 18.53 & 8.89 & 27.94 & 3.57 \\
\hline$A N D I B I N-A N D I G R Y$ & 240.88 & 9.91 & 39.50 & 1.10 \\
\hline$A N D I B I N-B I O$ & 154.24 & 5.62 & 106.18 & 1.10 \\
\hline ANDI BIN - CSA & 171.76 & 10.15 & 37.91 & 1.15 \\
\hline$A N D I B I N-C G 2$ & 150.54 & 6.38 & 94.26 & 1.13 \\
\hline ANDI BIN - L1 & 173.52 & 9.67 & 72.75 & 1.09 \\
\hline $\begin{array}{l}A N D I B I N-S M M \\
A N D I B I N-M F F\end{array}$ & 134.80 & 8.75 & 55.83 & 1.11 \\
\hline ANDI BIN - MFF & 189.75 & 8.95 & 40.86 & 1.03 \\
\hline$A N D I B I N-I I D$ & 26.25 & 9.05 & 31.50 & 3.44 \\
\hline$A N D I G R Y-B I O$ & 129.40 & 8.41 & 71.34 & 1.10 \\
\hline ANDI GRY - CSA & 149.24 & 8.14 & 36.20 & 1.07 \\
\hline$A N D I G R Y$ - CG2 & 150.66 & 9.05 & 56.88 & 1.02 \\
\hline$A N D I G R Y-L 1$ & 152.41 & 6.91 & 68.78 & 1.07 \\
\hline$A N D I G R Y-S M M$ & 124.64 & 8.39 & 42.48 & 1.13 \\
\hline$A N D I G R Y-M F F$ & 183.14 & 6.75 & 43.43 & 1.02 \\
\hline$A N D I G R Y-I I D$ & 20.43 & 8.91 & 30.22 & 1.06 \\
\hline$B I O-C S A$ & 267.14 & 9.94 & 128.17 & 1.26 \\
\hline$B I O-C G 2$ & 270.79 & 9.70 & 189.73 & 1.20 \\
\hline$B I O-L 1$ & 242.63 & 10.50 & 133.19 & 1.12 \\
\hline$B I O-S M M$ & 194.05 & 10.56 & 97.40 & 1.17 \\
\hline$B I O-M F F$ & 202.00 & 9.27 & 109.29 & 1.06 \\
\hline$B I O R$ - CSA R & 348.05 & 8.90 & 275.02 & 1.02 \\
\hline$B I O R-C G 2 R$ & 333.19 & 9.90 & 247.35 & 1.02 \\
\hline$B I O R-L 1 R$ & 330.59 & 0.00 & 231.09 & 0.00 \\
\hline$B I O-I I D$ & 23.30 & 8.39 & 33.73 & 1.24 \\
\hline$C S A-C G 2$ & 288.52 & 11.27 & 119.39 & 1.17 \\
\hline$C S A-L 1$ & 259.76 & 11.95 & 132.76 & 2.63 \\
\hline$C S A-S M M$ & 193.06 & 11.02 & 92.33 & 2.41 \\
\hline$C S A-M F F$ & 233.11 & 9.80 & 63.35 & 1.10 \\
\hline CSA R - CG2 R & 348.13 & 9.48 & 85.60 & 1.02 \\
\hline$C S A R-L 1 R$ & 320.97 & 0.00 & 176.75 & 0.00 \\
\hline$C S A-I I D$ & 20.85 & 7.65 & 29.83 & 1.94 \\
\hline$C G 2-L 1$ & 237.47 & 10.68 & 148.12 & 3.37 \\
\hline$C G 2$ - SMM & 183.25 & 9.81 & 136.43 & 1.23 \\
\hline$C G 2-M F F$ & 200.67 & 9.01 & 77.90 & 1.10 \\
\hline$C G 2 R-L 1 R$ & 325.06 & 0.00 & 214.50 & 0.00 \\
\hline$C G 2-I I D$ & 22.40 & 4.50 & 29.22 & 1.08 \\
\hline L1 - SMM & 194.19 & 10.46 & 111.26 & 1.14 \\
\hline$L 1-M F F$ & 182.92 & 7.64 & 79.59 & 1.09 \\
\hline$M F F-S M M$ & 136.34 & 8.34 & 56.30 & 1.06 \\
\hline$I I D-L 1$ & 21.33 & 9.07 & 35.39 & 1.11 \\
\hline IID - SMM & 19.90 & 7.89 & 29.47 & 1.09 \\
\hline IID - MFF & 23.68 & 7.88 & 31.36 & 1.04 \\
\hline
\end{tabular}




\section{A.6 SourceAFIS Cross-Examined Genuine/Imposter Score}

\section{Distributions}
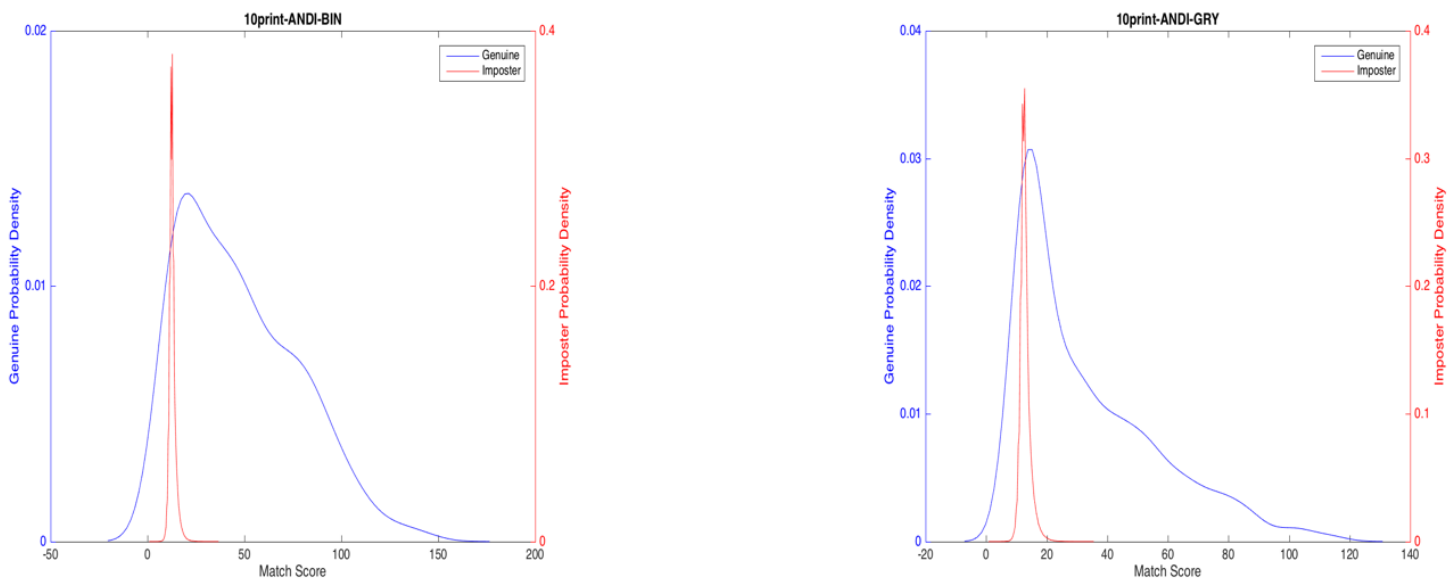

Figure A.6.1 (Left) SourceAFIS 10print - ANDI Binary (Right) SourceAFIS 10print - ANDI Greyscale
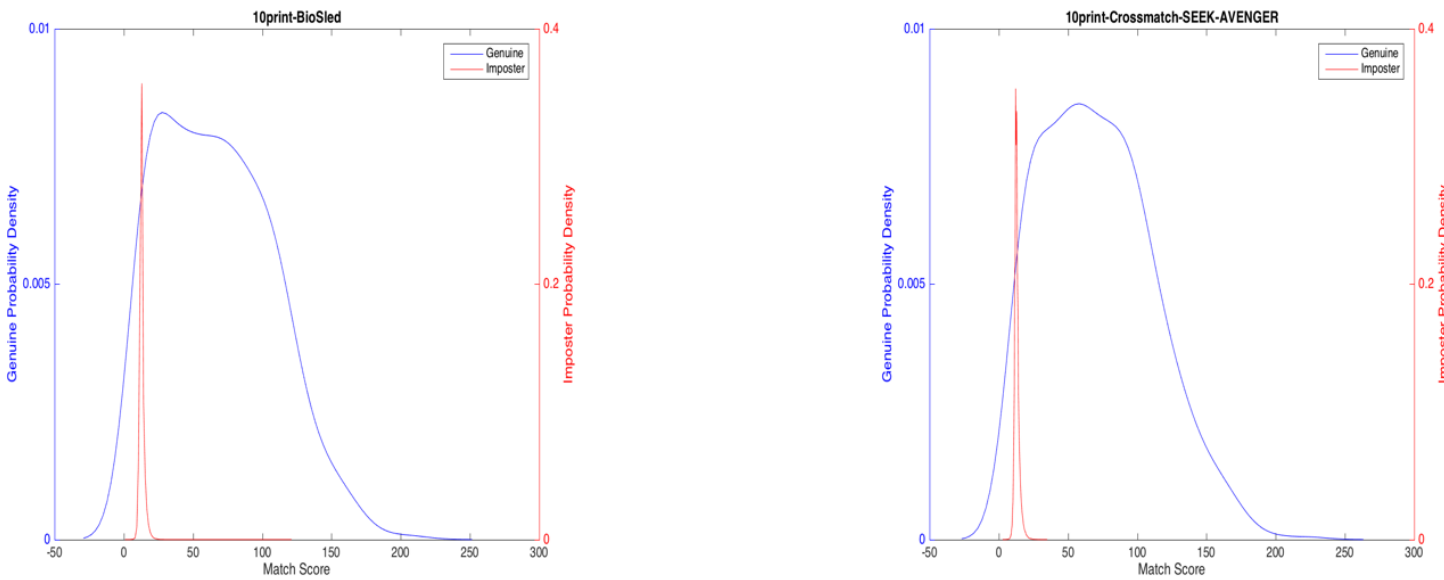

Figure A.6.2 (Left) SourceAFIS 10print - BioSled (Right) SourceAFIS 10print - Crossmatch Seek Avenger 

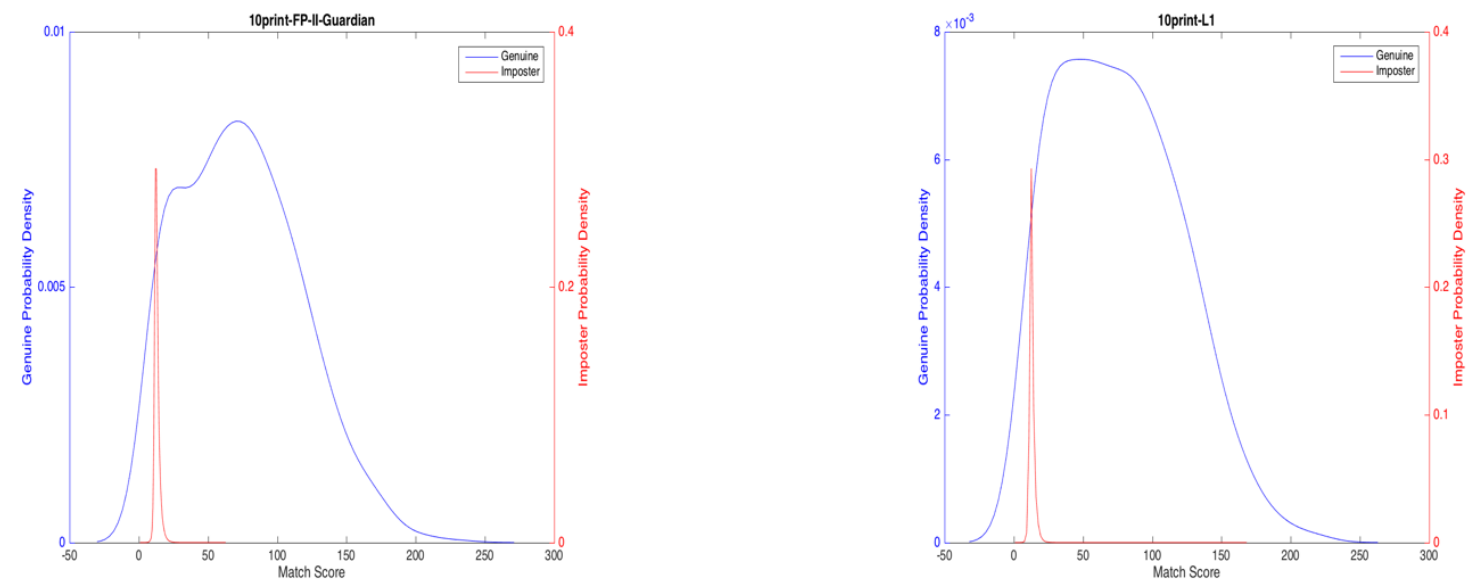

Figure A.6.3 (Left) SourceAFIS 10print - FP II Guardian (Right) SourceAFIS 10print - L1
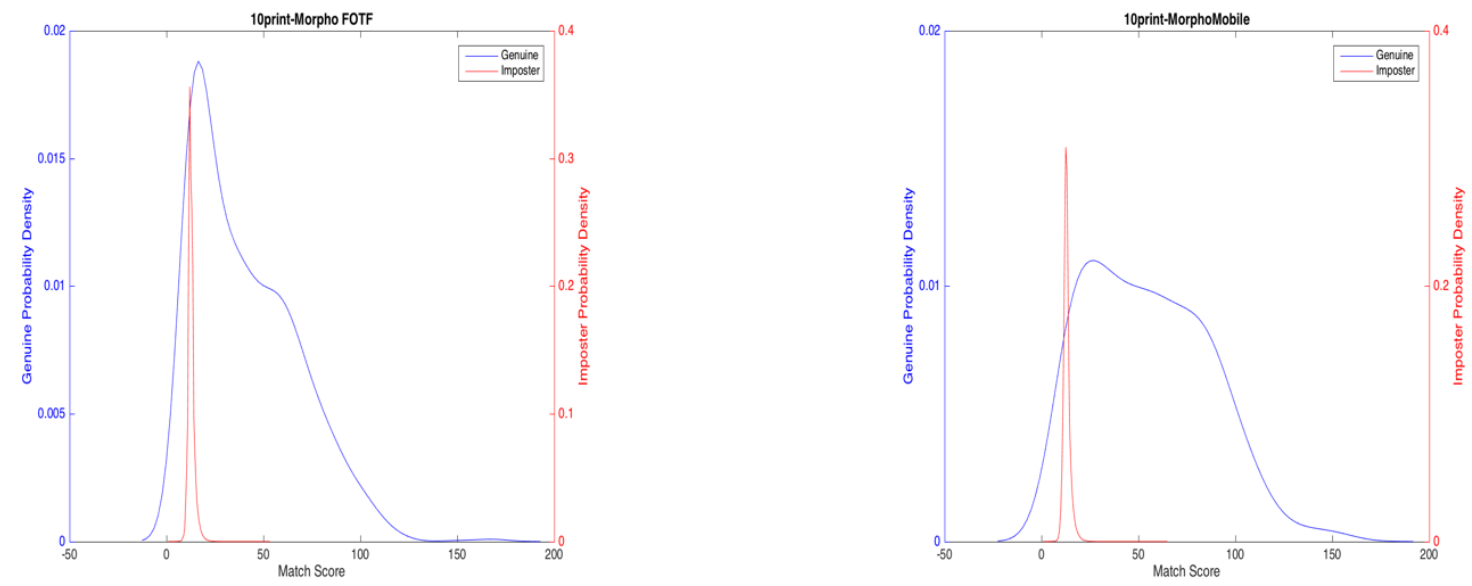

Figure A.6.4 (Left) SourceAFIS 10print - Morpho FOTF (Right) SourceAFIS 10print - MorphoMobile
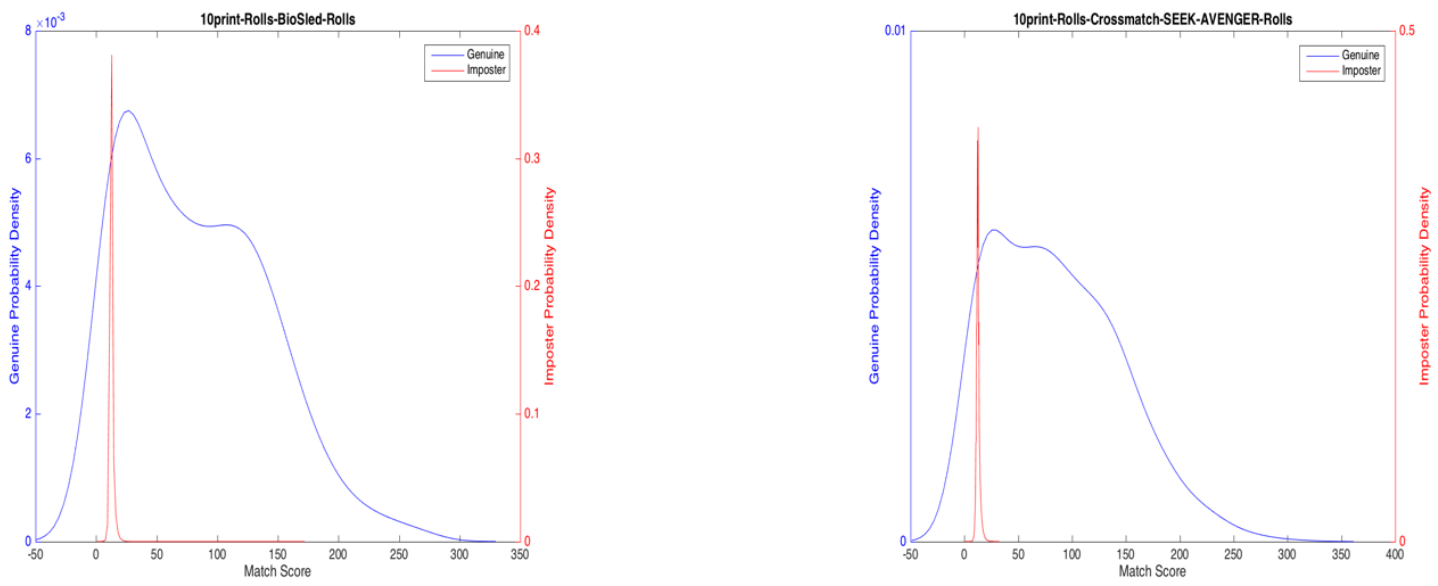

Figure A.6.5 (Left) SourceAFIS 10print Rolls - BioSled Rolls (Right) SourceAFIS 10print Rolls - Crossmatch Seek Avenger Rolls 

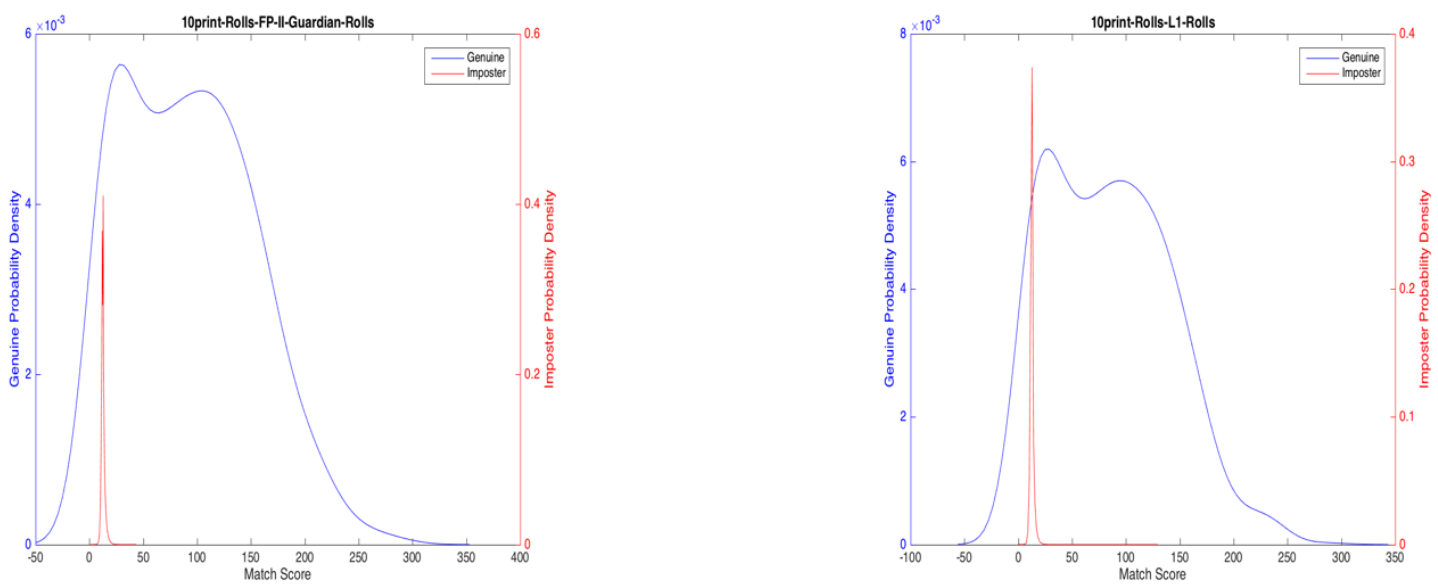

Figure A.6.6 (Left) SourceAFIS 10print Rolls - FP II Guardian Rolls (Right) SourceAFIS 10print Rolls - L1 Rolls
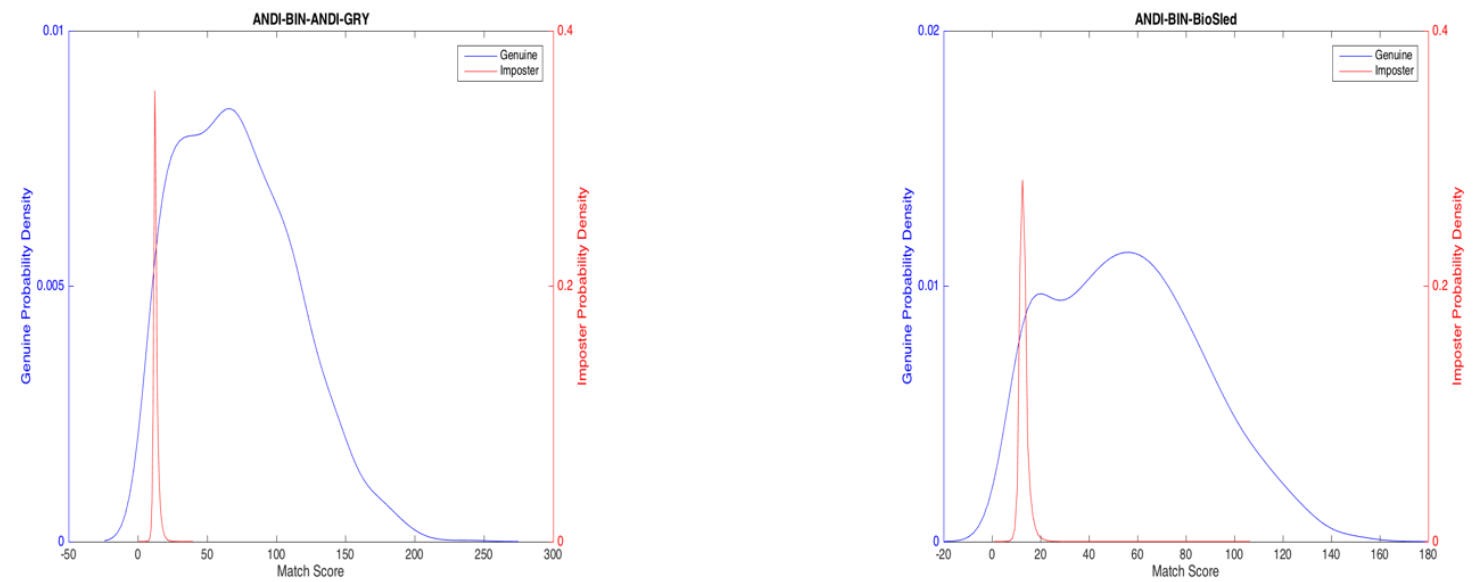

Figure A.6.7 (Left) SourceAFIS ANDI Binary - ANDI Greyscale (Right) SourceAFIS ANDI Binary - BioSled
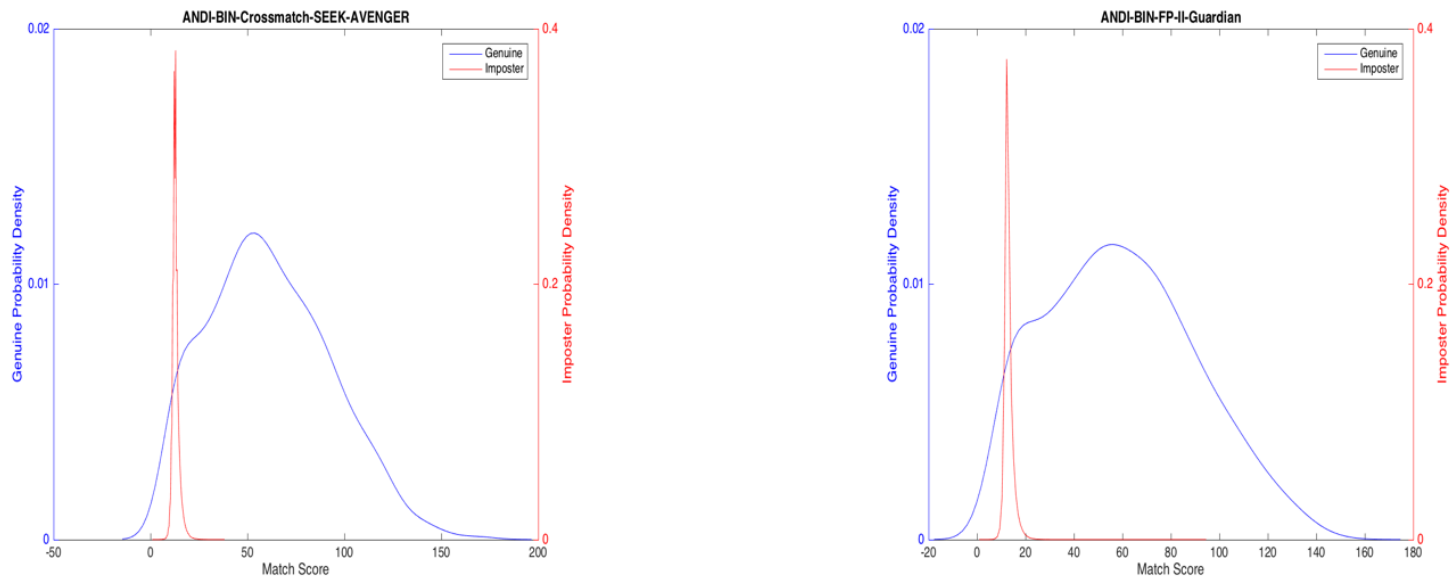

Figure A.6.8 (Left) SourceAFIS ANDI Binary - Crossmatch Seek Avenger (Right) SourceAFIS ANDI Binary - FP II Guardian 

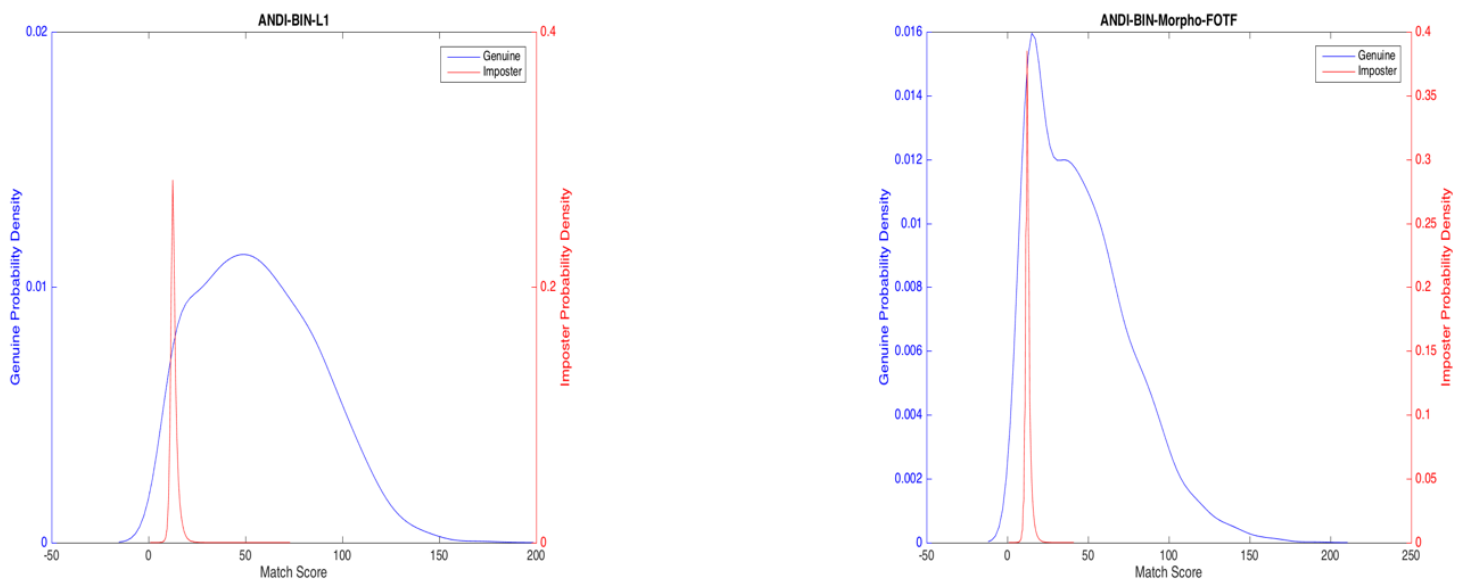

Figure A.6.9 (Left) SourceAFIS ANDI Binary - L1 (Right) SourceAFIS ANDI Binary - Morpho FOTF
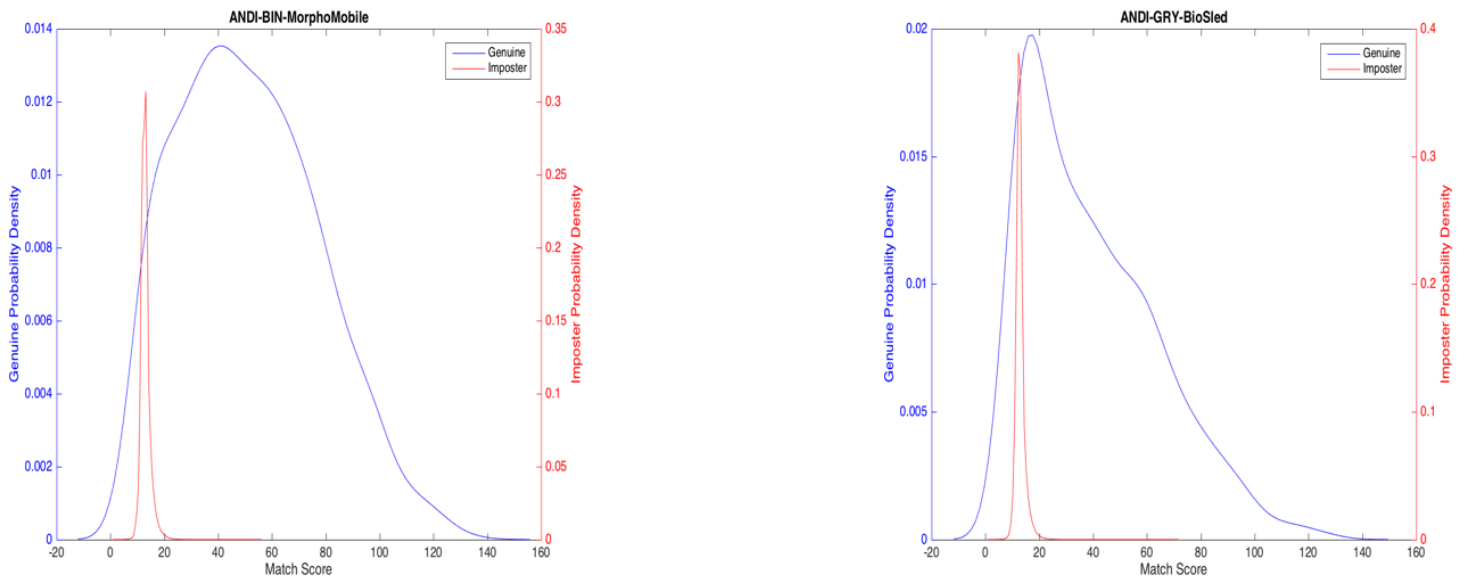

Figure A.6.10 (Left) SourceAFIS ANDI Binary - MorphoMobile (Right) SourceAFIS ANDI Greyscale - BioSled
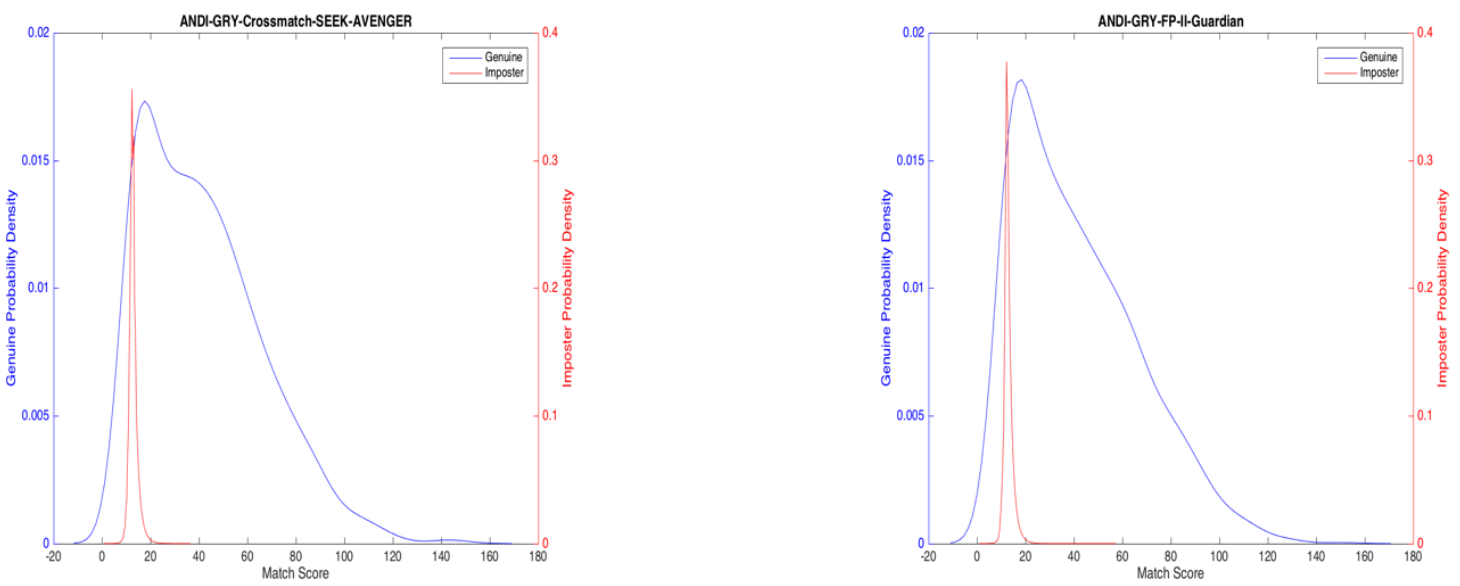

Figure A.6.11 (Left) SourceAFIS ANDI Greyscale - Crossmatch Seek Avenger (Right) SourceAFIS ANDI Greyscale - FP II Guardian 

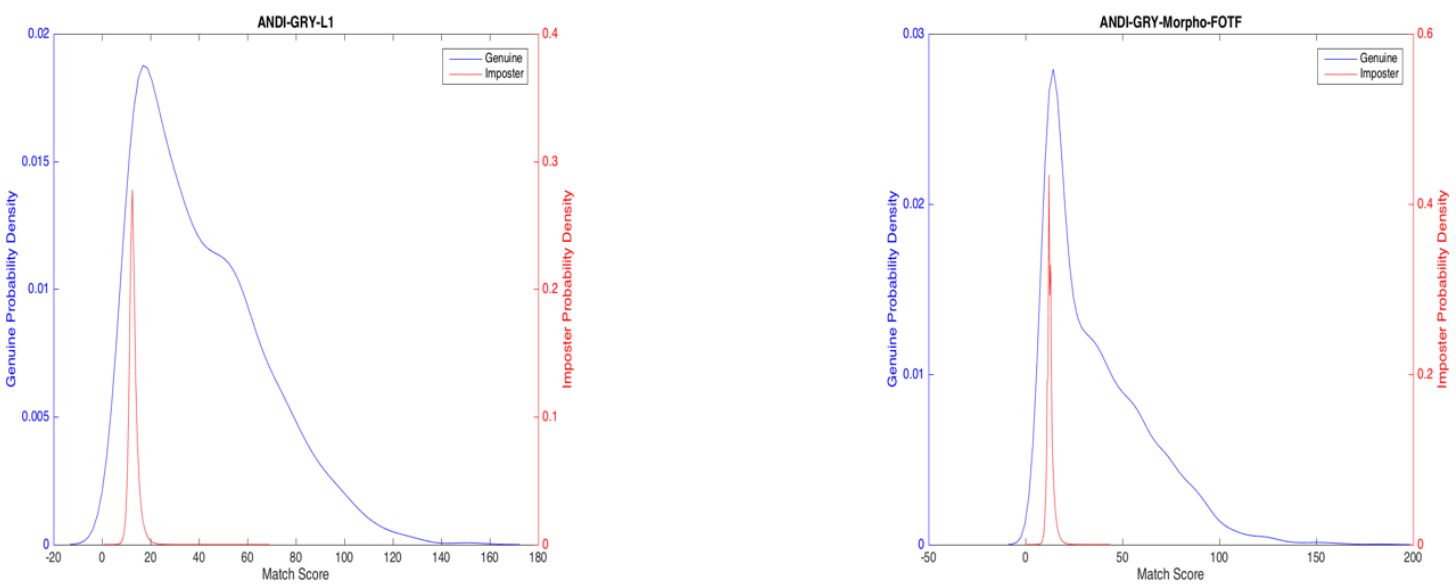

Figure A.6.12 (Left) SourceAFIS ANDI Greyscale - L1 (Right) SourceAFIS ANDI Greyscale - Morpho FOTF
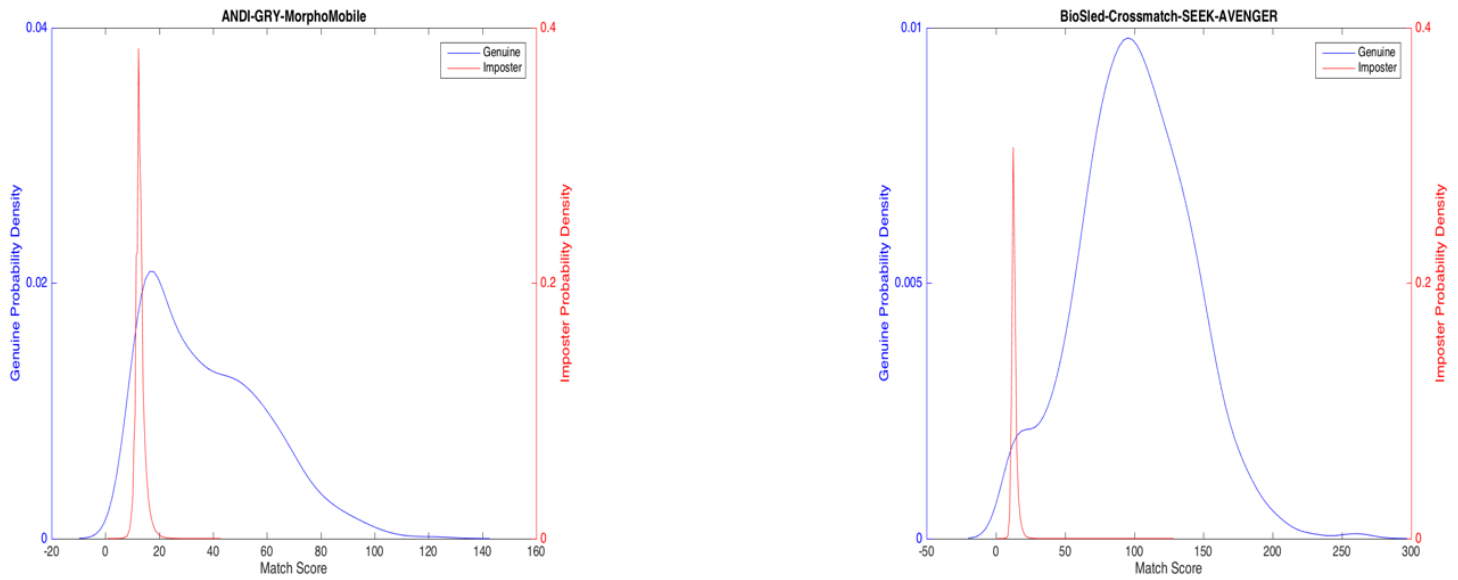

Figure A.6.13 (Left) SourceAFIS ANDI Greyscale - MorphoMobile (Right) SourceAFIS BioSled - Crossmatch Seek Avenger
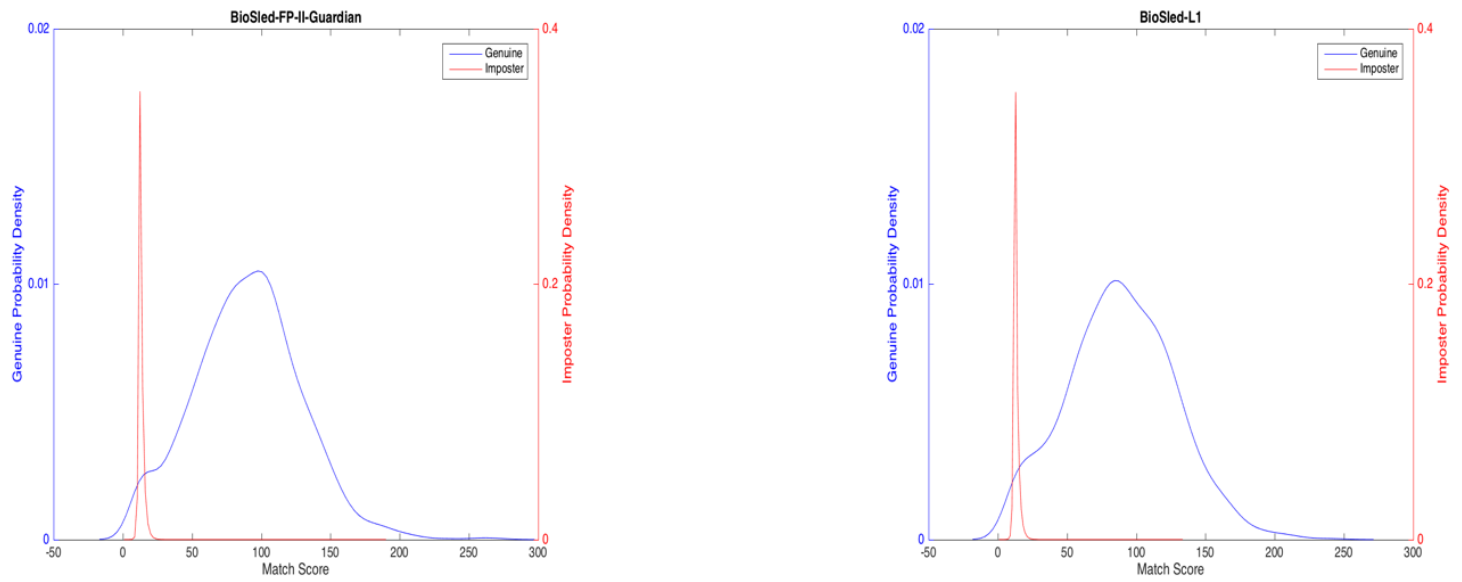

Figure A.6.14 (Left) SourceAFIS BioSled - FP II Guardian (Right) BioSled - L1 

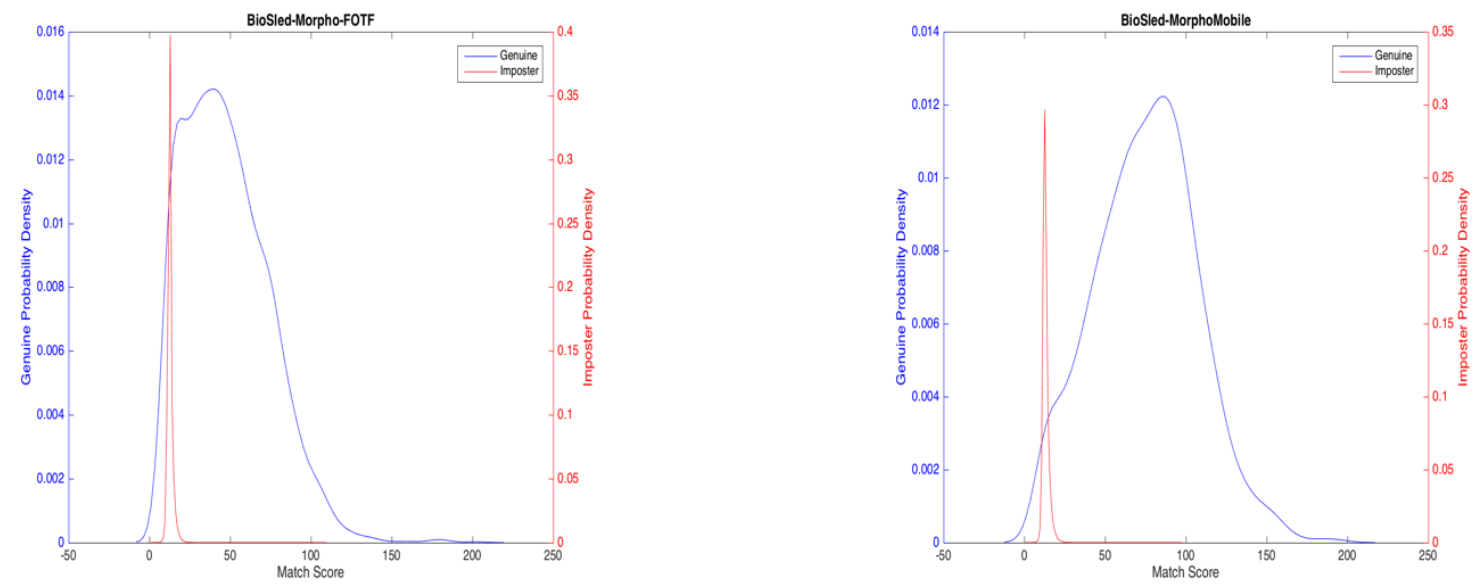

Figure A.6.15 (Left) SourceAFIS BioSled - Morpho FOTF (Right) SourceAFIS BioSled - MorphoMobile
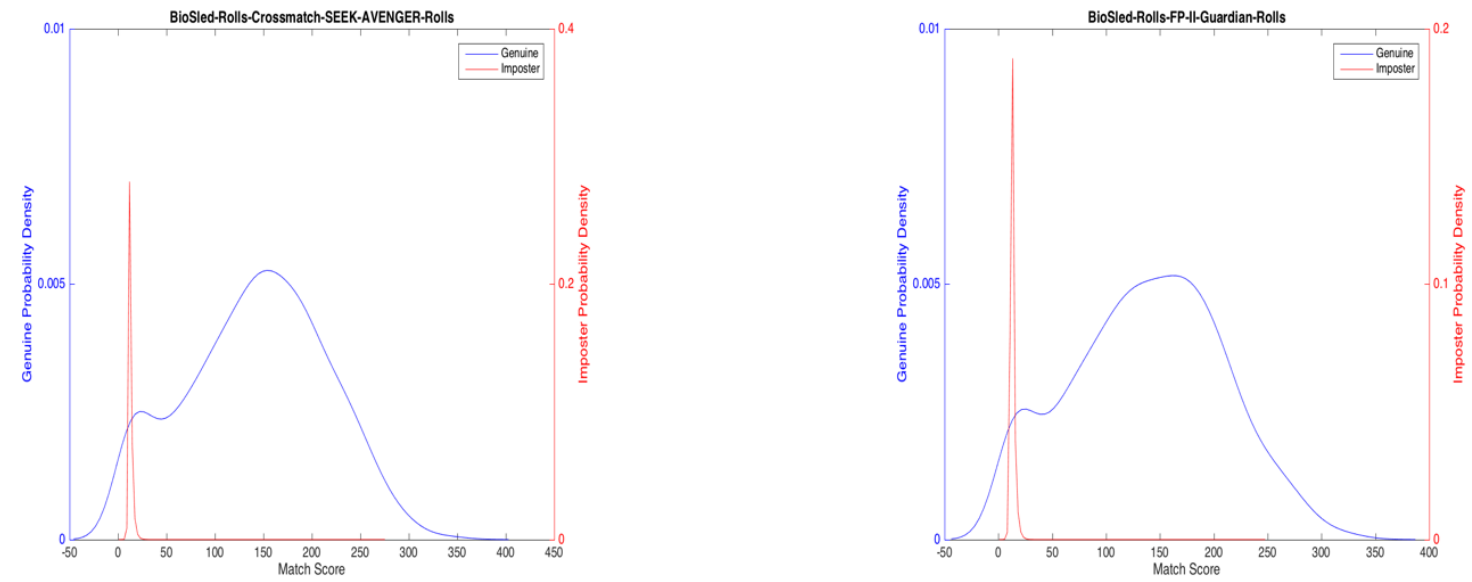

Figure A.6.16 (Left) SourceAFIS BioSled Rolls - Crossmatch Seek Avenger Rolls (Right) SourceAFIS BioSled Rolls - FP II Guardian Rolls
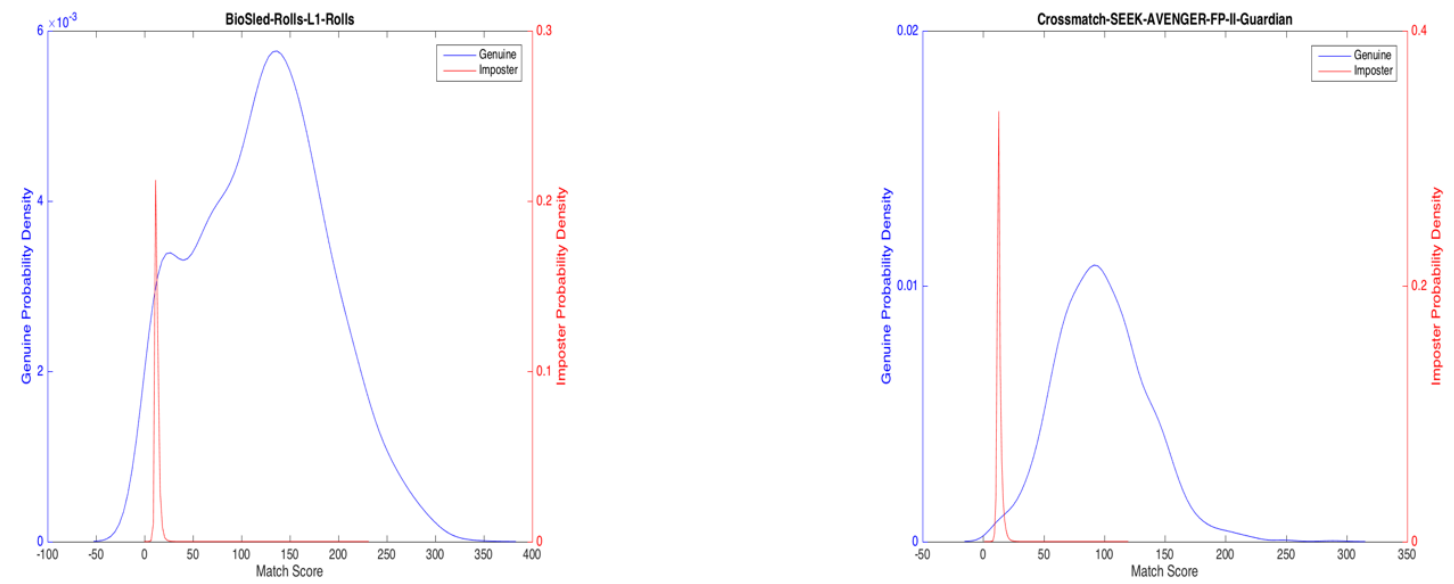

Figure A.6.17 (Left) SourceAFIS BioSled Rolls - L1 Rolls (Right) SourceAFIS Crossmatch Seek Avenger - FP II Guardian 

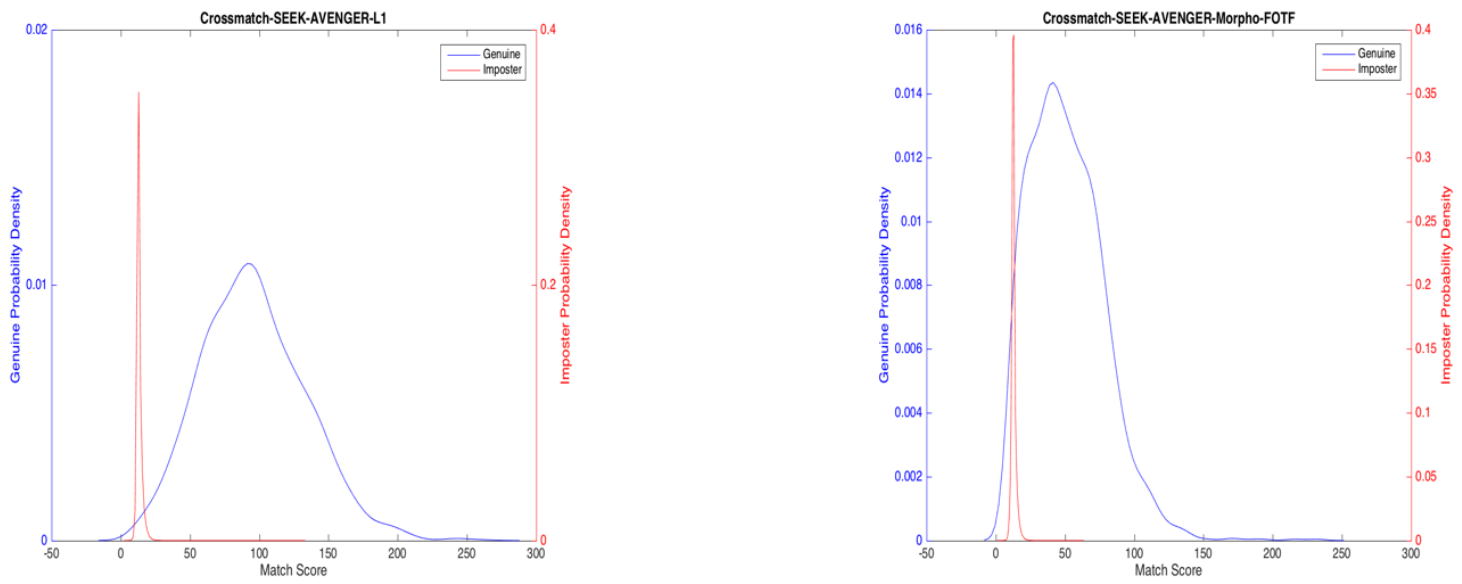

Figure A.6.18 (Left) SourceAFIS Crossmatch Seek Avenger- L1 (Right) SourceAFIS Crossmatch Seek Avenger Morpho FOTF
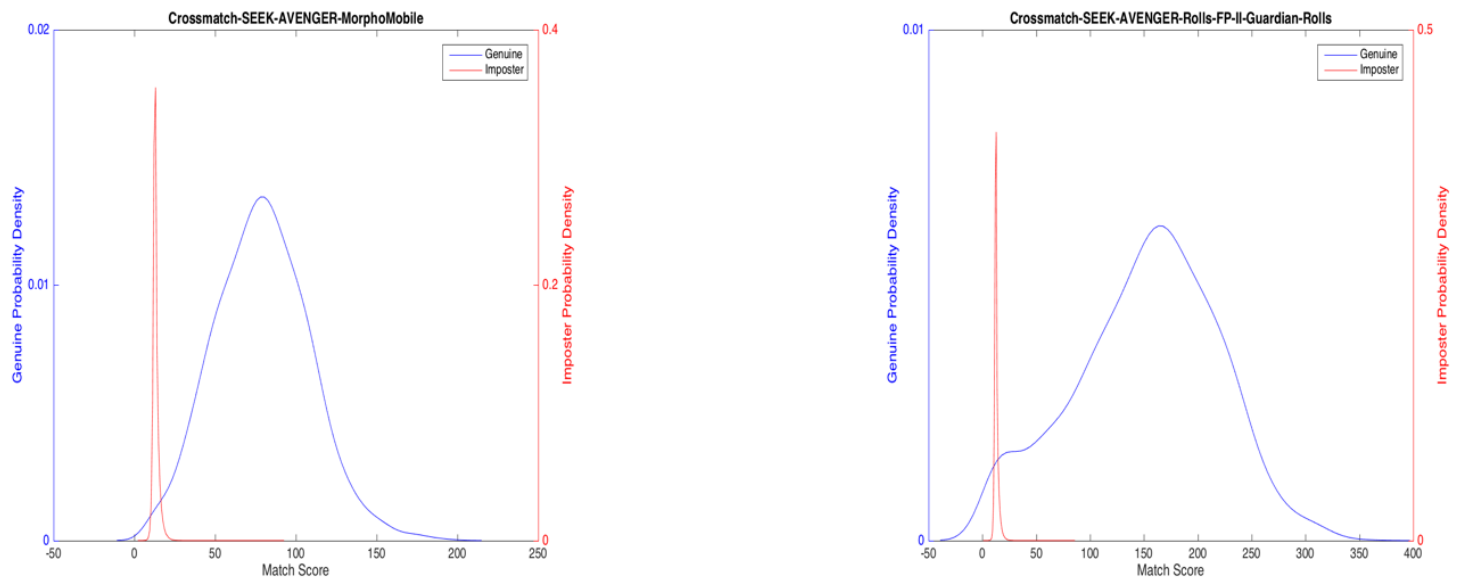

Figure A.6.19 (Left) SourceAFIS Crossmatch Seek Avenger - MorphoMobile (Right) SourceAFIS Crossmatch Seek Avenger Rolls - FP II Guardian Rolls 

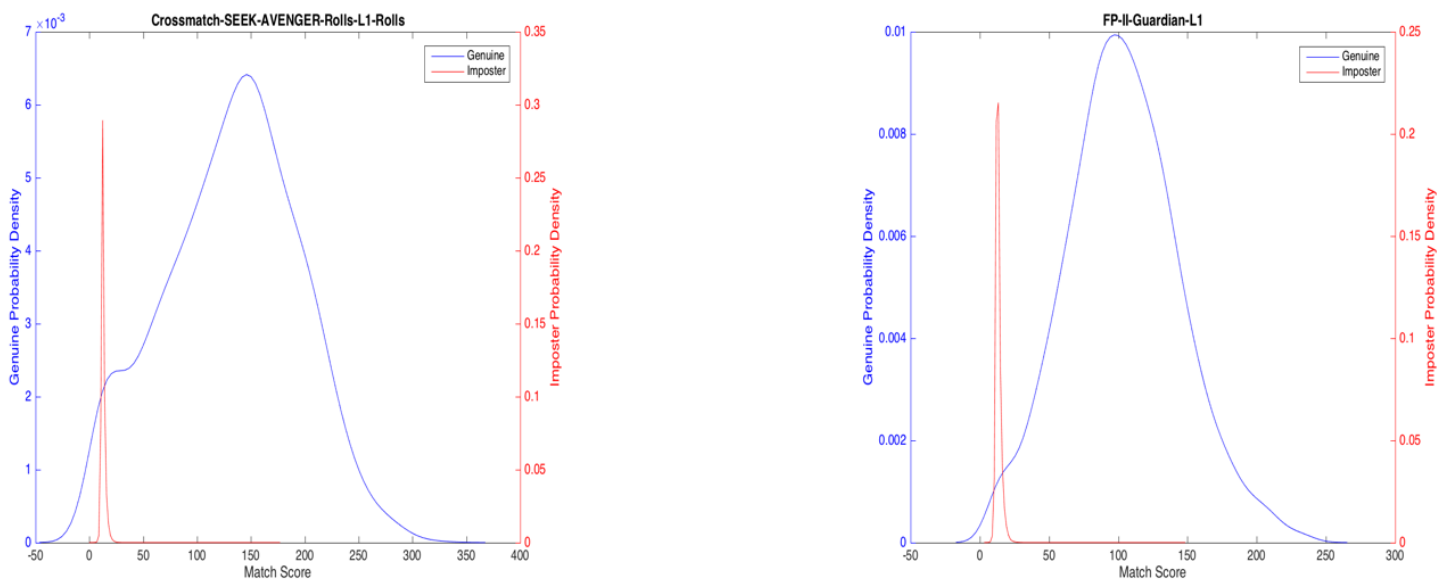

Figure A.6.20 (Left) SourceAFIS Crossmatch Seek Avenger Rolls - L1 Rolls (Right) SourceAFIS FP II Guardian L1
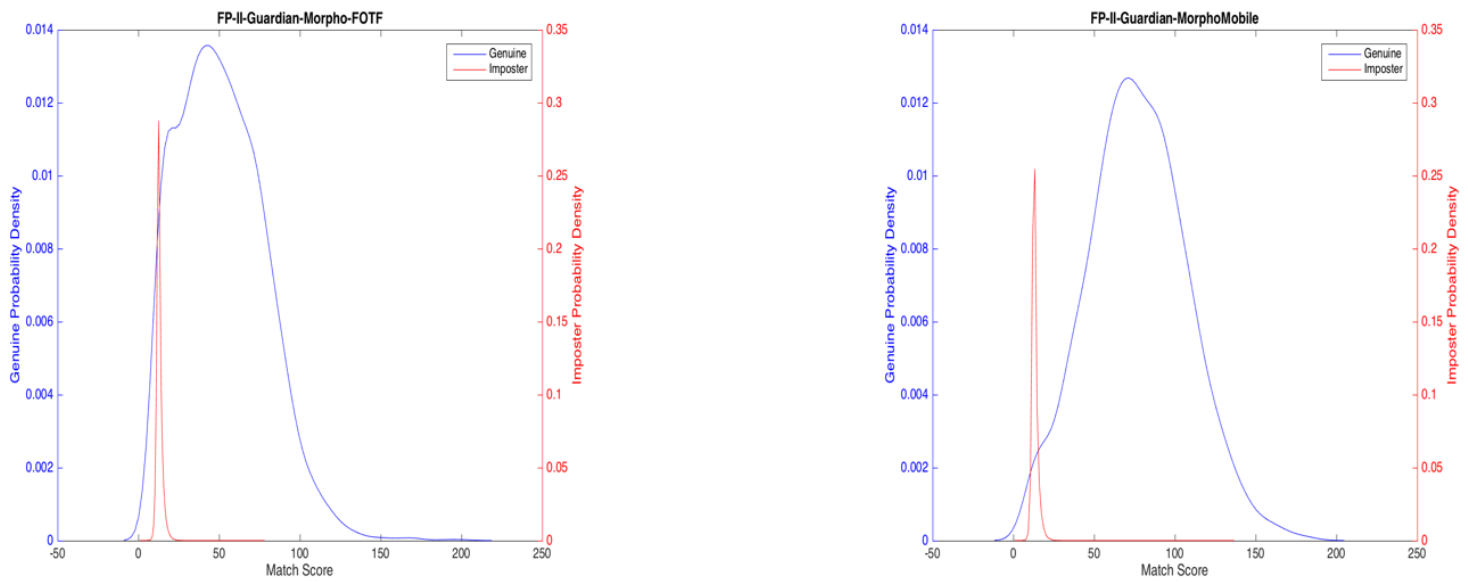

Figure A.6.21 (Left) SourceAFIS FP II Guardian - Morpho FOTF (Right) SourceAFIS FP II Guardian MorphoMobile
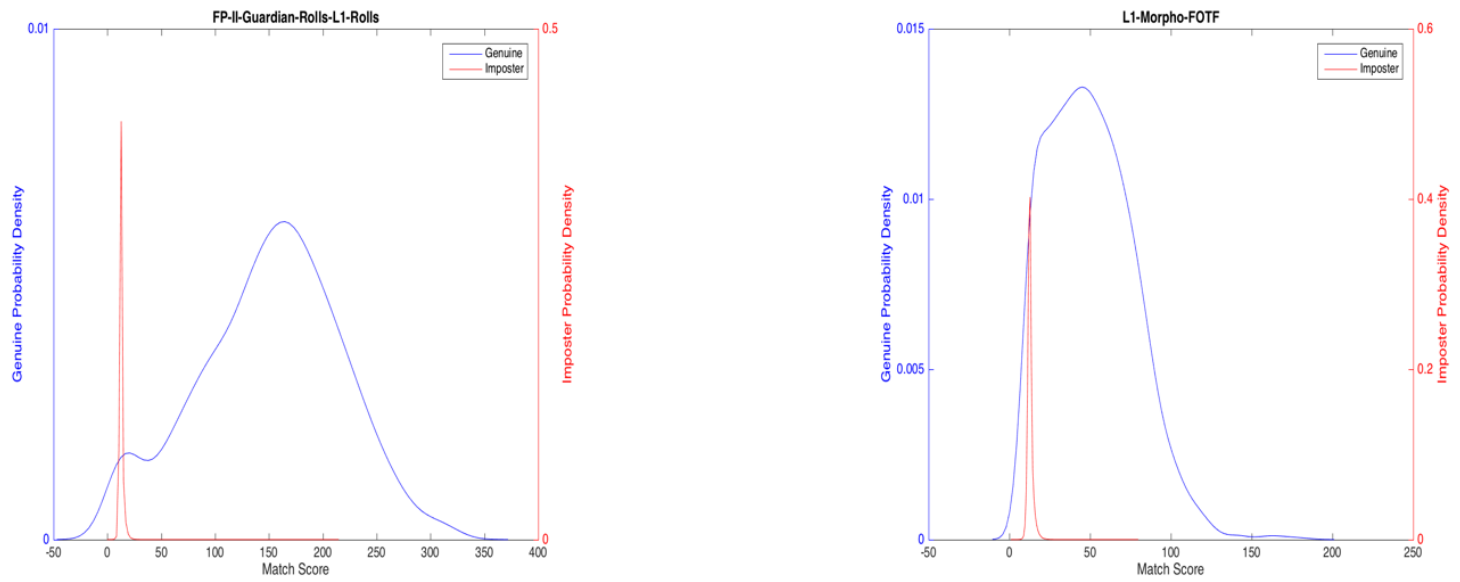

Figure A.6.22 (Left) SourceAFIS FP II Guardian Rolls - L1 Rolls (Right) SourceAFIS L1 - Morpho FOTF 

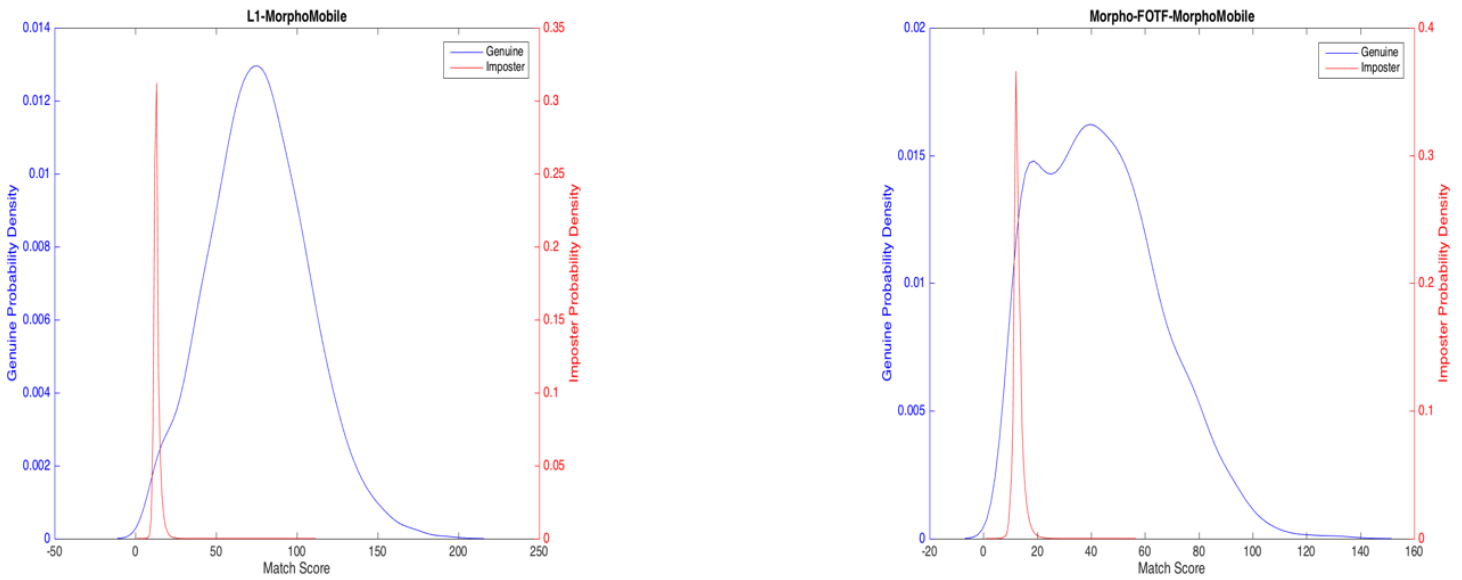

Figure A.6.23 (Left) SourceAFIS L1 - MorphoMobile (Right) SourceAFIS Morpho FOTF - MorphoMobile 


\section{A.7 NBIS ROC Curves and Area Under Curve}

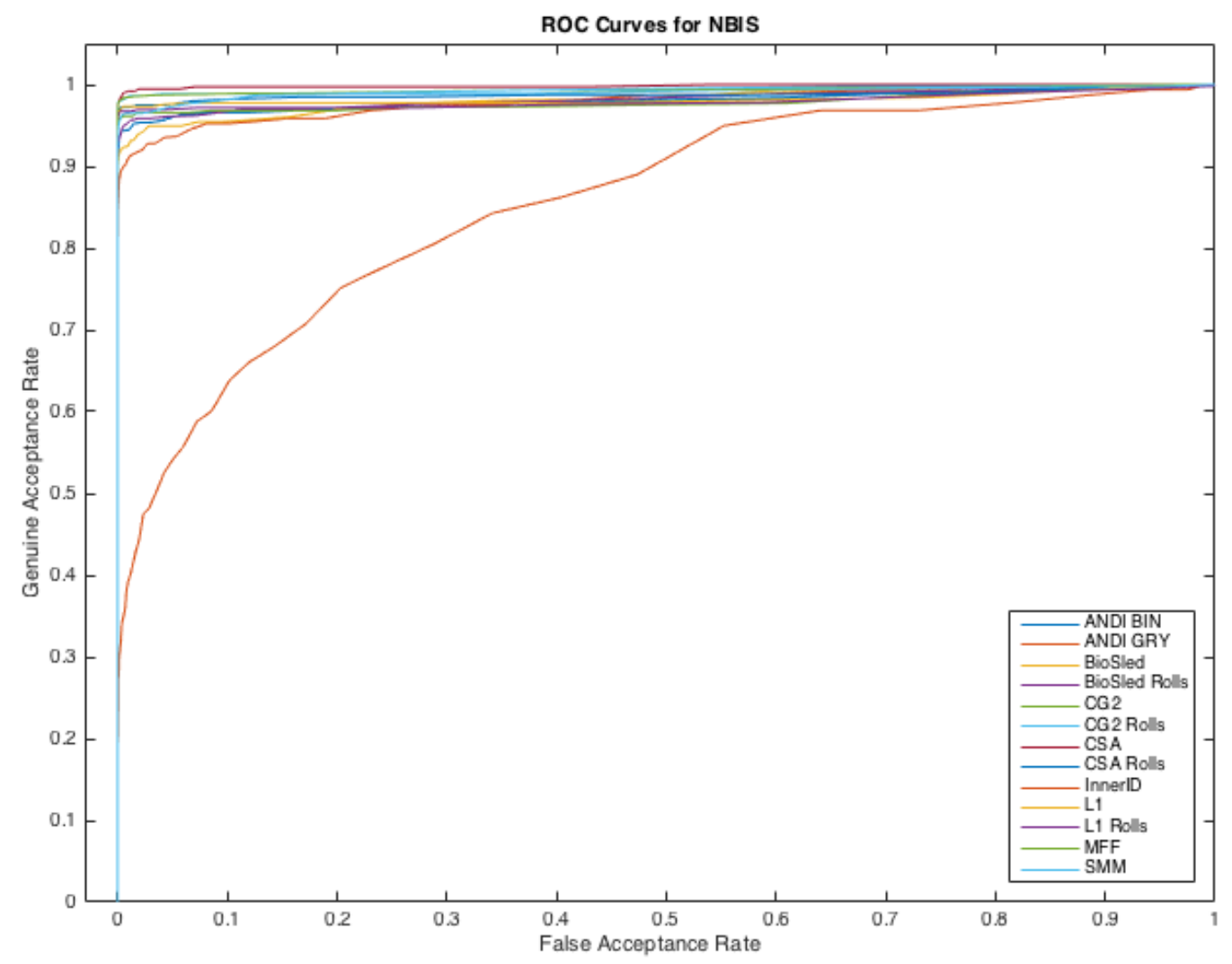

Figure A.7.1 NBIS ROC Curves for each device 


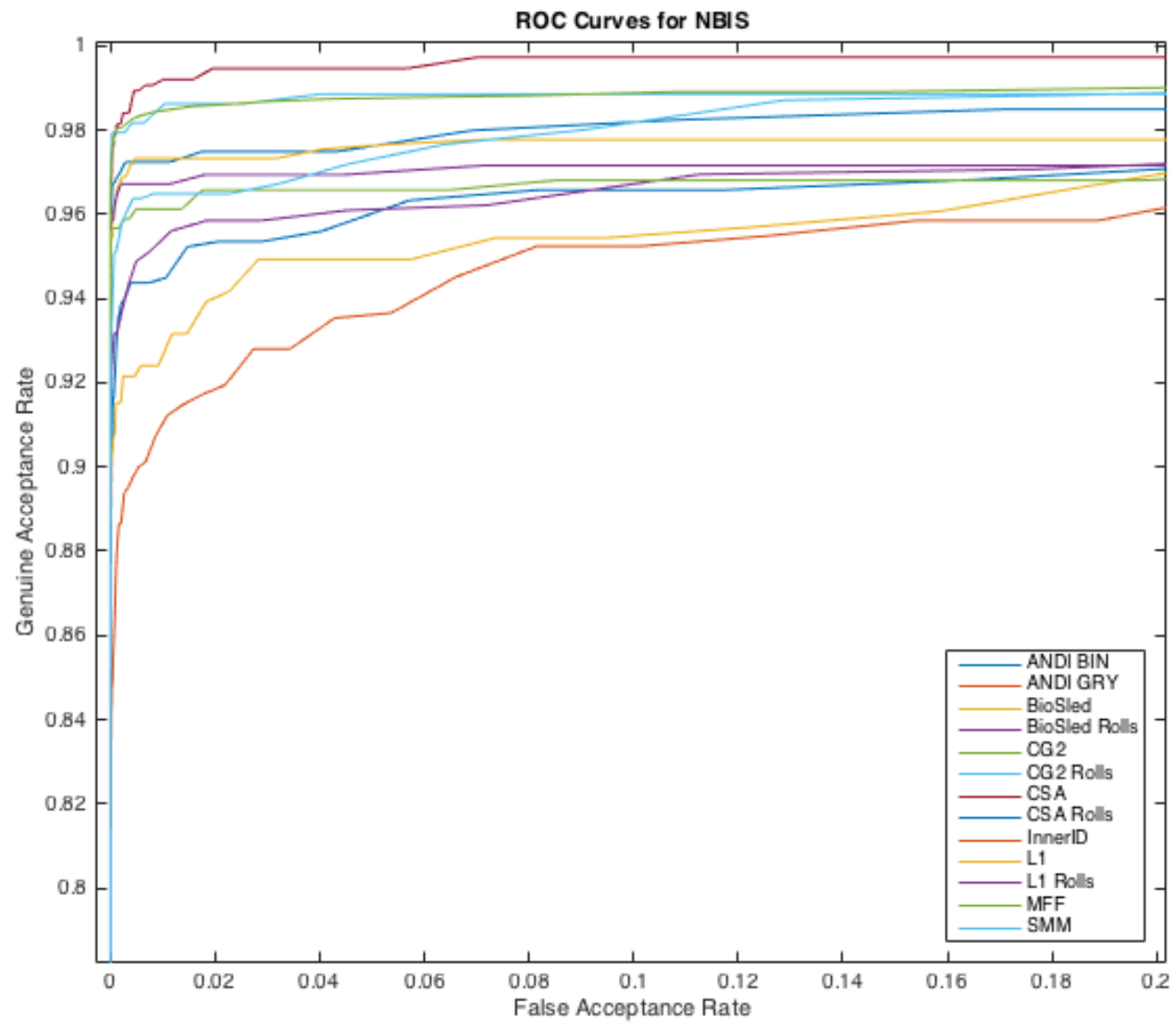

Figure A.7.2 Zoomed In NBIS ROC Curves for each device

Table A.13 NBIS Area Under the Curve for ROC graphs.

\begin{tabular}{|c|c|}
\hline Device & Area Under Curve \\
\hline ANDI BIN & 0.981396 \\
\hline$A N D I G R Y$ & 0.979449 \\
\hline BioSled & 0.982126 \\
\hline BioSled Rolls & 0.982929 \\
\hline CG2 & 0.979025 \\
\hline CG2 Rolls & 0.992307 \\
\hline CSA & 0.998439 \\
\hline CSA Rolls & 0.987915 \\
\hline InnerID & 0.856881 \\
\hline$L 1$ & 0.982475 \\
\hline L1 Rolls & 0.979738 \\
\hline$M F F$ & 0.993983 \\
\hline MorphoMobile & 0.99231 \\
\hline
\end{tabular}



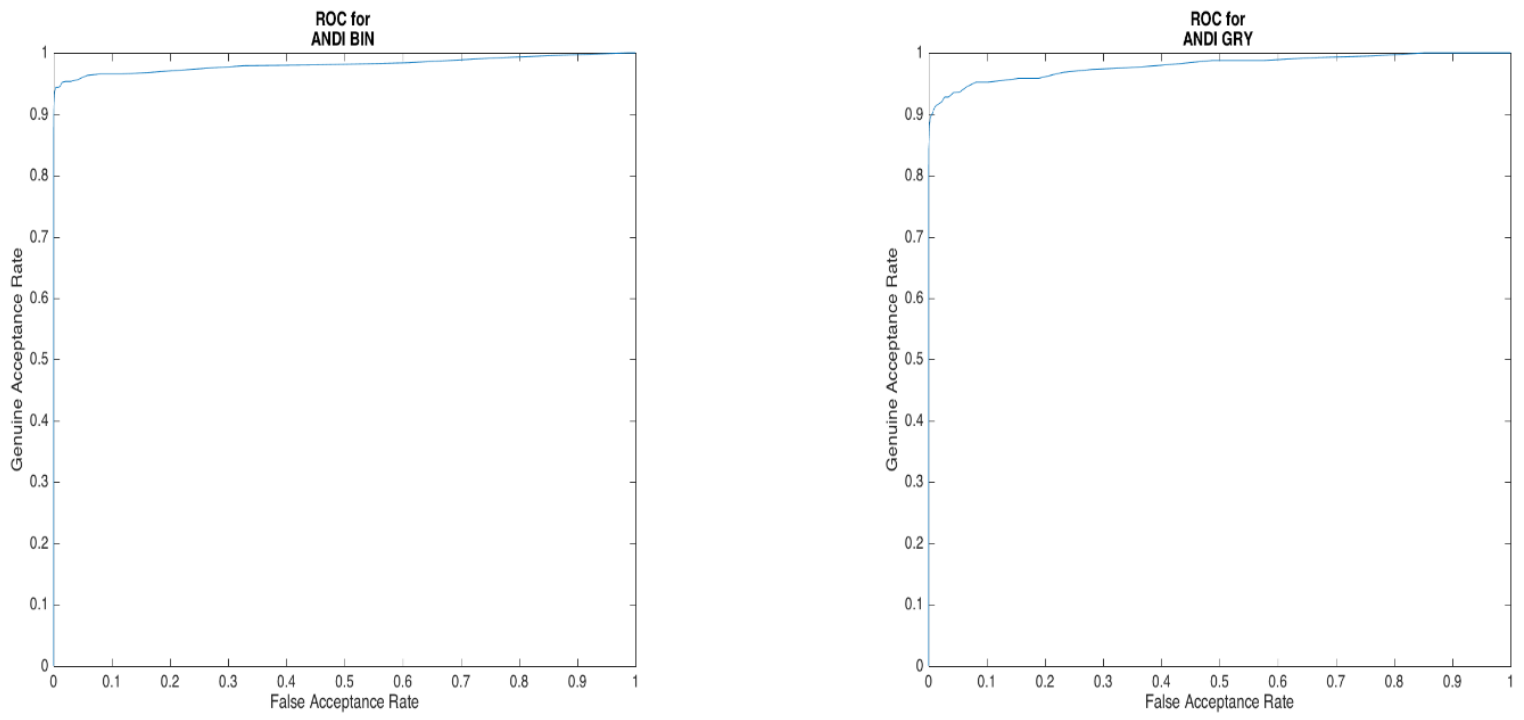

Figure A.7.3 (Left) NBIS ANDI Binary ROC Curve (Right) NBIS ANDI Greyscale ROC Curve
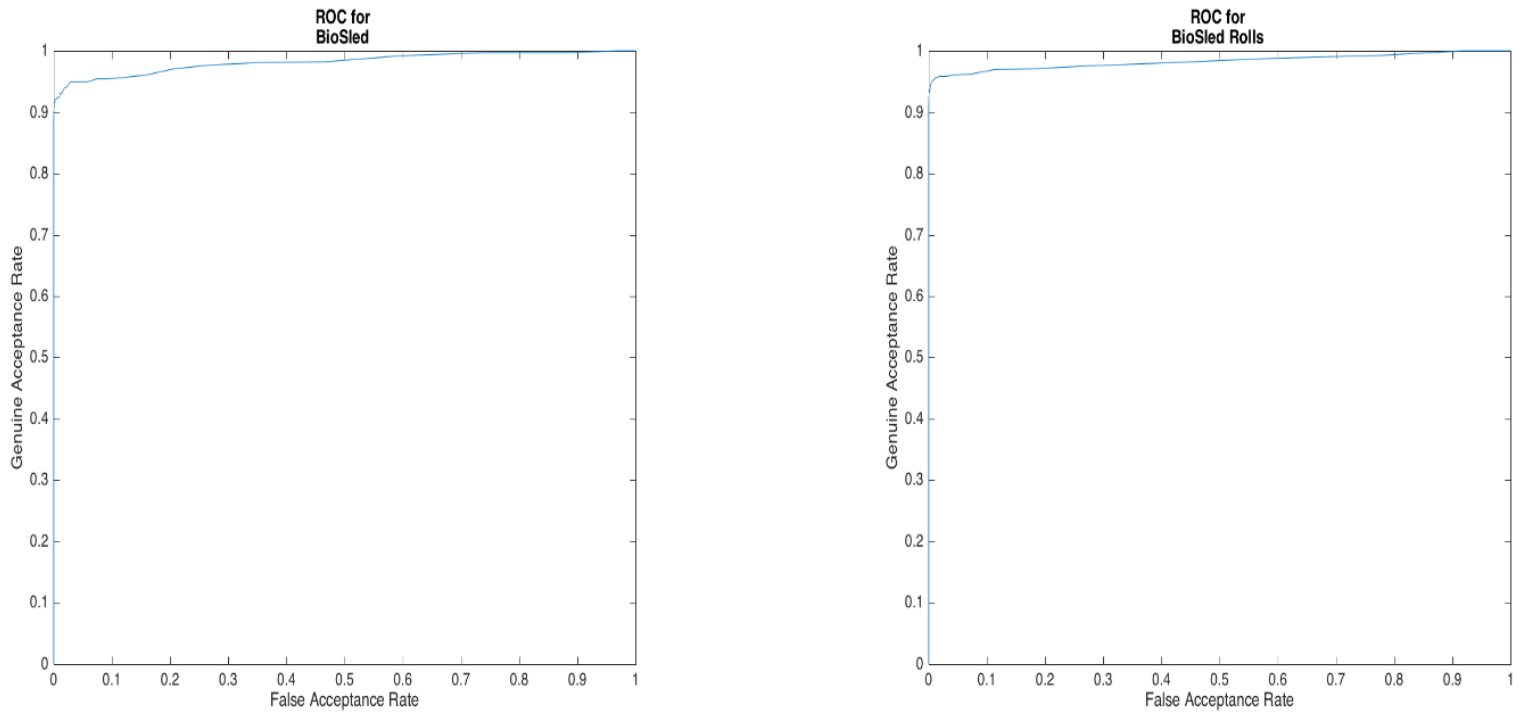

Figure A.7.4 (Left) NBIS BioSled ROC Curve (Right) NBIS BioSled Rolls ROC Curve 

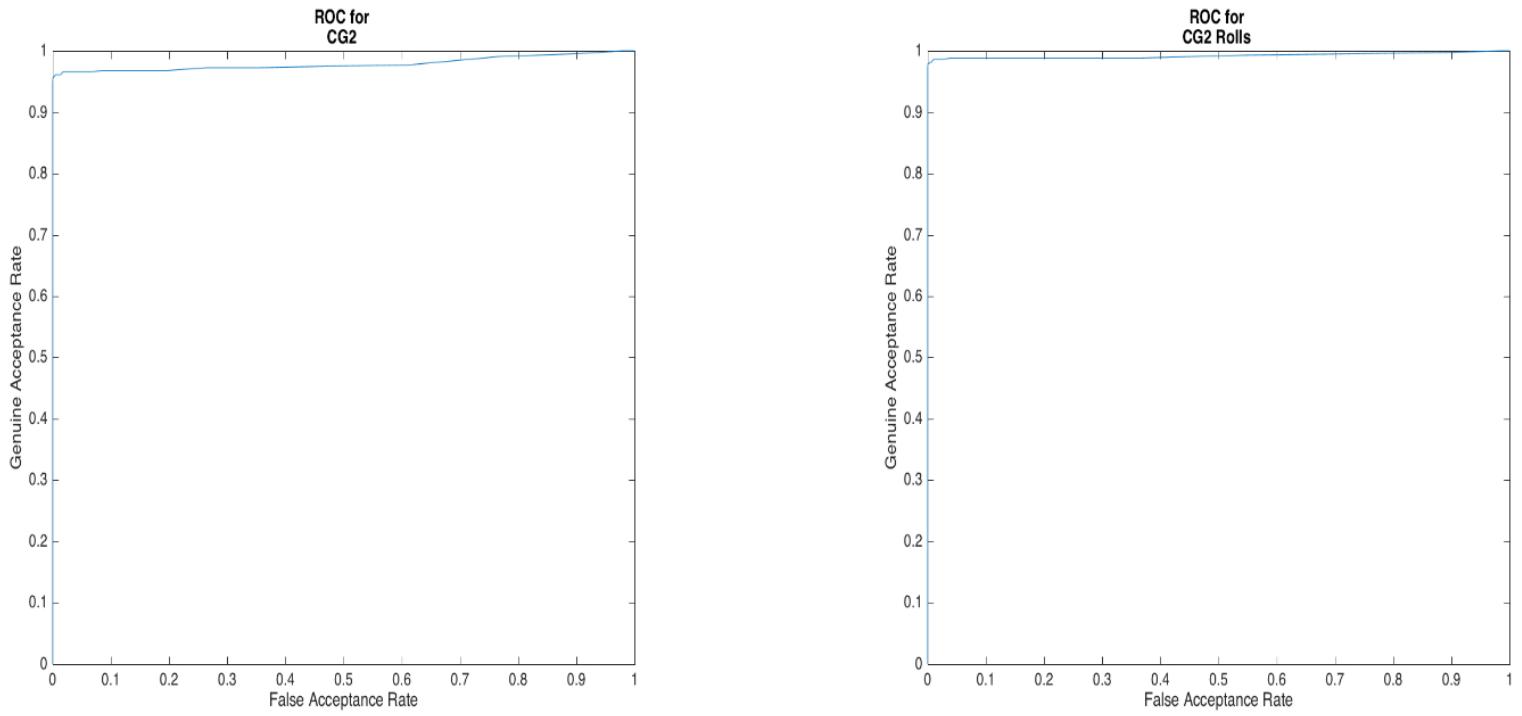

Figure A.7.5 (Left) NBIS FP II Guardian ROC Curve (Right) NBIS FP II Guardian Rolls ROC Curve
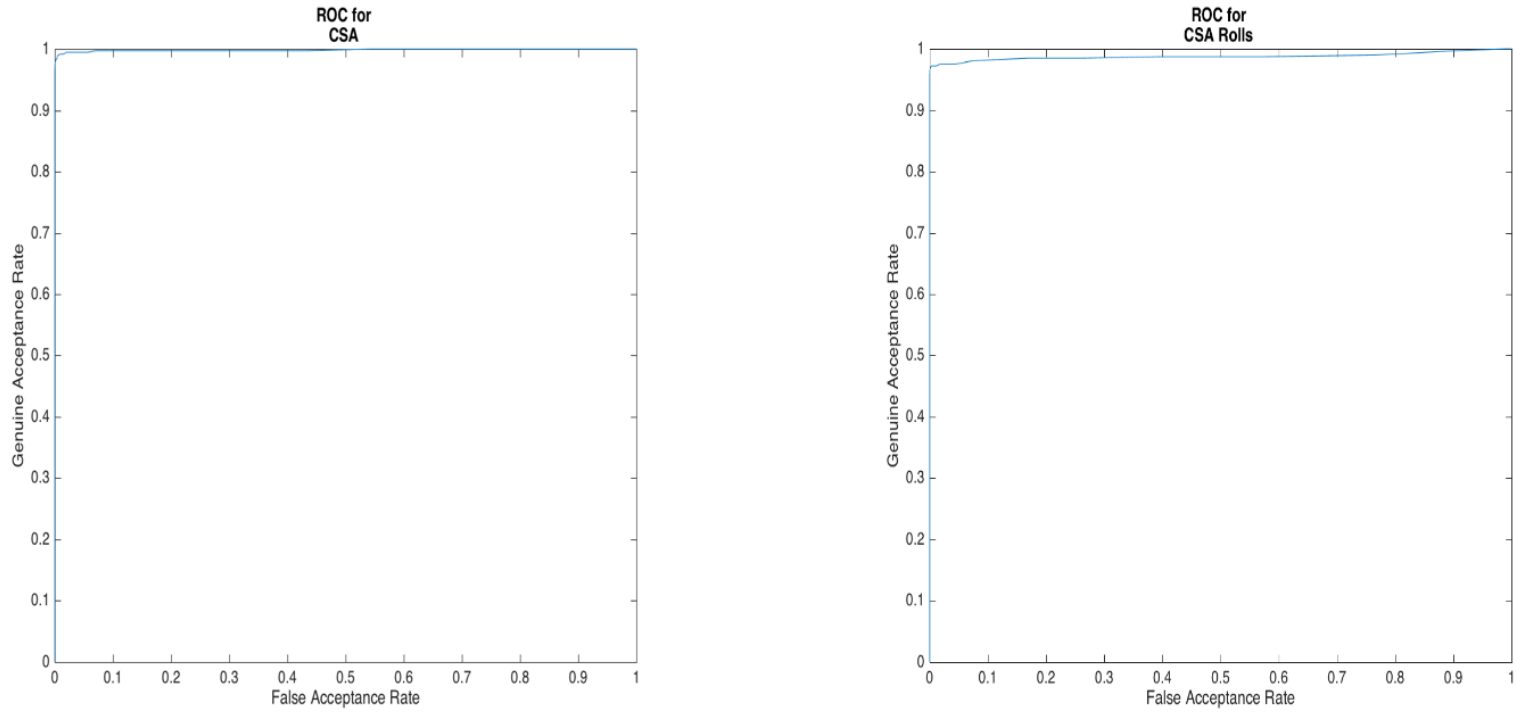

Figure A.7.6 (Left) NBIS Crossmatch Seek Avenger ROC Curve (Right) NBIS Crossmatch Seek Avenger Rolls ROC Curve 

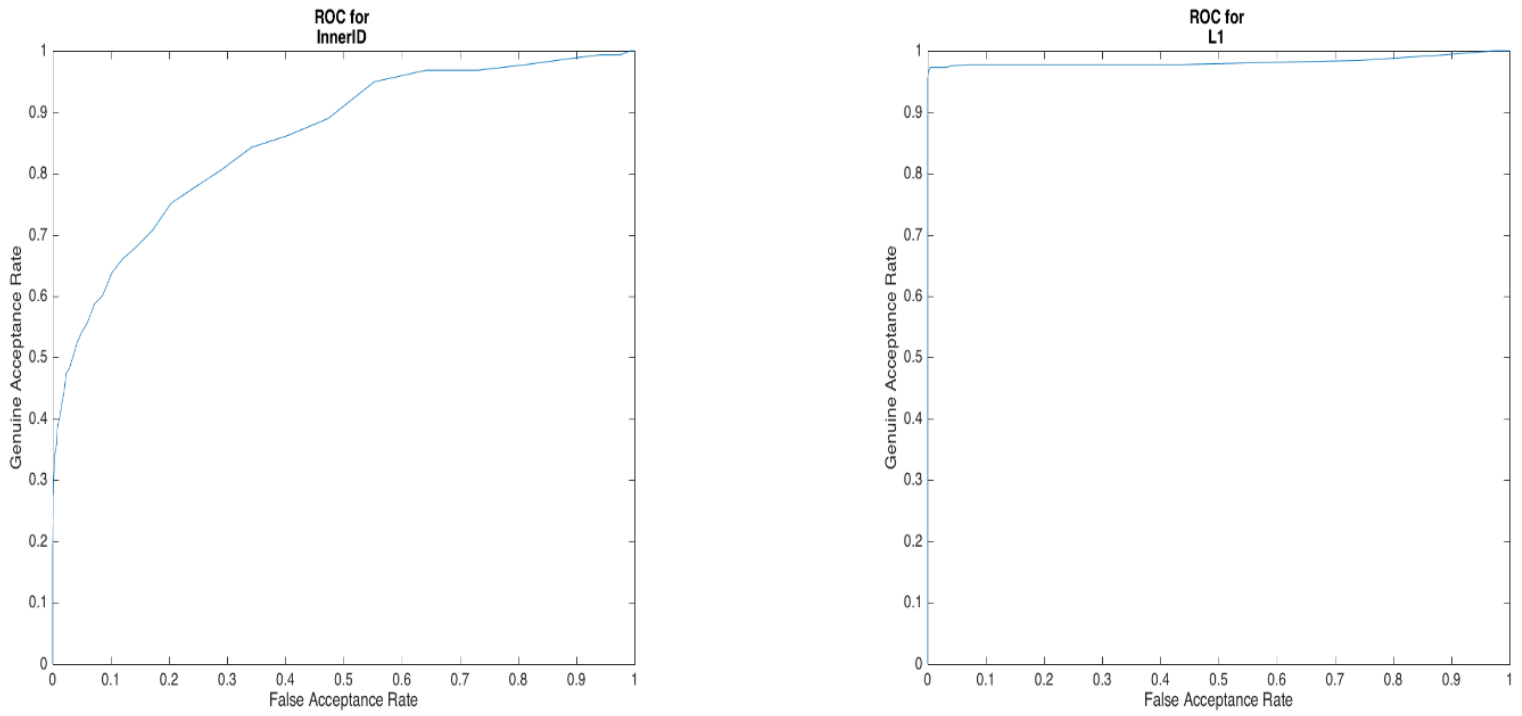

Figure A.7.7 (Left) NBIS InnerID ROC Curve (Right) NBIS L1 Curve
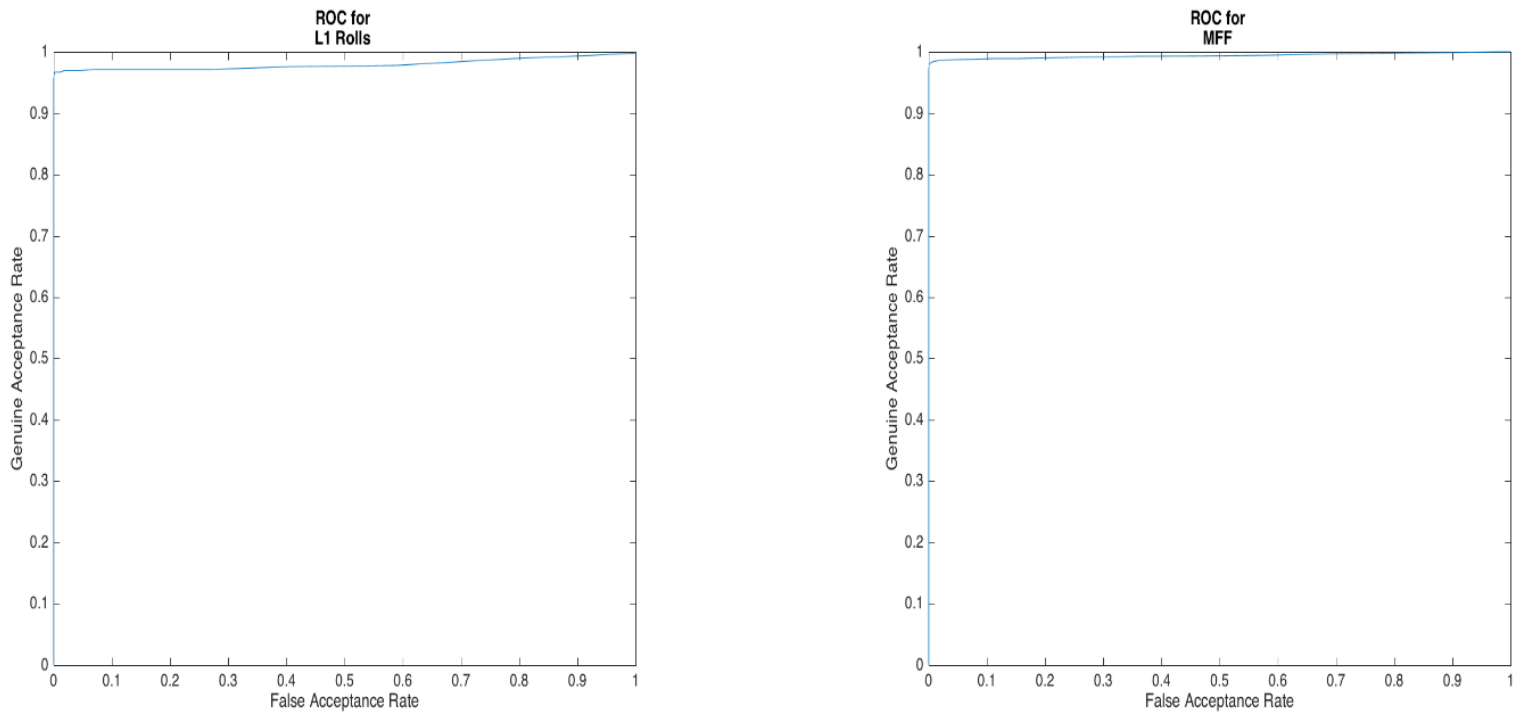

Figure A.7.8 (Left) NBIS L1 Rolls ROC Curve (Right) NBIS Morpho FOTF ROC Curve 


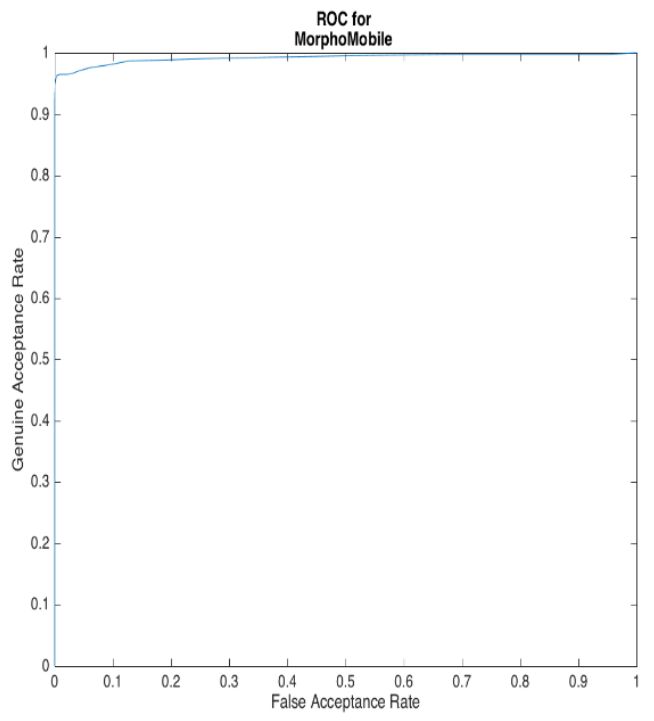

Figure A.7.9 NBIS MorphoMobile ROC Curve 


\section{A.8 Neurotech ROC Curves and Area Under Curve}

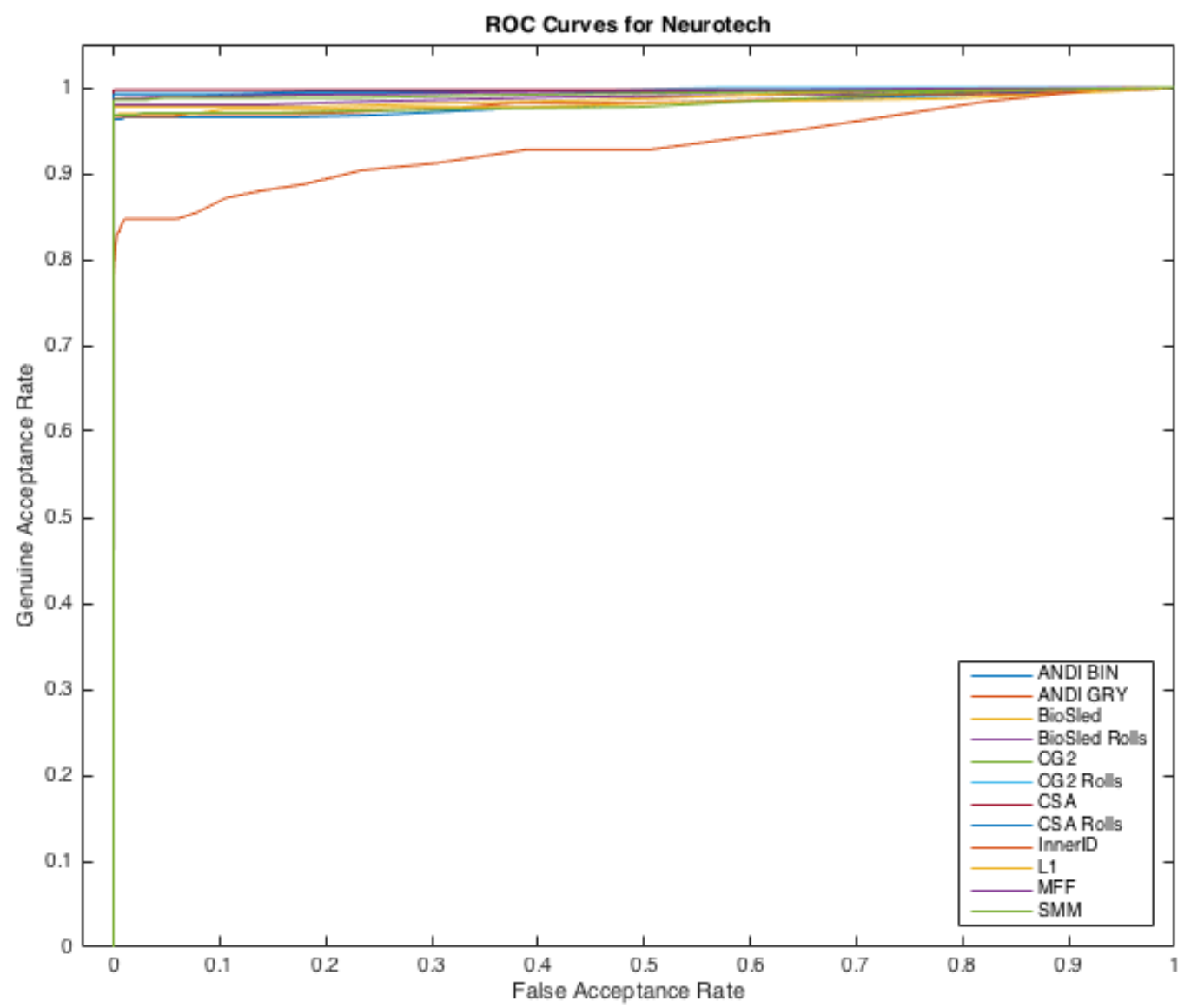

Figure A.8.1 Neurotech ROC Curves for each Device 


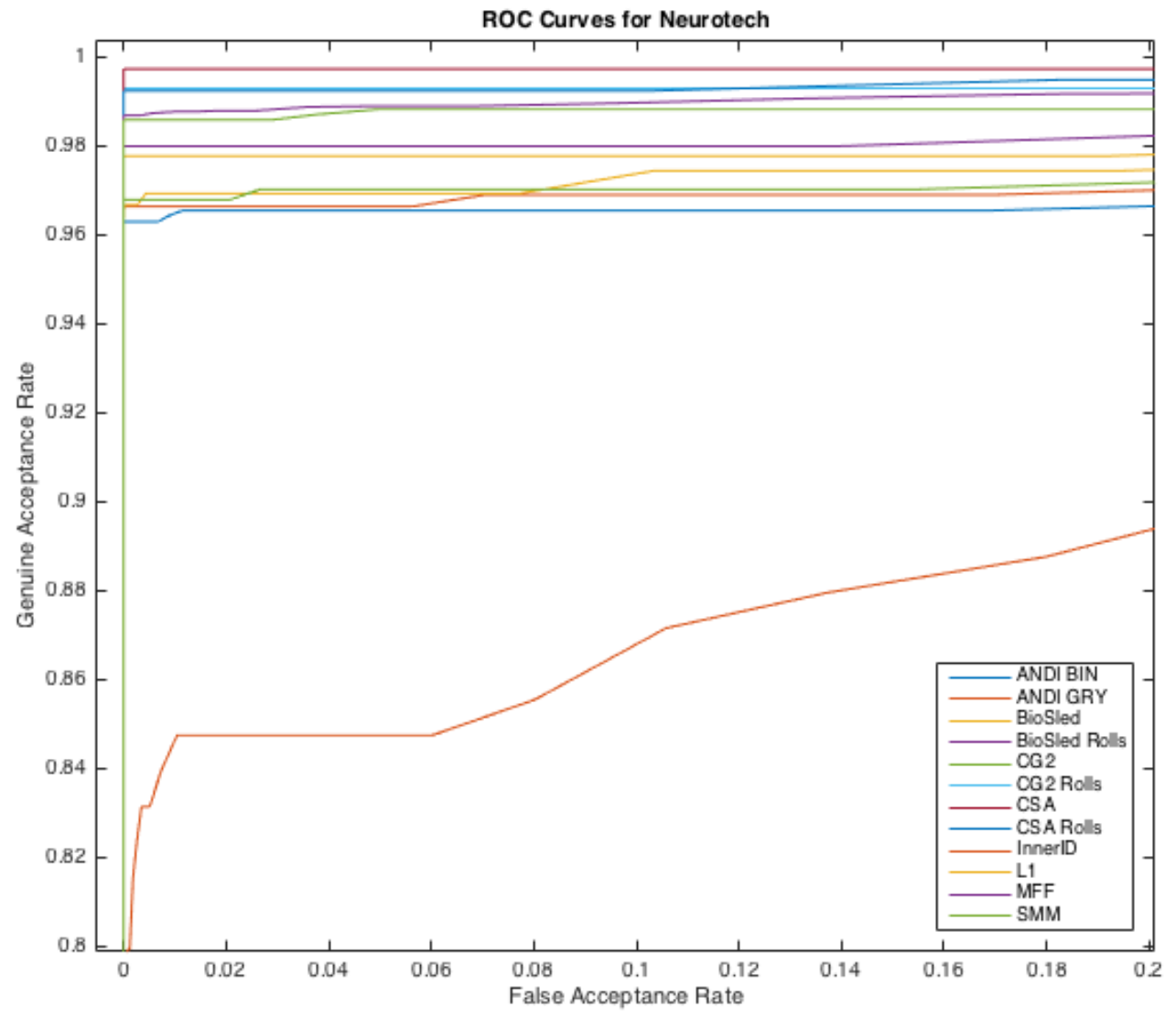

Figure A.8.2 Zoomed In Neurotech ROC Curves for each Device

Table A.14 Neurotech Area Under the Curve for ROC graphs.

\begin{tabular}{|r|l|}
\hline Neurotech Device & Area Under Curve \\
\hline ANDI BIN & 0.98037 \\
ANDI GRY & 0.982468 \\
BioSled & 0.981787 \\
BioSled Rolls & 0.988932 \\
CG2 & 0.982212 \\
CG2 Rolls & 0.996949 \\
CSA & 0.997675 \\
CSA Rolls & 0.99515 \\
InnerID & 0.93257 \\
L1 & 0.987632 \\
MFF & 0.99526 \\
MorphoMobile & 0.992997 \\
\hline &
\end{tabular}



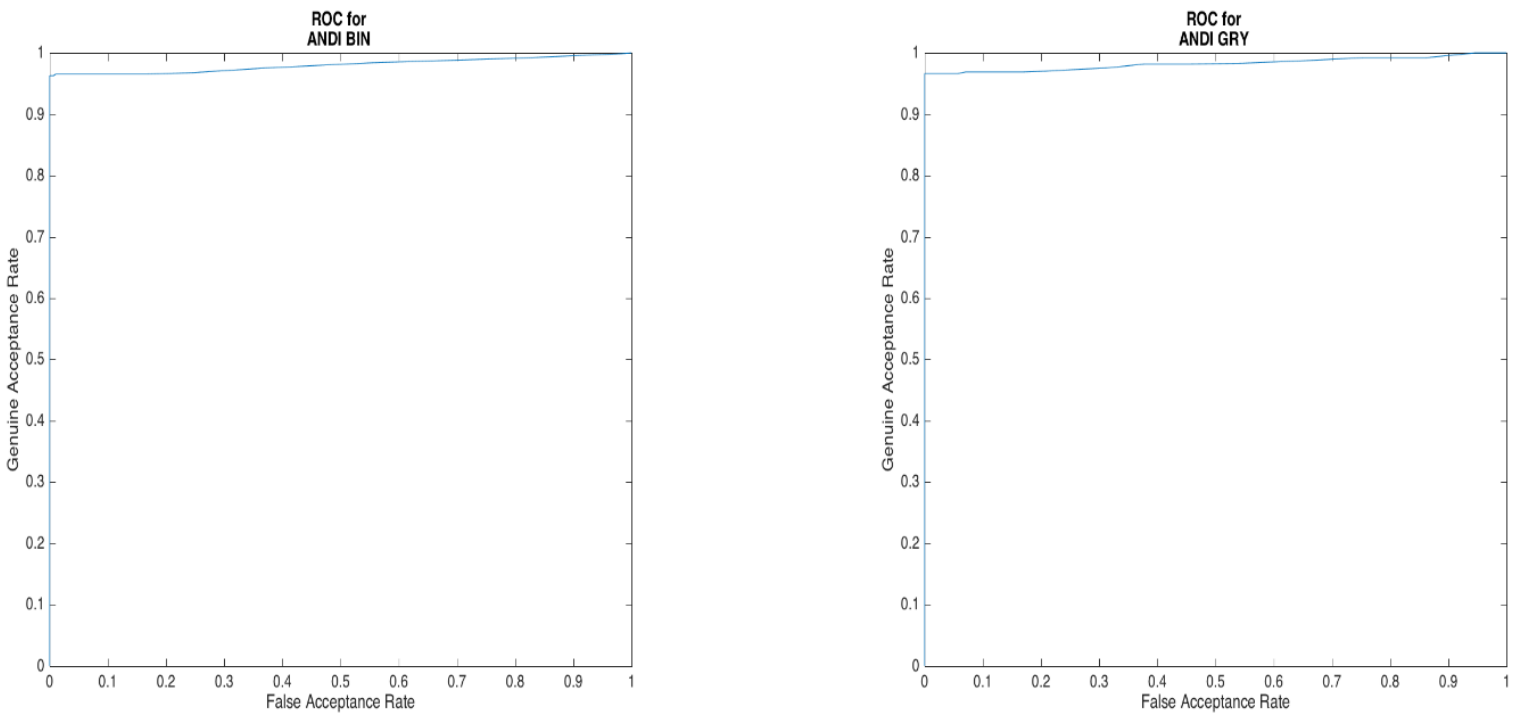

Figure A.8.3 (Left) Neurotech ANDI Binary ROC Curve (Right) Neurotech ANDI Greyscale ROC Curve
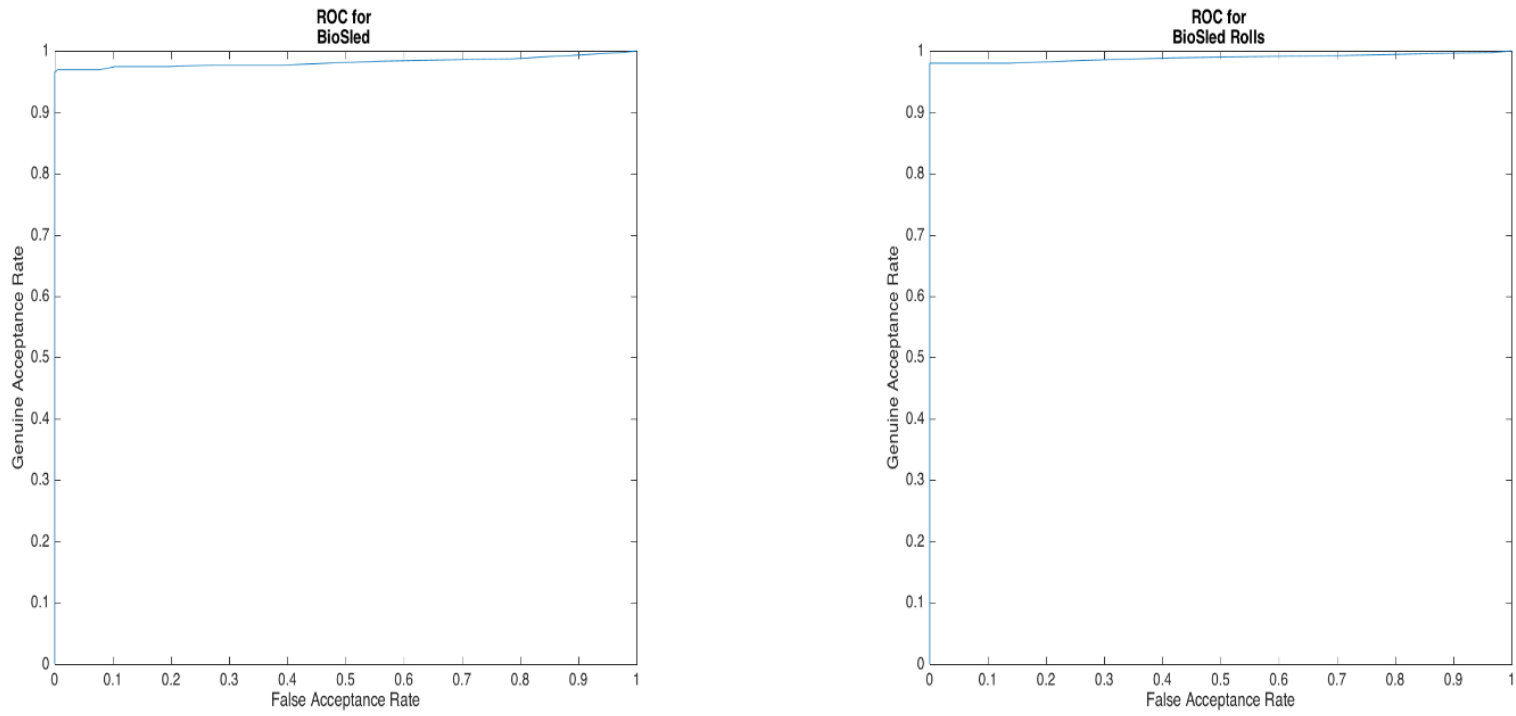

Figure A.8.4 (Left) Neurotech BioSled ROC Curve (Right) Neurotech BioSled Rolls ROC Curve 

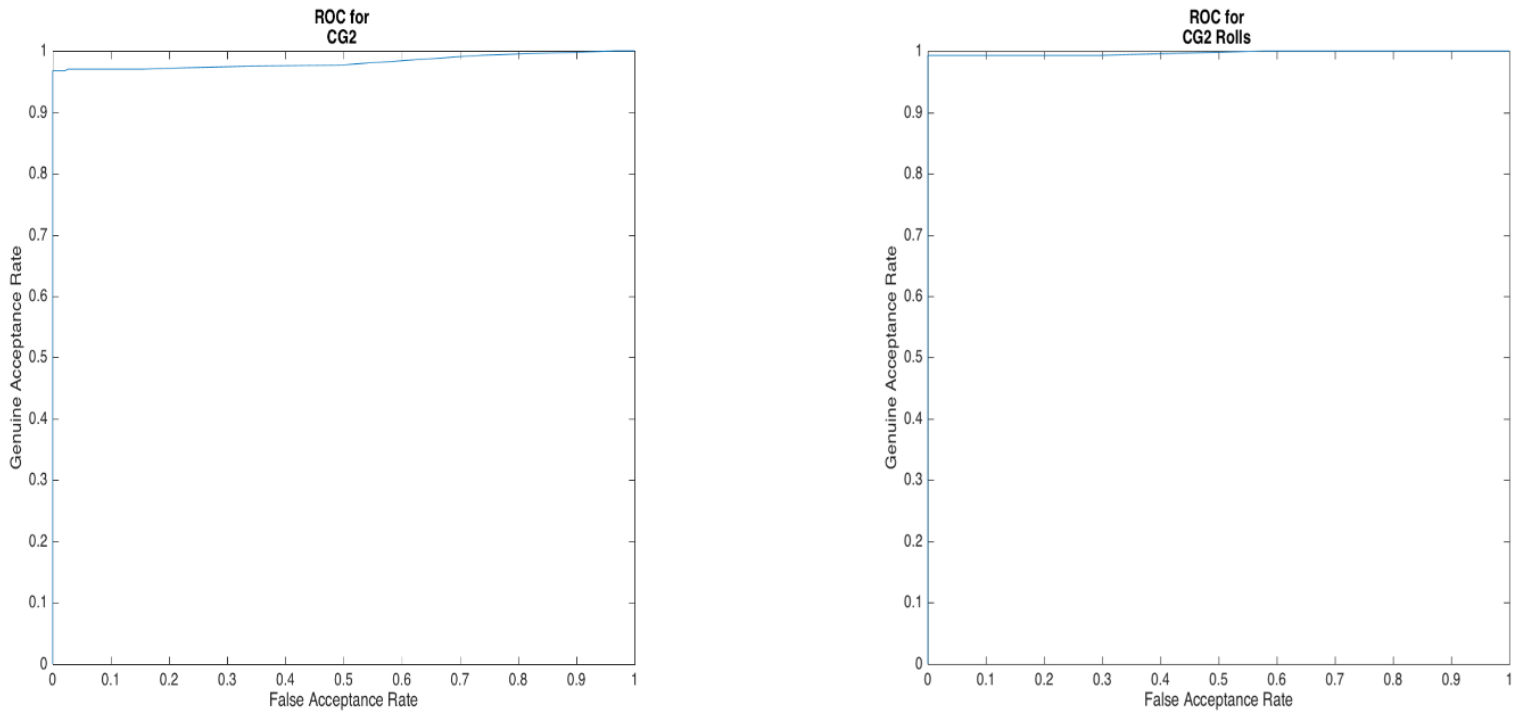

Figure A.8.5 (Left) Neurotech FP II Guardian ROC Curve (Right) Neurotech FP II Guardian Rolls ROC Curve
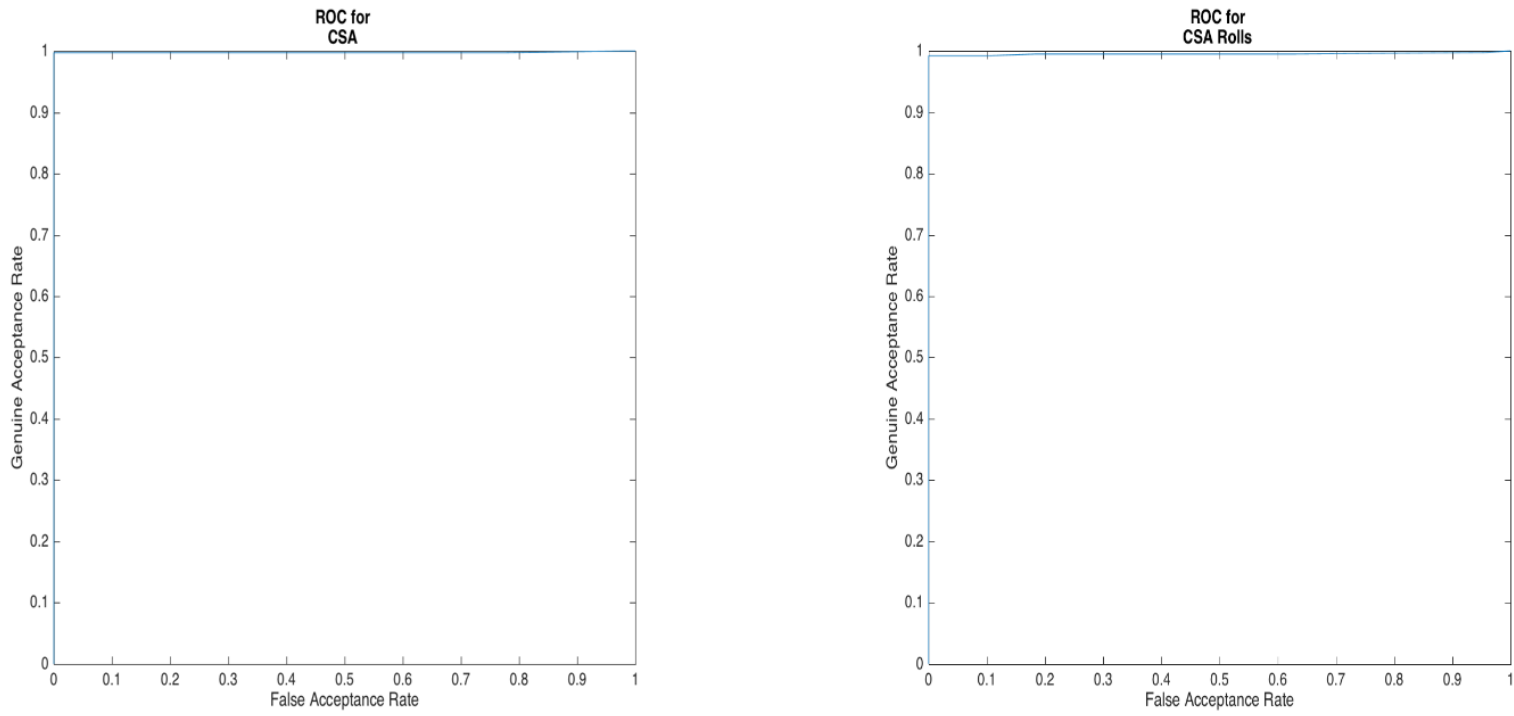

Figure A.8.6 (Left) Neurotech Crossmatch Seek Avenger ROC Curve (Right) Neurotech Crossmatch Seek Avenger Rolls ROC Curve 

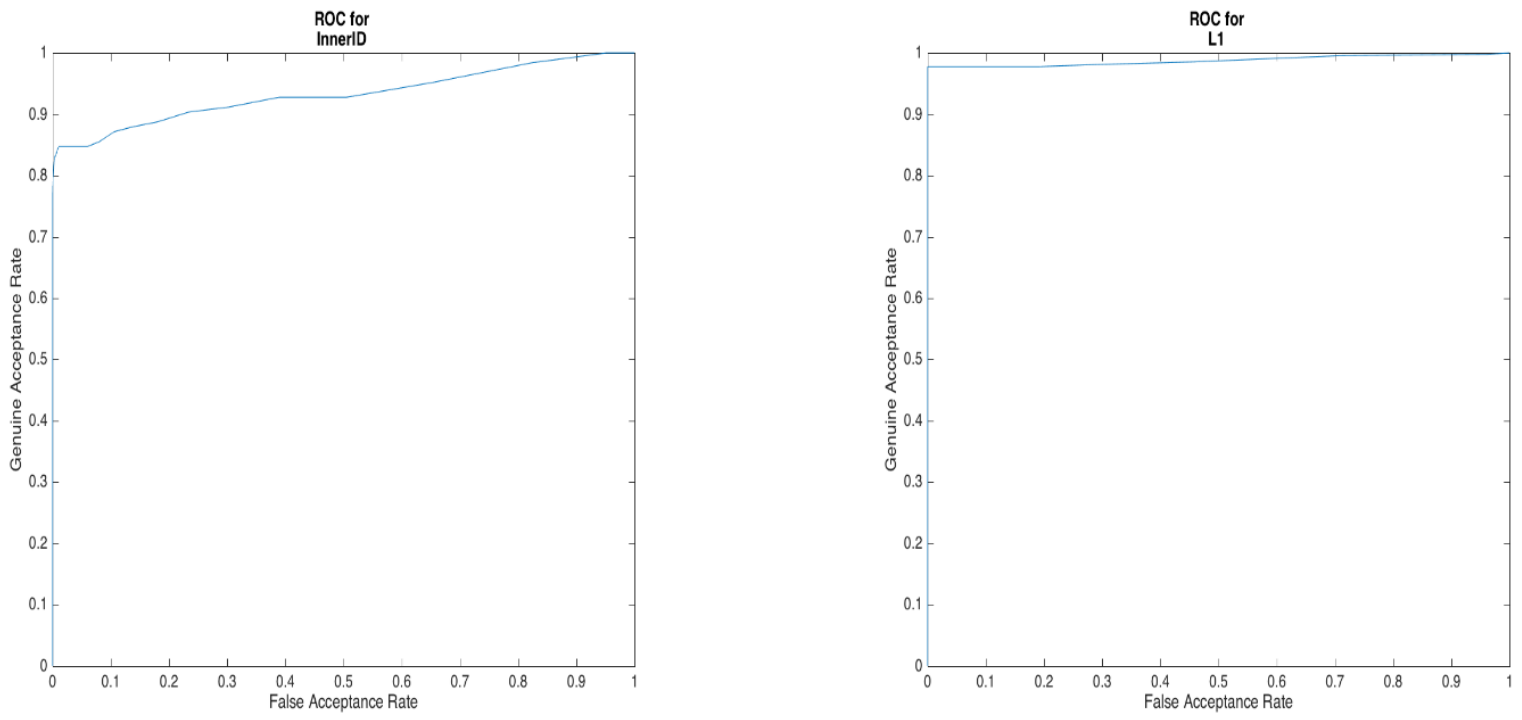

Figure A.8.7 (Left) Neurotech InnerID ROC Curve (Right) Neurotech L1 ROC Curve
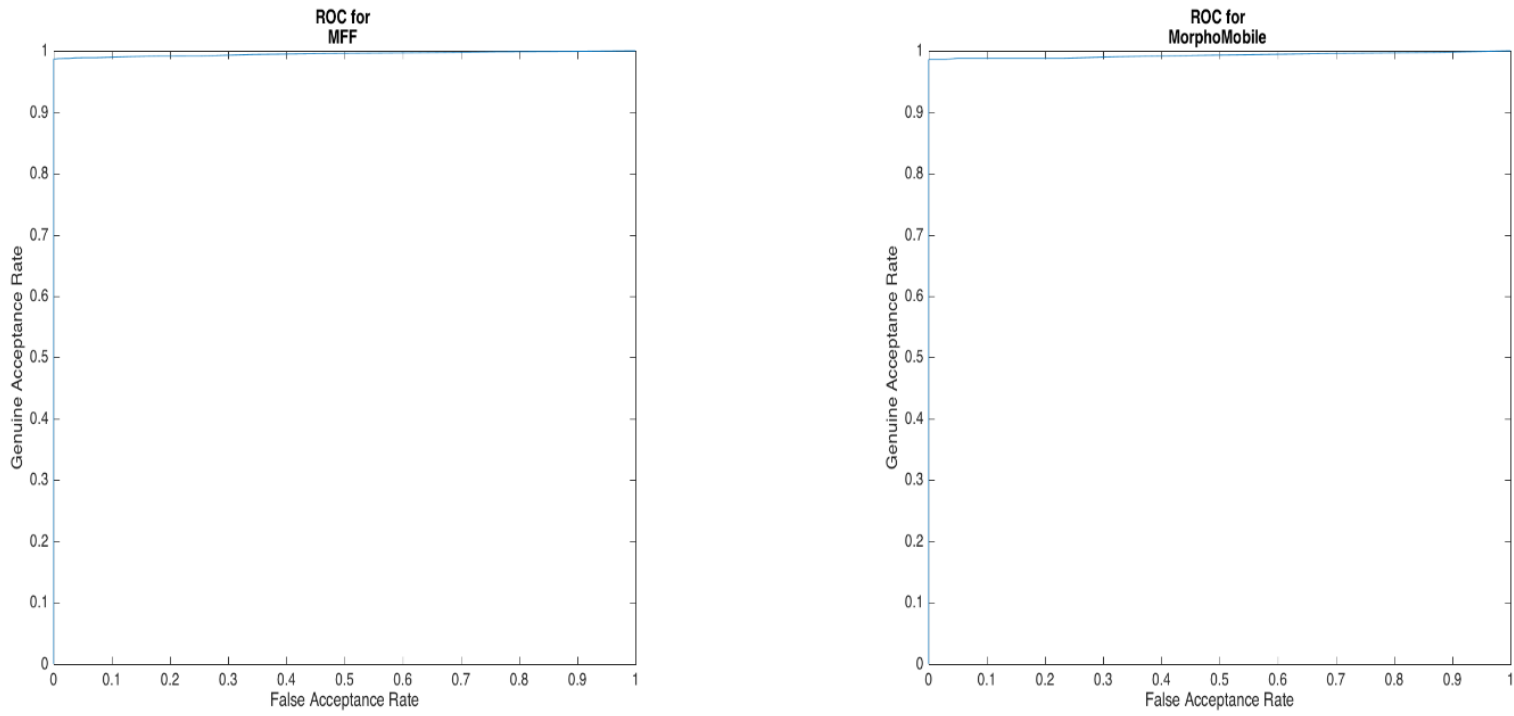

Figure A.8.8 (Left) Neurotech Morpho FOTF ROC Curve (Right) Neurotech MorphoMobile ROC Curve 


\section{A.9 SourceAFIS ROC Curves and Area Under Curve}

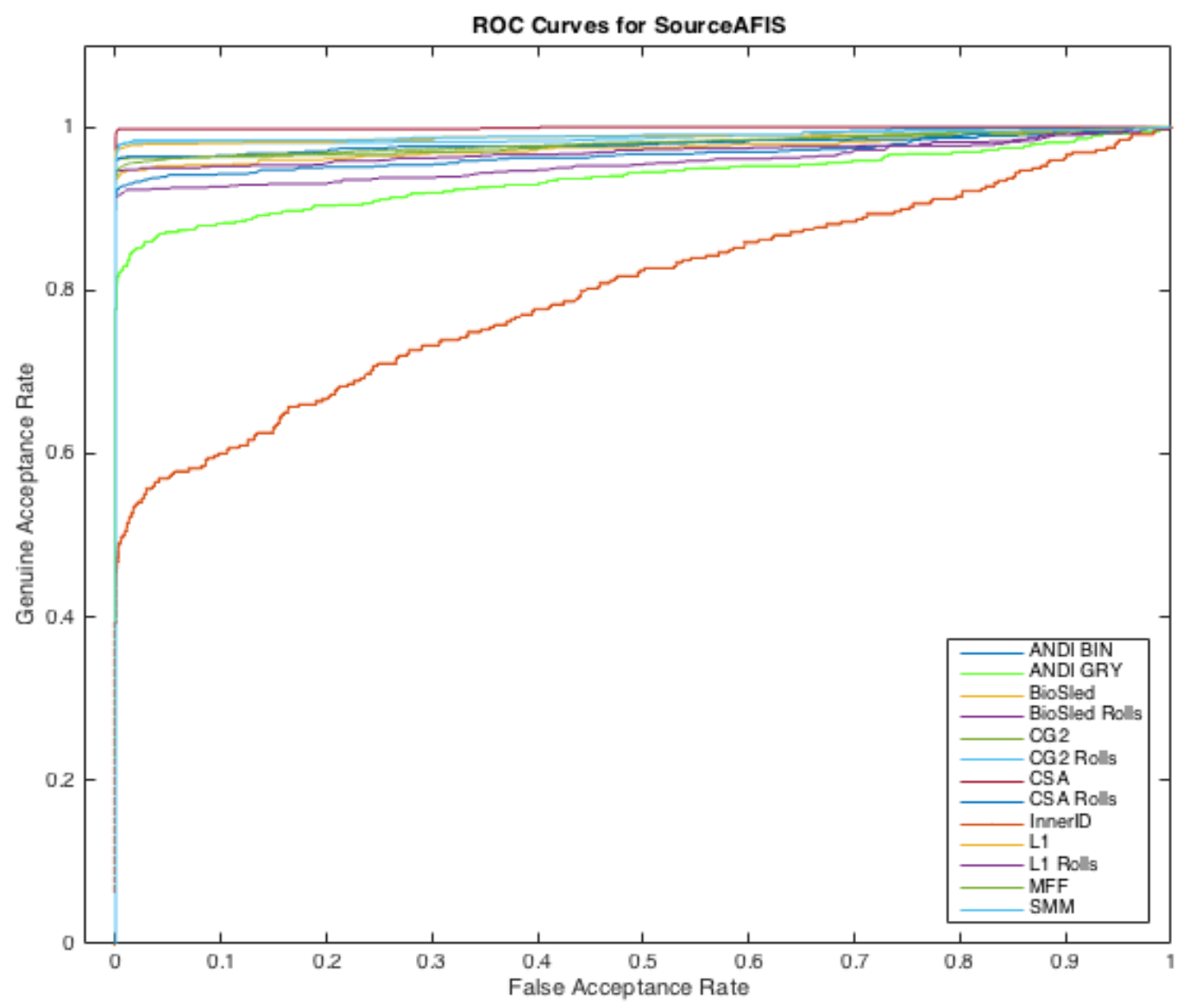

Figure A.9.1 SourceAFIS ROC Curves For each Device 


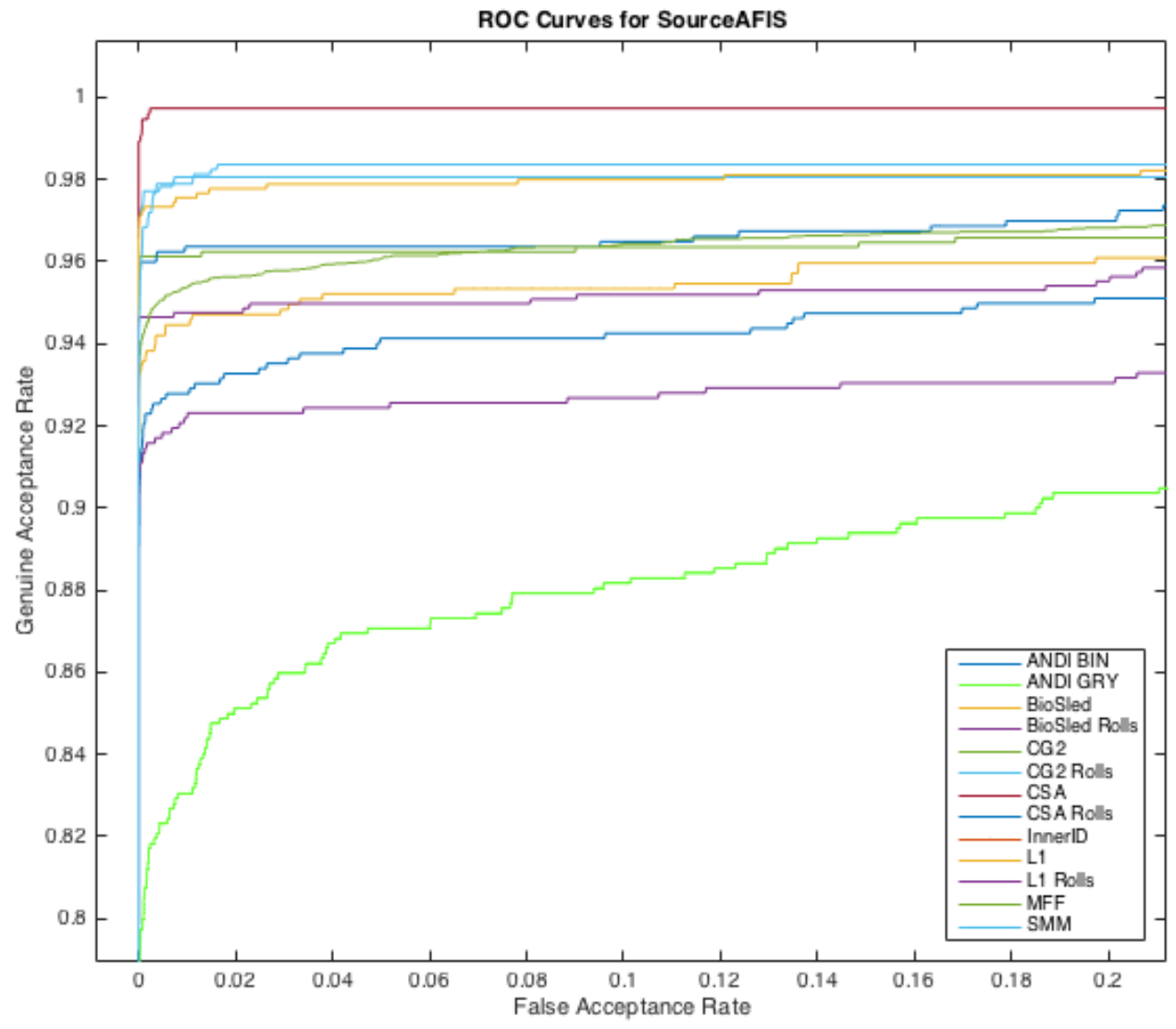

Figure A.9.2 Zoomed In SourceAFIS ROC Curves For each Device

Table A.15 SourceAFIS Area Under the Curve for ROC graphs.

\begin{tabular}{|c|c|}
\hline SourceAFIS Device & Area Under Curve \\
\hline ANDI BIN & 0.966689 \\
\hline$A N D I G R Y$ & 0.936297 \\
\hline Biosled & 0.974903 \\
\hline BioSled Rolls & 0.955877 \\
\hline CG2 & 0.978741 \\
\hline CG2 Rolls & 0.988263 \\
\hline CSA & 0.999 \\
\hline CSA Rolls & 0.97992 \\
\hline InnerID & 0.79834 \\
\hline$L 1$ & 0.98811 \\
\hline L1 Rolls & 0.970133 \\
\hline$M F F$ & 0.98041 \\
\hline MorphoMobile & 0.989864 \\
\hline
\end{tabular}



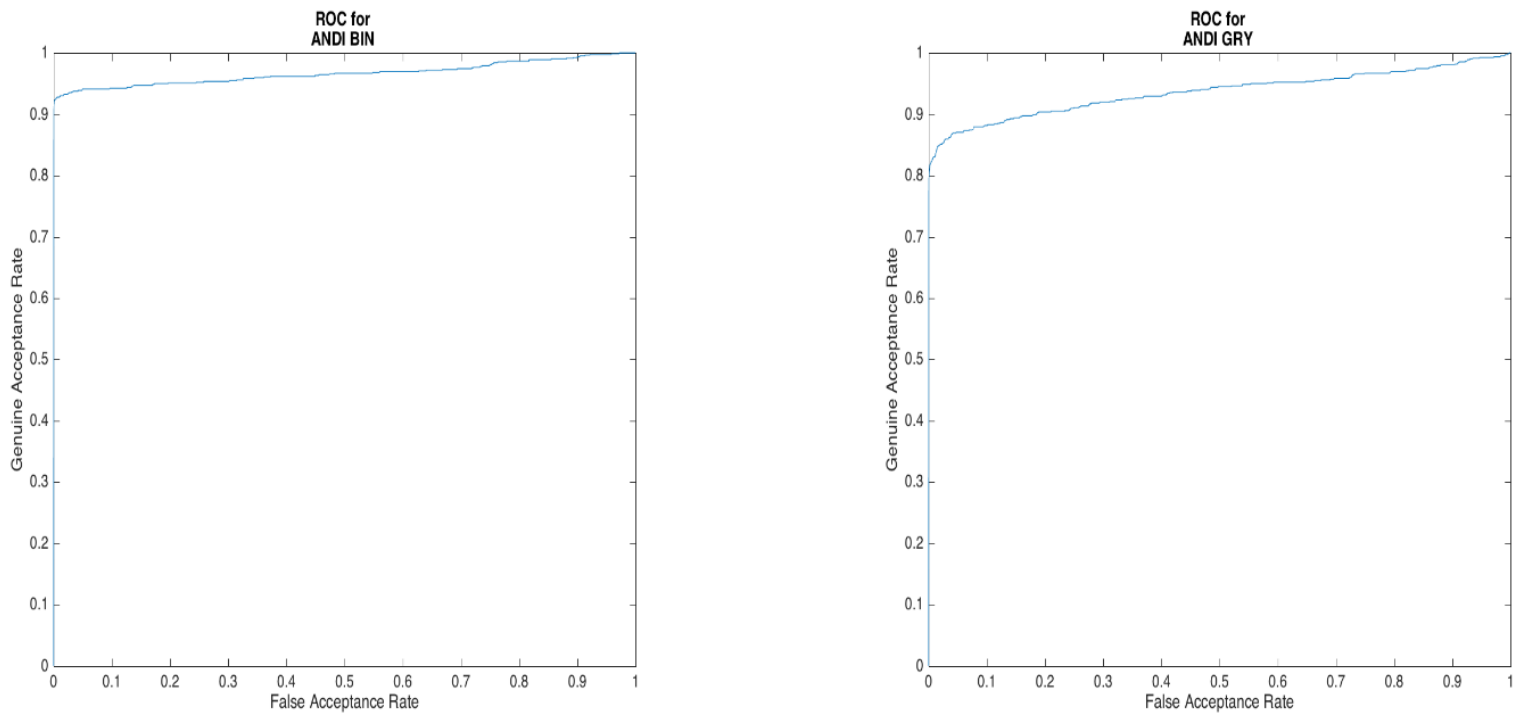

Figure A.9.3 (Left) SourceAFIS ANDI Binary ROC Curve (Right) SourceAFIS ANDI Greyscale ROC Curve
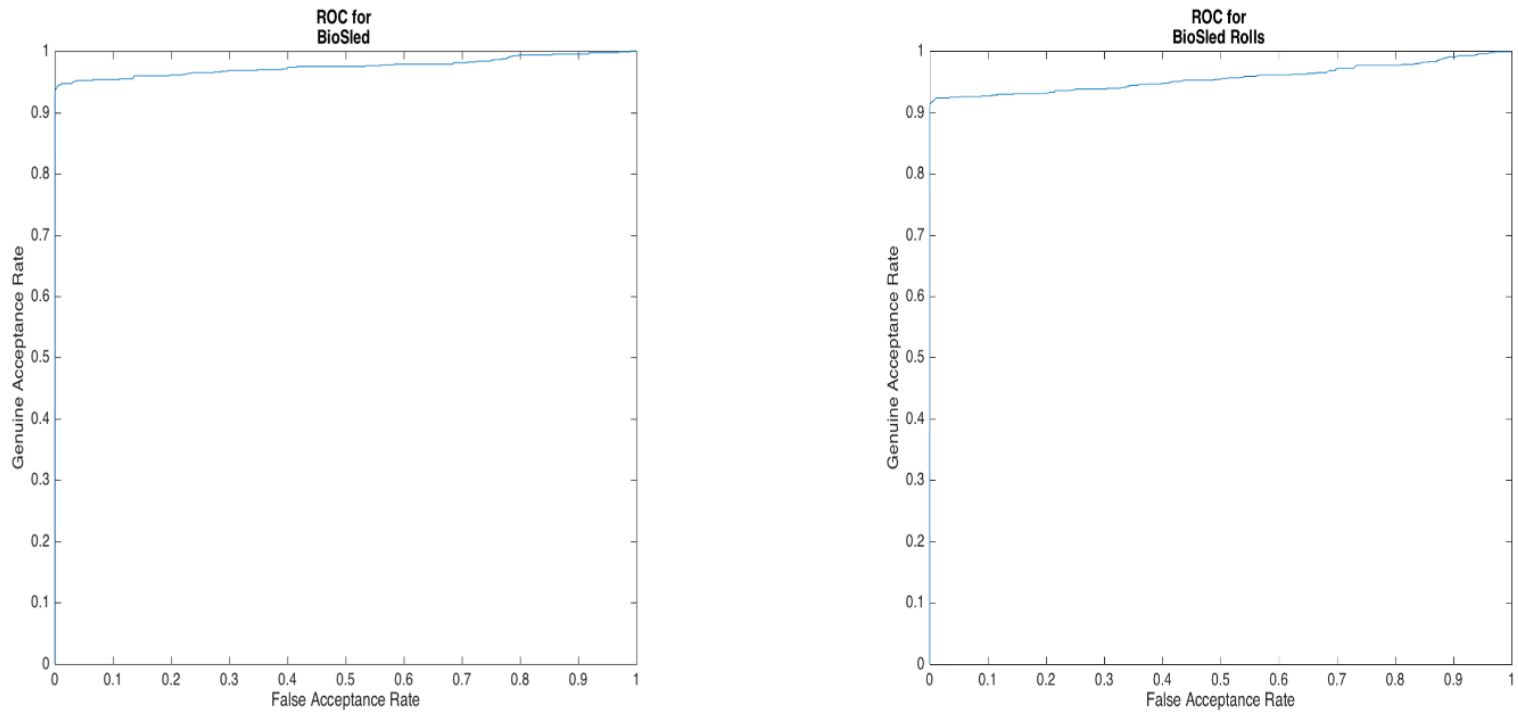

Figure A.9.4 (Left) SourceAFIS BioSled ROC Curve (Right) SourceAFIS BioSled Rolls ROC Curve 

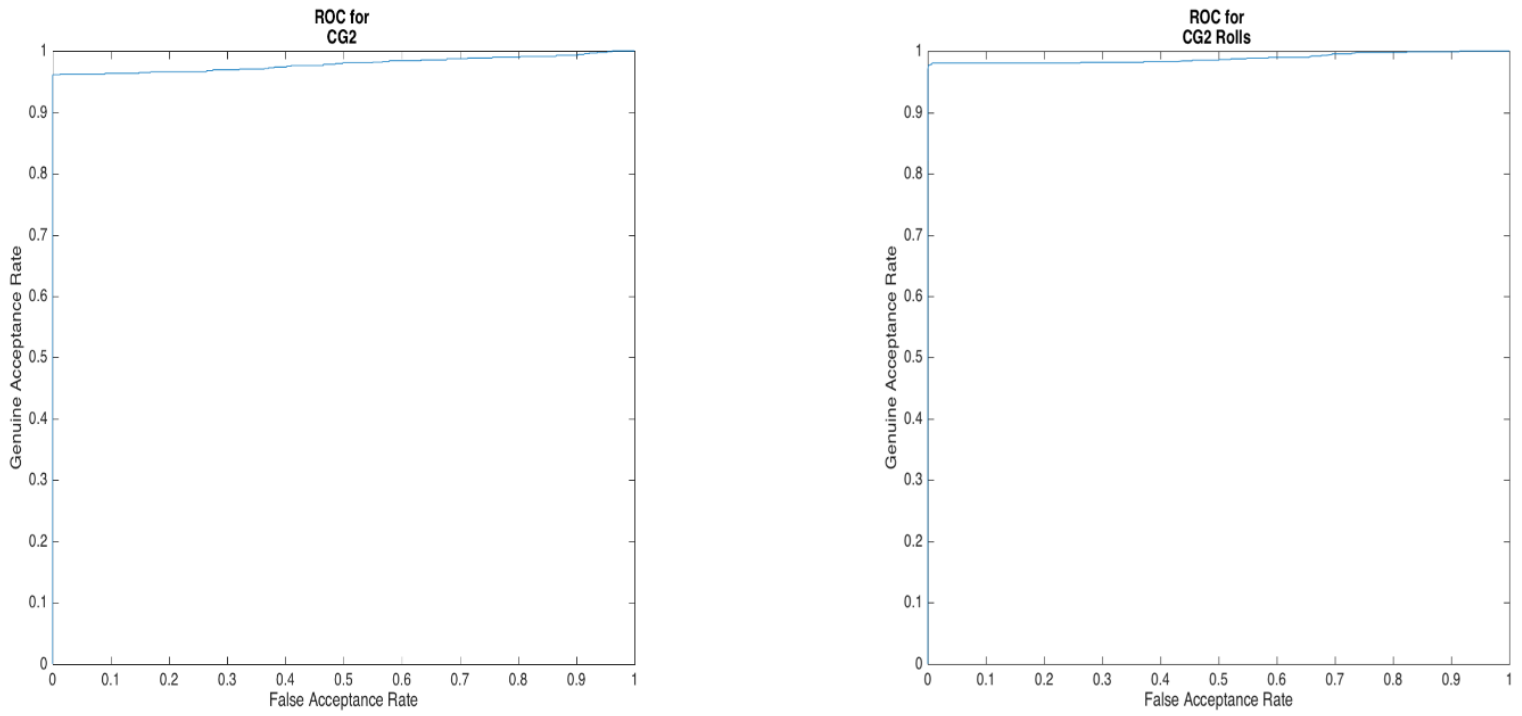

Figure A. 9.5(Left) SourceAFIS FP II Guardian ROC Curve (Right) SourceAFIS FP II Guardian Rolls ROC Curve
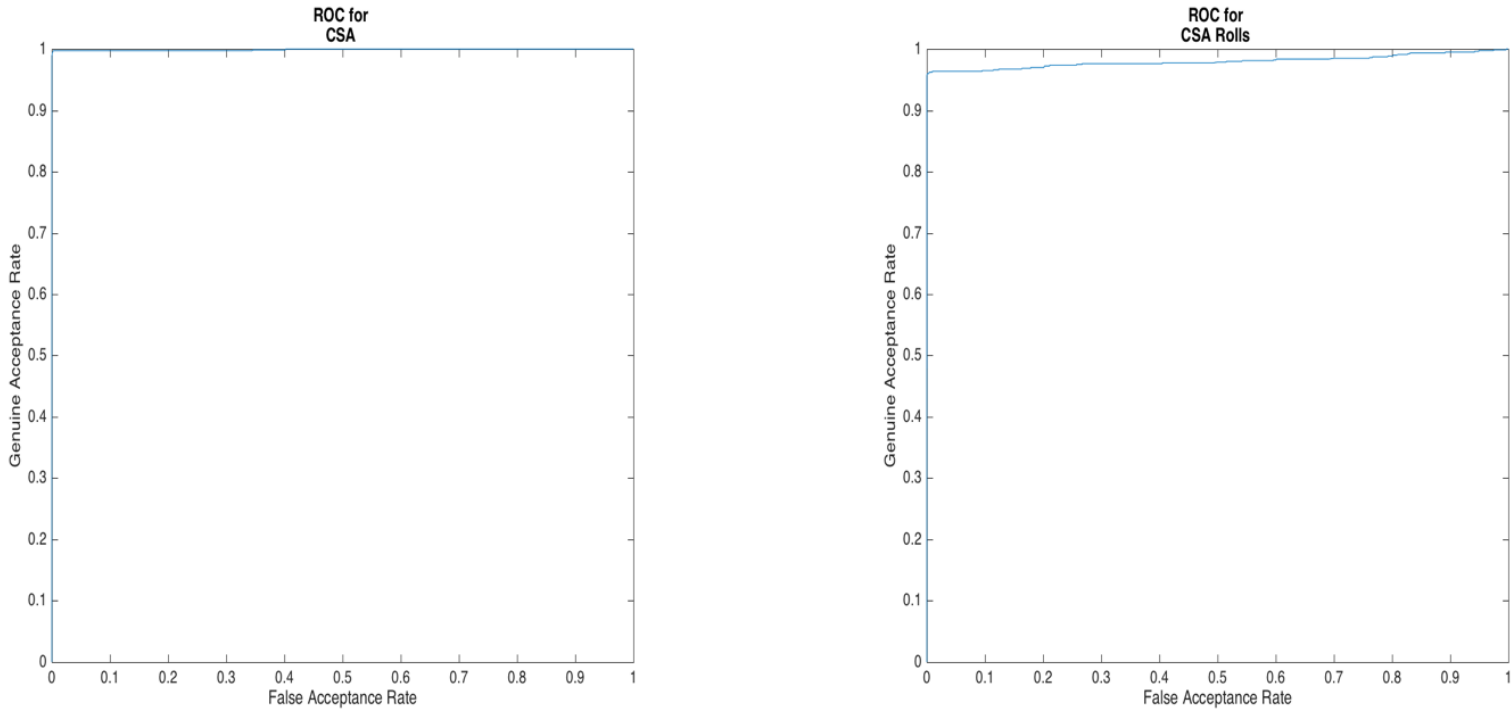

Figure A.9.6 (Left) SourceAFIS Crossmatch Seek Avenger ROC Curve (Right) SourceAFIS Crossmatch Seek Avenger Rolls ROC Curve 

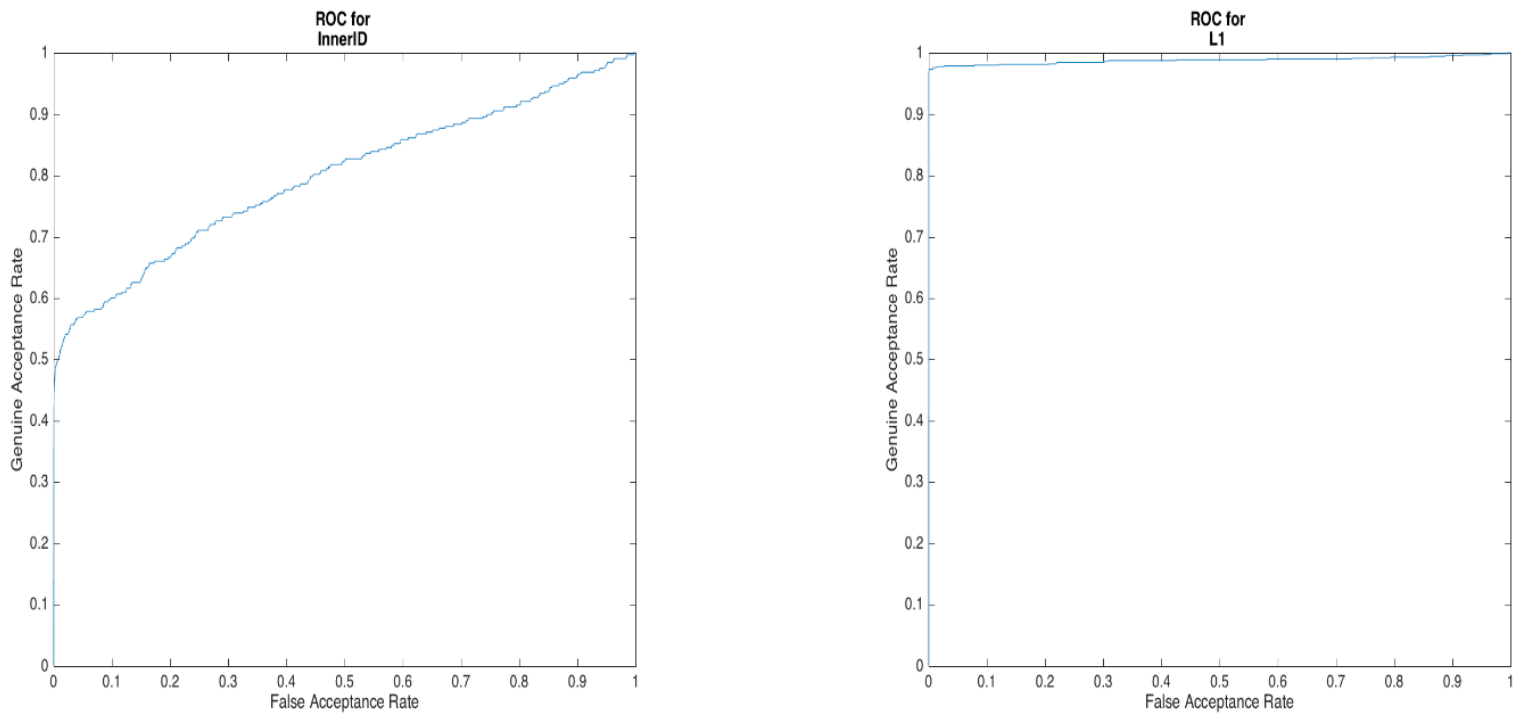

Figure A.9.7 (Left) SourceAFIS InnerID ROC Curve (Right) SourceAFIS L1 ROC Curve
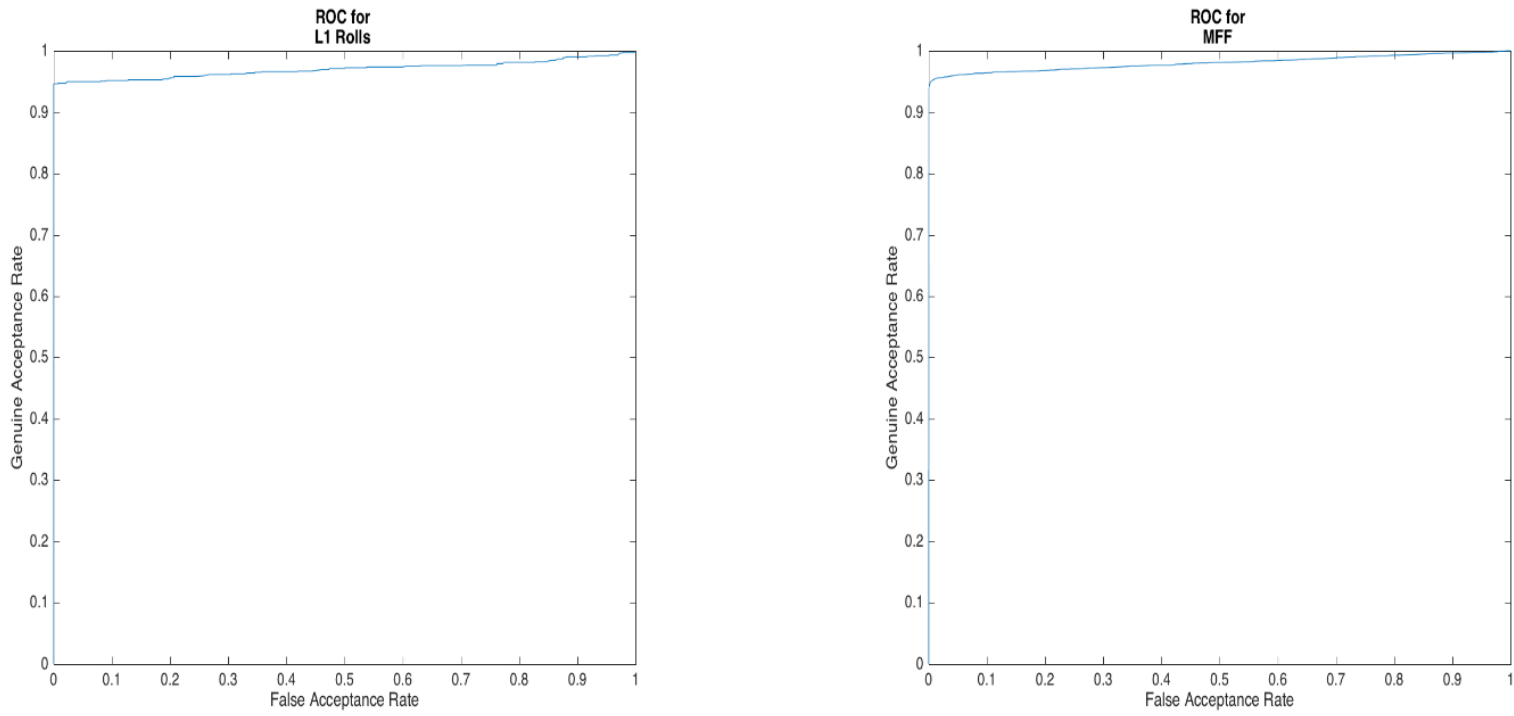

Figure A.9.8 (Left) SourceAFIS L1 Rolls ROC Curve (Right) SourceAFIS Morpho FOTF ROC Curve 


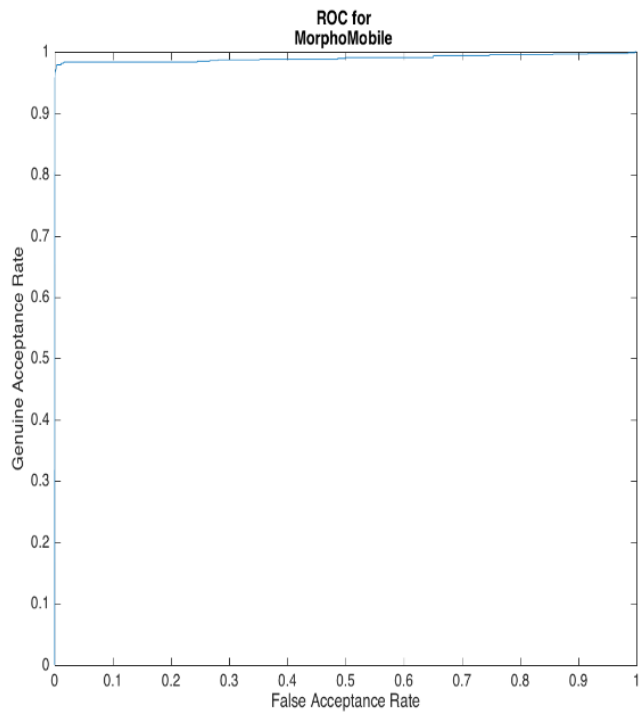

Figure A.9.9 SourceAFIS MorphoMobile ROC Curve

\section{A.10 Equal Error Rates For Each Matcher}


Table A.16 Equal Error Rates for Each Matching Run

\begin{tabular}{|c|c|c|c|}
\hline Match & NBIS EER Value & SourceAFIS EER & Neurotech EER \\
\hline $10 p-10 p$ & 0.0000 & 0.0000 & 0.0000 \\
\hline $10 p-A N D I B I N$ & 0.1207 & 0.1500 & 0.0411 \\
\hline 10p-ANDI GRY & 0.1495 & 0.2401 & 0.0309 \\
\hline $10 p-B I O$ & 0.0778 & 0.1034 & 0.0316 \\
\hline $10 p-C S A$ & 0.0587 & 0.0660 & 0.0123 \\
\hline $10 p-C G 2$ & 0.0558 & 0.0911 & 0.0107 \\
\hline $10 p-L 1$ & 0.0561 & 0.0748 & 0.0100 \\
\hline $10 p-M F F$ & 0.0980 & 0.1829 & 0.0269 \\
\hline $10 p-S M M$ & 0.0806 & 0.1111 & 0.0273 \\
\hline $10 p-I I D$ & 0.4660 & 0.4861 & \\
\hline$A N D I B I N-A N D I B I N$ & 0.0231 & 0.0309 & 0.0171 \\
\hline$A N D I B I N-A N D I G R Y$ & 0.0401 & 0.0782 & 0.0171 \\
\hline$A N D I B I N-B I O$ & 0.0997 & 0.1105 & 0.0479 \\
\hline$A N D I B I N-C S A$ & 0.0723 & 0.0782 & 0.0280 \\
\hline ANDI BIN-CG2 & 0.0769 & 0.0899 & 0.0328 \\
\hline$A N D I B I N-L 1$ & 0.0878 & 0.0996 & 0.0335 \\
\hline$A N D I B I N-M F F$ & 0.1048 & 0.1641 & 0.0377 \\
\hline ANDI BIN-SMM & 0.0919 & 0.0859 & 0.0370 \\
\hline$A N D I B I N-\| I D$ & 0.4714 & 0.4767 & 0.4298 \\
\hline ANDI GRY-ANDI GRY & 0.0345 & 0.0612 & 0.0166 \\
\hline$A N D I G R Y-B I O$ & 0.1549 & 0.1698 & 0.0491 \\
\hline$A N D I G R Y-C S A$ & 0.1178 & 0.1420 & 0.0281 \\
\hline ANDI GRY-CG2 & 0.1155 & 0.1487 & 0.0352 \\
\hline$A N D I G R Y-L 1$ & 0.1249 & 0.1640 & 0.0304 \\
\hline ANDI GRY-MFF & 0.1302 & 0.2232 & 0.0260 \\
\hline ANDI GRY-SMM & 0.1366 & 0.1523 & 0.0357 \\
\hline$A N D I G R Y-\| D$ & 0.4727 & 0.4667 & 0.4841 \\
\hline$B I O-B I O$ & 0.0256 & 0.0238 & 0.0151 \\
\hline$B I O-C S A$ & 0.0378 & 0.0359 & 0.0227 \\
\hline$B 1 O-C G 2$ & 0.0483 & 0.0387 & 0.0229 \\
\hline$B I O-L 1$ & 0.0452 & 0.0392 & 0.0238 \\
\hline$B I O-M F F$ & 0.0950 & 0.1007 & 0.0251 \\
\hline BIO-SMM & 0.0466 & 0.0438 & 0.0232 \\
\hline$B I O-I I D$ & 0.4622 & 0.4748 & 0.4342 \\
\hline CSA-CSA & 0.0049 & 0.0020 & 0.0013 \\
\hline$C S A-C G 2$ & 0.0154 & 0.0137 & 0.0059 \\
\hline CSA-L1 & 0.0181 & 0.0107 & 0.0032 \\
\hline$C S A-M F F$ & 0.0645 & 0.0706 & 0.0134 \\
\hline CSA-SMM & 0.0219 & 0.0166 & 0.0053 \\
\hline$C S A-I I D$ & 0.4490 & 0.4798 & 0.4507 \\
\hline CG2-CG2 & 0.0172 & 0.0189 & 0.0161 \\
\hline$C G 2-L 1$ & 0.0217 & 0.0192 & 0.0129 \\
\hline$C G 2-M F F$ & 0.0566 & 0.0785 & 0.0159 \\
\hline CG2-SMM & 0.0339 & 0.0299 & 0.0114 \\
\hline$C G 2-I I D$ & 0.4700 & 0.4886 & 0.4572 \\
\hline$L 1-L 1$ & 0.0134 & 0.0112 & 0.0112 \\
\hline L1-MFF & 0.0622 & 0.0877 & 0.0129 \\
\hline L1-SMM & 0.0291 & 0.0265 & 0.0089 \\
\hline$M F F-M F F$ & 0.0111 & 0.0295 & 0.0078 \\
\hline MFF-SMM & 0.0837 & 0.0970 & 0.0166 \\
\hline$S M M-S M M$ & 0.0175 & 0.0108 & 0.0070 \\
\hline$I I D-L 1$ & 0.4477 & 0.4817 & 0.4446 \\
\hline IID-MFF & 0.4816 & 0.4960 & 0.4601 \\
\hline IID-SMM & 0.4778 & 0.4810 & 0.4591 \\
\hline$\|D-\| D$ & 0.1392 & 0.1654 & 0.0678 \\
\hline
\end{tabular}


Table A.17 Equal Error Rates For Each Device

\begin{tabular}{|l|lll|}
\hline \multicolumn{4}{|c|}{ EER VALUES FOR THE GENUINE/IMPOSTER RUNS OF EACH DEVICE } \\
\hline MATCH & NBIS & Neurotech & SourceAFIS \\
Andi Binary & 0.0429 & 0.0346 & 0.0587 \\
Andi Greyscale & 0.0594 & 0.0336 & 0.1159 \\
BioSled & 0.0506 & 0.0307 & 0.0479 \\
BioSled Rolls & 0.0398 & 0.0201 & 0.0744 \\
CG2 & 0.0342 & 0.0298 & 0.0376 \\
CG2 Rolls & 0.0137 & 0.0070 & 0.0194 \\
CSA & 0.0088 & 0.0027 & 0.0027 \\
CSA Rolls & 0.0251 & 0.0076 & 0.0363 \\
InnerID & 0.2308 & 0.1239 & 0.2770 \\
L1 & 0.0267 & 0.0223 & 0.0222 \\
Morpho FOTF & 0.0145 & 0.0123 & 0.0405 \\
MorphoMobile & 0.0326 & 0.0141 & 0.0164 \\
\hline
\end{tabular}

Table A.18 Equal Error Rates For Each Rolled Matching Run

\begin{tabular}{|l|ccc|}
\hline \multicolumn{4}{|c|}{ EER VALUES FOR EACH ROLLS MATCHING RUN } \\
MATCH & NBIS & Neurotech & SourceAFIS \\
10p Rolls-10p Rolls & 0.0024 & 0.0000 & 0.0000 \\
10p Rolls-BIO Rolls & 0.0848 & 0.0232 & 0.1567 \\
10p Rolls-CSA Rolls & 0.0678 & 0.0136 & 0.1381 \\
10p Rolls-CG2 Rolls & 0.0753 & 0.0099 & 0.1254 \\
10p Rolls-L1 Rolls & 0.0755 & 0.0127 & 0.1300 \\
BIO Rolls-BIO Rolls & 0.0206 & 0.0099 & 0.0430 \\
BIO Rolls-CSA Rolls & 0.0462 & 0.0149 & 0.0707 \\
BIO Rolls-CG2 Rolls & 0.0452 & 0.0127 & 0.0662 \\
BIO Rolls-L1 Rolls & 0.0469 & 0.0142 & 0.0856 \\
CSA Rolls-CSA Rolls & 0.0130 & 0.0038 & 0.0174 \\
CSA Rolls-CG2 Rolls & 0.0230 & 0.0038 & 0.0396 \\
CSA Rolls-L1 Rolls & 0.0244 & 0.0056 & 0.0529 \\
CG2 Rolls-CG2 Rolls & 0.0080 & 0.0035 & 0.0096 \\
CG2 Rolls-L1 Rolls & 0.0245 & 0.0058 & 0.0456 \\
L1 Rolls-L1 Rolls & 0.0162 & & 0.0259 \\
\hline
\end{tabular}




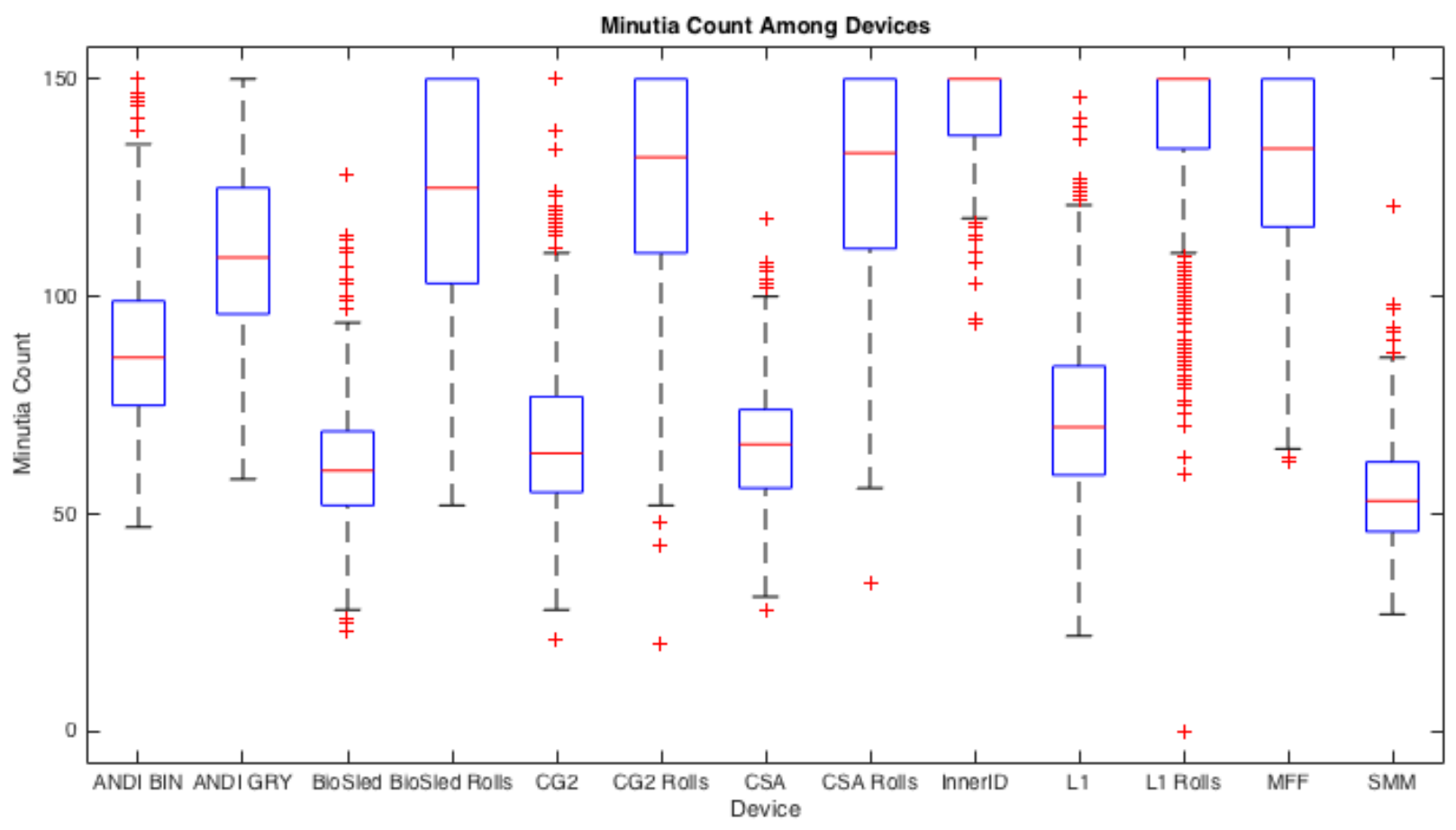

Figure A Minutiae Boxplots For Each Device 
Appendix B

Gender Based

Study 
This appendix shows all of the results from the Gender Based Demographic Study. The genuine and imposter distributions for each matching run and gender are shown in this section. The Receiver Operating Characteristic Curves or ROC Curves are shown for each of the devices used in this collection, a table of values is shown to identify the area under the curve for each ROC curve. All of the results stated above are shown for each of the three matchers used. At the end of this appendix there are box plots that represent the number of minutiae found in images from each device, this data came from the NBIS matcher.

\section{B.1 NBIS Genuine/Imposter Score Distributions}

Table B.1 NBIS Male Genuine and Imposter KLD, Minimum and Maximum Scores

\begin{tabular}{|r|l|l|l|l|l|l|}
\hline NBIS Male Device & $\begin{array}{l}\text { Genuine } \\
\text { KLD }\end{array}$ & $\begin{array}{l}\text { Imposter } \\
\text { KLD }\end{array}$ & $\begin{array}{l}\text { Max } \\
\text { Genuine }\end{array}$ & $\begin{array}{l}\text { Min } \\
\text { Genuine }\end{array}$ & $\begin{array}{l}\text { Max } \\
\text { Imposter }\end{array}$ & $\begin{array}{l}\text { Min } \\
\text { Imposter }\end{array}$ \\
\hline ANDI BIN & 0.0282 & 1.6236 & 319 & 4 & 34 & 0 \\
ANDI GRY & 0.0373 & 0.0893 & 322 & 8 & 70 & 0 \\
BioSled & 0.0328 & 0.0836 & 298 & 4 & 245 & 0 \\
BioSled Rolls & 0.0327 & 0.4227 & 285 & 5 & 184 & 0 \\
CG2 & 0.0272 & 0.1348 & 358 & 4 & 159 & 0 \\
CG2 Rolls & 0.0163 & 0.9924 & 452 & 4 & 262 & 0 \\
CSA & 0.0148 & 1.0866 & 499 & 17 & 48 & 0 \\
CSA Rolls & 0.0217 & 6.4243 & 506 & 5 & 30 & 0 \\
InnerID & 0.0411 & 0.2325 & 176 & 11 & 62 & 4 \\
L1 & 0.0219 & 0.1464 & 328 & 4 & 137 & 0 \\
L1 Rolls & 0.0155 & 0.2235 & 449 & 5 & 143 & 0 \\
MFF & 0.0543 & 0.5960 & 323 & 5 & 48 & 0 \\
\cline { 2 - 4 } & 0.0248 & 0.1028 & 278 & 3 & 201 & 0 \\
\hline Morphome & & & & & & \\
\hline
\end{tabular}


Table B.2 NBIS Female Genuine and Imposter KLD, Minimum and Maximum Scores

\begin{tabular}{|r|l|l|l|l|l|l|}
\hline NBIS Female Device & $\begin{array}{l}\text { Genuine } \\
\text { KLD }\end{array}$ & $\begin{array}{l}\text { Imposter } \\
\text { KLD }\end{array}$ & $\begin{array}{l}\text { Max } \\
\text { Genuine }\end{array}$ & $\begin{array}{l}\text { Min } \\
\text { Genuine }\end{array}$ & $\begin{array}{l}\text { Max } \\
\text { Imposter }\end{array}$ & $\begin{array}{l}\text { Min } \\
\text { Imposter }\end{array}$ \\
\hline ANDI BIN & 0.0316 & 0.7008 & 275 & 6 & 44 & 0 \\
ANDI GRY & 0.0214 & 0.2379 & 358 & 9 & 62 & 3 \\
BioSled & 0.0434 & 3.8267 & 304 & 10 & 57 & 0 \\
BioSled Rolls & 0.0176 & 5.4024 & 317 & 6 & 30 & 0 \\
CG2 & 0.0356 & 7.8629 & 310 & 4 & 50 & 0 \\
CG2 Rolls & 0.0388 & 2.2804 & 367 & 13 & 36 & 0 \\
CSA & 0.1378 & 0.3865 & 280 & 8 & 63 & 0 \\
CSA Rolls & 0.0614 & 3.5796 & 356 & 4 & 31 & 0 \\
InnerID & 0.2519 & 0.1381 & 132 & 7 & 60 & 4 \\
L1 & 0.0221 & 9.0137 & 297 & 4 & 52 & 0 \\
L1 Rolls & 0.0394 & 6.0952 & 384 & 0 & 31 & 0 \\
MFF & 0.0169 & 1.1263 & 360 & 8 & 47 & 3 \\
\hline MorphoMobile & 0.0461 & 1.7152 & 235 & 8 & 38 & 0 \\
\hline
\end{tabular}
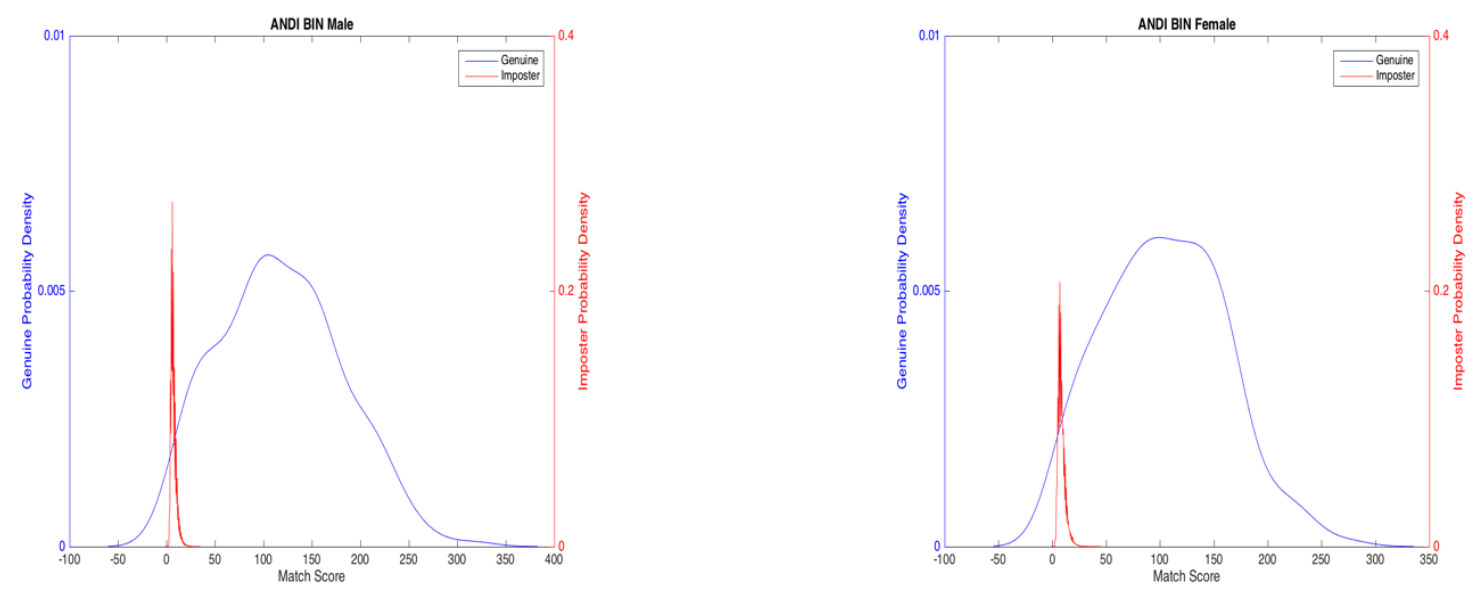

Figure B.1.1 NBIS ANDI Binary Male (Left) Female (Right) 

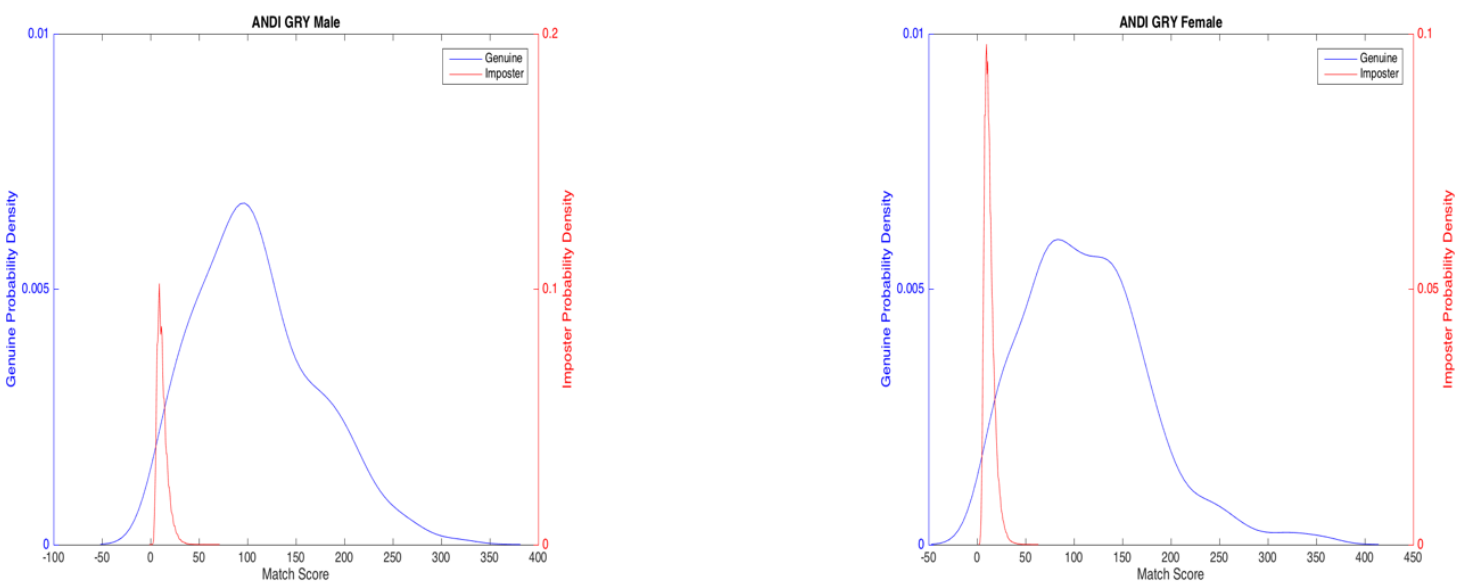

Figure B.1.2 NBIS ANDI Greyscale Male (Left) Female (Right)
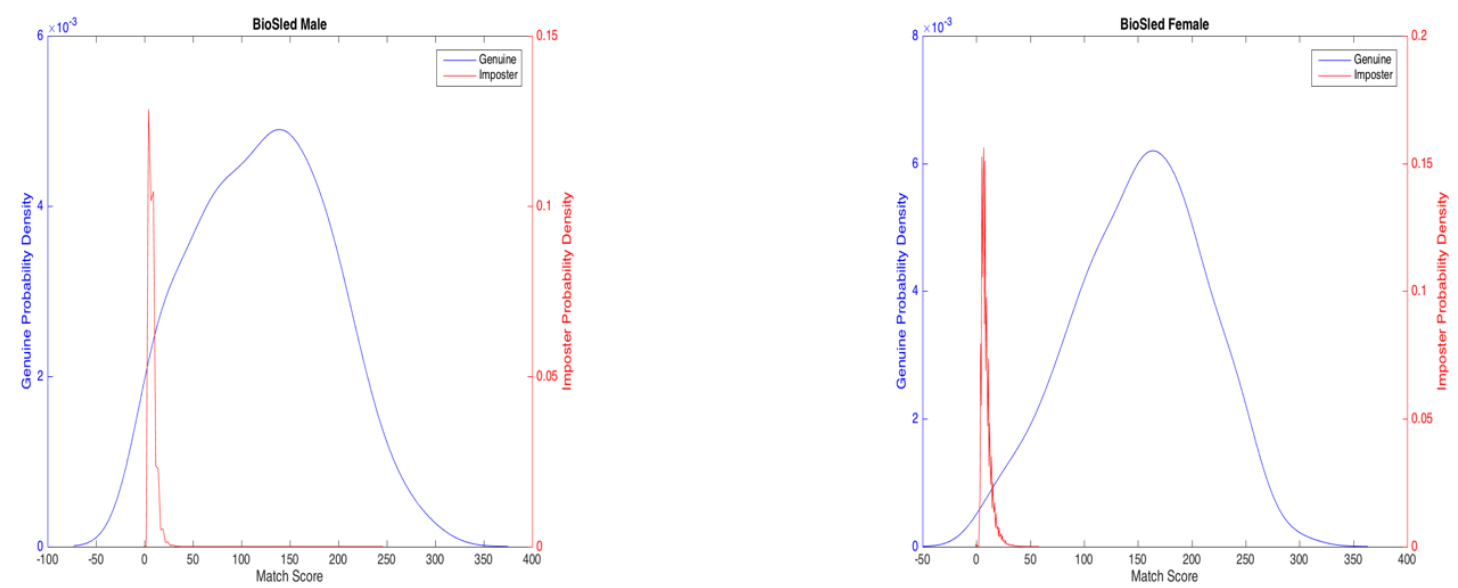

Figure B.1.3 NBIS BioSled Male (Left) Female (Right)
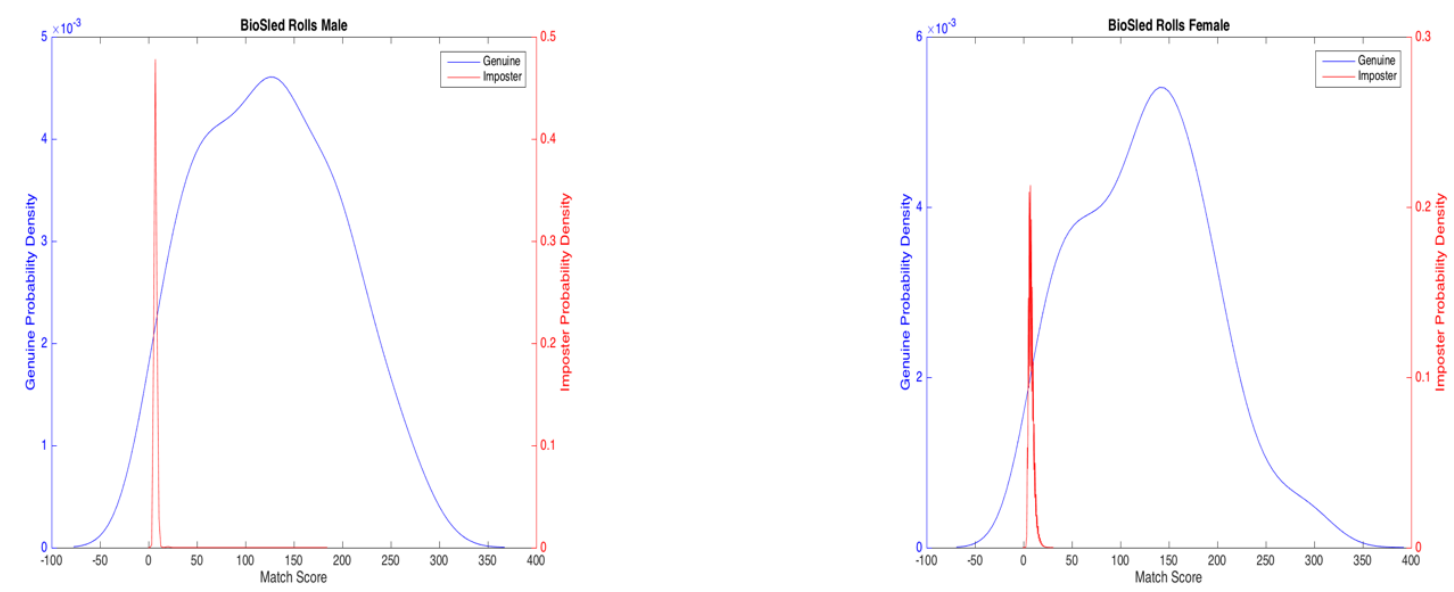

Figure B.1.4 NBIS BioSled Rolls Male (Left) Female (Right) 

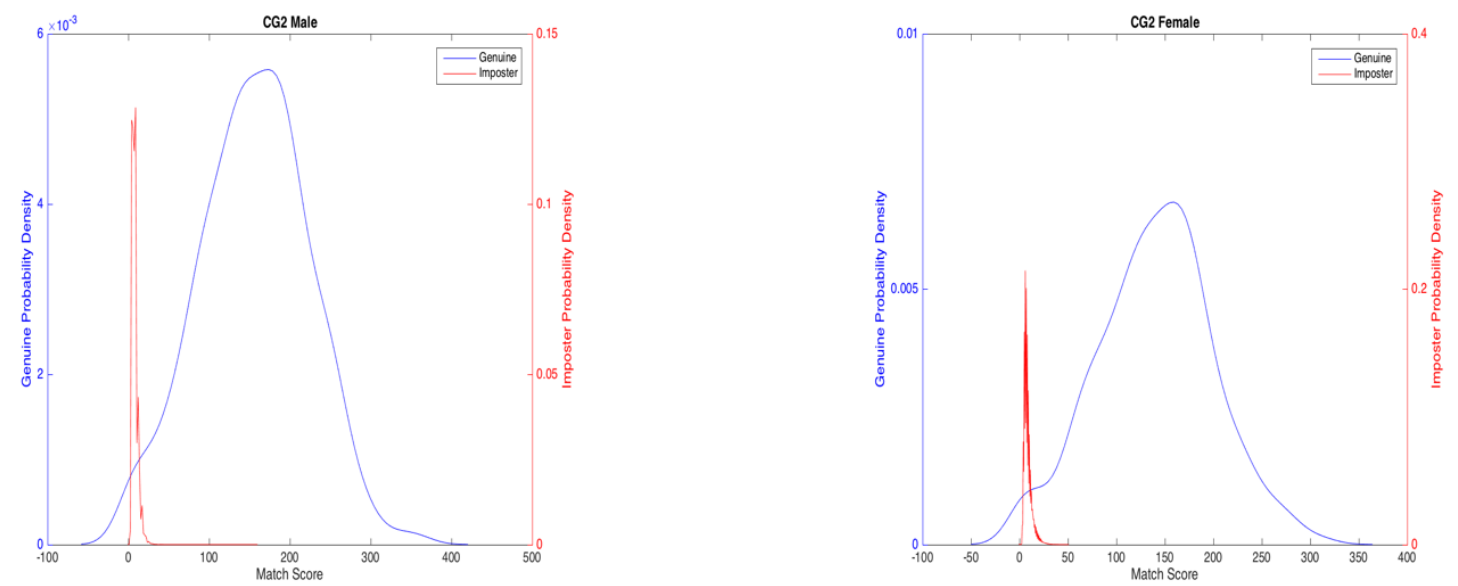

Figure B.1.5 NBIS FP II Guardian Male (Left) Female (Right)
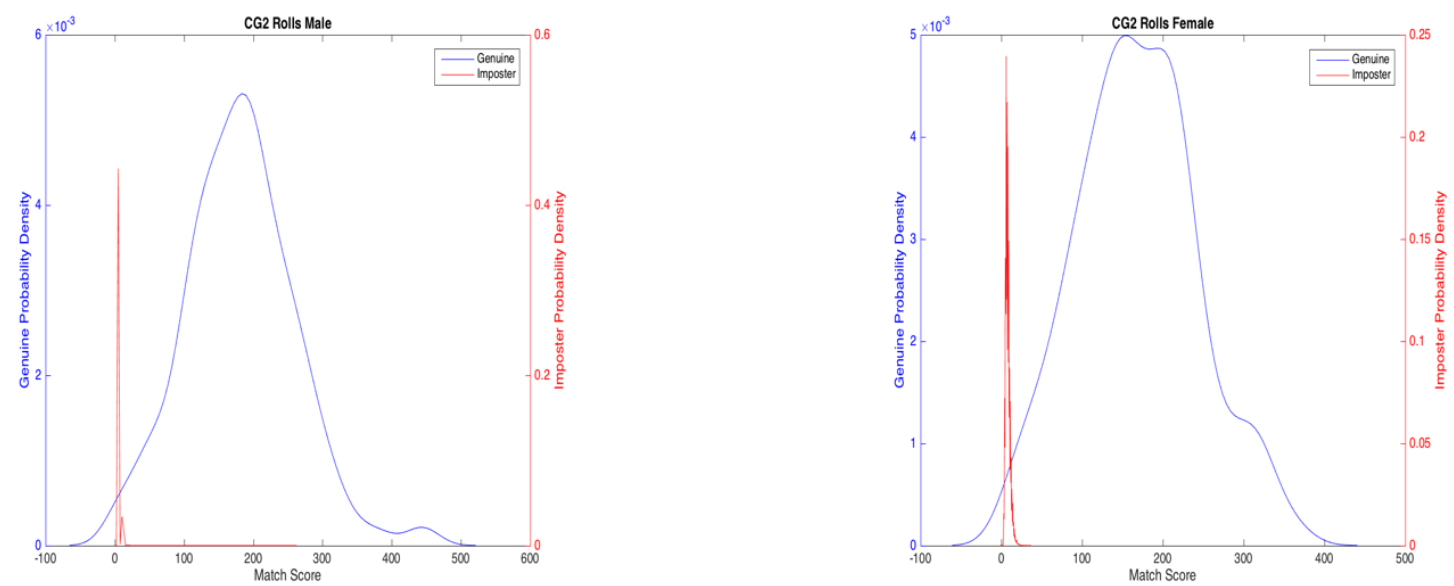

Figure B.1.6 NBIS FP II Guardian Rolls Male (Left) Female (Right)
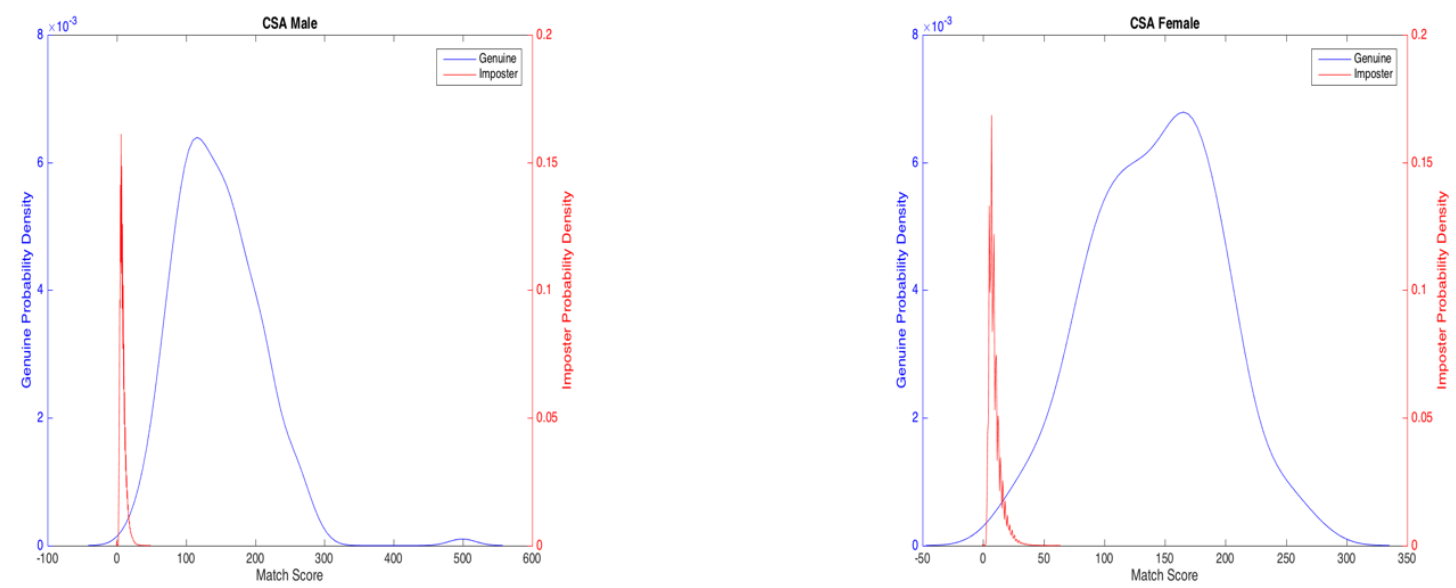

Figure B.1.7 NBIS Crossmatch Seek Avenger Male (Left) Female (Right) 

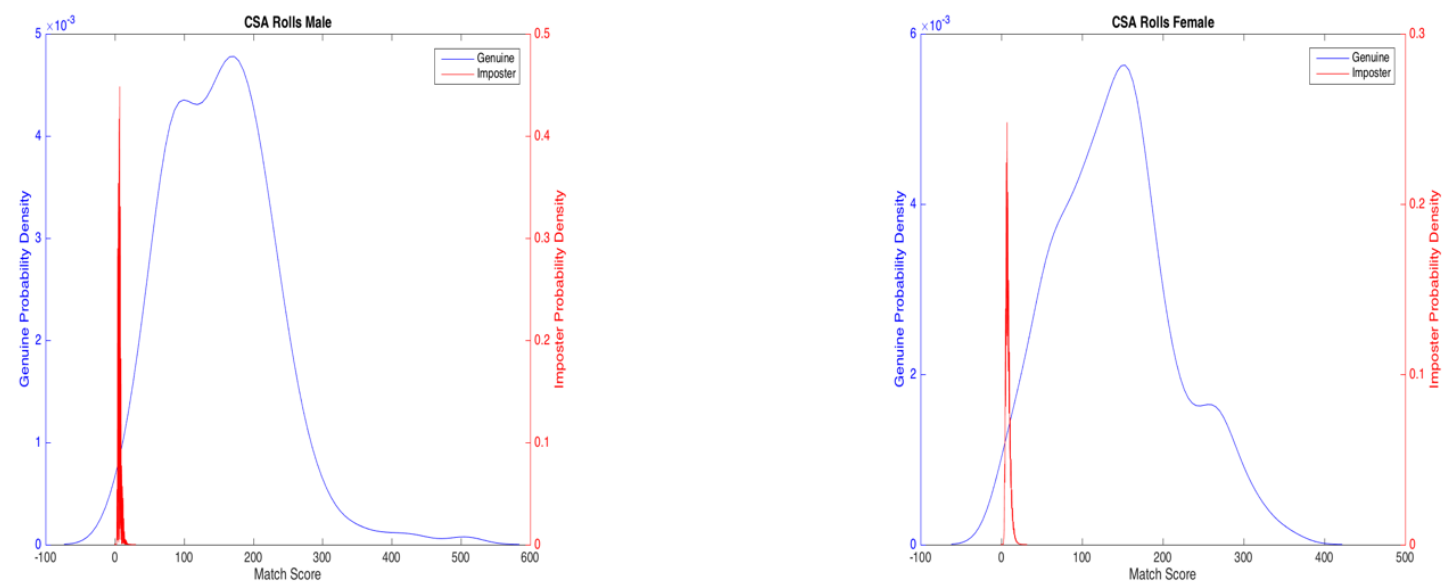

Figure B.1.8 NBIS Crossmatch Seek Avenger Rolls Male (Left) Female (Right)
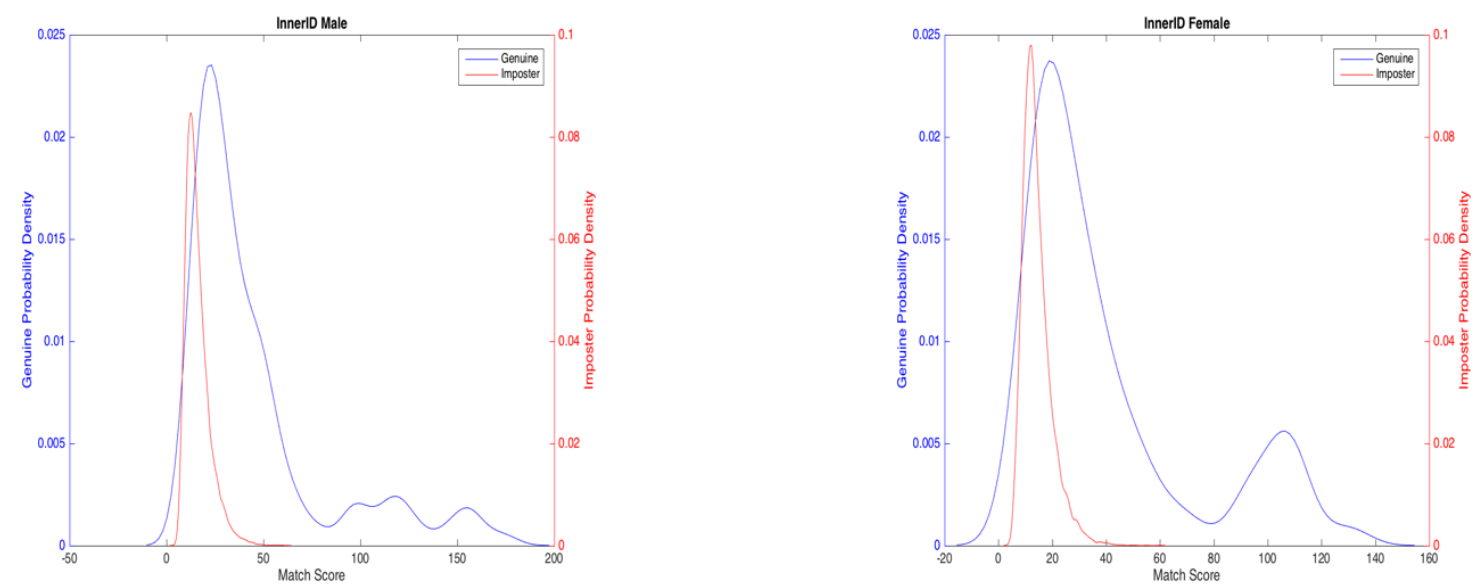

Figure B.1.9 NBIS InnerID Male (Left) Female (Right)
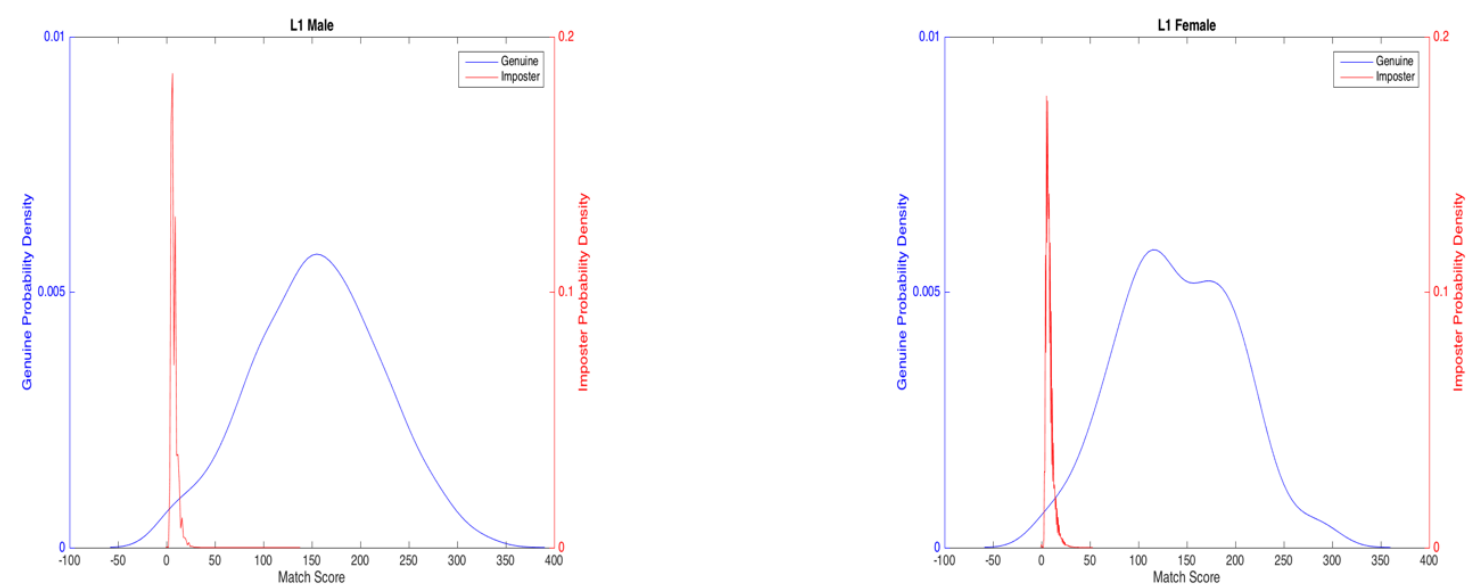

Figure B.1.10 NBIS L1 Male (Left) Female (Right) 

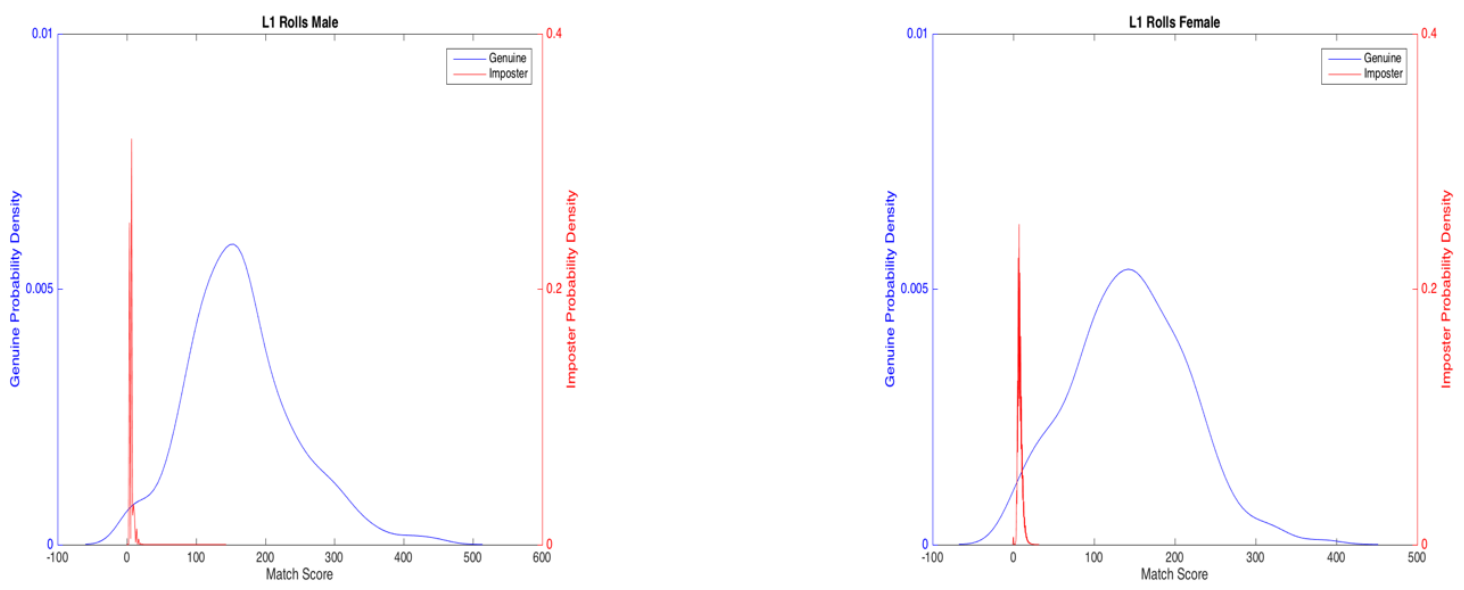

Figure B.1.11 NBIS L1 Rolls Male (Left) Female (Right)
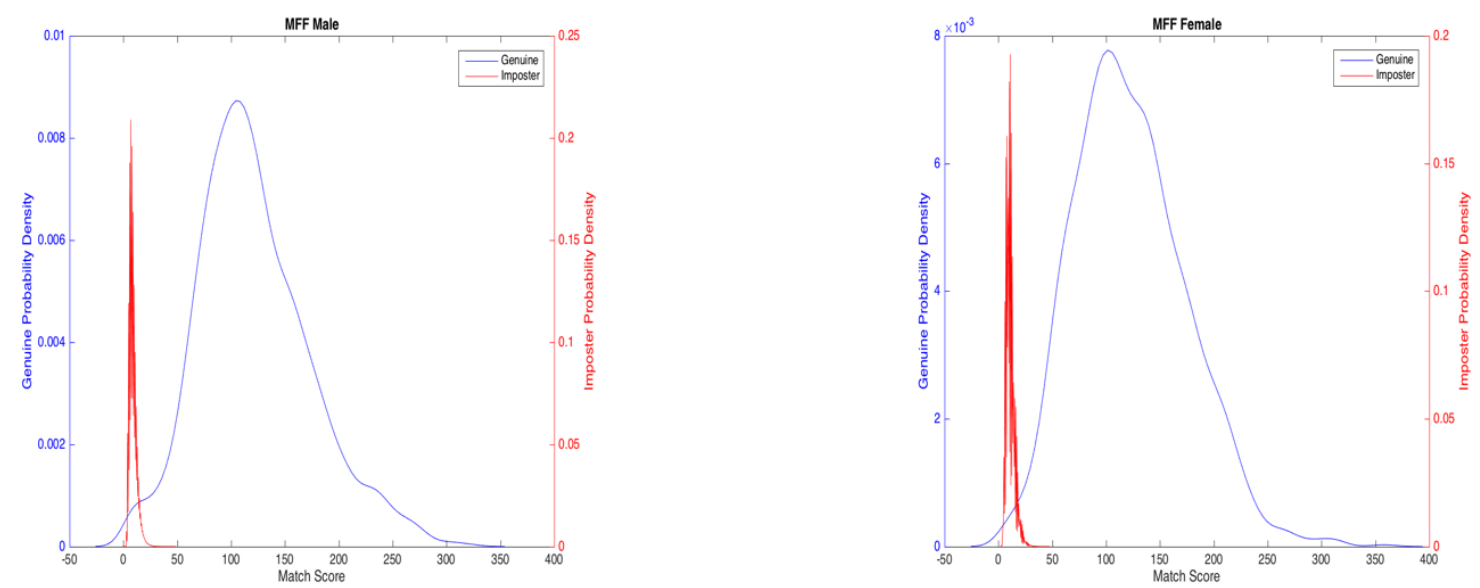

Figure B.1.12 NBIS Morpho FOTF Rolls Male (Left) Female (Right)
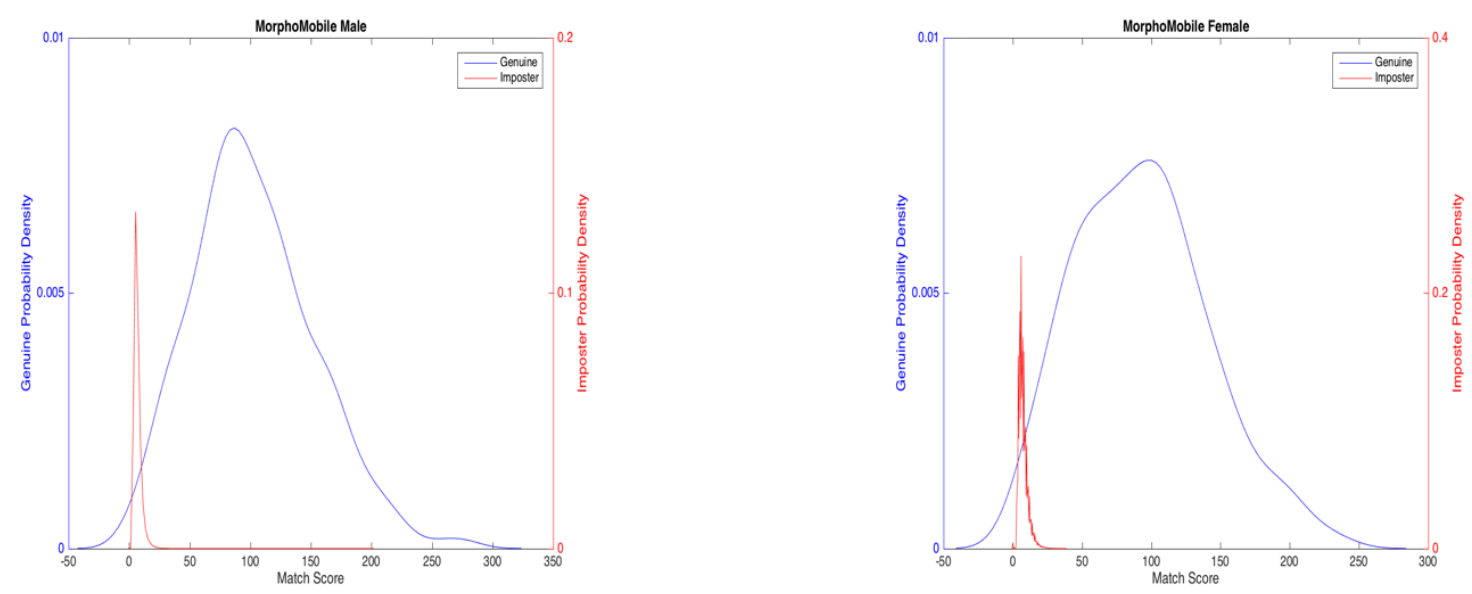

Figure B.1.13 NBIS MorphoMobile Rolls Male (Left) Female (Right) 


\section{B.2 Neurotech Genuine/Imposter Score Distributions}

Table B.3 Neurotech Male Genuine and Imposter KLD, Minimum and Maximum Scores

\begin{tabular}{|c|c|c|c|c|c|c|}
\hline $\begin{array}{r}\text { Neurotech Male } \\
\text { Device }\end{array}$ & $\begin{array}{l}\text { Genuine } \\
\text { KLD }\end{array}$ & $\begin{array}{l}\text { Imposter } \\
\text { KLD }\end{array}$ & $\begin{array}{l}\text { Max } \\
\text { Genuine }\end{array}$ & $\begin{array}{l}\text { Min } \\
\text { Genuine }\end{array}$ & $\begin{array}{l}\text { Max } \\
\text { Imposter }\end{array}$ & $\begin{array}{l}\text { Min } \\
\text { Imposter }\end{array}$ \\
\hline$A N D I B I N$ & 0.0073 & 5.9406 & 986 & 0 & 35 & 0 \\
\hline$A N D I G R Y$ & 0.0044 & 0.1447 & 971 & 3 & 45 & 0 \\
\hline Biosled & 0.0148 & 0.0063 & 951 & 0 & 539 & 0 \\
\hline BioSled Rolls & 0.0080 & 0.0110 & 968 & 0 & 656 & 0 \\
\hline$C G 2$ & 0.0041 & 0.0099 & 1000 & 1 & 641 & 0 \\
\hline CG2 Rolls & 0.0051 & 0.0052 & 1188 & 2 & 658 & 0 \\
\hline CSA & 0.0363 & 5.0927 & 1267 & 201 & 49 & 0 \\
\hline CSA Rolls & 0.0046 & 0.8327 & 1682 & 0 & 26 & 0 \\
\hline InnerID & 0.0135 & 0.3826 & 508 & 3 & 35 & 0 \\
\hline$L 1$ & 0.0052 & 0.0005 & 925 & 0 & 681 & 0 \\
\hline MFF & 0.0087 & 2.2364 & 952 & 2 & 34 & 0 \\
\hline MorphoMobile & 0.0144 & 0.0235 & 681 & 3 & 465 & 0 \\
\hline
\end{tabular}

Table B.4 Neurotech Female Genuine and Imposter KLD, Minimum and Maximum Scores

\begin{tabular}{|c|c|c|c|c|c|c|}
\hline $\begin{array}{c}\text { Neurotech Female } \\
\text { Device }\end{array}$ & $\begin{array}{l}\text { Genuine } \\
\text { KLD }\end{array}$ & $\begin{array}{l}\text { Imposter } \\
\text { KLD }\end{array}$ & $\begin{array}{l}\text { Max } \\
\text { Genuine }\end{array}$ & $\begin{array}{l}\text { Min } \\
\text { Genuine }\end{array}$ & $\begin{array}{l}\text { Max } \\
\text { Imposter }\end{array}$ & $\begin{array}{l}\text { Min } \\
\text { Imposter }\end{array}$ \\
\hline$A N D I B I N$ & 0.0102 & 3.1744 & 868 & 1 & 41 & 0 \\
\hline$A N D I G R Y$ & 0.0041 & 0.2866 & 1003 & 3 & 45 & 0 \\
\hline Biosled & 0.0251 & 0.3071 & 906 & 5 & 45 & 0 \\
\hline BioSled Rolls & 0.0073 & 0.0299 & 993 & 3 & 30 & 0 \\
\hline CG2 & 0.0094 & 0.0807 & 939 & 1 & 218 & 0 \\
\hline CG2 Rolls & 0.0103 & 0.0210 & 1102 & 175 & 29 & 0 \\
\hline CSA & 0.0641 & 0.6460 & 859 & 1 & 58 & 0 \\
\hline CSA Rolls & 0.0309 & 1.2743 & 1035 & 1 & 26 & 0 \\
\hline InnerID & 0.0516 & 2.4966 & 384 & 2 & 30 & 0 \\
\hline$L 1$ & 0.0054 & 0.0032 & 913 & 2 & 48 & 0 \\
\hline$M F F$ & 0.0218 & 0.6446 & 836 & 4 & 58 & 0 \\
\hline MorphoMobile & 0.0116 & 0.2484 & 705 & 1 & 48 & 0 \\
\hline
\end{tabular}



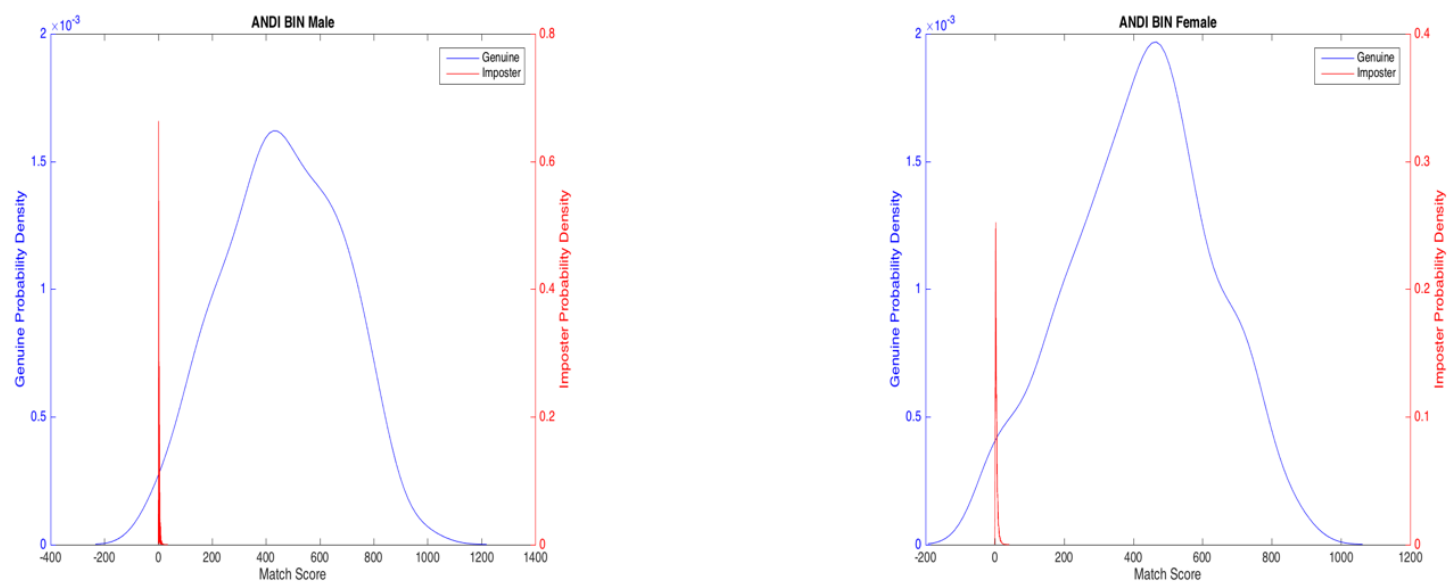

Figure B.2.1 Neurotech ANDI Binary Male (Left) Female (Right)
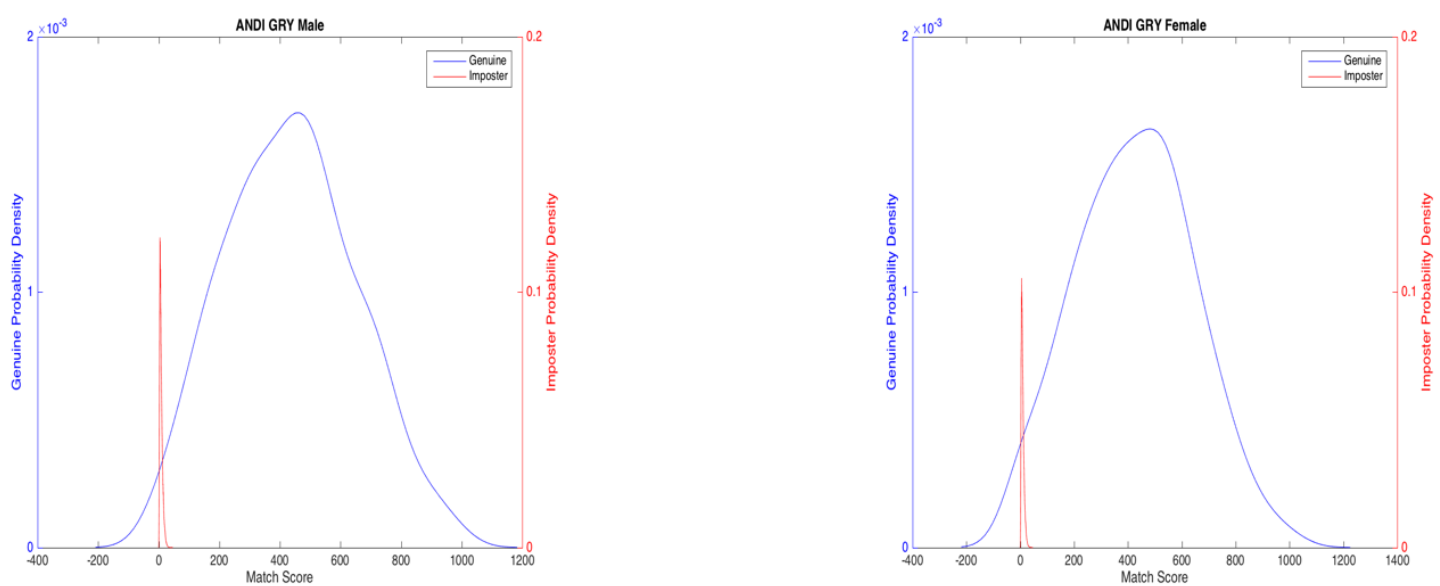

Figure B.2.2 Neurotech ANDI Greyscale Male (Left) Female (Right)
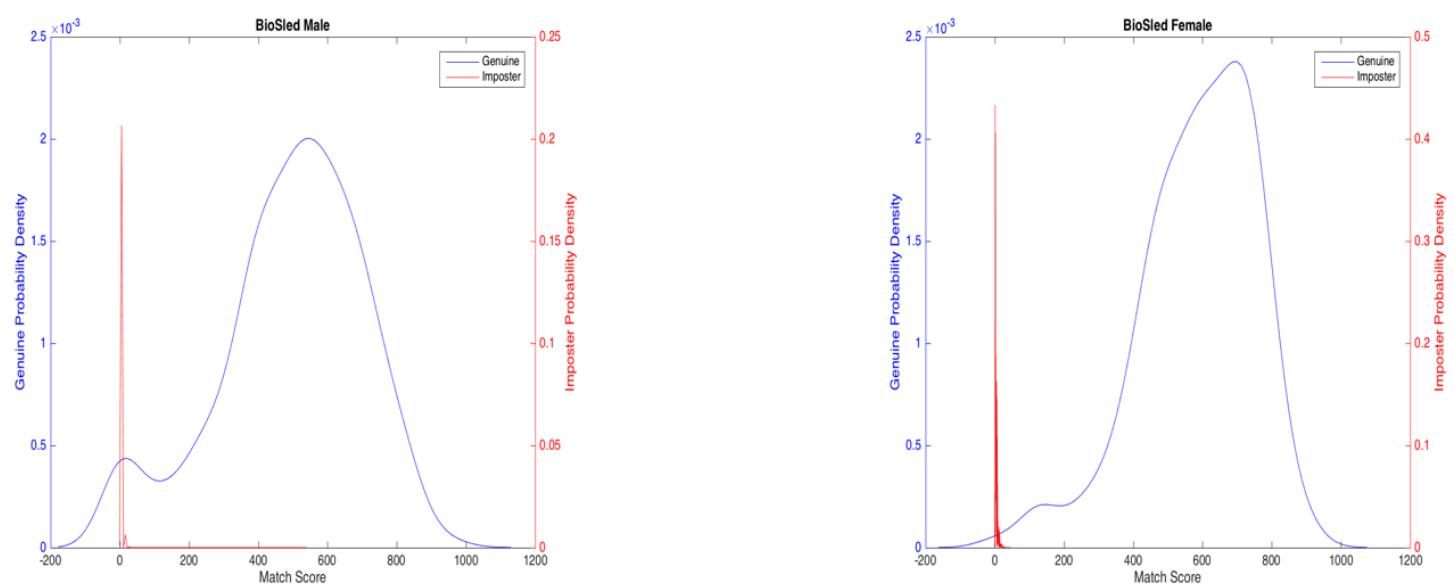

Figure B.2.3 Neurotech BioSled Male (Left) Female (Right) 

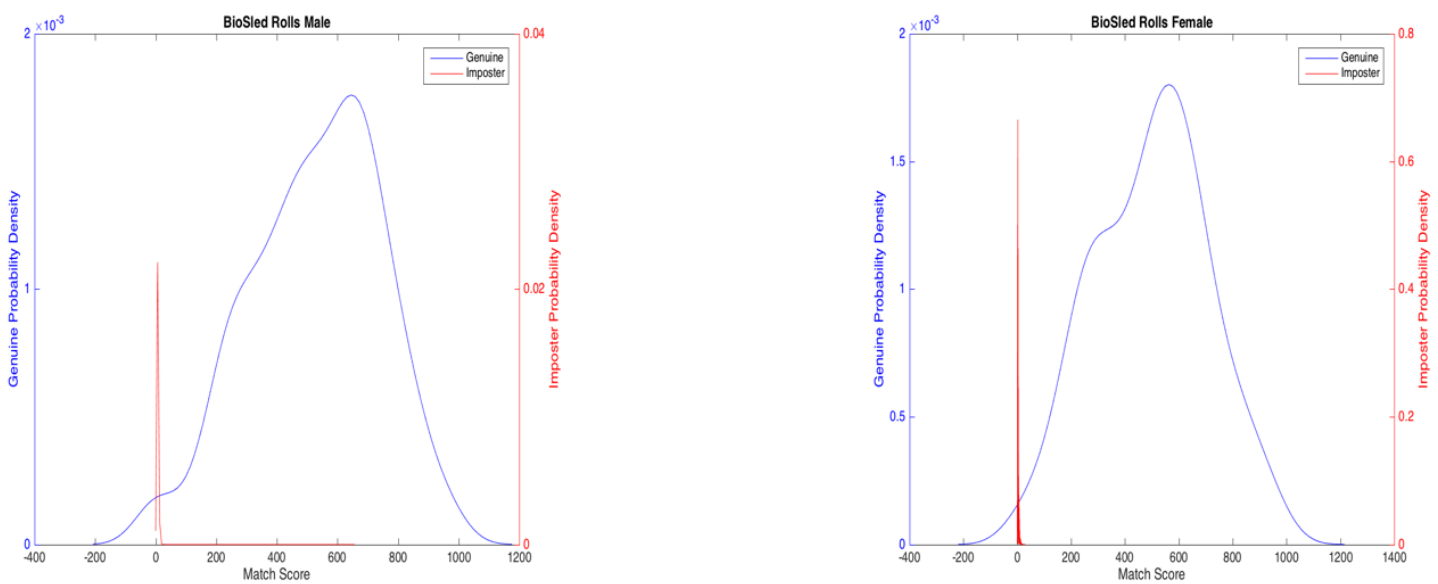

Figure B.2.4 Neurotech BioSled Rolls Male (Left) Female (Right)
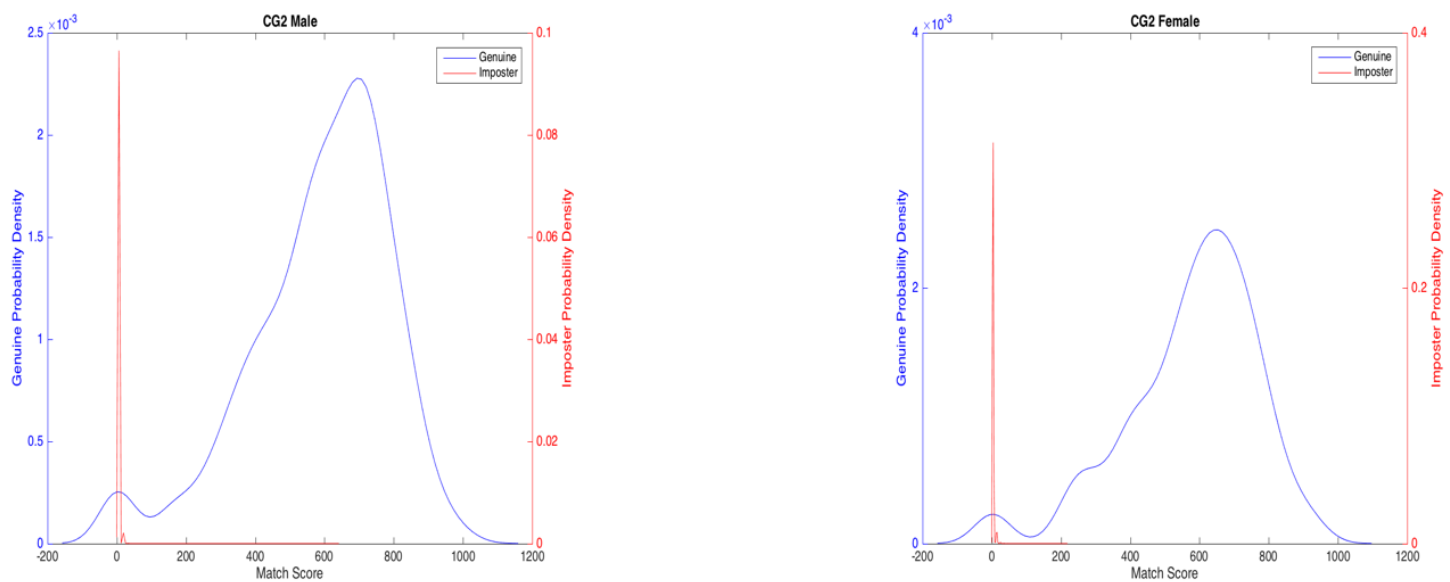

Figure B.2.5 Neurotech FP II Guardian Male (Left) Female (Right)
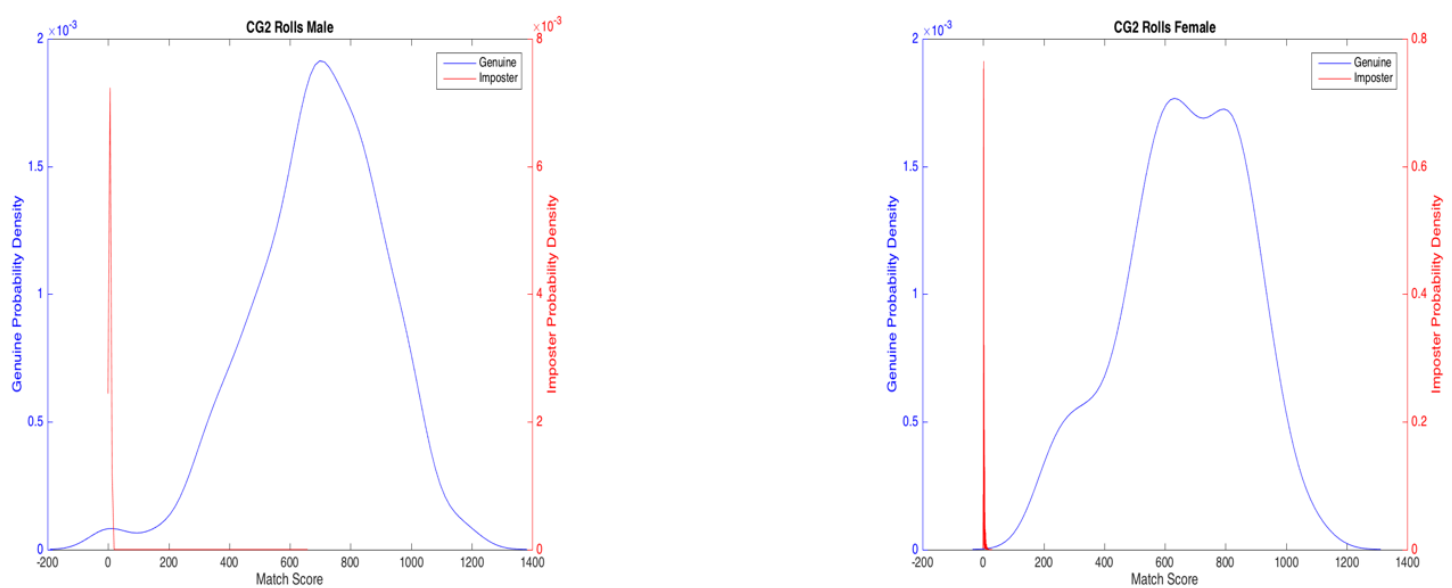

Figure B.2.6 Neurotech FP II Guardian Rolls Male (Left) Female (Right) 

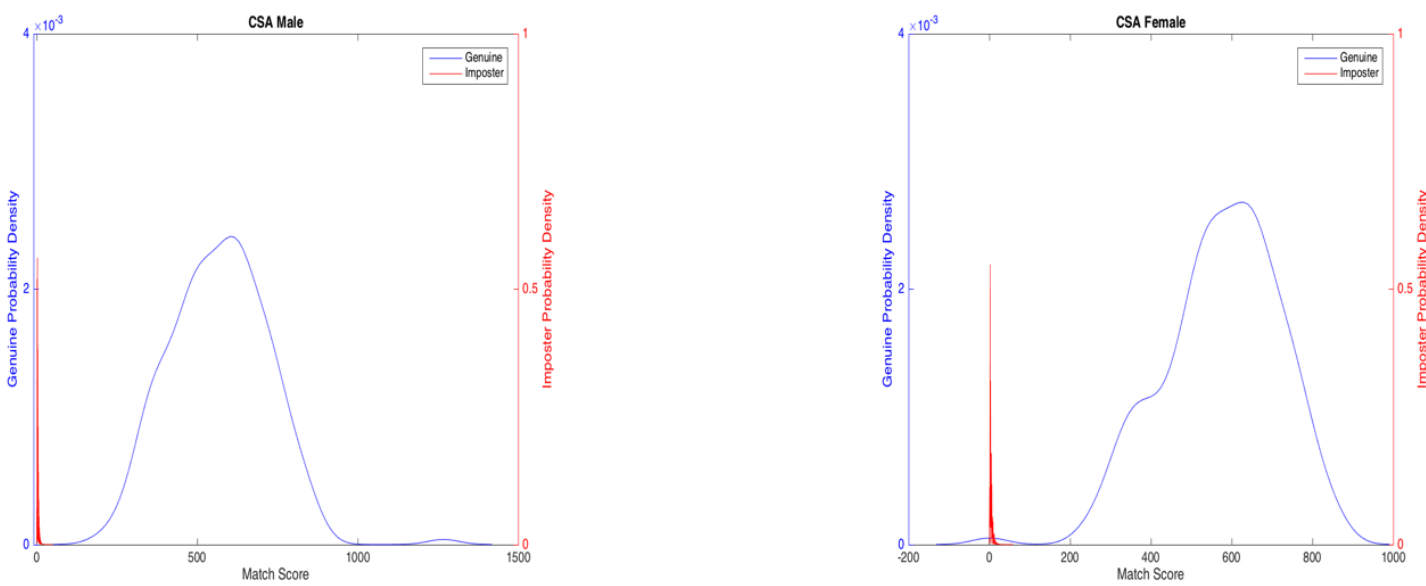

Figure B.2.7 Neurotech Crossmatch Seek Avenger Male (Left) Female (Right)
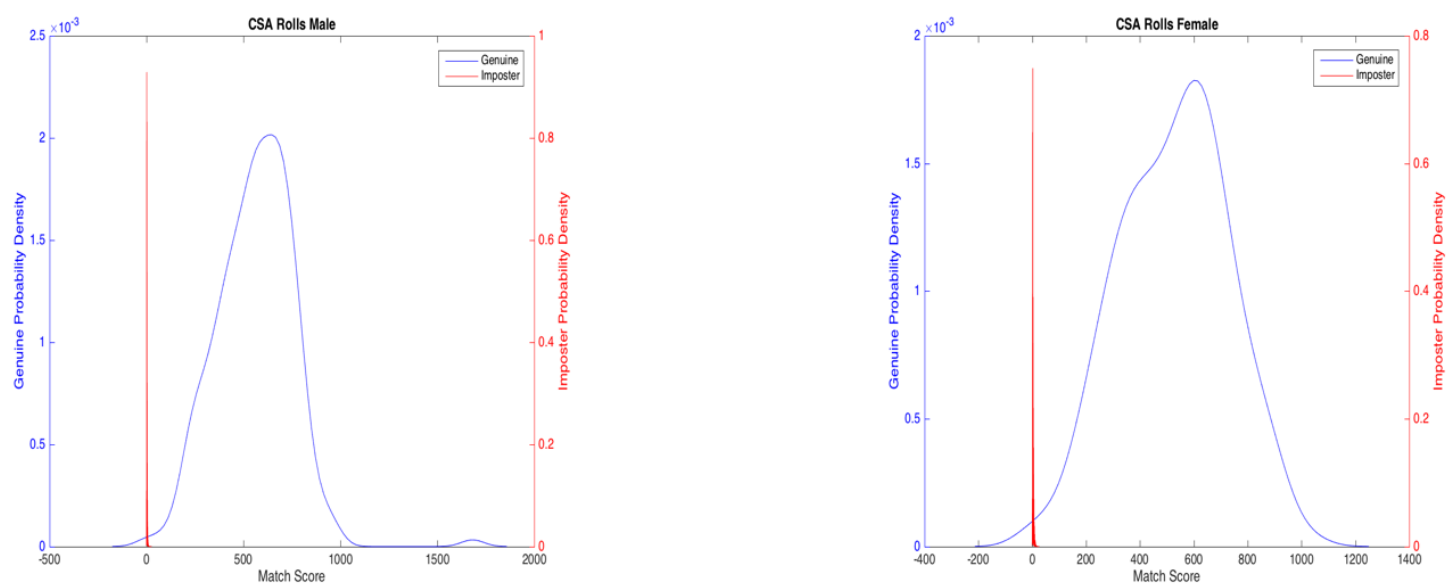

Figure B.2.8 Neurotech Crossmatch Seek Avenger Rolls Male (Left) Female (Right)
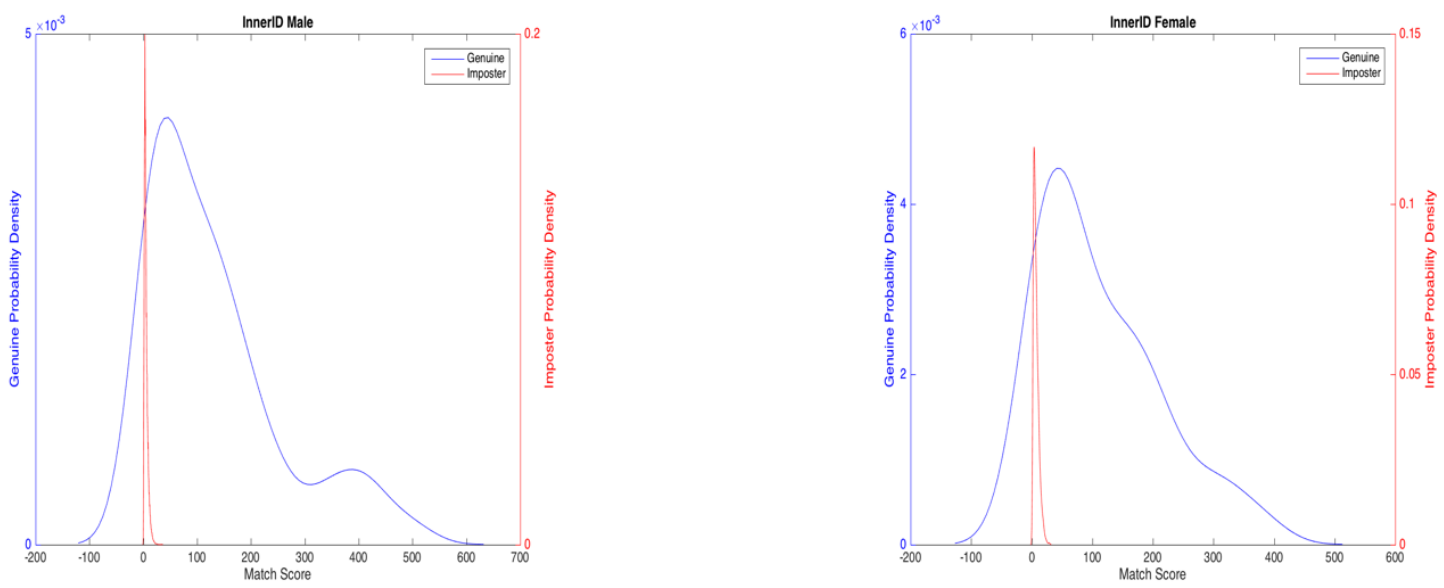

Figure B.2.9 Neurotech InnerID Male (Left) Female (Right) 

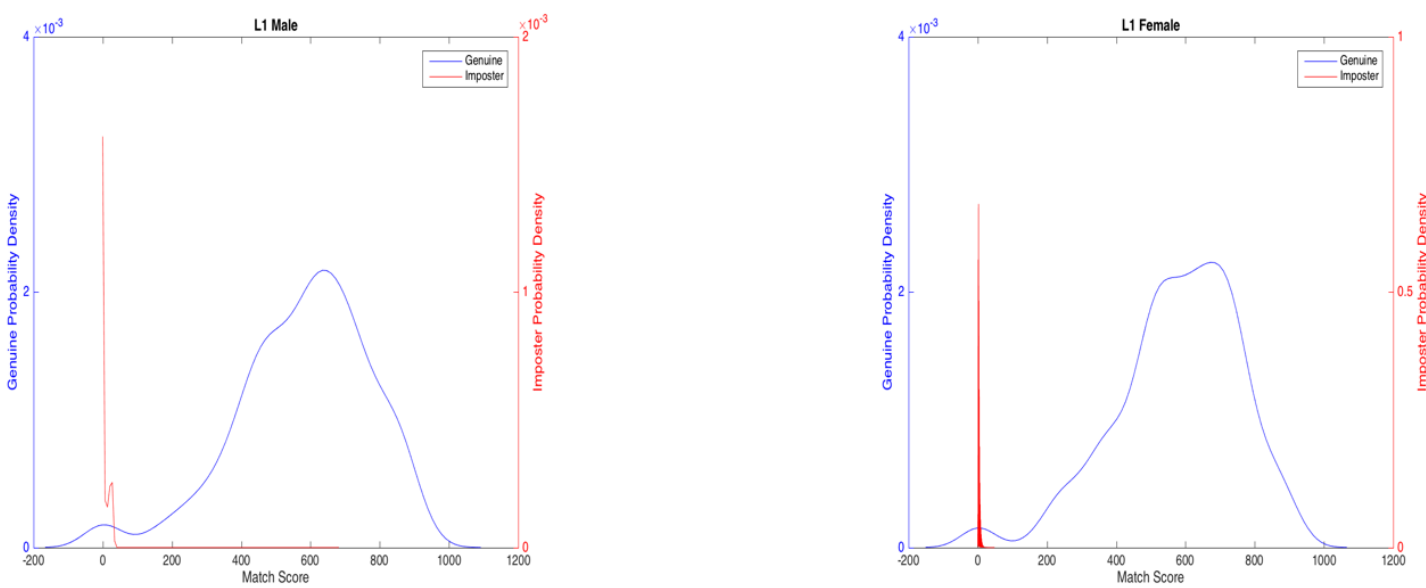

Figure B.2.10 Neurotech L1 Male (Left) Female (Right)
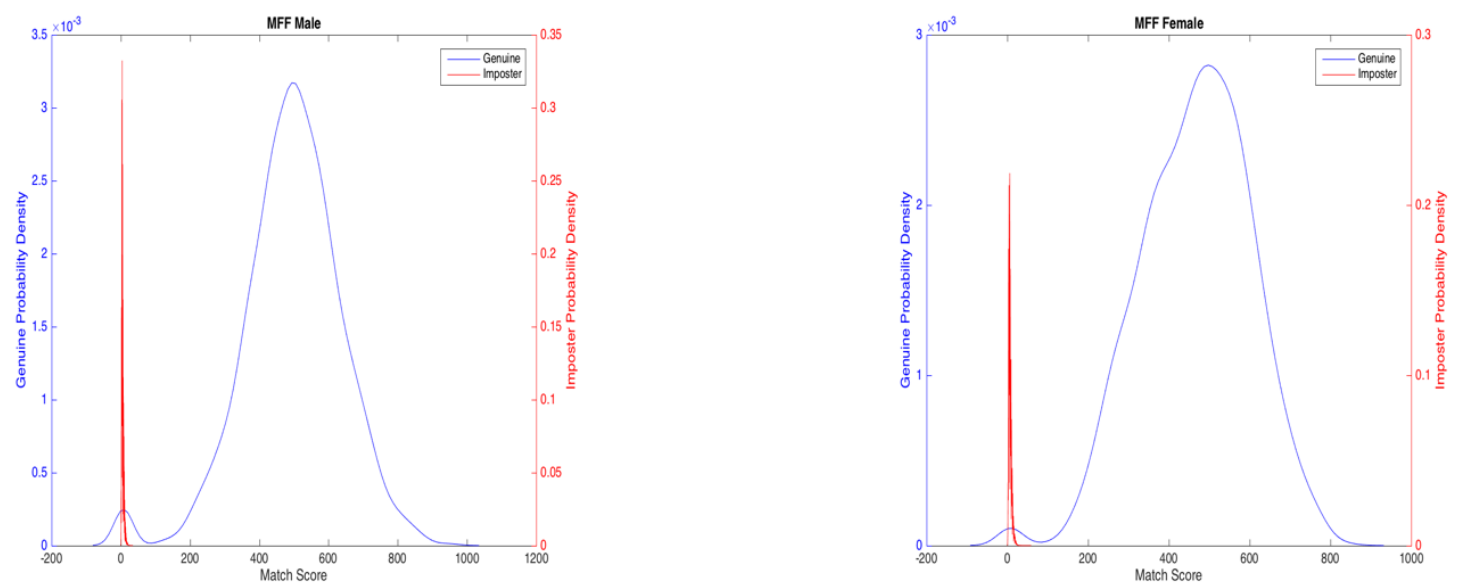

Figure B.2.11 Neurotech Morpho FOTF Male (Left) Female (Right)
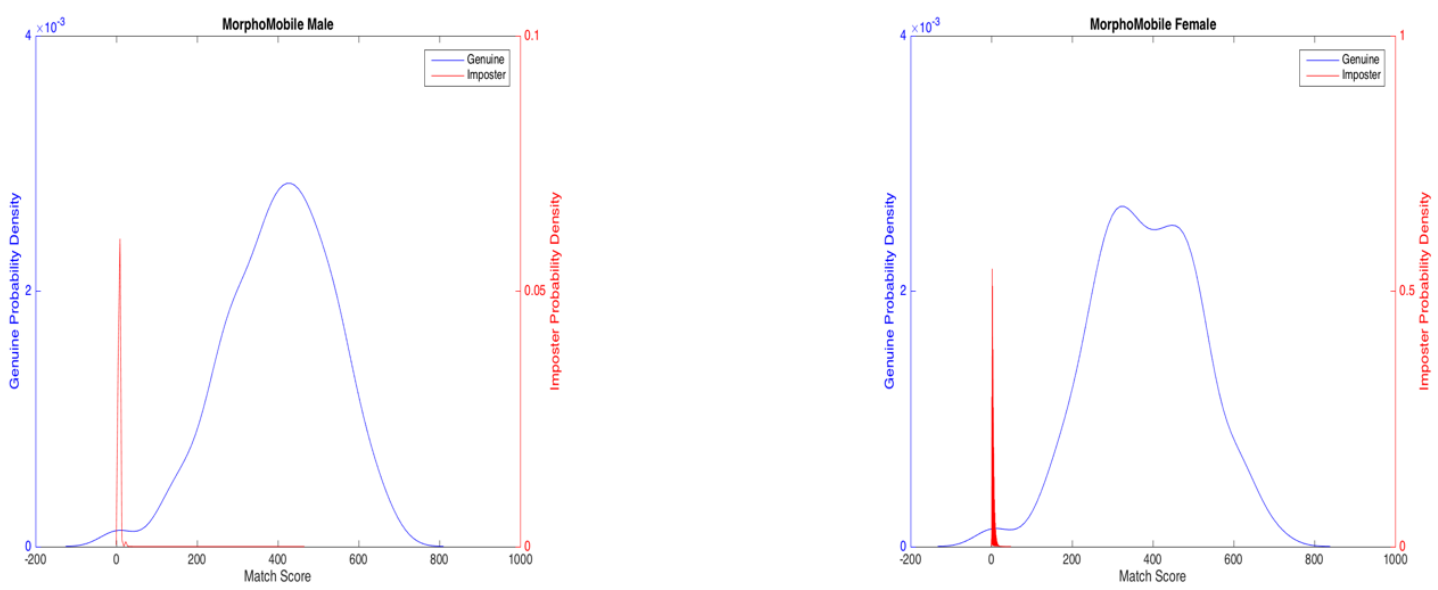

Figure B.2.12 Neurotech MorphoMobile Male (Left) Female (Right) 


\section{B.3 SourceAFIS Genuine/Imposter Score Distributions}

Table B.5 SourceAFIS Male Genuine and Imposter KLD, Minimum and Maximum Scores

\begin{tabular}{|c|c|c|c|c|c|c|}
\hline $\begin{array}{r}\text { SourceAFIS Male } \\
\text { Device }\end{array}$ & $\begin{array}{l}\text { Genuine } \\
\text { KLD }\end{array}$ & $\begin{array}{l}\text { Imposter } \\
\text { KLD }\end{array}$ & $\begin{array}{l}\text { Max } \\
\text { Genuine }\end{array}$ & $\begin{array}{l}\text { Min } \\
\text { Genuine }\end{array}$ & $\begin{array}{l}\text { Max } \\
\text { Imposter }\end{array}$ & $\begin{array}{l}\text { Min } \\
\text { Imposter }\end{array}$ \\
\hline$A N D I B I N$ & 0.0328 & 0.0849 & 258.72 & 11.14 & 41.22 & 2.40 \\
\hline$A N D I G R Y$ & 0.0269 & 2.5543 & 227.51 & 8.98 & 34.60 & 4.43 \\
\hline Biosled & 0.0359 & 1.0288 & 248.36 & 9.96 & 141.30 & 3.65 \\
\hline Biosled Rolls & 0.0265 & 0.0113 & 329.20 & 4.48 & 219.31 & 1.02 \\
\hline CG2 & 0.0491 & 0.0202 & 248.38 & 10.82 & 120.65 & 2.58 \\
\hline CG2 Rolls & 0.0255 & 0.3119 & 364.86 & 11.92 & 214.73 & 2.37 \\
\hline CSA & 0.0516 & 1.8634 & 284.96 & 33.32 & 33.18 & 2.56 \\
\hline CSA Rolls & 0.0204 & 1.6716 & 517.80 & 9.01 & 29.68 & 5.29 \\
\hline InnerID & 0.2466 & 0.0653 & 148.15 & 9.77 & 32.62 & 3.67 \\
\hline$\angle 1$ & 0.0511 & 0.0240 & 288.08 & 10.70 & 89.70 & 1.21 \\
\hline L1 Rolls & 0.0202 & 1.2847 & 341.77 & 9.87 & 35.18 & 1.02 \\
\hline$M F F$ & 0.0313 & 1.9772 & 289.72 & 9.97 & 33.23 & 1.02 \\
\hline MorphoMobile & 0.0440 & 0.0222 & 172.81 & 10.15 & 99.94 & 4.51 \\
\hline
\end{tabular}

Table B.6 SourceAFIS Female Genuine and Imposter KLD, Minimum and Maximum Scores

\begin{tabular}{|r|l|l|l|l|l|l|}
\hline $\begin{array}{r}\text { SourceAFIS Female } \\
\text { Device }\end{array}$ & $\begin{array}{l}\text { Genuine } \\
\text { KLD }\end{array}$ & $\begin{array}{l}\text { Imposter } \\
\text { KLD }\end{array}$ & $\begin{array}{l}\text { Max } \\
\text { Genuine }\end{array}$ & $\begin{array}{l}\text { Min } \\
\text { Genuine }\end{array}$ & $\begin{array}{l}\text { Max } \\
\text { Imposter }\end{array}$ & $\begin{array}{c}\text { Min } \\
\text { Imposter }\end{array}$ \\
\hline ANDI BIN & 0.0309 & 2.5337 & 222.20 & 10.45 & 35.35 & 2.87 \\
\cline { 2 - 2 } & 0.0737 & 0.1235 & 181.90 & 9.50 & 35.99 & 1.05 \\
\cline { 2 - 7 } BioSled & 0.1505 & 6.9133 & 210.80 & 10.65 & 37.64 & 1.13 \\
\cline { 2 - 7 } BioSled Rolls & 0.0275 & 42.0973 & 331.39 & 10.26 & 38.51 & 1.02 \\
CG2 & 0.0228 & 1.4555 & 230.58 & 11.39 & 86.49 & 3.61 \\
\cline { 2 - 7 } CG2 Rolls & 0.0294 & 16.7425 & 356.58 & 11.06 & 36.67 & 1.02 \\
\cline { 3 - 7 } CSA & 0.1705 & 0.4403 & 209.85 & 13.24 & 35.92 & 2.98 \\
\cline { 2 - 7 } CSA Rolls & 0.0719 & 0.1603 & 319.62 & 10.27 & 35.01 & 1.03 \\
\cline { 3 - 7 } InnerID & 0.2600 & 2.0087 & 116.29 & 8.88 & 31.22 & 5.57 \\
\cline { 2 - 7 } L1 & 0.0621 & 6.6097 & 239.31 & 9.57 & 33.07 & 3.16 \\
\cline { 2 - 7 } L1 Rolls & 0.0205 & 1.6524 & 323.08 & 0.00 & 32.98 & 0.00 \\
\cline { 2 - 7 } MFF & 0.0492 & 0.0939 & 235.71 & 9.18 & 36.04 & 1.02 \\
\cline { 2 - 7 } & 0.0364 & 6.4280 & 168.69 & 9.08 & 33.70 & 4.47 \\
\hline MorphoMobile
\end{tabular}



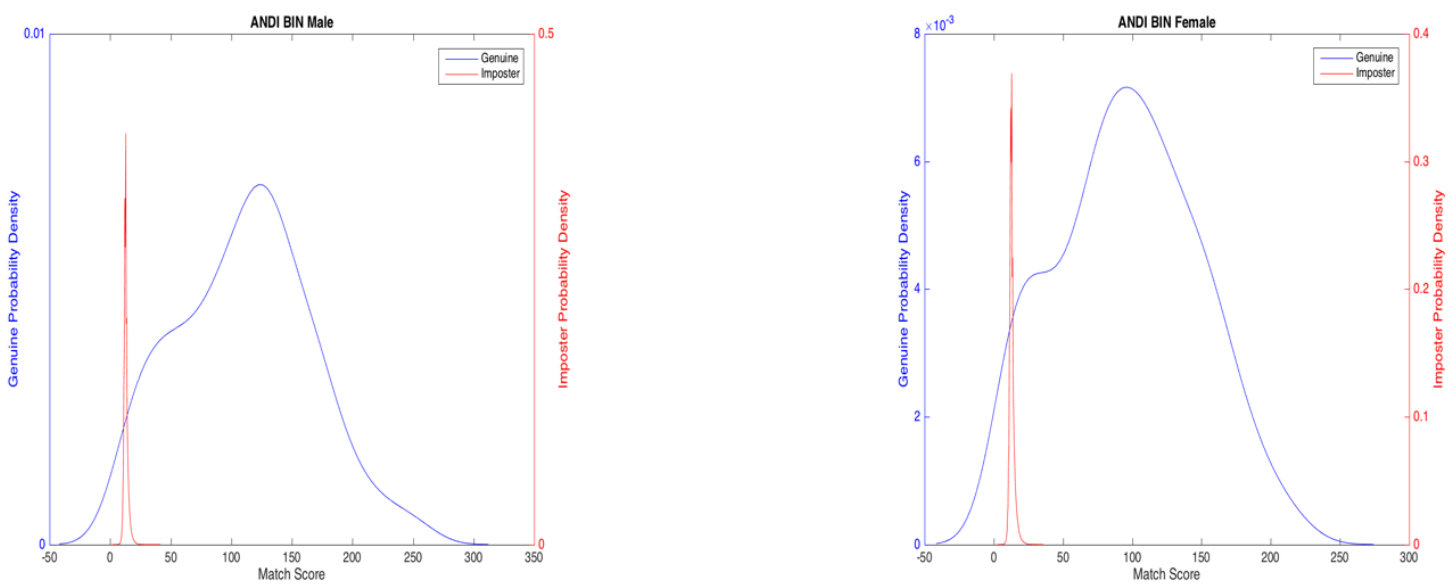

Figure B.3.1 SourceAFIS ANDI Binary Male (Left) Female (Right)
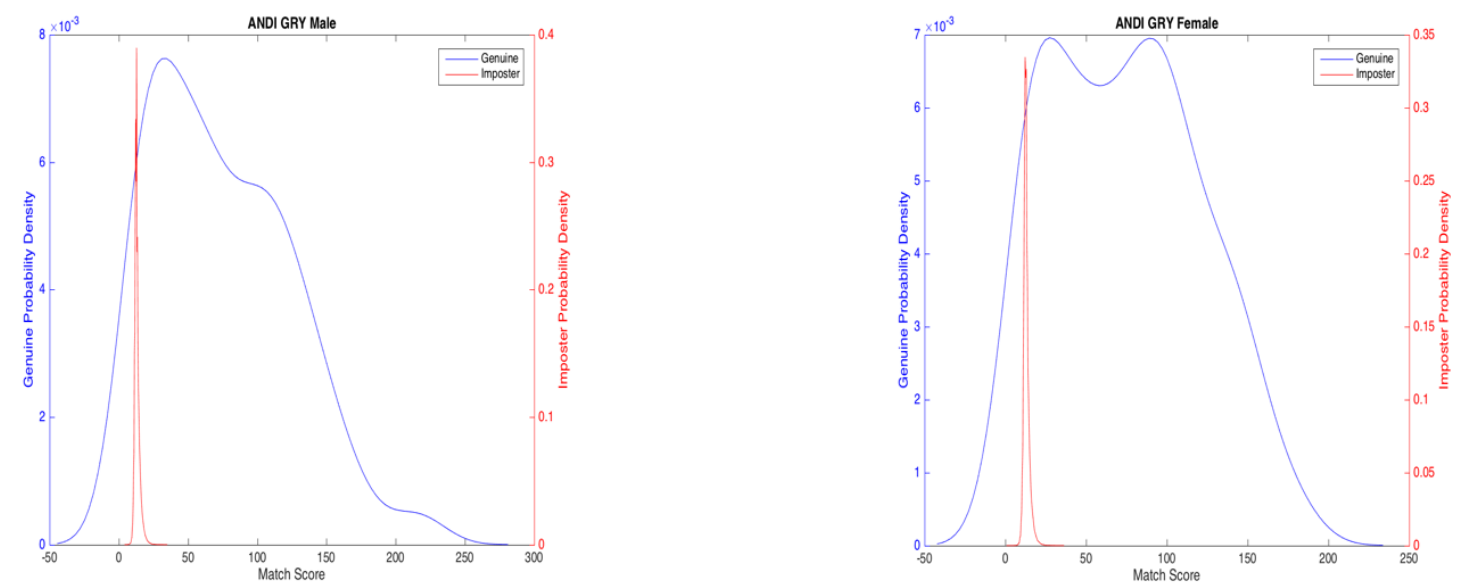

Figure B.3.2 SourceAFIS ANDI Greyscale Male (Left) Female (Right)
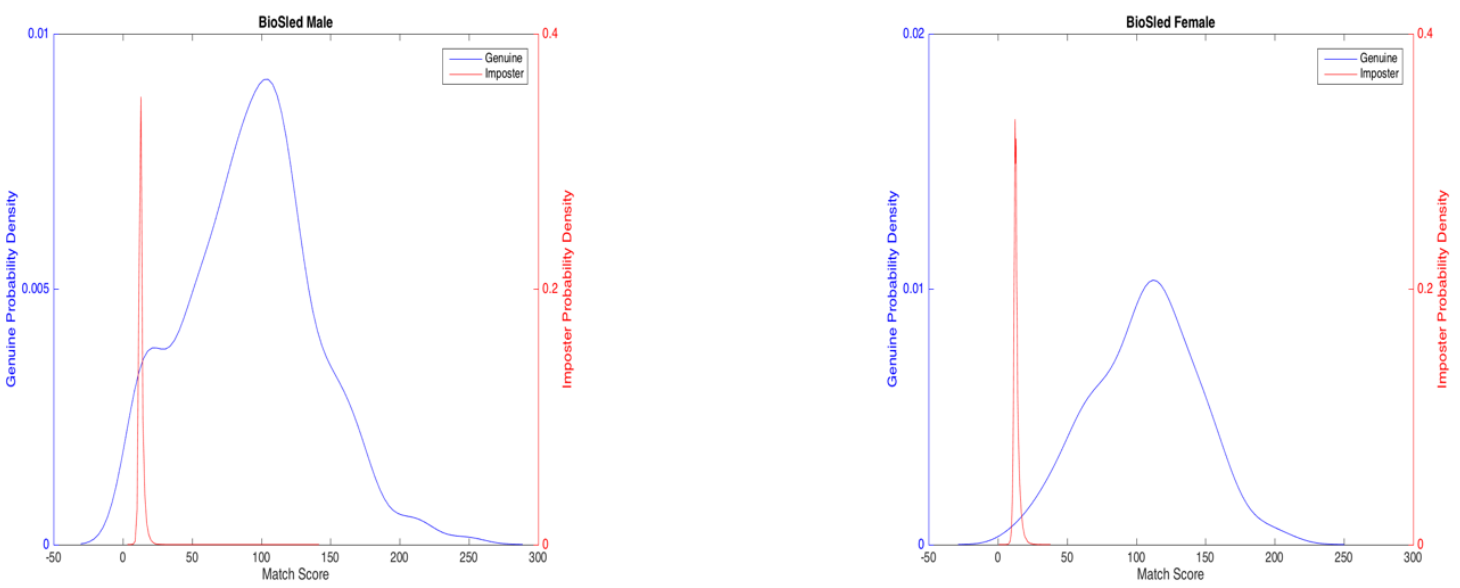

Figure B.3.3 SourceAFIS BioSled Male (Left) Female (Right) 

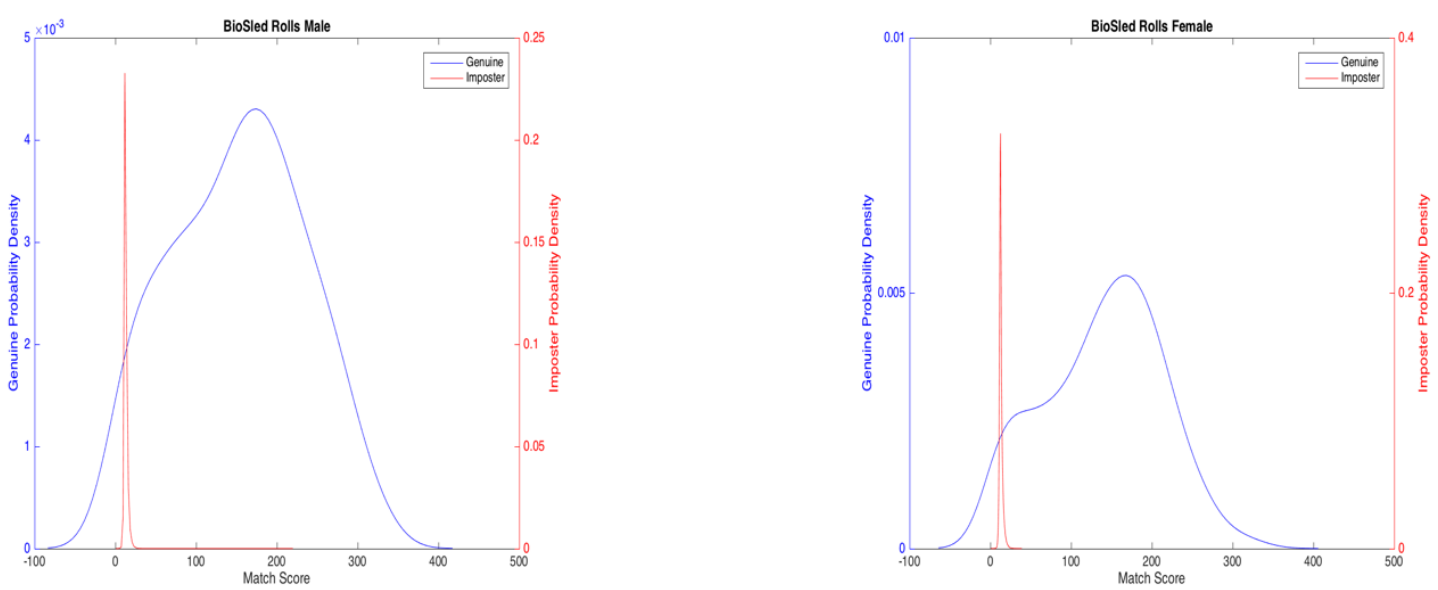

Figure B.3.4 SourceAFIS BioSled Rolls Male (Left) Female (Right)
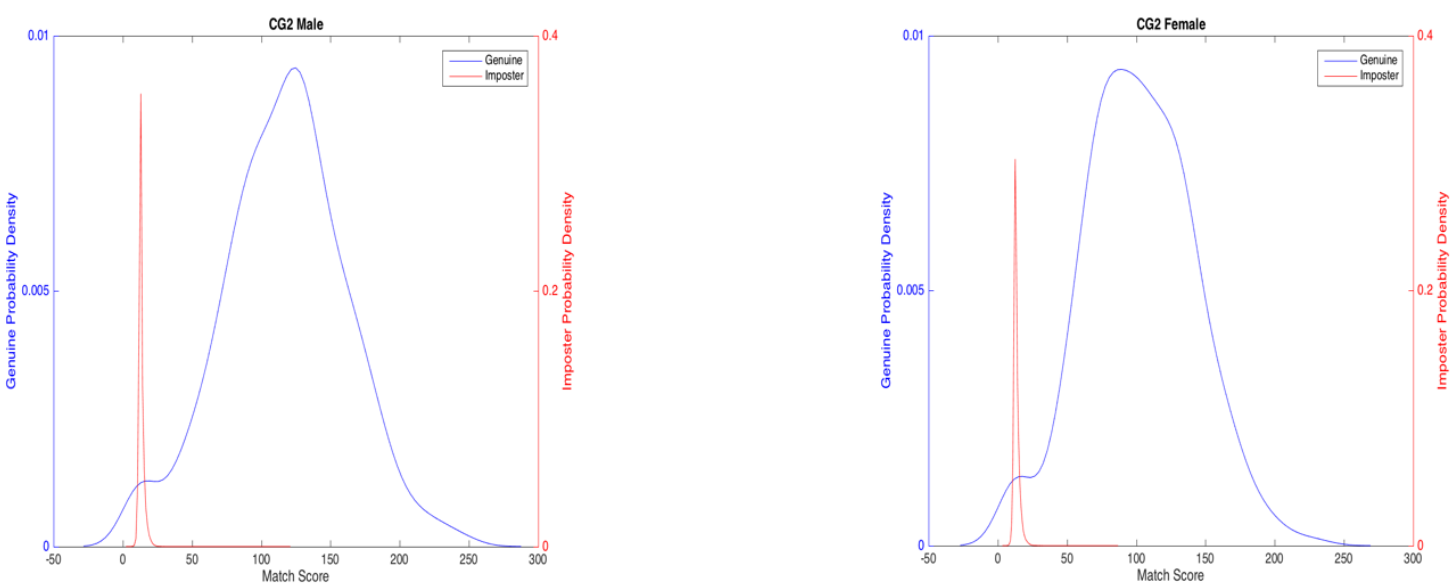

Figure B.3.5 SourceAFIS FP II Guardian Male (Left) Female (Right)
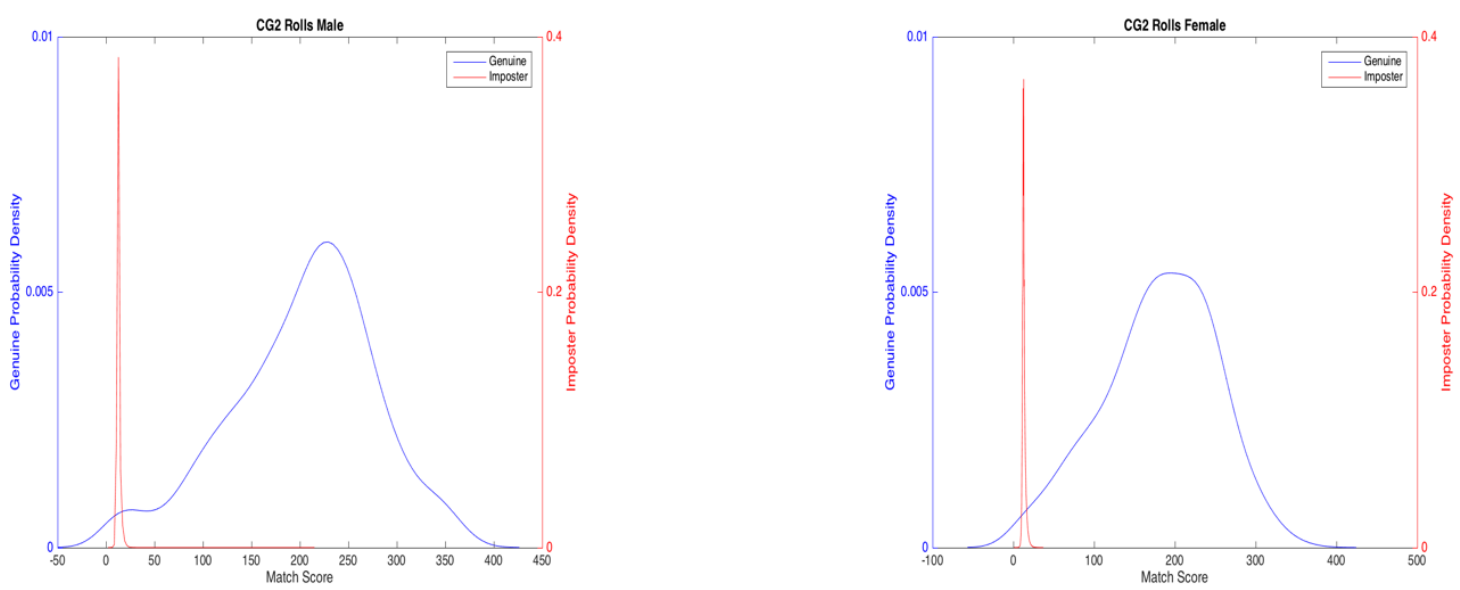

Figure B.3.6 SourceAFIS FP II Guardian Male (Left) Female (Right) 

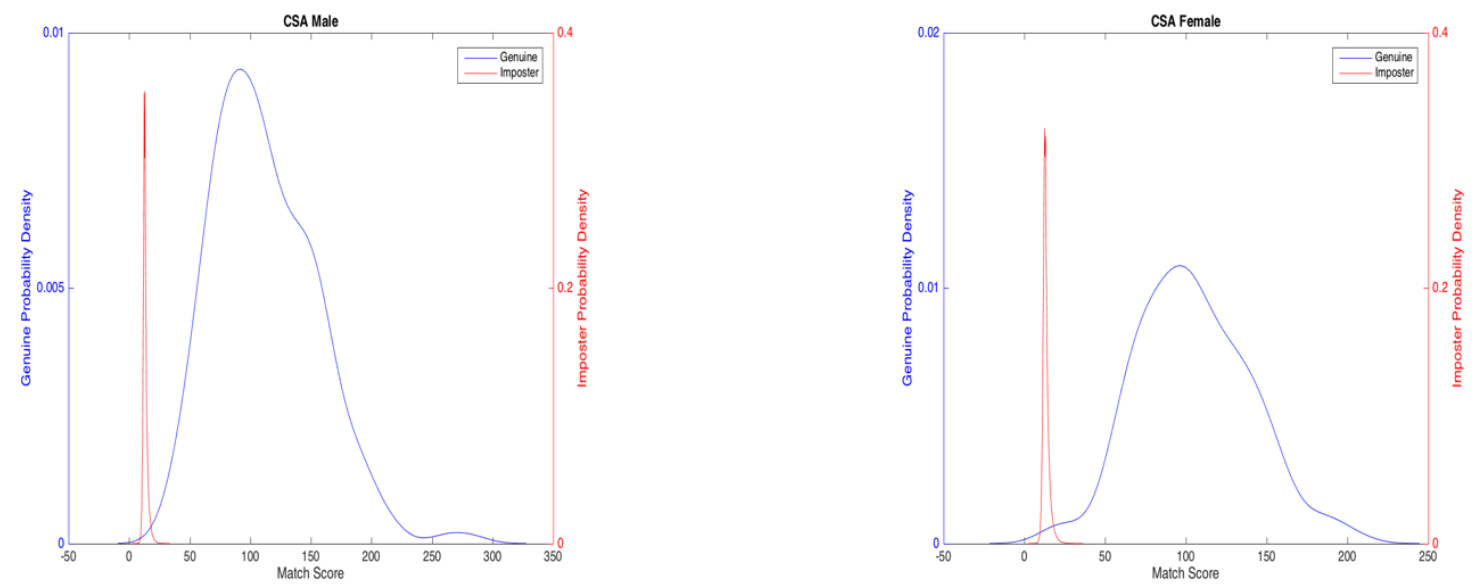

Figure B.3.7 SourceAFIS Crossmatch Seek Avenger Male (Left) Female (Right)
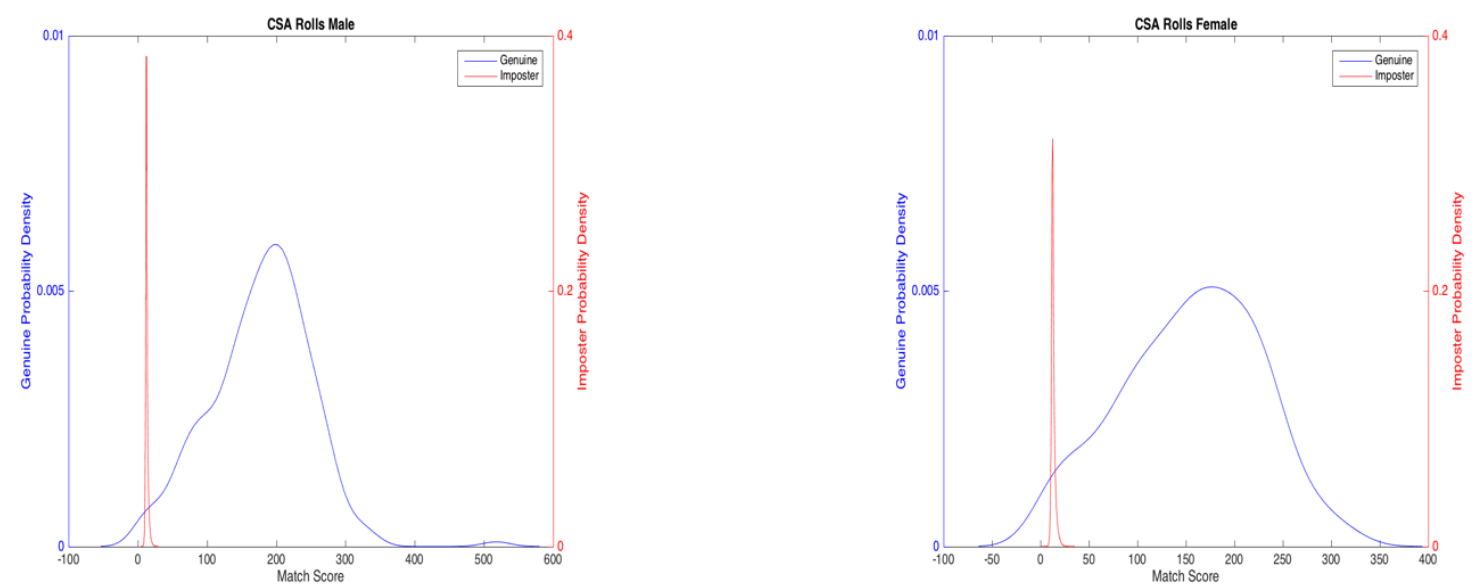

Figure B.3.8 SourceAFIS Crossmatch Seek Avenger Rolls Male (Left) Female (Right)
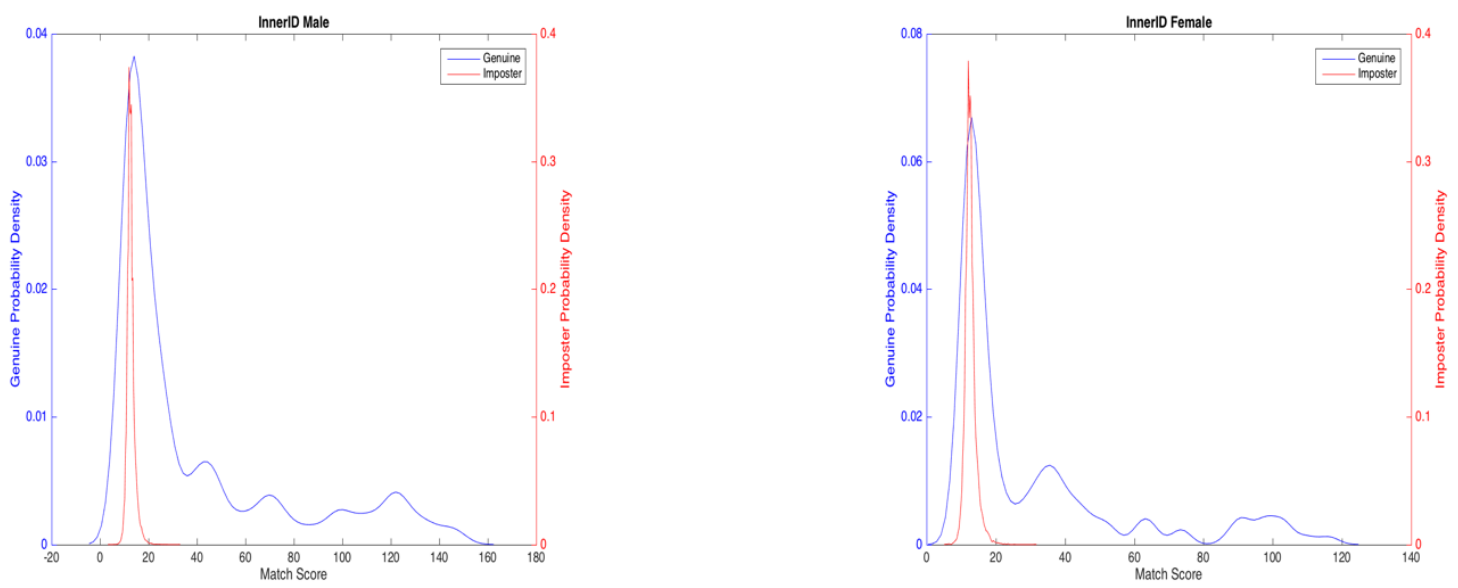

Figure B.3.9 SourceAFIS InnerID Male (Left) Female (Right) 

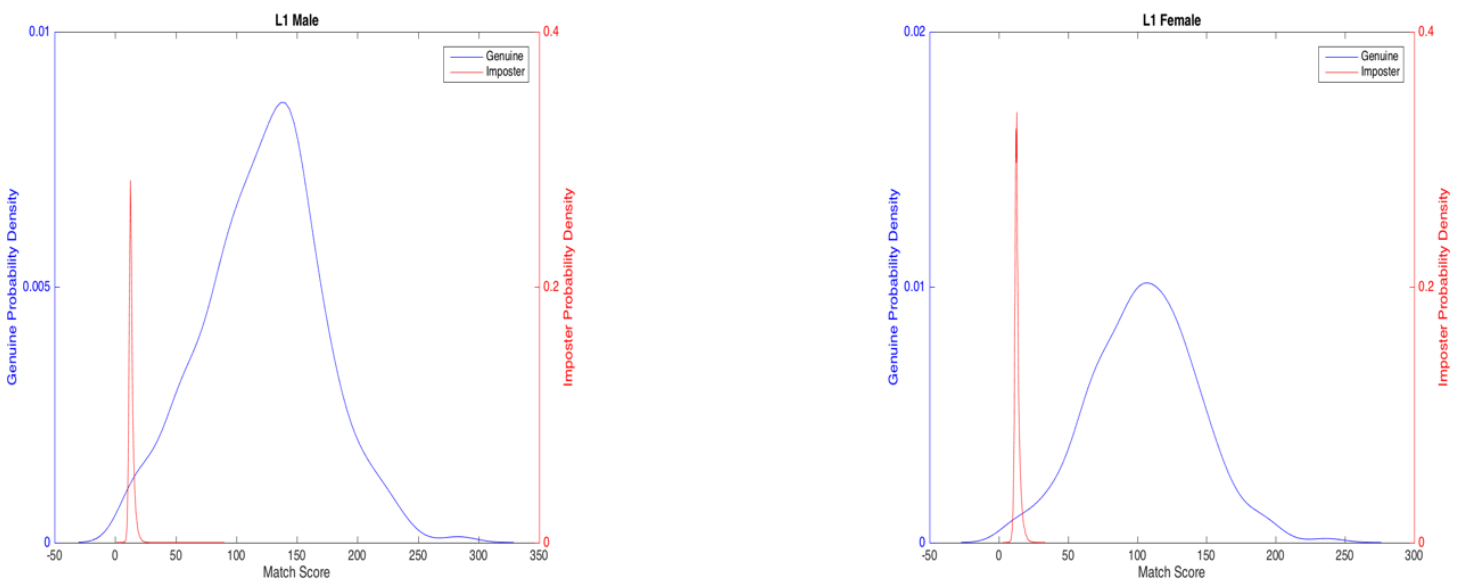

Figure B.3.10 SourceAFIS L1 Male (Left) Female (Right)
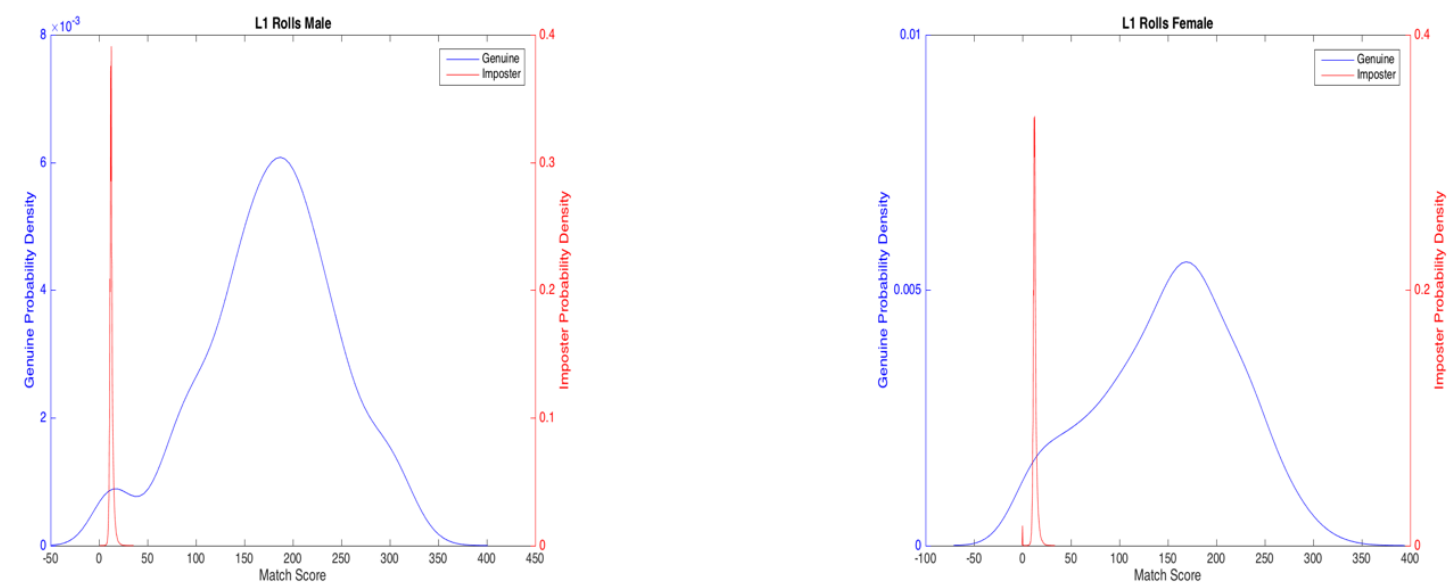

Figure B.3.11 SourceAFIS L1 Rolls Male (Left) Female (Right)
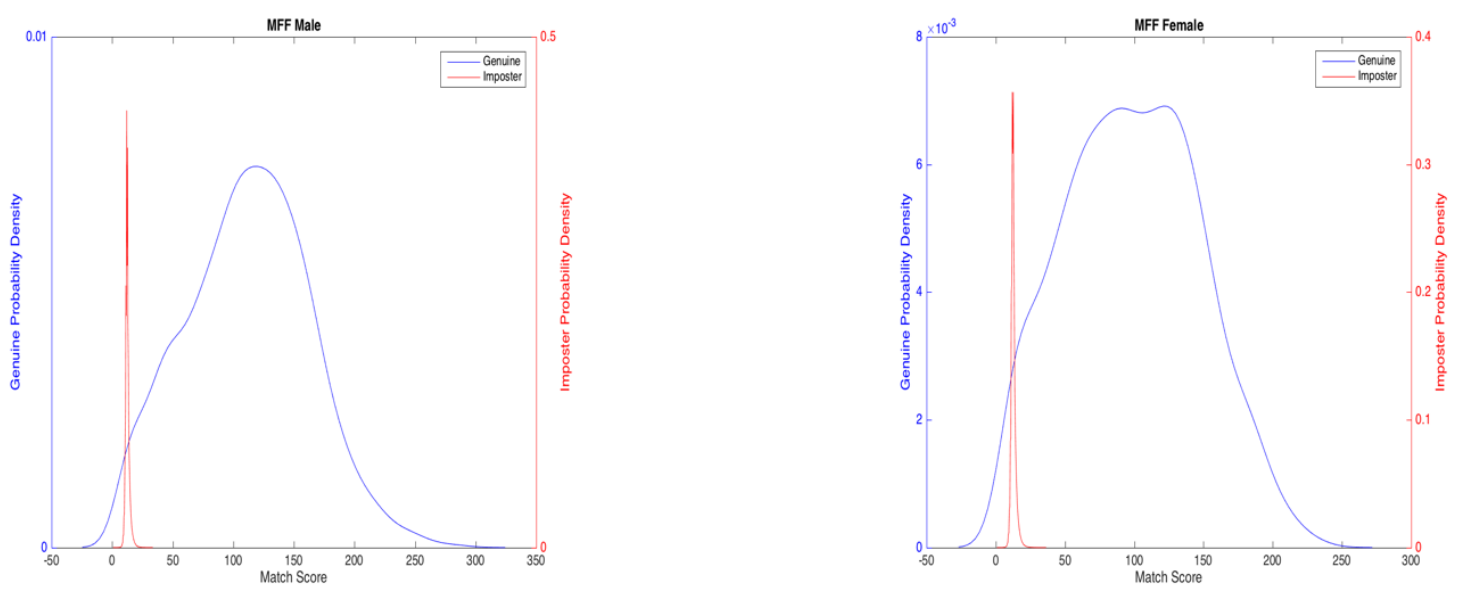

Figure B.3.12 SourceAFIS Morpho FOTF Male (Left) Female (Right) 

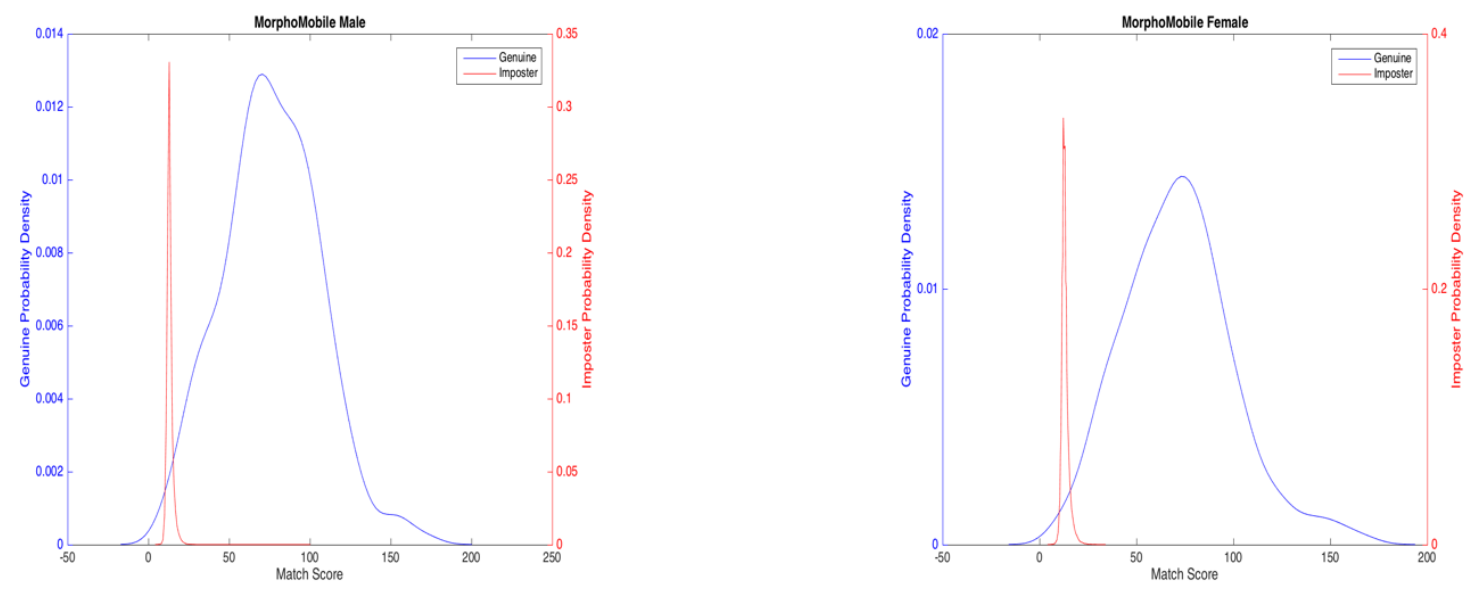

Figure B.3.13 SourceAFIS MorphoMobile Male (Left) Female (Right)

\section{B.4 NBIS ROC Curves and Area Under Curve}

Table B.7 NBIS Area Under the Curve for ROC Plots

\begin{tabular}{|c|c|c|}
\hline Device & Male Area Under Curve & Female Area Under Curve \\
\hline ANDI BIN & 0.9826 & 0.9773 \\
\hline$A N D I G R Y$ & 0.9784 & 0.9808 \\
\hline Biosled & 0.9731 & 0.9958 \\
\hline Biosled Rolls & 0.9783 & 0.9891 \\
\hline CG2 & 0.9821 & 0.9752 \\
\hline CG2 Rolls & 0.9872 & 0.9996 \\
\hline CSA & 0.9998 & 0.9967 \\
\hline CSA Rolls & 0.9893 & 0.9861 \\
\hline InnerID & 0.8745 & 0.8440 \\
\hline$L 1$ & 0.9815 & 0.9836 \\
\hline$M F F$ & 0.9854 & 0.9739 \\
\hline MorphoMobile & 0.9932 & 0.9953 \\
\hline
\end{tabular}



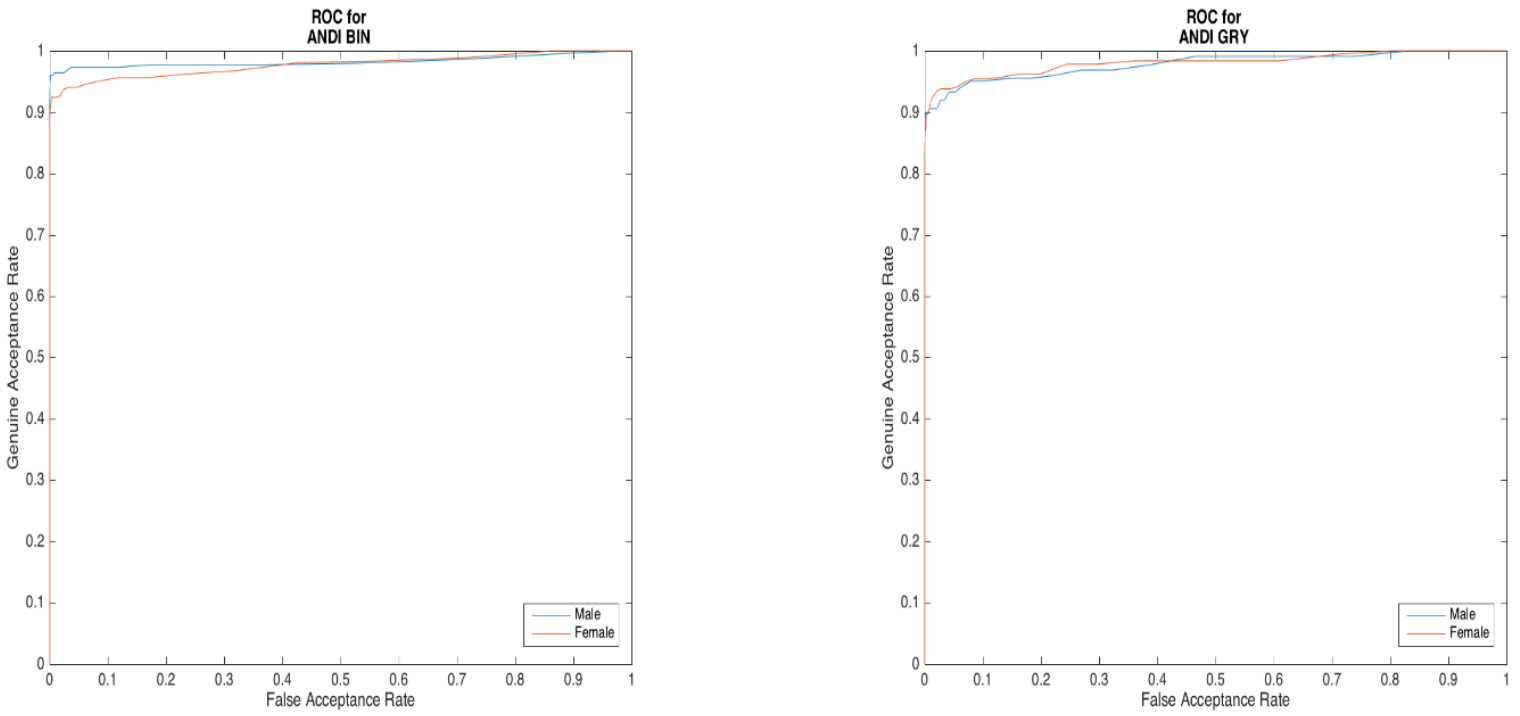

Figure B.4.1 NBIS ANDI Binary Gender ROC Curves (Left) NBIS ANDI Greyscale Gender ROC Curves (Right)
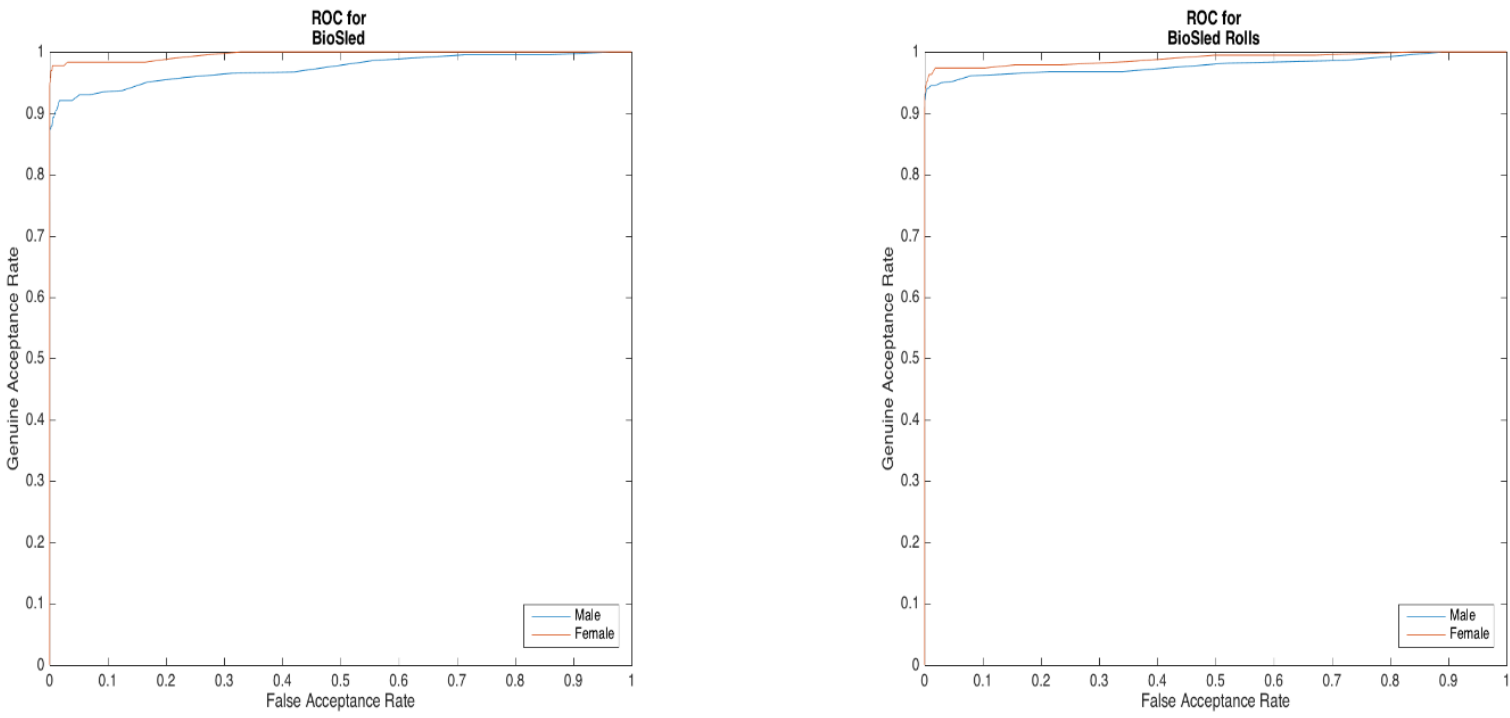

Figure B.4.2 NBIS BioSled Gender ROC Curves (Left) NBIS BioSled Rolls Gender ROC Curves (Right) 

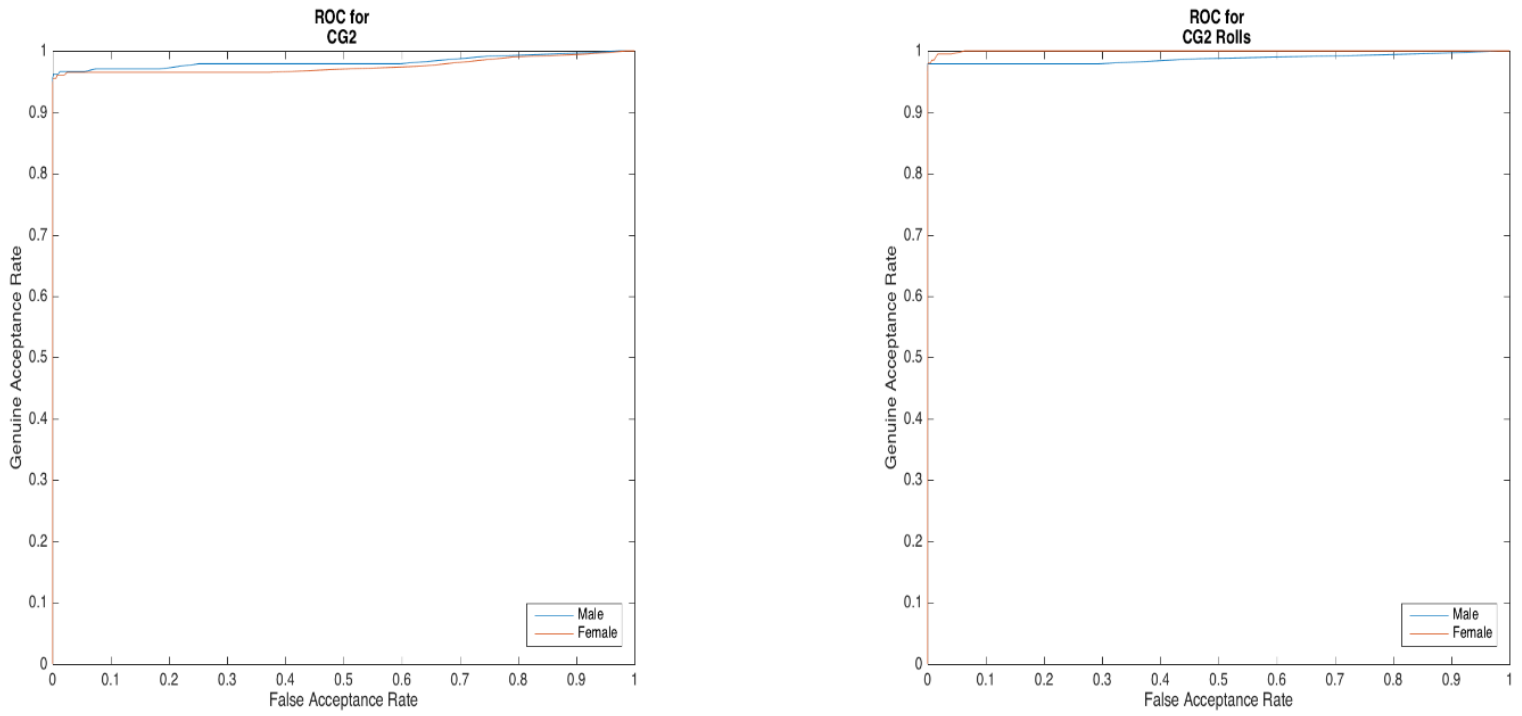

Figure B.4.3 NBIS FP II Guardian Gender ROC Curves (Left) NBIS FP II Guardian Rolls Gender ROC Curves (Right)
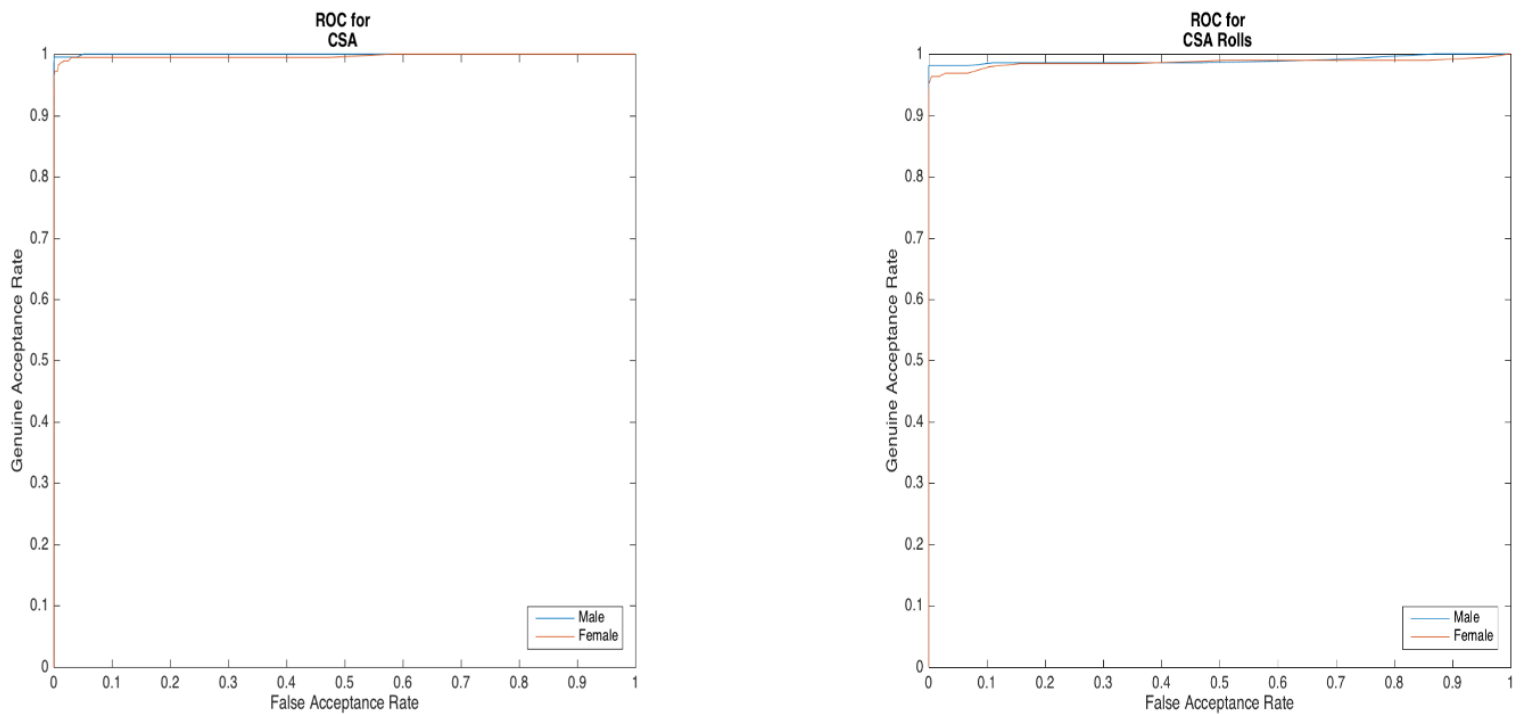

Figure B.4.4 NBIS Crossmatch Seek Avenger Gender ROC Curves (Left) NBIS Crossmatch Seek Avenger Rolls Gender ROC Curves (Right) 

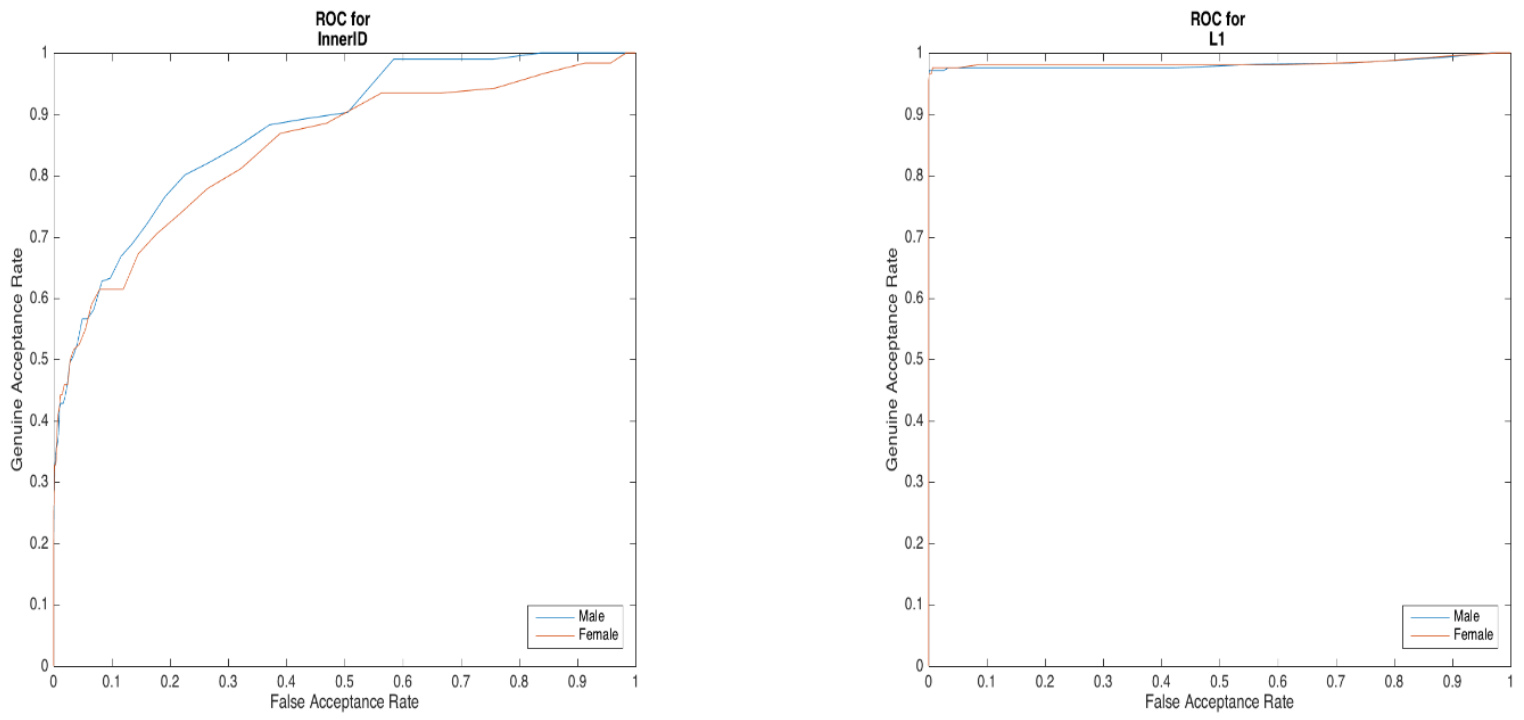

Figure B.4.5 NBIS InnerID Gender ROC Curves (Left) NBIS L1 Gender ROC Curves (Right)
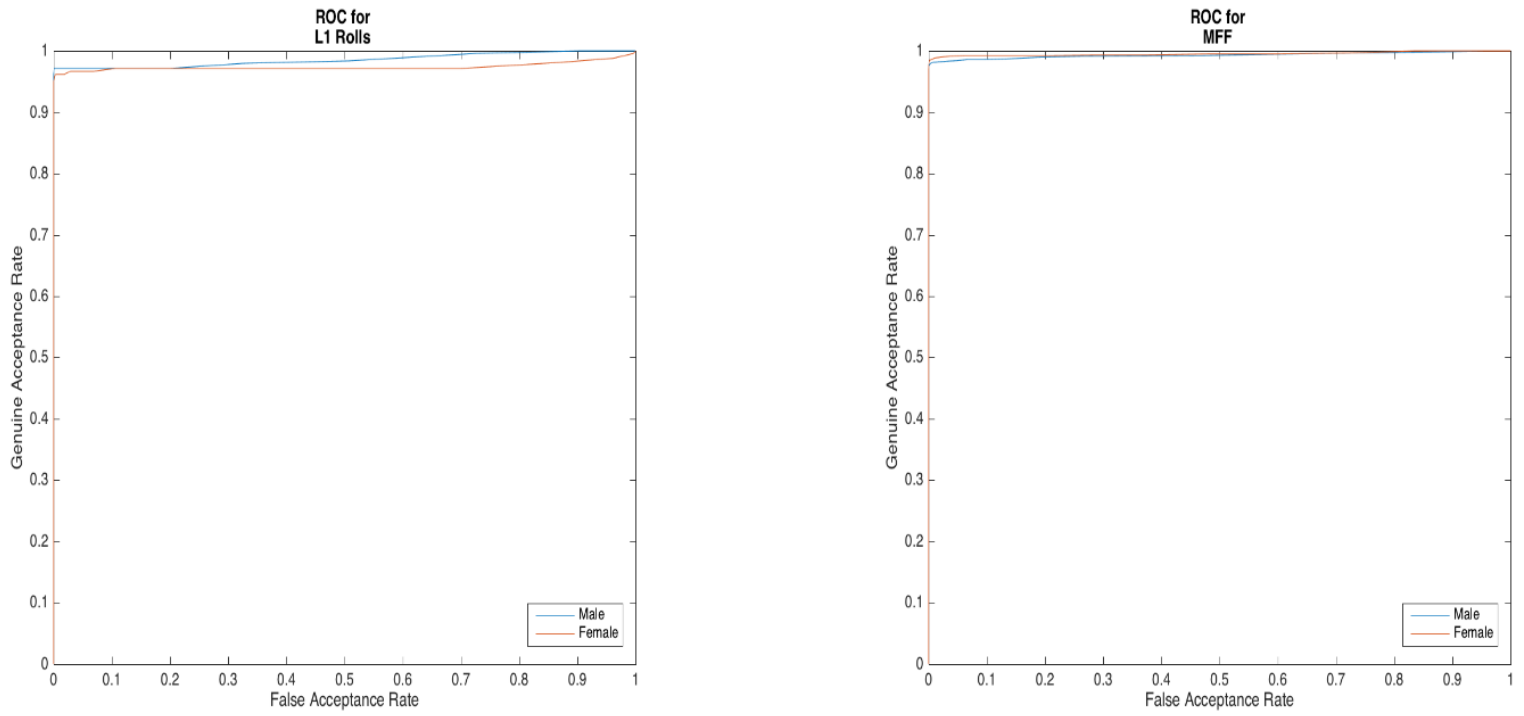

Figure B.4.6 NBIS L1 Rolls Gender ROC Curves (Left) NBIS Morpho FOTF Gender ROC Curves (Right) 


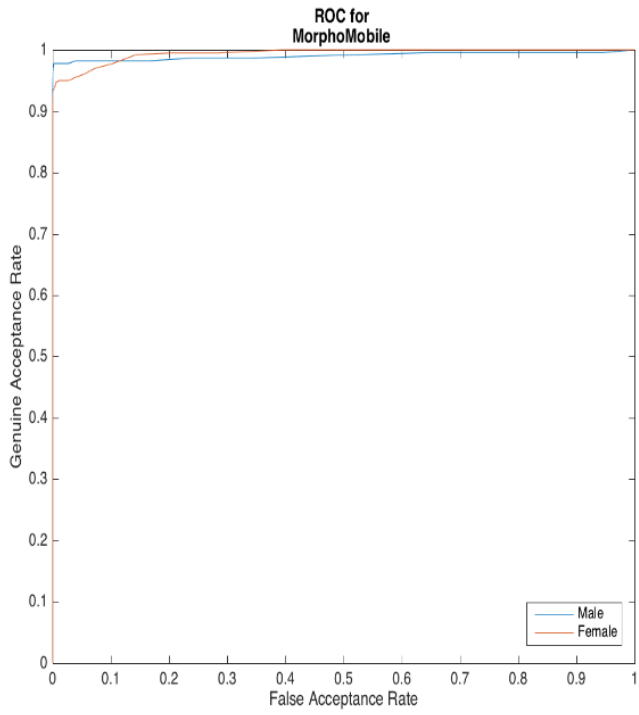

Figure B.4.7 NBIS MorphoMobile Gender ROC Curves 


\section{B.5 Neurotech ROC Curves and Area Under Curve}

Table B. 8 Neurotech Area Under the Curve for ROC Plots

\begin{tabular}{|c|c|c|}
\hline $\begin{array}{r}\text { Neurotech } \\
\text { Device }\end{array}$ & Male Area Under Curve & Female Area Under Curve \\
\hline ANDI BIN & 0.9851 & 0.9715 \\
\hline ANDI GRY & 0.9880 & 0.9752 \\
\hline BioSled & 0.9682 & 0.9986 \\
\hline BioSled Rolls & 0.9823 & 0.9965 \\
\hline CG2 & 0.9817 & 0.9828 \\
\hline CG2 Rolls & 0.9950 & 1.0000 \\
\hline CSA & 1.0000 & 0.9950 \\
\hline CSA Rolls & 0.9953 & 0.9946 \\
\hline InnerID & 0.9422 & 0.9089 \\
\hline$L 1$ & 0.9853 & 0.9904 \\
\hline$M F F$ & 0.9940 & 0.9971 \\
\hline MorphoMobile & 0.9948 & 0.9911 \\
\hline
\end{tabular}
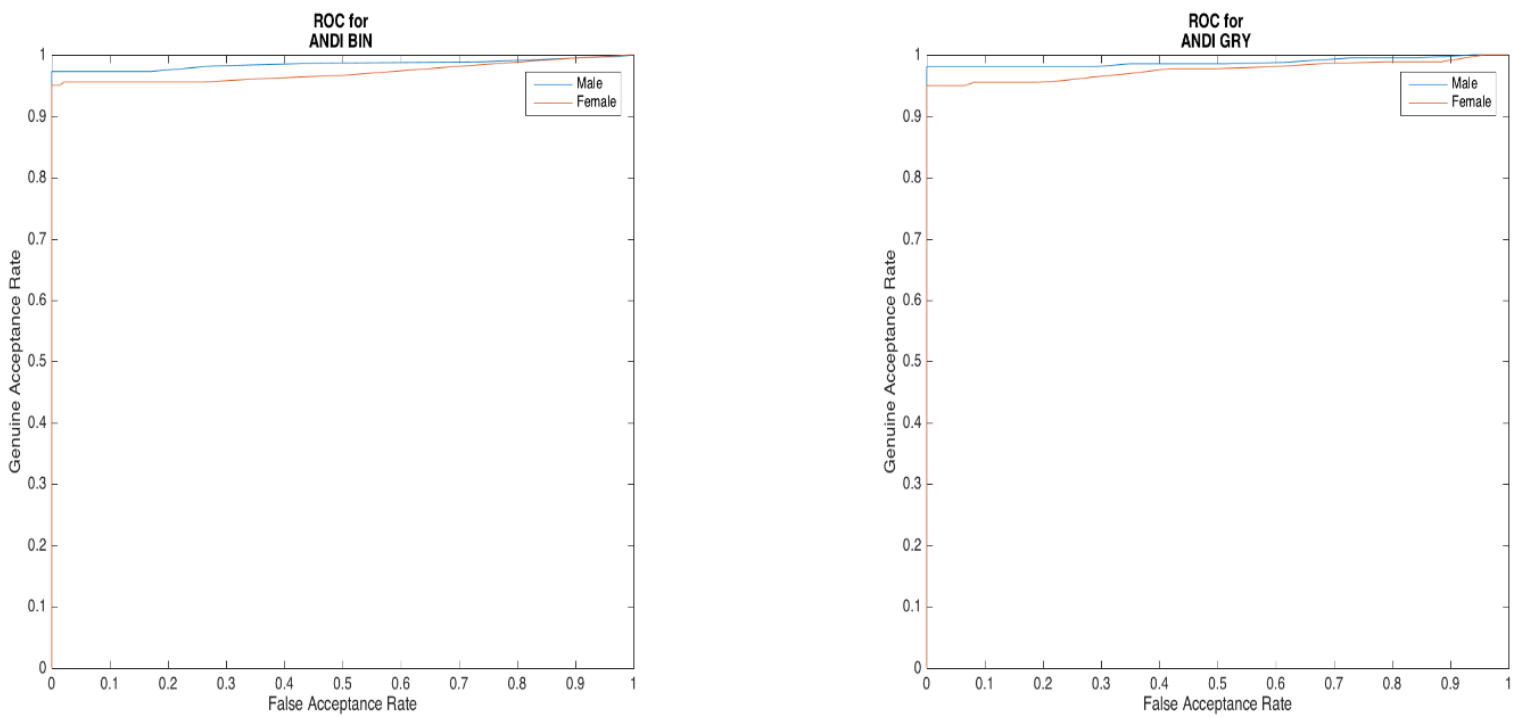

Figure B.5.1 Neurotech ANDI Binary Gender ROC Curves (Left) Neurotech ANDI Greyscale Gender ROC Curves (Right) 

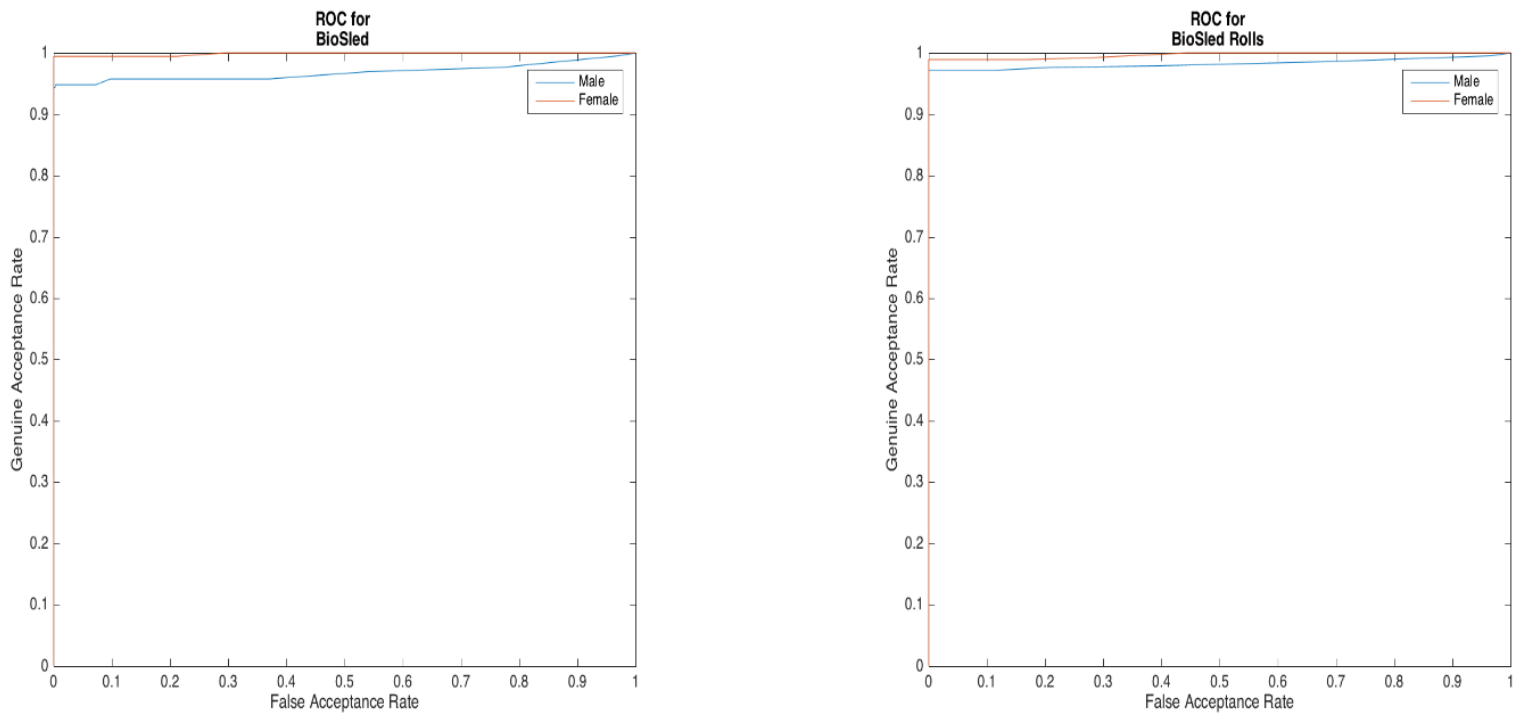

Figure B.5.2 Neurotech BioSled Gender ROC Curves (Left) Neurotech BioSled Rolls Gender ROC Curves (Right)
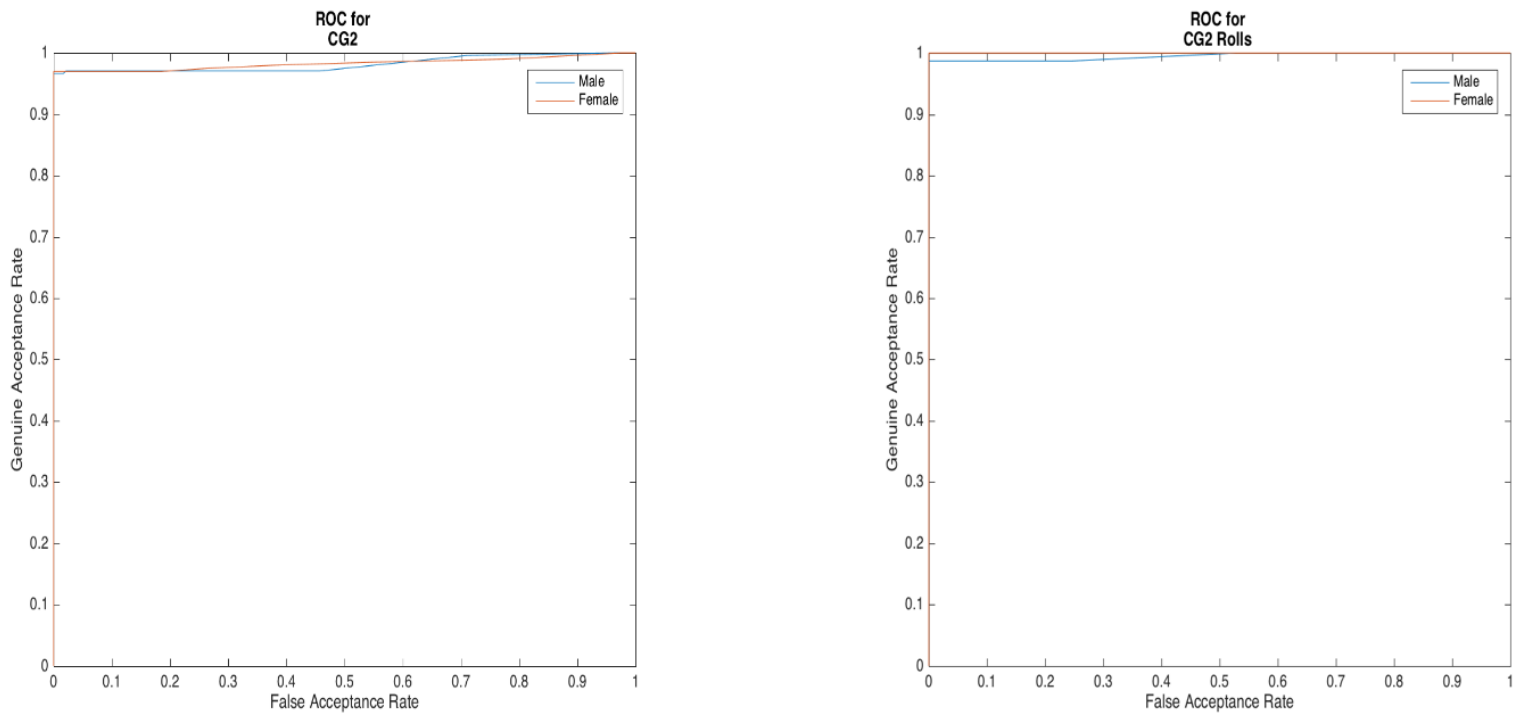

Figure B.5.3 Neurotech FP II Guardian Gender ROC Curves (Left) Neurotech FP II Guardian Rolls Gender ROC Curves (Right) 

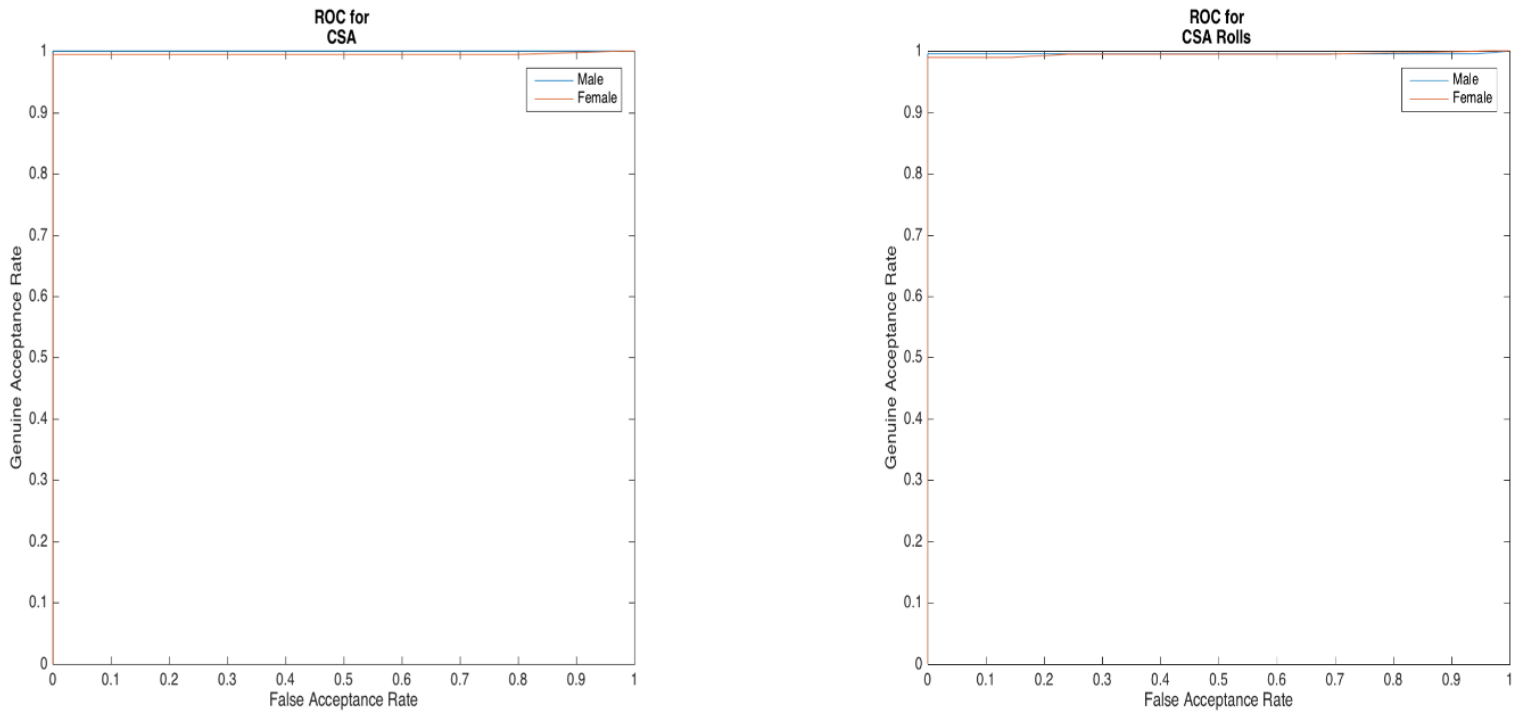

Figure B.5.4 Neurotech Crossmatch Seek Avenger Gender ROC Curves (Left) Neurotech Crossmatch Seek Avenger Rolls Gender ROC Curves (Right)
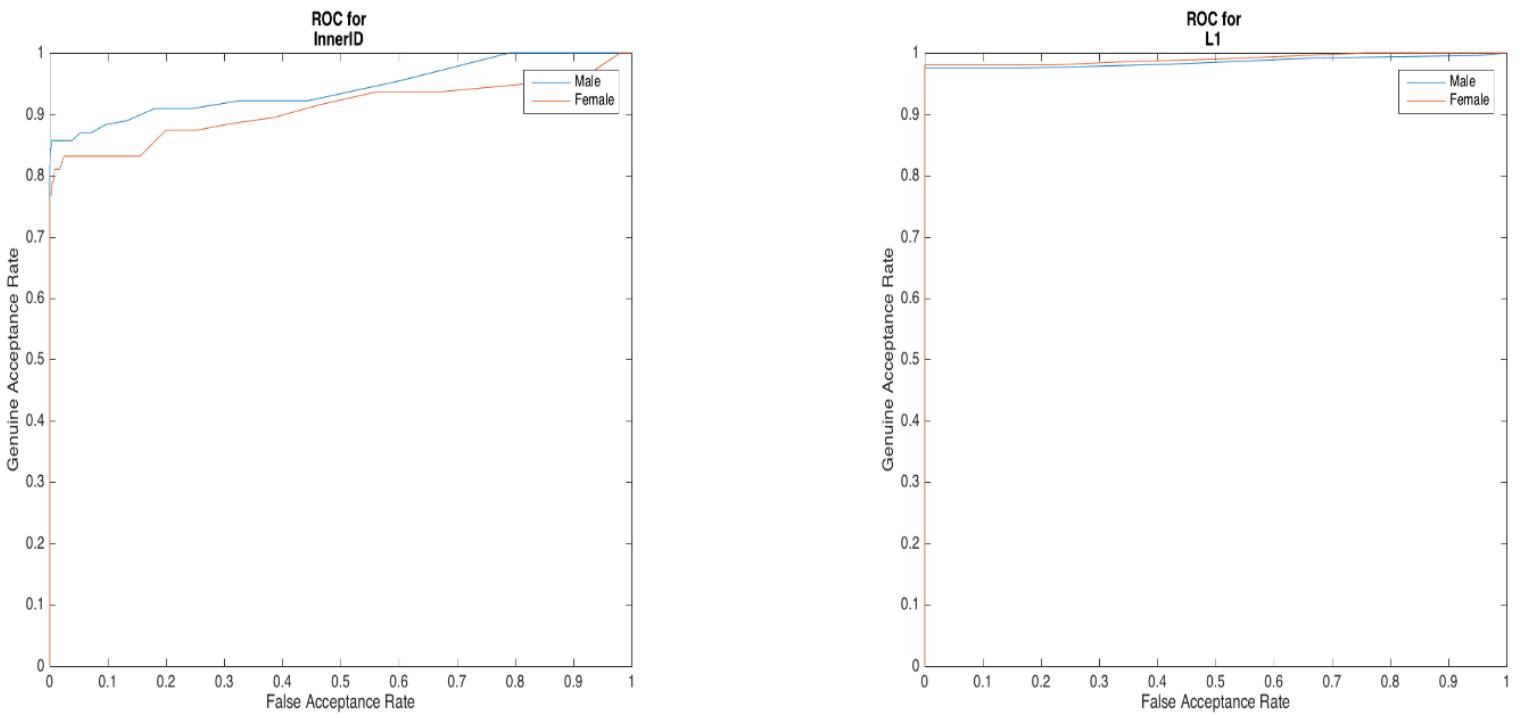

Figure B.5.5 Neurotech InnerID Gender ROC Curves (Left) Neurotech L1 Gender ROC Curves (Right) 

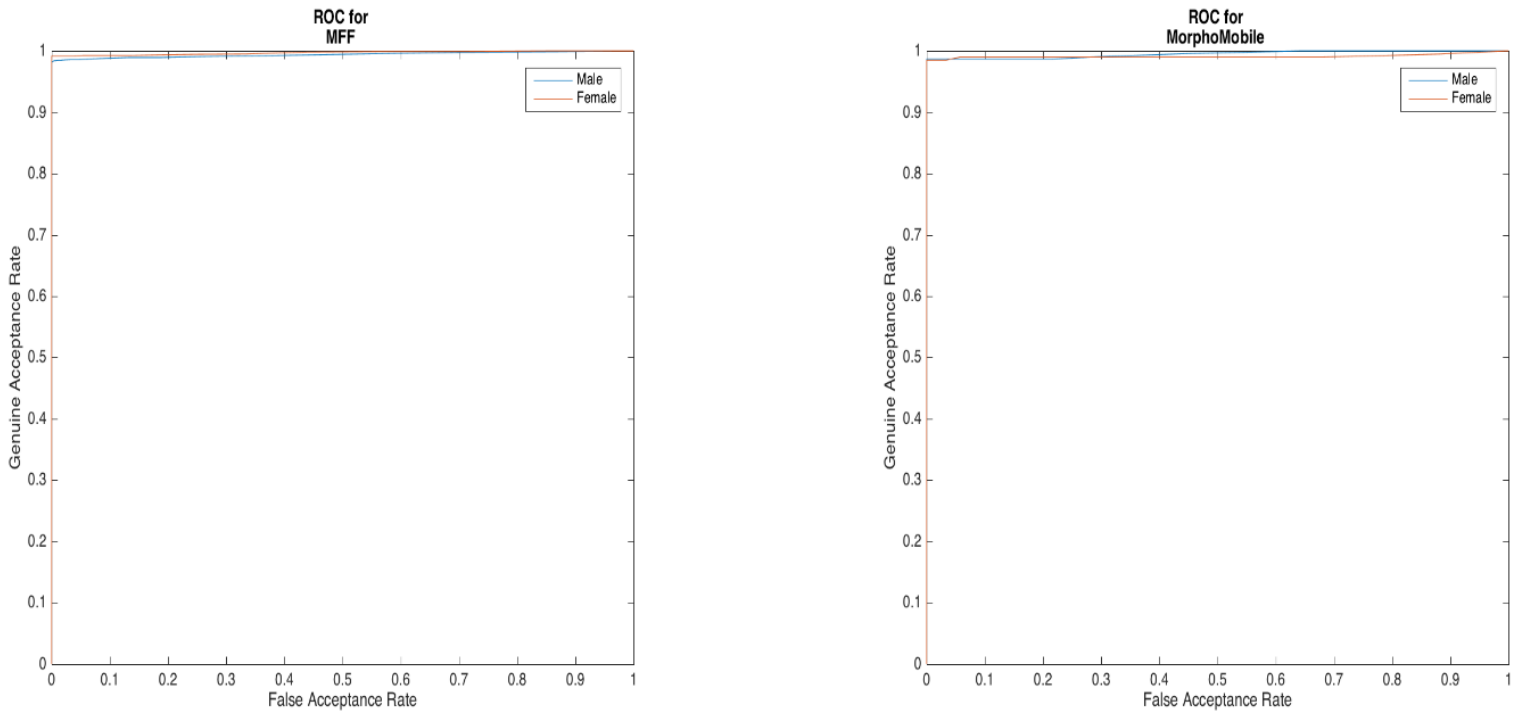

Figure B.5.6 Neurotech Morpho FOTF Gender ROC Curves (Left) Neurotech MorphoMobile Gender ROC Curves (Right) 


\section{B.6 SourceAFIS ROC Curves and Area Under Curve}

Table B.9 SourceAFIS Area Under the Curve for ROC Plots

\begin{tabular}{|c|c|c|}
\hline SourceAFIS Device & Male Area Under Curve & Female Area Under Curve \\
\hline ANDI BIN & 0.9760 & 0.9554 \\
\hline$A N D I G R Y$ & 0.9389 & 0.9340 \\
\hline Biosled & 0.9609 & 0.9927 \\
\hline BioSled Rolls & 0.9584 & 0.9526 \\
\hline CG2 & 0.9779 & 0.9798 \\
\hline CG2 Rolls & 0.9892 & 0.9872 \\
\hline CSA & 1.0000 & 0.9978 \\
\hline CSA Rolls & 0.9849 & 0.9742 \\
\hline InnerID & 0.8209 & 0.7635 \\
\hline$L 1$ & 0.9897 & 0.9863 \\
\hline L1 Rolls & 0.9758 & 0.9634 \\
\hline$M F F$ & 0.9857 & 0.9745 \\
\hline MorphoMobile & 0.9902 & 0.9895 \\
\hline
\end{tabular}
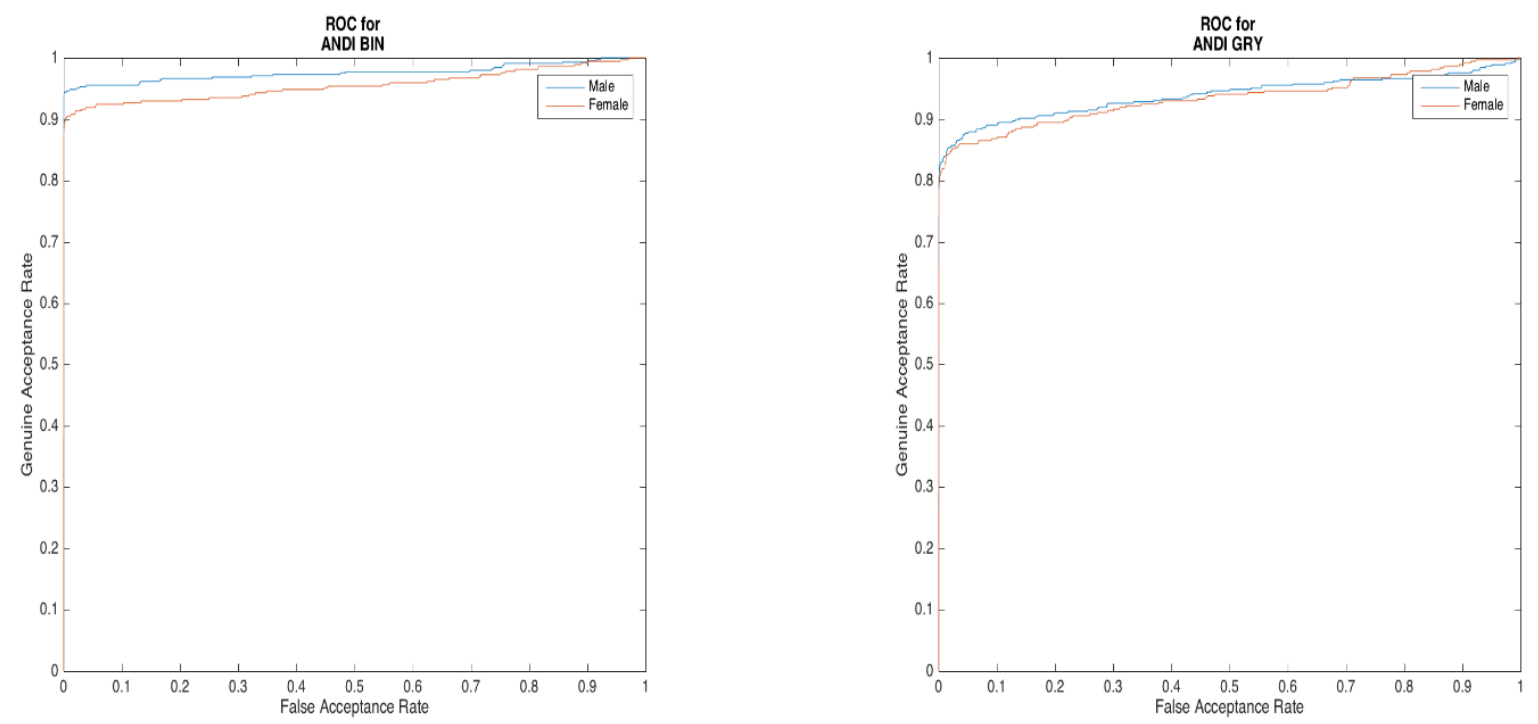

Figure B.6.1 SourceAFIS ANDI Binary Gender ROC Curves (Left) SourceAFIS ANDI Greyscale Gender ROC Curves (Right) 

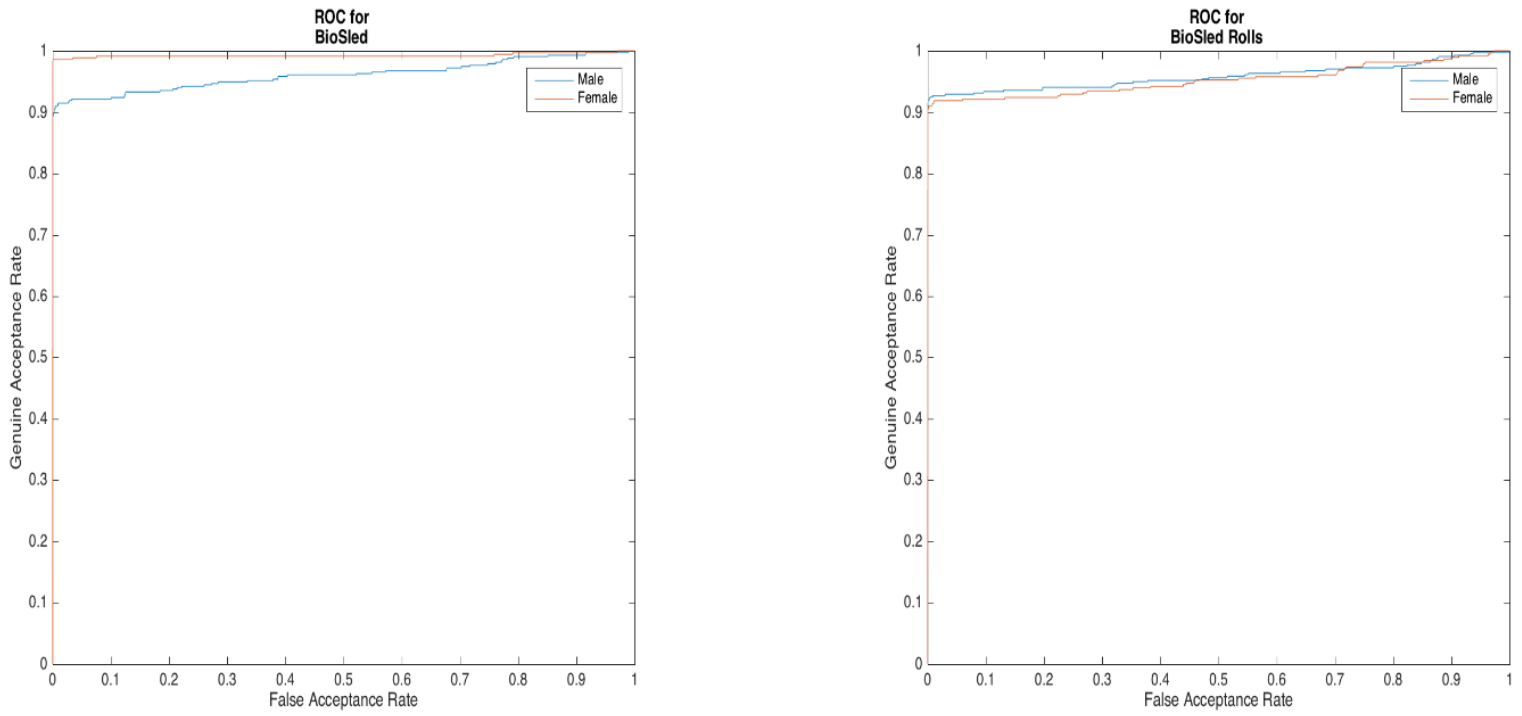

Figure B.6.2 SourceAFIS BioSled Gender ROC Curves (Left) SourceAFIS BioSled Rolls Gender ROC Curves (Right)
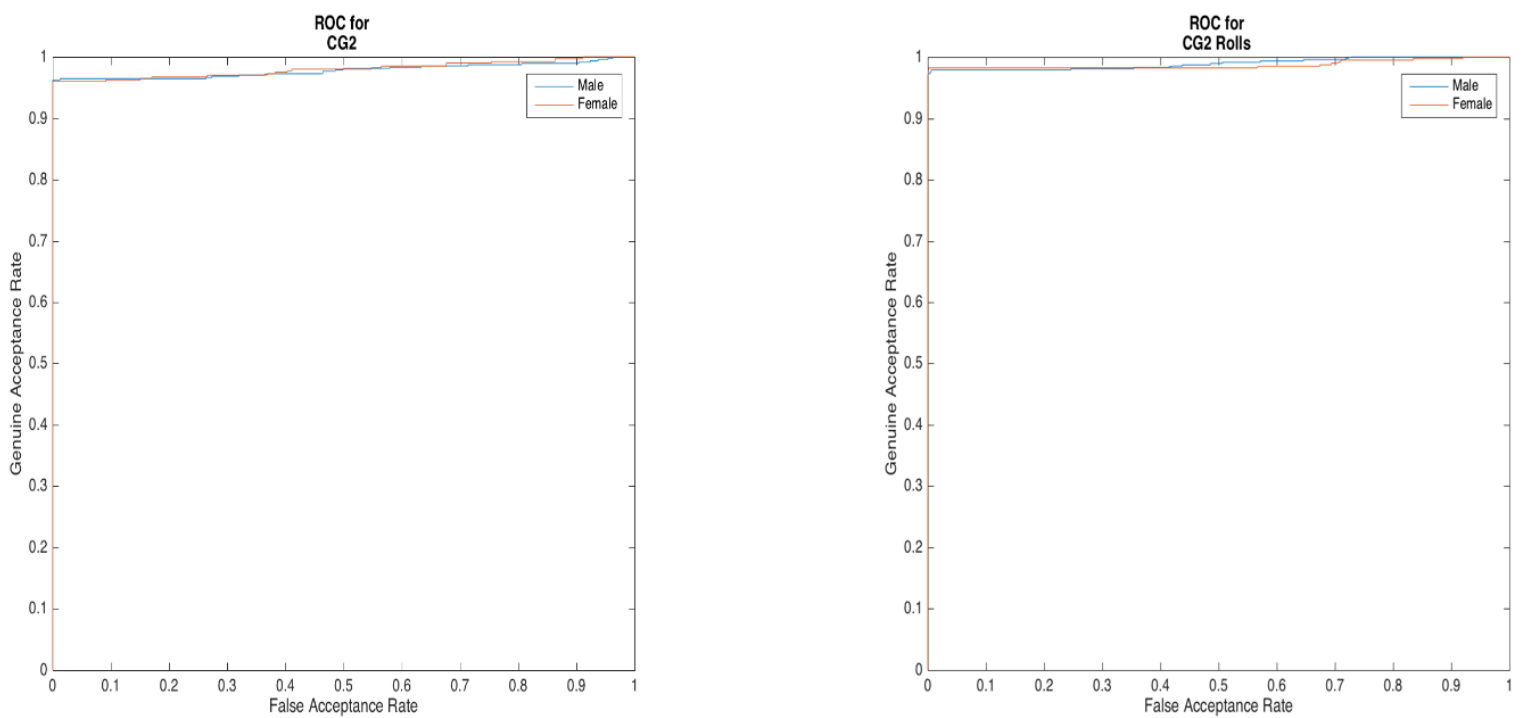

Figure B.6.3 SourceAFIS FP II Guardian Gender ROC Curves (Left) SourceAFIS FP II Guardian Rolls Gender ROC Curves (Right) 

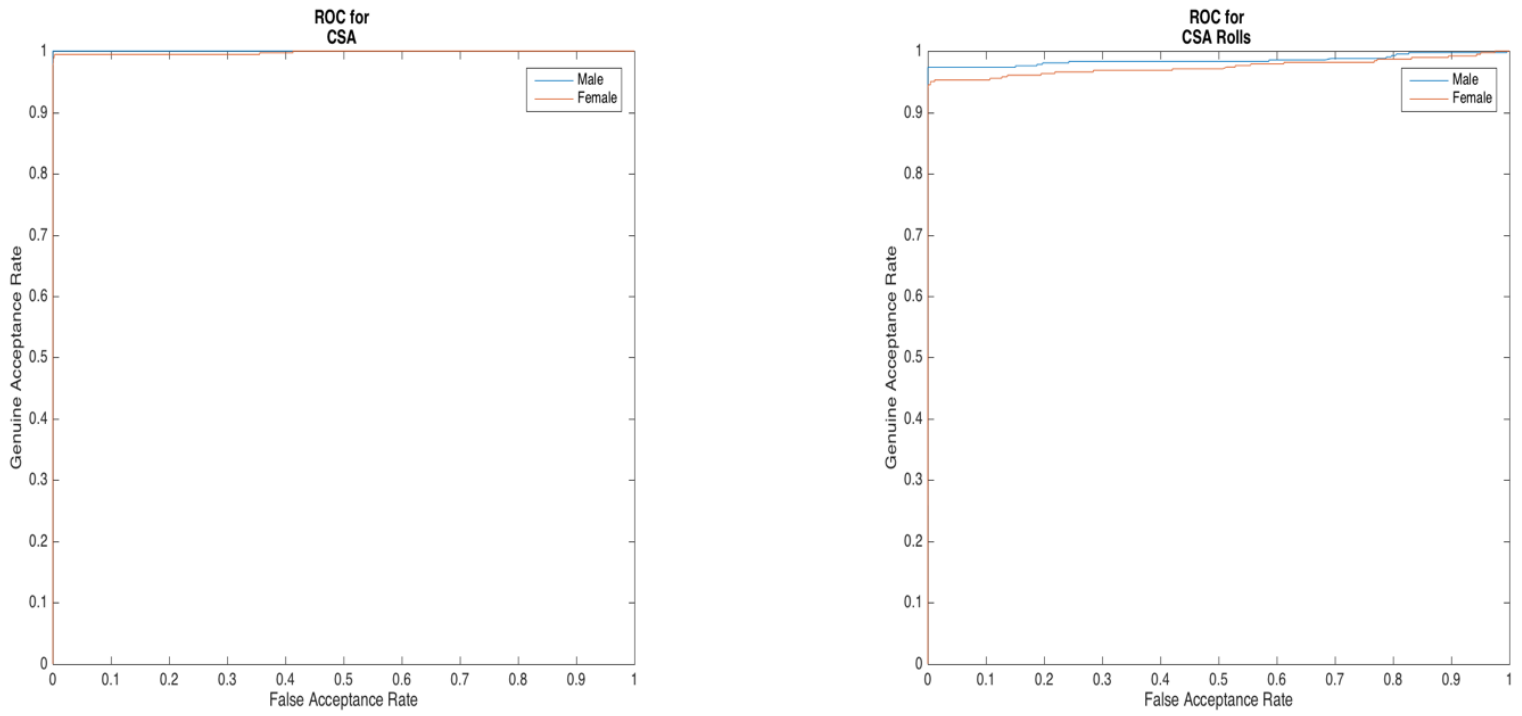

Figure B.6.4 SourceAFIS Crossmatch Seek Avenger Gender ROC Curves (Left) SourceAFIS Crossmatch Seek Avenger Gender ROC Curves (Right)
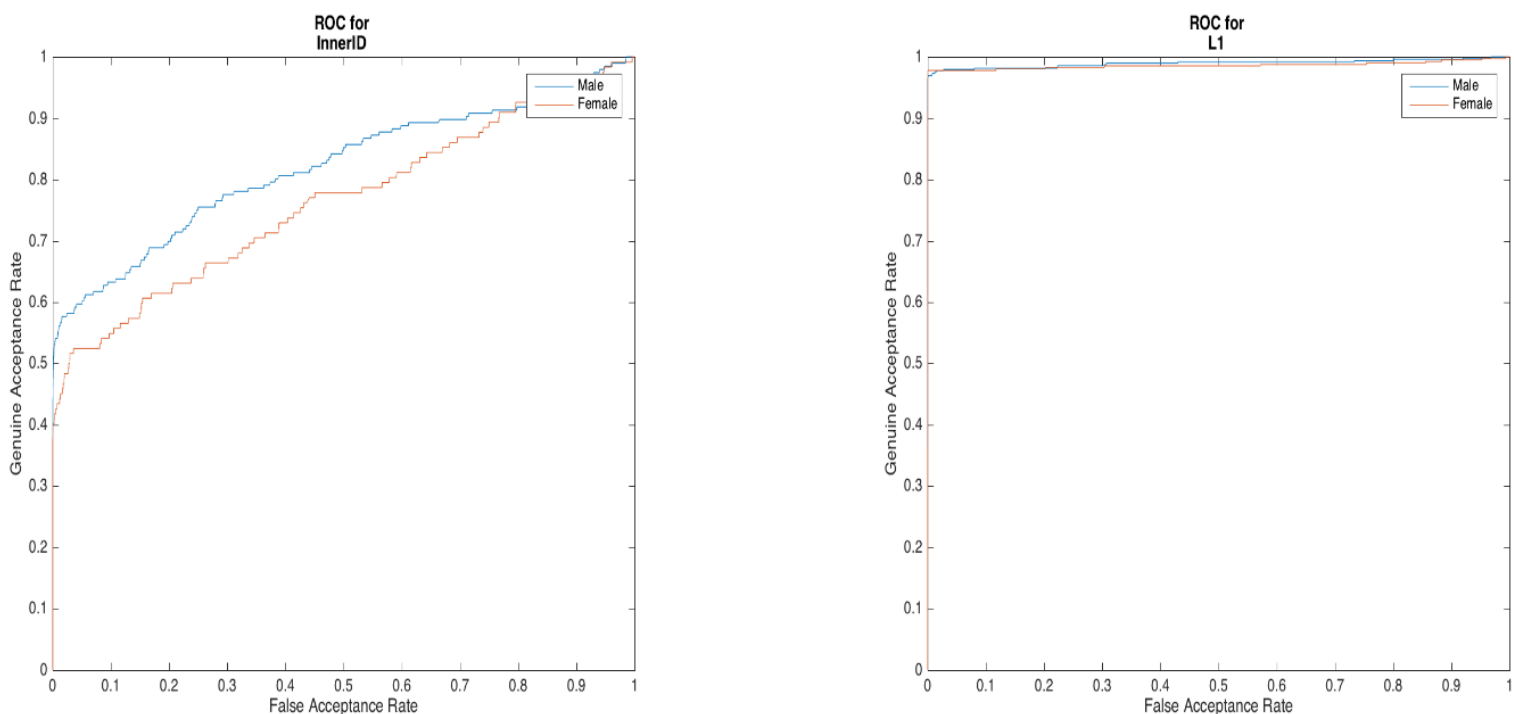

Figure B.6.5 SourceAFIS InnerID Gender ROC Curves (Left) SourceAFIS L1 Gender ROC Curves (Right) 

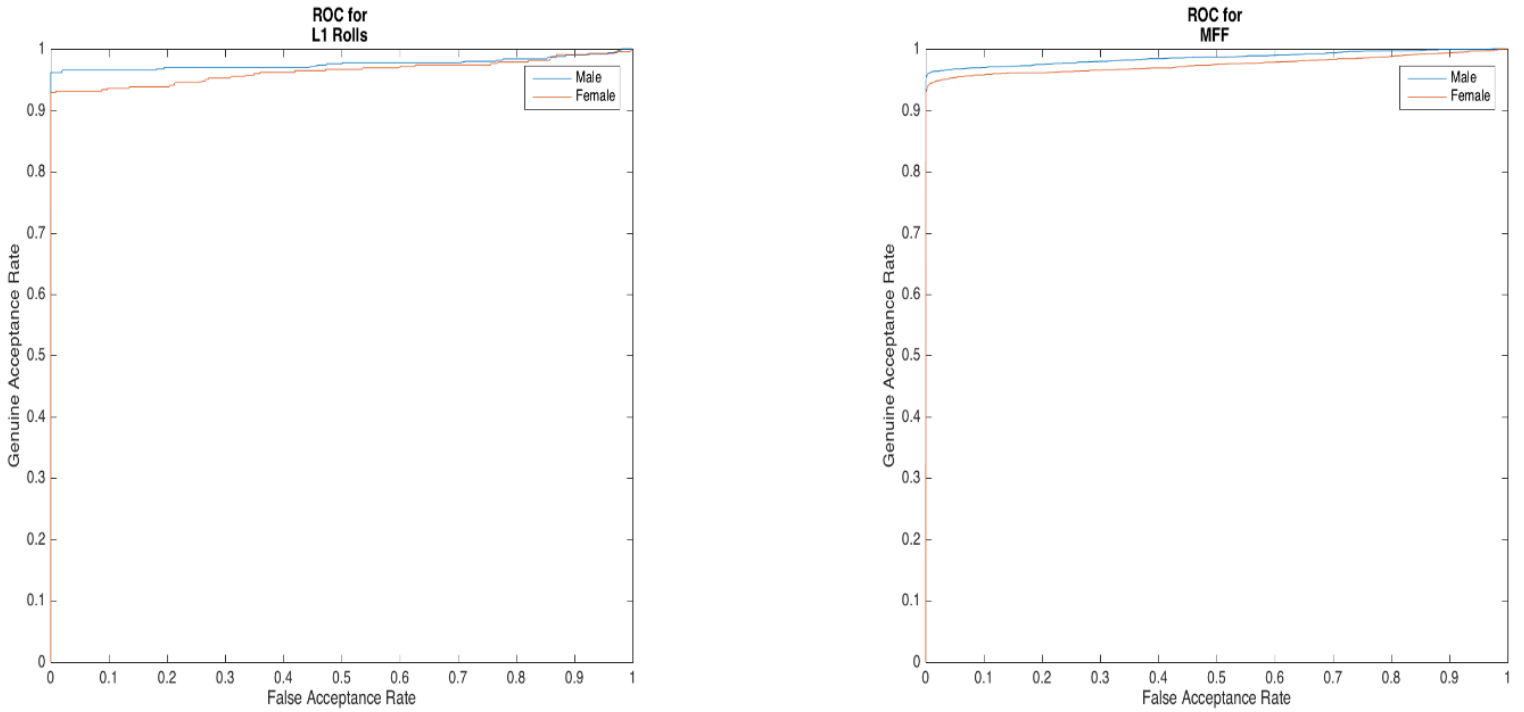

Figure B.6.6 SourceAFIS L1 Rolls Gender ROC Curves (Left) SourceAFIS Morpho FOTF Gender ROC Curves (Right)

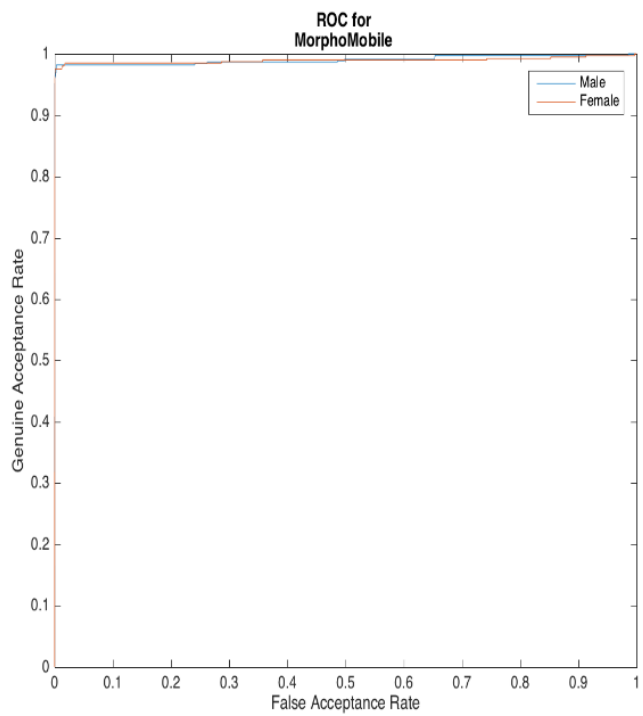

Figure B.6.7 SourceAFIS MorphoMobile Gender ROC Curves 


\section{B.7 Minutiae Box Plots by Device and Gender}
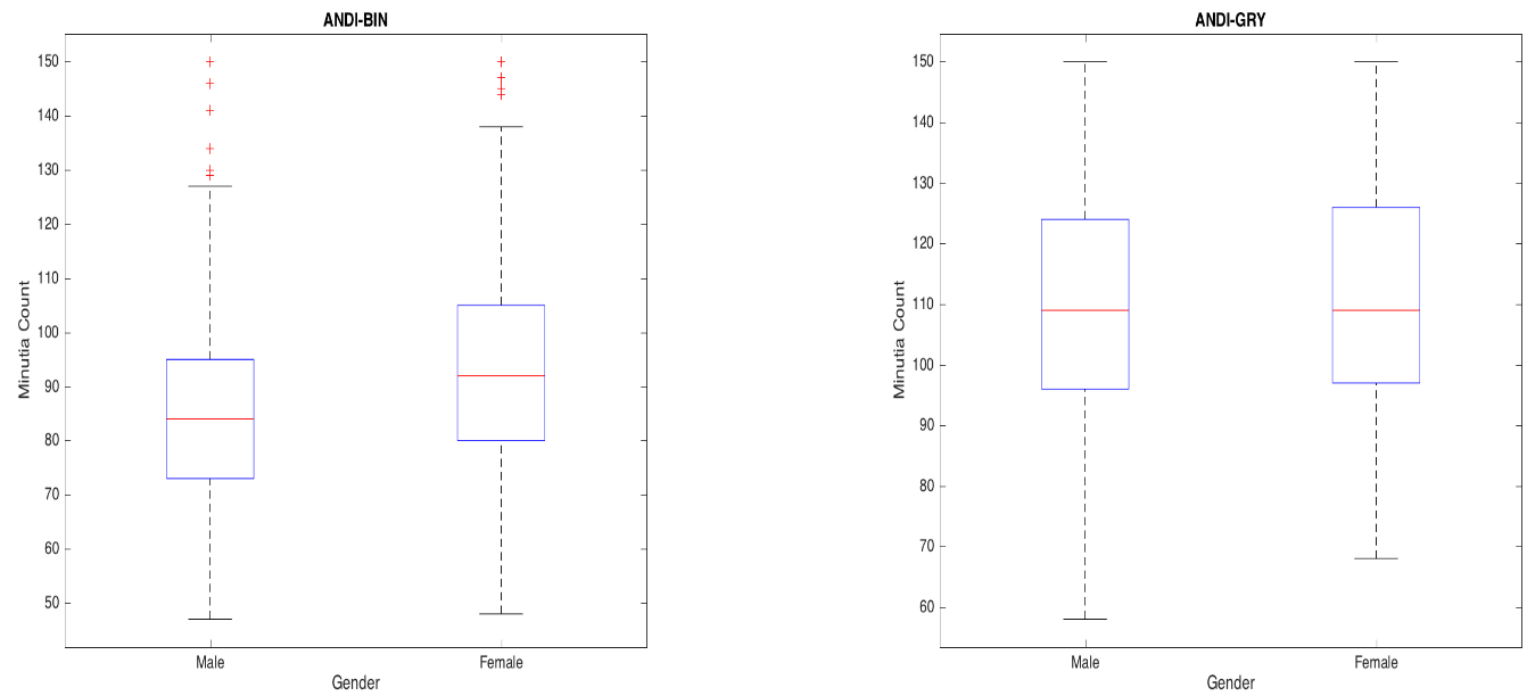

Figure B.7.1 Minutiae Box Plot for ANDI Binary by Gender (Left) Minutiae Box Plot for ANDI Greyscale by Gender (Right)
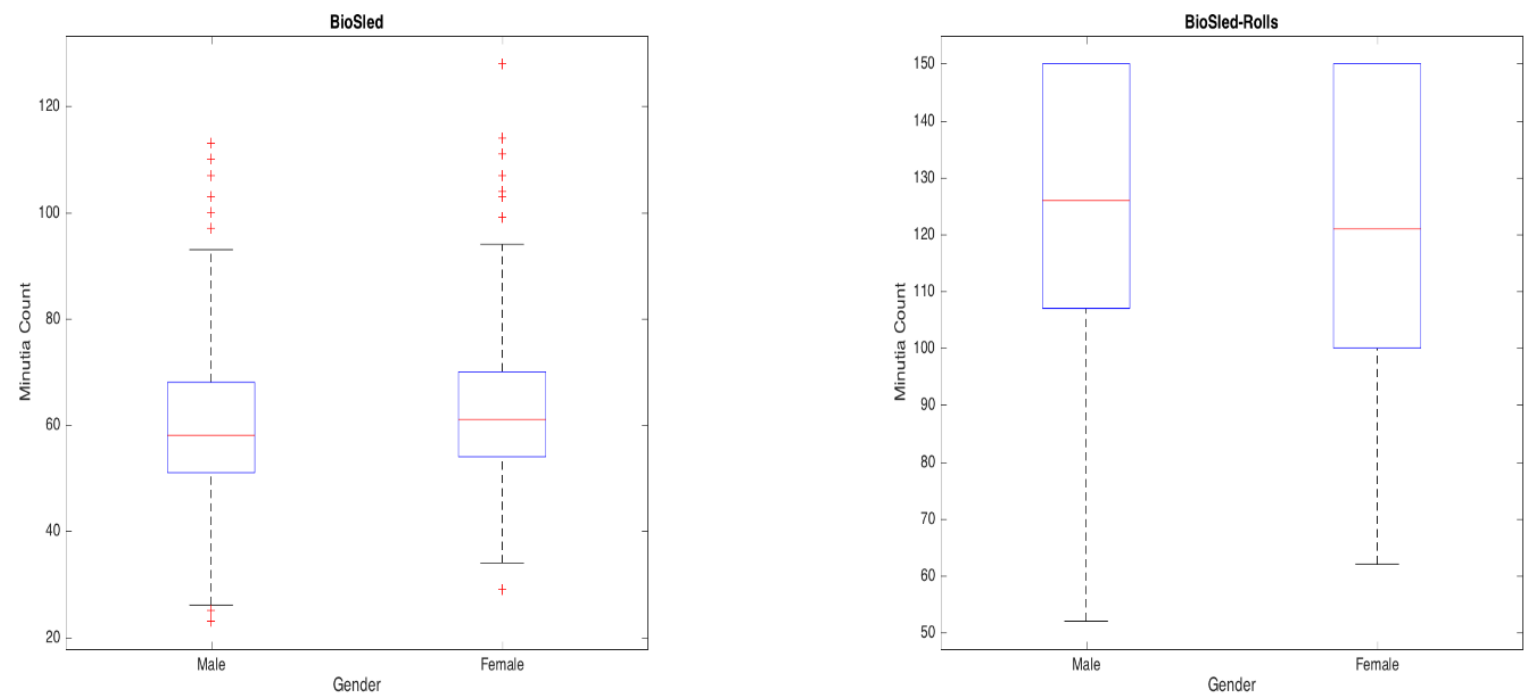

Figure B.7.2 Minutiae Box Plot for BioSled by Gender (Left) Minutiae Box Plot for BioSled Rolls by Gender (Right) 

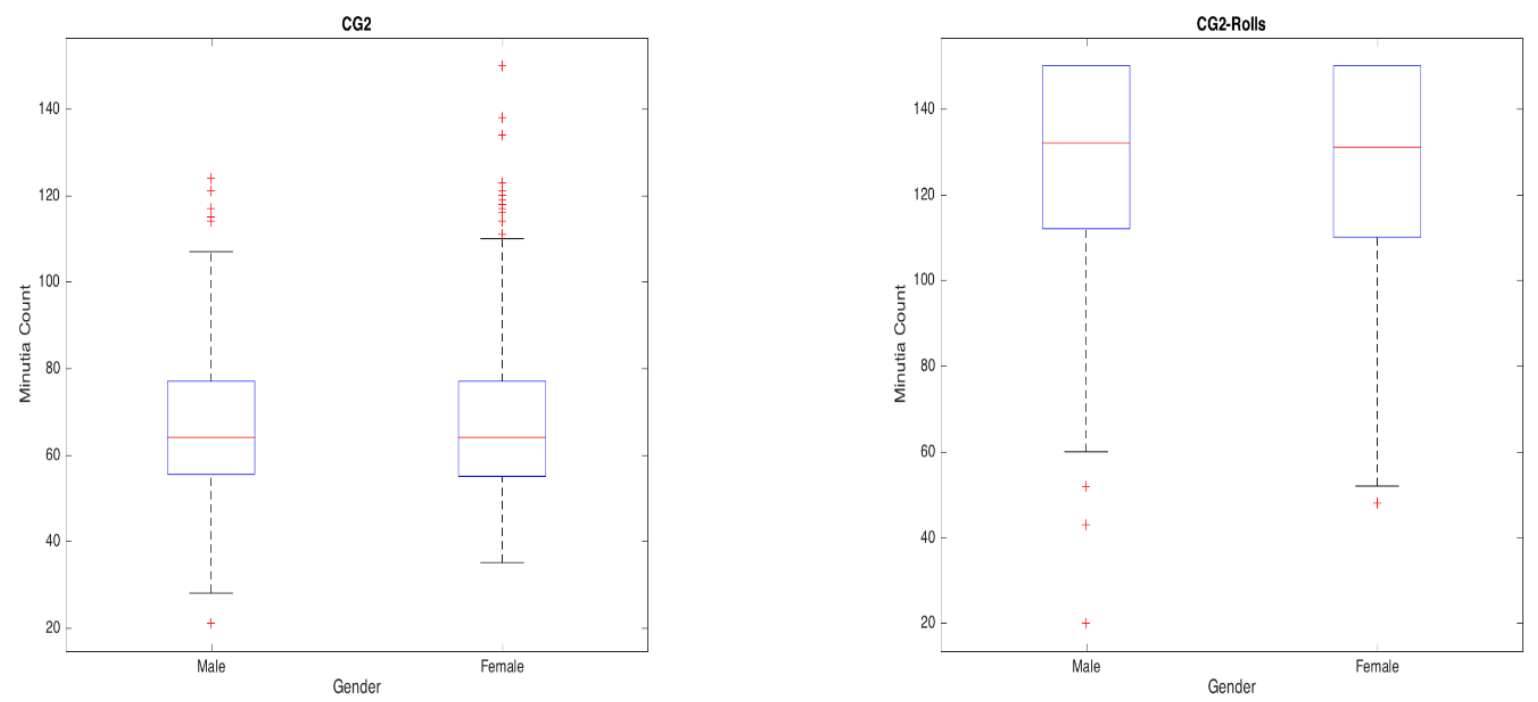

Figure B.7.3 Minutiae Box Plot for FP II Guardian by Gender (Left) Minutiae Box Plot for FP II Guardian Rolls by Gender (Right)
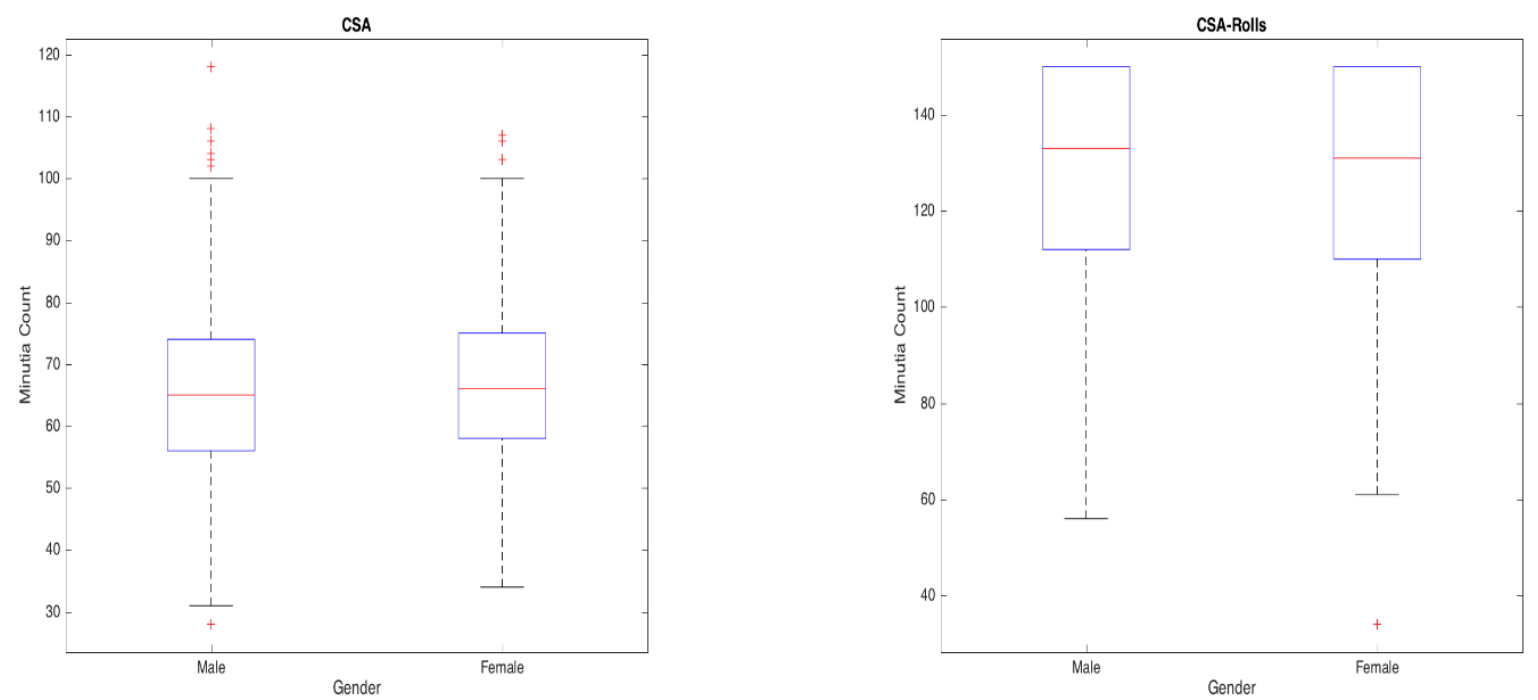

Figure B.7.4 Minutiae Box Plot for Crossmatch Seek Avenger by Gender (Left) Minutiae Box Plot for Crossmatch Seek Avenger Rolls by Gender (Right) 

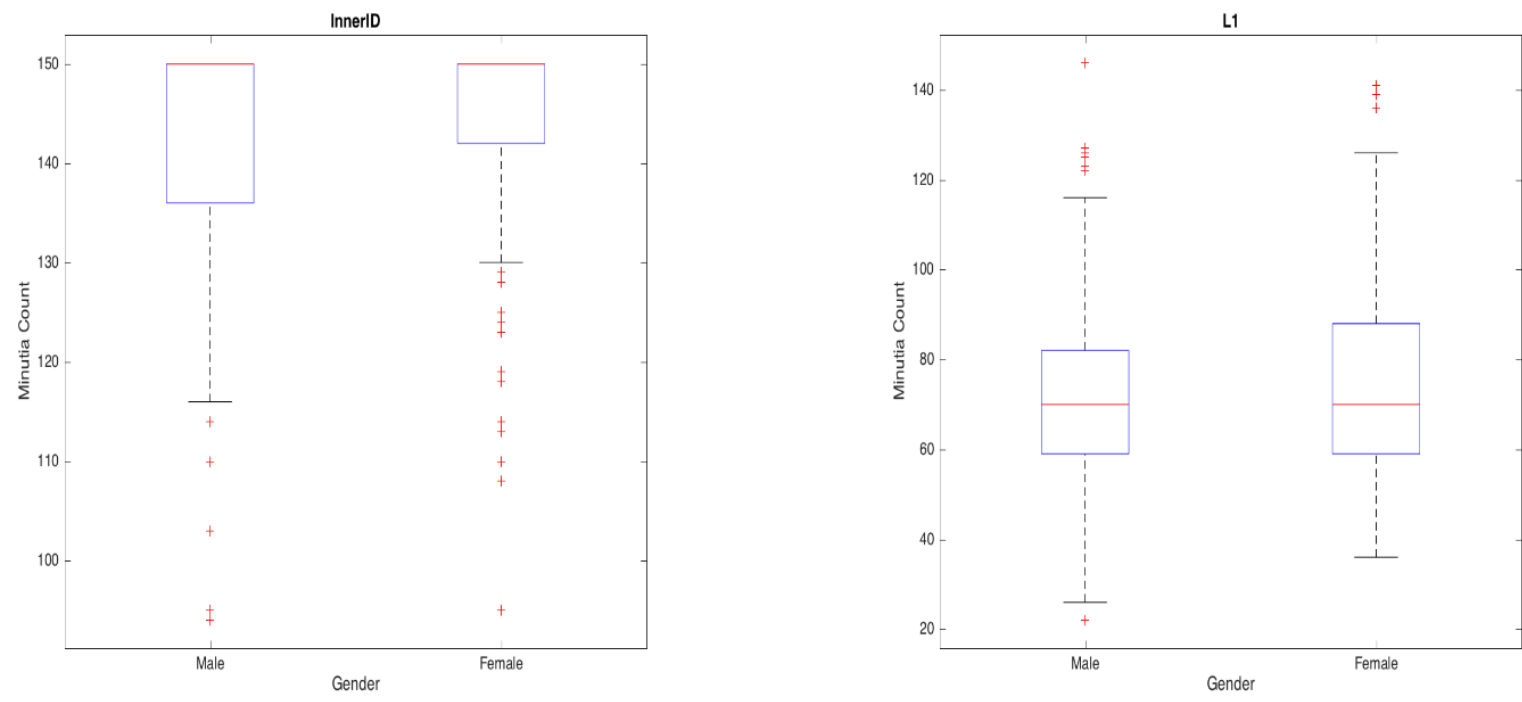

Figure B.7.5 Minutiae Box Plot for InnerID by Gender (Left) Minutiae Box Plot for L1 by Gender (Right)
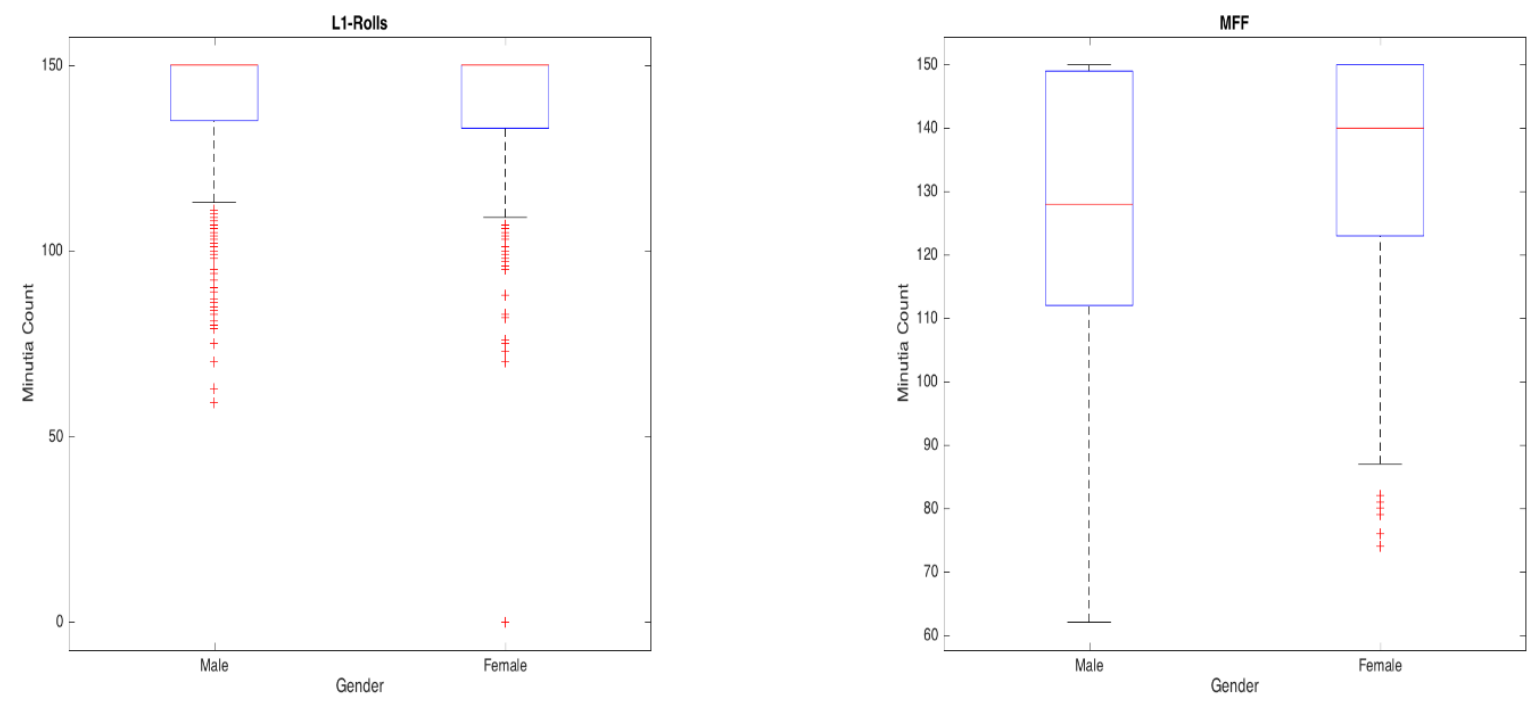

Figure B.7.6 Minutiae Box Plot for L1 Rolls by Gender (Left) Minutiae Box Plot for Morpho FOTF by Gender (Right) 


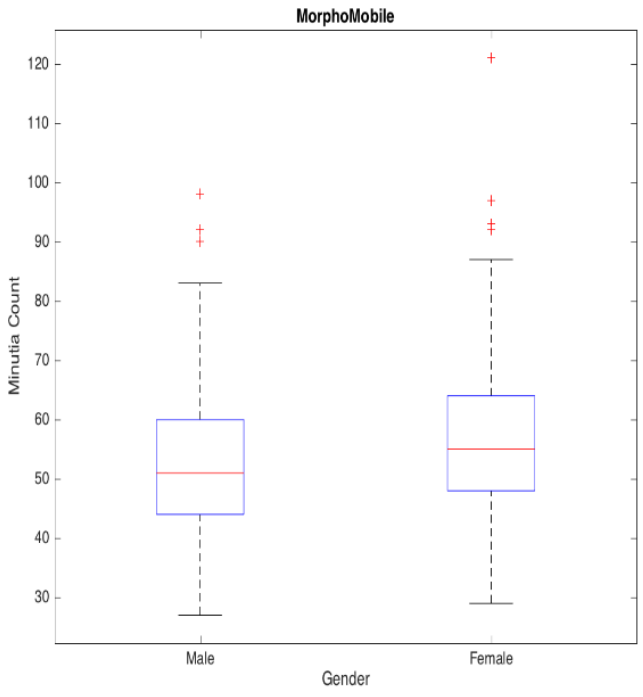

Figure B.7.7 Minutiae Box Plot for MorphoMobile by Gender

\section{B.8 Equal Error Rates For Gender Based Study}

Table B.10 NBIS Gender Based EER Values

\begin{tabular}{|r|ll|}
\hline NBIS & \multicolumn{2}{|c|}{ EER Value } \\
\hline Device & Male & Female \\
ANDI BIN & 0.0310 & 0.0561 \\
ANDI GRY & 0.0607 & 0.0577 \\
BioSled & 0.0696 & 0.0222 \\
BioSled Rolls & 0.0480 & 0.0262 \\
CG2 & 0.0335 & 0.0350 \\
CG2 Rolls & 0.0210 & 0.0125 \\
CSA & 0.0050 & 0.0139 \\
CSA Rolls & 0.0192 & 0.0314 \\
InnerID & 0.2125 & 0.2412 \\
L1 & 0.0276 & 0.0244 \\
MFF & 0.0176 & 0.0113 \\
MorphoMobile & 0.0219 & 0.0433 \\
\hline
\end{tabular}


Table B.11 Neurotech Gender Based EER Values

\begin{tabular}{|r|ll|}
\hline Neurotech & \multicolumn{2}{|c|}{ EER Value } \\
\hline Device & Male & Female \\
ANDI BIN & 0.0268 & 0.0441 \\
ANDI GRY & 0.0193 & 0.0501 \\
BioSled & 0.0518 & 0.0056 \\
BioSled Rolls & 0.0281 & 0.0108 \\
CG2 & 0.0294 & 0.0303 \\
CG2 Rolls & 0.0128 & 0.0000 \\
CSA & 0.0000 & 0.0057 \\
CSA Rolls & 0.0048 & 0.0107 \\
InnerID & 0.1138 & 0.1620 \\
L1 & 0.0245 & 0.0196 \\
MFF & 0.0154 & 0.0082 \\
MorphoMobile & 0.0132 & 0.0152 \\
\hline
\end{tabular}

Table B.12 SourceAFIS Gender Based EER Values

\begin{tabular}{|r|ll|}
\hline SourceAFIS & \multicolumn{2}{|c|}{$E E R$ Value } \\
\hline Device & Male & Female \\
ANDI BIN & 0.0446 & 0.0757 \\
ANDI GRY & 0.1049 & 0.1210 \\
BioSled & 0.0783 & 0.0139 \\
BioSled Rolls & 0.0708 & 0.0785 \\
CG2 & 0.0356 & 0.0400 \\
CG2 Rolls & 0.0210 & 0.0175 \\
CSA & 0.0000 & 0.0056 \\
CSA Rolls & 0.0264 & 0.0471 \\
InnerID & 0.2500 & 0.3197 \\
L1 & 0.0224 & 0.0220 \\
MFF & 0.0346 & 0.0470 \\
MorphoMobile & 0.0175 & 0.0175 \\
\hline
\end{tabular}


Appendix $\mathrm{C}$

Age Based Study 
This appendix shows all of the results from the Age Based Demographic Study. The data was broken into 4 categories, ages 18-19, 20-29, 30-39, and 40 and over. The largest group is made up of age 20 to 29 year old subjects due to the environment where the collection was held. The age breakdowns can be seen in Figure 3.6. The results that can be seen in this section include: genuine and imposter distributions, Kullback Leibler Divergence measures, maximum and minimum match scores, ROC Curves, area under the curve, and Equal Error Rates for each matching run. All of the results stated above are shown for each of the three matchers used. At the end of this appendix there are box plots that represent the number of minutiae found in images from each device, this data came from the NBIS matcher.

\section{C.1 NBIS Genuine/Imposter Score Distributions}

Table C.1 NBIS 18-19 Genuine and Imposter KLD, Minimum and Maximum Scores

\begin{tabular}{|c|c|c|c|c|c|c|}
\hline \multicolumn{7}{|l|}{ NBIS 18-19 } \\
\hline Device & $\begin{array}{l}\text { Genuine } \\
\text { KLD }\end{array}$ & $\begin{array}{l}\text { Imposter } \\
\text { KLD }\end{array}$ & $\begin{array}{l}\text { Max } \\
\text { Genuine }\end{array}$ & $\begin{array}{l}\text { Min } \\
\text { Genuine }\end{array}$ & $\begin{array}{l}\text { Max } \\
\text { Imposter }\end{array}$ & $\begin{array}{l}\text { Min } \\
\text { Imposter }\end{array}$ \\
\hline ANDI BIN & 0.0838 & 0.8833 & 241 & 8 & 36 & 3 \\
\hline$A N D I G R Y$ & 0.0343 & 0.6518 & 332 & 16 & 40 & 4 \\
\hline Biosled & 0.0408 & 1.9944 & 298 & 11 & 47 & 0 \\
\hline Biosled Rolls & 0.0437 & 3.4163 & 277 & 20 & 27 & 0 \\
\hline CG2 & 0.1168 & 3.1837 & 246 & 4 & 40 & 0 \\
\hline CG2 Rolls & 0.0633 & 2.1104 & 359 & 31 & 22 & 0 \\
\hline CSA & 0.0394 & 1.7727 & 499 & 30 & 46 & 0 \\
\hline CSA Rolls & 0.0348 & 4.3977 & 506 & 10 & 28 & 3 \\
\hline InnerID & 0.6693 & 1.1118 & 118 & 10 & 49 & 5 \\
\hline$L 1$ & 0.0604 & 3.4227 & 297 & 16 & 47 & 0 \\
\hline L1 Rolls & 0.0529 & 2.4702 & 449 & 0 & 28 & 0 \\
\hline$M F F$ & 0.0320 & 1.8875 & 310 & 24 & 51 & 3 \\
\hline MorphoMobile & 0.0427 & 0.8768 & 278 & 11 & 35 & 0 \\
\hline
\end{tabular}


Table C.2 NBIS 20-29 Genuine and Imposter KLD, Minimum and Maximum Scores

\begin{tabular}{|c|c|c|c|c|c|c|c|}
\hline \multicolumn{8}{|l|}{ NBIS 20-29 } \\
\hline Device & $\begin{array}{l}\text { Genuine } \\
\text { KLD }\end{array}$ & $\begin{array}{l}\text { Imposter } \\
\text { KLD }\end{array}$ & $\begin{array}{l}\text { Max } \\
\text { Genuine }\end{array}$ & $\begin{array}{l}\text { Min } \\
\text { Genuine }\end{array}$ & $\begin{array}{l}\text { Max } \\
\text { Imposter }\end{array}$ & $\begin{array}{l}\text { Min } \\
\text { Imposter }\end{array}$ & $\begin{array}{l}\text { Genuine } \\
\text { KLD }\end{array}$ \\
\hline$A N D I B I N$ & 0.0114 & 0.1265 & 319 & 5 & 44 & 0 & 0.45367 \\
\hline ANDI GRY & 0.0131 & 0.2460 & 358 & 8 & 64 & 0 & 0.325301 \\
\hline Biosled & 0.0065 & 0.0128 & 304 & 4 & 245 & 0 & 10.166853 \\
\hline BioSled Rolls & 0.0136 & 0.6659 & 317 & 5 & 184 & 0 & 2.039956 \\
\hline CG2 & 0.0132 & 0.0120 & 358 & 4 & 159 & 0 & 20.75243 \\
\hline CG2 Rolls & 0.0071 & 0.0106 & 452 & 4 & 262 & 0 & 0.031799 \\
\hline CSA & 0.1228 & 0.8538 & 289 & 17 & 61 & 0 & 0.615569 \\
\hline CSA Rolls & 0.0452 & 7.4043 & 435 & 4 & 32 & 0 & 0.566192 \\
\hline InnerID & 0.0688 & 0.7238 & 176 & 7 & 73 & 4 & 0.687748 \\
\hline L1 & 0.0092 & 0.0190 & 328 & 4 & 137 & 0 & 0.546715 \\
\hline L1 Rolls & 0.0258 & 0.0337 & 422 & 4 & 143 & 0 & 0.084613 \\
\hline MFF & 0.0137 & 0.1676 & 360 & 5 & 48 & 0 & 0.330587 \\
\hline MorphoMobile & 0.0373 & 3.5694 & 261 & 3 & 44 & 0 & 0.550937 \\
\hline
\end{tabular}

Table C.3 NBIS 30-39 Genuine and Imposter KLD, Minimum and Maximum Scores

\begin{tabular}{|c|c|c|c|c|c|c|c|}
\hline \multicolumn{8}{|l|}{ NBIS 30-39 } \\
\hline Device & $\begin{array}{l}\text { Genuine } \\
\text { KLD }\end{array}$ & $\begin{array}{l}\text { Imposter } \\
\text { KLD }\end{array}$ & $\begin{array}{l}\text { Max } \\
\text { Genuine }\end{array}$ & $\begin{array}{l}\text { Min } \\
\text { Genuine }\end{array}$ & $\begin{array}{l}\text { Max } \\
\text { Imposter }\end{array}$ & $\begin{array}{l}\text { Min } \\
\text { Imposter }\end{array}$ & $\begin{array}{l}\text { Genuine } \\
\text { KLD }\end{array}$ \\
\hline ANDI BIN & 0.0489 & 1.0251 & 275 & 6 & 27 & 3 & 0.397235 \\
\hline ANDI GRY & 0.0730 & 0.8623 & 281 & 10 & 48 & 3 & 0.404862 \\
\hline Biosled & 0.0554 & 1.9477 & 267 & 9 & 27 & 0 & 0.374085 \\
\hline BioSled Rolls & 0.0471 & 1.4452 & 289 & 27 & 21 & 3 & 0.570223 \\
\hline CG2 & 0.0786 & 1.2275 & 259 & 8 & 29 & 3 & $0.33427 \epsilon$ \\
\hline CG2 Rolls & 0.0552 & 0.9662 & 336 & 29 & 22 & 3 & 0.392907 \\
\hline CSA & 0.2237 & 3.2299 & 244 & 8 & 34 & 0 & $0.32100 \mathrm{~s}$ \\
\hline CSA Rolls & 0.0762 & 3.0186 & 292 & 36 & 22 & 3 & 0.57702 \\
\hline InnerID & 0.6002 & 2.8443 & 29 & 14 & 27 & 10 & 1.470773 \\
\hline$L 1$ & 0.0559 & 4.0166 & 222 & 54 & 24 & 0 & 0.468441 \\
\hline L1 Rolls & 0.1650 & 2.7238 & 221 & 4 & 23 & 3 & 0.640702 \\
\hline MFF & 0.1250 & 1.0947 & 262 & 8 & 43 & 3 & 0.471992 \\
\hline MorphoMobile & 0.1000 & 0.7262 & 200 & 7 & 28 & 0 & 0.463784 \\
\hline
\end{tabular}


Table C.4 NBIS 40 and up Genuine and Imposter KLD, Minimum and Maximum Scores

\begin{tabular}{|c|c|c|c|c|c|c|c|}
\hline \multicolumn{8}{|l|}{ NBIS 40 and up } \\
\hline Device & $\begin{array}{l}\text { Genuine } \\
\text { KLD }\end{array}$ & $\begin{array}{l}\text { Imposter } \\
\text { KLD }\end{array}$ & $\begin{array}{l}\text { Max } \\
\text { Genuine }\end{array}$ & $\begin{array}{l}\text { Min } \\
\text { Genuine }\end{array}$ & $\begin{array}{l}\text { Max } \\
\text { Imposter }\end{array}$ & $\begin{array}{l}\text { Min } \\
\text { Imposter }\end{array}$ & $\begin{array}{l}\text { Genuine } \\
\text { KLD }\end{array}$ \\
\hline ANDI BIN & 0.1035 & 0.9506 & 225 & 14 & 41 & 3 & 0.310583 \\
\hline ANDI GRY & 0.1147 & 0.9976 & 251 & 22 & 48 & 6 & 0.384789 \\
\hline Biosled & 0.0398 & 1.6229 & 241 & 19 & 27 & 0 & 0.42708 \\
\hline BioSled Rolls & 0.0861 & 2.5945 & 170 & 9 & 18 & 0 & 0.831423 \\
\hline CG2 & 0.0984 & 2.7650 & 209 & 5 & 23 & 0 & 0.494974 \\
\hline CG2 Rolls & 0.0985 & 1.6706 & 327 & 7 & 25 & 4 & 0.293415 \\
\hline CSA & 0.0485 & 5.4184 & 242 & 23 & 23 & 0 & 0.586351 \\
\hline CSA Rolls & 0.0991 & 3.9052 & 337 & 5 & 20 & 3 & 0.616656 \\
\hline InnerID & 0.6008 & 1.7907 & 30 & 17 & 32 & 10 & 1.62096 \\
\hline L1 & 0.0718 & 2.9361 & 180 & 14 & 22 & 0 & 0.497768 \\
\hline L1 Rolls & 0.0499 & 4.7252 & 235 & 7 & 23 & 3 & 0.569319 \\
\hline MFF & 0.0436 & 1.1245 & 272 & 18 & 41 & 4 & 0.571957 \\
\hline MorphoMobile & 0.1182 & 0.6724 & 137 & 11 & 25 & 0 & 0.644492 \\
\hline
\end{tabular}




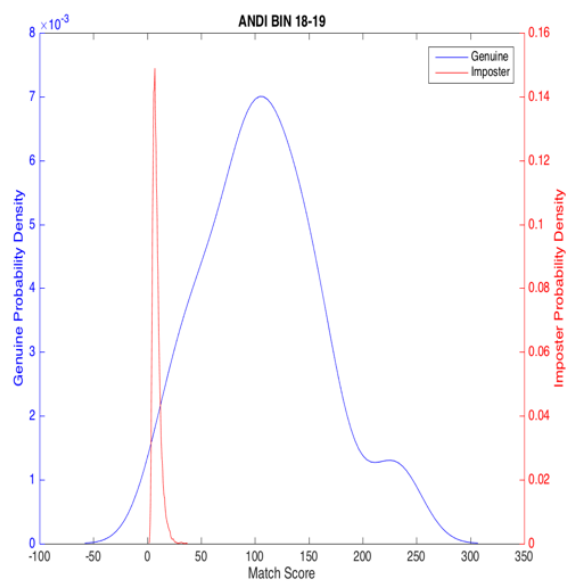

(a)

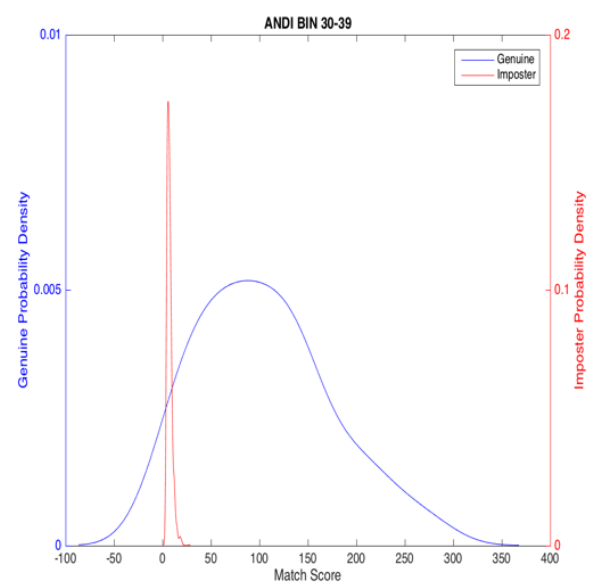

(c)

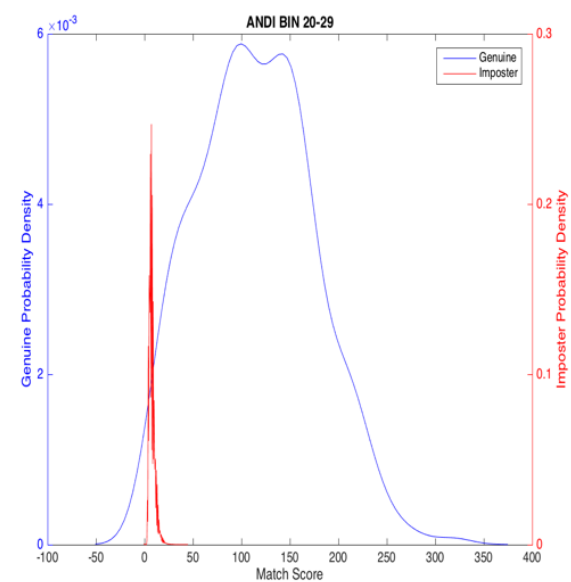

(b)

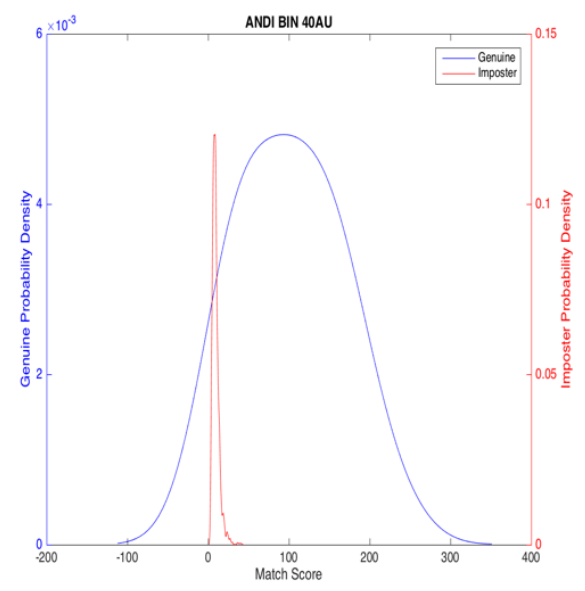

(d)

Figure C.1.1 NBIS ANDI Binary (a) Ages 18-19 (b) Ages 20-29 (c) Ages 30-39 (d) Ages 40 and up 


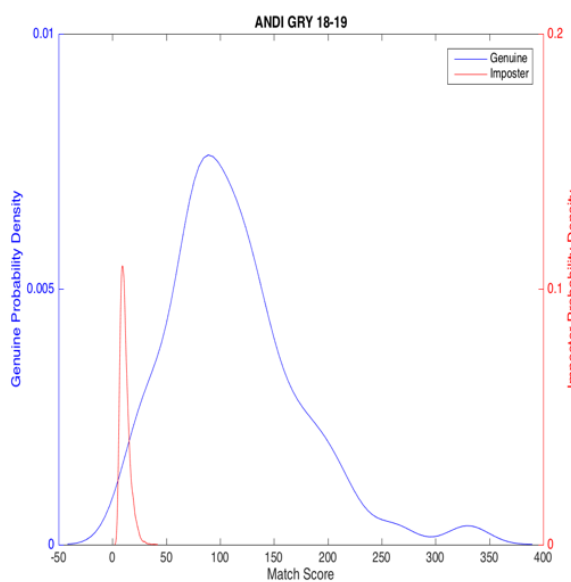

(a)

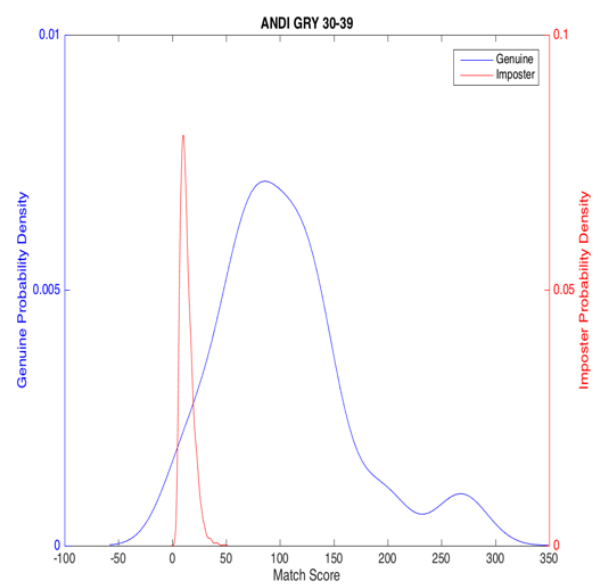

(c)

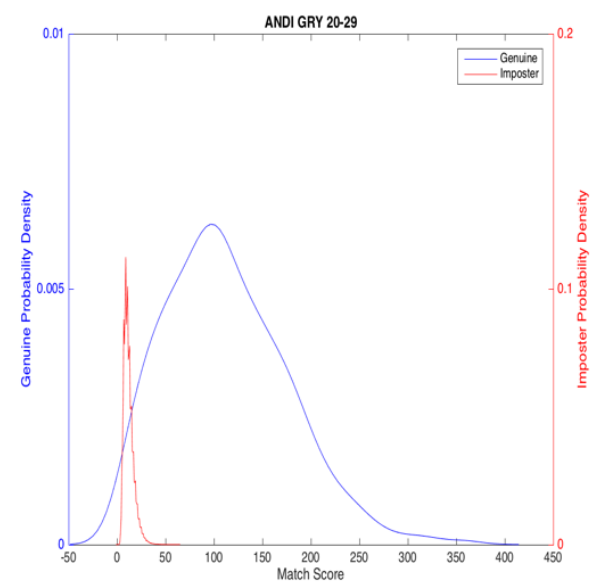

(b)

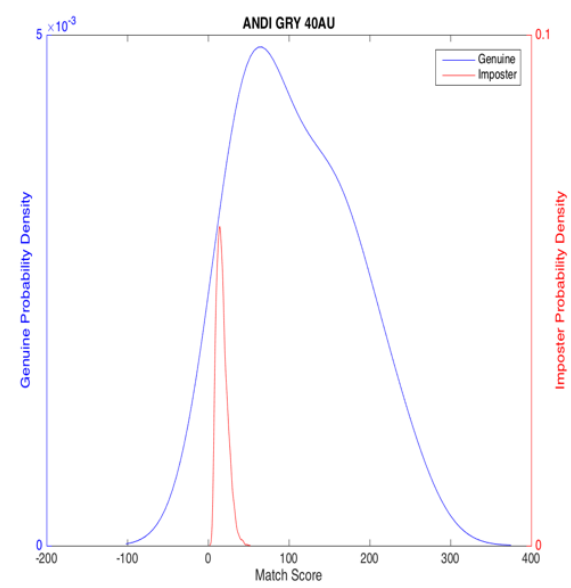

(d)

Figure C.1.2 NBIS ANDI Greyscale (a) Ages 18-19 (b) Ages 20-29 (c) Ages 30-39 (d) Ages 40 and up 


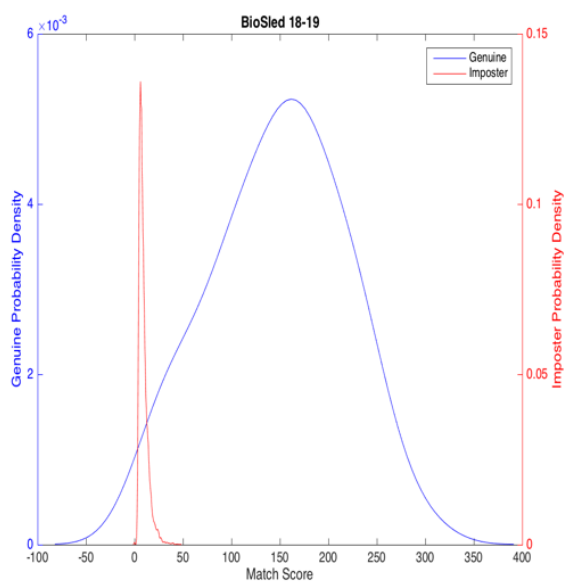

(a)

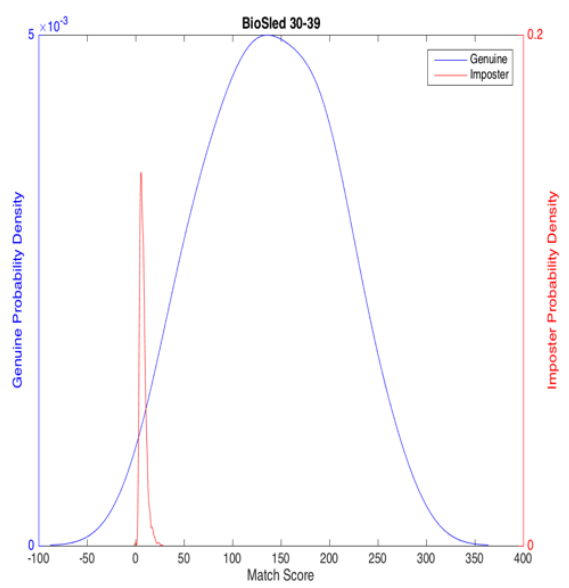

(c)

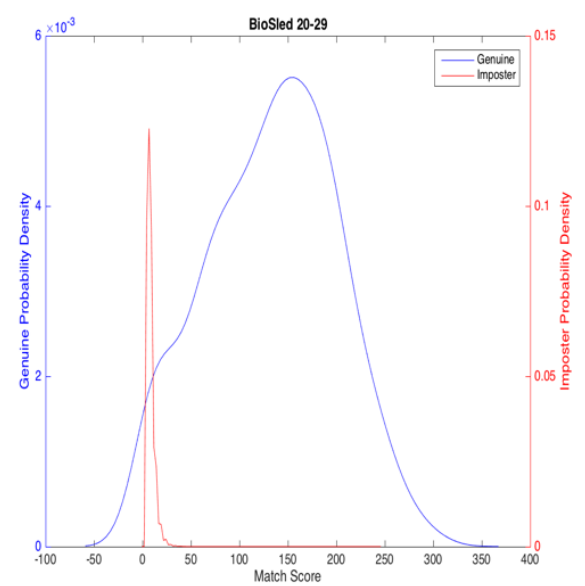

(b)

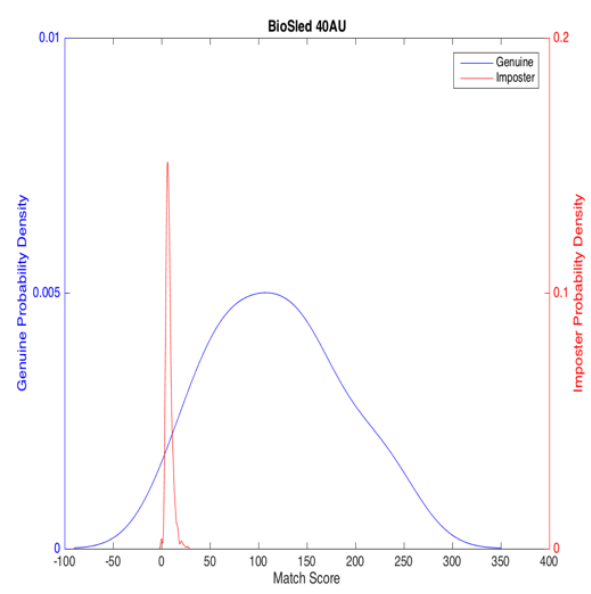

(d)

Figure C.1.3 NBIS BioSled (a) Ages 18-19 (b) Ages 20-29 (c) Ages 30-39 (d) Ages 40 and up 


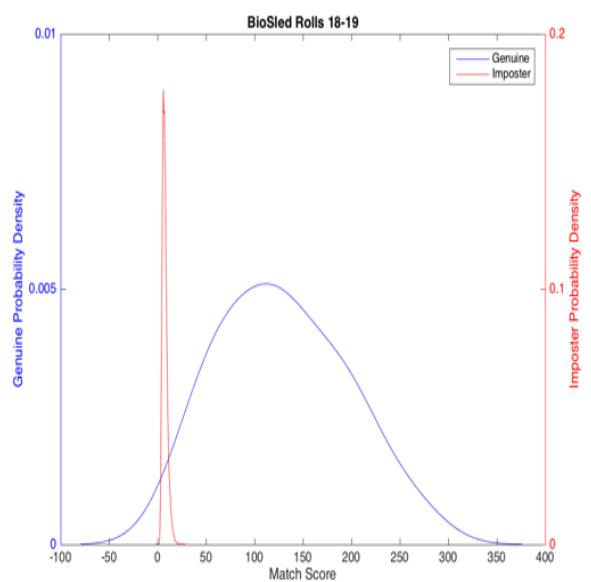

(a)

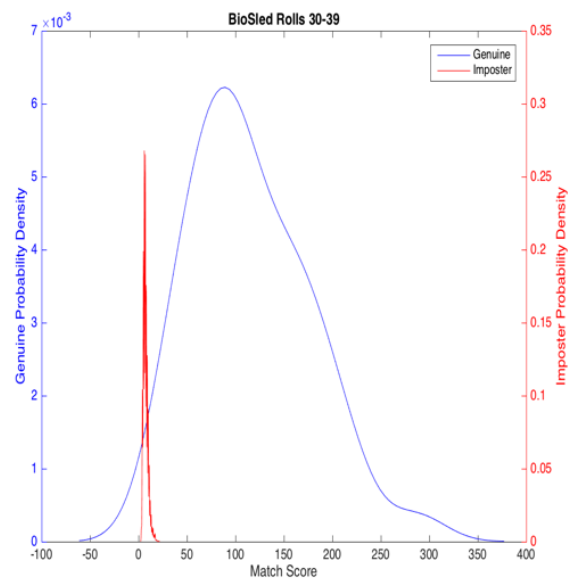

(c)

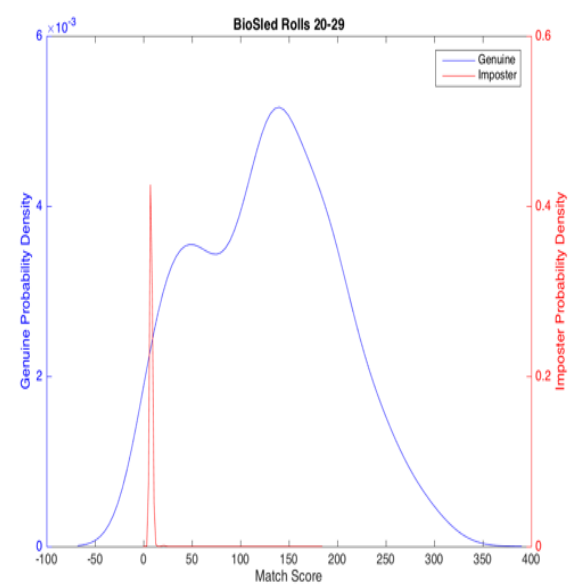

(b)

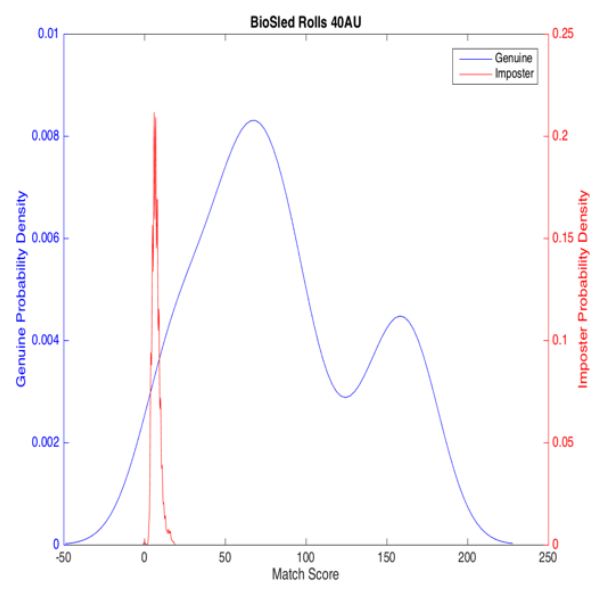

(d)

Figure C.1.4 NBIS BioSled Rolls (a) Ages 18-19 (b) Ages 20-29 (c) Ages 30-39 (d) Ages 40 and up 


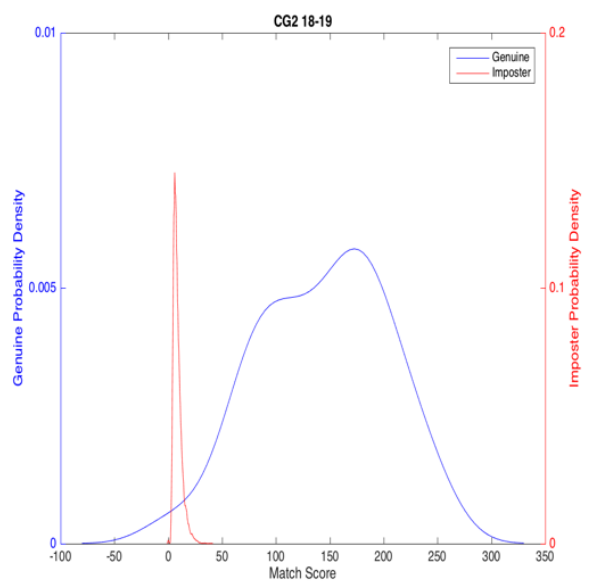

(a)

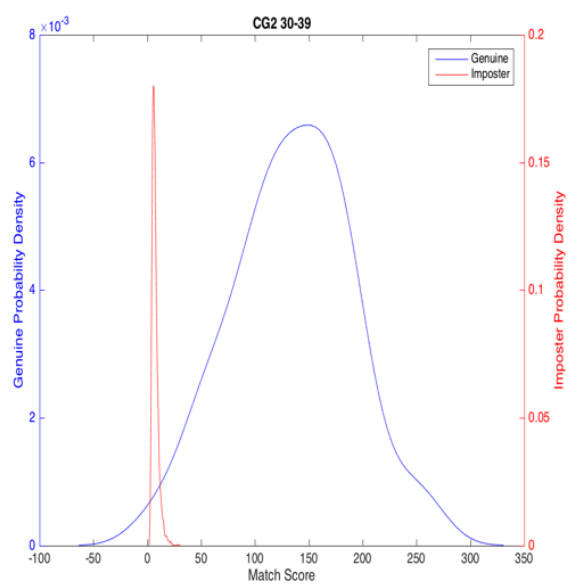

(c)

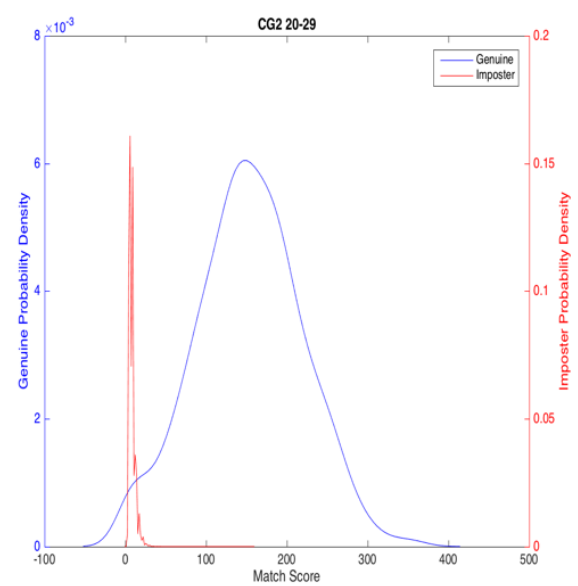

(b)

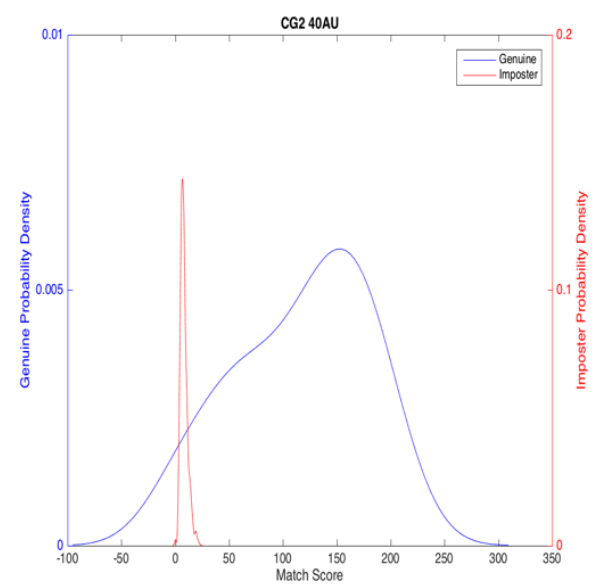

(d)

Figure C.1.5 NBIS FP II Guardian (a) Ages 18-19 (b) Ages 20-29 (c) Ages 30-39 (d) Ages 40 and up 


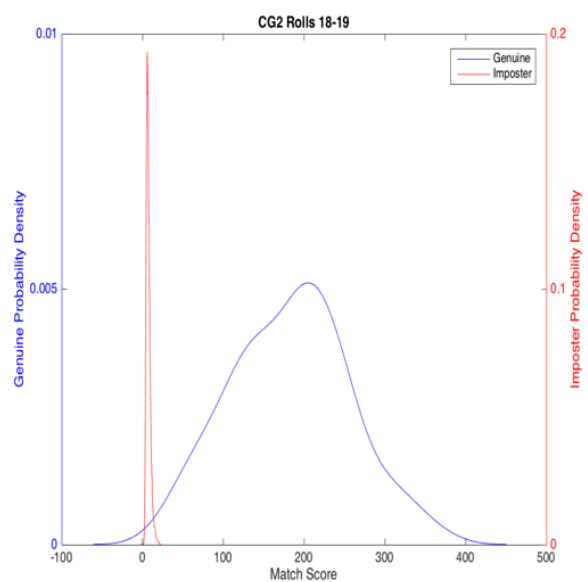

(a)

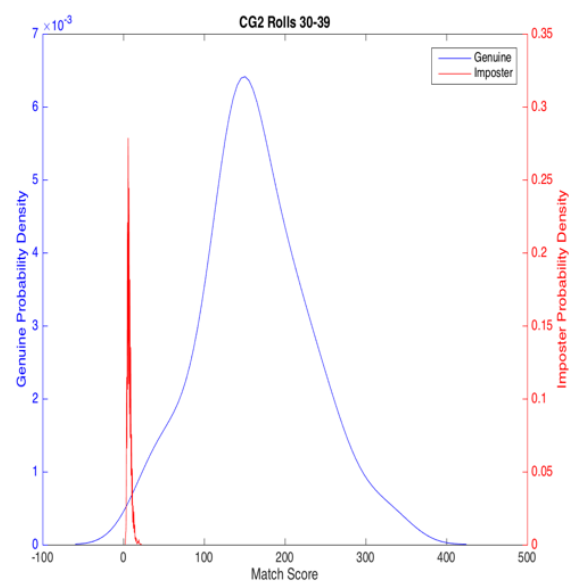

(c)

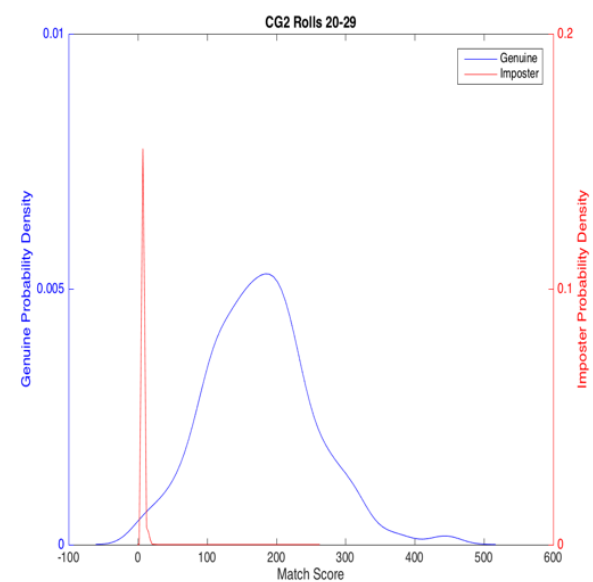

(b)

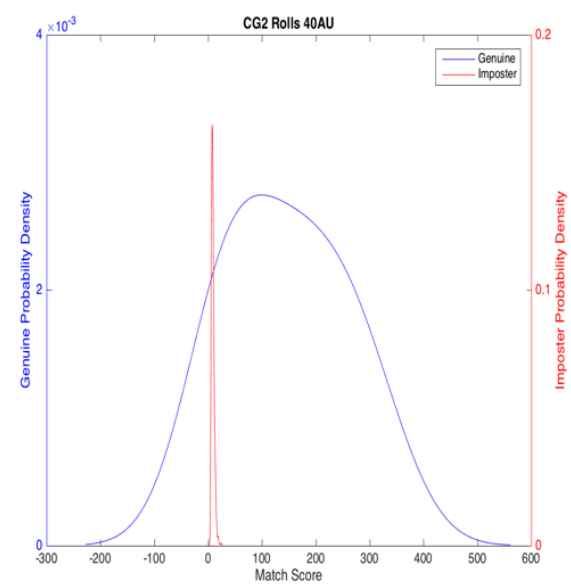

(d)

Figure C.1.6 NBIS FP II Guardian Rolls (a) Ages 18-19 (b) Ages 20-29 (c) Ages 30-39 (d) Ages 40 and up 


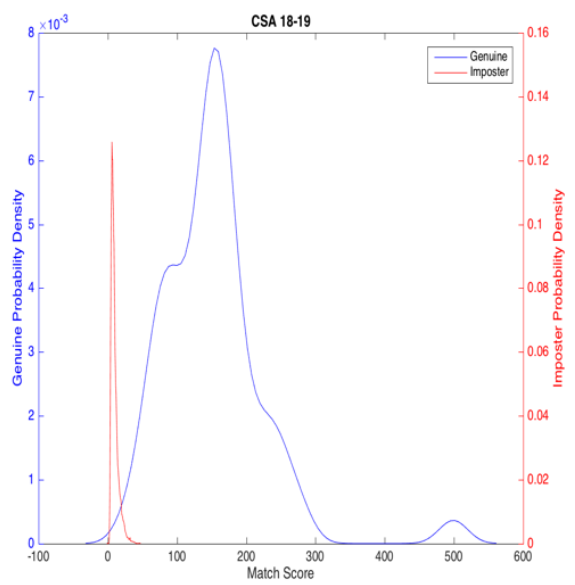

(a)

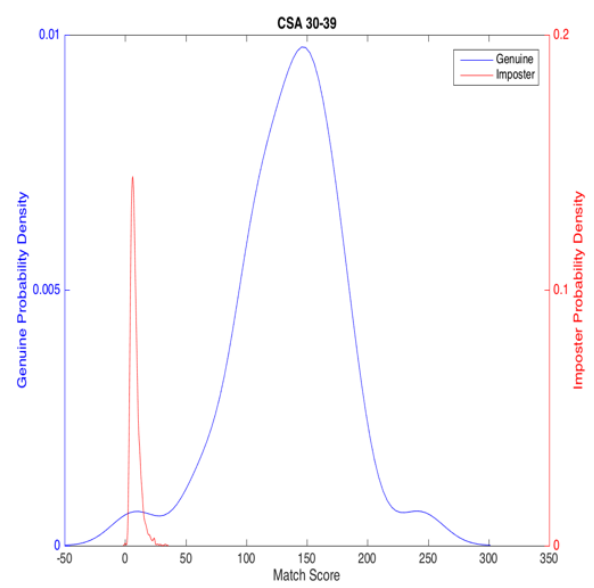

(c)

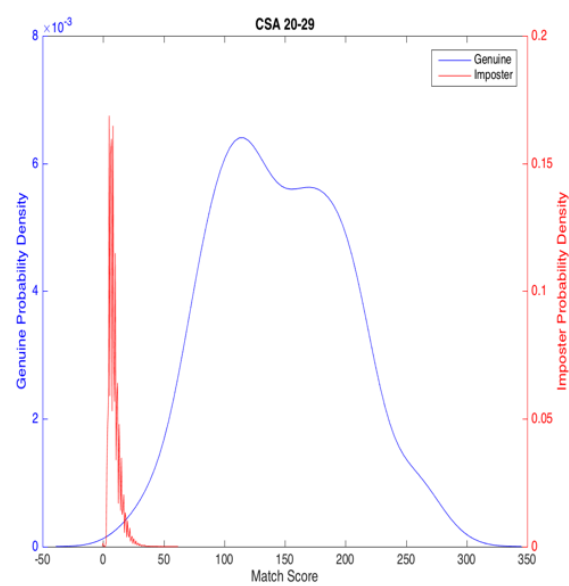

(b)

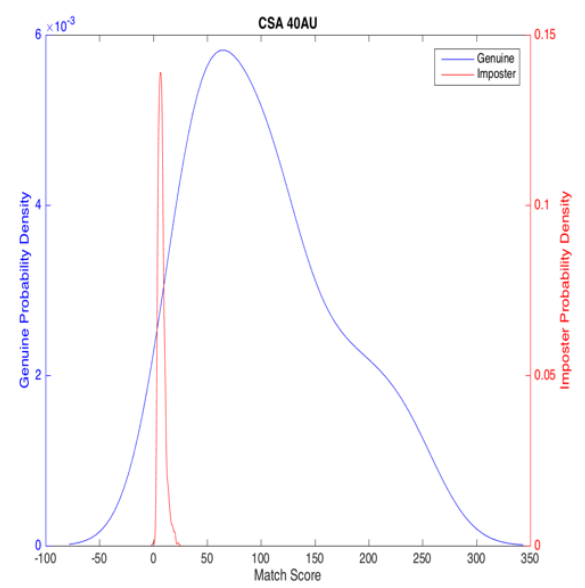

(d)

Figure C.1.7 NBIS Crossmatch Seek Avenger (a) Ages 18-19 (b) Ages 20-29 (c) Ages 30-39 (d) Ages 40 and up 


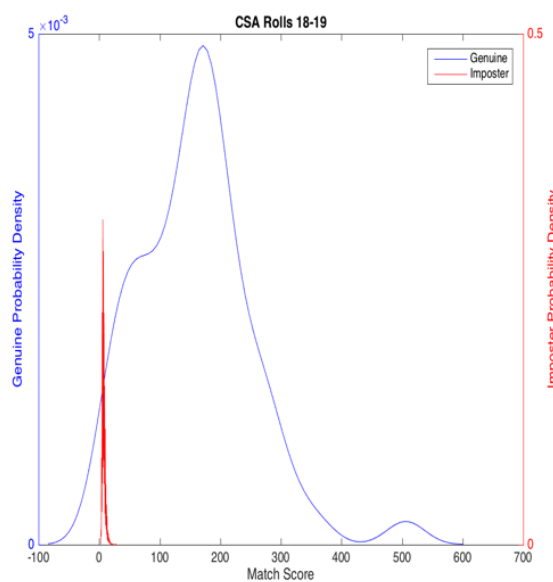

(a)

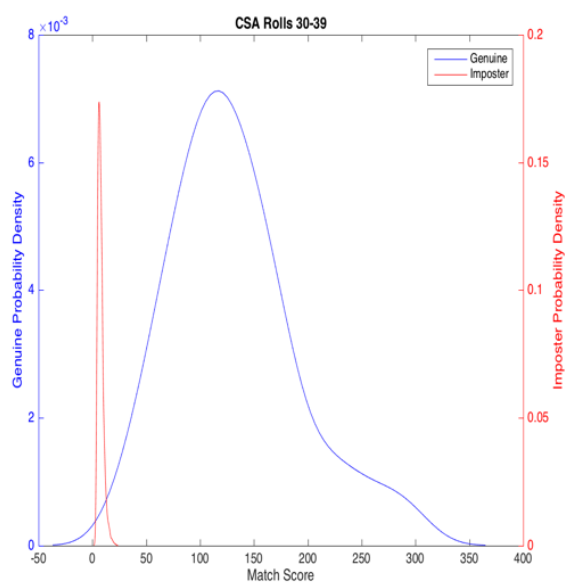

(c)

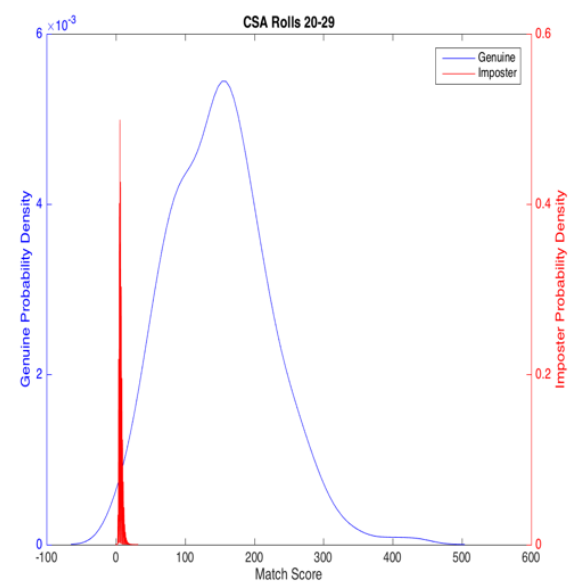

(b)

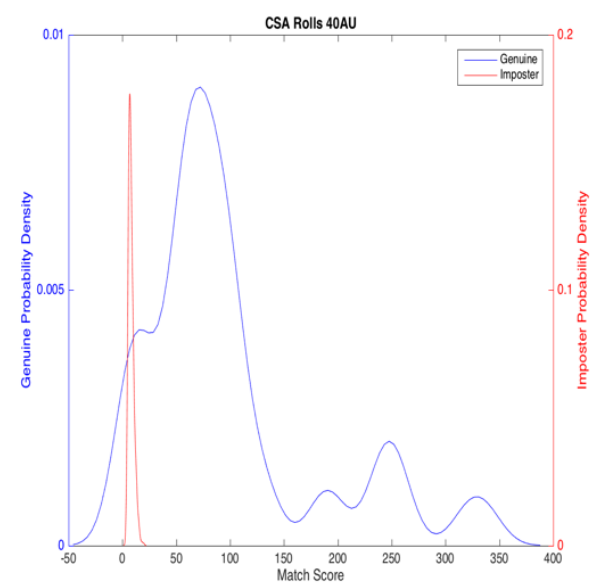

(d)

Figure C.1.8 NBIS Crossmatch Seek Avenger Rolls (a) Ages 18-19 (b) Ages 20-29 (c) Ages 30-39 (d) Ages 40 and up 


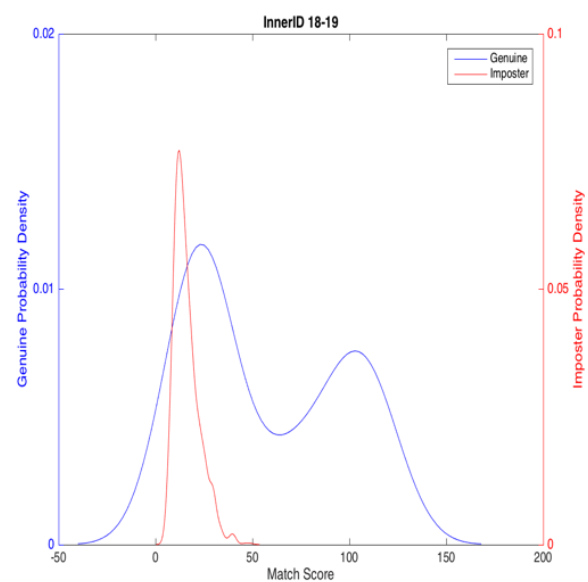

(a)

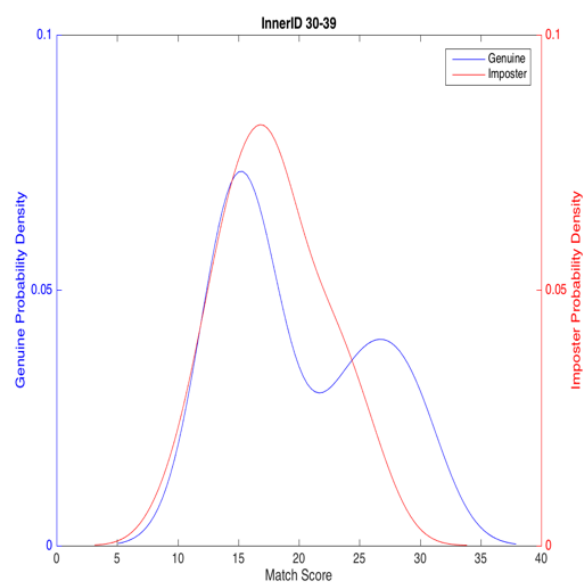

(c)

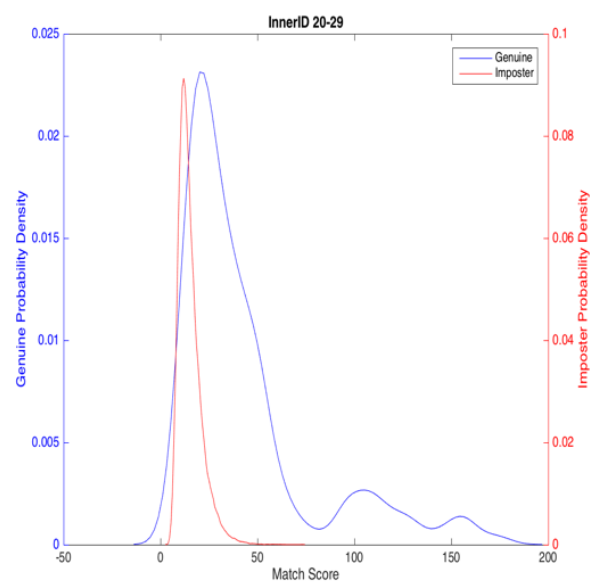

(b)

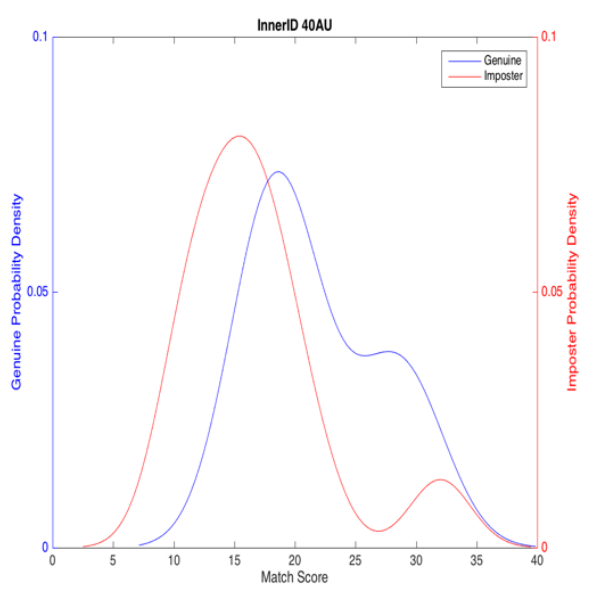

(d)

Figure C.1.9 NBIS InnerID (a) Ages 18-19 (b) Ages 20-29 (c) Ages 30-39 (d) Ages 40 and up 


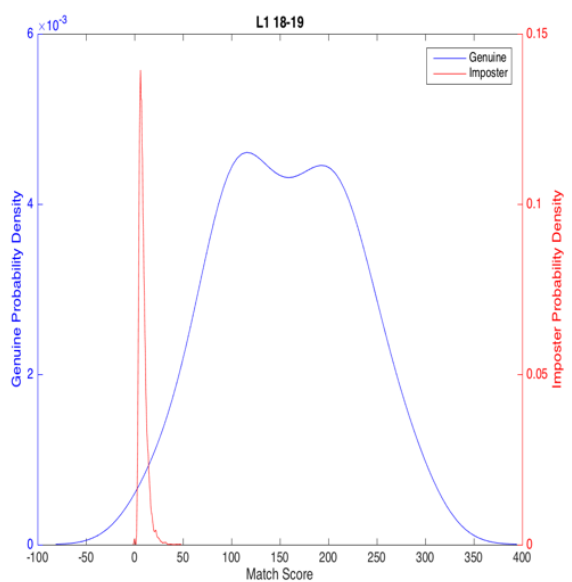

(a)

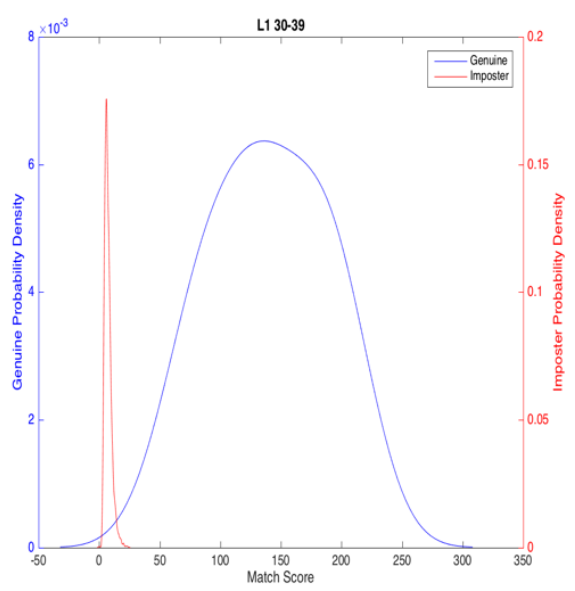

(c)

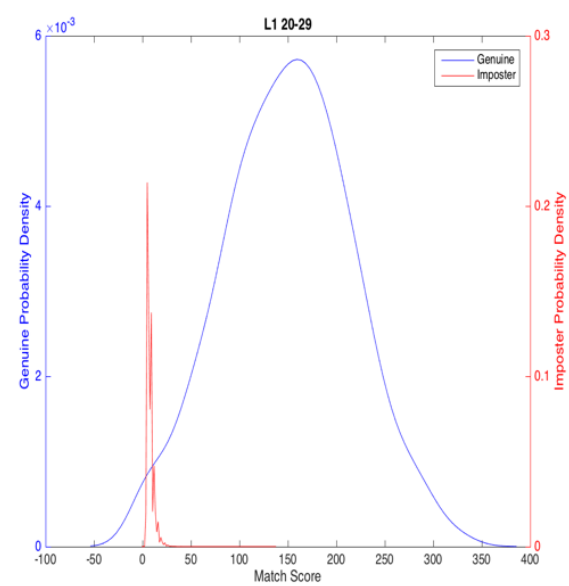

(b)

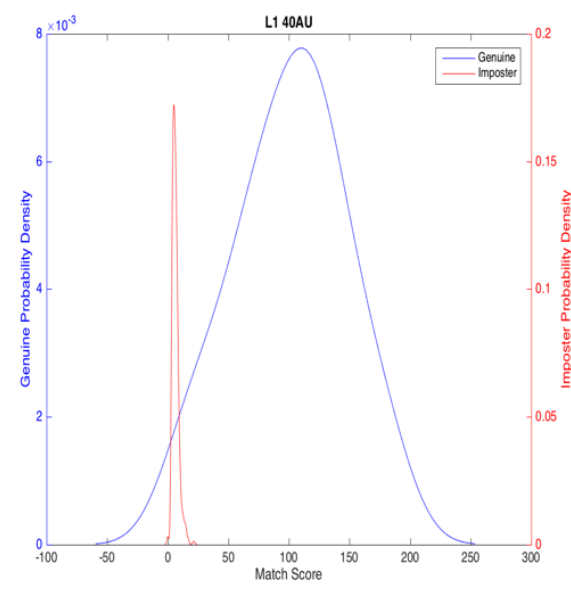

(d)

Figure C.1.10 NBIS L1 (a) Ages 18-19 (b) Ages 20-29 (c) Ages 30-39 (d) Ages 40 and up 


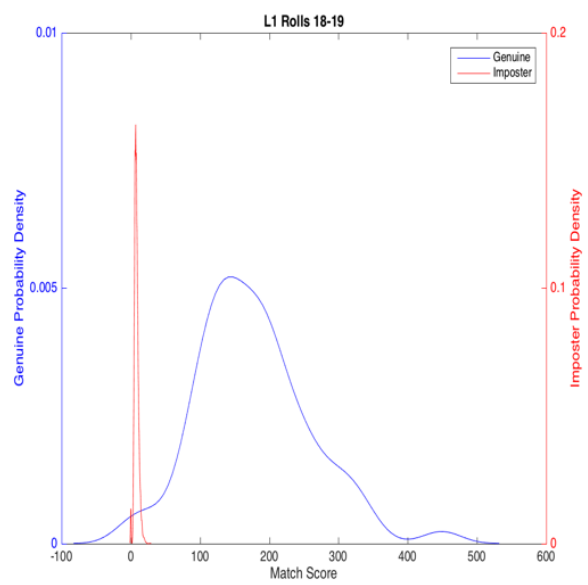

(a)

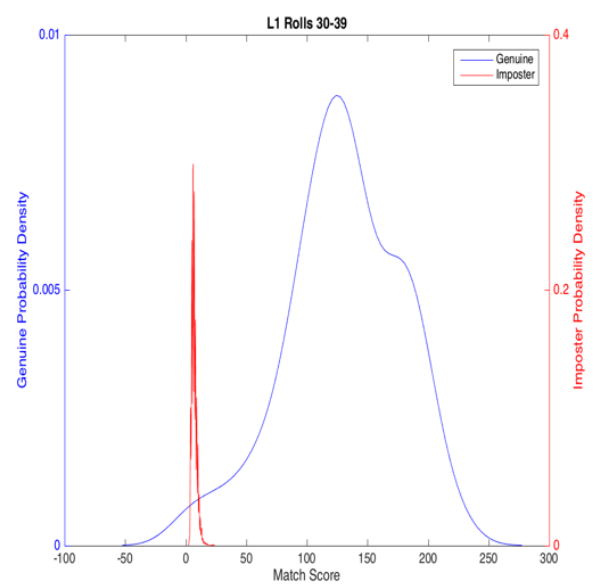

(c)

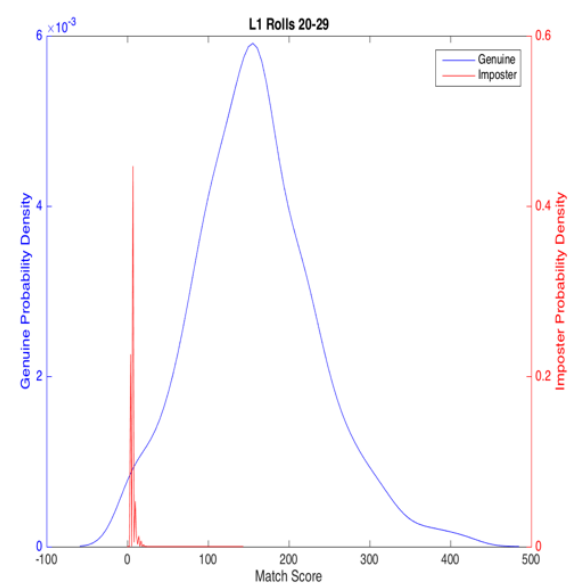

(b)

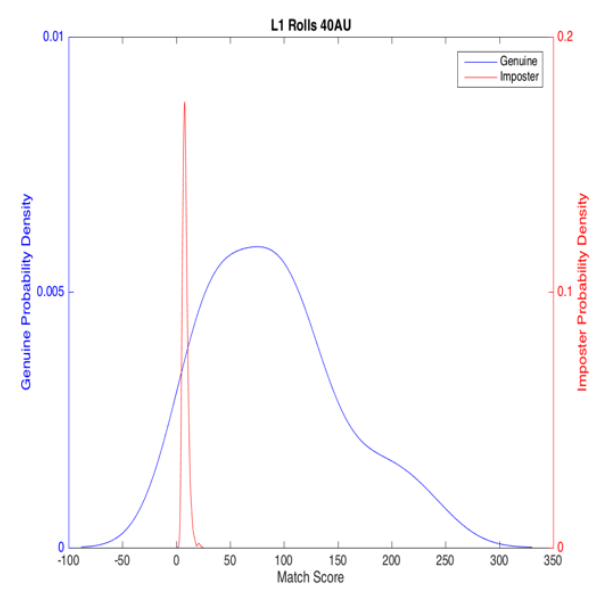

(d)

Figure C.1.11 NBIS L1 Rolls (a) Ages 18-19 (b) Ages 20-29 (c) Ages 30-39 (d) Ages 40 and up 


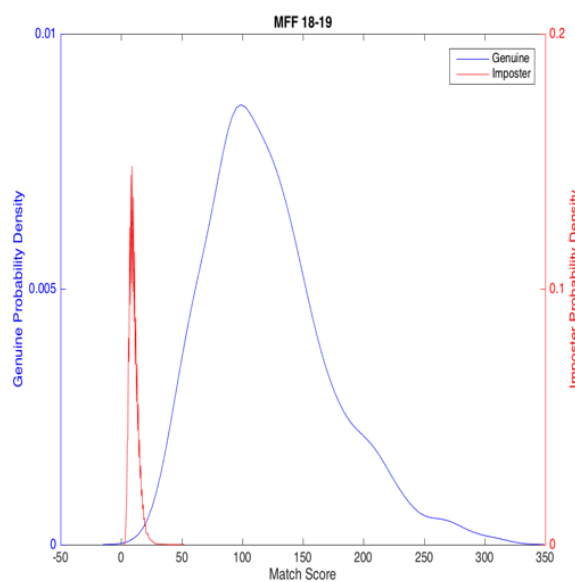

(a)

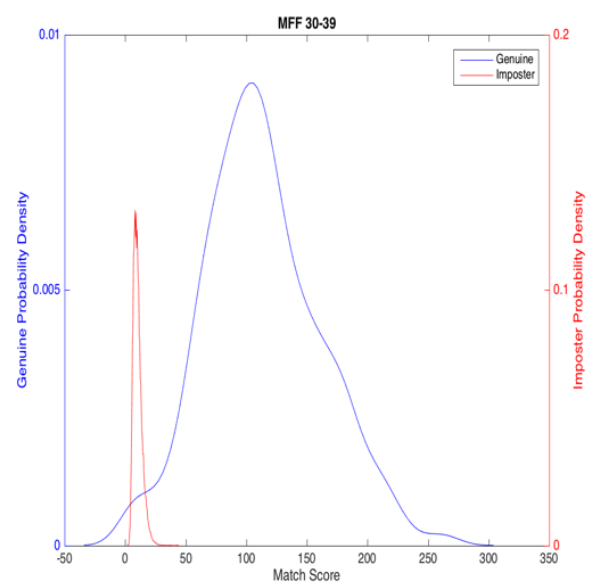

(c)

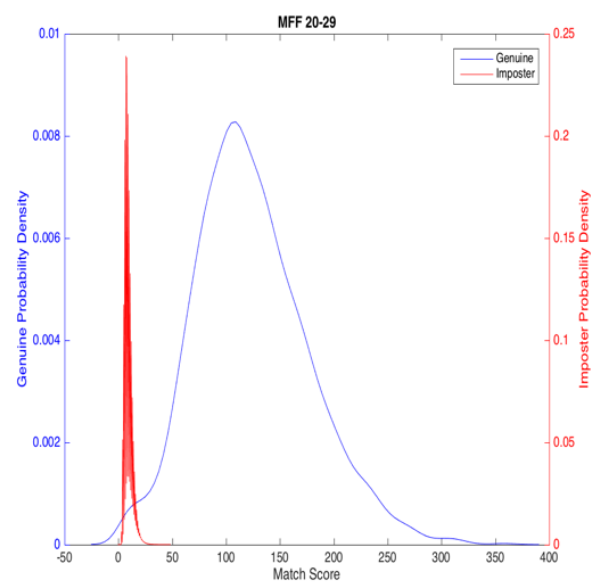

(b)

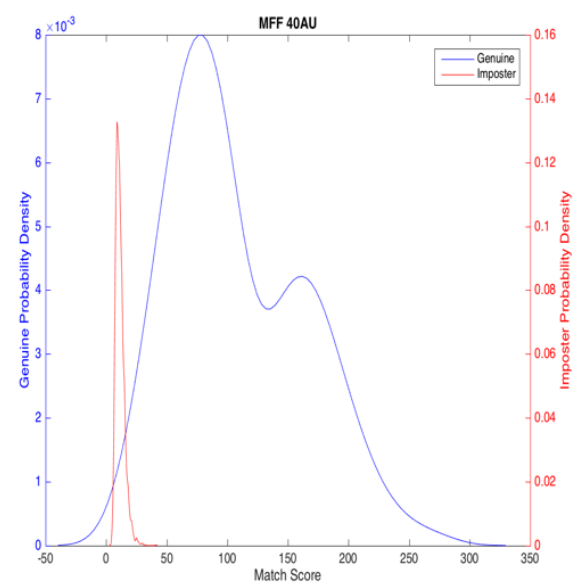

(d)

Figure C.1.12 NBIS Morpho FOTF (a) Ages 18-19 (b) Ages 20-29 (c) Ages 30-39 (d) Ages 40 and up 


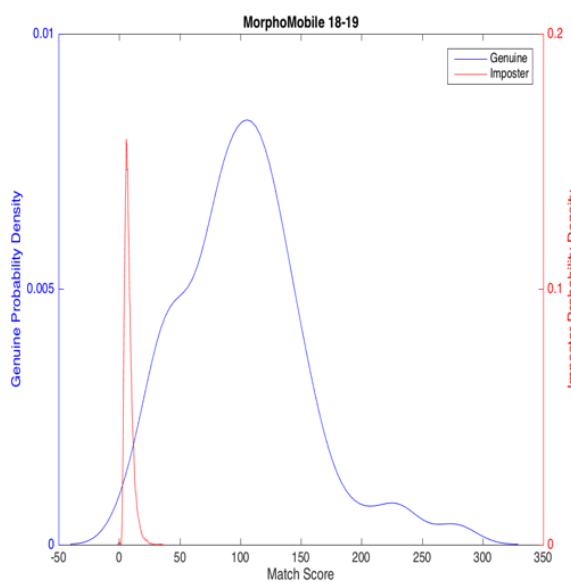

(a)

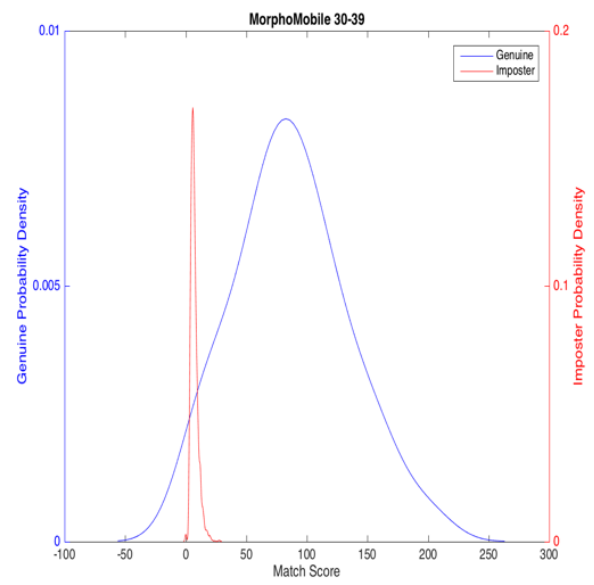

(c)

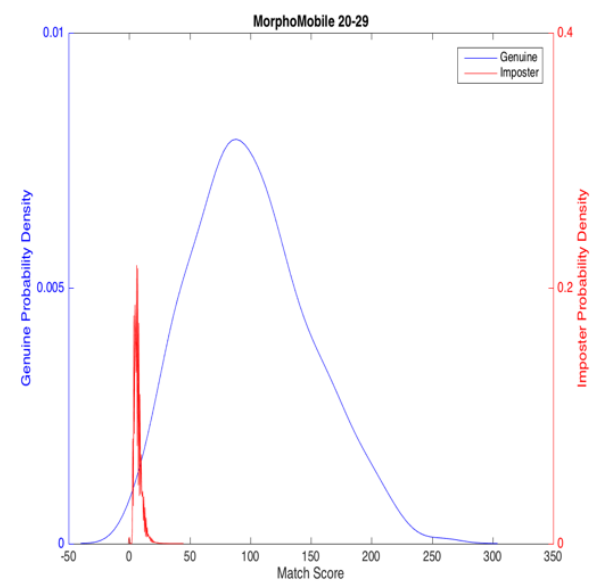

(b)

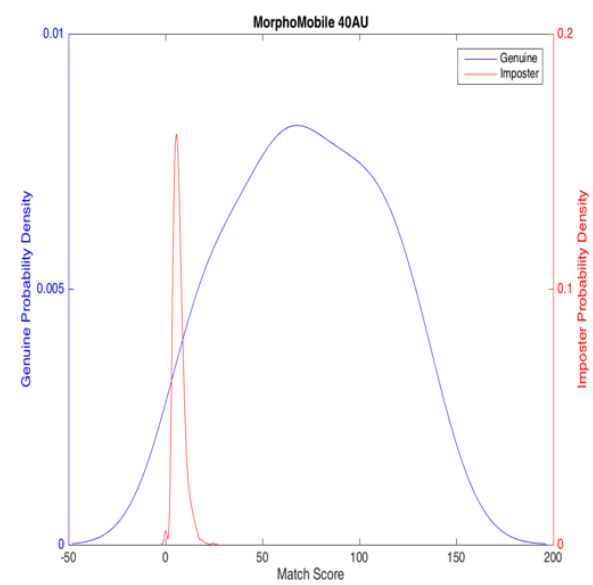

(d)

Figure C.1.13 NBIS MorphoMobile (a) Ages 18-19 (b) Ages 20-29 (c) Ages 30-39 (d) Ages 40 and up 


\section{C.2 Neurotech Genuine/Imposter Score Distributions}

Table C.5 Neurotech 18-19 Genuine and Imposter KLD, Minimum and Maximum Scores

\begin{tabular}{|c|c|c|c|c|c|c|}
\hline \multicolumn{7}{|l|}{ Neurotech 18-19 } \\
\hline Device & $\begin{array}{l}\text { Genuine } \\
\text { KLD }\end{array}$ & $\begin{array}{l}\text { Imposter } \\
\text { KLD }\end{array}$ & $\begin{array}{l}\text { Max } \\
\text { Genuine }\end{array}$ & $\begin{array}{l}\text { Min } \\
\text { Genuine }\end{array}$ & \begin{tabular}{|l|} 
Max \\
Imposter
\end{tabular} & $\begin{array}{l}\text { Min } \\
\text { Imposter }\end{array}$ \\
\hline ANDI BIN & 0.0230 & 3.6518 & 809 & 4 & 26 & 0 \\
\hline$A N D I G R Y$ & 0.0174 & 1.2327 & 863 & 9 & 36 & 0 \\
\hline BioSled & 0.0138 & 0.4548 & 860 & 107 & 36 & 0 \\
\hline BioSled Rolls & 0.0167 & 0.0106 & 914 & 212 & 20 & 0 \\
\hline$C G 2$ & 0.0118 & 0.1391 & 927 & 4 & 31 & 0 \\
\hline CG2 Rolls & 0.0109 & 0.0156 & 1018 & 230 & 20 & 0 \\
\hline$C S A$ & 0.0617 & 2.1182 & 1267 & 267 & 35 & 0 \\
\hline CSA Rolls & 0.0115 & 4.1534 & 1682 & 86 & 26 & 0 \\
\hline L1 & 0.0203 & 0.0037 & 913 & 224 & 37 & 0 \\
\hline$M F F$ & 0.0224 & 2.8592 & 836 & 148 & 31 & 0 \\
\hline MorphoMobile & 0.0139 & 0.1080 & 681 & 1 & 39 & 0 \\
\hline
\end{tabular}

Table C.6 Neurotech 20-29 Genuine and Imposter KLD, Minimum and Maximum Scores

\begin{tabular}{|r|l|l|l|l|l|l|}
\hline \multicolumn{9}{|l|}{ Neurotech 20-29 } & & & \\
Device & $\begin{array}{l}\text { Genuine } \\
\text { KLD }\end{array}$ & $\begin{array}{l}\text { Imposter } \\
\text { KLD }\end{array}$ & $\begin{array}{l}\text { Max } \\
\text { Genuine }\end{array}$ & $\begin{array}{l}\text { Min } \\
\text { Genuine }\end{array}$ & $\begin{array}{l}\text { Max } \\
\text { Imposter }\end{array}$ & $\begin{array}{l}\text { Min } \\
\text { Imposter }\end{array}$ \\
\cline { 2 - 7 } ANDI BIN & 0.0022 & 0.1658 & 986 & 1 & 41 & 0 \\
ANDI GRY & 0.0029 & 0.9697 & 1003 & 3 & 45 & 0 \\
\cline { 2 - 7 } BioSled & 0.0033 & 0.0120 & 951 & 0 & 539 & 0 \\
BioSled Rolls & 0.0073 & 0.0012 & 968 & 0 & 656 & 0 \\
\cline { 2 - 7 } CG2 & 0.0036 & 0.0090 & 1000 & 1 & 641 & 0 \\
CG2 Rolls & 0.0028 & 0.0015 & 1188 & 2 & 658 & 0 \\
\cline { 2 - 7 } CSA & 0.0153 & 5.0395 & 863 & 241 & 49 & 0 \\
\cline { 2 - 7 } Rolls & 0.0377 & 9.8309 & 1035 & 0 & 29 & 0 \\
L1 & 0.0034 & 0.0002 & 925 & 0 & 681 & 0 \\
\cline { 2 - 7 } MFF & 0.0059 & 0.9825 & 952 & 3 & 58 & 1 \\
\cline { 2 - 6 } MorphoMile & 0.0044 & 0.4012 & 705 & 4 & 44 & 0 \\
\hline
\end{tabular}


Table C.7 NBIS 30-39 Genuine and Imposter KLD, Minimum and Maximum Scores

\begin{tabular}{|r|l|l|l|l|l|l|}
\hline Neurotech 30-39 & \multicolumn{1}{l|}{} & \\
Device & Genuine & Imposter & Max & Min & Max & Min \\
& KLD & KLD & Genuine & Genuine & Imposter & Imposter \\
\cline { 2 - 7 } ANDI BIN & 0.0150 & 3.4460 & 842 & 2 & 21 & 0 \\
ANDI GRY & 0.0101 & 0.6944 & 903 & 6 & 41 & 1 \\
\cline { 2 - 7 } BioSled & 0.0300 & 0.9620 & 742 & 3 & 33 & 0 \\
BioSled Rolls & 0.0434 & 0.0183 & 993 & 181 & 18 & 0 \\
CG2 & 0.0170 & 0.2309 & 875 & 141 & 27 & 0 \\
CG2 Rolls & 0.0324 & 0.0102 & 1072 & 229 & 18 & 0 \\
CSA & 0.1189 & 1.4025 & 793 & 1 & 41 & 0 \\
CSA Rolls & 0.0207 & 6.1675 & 760 & 219 & 17 & 0 \\
L1 & 0.0362 & 0.0039 & 891 & 164 & 23 & 0 \\
MFF & 0.0252 & 1.9548 & 801 & 4 & 33 & 1 \\
\cline { 2 - 7 } MorphoMobile & 0.0191 & 0.3839 & 595 & 3 & 30 & 0 \\
\hline
\end{tabular}

Table C. 8 NBIS 40 and up Genuine and Imposter KLD, Minimum and Maximum Scores

\begin{tabular}{|r|l|l|l|l|l|l|}
\hline Neurotech 40 and up & \multicolumn{1}{l|}{} & \\
\hline Device & $\begin{array}{l}\text { Genuine } \\
\text { KLD }\end{array}$ & $\begin{array}{l}\text { Imposter } \\
\text { KLD }\end{array}$ & $\begin{array}{l}\text { Max } \\
\text { Genuine }\end{array}$ & $\begin{array}{l}\text { Min } \\
\text { Genuine }\end{array}$ & $\begin{array}{l}\text { Imposter } \\
\text { Imposter }\end{array}$ \\
\cline { 2 - 7 } ANDI BIN & 0.0232 & 3.8684 & 749 & 132 & 25 & 0 \\
ANDI GRY & 0.0334 & 3.1249 & 820 & 61 & 32 & 1 \\
BioSled & 0.0140 & 1.2292 & 768 & 126 & 21 & 0 \\
BioSled Rolls & 0.0079 & 0.0277 & 762 & 3 & 24 & 0 \\
CG2 & 0.0388 & 0.3270 & 748 & 1 & 29 & 0 \\
CG Rolls & 0.0178 & 0.0164 & 1072 & 216 & 10 & 0 \\
CSA & 0.0193 & 5.4521 & 749 & 201 & 18 & 0 \\
CSA Rolls & 0.0175 & 9.6163 & 891 & 73 & 12 & 0 \\
L1 & 0.0289 & 0.0048 & 759 & 342 & 29 & 0 \\
MFF & 0.0292 & 2.4726 & 671 & 208 & 25 & 2 \\
\cline { 2 - 7 } & 0.0229 & 0.4160 & 555 & 139 & 22 & 0 \\
\hline MorphoMobile & & & & & & \\
\hline
\end{tabular}




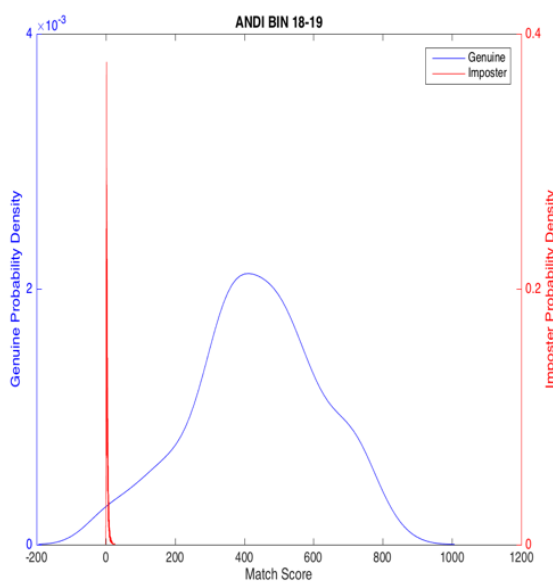

(a)

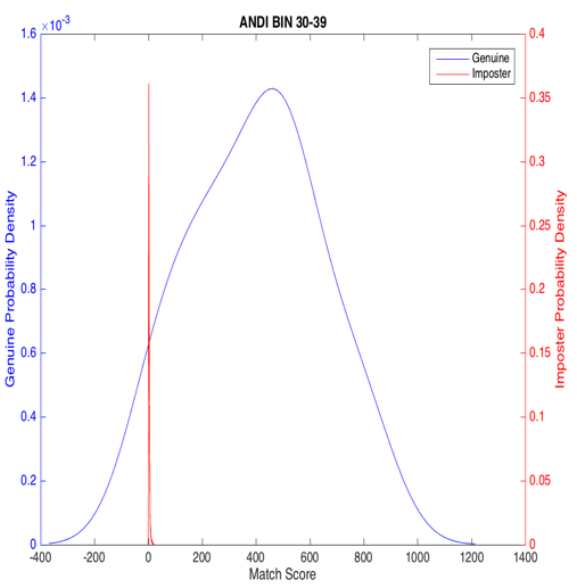

(c)

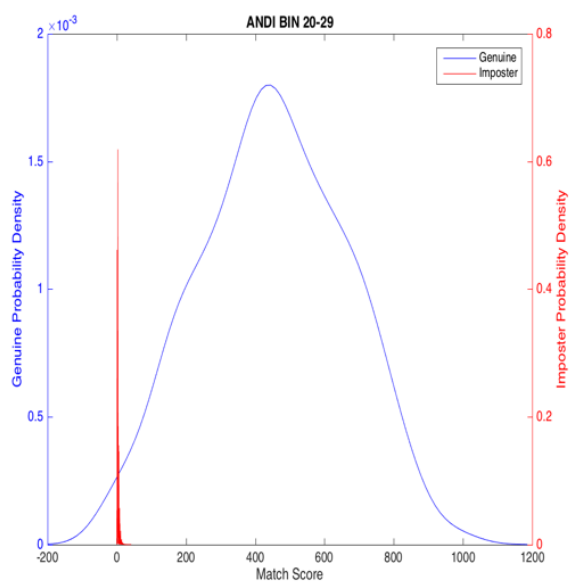

(b)

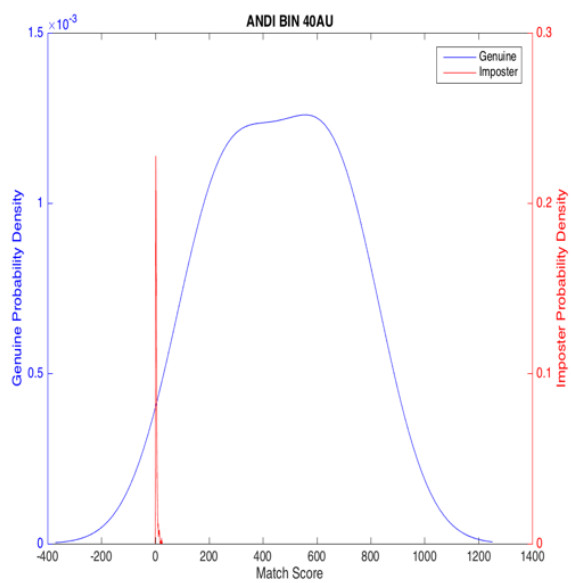

(d)

Figure C.2.1 Neurotech ANDI Binary (a) Ages 18-19 (b) Ages 20-29 (c) Ages 30-39 (d) Ages 40 and up 


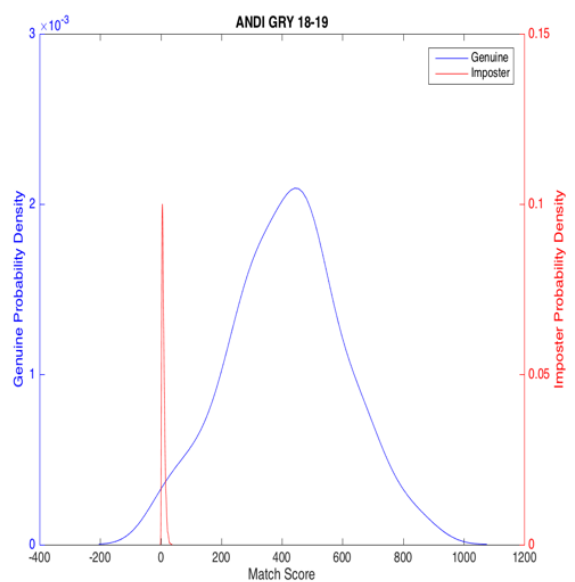

(a)

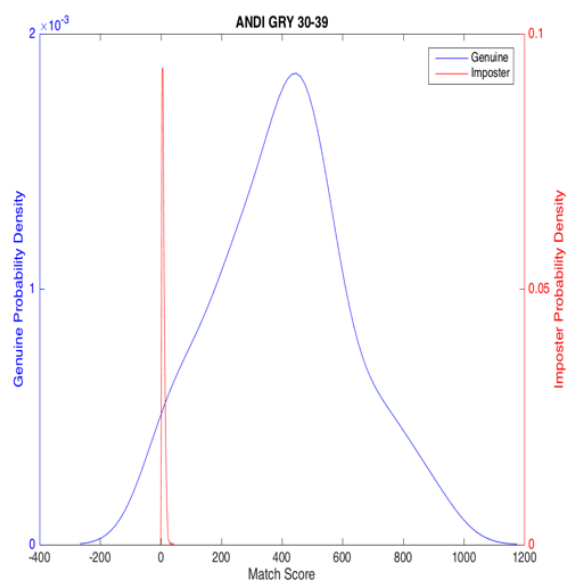

(c)

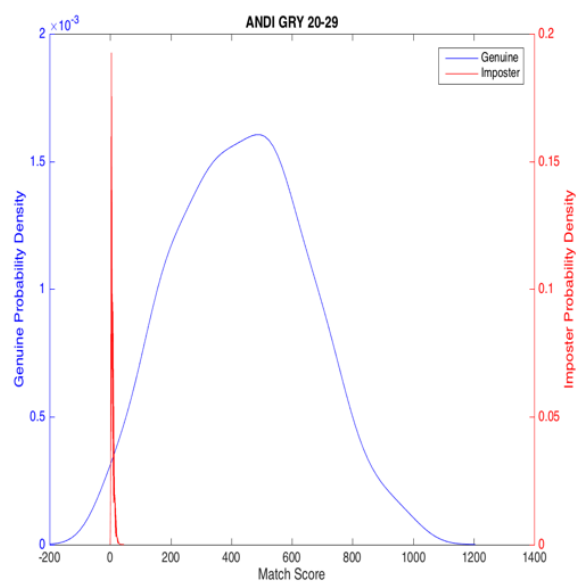

(b)

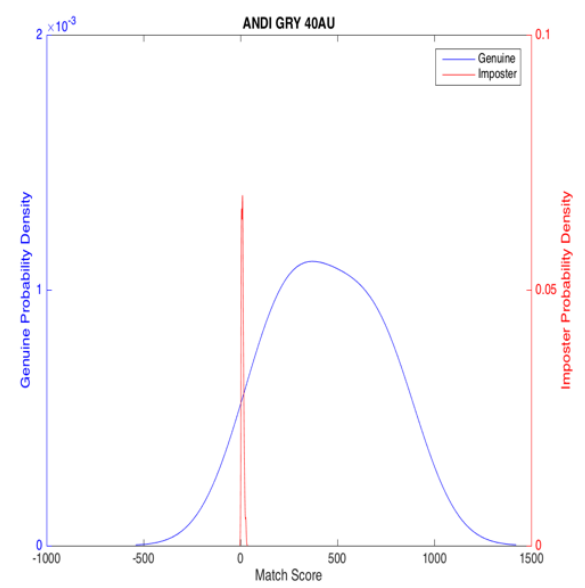

(d)

Figure C.2.2 Neurotech ANDI Greyscale (a) Ages 18-19 (b) Ages 20-29 (c) Ages 30-39 (d) Ages 40 and up 


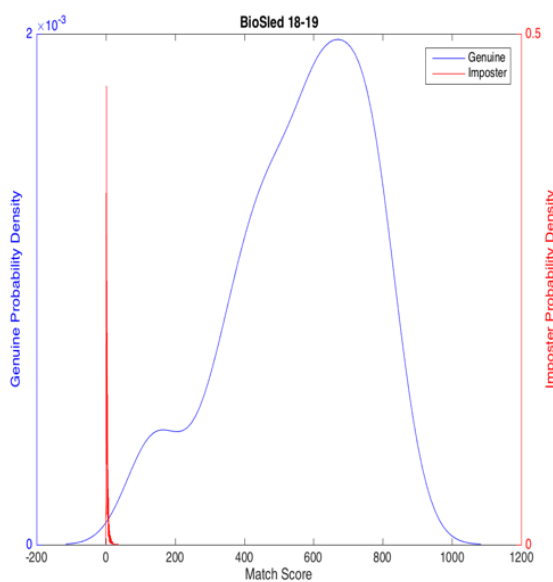

(a)

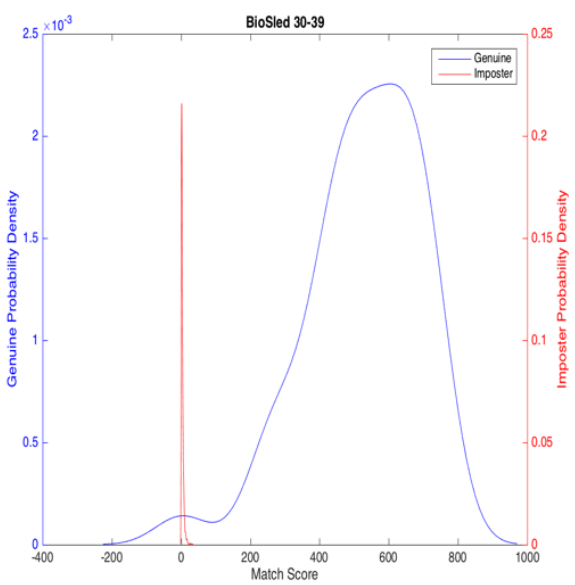

(c)

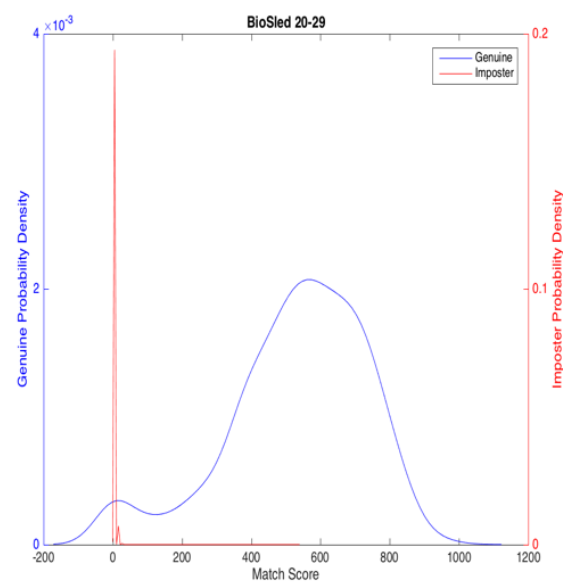

(b)

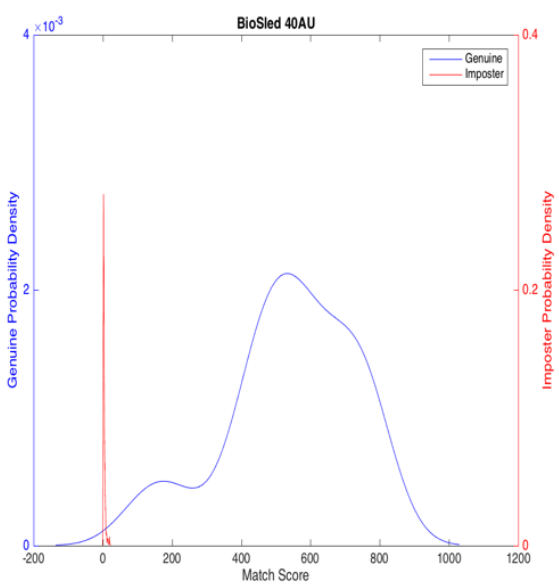

(d)

Figure C.2.3 Neurotech BioSled (a) Ages 18-19 (b) Ages 20-29 (c) Ages 30-39 (d) Ages 40 and up 


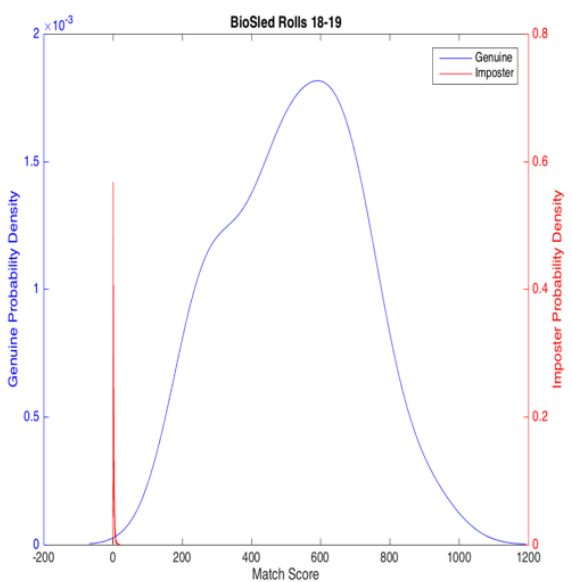

(a)

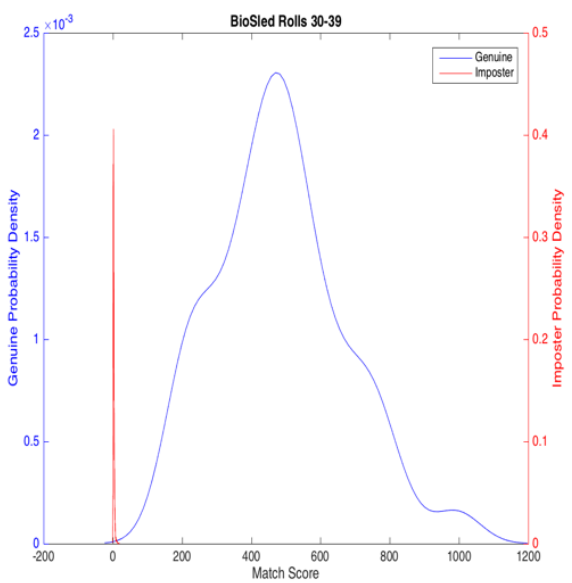

(c)

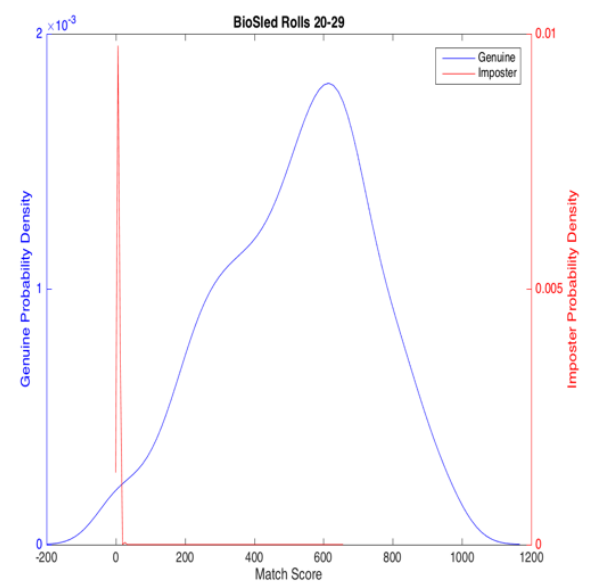

(b)

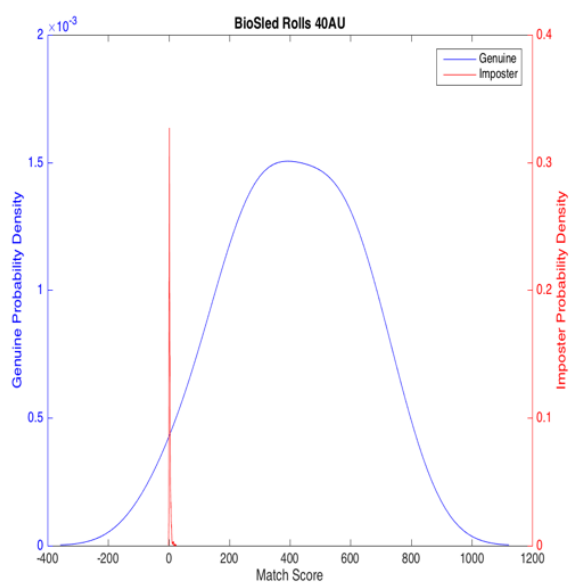

(d)

Figure C.2.4 Neurotech BioSled Rolls (a) Ages 18-19 (b) Ages 20-29 (c) Ages 30-39 (d) Ages 40 and up 


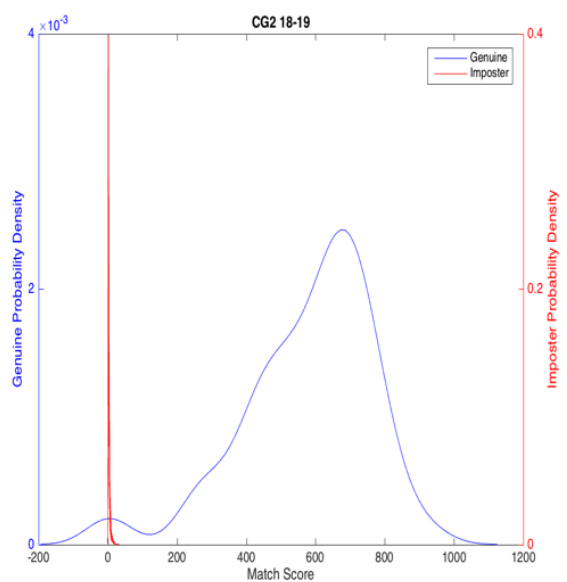

(a)

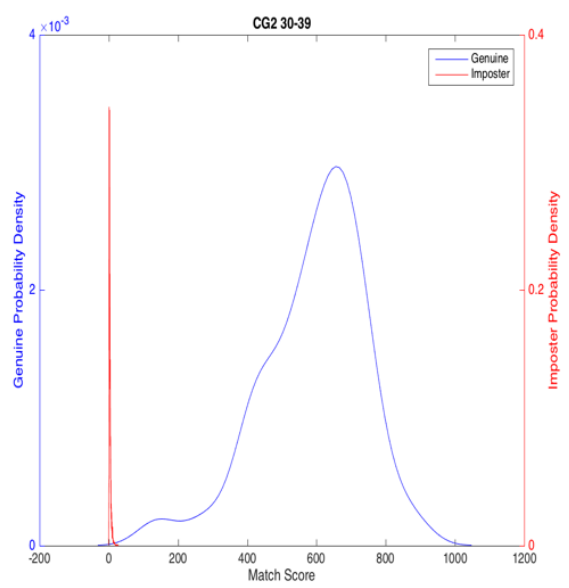

(c)

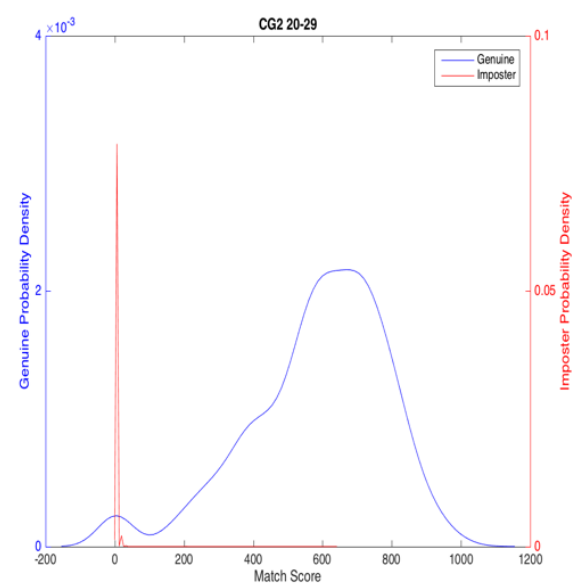

(b)

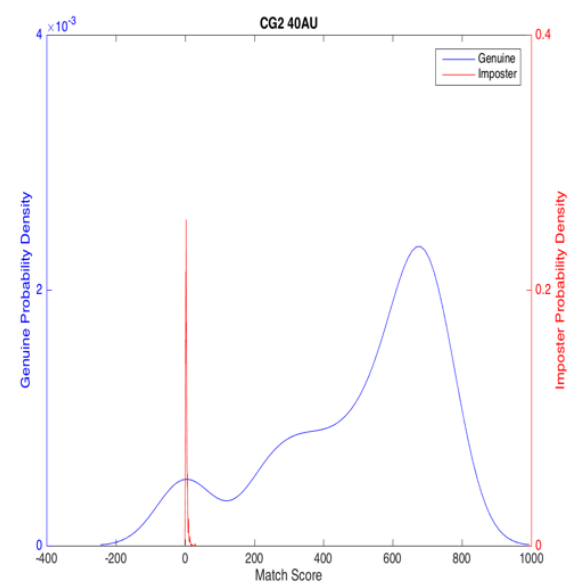

(d)

Figure C.2.5 Neurotech FP II Guardian (a) Ages 18-19 (b) Ages 20-29 (c) Ages 30-39 (d) Ages 40 and up 


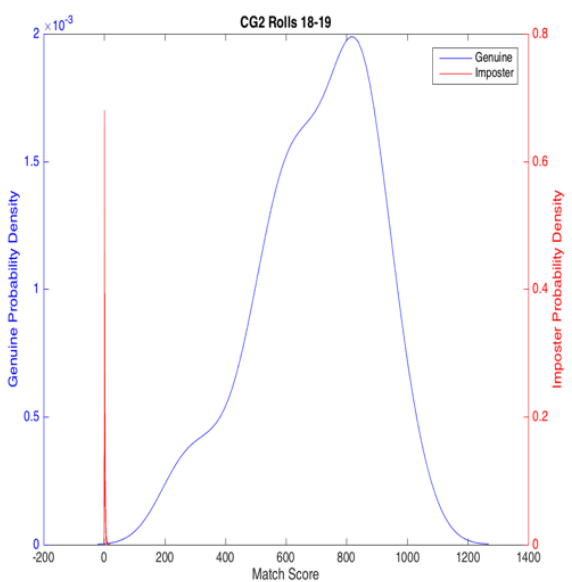

(a)

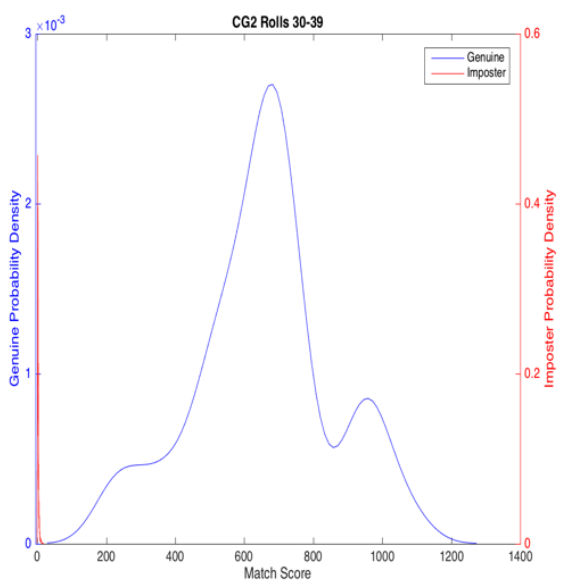

(c)

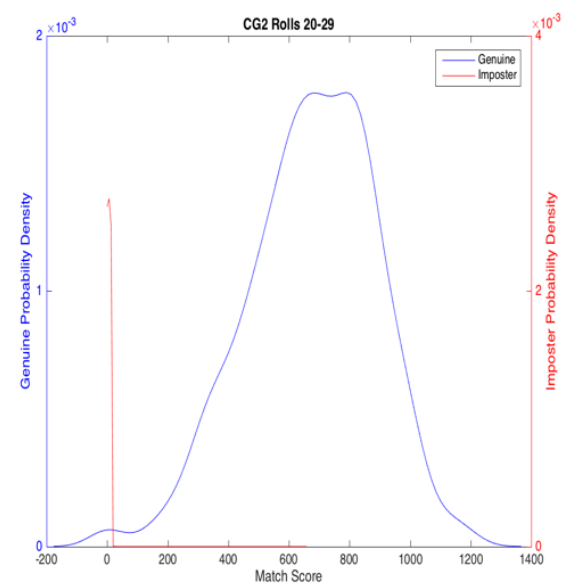

(b)

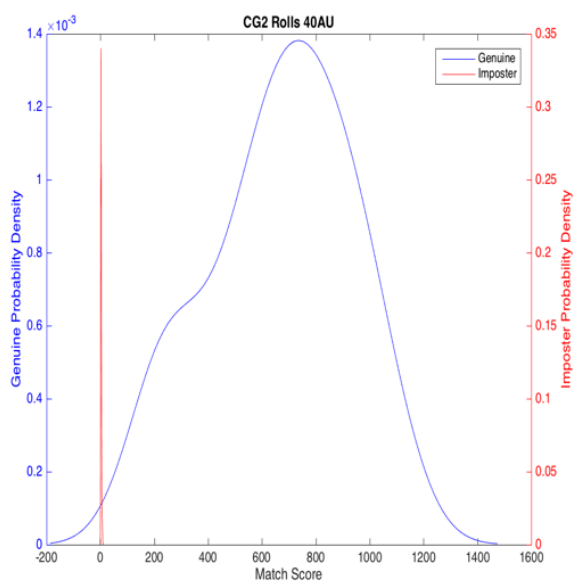

(d)

Figure C.2.6 Neurotech FP II Guardian Rolls (a) Ages 18-19 (b) Ages 20-29 (c) Ages 30-39 (d) Ages 40 and up 


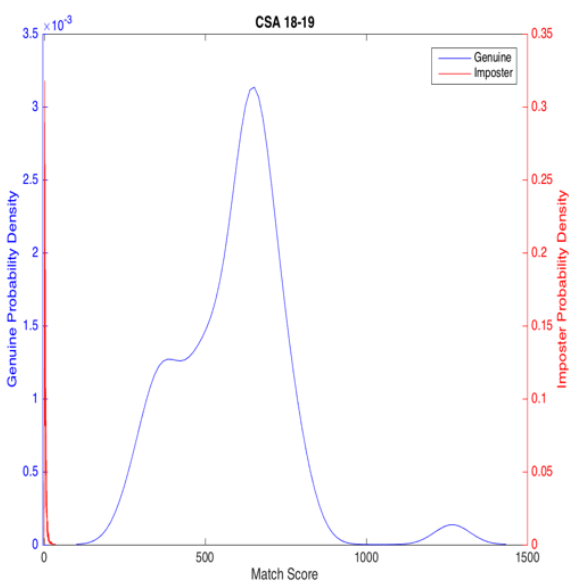

(a)

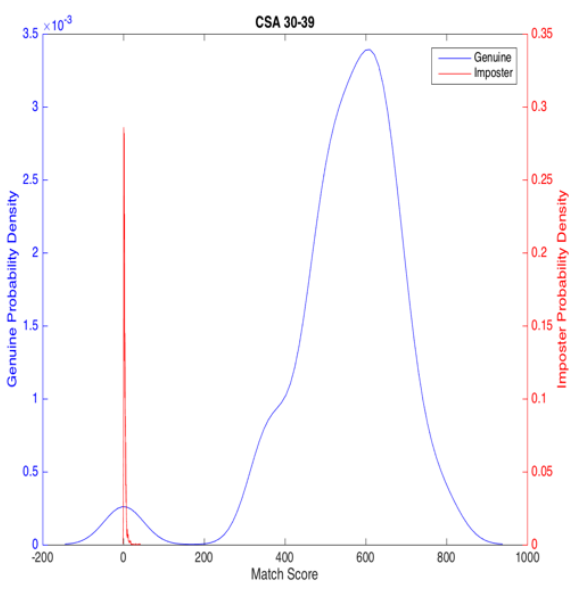

(c)

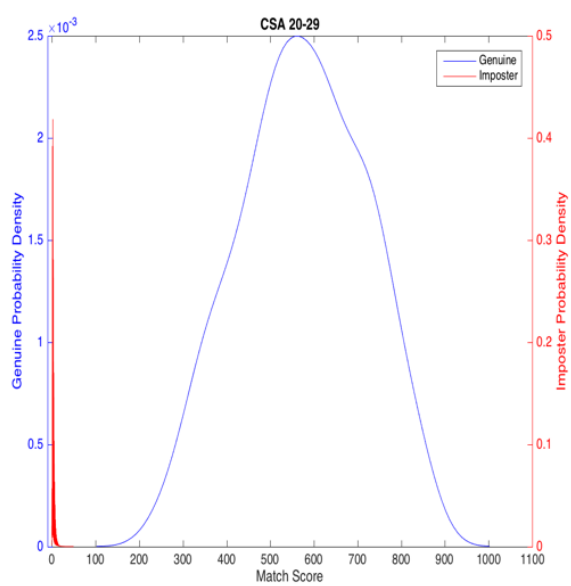

(b)

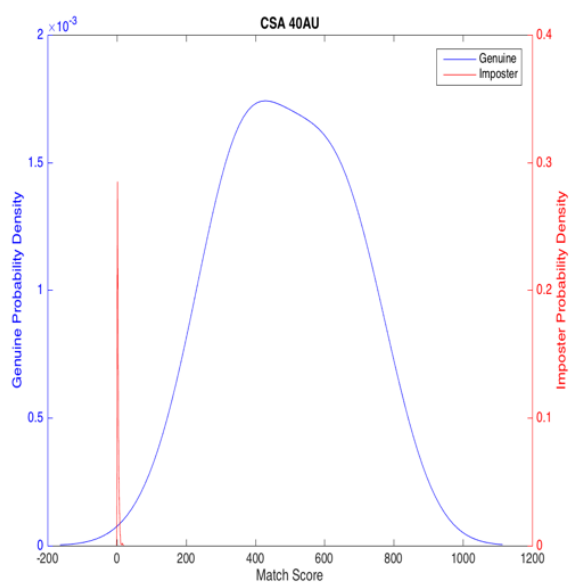

(d)

Figure C.2.7 Neurotech Crossmatch Seek Avenger (a) Ages 18-19 (b) Ages 20-29 (c) Ages 30-39 (d) Ages 40 and up 


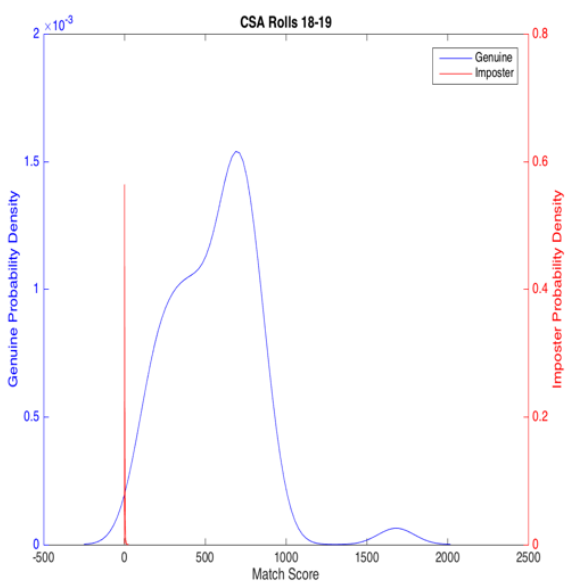

(a)

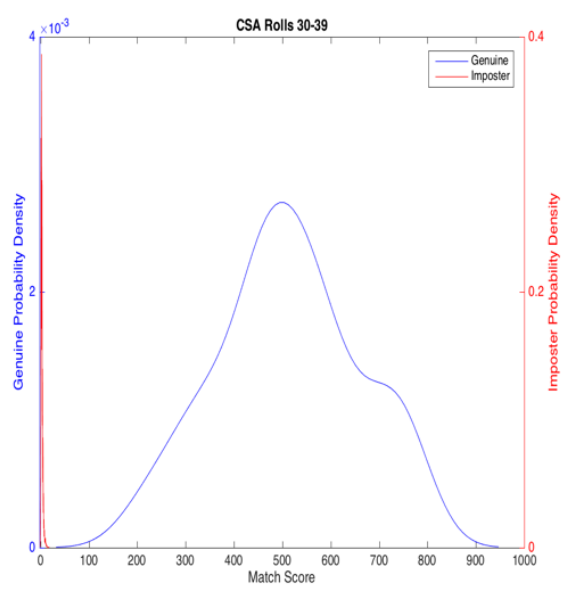

(c)

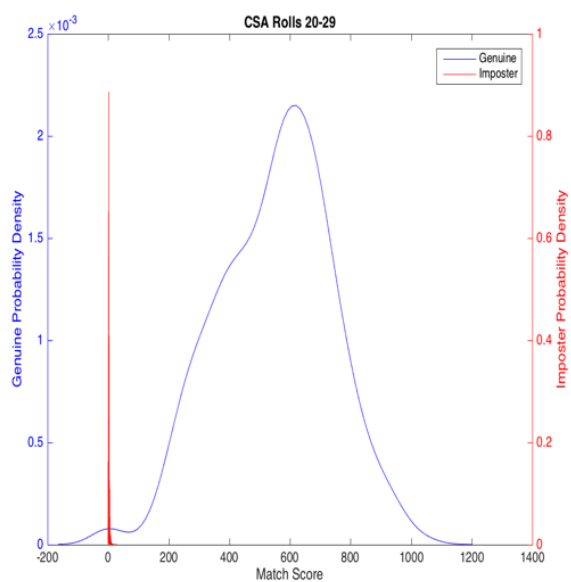

(b)

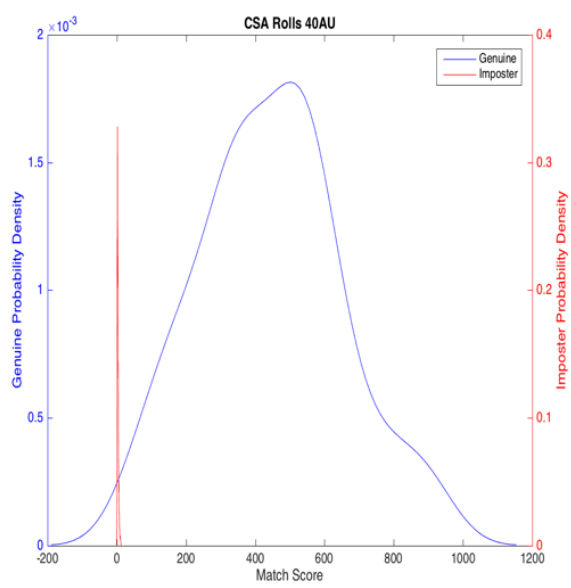

(d)

Figure C.2.8 Neurotech Crossmatch Seek Avenger Rolls (a) Ages 18-19 (b) Ages 20-29 (c) Ages 30-39 (d) Ages 40 and up 


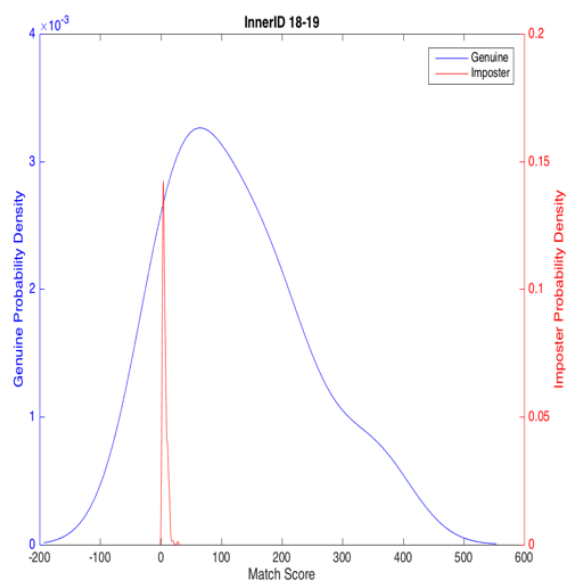

(a)

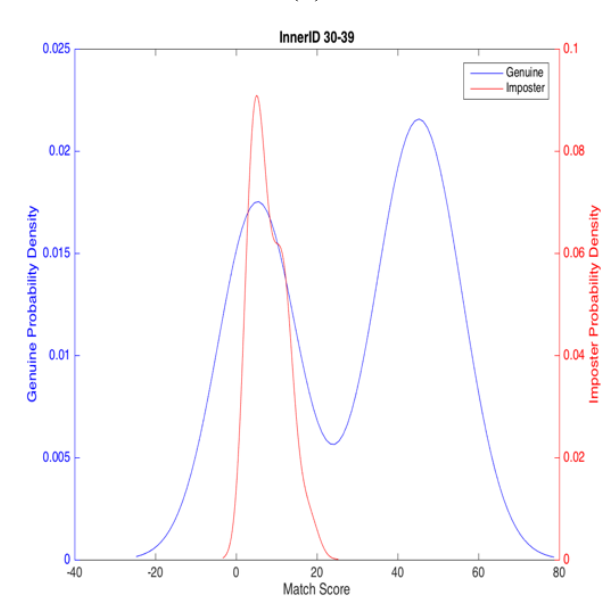

(c)

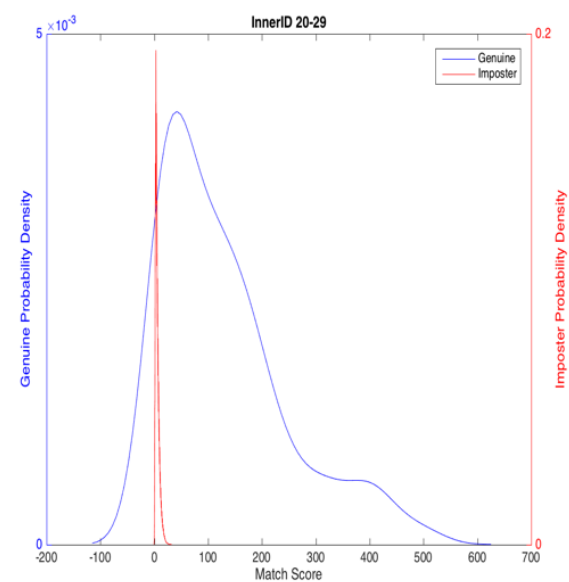

(b)

Figure C.2.9 Neurotech InnerID (a) Ages 18-19 (b) Ages 20-29 (c) Ages 30-39 (d) Ages 40 and up (Not Available) 


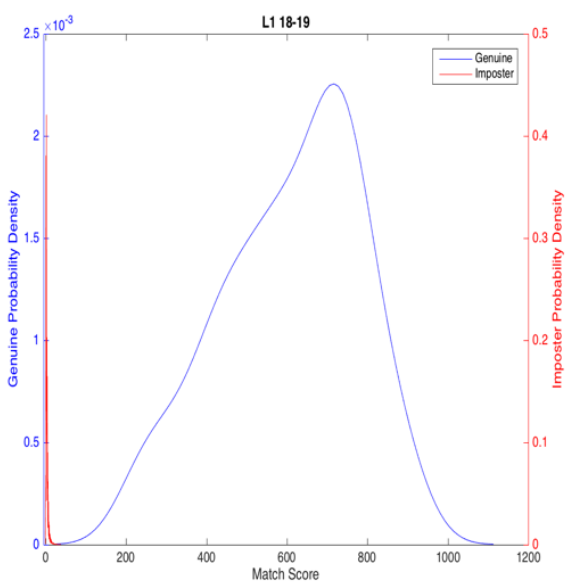

(a)

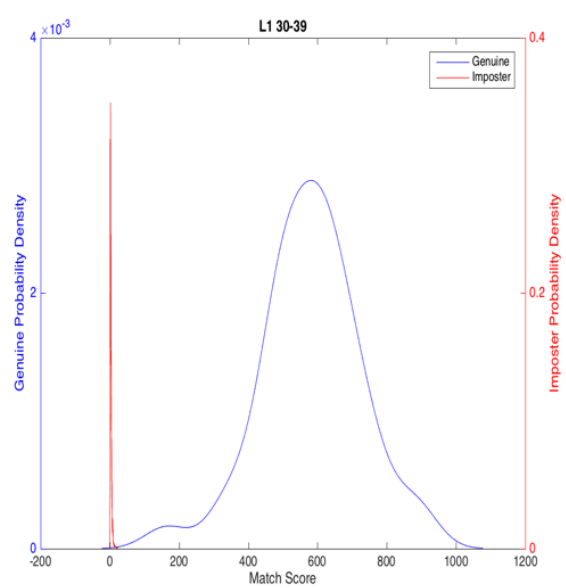

(c)

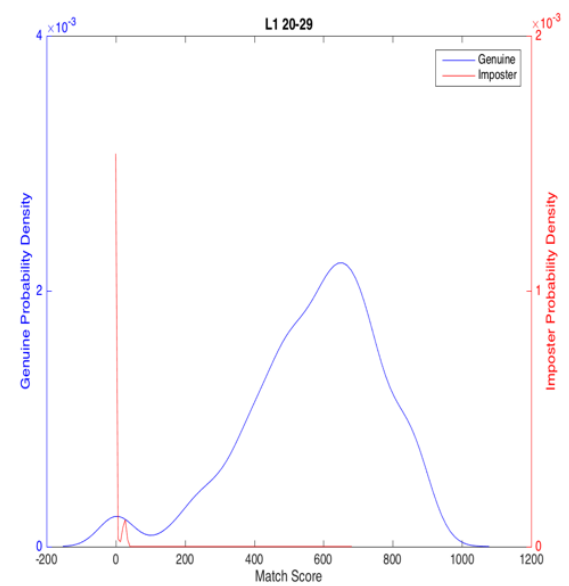

(b)

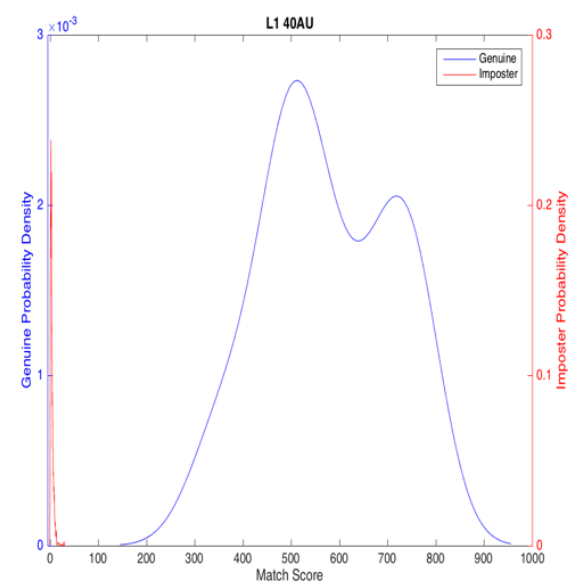

(d)

Figure C.2.10 Neurotech L1 (a) Ages 18-19 (b) Ages 20-29 (c) Ages 30-39 (d) Ages 40 and up 


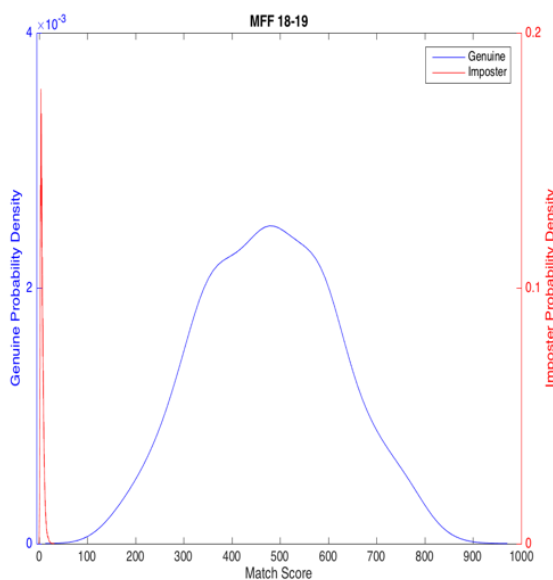

(a)

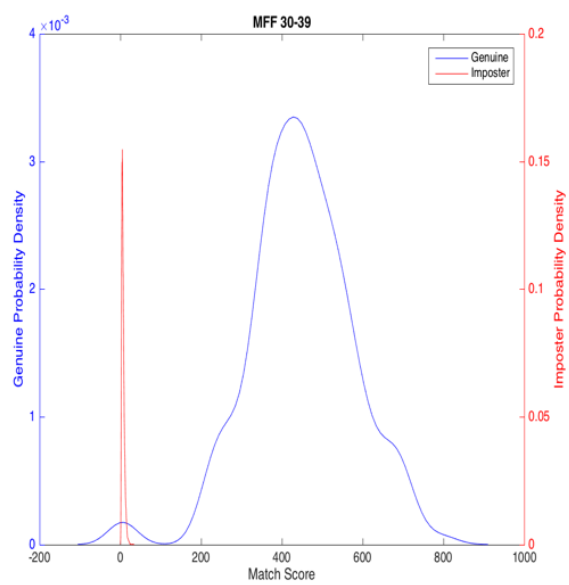

(c)

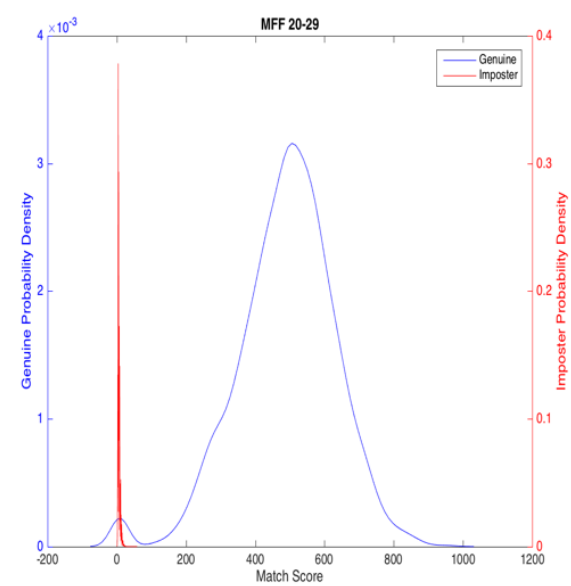

(b)

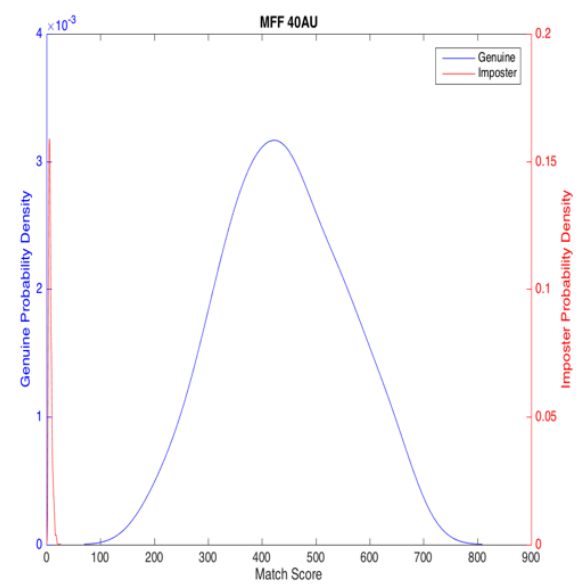

(d)

Figure C.2.11 Neurotech Morpho FOTF (a) Ages 18-19 (b) Ages 20-29 (c) Ages 30-39 (d) Ages 40 and up 


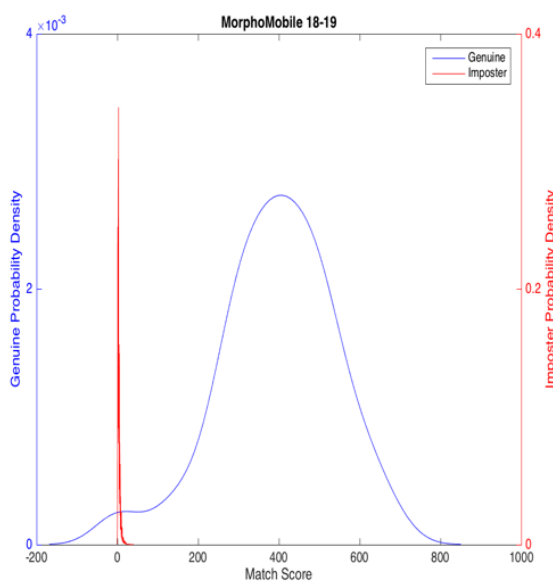

(a)

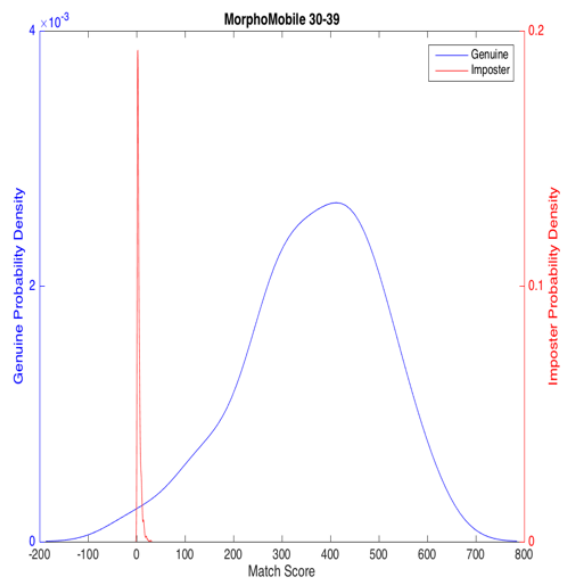

(c)

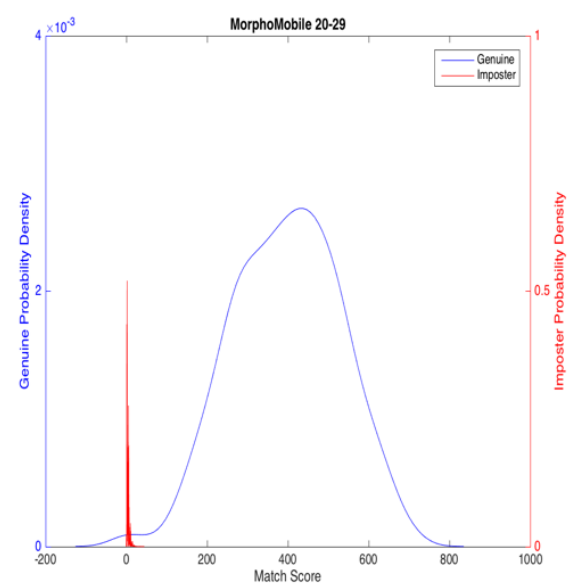

(b)

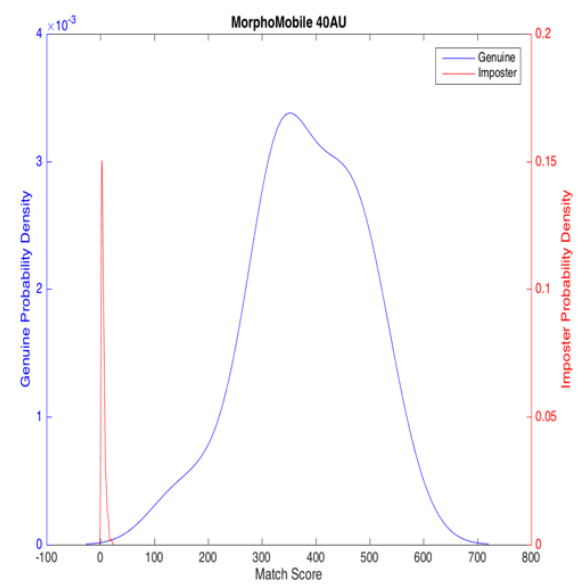

(d)

Figure C.2.12 Neurotech MorphoMobile (a) Ages 18-19 (b) Ages 20-29 (c) Ages 30-39 (d) Ages 40 and up 


\section{C.3 SourceAFIS Genuine/Imposter Score Distributions}

Table C.9 SourceAFIS 18-19 Genuine and Imposter KLD, Minimum and Maximum Scores

\begin{tabular}{|c|c|c|c|c|c|c|}
\hline \multicolumn{7}{|c|}{ SourceAFIS 18-19 } \\
\hline Device & $\begin{array}{l}\text { Genuine } \\
\text { KLD }\end{array}$ & $\begin{array}{l}\text { Imposter } \\
\text { KLD }\end{array}$ & $\begin{array}{l}\text { Max } \\
\text { Genuine }\end{array}$ & $\begin{array}{l}\text { Min } \\
\text { Genuine }\end{array}$ & $\begin{array}{l}\text { Max } \\
\text { Imposter }\end{array}$ & $\begin{array}{l}\text { Min } \\
\text { Imposter }\end{array}$ \\
\hline$A N D I B I N$ & 0.0360 & 1.1649 & 259.7252 & 11.85273 & 30.19959 & 5.878553 \\
\hline$A N D I G R Y$ & 0.0584 & 6.9912 & 216.3056 & 10.55984 & 38.68415 & 6.92837 \\
\hline BioSled & 0.0944 & 3.9879 & 214.9599 & 13.20869 & 31.48004 & 6.289892 \\
\hline BioSled Rolls & 0.0369 & 4.3084 & 305.3576 & 10.55244 & 26.79964 & 5.206804 \\
\hline$C G 2$ & 0.1343 & 4.4908 & 190.5806 & 9.11262 & 35.63965 & 5.668835 \\
\hline CG2 Rolls & 0.0708 & 8.6481 & 322.159 & 10.87964 & 34.52416 & 4.572073 \\
\hline$C S A$ & 0.0368 & 2.3131 & 261.0499 & 21.84235 & 32.15683 & 6.654526 \\
\hline CSA Rolls & 0.0461 & 1.8668 & 518.3488 & 11.35223 & 29.48184 & 6.068511 \\
\hline InnerID & 1.3770 & 3.9396 & 117.0034 & 9.712775 & 19.33523 & 7.380855 \\
\hline$L 1$ & 0.0755 & 3.0207 & 238.0814 & 23.20657 & 29.80924 & 7.061163 \\
\hline L1 Rolls & 0.1159 & 4.8319 & 291.972 & 0 & 30.03865 & 0 \\
\hline$M F F$ & 0.0866 & 1.1763 & 252.8406 & 10.53184 & 30.18343 & 5.116627 \\
\hline MorphoMobile & 0.1229 & 4.1186 & 156.4674 & 9.806087 & 31.98695 & 6.877412 \\
\hline
\end{tabular}

Table C.10 SourceAFIS 20-29 Genuine and Imposter KLD, Minimum and Maximum Scores

\begin{tabular}{|c|c|c|c|c|c|c|}
\hline \multicolumn{7}{|c|}{ SourceAFIS 20-29 } \\
\hline Device & $\begin{array}{l}\text { Genuine } \\
\text { KLD }\end{array}$ & $\begin{array}{l}\text { Imposter } \\
\text { KLD }\end{array}$ & $\begin{array}{l}\text { Max } \\
\text { Genuine }\end{array}$ & $\begin{array}{l}\text { Min } \\
\text { Genuine }\end{array}$ & $\begin{array}{l}\text { Max } \\
\text { Imposter }\end{array}$ & $\begin{array}{l}\text { Min } \\
\text { Imposter }\end{array}$ \\
\hline$A N D I B I N$ & 0.0139 & 3.7860 & 258.9763 & 11.24244 & 37.96463 & 1.1505 \\
\hline$A N D I G R Y$ & 0.0255 & 3.2567 & 227.3911 & 8.953438 & 41.80738 & 1.068082 \\
\hline BioSled & 0.0158 & 0.4477 & 247.8092 & 10.44588 & 140.6996 & 2.44783 \\
\hline BioSled Rolls & 0.0112 & 0.0946 & 336.4065 & 9.458447 & 214.1273 & 1.01918 \\
\hline$C G 2$ & 0.0367 & 1.2740 & 244.6543 & 8.773584 & 155.1426 & 2.002312 \\
\hline CG2 Rolls & 0.0122 & 0.0198 & 364.865 & 11.4828 & 214.8845 & 1.072709 \\
\hline$C S A$ & 0.0331 & 1.6879 & 285.5363 & 25.56576 & 37.04658 & 3.415862 \\
\hline CSA Rolls & 0.0927 & 0.6877 & 336.2823 & 8.577291 & 34.38301 & 1.021923 \\
\hline InnerID & 0.0795 & 0.7772 & 148.194 & 9.427563 & 31.8045 & 3.630311 \\
\hline$L 1$ & 0.0149 & 0.4659 & 287.0937 & 9.631104 & 89.61704 & 2.009291 \\
\hline L1 Rolls & 0.0119 & 1.3526 & 341.7931 & 9.870334 & 35.23992 & 1.0158 \\
\hline$M F F$ & 0.0243 & 1.9716 & 290.2585 & 8.428964 & 39.19853 & 1.0158 \\
\hline MorphoMobile & 0.0235 & 10.5725 & 172.8451 & 11.19441 & 33.69872 & 1.265244 \\
\hline
\end{tabular}


Table C.11 SourceAFIS 30-39 Genuine and Imposter KLD, Minimum and Maximum Scores

\begin{tabular}{|c|c|c|c|c|c|c|}
\hline \multicolumn{7}{|c|}{ SourceAFIS 30-39 } \\
\hline Device & $\begin{array}{l}\text { Genuine } \\
\text { KLD }\end{array}$ & $\begin{array}{l}\text { Imposter } \\
\text { KLD }\end{array}$ & $\begin{array}{l}\text { Max } \\
\text { Genuine }\end{array}$ & $\begin{array}{l}\text { Min } \\
\text { Genuine }\end{array}$ & $\begin{array}{l}\text { Max } \\
\text { Imposter }\end{array}$ & $\begin{array}{l}\text { Min } \\
\text { Imposter }\end{array}$ \\
\hline ANDI BIN & 0.0889 & 5.4711 & 198.5667 & 12.04636 & 25.95447 & 4.662342 \\
\hline$A N D I G R Y$ & 0.1356 & 0.9623 & 147.5204 & 10.5444 & 27.5129 & 4.930195 \\
\hline BioSled & 0.0872 & 18.7870 & 213.4055 & 13.12 & 25.72716 & 4.65225 \\
\hline BioSled Rolls & 0.0423 & 2.1651 & 284.5245 & 11.3334 & 25.43212 & 6.607458 \\
\hline$C G 2$ & 0.1037 & 4.5059 & 210.5107 & 12.60935 & 30.83772 & 6.081194 \\
\hline CG2 Rolls & 0.0461 & 2.9185 & 293.6561 & 8.773874 & 28.32818 & 3.84902 \\
\hline$C S A$ & 0.3016 & 1.0281 & 171.7189 & 11.71629 & 27.61703 & 5.962965 \\
\hline CSA Rolls & 0.0652 & 3.2166 & 257.2393 & 51.99955 & 28.55286 & 7.618226 \\
\hline InnerID & 1.3632 & 1.9192 & 15.35044 & 10.02823 & 17.53011 & 10.17242 \\
\hline L1 & 0.1115 & 2.5975 & 183.8853 & 45.33661 & 26.11816 & 7.516325 \\
\hline L1 Rolls & 0.0743 & 2.5510 & 244.6956 & 12.48463 & 29.49694 & 5.333611 \\
\hline$M F F$ & 0.1043 & 0.9298 & 195.1617 & 11.24871 & 29.006 & 5.155389 \\
\hline MorphoMobile & 0.1931 & 5.1173 & 104.4118 & 12.4216 & 29.11408 & 6.372153 \\
\hline
\end{tabular}

Table C.12 SourceAFIS 40 and up Genuine and Imposter KLD, Minimum and Maximum Scores

\begin{tabular}{|c|c|c|c|c|c|c|}
\hline \multicolumn{7}{|c|}{ SourceAFIS 40 and up } \\
\hline Device & $\begin{array}{l}\text { Genuine } \\
\text { KLD }\end{array}$ & $\begin{array}{l}\text { Imposter } \\
\text { KLD }\end{array}$ & $\begin{array}{l}\text { Max } \\
\text { Genuine }\end{array}$ & $\begin{array}{l}\text { Min } \\
\text { Genuine }\end{array}$ & $\begin{array}{l}\text { Max } \\
\text { Imposter }\end{array}$ & $\begin{array}{l}\text { Min } \\
\text { Imposter }\end{array}$ \\
\hline ANDI BIN & 0.0760 & 2.7526 & 209.7784 & 11.5091 & 24.01522 & 6.774823 \\
\hline$A N D I G R Y$ & 0.1072 & 1.7703 & 150.2118 & 10.76227 & 24.582 & 8.93766 \\
\hline BioSled & 0.1062 & 1.6458 & 172.6986 & 29.25277 & 25.50017 & 9.102567 \\
\hline BioSled Rolls & 0.0501 & 1.8843 & 251.6365 & 11.20195 & 24.24384 & 6.865717 \\
\hline$C G 2$ & 0.1089 & 5.8069 & 190.3472 & 12.61516 & 23.62895 & 6.888422 \\
\hline CG2 Rolls & 0.0882 & 2.1999 & 263.4852 & 10.74035 & 24.87952 & 5.531532 \\
\hline$C S A$ & 0.1147 & 1.4563 & 159.6051 & 25.41742 & 22.98182 & 8.734149 \\
\hline CSA Rolls & 0.0540 & 2.0094 & 264.1351 & 12.81646 & 28.26393 & 6.489259 \\
\hline InnerID & 1.3822 & 2.4455 & 14.55067 & 11.29338 & 16.08005 & 10.46509 \\
\hline$L 1$ & 0.0675 & 3.1641 & 193.4362 & 28.72438 & 24.41692 & 7.669943 \\
\hline L1 Rolls & 0.0675 & 1.5156 & 205.0054 & 11.37656 & 24.54967 & 4.833644 \\
\hline$M F F$ & 0.0611 & 2.3292 & 190.9 & 9.912061 & 23.76718 & 5.180087 \\
\hline MorphoMobile & 0.1420 & 14.1331 & 102.6575 & 12.29423 & 22.09316 & 3.040535 \\
\hline
\end{tabular}




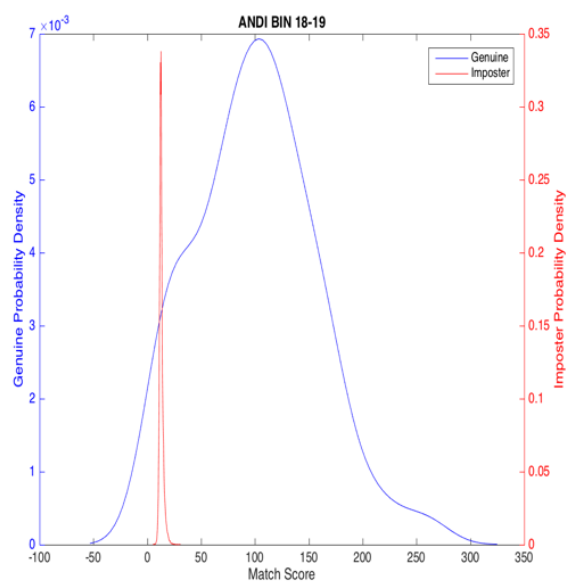

(a)

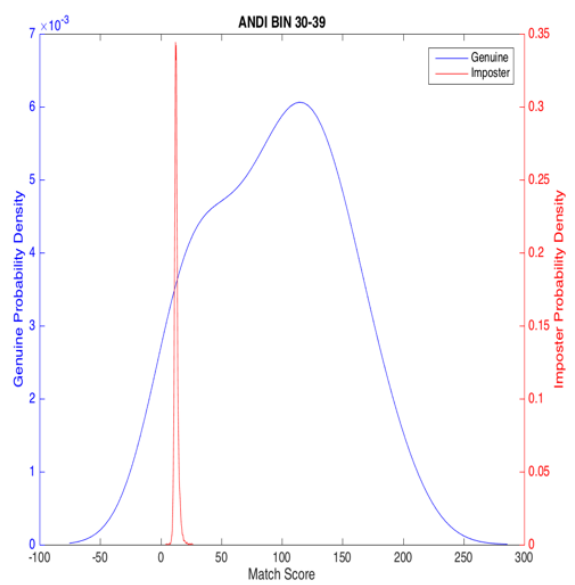

(c)

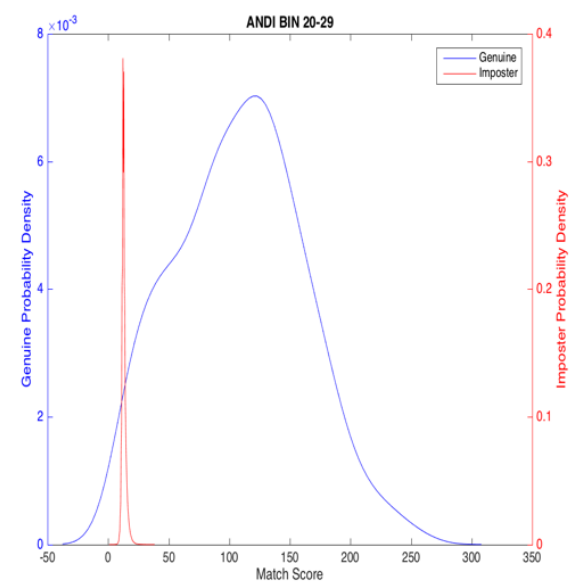

(b)

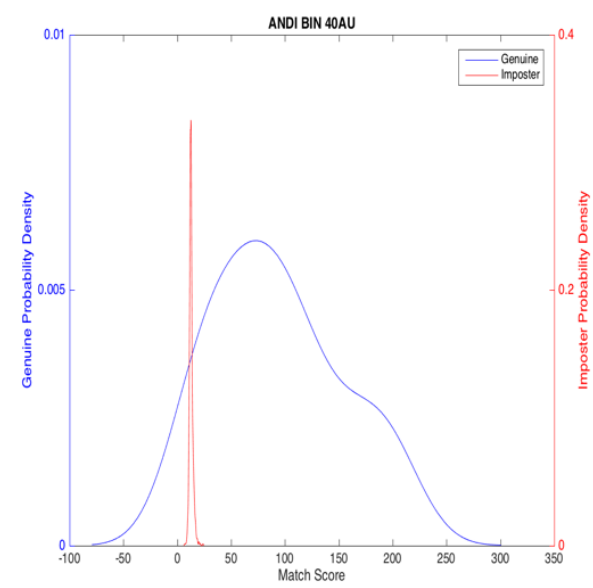

(d)

Figure C.3.1 SourceAFIS ANDI Binary (a) Ages 18-19 (b) Ages 20-29 (c) Ages 30-39 (d) Ages 40 and up 


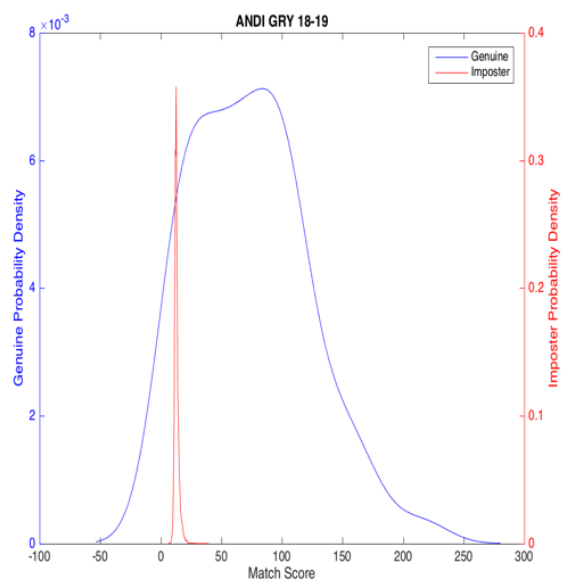

(a)

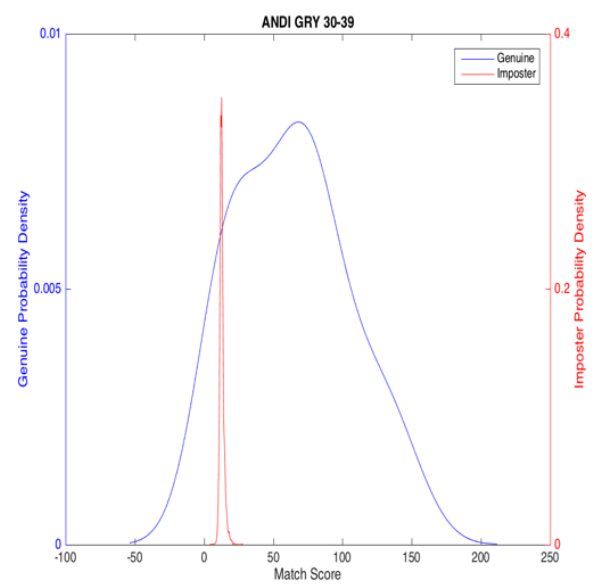

(c)

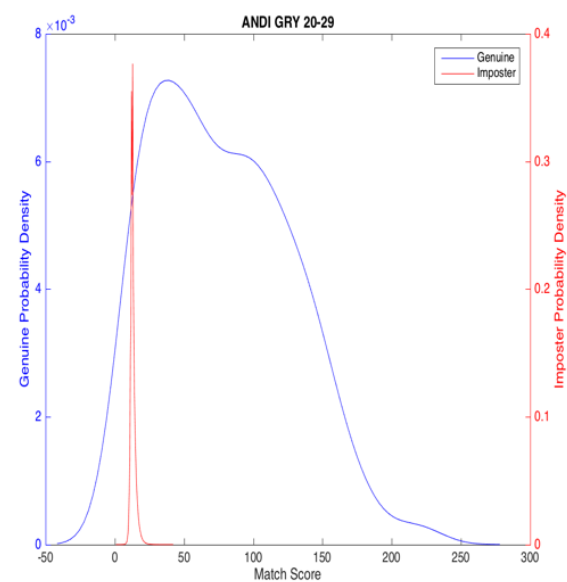

(b)

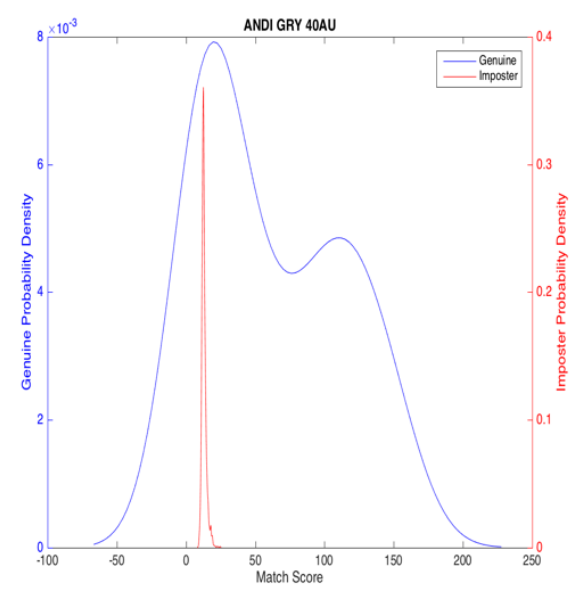

(d)

Figure C.3.2 SourceAFIS ANDI Greyscale (a) Ages 18-19 (b) Ages 20-29 (c) Ages 30-39 (d) Ages 40 and up 


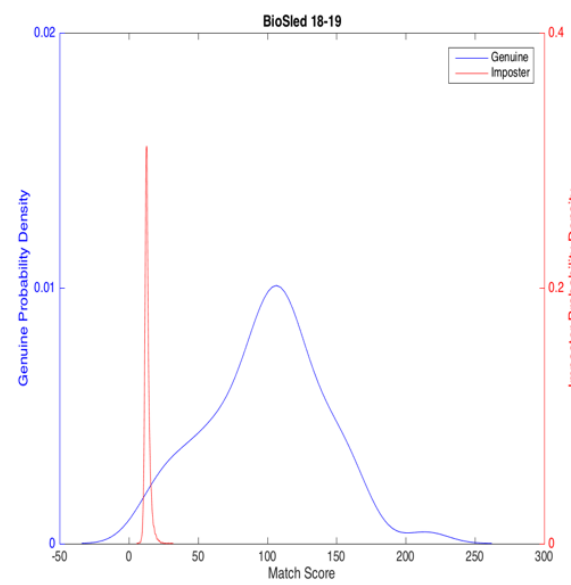

(a)

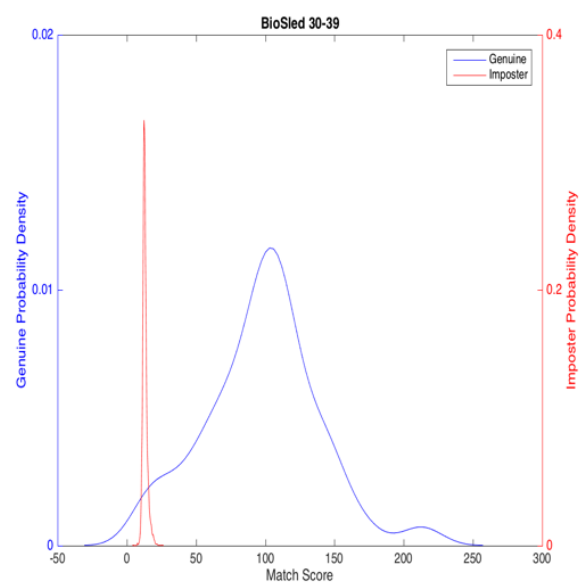

(c)

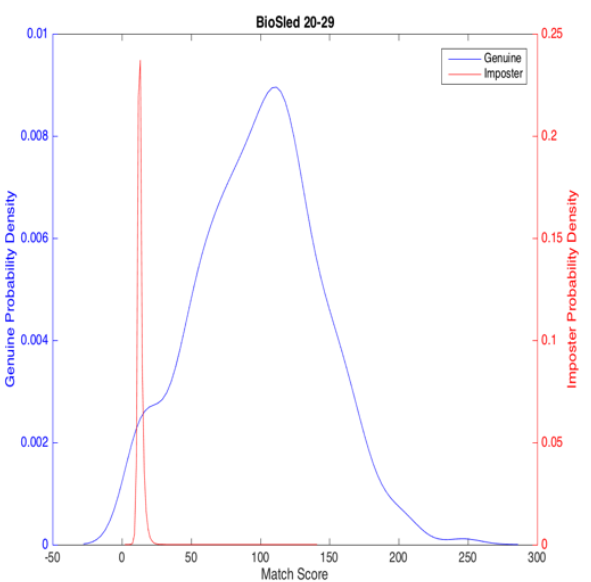

(b)

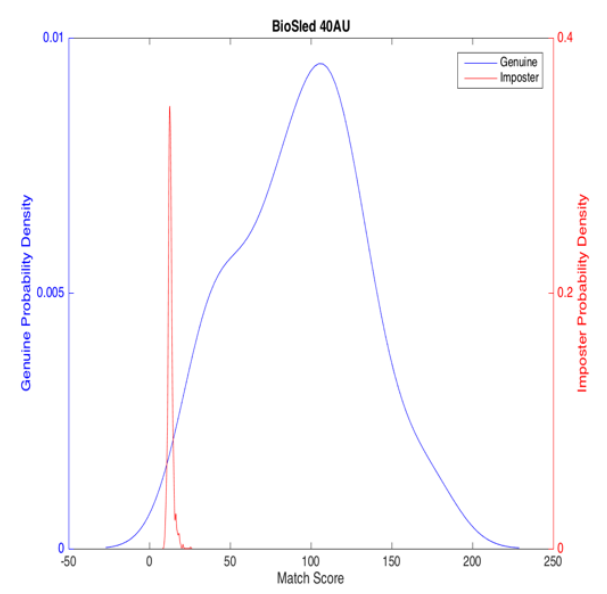

(d)

Figure C.3.3 SourceAFIS BioSled (a) Ages 18-19 (b) Ages 20-29 (c) Ages 30-39 (d) Ages 40 and up 


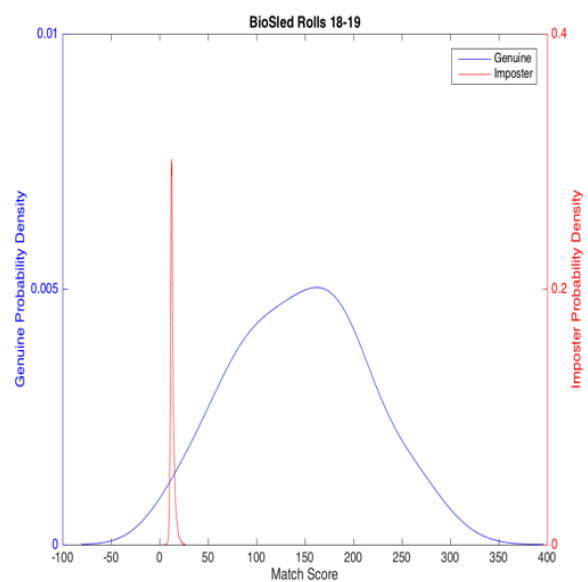

(a)

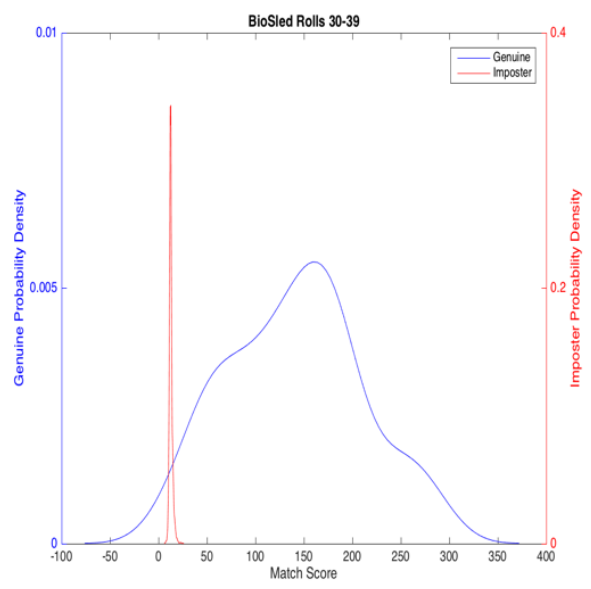

(c)

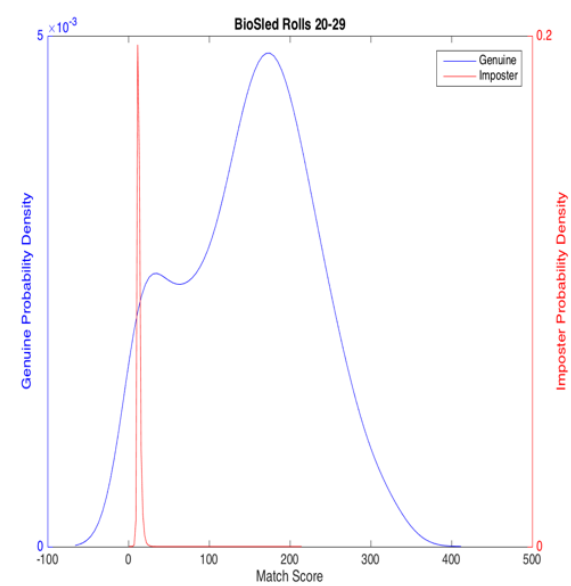

(b)

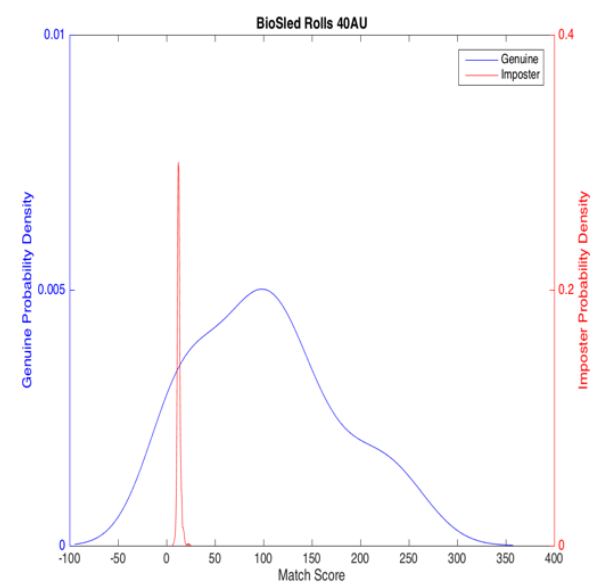

(d)

Figure C.3.4 SourceAFIS BioSled Rolls (a) Ages 18-19 (b) Ages 20-29 (c) Ages 30-39 (d) Ages 40 and up 


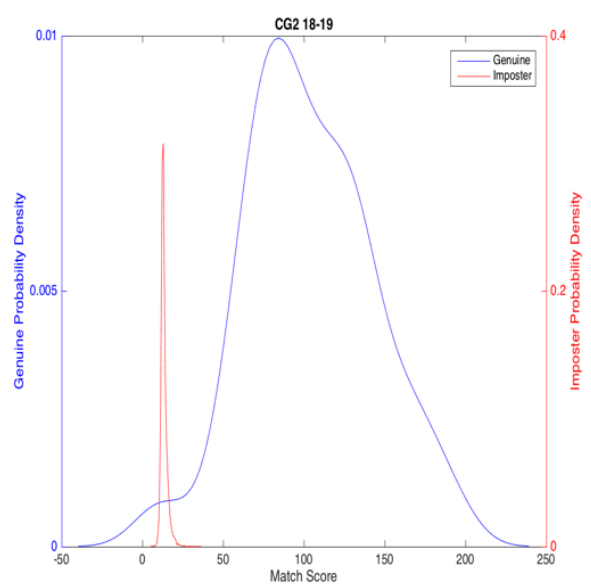

(a)

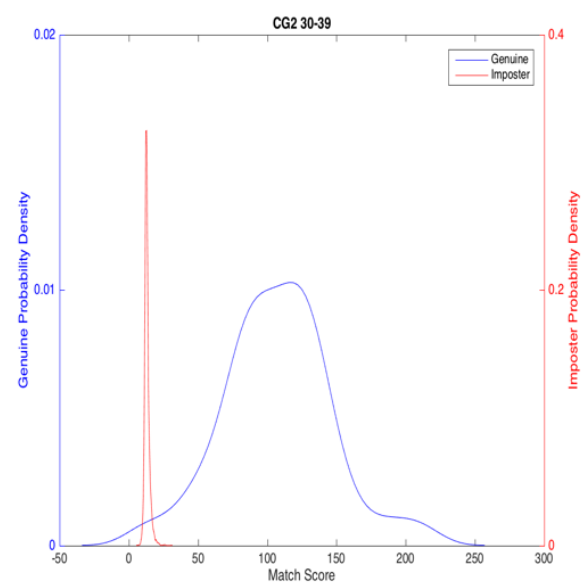

(c)

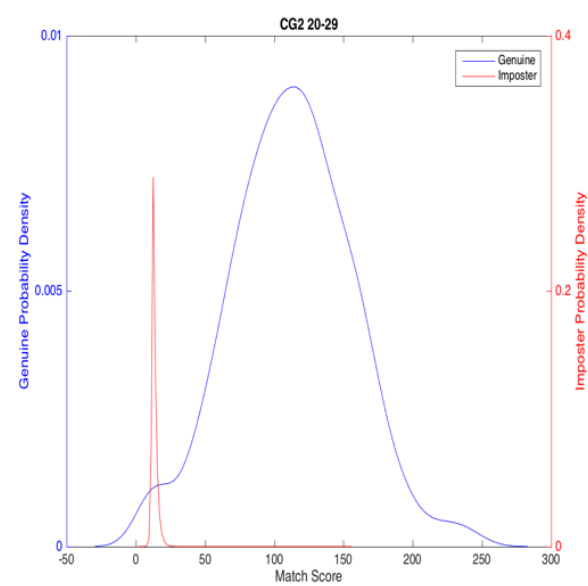

(b)

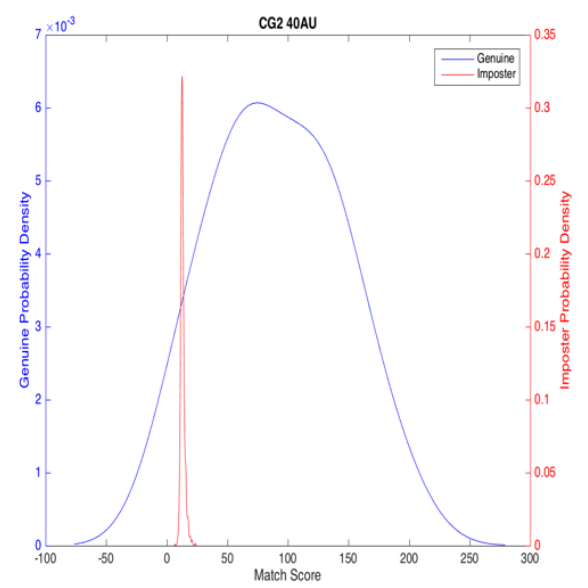

(d)

Figure C.3.5 SourceAFIS FP II Guardian (a) Ages 18-19 (b) Ages 20-29 (c) Ages 30-39 (d) Ages 40 and up 


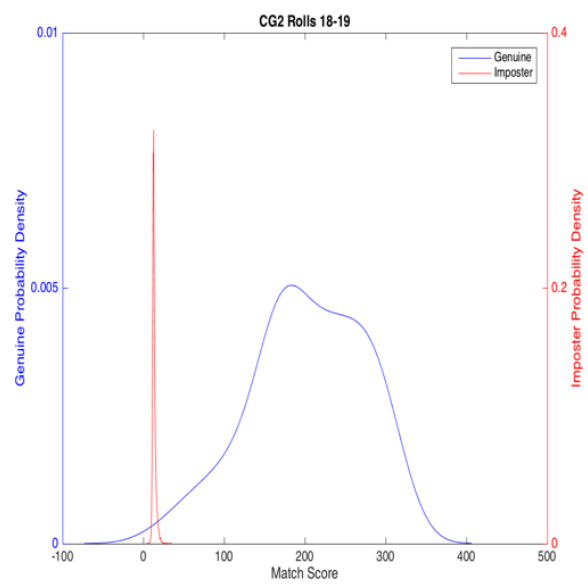

(a)

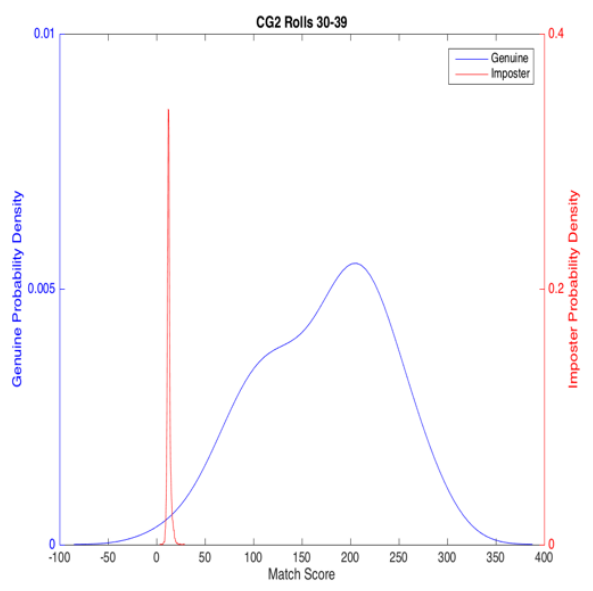

(c)

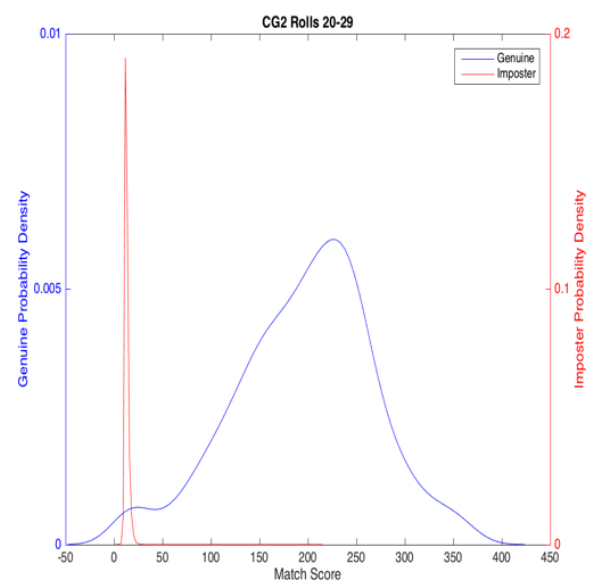

(b)

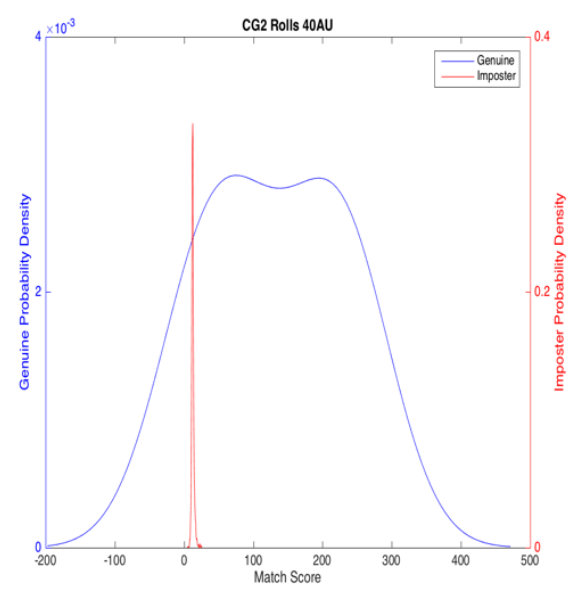

(d)

Figure C.3.6 SourceAFIS FP II Guardian Rolls (a) Ages 18-19 (b) Ages 20-29 (c) Ages 30-39 (d) Ages 40 and up 


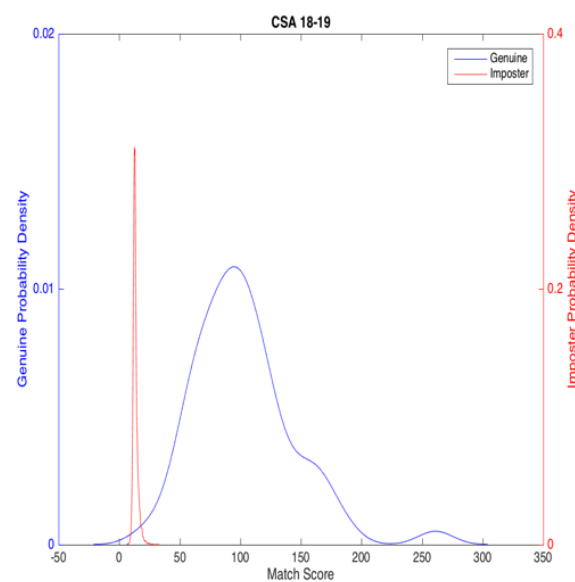

(a)

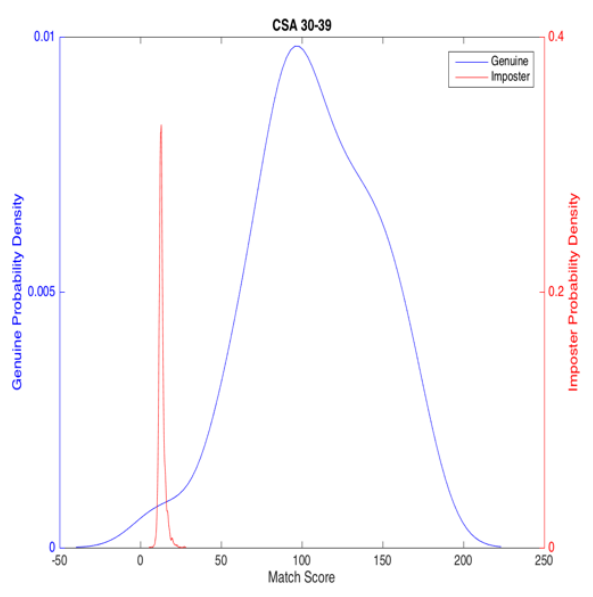

(c)

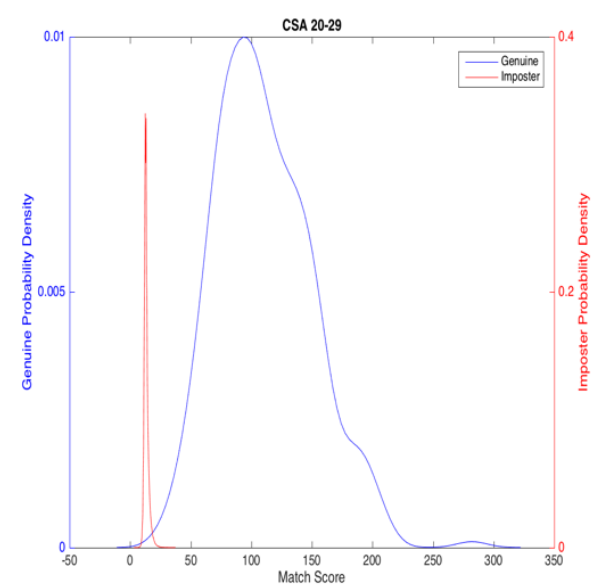

(b)

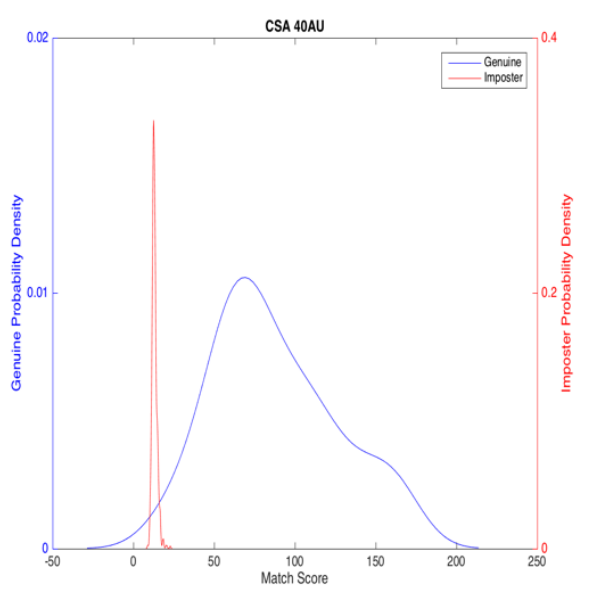

(d)

Figure C.3.7 SourceAFIS Crossmatch Seek Avenger (a) Ages 18-19 (b) Ages 20-29 (c) Ages 30-39 (d) Ages 40 and up 


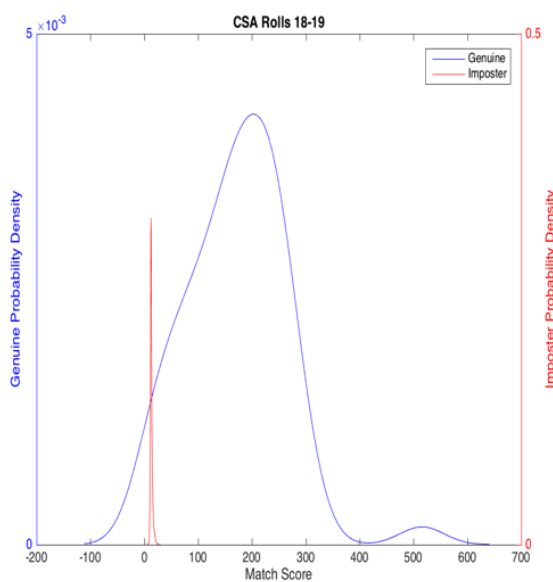

(a)

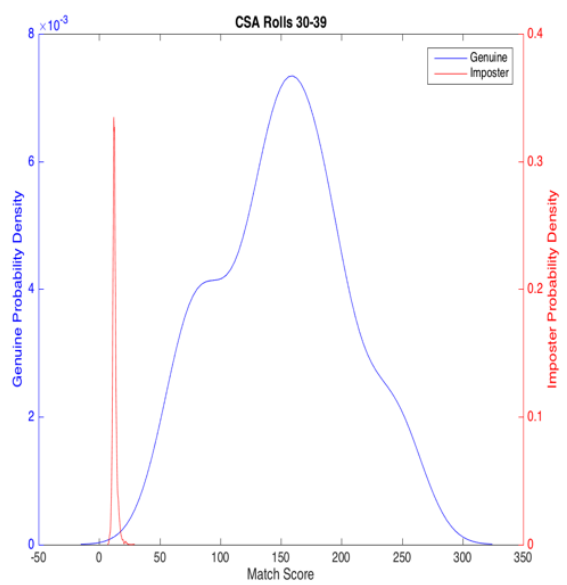

(c)

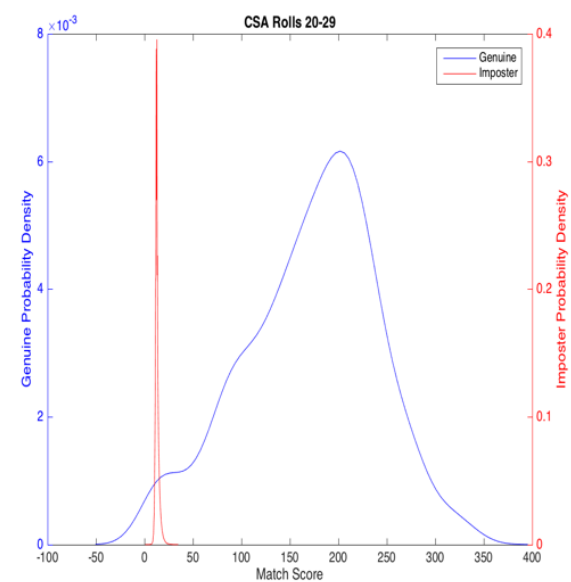

(b)

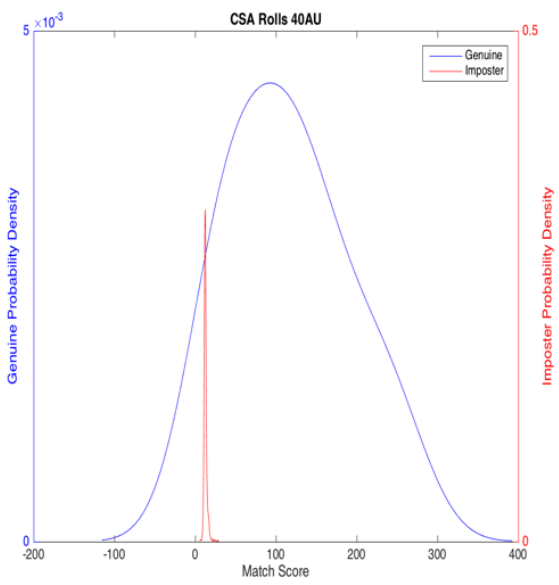

(d)

Figure C.3.8 SourceAFIS Crossmatch Seek Avenger Rolls (a) Ages 18-19 (b) Ages 20-29 (c) Ages 30-39 (d) Ages 40 and up 


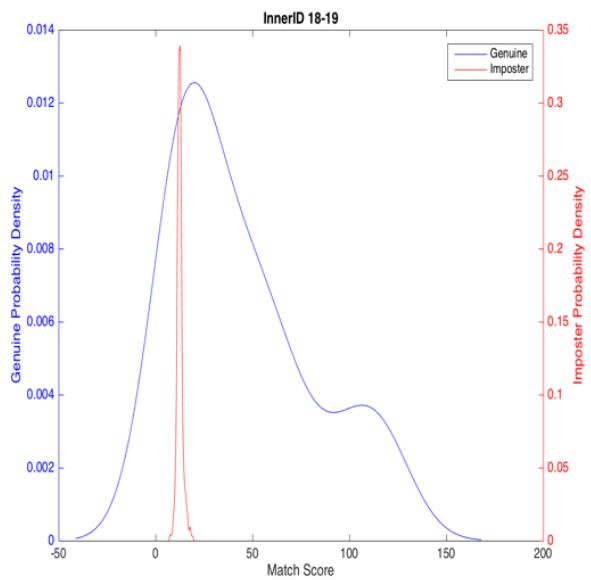

(a)

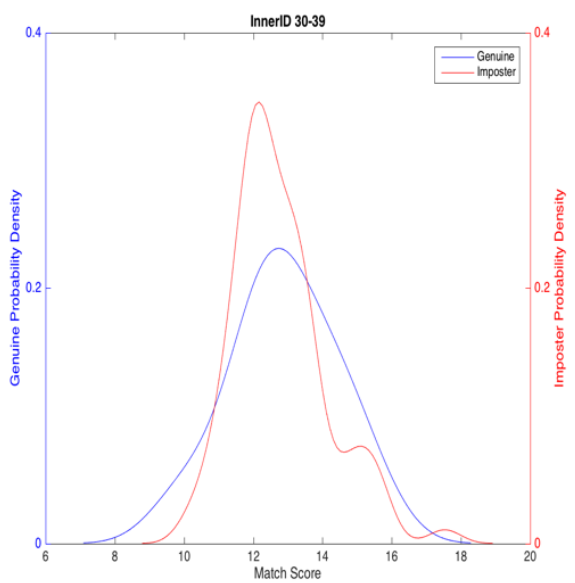

(c)

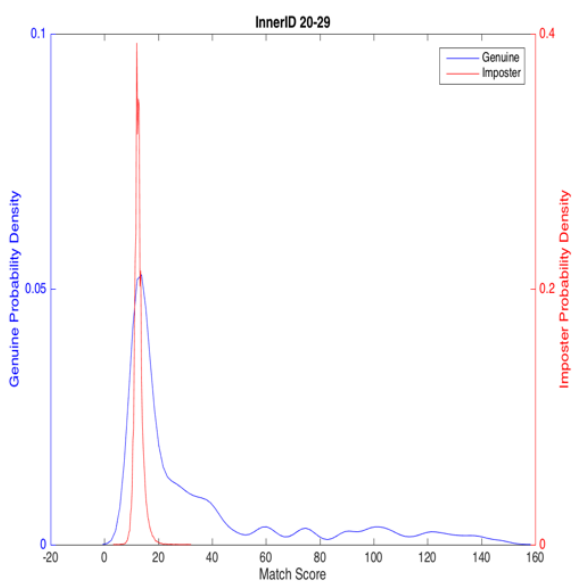

(b)

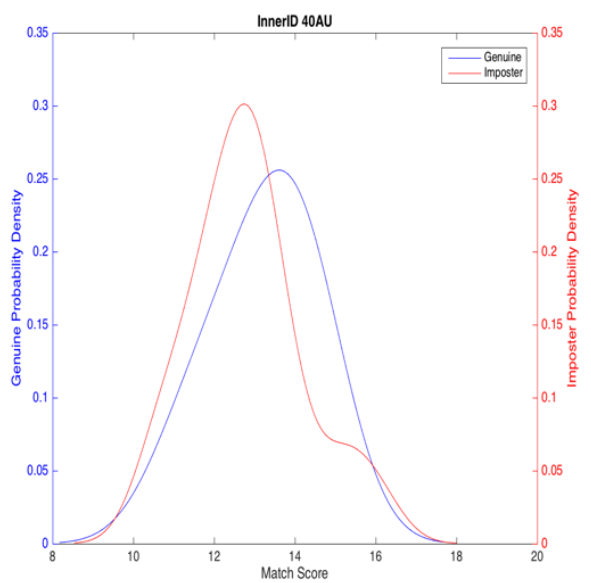

(d)

Figure C.3.9 SourceAFIS InnerID (a) Ages 18-19 (b) Ages 20-29 (c) Ages 30-39 (d) Ages 40 and up 


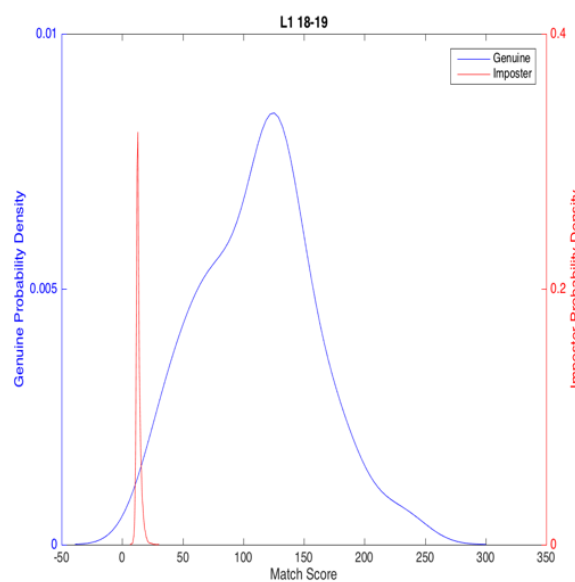

(a)

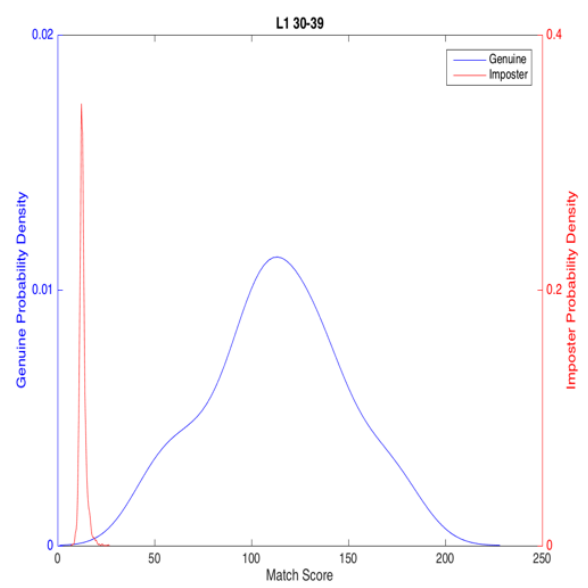

(c)

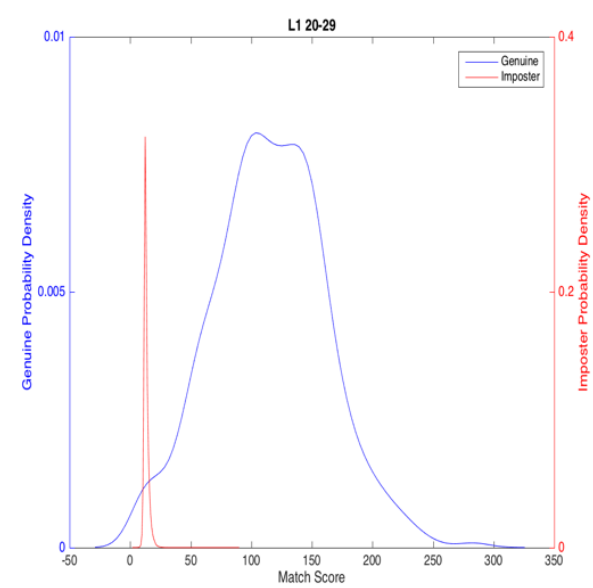

(b)

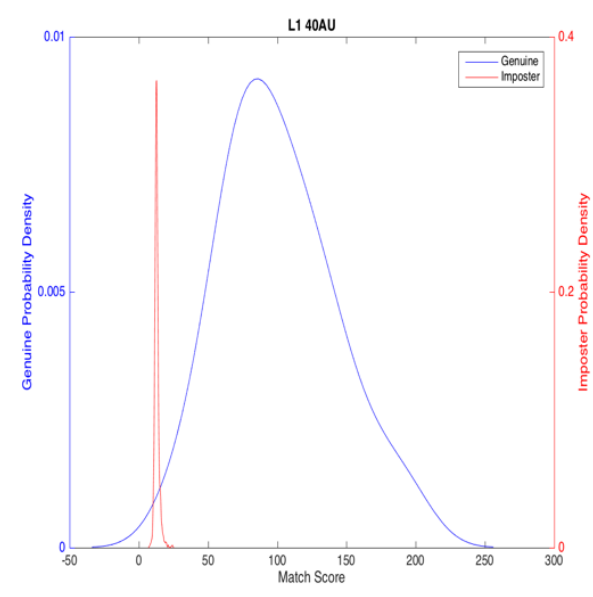

(d)

Figure C.3.10 SourceAFIS L1 (a) Ages 18-19 (b) Ages 20-29 (c) Ages 30-39 (d) Ages 40 and up 


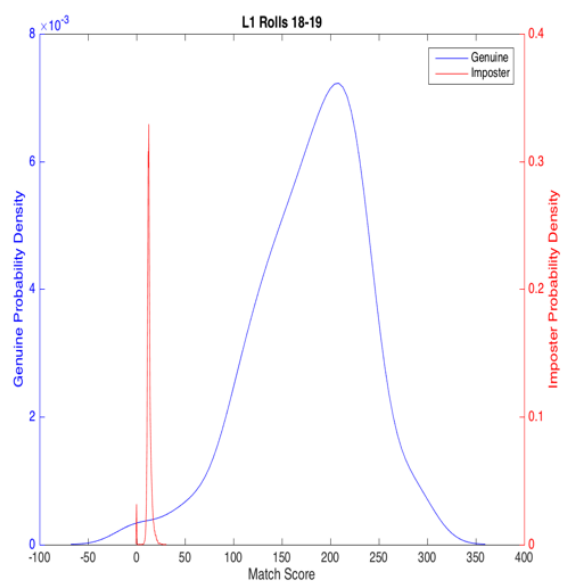

(a)

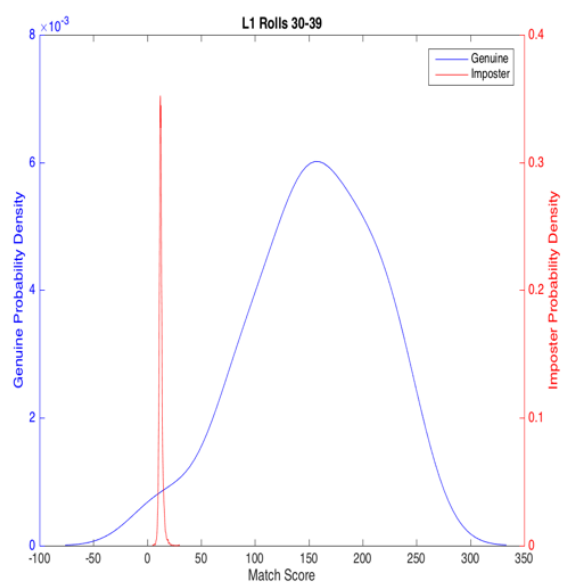

(c)

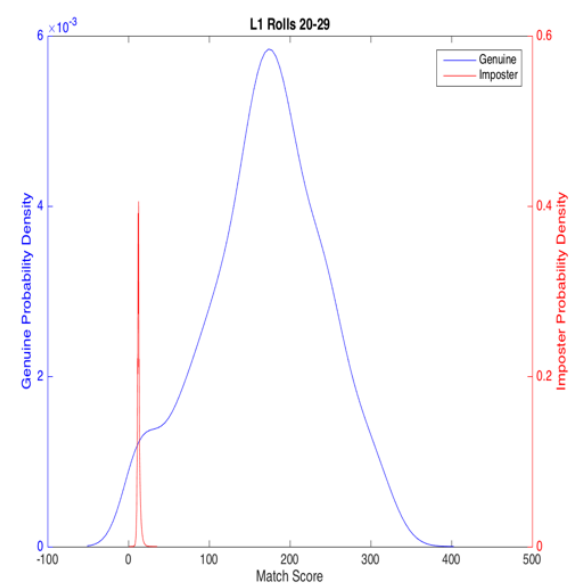

(b)

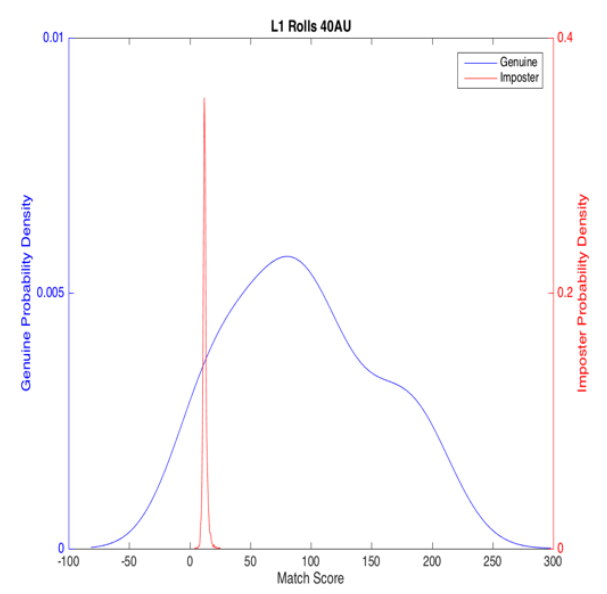

(d)

Figure C.3.11 SourceAFIS L1 Rolls (a) Ages 18-19 (b) Ages 20-29 (c) Ages 30-39 (d) Ages 40 and up 


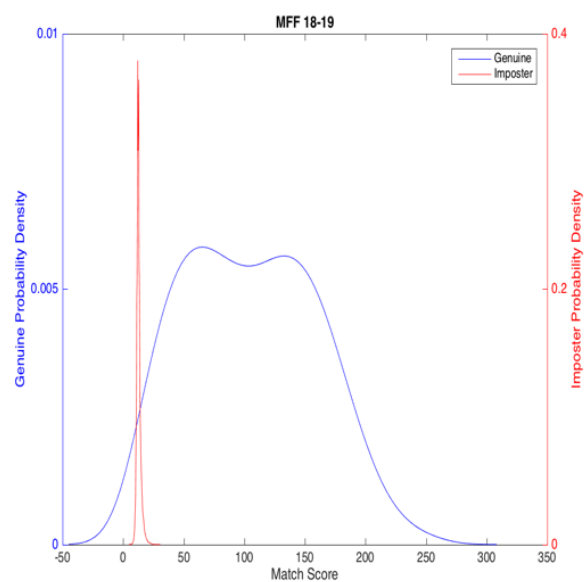

(a)

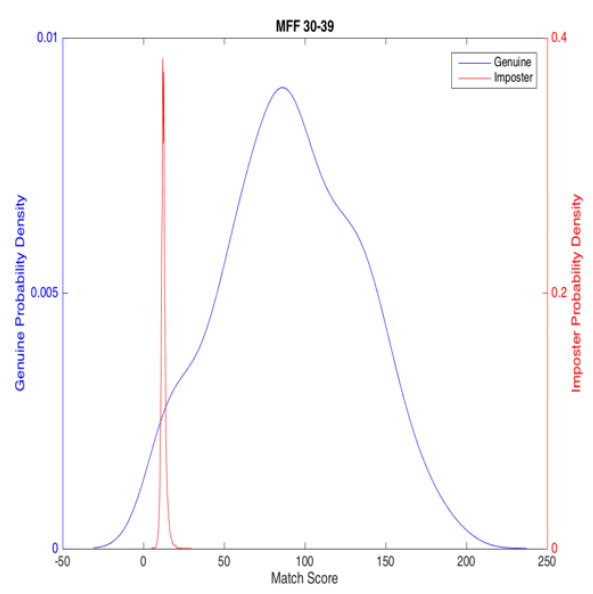

(c)

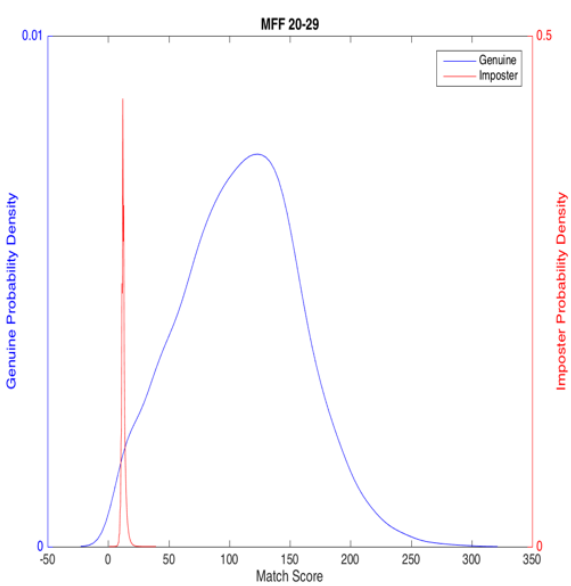

(b)

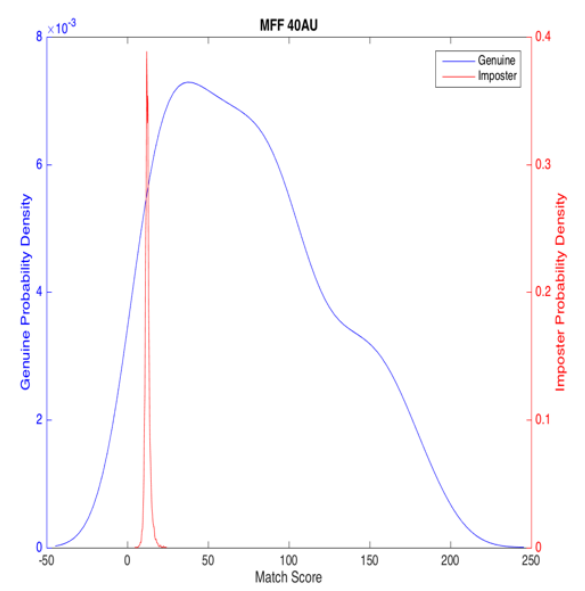

(d)

Figure C.3.12 SourceAFIS Morpho FOTF (a) Ages 18-19 (b) Ages 20-29 (c) Ages 30-39 (d) Ages 40 and up 


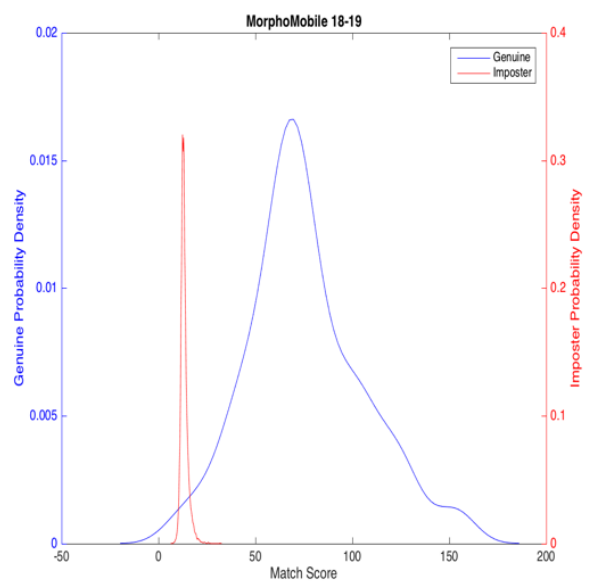

(a)

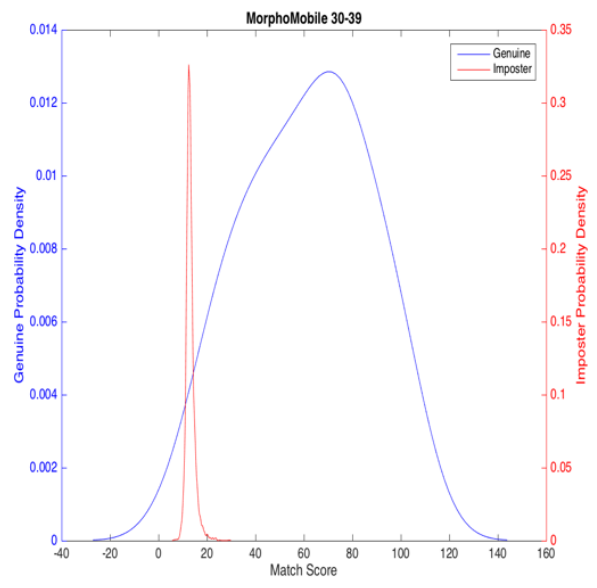

(c)

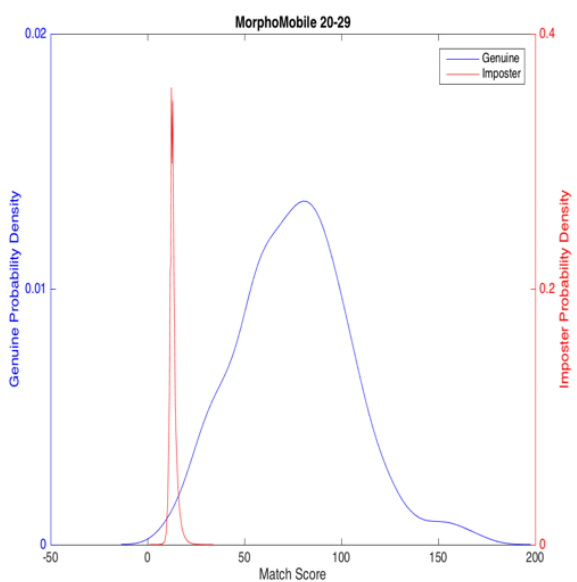

(b)

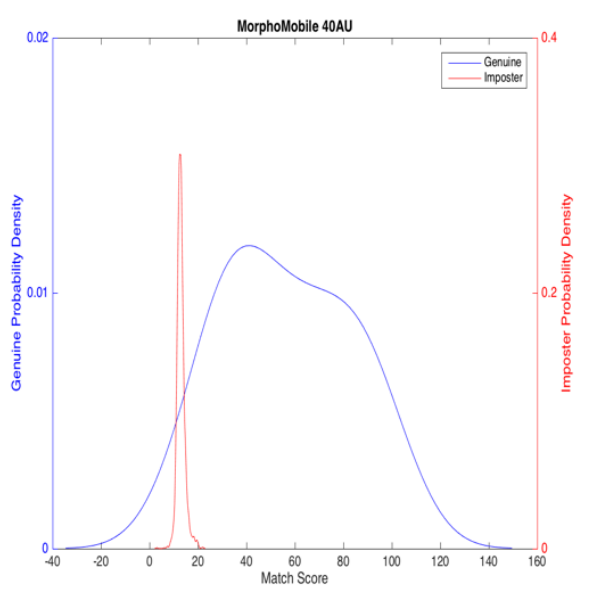

(d)

Figure C.3.13 SourceAFIS MorphoMobile (a) Ages 18-19 (b) Ages 20-29 (c) Ages 30-39 (d) Ages 40 and up 


\section{C.4 NBIS ROC Curves and Area Under Curve}

Table C.13 NBIS 18-19 Area under Curve

\begin{tabular}{|l|l|}
\hline NBIS 18-19 & \\
\hline Device & Area Under Curve \\
\hline ANDI BIN & 0.988839 \\
\hline ANDI GRY & 0.99552 \\
\hline BioSled & 0.994623 \\
\hline BioSled Rolls & 0.999971 \\
\hline CG2 & 0.972421 \\
\hline CG2 Rolls & 1 \\
\hline CSA & 0.999838 \\
\hline CSA Rolls & 0.996351 \\
\hline InnerID & 0.87304 \\
\hline L1 & 0.998894 \\
\hline L1 Rolls & 0.983324 \\
\hline MFF & 0.99998 \\
\hline MorphoMobile & 0.996163 \\
\hline
\end{tabular}

Table C.14 NBIS 20-29 Area under Curve

\begin{tabular}{|l|l|}
\hline NBIS 20-29 & \\
\hline Device & Area Under Curve \\
\hline ANDI BIN & 0.986152 \\
\hline ANDI GRY & 0.980966 \\
\hline BioSled & 0.977594 \\
\hline BioSled Rolls & 0.97685 \\
\hline CG2 & 0.982419 \\
\hline CG2 Rolls & 0.991015 \\
\hline CSA & 0.999744 \\
\hline CSA Rolls & 0.987361 \\
\hline InnerID & 0.870499 \\
\hline L1 & 0.976552 \\
\hline L1 Rolls & 0.979743 \\
\hline MFF & 0.993658 \\
\hline MorphoMobile & 0.992372 \\
\hline
\end{tabular}


Table C.15 NBIS 30-39 Area under Curve

\begin{tabular}{|l|l|}
\hline NBIS 30-39 & \\
\hline Device & Area Under Curve \\
\hline ANDI BIN & 0.960701 \\
\hline ANDI GRY & 0.954972 \\
\hline BioSled & 0.992645 \\
\hline BioSled Rolls & 1 \\
\hline CG2 & 0.991879 \\
\hline CG2 Rolls & 1 \\
\hline CSA & 0.987423 \\
\hline CSA Rolls & 1 \\
\hline InnerID & 0.57375 \\
\hline L1 & 1 \\
\hline L1 Rolls & 0.976454 \\
\hline MFF & 0.984673 \\
\hline MorphoMobile & 0.983952 \\
\hline
\end{tabular}

Table C.16 NBIS 40 and up Area under Curve

\begin{tabular}{|l|l|}
\hline NBIS 40 and up & \\
\hline Device & Area Under Curve \\
\hline ANDI BIN & 0.990047 \\
\hline ANDI GRY & 0.97675 \\
\hline BioSled & 0.999112 \\
\hline BioSled Rolls & 0.989228 \\
\hline CG2 & 0.922401 \\
\hline CG2 Rolls & 0.964758 \\
\hline CSA & 0.999952 \\
\hline CSA Rolls & 0.948507 \\
\hline InnerID & 0.791667 \\
\hline L1 & 0.999047 \\
\hline L1 Rolls & 0.970189 \\
\hline MFF & 0.999351 \\
\hline MorphoMobile & 0.994694 \\
\hline
\end{tabular}



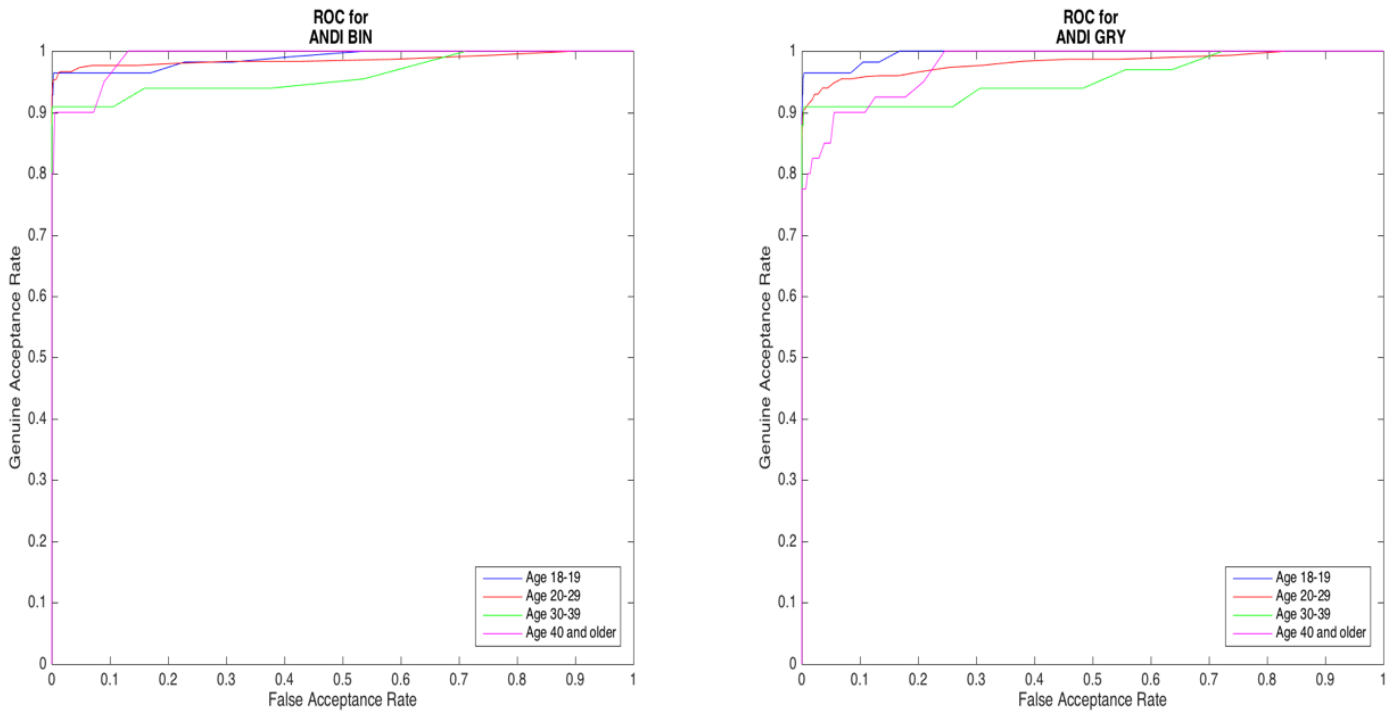

Figure C.4.1 NBIS ANDI Binary Age Demographic ROC Curves (Left) NBIS ANDI Greyscale Age Demographic ROC Curves (Right)
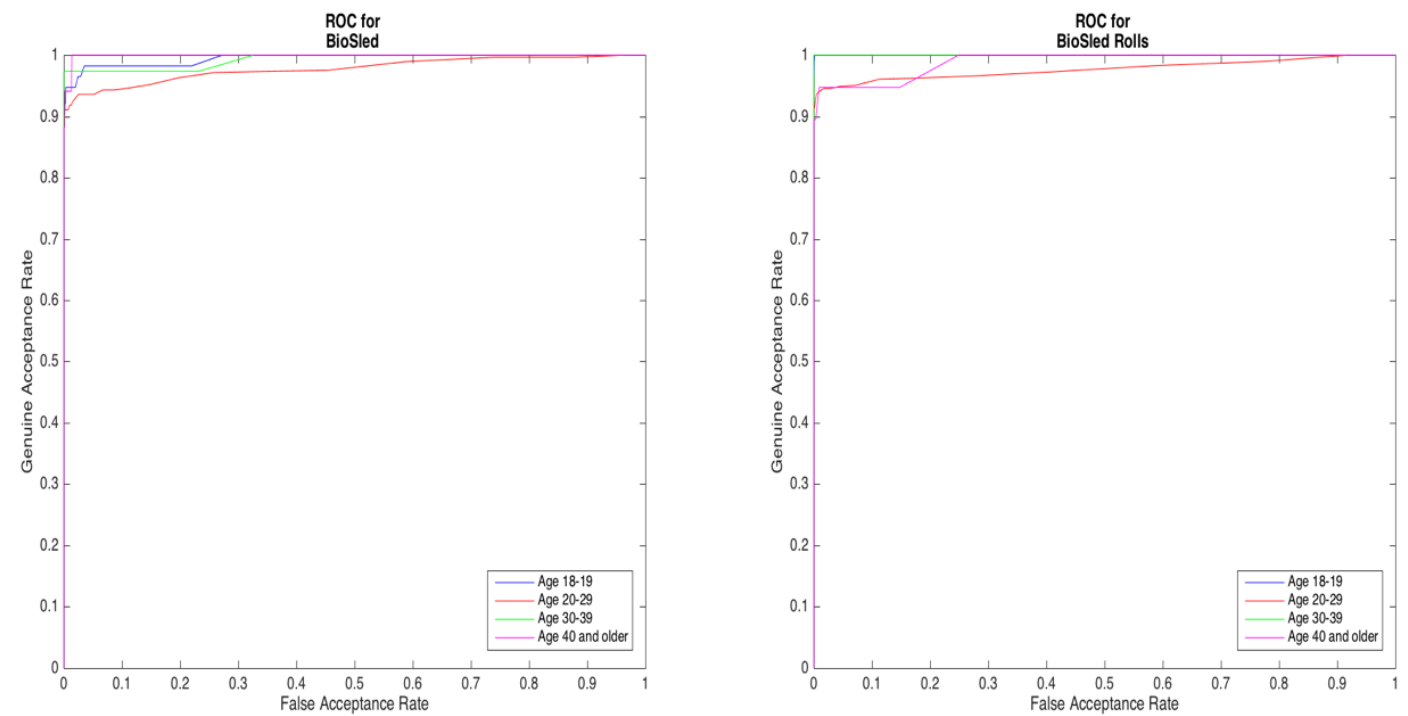

Figure C.4.2 NBIS BioSled Age Demographic ROC Curves (Left) NBIS BioSled Rolls Age Demographic ROC Curves (Right) 

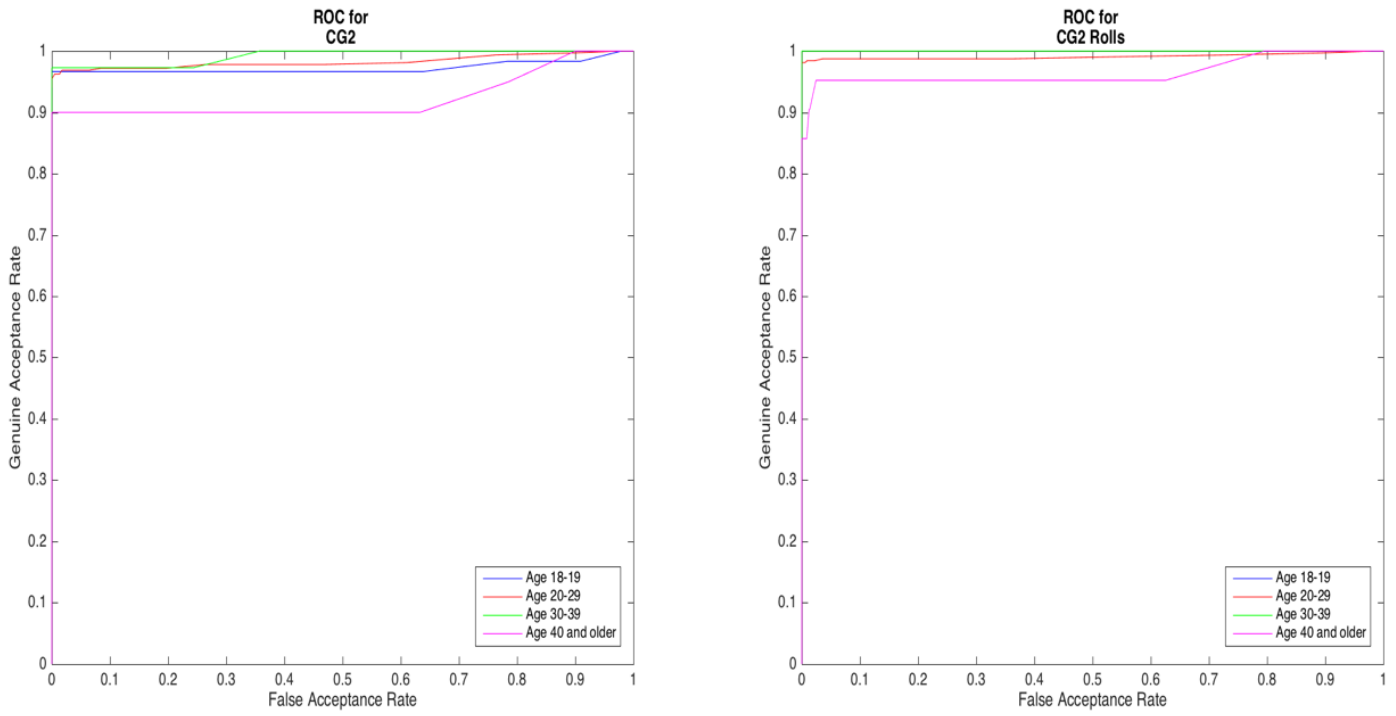

Figure C.4.3 NBIS FP II Guardian Age Demographic ROC Curves (Left) NBIS FP II Guardian Rolls Age Demographic ROC Curves (Right)
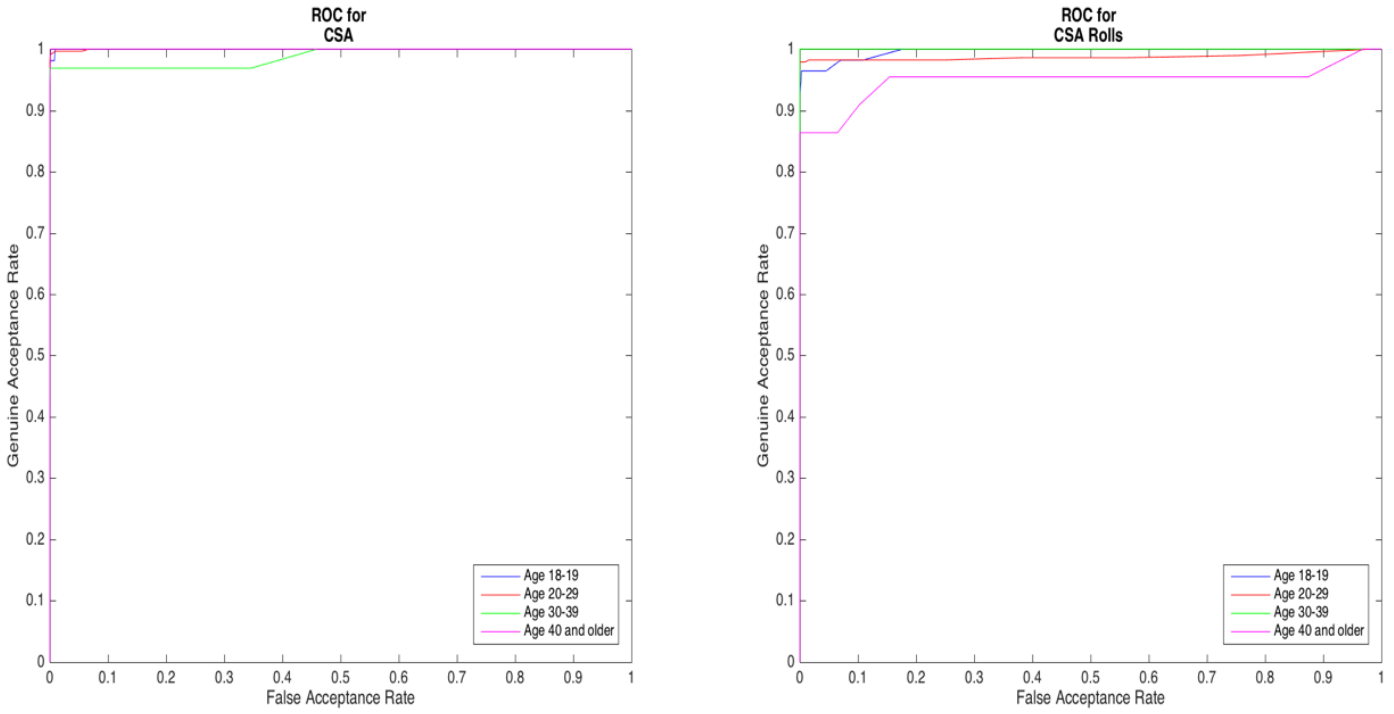

Figure C.4.4 NBIS Crossmatch Seek Avenger Age Demographic ROC Curves (Left) NBIS Crossmatch Seek Avenger Rolls Age Demographic ROC Curves (Right) 

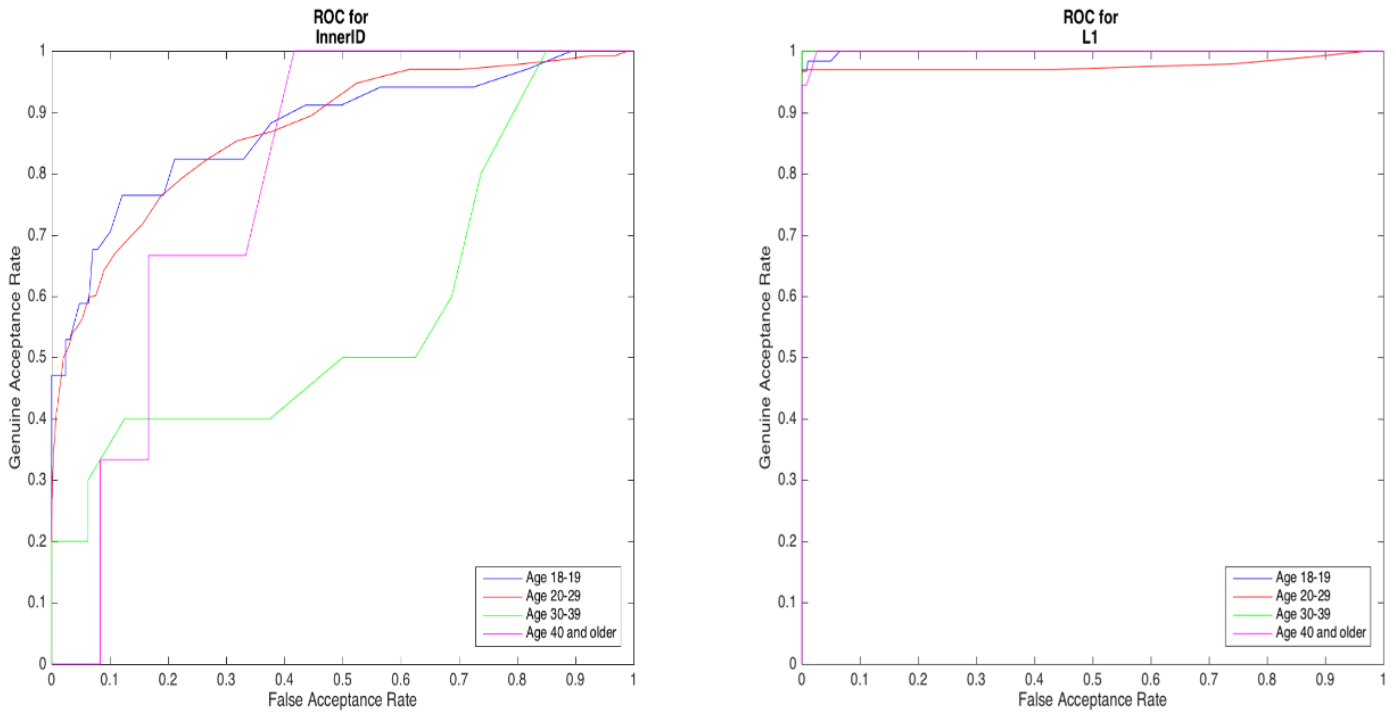

Figure C.4.5 NBIS InnerID Age Demographic ROC Curves (Left) NBIS L1 Age Demographic ROC Curves (Right)
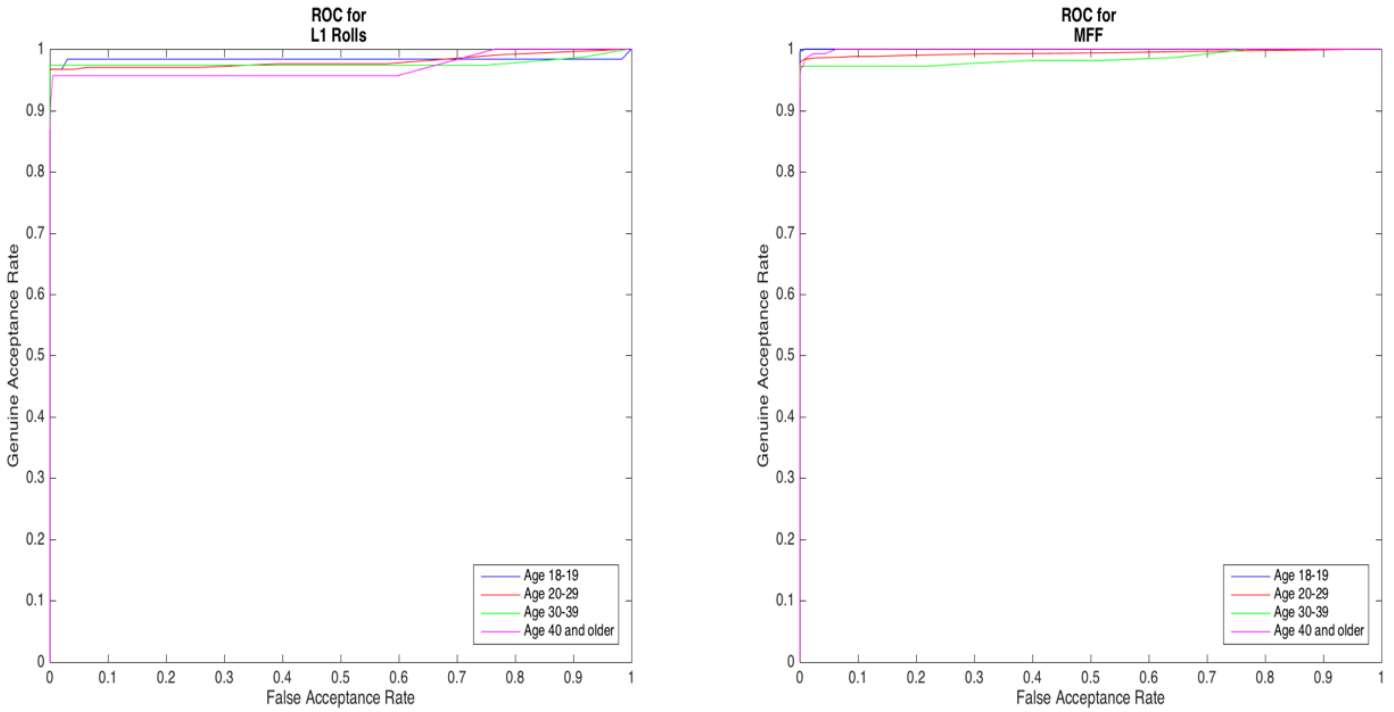

Figure C.4.6 NBIS L1 Rolls Age Demographic ROC Curves (Left) NBIS Morpho FOTF Age Demographic ROC Curves (Right) 


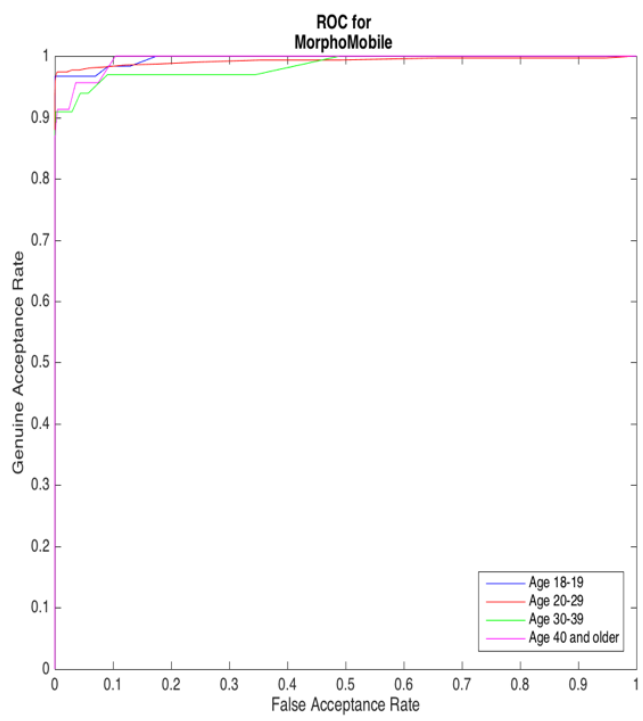

Figure C.4.7 NBIS MorphoMobile Age Demographic ROC Curves

\section{C.5 Neurotech ROC Curves and Area Under Curve}

Table C.17 Neurotech 18-19 Area under Curve

\begin{tabular}{|l|l|}
\hline Neurotech 18-19 & \\
\hline Device & Area Under Curve \\
\hline ANDI BIN & 0.989207 \\
\hline ANDI GRY & 0.988166 \\
\hline BioSled & 1 \\
\hline BioSled Rolls & 1 \\
\hline CG2 & 0.993148 \\
\hline CG2 Rolls & 1 \\
\hline CSA & 1 \\
\hline CSA Rolls & 1 \\
\hline L1 & 1 \\
\hline MFF & 1 \\
\hline MorphoMobile & 0.972874 \\
\hline
\end{tabular}


Table C.18 Neurotech 20-29 Area under Curve

\begin{tabular}{|l|l|}
\hline Neurotech 20-29 & \\
\hline Device & Area Under Curve \\
\hline ANDI BIN & 0.984873 \\
\hline ANDI GRY & 0.98276 \\
\hline BioSled & 0.976493 \\
\hline BioSled Rolls & 0.985896 \\
\hline CG2 & 0.982915 \\
\hline CG2 Rolls & 0.99611 \\
\hline CSA & 1 \\
\hline CSA Rolls & 0.993444 \\
\hline L1 & 0.983861 \\
\hline MFF & 0.995235 \\
\hline MorphoMobile & 0.997851 \\
\hline
\end{tabular}

Table C.19 Neurotech 30-39 Area under Curve

\begin{tabular}{|l|l|}
\hline Neurotech 30-39 & \\
\hline Device & Area Under Curve \\
\hline ANDI BIN & 0.963432 \\
\hline ANDI GRY & 0.966645 \\
\hline BioSled & 0.988278 \\
\hline BioSled Rolls & 1 \\
\hline CG2 & 1 \\
\hline CG2 Rolls & 1 \\
\hline CSA & 0.973109 \\
\hline CSA Rolls & 1 \\
\hline L1 & 1 \\
\hline MFF & 0.991565 \\
\hline MorphoMobile & 0.981169 \\
\hline
\end{tabular}


Table C.20 Neurotech 40 and up Area under Curve

\begin{tabular}{|l|l|}
\hline Neurotech 40 and up & \\
\hline Device & Area Under Curve \\
\hline ANDI BIN & 1 \\
\hline ANDI GRY & 1 \\
\hline BioSled & 1 \\
\hline BioSled Rolls & 0.976248 \\
\hline CG2 & 0.904583 \\
\hline CG2 Rolls & 1 \\
\hline CSA & 1 \\
\hline CSA Rolls & 1 \\
\hline L1 & 1 \\
\hline MFF & 1 \\
\hline MorphoMobile & 1 \\
\hline
\end{tabular}



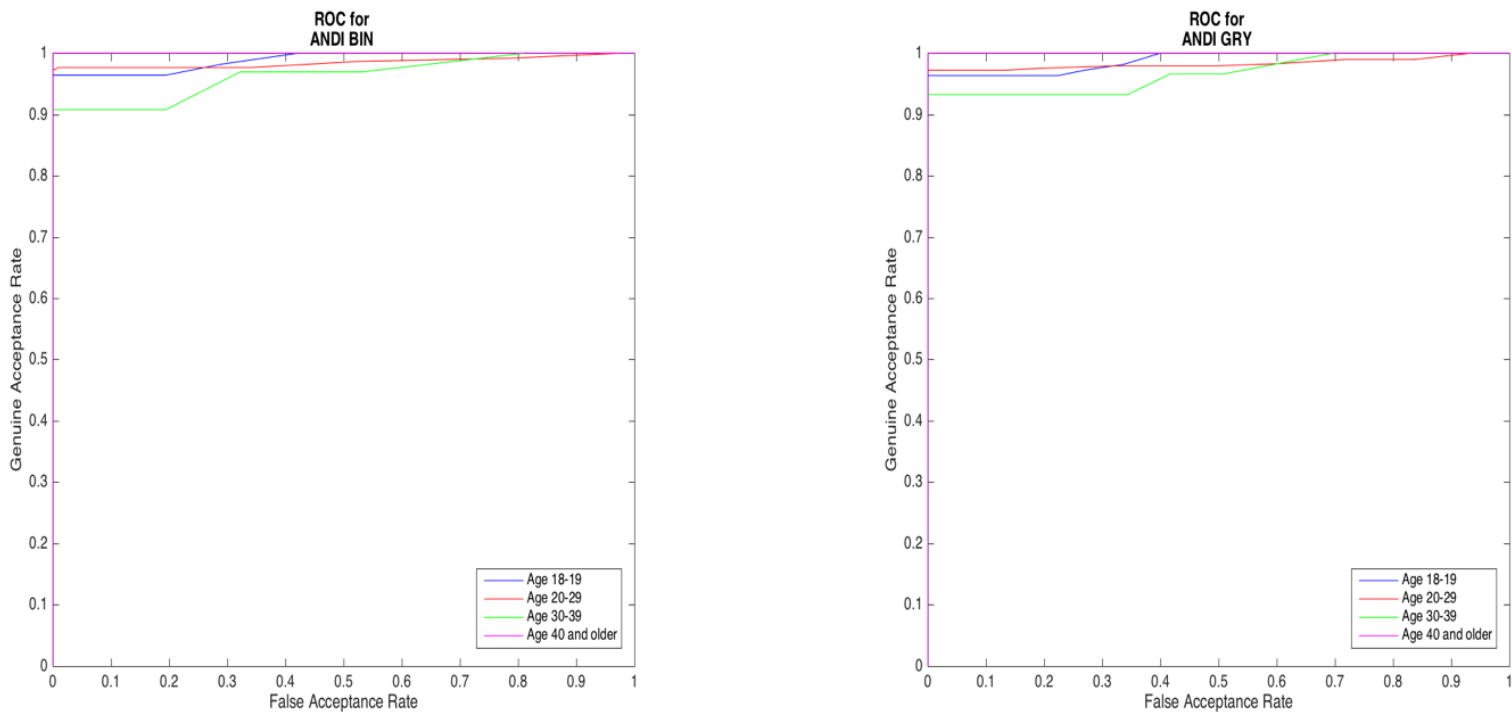

Figure C.5.1 Neurotech ANDI Binary Age Demographic ROC Curves (Left) Neurotech ANDI Greyscale Age Demographic ROC Curves (Right)
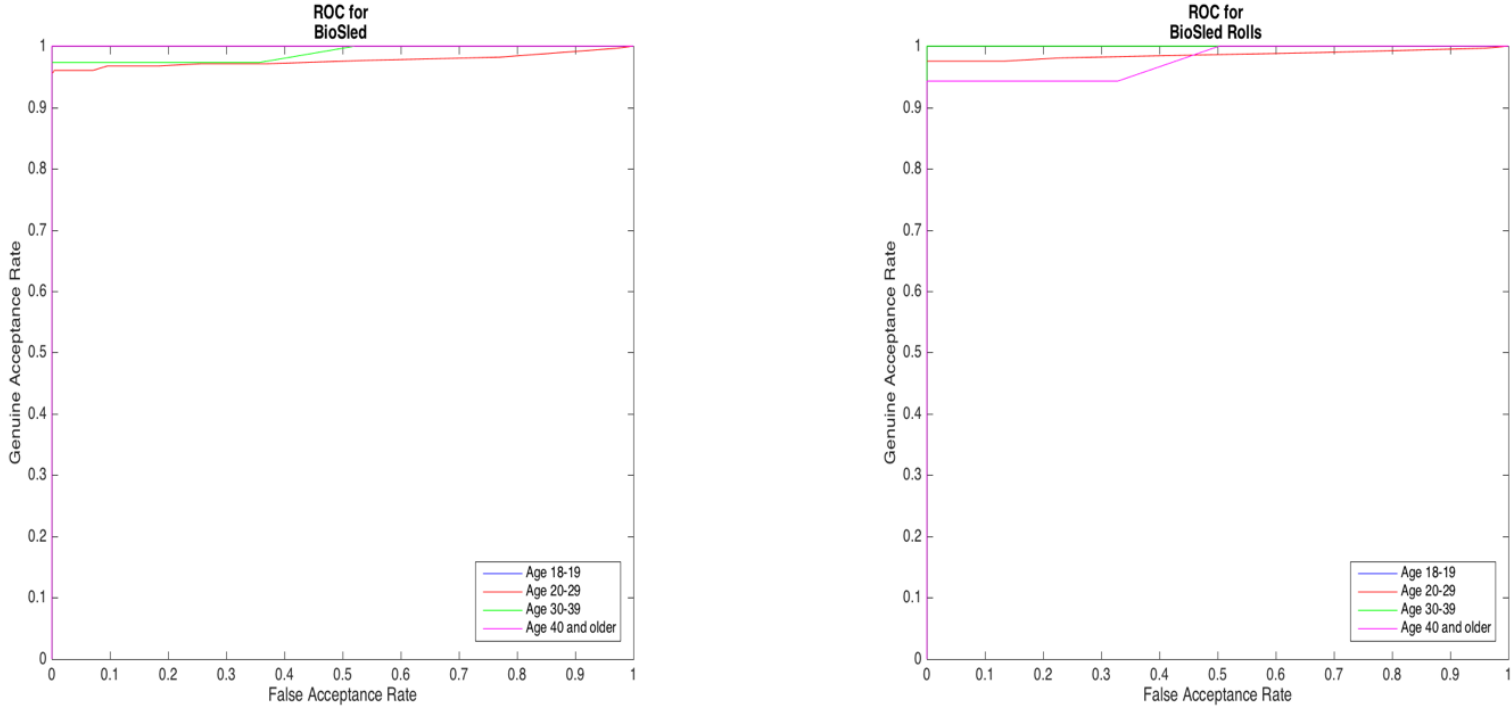

Figure C.5.2 Neurotech BioSled Age Demographic ROC Curves (Left) Neurotech BioSled Rolls Age Demographic ROC Curves (Right) 

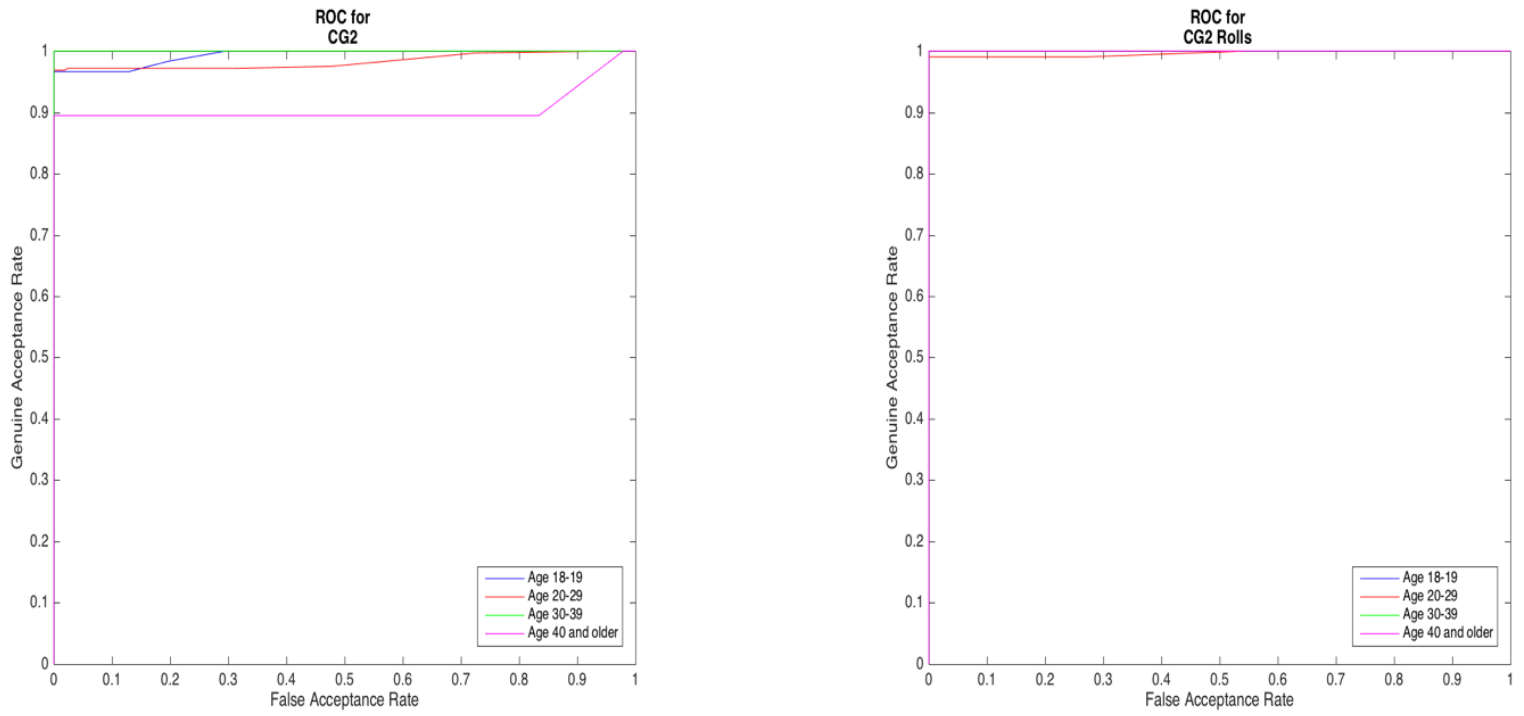

Figure C.5.3 Neurotech FP II Guardian Age Demographic ROC Curves (Left) Neurotech FP II Guardian Rolls Age Demographic ROC Curves (Right)
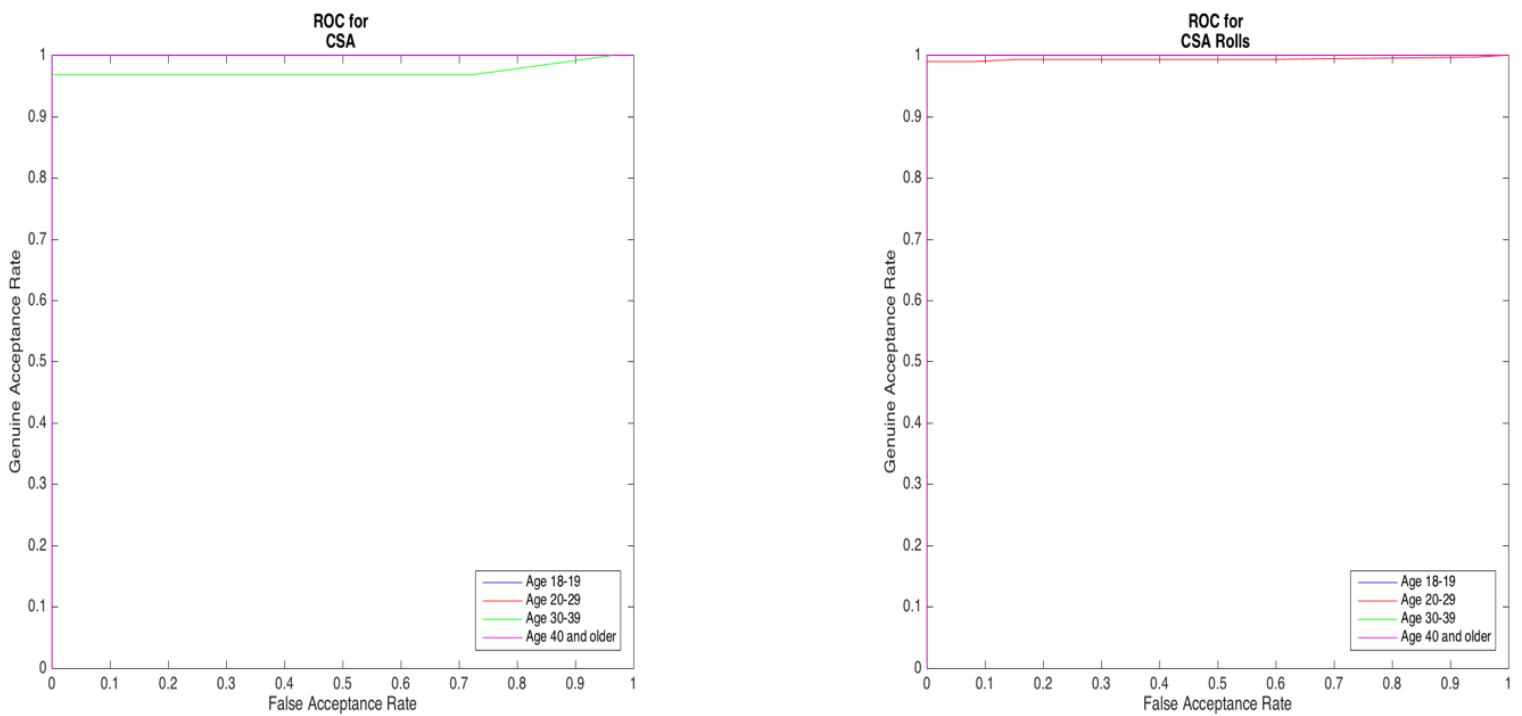

Figure C.5.4 Neurotech Crossmatch Seek Avenger Age Demographic ROC Curves (Left) Neurotech Crossmatch Seek Avenger Rolls Age Demographic ROC Curves (Right) 

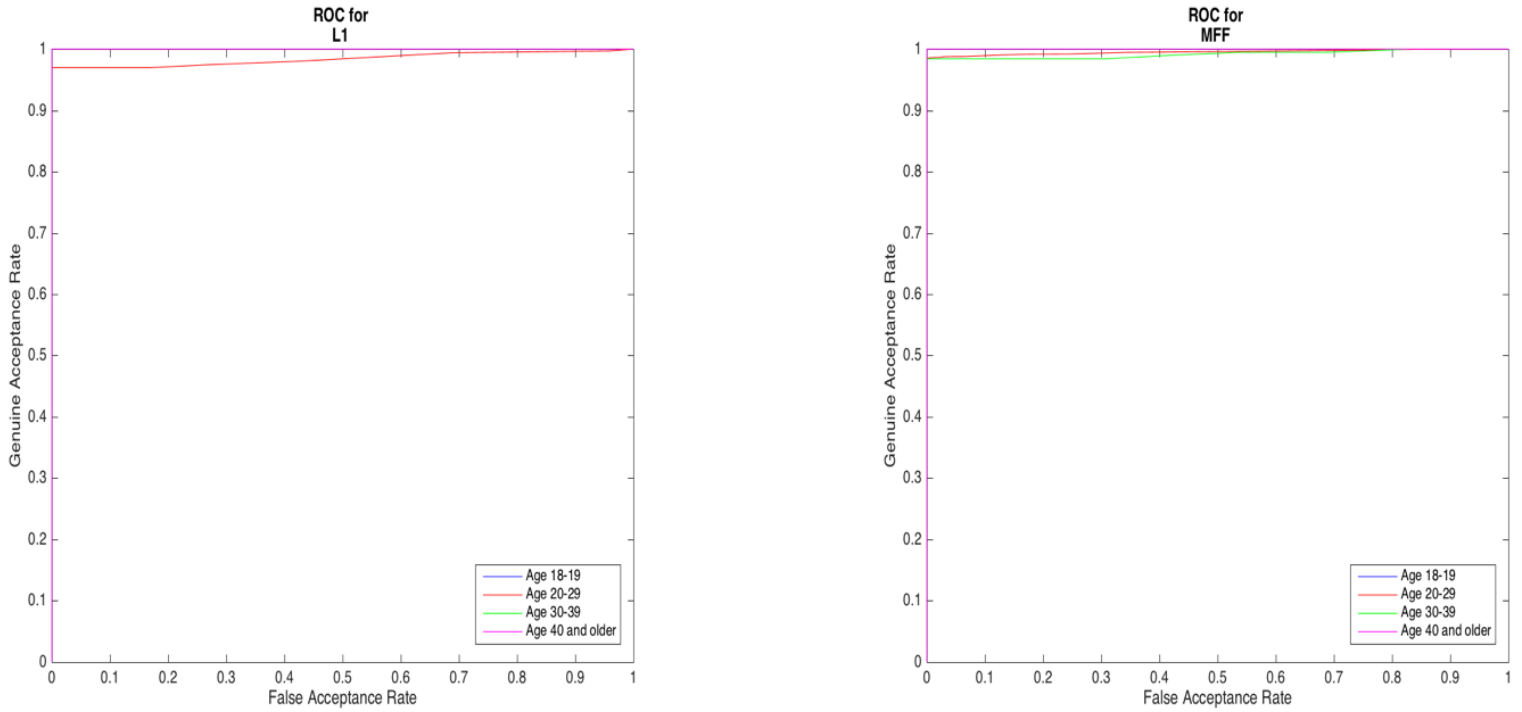

Figure C.5.5 Neurotech L1 Age Demographic ROC Curves (Left) Neurotech Morpho FOTF Age Demographic ROC Curves (Right)

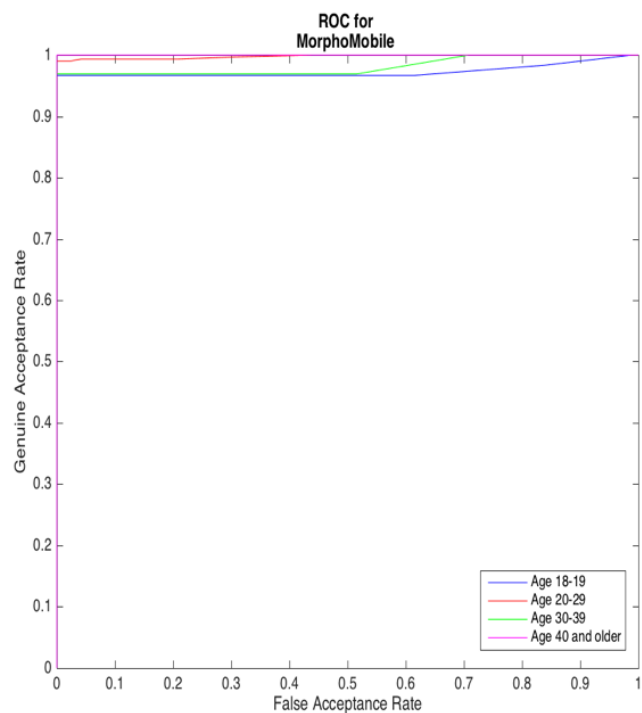

Figure C.5.6 Neurotech MorphoMobile Age Demographic ROC Curves 


\section{C.6 SourceAFIS ROC Curves and Area Under Curve}

Table C.21 SourceAFIS 18-19 Area under Curve

\begin{tabular}{|l|l|}
\hline SourceAFIS 18-19 & \\
\hline Device & Area Under Curve \\
\hline ANDI BIN & 0.964563 \\
\hline ANDI GRY & 0.920342 \\
\hline BioSled & 0.98982 \\
\hline BioSled Rolls & 0.980301 \\
\hline CG2 & 0.974147 \\
\hline CG2 Rolls & 0.991968 \\
\hline CSA & 0.999921 \\
\hline CSA Rolls & 0.957505 \\
\hline InnerID & 0.844872 \\
\hline L1 & 0.999979 \\
\hline L1 Rolls & 0.983741 \\
\hline MFF & 0.986496 \\
\hline MorphoMobile & 0.983584 \\
\hline
\end{tabular}

Table C.22 SourceAFIS 20-29 Area under Curve

\begin{tabular}{|l|l|}
\hline SourceAFIS 20-29 & \\
\hline Device & Area Under Curve \\
\hline ANDI BIN & 0.983914 \\
\hline ANDI GRY & 0.953698 \\
\hline BioSled & 0.969801 \\
\hline BioSled Rolls & 0.952522 \\
\hline CG2 & 0.981794 \\
\hline CG2 Rolls & 0.990839 \\
\hline CSA & 0.999998 \\
\hline CSA Rolls & 0.984035 \\
\hline InnerID & 0.783963 \\
\hline L1 & 0.980878 \\
\hline L1 Rolls & 0.973318 \\
\hline MFF & 0.987444 \\
\hline MorphoMobile & 0.993096 \\
\hline
\end{tabular}


Table C.23 SourceAFIS 30-39 Area under Curve

\begin{tabular}{|l|l|}
\hline SourceAFIS 30-39 & \\
\hline Device & Area Under Curve \\
\hline ANDI BIN & 0.960774 \\
\hline ANDI GRY & 0.892799 \\
\hline BioSled & 0.990629 \\
\hline BioSled Rolls & 0.988284 \\
\hline CG2 & 0.985936 \\
\hline CG2 Rolls & 0.986558 \\
\hline CSA & 0.980732 \\
\hline CSA Rolls & 1 \\
\hline InnerID & 0.5375 \\
\hline L1 & 1 \\
\hline L1 Rolls & 0.987974 \\
\hline MFF & 0.980062 \\
\hline MorphoMobile & 0.980289 \\
\hline
\end{tabular}

Table C.24 SourceAFIS 40 and up Area under Curve

\begin{tabular}{|l|l|}
\hline SourceAFIS 40 and up & \\
\hline Device & Area Under Curve \\
\hline ANDI BIN & 0.93168 \\
\hline ANDI GRY & 0.858469 \\
\hline BioSled & 1 \\
\hline BioSled Rolls & 0.857277 \\
\hline CG2 & 0.951094 \\
\hline CG2 Rolls & 0.883476 \\
\hline CSA & 1 \\
\hline CSA Rolls & 0.974026 \\
\hline InnerID & 0.631944 \\
\hline L1 & 1 \\
\hline L1 Rolls & 0.931319 \\
\hline MFF & 0.92585 \\
\hline MorphoMobile & 0.973707 \\
\hline
\end{tabular}



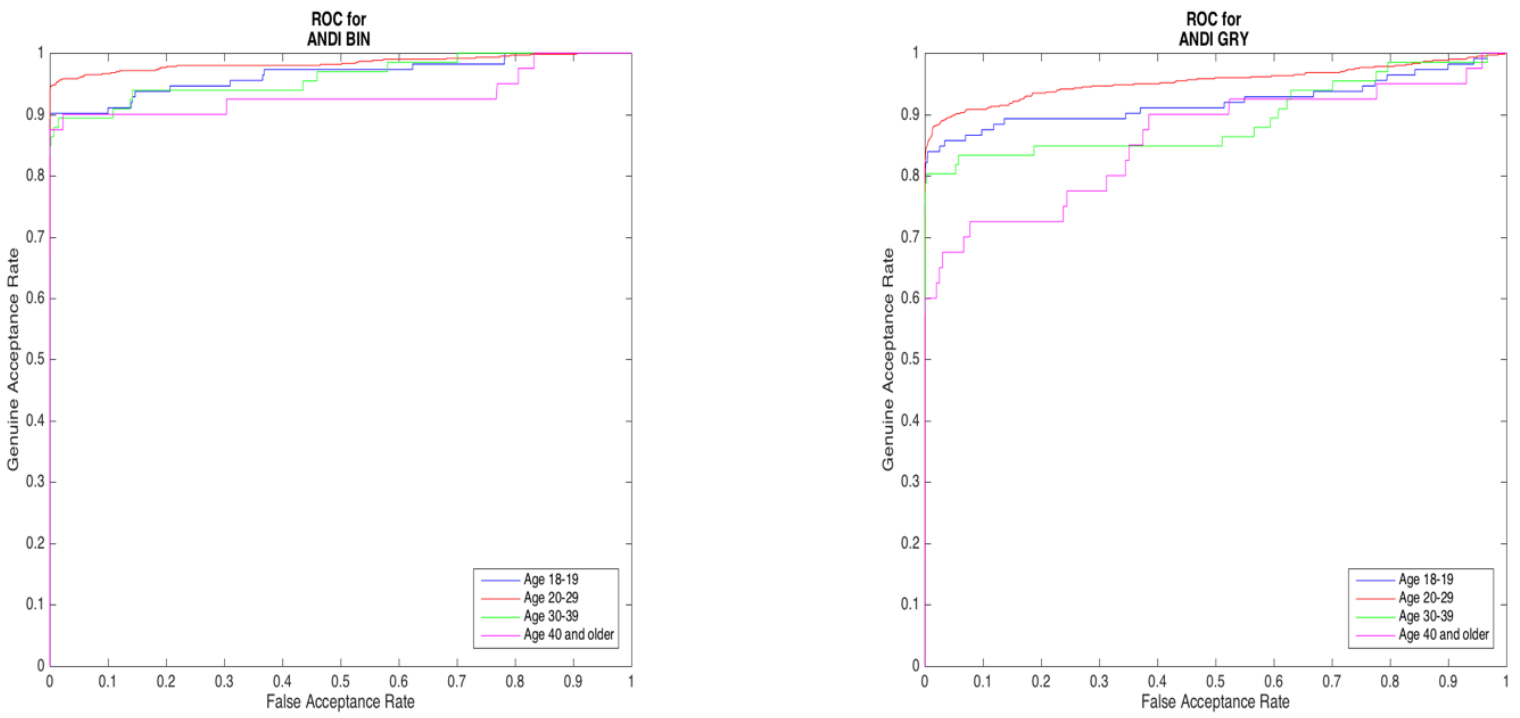

Figure C.6.1 SourceAFIS ANDI Binary Age Demographic ROC Curves (Left) SourceAFIS ANDI Greyscale Age Demographic ROC Curves (Right)
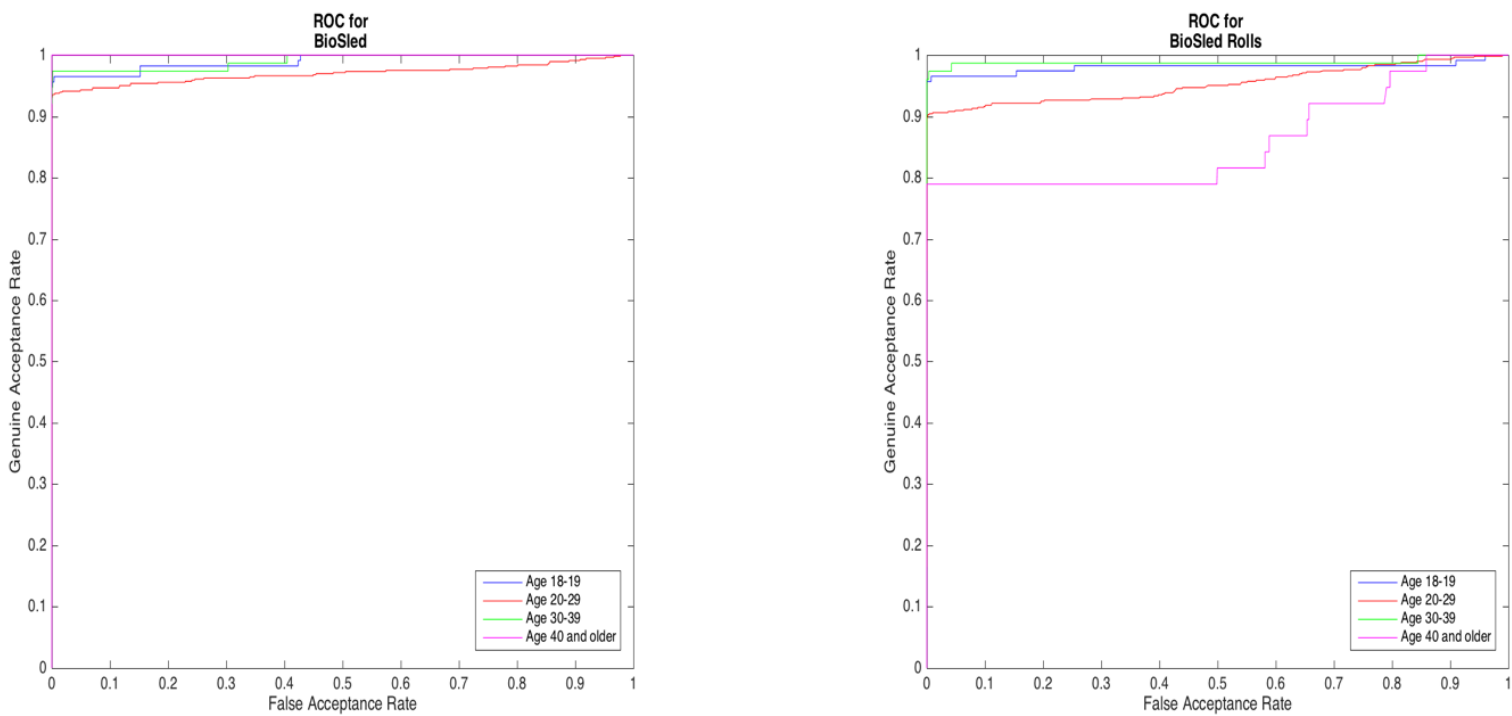

Figure C.6.2 SourceAFIS BioSled Age Demographic ROC Curves (Left) SourceAFIS BioSled Rolls Age Demographic ROC Curves (Right) 

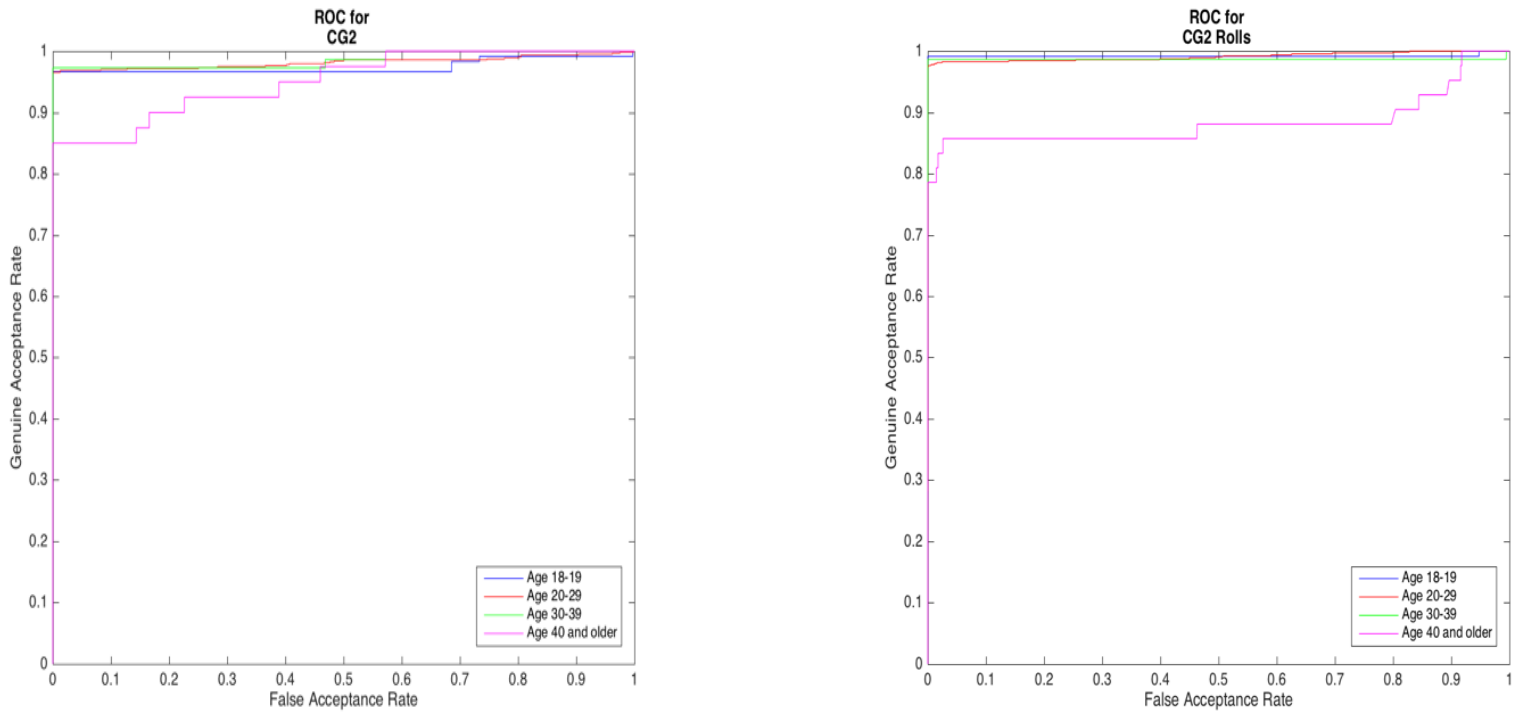

Figure C.6.3 SourceAFIS FP II Guardian Age Demographic ROC Curves (Left) SourceAFIS FP II Guardian Rolls Age Demographic ROC Curves (Right)
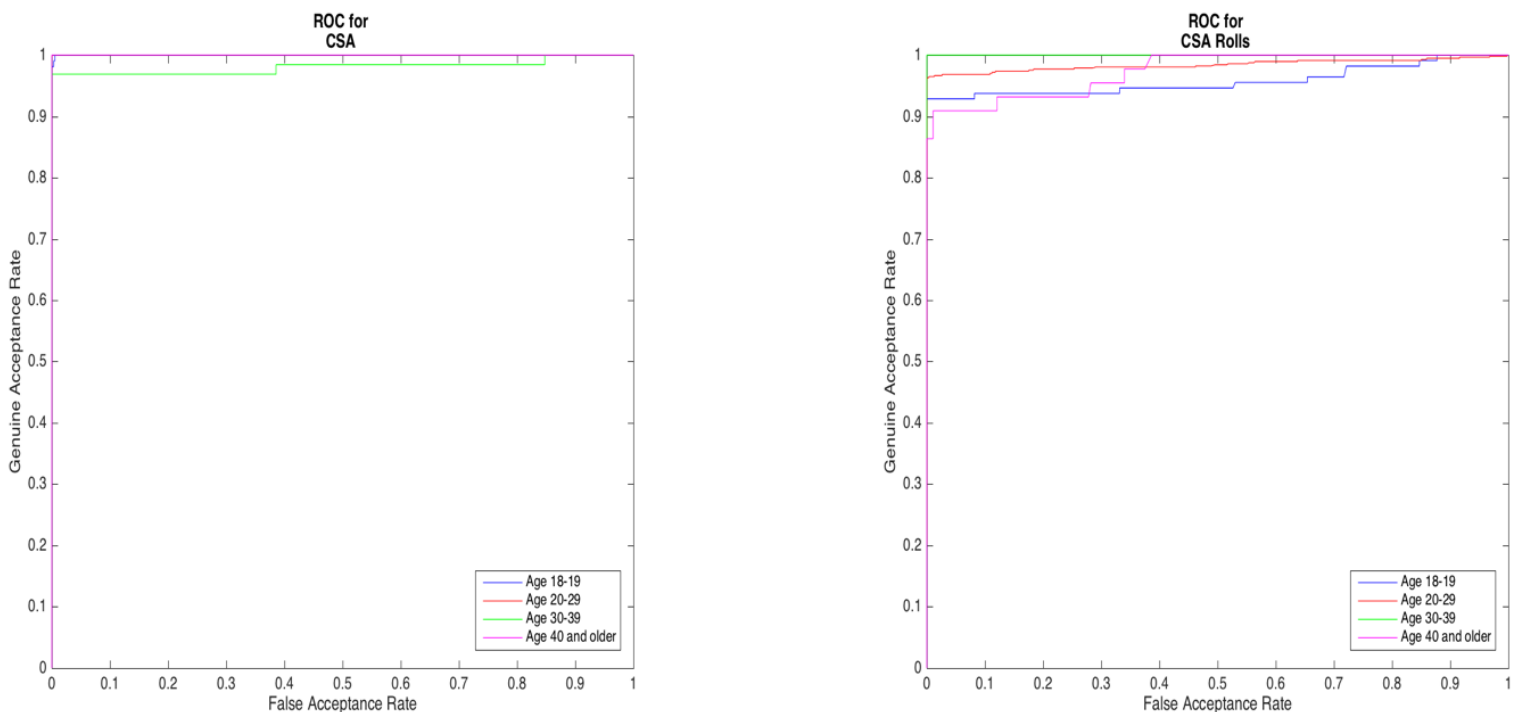

Figure C.6.4 SourceAFIS Crossmatch Seek Avenger Age Demographic ROC Curves (Left) SourceAFIS Crossmatch Seek Avenger Age Demographic ROC Curves (Right) 

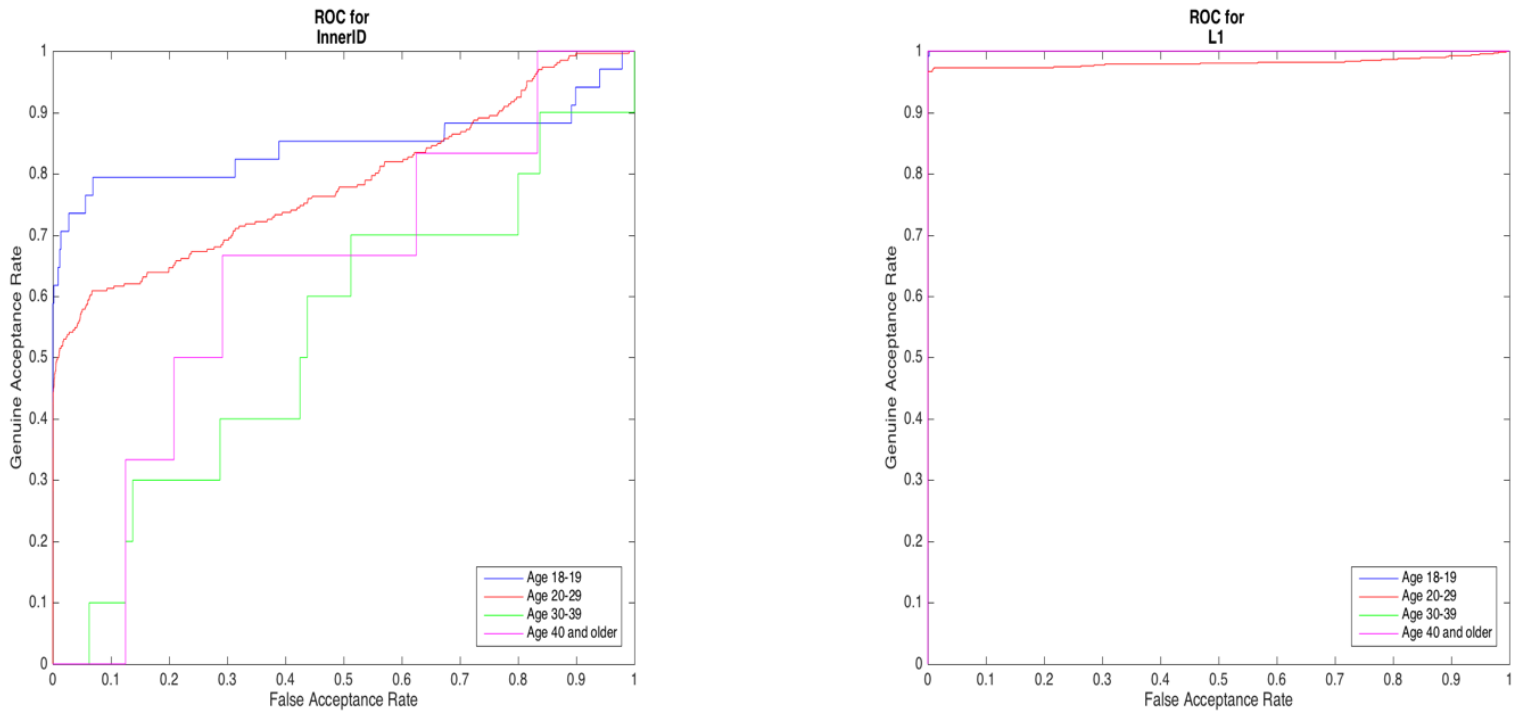

Figure C.6.5 SourceAFIS InnerID Age Demographic ROC Curves (Left) SourceAFIS L1 Age Demographic ROC Curves (Right)
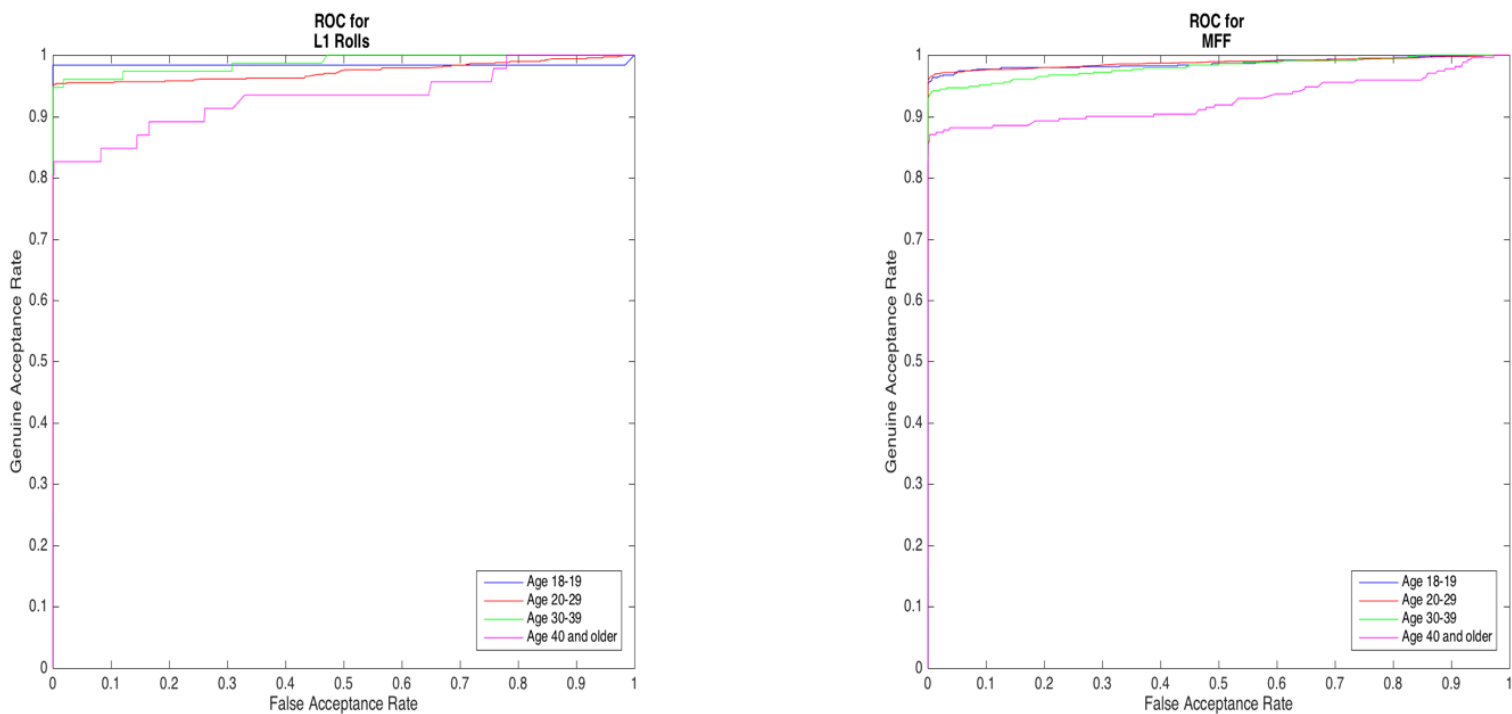

Figure C.6.6 SourceAFIS L1 Rolls Age Demographic ROC Curves (Left) SourceAFIS Morpho FOTF Age Demographic ROC Curves (Right) 


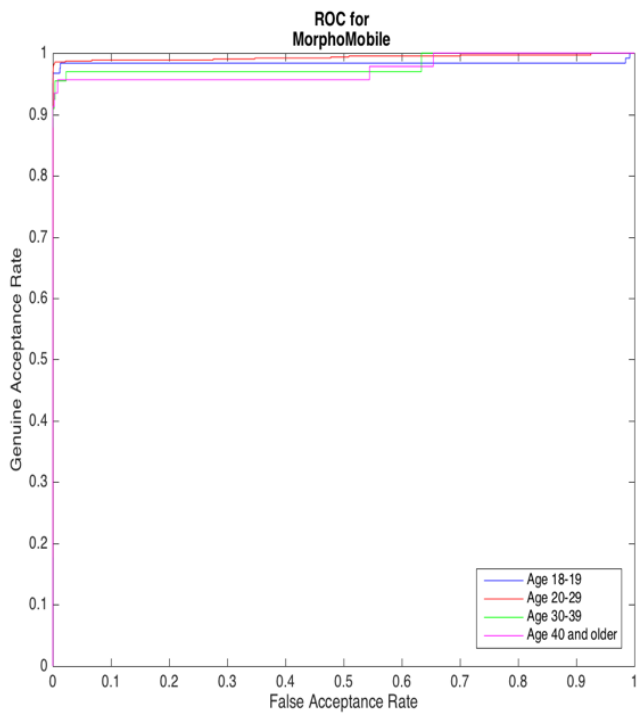

Figure C.6.7 SourceAFIS MorphoMobile Age Demographic ROC Curves 


\section{C.7 Minutiae Box Plots by Age Range and Device}
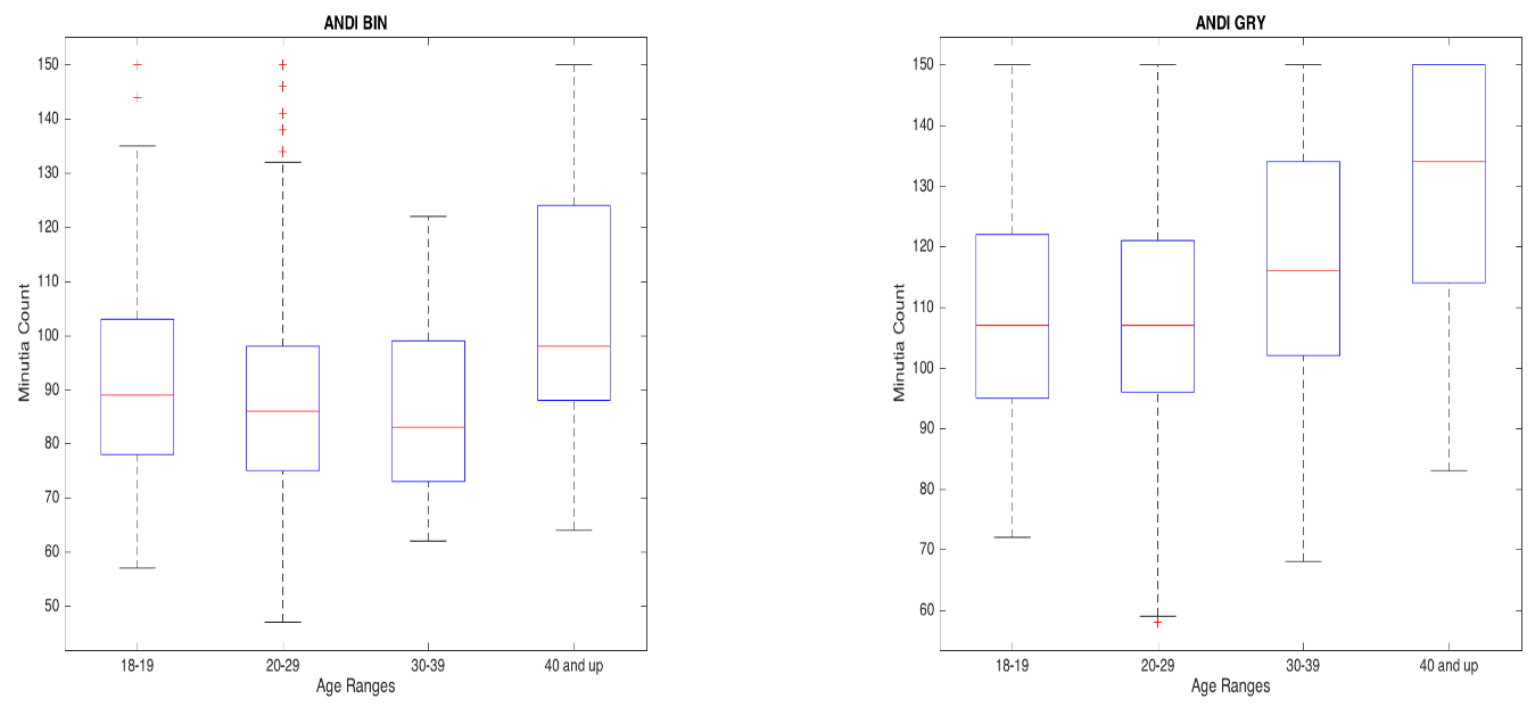

Figure C.7.1 (Left)AND Binary Box Plot (Right) ANDI Greyscale Box Plot
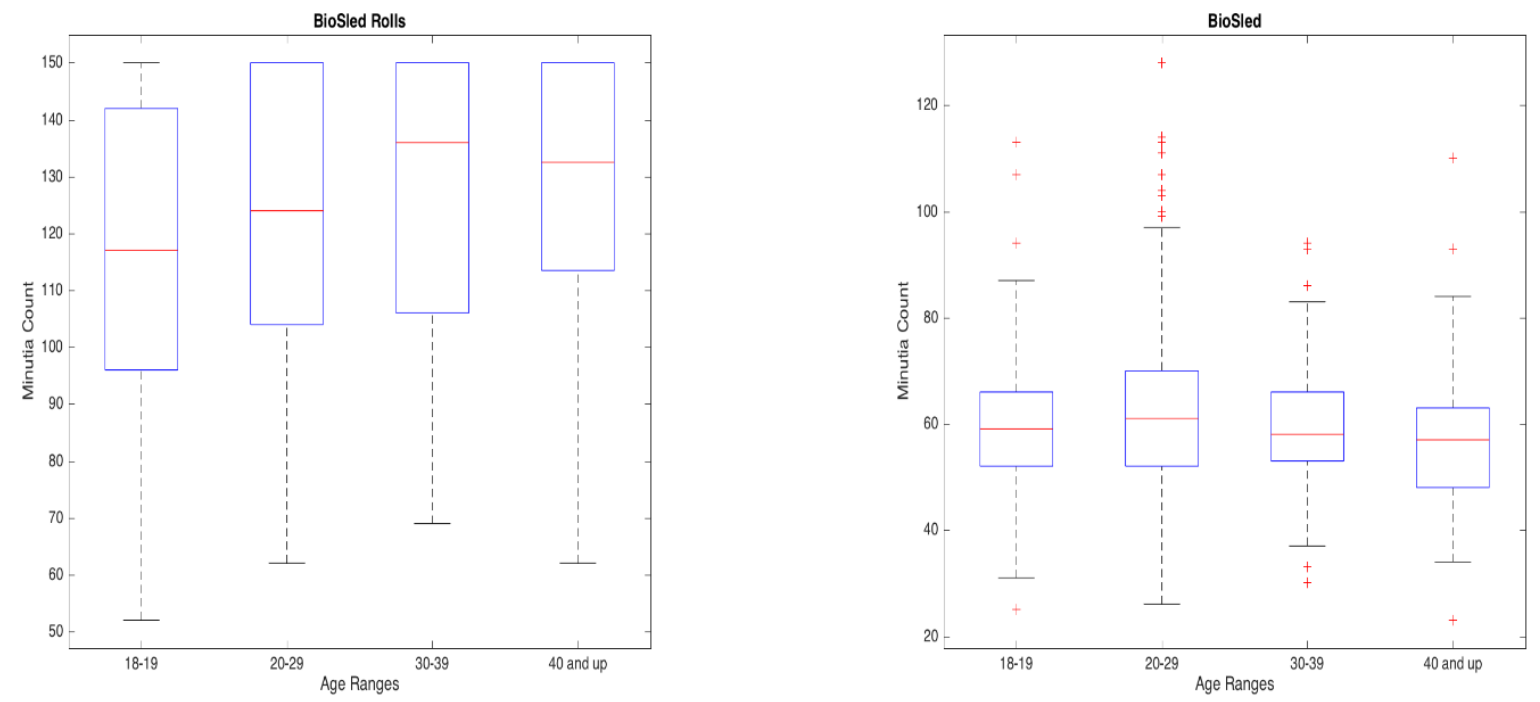

Figure C.7.2 (Left) BioSled Rolls Box Plot (Right) BioSled Box Plot 

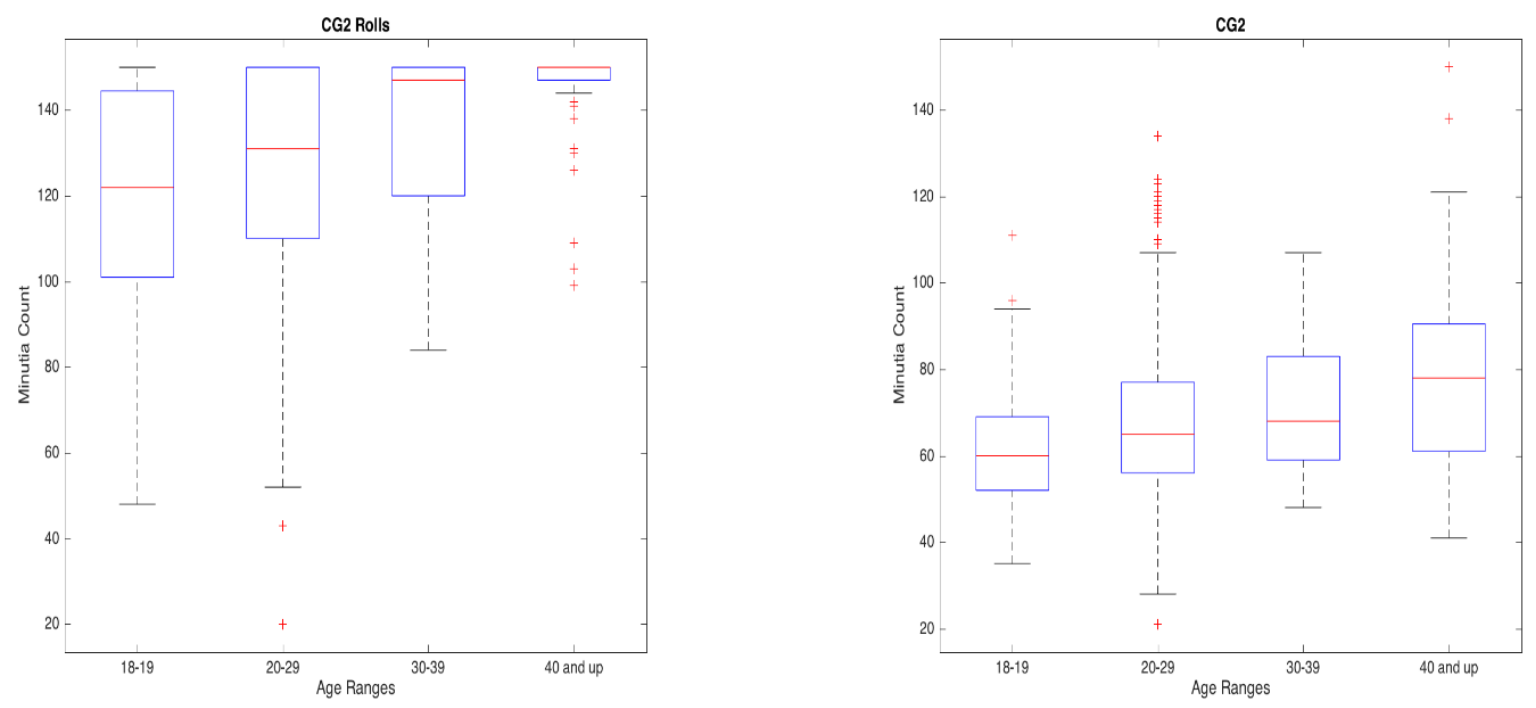

Figure C.7.3 (Left)FP II Guardian Rolls Box Plot (Right) FP II Guardian Box Plot
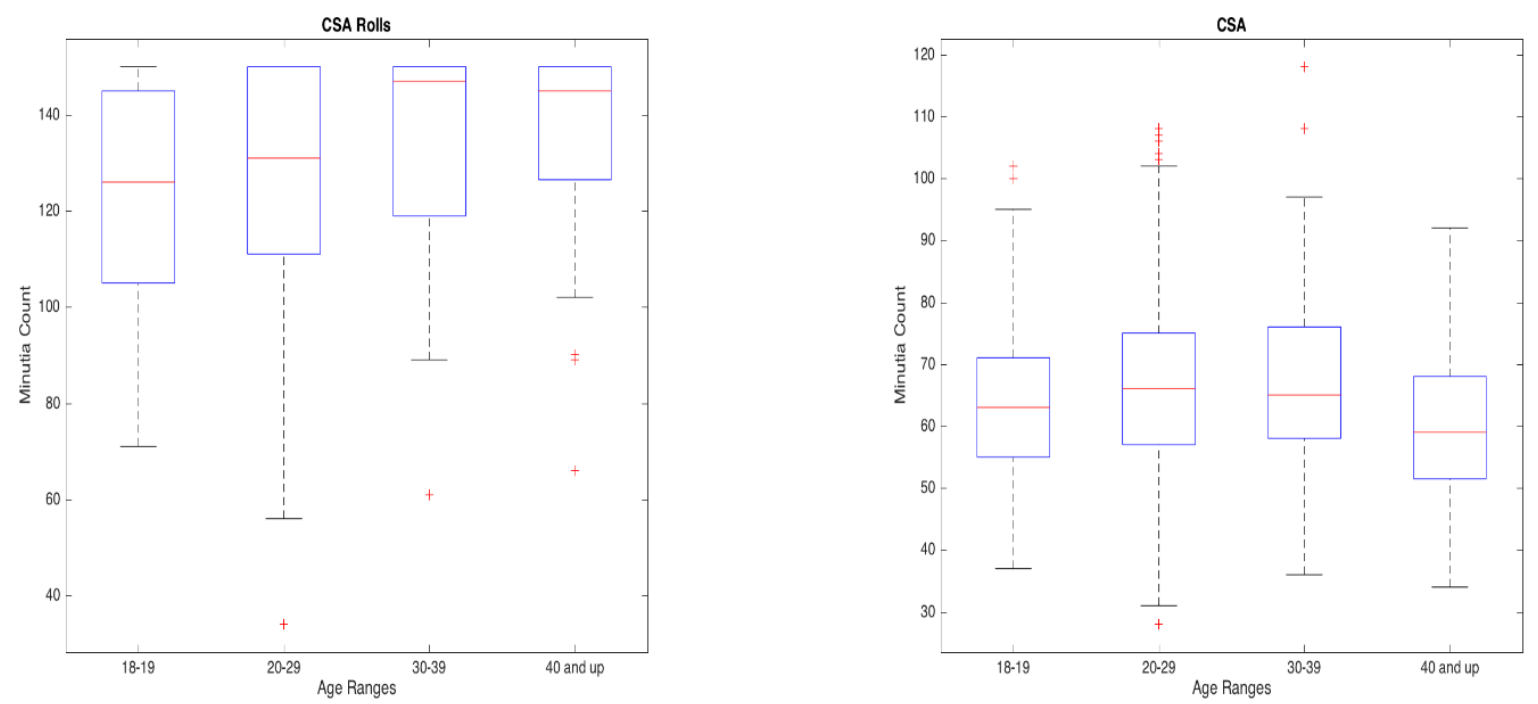

Figure C.7.4 (Left)Crossmatch Seek Avenger Rolls Box Plot (Right) Crossmatch Seek Avenger Box Plot 

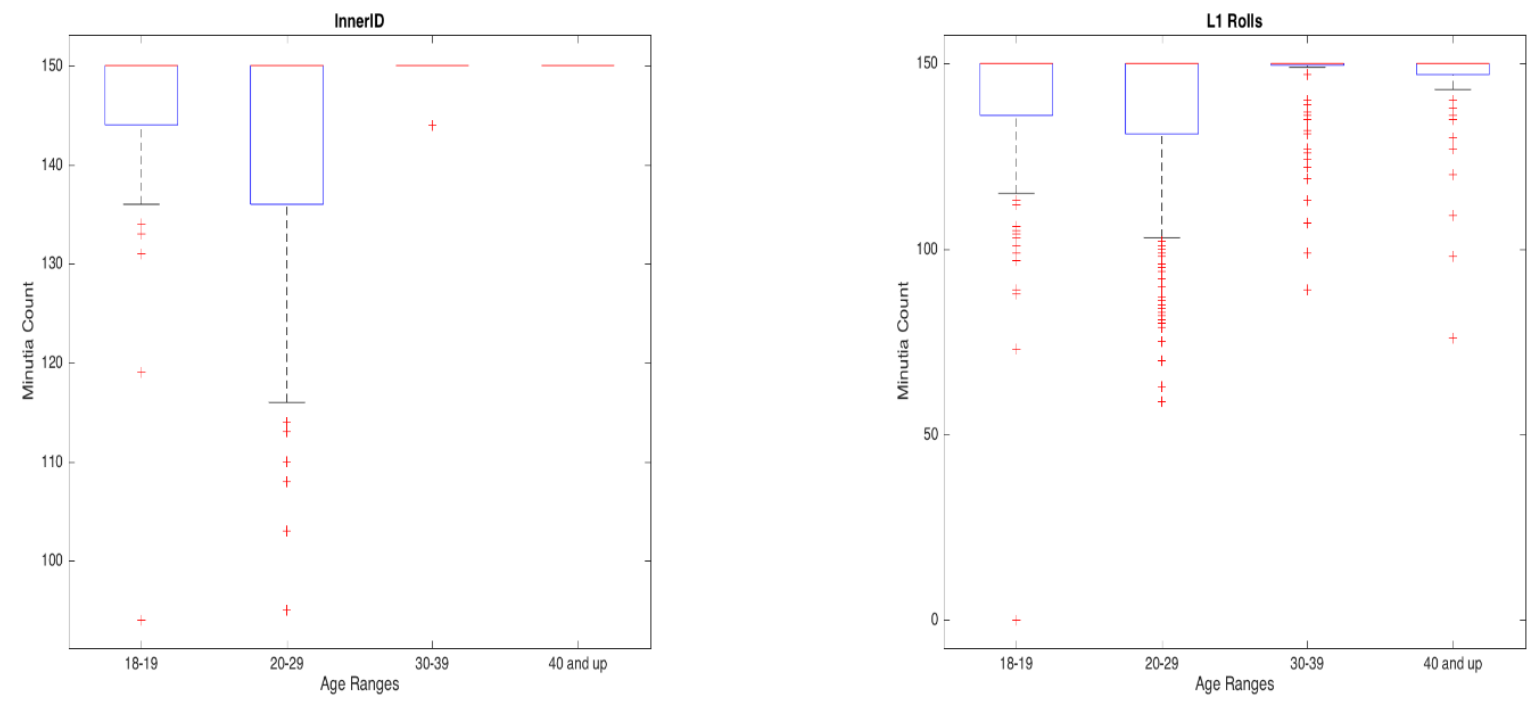

Figure C.7.5 (Left)InnerID Box Plot (Right) L1 Rolls Box Plot
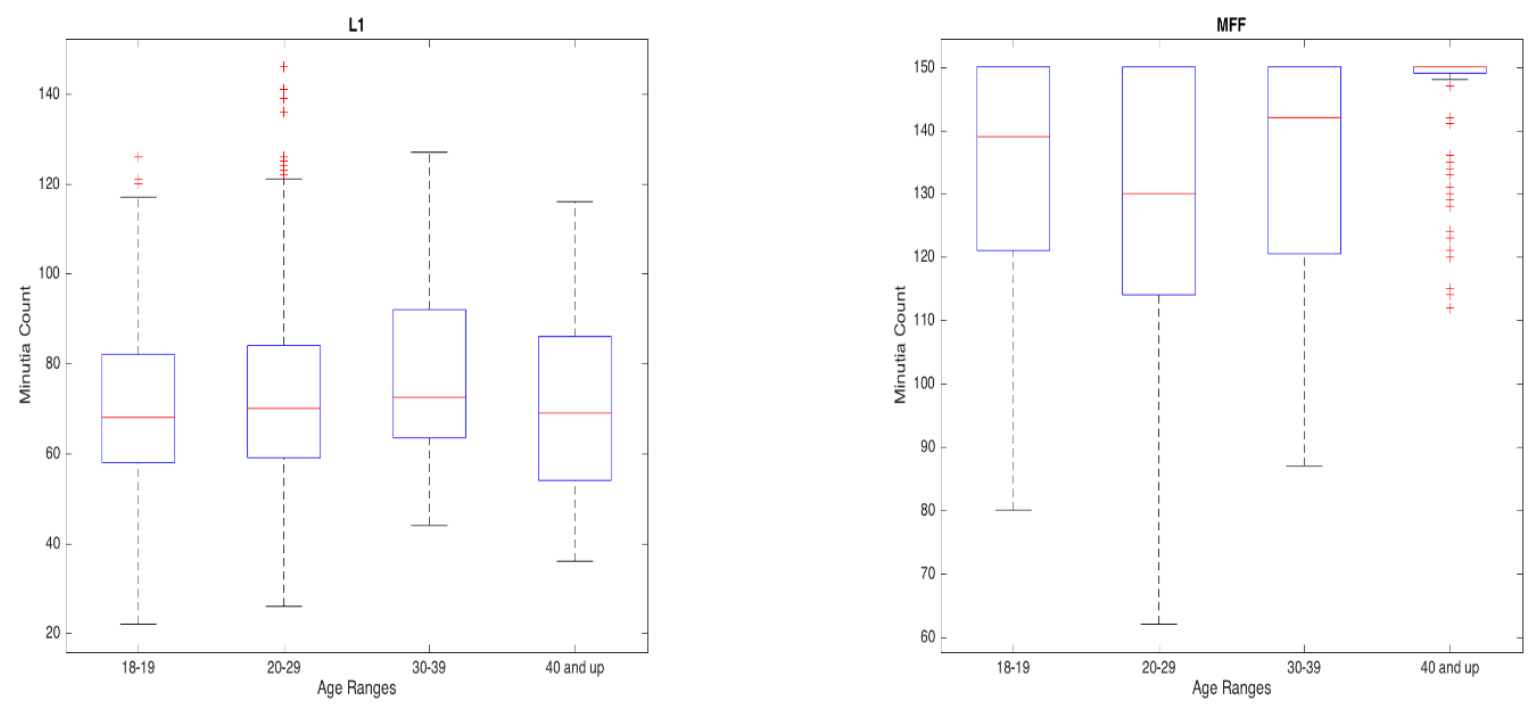

Figure C.7.6 (Left)L1 Box Plot (Right) Morpho FOTF Box Plot 


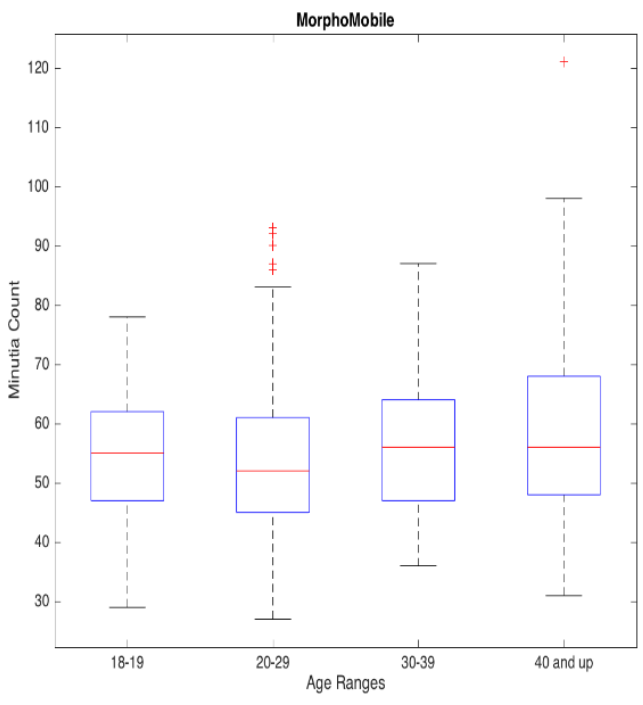

Figure C.7.7 MorphoMobile Box Plots

\section{C.8 Equal Error Rates for Age Based Study}

Table C.25 NBIS Equal Error Rates For Age Based Study

\begin{tabular}{|r|ll|ll|}
\hline NBIS & \multicolumn{4}{|c|}{ EER Value } \\
Device & $\mathbf{1 8 - 1 9}$ & $\mathbf{2 0 - 2 9}$ & $\mathbf{3 0 - 3 9}$ & $\mathbf{4 0}$ and up \\
ANDI BIN & 0.0357 & 0.0334 & 0.0909 & 0.0794 \\
ANDI GRY & 0.0357 & 0.0533 & 0.0909 & 0.1000 \\
BioSled & 0.0306 & 0.0602 & 0.0263 & 0.0137 \\
BioSled Rolls & 0.0014 & 0.0508 & 0.0000 & 0.0526 \\
CG2 & 0.0333 & 0.0313 & 0.0270 & 0.1000 \\
CG2 Rolls & 0.0000 & 0.0157 & 0.0000 & 0.0476 \\
CSA & 0.0086 & 0.0055 & 0.0313 & 0.0016 \\
CSA Rolls & 0.0357 & 0.0175 & 0.0000 & 0.0971 \\
InnerID & 0.2028 & 0.2148 & 0.5000 & 0.3333 \\
L1 & 0.0164 & 0.0302 & 0.0000 & 0.0198 \\
L1 Rolls & 0.0251 & 0.0329 & 0.0263 & 0.0435 \\
MFF & 0.0027 & 0.0159 & 0.0278 & 0.0131 \\
MorphoMobile & 0.0328 & 0.0246 & 0.0590 & 0.0435 \\
\hline
\end{tabular}


Table C.26 Neurotech Equal Error Rates For Age Based Study

\begin{tabular}{|r|ll|ll|}
\hline Neurotech & \multicolumn{4}{|c|}{ EER Value } \\
\hline Device & $\mathbf{1 8 - 1 9}$ & $\mathbf{2 0 - 2 9}$ & $\mathbf{3 0 - 3 9}$ & $\mathbf{4 0}$ and up \\
ANDI BIN & 0.0360 & 0.0235 & 0.0923 & 0.0000 \\
ANDI GRY & 0.0367 & 0.0278 & 0.0678 & 0.0000 \\
BioSled & 0.0000 & 0.0396 & 0.0267 & 0.0000 \\
BioSled Rolls & 0.0000 & 0.0246 & 0.0000 & 0.0571 \\
CG2 & 0.0336 & 0.0282 & 0.0000 & 0.1053 \\
CG2 Rolls & 0.0000 & 0.0095 & 0.0000 & 0.0000 \\
CSA & 0.0000 & 0.0000 & 0.0317 & 0.0000 \\
CSA Rolls & 0.0000 & 0.0106 & 0.0000 & 0.0000 \\
L1 & 0.0000 & 0.0303 & 0.0000 & 0.0000 \\
MFF & 0.0000 & 0.0136 & 0.0159 & 0.0000 \\
MorphoMobile & 0.0331 & 0.0097 & 0.0308 & 0.0000 \\
\hline
\end{tabular}

Table C.27 SourceAFIS Equal Error Rates For Age Based Study

\begin{tabular}{|r|ll|ll|}
\hline SourceAFIS & \multicolumn{4}{|c|}{ EER Value } \\
\hline Device & $\mathbf{1 8 - 1 9}$ & $\mathbf{2 0 - 2 9}$ & $\mathbf{3 0 - 3 9}$ & $\mathbf{4 0}$ and up \\
ANDI BIN & 0.0982 & 0.0419 & 0.1061 & 0.1000 \\
ANDI GRY & 0.1184 & 0.0920 & 0.1667 & 0.2444 \\
BioSled & 0.0351 & 0.0569 & 0.0263 & 0.0000 \\
BioSled Rolls & 0.0345 & 0.0870 & 0.0263 & 0.2105 \\
CG2 & 0.0333 & 0.0313 & $\mathrm{~N} / \mathrm{A}$ & 0.1434 \\
CG2 Rolls & 0.0085 & 0.0188 & $\mathrm{~N} / \mathrm{A}$ & 0.1429 \\
CSA & 0.0052 & 0.0007 & 0.0313 & 0.0000 \\
CSA Rolls & 0.0714 & 0.0316 & 0.0000 & 0.0909 \\
InnerID & 0.2059 & 0.3045 & 0.4375 & 0.3333 \\
L1 & 0.0024 & 0.0272 & 0.0000 & 0.0000 \\
L1 Rolls & 0.0164 & 0.0449 & 0.0395 & 0.1444 \\
MFF & 0.0325 & 0.0283 & 0.0532 & 0.1148 \\
MorphoMobile & 0.0164 & 0.0146 & 0.0303 & N/A \\
\hline
\end{tabular}


Appendix D

Ethnicity Based

Study 
This section is made up of the results derived from dividing the data into categories by ethnicity. The results that can be seen in this section include Genuine/Imposter Distributions for each device, Kullback Leibler Divergence measurements, minimum and maximum scores for each matching run, ROC Curves, and Area under the curve measurements. Each of these results were produced with each of the three matchers. The ethnicity categories of "Other Pacific Islander" and "Unknown" were too small to produce any distributions or any other results analysis. At the end of this appendix there are Minutiae Box Plots that are categorized by device, this data was obtained from the NBIS matching algorithm.

\section{D.1 NBIS Genuine/Imposter Score Distributions}

Table D.1 NBIS African Genuine and Imposter KLD, Maximum and Minimum Scores

\begin{tabular}{|c|c|c|c|c|c|c|}
\hline \multicolumn{7}{|l|}{ NBIS African } \\
\hline Device & $\begin{array}{l}\text { Genuine } \\
\text { KLD }\end{array}$ & $\begin{array}{l}\text { Imposter } \\
\text { KLD }\end{array}$ & $\begin{array}{l}\text { Max } \\
\text { Genuine }\end{array}$ & $\begin{array}{l}\text { Min } \\
\text { Genuine }\end{array}$ & $\begin{array}{l}\text { Max } \\
\text { Imposter }\end{array}$ & $\begin{array}{l}\text { Min } \\
\text { Imposter }\end{array}$ \\
\hline$A N D I B I N$ & 0.0783 & 3.7148 & 214 & 61 & 11 & 3 \\
\hline$A N D I G R Y$ & 0.0510 & 1.7698 & 240 & 40 & 22 & 4 \\
\hline Biosled & 1.1091 & 1.3129 & 209 & 6 & 30 & 4 \\
\hline BioSled Rolls & 0.0779 & 1.8666 & 208 & 80 & 13 & 4 \\
\hline CG2 & 0.2033 & 1.9118 & 202 & 92 & 16 & 5 \\
\hline CG2 Rolls & 0.0749 & 1.6157 & 240 & 142 & 13 & 3 \\
\hline CSA & 0.0744 & 1.1948 & 194 & 84 & 35 & 4 \\
\hline CSA Rolls & 0.1097 & 4.4832 & 187 & 83 & 11 & 3 \\
\hline$L 1$ & 0.1288 & 2.8118 & 227 & 49 & 18 & 3 \\
\hline L1 Rolls & 0.1106 & 3.7102 & 341 & 92 & 13 & 3 \\
\hline MFF & 0.1435 & 2.3449 & 143 & 6 & 14 & 4 \\
\hline$S M M$ & 0.0581 & 0.4241 & 178 & 37 & 13 & 4 \\
\hline
\end{tabular}


Table D.2 NBIS African American Genuine and Imposter KLD, Maximum and Minimum Scores

\begin{tabular}{|r|r|r|r|r|r|r|}
\hline \multicolumn{1}{|c|}{ NBIS African American } \\
\hline Device & Genuine KLD & Imposter KLD & $\begin{array}{l}\text { Max } \\
\text { Genuine }\end{array}$ & $\begin{array}{l}\text { Min } \\
\text { Genuine }\end{array}$ & $\begin{array}{l}\text { Max } \\
\text { Imposter }\end{array}$ & $\begin{array}{l}\text { Min } \\
\text { Imposter }\end{array}$ \\
\cline { 2 - 8 } ANDI BIN & 0.0958 & 2.1134 & 319 & 29 & 21 & 3 \\
ANDI GRY & 0.0829 & 0.8404 & 278 & 15 & 37 & 4 \\
\cline { 2 - 8 } BioSled & 0.0744 & 1.6203 & 223 & 7 & 37 & 3 \\
BioSled Rolls & 0.0757 & 1.9739 & 199 & 30 & 18 & 3 \\
\cline { 2 - 8 } CG2 & 0.1037 & 1.4777 & 260 & 4 & 34 & 3 \\
\cline { 2 - 8 } CG2 Rolls & 0.0621 & 0.9826 & 276 & 68 & 17 & 3 \\
\cline { 2 - 8 } CSA & 0.1006 & 1.3257 & 183 & 30 & 36 & 3 \\
\cline { 2 - 8 } CSA Rolls & 0.0769 & 3.1626 & 236 & 45 & 20 & 3 \\
\cline { 2 - 8 } L1 & 0.1638 & 3.5862 & 239 & 29 & 28 & 0 \\
\cline { 2 - 8 } L1 Rolls & 0.1393 & 2.2078 & 234 & 31 & 19 & 3 \\
MFF & 0.0915 & 1.0985 & 216 & 12 & 36 & 3 \\
\cline { 2 - 8 } SMM & 0.1340 & 0.5541 & 139 & 40 & 22 & 0 \\
\hline
\end{tabular}

Table D.3 NBIS American Indian Genuine and Imposter KLD, Maximum and Minimum Scores

\begin{tabular}{|c|c|c|c|c|c|c|}
\hline \multicolumn{7}{|c|}{ NBIS American Indian } \\
\hline Device & Genuine KLD & Imposter KLD & $\begin{array}{l}\text { Max } \\
\text { Genuine }\end{array}$ & $\begin{array}{l}\text { Min } \\
\text { Genuine }\end{array}$ & $\begin{array}{l}\text { Max } \\
\text { Imposter }\end{array}$ & $\begin{array}{l}\text { Min } \\
\text { Imposter }\end{array}$ \\
\hline ANDI BIN & 0.1323 & 3.4101 & 148 & 60 & 9 & 4 \\
\hline$A N D I G R Y$ & 0.4058 & 2.3173 & 179 & 84 & 19 & 7 \\
\hline Biosled & 0.2299 & 1.6737 & 235 & 200 & 17 & 8 \\
\hline BioSled Rolls & 0.1608 & 1.8898 & 225 & 52 & 12 & 8 \\
\hline CG2 & 0.1522 & 1.6136 & 177 & 110 & 17 & 3 \\
\hline CG2 Rolls & 16.2262 & 1.5360 & 239 & 4 & 15 & 3 \\
\hline CSA & 0.6285 & 3.3994 & 185 & 185 & 12 & 6 \\
\hline CSA Rolls & 0.4880 & 7.7471 & 83 & 80 & 10 & 7 \\
\hline$L 1$ & 0.0855 & 2.4008 & 154 & 98 & 13 & 5 \\
\hline L1 Rolls & 1.0198 & 3.2321 & 156 & 64 & 18 & 3 \\
\hline MFF & 0.1508 & 2.7805 & 131 & 39 & 19 & 8 \\
\hline$S M M$ & 0.7334 & 0.5920 & & & & \\
\hline
\end{tabular}


Table D.4 NBIS Asian Genuine and Imposter KLD, Maximum and Minimum Scores

\begin{tabular}{|c|c|c|c|c|c|c|}
\hline \multicolumn{7}{|l|}{ NBIS Asian } \\
\hline Device & Genuine KLD & Imposter KLD & $\begin{array}{l}\text { Max } \\
\text { Genuine }\end{array}$ & $\begin{array}{l}\text { Min } \\
\text { Genuine }\end{array}$ & $\begin{array}{l}\text { Max } \\
\text { Imposter }\end{array}$ & $\begin{array}{l}\text { Min } \\
\text { Imposter }\end{array}$ \\
\hline$A N D I B I N$ & 0.0840 & 0.9661 & 279 & 6 & 37 & 3 \\
\hline$A N D I G R Y$ & 0.1544 & 0.7247 & 219 & 12 & 41 & 3 \\
\hline Biosled & 0.0697 & 2.2829 & 210 & 9 & 34 & 0 \\
\hline BioSled Rolls & 0.0348 & 1.6884 & 294 & 11 & 27 & 3 \\
\hline CG2 & 0.0359 & 3.6145 & 335 & 6 & 31 & 0 \\
\hline CG2 Rolls & 0.0609 & 1.0781 & 452 & 5 & 25 & 3 \\
\hline CSA & 0.1235 & 0.7760 & 248 & 33 & 38 & 3 \\
\hline CSA Rolls & 0.0646 & 3.4451 & 292 & 24 & 29 & 3 \\
\hline$L 1$ & 0.0797 & 4.1767 & 292 & 64 & 34 & 0 \\
\hline L1 Rolls & 0.0824 & 3.1502 & 271 & 65 & 20 & 3 \\
\hline MFF & 0.0782 & 1.1225 & 323 & 10 & 40 & 3 \\
\hline$S M M$ & 0.0992 & 0.8352 & 200 & 7 & 28 & 0 \\
\hline
\end{tabular}

Table D.5 NBIS Asian Indian Genuine and Imposter KLD, Maximum and Minimum Scores

\begin{tabular}{|c|c|c|c|c|c|c|}
\hline \multicolumn{7}{|c|}{ NBIS Asian Indian } \\
\hline Device & Genuine KLD & Imposter KLD & $\begin{array}{l}\text { Max } \\
\text { Genuine }\end{array}$ & $\begin{array}{l}\text { Min } \\
\text { Genuine }\end{array}$ & $\begin{array}{l}\text { Max } \\
\text { Imposter }\end{array}$ & $\begin{array}{l}\text { Min } \\
\text { Imposter }\end{array}$ \\
\hline$A N D I B I N$ & 0.1264 & 1.1123 & 226 & 14 & 27 & 3 \\
\hline$A N D I G R Y$ & 0.1199 & 0.6402 & 315 & 12 & 45 & 4 \\
\hline Biosled & 0.0495 & 1.6303 & 304 & 8 & 27 & 0 \\
\hline Biosled Rolls & 0.0640 & 1.1785 & 262 & 5 & 25 & 3 \\
\hline CG2 & 0.0854 & 3.7023 & 294 & 20 & 27 & 0 \\
\hline CG2 Rolls & 0.0377 & 0.7534 & 352 & 68 & 36 & 3 \\
\hline CSA & 0.1555 & 4.0159 & 271 & 26 & 30 & 0 \\
\hline CSA Rolls & 0.1786 & 4.2146 & 303 & 4 & 28 & 3 \\
\hline$\angle 1$ & 0.0133 & 4.0681 & 289 & 29 & 30 & 0 \\
\hline L1 Rolls & 0.0592 & 2.9283 & 340 & 47 & 23 & 3 \\
\hline$M F F$ & 0.1142 & 1.1213 & 316 & 5 & 37 & 3 \\
\hline$S M M$ & 0.1598 & 0.7430 & 220 & 6 & 28 & 0 \\
\hline
\end{tabular}


Table D.6 NBIS Caucasian Genuine and Imposter KLD, Maximum and Minimum Scores

\begin{tabular}{|c|c|c|c|c|c|c|}
\hline \multicolumn{7}{|c|}{ NBIS Caucasian } \\
\hline Device & Genuine KLD & Imposter KLD & $\begin{array}{l}\text { Max } \\
\text { Genuine }\end{array}$ & $\begin{array}{l}\text { Min } \\
\text { Genuine }\end{array}$ & $\begin{array}{l}\text { Max } \\
\text { Imposter }\end{array}$ & $\begin{array}{l}\text { Min } \\
\text { Imposter }\end{array}$ \\
\hline ANDI BIN & 0.0451 & 1.2735 & 265 & 4 & 41 & 0 \\
\hline ANDI GRY & 0.0177 & 0.2685 & 358 & 8 & 62 & 3 \\
\hline Biosled & 0.0126 & 0.0685 & 298 & 4 & 245 & 0 \\
\hline Biosled Rolls & 0.0147 & 0.4063 & 317 & 5 & 172 & 0 \\
\hline CG2 & 0.0188 & 0.5531 & 358 & 4 & 131 & 0 \\
\hline CG2 Rolls & 0.0203 & 1.6604 & 442 & 7 & 29 & 0 \\
\hline CSA & 0.0130 & 0.7696 & 499 & 23 & 54 & 0 \\
\hline CSA Rolls & 0.0114 & 10.4262 & 506 & 5 & 29 & 0 \\
\hline$L 1$ & 0.0191 & 0.1701 & 328 & 6 & 137 & 0 \\
\hline L1 Rolls & 0.0127 & 0.2256 & 449 & 0 & 143 & 0 \\
\hline MFF & 0.0133 & 2.6603 & 360 & 8 & 51 & 0 \\
\hline$S M M$ & 0.0220 & 1.7941 & 278 & 8 & 37 & 0 \\
\hline
\end{tabular}

Table D.7 NBIS Hispanic Genuine and Imposter KLD, Maximum and Minimum Scores

\begin{tabular}{|c|c|c|c|c|c|c|}
\hline \multicolumn{7}{|c|}{ NBIS Hispanic } \\
\hline Device & Genuine KLD & Imposter KLD & $\begin{array}{l}\text { Max } \\
\text { Genuine }\end{array}$ & $\begin{array}{l}\text { Min } \\
\text { Genuine }\end{array}$ & $\begin{array}{l}\text { Max } \\
\text { Imposter }\end{array}$ & $\begin{array}{l}\text { Min } \\
\text { Imposter }\end{array}$ \\
\hline$A N D I B I N$ & 0.1275 & 3.9449 & 230 & 7 & 29 & 0 \\
\hline ANDI GRY & 0.1196 & 0.5169 & 254 & 10 & 47 & 3 \\
\hline Biosled & 0.0451 & 1.8945 & 250 & 11 & 71 & 0 \\
\hline Biosled Rolls & 0.0863 & 0.2200 & 232 & 5 & 143 & 3 \\
\hline CG2 & 0.0451 & 1.5253 & 275 & 45 & 46 & 3 \\
\hline CG2 Rolls & 0.0720 & 2.0993 & 302 & 28 & 27 & 0 \\
\hline CSA & 0.1817 & 1.2669 & 223 & 17 & 61 & 0 \\
\hline CSA Rolls & 0.0300 & 3.1587 & 394 & 24 & 24 & 3 \\
\hline$\angle 1$ & 0.0292 & 3.6985 & 277 & 43 & 46 & 0 \\
\hline L1 Rolls & 0.0485 & 2.9663 & 329 & 49 & 27 & 3 \\
\hline$M F F$ & 0.1302 & 1.1028 & 245 & 19 & 36 & 3 \\
\hline$S M M$ & 0.0447 & 0.7097 & 233 & 24 & 31 & 0 \\
\hline
\end{tabular}


Table D.8 NBIS Middle Eastern Genuine and Imposter KLD, Maximum and Minimum Scores

\begin{tabular}{|c|c|c|c|c|c|c|}
\hline \multicolumn{7}{|c|}{ NBIS Middle Eastern } \\
\hline Device & Genuine KLD & Imposter KLD & $\begin{array}{l}\text { Max } \\
\text { Genuine }\end{array}$ & $\begin{array}{l}\text { Min } \\
\text { Genuine }\end{array}$ & $\begin{array}{l}\text { Max } \\
\text { Imposter }\end{array}$ & $\begin{array}{l}\text { Min } \\
\text { Imposter }\end{array}$ \\
\hline$A N D I B I N$ & 0.1897 & 0.9501 & 275 & 6 & 32 & 3 \\
\hline$A N D I G R Y$ & 0.1799 & 0.4859 & 282 & 10 & 49 & 4 \\
\hline Biosled & 0.0942 & 1.3721 & 238 & 10 & 35 & 0 \\
\hline BioSled Rolls & 0.0573 & 2.0614 & 289 & 25 & 19 & 3 \\
\hline CG2 & 0.0991 & 3.0393 & 275 & 8 & 45 & 0 \\
\hline CG2 Rolls & 0.0197 & 1.0196 & 379 & 13 & 20 & 3 \\
\hline CSA & 0.2012 & 3.0568 & 244 & 8 & 33 & 0 \\
\hline CSA Rolls & 0.1629 & 3.0872 & 265 & 5 & 19 & 3 \\
\hline$L 1$ & 0.0284 & 1.2655 & 285 & 33 & 33 & 3 \\
\hline L1 Rolls & 0.0442 & 2.7705 & 311 & 30 & 23 & 3 \\
\hline MFF & 0.1744 & 1.1906 & 262 & 9 & 42 & 3 \\
\hline$S M M$ & 0.1195 & 0.6415 & 177 & 12 & 25 & 0 \\
\hline
\end{tabular}

Table D.9 NBIS Other Genuine and Imposter KLD, Maximum and Minimum Scores

\begin{tabular}{|c|c|c|c|c|c|c|}
\hline \multicolumn{7}{|l|}{ NBIS Other } \\
\hline Device & Genuine KLD & Imposter KLD & $\begin{array}{l}\text { Max } \\
\text { Genuine }\end{array}$ & $\begin{array}{l}\text { Min } \\
\text { Genuine }\end{array}$ & $\begin{array}{l}\text { Max } \\
\text { Imposter }\end{array}$ & $\begin{array}{l}\text { Min } \\
\text { Imposter }\end{array}$ \\
\hline$A N D I B I N$ & 0.1411 & 1.1652 & 213 & 9 & 29 & 3 \\
\hline$A N D I G R Y$ & 0.1260 & 0.9902 & 213 & 21 & 37 & 3 \\
\hline Biosled & 0.0306 & 0.8462 & 286 & 7 & 36 & 3 \\
\hline BioSled Rolls & 0.0979 & 2.8849 & 265 & 11 & 23 & 3 \\
\hline CG2 & 0.0582 & 3.5313 & 303 & 25 & 33 & 0 \\
\hline CG2 Rolls & 0.1028 & 1.0031 & 313 & 16 & 23 & 3 \\
\hline CSA & 0.0718 & 0.5612 & 259 & 77 & 52 & 3 \\
\hline CSA Rolls & 0.0518 & 3.2520 & 435 & 45 & 26 & 3 \\
\hline$L 1$ & 0.0657 & 3.3504 & 299 & 4 & 29 & 0 \\
\hline L1 Rolls & 0.0783 & 3.0633 & 404 & 4 & 21 & 3 \\
\hline MFF & 0.0935 & 1.3848 & 283 & 28 & 33 & 3 \\
\hline$S M M$ & 0.0762 & 0.6609 & 261 & 3 & 26 & 0 \\
\hline
\end{tabular}




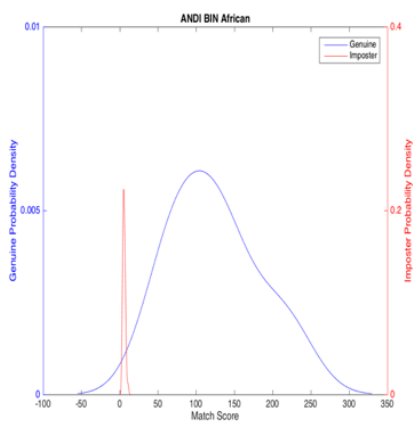

(a)

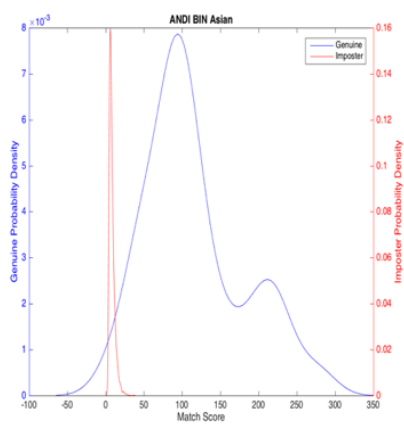

(d)

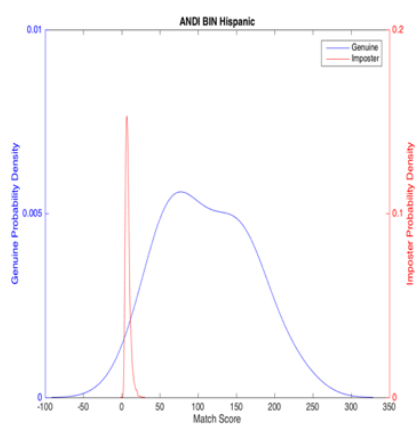

(g)

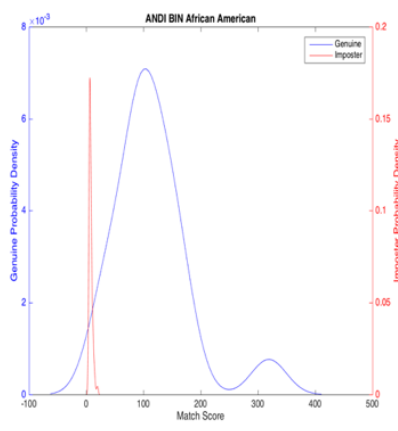

(b)

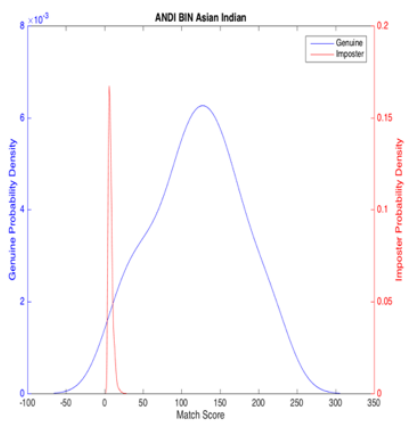

(e)

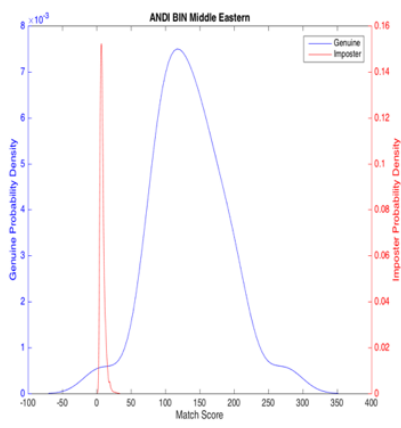

(h)

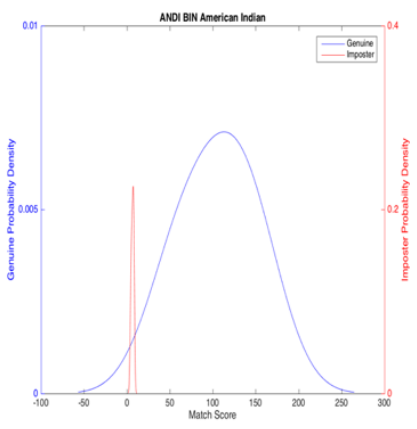

(c)

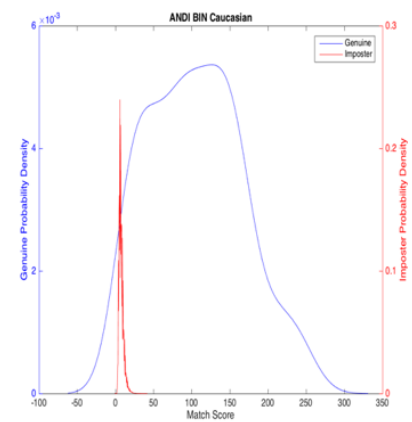

(f)

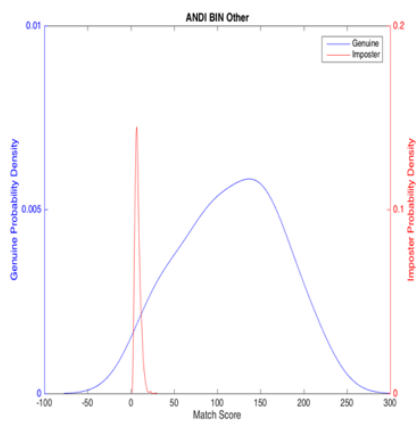

(i)

Figure D.1.1 NBIS ANDI Binary (a) African (b) African American (c) American Indian (d) Asian (e) Asian Indian (f) Caucasian (g) Hispanic (h) Middle Eastern (i) Other 


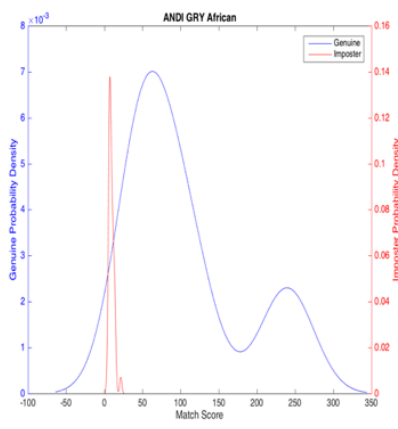

(a)

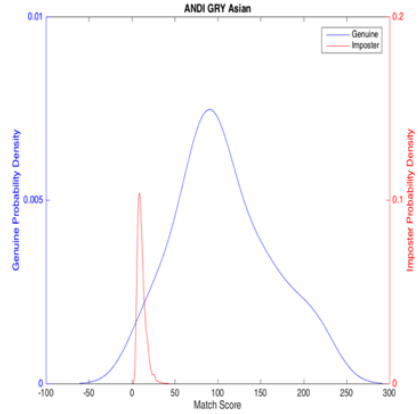

(d)

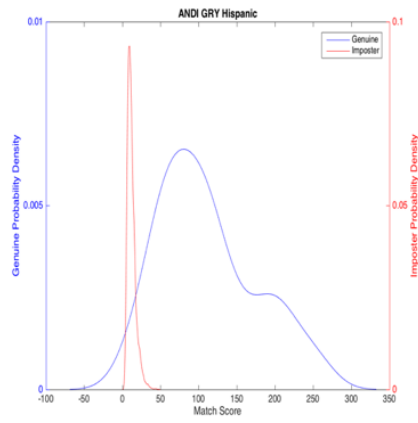

(g)

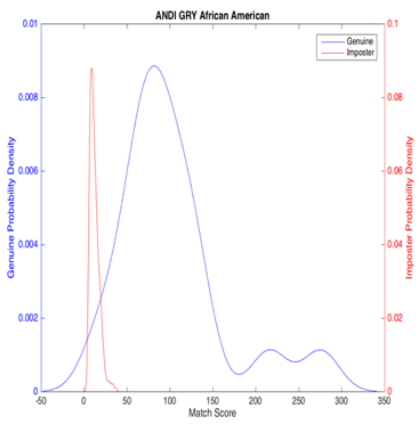

(b)

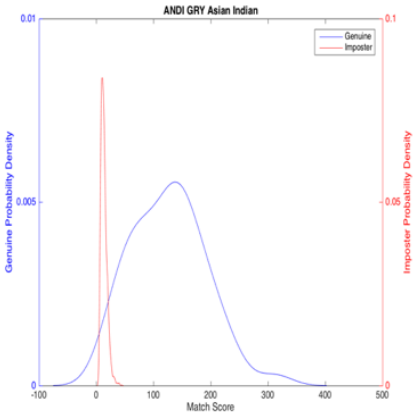

(e)

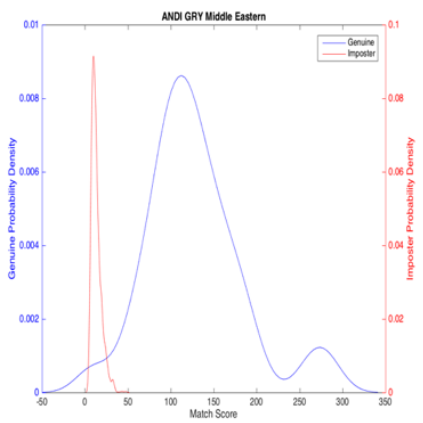

(h)

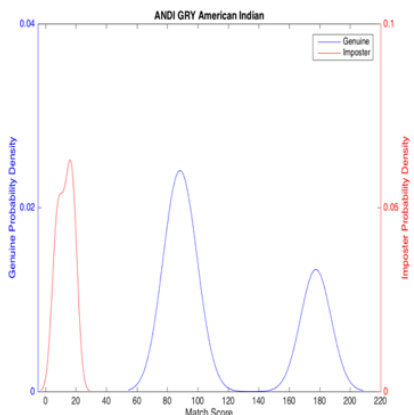

(c)

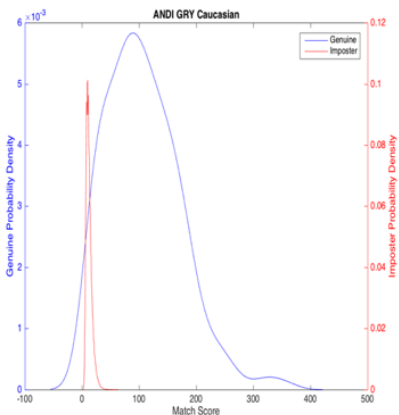

(f)

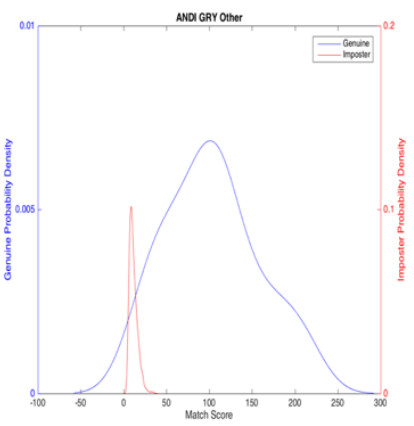

(i)

Figure D.1.2 NBIS ANDI Greyscale (a) African (b) African American (c) American Indian (d) Asian (e) Asian Indian (f) Caucasian (g) Hispanic (h) Middle Eastern (i) Other 


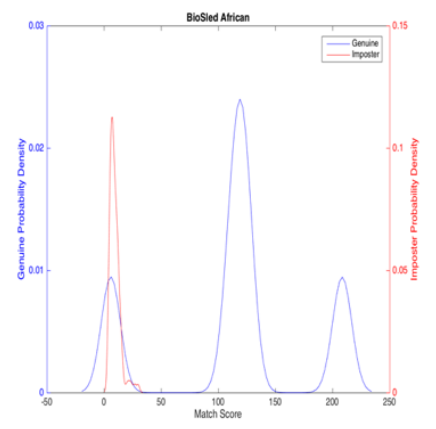

(a)

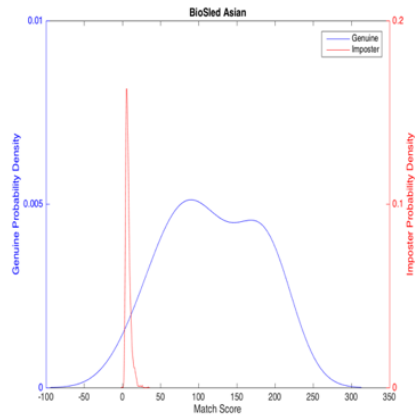

(d)

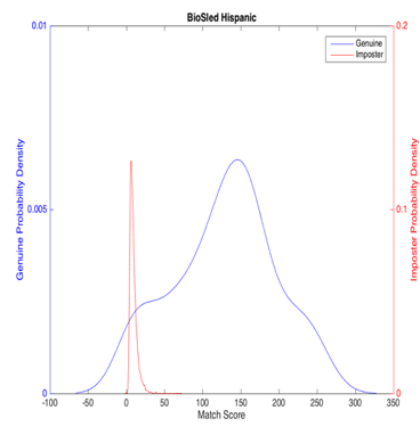

(g)

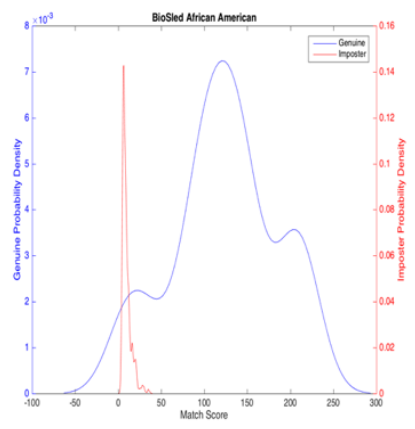

(b)

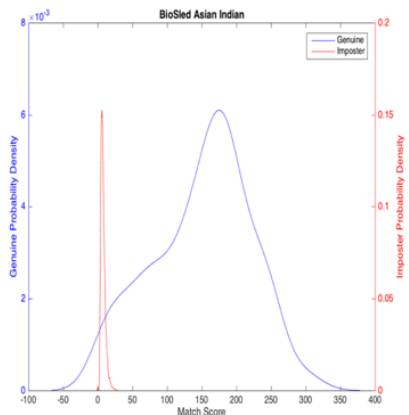

(e)

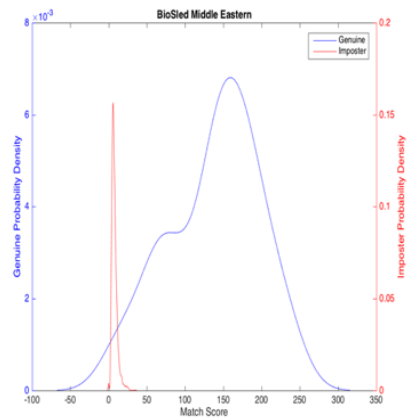

(h)

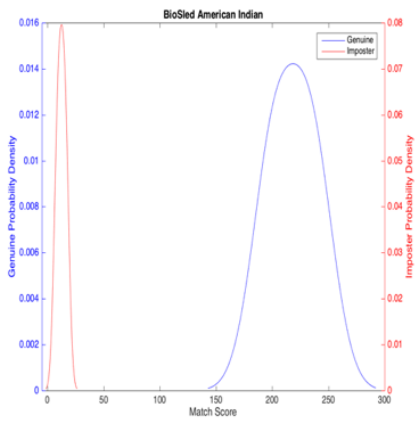

(c)

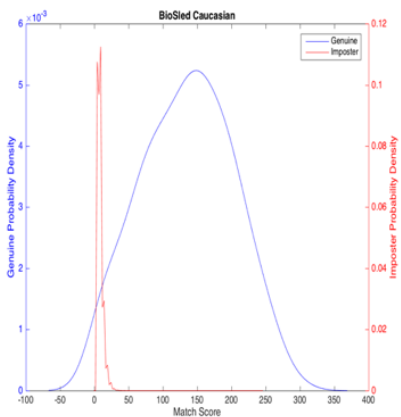

(f)

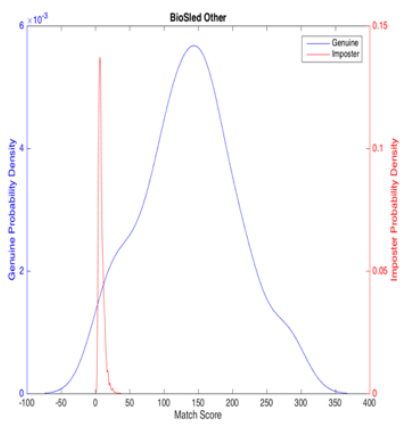

(i)

Figure D.1.3 NBIS BioSled (a) African (b) African American (c) American Indian (d) Asian (e) Asian Indian (f) Caucasian (g) Hispanic (h) Middle Eastern (i) Other 


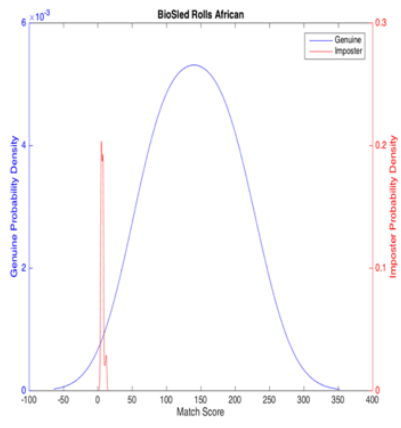

(a)

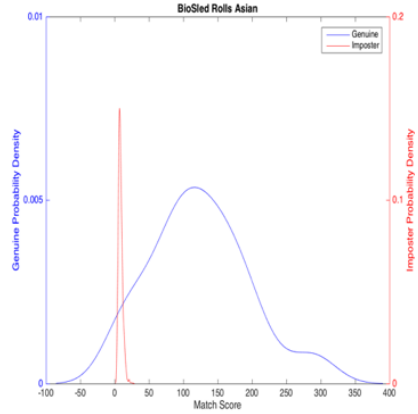

(d)

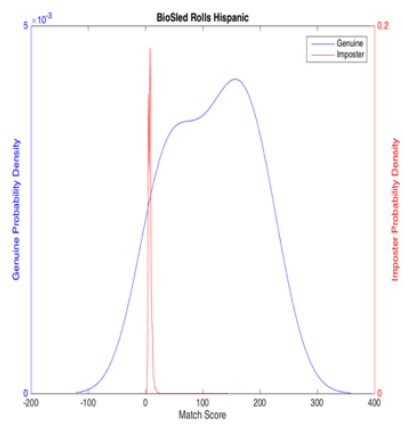

(g)

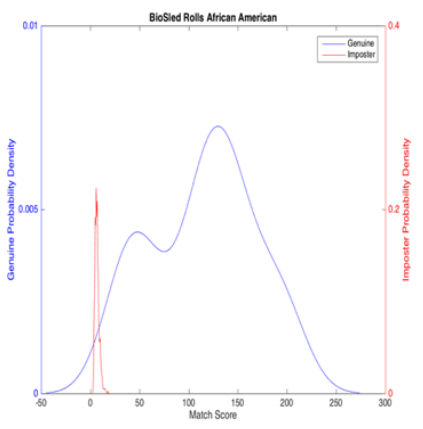

(b)

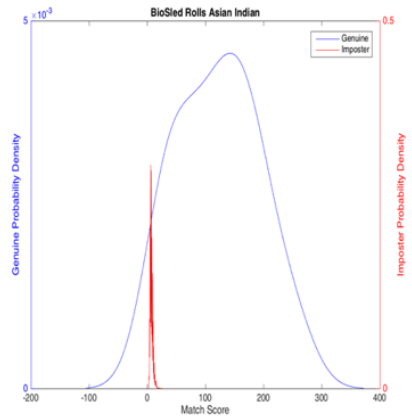

(e)

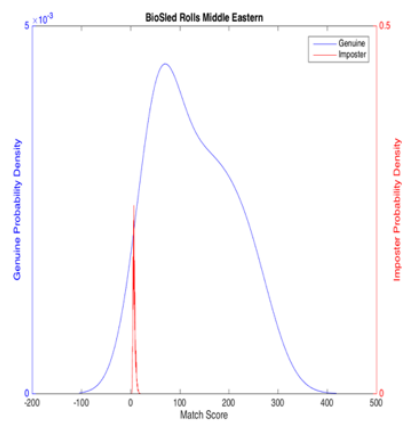

(h)

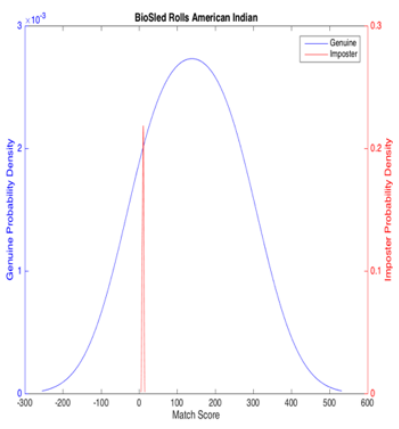

(c)

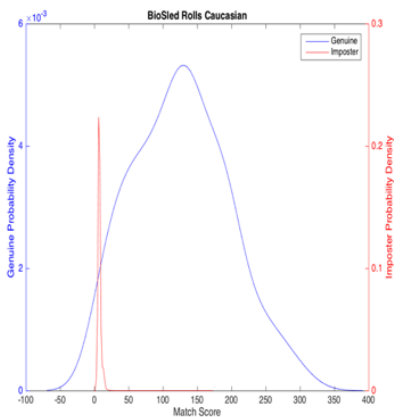

(f)

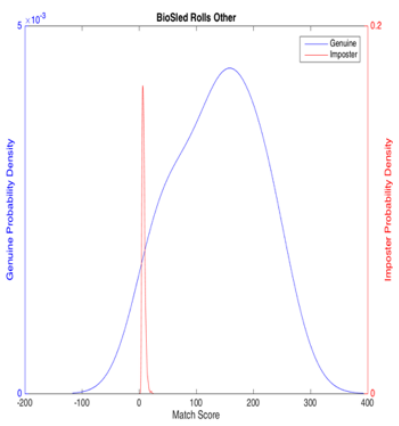

(i)

Figure D.1.4 NBIS BioSled Rolls (a) African (b) African American (c) American Indian (d) Asian (e) Asian Indian (f) Caucasian (g) Hispanic (h) Middle Eastern (i) Other 


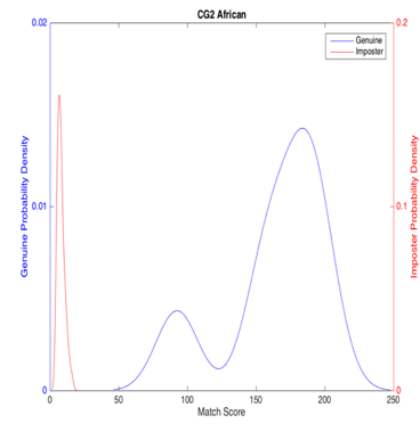

(a)

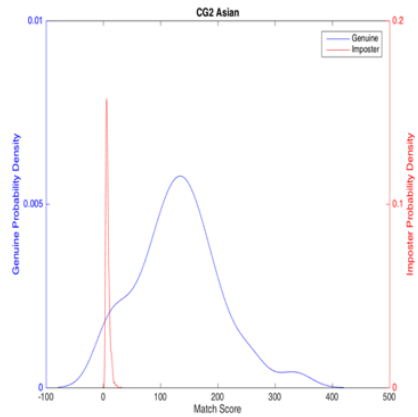

(d)

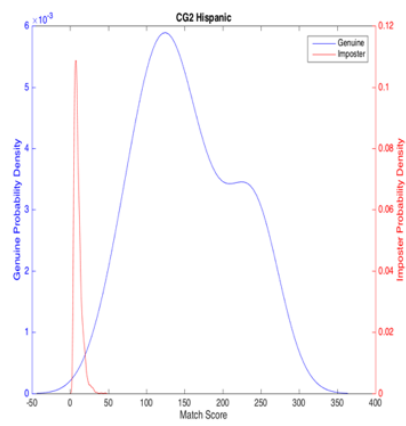

(g)

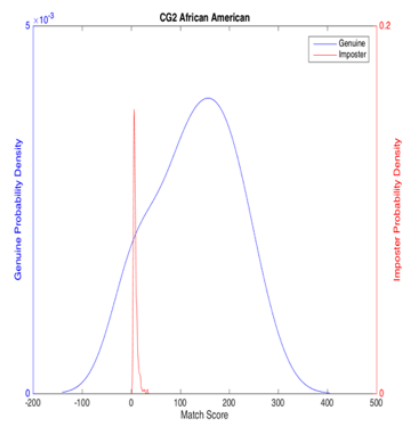

(b)

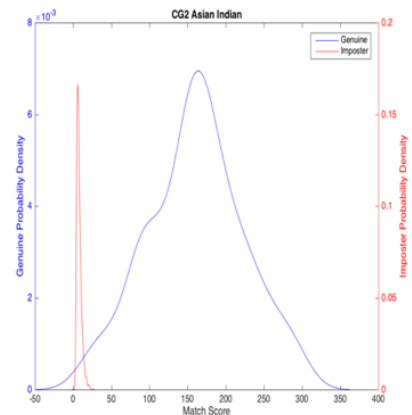

(e)

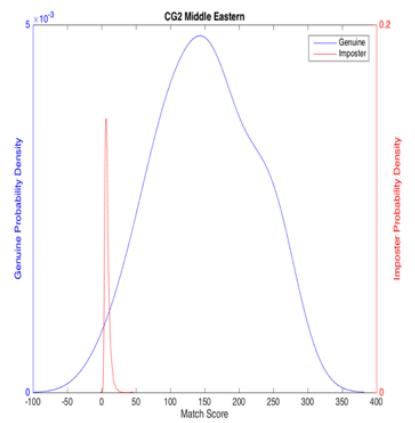

(h)

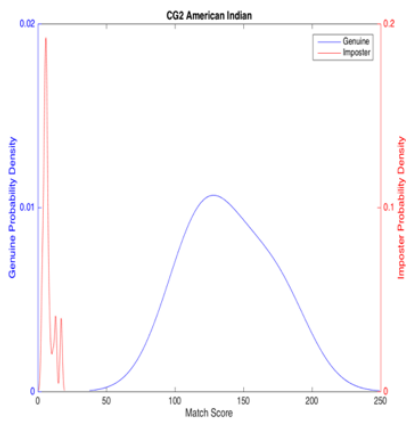

(c)

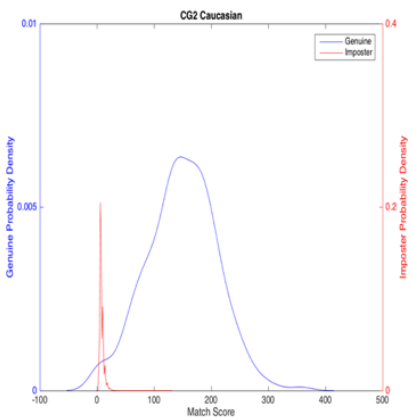

(f)

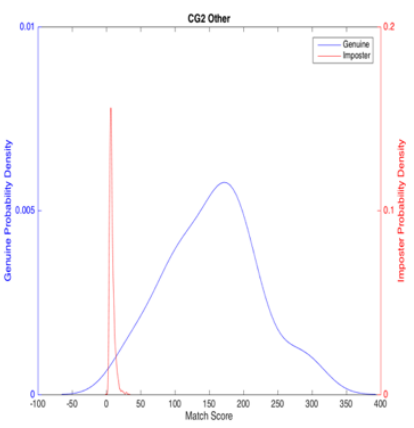

(i)

Figure D.1.5 NBIS FP II Guardian (a) African (b) African American (c) American Indian (d) Asian (e) Asian Indian (f) Caucasian (g) Hispanic (h) Middle Eastern (i) Other 


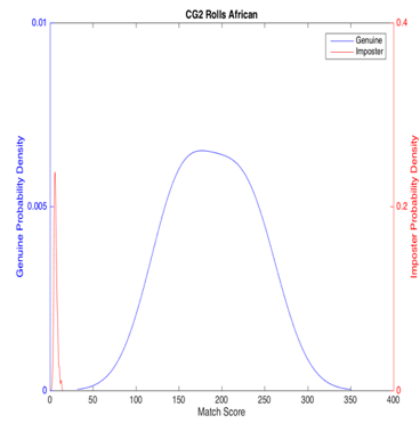

(a)

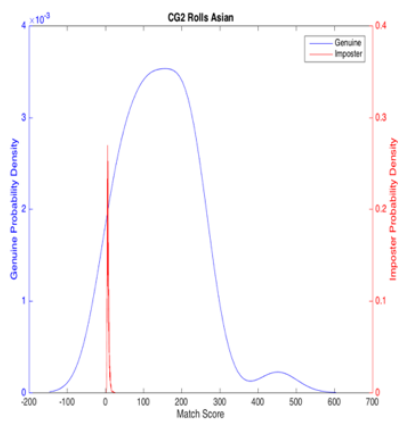

(d)

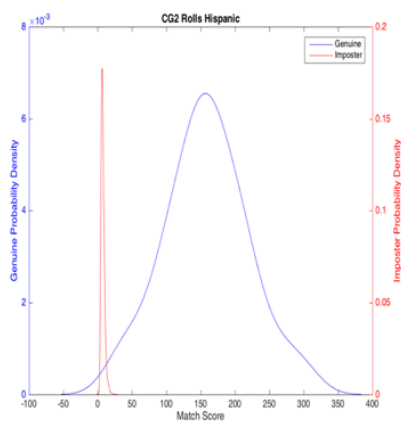

(g)

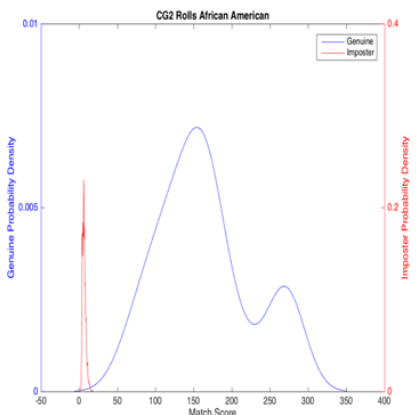

(b)

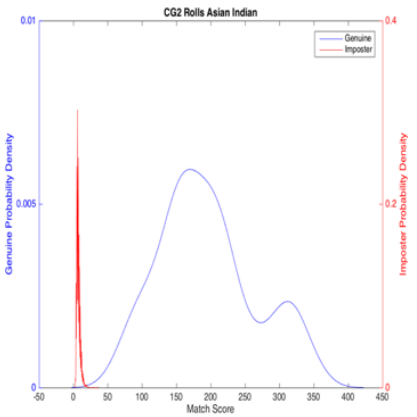

(e)

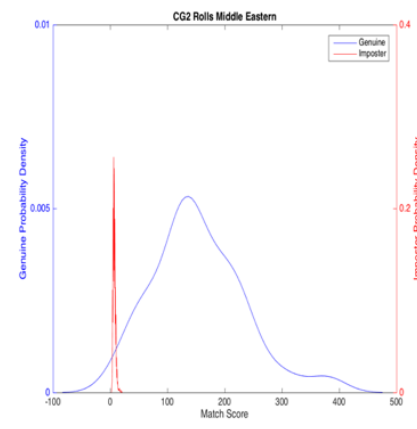

(h)

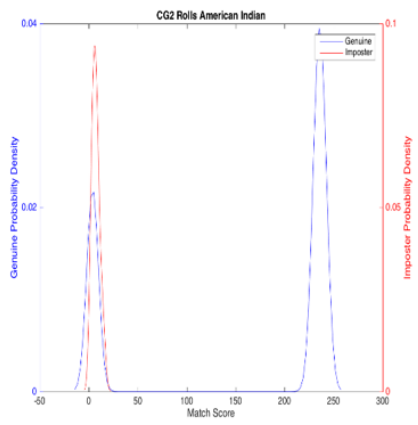

(c)

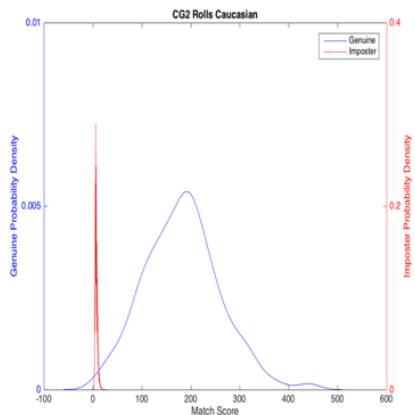

(f)

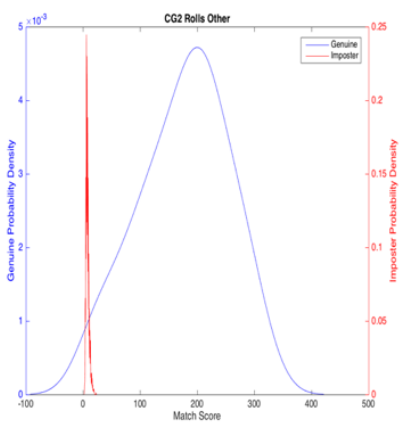

(i)

Figure D.1.6 NBIS FP II Guardian Rolls (a) African (b) African American (c) American Indian (d) Asian (e) Asian Indian (f) Caucasian (g) Hispanic (h) Middle Eastern (i) Other 


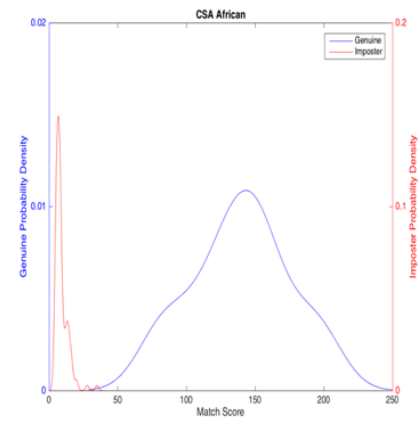

(a)

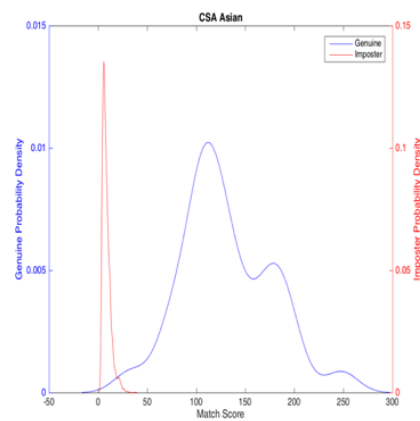

(d)

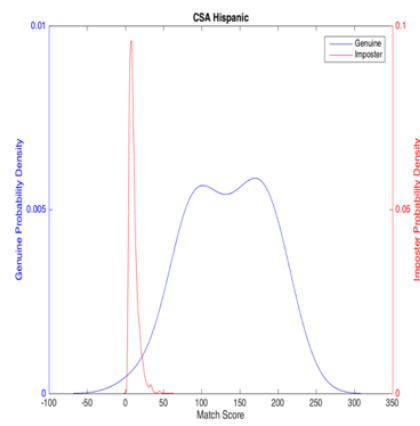

(g)

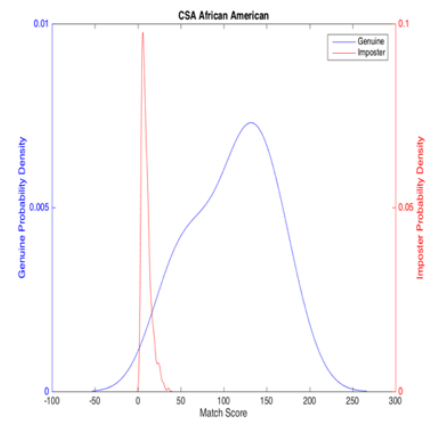

(b)

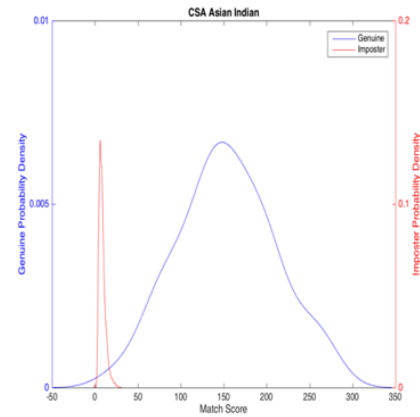

(e)

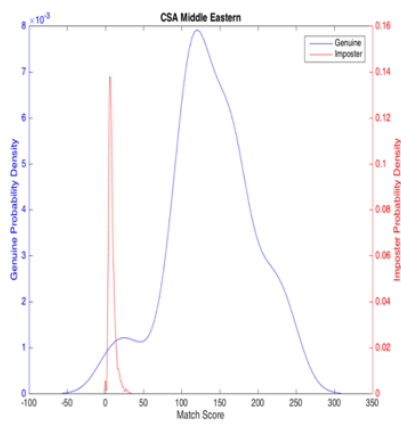

(h)

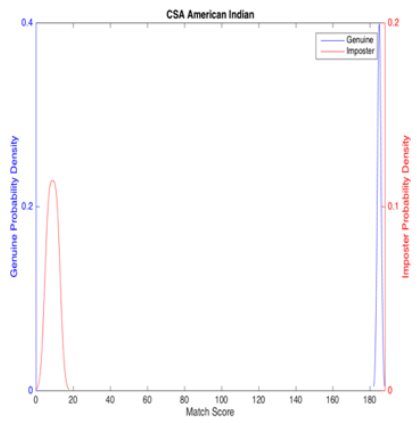

(c)

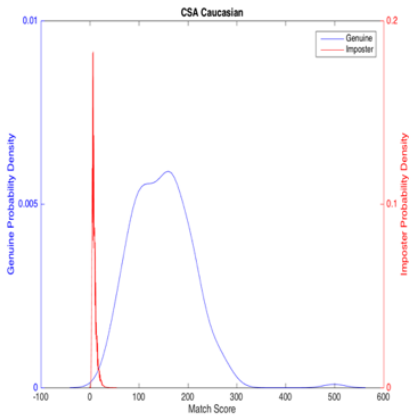

(f)

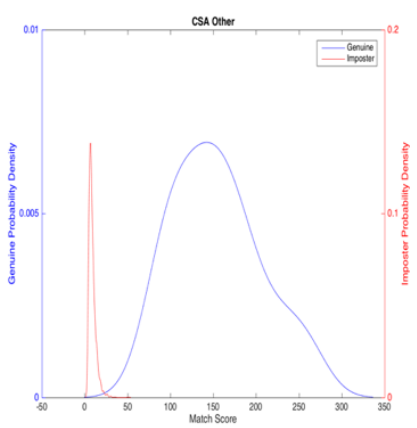

(i)

Figure D.1.7 NBIS Crossmatch Seek Avenger (a) African (b) African American (c) American Indian (d) Asian (e) Asian Indian (f) Caucasian (g) Hispanic (h) Middle Eastern (i) Other 


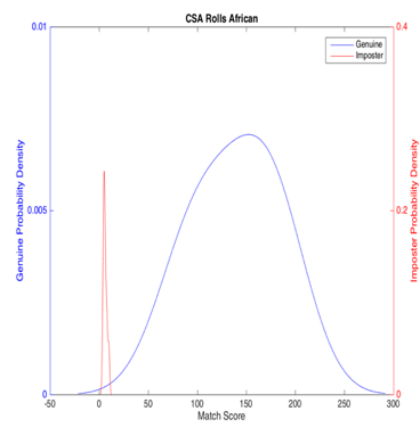

(a)

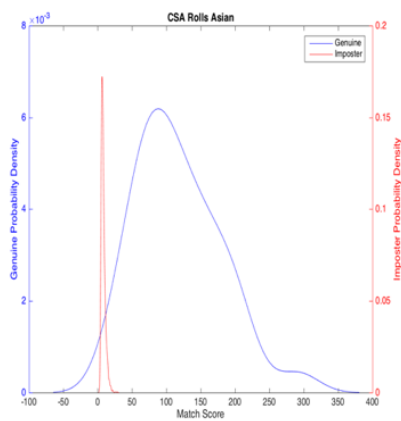

(d)

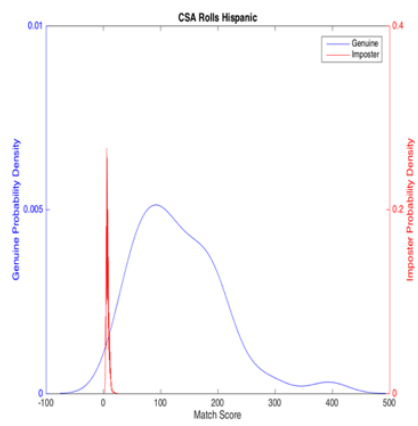

(g)

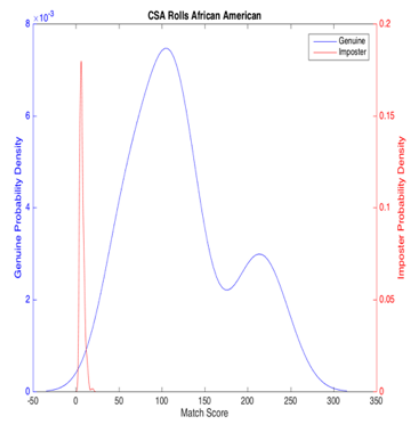

(b)

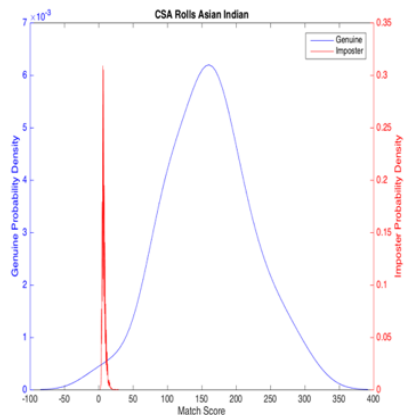

(e)

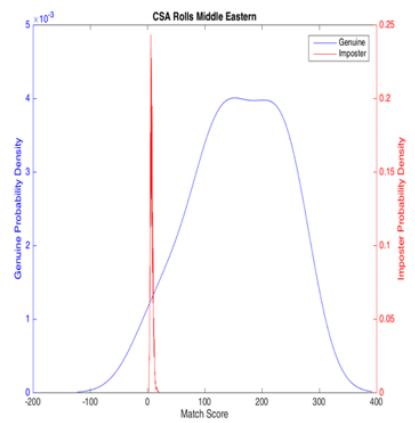

(h)

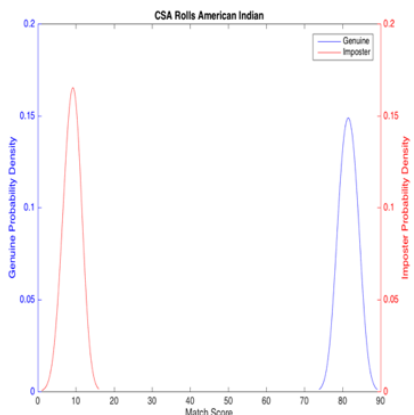

(c)

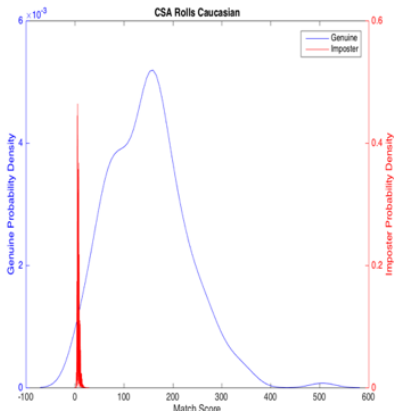

(f)

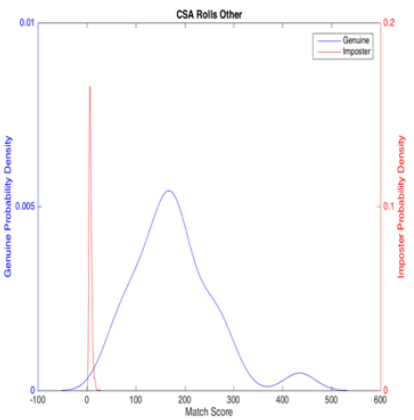

(i)

Figure D.1.8 Crossmatch Seek Avenger Rolls (a) African (b) African American (c) American Indian (d) Asian (e) Asian Indian (f) Caucasian (g) Hispanic (h) Middle Eastern (i) Other 


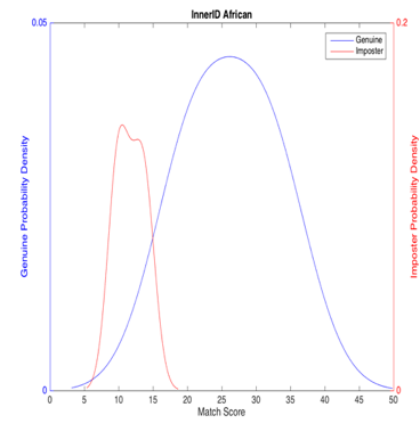

(a)

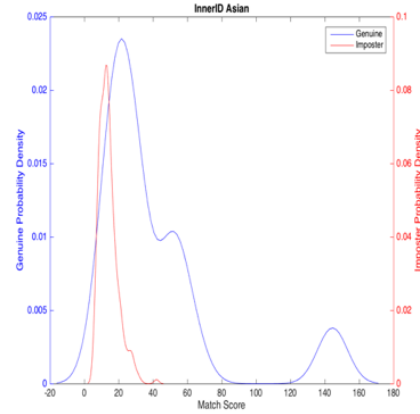

(d)

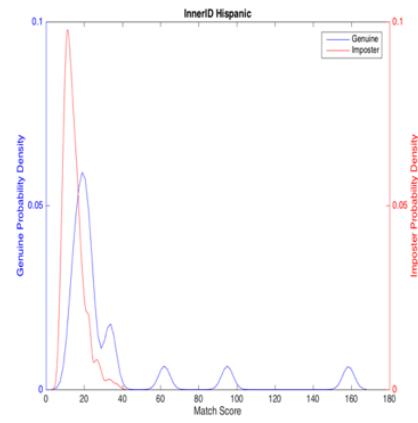

(g)

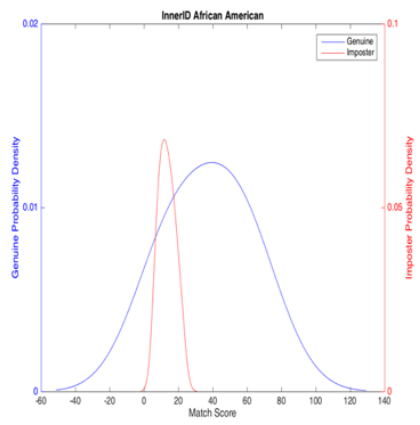

(b)

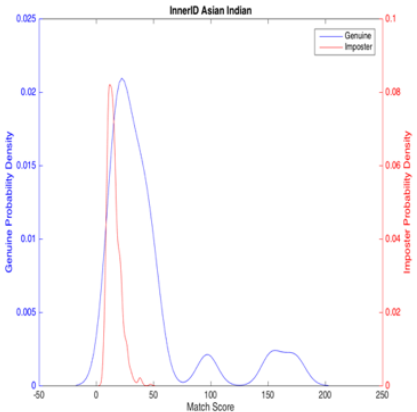

(e)

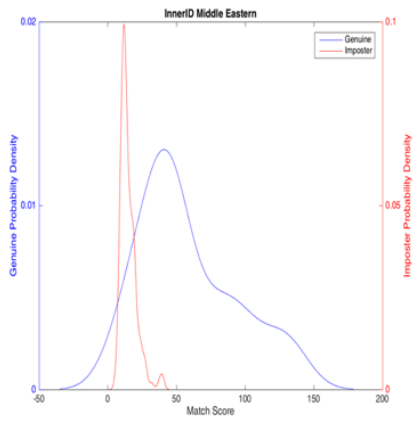

(h) (c)

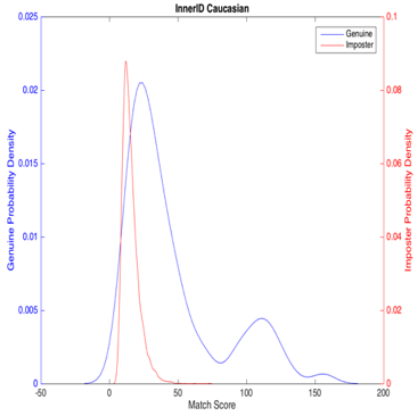

(f)

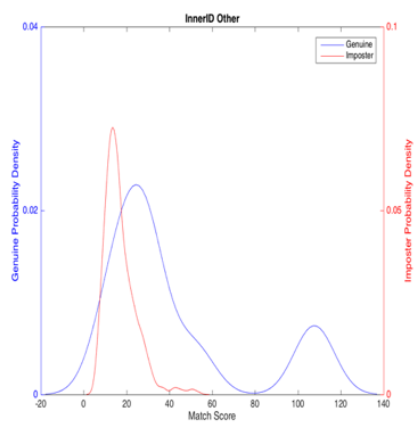

(i)

Figure D.1.9 NBIS InnerID (a) African (b) African American (c) American Indian (Not Available due to InnerID being removed from collection midway through) (d) Asian (e) Asian Indian (f) Caucasian (g) Hispanic (h) Middle Eastern (i) Other 


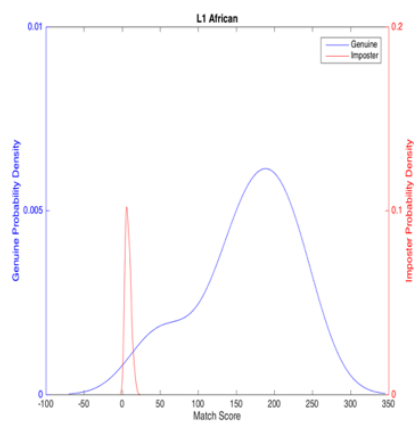

(a)

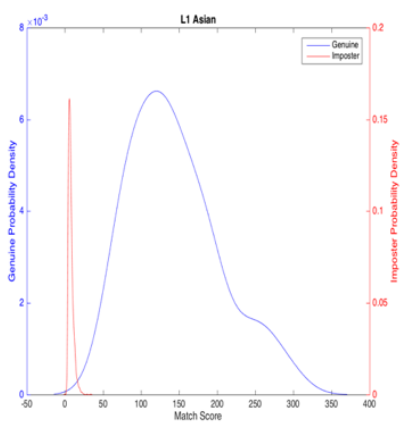

(d)

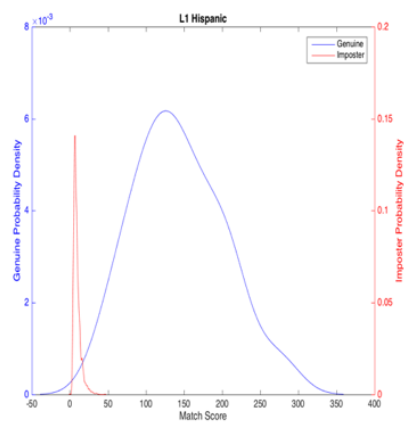

(g)

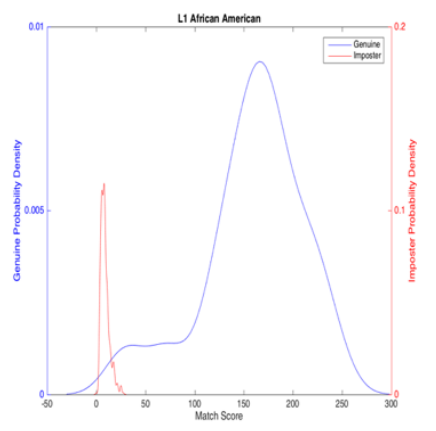

(b)

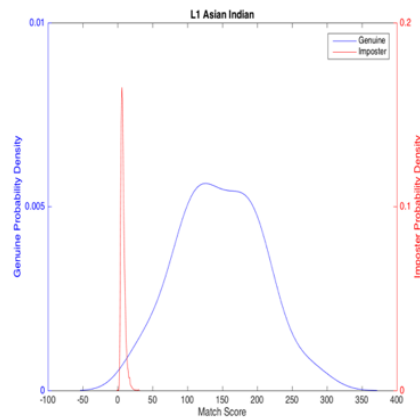

(e)

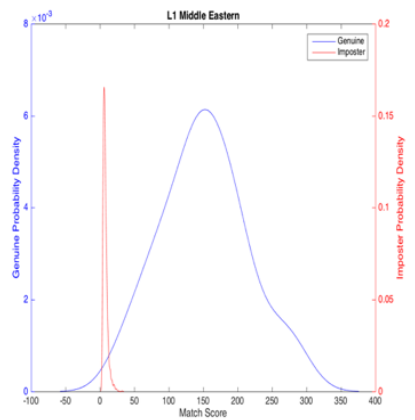

(h)

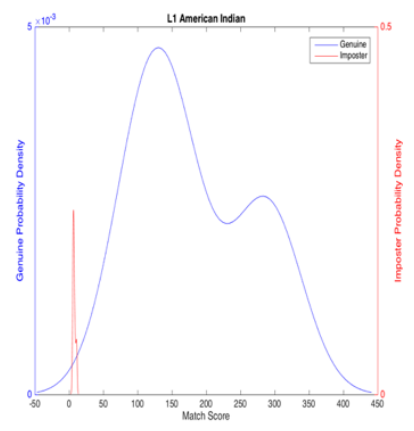

(c)

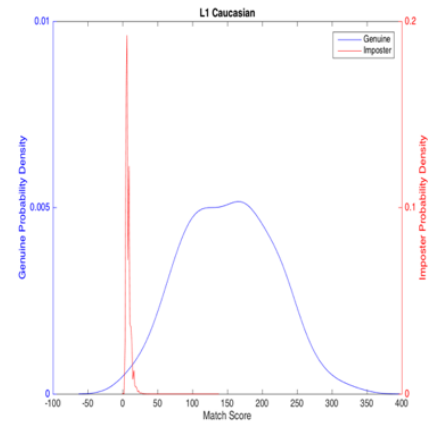

(f)

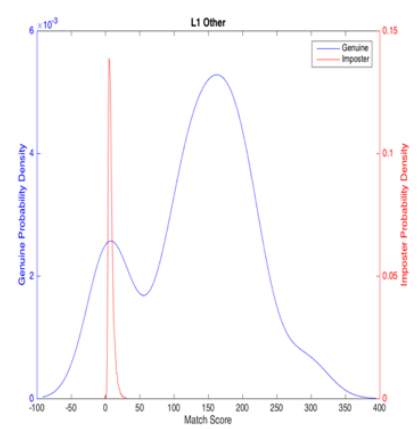

(i)

Figure D.1.10 NBIS L1 (a) African (b) African American (c) American Indian (d) Asian (e) Asian Indian (f) Caucasian (g) Hispanic (h) Middle Eastern (i) Other 


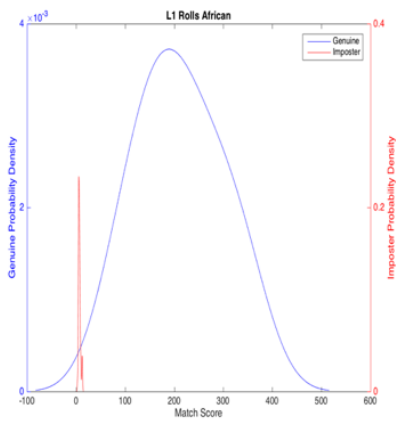

(a)

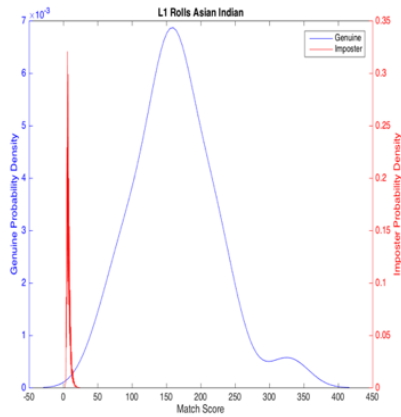

(e)

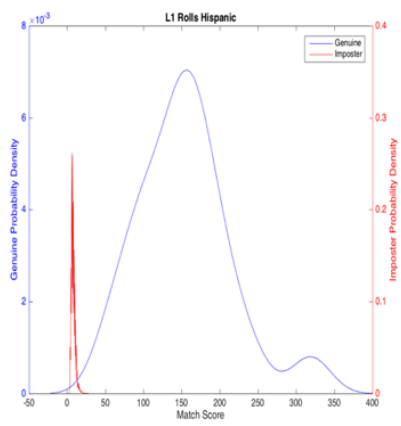

(g)

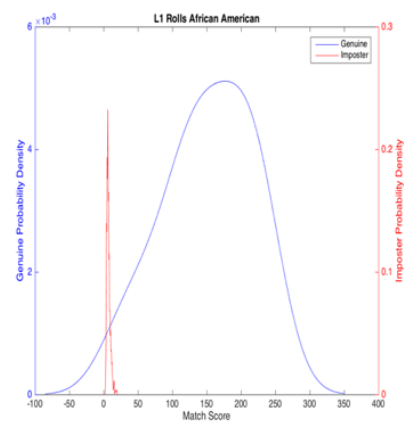

(b)

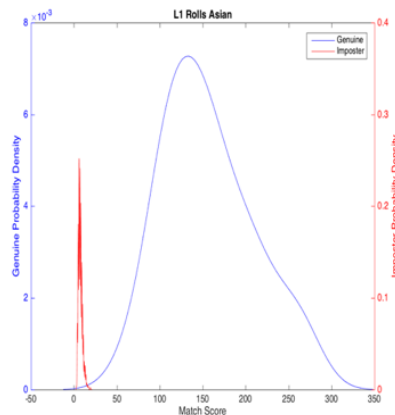

(d)

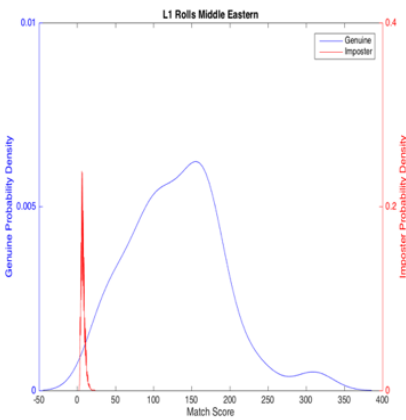

(h)

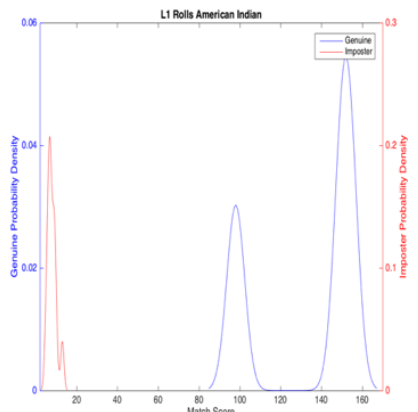

(c)

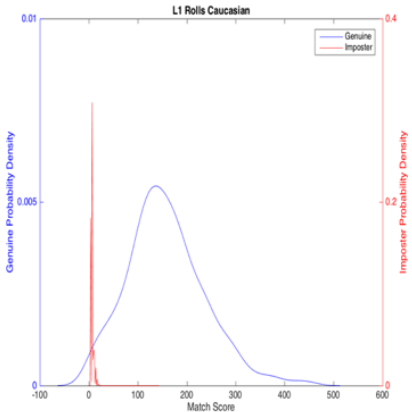

(f)

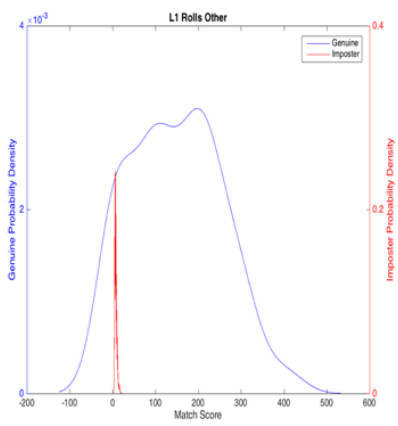

(i)

Figure D.1.11 L1 Rolls (a) African (b) African American (c) American Indian (d) Asian (e) Asian Indian (f) Caucasian (g) Hispanic (h) Middle Eastern (i) Other 


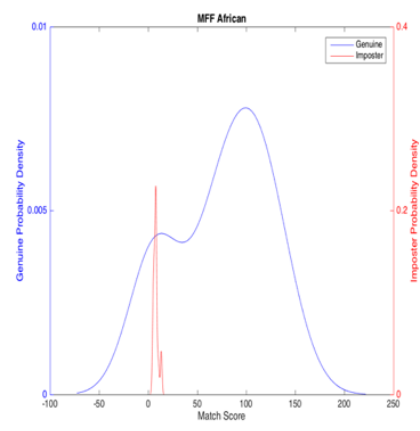

(a)

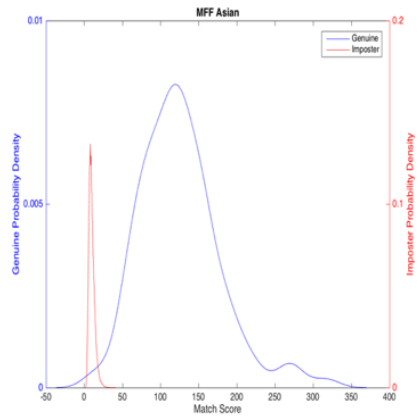

(d)

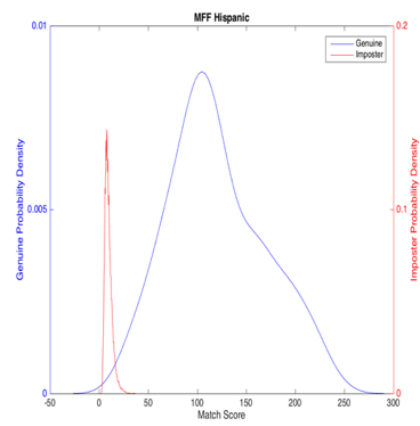

(g)

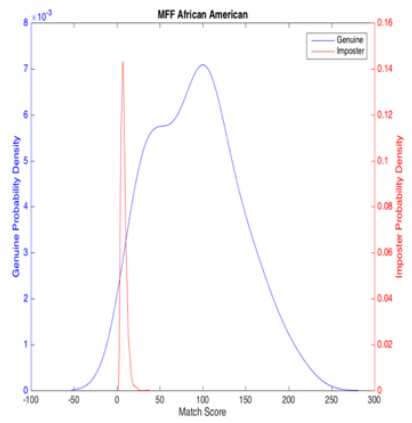

(b)

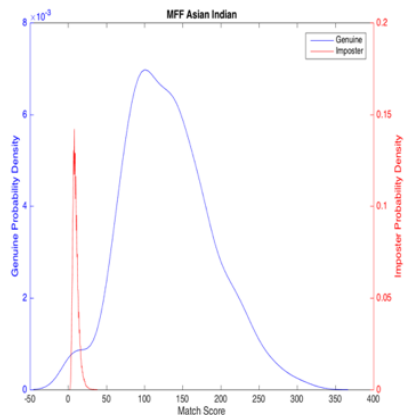

(e)

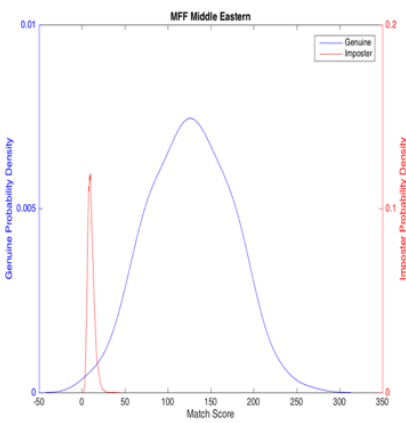

(h)

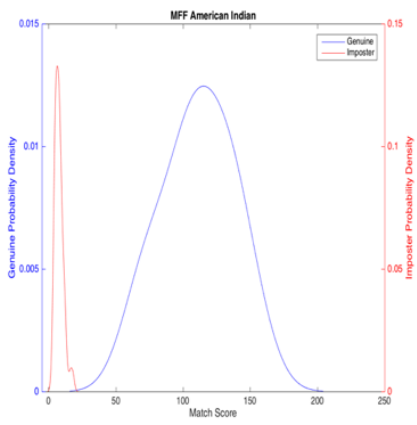

(c)

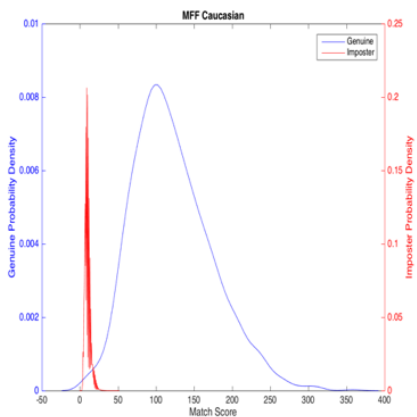

(f)

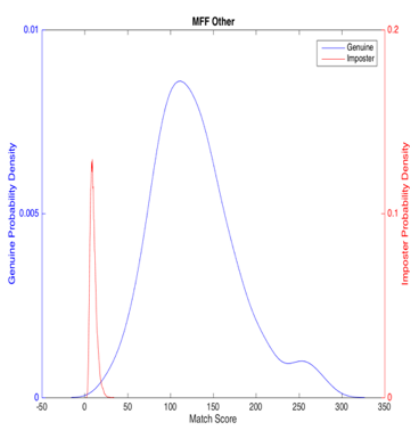

(i)

Figure D.1.12 NBIS Morpho FOTF (a) African (b) African American (c) American Indian (d) Asian (e) Asian Indian (f) Caucasian (g) Hispanic (h) Middle Eastern (i) Other 


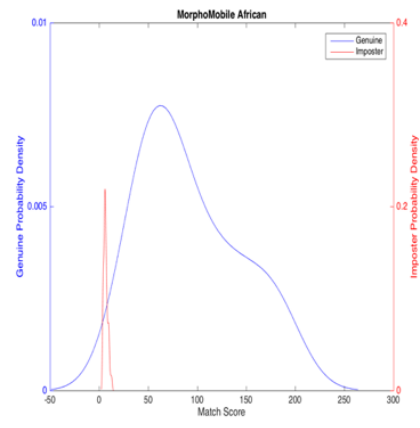

(a)

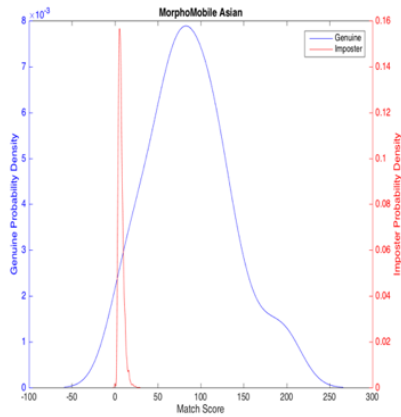

(d)

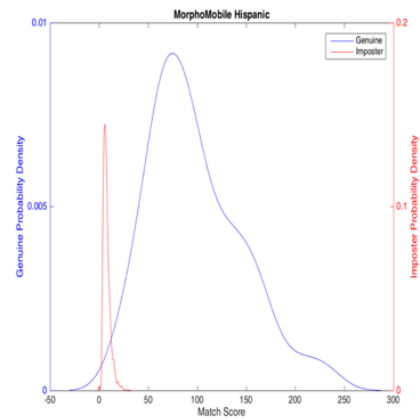

(g)

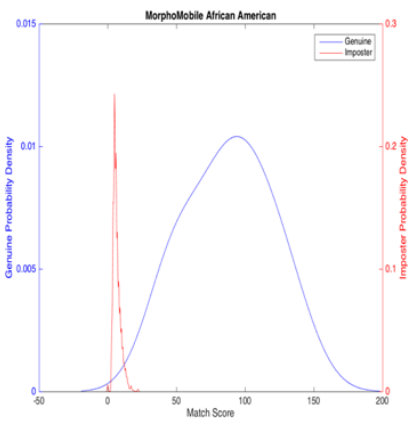

(b)

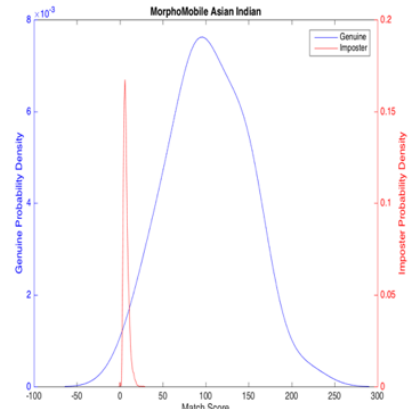

(e)

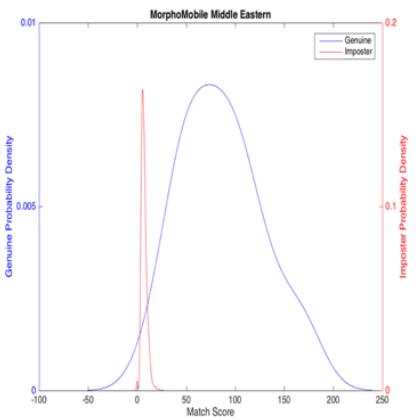

(h)

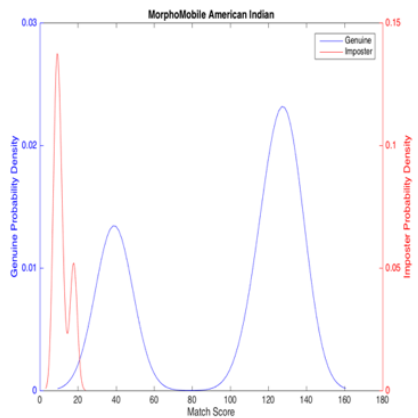

(c)

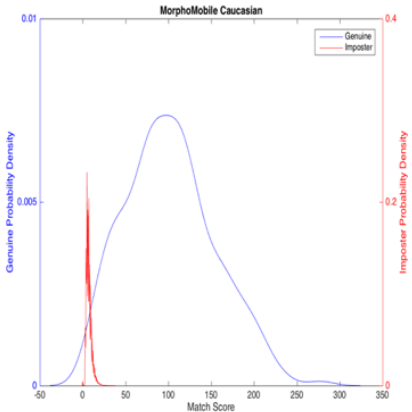

(f)

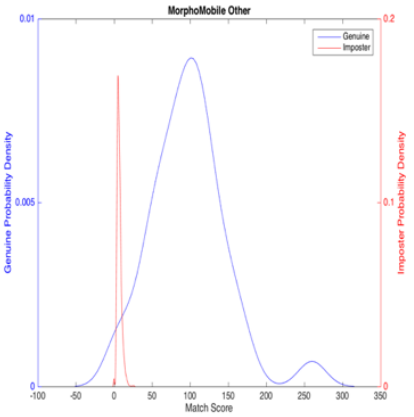

(i)

Figure D.1.13 NBIS MorphoMobile (a) African (b) African American (c) American Indian (d) Asian (e) Asian Indian (f) Caucasian (g) Hispanic (h) Middle Eastern (i) Other 


\section{D.2 Neurotech Genuine/Imposter Score Distributions}

Table D.10 Neurotech African Genuine and Imposter KLD, Maximum and Minimum Scores

\begin{tabular}{|c|c|c|c|c|c|c|}
\hline \multicolumn{7}{|c|}{ Neurotech African } \\
\hline Device & $\begin{array}{l}\text { Genuine } \\
\text { KLD }\end{array}$ & $\begin{array}{l}\text { Imposter } \\
\text { KLD }\end{array}$ & $\begin{array}{l}\text { Max } \\
\text { Genuine }\end{array}$ & $\begin{array}{l}\text { Min } \\
\text { Genuine }\end{array}$ & $\begin{array}{l}\text { Max } \\
\text { Imposter }\end{array}$ & $\begin{array}{l}\text { Min } \\
\text { Imposter }\end{array}$ \\
\hline$A N D I B I N$ & 0.0097 & 11.8608 & 813 & 324 & 4 & 0 \\
\hline$A N D I G R Y$ & 0.0311 & 2.5344 & 863 & 156 & 16 & 1 \\
\hline Biosled & 0.1777 & 1.294 & 714 & 3 & 16 & 0 \\
\hline $\begin{array}{r}\text { Biosled } \\
\text { Rolls }\end{array}$ & 0.0744 & 0.0389 & 647 & 333 & 8 & 0 \\
\hline CG2 & 0.0442 & 0.382 & 754 & 383 & 9 & 0 \\
\hline CG2 Rolls & 0.0462 & 0.0045 & 977 & 498 & 6 & 0 \\
\hline CSA & 0.074 & 8.7876 & 701 & 465 & 7 & 0 \\
\hline CSA Rolls & 0.0204 & 11.2825 & 795 & 362 & 7 & 0 \\
\hline$L 1$ & 0.158 & 0.0027 & 848 & 323 & 12 & 0 \\
\hline$S M M$ & 0.0151 & 0.4388 & 527 & 231 & 9 & 0 \\
\hline
\end{tabular}

Table D.11 Neurotech African American Genuine and Imposter KLD, Maximum and Minimum Scores

\begin{tabular}{|c|c|c|c|c|c|c|}
\hline \multicolumn{7}{|c|}{ Neurotech African American } \\
\hline Device & $\begin{array}{l}\text { Genuine } \\
\text { KLD }\end{array}$ & $\begin{array}{l}\text { Imposter } \\
\text { KLD }\end{array}$ & $\begin{array}{l}\text { Max } \\
\text { Genuine }\end{array}$ & $\begin{array}{l}\text { Min } \\
\text { Genuine }\end{array}$ & $\begin{array}{l}\text { Max } \\
\text { Imposter }\end{array}$ & $\begin{array}{l}\text { Min } \\
\text { Imposter }\end{array}$ \\
\hline$A N D I B I N$ & 0.0149 & 5.2858 & 838 & 132 & 14 & 0 \\
\hline$A N D I G R Y$ & 0.012 & 2.4253 & 754 & 61 & 30 & 0 \\
\hline Biosled & 0.019 & 0.9989 & 744 & 1 & 21 & 0 \\
\hline $\begin{array}{r}\text { BioSled } \\
\text { Rolls }\end{array}$ & 0.0172 & 0.0317 & 740 & 187 & 10 & 0 \\
\hline CG2 & 0.0316 & 0.0974 & 848 & 1 & 193 & 0 \\
\hline CG2 Rolls & 0.0208 & 0.0052 & 897 & 322 & 11 & 0 \\
\hline CSA & 0.0186 & 5.251 & 737 & 359 & 16 & 0 \\
\hline CSA Rolls & 0.0244 & 9.1188 & 720 & 224 & 10 & 0 \\
\hline$L 1$ & 0.0152 & 0.0029 & 757 & 217 & 19 & 0 \\
\hline SMM & 0.0405 & 0.3605 & 579 & 286 & 23 & 0 \\
\hline
\end{tabular}


Table D.12 Neurotech American Indian Genuine and Imposter KLD, Maximum and Minimum Scores

\begin{tabular}{|c|c|c|c|c|c|c|}
\hline \multicolumn{7}{|c|}{ Neurotech American Indian } \\
\hline Device & $\begin{array}{l}\text { Genuine } \\
\text { KLD }\end{array}$ & $\begin{array}{l}\text { Imposter } \\
\text { KLD }\end{array}$ & $\begin{array}{l}\text { Max } \\
\text { Genuine }\end{array}$ & $\begin{array}{l}\text { Min } \\
\text { Genuine }\end{array}$ & $\begin{array}{l}\text { Max } \\
\text { Imposter }\end{array}$ & $\begin{array}{l}\text { Min } \\
\text { Imposter }\end{array}$ \\
\hline$A N D I B I N$ & 0.008 & 10.1086 & 666 & 321 & 6 & 1 \\
\hline ANDI GRY & 0.0299 & 2.9429 & 798 & 464 & 10 & 7 \\
\hline Biosled & 0.2007 & 0.9628 & 501 & 486 & 7 & 4 \\
\hline $\begin{array}{r}\text { Biosled } \\
\text { Rolls }\end{array}$ & 0.3719 & 0.0142 & 679 & 254 & 4 & 2 \\
\hline CG2 & 0.1273 & 0.4346 & 680 & 360 & 17 & 1 \\
\hline CG2 Rolls & 0.0367 & 0.0117 & 848 & 143 & 24 & 1 \\
\hline CSA & 0.3452 & 7.3243 & 587 & 587 & 12 & 5 \\
\hline CSA Rolls & 0.256 & 13.0463 & 339 & 339 & 4 & 2 \\
\hline$L 1$ & 0.042 & 0.0021 & 618 & 307 & 11 & 1 \\
\hline$M F F$ & 0.0272 & 4.5010 & 610 & 228 & 20 & 2 \\
\hline$S M M$ & 0.4359 & 0.405 & 301 & 227 & 14 & 1 \\
\hline
\end{tabular}

Table D.13 Neurotech Asian Genuine and Imposter KLD, Maximum and Minimum Scores

\begin{tabular}{|c|c|c|c|c|c|c|}
\hline \multicolumn{7}{|c|}{ Neurotech Asian } \\
\hline Device & $\begin{array}{l}\text { Genuine } \\
\text { KLD }\end{array}$ & $\begin{array}{l}\text { Imposter } \\
\text { KLD }\end{array}$ & $\begin{array}{l}\text { Max } \\
\text { Genuine }\end{array}$ & $\begin{array}{l}\text { Min } \\
\text { Genuine }\end{array}$ & $\begin{array}{l}\text { Max } \\
\text { Imposter }\end{array}$ & $\begin{array}{l}\text { Min } \\
\text { Imposter }\end{array}$ \\
\hline$A N D I B I N$ & 0.0171 & 3.073 & 842 & 4 & 24 & 0 \\
\hline ANDI GRY & 0.0139 & 0.7672 & 867 & 9 & 33 & 1 \\
\hline Biosled & 0.0071 & 0.7864 & 763 & 3 & 25 & 0 \\
\hline $\begin{array}{r}\text { Biosled } \\
\text { Rolls }\end{array}$ & 0.0343 & 0.023 & 942 & 180 & 21 & 0 \\
\hline$C G 2$ & 0.0219 & 0.29 & 935 & 2 & 21 & 0 \\
\hline CG2 Rolls & 0.0156 & 0.0118 & 1102 & 2 & 22 & 0 \\
\hline CSA & 0.0267 & 4.9202 & 735 & 325 & 27 & 0 \\
\hline CSA Rolls & 0.0184 & 5.9803 & 700 & 179 & 19 & 0 \\
\hline L1 & 0.0476 & 0.0046 & 863 & 287 & 27 & 0 \\
\hline MFF & 0.0438 & 3.1822 & 827 & 4 & 28 & 0 \\
\hline$S M M$ & 0.0219 & 0.1911 & 672 & 3 & 27 & 0 \\
\hline
\end{tabular}


Table D.14 Neurotech Asian Indian Genuine and Imposter KLD, Maximum and Minimum Scores

\begin{tabular}{|c|c|c|c|c|c|c|}
\hline \multicolumn{7}{|c|}{ Neurotech Asian Indian } \\
\hline Device & $\begin{array}{l}\text { Genuine } \\
\text { KLD }\end{array}$ & $\begin{array}{l}\text { Imposter } \\
\text { KLD }\end{array}$ & $\begin{array}{l}\text { Max } \\
\text { Genuine }\end{array}$ & $\begin{array}{l}\text { Min } \\
\text { Genuine }\end{array}$ & $\begin{array}{l}\text { Max } \\
\text { Imposter }\end{array}$ & $\begin{array}{l}\text { Min } \\
\text { Imposter }\end{array}$ \\
\hline ANDI BIN & 0.0179 & 3.4657 & 810 & 55 & 40 & r $=5$ \\
\hline ANDI GRY & 0.0115 & 1.506 & 971 & 58 & 36 & $c$ \\
\hline Biosled & 0.029 & 0.6308 & 811 & 0 & 33 & $c$ \\
\hline $\begin{array}{r}\text { Biosled } \\
\text { Rolls }\end{array}$ & 0.0062 & 0.0119 & 924 & 1 & 28 & $c$ \\
\hline CG2 & 0.0242 & 0.1915 & 939 & 159 & 30 & $c$ \\
\hline CG2 Rolls & 0.0166 & 0.0147 & 1072 & 314 & 29 & $c$ \\
\hline CSA & 0.0206 & 2.4215 & 835 & 275 & 35 & c \\
\hline CSA Rolls & 0.0805 & 4.7043 & 888 & 1 & 26 & $c$ \\
\hline$L 1$ & 0.0309 & 0.0042 & 893 & 141 & 33 & c \\
\hline MFF & 0.0325 & 2.7939 & 848 & 2 & 33 & $c$ \\
\hline$S M M$ & 0.0186 & 0.1396 & 648 & 5 & 31 & $\mathrm{c}$ \\
\hline
\end{tabular}

Table D.15 Neurotech Caucasian Genuine and Imposter KLD, Maximum and Minimum Scores

\begin{tabular}{|c|c|c|c|c|c|c|}
\hline \multicolumn{7}{|c|}{ Neurotech Caucasian } \\
\hline Device & $\begin{array}{l}\text { Genuine } \\
\text { KLD }\end{array}$ & $\begin{array}{l}\text { Imposter } \\
\text { KLD }\end{array}$ & $\begin{array}{l}\text { Max } \\
\text { Genuine }\end{array}$ & $\begin{array}{l}\text { Min } \\
\text { Genuine }\end{array}$ & $\begin{array}{l}\text { Max } \\
\text { Imposter }\end{array}$ & $\begin{array}{l}\text { Min } \\
\text { Imposter }\end{array}$ \\
\hline$A N D I B I N$ & 0.0054 & 4.9975 & 986 & 0 & 38 & 0 \\
\hline ANDI GRY & 0.0051 & 0.1466 & 1003 & 3 & 45 & 0 \\
\hline Biosled & 0.0086 & 0.1891 & 951 & 0 & 518 & 0 \\
\hline $\begin{array}{r}\text { BioSled } \\
\text { Rolls }\end{array}$ & 0.0042 & 0.0155 & 968 & 0 & 601 & 0 \\
\hline$C G 2$ & 0.0066 & 0.1977 & 1000 & 1 & 400 & 0 \\
\hline CG2 Rolls & 0.0187 & 0.0238 & 1188 & 230 & 28 & 0 \\
\hline CSA & 0.0305 & 5.8025 & 1267 & 201 & 45 & 0 \\
\hline CSA Rolls & 0.0049 & 0.8022 & 1682 & 0 & 26 & 0 \\
\hline$L 1$ & 0.009 & 0.0005 & 913 & 1 & 681 & 0 \\
\hline$M F F$ & 0.0230 & 1.3482 & 863 & 4 & 39 & 0 \\
\hline$S M M$ & 0.0078 & 0.2404 & 691 & 1 & 48 & 0 \\
\hline
\end{tabular}


Table D.16 Neurotech Hispanic Genuine and Imposter KLD, Maximum and Minimum Scores

\begin{tabular}{|c|c|c|c|c|c|c|}
\hline \multicolumn{7}{|c|}{ Neurotech Hispanic } \\
\hline Device & $\begin{array}{l}\text { Genuine } \\
\text { KLD }\end{array}$ & $\begin{array}{l}\text { Imposter } \\
\text { KLD }\end{array}$ & $\begin{array}{l}\text { Max } \\
\text { Genuine }\end{array}$ & $\begin{array}{l}\text { Min } \\
\text { Genuine }\end{array}$ & $\begin{array}{l}\text { Max } \\
\text { Imposter }\end{array}$ & $\begin{array}{l}\text { Min } \\
\text { Imposter }\end{array}$ \\
\hline$A N D I B I N$ & 0.0159 & 3.4466 & 975 & 1 & 21 & $\mathrm{c}$ \\
\hline$A N D I G R Y$ & 0.0162 & 0.5137 & 915 & 3 & 41 & c \\
\hline Biosled & 0.0298 & 0.0173 & 747 & 1 & 397 & $c$ \\
\hline $\begin{array}{r}\text { BioSled } \\
\text { Rolls }\end{array}$ & 0.0167 & 0.0226 & 835 & 1 & 520 & $c$ \\
\hline CG2 & 0.0383 & 0.1664 & 908 & 239 & 34 & $c$ \\
\hline CG2 Rolls & 0.0163 & 0.016 & 1018 & 174 & 28 & $c$ \\
\hline CSA & 0.0166 & 2.2627 & 804 & 254 & 34 & c \\
\hline CSA Rolls & 0.0163 & 4.6099 & 946 & 142 & 17 & c \\
\hline$L 1$ & 0.024 & 0.0034 & 863 & 164 & 28 & c \\
\hline$M F F$ & 0.0232 & 2.8467 & 813 & 201 & 27 & c \\
\hline$S M M$ & 0.0307 & 0.1323 & 647 & 187 & 44 & $\mathrm{C}$ \\
\hline
\end{tabular}

Table D.17 Neurotech Middle Eastern Genuine and Imposter KLD, Maximum and Minimum Scores

\begin{tabular}{|c|c|c|c|c|c|c|}
\hline \multicolumn{7}{|c|}{ Neurotech Middle Eastern } \\
\hline Device & $\begin{array}{l}\text { Genuine } \\
\text { KLD }\end{array}$ & $\begin{array}{l}\text { Imposter } \\
\text { KLD }\end{array}$ & $\begin{array}{l}\text { Max } \\
\text { Genuine }\end{array}$ & $\begin{array}{l}\text { Min } \\
\text { Genuine }\end{array}$ & $\begin{array}{l}\text { Max } \\
\text { Imposter }\end{array}$ & $\begin{array}{l}\text { Min } \\
\text { Imposter }\end{array}$ \\
\hline ANDI BIN & 0.0567 & 2.7733 & 847 & 2 & 26 & 0 \\
\hline$A N D I G R Y$ & 0.0509 & 1.7713 & 903 & 6 & 27 & 1 \\
\hline Biosled & 0.0299 & 0.7524 & 854 & 109 & 29 & 0 \\
\hline $\begin{array}{r}\text { Biosled } \\
\text { Rolls }\end{array}$ & 0.0219 & 0.0228 & 993 & 180 & 17 & 0 \\
\hline CG2 & 0.0211 & 0.2659 & 875 & 141 & 19 & 0 \\
\hline CG2 Rolls & 0.0274 & 0.0091 & 1000 & 308 & 20 & 0 \\
\hline CSA & 0.1126 & 3.3431 & 808 & 1 & 19 & 0 \\
\hline CSA Rolls & 0.0375 & 7.193 & 863 & 148 & 12 & 0 \\
\hline$L 1$ & 0.0399 & 0.0036 & 891 & 288 & 19 & 0 \\
\hline$M F F$ & 0.0636 & 1.9447 & 727 & 7 & 28 & 1 \\
\hline$S M M$ & 0.0162 & 0.1793 & 542 & 122 & 35 & 0 \\
\hline
\end{tabular}


Table D.18 Neurotech Other Genuine and Imposter KLD, Maximum and Minimum Scores

\begin{tabular}{|c|c|c|c|c|c|c|}
\hline \multicolumn{7}{|c|}{ Neurotech Other } \\
\hline Device & $\begin{array}{l}\text { Genuine } \\
\text { KLD }\end{array}$ & $\begin{array}{l}\text { Imposter } \\
\text { KLD }\end{array}$ & $\begin{array}{l}\text { Max } \\
\text { Genuine }\end{array}$ & $\begin{array}{l}\text { Min } \\
\text { Genuine }\end{array}$ & $\begin{array}{l}\text { Max } \\
\text { Imposter }\end{array}$ & $\begin{array}{l}\text { Min } \\
\text { Imposter }\end{array}$ \\
\hline$A N D I B I N$ & 0.0309 & 2.9675 & 774 & 2 & 22 & 0 \\
\hline$A N D I G R Y$ & 0.0302 & 0.4649 & 767 & 17 & 37 & 1 \\
\hline Biosled & 0.0166 & 1.2776 & 906 & 2 & 27 & 0 \\
\hline $\begin{array}{r}\text { Biosled } \\
\text { Rolls }\end{array}$ & 0.0145 & 0.0199 & 965 & 107 & 23 & 0 \\
\hline CG2 & 0.0203 & 0.2719 & 925 & 228 & 31 & 0 \\
\hline CG2 Rolls & 0.0222 & 0.0103 & 1002 & 175 & 21 & 0 \\
\hline CSA & 0.0237 & 3.0206 & 852 & 383 & 30 & 0 \\
\hline CSA Rolls & 0.0349 & 4.8668 & 905 & 191 & 20 & 0 \\
\hline$L 1$ & 0.0379 & 0.0041 & 925 & 0 & 28 & 0 \\
\hline$M F F$ & 0.0556 & 2.8755 & 952 & 165 & 32 & 0 \\
\hline$S M M$ & 0.0192 & 0.2111 & 705 & 4 & 34 & 0 \\
\hline
\end{tabular}




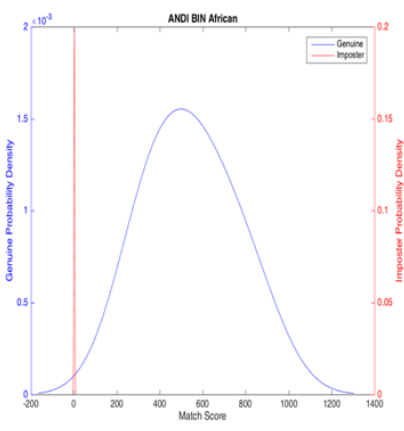

(a)

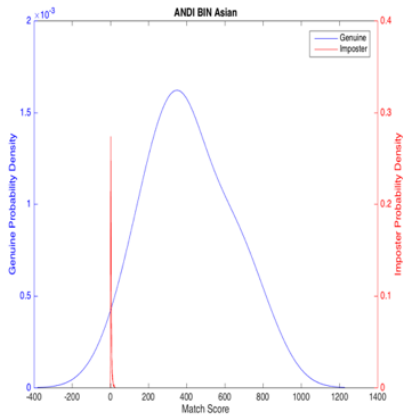

(d)

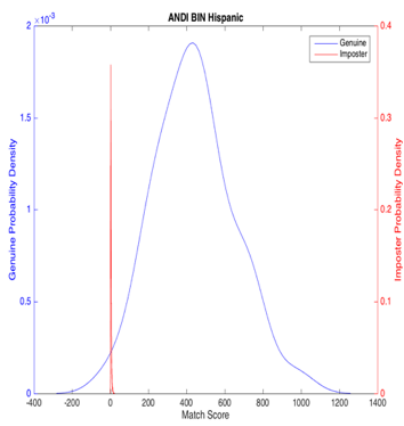

(g)

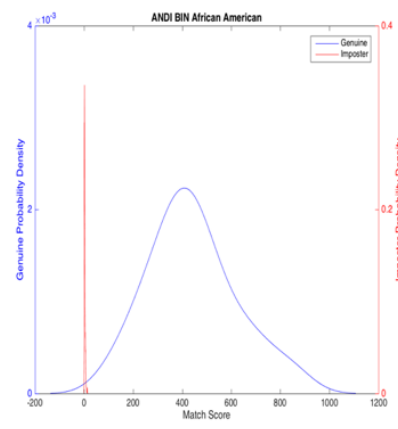

(b)

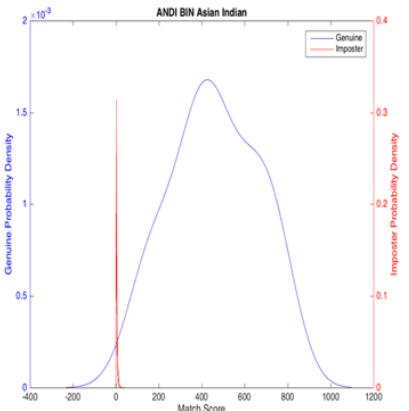

(e)

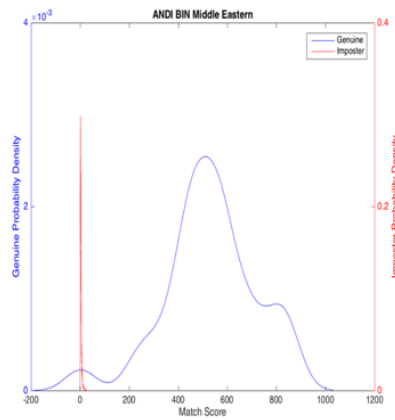

(h)

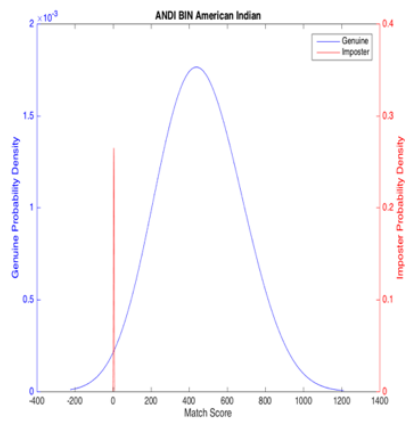

(c)

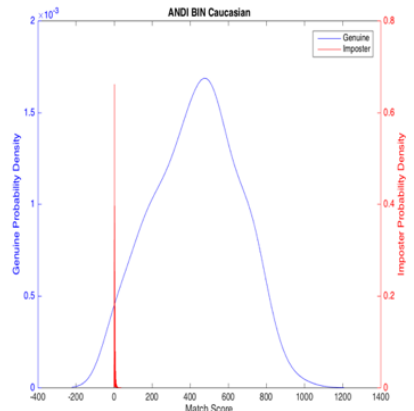

(f)

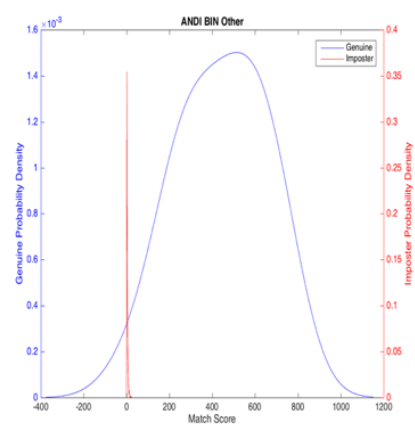

(i)

Figure D.2.1 Neurotech ANDI Binary (a) African (b) African American (c) American Indian (d) Asian (e) Asian Indian (f) Caucasian (g) Hispanic (h) Middle Eastern (i) Other 


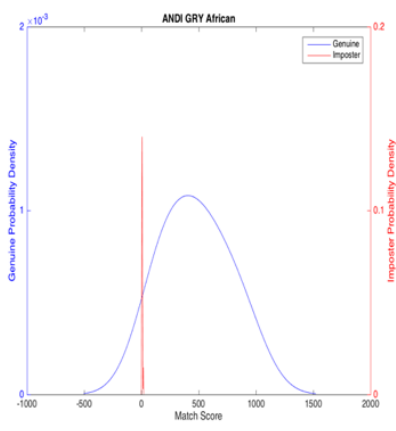

(a)

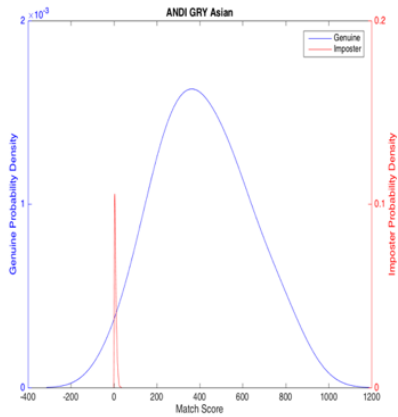

(d)

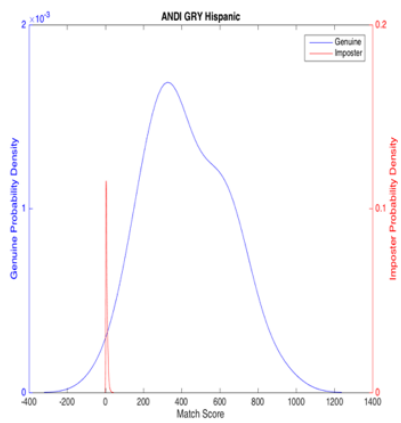

(g)

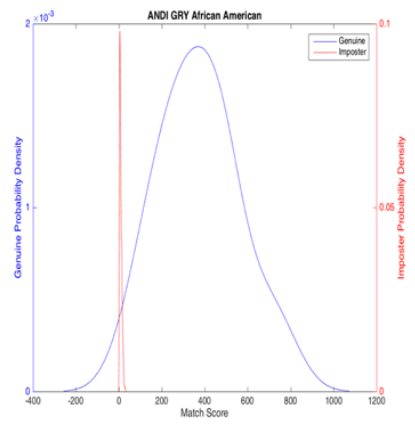

(b)

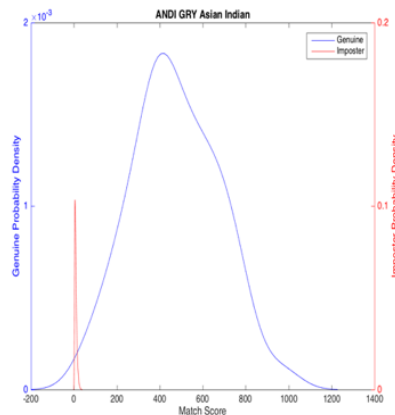

(e)

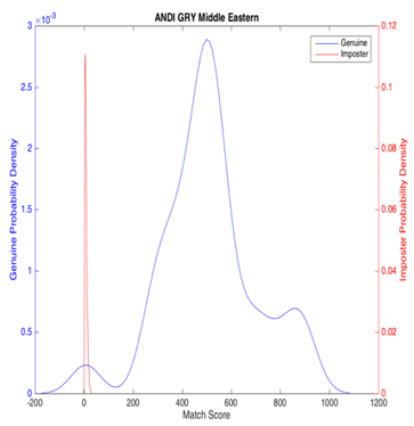

(h)

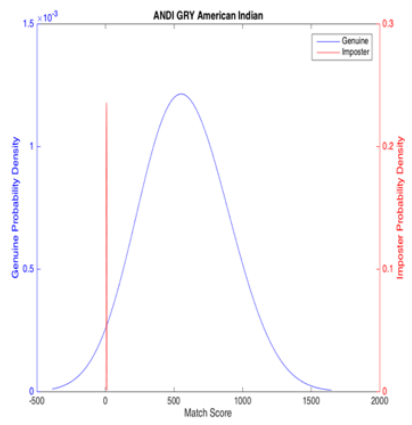

(c)

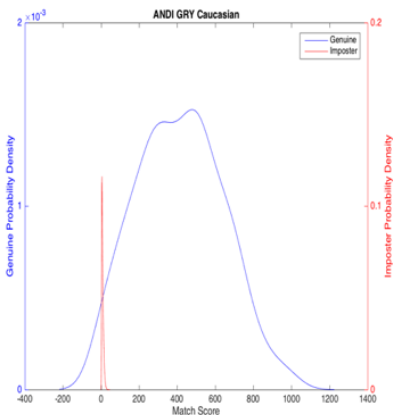

(f)

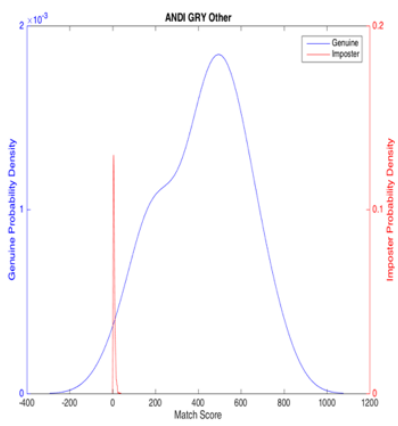

(i)

Figure D.2.2 Neurotech ANDI Greyscale (a) African (b) African American (c) American Indian (d) Asian (e) Asian Indian (f) Caucasian (g) Hispanic (h) Middle Eastern (i) Other 


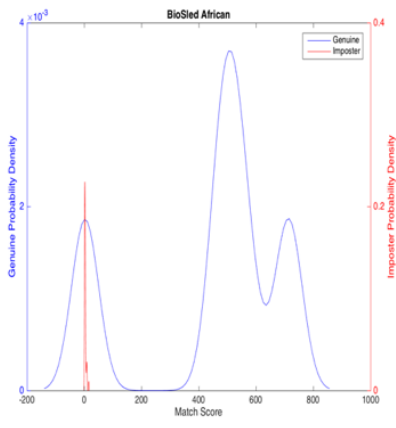

(a)

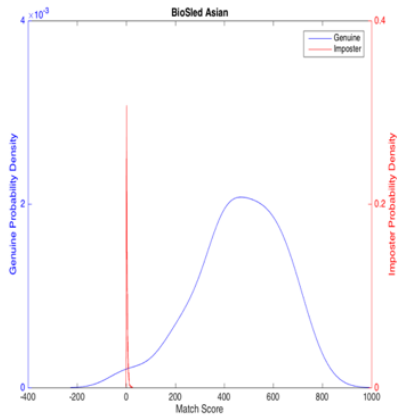

(d)

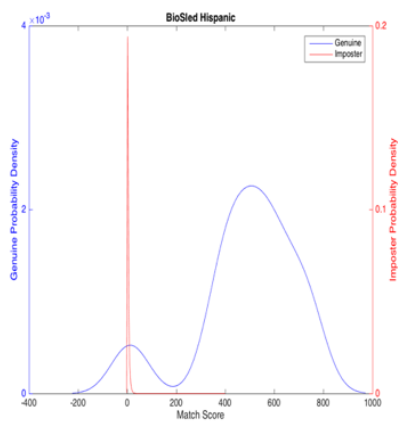

(g)

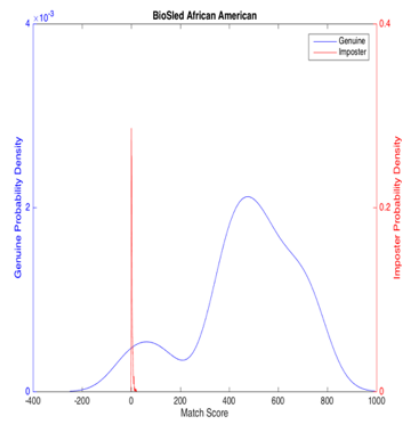

(b)

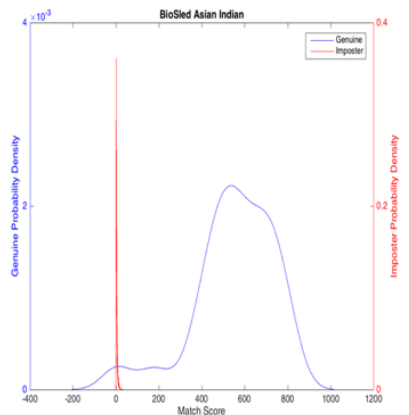

(e)

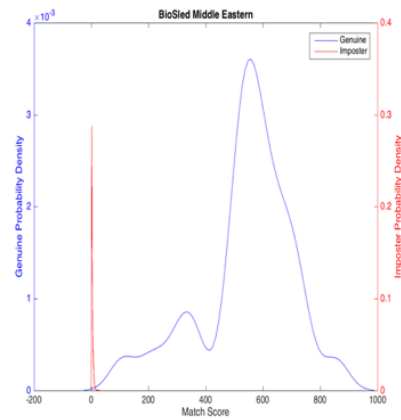

(h)

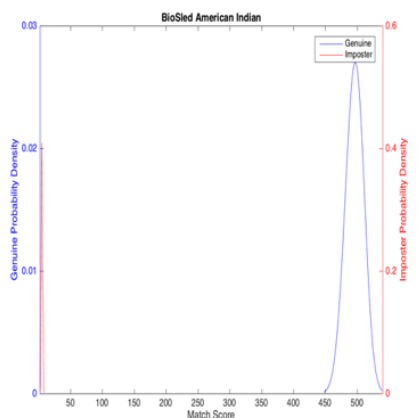

(c)

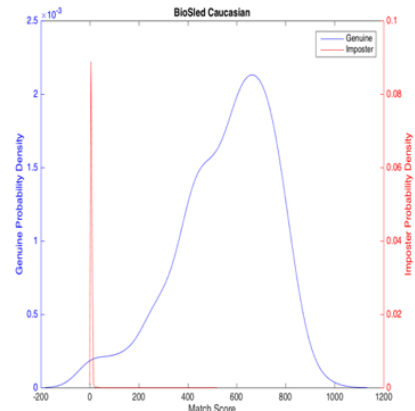

(f)

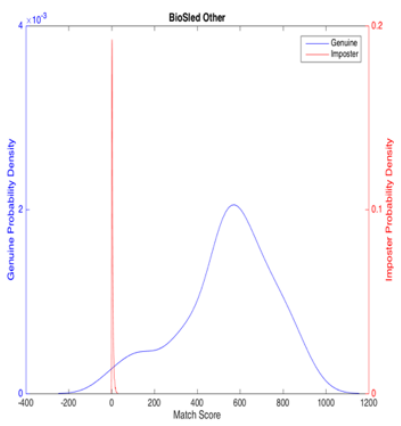

(i)

Figure D.2.3 Neurotech BioSled (a) African (b) African American (c) American Indian (d) Asian (e) Asian Indian (f) Caucasian (g) Hispanic (h) Middle Eastern (i) Other 


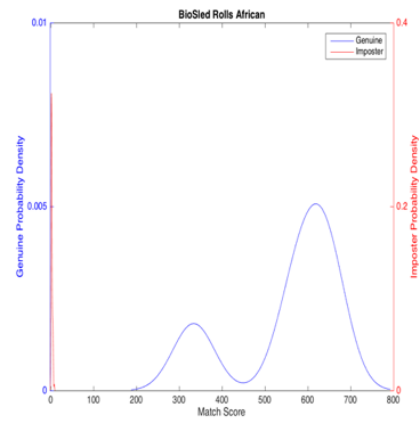

(a)

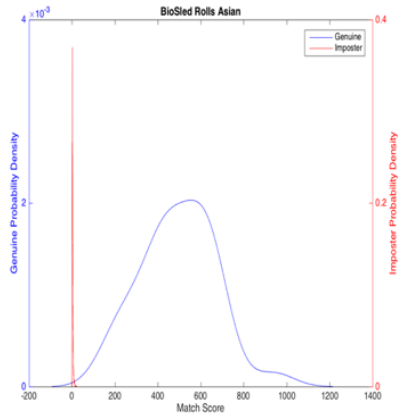

(d)

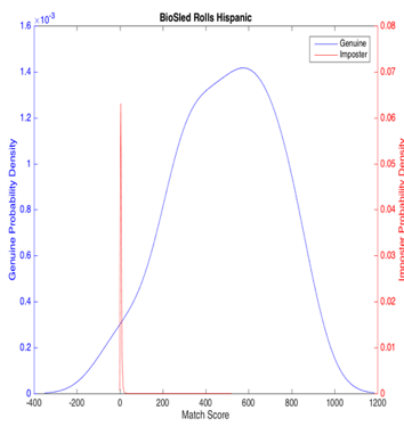

(g)

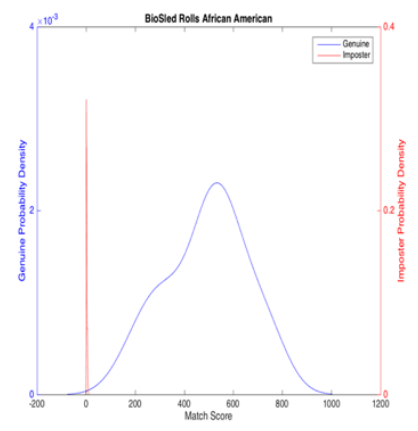

(b)

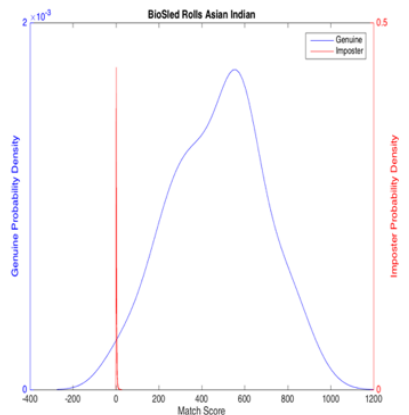

(e)

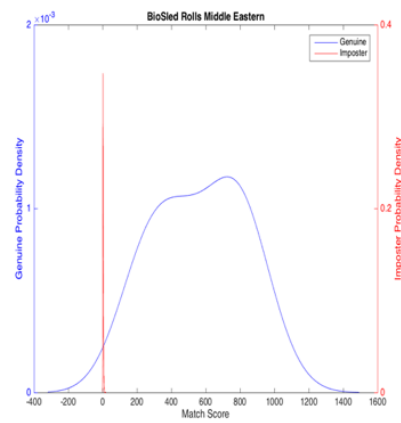

(h)

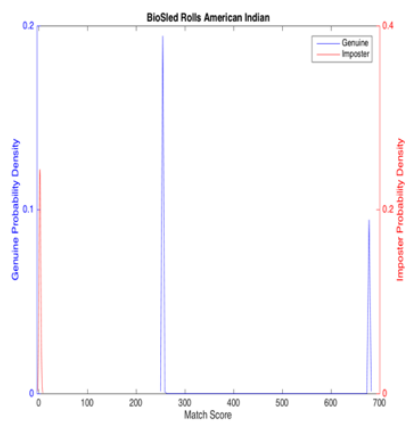

(c)

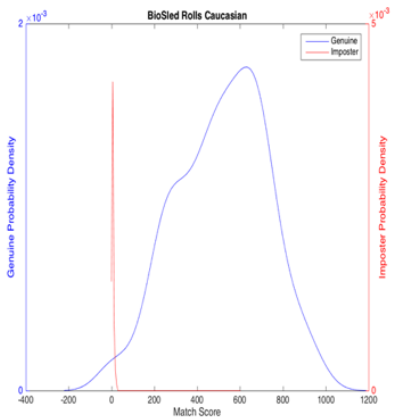

(f)

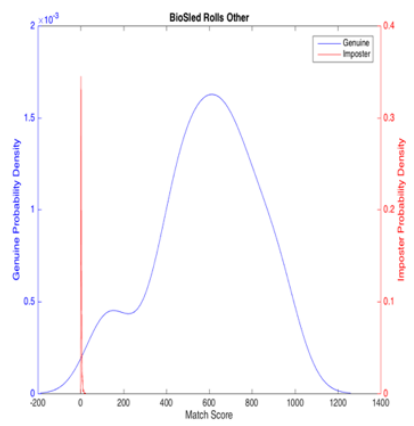

(i)

Figure D.2.4 Neurotech BioSled Rolls (a) African (b) African American (c) American Indian (d) Asian (e) Asian Indian (f) Caucasian (g) Hispanic (h) Middle Eastern (i) Other 


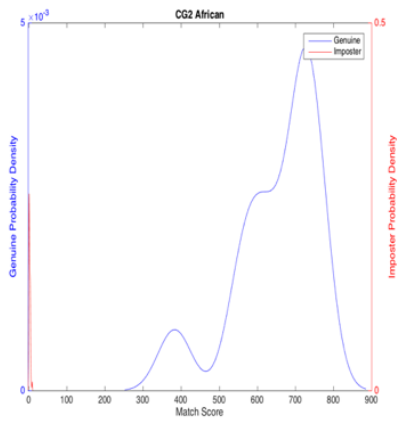

(a)

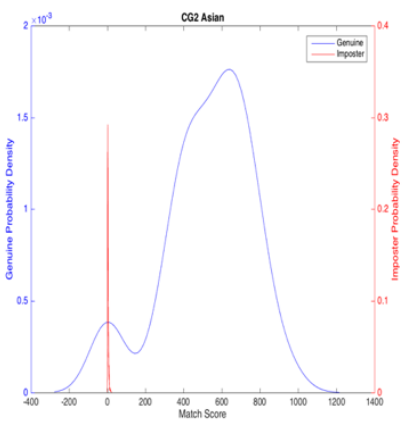

(d)

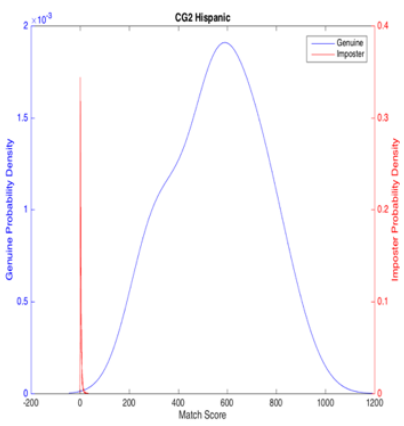

(g)

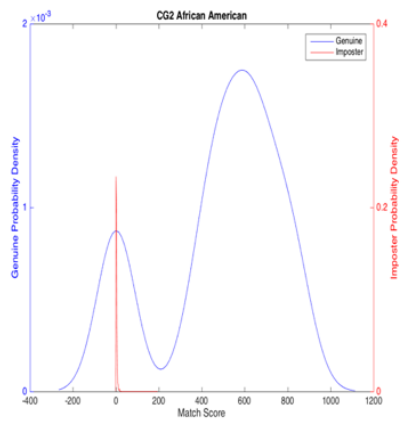

(b)

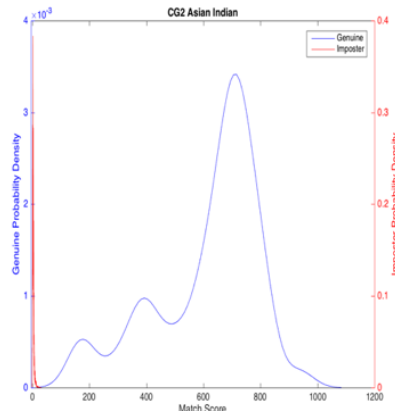

(e)

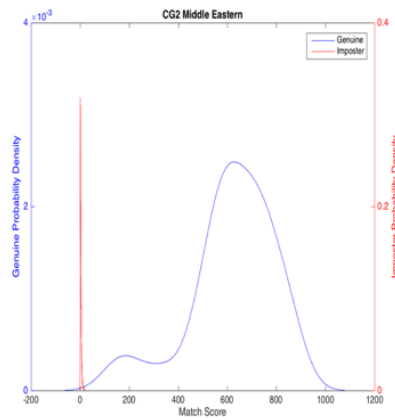

(h)

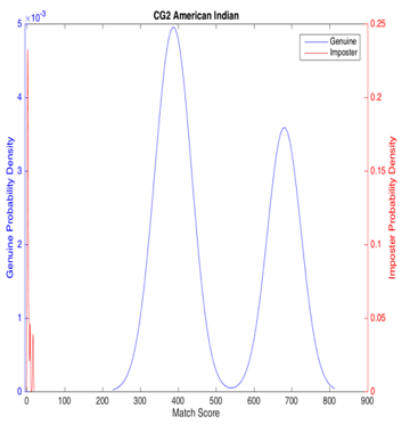

(c)

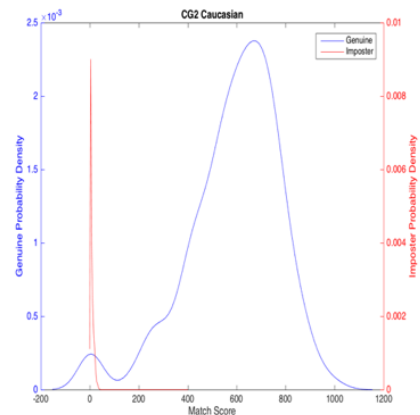

(f)

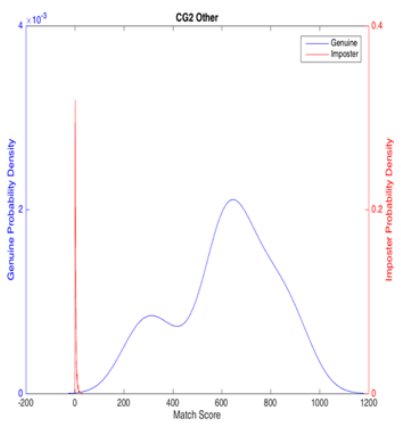

(i)

Figure D.2.5 Neurotech FP II Guardian (a) African (b) African American (c) American Indian (d) Asian (e) Asian Indian (f) Caucasian (g) Hispanic (h) Middle Eastern (i) Other 


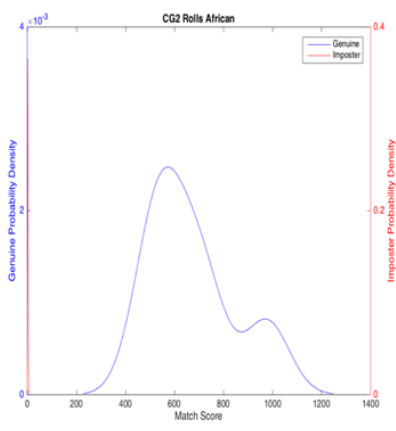

(a)

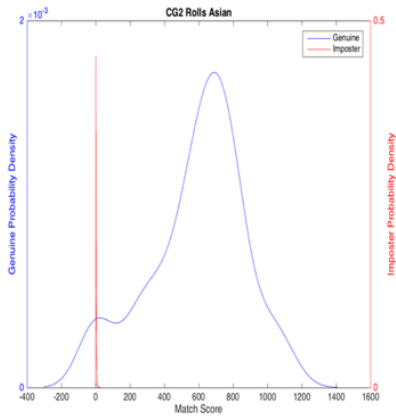

(d)

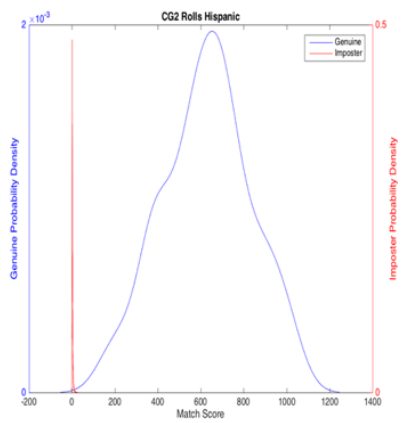

(g)

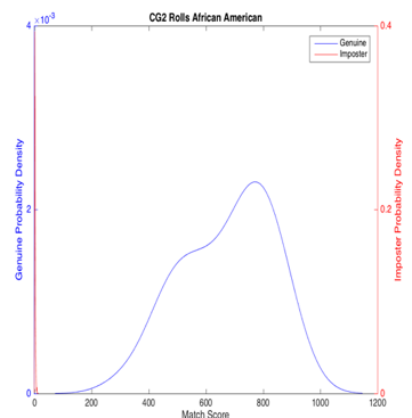

(b)

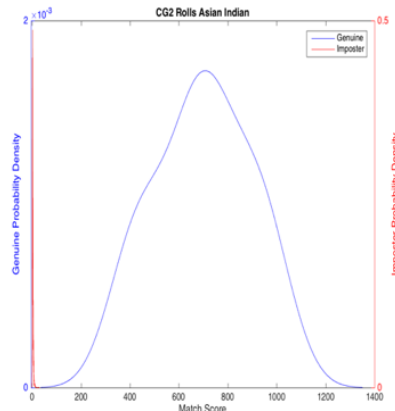

(e)

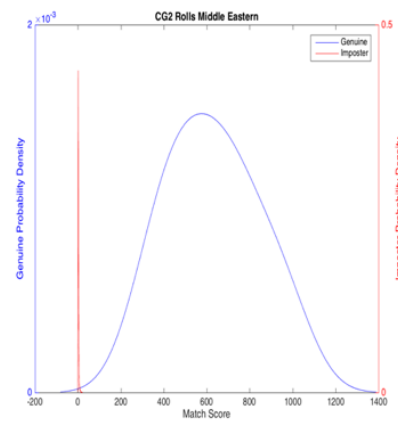

(h)

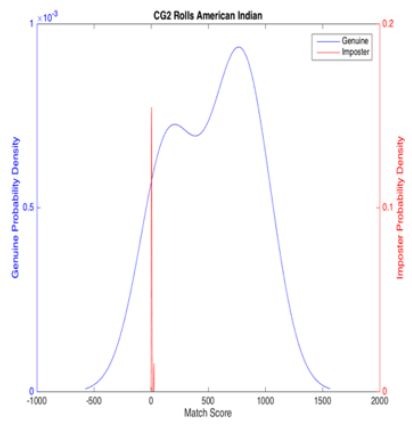

(c)

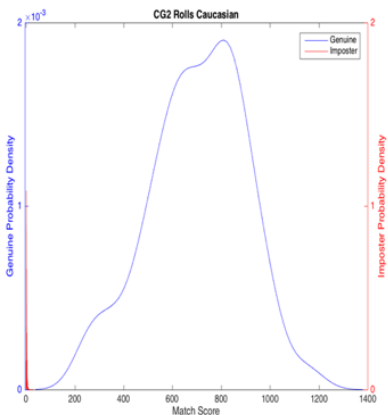

(f)

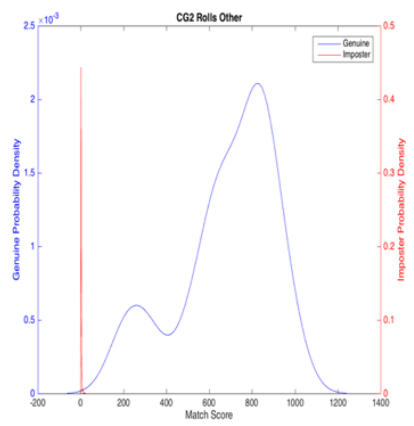

(i)

Figure D.2.6 Neurotech FP II Guardian Rolls (a) African (b) African American (c) American Indian (d) Asian (e) Asian Indian (f) Caucasian (g) Hispanic (h) Middle Eastern (i) Other 


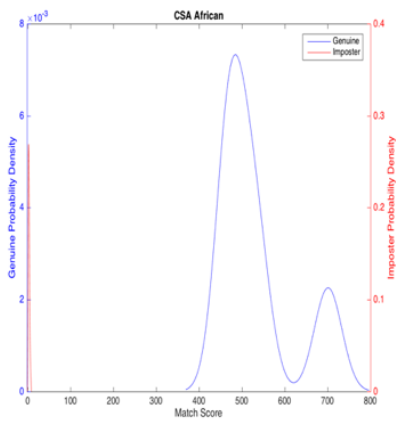

(a)

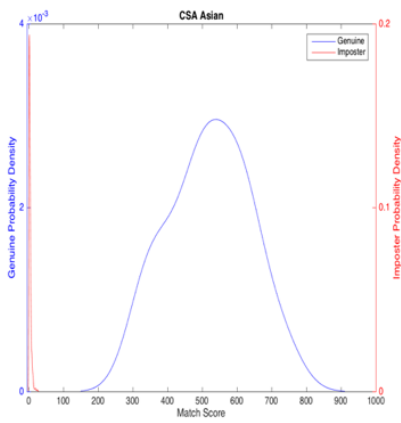

(d)

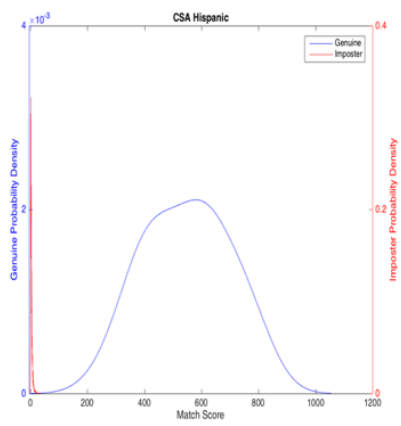

(g)

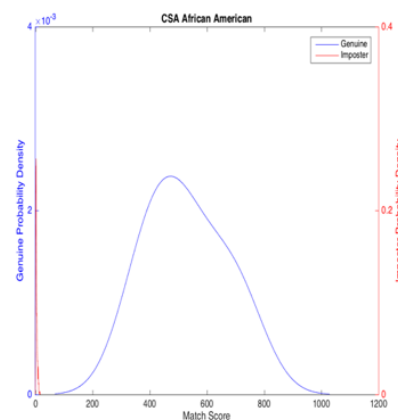

(b)

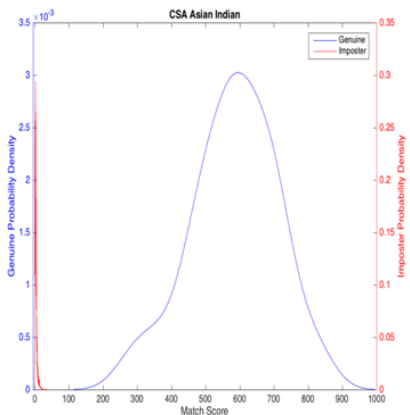

(e)

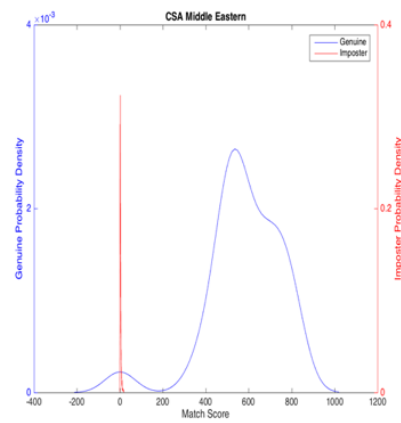

(h)

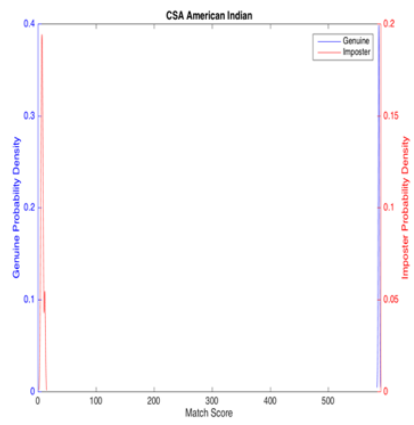

(c)

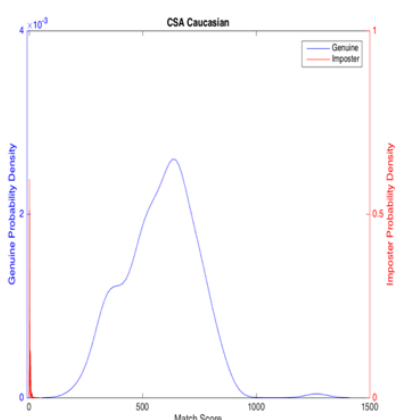

(f)

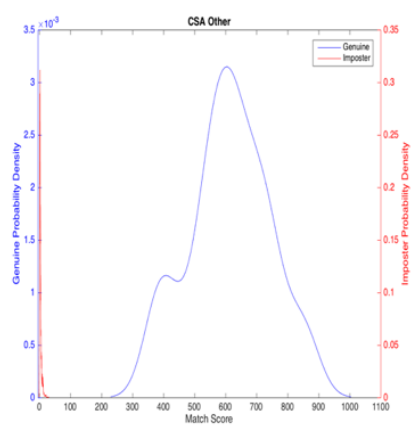

(i)

Figure D.2.7 Neurotech Crossmatch Seek Avenger (a) African (b) African American (c) American Indian (d) Asian (e) Asian Indian (f) Caucasian (g) Hispanic (h) Middle Eastern (i) Other 


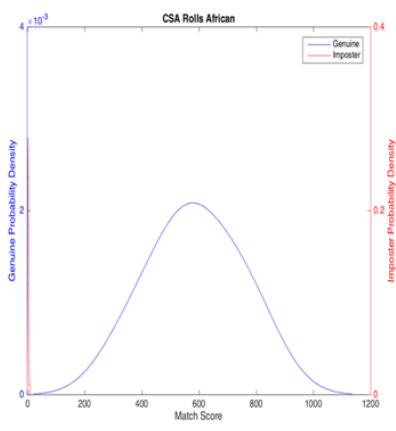

(a)

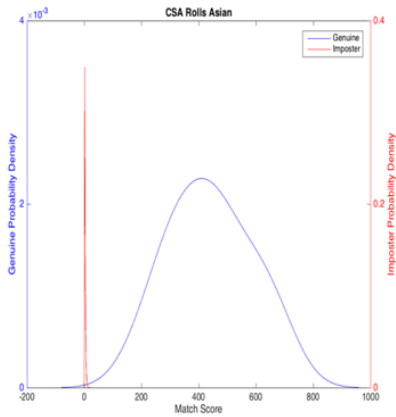

(d)

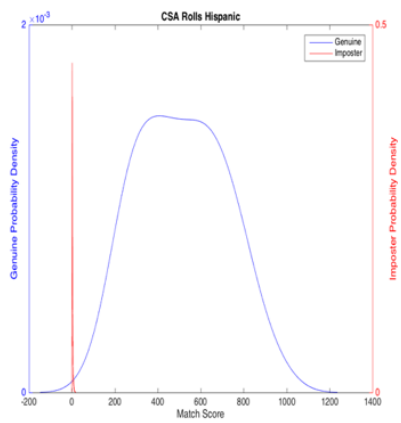

(g)

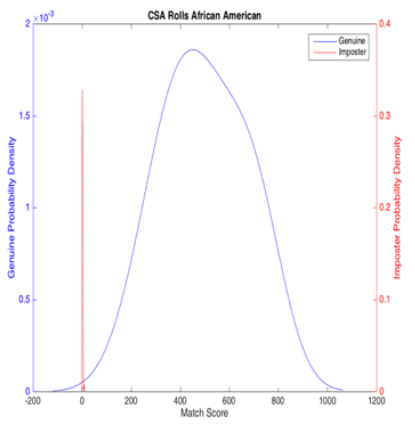

(b)

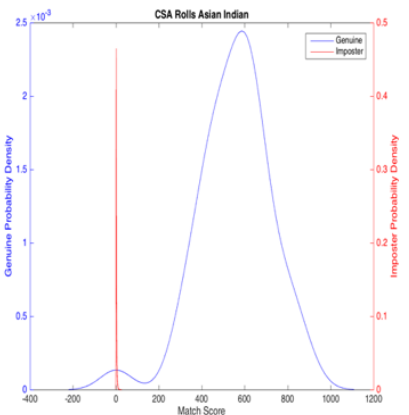

(e)

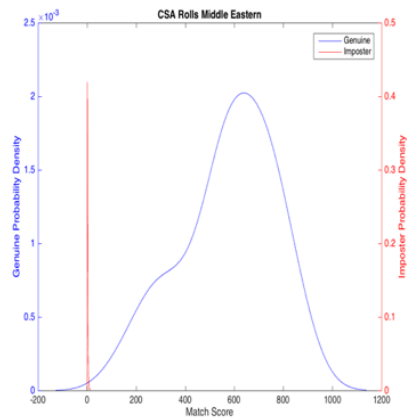

(h)

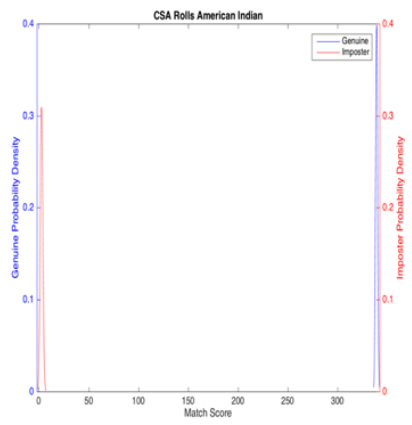

(c)

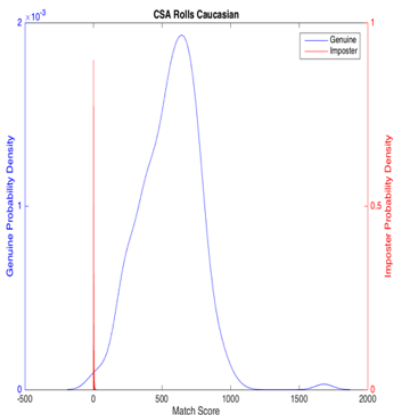

(f)

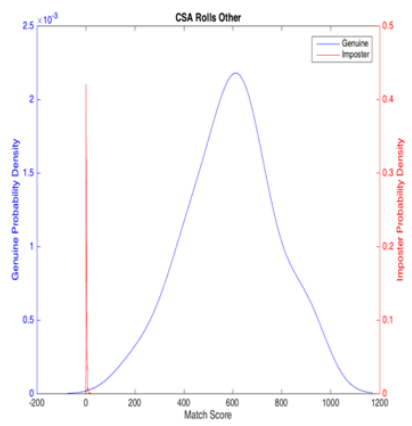

(i)

Figure D.1.8 Neurotech Crossmatch Seek Avenger Rolls (a) African (b) African American (c) American Indian (d) Asian (e) Asian Indian (f) Caucasian (g) Hispanic (h) Middle Eastern (i) Other 
(a)

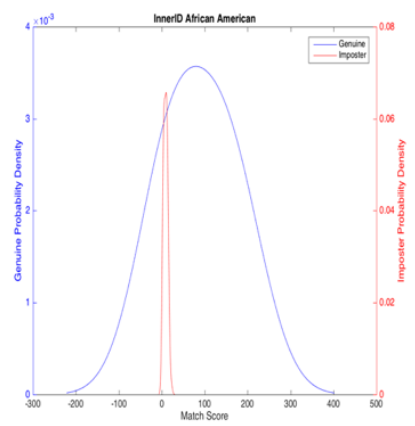

(b)

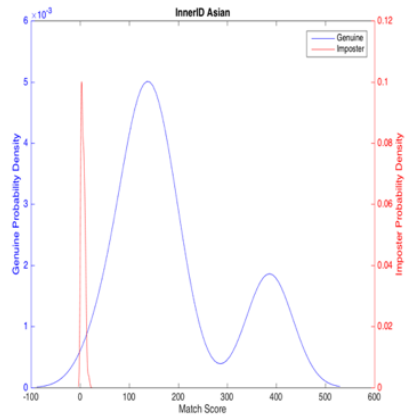

(d)

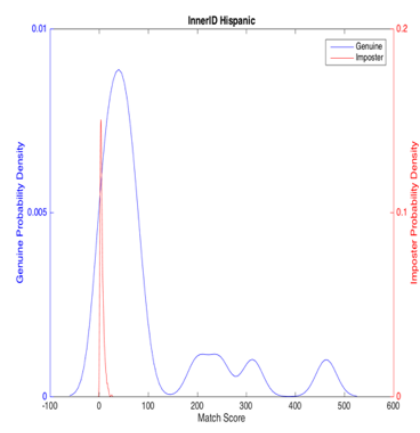

(g)

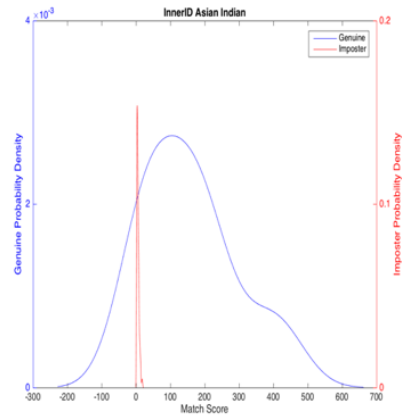

(e)

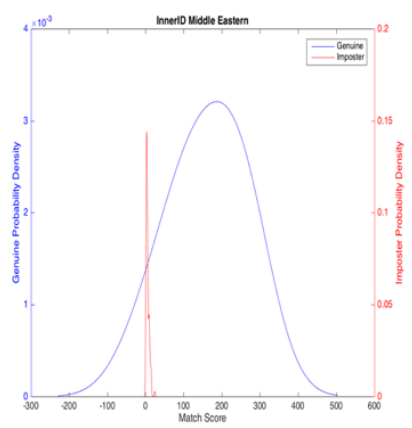

(h) (c)

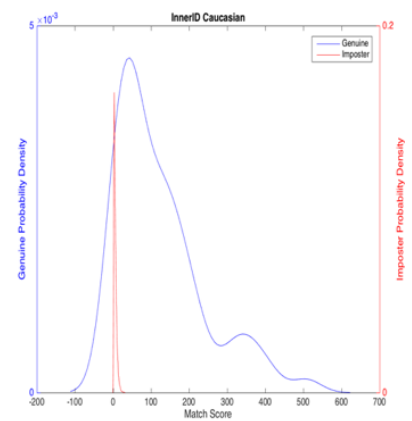

(f)

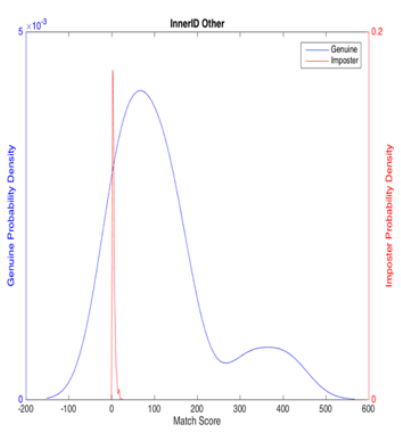

(i)

Figure D.2.9 Neurotech InnerID (a) African (b) African American (c) American Indian (d) Asian (e) Asian Indian (f) Caucasian (g) Hispanic (h) Middle Eastern (i) Other

(Some Results aren't shown because of this device's low quality images and stopping operation midway through collection) 


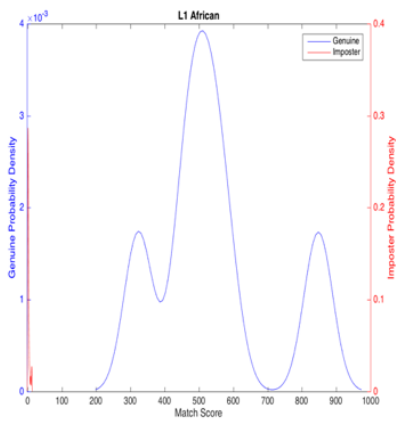

(a)

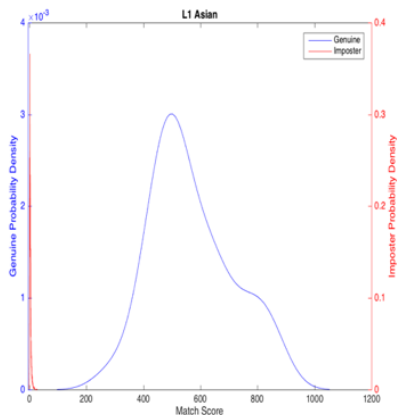

(d)

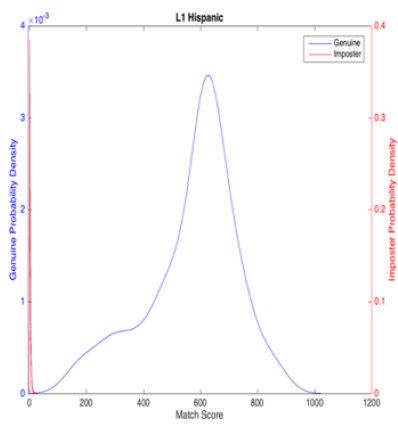

(g)

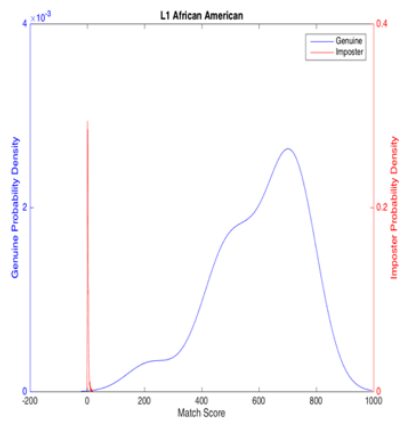

(b)

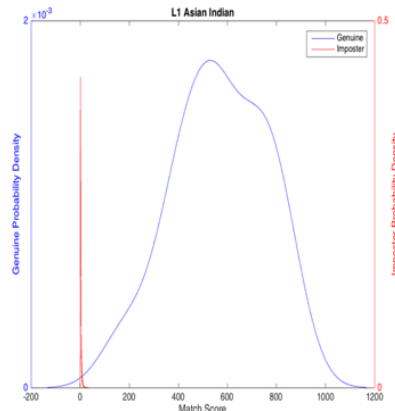

(e)

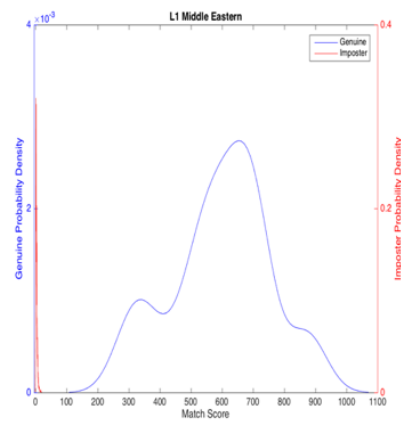

(h)

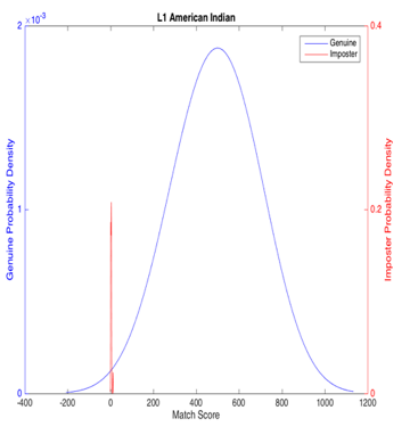

(c)

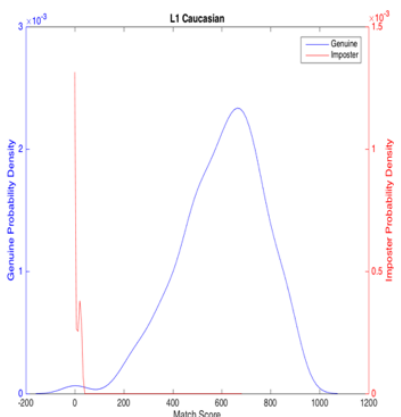

(f)

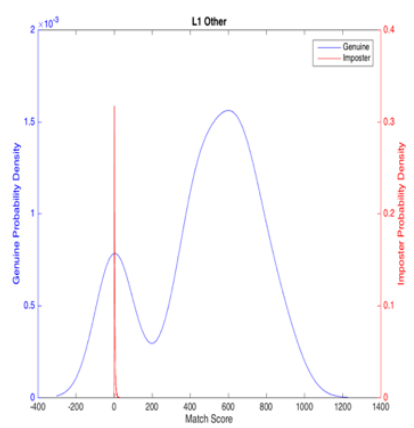

(i)

Figure D.2.10 Neurotech L1 (a) African (b) African American (c) American Indian (d) Asian (e) Asian Indian (f) Caucasian (g) Hispanic (h) Middle Eastern (i) Other 
(a)

(b)

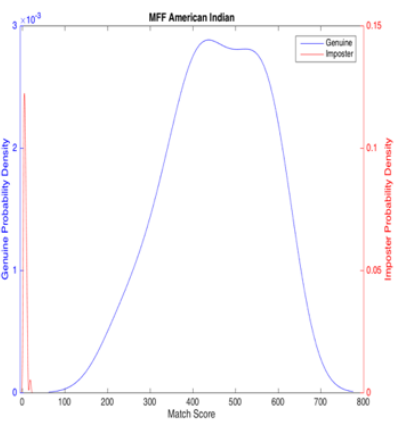

(e)

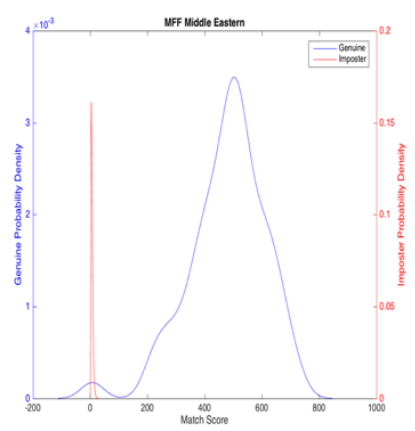

(h)

$(\mathrm{g})$

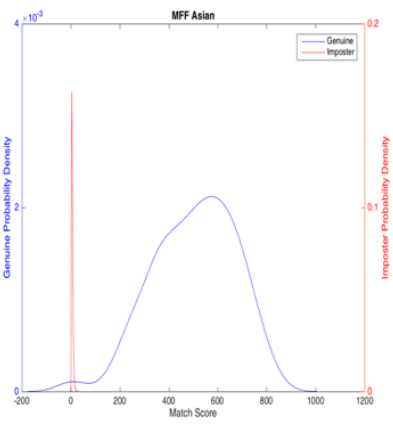

(c)

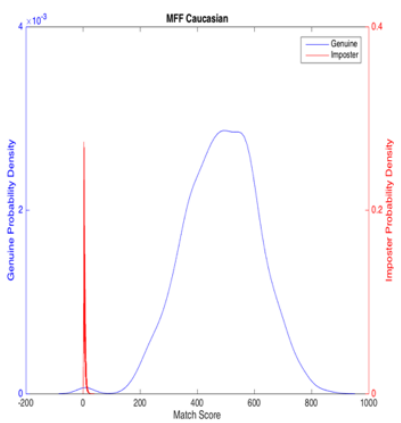

(f)

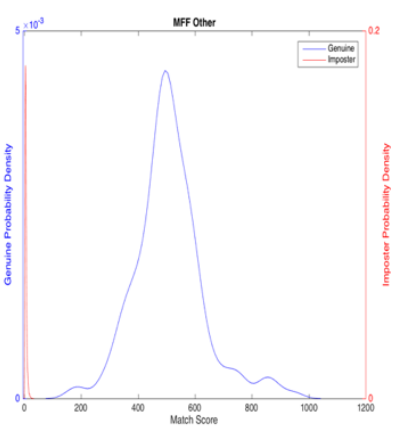

(i)

Figure D.2.11 Neurotech Morpho FOTF (a) African (b) African American (c) American Indian (d) Asian (e) Asian Indian (f) Caucasian (g) Hispanic (h) Middle Eastern (i) Other

(This device had trouble capturing darker complexions and the Neurotech matcher removed images that were not up to a certain quality so that is why this device could not show distributions for the ethnicities African and African American. 


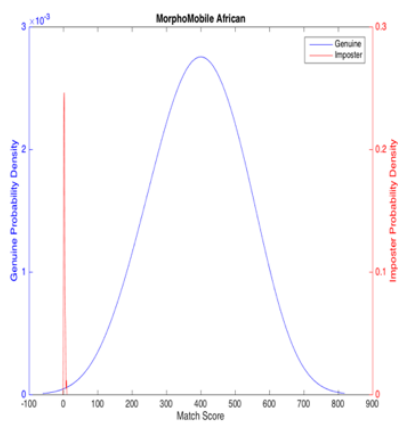

(a)

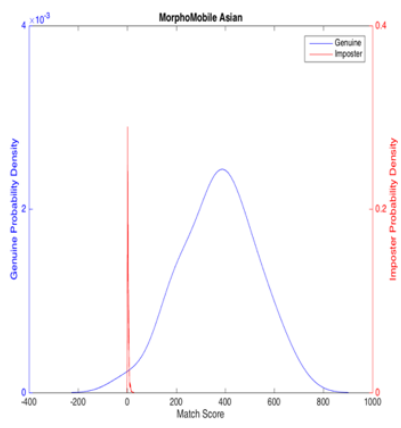

(d)

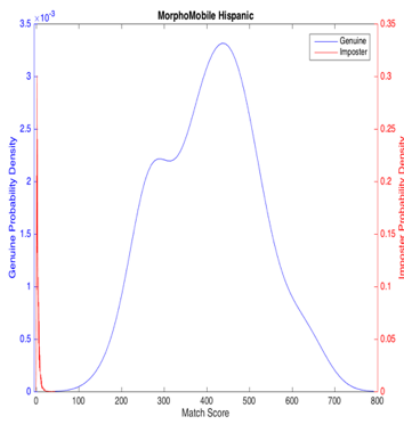

(g)

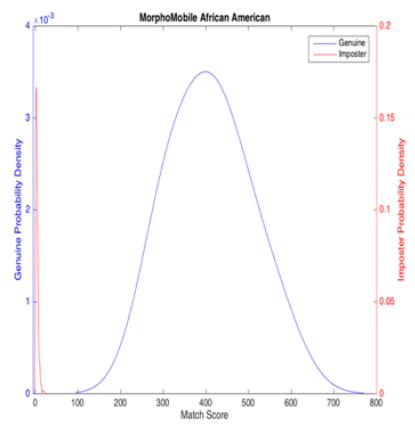

(b)

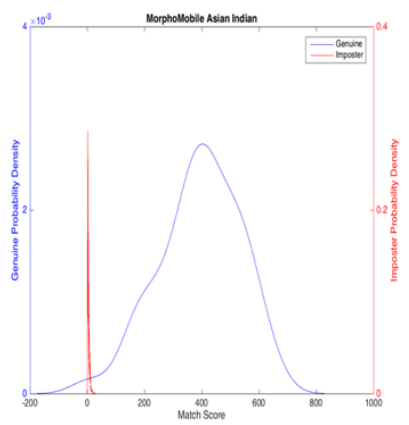

(e)

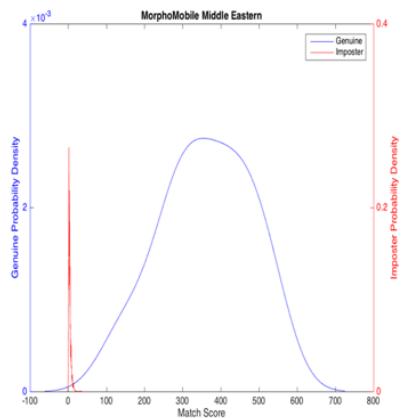

(h) (c)

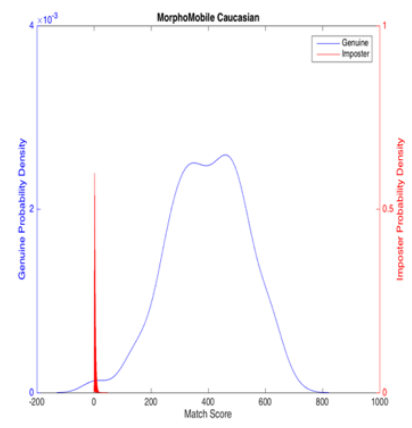

(f)

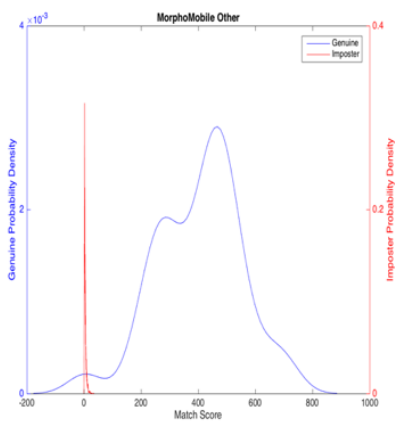

(i)

Figure D.2.12 Neurotech MorphoMobile (a) African (b) African American (c) American Indian (d) Asian (e) Asian Indian (f) Caucasian (g) Hispanic (h) Middle Eastern (i) Other 


\section{D.3 SourceAFIS Genuine/Imposter Score Distributions}

Table D.19 SourceAFIS African Genuine and Imposter KLD, Maximum and Minimum Scores

\begin{tabular}{|c|c|c|c|c|c|c|}
\hline \multicolumn{7}{|c|}{ SourceAFIS African } \\
\hline Device & $\begin{array}{l}\text { Genuine } \\
\text { KLD }\end{array}$ & $\begin{array}{l}\text { Imposter } \\
\text { KLD }\end{array}$ & $\begin{array}{l}\text { Max } \\
\text { Genuine }\end{array}$ & $\begin{array}{l}\text { Min } \\
\text { Genuine }\end{array}$ & $\begin{array}{l}\text { Max } \\
\text { Imposter }\end{array}$ & $\begin{array}{l}\text { Min } \\
\text { Imposter }\end{array}$ \\
\hline$A N D I B I N$ & 0.2958 & 2.7067 & 259.1137 & 26.29867 & 19.56224 & 9.071217 \\
\hline$A N D I G R Y$ & 0.4007 & 1.5936 & 219.8125 & 13.64682 & 19.78427 & 10.34025 \\
\hline Biosled & 0.4054 & 2.0859 & 116.7156 & 18.48837 & 23.66089 & 8.728425 \\
\hline $\begin{array}{r}\text { Biosled } \\
\text { Rolls }\end{array}$ & 0.0877 & 1.3681 & 232.7462 & 90.15849 & 18.7267 & 9.510126 \\
\hline$C G 2$ & 0.1495 & 3.1481 & 126.3058 & 52.07077 & 19.61867 & 8.699016 \\
\hline CG2 Rolls & 0.1186 & 1.4545 & 284.1035 & 136.0848 & 18.56935 & 8.164021 \\
\hline CSA & 0.1544 & 1.8807 & 145.7916 & 83.77911 & 25.81906 & 9.968618 \\
\hline CSA Rolls & 0.0627 & 1.3722 & 331.1613 & 140.573 & 21.13575 & 9.915087 \\
\hline L1 & 0.1761 & 1.9177 & 191.7825 & 55.52259 & 20.45505 & 9.566579 \\
\hline L1 Rolls & 0.0652 & 2.1790 & 324.0332 & 98.4247 & 18.31746 & 8.345504 \\
\hline$M F F$ & 0.0991 & 3.9406 & 137.2591 & 11.3185 & 17.39243 & 6.982936 \\
\hline$S M M$ & 0.1763 & 2.2796 & 115.0201 & 38.36327 & 22.87316 & 9.75632 \\
\hline
\end{tabular}

Table D.20 SourceAFIS African American Genuine and Imposter KLD, Maximum and Minimum Scores

\begin{tabular}{|c|c|c|c|c|c|c|}
\hline \multicolumn{7}{|c|}{ SourceAFIS African American } \\
\hline Device & $\begin{array}{l}\text { Genuine } \\
\text { KLD }\end{array}$ & $\begin{array}{l}\text { Imposter } \\
\text { KLD }\end{array}$ & $\begin{array}{l}\text { Max } \\
\text { Genuine }\end{array}$ & $\begin{array}{l}\text { Min } \\
\text { Genuine }\end{array}$ & $\begin{array}{l}\text { Max } \\
\text { Imposter }\end{array}$ & $\begin{array}{l}\text { Min } \\
\text { Imposter }\end{array}$ \\
\hline$A N D I B I N$ & 0.1160 & 1.6159 & 238.8023 & 33.84354 & 26.73114 & 6.897929 \\
\hline$A N D I G R Y$ & 0.1484 & 1.7079 & 152.3309 & 12.71153 & 26.36873 & 8.367515 \\
\hline Biosled & 0.1706 & 2.0052 & 198.0786 & 9.413997 & 22.15639 & 9.434989 \\
\hline $\begin{array}{r}\text { BioSled } \\
\text { Rolls }\end{array}$ & 0.0599 & 1.8500 & 274.1496 & 30.08374 & 26.01874 & 7.327523 \\
\hline$C G 2$ & 0.2349 & 0.4904 & 167.3741 & 11.76633 & 56.35846 & 8.749595 \\
\hline CG2 Rolls & 0.0525 & 1.0825 & 305.1128 & 58.29882 & 25.08028 & 8.702084 \\
\hline CSA & 0.2122 & 1.8233 & 136.6192 & 33.32489 & 20.97421 & 9.594802 \\
\hline CSA Rolls & 0.1688 & 1.5454 & 275.242 & 16.3768 & 21.24726 & 9.086631 \\
\hline$L 1$ & 0.1865 & 3.9513 & 213.8614 & 46.14794 & 23.99998 & 8.074486 \\
\hline L1 Rolls & 0.0430 & 1.5083 & 324.7177 & 10.10076 & 23.43524 & 6.591611 \\
\hline$M F F$ & 0.0971 & 6.1422 & 170.7587 & 11.29338 & 24.38368 & 2.894054 \\
\hline$S M M$ & 0.2903 & 4.0777 & 100.2059 & 29.83898 & 19.79535 & 8.270656 \\
\hline
\end{tabular}


Table D.21 SourceAFIS American Indian Genuine and Imposter KLD, Maximum and Minimum Scores

\begin{tabular}{|c|c|c|c|c|c|c|}
\hline \multicolumn{7}{|c|}{ SourceAFIS American Indian } \\
\hline Device & $\begin{array}{l}\text { Genuine } \\
\text { KLD }\end{array}$ & $\begin{array}{l}\text { Imposter } \\
\text { KLD }\end{array}$ & $\begin{array}{l}\text { Max } \\
\text { Genuine }\end{array}$ & $\begin{array}{l}\text { Min } \\
\text { Genuine }\end{array}$ & $\begin{array}{l}\text { Max } \\
\text { Imposter }\end{array}$ & $\begin{array}{l}\text { Min } \\
\text { Imposter }\end{array}$ \\
\hline$A N D I B I N$ & 0.1132 & 4.7527 & 142.8334 & 64.82221 & 16.79907 & 9.212389 \\
\hline$A N D I G R Y$ & 0.2532 & 1.3756 & 122.2119 & 32.08049 & 17.0113 & 11.38951 \\
\hline Biosled & 1.6077 & 1.8946 & 129.9908 & 129.2516 & 15.70051 & 12.04497 \\
\hline $\begin{array}{r}\text { BioSled } \\
\text { Rolls }\end{array}$ & 0.1340 & 1.8281 & 274.3735 & 92.58033 & 19.93548 & 11.77341 \\
\hline$C G 2$ & 11.5257 & 3.1644 & 131.4841 & 90.385 & 16.02594 & 10.15785 \\
\hline CG2 Rolls & 0.2110 & 1.2978 & 318.4995 & 13.73975 & 15.39719 & 10.69792 \\
\hline CSA & 1.4274 & 2.5726 & 140.2756 & 139.9248 & 15.67848 & 12.68303 \\
\hline CSA Rolls & 1.1587 & 1.6133 & 133.718 & 133.6721 & 18.78525 & 12.4854 \\
\hline$L 1$ & 0.3275 & 2.1223 & 178.5928 & 147.2782 & 17.46229 & 10.76368 \\
\hline L1 Rolls & 0.3340 & 1.8069 & 169.3093 & 20.85485 & 17.94952 & 9.443677 \\
\hline$M F F$ & 0.2095 & 1.8234 & 71.79872 & 41.32655 & 17.834 & 11.50585 \\
\hline
\end{tabular}

Table D.22 SourceAFIS Asian Genuine and Imposter KLD, Maximum and Minimum Scores

\begin{tabular}{|c|c|c|c|c|c|c|}
\hline \multicolumn{7}{|c|}{ SourceAFIS Asian } \\
\hline Device & $\begin{array}{l}\text { Genuine } \\
\text { KLD }\end{array}$ & $\begin{array}{l}\text { Imposter } \\
\text { KLD }\end{array}$ & $\begin{array}{l}\text { Max } \\
\text { Genuine }\end{array}$ & $\begin{array}{l}\text { Min } \\
\text { Genuine }\end{array}$ & $\begin{array}{l}\text { Max } \\
\text { Imposter }\end{array}$ & $\begin{array}{l}\text { Min } \\
\text { Imposter }\end{array}$ \\
\hline$A N D I B I N$ & 0.0840 & 4.7585 & 194.8807 & 11.06559 & 22.97893 & 5.939764 \\
\hline$A N D I G R Y$ & 0.1145 & 5.1985 & 174.2071 & 12.11068 & 22.63984 & 4.346674 \\
\hline Biosled & 0.1331 & 2.9401 & 163.281 & 13.01383 & 24.57107 & 7.623753 \\
\hline $\begin{array}{r}\text { Biosled } \\
\text { Rolls }\end{array}$ & 0.0268 & 1.5402 & 298.9304 & 11.42592 & 26.4133 & 7.951399 \\
\hline CG2 & 0.1013 & 4.2584 & 201.406 & 10.05764 & 25.60952 & 7.072711 \\
\hline CG2 Rolls & 0.0552 & 2.3071 & 288.6129 & 10.53184 & 27.77499 & 6.210144 \\
\hline CSA & 0.2142 & 1.0064 & 151.9046 & 40.32472 & 26.87905 & 6.870134 \\
\hline CSA Rolls & 0.0797 & 4.0234 & 256.5767 & 12.10818 & 32.35595 & 6.524864 \\
\hline$L 1$ & 0.2285 & 3.3654 & 172.7331 & 11.1369 & 26.66762 & 6.883381 \\
\hline L1 Rolls & 0.0987 & 1.2757 & 247.3902 & 55.72922 & 26.01221 & 5.831905 \\
\hline$M F F$ & 0.1283 & 2.5673 & 252.5184 & 9.009671 & 32.68176 & 5.042907 \\
\hline$S M M$ & 0.0760 & 6.0662 & 159.7176 & 12.4216 & 21.77014 & 7.413802 \\
\hline
\end{tabular}


Table D.23 SourceAFIS Asian Indian Genuine and Imposter KLD, Maximum and Minimum Scores

\begin{tabular}{|c|c|c|c|c|c|c|}
\hline \multicolumn{7}{|c|}{ SourceAFIS Asian Indian } \\
\hline Device & $\begin{array}{l}\text { Genuine } \\
\text { KLD }\end{array}$ & $\begin{array}{l}\text { Imposter } \\
\text { KLD }\end{array}$ & $\begin{array}{l}\text { Max } \\
\text { Genuine }\end{array}$ & $\begin{array}{l}\text { Min } \\
\text { Genuine }\end{array}$ & $\begin{array}{l}\text { Max } \\
\text { Imposter }\end{array}$ & $\begin{array}{l}\text { Min } \\
\text { Imposter }\end{array}$ \\
\hline$A N D I B I N$ & 0.0501 & 3.6349 & 238.4998 & 13.34194 & 25.44888 & 5.948489 \\
\hline ANDI GRY & 0.1811 & 1.6904 & 163.7371 & 10.82603 & 33.1993 & 4.561862 \\
\hline Biosled & 0.1587 & 3.2662 & 213.3672 & 11.49542 & 32.63971 & 6.999399 \\
\hline $\begin{array}{r}\text { BioSled } \\
\text { Rolls }\end{array}$ & 0.0287 & 59.8285 & 303.5761 & 11.1294 & 30.26171 & 2.765737 \\
\hline CG2 & 0.1493 & 2.9030 & 229.8958 & 15.6628 & 32.53723 & 7.979208 \\
\hline CG2 Rolls & 0.0658 & 3.8008 & 335.2613 & 50.97131 & 33.62403 & 4.382007 \\
\hline CSA & 0.2086 & 1.0524 & 213.7571 & 25.56576 & 28.16516 & 6.971332 \\
\hline CSA Rolls & 0.1789 & 1.6509 & 278.3809 & 11.35834 & 27.82566 & 6.459023 \\
\hline$L 1$ & 0.1051 & 2.0692 & 245.6121 & 18.29633 & 35.7662 & 6.872118 \\
\hline L1 Rolls & 0.0603 & 2.1540 & 275.835 & 11.6884 & 25.35682 & 3.819754 \\
\hline$M F F$ & 0.0915 & 0.9591 & 236.7135 & 10.92929 & 29.63063 & 3.892233 \\
\hline$S M M$ & 0.2404 & 7.7016 & 150.498 & 10.04467 & 28.08446 & 5.794656 \\
\hline
\end{tabular}

Table D.24 SourceAFIS Caucasian Genuine and Imposter KLD, Maximum and Minimum Scores

\begin{tabular}{|c|c|c|c|c|c|c|}
\hline \multicolumn{7}{|c|}{ SourceAFIS Caucasian } \\
\hline Device & $\begin{array}{l}\text { Genuine } \\
\text { KLD }\end{array}$ & $\begin{array}{l}\text { Imposter } \\
\text { KLD }\end{array}$ & $\begin{array}{l}\text { Max } \\
\text { Genuine }\end{array}$ & $\begin{array}{l}\text { Min } \\
\text { Genuine }\end{array}$ & $\begin{array}{l}\text { Max } \\
\text { Imposter }\end{array}$ & $\begin{array}{l}\text { Min } \\
\text { Imposter }\end{array}$ \\
\hline ANDI BIN & 0.0217 & 4.9814 & 258.8593 & 9.766249 & 33.64554 & 2.022266 \\
\hline$A N D I G R Y$ & 0.0348 & 5.4394 & 222.0455 & 9.902842 & 40.35332 & 4.321751 \\
\hline Biosled & 0.0809 & 0.5678 & 216.0775 & 10.76042 & 133.0017 & 3.549333 \\
\hline $\begin{array}{r}\text { Biosled } \\
\text { Rolls }\end{array}$ & 0.0195 & 0.3038 & 332.5888 & 10.61221 & 214.1114 & 3.359193 \\
\hline CG2 & 0.0453 & 1.9521 & 229.018 & 9.126302 & 150.8312 & 4.068837 \\
\hline CG2 Rolls & 0.0223 & 6.5154 & 365.2294 & 10.53184 & 37.59151 & 1.0158 \\
\hline CSA & 0.0317 & 0.6262 & 261.1282 & 25.35305 & 32.90313 & 4.415732 \\
\hline CSA Rolls & 0.0125 & 1.1713 & 518.2886 & 11.45706 & 36.75151 & 1.035972 \\
\hline$L 1$ & 0.0174 & 1.1054 & 280.5571 & 12.77533 & 89.70628 & 3.698551 \\
\hline L1 Rolls & 0.0356 & 2.1613 & 324.1864 & 0 & 38.23815 & 0 \\
\hline$M F F$ & 0.0555 & 0.4224 & 254.9791 & 9.659011 & 36.71928 & 1.0158 \\
\hline$S M M$ & 0.0440 & 13.3578 & 166.9086 & 9.806087 & 34.43536 & 1.19943 \\
\hline
\end{tabular}


Table D.25 SourceAFIS Hispanic Genuine and Imposter KLD, Maximum and Minimum Scores

\begin{tabular}{|c|c|c|c|c|c|c|}
\hline \multicolumn{7}{|c|}{ SourceAFIS Hispanic } \\
\hline Device & $\begin{array}{l}\text { Genuine } \\
\text { KLD }\end{array}$ & $\begin{array}{l}\text { Imposter } \\
\text { KLD }\end{array}$ & $\begin{array}{l}\text { Max } \\
\text { Genuine }\end{array}$ & $\begin{array}{l}\text { Min } \\
\text { Genuine }\end{array}$ & $\begin{array}{l}\text { Max } \\
\text { Imposter }\end{array}$ & $\begin{array}{l}\text { Min } \\
\text { Imposter }\end{array}$ \\
\hline ANDI BIN & 0.0701 & 1.9296 & 233.7107 & 12.51677 & 29.45402 & 5.533568 \\
\hline ANDI GRY & 0.0884 & 1.1892 & 226.3276 & 10.56398 & 28.44455 & 6.006702 \\
\hline Biosled & 0.1920 & 1.8107 & 163.2991 & 11.87605 & 49.03751 & 6.748479 \\
\hline $\begin{array}{r}\text { BioSled } \\
\text { Rolls }\end{array}$ & 0.0685 & 0.1880 & 264.4757 & 10.53184 & 179.3929 & 5.404606 \\
\hline$C G 2$ & 0.0971 & 6.6522 & 179.4396 & 33.96733 & 32.02315 & 4.746463 \\
\hline CG2 Rolls & 0.0714 & 3.2236 & 310.4168 & 11.92265 & 27.33675 & 4.081307 \\
\hline CSA & 0.1413 & 2.8328 & 166.0101 & 40.81303 & 32.3677 & 6.931957 \\
\hline CSA Rolls & 0.0894 & 2.3991 & 318.4915 & 13.7747 & 29.25764 & 6.812571 \\
\hline$L 1$ & 0.1326 & 2.5505 & 227.5417 & 54.56351 & 27.07703 & 7.757417 \\
\hline L1 Rolls & 0.0691 & 2.0646 & 284.7716 & 12.18746 & 28.70368 & 5.735529 \\
\hline$M F F$ & 0.1329 & 3.5195 & 242.7262 & 10.3637 & 35.07605 & 4.917602 \\
\hline$S M M$ & 0.0796 & 17.7029 & 152.8738 & 24.03162 & 32.02045 & 4.576688 \\
\hline
\end{tabular}

Table D.26 SourceAFIS Middle Eastern Genuine and Imposter KLD, Maximum and Minimum Scores

\begin{tabular}{|c|c|c|c|c|c|c|}
\hline \multicolumn{7}{|c|}{ SourceAFIS Middle Eastern } \\
\hline Device & $\begin{array}{l}\text { Genuine } \\
\text { KLD }\end{array}$ & $\begin{array}{l}\text { Imposter } \\
\text { KLD }\end{array}$ & $\begin{array}{l}\text { Max } \\
\text { Genuine }\end{array}$ & $\begin{array}{l}\text { Min } \\
\text { Genuine }\end{array}$ & $\begin{array}{l}\text { Max } \\
\text { Imposter }\end{array}$ & $\begin{array}{l}\text { Min } \\
\text { Imposter }\end{array}$ \\
\hline$A N D I B I N$ & 0.1821 & 1.6499 & 218.4843 & 12.04636 & 27.98296 & 6.334945 \\
\hline$A N D I G R Y$ & 0.2166 & 3.6323 & 187.8634 & 11.47555 & 26.27157 & 3.627262 \\
\hline Biosled & 0.2231 & 2.9841 & 168.724 & 14.43362 & 26.5876 & 8.149198 \\
\hline $\begin{array}{r}\text { BioSled } \\
\text { Rolls }\end{array}$ & 0.1069 & 2.4911 & 327.6002 & 9.770303 & 25.53419 & 7.505122 \\
\hline CG2 & 0.1805 & 3.7760 & 210.9301 & 11.47672 & 27.88251 & 6.963401 \\
\hline CG2 Rolls & 0.0553 & 1.8371 & 308.9363 & 10.13526 & 21.98601 & 6.707691 \\
\hline CSA & 0.2585 & 1.2818 & 216.3997 & 11.83961 & 25.29209 & 6.895127 \\
\hline CSA Rolls & 0.1423 & 1.1311 & 275.2286 & 10.59432 & 24.75159 & 7.287093 \\
\hline$\angle 1$ & 0.1101 & 3.7773 & 229.4577 & 34.61526 & 23.36124 & 7.078579 \\
\hline L1 Rolls & 0.0589 & 1.3476 & 300.8031 & 10.3993 & 25.04766 & 6.524864 \\
\hline MFF & 0.1368 & 1.1941 & 222.0757 & 10.66438 & 26.32177 & 5.368564 \\
\hline$S M M$ & 0.2942 & 14.0849 & 115.637 & 10.43807 & 27.00021 & 4.776016 \\
\hline
\end{tabular}


Table D.27 SourceAFIS Other Genuine and Imposter KLD, Maximum and Minimum Scores

\begin{tabular}{|c|c|c|c|c|c|c|}
\hline \multicolumn{7}{|c|}{ SourceAFIS Other } \\
\hline Device & $\begin{array}{l}\text { Genuine } \\
\text { KLD }\end{array}$ & $\begin{array}{l}\text { Imposter } \\
\text { KLD }\end{array}$ & $\begin{array}{l}\text { Max } \\
\text { Genuine }\end{array}$ & $\begin{array}{l}\text { Min } \\
\text { Genuine }\end{array}$ & $\begin{array}{l}\text { Max } \\
\text { Imposter }\end{array}$ & $\begin{array}{l}\text { Min } \\
\text { Imposter }\end{array}$ \\
\hline$A N D I B I N$ & 0.0872 & 1.1178 & 216.2899 & 12.81646 & 30.53337 & 5.857705 \\
\hline ANDI GRY & 0.1441 & 1.4404 & 175.0319 & 11.39268 & 26.73659 & 7.282349 \\
\hline Biosled & 0.0888 & 2.9397 & 248.3664 & 13.89209 & 28.31911 & 7.980638 \\
\hline $\begin{array}{r}\text { Biosled } \\
\text { Rolls }\end{array}$ & 0.1010 & 1.4833 & 320.201 & 11.56863 & 26.92059 & 8.289468 \\
\hline$C G 2$ & 0.0951 & 3.8648 & 244.837 & 38.79229 & 24.80103 & 7.655017 \\
\hline CG2 Rolls & 0.0355 & 1.9505 & 363.1709 & 12.8476 & 28.51096 & 6.200431 \\
\hline CSA & 0.0519 & 0.8744 & 279.6029 & 52.56234 & 28.43942 & 6.117246 \\
\hline CSA Rolls & 0.0609 & 0.9662 & 317.2518 & 86.21761 & 27.25427 & 5.039689 \\
\hline$L 1$ & 0.0997 & 2.0550 & 243.2939 & 6.561505 & 26.2432 & 8.264335 \\
\hline L1 Rolls & 0.0847 & 4.0564 & 339.6964 & 10.20002 & 30.91195 & 6.258831 \\
\hline MFF & 0.0638 & 1.3916 & 291.2537 & 10.16441 & 30.48556 & 2.927113 \\
\hline$S M M$ & 0.0784 & 11.1336 & 171.836 & 10.90741 & 25.3558 & 3.236016 \\
\hline
\end{tabular}




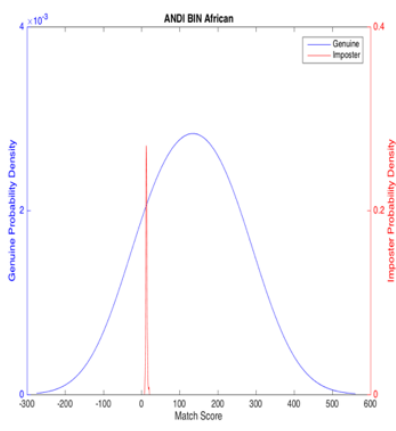

(a)

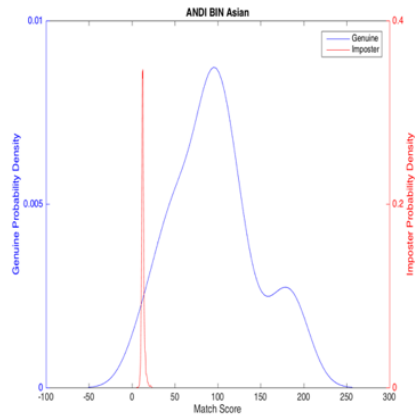

(d)

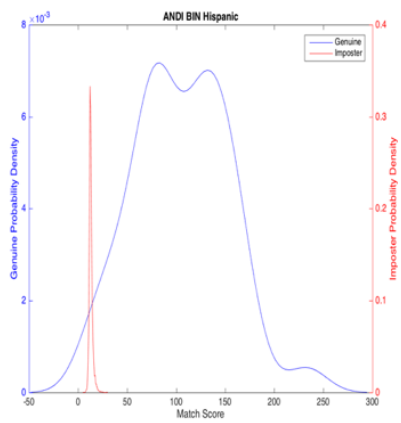

(g)

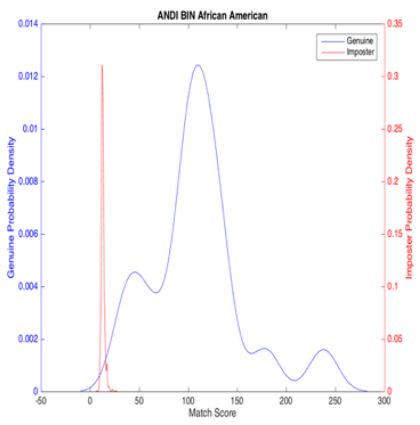

(b)

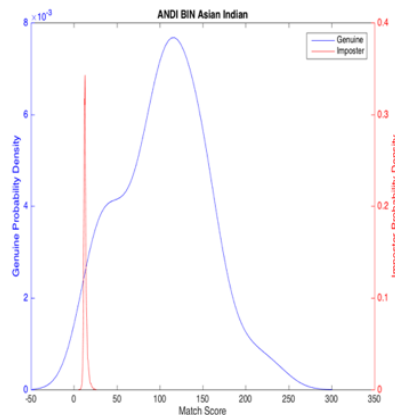

(e)

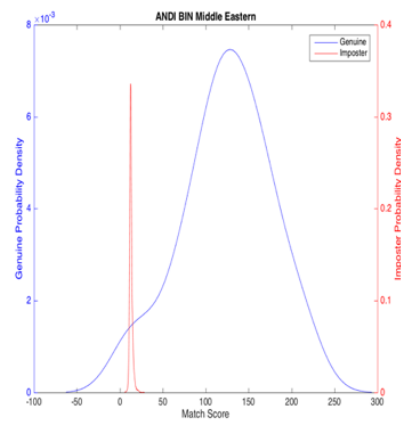

(h)

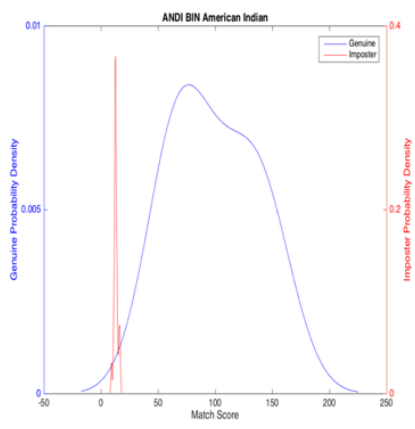

(c)

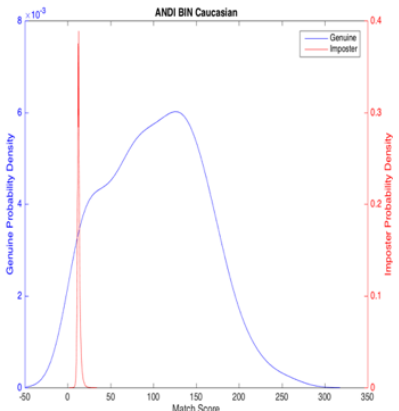

(f)

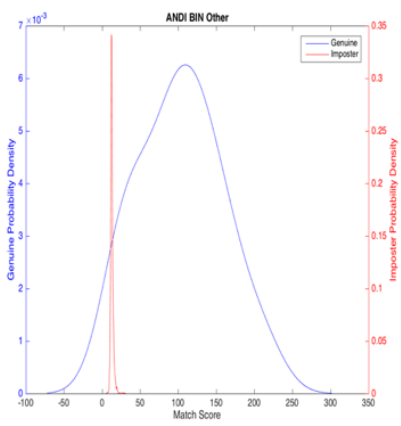

(i)

Figure D.3.1 SourceAFIS ANDI Binary (a) African (b) African American (c) American Indian (d) Asian (e) Asian Indian (f) Caucasian (g) Hispanic (h) Middle Eastern (i) Other 


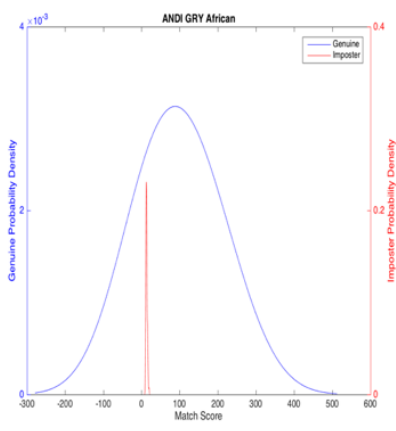

(a)

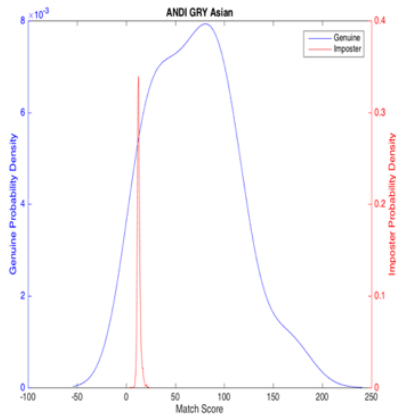

(d)

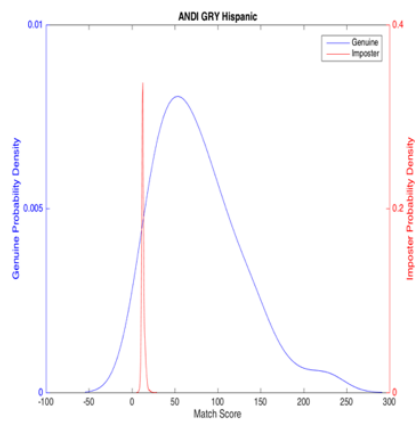

(g)

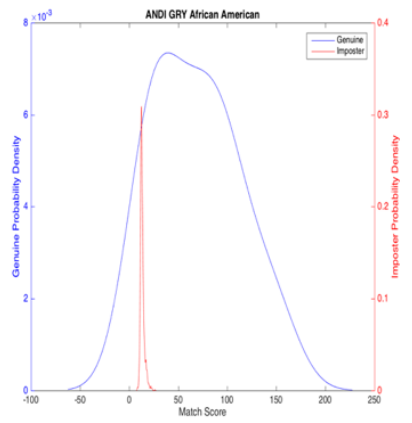

(b)

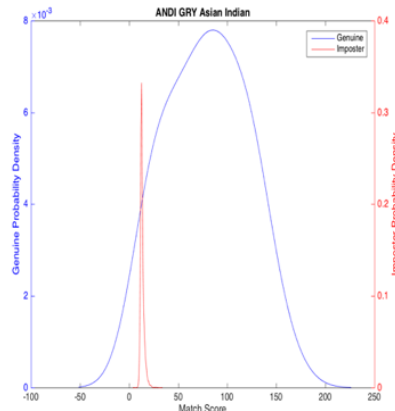

(e)

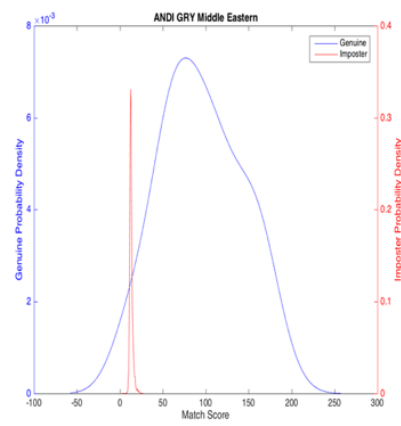

(h)

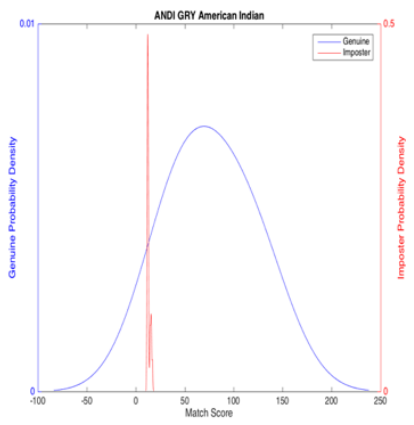

(c)

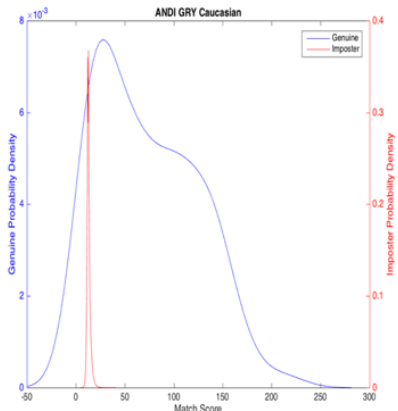

(f)

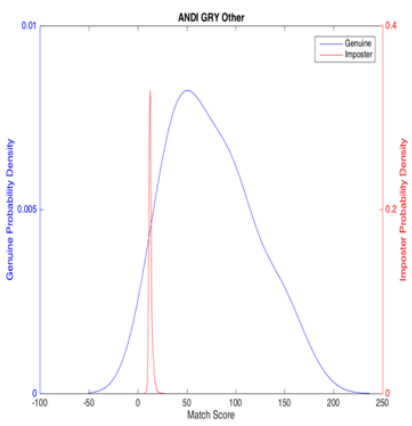

(i)

Figure D.3.2 SourceAFIS ANDI Greyscale (a) African (b) African American (c) American Indian (d) Asian (e) Asian Indian (f) Caucasian (g) Hispanic (h) Middle Eastern (i) Other 


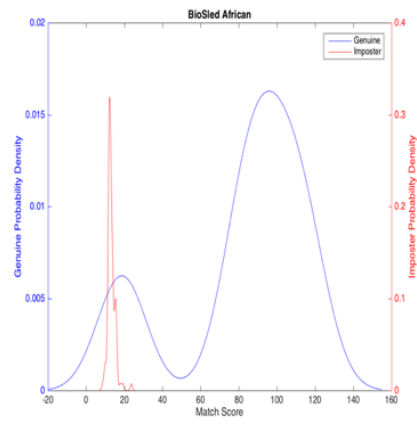

(a)

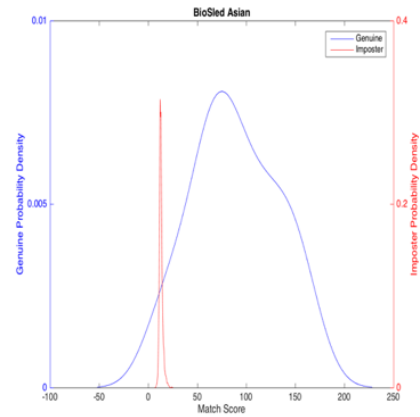

(d)

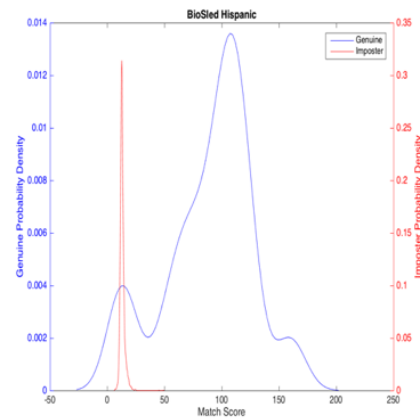

(g)

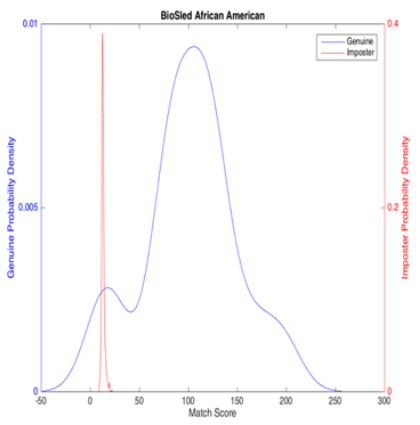

(b)

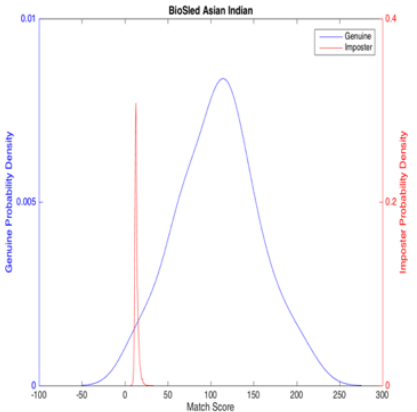

(e)

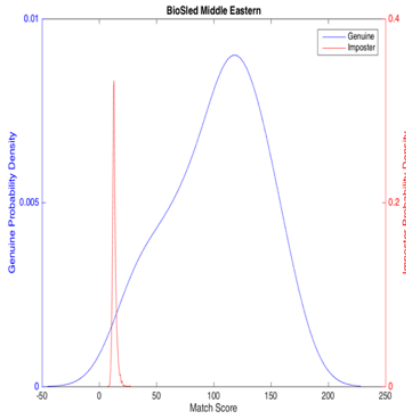

(h)

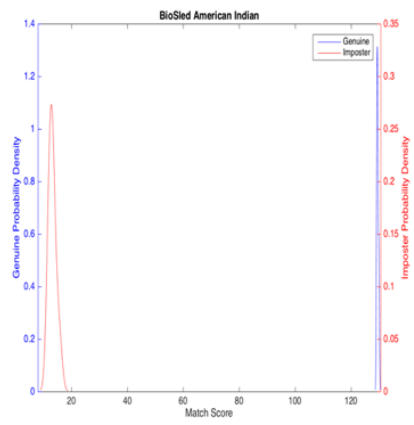

(c)

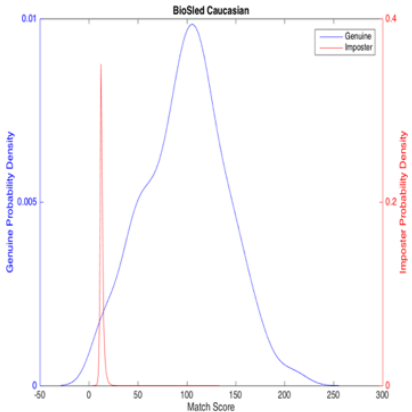

(f)

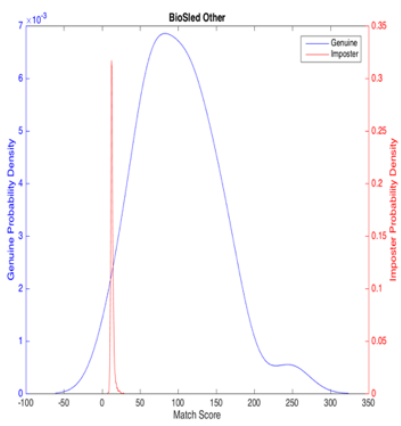

(i)

Figure D.3.3 SourceAFIS BioSled (a) African (b) African American (c) American Indian (d) Asian (e) Asian Indian (f) Caucasian (g) Hispanic (h) Middle Eastern (i) Other 


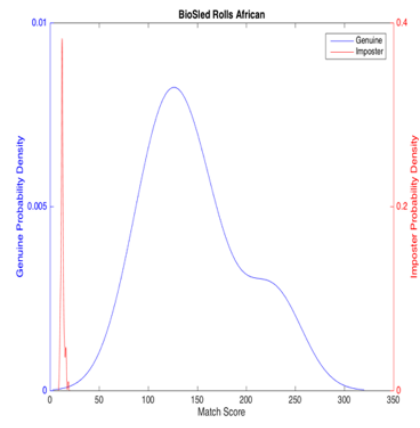

(a)

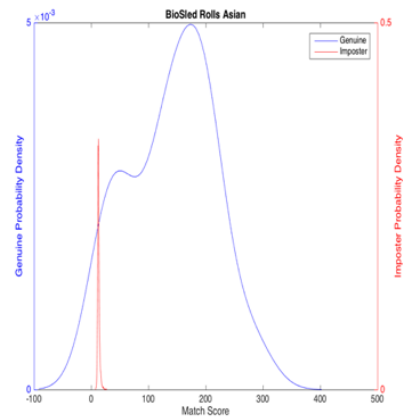

(d)

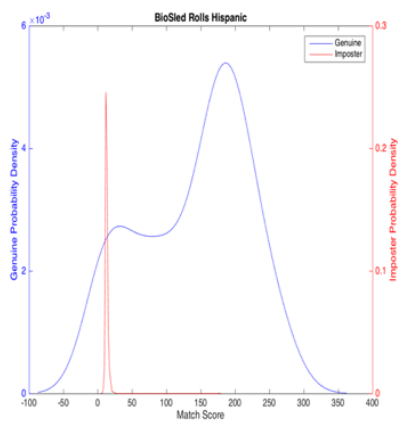

(g)

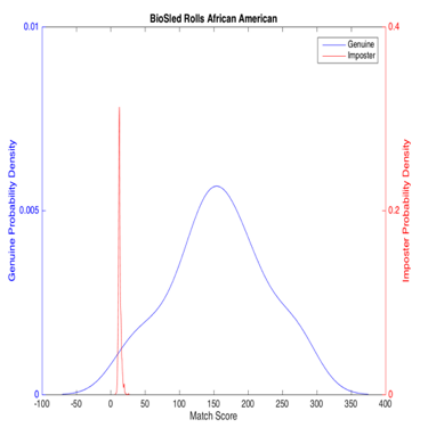

(b)

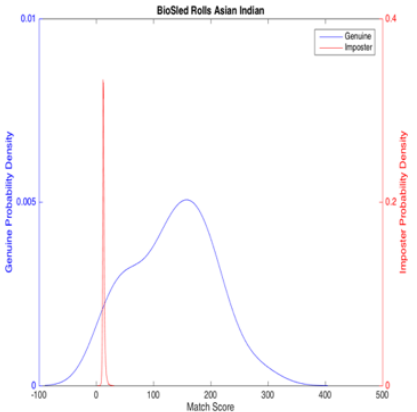

(e)

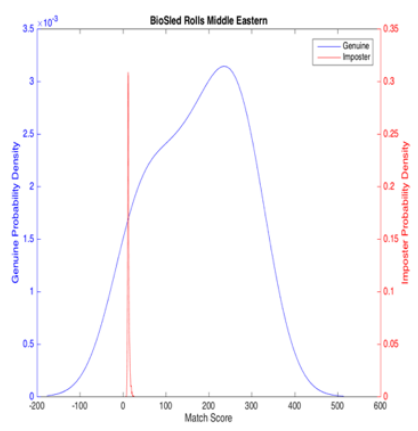

(h)

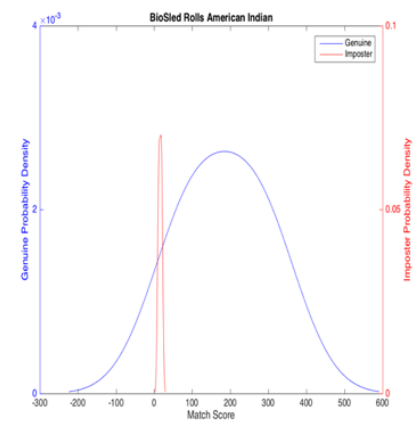

(c)

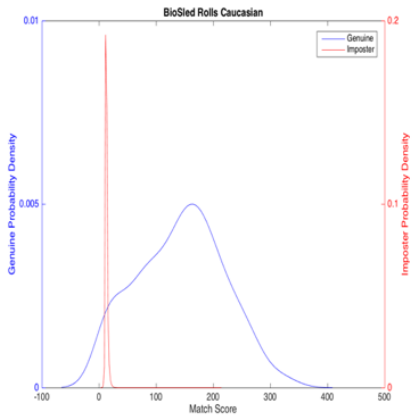

(f)

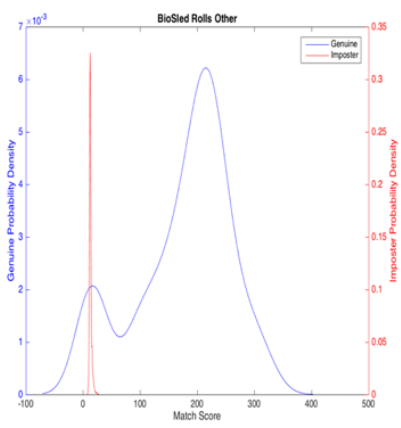

(i)

Figure D.3.4 SourceAFIS BioSled Rolls (a) African (b) African American (c) American Indian (d) Asian (e) Asian Indian (f) Caucasian (g) Hispanic (h) Middle Eastern (i) Other 


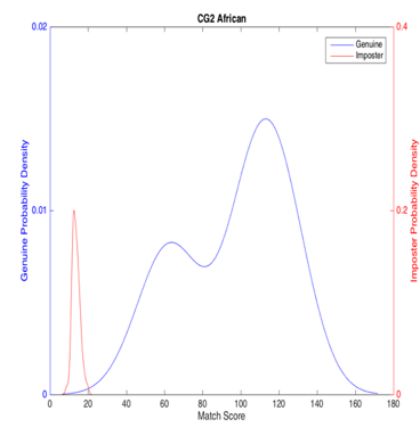

(a)

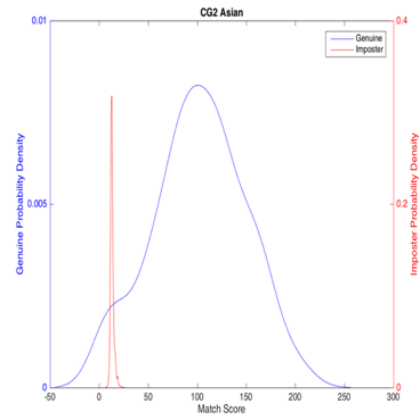

(d)

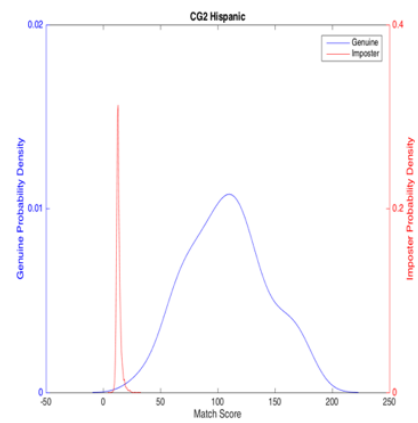

(g)

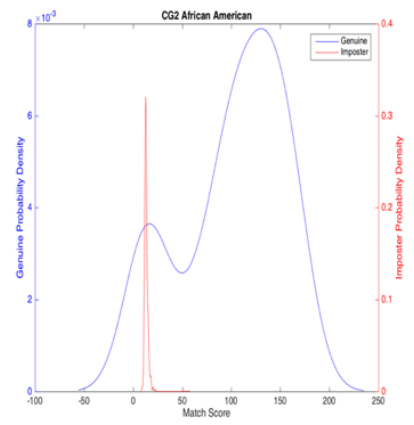

(b)

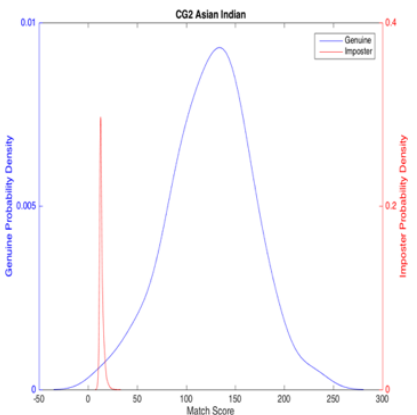

(e)

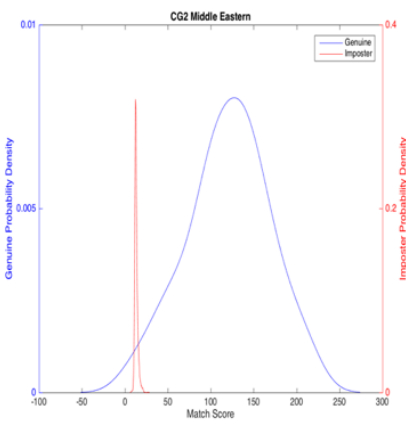

(h)

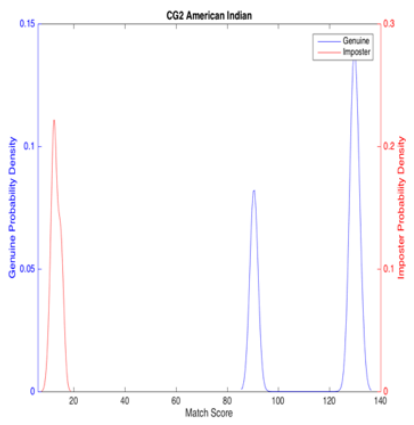

(c)

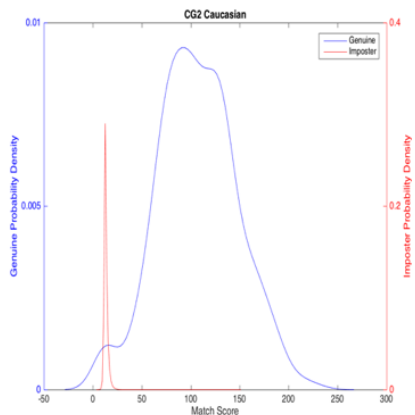

(f)

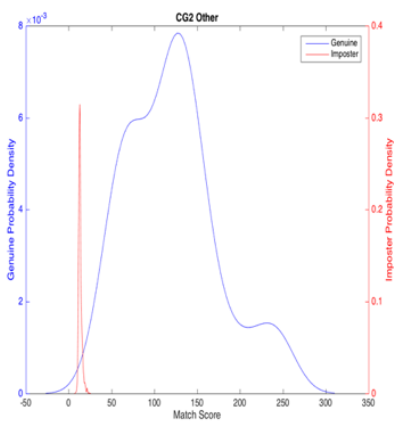

(i)

Figure D.3.5 SourceAFIS FP II Guardian (a) African (b) African American (c) American Indian (d) Asian (e) Asian Indian (f) Caucasian (g) Hispanic (h) Middle Eastern (i) Other 


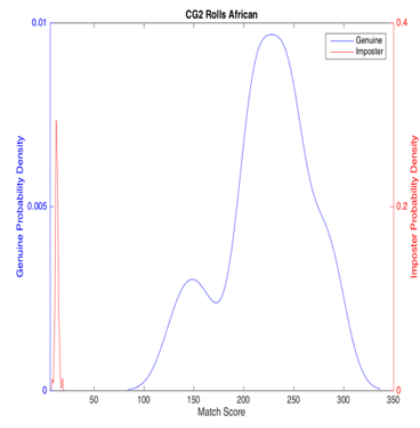

(a)

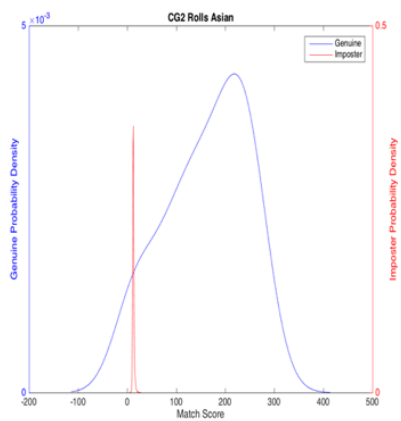

(d)

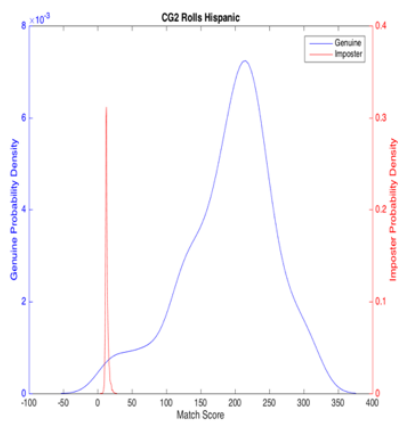

(g)

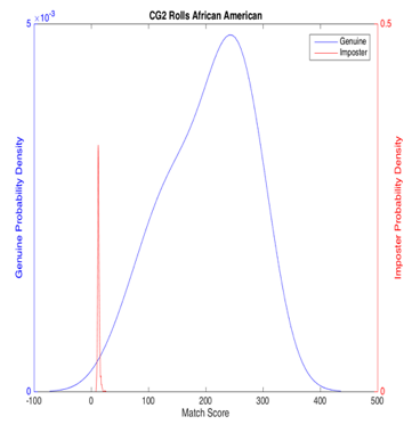

(b)

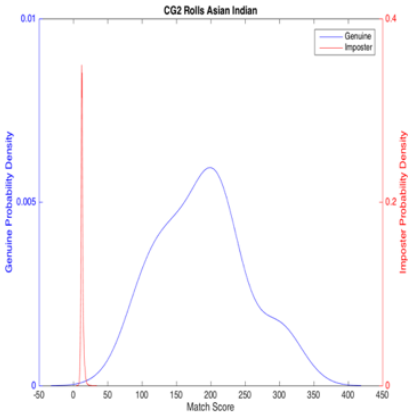

(e)

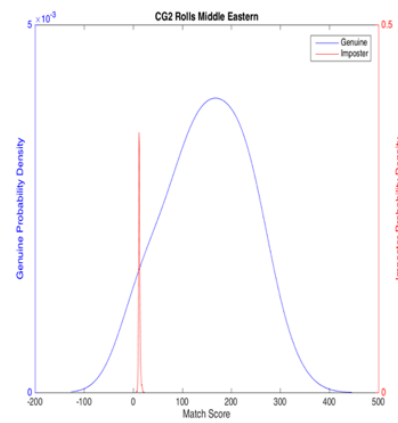

(h)

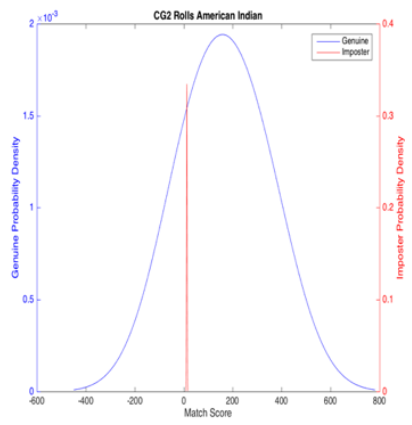

(c)

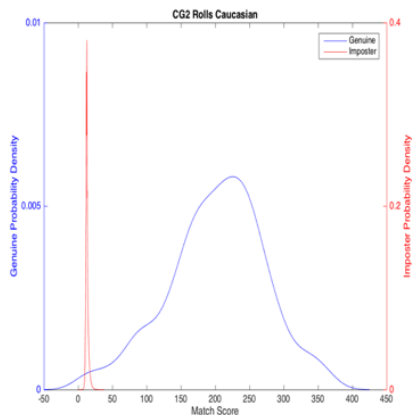

(f)

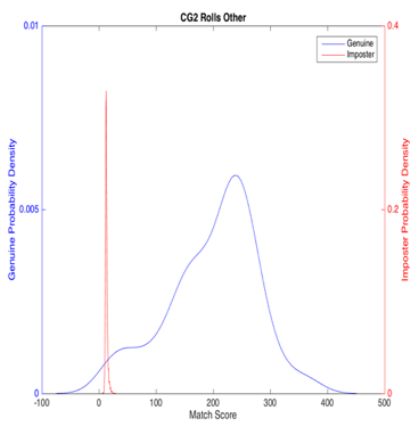

(i)

Figure D.3.6 SourceAFIS FP II Guardian Rolls (a) African (b) African American (c) American Indian (d) Asian (e) Asian Indian (f) Caucasian (g) Hispanic (h) Middle Eastern (i) Other 


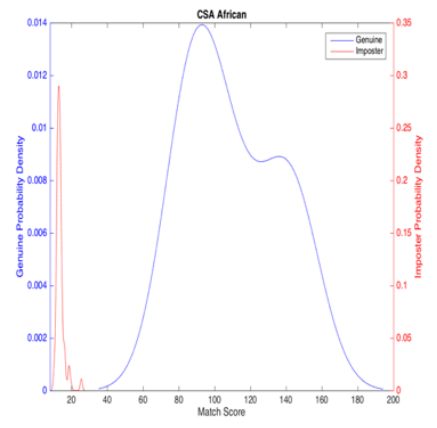

(a)

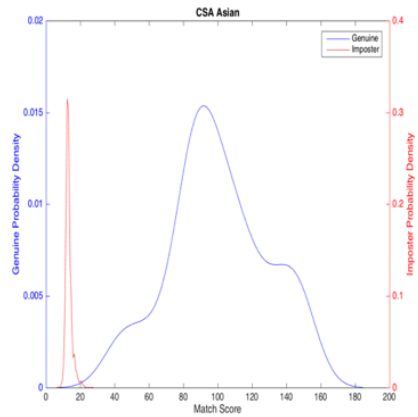

(d)

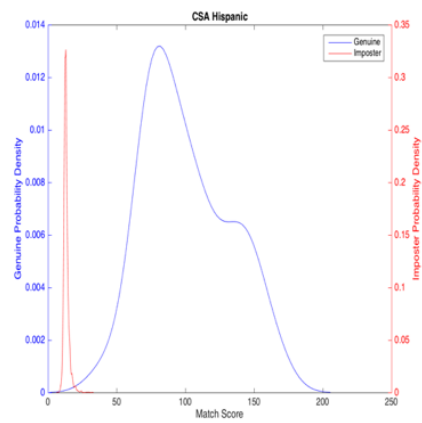

(g)

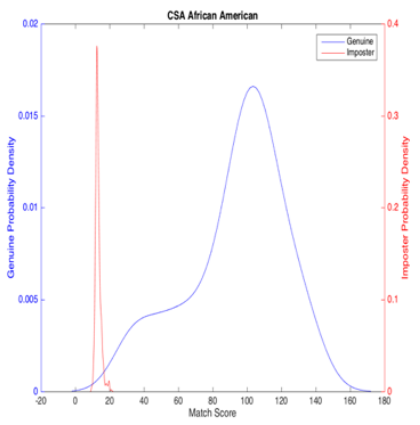

(b)

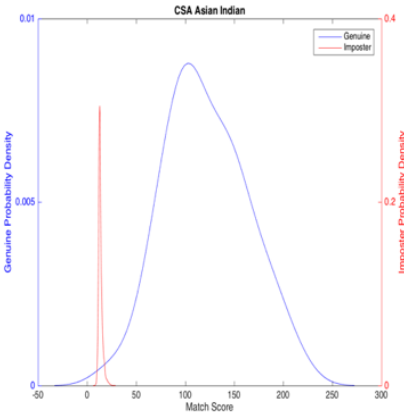

(e)

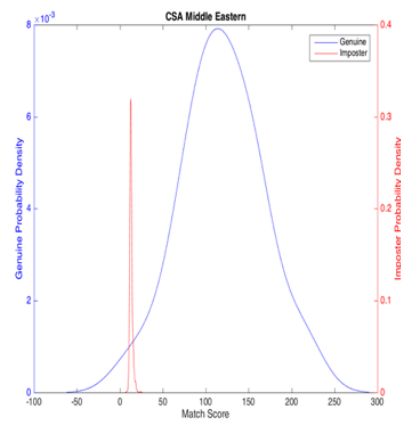

(h)

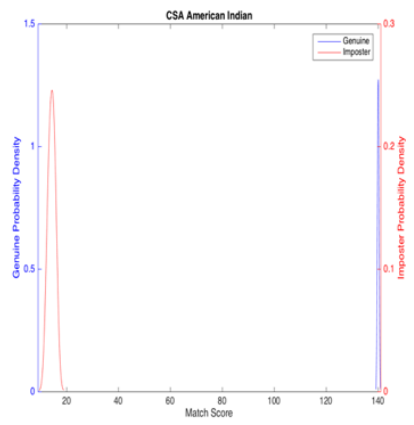

(c)

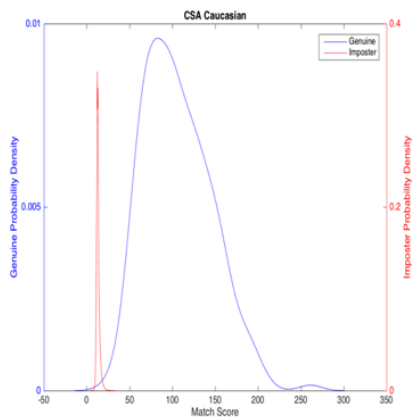

(f)

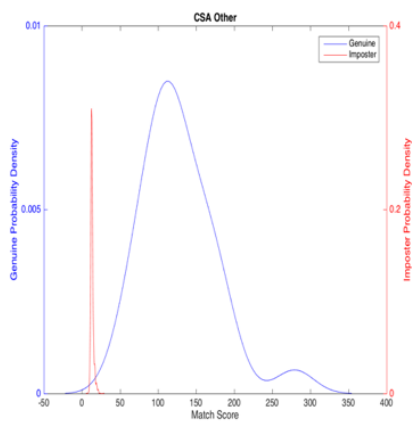

(i)

Figure D.3.7 SourceAFIS Crossmatch Seek Avenger (a) African (b) African American (c) American Indian (d) Asian (e) Asian Indian (f) Caucasian (g) Hispanic (h) Middle Eastern (i) Other 


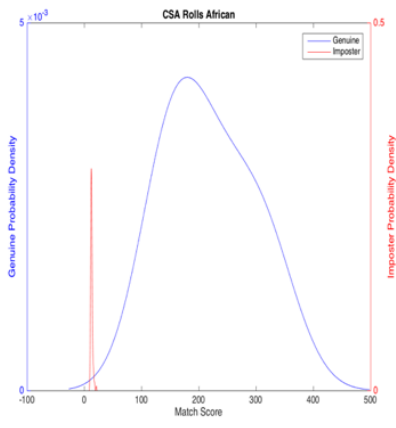

(a)

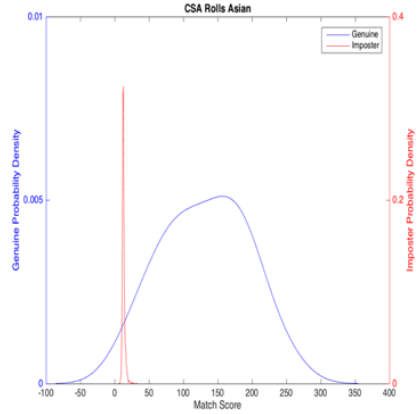

(d)

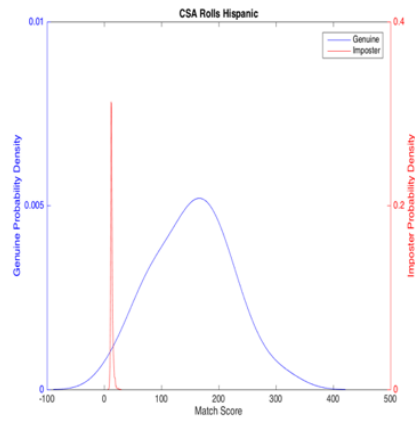

(g)

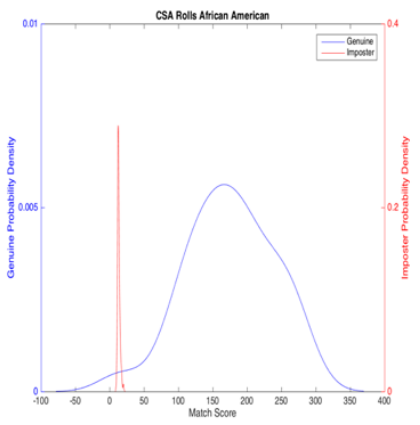

(b)

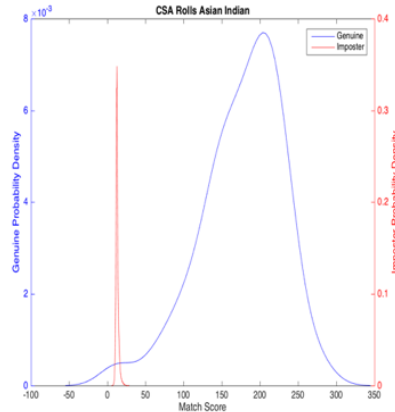

(e)

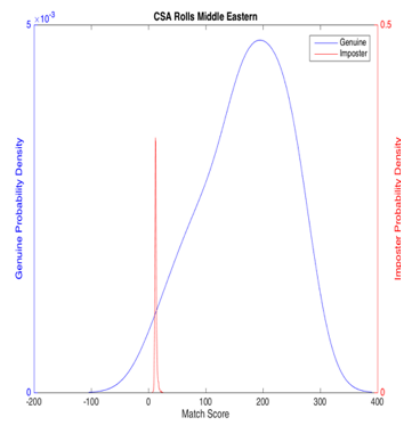

(h)

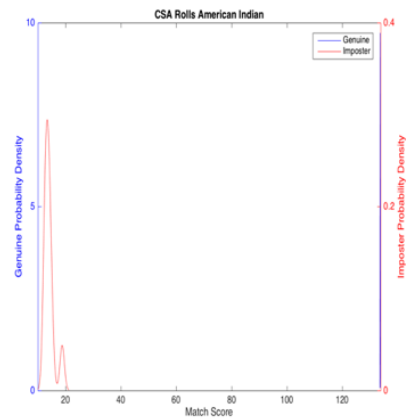

(c)

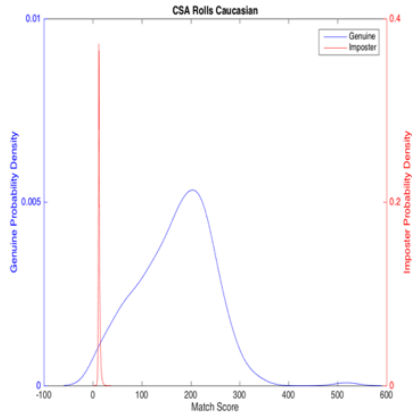

(f)

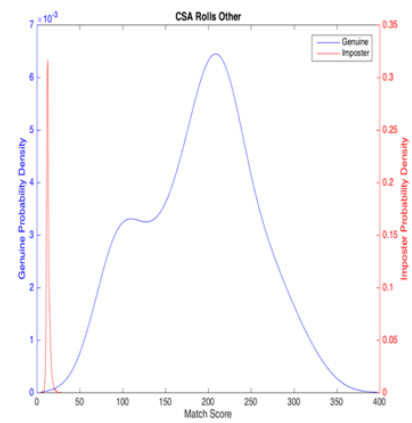

(i)

Figure D.3.8 Crossmatch Seek Avenger Rolls (a) African (b) African American (c) American Indian (d) Asian (e) Asian Indian (f) Caucasian (g) Hispanic (h) Middle Eastern (i) Other 


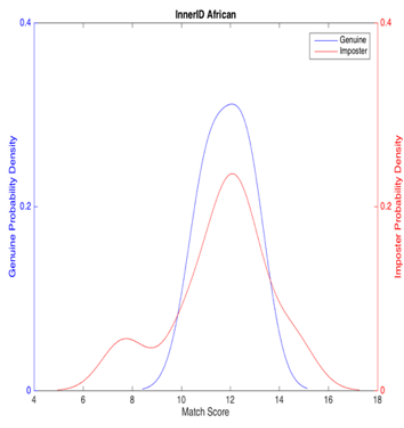

(a)

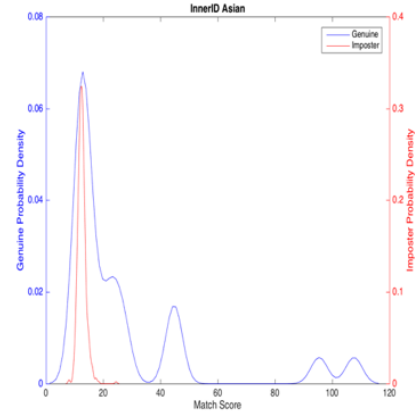

(d)

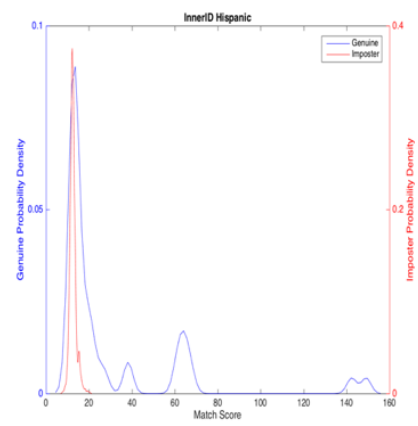

$(\mathrm{g})$

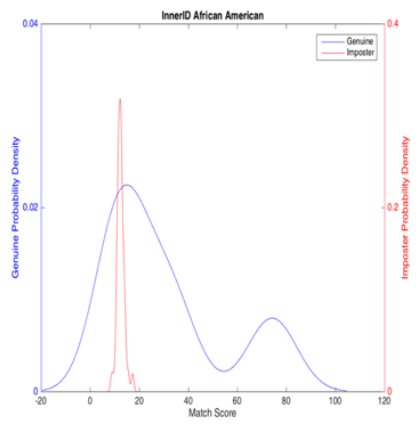

(b)

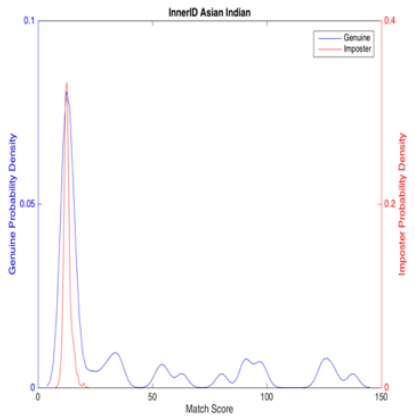

(e)

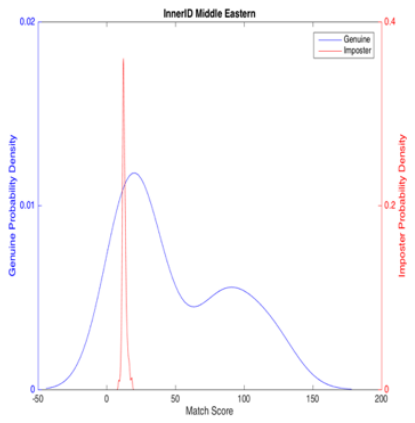

(h) (c)

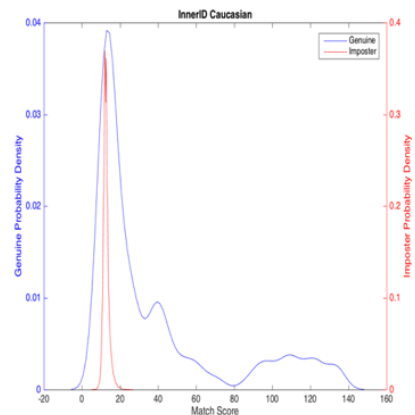

(f)

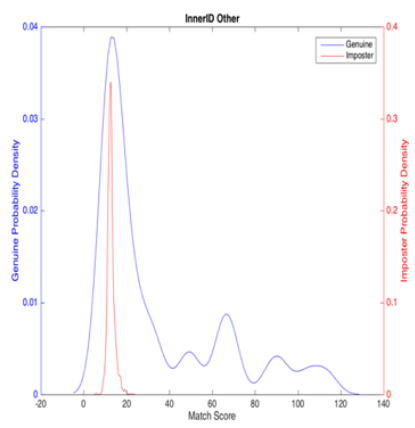

(i)

Figure D.3.9 SourceAFIS InnerID (a) African (b) African American (c) American Indian (d) Asian (e) Asian Indian (f) Caucasian (g) Hispanic (h) Middle Eastern (i) Other

This Device was removed from the collection midway through when it stopped working. What images were produced are low quality, i.e. the reason for the outlying distributions. 


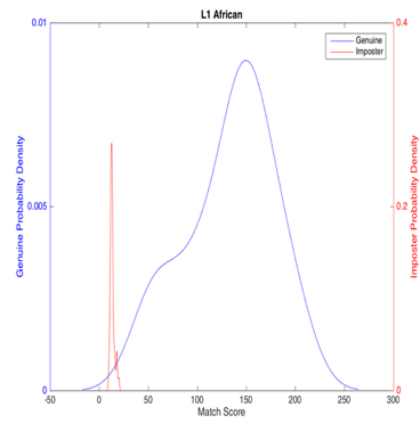

(a)

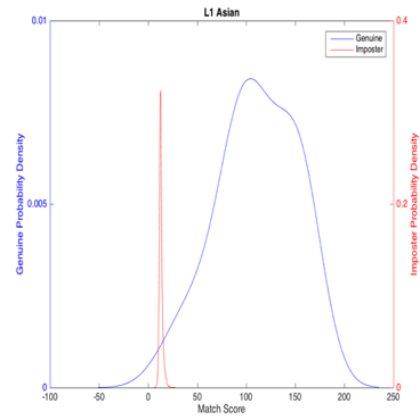

(d)

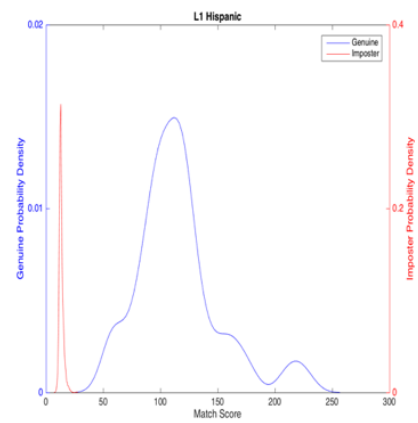

(g)

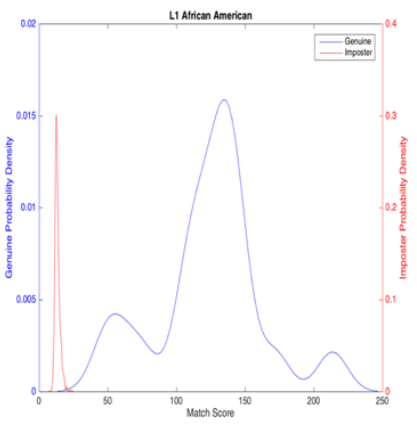

(b)

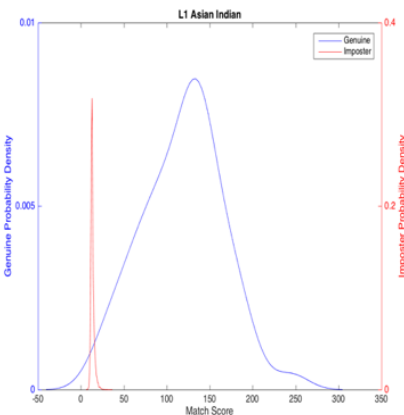

(e)

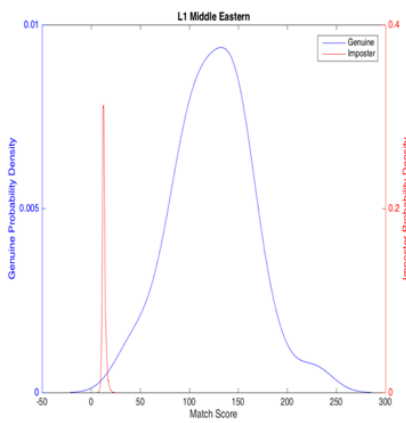

(h)

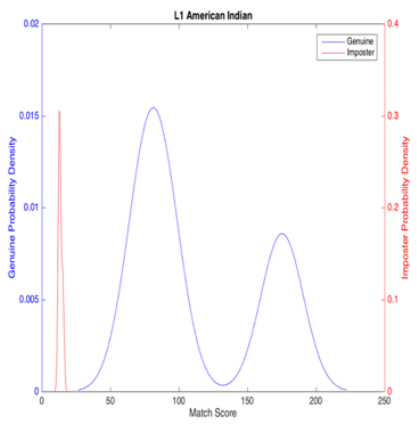

(c)

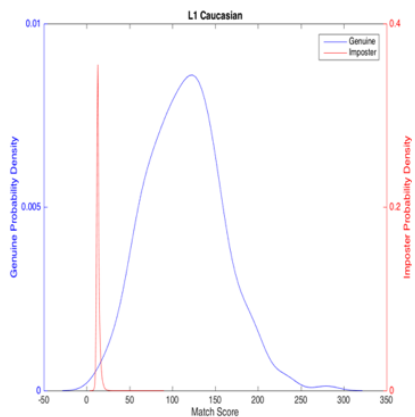

(f)

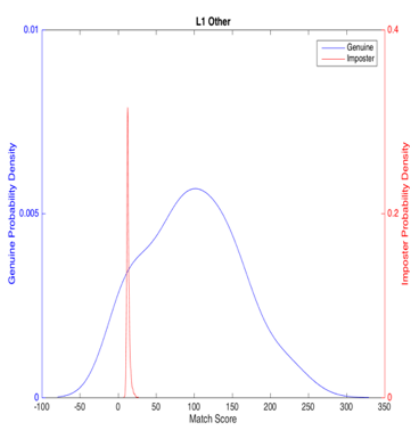

(i)

Figure D.3.10 SourceAFIS L1 (a) African (b) African American (c) American Indian (d) Asian (e) Asian Indian (f) Caucasian (g) Hispanic (h) Middle Eastern (i) Other 


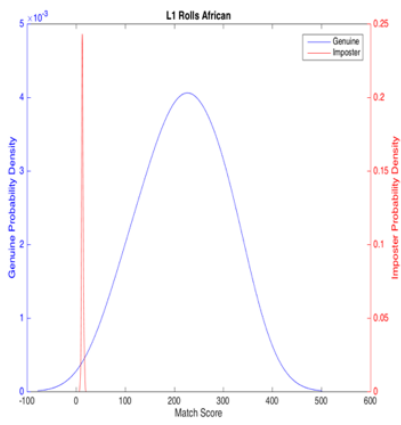

(a)

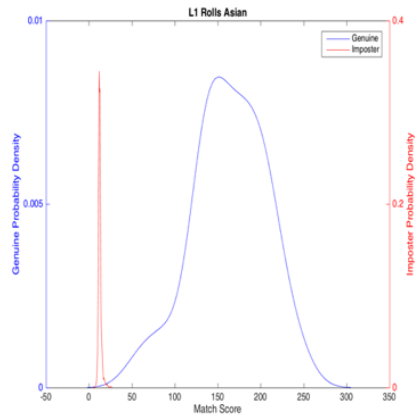

(d)

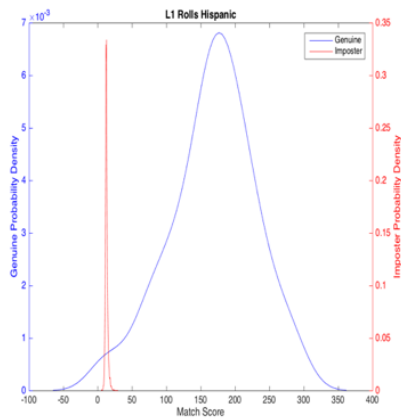

(g)

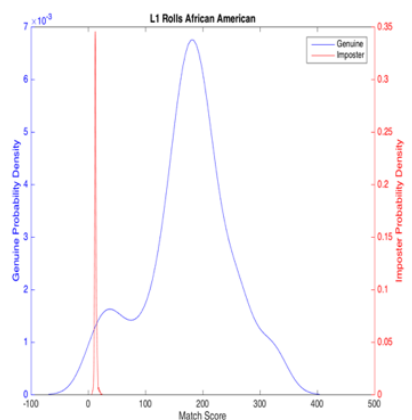

(b)

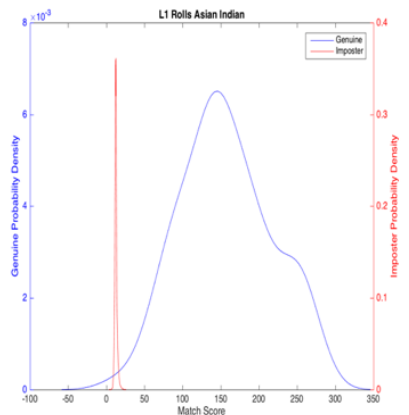

(e)

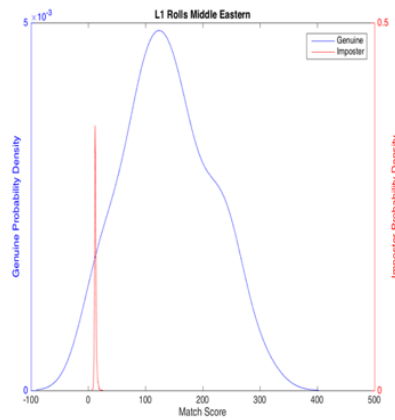

(h)

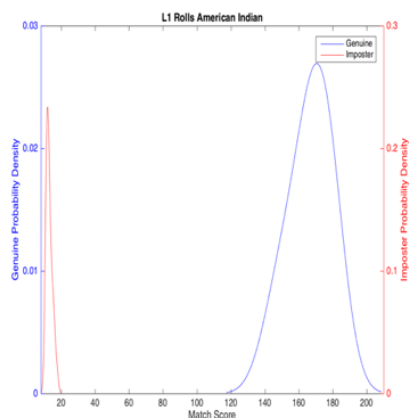

(c)

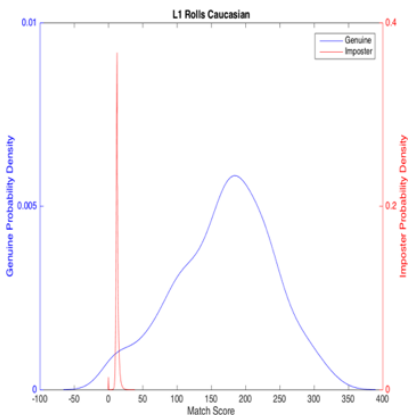

(f)

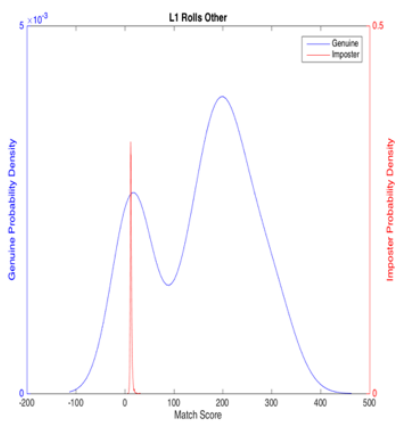

(i)

Figure D.3.11 SourceAFIS L1 Rolls (a) African (b) African American (c) American Indian (d) Asian (e) Asian Indian (f) Caucasian (g) Hispanic (h) Middle Eastern (i) Other 


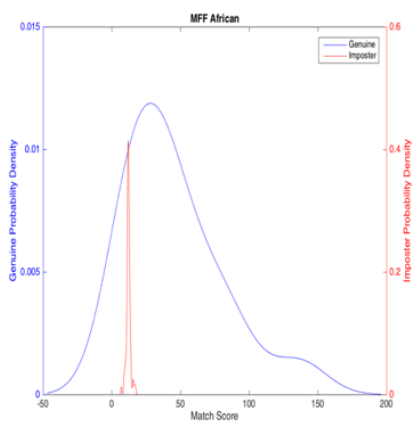

(a)

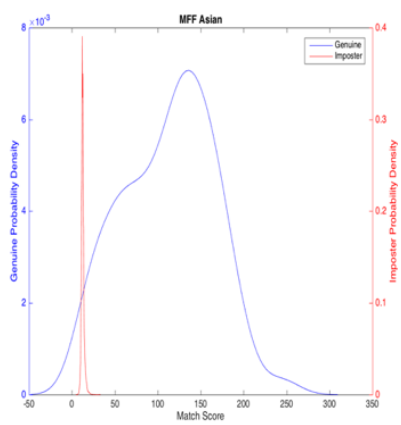

(d)

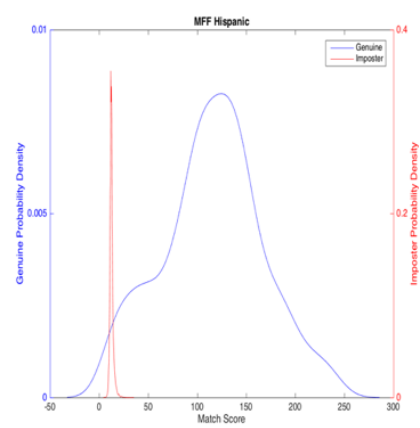

(g)

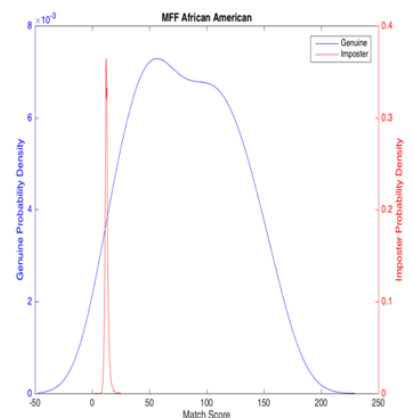

(b)

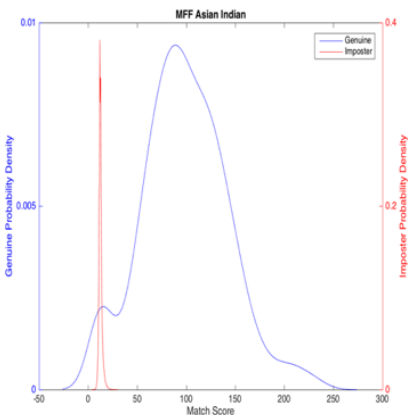

(e)

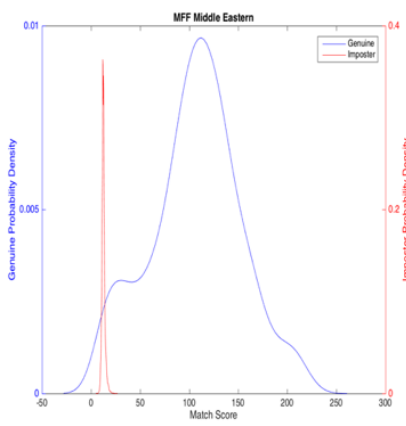

(h)

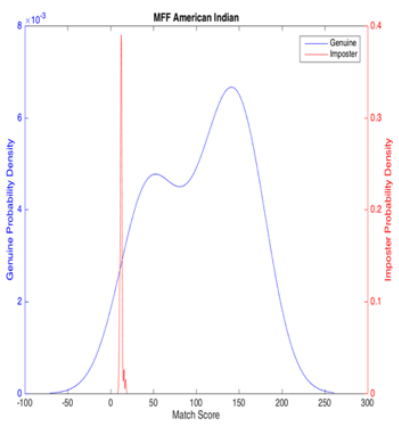

(c)

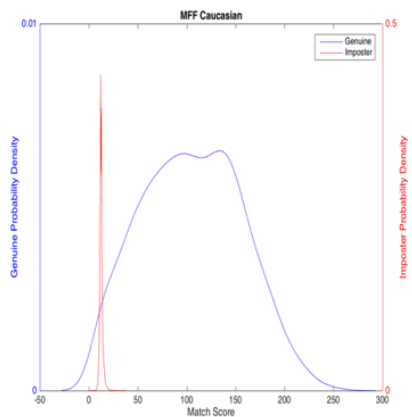

(f)

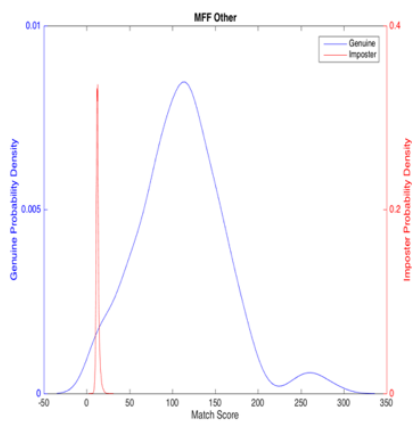

(i)

Figure D.3.12 SourceAFIS Morpho FOTF (a) African (b) African American (c) American Indian (d) Asian (e) Asian Indian (f) Caucasian (g) Hispanic (h) Middle Eastern (i) Other 


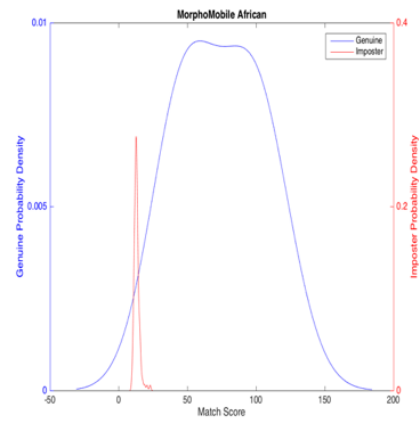

(a)

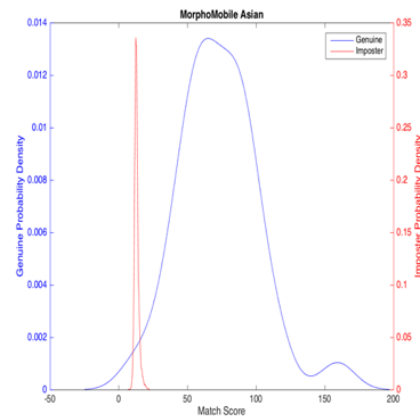

(d)

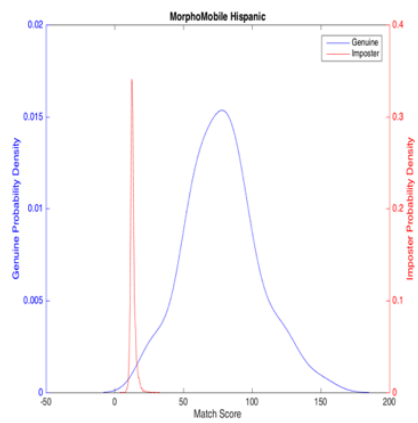

(g)

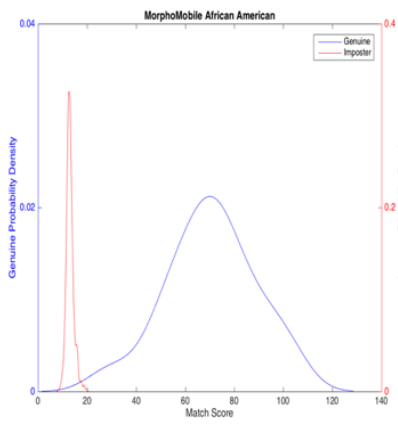

(b)

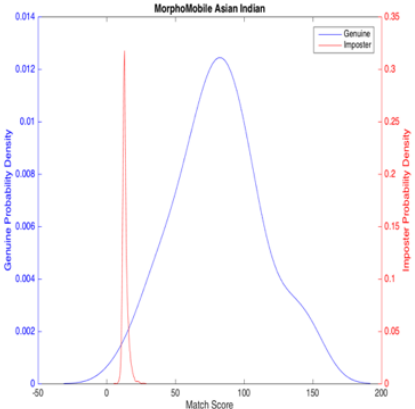

(e)

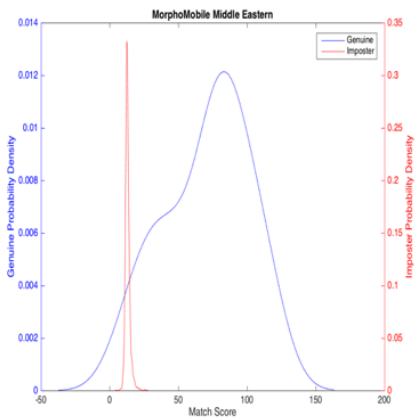

(h)

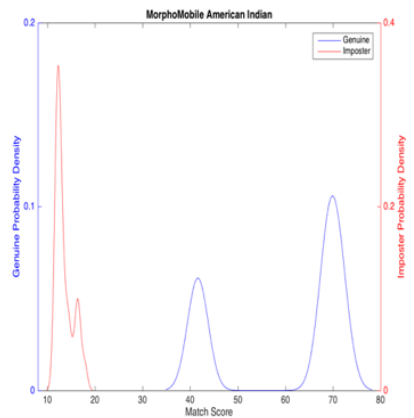

(c)

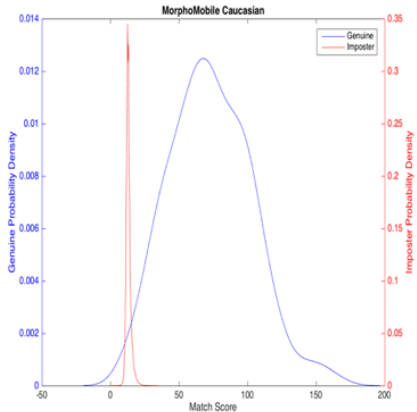

(f)

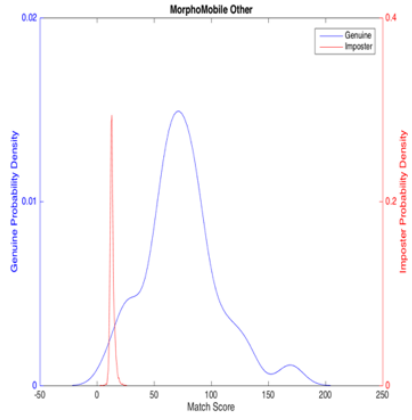

(i)

Figure D.3.13 SourceAFIS MorphoMobile (a) African (b) African American (c) American Indian (d) Asian (e) Asian Indian (f) Caucasian (g) Hispanic (h) Middle Eastern (i) Other 


\section{D.4 NBIS ROC Curves and Area Under Curve}

Table D.28 NBIS Area Under Curve for Each Ethnicity

\begin{tabular}{|c|c|c|c|c|c|c|c|c|c|}
\hline \multicolumn{10}{|c|}{ NBIS Area Under the Curve } \\
\hline & African & $\begin{array}{l}\text { African } \\
\text { American }\end{array}$ & $\begin{array}{l}\text { American } \\
\text { Indian }\end{array}$ & Asian & $\begin{array}{l}\text { Asian } \\
\text { Indian }\end{array}$ & Caucasian & Hispanic & $\begin{array}{l}\text { Middle } \\
\text { Eastern }\end{array}$ & Other \\
\hline ANDI BIN & 1.0000 & 1.0000 & 1.0000 & 0.9807 & 0.9987 & 0.9751 & 0.9845 & 0.9748 & 0.9889 \\
\hline $\begin{array}{c}A N D I \\
G R Y\end{array}$ & 1.0000 & 0.9844 & 1.0000 & 0.9834 & 0.9883 & 0.9742 & 0.9833 & 0.9777 & 0.9958 \\
\hline Biosled & 0.8410 & 0.9549 & 1.0000 & 0.9918 & 0.9907 & 0.9816 & 0.9784 & 0.9930 & 0.9807 \\
\hline $\begin{array}{c}\text { BioSled } \\
\text { Rolls }\end{array}$ & 1.0000 & 1.0000 & 1.0000 & 0.9905 & 0.9688 & 0.9870 & 0.9258 & 1.0000 & 0.9961 \\
\hline CG2 & 1.0000 & 0.8477 & 1.0000 & 0.9736 & 0.9999 & 0.9795 & 1.0000 & 0.9869 & 0.9998 \\
\hline CG2 Rolls & 1.0000 & 1.0000 & 0.7500 & 0.9443 & 1.0000 & 0.9979 & 1.0000 & 0.9994 & 0.9995 \\
\hline CSA & 1.0000 & 0.9995 & 1.0000 & 1.0000 & 1.0000 & 0.9999 & 0.9963 & 0.9830 & 1.0000 \\
\hline CSA Rolls & 1.0000 & 1.0000 & 1.0000 & 0.9999 & 0.9763 & 0.9900 & 1.0000 & 0.9416 & 1.0000 \\
\hline$\| I D$ & 1.0000 & 0.8413 & 1.0000 & 0.8823 & 0.8592 & 0.8676 & 0.8478 & 0.9444 & 0.8100 \\
\hline L1 & 1.0000 & 1.0000 & 1.0000 & 1.0000 & 1.0000 & 0.9950 & 1.0000 & 1.0000 & 0.8407 \\
\hline L1 Rolls & 1.0000 & 1.0000 & 1.0000 & 1.0000 & 1.0000 & 0.9867 & 1.0000 & 1.0000 & 0.8445 \\
\hline$M F F$ & 0.8683 & 0.9956 & 1.0000 & 0.9972 & 0.9795 & 0.9965 & 0.9998 & 0.9934 & 1.0000 \\
\hline$S M M$ & 1.0000 & 1.0000 & 1.0000 & 0.9835 & 0.9873 & 0.9955 & 0.9999 & 0.9979 & 0.9664 \\
\hline
\end{tabular}

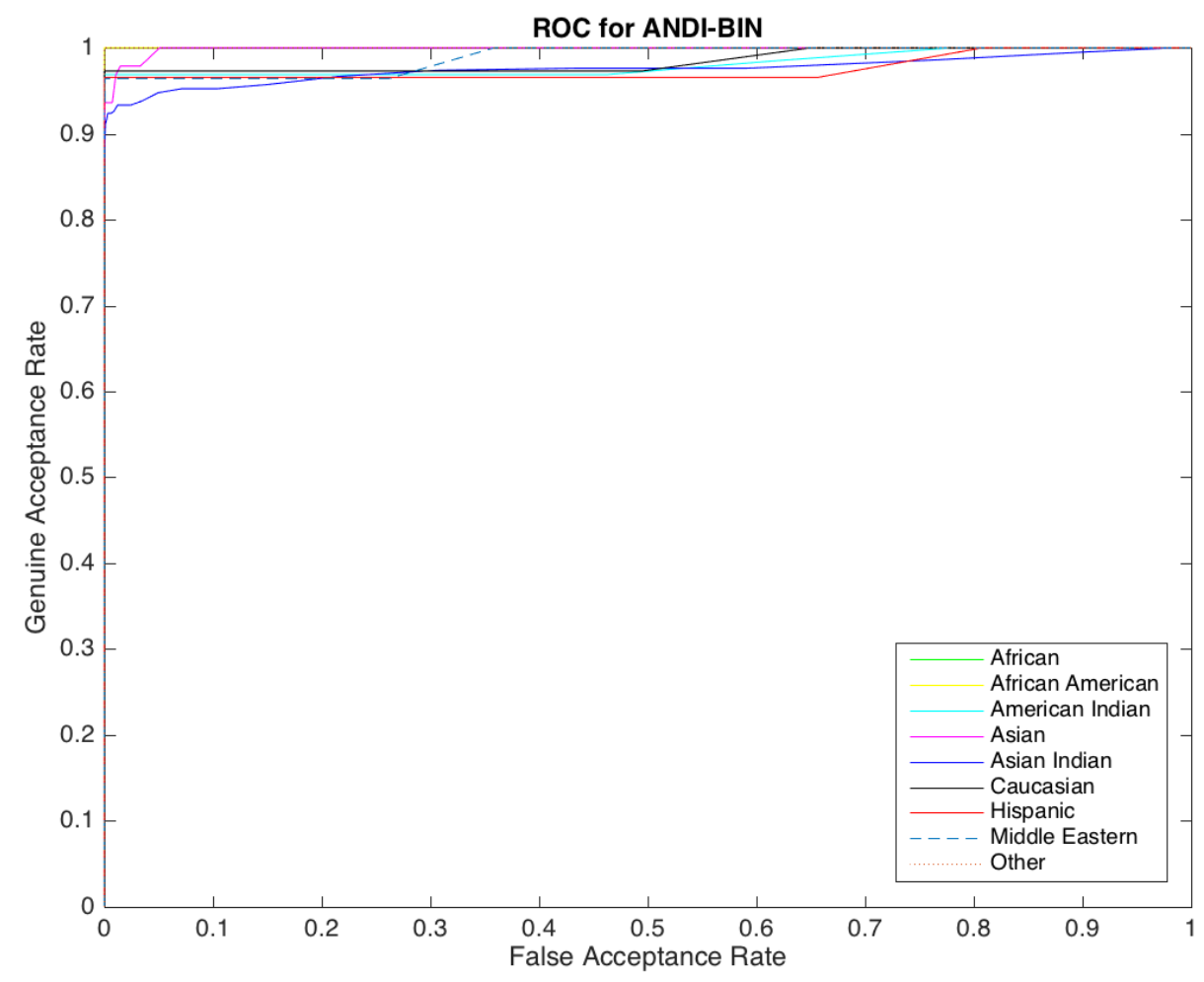

Figure D.4.1 NBIS ANDI Binary Ethnicity Based ROC Curves 


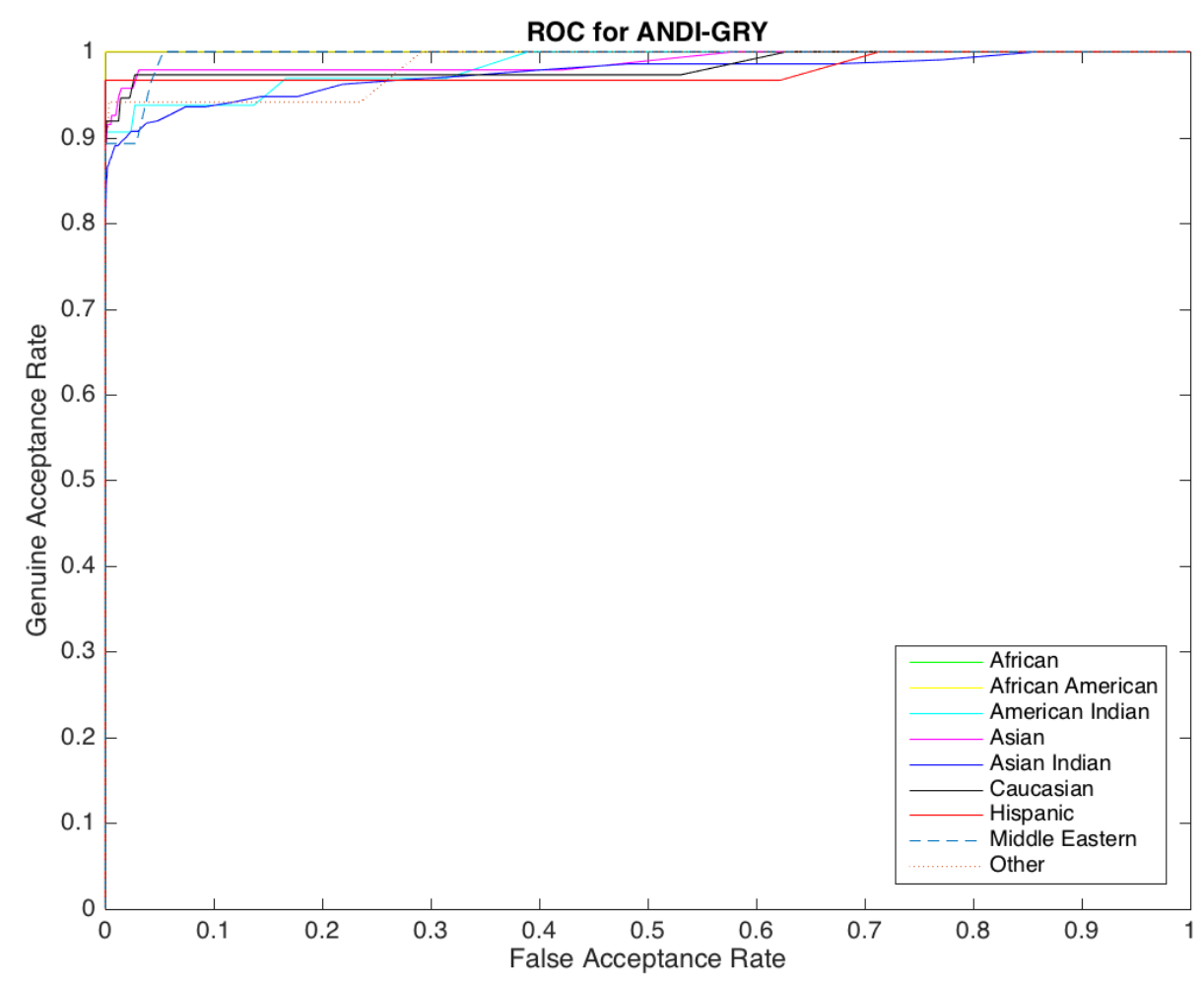

Figure D.4.2 NBIS ANDI Greyscale Ethnicity Based ROC Curves

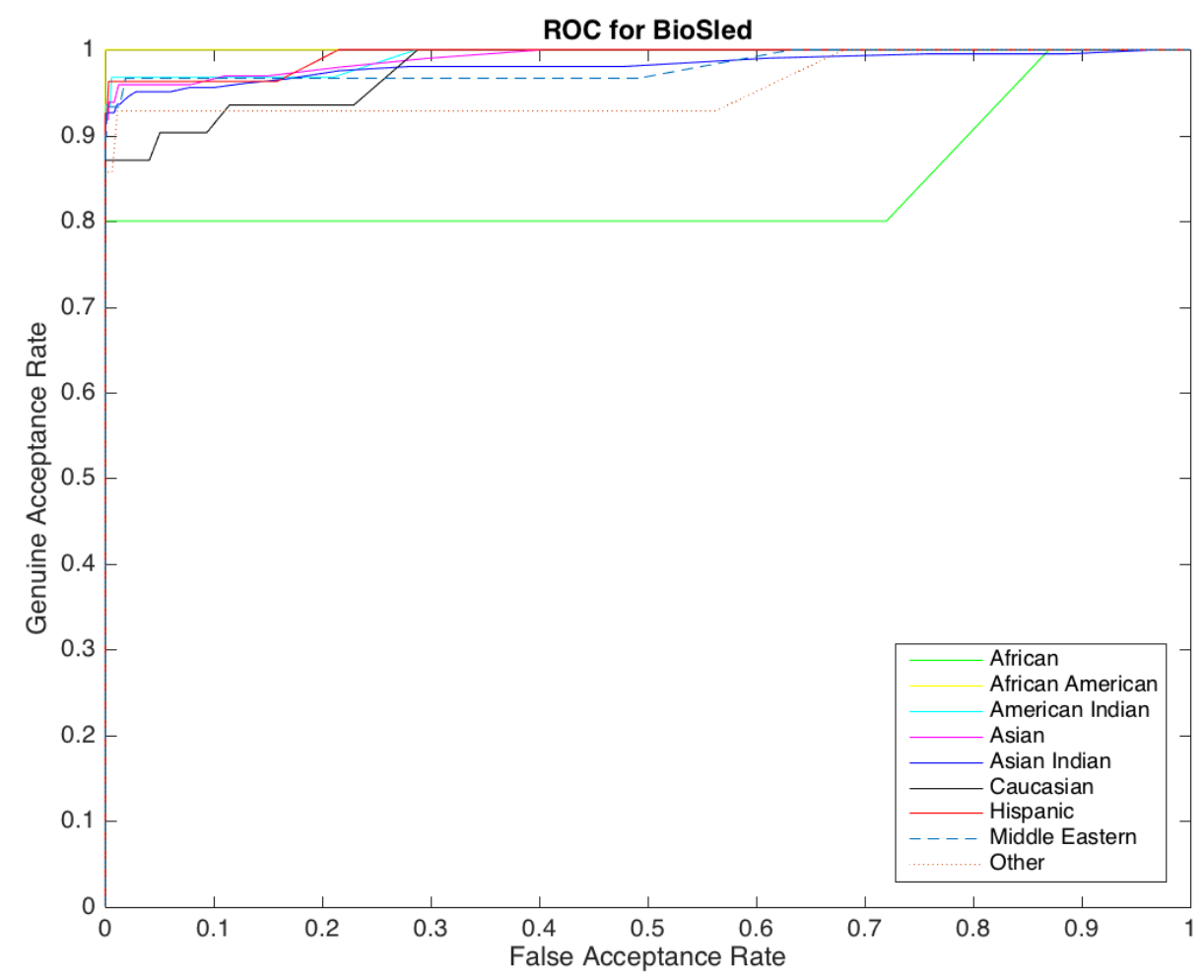

Figure D.4.3 NBIS BioSled Ethnicity Based ROC Curves 


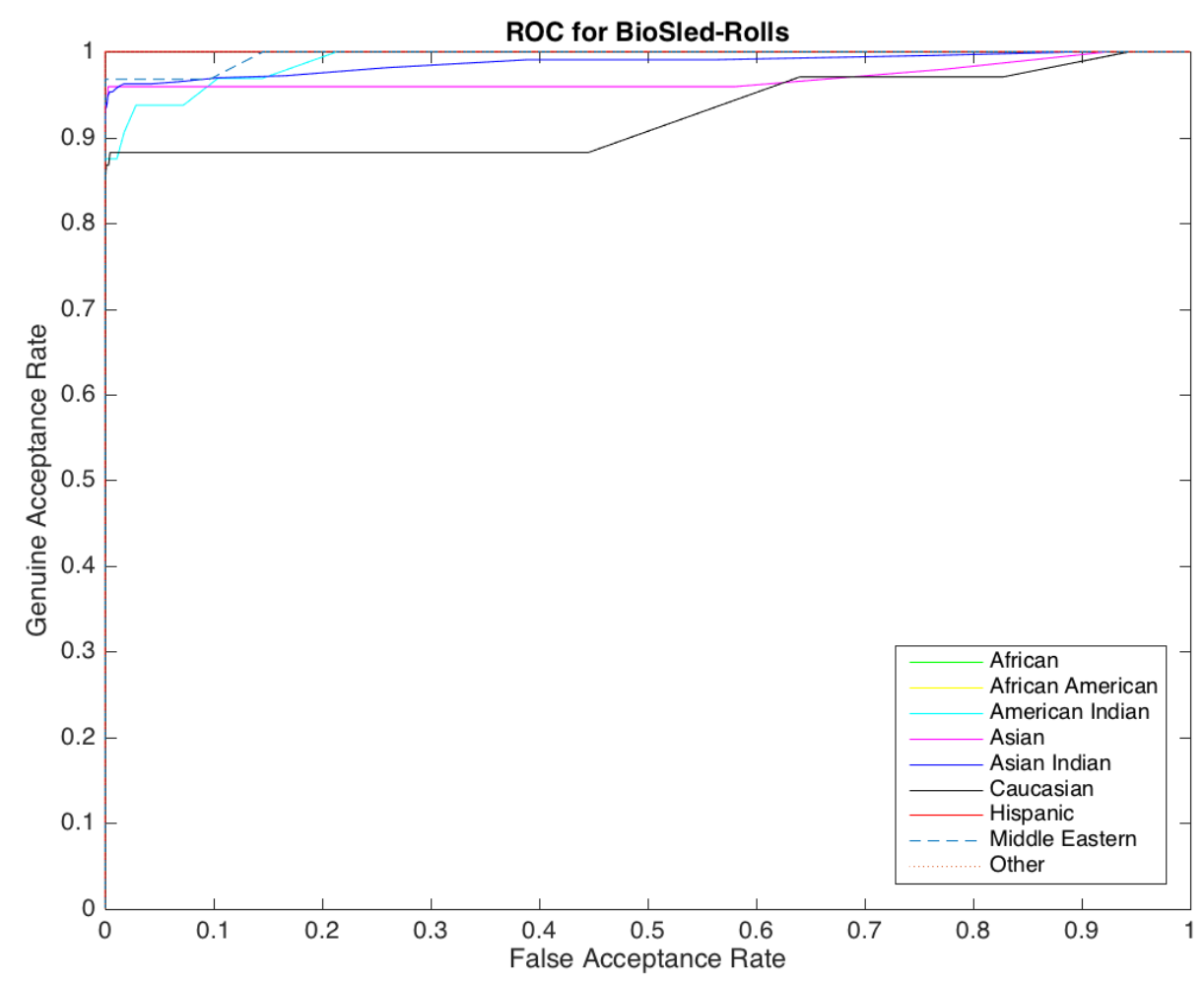

Figure D.4.4 NBIS BioSled Rolls Ethnicity Based ROC Curves

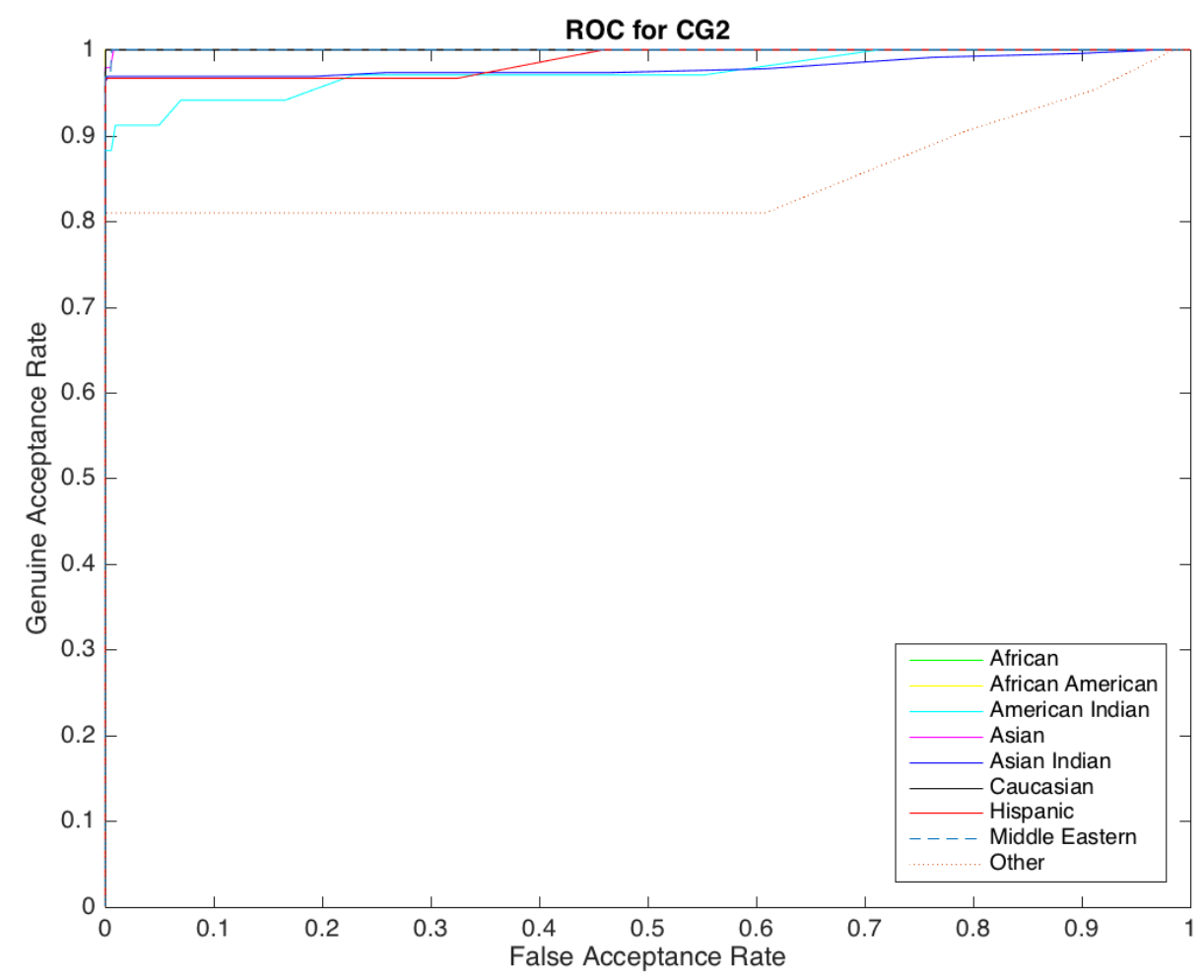

Figure D.4.5 NBIS FP II Guardian Ethnicity Based ROC Curves 


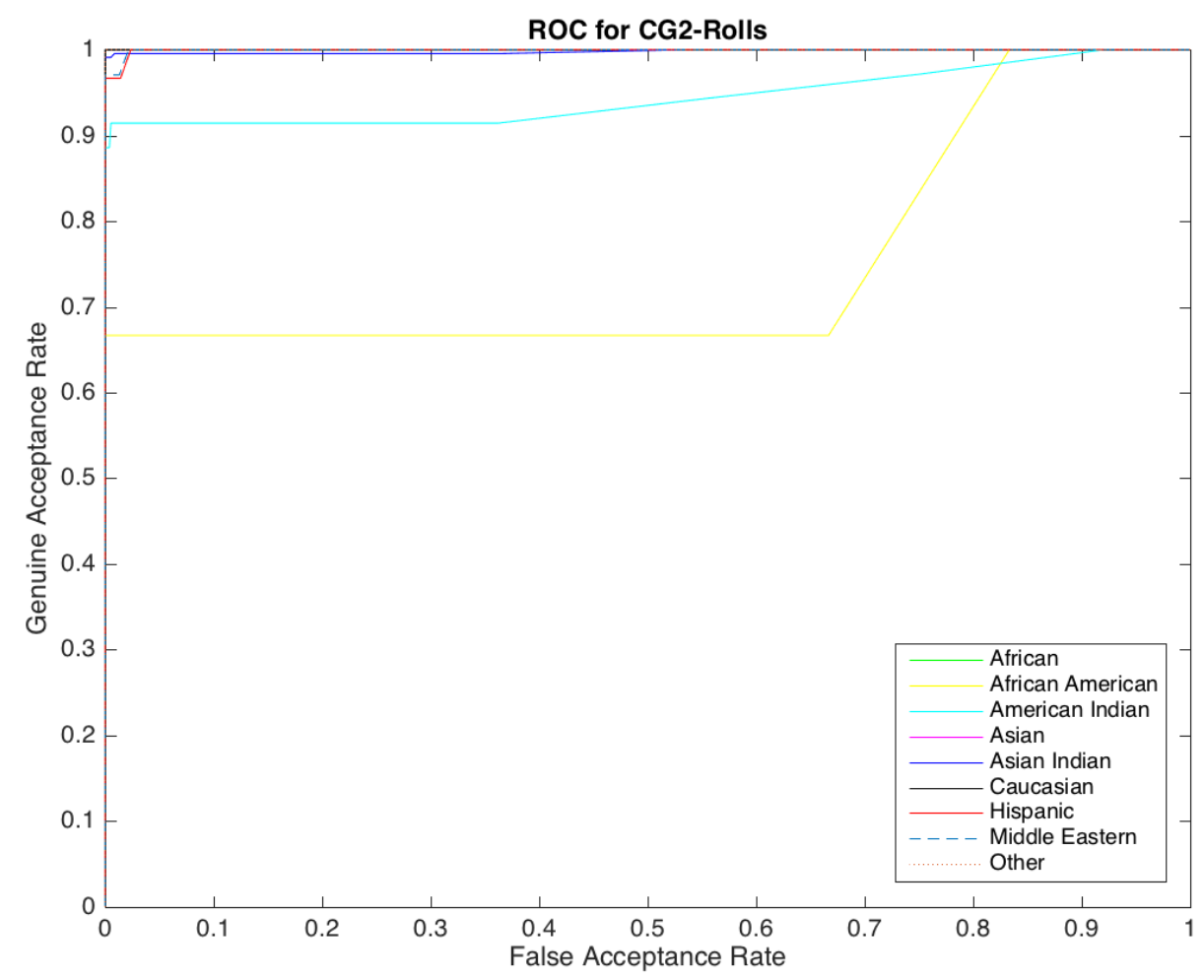

Figure D.4.6 NBIS FP II Guardian Rolls Ethnicity Based ROC Curves

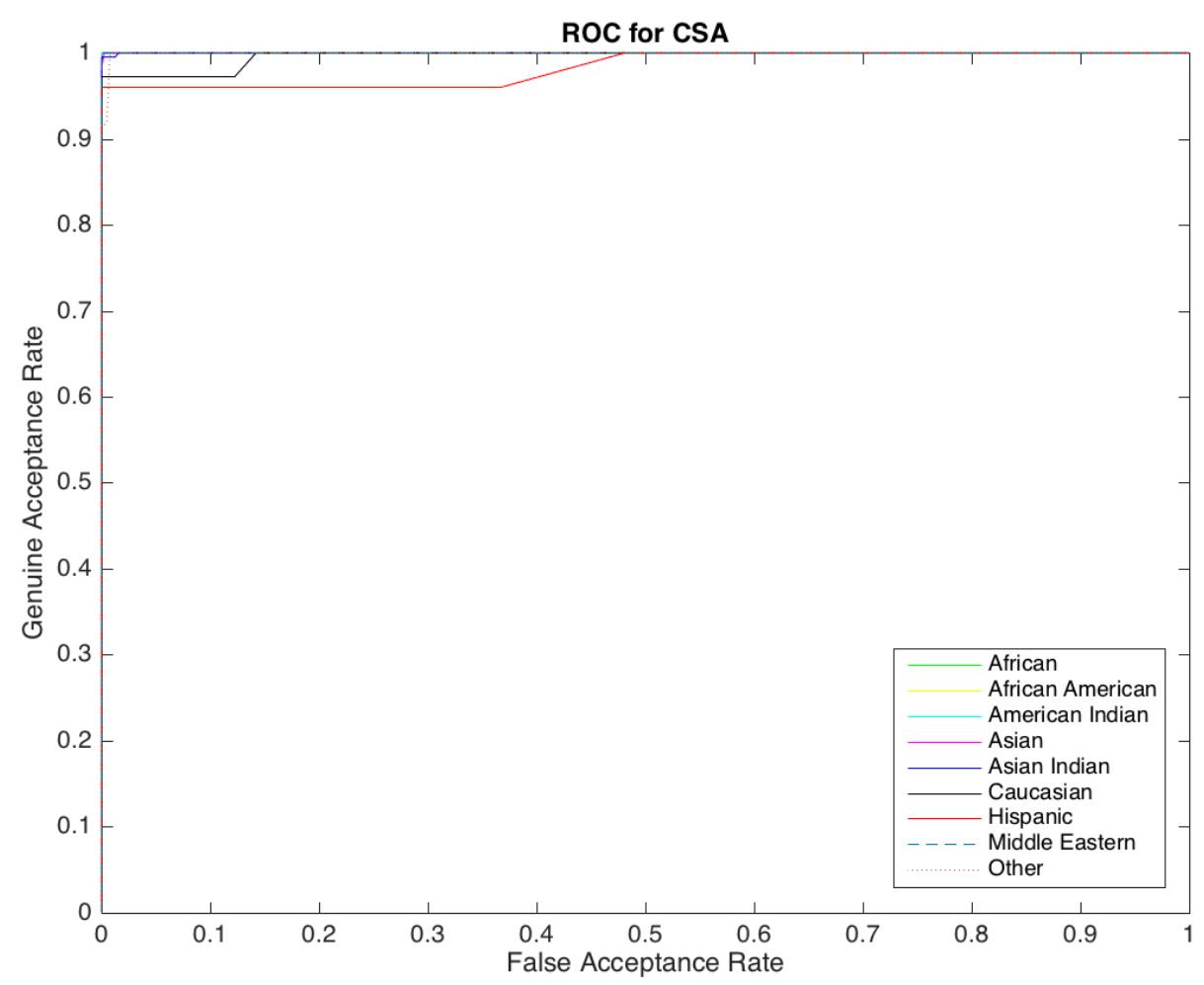

Figure D.4.7 NBIS Crossmatch Seek Avenger Ethnicity Based ROC Curves 


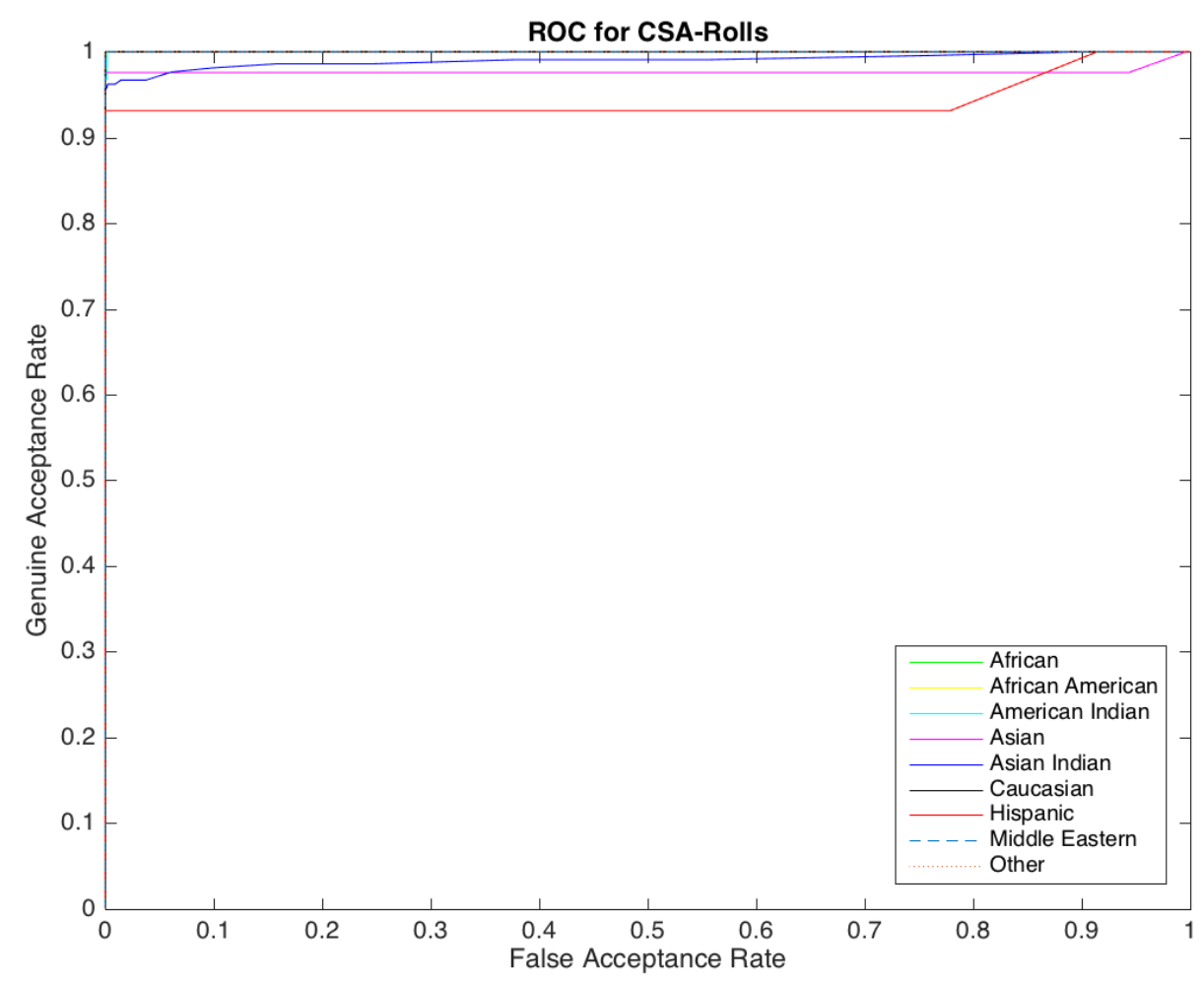

Figure D.4.8 NBIS Crossmatch Seek Avenger Rolls Ethnicity Based ROC Curves

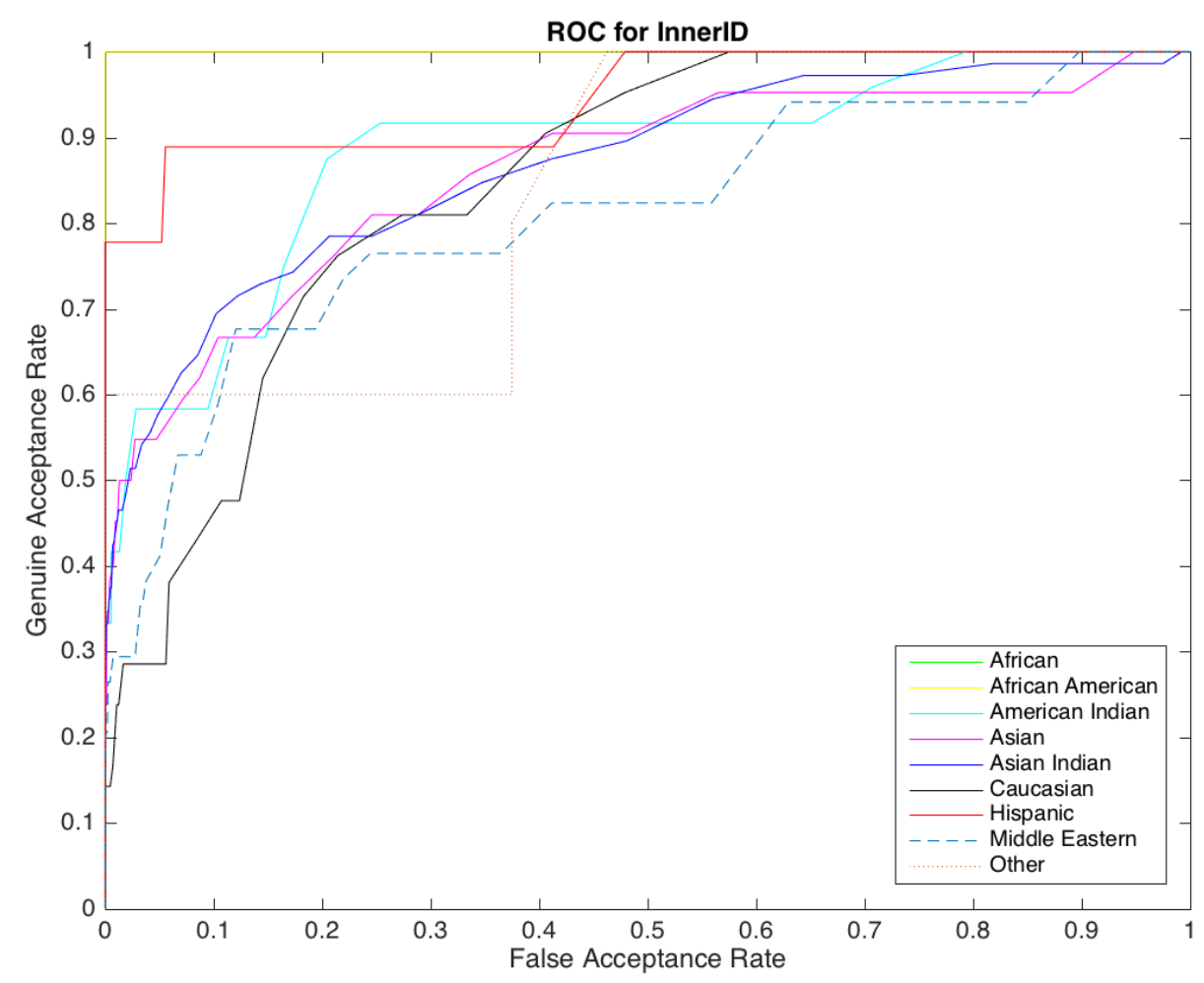

Figure D.4.9 NBIS InnerID Ethnicity Based ROC Curves 


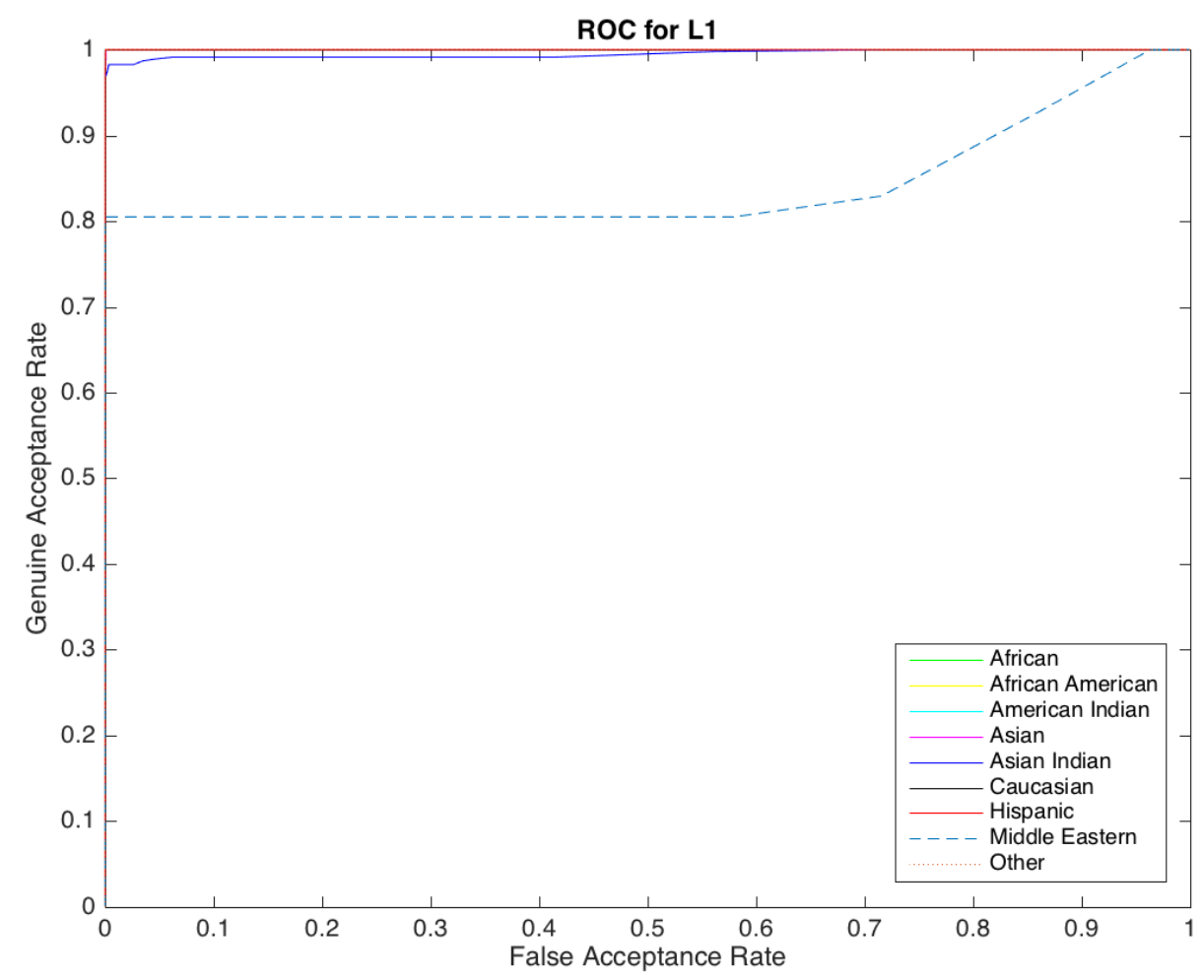

Figure D.4.10 NBIS L1 Ethnicity Based ROC Curves

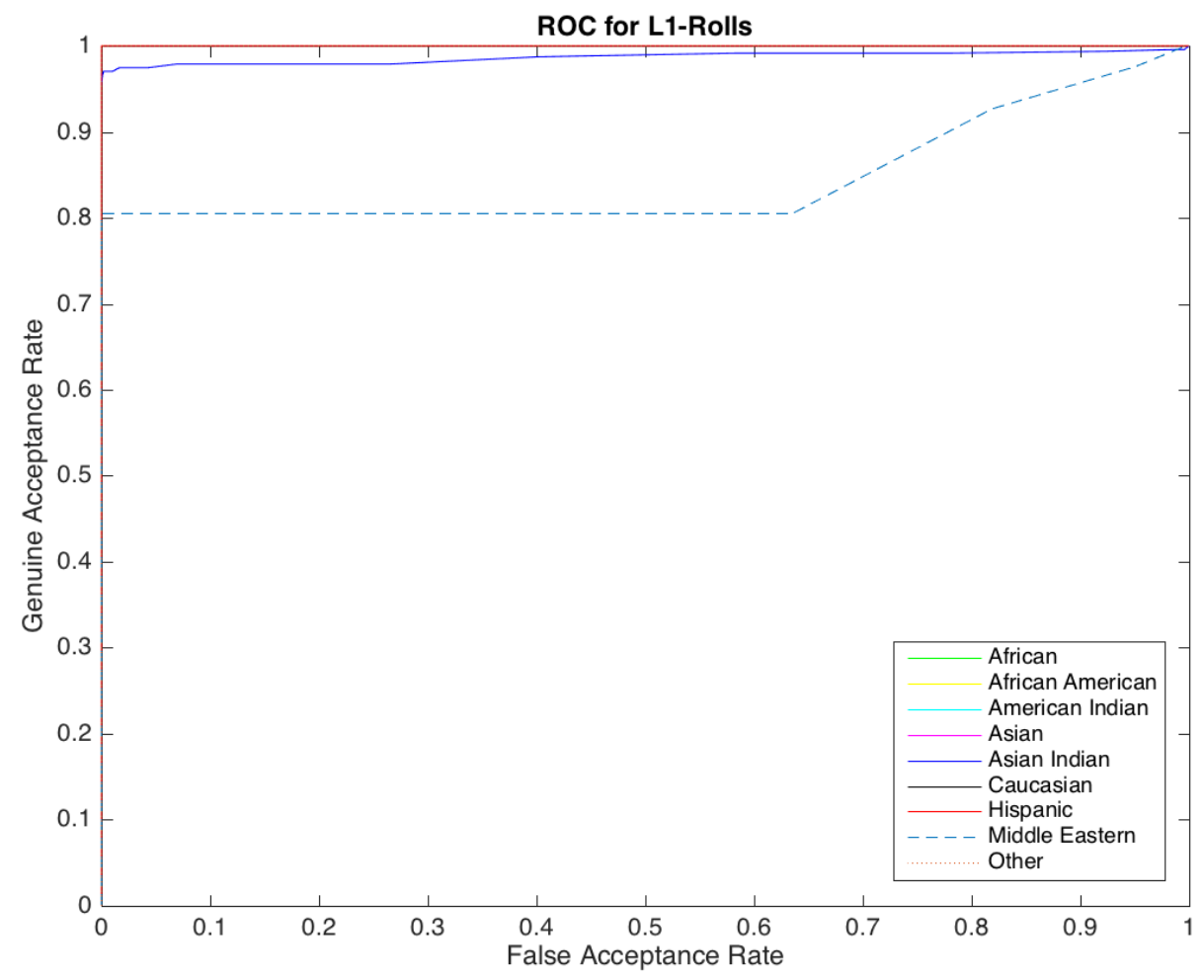

Figure D.4.11 NBIS L1 Rolls Ethnicity Based ROC Curves 


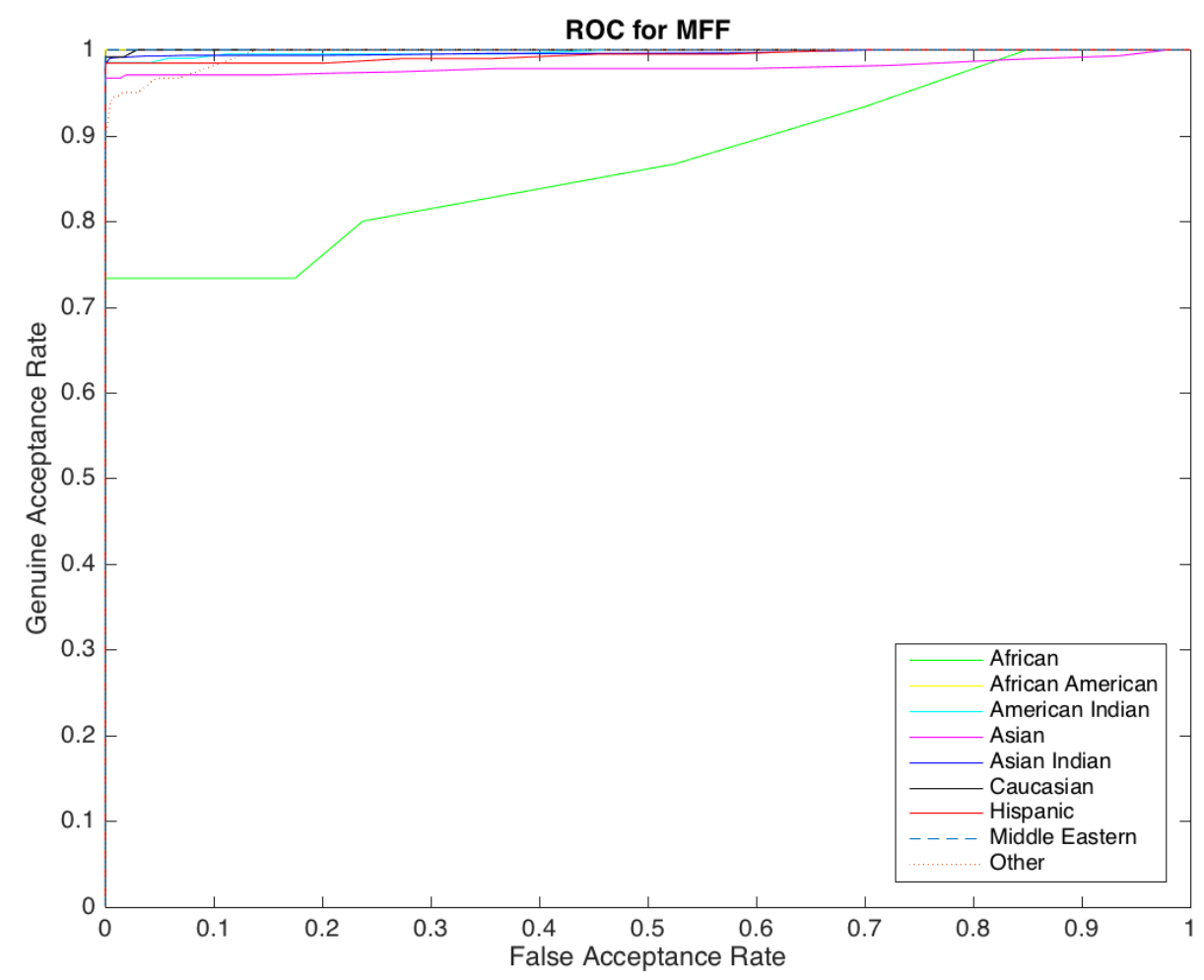

Figure D.4.12 NBIS Morpho FOTF Ethnicity Based ROC Curves

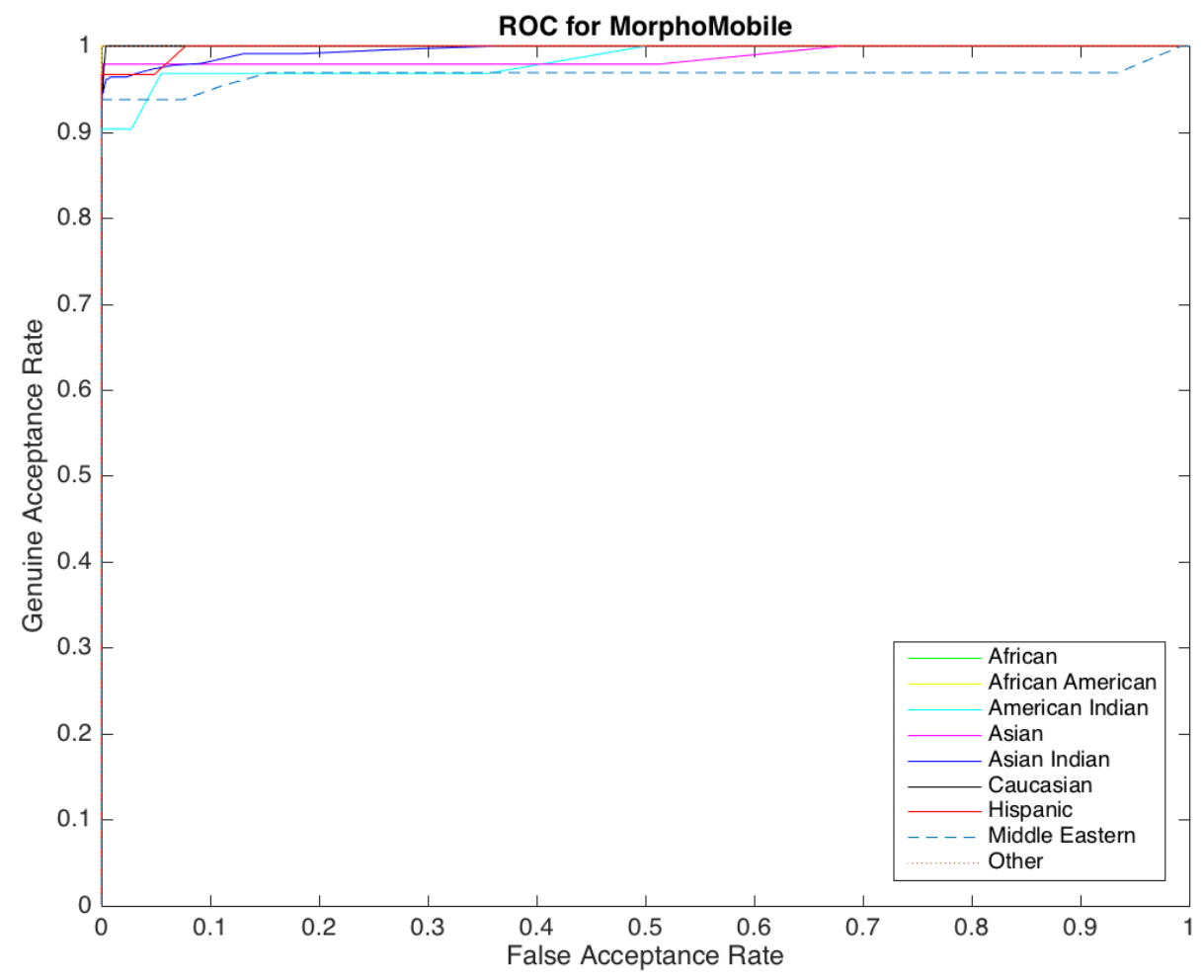

Figure D.4.13 NBIS MorphoMobile Ethnicity Based ROC Curves 


\section{D.5 Neurotech ROC Curves and Area Under Curve}

Table D.29 Neurotech Area Under Curve for Each Ethnicity

\begin{tabular}{|c|c|c|c|c|c|c|c|c|c|}
\hline \multicolumn{10}{|c|}{ Neurotech Area Under Curve } \\
\hline & irican & $\begin{array}{c}\text { African } \\
\text { American }\end{array}$ & $\begin{array}{c}\text { American } \\
\text { Indian }\end{array}$ & Asian & $\begin{array}{l}\text { Asian } \\
\text { Indian }\end{array}$ & Caucasian & Hispanic & $\begin{array}{l}\text { Middle } \\
\text { Eastern }\end{array}$ & Other \\
\hline ANDI BIN & 1.0000 & 1.0000 & 1.0000 & 0.9886 & 1.0000 & 9746 & 9753 & 0.9768 & 0.9815 \\
\hline ANDI GRY & 1.0000 & 1.0000 & 1.0000 & 0.9896 & 1.0000 & 0.9749 & 0.9768 & 0.9816 & 0.9985 \\
\hline BioSled & 0.9011 & 0280 & 0000 & 9851 & 0780 & & 8 & 0 & 0756 \\
\hline $\begin{array}{l}\text { BioSled } \\
\text { Rolls }\end{array}$ & 1.0000 & 000 & 00 & 1.0000 & 0.9825 & 94 & 9602 & 1.0000 & 1.0000 \\
\hline CG2 & 1.0000 & 0.8573 & 1.0000 & 0.9410 & 1.0000 & 0.9870 & 1.0000 & 1.0000 & 1.0000 \\
\hline CG2 Rolls & 1.0000 & 1.0000 & 1.0000 & 0.9574 & 1.0000 & 1.0000 & 1.0000 & 1.0000 & 1.0000 \\
\hline CSA & 1.0000 & 1.0000 & 1.0000 & 1.0000 & 1.0000 & 1.0000 & 1.0000 & 0.9665 & 1.0000 \\
\hline CSA Rolls & 1.0000 & 1.0000 & 000 & 1.0000 & 0.9797 & 0.9947 & 1.0000 & 1.0000 & 1.0000 \\
\hline IID & 1.0000 & 0.7963 & 1.0000 & 1.0000 & 0.9728 & 0.9225 & 0.9288 & 0.9180 & 0.9493 \\
\hline L1 & 1.0000 & 1.0000 & 1.0000 & 1.0000 & 1.0000 & 0.9939 & 1.0000 & 1.0000 & 0.8938 \\
\hline MFF & & & 1.0000 & 0.9934 & 0.9798 & 0.9987 & 1.0000 & 0.9949 & 1.0000 \\
\hline SMM & 1.0000 & 1.0000 & 1.0000 & 0.9819 & 0.9940 & 0.9922 & 1.0000 & 1.0000 & 0.9875 \\
\hline
\end{tabular}

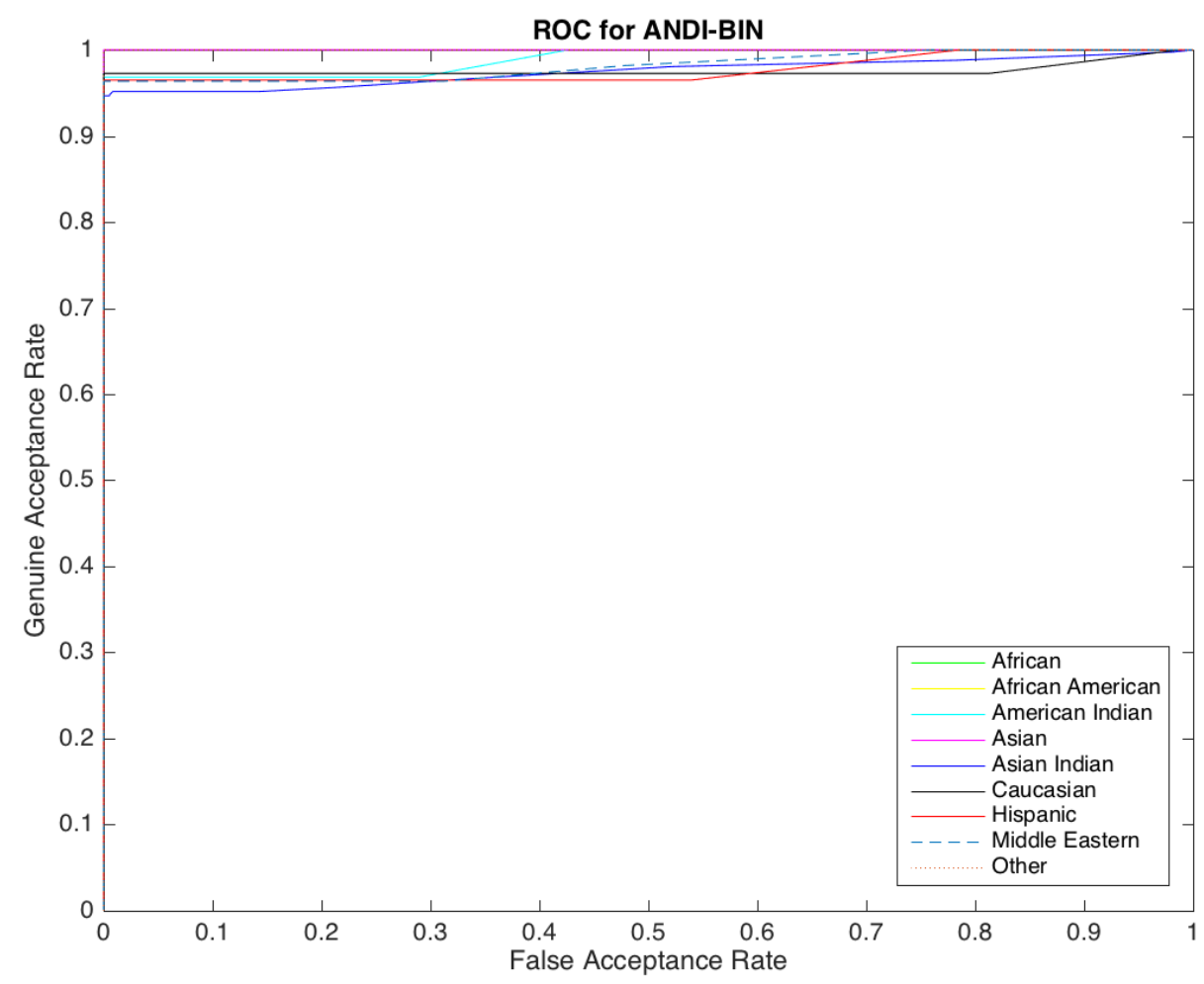

Figure D.5.1 Neurotech ANDI Binary Ethnicity Based ROC Curves 


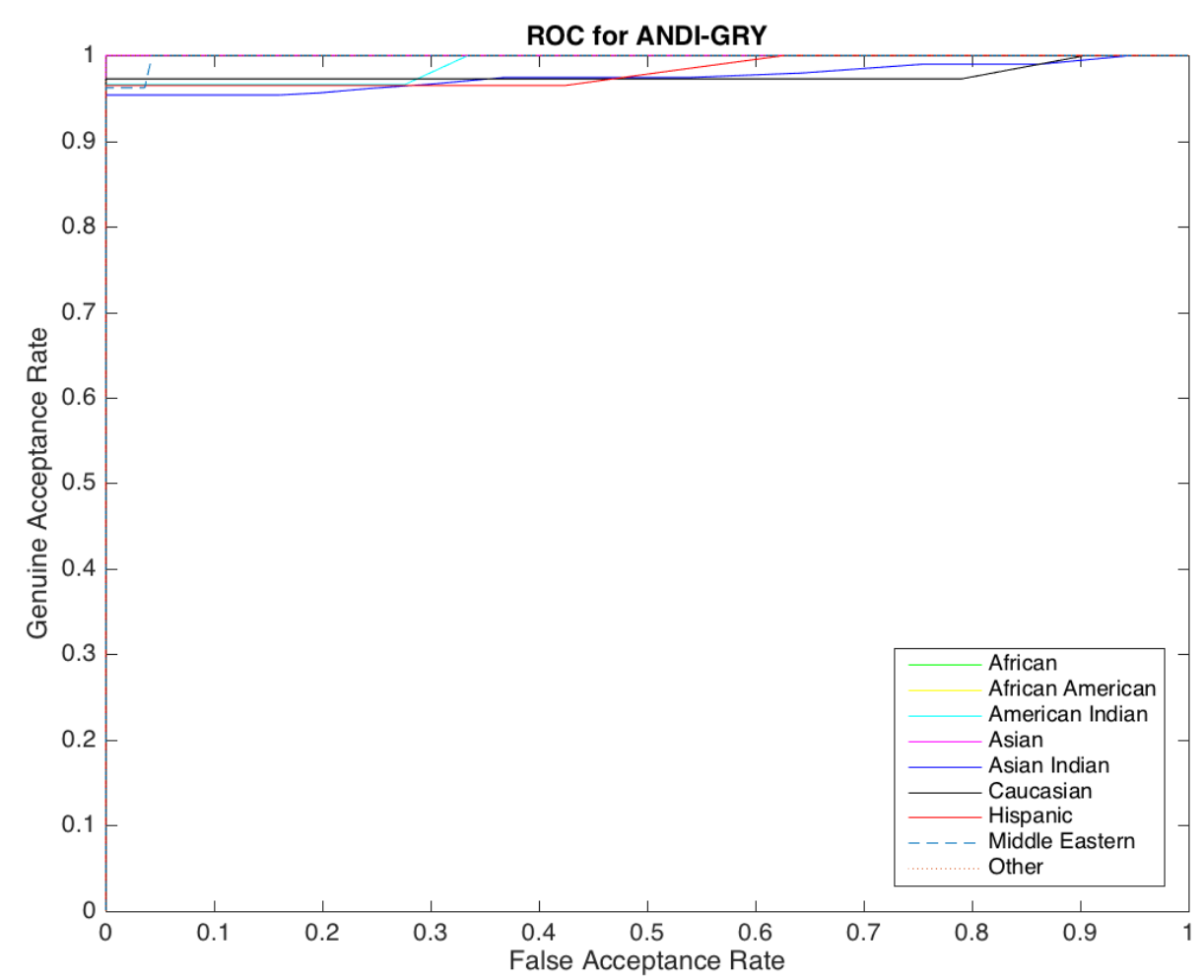

Figure D.5.2 Neurotech ANDI Greyscale Ethnicity Based ROC Curves

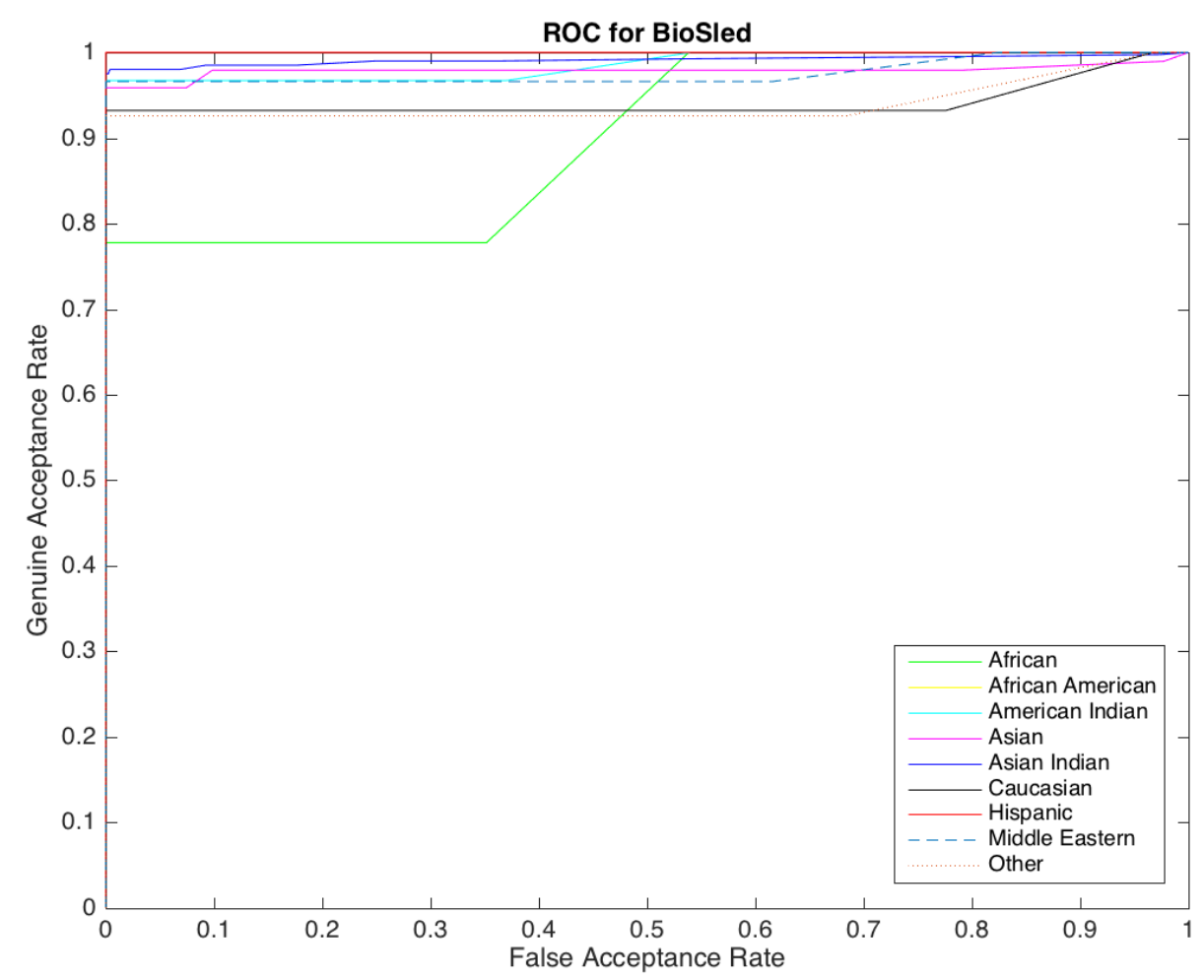

Figure D.5.3 Neurotech BioSled Ethnicity Based ROC Curves 


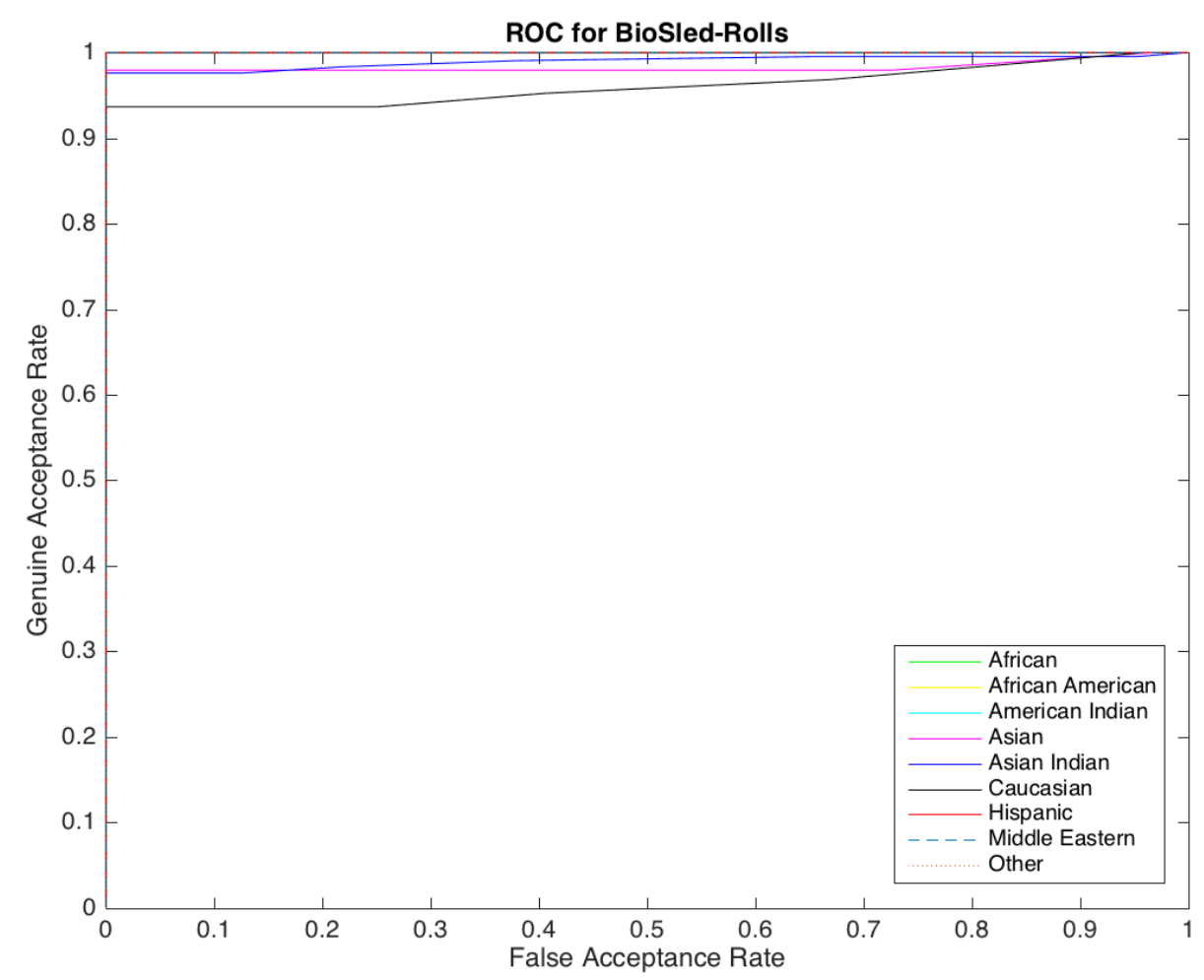

Figure D.5.4 Neurotech BioSled Rolls Ethnicity Based ROC Curves

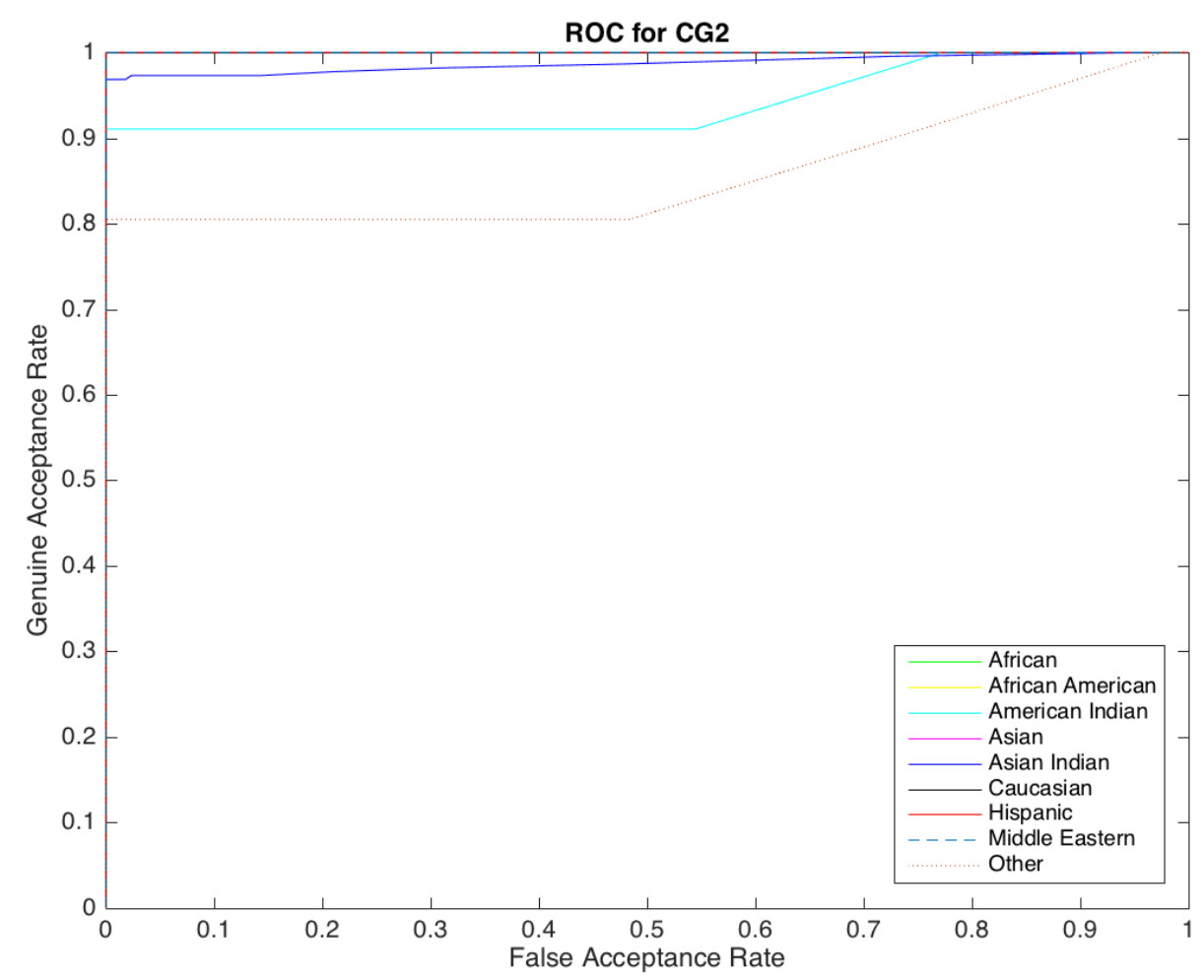

Figure D.5.5 Neurotech FP II Guardian Ethnicity Based ROC Curves 


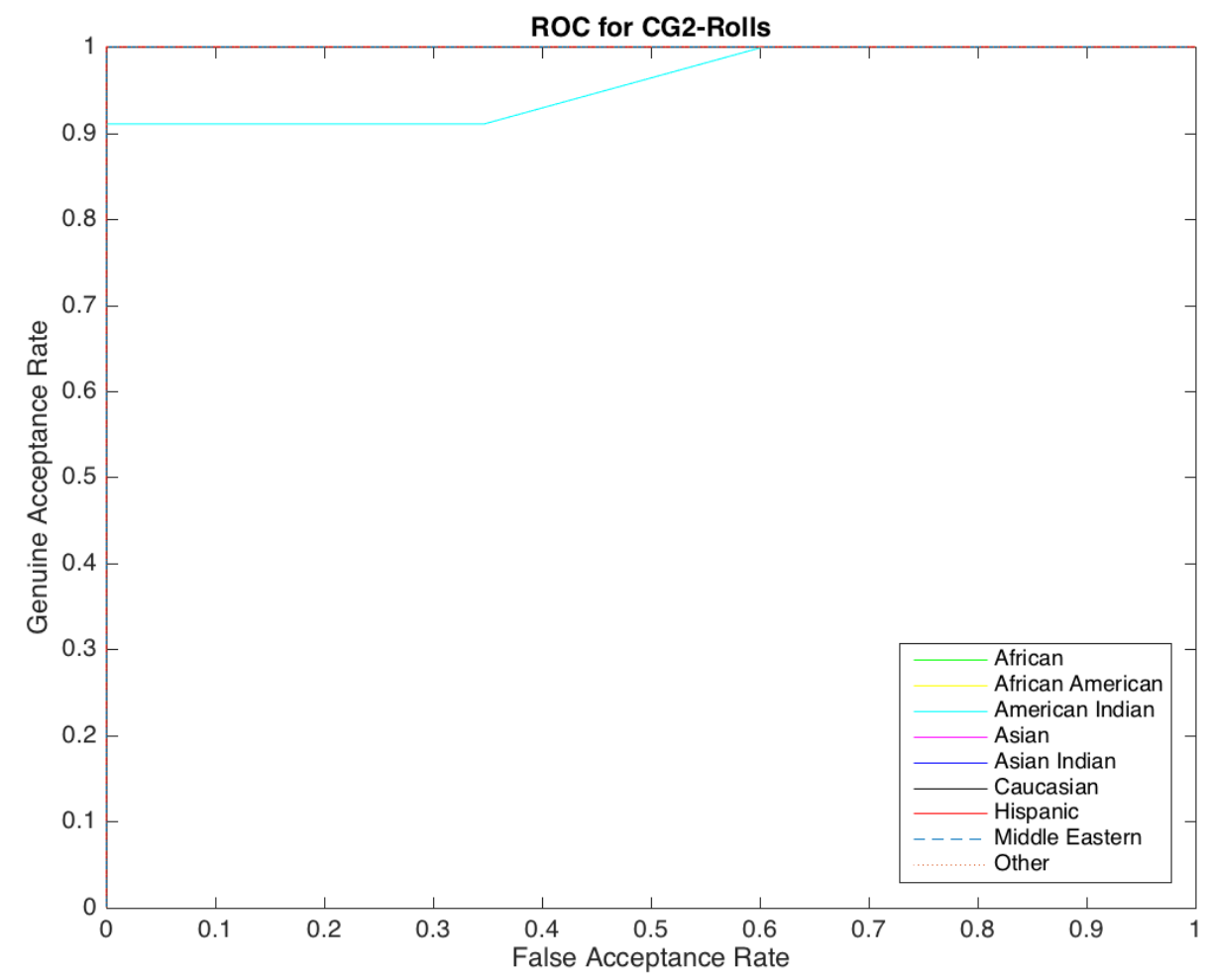

Figure D.5.6 Neurotech FP II Guardian Rolls Ethnicity Based ROC Curves

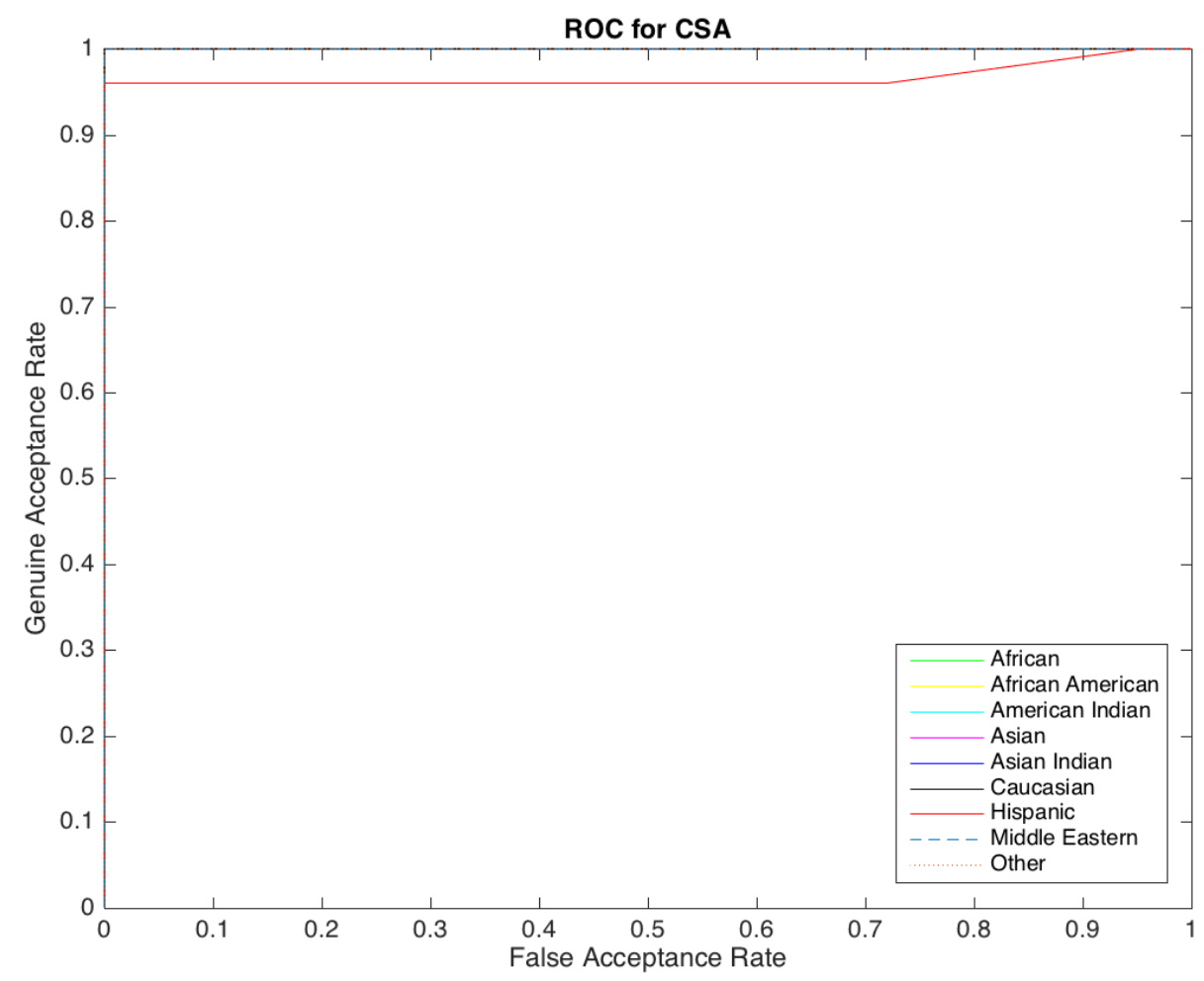

Figure D.5.7 Neurotech Crossmatch Seek Avenger Ethnicity Based ROC Curves 


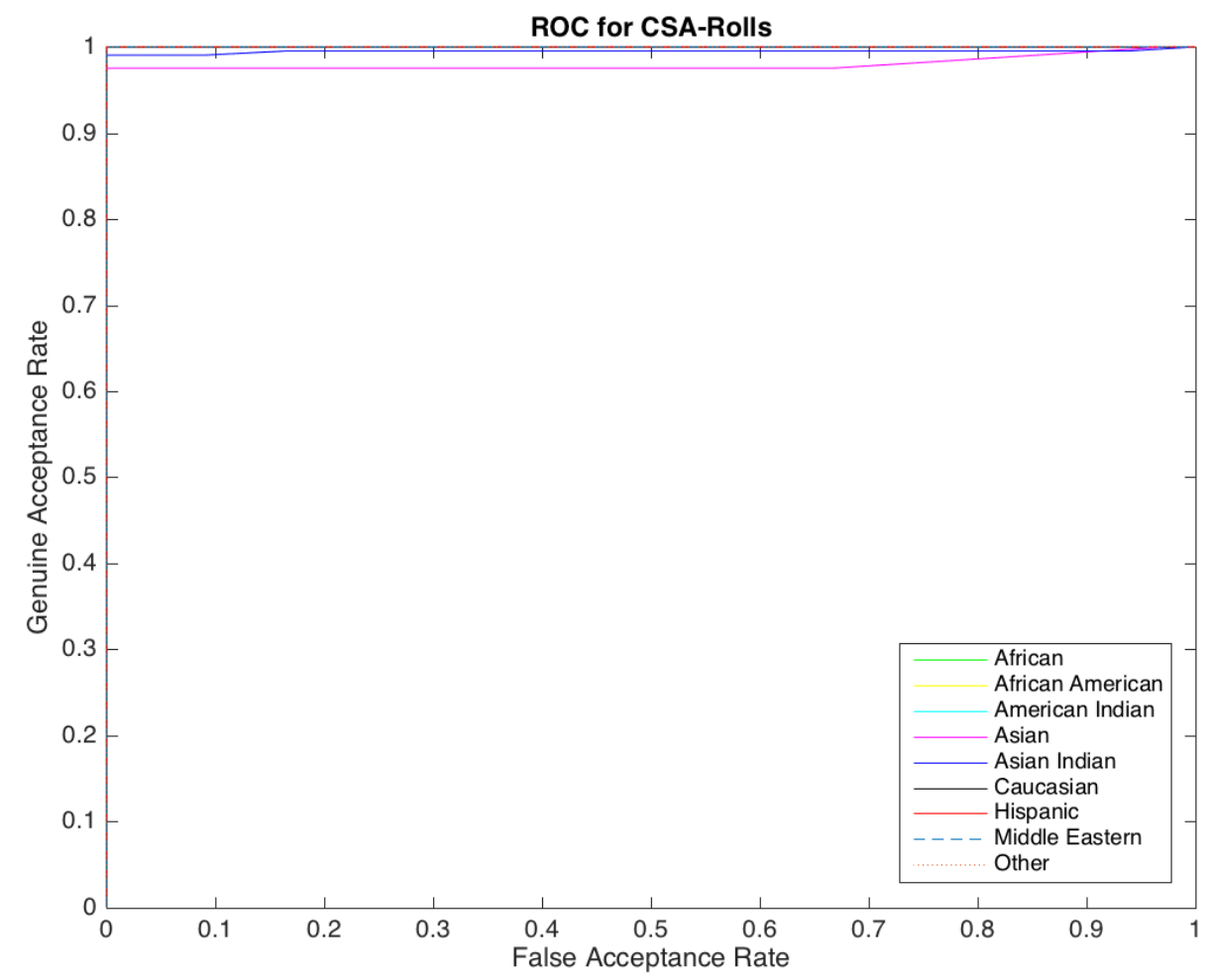

Figure D.5.8 Neurotech Crossmatch Seek Avenger Rolls Ethnicity Based ROC Curves

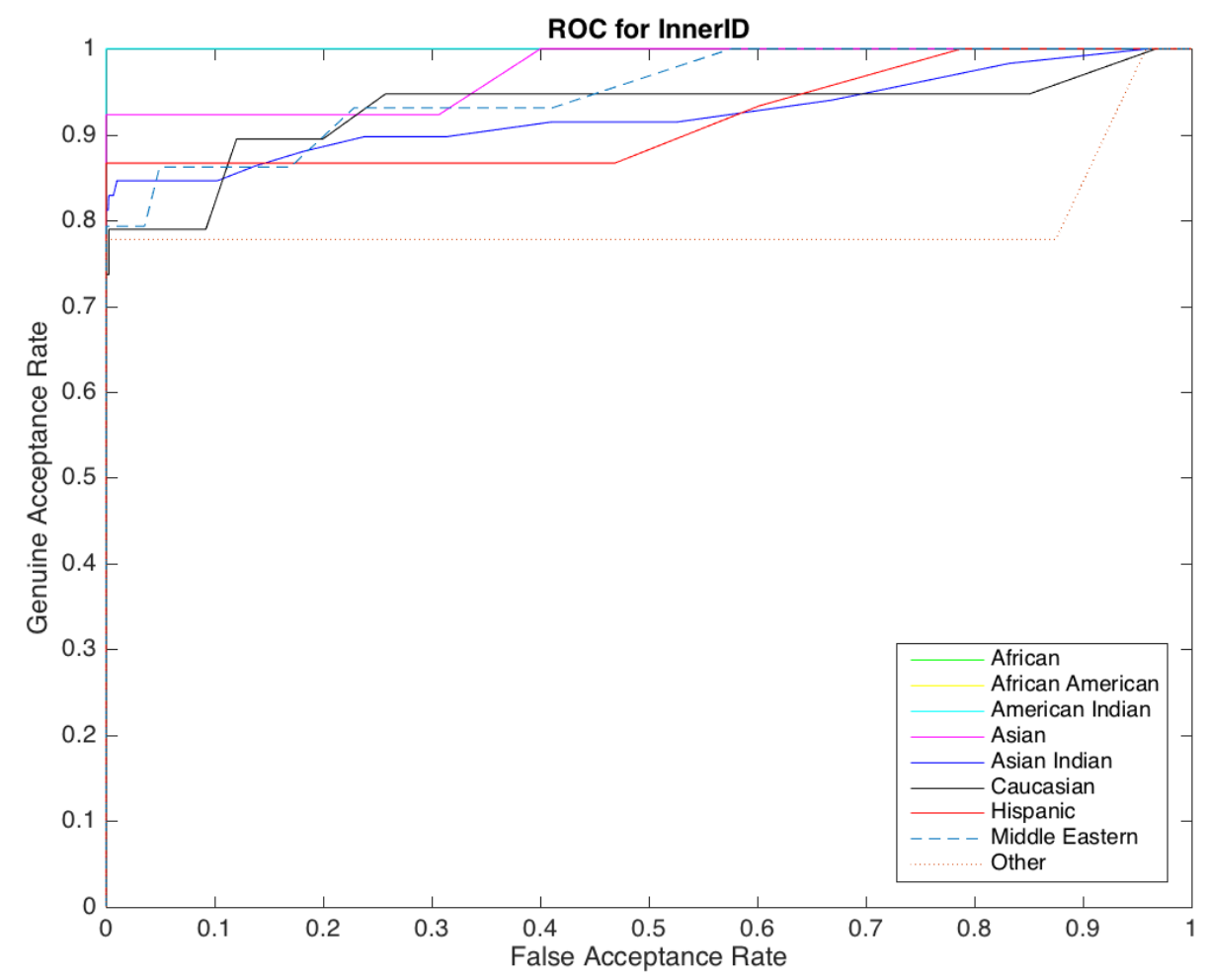

Figure D.5.9 Neurotech InnerID Ethnicity Based ROC Curves 


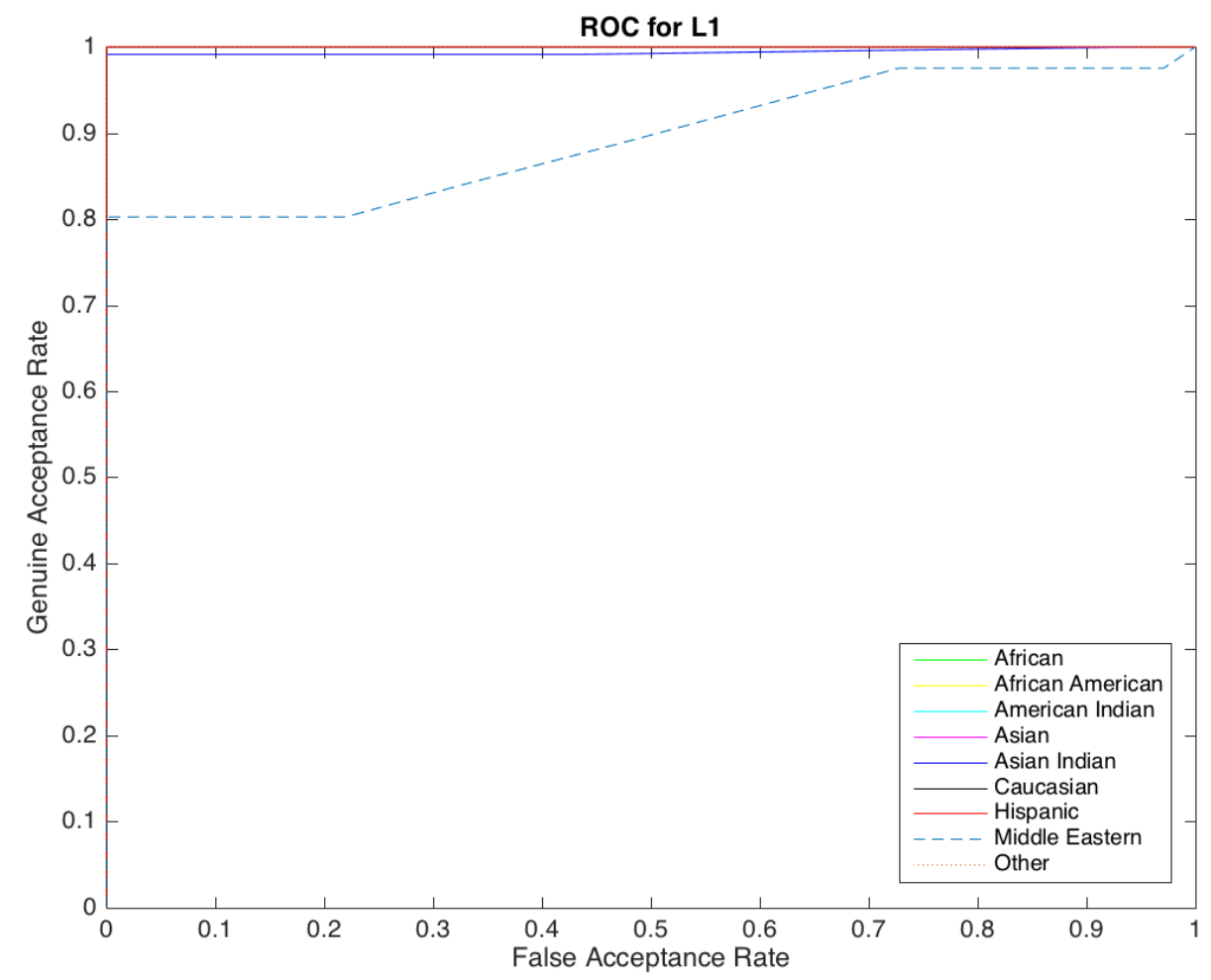

Figure D.5.10 Neurotech L1 Ethnicity Based ROC Curves

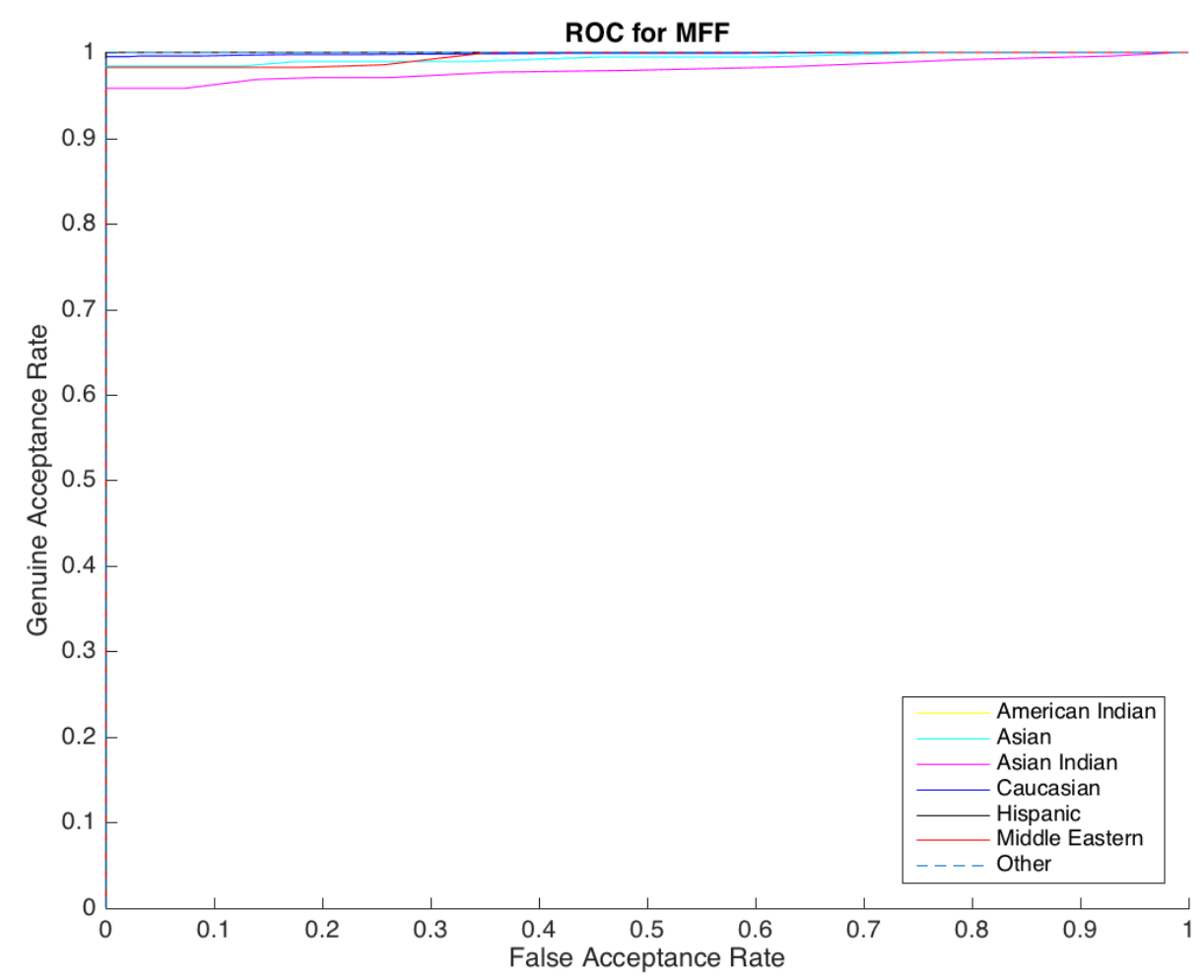

Figure D.5.11 Neurotech Morpho FOTF Ethnicity Based ROC Curves 


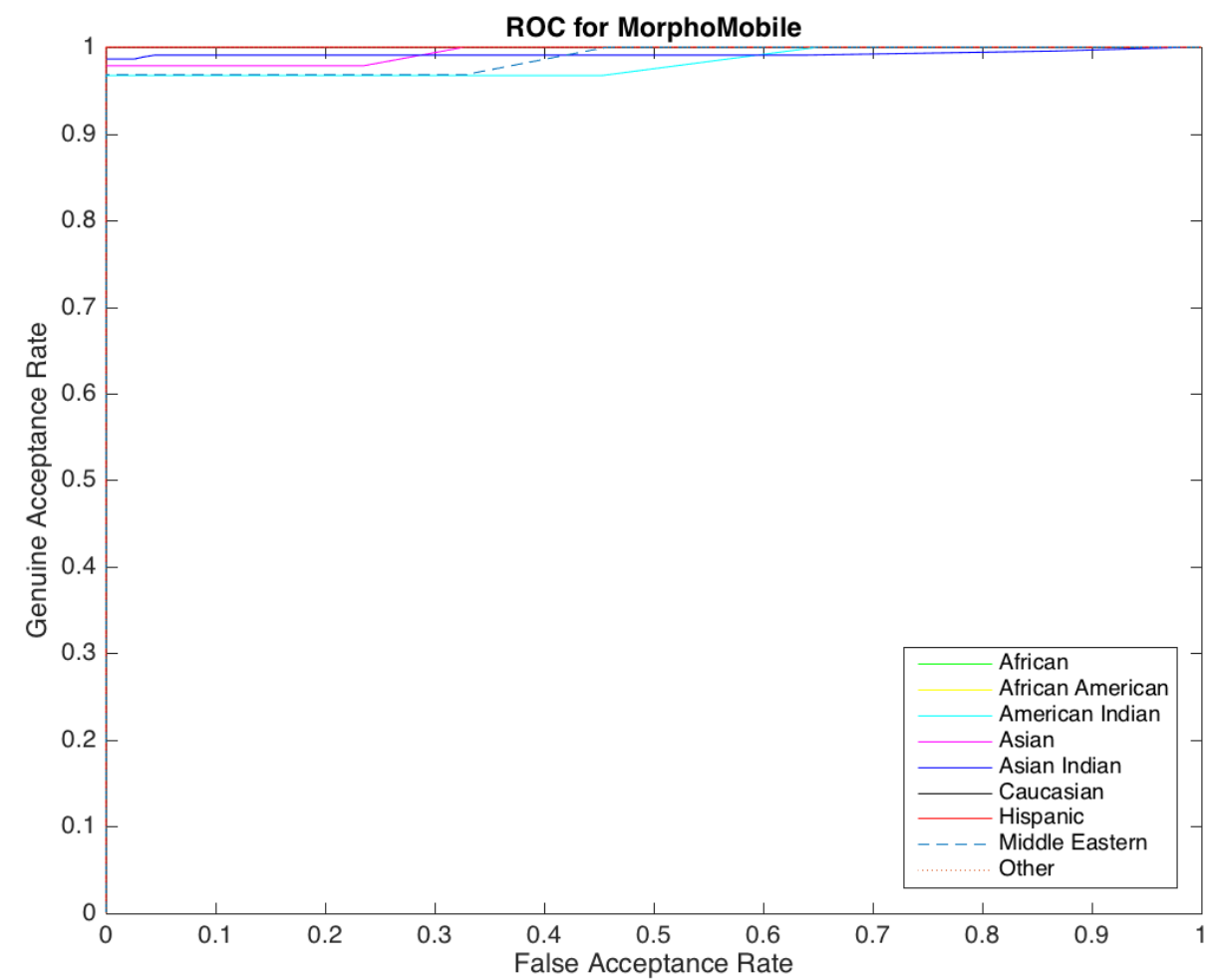

Figure D.5.12 Neurotech MorphoMobile Ethnicity Based ROC Curves 


\section{D.6 SourceAFIS ROC Curves and Area Under Curve}

Table D.30 SourceAFIS Area Under Curve for Each Ethnicity

\begin{tabular}{|c|c|c|c|c|c|c|c|c|c|}
\hline \multicolumn{10}{|c|}{ SourceAFIS Area Under Curve } \\
\hline & rican & $\begin{array}{c}\text { African } \\
\text { American }\end{array}$ & $\begin{array}{c}\text { American } \\
\text { Indian }\end{array}$ & Asian & $\begin{array}{l}\text { Asian } \\
\text { Indian }\end{array}$ & Caucasian & Hispanic & $\begin{array}{l}\text { Middle } \\
\text { Eastern }\end{array}$ & Other \\
\hline ANDI BIN & 1.0000 & 1.0000 & 1.0000 & 9690 & 0.9930 & .9540 & 9864 & 0.9669 & 0.9843 \\
\hline ANDI GRY & 0.9075 & 9728 & 1.0000 & 9578 & .9749 & 0.9174 & 9667 & 0.9571 & 9567 \\
\hline BioSled & 0.9960 & 0.9357 & 1.0000 & 0.9748 & 0.9707 & 0.9798 & 0.9419 & 0.9966 & 0.9921 \\
\hline $\begin{array}{l}\text { BioSled } \\
\text { Rolls }\end{array}$ & 1.0000 & 0 & 0 & 0 & 3 & 30 & 31 & 384 & 12 \\
\hline CG2 & 1.0000 & & & 9362 & & & & 0.9767 & 0000 \\
\hline CG2 Rolls & 1.0000 & 1.0000 & 0.9653 & 0.9242 & 1.0000 & 0.9904 & 0.9904 & 0.9393 & 0.9938 \\
\hline CSA & 1.0000 & 1.0000 & 1.0000 & 1.0000 & 1.0000 & 1.0000 & 1.0000 & 0.9770 & 1.0000 \\
\hline CSA Rolls & 1.0000 & 0.9977 & 1.0000 & 0.9801 & 0.9870 & 0.9841 & 0.9965 & 0.9720 & 1.0000 \\
\hline IID & 0.5313 & 0.7225 & 1.0000 & 0.7682 & 0.7171 & 0.8074 & 0.7880 & 0.9064 & 0.7400 \\
\hline L1 & 1.0000 & 1.0000 & 1.0000 & 0.9862 & 0.9995 & 0.9978 & 1.0000 & 1.0000 & 0.8552 \\
\hline L1 Rolls & 1.0000 & 0.9714 & 1.0000 & 1.0000 & 0.9924 & 0.9693 & 0.9845 & 0.9742 & 0.8898 \\
\hline MFF & 0.8896 & 0.9759 & 1.0000 & 0.9826 & 0.9698 & 0.9807 & 0.9837 & 0.9839 & 0.9782 \\
\hline SMM & 1.0000 & 1.0000 & 1.0000 & 0.9799 & 0.9816 & 0.9881 & 0.9999 & 0.9675 & 0.9705 \\
\hline
\end{tabular}

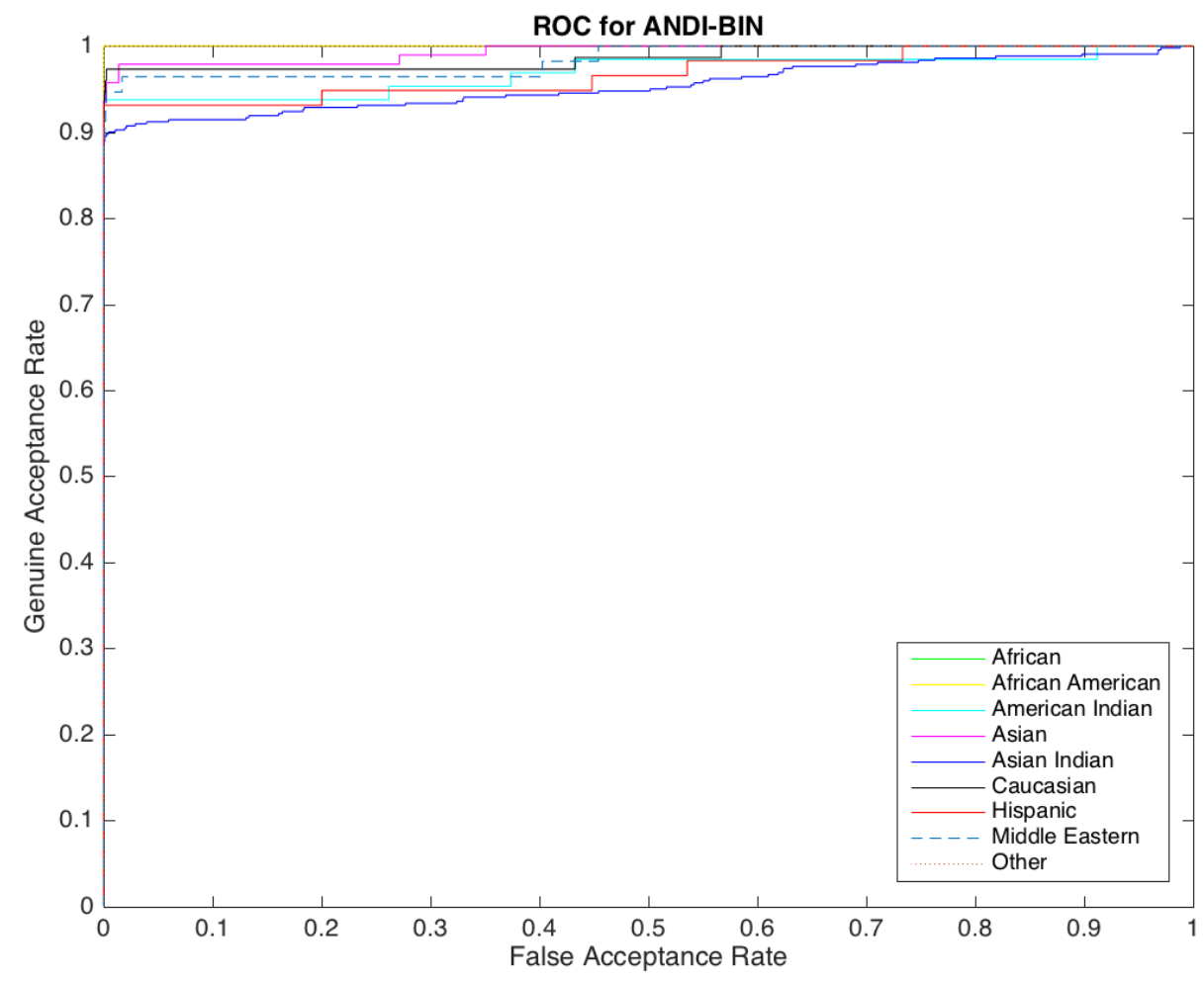


Figure D.6.1 SourceAFIS ANDI Binary Ethnicity Based ROC Curves

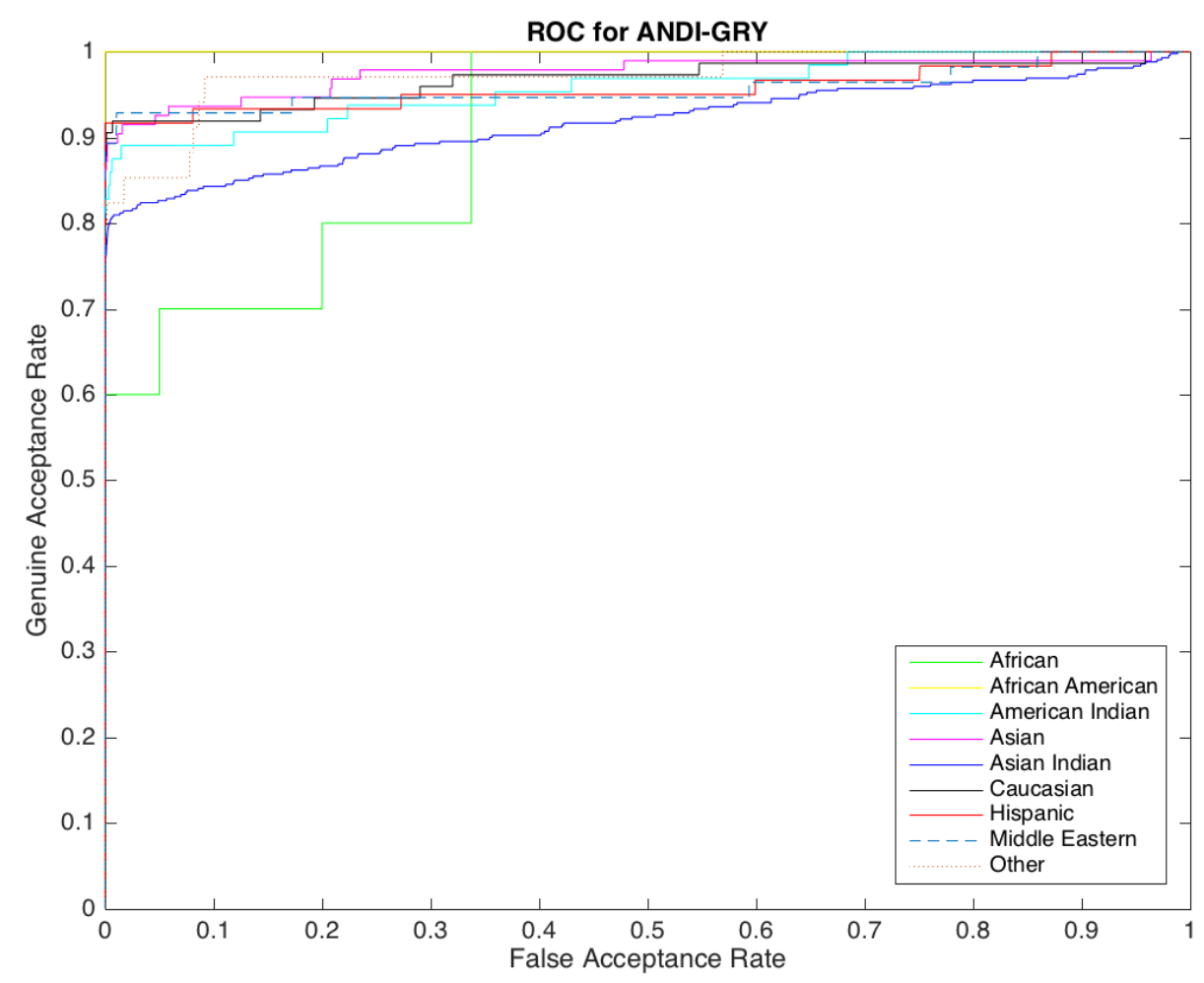

Figure D.6.2 SourceAFIS ANDI Greyscale Ethnicity Based ROC Curves 


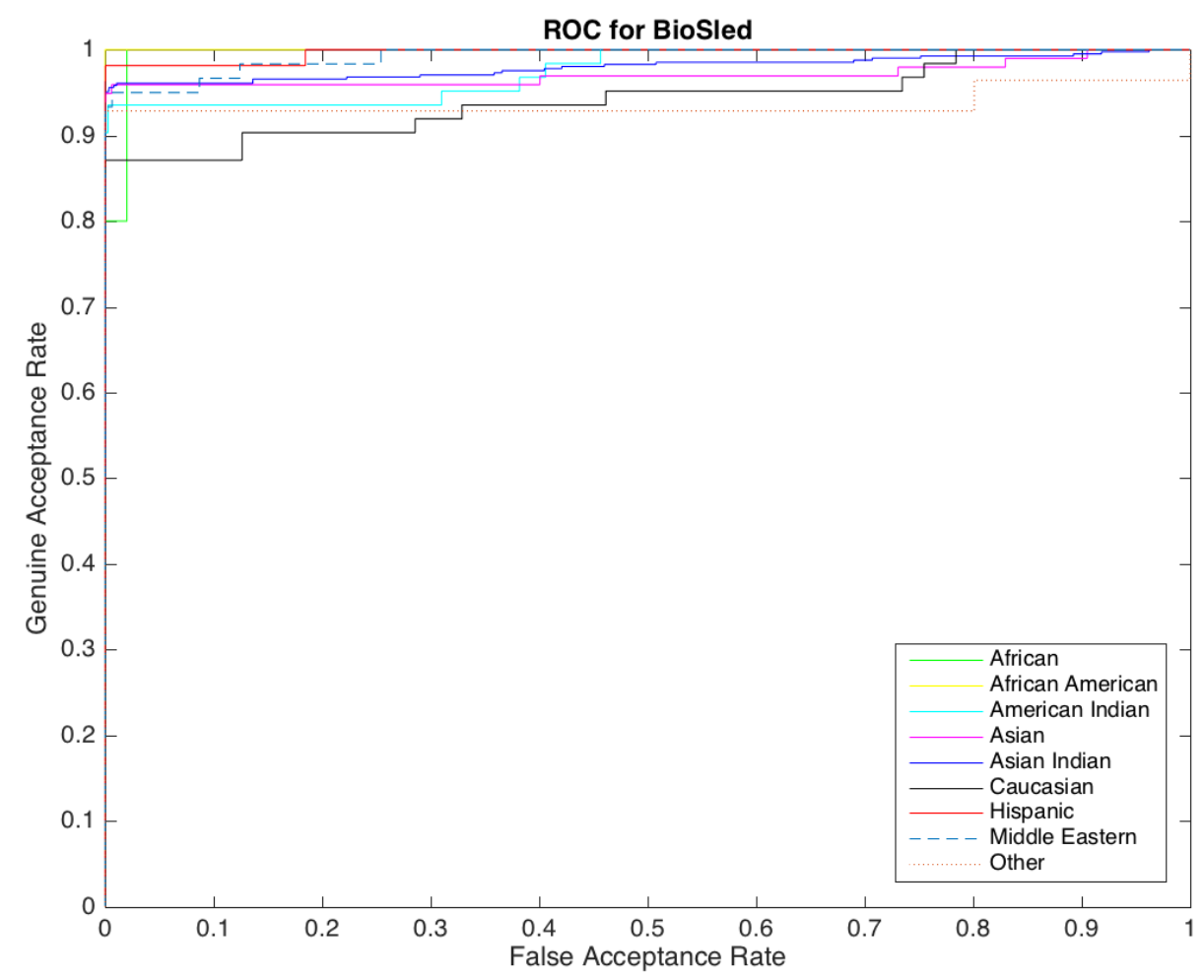

Figure D.6.3 SourceAFIS BioSled Ethnicity Based ROC Curves

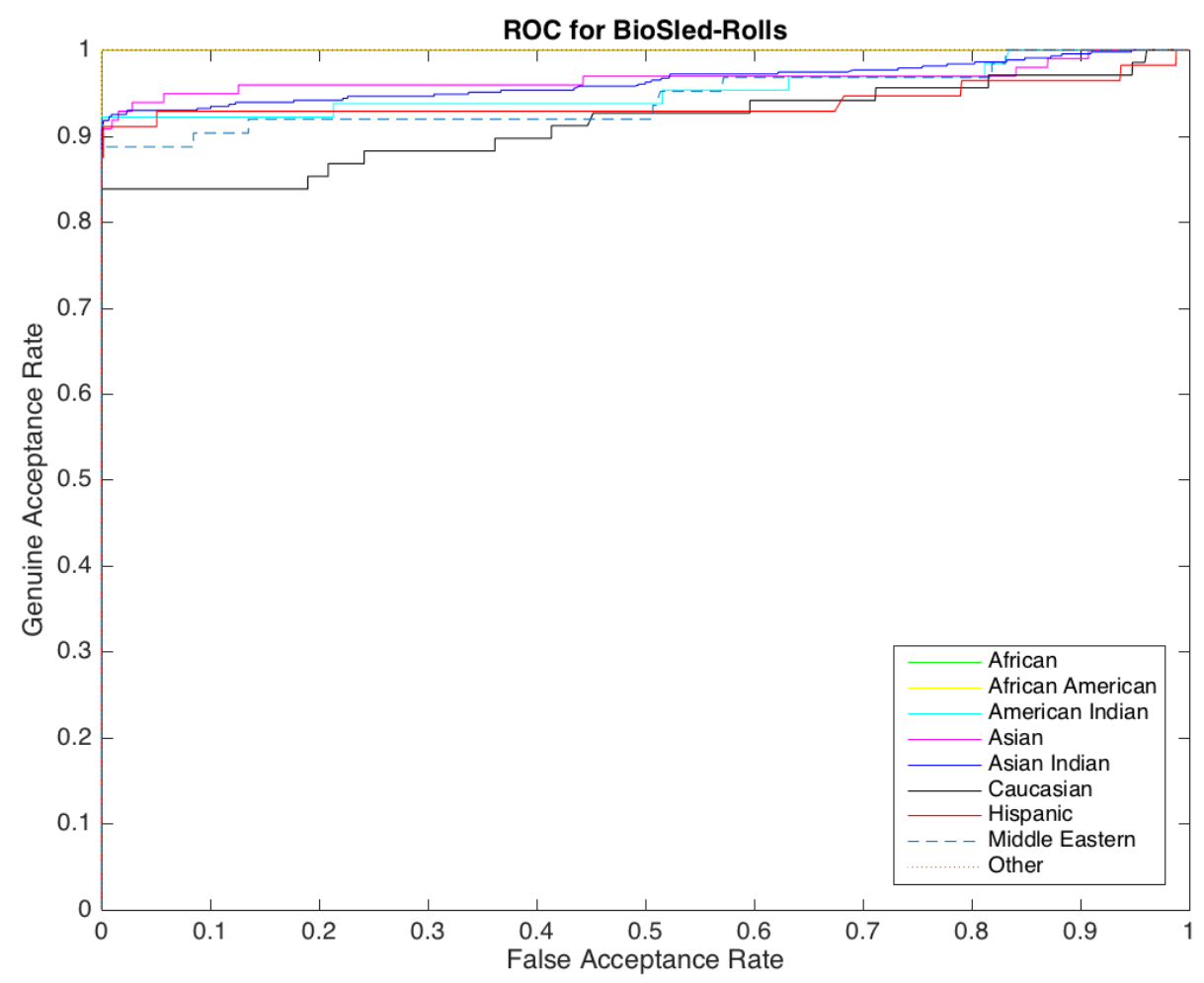

Figure D.6.4 SourceAFIS BioSled Rolls Ethnicity Based ROC Curves 


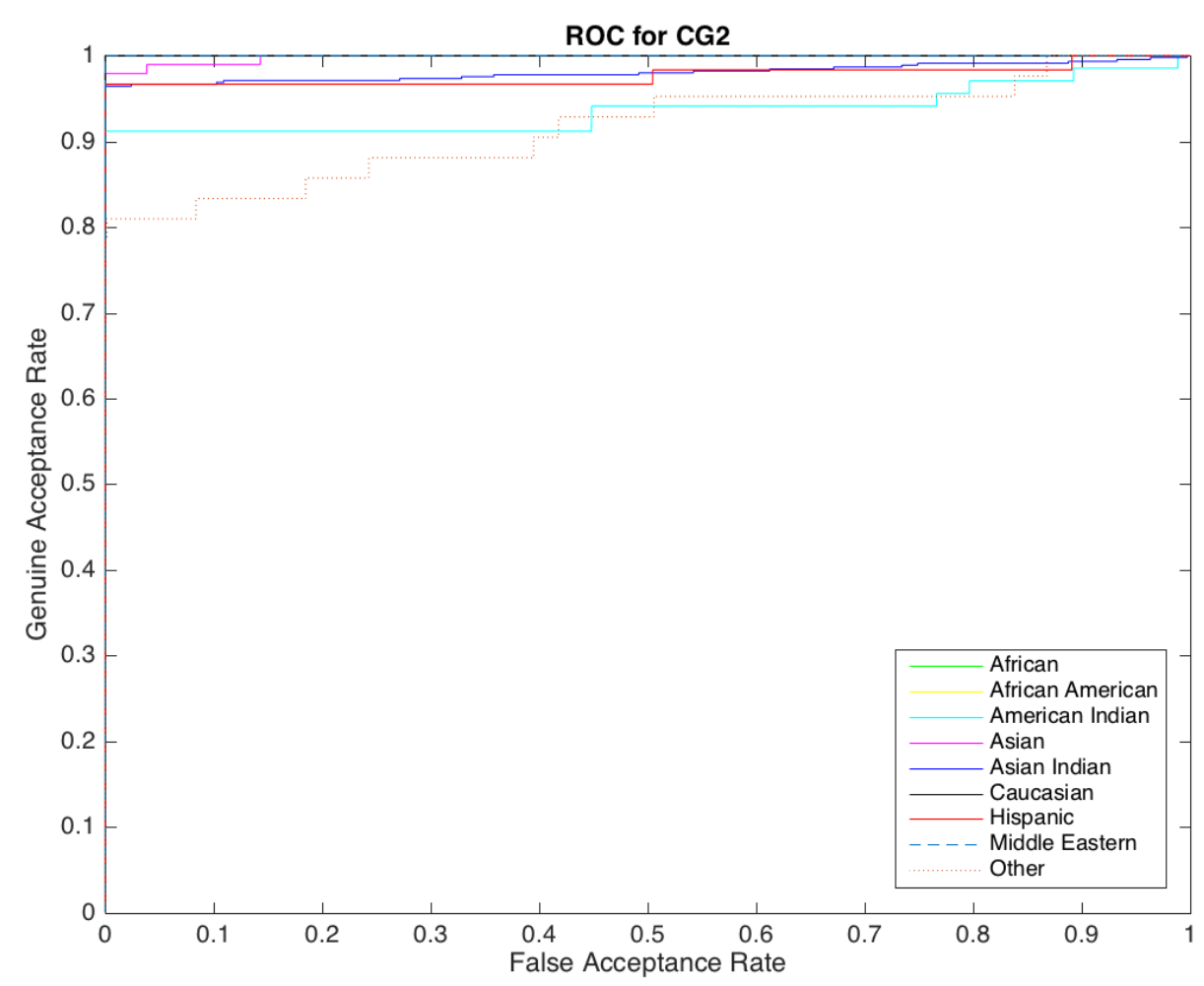

Figure D.6.5 SourceAFIS FP II Guardian Ethnicity Based ROC Curves

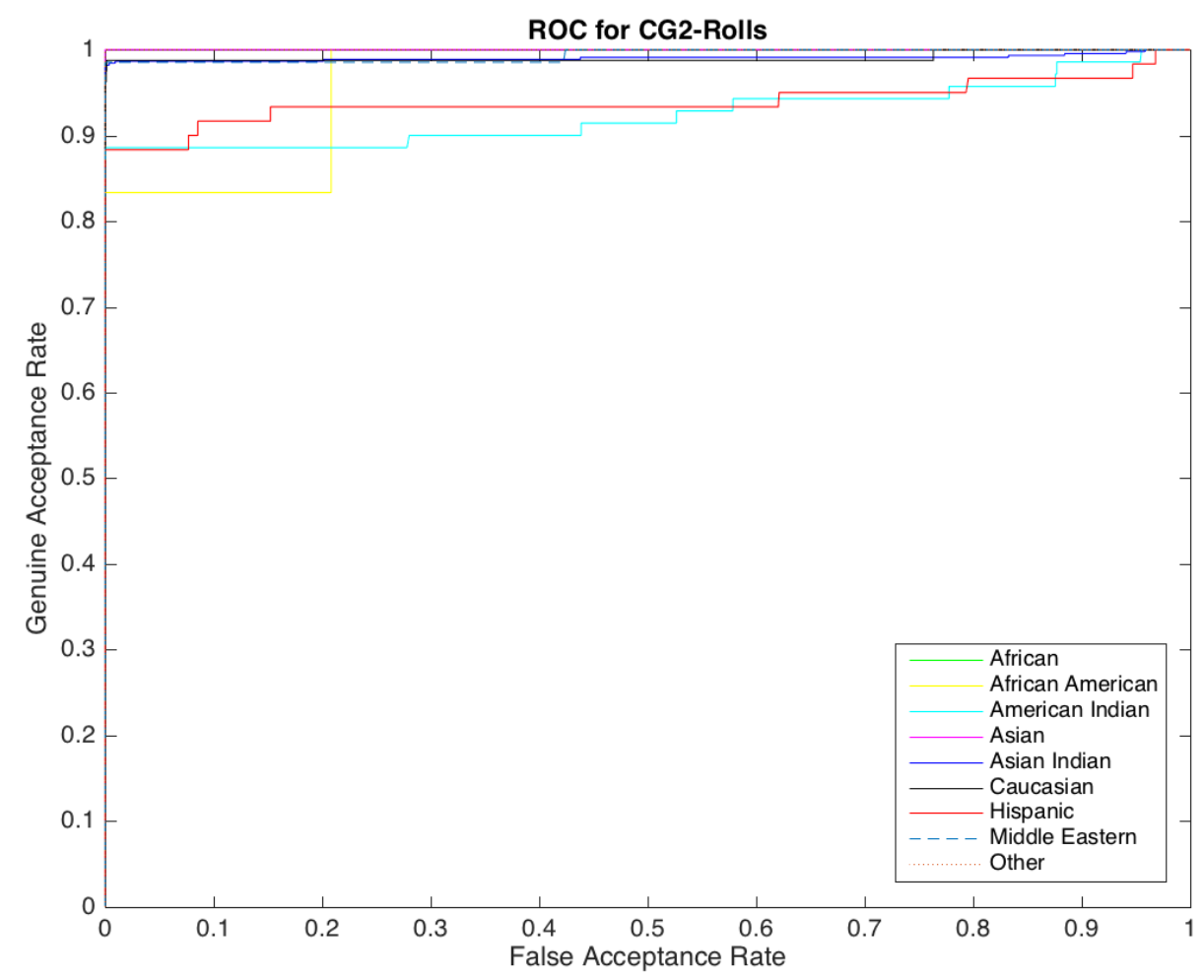

Figure D.6.6 SourceAFIS FP II Guardian Rolls Ethnicity Based ROC Curves 


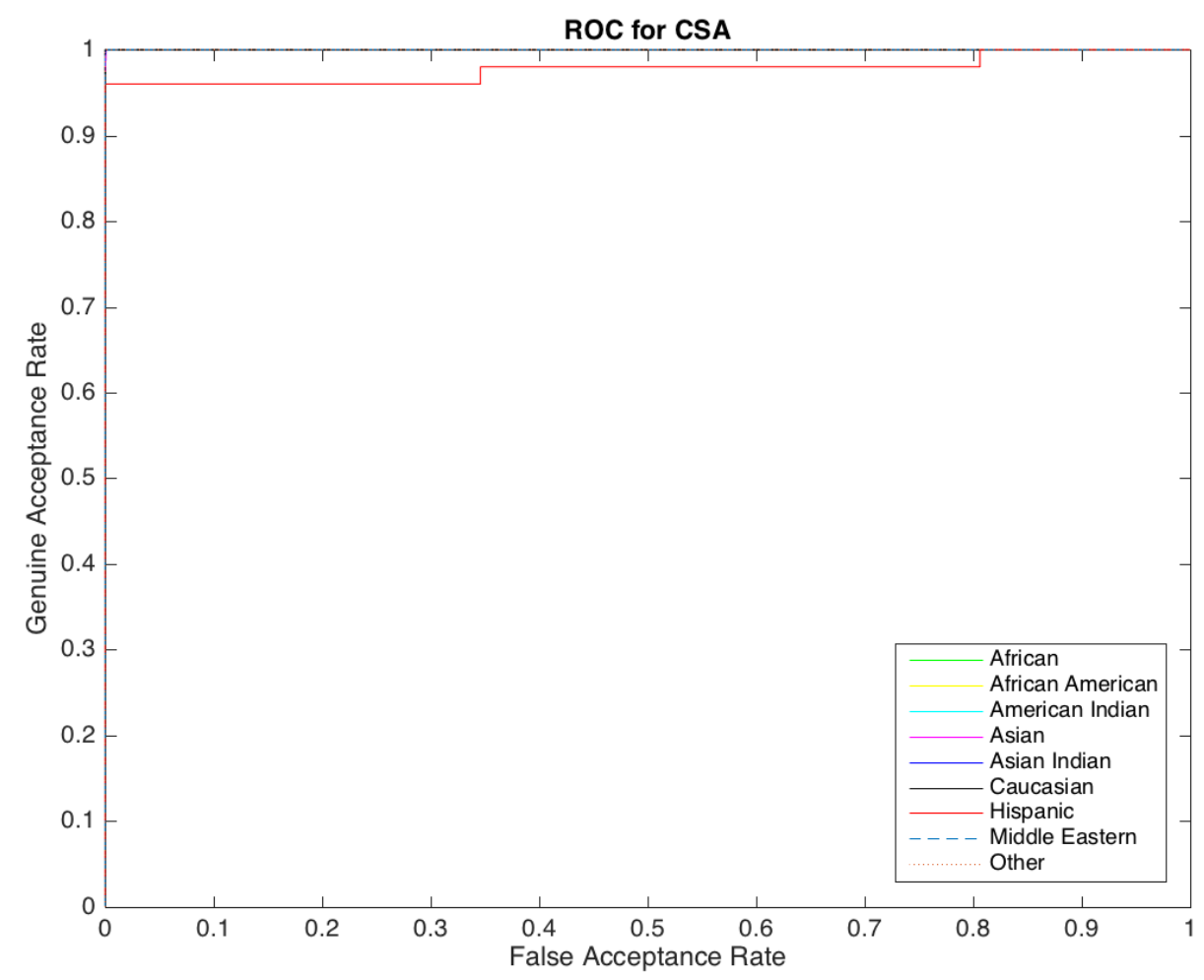

Figure D.6.7 SourceAFIS Crossmatch Seek Avenger Ethnicity Based ROC Curves

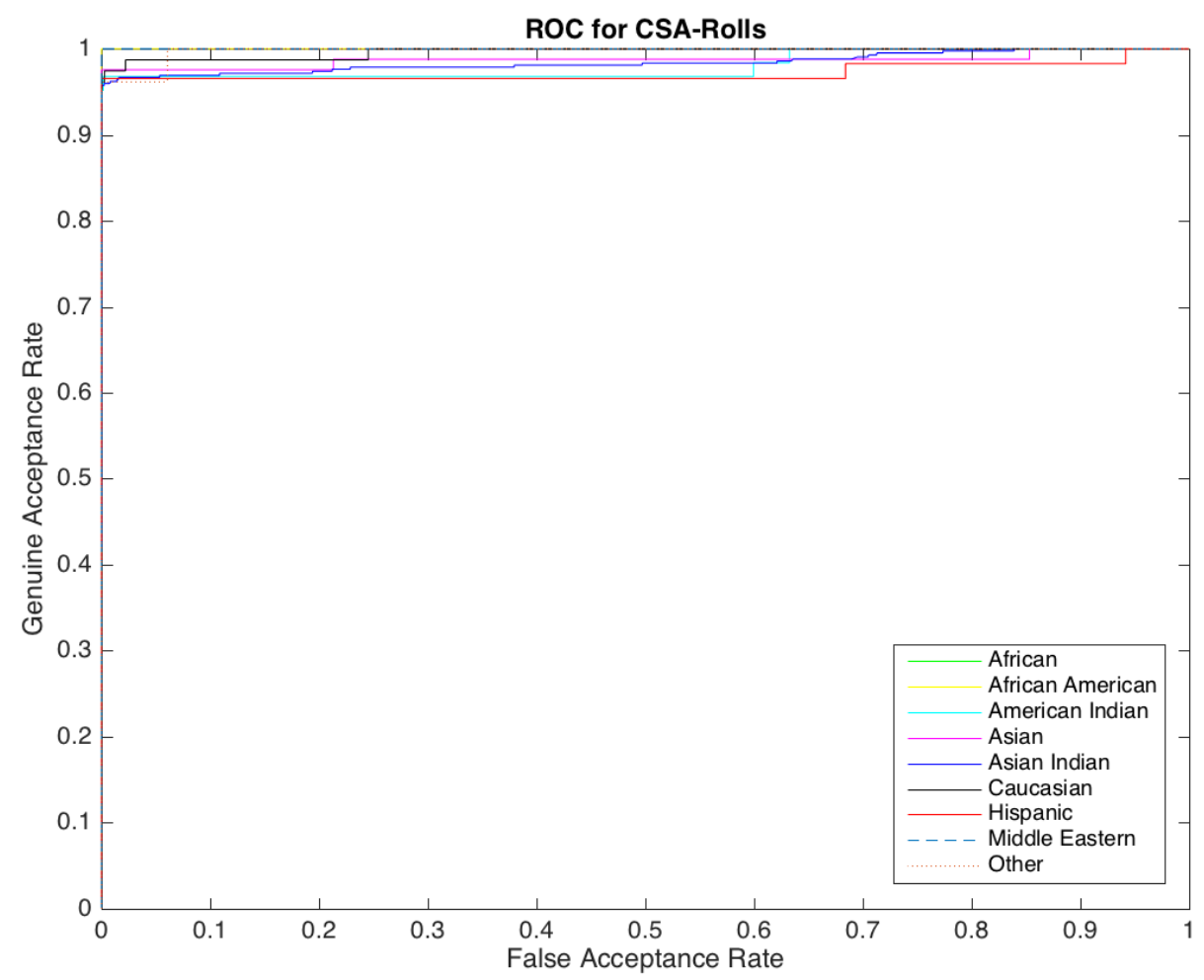

Figure D.6.8 SourceAFIS Crossmatch Seek Avenger Rolls Ethnicity Based ROC Curves 


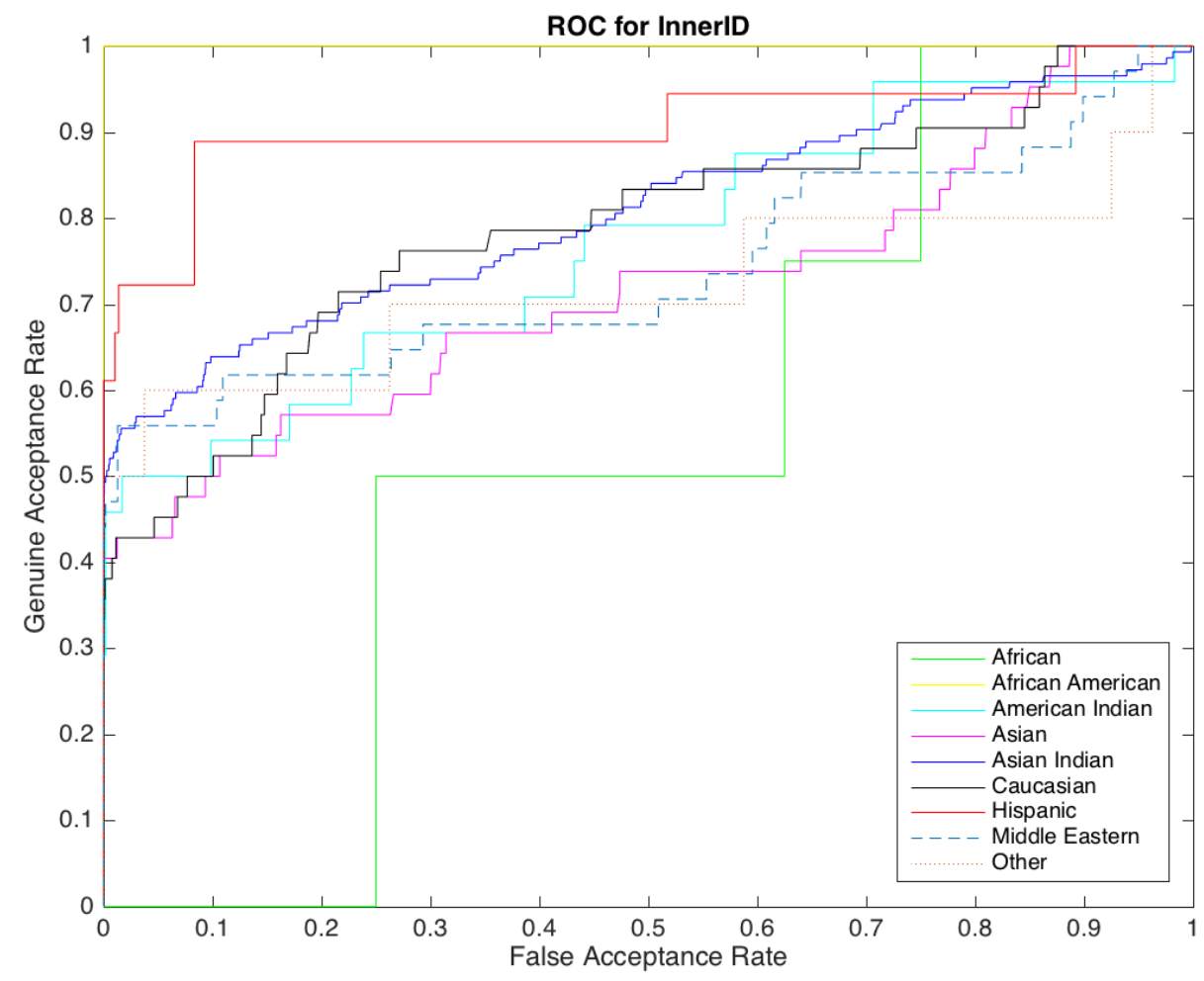

Figure D.6.9 SourceAFIS InnerID Ethnicity Based ROC Curves

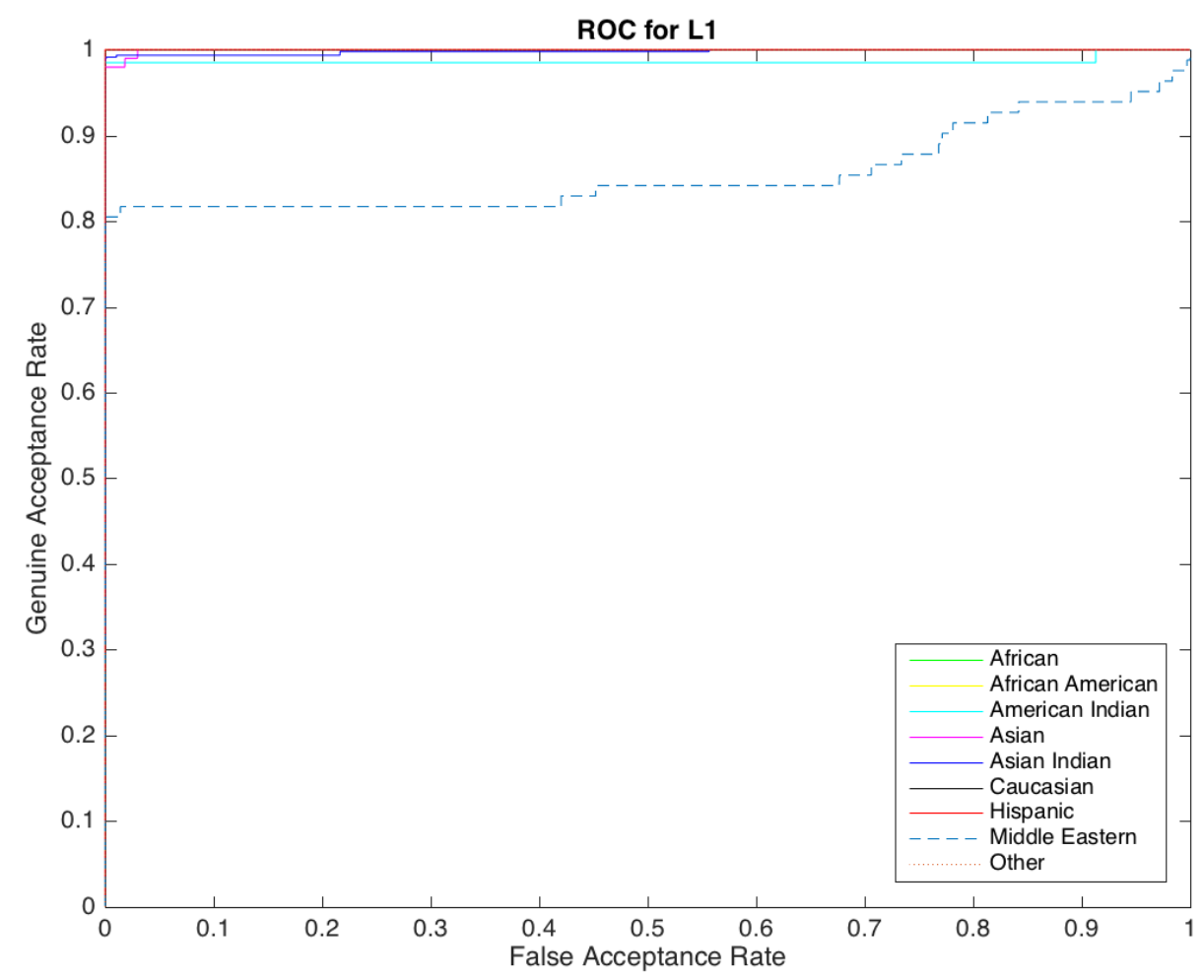

Figure D.6.10 SourceAFIS L1 Ethnicity Based ROC Curves 


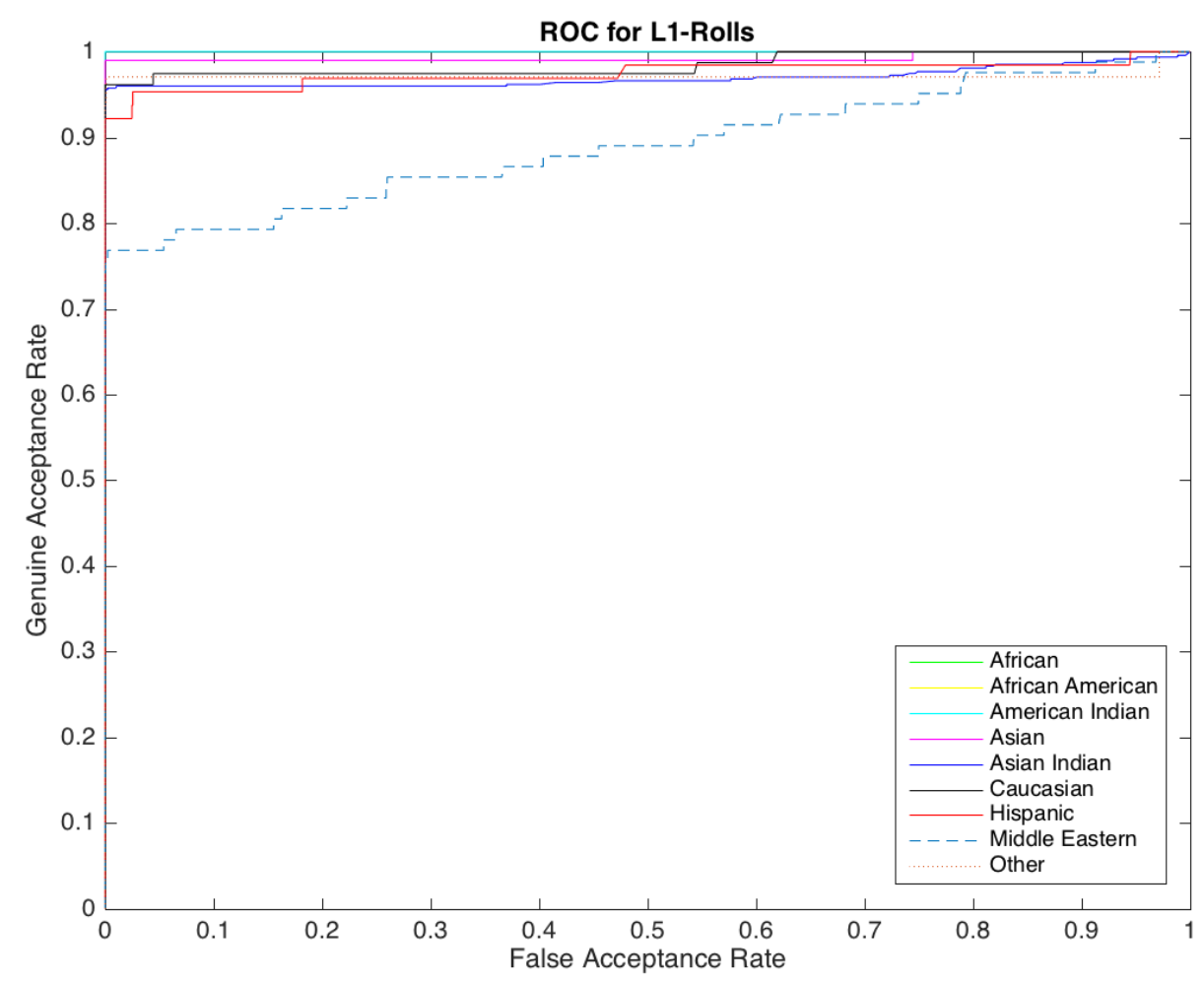

Figure D.6.11 SourceAFIS L1 Rolls Ethnicity Based ROC Curves

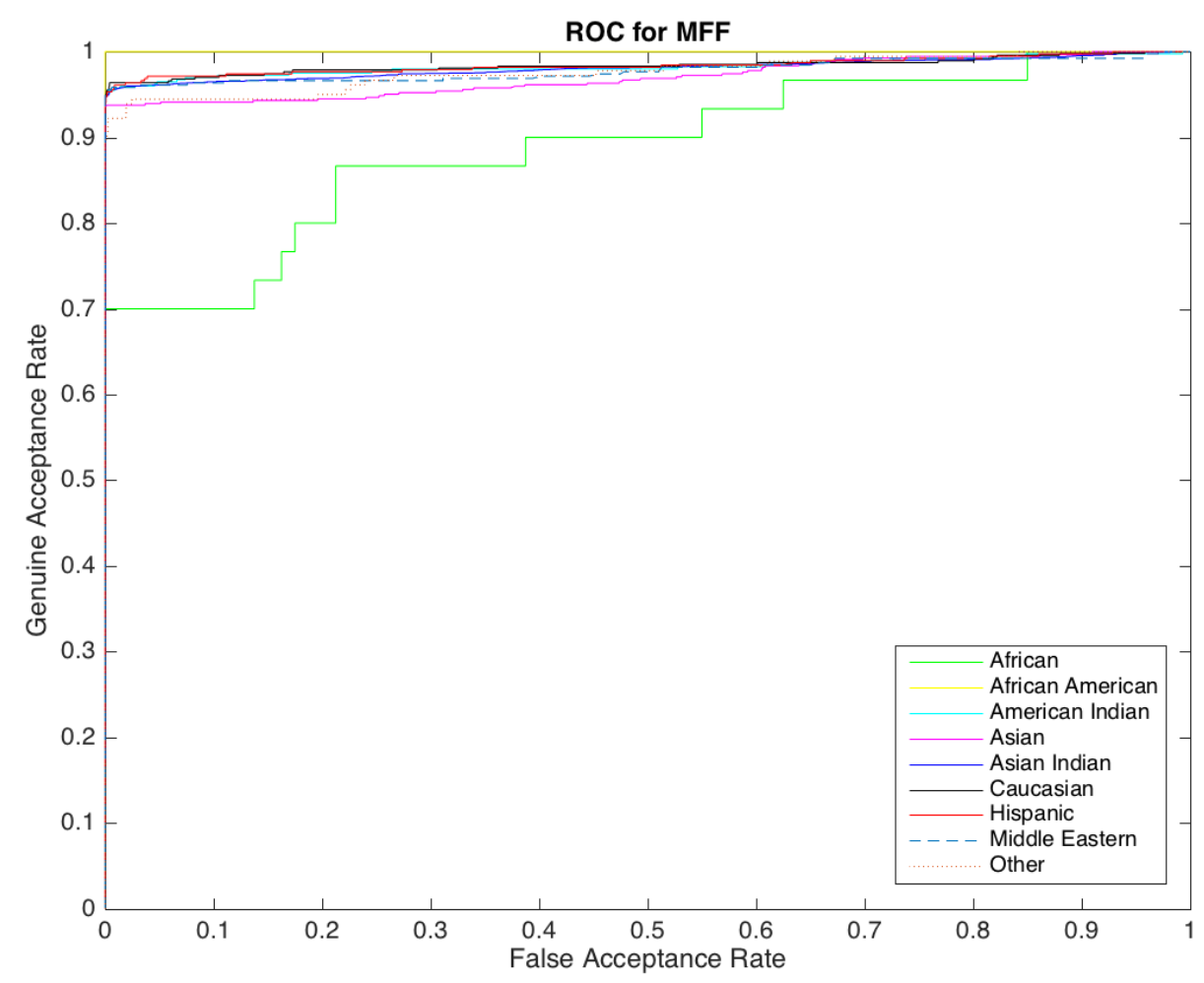

Figure D.6.12 SourceAFIS Morpho FOTF Ethnicity Based ROC Curves 


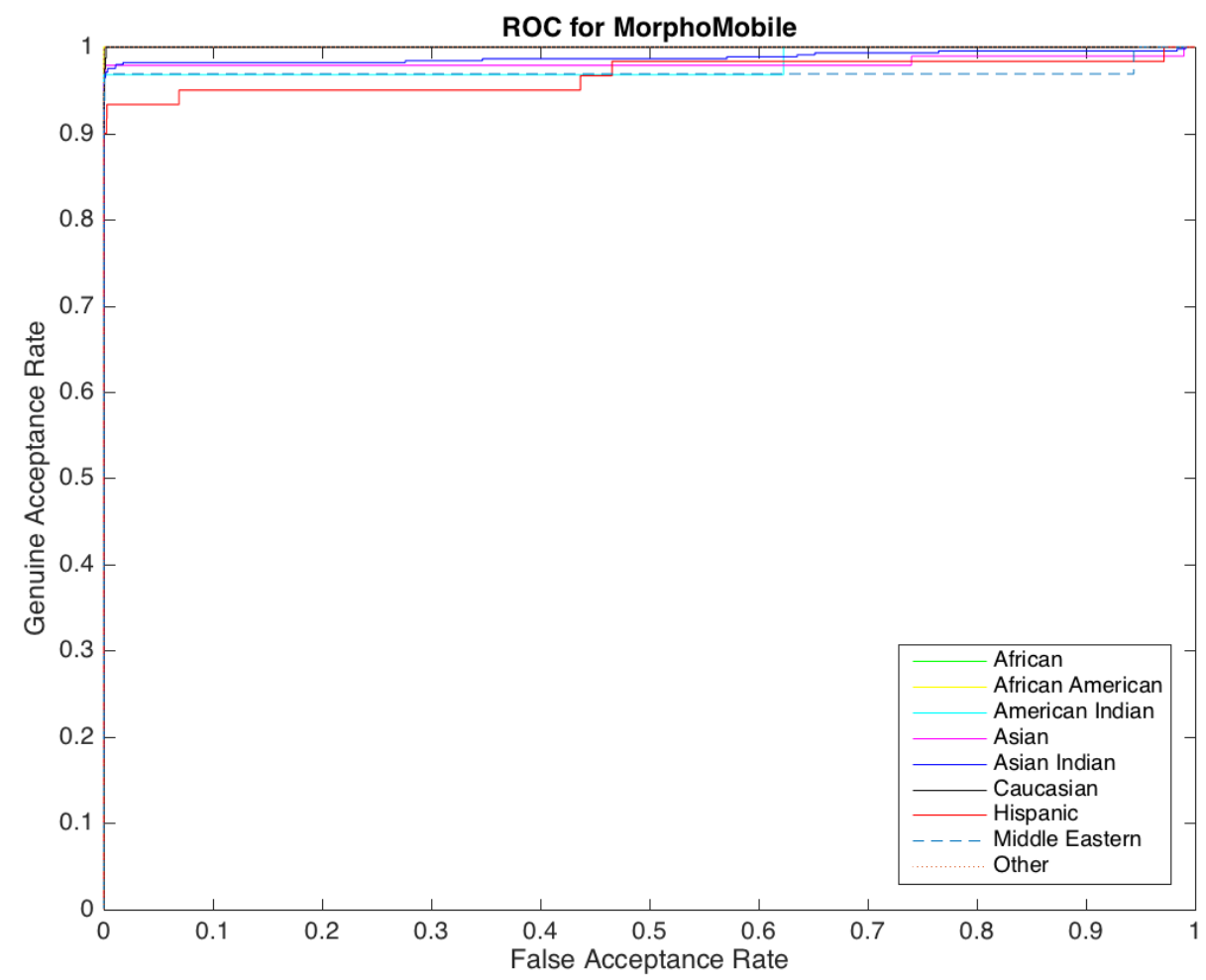

Figure D.6.13 SourceAFIS MorphoMobile Ethnicity Based ROC Curves 


\section{D.7 Minutiae Box Plots by Ethnicity and Device}

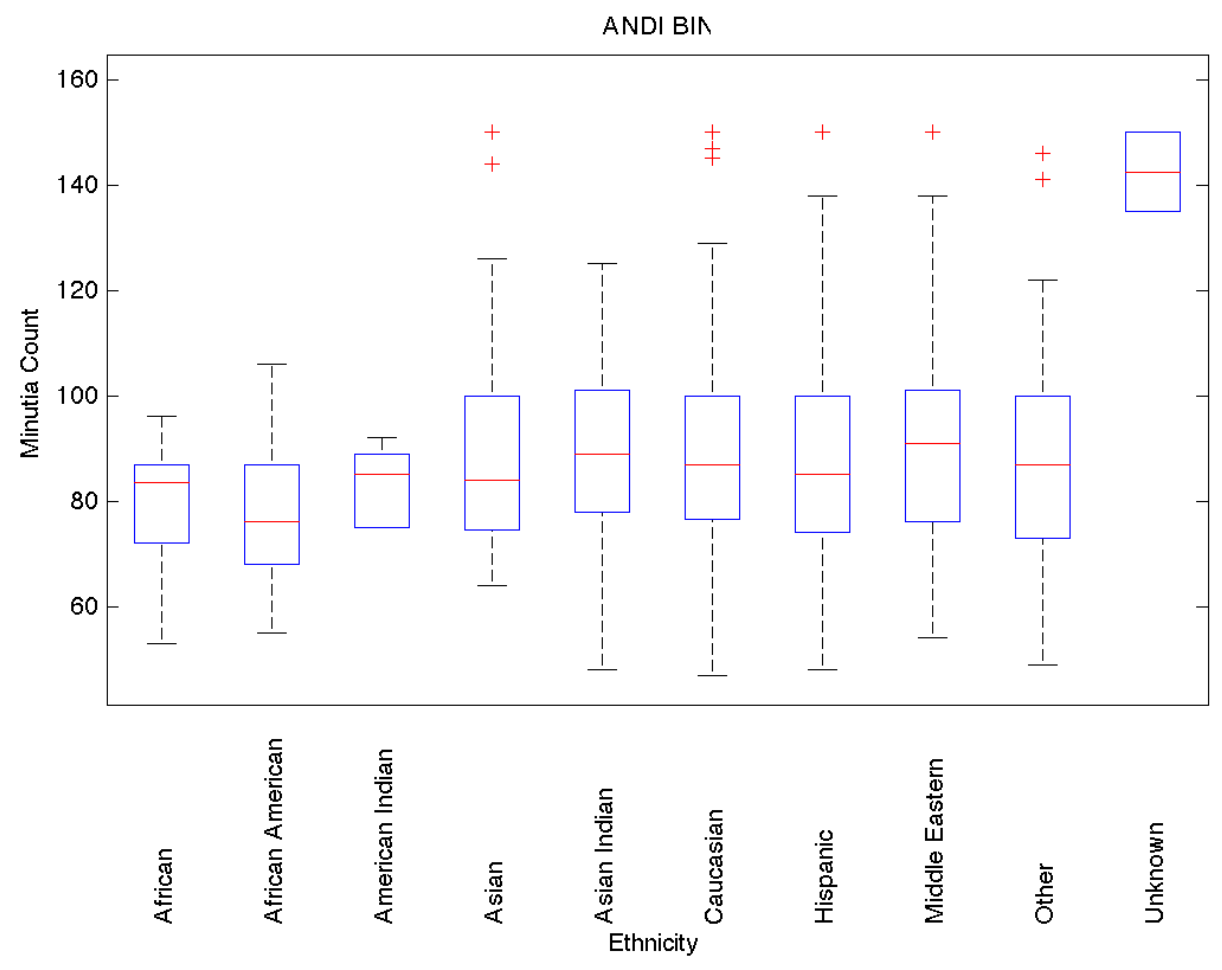

Figure D.7.1 ANDI Binary Ethnicity Based Minutiae Box Plots 


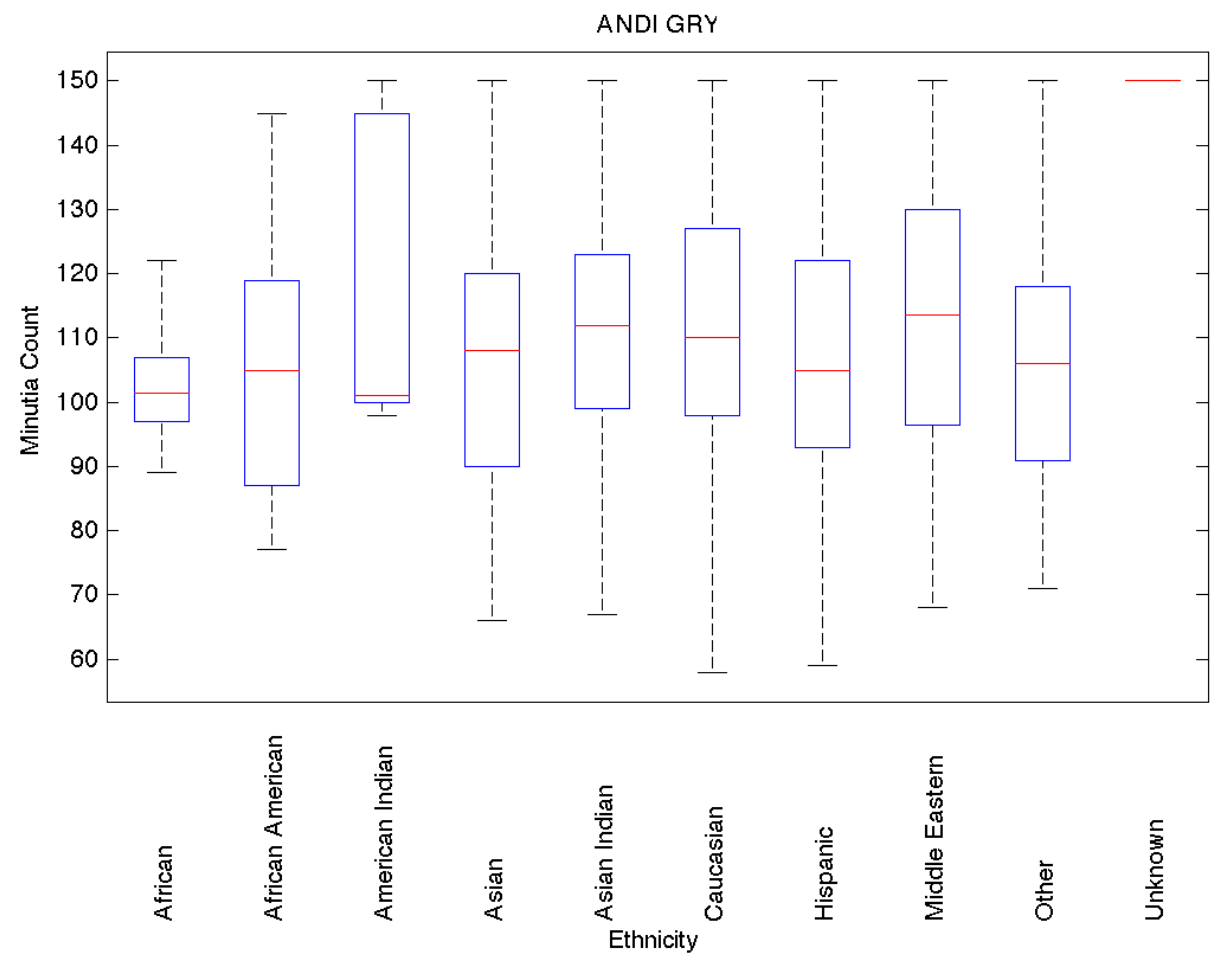

Figure D.7.2 ANDI Greyscale Ethnicity Based Minutiae Box Plots

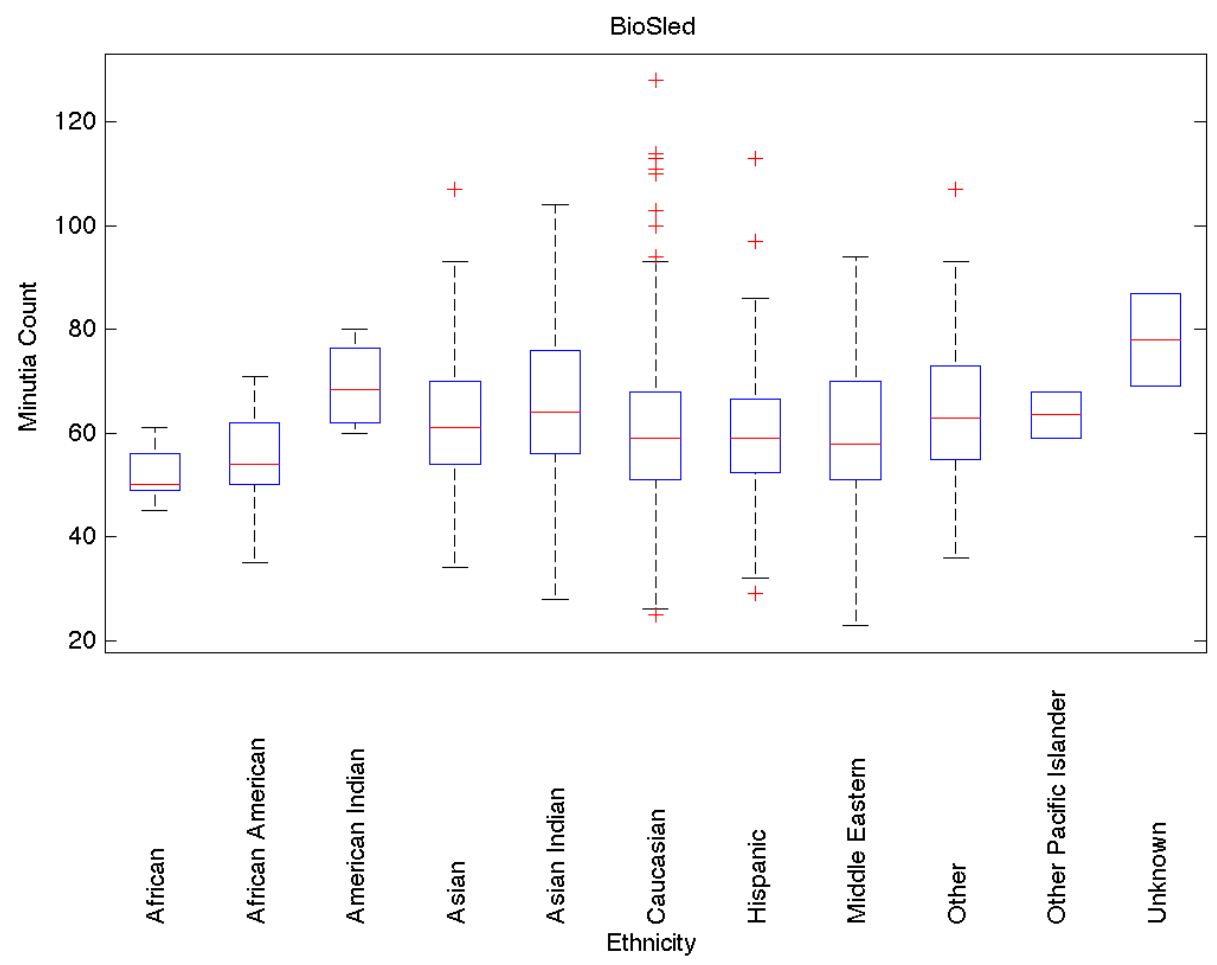

Figure D.7.3 BioSled Ethnicity Based Minutiae Box Plots 


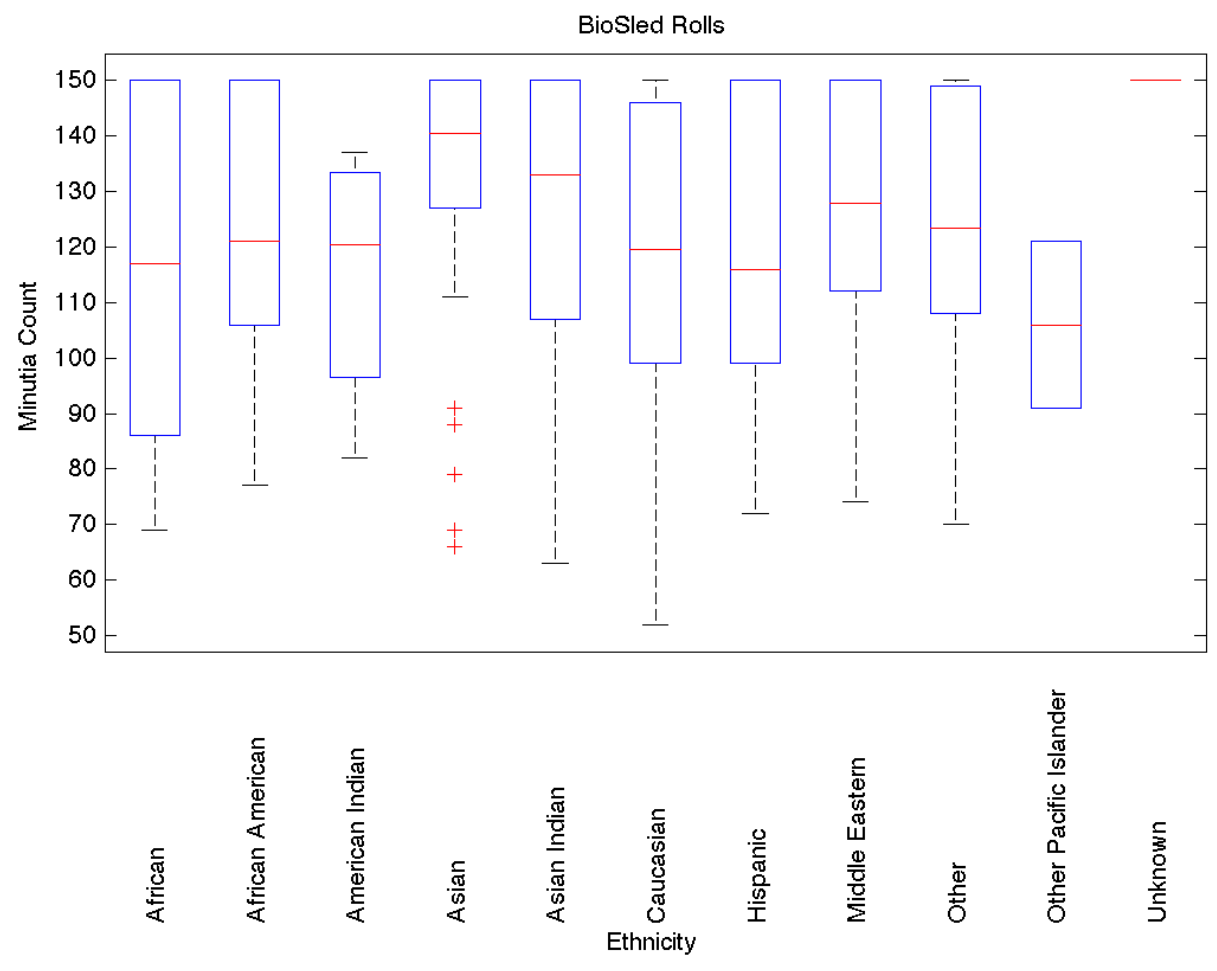

Figure D.7.4 BioSled Rolls Ethnicity Based Minutiae Box Plots

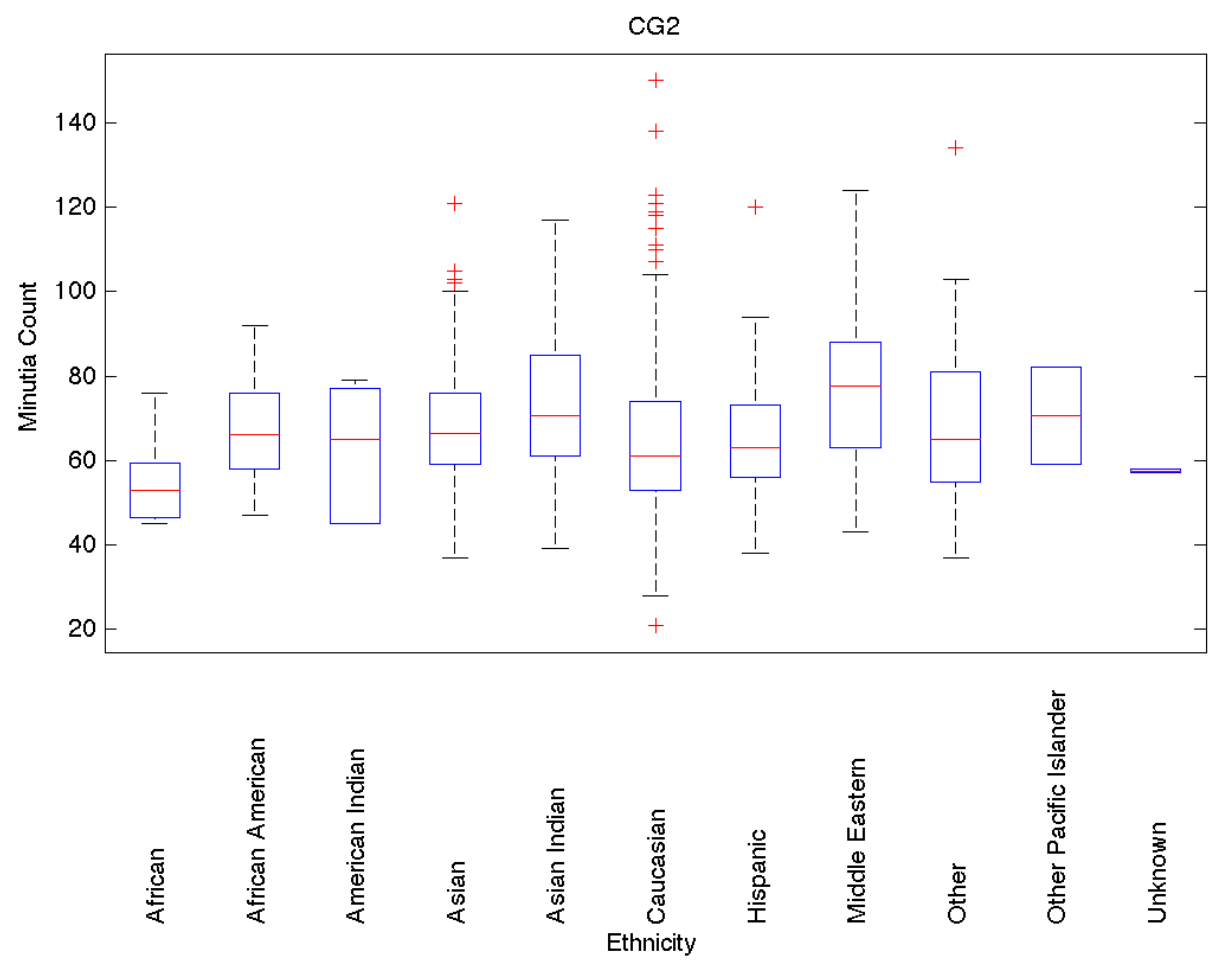

Figure D.7.5 FP II Guardian Ethnicity Based Minutiae Box Plots 


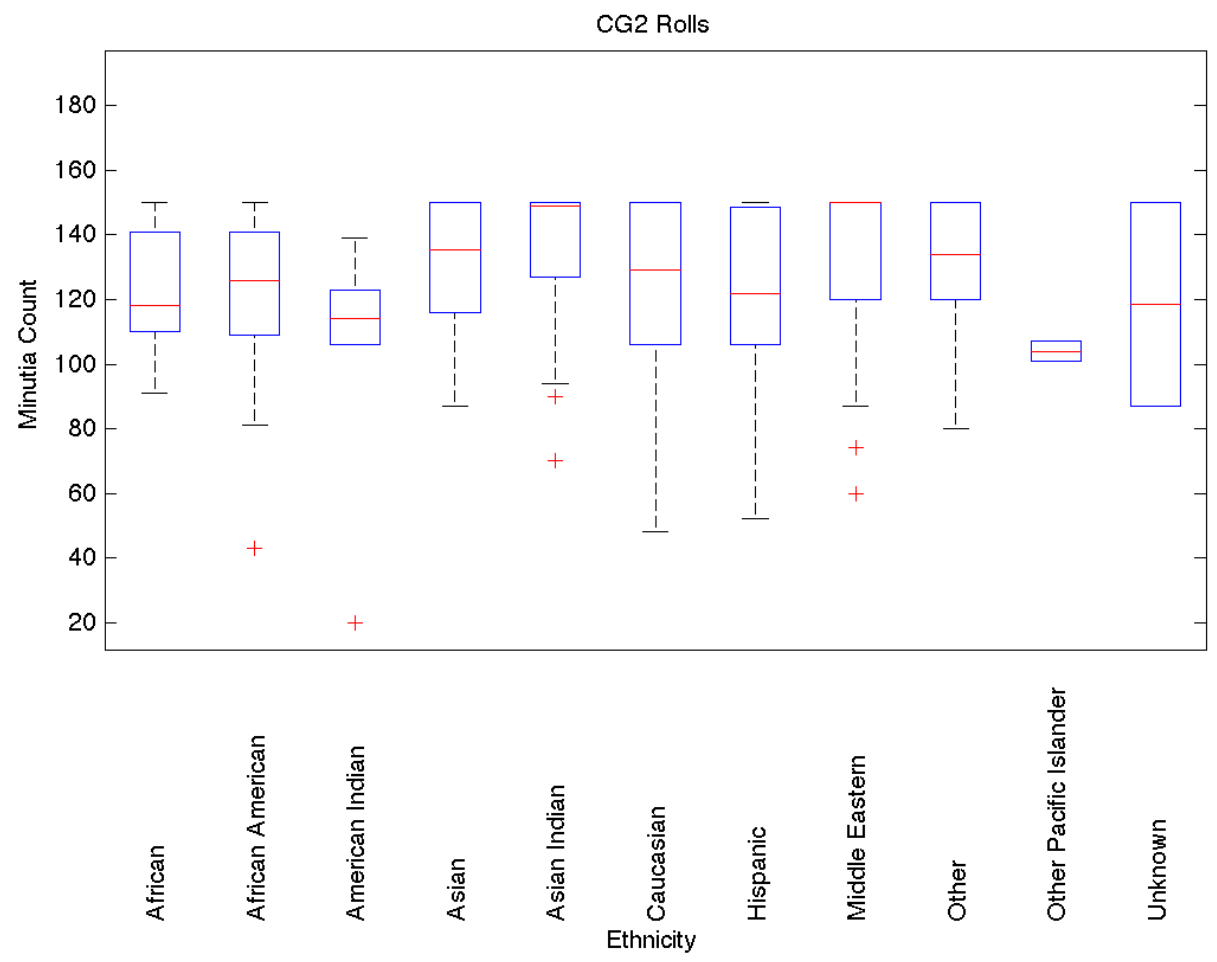

Figure D.7.6 FP II Guardian Rolls Ethnicity Based Minutiae Box Plots

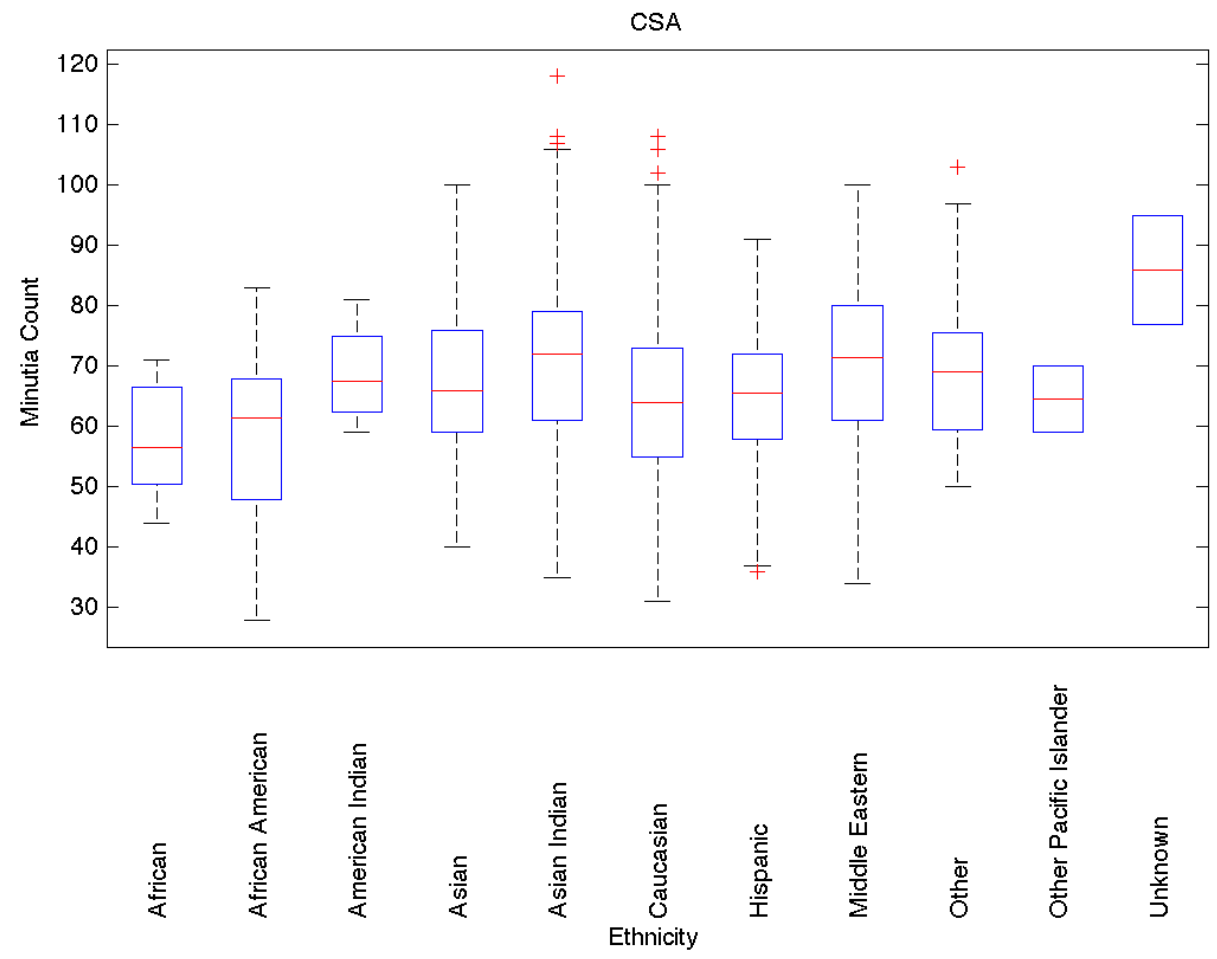

Figure D.7.7 Crossmatch Seek Avenger Ethnicity Based Minutiae Box Plots 


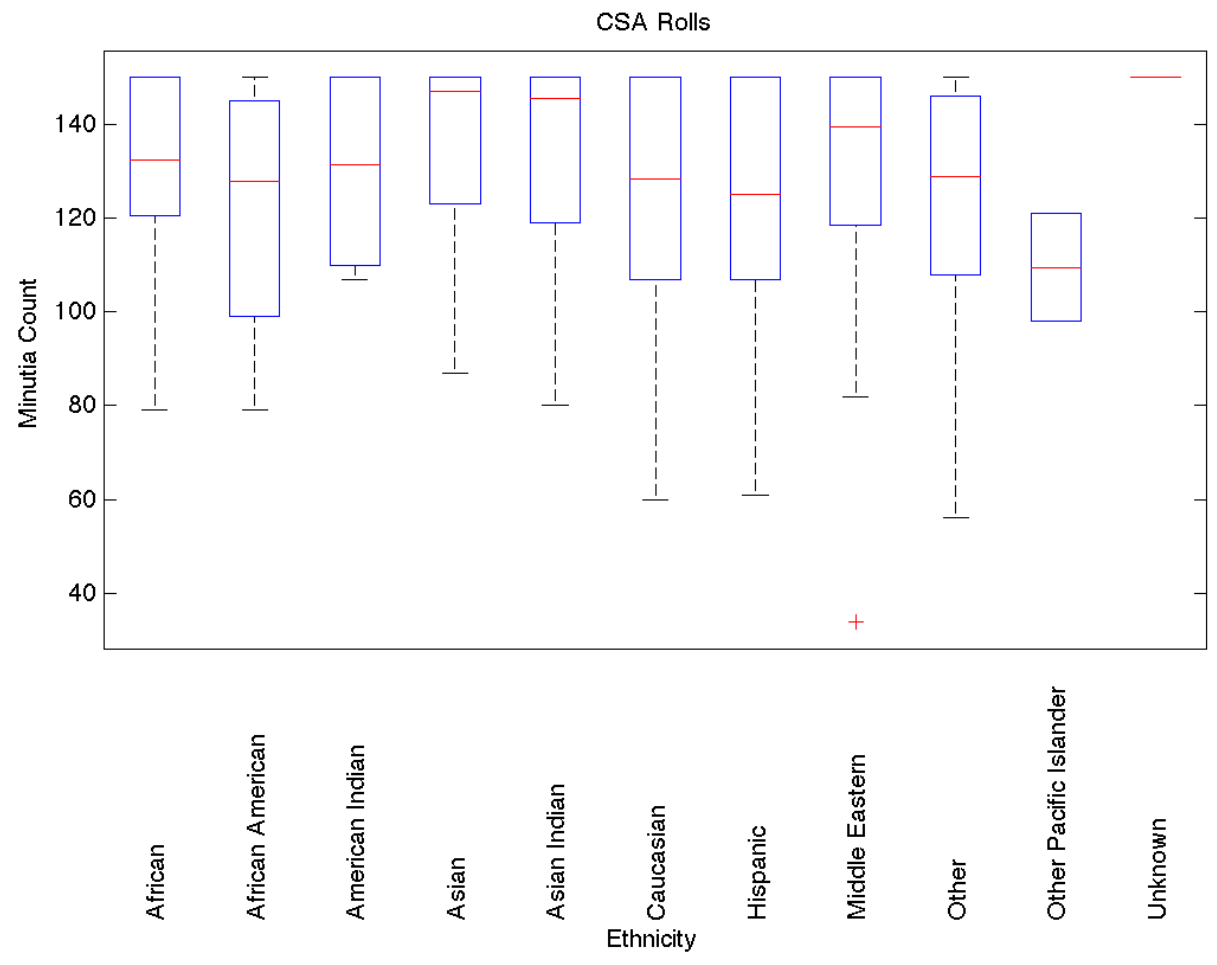

Figure D.7.8 Crossmatch Seek Avenger Rolls Ethnicity Based Minutiae Box Plots

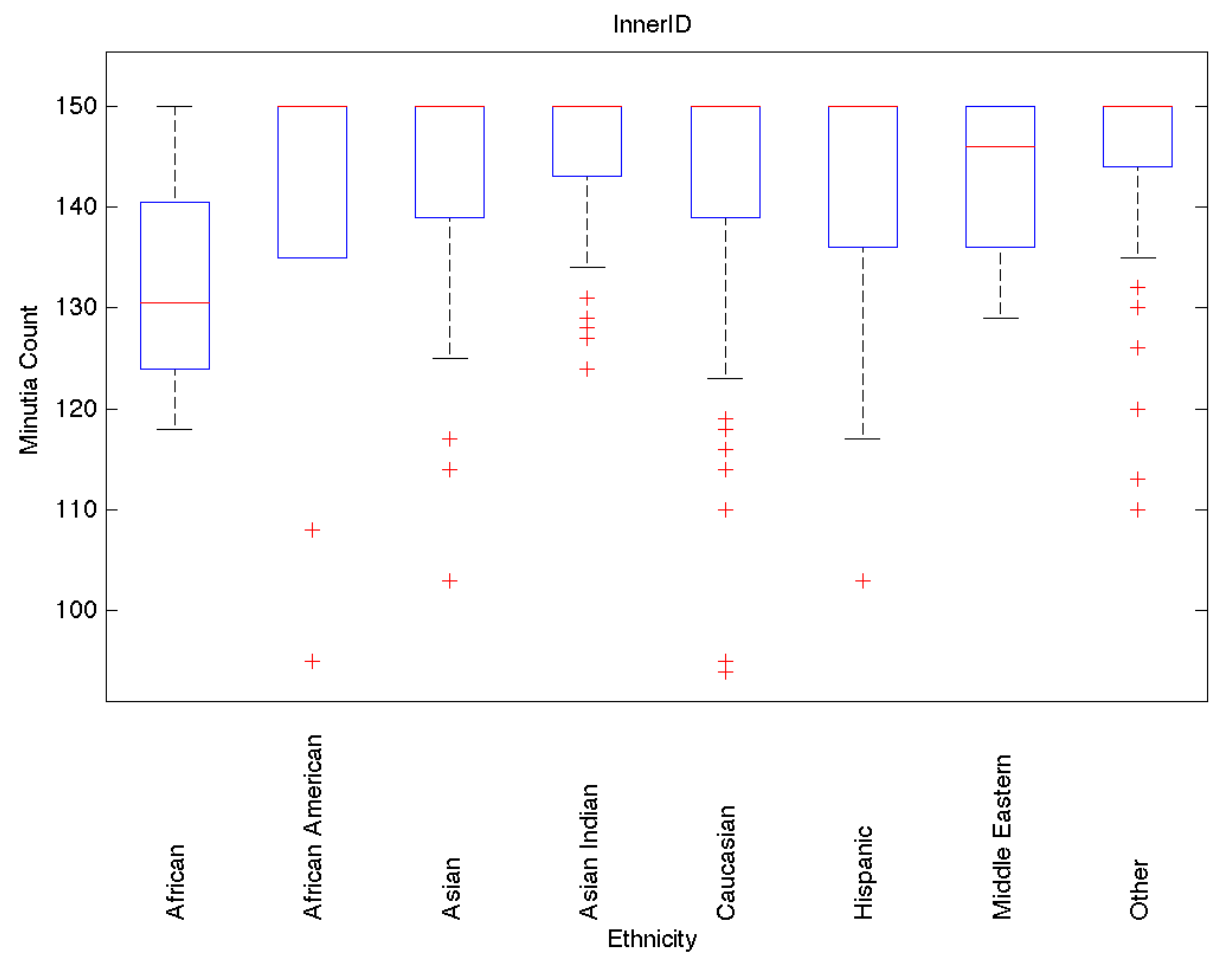

Figure D.7.9 InnerID Ethnicity Based Minutiae Box Plots 


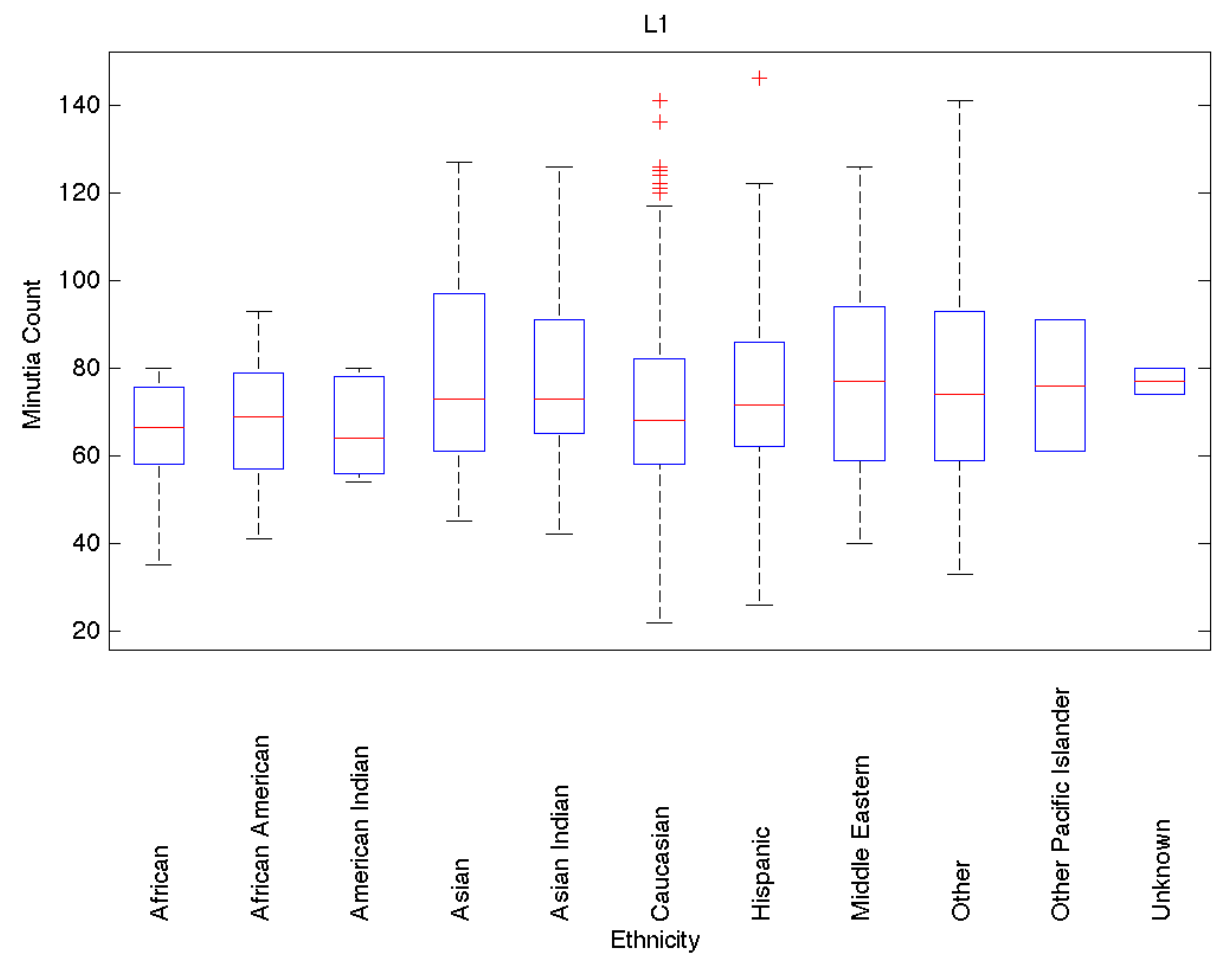

Figure D.7.10 L1 Ethnicity Based Minutiae Box Plots

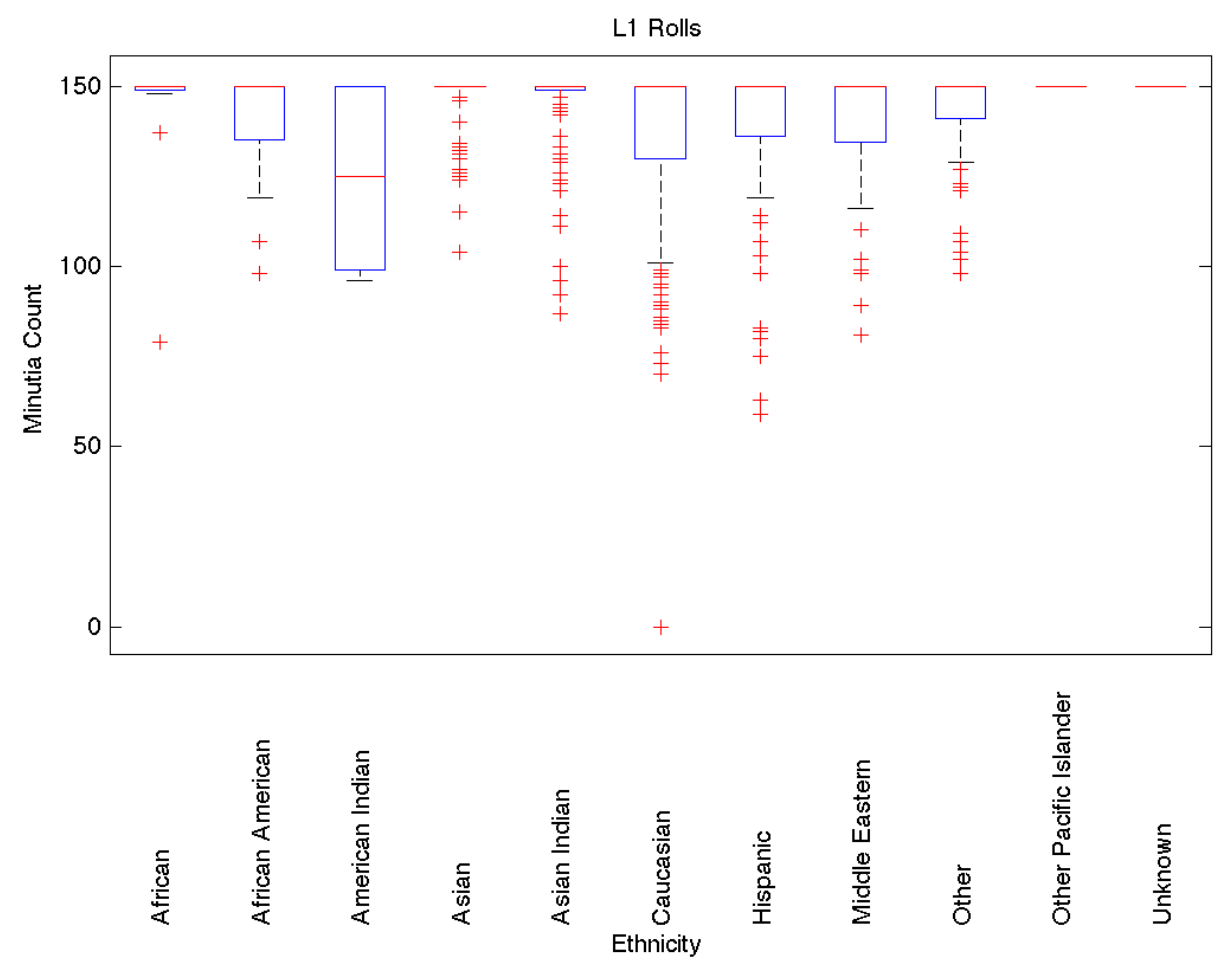

Figure D.7.11 L1 Rolls Ethnicity Based Minutiae Box Plots 


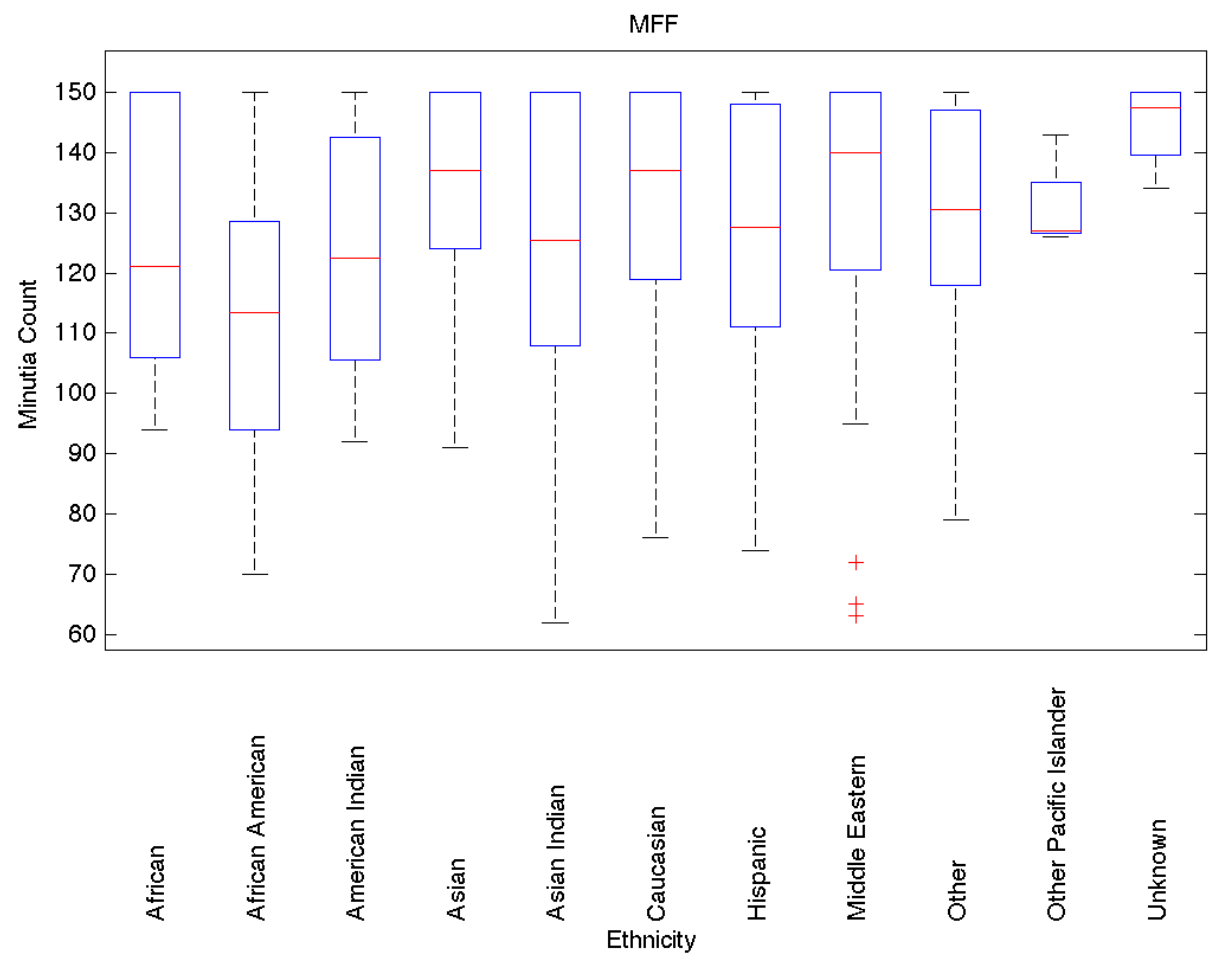

Figure D.7.12 Morpho FOTF Ethnicity Based Minutiae Box Plots

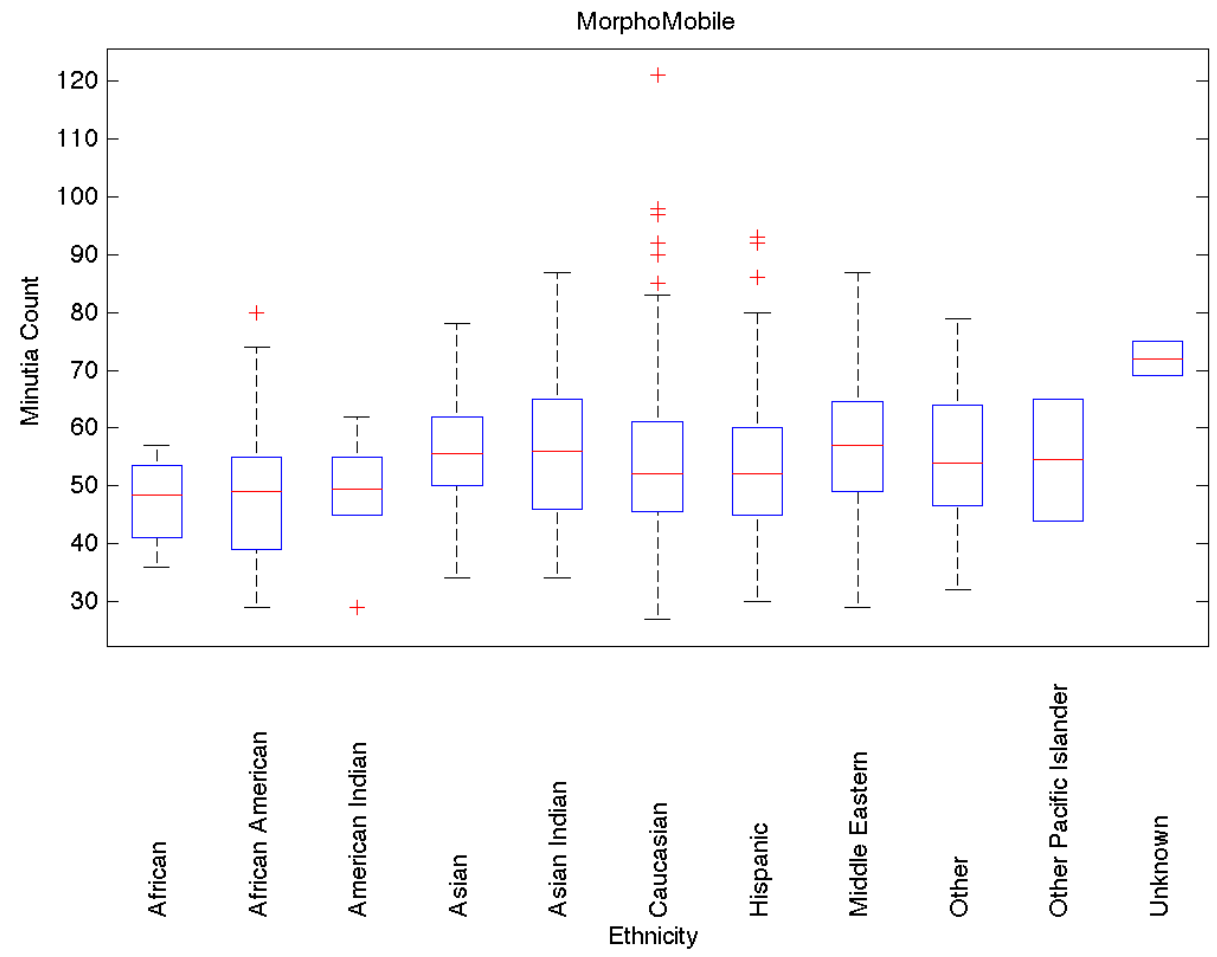

Figure D.7.13 MorphoMobile Ethnicity Based Minutiae Box Plots 


\section{References}

[1] P. Wiggins and L. Ericson, "Contactless Fingerprint Technologies Assessment (Version 2)," ncjrs.gov, 2014.

[2] L. Ericson and S. Shine, "Evaluation of Contactless versus Contact Fingerprint Data, Phase 2 (Version 1.1)," ncjrs.gov.

[3] "FBI-Certified Scanning and Digitization of Fingerprint Cards," [Online]. Available: http://www.aware.com/biometrics/accuscan/. [Accessed 24 October 2016].

[4] K. R. Moses, P. Higgins, M. McCabe, S. Prabhakar and S. Swann, "Automated Fingerprint Identification System (AFIS)," in Fingerprint Sourcebook, Rockville, MD, National Institute of Justice/NCJRS, 2010.

[5] "Wikipedia," Wikipedia, [Online]. Available: https://en.wikipedia.org/wiki/Interoperability. [Accessed 17 January 2017].

[6] Understanding Biometrics, Griaule Biometrics, 2008.

[7] N. Nain, B. Bhadviya, B. Gautam, D. Kumar and D. B. M, "A Fast Fingerprint Classification Algorithm by Tracing Ridge-Flow Patterns," in Signal Image Technology and Internet Based Systems, 2008. SITIS '08. IEEE Conference on Signal Image Technology and Internet Based Systems, 2008.

[8] "Fingerprint Recognition," Wikipedia, [Online]. Available: https://en.wikipedia.org/wiki/Fingerprint_recognition. [Accessed 24 October 2016].

[9] P. Reid, Biometrics for Network Security (Prentice Hall Series in Computer Networking and Distributed), Upper Saddle River, NJ: Prentice Hall PTR, 2003.

[10] P. T. Higgins, K. M. Higgins, L. K. Fox and P. Komarinski, Automated Fingerprint Identification Systems (AFIS), Burlington, MA: Elsevier Academic Press, 2005.

[11] National Science and Technology Council (NSTC) Committee on Technology, Committee on Homeland and National Security, Subcommittee on Biometrics, "Fingerprint Recognition," in Introduction to Biometrics, Biometrics.gov, 2006.

[12] A. A. Paulino, A. K. Jain and J. Feng, "Latent Fingerprint Matching: Fusion of Manually Marked and Derived Minutiae," in 2010 23rd SIBGRAPI - Conference on Graphics, Patterns and Images, 2010.

[13] "Fingerprint Feature Extraction," Syahrul, 13 April 2012. [Online]. Available: http://syhrl.blogspot.com/2012/04/036-fyp-minutiae.html. [Accessed 24 October 2016].

[14] J. Zhou, F. Chen, N. Wu and C. Wu, "Crease detection from fingerprint images and its applications in elderly people," Pattern Recognition, vol. 42, no. 5, pp. 896-906, May 2009. 
[15] Y. Wang, L. G. Hassebrook and D. L. Lau, "Data Acquisition and Processing of 3-D Fingerprints," University of Kentucky, Lexington, KY, 2009.

[16] Y. Wang, Q. Hao, A. Fatehpuria, L. G. Hassebrook and D. L. Lau, "Quality and Matching Performance Analysis of Three-dimensional Unraveled Fingerprints," University of Kentucky, Lexington, KY, 2010.

[17] A. Kumar and C. Kwong, "Towards Contactless, Low-Cost and Accurate 3D Fingerprint Identification," in The IEEE Conference on Computer Vision and Pattern Recognition (CVPR), 2013.

[18] Y. Wang, D. L. Lau and L. G. Hassebrook, "Fit-sphere Unwrapping and Performance Analysis of 3D Fingerprints," University of Kentucky, Lexington, KY, 2010.

[19] Y. Wang, Q. Hao, A. Fatehpuria, L. G. Hassebrook and D. L. Lau, "Data Aquisition and Quality Analysis of 3-Dimensional Fingerprints," University of Kentucky, Lexington, KY.

[20] Q. Zhao, A. Jain and G. Abramovich, "3D to 2D Fingerprints: Unrolling and Distortion Correction," Biometrics (IJCB), no. 11-13, 2011.

[21] H. C. Lee and R. E. Gaensslen, Eds., Advances in Fingerprint Technology, 2nd Edition ed., Boca Raton, FL: CRC Press, 2001.

[22] N. K. Ratha and V. Govindaraju, Eds., Advances in Biometrics Sensors, Algorithms and Systems, London: Springer-Verlag , 2008.

[23] S. Mil'shtein and C. Buzawa, "Wireless Communication Supported by Contactless Fingerprints," in Technologies for Homeland Security (HST), 2016 IEEE Symposium on Technologies for Homeland Security (HST), 2016.

[24] T. Dunstone and N. Yager, Eds., Biometric System and Data Analysis Design, Evaluation, and Data Mining, New York, NY: Springer Science + Business Media, LLC, 2009.

[25] G. Parziale and Y. Chen, Handbook of Remote Biometrics, M. Tistarelli, S. Z. Li and R. Chellappa, Eds., London: Springer London, 2009.

[26] C. R. Blomeke, S. K. Modi and S. J. Elliot, "Investigating The Relationship Between Fingerprint Image Quality and Skin Characteristics," Purdue University, West Lafayette, IN.

[27] X. Xia and L. O'Gorman, "Innovations In Fingerprint Capture Devices," Pattern Recognition, vol. 36, pp. 361-369, 2003.

[28] W. Zhou, J. Hu, I. Petersen, S. Wang and M. Bennamoun, "A Benchmark 3D Fingerprint Database," in 2014 11th International Conference on Fuzzy Systems and Knowledge Discovery, 2014.

[29] A. Pillai and S. Mil'shtein, "Can Contactless Fingerprints be Compared to Existing Database?," in Homeland Security (HST), 2012 IEEE Conference on Technologies for , 2012. 
[30] A. Ross, S. C. Dass and A. K. Jain, "Estimating Fingerprint Deformation," in Internation Conference on Biometric Authentication (ICBA), Hong Kong, 2004.

[31] Y. Chen, G. Parziale, E. Diaz-Santana and A. K. Jain, "3D Touchless Fingerprints: Compatibility with Legacy Rolled Images," in Biometric Consortium Conference, 2006 Biometrics Symposium: Special Session on Research at the Biometric Consortium Conference, Baltimore, MD, 2006.

[32] N. B. Nill, D. J. Braunegg and B. R. Paine, "Computer Program to Determine the Sine Wave Modulation Transfer Function (MTF) of Imaging Devices," The MITRE Corporation, Bedford, MA, 1996.

[33] M. E. Bravo-Zanoguera, J. Rivera-Castillo, M. Vera-Perez and M. A. Reyna Carranza, "Use of the Modulation Transfer Function to Measure Quality of Digital Cameras," in Proceedings of the 16th IEEE International Conference on Electronics, Communications and Computers (CONIELECOMP 2006), 2006.

[34] "CRADA Program: Contactless Fingerprint Capture Device Measurement," NIST, 26 January 2015. [Online]. Available: https://www.nist.gov/itl/iad/image-group/crada-program-contactlessfingerprint-capture-device-measurement. [Accessed 24 October 2016].

[35] S. Orandi, M. Garris, B. Wing, E. Tabassi, P. Grother, C. Watson, R. Michaels and M. Theofanos, "NIST Fingeprint Testing and Standards," biometrics.nist.gov, 2013.

[36] S. Furman, B. Stanton, M. Theofanos, J. M. Libert and J. Grantham, "nist.gov," National Institute of Standards and Technology, March 2017. [Online]. Available: http://nvlpubs.nist.gov/nistpubs/ir/2017/NIST.IR.8171.pdf.

[37] C. Centamore, "AIRprint - Rapid, Long Range Fingerprinting Technology," Advanced Optical Systems, Inc. , 26 November 2012. [Online]. Available: http://www.aosinc.com/index.php/products/biometrics-new/airprint-new. [Accessed 24 October 2016].

[38] P. Wiggins and L. Ericson, "University of Massachusetts Lowell 3D Contactless Fingerprint Scanner: Technology Evaluation (Version 2)," ncjrs.com, Fairmont, WV, 2014.

[39] Safran Morpho MorphoTrak, "MorphoTrak Finger-On-The-Fly Specsheet," MorphoTrak.com, Alexandria, VA, 2014.

[40] TBS, "TBS Touchless Fingerprinting 3D Enroll Technical Datasheet," Touchless Biometrics Systems, Switzerland.

[41] IDair, "ONEprint Touchless Fingerprint System Technical Datasheet," idairco.com, Huntsville, AL.

[42] NEC, "Multi-modal Identification Solution Contactless Hybrid Fingerprint Scanner Technical Datasheet," au.nec.com.

[43] Advanced Optical Systems, INC. AOS, "ANDI OTG Automated Non-Contact Distance Identity Technical Datasheet," Advanced Optical Systems, Inc., Huntsville, AL, 2014. 
[44] "ANDI OTG Recieves FBI Certification," ANDI OTG, 30 November 2015. [Online]. Available: http://www.andiotg.com/single-post/2015/11/30/ANDI-OTG-Receives-FBI-Certification. [Accessed 24 October 2016].

[45] "FBI Certifies MorphoWave Desktop Contactless Fingerprint Scanner," Safran Morpho, 5 January 2016. [Online]. Available: http://www.morpho.com/en/media/20160105_fbi-certifiesmorphowave-desktop-contactless-fingerprint-scanner. [Accessed 24 October 2016].

[46] A. Ross and A. Jain, "Biometric Sensor Interoperability: A Case Study in Fingerprints," in ECCV Workshop on Biometric Authentication (BioAW), Prague, Czech Republic, 2004.

[47] E. Marasco, L. Lugini, B. Cukic and B. Thirimachos, "Minimizing the Impact of Low Interoperability between Optical Fingerprints Sensors," in Biometrics: Theory, Applications and Systems (BTAS), Arlington, VA, 2013.

[48] L. Lugini, E. Marasco, B. Cukic and I. Gashi, "Interoperability in fingerprint recognition: A large-scale empirical study," in Dependable Systems and Networks Workshop, Budapest, Hungary, 2013.

[49] "Sherlock," Neurotechnology, [Online]. Available: http://www.neurotechnology.com/fingerprintscanner-integrated-biometrics-sherlock.html. [Accessed 24 October 2016].

[50] Integrated Biometrics, "The Differences and benefits of "LES" fingerprint sensor technology vs. Optical based FAP 45, FBI appendix "F" certified fingerprint sensors.," Integrated Biometrics LLC, Spartanburg, SC.

[51] Integrated Biometrics, "Sherlock Technical Datasheet," Integrated Biometrics.

[52] S. Thies, Interviewee, Interview with Stephen Thies, CEO Integrated Biometrics. [Interview]. 19 November 2013.

[53] Northrop Grumman, "Northrop Grumman BioSled Technical Datasheet," Northrop Grumman, 2016.

[54] CrossMatch, "CrossMatch SEEK Avenger Rugged Multimodal Handheld Technical Datasheet," Cross Match Technologies Inc., 2014.

[55] D. Maltoni, "Fingerprint Sensing," in Handbook of Fingerprint Recognition, Springer, 2009, pp. 57 95.

[56] K. Antonelli, G. Vanderkooy, T. Vlaar and G. Immega, "Fingerprint Image Optical Input Apparatus". United States Patent US6259108 B1, 10 July 2001.

[57] S. J. Borza, "Imaging Device, Especially for Optical Fingerprinting". US Patent US6429927 B1, 6 August 2002.

[58] F. Chen, "3D fingerprint and palm print data model and capture devices using multi structured lights and cameras". United States of America Patent US 7609865 B2, 27 October 2009. 
[59] L. G. Hassebrook, D. L. Lau and H. G. Dietz, "System and method for 3D imaging using structured light illumination". United States of America Patent US8224064 B1, 17 Jul 2012.

[60] R. D. Labati, A. Genovese, V. Piuri and F. Scotti, "Fast 3-D Fingertip Reconstruction Using a Single Two-View Structured Light Acquisition," in Biometric Measurements and Systems for Security and Medical Applications (BIOMS) 2011 IEEE Workshop, 2011.

[61] G. Abramovich, K. George Harding, Q. Hu, S. Manickam, M. Ganesh and C. Allen Nafis, "Method and system for contactless fingerprint detection and verification". United States of America Patent US8406487 B2, 26 March 2013.

[62] N. Engheta, E. Pugh, S.-S. Lin and K. Yemelyanov, "Polarization and Reflection Based Non-Contact Latent Fingerprint Imaging and Lifting". United States of America Patent US20070280513 A1, 6 December 2007.

[63] S. Prabhakar and A. K. Jain, "Fingerprint Matching," in Automatic Fingerprint Recognition Systems, N. Ratha and R. Bolle, Eds., New York, NY: Springer Science \& Media, 2004.

[64] A. K. Jain, J. Feng and K. Nandakumar, "Fingerprint Matching," Computer, vol. 43, no. 2, February 2010.

[65] M. Adhiyaman and D. Ezhilmaran, "Fingerprint Matching and Similarity Checking System using Minutia Based Technique," in 2015 IEEE International Conference on Engineering and Technology (ICETECH), Coimbatore, TN, India, 2015.

[66] A. K. Jain, A. Ross and S. Prabhakar, "An Introduction to Biometric Recognition," IEEE Transactions on Circuits and Systems For Video Technology, vol. 14, no. 1, pp. 4-20, January 2004.

[67] R. Cappelli, M. Ferrara and D. Maltoni, "Minutiae-Based Fingerprint Matching," in Cross Disciplinary Biometric Systems, J. Kacprzyk and L. C. Jain, Eds., Berlin, NY: Springer, 2012.

[68] A. Saleh, A. Bahaa and A. Wahdan, "Fingerprint Recognition," in Advanced Biometric Technologies, G. Chetty and J. Yang, Eds., InTech, 2011, pp. 201-224.

[69] "Fingerprint Matching," in Handbook of Fingerprint Recognition, Second ed., London, SpringerVerlag, 2009, pp. 167-233.

[70] C. Ntantogian, S. Malliaros and C. Xenakis, "Gaithashing: A two-factor authentication scheme based on gait features," Computers \& Security, April 2015.

[71] "KL-Divergence," [Online]. Available: http://web.engr.illinois.edu/ hanj/cs412/bk3/KLdivergence.pdf. [Accessed 18 January 2017].

[72] M. E. Schuckers, Computational Methods in Biometric Authentication, Springer, 2010.

[73] "Plotting and Interpretating an ROC Curve," [Online]. Available: http://gim.unmc.edu/dxtests/roc2.htm. [Accessed 20 February 2017]. 
[74] NIST, "Biometric Quality Homepage," NIST, [Online]. Available: https://www.nist.gov/programsprojects/biometric-quality-homepage. [Accessed 17 April 2017].

[75] NIST, "Development of NFIQ 2.0," NIST, 28 April 2016. [Online]. Available: https://www.nist.gov/services-resources/software/development-nfiq-20. [Accessed 26 April 2017].

[76] Neurotechnology, "www.neurotechnology.com," Neurotechnology, 2016. [Online]. Available: http://www.neurotechnology.com/megamatcher.html. [Accessed 9 November 2016].

[77] NIST, "NIST Biometric Image Software nbis," NIST , [Online]. Available: https://www.nist.gov/services-resources/software/nist-biometric-image-software-nbis. [Accessed 23 March 2017].

[78] "SourceAFIS," [Online]. Available: http://www.sourceafis.org/blog/. [Accessed 23 March 2017].

[79] P. Komarinski, P. T. Higgins, K. M. Higgins and L. K. Fox, Automated Fingerprint Identification Systems (AFIS), Burlington, MA: Elsevier Academic Press, 2005.

[80] Safran Morpho, "MorphoWave Desktop Safran Identity \& Security," Safran Morpho, [Online]. Available: http://www.morpho.com/en/public-security/smart-borders/automatedsolutions/acquisition-devices/morphowave-desktop. [Accessed 24 October 2016].

[81] L. Ericson and S. Shine, "Evaluation of Contactless versus Contact Fingerprint Data, Phase 2 (Version 1.1)," ncjrs.gov. 